\title{
Measurement and Modeling of Advanced Coal Conversion Processes Volume II
}

\section{SECIVED Nov 211995 OSTI}

\section{User's Manual \\ 93-PCGC-2: Pulverized Coal Gasification and Combustion Model (2-Dimensional) with a Generalized Coal Reactions Submodel (FG-DVC)}

Peter R. Solomon

Michael A. Serio

David G. Hamblen

Ziaul Huque

Andrew M. Eaton

Sylvie Charpenay

Zhen Zhong Yu
L. Douglas Smoot

B. Scott Brewster

Richard D. Boardman

Susana K. Berrondo

Yuxin Zhao

Philip E. Best

June 1993

Work Performed Under Contract No.: DE-AC21-86MC23075

For

U.S. Department of Energy

Office of Fossil Energy

Morgantown Energy Technology Center

Morgantown, West Virginia

By

Advanced Fuel Research, Inc.

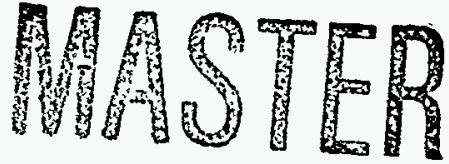

East Hartford, Connecticut

and

Brigham Young University

Provo, Utah 
DISCLAIMER

This report was prepared as an account of work sponsored by an agency of the United States Government. Neither the United States Government nor any agency thereof, nor any of their employees, makes any warranty, express or implied, or assumes any legal liability or responsibility for the accuracy, completeness, or usefulness of any information, apparatus, product, or process disclosed, or represents that its use would not infringe privately owned rights. Reference herein to any specific commercial product, process, or service by trade name, trademark, manufacturer, or otherwise does not necessarily constitute or imply its endorsement, recommendation, or favoring by the United States Government or any agency thereof. The views and opinions of authors expressed herein do not necessarily state or reflect those of the United States Government or any agency thereof.

This report has been reproduced directly from the best available copy.

Available to DOE and DOE contractors from the Office of Scientific and Technical Information, 175 Oak Ridge Turnpike, Oak Ridge, TN 37831; prices available at (615) 576-8401.

Available to the public from the National Technical Information Service, U.S. Department of Commerce, 5285 Port Royal Road, Springfield, VA 22161; phone orders accepted at (703) 487-4650. 



\section{Measurement and Modeling of Advanced Coal Conversion Processes Volume II}

\section{User's Manual \\ 93-PCGC-2: Pulverized Coal Gasification and Combustion Model (2-Dimensional) with a Generalized Coal Reactions Submodel (FG-DVC)}

\author{
Peter R. Solomon \\ Michael A. Serio \\ David G. Hamblen \\ Ziaul Huque \\ Andrew M. Eaton \\ Sylvie Charpenay \\ Zhen Zhong Yu
}

L. Douglas Smoot

B. Scott Brewster

Richard D. Boardman

Susana K. Berrondo

Yuxin Zhao

Philip E. Best

Work Performed Under Contract No.: DE-AC21-86MC23075

For

U.S. Department of Energy

Office of Fossil Energy

Morgantown Energy Technology Center

P.O. Box 880

Morgantown, West Virginia 26507-0880

By

Advanced Fuel Research, Inc.

87 Church Street

East Hartford, Connecticut 06108 and

Brigham Young University

Provo, Utah 84602 


\section{Abstract}

A two-dimensional, steady-state model for describing a variety of reactive and nonreactive flows, including pulverized coal combustion and gasification, is presented. Recent code revisions and additions are described. The model, referred to as 93PCGC-2, is applicable to cylindrical, axi-symmetric systems. Turbulence is accounted for in both the fluid mechanics equations and the combustion scheme. Radiation from gases, walls, and particles is taken into account using a discrete ordinates method. The particle phase is modeled in a Lagrangian framework, such that mean paths of particle groups are followed. A new coal-general devolatilization submodel (FG-DVC) with coal swelling and char reactivity submodels has been added. The heterogeneous reaction scheme allows for both diffusion and chemical reaction. Major gas-phase reactions are modeled assuming local instantaneous equilibrium, and thus the reaction rates are limited by the turbulent rate of mixing. A thermal and fuel $\mathrm{NO}_{\mathrm{x}}$ finite rate chemistry submodel is included which integrates chemical kinetics and the statistics of the turbulence. A sorbent injection submodel with sulfur capture is included. The gas phase is described by elliptic partial differential equations that are solved by an iterative line-by-line technique. Underrelaxation is used to achieve numerical stability. Both combustion and gasification environments are permissible. User information and theory are presented, along with sample problems. 


\section{Preface}

Considerable effort has been made to produce a computer code which will converge to a solution under as wide a variety of input conditions as possible. First elements of this code have been operating at Brigham Young University since 1977, with improvements having been made continuously since that time. Hundreds of predictions have been made with the code; however, the complex nature of the physical processes being modeled results in a numerical computer program that is sensitive to input conditions. Users are cautioned that arbitrary sets of input parameters will not necessarily produce converged solutions. A thorough understanding of the model equations and the numerical solution technique will give the user a better understanding of how to adjust appropriate numerical parameters to obtain a converged solution. 93-PCGC-2 is still considered to be a "specialist usable" code.

This code has been developed to apply to a variety of reacting and nonreacting flows, including pulverized coal combustion and coal gasification. Gasification can be interpreted as fuel-rich combustion, with little or no differences in the basic governing processes. Recent extensions have included the ability to predict laminar and near-laminar systems.

Key improvements and changes in the code include the following: 1) coalgeneral devolatilization submodel (FG-DVC) with coal swelling and char reactivity submodels; 2) robust, user-friendly energy equation option; 3) laminar-flow option and laminarization with turbulence; 4) generalized solids feeding in inlets other than the primary; 5) condensed-phase equilibrium algorithm; 6) thermal NO submodel; 7) sorbent particle injection with sulfur capture; and 8) graphical pre-processor. In order to differentiate among various versions of the code, the version associated with this user's manual is referred to as 93-PCGC-2, the "93" indicating the year of release. The code is referred to herein as simply "PCGC-2." 


\section{Acknowledgments}

This document is the user's manual for the improved computer model for Pulverized Coal Gasification and Combustion in Two Dimensions (93-PCGC-2). The code is so named in order to differentiate it from the earlier version, 87-PCGC2 , and because it was completed in 1993 . Work on this code was initiated originally under contracts from the U.S. Department of Energy and the Electric Power Research Institute in December of 1974. Research and development on this technology has continued since that time.

Work during 1982 through 1984 was supported by the U.S. Department of Energy, Morgantown Energy Technology Center (METC), Morgantown, West Virginia, under Contract No. DE-AC21-81MC-16518 with Mr. Gary R. Friggens and subsequently Dr. Holmes A. Webb, Jr., and then Dr. Lily Herskovits as Technical Project Officers; Grant No. DE-FG22-80PC-30306 with Mr. James Hickerson as Technical Project Officer; and Contract No. DE-AC21-80MC-14368 with Mr. Kenneth Markel as Technical Project Officer, The Electric Power Research Institute, Palo Alto, California, under Contract No. RP-364-3 with Mr. John Dimmer as Contract Officer. Work during the period from 1985 to April 30, 1987 was sponsored in part by METC under Contract No. DE-AC21-85MC-22059. Mr. Gary Friggens was the DOE technical project officer until the summer of 1986 when Mr. Leland E. Paulson became the technical project officer for DOE.

Work from 1985 to 1987 was also sponsored by Technical Associates and Affiliates of the University's Advanced Combustion Engineering Research Center who were in alphabetical order: Advanced Fuel Research, Babcock and Wilcox, Chevron Research Co., Combustion Engineering Co., Consolidation Coal Co., Convex Computer Corp., Corning Glass Works, Dow Chemical U.S.A., Electric Power Research Institute, Empire State Electric Energy Research Corp., Foster Wheeler Development Co., Gas Research Institute, General Electric Corp., General Motors Corp., Idaho National Engineering Laboratory, International Fuel Cells, Morgantown Energy Technology Center (METC), Pittsburgh Energy Technology Center, Pyropower Corp., Questar Development Corp., Shell Development Co., 
93-PCGC-2 with FG-DVC

Southern California Edison Co., Tennessee Valley Authority, The National Science Foundation, The State of Utah, TRW, Inc. and Utah Power and Light Co.

During the last six years, work continued jointly by BYU and Advanced Fuel Research, Inc. (AFR) under sponsorship of METC. Mr. Justin Beeson, Dr. Richard Johnson, and Dr. Norman Holcomb were Contracting Office Technical Representatives (COTRs). Financial cost sharing from the Research Division of Brigham Young University for development of this code during the entire period of development is gratefully acknowledged. 


\section{Table of Contents}

ABSTRACT

PART ONE: MODEL DESCRIPTION AND EVALUATION

\section{CHAPTER}

1 INTRODUCTION

Code Development .............................................1-2

Code Improvements and Revisions ..............................1-2

Limitations ...................................................1-4

Document Format .............................................1-5

Code Availability and Training .................................

2 THEORETICAL MODEL DEVELOPMENT......................2-1

Model Basis......................................................2-1

Gas Differential Equations ......................................2-5

Gas-Phase Fluid Mechanics.........................................2-8

Turbulence Model ................................................2-11

Particle Phase Mechanics..........................................2-16

Gas Phase Reactions...........................................2-21

Particle Phase Reactions.........................................2-36

Radiation ..................................................2-52

Nitrogen Pollutants............................................2-68 
Sulfur Pollutants and Sorbent Reactions Submodel................. 2-80

3 .SOLUTION TECHNIQUE ........................................3-1

Approach ...........................................................3-1

Finite Difference Scheme............................................3-7

Boundary Conditions ...............................................3-59

Generalized Geometry .............................................3-65

Additional Inlets .................................................. 3-73

Gas Phase Properties ............................................ 3-77

Radiation ..................................................... 3-79

Integration of Particle Equations......................................3-81

Particle-Gas Coupling .......................................... 3-85

Nitrogen Pollutants Submodel.................................. 3-91

Sulfur/Sorbent Reactions Submodel ...........................3-97

4 GENERALIZED PARTICLE REACTIONS (FG-DVC)...........4-1

FG-DVC Model Basis ...........................................4-1

Depolymerization-Vaporization-Crosslinking (DVC)

Formulation.....................................................4-4

Functional Group (FG) Model Formulation ......................4-9

Percolation Theory ............................................... 4-10

Coal Viscosity Model ..........................................4 4-11

Coal Swelling Model..............................................4 4-13

Char Reactivity Model ..........................................4-17

Coal Sulfur and Nitrogen Devolatilization Model ................ 4-20

Solution Technique..................................................4-25

Model Parameters ...............................................4-29

References....................................................4-31

\section{PART TWO: CODE USER'S MANUAL}

5 USER GUIDE................................................5-1

General Description .............................................5-1 
Description of Subroutines.........................................5-3

Program Input ...................................................5-7

Program Output.......................................................5-18

Code Operation .............................................5-22

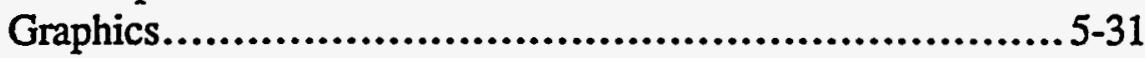

6 FG-DVC SUBMODEL DESCRIPTIONS .......................6-1

FG-DVC Subroutines.................................................6-1

The Coal Composition File...............................................6-3

The Kinetics File ..............................................6-3

The Polymer File .................................................6-4

The Mass Bin File .............................................6-5

The Reactivity Input File ........................................6-5

FG-DVC Input Data Creation and Maintenance ..................6-6

7 IMPLEMENTATION GUIDE.......................................

Code Files.......................................................7-1

FORTRAN and Machine Incompatibilities........................7-4

8 SAMPLE PROBLEMS ........................................

Gaseous Combustion............................................8-1

Coal Combustion with $\mathrm{NO}_{\mathrm{x}}$ Formation...............................8-1 Coal Gasification with Sidewall Sorbent Injection and Sulfur Capture ...................................................8-6

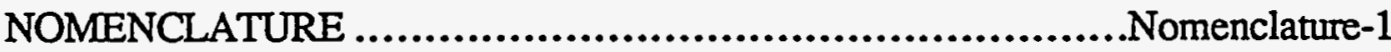

Greek Symbols ...........................................Nomenclature-7

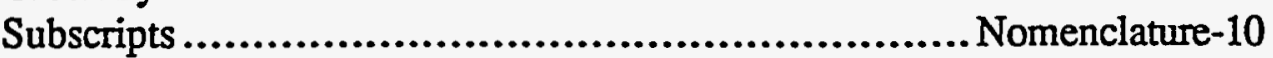

Superscripts............................................. Nomenclature-13

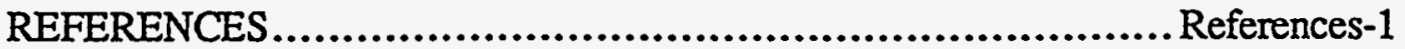




\section{APPENDICES}

A Description of Subroutines........................................A-1

Main Program......................................................... A-1

Auxiliary Subroutines.............................................A-1

Gas-Phase Fluid Mechanics....................................... A-3

Gas-Phase Chemistry ...........................................A-6

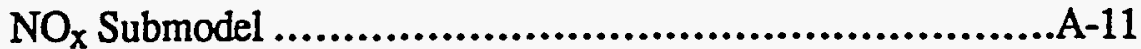

Plotting ........................................................

$\mathrm{SO}_{\mathrm{x}} /$ Sorbents Submodel..............................................A-14

Particle Phase .................................................A-15

Radiation Submodel.............................................17

B Description of Input Data....................................... B-1

C Sample Script Files for UNIX Operation Systems................ C-1

Script File No. 1 - pcf .......................................... C-2

Script File No. 2 - pcgc2.com ................................... -6

Script File No. 3 - .cshrc (partial listing) ..........................

D Sample Make File for Program Maintenance on UNIX

System s..................................................

E Sample Computer Files for Gaseous Combustion ................ E-1

Main Data File (cpr.dat, PCGCIN) .............................. E-1

Inlet Profile Data File (cpr.inl, INLET)................................. E-3

Thermo Data File (cpr.thm, THERMO) .......................... E-3

F Sample Computer Files for Coal Combustion with $\mathrm{NO}_{\mathrm{x}}$

Formation ................................................. F-1

Main Data File (cprcoal.dat, PCGCIN)............................... F-1

Grid Data File (cprcoal.grd, GRIDS)............................ F-3 
$\mathrm{NO}_{\mathbf{x}}$ Data File (cprcoal.nox, NOXIN)

Log File (cprcoal.log).................................................. F-7

G Sample Computer Files for Coal Gasification with Sidewall Sorbent Injection and Sulfur Capture ..............................G-1

Main Data File (ill.dat, PCGCIN) .................................G-1

Inlet Profile Data File (ill.inl, INLET) ...........................G-3

Thermo Data File (ill.thm, THERMO).................................G-4

$\mathrm{SO}_{\mathrm{x}}$ Data File (ill.sox, SOXIN) ..............................G-5

Grid Data File (ill.grd, GRIDS) ................................G-6

H Major FORTRAN Variables ....................................

I Sample Calculations................................................ I-1

Char Oxidation Parameters...................................... I-1

J General Model of Coal Devolatilization.................................J-1

$\mathrm{K} \quad$ Can Coal Science Be Predictive .................................

L Network Models of Coal Thermal Decomposition ................ L-1

M An Empirical Model for Coal Fluidity Based on a Macromolecular Network Pyrolysis Model........................................M-1

N Analysis of the Argonne Premium Coal Samples by Thermogravimetric Fourier Transform Infrared Spectroscopy ... N-1

O Analysis of Coal by Thermogravimetry-Fourier Transform Infrared Spectroscopy and Pyrolysis Modeling .................... O-1

USER'S FORUM (Comments reporting form) …...................... Forum -1 
93-PCGC-2 with FG-DVC

\section{List of Figures}

2-1 Two-dimensional reactor schematic......................................2-2

2-2 Submodels in PCGC-2 ..............................................2-4

2-3 Schematic of coal particle/droplet illustrating components and reaction processes ............................................................2-38

2-4 Directional intensities reaching a volume element ..................... 2-54

2-5 The direction cosines......................................................2-55

2-6 The absorption, scattering, and extinction efficiencies of char particles at wavelength of $2 \mu \mathrm{m}$ and refractive index of $1.93[1-\mathrm{i}(0.53)] \ldots \ldots . \ldots . . .2-65$

2-7 Kinetic mechanisms for prediction of fuel and thermal NO..............2-69

2-8 Conceptual view of the shrinking-core grain model......................2-83

3-1 Information flow diagram ...............................................3-3

3-2 Sample grid pattem for PCGC-2 ......................................3-5

3-3 The boomerang-shaped structure enclosing the points of definition of $u, v$, $p$, and $\phi$ corresponding to FORTRAN index I,J.........................3-12

3-4 Illustration of the grid symbols for a computational cell...................3-13

3-5 FORTRAN symbols for p-cell......................................3-15

3-6 FORTRAN symbols for $u$-cell......................................3-16

3-7 FORTRAN symbols for $v$-cell......................................3-17 
3-8 Illustration of the main cell finite-difference grid showing the node point labeling

3-9 A typical $u$-cell showing adjacent velocities...............................3-38

3-10 A typical $v$-cell showing adjacent velocities..............................3-39

3-11 A typical main boundary cell.......................................... 3-56

3-12 Sample reactor configurations possible with modified PCGC-2 ........ 3-66

3-13 Example of saw-tooth representation of reactor wall..........................3-67

3-14 Arbitrary grid cell showing location of X, R., I and J................... 3-68

3-15 Arbitrary $u$-cell showing locations of cells affected by intrusion at

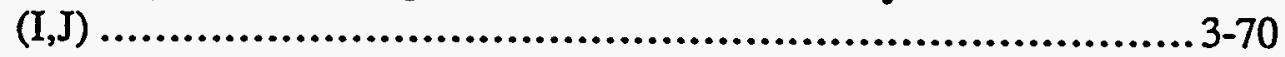

3-16 Arbitrary $v$-cell showing locations of cells affected by intrusion at

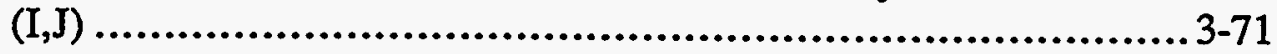

3-17 Example feed stream configurations .................................. 3-74

3-18 Axial grid locations for west wall inlets ............................. 3-75

3-19 Radial grid locations for north wall inlets ............................. 3-76

3-20 Information flow diagram for mixed Eulerian-Lagrangian particle

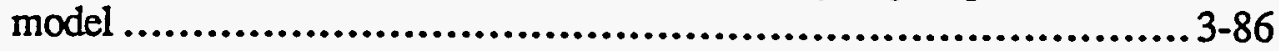

3-21 $\mathrm{NO}_{\mathrm{x}}$ model logical sequencing............................................3-93

3-22 Expansion of shaded box in Fig. 3-21 showing alternative mechanisms and options available in the revised $\mathrm{NO}_{\mathrm{x}}$ model ..........................3-95

3-23 Information flow diagram for the sorbent/sulfur capture submodel...... 3-98 
4-1 Bethe lattice for two- $\sigma$ model with $\sigma_{1}=\sigma_{2}=1 \ldots \ldots \ldots \ldots \ldots \ldots \ldots \ldots \ldots \ldots . . . \ldots \ldots 1$

4-2 Schematic diagrams of sulfur devolatilization................................4-42

4-3 A schematic diagram of sulfur devolatilization in oxidation conditions.. 4-43

4-4 A schematic diagram of nitrogen gas distribution ........................ 4-44

5-1 Information flow diagram for combined Eulerian-Lagrangian particle model ...................................................................5-5

5-2 Menu bar and help window ......................................5-32

5-3 Pull-down menu for reading and saving input files ...................5-32

5-4 Window for specifying filename for reading input files.................5-33

5-5 Window for specifying simulation title .................................5-34

5-6 Window for specifying reactor geometry.....................................5-35

5-7 Window for specifying primary inlet parameters....................... 5-36

5-8 Window for selecting chemical species to add..........................5-37

5-9 Window for specifying grid generation parameters ......................5-38

5-10 Message window for generating the calculational grid.......................5-39

5-11 Pull-down menu for selecting coal or sorbent particle properties.......... 5-40

5-12 Window for specifying coal particle input data .......................5-41

5-13 Window for specifying elemental coal composition for coals not included in the Argonne data bank ........................................5-42 
5-14 Information window displaying results of coal data file generation program.......................................................................5-43

5-15 Window for modifying coal composition data files ....................5-44

5-16 Window for modifying coal kinetics data files.................................5-44

5-17 Window for specifying sorbent particle input data .....................5-45

5-18 Window for specifying filename to save input data .....................5-46

5-19 Prompting window for saving data files.....................................5-46

5-20 Sample plot of gas temperature .................................. 5-52

5-21 Sample U-V velocity vector plot............................................5-61

5-22 Near-burner $U-V$ velocity vector plot..................................5-65

5-23 Near-burner U-V velocity vector plot with two types of arrows representing two velocity scales.........................................5-66

6-1 FG-DVC subroutines ......................................... 6-12

6-2 A list of FORTRAN variables of the input data for FG-DVC.............. 6-16

6-3 FG-DVC subroutine called from COAL2 ........................... 6-21

6-4 An example of the coal composition file............................... 6-22

6-5 An example of the kinetic file ........................................ 6-24

6-6 The data structure of the polymer file ................................. 6-29

6-7 The mesh and the nodal points used for interpolations ................ 6-30 


\section{List of Tables}

2-1 Gas phase differential equations

2-2 Turbulence model constants..............................................2-14

2-3. Turbulent combustion model constants.............................. 2-25

2-4 Physical properties for particle model............................................ 2-42

2-5 Convective and conductive heat transfer for particle model .............. 2-46

2-6 Particle reactions for particle model .......................................2-48

2-7 Devolatilization rate expressions and parameters .........................2-50

2-8 Coefficients for char reaction with $\mathrm{O}_{2}, \mathrm{CO}_{2}$, and $\mathrm{H}_{2} \mathrm{O} \ldots \ldots \ldots \ldots \ldots \ldots . . . . .2-52$

2-9 The $\mathrm{S}_{2}$ quadrature for axisymmetric cylindrical enclosures ............... 2-58

2-10 The $\mathrm{S}_{4}$ quadrature for axisymmetric cylindrical enclosures ..............2-58

2-11 Coefficients for flux sums.....................................................2-63

2-12 Reaction expression alternatives for the generalized nitric oxide

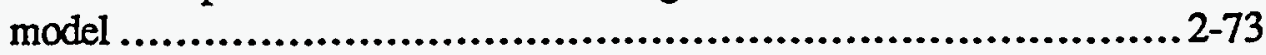

2-13 Equation set for a generalized NO model................................2 2-79

3-1 PCGC-2 boundary conditions ............................................. 3-64

3-2 Use of FORTRAN input variables INRDGD and INTRUS ............. 3-72

3-3 Finite difference for the radiation flux sums ............................ 3-80 


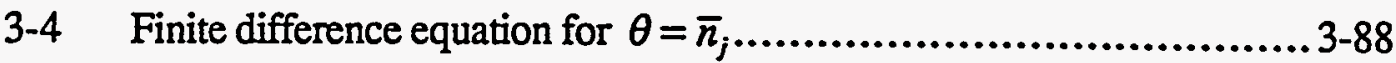

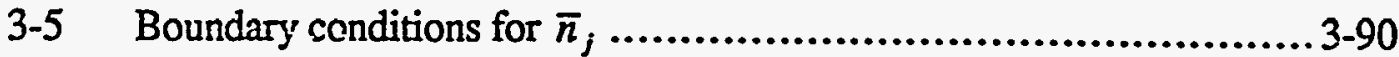

4-1 Nomenclature of the variables in Chapter 4 ............................. 4-32

4-2 Swelling model parameters.................................................4-37

4-3 Sulfur devolatilization: correspondence between pools and gases.......4-38

4-4 Elemental Compositions of the eight Argonne coals and the three PSOC coals ............................................................. 4-39

4-5 Kinetic data of major functional group pools .......................... 4-40

5-1 Summary of input for PCGC-2 2............................................5-2

$5-2 \quad$ Input data.......................................................... $5-8$

5-3 Program output control .................................................5-19

5-4 Outline of output from PCGC-2 calculation...................................5-20

5-5 PCGC-2 output files .............................................5-21

5-6 Spreadsheet output from PCPLOT...................................5-57

6-1 qo's and $\mathrm{ph}_{\mathrm{h}}$ 's of the eight Argonne coals........................... 6-31

7-1 Files opened by PCGC-2...................................................

8-1 Key input data for natural gas combustion case ............................8-2

8-2 Key input data for pulverized coal combustion case .......................8.8

8-3 Parameters for coal gasification case.....................................8-6 
93-PCGC-2 with FG-DVC 


\section{Chapter One}

\section{Introduction}

This manual presents the details of the improved and revised two-dimensional computer code referred to as 93-PCGC-2 (Pulverized Coal Gasification and

Combustion: 2-Dimensional), which can be used to describe a variety of reacting and non-reacting flows, including pulverized coal combustion and gasification. This version of PCGC-2 has also been used to describe the combustion of gaseous diffusion flames and isothermal, two-phase fluid mechanics. Previous versions of the code have also been applied to liquid sprays, coal slurries, and other oxidation/reduction reaction chambers.

The modeling scheme is based on an Eulerian framework for the gas phase and a mixed Eulerian/Lagrangian framework for the particle phase. The approach emphasizes the turbulent fluid mechanics and the turbulent combustion processes in a turbulent flame. Most gas-phase reactions are assumed to be mixing-limited, so that gaseous combustion is modeled with a statistical probability density function based on mixture fractions. A two-equation $(k-\varepsilon)$ turbulence model is used for closure. The particle phase includes the effects of aerodynamic particle drag and turbulent particle dispersion. Particle reactions are considered to be slow with respect to the gas-phase turbulence time scale. Rate processes for slurry liquid vaporization, coal devolatilization, and char reaction are included, based on time- 
mean gaseous properties. Particle and gas radiation, with anisotropic and multiple scattering, are included.

The description that follows applies to entrained-flow, pulverized-coal reaction processes, where particles are small $(<150 \mu \mathrm{m})$ and heating rates are high $\left(10^{3}-10^{6} \mathrm{~K} \mathrm{~s}^{-1}\right)$. This treatment would not necessarily apply to fixed- or fluidizedbed processes without modification.

\section{Code Development}

Formulation, numerical solution and evaluation of 93-PCGC-2, or just PCGC-2 as it is commonly called herein, has been completed over the past $12-13$ years. Development has reached a point where the code is being applied to systems of practical interest. However, appropriate use of this comprehensive code requires a thorough understanding of the basic code foundations and operation. Our experience suggests that a person with an advanced (i.e. graduate) engineering degree and a strong background in fluid mechanics, heat and mass transfer, thermodynamics, and numerical analysis requires at least six months to a year (full time) to effectively understand and use the code. Results can be obtained faster with the guidance of experienced users.

\section{Code Improvements and Revisions}

The 1993 release of PCGC-2 contains several improvements over the previous (1987) release, including a generalized coal reactions submodel with a database of coal reaction parameters for the eight coals in the Argonne Premium Coal Sample Program, improved applicability to fuel-rich systems (e.g. gasification) due to a new condensed-phase chemical equilibrium submodel, an extended pollutant submodel, generalized solids feeding with capability for sorbent injection and sulfur capture, a laminar-flow option and laminarization with turbulence, effects of gas buoyancy, improved robustness and user-friendliness, and improved code graphics. Each of these improvements is briefly described below. 
The generalized coal reactions submodel was developed by Solomon and coworkers (Solomon et al., 1988) at Advanced Fuel Research, Inc., and is referred to herein and in the literature as the FG-DVC model. It is basically a combination of two submodels, a function group (FG) submodel for predicting evolution of light gases and tar, and a char formation submodel based on the processes of char depolymerization, tar vaporization, and char cross-linking (DVC). Information from FG-DVC is also used in the particle submodel to predict swelling and char reactivity. A database of submodel parameters for the eight standard Argonne coals is provided with the code, so that the user need only specify the type of coal he or she wishes to use. Coals other than the Argonne coals are accommodated through an interpolation scheme.

The new condensed-phase equilibrium submodel was based on the METCEC code. It was developed by METC based on the NASA-Lewis equilibrium code CEC. It's incorporation into 93-PCGC-2 has improved code predictions for fuel-rich cases where solid carbon is a significant species for very fuel-rich stoichiometric mixtures. The new submodel was incorporated as an option, preserving the older CREE chemical equilibrium option which is faster and which is still applicable to gaseous combustion and fuel-lean coal systems.

The full energy equation option is considerably more robust, user-friendly, and accurate, than in the previous version of PCGC-2. Full energy equation solutions can now be accomplished fairly routinely. The basic theory regarding the treatment of enthalpy in turbulent flows has remained unchanged, but the particlegas interaction term is calculated more accurately, and the table look-up scheme for gas properties is more accurate, robust, efficient, and user-friendly.

The pollutant submodel was extended to calculate fuel $\mathrm{NO}_{\mathrm{x}}$ with additional mechanisms that are applicable to fuel-rich (gasification) conditions. In addition, capability was added for calculating thermal $\mathrm{NO}_{\mathrm{x}}$. A sorbent reactions submodel was added to calculate the capture of $\mathrm{SO}_{2}$ or $\mathrm{H}_{2} \mathrm{~S}$ by injected sorbent particles.

The solids feed submodel was generalized to allow solids feeding in the secondary and additional (e.g. sidewall) inlets. In addition, capability was added 
for injecting and tracking sorbent particles and their reactions after the main combusting coal particle/gas flowfield has been computed.

An option was added to PCGC-2 for modeling laminar flow. This option still assumes gas mixing to be rate limiting (i.e. infinite-rate gas kinetics). A laminarization extension was also added to the $k-\varepsilon$ turbulence submodel to calculate transitional flow. A gas buoyancy term was added to the axial gas momentum equation to allow free convection effects to be included in these types of flows.

Significant improvements were also been made in the area of code graphics. An X-windows-based pre-processor, which uses software available for license under an independent arrangement, was developed for quickly generating code input files for "standard" cases. A standard case is defined as one which has no additional inlets (i.e. only a primary and a secondary), no intrusions (reactor diameter is constant except for the quarl region), and coal properties can be modeled by one of the Argonne coals. The pre-processor provides default values for most of the code options and input parameters, and is a considerable step forward in making the code usable by a person who has a technical background, but no experience with PCGC-2. In addition to the pre-processor, an improved postprocessor, which also uses software available for license under an independent arrangement, provides plotting capability for gas and particle properties, as well as results of pollutant calculations. There is also an option for converting output data into a format compatible with spreadsheet plotting programs.

\section{Limitations}

The following limitations apply to PCGC-2.

- The flowfield, geometry (e.g. inlets and intrusions), and body forces (gravity) are axisymmetric.

- The flow is subsonic (dilatation is ignored). The maximum Mach number should be less than about 0.3 . 
- Chemical reaction rates are limited by turbulent mixing and not by kinetics (with the exception of nitrogen oxides).

- Particles are dispersed in the gas phase, and particle-particle interactions are ignored.

These assumptions are discussed in the next chapter.

The submodels dealing with turbulence issues are at best tenuous. Specifically, the turbulence $(k-\varepsilon)$, turbulent particle dispersion, and turbulencechemistry interactions, are not yet capable of producing accurate results in all cases.

\section{Document Format}

This users manual is organized into two parts. Part One documents the theory (Chapter 2), the solution of the model equations (Chapter 3), and the generalized particle reactions submodel (Chapter 4). An understanding of the theoretical foundations of the code is prerequisite to its correct application, and potential users are encouraged to study this material carefully. Part Two describes the use of the code (Chapters 5 and 6), it's implementation on computer hardware (Chapter 7), and sample problems illustrating code application (Chapter 8). While significant effort has been expended to make the code user-friendly and robust, it must be recognized that PCGC-2 is still very much a "user-specialist" code requiring a significant amount of experience beyond simple theoretical understanding for efficient management and use. Laboratories interested in extensive use of PCGC-2 should plan on committing a technical person full-time for approximately six months to a year to learn to use this code. Training is available as described below.

\section{Code Availability and Training}

The 1993 version of PCGC-2 with FG-DVC submodel was developed jointly by Brigham Young University and Advanced Fuel Research, Inc. under contract to the 
Introduction

Department of Energy (Contract No. DE-AC21-86MC23075). It is a shared product of the Advanced Combustion Engineering Research Center and is subject to Center distribution policy for shared products. The code will be available starting approximately May 1, 1993.

Organizations wishing to obtain the code should contact

Dr. Andrew M. Eaton

ACERC Software Specialist

75 CTB

Brigham Young University

Provo, Utah 84602

(801) 378-5008

Fax: (801) 378-3831

The source code and sample problems are available on magnetic tape. The code has been developed on Convex and Sun Microsystems computers with the UNIX operating system. The code is not guaranteed to run on any particular computer system, however, and users should plan on making modifications, particularly if their system is not one of the above. The extent of the required modifications varies with each installation.

Training in the use of the code is also available through the Advanced Combustion Engineering Research Center (ACERC). This training is best accomplished on an individual basis. Interested scientists and engineers are encouraged to visit the laboratory for a short period (4-6 weeks) during which time they will receive assistance in learning to use the code and apply it to a problem of interest. All travel and living expenses during this period will be the responsibility of the visiting scientist/engineer ${ }^{1}$. On-campus housing may be available, especially during the non-academic (summer) months. BYU will provide office space and computer resources and assist in making suitable housing arrangements.

\footnotetext{
${ }^{1}$ Industrial Associate trainees can request partial financial support from ACERC for their visit to the Center.
} 


\section{Chapter Two}

\section{Theoretical Development}

The mathematical modeling of turbulent combustion and gasification has been recognized as a difficult problem not only due to the numerical challenges associated with solving the differential equation set but also the challenges of physically describing the important chemical and physical processes, such as chemistry-turbulence interactions, turbulent particle dispersion, particle reactions, etc. PCGC-2 uses currently available mathematical technology to combine knowledge of fluid mechanics with a reasonable approach to the reaction processes. Care has been taken to maintain the scope of the simplifying approximations on a consistent level.

\section{Model Basis}

PCGC-2 has been applied to non-reacting flow systems, gaseous diffusion flames, pulverized coal-fired combustion, entrained-flow gasification, droplet combustion, and slurry combustion or gasification (including coal-water mixtures) in axisymmetric coordinates. Variations in the properties are considered only in the axial and radial directions. Symmetry is assumed in the angular direction. A 


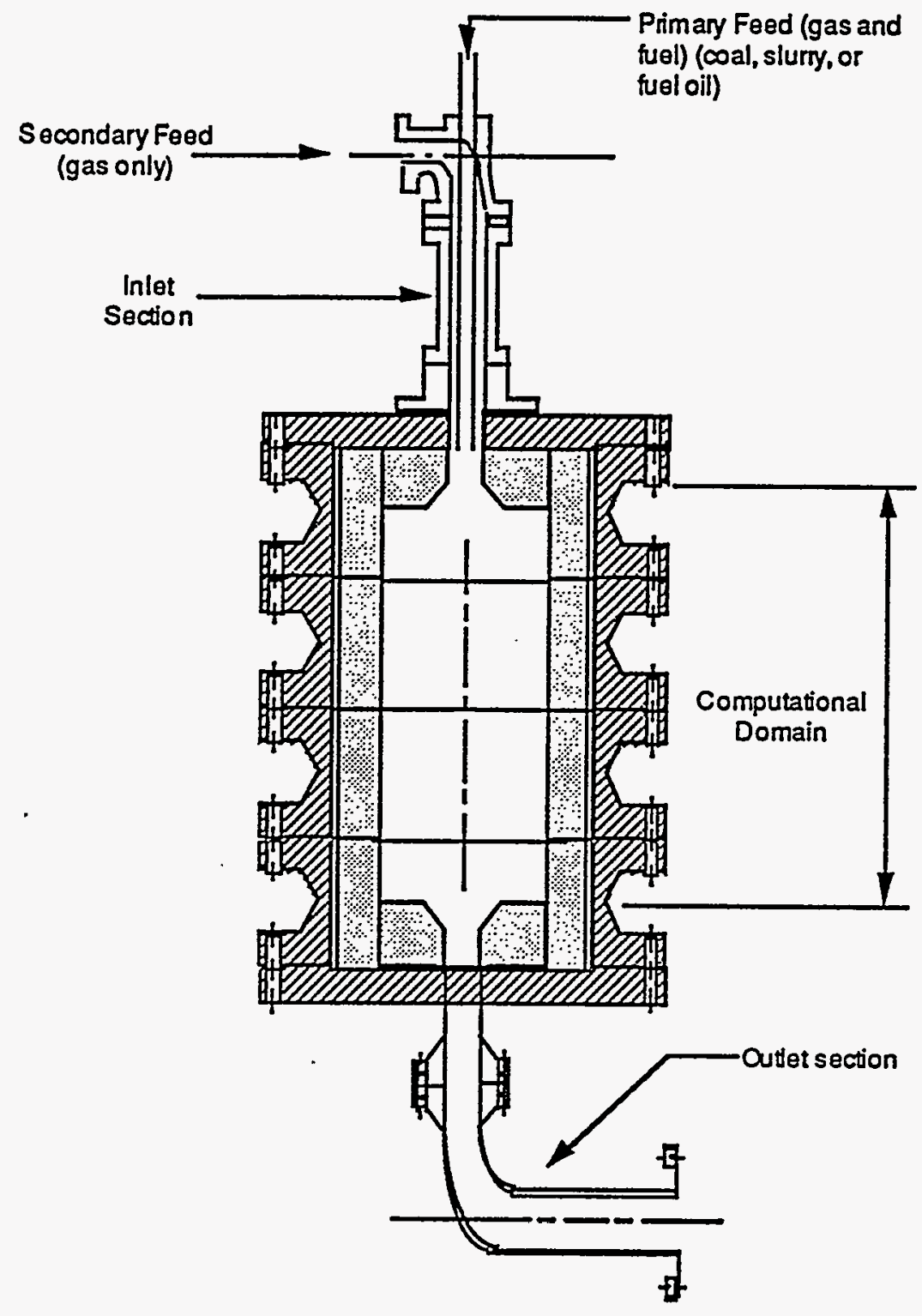

Figure 2-1. Two-dimensional reactor schematic. 
schematic of a typical reactor modeled by PCGC-2 is shown in Figure 2-1 (Smith, 1979; Thurgood, 1979). This particular cylindrical reactor is coaxial, with coal entering the reactor in the central (primary) stream and the majority of the oxidizer entering in the outer (secondary) stream or annulus. The code has been generalized to include multiple inlets that can be located along any of the walls and to include arbitrary shapes within the axisymmetric assumption. Typical pulverized coal particles range in size from two micrometers (microns) to one hundred fifty micrometers. Slurry droplets can reach several hundred micrometers. The model predicts the mean gas field properties for axisymmetric, steady-state, turbulent flames (i.e., local velocity, temperature, density, and species composition). Particle properties are also computed, such as coal burnout, particle or droplet velocity and temperature, and coal/droplet component composition. Figure 2-2 illustrates the various submodels incorporated into PCGC-2.

The gas phase is assumed to be a turbulent, reacting continuum field that can be described locally by general conservation equations. The flow is assumed to be time-steady. Gas properties (i.e., density, temperature, species composition) are assumed to fluctuate randomly according to a probability density function (PDF) characteristic of the turbulence. Gas-phase body forces are neglected. The gas is assumed to be Newtonian and dilatation is neglected.

The gas field is treated in an Eulerian framework. Turbulence is modeled by breaking the variables into fluctuating and time-mean components in the conventional manner (Hinze, 1967; Tennekes and Lumley, 1972). The twoequation $k-\varepsilon$ model is used for closure (Launder and Spalding, 1972). PCGC-2 is coded to handle either Reynolds- or Favre-averaging. The effect of particles on the gas-phase turbulence is modeled with an empirical correlation. Gas-phase reactions are assumed to be limited by mixing rates for major species and not by kinetics; gaseous properties are calculated assuming local instantaneous equilibrium. For non-equilibrium reactions, such as occur in methane flames or in coal gasifiers, partial (frozen) equilibrium can be used to describe mixing-limited reactions.

The pulverized coal flame radiation field is a multicomponent, non-uniform, emitting, absorbing, scattering gas-particle system (Smith et al., 1981). The coal particles cause anisotropic and multiple scattering. The flame may be surrounded 


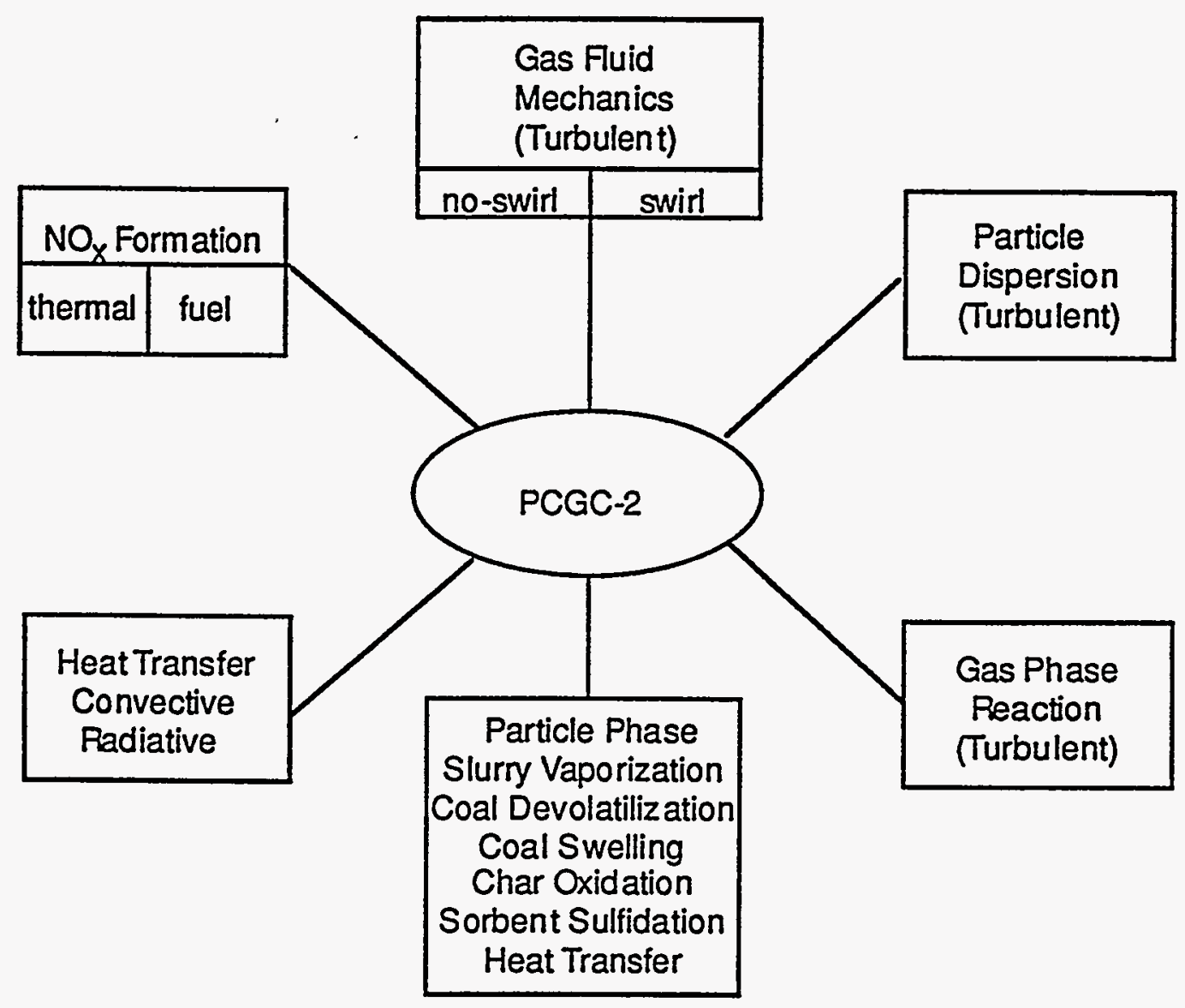

Figure 2-2. Submodels in PCGC-2.

by non-uniform, emitting, reflecting, absorbing surfaces. Optical coefficients for the gas, walls, and particles are difficult to obtain in flames (Sarofim and Hottel, 1978) and PCGC-2 uses the recommendations of Varma and Pratt (1978) to calculate the optical coefficients necessary for a pulverized coal flame. An Eulerian framework is used to model the radiation, which facilitates incorporation of radiation properties into gas-phase equations. The Lagrangian particle field does 
not mesh completely with the Eulerian radiation equations, since a bulk particle density is difficult to obtain from Lagrangian systems.

The particle phase is not considered a continuum as is the gas phase (Smith et al., 1981; Fletcher, 1980). Different particles at the same location may exhibit different properties cue to their different histories. In PCGC-2, Lagrangian treatment of the particles is performed, representing the particle field as a series of mean trajectories. Particle properties can be obtained along the mean trajectories by integration of the governing particle equations. The effect of turbulent fluctuations on the trajectories of the particles is treated in a mixed Eulerian-Lagrangian framework. The effect of turbulent fluctuations in the gas phase on particle reaction rates is ignored.

PCGC-2 assumes that the off-gas from the coal is of constant elemental composition. The particle reaction submodel is discussed by Smith (Smith, 1979) (see also Smoot and Pratt, 1979; Fletcher, 1983). Particles are defined to consist of slurry liquid, raw coal, char, and ash. Ash is inert by definition; volatile mineral matter is considered as part of the volatile matter of the coal. Coal reaction rates can be characterized by multiple parallel reaction rates with fixed activation energies, or by a general coal reactions submodel (FG-DVC) based on a functional group description of the coal for gas evolution, and a network description for depolymerization, tar vaporization, and cross-linking. In the former case, coal particle swelling is predicted empirically. In the latter case, it can be predicted from gas evolution rate and viscosity of the melt (see Chapter 4). The particles are assumed to be isothermal. The parameters which describe the coal reaction rates are part of the input to the code. Default values can also be supplied by the code for some of the parameters, based on coal type.

\section{Gas Differential Equations}

All of the gas-phase equations are Eulerian, steady-state, second-order, non-linear, elliptical partial differential equations. Each of these may be conveniently cast into one finite difference form, so that only one solution technique is required. Table 2 1 contains a summary of this form for all of the gas phase equations. Note that the particles are accounted for only by appropriate particle source terms for mass $\left(S_{p}^{m}\right)$, 
momentum $\left(S_{p}^{u}\right.$ and $\left.S_{p}^{v}\right)$, and enthalpy $\left(S_{p}^{h}\right)$. These terms represent the addition of mass, momentum, and energy to the gas phase from the particles. The source terms are found along a particle trajectory by taking the difference in the particle properties on each side of a computational cell and dividing by the cell volume (see Chapter III).

The following sections describe each of the equations in Table 2-1 in more detail. The equations for the radiation field and the particle number-density field do not fit the form of the gas-phase equations and are discussed separately. The method used to solve these equations is discussed in Chapter 3.

TABLE 2-1

\section{GAS PHASE DIFFERENTIAL EQUATIONS}

$\frac{\partial}{\partial x}(\bar{\rho} \bar{u} \phi)+\frac{1}{r} \frac{\partial}{\partial r}(r \bar{p} \bar{v} \phi)-\frac{\partial}{\partial x}\left(\Gamma_{\phi} \frac{\partial \phi}{\partial r}\right)-\frac{1}{r} \frac{\partial}{\partial r}\left(r \Gamma_{\phi} \frac{\partial \phi}{\partial r}\right)=S_{\phi}$

$\phi \quad \Gamma_{\text {. }}$

$S_{\phi}$

10

$S_{p}^{m}$

$\bar{u} \quad \mu_{e}$

$-\frac{\partial \bar{p}}{\partial x}+\frac{\partial}{\partial x}\left(\mu_{\epsilon} \frac{\partial \bar{u}}{\partial x}\right)$

$+\frac{1}{r} \frac{\partial}{\partial r}\left(r \mu_{c} \frac{\partial \bar{v}}{\partial x}\right)\left\{\begin{array}{ll}+\bar{\rho} g \text { (down - fired) } \\ -\bar{\rho} g \text { (up - fired) } \\ +0 \text { (horizontally fired) }\end{array}\right\}+S_{p}^{u}+\bar{u} S_{p}^{m}$

$\bar{v} \quad \mu_{e} \quad-\frac{\partial \bar{p}}{\partial r}+\frac{\partial}{\partial x}\left(\mu_{e} \frac{\partial \bar{u}}{\partial r}\right)+\frac{1}{r} \frac{\partial}{\partial r}\left(r \mu_{e} \frac{\partial \bar{v}}{\partial r}\right)-2 \mu_{e} \frac{\bar{v}}{r^{2}}+\frac{\bar{\rho}}{r} \bar{w} \bar{w}+S_{p}^{\nu}+\bar{v} S_{p}^{m}$

$\tilde{w} \quad \mu_{c} \quad-\frac{\rho \tilde{w} \bar{w}}{r}-\frac{\bar{w}}{r^{2}} \frac{\partial}{\partial r}\left(r \mu_{c}\right)$ 
TABLE 2-1 (continued)

$\frac{\partial}{\partial x}(\bar{\rho} \bar{u} \phi)+\frac{1}{r} \frac{\partial}{\partial r}(r \bar{p} \bar{v} \phi)-\frac{\partial}{\partial x}\left(\Gamma_{\phi} \frac{\partial \phi}{\partial r}\right)-\frac{1}{r} \frac{\partial}{\partial r}\left(r \Gamma_{\phi} \frac{\partial \phi}{\partial r}\right)=S_{\phi}$

$\phi \quad \Gamma_{\bullet}$

$S_{\phi}$

$k \quad \mu_{\varepsilon} / \sigma_{k} \quad \Phi-\bar{\rho} \varepsilon$

$\varepsilon \quad \mu_{c} / \sigma_{\varepsilon} \quad \frac{\varepsilon}{k}\left[C_{l} \Phi-C_{2} \bar{\rho} \varepsilon\right]$

$\begin{array}{lll}\tilde{f}_{p} & \mu_{e} / \sigma_{f} & S_{p}^{\prime}\end{array}$

$g_{f} \quad \mu_{e} / \sigma_{g} \quad \frac{C_{g} \mu_{c}}{\sigma_{g}}\left[\left(\frac{\partial \bar{f}}{\partial x}\right)^{2}+\left(\frac{\partial \bar{f}}{\partial r}\right)^{2}\right]-\frac{C_{g 2} \bar{\rho} \varepsilon g_{f}}{k}$

$\bar{\eta} \quad \mu_{e} / \sigma_{\eta} \quad S_{p}^{\eta}$

$g_{\eta} \quad \mu_{e} / \sigma_{g} \quad \frac{C_{g g} \mu_{e}}{\sigma_{g}}\left[\left(\frac{\partial \tilde{\eta}}{\partial x}\right)^{2}+\left(\frac{\partial \tilde{\eta}}{\partial r}\right)^{2}\right]-\frac{C_{g} \bar{\rho} \varepsilon g_{n}}{k}$

$\bar{h} \quad \mu_{e} / \sigma_{h} \quad q_{r g}^{\prime}+\tilde{u} \frac{\partial \bar{p}}{\partial x}+\bar{\nu} \frac{\bar{p}}{\partial r}+S_{p}^{h}$

where $\Phi=\mu_{c}\left\{\left[\left\{\left(\frac{\partial \bar{u}}{\partial x}\right)^{2}+\left(\frac{\partial \bar{v}}{\partial r}\right)^{2}+\left(\frac{\tilde{v}}{r}\right)^{2}\right]+\left[\frac{\partial \bar{u}}{\partial r}+\frac{\partial \bar{v}}{\partial x}\right]^{2}+\left[r \frac{\partial}{\partial r}\left(\frac{\bar{w}}{r}\right)\right]^{2}+\left(\frac{\partial \bar{w}}{\partial x}\right)^{2}\right\}\right.$

$$
\mu_{e}=\mu^{t}+\mu^{\ell}
$$




$$
\mu^{z}=C_{\mu} \bar{\rho} k^{2} / \varepsilon \quad S_{p}^{m}=S_{p}^{f}+S_{p}^{n}
$$

\section{Gas-Phase Fluid Mechanics}

The instantaneous gas-phase equations of continuity and momentum (Bird et al., 1960) are presented here with the particle reaction source terms (Smoot and Pratt, 1979). Equations for turbulence properties, mixture fractions, and energy can be similarly derived. The equations of continuity and motion are:

$$
\begin{gathered}
\frac{D \rho}{D t}=-\rho(\vec{\nabla} \cdot \bar{v})+S_{p}^{m} \\
\rho \frac{D \vec{v}}{D t}=-\vec{\nabla} p-\vec{\nabla} \cdot \bar{\tau}+\rho \vec{g}+\vec{S}_{p}^{v}
\end{gathered}
$$

where $\mathrm{D} / \mathrm{Dt}$ is the substantial derivative. The $\mathrm{S}_{\mathrm{p}}$ terms represent the coupling between the gas phase and the particle phase, i.e. the particle source/sink terms. All variables are defined in the Nomenclature.

The equations are reduced with following simplifications: 1) cylindrical coordinates, 2) 2-dimensional axisymmetric geometry, 3) body forces only in the axial direction, 4) steady-state, and 5) Newtonian fluid. Equations 2-1 and 2-2 become:

$$
\begin{gathered}
\frac{\partial}{\partial x}(\rho u)+\frac{1}{r} \frac{\partial}{\partial r}(r \rho v)=S_{p}^{m} \\
\frac{\partial}{\partial x}(\rho u u)+\frac{1}{r} \frac{\partial}{\partial r}(r \rho v u)-\frac{\partial}{\partial x}\left(\mu \frac{\partial u}{\partial x}\right)-\frac{1}{r} \frac{\partial}{\partial r}\left(\mu r \frac{\partial u}{\partial r}\right) \\
=-\frac{\partial p}{\partial x}+\frac{\partial}{\partial x}\left(\mu \frac{\partial u}{\partial x}\right)+\frac{1}{r} \frac{\partial}{\partial r}\left(\mu r \frac{\partial v}{\partial x}\right)-\frac{\partial}{\partial x}\left(\frac{2}{3} \mu d i v \bar{v}\right)+\psi \rho g+S_{p}^{u}+u_{p g} S_{p}^{m}
\end{gathered}
$$




$$
\begin{aligned}
& \frac{\partial}{\partial x}(\rho u v)+\frac{I}{r} \frac{\partial}{\partial r}(r \rho v v)-\frac{\partial}{\partial x}\left(\mu \frac{\partial v}{\partial x}\right)-\frac{1}{r} \frac{\partial}{\partial r}\left(\mu r \frac{\partial v}{\partial r}\right) \\
& =-\frac{\partial p}{\partial r}+\frac{\partial}{\partial x}\left(\mu \frac{\partial u}{\partial r}\right)+\frac{1}{r} \frac{\partial}{\partial r}\left(\mu r \frac{\partial v}{\partial r}\right)-2 \frac{\mu v}{r^{2}} \\
& -\frac{1}{r} \frac{\partial}{\partial r}\left(\frac{2}{3} \mu r \operatorname{div} \bar{v}\right)+\frac{2}{3} \frac{\mu}{r} \operatorname{div} \bar{v}+S_{p}^{v}+v_{p g} S_{p}^{m} \\
& \frac{\partial}{\partial x}(\rho u w)+\frac{1}{r} \frac{\partial}{\partial r}(r \rho v w)-\frac{\partial}{\partial x}\left(\mu \frac{\partial w}{\partial x}\right)-\frac{1}{r} \frac{\partial}{\partial r}\left(\mu r \frac{\partial w}{\partial r}\right) \\
& =-\frac{\rho v w}{r}-\left(\frac{w}{r^{2}}\right) \frac{\partial}{\partial r}(r \mu)
\end{aligned}
$$

where $\psi$ is equal to 1 for downfired, -1 for upfired, and 0 for horizontally fired.

One more approximation is made before arriving at the equations used in PCGC-2. The div $\vec{v}$ term in Eqns. 2-4 and 2-5 are associated with the dilatation of the fluid. These terms describe the volume change of a fluid element due to expansion or compression. For perfectly incompressible fluids, the equation of continuity can be used to prove that those terms are identically zero. For a compressible fluid, the effect is minimal at low velocities (less than 0.25 Mach No.) and is often neglected (Bird et al., 1960; Smoot and Pratt, 1979). This convention is also followed in PCGC-2.

It is useful to understand the significance of each term in the equation set. The first two terms in the equation of continuity (Eqn. 2-3) represent the net rate of mass addition to the volume element by convection from the axial and radial components respectively. The $S_{p}^{m}$ term represents the net rate of mass addition per unit volume due to particle reaction. The first two terms in the momentum equations (Eqns. 2-4, 5 and 6) are the convection terms. They represent the net rate of convected momentum to the volume element attributed to the axial and radial components. Equation 2-4 describes the momentum contributed by the axial component of the velocity, Eqn. $2-5$ describes that contributed by the radial component, and Eqn. 2-6 describes that contributed by the tangential component. The third and fourth terms in each of these equations may conveniently be thought 
of as the corresponding diffusion terms. When turbulence is modeled using Reynolds stresses, they are not strictly molecular diffusion terms because of the contribution to the momentum due to turbulent transfer. The diffusion appearance of second-order derivatives makes it natural to refer to them in this way. The first term on the right-hand side of the axial and radial momentum equations represents the pressure force on the volume element. All other terms on the right-hand side of these equations are considered source or sink terms, representing other sources or sinks of momentum. The $S_{p}^{u}$ and $S_{p}^{\nu}$ terms represent the aerodynamic drag effects of the particles on the axial and radial components of momentum. The $u_{p g} S_{p}^{m}$ and $v_{p g} S_{p}^{m}$ terms represent the momentum exchange due to particle mass efflux. In this version of the code, $u_{p g}$ and $v_{p g}$ are assumed to be equal to the gas velocity and $S_{p}^{w}$ and $w_{p g} s_{p}^{m}$ are neglected. These assumptions will be relaxed in future versions of the code. The reasons for these physical meanings for each term are more apparent because each differential equation is cast into the standard form of Table 2-1. The numerical method for solving these equations is discussed Chapter 3.

Equations 2-3 through 2-6 must be solved for $u, v, w$, and $p$. Historically, the pressure term caused numerical solution difficulties, and thus a transformation was used to change the four variables and four partial differential equations into two partial differential equations and two variables, namely, the stream function and vorticity. The pressure term was then eliminated. This approach had difficulties with defining the physical boundary conditions. The numerical approach discussed in Chapter 3 obviates the difficulties with the pressure term, and thus the primitive variable formulation $(u, v, w, p)$ is solved in PCGC-2.

The Eulerian equation set, along with the appropriate boundary conditions (see Chapter 3), is sufficient to permit solution for the flow field, provided that the density and viscosity are known everywhere in the field. In an isothermal, constant-density fluid, these variables are known a priori. However, in the combustion system of interest, these variables are position dependent. It is through these terms alone that coupling of the combustion model to the fluid mechanics model occurs. This subject is discussed later in this chapter. Also, the problem of time-averaging changes the equation set somewhat. This is the topic of the next section. 


\section{Turbulence Model}

\section{The Closure Problem}

In laminar flows, velocity gradients are sufficiently mild that the fluid can dynamically adjust to imposed shear stresses through molecular (viscous) forces. When imposed shear forces are too great for the fluid to adjust through molecular processes, the fluid is "tom" into largely coherent regions (turbulent eddies), which can rotate and thus relieve the shear forces caused by the imposed velocity differences. A review of turbulence and its associated closure problem can be found in Tennekes and Lumley (1972), and for applications to coal combustion modeling, in Smoot and Smith (1985). A particularly pertinent review of turbulence models and their applications to swirling flow is given by Sloan et al. (1986).

\section{k- $\varepsilon$ Turbulence Model Used in PCGC-2}

PCGC-2 uses the approach to this closure problem credited to Boussinesq (1877) which is derived by analogy to the laminar Newtonian viscosity. It is assumed that

$$
\overline{u^{\prime} v^{\prime}}=-v_{s}^{s}\left(\frac{\partial \bar{u}}{\partial r}+\frac{\partial \bar{v}}{\partial x}\right)
$$

where $v_{g}^{t}$ is the turbulent kinematic eddy viscosity. The correlation of fluctuating variables is thus expressed in terms of the mean field variables. Although Eqn. 2-7 is convenient, it does not have a sound theoretical basis. However, this approach has been widely used in turbulent gaseous systems, and is therefore applied in PCGC-2 for lack of a better model (Smith, 1979; Tennekes and Lumley, 1972; Pratt, 1976). A review of the accuracy of the approach in these applications with comparisons to more sophisticated approaches is given by Sloan, et al. (1986). The turbulence closure model used in PCGC-2 (Launder and Spalding, 1972) relates the turbulent kinetic energy $(k)$ and its rate of dissipation $(\varepsilon)$ by: 


$$
v_{g}^{s}=\frac{C_{\mu} k^{2}}{\varepsilon}
$$

where:

$$
k=\frac{\left(\overline{u^{\prime 2}}+\overline{v^{\prime 2}}+\overline{w^{\prime 2}}\right)}{2}
$$

Transport equations for $k$ and $\varepsilon$ can be derived from basic principles (Launder and Spalding, 1972), although some terms must be modeled empirically. The $k$ and $\varepsilon$ equations used in PCGC-2 are:

$$
\begin{aligned}
& \frac{\partial}{\partial x}(\bar{\rho} \bar{\mu} k)+\frac{1}{r} \frac{\partial}{\partial r}(r \bar{\rho} \bar{v} k)-\frac{\partial}{\partial x}\left(\frac{\mu_{e}}{\sigma_{k}} \frac{\partial k}{\partial x}\right)-\frac{1}{r} \frac{\partial}{\partial r}\left(r \frac{\mu_{e}}{\sigma_{k}} \frac{\partial k}{\partial r}\right) \\
& =\mu_{e}\left\{2\left[\left(\frac{\partial \bar{\mu}}{\partial x}\right)^{2}+\left(\frac{\partial \bar{v}}{\partial r}\right)^{2}+\left(\frac{\bar{v}}{r}\right)^{2}\right]+\left(\frac{\partial \bar{\mu}}{\partial r}+\frac{\partial \bar{v}}{\partial x}\right)^{2}+\left(r \frac{\partial}{\partial r} \frac{\bar{w}}{r}\right)^{2}+\left(\frac{\partial \bar{w}}{\partial x}\right)^{2}\right\}-\bar{\rho} \varepsilon \\
& \frac{\partial}{\partial x}(\bar{\rho} \tilde{\mu} \varepsilon)+\frac{1}{r} \frac{\partial}{\partial r}(r \bar{\rho} \bar{v} \varepsilon)-\frac{\partial}{\partial x}\left(\frac{\mu_{e}}{\sigma_{e}} \frac{\partial \varepsilon}{\partial x}\right)-\frac{1}{r} \frac{\partial}{\partial r}\left(\frac{\mu_{e}}{\sigma_{e}} \frac{\partial \varepsilon}{\partial r}\right) \\
& =\left(\frac{\varepsilon C_{1}}{k}\right) \mu_{e}\left\{2\left[\left(\frac{\partial \bar{\mu}}{\partial x}\right)^{2}+\left(\frac{\partial \bar{v}}{\partial r}\right)^{2}+\left(\frac{\bar{v}}{r}\right)^{2}\right]+\left(\frac{\partial \bar{\mu}}{\partial r}+\frac{\partial \bar{v}}{\partial x}\right)^{2}\right. \\
& \left.+\left(r \frac{\partial}{\partial r} \frac{\tilde{w}}{r}\right)^{2}+\left(\frac{\partial \bar{w}}{\partial x}\right)^{2}\right\}-\frac{\varepsilon^{2} C_{2} \bar{\rho}}{k}
\end{aligned}
$$

where:

$$
\mu_{c}=\mu^{2}+\mu^{t}
$$


and:

$$
\mu^{b}=\bar{\rho} \mu_{8}^{s}
$$

The constants $C_{\mu}, C_{1}, C_{2}, \sigma_{k}$ and $\sigma_{\varepsilon}$ are "universal" constants and have been optimized by comparison with experimental observations of both isothermal and reacting flows (Kahlil et al., 1975). The recommended values of these constants are found in Table 2-2. The $k-\varepsilon$ model is the most popular method for turbulence closure for both gaseous and particle-laden systems (Bilger, 1974; Spalding, 1975; Smoot and Smith, 1987). Models which account for combustiongenerated turbulence, coherent structures, and gas-particle interaction (Bilger, 1976; Spalding, 1975) are needed.

TABLE 2-2

TURBULENCE MODEL CONSTANTS

\begin{tabular}{cl} 
Constant & Value \\
\hline$C_{\mu}$ & 0.09 \\
$C_{1}$ & 1.44 \\
$C_{2}$ & 1.92 \\
$\sigma_{\mathrm{k}}$ & 0.9 \\
$\sigma_{\varepsilon}$ & 1.22
\end{tabular}

Melville and Bray (1979) note that the presence of particles has only a slight effect on the gas-phase turbulence. They suggest a correlation using the ratio of the mean particle bulk density to the mean gas density, as follows:

$$
v_{g \text { particles }}^{l}=v_{g \text { no part. }}^{l}\left(1+\frac{\bar{\rho}_{b}}{\bar{\rho}_{z}}\right)^{-0.5}
$$

According to this equation, the amount of turbulence decreases as the bulk particle density increases. PCGC-2 is coded to allow for this empirical correction of gas- 
phase turbulence due to particles. The correction is limited to a minimum value of 0.95 .

\section{Laminarization and laminar flow}

Some 2-D reactors have zones where the local Reynolds number is low and laminar effects are significant. An option is therefore provided in PCGC-2 for including laminarization effects in the $\mathrm{k}-\varepsilon$ model according to the extension of Jones and Launder $(1972,1973)$. This extension includes the following modifications:

(i) Viscous diffusion of $\mathrm{k}$ and $\varepsilon$ are included.

(ii) The terms containing the $C$ 's in eqns. 2-8 and 2-11 become dependent upon the Reynolds number of turbulence.

(iii) Terms are added to account for the fact that the dissipation processes are not isotropic.

The complete form of the turbulence model with the laminarization extension is given below.

Turbulence energy

$$
\begin{aligned}
& \frac{\partial}{\partial x}(\bar{\rho} \bar{\mu} k)+\frac{1}{r} \frac{\partial}{\partial r}(r \bar{\rho} \bar{v} k)-\frac{\partial}{\partial x}\left(\frac{\mu_{e}}{\sigma_{k}} \frac{\partial k}{\partial x}\right)-\frac{1}{r} \frac{\partial}{\partial r}\left(r \frac{\mu_{e}}{\sigma_{k}} \frac{\partial k}{\partial r}\right) \\
& =\mu_{e}\left\{2\left[\left(\frac{\partial \bar{\mu}}{\partial x}\right)^{2}+\left(\frac{\partial \bar{v}}{\partial r}\right)^{2}+\left(\frac{\bar{v}}{r}\right)^{2}\right]+\left(\frac{\partial \bar{\mu}}{\partial r}+\frac{\partial \bar{v}}{\partial x}\right)^{2}+\left(r \frac{\partial}{\partial r} \frac{\bar{w}}{r}\right)^{2}+\left(\frac{\partial \bar{w}}{\partial x}\right)^{2}\right\} \\
& -\bar{\rho} \varepsilon-2 \mu^{\ell}\left(\frac{\partial k^{\frac{1}{2}}}{\partial x}+\frac{\partial k^{\frac{1}{2}}}{\partial r}\right)^{2}
\end{aligned}
$$

Energy dissipation 


$$
\begin{aligned}
& \frac{\partial}{\partial x}(\bar{\rho} \bar{\mu} \varepsilon)+\frac{1}{r} \frac{\partial}{\partial r}(r \bar{\rho} \bar{v} \varepsilon)-\frac{\partial}{\partial x}\left(\frac{\mu_{e}}{\sigma_{e}} \frac{\partial \varepsilon}{\partial x}\right)-\frac{l}{r} \frac{\partial}{\partial r}\left(\frac{\mu_{e}}{\sigma_{e}} \frac{\partial \varepsilon}{\partial r}\right) \\
& =\left(\frac{\varepsilon C_{1} f_{1}}{k}\right) \mu_{e}\left\{2\left[\left(\frac{\partial \bar{\mu}}{\partial x}\right)^{2}+\left(\frac{\partial \bar{v}}{\partial r}\right)^{2}+\left(\frac{\bar{v}}{r}\right)^{2}\right]+\left(\frac{\partial \bar{\mu}}{\partial r}+\frac{\partial \bar{v}}{\partial x}\right)^{2}+\left(r \frac{\partial}{\partial r} \frac{\bar{w}}{r}\right)^{2}+\left(\frac{\partial \bar{w}}{\partial x}\right)^{2}\right\} \\
& -\frac{\varepsilon^{2} C_{2} f_{2} \bar{\rho}}{k}+2.0 \mu^{2} \mu^{2}\left(\frac{\partial^{2} u}{\partial x^{2}}+\frac{\partial^{2} u}{\partial r^{2}}+\frac{\partial^{2} v}{\partial x^{2}}+\frac{\partial^{2} v}{\partial r^{2}}+\frac{\partial^{2} w}{\partial x^{2}}+\frac{\partial^{2} w}{\partial r^{2}}\right)^{2}
\end{aligned}
$$

Turbulent viscosity formula

$$
v_{g}^{t}=\frac{C_{\mu} f_{\mu} k^{2}}{\varepsilon}
$$

In the above equation set, the $C$ 's and $\sigma$ 's retain the values assigned to them in Table 2-2; the influence of Reynolds number mentioned under (ii) above is introduced by way of the $f$ 's, which are assigned the following forms:

$$
\begin{aligned}
& f_{1}=1.0 \\
& f_{2}=1.0-0.3 \exp \left[\left(-R e^{t}\right)^{2}\right] \\
& f_{\mu}=\exp \left(-\frac{2.5}{1+\frac{R e}{50}}\right)
\end{aligned}
$$

where $R e \equiv \rho k^{2} / \mu \varepsilon$ is interpreted as the Reynolds number of turbulence.

For purely laminar reactors, the $k-\varepsilon$ calculations may be turned completely off in the model, thus allowing diffusion to be calculated according to purely molecular forces. 


\section{Particle Phase Mechanics}

\section{Background}

A distinguishing feature among pulverized coal models is the treatment of the dispersed particle phase. In large-scale furnaces there are large recirculation zones. Swirling inlet flows can also have a major influence on the particle motion. Gas molecules tend to equilibrate locally. However, particles exist locally with different properties due to the varying history of each particle.

The approach used in PCGC-2 is based on the PSI-CELL technique of Crowe, et al. (1977). Care is taken to account for all modes of gas-particle coupling. The method does not account for particle-particle interactions and thus would not be applicable to highly loaded, dense-phase flow. It is intended to be applied to dispersed-flow systems. The model is based on calculating the trajectories or paths of representative particles through the gas phase (continuum) field in a Lagrangian fashion. These particles are used to calculate sources of mass, momentum, and energy to the gas phase. These source terms were introduced in the previous section. Crowe, et al. (1977) discuss the basic concept, the derivation of the gas and particle equations, and calculation of the source terms. Briefly, mean particle velocities, trajectories, temperatures, and compositions are obtained by integrating representative equations for the mean motion, energy, and component continuity for an ensemble of particles in the gas flow field. Total momentum, energy, and mass of the particle cloud are recorded on crossing cell boundaries. The net difference in the particle properties between leaving and entering any given cell then provides the particle source terms for the gas flow equations. While the mean location of the particle ensemble is being tracked, the statistical spatial distribution about this mean is currently modeled only by a delta function at the mean. Work is currently underway to include the variance (Baxter, 1987). 


\section{Particle Momentum Equation}

The particle momentum equation for a single particle in the Lagrangian framework is given by:

$$
\alpha_{j} d\left(\vec{v}_{j}\right) / d t=\vec{f}_{j}+\alpha_{j} \bar{g}
$$

The reaction rate term is accounted for by assuming the particle mass leaves the particle with equal mass and velocity in all directions. The drag term $\left(\bar{f}_{p}\right)$ is related to the difference in gas and particle velocities by:

$$
\vec{f}_{j}=\Gamma_{d}\left(\vec{v}_{g}-\vec{v}_{j}\right)
$$

where $\Gamma_{d}$ is some time constant based on the drag coefficient $\left(C_{d}\right)$, as shown below:

$$
\Gamma_{d}=\frac{\left(\frac{\pi D_{j}^{2}}{4}\right) \rho_{8} C_{d}\left\|\vec{v}_{j}-\vec{v}_{8}\right\|}{2}
$$

For spherical particles, the following correlation is suggested for the drag coefficient (Wallis, 1969):

$$
C_{d o}=\left(\frac{24}{R e}\right)\left(1+0.15 R e^{0.687}\right)
$$

It has been shown that high particle reaction rates can alter the drag coefficient (Bird et al., 1960). The relationship used in PCGC-2 is that suggested by Bailey et al. (1970):

$$
C_{d}=\frac{C_{d o}}{1+B_{m}}
$$




$$
B_{m}=\sum \frac{r_{i}}{k_{i}}
$$

where $B_{m}$ is the particle blowing parameter for mass transfer, which will be discussed later in this chapter.

In a turbulent system, the equation of motion for an individual particle becomes more complicated. The approach taken to account for turbulence in Eqn. 2-19 is to decompose the particle velocity into convective and diffusive components:

$$
\vec{v}_{j}=\vec{v}_{j c}+\vec{v}_{j d}
$$

The convective component of velocity $\left(\vec{v}_{j c}\right)$ is defined as the velocity that would arise in the absence of turbulence, or the ballistic velocity based on the mean gas velocity. Therefore, Eqn. $2-19$ is used to find $\vec{v}_{j c}$ along a trajectory by numerical integration. The diffusive component of velocity $\left(\vec{v}_{j d}\right)$ is added to account for the turbulence effects on particle motion.

In the Lagrangian framework, the diffusive velocity $\vec{v}_{j d}$ is modeled using the mean particle bulk density gradient:

$$
\vec{v}_{j d} \bar{\rho}_{j b}=-\Gamma_{j} \bar{\nabla} \bar{\rho}_{j b}
$$

Equation 2-26 can be viewed as a particle diffusivity equation, since the left-hand side can be thought of as a turbulent mass flux (relative to the convective velocity):

$$
\bar{j}_{j}=\left(\bar{v}_{j}-\bar{v}_{j c}\right) \bar{\rho}_{j b}=\bar{v}_{j d} \bar{\rho}_{j b}
$$

Equation 2-26 now defines the transport coefficient $\Gamma_{j}$ as a turbulent particle diffusivity, which can also be expressed as:

$$
\Gamma_{j}=D_{j}^{t}=\frac{v_{j}^{t}}{\sigma_{j}^{s}}
$$


where $D_{j}^{t}$ is the turbulent particle diffusivity, $v_{j}^{t}$ is the turbulent particle kinematic viscosity, and $\sigma_{j}^{b}$ is the turbulent particle Schmidt number. Much research is currently being conducted by the scientific community on how to obtain the turbulent particle kinematic viscosity $\left(v_{j}\right)$.

An expression for $v_{j}^{t}$ should account for particle size, so that larger particles are not affected by the turbulence as much as are smaller particles. The $v_{j}^{t}$ expression should also include some term to account for the level of turbulence intensity. The model selected for use in PCGC-2 is that of Melville and Bray (1979), which relates $v_{j}^{t}$ to $v_{g}^{t}$ as follows:

$$
v_{j}^{s}=\frac{v_{g}^{l}}{\left[I+\left(t_{j} / t^{t}\right)\right]}
$$

The particle relaxation time $\left(t_{j}\right)$ is related to the Stokesian particle drag (Melville and Bray, 1979; Longwell and Weiss, 1980; Lockwood et al., 1980; Lilly, 1973) by:

$$
t_{j}=\frac{\alpha_{j}}{3 \pi \mu_{g} d_{j}}
$$

The turbulence time scale $\left(t^{n}\right)$ is related to the local turbulence by:

$$
t^{\prime}=\frac{v_{8}^{\prime}}{\overline{u_{8}^{\prime 2}}}
$$
Assuming isotropic turbulence $\left(\overline{u_{g}^{2}}=2 / 3 k\right)$ and substituting Eq. $2-17$ for $v_{g}^{t}$,
Eqn. $2-31$ becomes

$$
t^{t}=\frac{3 C_{\mu} k}{2 \varepsilon}
$$

The value of the turbulent particle Schmidt number $\sigma_{j}^{t}$ is not well characterized. Comparisons of predictions to data can be used to empirically determine this parameter. Best results were obtained by Fletcher (1983) using $\sigma_{j}^{t}=$ 0.35 . This value compares well with the results of Lilly (1973), where $\sigma_{j}^{t}=0.34$, 
but not as well with the results of Melville and Bray (1979), who recommended a value of 0.7 .

Several other particle dispersion models are also found in the current literature. Lockwood, et al. (1980) used an empirical approach to get $v_{j d}$ from $u^{2}$ and $v^{2}$. Longwell and Weiss (1980) considered that the ratio of particle eddy diffusivity to gas eddy diffusivity followed a sinusoidal pattern involving $t j$ and $t^{t}$. Lilly (1973) gives evidence that $v_{f}^{t} / v_{g}^{t}$ decreases as $t j / t^{t}$ increases, especially for small particles $(6$ to $16 \mu \mathrm{m})$. Lilly's results are surprising, since the large particles were more dispersed than the smaller particles. The model of Melville and Bray (1979) was selected here because it seemed to be more complete than the other models and is applicable to the size range of interest (10 to $100 \mu \mathrm{m}$ ).

\section{Bulk Particle Density}

The bulk particle density is needed to calculate the radiation field (discussed later) and to calculate particle velocities (Eqn. 2-26). However, the Eulerian bulk particle density field cannot be calculated directly from the Lagrangian particle trajectory information. Methods used for obtaining this gradient by counting particles and smoothing Lagrangian density information have not been completely acceptable. The bulk particle mass, and number densities are related as follows:

$$
\bar{\rho}_{b}=\alpha_{j} \bar{n}_{j}
$$

The number density $\left(\bar{n}_{j}\right)$ is easier to calculate than the mass density $\left(\bar{\rho}_{b}\right)$ because the number of particles represented by each trajectory is constant throughout the computational domain. The mass density changes due to particle reaction. Equation 2-26 then becomes:

$$
\vec{v}_{j d} \bar{n}_{j}=\Gamma_{j} \bar{\nabla} \bar{n}_{j}
$$

Equation 2-34 is thought to approximate $\vec{v}_{j d}$ just as well as Eqn. 2-26. However, $\bar{n}_{j}$ cannot be calculated from the Lagrangian particle-phase information, and can only be approximated using the Eulerian gas-phase information. The Eulerian equation of continuity for the particle number density in turbulent flow (steady-state) is: 


$$
\frac{\partial}{\partial x}\left(\bar{u}_{j} \bar{n}_{j}\right)+\frac{1}{r} \frac{\partial}{\partial r}\left(r \bar{v}_{j} \bar{n}_{j}\right)-\frac{\partial}{\partial x}\left(\Gamma_{j} \frac{\partial \bar{n}_{j}}{\partial x}\right)-\frac{1}{r} \frac{\partial}{\partial r}\left(r \Gamma_{i} \frac{\partial \bar{n}_{j}}{\partial r}\right)=0
$$

The diffusion coefficient $\left(\Gamma_{j}\right)$ in Eqn. 2-35 is the same as in Eqn. 2-28, and can be approximated on an Eulerian basis assuming an average size for the particle as it reacts. The equation, however, requires the Eulerian particle velocity field for its solution. For the purposes of Eqn. 2-35 the gas-phase velocity components are used to approximate $u_{j}$ and $v_{j}$. With the small particles of interest to pulverized coal conversion processes, the particle and gas velocities are similar. Reynolds numbers for the particles relative to the gas are often less than 0.01 . This approximation is only used for the purposes of Eq. 2-35, since the Lagrangian solution yields the particle velocity including the effects of particle drag.

\section{Gas Phase Reactions}

\section{The Turbulent Reaction Rate}

The importance of properly accounting for the turbulent interactions with the combustion chemistry in diffusion flames cannot be overemphasized (Bilger, 1974). In writing conservation equations for individual species in turbulent reacting systems, time-averaging is required as has been shown earlier. The instantaneous form of these equations can be found in several sources (Bird et al., 1960; Smoot and Pratt, 1979; Williams, 1965). All of these species conservation equations contain reaction rate terms of the form.

$$
r_{i}=m_{i} m_{j} \rho^{2} A \exp \left(-\frac{E}{R T}\right)
$$

Proper time-averaging of such terms is accomplished by decomposing the instantaneous variables into their main and fluctuating components, giving rise to terms of the form: 


$$
\begin{aligned}
& \bar{r}_{i}=\overline{m_{i} m_{j} \rho^{2} A \exp \left(-\frac{E}{R T}\right)}=\rho^{-2} \bar{m}_{i} \bar{m}_{j} A \exp \left(-\frac{E}{R T}\right)\left\{1+\frac{\overline{\rho^{\prime 2}}}{\rho^{-2}}+\frac{m_{i} m_{j}}{\bar{m}_{i} \bar{m}_{j}}\right. \\
& \left.+\frac{\overline{2 \rho m_{i}^{\prime}}}{\bar{\rho} \bar{m}_{i}}+2 \frac{\overline{\rho m_{j}}}{\bar{\rho} \bar{m}_{j}}+\frac{E}{R \bar{T}}\left[\overline{\frac{m_{i} T^{\prime}}{\bar{m}_{i} \bar{T}}}+\frac{\overline{m_{j} T}}{\bar{m}_{j} \bar{T}}+\left(\frac{E}{2 R \bar{T}}-1\right) \overline{\frac{T^{\prime 2}}{\bar{T}^{2}}}\right]+\ldots\right\}
\end{aligned}
$$

The other terms which appear involve triple correlations of the fluctuating variables. It is to be remembered that for any realistic reaction scheme, scores of these equations must necessarily appear.

Historically, two approaches have been used to solve this problem. At one extreme, complex finite-rate chemistry is considered but turbulent mixing is disregarded. Bowman (1975) and Caretto (1976) reviewed this literature. At the other extreme, turbulent mixing is treated in detail as described earlier, but local instantaneous (infinite rate) chemistry is assumed. Bilger (1974) reviewed this literature. Very few attempts have been made to combine finite-rate chemistry and detailed turbulent mixing. Borghi (1974) applied turbulent chemical reaction rate calculations to a CO-containing plume. He introduced the concept of the contact index. Spalding (1978) proposed a combined Eulerian/Lagrangian formulation. Even with these difficulties, in many situations simplified combustion models can predict gaseous turbulent combustion processes with acceptable engineering accuracy (Spalding, 1978; Gosman et al., 1978; Libby, 1976; Spalding, 1971). Each of these references suggests different forms of a combustion model. Simplifications are usually made by assigning properties to the bulk fuel and oxidizer streams.

\section{The Mixture Fraction}

In gaseous diffusion flames, the fuel and oxidizer are fed in separate streams. They enter the reactor in separate eddies which must be intimately contacted on a molecular level before reaction can occur. The assumption is then made that this micro-mixing process is rate-limiting, not the kinetic process. This allows the chemistry to be computed from equilibrium. Only one differential equation is required to describe the degree of "mixedness" or "unmixedness" at a point, a great 
simplification as compared to the immense system of equations described above for the kinetic scheme.

For cases where there are two identifiable streams or states that have uniform properties, it is convenient to describe a conservative scalar $f$, the mixture fraction:

$$
f=\frac{M_{p}}{M_{p}+M_{s}}=\text { mass fraction of fluid atoms originating in primary stream }
$$

The advantage of the mixture fraction approach lies in its ability to calculate any conserved scalar $(s)$ from the local value of $f$ :

$$
s=f s_{p}+(1-f) s_{s}
$$

An example of a conserved scalar is the mass fraction of a given element.

The following sections develop an approach to comprehensive calculations which use the concept of mixture fractions. The application of this concept requires that the turbulent diffusivities of all gas phase species be equal and that their boundary conditions be the same. These properties taken together constitute what is sometimes called Crocco similarity (Smith, 1979). The mixture fraction approach greatly reduces the number of conserved scalars required to describe a combustion system.

\section{Scalar Transport}

The differential equation for the transport of a scalar variable is shown here in its Favre-averaged form:

$$
\frac{\partial}{\partial x}(\bar{\rho} \vec{u} \vec{f})+\frac{I}{r} \frac{\partial}{\partial r}(r \bar{\rho} \vec{v} \tilde{f})-\frac{\partial}{\partial x}\left(\frac{\mu_{e}}{\sigma_{f}} \frac{\partial \bar{f}}{\partial x}\right)-\frac{1}{r} \frac{\partial}{\partial r}\left(\frac{r \mu_{e}}{\sigma_{f}} \frac{\partial \bar{f}}{\partial r}\right)=0
$$

The solution of this equation, together with the fluid mechanics model, will prescribe the mean fluid values for the flow and the mixing, provided the appropriate mean density is available. The advantage of knowing $\vec{f}$ is that with the 
aid of a probability density function (pdf) for $f$, the time-mean values may be found for any arbitrary value $\beta$ which is only a function of $f$.

$$
\bar{\beta}=\alpha_{p} \beta_{p}+\alpha_{s} \beta_{s}+\int_{0}^{r} \beta(f) P(f) d f
$$

The mean of the distribution of the mixture fraction (first moment about the origin) is given by Eqn. 2-40. The variance (second moment about the mean, or the mean square fluctuation) of the mixture fraction is defined as:

$$
g_{f}=\overline{(f-\tilde{f})^{2}}=\frac{I}{T} \int_{0}^{T}[f(t)-\tilde{f}]^{2} d t
$$

where $\mathrm{T}$ is large compared with the time scale of the local turbulence. Launder and Spalding (1972) show how a transport equation for $g_{f}$ can be derived and appropriate terms modeled in a manner analogous to, and consistent with the other two equations in the $k-\varepsilon$ turbulence model. The resulting equation is:

$$
\begin{aligned}
& \frac{\partial}{\partial x}\left(\bar{\rho} \tilde{u} g_{f}\right)+\frac{1}{r} \frac{\partial}{\partial r}\left(r \bar{\rho} \bar{v} g_{f}\right)-\frac{\partial}{\partial x}\left(\frac{\mu_{e}}{\sigma_{g}} \frac{\partial g}{\partial x} f\right)-\frac{1}{r} \frac{\partial}{\partial r}\left(\frac{r \mu_{e}}{\sigma_{g}} \frac{\partial g}{\partial r} r\right) \\
& =\frac{C_{g l} \mu_{e}}{\sigma_{g}}\left[\left(\frac{\partial \bar{f}}{\partial x}\right)^{2}+\left(\frac{\partial \bar{f}}{\partial r}\right)^{2}\right]-C_{g 2} \frac{\bar{\rho} \varepsilon g}{k}
\end{aligned}
$$

Appropriate constants for the combustion submodel, introduced by Eqns. $2-40$ and 2-42, are given in Table 2-3. 
TABLE 2-3

\section{TURBULENT COMBUSTION MODEL CONSTANTS}

\begin{tabular}{cc} 
Constant & $\frac{\text { Value }}{2.8}$ \\
\hline$C_{g l}$ & 1.92 \\
$C_{g 2}$ & 0.9 \\
$\sigma_{f}$ & 0.9 \\
$\sigma_{g}$ & 0.9 \\
$\sigma_{h l}$ & 0.8 \\
$\sigma_{b}$ & 9.793 \\
$E^{*}$ & \\
\hline
\end{tabular}

\footnotetext{
*These constants arise from boundary conditions
}

Besides $\bar{f}$ and $g_{f}$, the pdf shape is needed. In regions of small $g_{f}$ the shape of the pdf makes little difference. The pdf shape is determined from experimentally observed fluctuations in coal flames. Smith (1979), for example, used a top-hat (uniform) distribution, while forms of the Gaussian distribution and the Beta distribution have also been used. Kent and Bilger (1977) used a "clipped" Gaussian distribution which accounts for intermittency, and noted significant sensitivity of the predictions to the form of the pdf, particularly for nitric oxide predictions. Other references pertaining to pdf shape are found in Smith (1979), Fletcher (1980), Kuo (1986), Lockwood and Naguib (1975), and Kent and Bilger (1976).

PCGC-2 is presently coded to use the clipped Gaussian distribution, (i.e. adjusted for intermittency) similar to that used in the last two references. The parameters needed to calculate this Gaussian pdf are as follows: The continuous portion of the probability curve is given by

$$
P(f)=\left(2 \pi G_{f}\right)^{-1 / 2} \exp \left(-Z_{f}^{2} / 2\right)
$$

where

$$
Z_{f}=\frac{(f-F)}{\sqrt{G_{f}}}
$$


and $F$ and $G_{f}$ come from:

$$
\tilde{f}=\alpha_{p}+\frac{1}{\sqrt{2 \pi G_{f}}} \int_{0+}^{l-} f \exp \left[-\frac{(f-F)^{2}}{2 G_{f}}\right] d f
$$

and

$$
g_{f}=\alpha_{p}-(\bar{f})^{2}+\frac{1}{\sqrt{2 \pi G_{f}}} \int_{0^{+}}^{1-} f^{2} \exp \left[-\frac{(f-F)^{2}}{2 G_{f}}\right] d f
$$

The area under the clipped portions are given by

$$
\alpha_{\rho}=\frac{1}{\sqrt{2 \pi}} \int_{\frac{l-F}{\sqrt{\sigma_{f}}}} \exp \left(-\frac{Z_{f}^{2}}{2}\right) d Z_{f}
$$

and

$$
\alpha_{s}=\frac{1}{\sqrt{2 \pi}} \int_{-\infty}^{-\frac{F}{\sqrt{G_{f}}}} \exp \left(-\frac{Z_{f}^{2}}{2}\right) d Z_{f}
$$

where $\alpha_{p}$ and $\alpha_{s}$ are the intermittency of the primary and secondary streams, respectively.

\section{Chemical Equilibrium}

In a combustor which is assumed locally adiabatic (no gas radiation, external heat loss, or compressible heating/cooling), the standard enthalpy is a conserved scalar, and thus with the assumption of equal diffusivities, the instantaneous local enthalpy $h$ may be calculated directly from $f$.

$$
h=f h_{p}+(1-f) h_{s}
$$


If the local turbulent diffusivities are equal, the elemental mass fractions $\left(b_{k}\right)$ are also conserved scalars and are given by:

$$
b_{k}=f b_{k p}+(1-f) b_{k s}
$$

Equations 2-49 and 2-50 are not dependent on the assumption of chemical equilibrium but only on the equality of diffusivities, and in the case of Eqn. 2-49, on the assumption of locally adiabatic operation. These assumptions comprise what is called Crocco similarity (Smith, 1979). For non-adiabatic operation, the energy equation (Eqn. 2-89) must be solved.

If local, instantaneous, equilibrium is assumed (micro-mixing is limiting rather than chemical kinetics), all thermodynamic properties can be obtained. For a Gibbs free energy reduction scheme, the required parameters are the energy level, pressure, and elemental composition. The equilibrium properties are then functions of $f$ alone:

$$
\begin{gathered}
T=T\left(b_{k}, h\right)=T\left[b_{k}(f), h(f)\right]=T(f) \\
\rho=\rho\left(b_{k}, h\right)=\rho\left[b_{k}(f), h(f)\right]=\rho(f) \\
y_{i}=y_{i}\left(b_{k}, h\right)=y_{i}\left[b_{k}(f), h(f)\right]=y_{i}(f)
\end{gathered}
$$

The turbulent flow mean compositions and other properties are obtained by weighting with the pdf of $f$, as in Eqn. 2-41:

Thus

$$
\begin{gathered}
\bar{y}_{i}=\alpha_{p} y_{i p}+\alpha_{s} y_{i s}+\int_{0+}^{t-} y_{i}(f) P(f) d f \\
\bar{\rho}=\alpha_{p} \rho_{p}+\alpha_{s} \rho_{s}+\int_{0+}^{t-} \rho(f) P(f) d f
\end{gathered}
$$




$$
\tilde{T}=\alpha_{p} T_{p}+\alpha_{s} T_{s}+\int_{0+}^{l-} T(f) P(f) d f
$$

This approach to find the mean properties is dependent on the enthalpy $(h)$ and the element mass fractions $\left(b_{k}\right)$ being functions only of the mixture fraction $(f)$. When heat losses (due to radiation, external heat loss, or compression/expansion) are significant, then Crocco similarity must be abandoned for the conservation equation in $h$ (Eqn. 2-89). In this situation, Eqns. 2-51 through 2-53 are no longer valid, and in general, the properties become functions of both $f$ and $h$ :

$$
\beta=\beta\left(b_{k}, h\right)=\beta\left[b_{k}(f), h\right]=\beta(f, h)
$$

Favre-averaged properties are obtained by convolution with a joint pdf:

$$
\tilde{\beta}=\iint_{h f}(f, h) P(f, h) d f d h
$$

This equation is valid for locally adiabatic operation as well, in which case the enthalpy is a function of $f$ alone, and Eqn. 2-58 reduces to Eqn. 2-41.

The problem of obtaining $P(f, h)$ is greatly simplified by partitioning the enthalpy into: 1) the energy which would be convected if there were no local heat losses or gains $\left(h_{f}\right)$, and 2$)$ the residual energy due to the local heat losses/gains $\left(h_{r}\right)$.

$$
\begin{gathered}
h=h_{f}+h_{r} \\
h=\left[f h_{p}+(1-f) h_{s}\right]+h_{r}
\end{gathered}
$$

In practice, the residual enthalpy can be calculated directly from the known field values of $h$ and $f$ from the inlet conditions:

$$
\tilde{h}_{r}=\tilde{h}-\tilde{f} h_{p}-(I-\tilde{f}) h_{s}
$$


Finally, it is assumed that the effects of the fluctuations of $h_{r}$ are small compared with the effects of the fluctuations of $h f$ as implied by Eqn. 2-61.

$$
\dot{h_{r}}=0
$$

Thus

$$
\tilde{h}_{r}=h_{r}
$$

This means that the fluctuations are correlated by the single variable $f$ as before

$$
\tilde{\beta}=\alpha_{p} \beta_{p}+\alpha_{s} \beta_{s}+\int_{0+}^{l-} \beta\left(f, h_{r}\right) P(f) d f
$$

where $h_{r}$ is constant with respect to the fluctuations in $f$. Since $h_{r}$ will often be small relative to $h f$, this approximation is thought to be reasonable in many cases. Without the above assumption, it would be necessary to include transport equations for both $h$ and the mean square fluctuation of $h_{r}$. In addition, Eqn. (2-64) would need to be convoluted over the joint probability density function $P\left(f, h_{r}\right)$.

A special case arises if it is assumed that the heat loss from the reactor is uniform throughout the reactor. The total amount of sensible heat potentially in the reactor is

$$
Q_{s}=\int_{T_{s s}}^{T_{s}} C_{p} d T
$$

where $T_{a}$ is the adiabatic gas temperature and $T_{r e f}$ is the inlet gas temperature (ambient). The heat loss factor $(\gamma)$ is defined to be the fraction of the total sensible heat lost from the reactor:

$$
\gamma=\frac{h_{a}-h}{Q_{s}}
$$


where $h_{a}$ is the adiabatic enthalpy calculated from Eqn. 2-49. The enthalpy can now be calculated as a function of the heat loss:

$$
h=h_{a}-\gamma Q
$$

For a constant value of $\gamma$, Eqn. 2-67 maintains Crocco similarity while allowing for an approximation to non-adiabatic operation.

\section{Reacting Particles}

The gas model development to this point has assumed that no particles are present. When reacting particles are accounted for, it is convenient to define a new progress variable " $\eta$ " (similar to $f$ ) which describes the mass fraction of gas originating from the burning coal. This progress variable is called the coal-gas mixture fraction, and is defined as shown below:

$$
\eta=\frac{M_{c}}{M_{p}+M_{s}+M_{c}}=\text { mass fraction of fluid atoms originating from the coal (2-68) }
$$

If the only source of fuel for the combustion or gasification system of interest is from the coal particles or slurry droplets, and if all of the remaining transporting fluid is composed of the same fluid with the same energy level, then $\eta$ is the only required progress variable. Such is the case for practical combustors where the coal particles are transported with air in the primary stream, and the secondary stream is composed of air at the same temperature as the primary stream. In such systems the primary and secondary streams have exactly the same composition and energy level. In this limiting case, the coal gas mixture fraction $(\eta)$ is adequate to describe the progress of mixing in the chemical field since the components of the gas can be broken into only two parts, namely the coal offgas and the inlet gas.

A more general approach is adopted in PCGC-2. A mixture fraction is included to allow primary carrier gas fluid different than the secondary gas in either composition and/or enthalpy level. Alternatively, the mixture fraction may be used to track a vaporizing liquid (e.g. coal-oil, coal-water mixtures), which evolves at a different rate and has a different composition and heating value than the coal offgas. In either case, the gas phase must be broken into three components: 1) mass of gas 
originating from the primary gas or a slurry liquid, 2) gas originating from the secondary gas, and 3) the coal offgas. A minimum of two progress variables are required to describe the gas field at any one point in this approach. The variable $\eta$ is used to describe the mass fraction of coal offgas. In addition, the mixture fraction $f$ provides the information regarding the amount of primary carrier gas fluid or slurry liquid. Whether the variable $f$ is used to track the mixing of the primary and secondary streams or the mixing of vaporizing liquid in the combustion gases, there is a significant advantage in defining it as a mass ratio rather than a mass fraction. That is

$$
f=\frac{\dot{m}_{p}}{\dot{m}_{p}+\dot{m}_{s}}
$$

or, in the case of slurry combustion,

$$
f=\frac{\dot{m}_{\ell}}{\dot{m}_{\ell}+\dot{m}_{p+s}}
$$

In this case, $f$ is not the local mass fraction of gas originating from the primary stream or slurry liquid, but instead is the mass fraction of this quantity in the local mixture of primary plus secondary or vaporized slurry liquid plus inlet gas. Since the mixture fraction $f$ contains only information of the intermixing of the primary and secondary gas streams, or inlet gas with vaporized liquid, it is somewhat insensitive to local fluctuations of $\eta$, and thus is much more independent of $\eta$ than is the local mass fraction of primary fluid or slurry liquid. This statistical independence is useful in calculating the local chemical composition. Thus, $f$ and $\eta$ can be considered statistically independent to a first-order approximation.

Although $f$, as defined in Eqns. 2-69 or 2-70, is a useful quantity for the calculational procedure, the local mass fraction of primary fluid or vaporizing liquid is also an important parameter. This quantity is defined as $f_{p}$ :

$$
f_{p}=\frac{\dot{m}_{p}}{\dot{m}_{p}+\dot{m}_{s}+\dot{m}_{c}}
$$


or

$$
f_{p}=\frac{\dot{m}_{l}}{\dot{m}_{l}+\dot{m}_{p+s}+\dot{m}_{c}}
$$

where $f_{p}$ is the conserved scalar for the reacting particle or droplet-laden system. The variable $f$ as defined in Eqns. 2-69 or 2-70 is not a conserved scalar in reacting, particle-laden systems. The importance of $f_{p}$ is obvious from this conservative principle. Its mean value can be calculated from a Favre-averaged $\left(\tilde{f}_{p}\right)$ conservation equation which includes convection and turbulent diffusion terms. There is no source term for the $\tilde{f}_{p}$ equation when $f p$ is defined as in Eqn. 2-71. When $f_{p}$ is defined by Eqn. $2-72$, the source term is given by the mass of vaporizing liquid. From these definitions, the relationship between $f$ and $f_{p}$ is:

$$
f=\frac{f_{p}}{1-\eta}
$$

The progress variables $f$ and $\eta$ are useful quantities for partitioning the characteristics of the gas field. In order to obtain the mean temperature, density, composition, etc. of the gas field, we must understand the statistical distribution of these variables due to the turbulent fluctuations (i.e. the probability density function). In addition, each of the three components (coal offgas, primary carrier gas and secondary gas or slurry vapor) can react chemically to form new products.

In coal combustion/gasification systems, $\tilde{f}$ can be obtained from a nonconservative (i.e., with a source term) transport equation. Alternatively, $f_{p}$ can be calculated from a conservative transport equation and $f$ obtained through the relationship shown in Eqn. 2-73. Of course, Eqn. 2-73 relates the instantaneous values of $f$ and $f_{p}$. But if $f_{p}$ can be considered statistically independent of $\eta$, then the relationship also holds true in the mean:

$$
\tilde{f}=\frac{\tilde{f}_{p}}{1-\tilde{\eta}}
$$

The assumption of Crocco similarity applies here as well as in the case of gaseous diffusion flames. With the above assumptions, any local property that is a function of $f$ and $\eta$, can be calculated directly. 


$$
\beta=\beta_{c} \eta+(1-\eta)\left[f \beta_{p}+(1-f) \beta_{s}\right]
$$

The subscript $c$ stands for pure coal off-gas. Time-mean gas properties (i.e. species composition, density, and temperature) can be obtained for any values of $\eta$ and $f$ by convolution over a joint probability density function:

$$
\tilde{\beta}=\int_{-\infty}^{\infty} \beta(\eta, f) P(\eta, f) d \eta d f
$$

Note that Eqn. 2-76 is analogous to Eqn. 2-41. Assuming $\eta$ and $f$ are independent, the joint probability density function, $P(\eta, f)$, can be separated:

$$
P(\eta, f)=P(\eta) P(f)
$$

Intermittency can occur for both $\eta$ and $f$, and must be handled carefully (Fletcher, 1980). Intermediate steps to the final formulation are shown for completeness. By substituting Eqn. 2-77 into 2-76 and expanding:

$$
\begin{gathered}
\left.\tilde{\beta}=\int_{-\infty}^{\infty} \int_{-\infty}^{\infty} P(f) \beta(\eta, f) d f\right] P(\eta) d \eta \\
\tilde{\beta}=\int_{-\infty}^{\infty}\left[\alpha_{p} \beta(\eta, 1)+\alpha_{s} \beta(\eta, 0)+\int_{0+}^{1-} P(f) \beta(\eta, f) d f\right] P(\eta) d \eta
\end{gathered}
$$

Finally:

$$
\begin{aligned}
& \tilde{\beta}(\eta, f)=\alpha_{c} \beta_{c}+\alpha_{l}\left[\alpha_{p} \beta_{p}+\alpha_{s} \beta_{s}+\int_{0+}^{1+} P(f) \beta(0, f) d f\right] \\
& +\alpha_{p} \int_{0+}^{1-} P(\eta) \beta(\eta, 1) d \eta+\alpha_{s} \int_{0+}^{1-} P(\eta) \beta(\eta, 0) d \eta+\int_{0+0+}^{1-1-} \int_{0} P(\eta) P(f) \beta(\eta, f) d \eta d f
\end{aligned}
$$

where it has been recognized that 


$$
\alpha_{p}+\alpha_{s}+\int_{0+}^{1-} P(f) d f=1
$$

Intermittency of the coal off-gas stream, $\alpha_{c}$ and $\alpha_{1}$, are given by

and

$$
\alpha_{c}=\frac{1}{\sqrt{2 \pi}} \int_{\frac{1-H}{\sqrt{\sigma_{\eta}}}}^{\infty} \exp \left(-\frac{Z_{\phi}^{2}}{2}\right) d Z_{\phi}
$$

where $Z_{\phi}$ is given by $\quad Z_{\phi}=\frac{\eta-H}{\sqrt{G_{\eta}}}$

The subscript $I$ represents pure inlet gas from both the primary and secondary streams. $H$ and $G_{\eta}$ are determined from:

$$
\begin{gathered}
\tilde{\eta}=\alpha_{c}+\frac{1}{\sqrt{2 \pi G_{f}}} \int_{0_{+}}^{1-} \eta \exp \left[-\frac{(\eta-H)^{2}}{2 G_{\eta}}\right] d \eta \\
g_{\eta}=\alpha_{c}-(\bar{\eta})^{2}+\frac{1}{\sqrt{2 \pi G_{\eta}}} \int_{0_{+}}^{1-} \eta^{2} \exp \left[-\frac{(\eta-H)^{2}}{2 G_{\eta}}\right] d \eta
\end{gathered}
$$

Equations for $\tilde{\eta}$ and $g_{n}$ are necessary to obtain the pdf, and are derived in a manner which is analogous to the equations for $\tilde{f}$ and $g f$. 


$$
\begin{aligned}
& \frac{\partial(\bar{\rho} \tilde{u} \tilde{\eta})}{\partial x}+\frac{1}{r} \frac{\partial(r \bar{\rho} \tilde{v} \tilde{\eta})}{\partial r}-\frac{\partial}{\partial x}\left(\frac{\mu_{e} \partial \tilde{\eta}}{\sigma_{\eta} \partial x}\right)-\frac{1}{r} \frac{\partial}{\partial r}\left(r \frac{\mu_{e} \partial \tilde{\eta}}{\sigma_{\eta} \partial r}\right)=S_{p}^{\eta} \\
& \frac{\partial\left(\bar{\rho} \tilde{u} g_{\eta}\right)}{\partial x}+\frac{1}{r} \frac{\partial\left(r \bar{\rho} \tilde{v} g_{\eta}\right)}{\partial r}-\frac{\partial}{\partial x}\left(\frac{\mu_{e}}{\sigma_{g}} \frac{\partial g_{\eta}}{\partial x}\right)-\frac{1}{r} \frac{\partial}{\partial r}\left(r \frac{\mu_{e}}{\sigma_{g}} \frac{\partial g_{\eta}}{\partial r}\right) \\
& =\frac{C_{g 1} \mu_{e}}{\sigma_{g}}\left[\left(\frac{\partial \tilde{\eta}}{\partial x}\right)^{2}+\left(\frac{\partial \tilde{\eta}}{\partial r}\right)^{2}\right]-\frac{C_{g 2} \bar{\rho} \varepsilon g_{\eta}}{k}
\end{aligned}
$$

For lack of better constants, the "universal" constants from Table 2-3 (e.g. $\left.C_{g 1}, C_{g 2}\right)$ are used in Eqns. 2-87 and 2-88. These constants are semi-empirical, and need to be investigated further. The $S_{p}^{\eta}$ term in Eqn. 2-87 represents the local mass addition to the gas phase from coal reactions (one component of the total particle mass source term).

When the adiabatic assumption is relaxed, the enthalpy must be calculated from a transport equation. Gas properties will then be functions of $\eta, f$, and $h$, so that $(\eta, f)$ in Eqn. 2-77 must be replaced by $(\eta, f, h)$. Equation 2-77 must also be convoluted with $P(h)$ if fluctuations in $h$ are important (Smith, 1979). The gasphase energy conservation equation is listed here for completeness:

$$
\frac{\partial(\bar{\rho} \tilde{u} \tilde{h})}{\partial x}+\frac{1}{r} \frac{\partial(r \bar{\rho} \tilde{v} \tilde{h})}{\partial r}-\frac{\partial}{\partial x}\left(\frac{\mu_{e}}{\sigma_{h}} \frac{\partial \tilde{h}}{\partial x}\right)-\frac{1}{r} \frac{\partial}{\partial r}\left(r \frac{\mu_{e}}{\sigma_{h}} \frac{\partial \tilde{h}}{\partial r}\right)=q_{r g}^{\prime}+\tilde{u} \frac{\partial \bar{p}}{\partial x}+\tilde{v} \frac{\partial \bar{p}}{\partial r}+S_{p}^{h}(2
$$

The $q_{r g}^{\prime}$ term represents the net volumetric heat addition due to radiation. The $S_{p}^{h}$ term represents the heat given to the gas by the particles due to particle reaction and convection. The other two terms on the right-hand side represent the net heat addition due to expansion/contraction.

Formally, the gas phase properties are a function of the elemental composition, the total enthalpy and the local pressure. The enthalpy fluctuations due to the presence of the particles can be accounted for by partitioning the total gas enthalpy into the contributions by $\lambda$, by $f$, and by the residual enthalpy contributions. 
To this point, the elemental composition of the coal offgas has been assumed constant. This assumption could be relaxed, and each reaction involved (i.e., high activation energy pyrolysis, heterogeneous oxidation, etc.) could produce different gaseous elemental compositions by defining a new $\eta_{m}$ for each reaction. In this case, $\eta_{m}$ would be a local mass ratio of gas originating from the $m^{\text {th }}$ reaction, defined in such a way as to preserve (if possible) their statistical independence. The instantaneous local gaseous properties would then be obtained from $f, \eta_{1}, \eta_{2}, \ldots, \eta_{m}$. Intermittency of each mixture fraction would need to be properly included as before. Brewster et al. (1988) have formalized a general approach for an arbitrary number of mixture fractions, including intermittency and accounting for fluctuations, assuming mutual independence among the mixture fractions. The added complexity in solving transport equations for each new $\eta_{m}$ and for each new $g_{\eta}$, and then convolving the instantaneous properties over the probability density functions of all mixture fractions would significantly increase computational time and storage. The approximations involved in the statistical model might then nullify any advantage achieved by way of increased flexibility in specification of the coal offgas composition unless $\eta$ fluctuations were ignored.

\section{Particle Phase Reactions}

This section describes the modeling of single, reacting coal particles in pulverized coal combustion and gasification processes. The description includes vaporization, devolatilization, char oxidation, and gas-particle interchange of momentum, heat, and mass. The resulting model describes the response of a coal particle to its thermal, chemical, and physical environment.

Development of an analytical treatment of pulverized coal-char behavior in reacting systems is based largely on independent experimental observations and kinetic parameters deduced from these observations. The description that follows applies to pulverized coal reaction processes, where particles are small $(<150 \mu \mathrm{m})$, and heating rates are high $\left(10^{3}-10^{6} \mathrm{~K} \mathrm{~s}^{-1}\right)$. The treatment is intended for application to pulverized-coal furnaces and entrained-coal gasifiers and would not necessarily apply to fixed or fluidized-bed processes without modification. 
Particle reactions can be described by one of two approaches: 1) simple kinetics models for weight loss or 2) a comprehensive chemical model for devolatilization, particle swelling, and char reactivity (FG-DVC). The latter approach is described in Section?.

\section{Particle Continuity Equations}

In the case of simple weight-loss kinetics, the coal particle (droplet) is considered to consist of four components: Slurry liquid, coal, char, and ash. Ash is defined as that part of the particle (droplet) that is inert. Char is the residue left in the coal particle when the volatile products are released plus that portion of the particle which cannot undergo devolatilization.

The process by which each particle reacts is schematically presented in Fig. 2-3. The continuity equations for the components are listed below for the $j^{\text {th }}$ particle size:

$$
\begin{gathered}
\frac{d \alpha_{w j}}{d t}=-r_{w j} \\
\frac{d \alpha_{c j}}{d t}=r_{c j} \\
\frac{d \alpha_{h j}}{d t}=r_{h j} \\
\frac{d \alpha_{a j}}{d t}=0
\end{gathered}
$$

The slurry liquid vaporizes in accordance with classical expressions.

$$
\text { liquid } \rightarrow \text { vapor }
$$




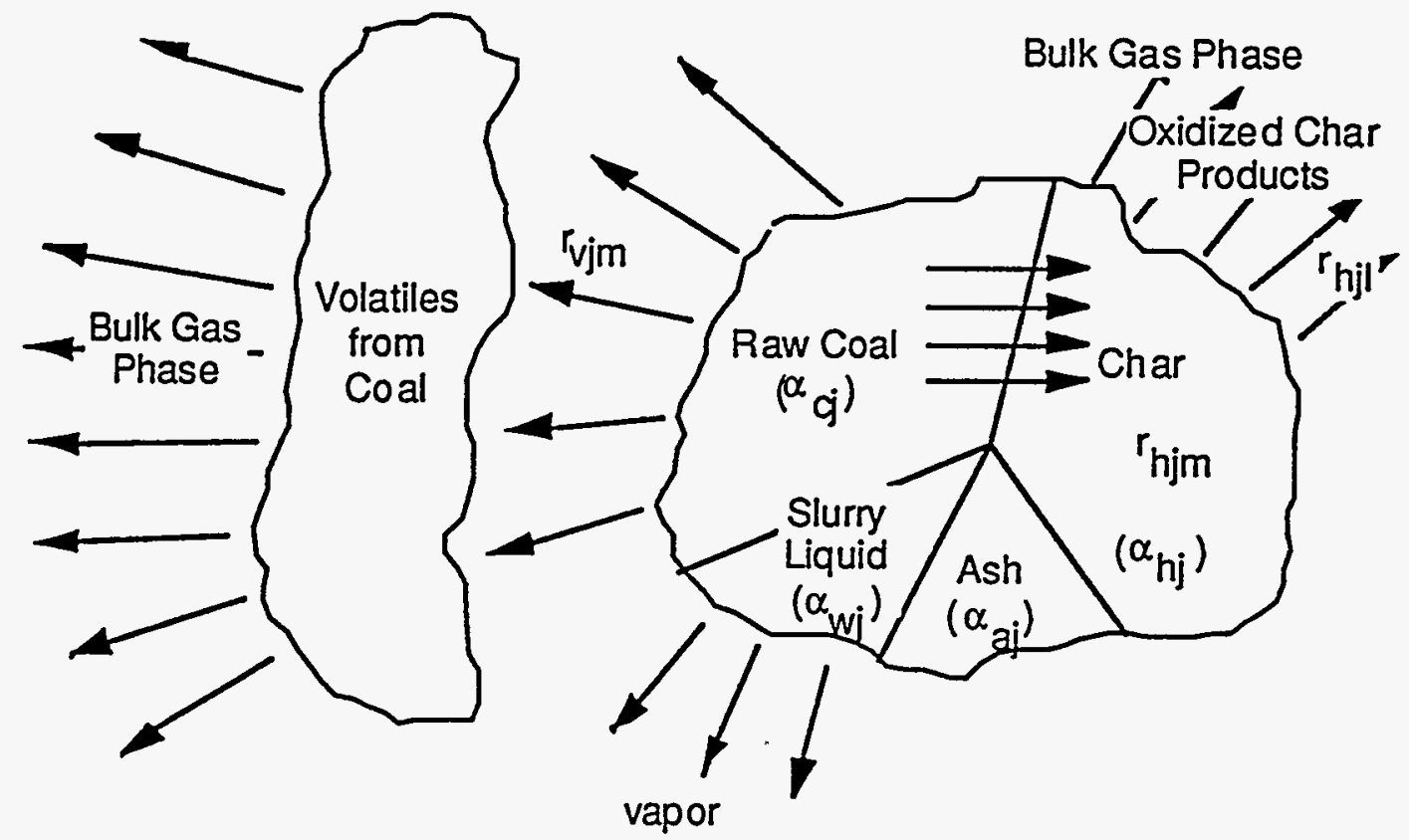

Fig. 2-3. Schematic of coal particle/droplet illustrating components and reaction processes.

The vaporization rate is modeled such that it can be controlled by either heat or mass transfer. In practice, the vaporization rate is nearly always controlled by the heat transfer rate. The mass transfer driving force, i.e. the difference between the vapor concentration at the droplet surface and that in the bulk gas, has a larger impact on the droplet temperature than on the vaporization rate. The fraction of the droplet surface covered with liquid is specified.

The raw coal, or the dry, ash-free portion of the coal, undergoes devolatilization to volatiles and char by one or more reactions ( $M$ in total number) of the form:

$$
(\text { raw coal })_{j} \stackrel{k_{j m}}{\longrightarrow} Y_{j m}(\text { volatiles })+\left(I-Y_{j m}\right)(\text { char })
$$


The volatiles react further in the gas phase. The char reacts heterogeneously, after diffusion of the reactant (i.e. $\mathrm{O}_{2}, \mathrm{CO}_{2}, \mathrm{H}_{2} \mathrm{O}, \mathrm{H}_{2}$ ) to the particle surface, by one or more reacrions ( $\mathrm{L}$ in total number) of the form:

$$
\left.\phi_{\ell}(\text { char })+(\text { oxidizer })_{\ell} \stackrel{k_{\ell}}{\longrightarrow} \text { (gaseous products) }\right)_{\ell}
$$

$\phi_{\ell}$ is the stoichiometric coefficient (moles carbon required per mole of oxidizer). Values of $\phi_{\ell}$ usually assumed for $\mathrm{O}_{2}, \mathrm{CO}_{2}, \mathrm{H}_{2} \mathrm{O}$, and $\mathrm{H}_{2}$, are $1.74,1.0,1.0$, and 0.5 , respectively. Hence, 15 percent $\mathrm{CO}_{2}$ is assumed to form heterogeneously for $\mathrm{O}_{2}$ oxidation, following the recommendations of Mitchell (1988). The oxidation reactions are assumed to be first-order with respect to the oxidizer concentration.

An overview of this coal submodel is presented in the following sections, and further detail is given elsewhere (Smoot and Pratt, 1979; Smith, 1979; Fletcher, 1980, Smoot and Smith, 1985).

\section{Particle Energy Equation}

The Lagrangian equation for particle energy is:

$$
\frac{d\left(\alpha_{j} h_{j}\right)}{d t}=Q_{r j}-Q_{j}-(1-\chi) r_{j} h_{j 8}
$$

where $Q_{r j}$ represents the net radiation to the particle, $Q_{j}$ represents convection and conduction from the particle to the gas phase, $h_{j g}$ is the enthalpy of the coal offgas and accounts for heats of reaction, and $\chi$ is the fraction of the volatile enthalpy that gets directly fed back to the particle from the volatiles combustion. At the present time, $\chi$ is an empirical parameter of the model. The third term on the right represents the net enthalpy lost to the gas by transfer of mass due to particle reaction. The heats of reaction for the heterogeneous reactions are given to the particle. Note that the radiative and convective heat transfer terms have opposite signs. That is, if both are heating the particle, radiation is positive and convection is negative. This is a consequence of sign conventions used in the code.

Physical Properties. This section discusses the equations proposed to obtain the particle and gas phase properties needed for the particle reactions submodel. The gas phase properties discussed are necessary in order to incorporate 
diffusion limitations on particle reaction rates. These properties are not determined from the statistical PDF approach since incorporation of turbulent fluctuating effects on such physical properties is not justifiable. Table 2-4 shows the calculation procedures used for the gas phase properties. These procedures are discussed elsewhere (Smith, 1979; Baxter, 1987) and may be found in standard text books on transport properties (e.g. Bird et al., 1960).

The physical characteristics of the $\mathrm{j}^{\text {th }}$ particle type are calculated as follows: During vaporization, slurry droplets maintain diameters which are essentially equal to their original diameter (Baxter, 1987). Fuel oil droplets decrease in diameter with constant density. The particle is allowed to swell linearly with the extent of devolatilization:

$$
\frac{d_{j}}{d_{j o}}=1+\gamma \frac{\alpha_{c j o}-\alpha_{c j}}{\alpha_{c j o}}
$$

The swelling coefficient $\gamma$ must be specified. This swelling during devolatilization is an experimentally observed phenomenon (Anson et al., 1971). The average particle diameter increase is often on the order of 10 percent for highly volatile bituminous coals (Smoot and Horton, 1978).

Char particles are assumed to burn out according to (Mitchell, 1989)

$$
\frac{d_{j}}{d_{j 0}}=\left(\frac{\alpha_{j}}{\alpha_{j 0}}\right)^{b} ; \frac{\rho_{j}}{\rho_{j 0}}=\left(\frac{\alpha_{j}}{\alpha_{j 0}}\right)^{a}
$$

where $\rho_{j o}, d_{j o}$, and $\alpha_{j o}$ are the density, diameter, and mass, respectively, of the $j^{\text {th }}$ particle size after complete devolatilization (i.e., when $\alpha_{c j}=0$ ). For spherical particles, $a+3 b=1.0$. For constant density burning, $a=0$, and for constant diameter burning, $a=1.0$. For values between 0 and 1 , both the size and diameter of the particle decrease with burnout. Following Mitchell's recommendation, $a$ is normally taken as 0.25 . Constant density burning is recommended for fuel oil combustion. 
TABLE 2-4

\section{FHYSICAL PROPERTIES FOR PARTICLE MODEL}

Description Equation

Gas species conductivity

$$
k_{i}=\left(C_{p i}+\frac{5 R}{4 M_{i}}\right) \mu_{i}
$$

No.

Gas mixture conductivity

$$
k_{g}=\sum_{i} \frac{X_{i} k_{i}}{\sum_{k} X_{k} \phi_{i k}}
$$

Gas species viscosity

$$
\mu_{i}=\frac{2.67 \times 10^{-6}\left(M_{i} T_{8}\right)^{1 / 2}}{\sigma_{i}^{2} \Omega_{\mu}}
$$

Interaction parameter

$$
\phi_{i k}=\frac{1}{2 \sqrt{2}}\left[1+\frac{M_{i}}{M_{k}}\right]^{1 / 2}\left[1+\left(\frac{\mu_{i}}{\mu_{k}}\right)^{1 / 2}\left(M_{k} M_{i}\right)^{1 / 4}\right]^{2}
$$

Gas mixture viscosity

$$
\mu_{g}=\sum_{i} \frac{X_{i} \mu_{i}}{\sum_{k} X_{k} \phi_{i k}}
$$

Species diffusivity

Mixture diffusivity

$$
D_{i k}=\frac{1.83 \times 10^{-12} T_{g}^{3 / 2}\left[\frac{1}{M_{k}}+\frac{1}{M_{i}}\right]^{1 / 2}}{p \sigma_{i k}^{2} \Omega_{d}}
$$

$$
D_{i m}=\frac{1-X_{i}}{\sum_{i \neq k} \frac{X_{k}}{D_{i k}}}
$$

The predicted particle temperature and reaction rates change with changing heat capacity. Particle heat capacity is calculated according to:

$$
C_{p j}=\frac{\alpha_{w j} C_{p w j}+\alpha_{h j} C_{p h j}+\alpha_{c j} C_{p c j}+\alpha_{a j} C_{p a j}}{\alpha_{j}}
$$

Coal and char heat capacity are calculated according to (Merrick, 1983): 


$$
c_{v}=\left(\frac{R}{a}\right)\left[g_{1}\left(\frac{380}{T}\right)-2 g_{1}\left(\frac{1800}{T}\right)\right]
$$

where $R$ is the universal gas constant $(8314.4 \mathrm{~J} / \mathrm{kg}-\mathrm{mol} / \mathrm{K}), a$ is the average atomic weight of the coal or char $(\mathrm{kg} / \mathrm{kg}-\mathrm{mol}), T$ is the temperature $(\mathrm{K})$, and $g_{I}$ is the following function:

$$
g_{1}(z)=\frac{e^{z}}{\left(\frac{e^{z}-1}{z}\right)^{2}}
$$

The average atomic weight of the coal is given by

$$
a=\frac{1}{\sum_{i}^{n} \frac{y_{i}}{\mu_{i}}}
$$

where $y_{i}$ is the mass fraction of element $i, \mu$ is the atomic weight of element $i$, and $n$ is the number of elements in the organic portion of the coal. Merrick compared his heat capacity model with experimental data and found agreement within about 10\% in the temperature range of $273-573 \mathrm{~K}$. The heat capacity of ash is taken as

$$
C_{v}=593.3+0.586 T
$$

where $T$ is in $K$. Predicted particle temperatures can differ by as much at $500 \mathrm{~K}$ depending on the choice of heat capacity models used (Baxter, 1988).

The equilibrium mole fraction of liquid at the temperature, $T$, of the droplet is calculated from the Clapeyron equation and Raoult's law: 


$$
x_{I}=\frac{P\left(T_{l}\right)}{P_{\text {tot }}} \exp \left[\int_{T_{t}}^{T} \frac{\int_{T^{o}}^{T} \Delta C_{p} d T^{\prime \prime}+\Delta h\left(T^{o}\right)}{R T^{\prime 2}} d T^{\prime}\right]
$$

$\Delta C_{p}$ is the difference in the vapor and liquid heat capacities, and $\Delta h\left(T^{\circ}\right)$ is the difference in specific enthalpies at the specified temperature $T^{o}$. All quantities needed for this equation are input variables. An option for an Antoine equation is also included.

The actual mole fraction of the vaporizing liquid at the surface of the droplet is not equal to the equilibrium value but is a function of it. If the droplet temperature is greater than or equal to its boiling temperature, the vaporization rate is given by an equation which does not depend on the surface concentration. The heat of vaporization as a function of temperature is calculated according to

$$
\Delta H_{v}^{T}=\int_{T^{\circ}}^{T} \Delta C_{p} d T^{*}+\Delta h\left(T^{\circ}\right)
$$

Convective and Conductive Heat Transfer. The particle energy is computed from Eqn. 2-97. Auxiliary relationships needed to describe the heat transfer processes are shown in Table 2-5. The temperature of the particle is obtained by integrating Eqn. 2-97 formally with the definition of $C_{p g}$ given in Eqn. 2-107 and $h_{f j}^{o}$ in Eqn. 2-126. The resulting nonlinear equation is solved with a combined bisection-regula falsi technique.

Equation 2-115 of Table 2-5 represents the heat transfer rate for a single particle between the $j^{\text {th }}$ particle and the gas phase. $N u_{j g}$ represents the mean conductive Nusselt number for heat transfer from spheres without the effects of mass transfer. The term enclosed between brackets is the mass transfer correction. The derivation of this term based on classical film theory is discussed elsewhere (Smith, 1979). A reasonable expression for $N u_{j g}$ is given in Eqn. 2-116 (Kreith, 1973). The blowing transpiration parameter for heat transfer $\left(B_{j}\right)$ is given in Eqn. 2-117. The relationship of this parameter to that for mass transfer is also discussed by Smith (1979). The definitions of $R e_{j g}$ and $P r_{g}$ are given in Eqns. 2-118 and 2- 
119. The resulting $Q_{j}$ is required for the particle differential energy equation (Eqn. 2-97).

Equations 2-120 through 2-123 show how the enthalpy of each of the particle constituents is calculated for use in the particle enthalpy calculation (Eqn. 2114). The functional form of the heat capacity correlation depends on input parameters, as discussed earlier. The enthalpy of the volatile matter from each of the $M$ devolatilization reactions is calculated from an energy balance as shown in Eqn. 2-124. The enthalpy of the particle products for use in Eqn. 2-97 is shown in Eqn. 2-125. The first three terms in Eqn. 2-125 represent the enthalpy of coal volatiles, oxidized char, and slurry liquid, respectively, assuming negligible heat of reaction for the devolatilization and char oxidation reactions and negligible heat of vaporization for the slurry liquid. The last three terms (multiplied by $\zeta$ ) represent the heats of reaction for devolatilization and char oxidation and the heat of vaporization of the slurry liquid, respectively, a fraction $(\zeta)$ of which is added to the product gas enthalpy. Since heats of reaction and vaporization are negative when heat is given off (exothermic) $\zeta$ represents the fraction of these heats which is given to the particle phase. When $\zeta$ equals one, the heats of reaction and vaporization are given entirely to the particle phase. When $\zeta$ equals zero, they are given entirely to the gas phase. The proper value of $\zeta$ is thought to be 1.0 .

\section{Particle Reactions}

This section discusses each of the reactions involved in the reaction model of the $j^{\text {th }}$ particle classification. The rate expression for each is presented and the effects of oxidizer diffusion and high rates of mass transfer or particle blowing are incorporated. As presented schematically in Fig. 2-3, the three overall mass loss processes for each particle are vaporization, devolatilization, and heterogeneous reaction. The corresponding equations are shown in Table 2-6. The model allows one vaporization reaction, $M$ devolatilization reactions, and $L$ char oxidation reactions of the form shown in Eqn. 2-96 per particle type. Each of these reaction sets will be discussed in turn. The total reaction rate of the $j^{\text {th }}$ particle classification per unit reactor volume is then the sum of each of these reactions, $(I+L+M)$ in total number, as shown in Eqn. 2-129. 


\section{TABLE 2-5}

CONVECTIVE AND CONDUCTIVE HEAT TRANSFER FOR PARTICLE MODEL

Type Equation

Particle enthalpy

$$
h_{j}=\frac{\alpha_{w j} h_{w j}+\alpha_{c j} h_{c j} \alpha_{h j} h_{h j}+\alpha_{a j} h_{a j}}{\alpha_{j}}
$$

Particle-gas heat transfer

$$
Q_{j}=N u_{j g} \pi\left(\frac{B_{j}}{\exp B_{j}-1}\right) K_{g}\left(T_{j}-T_{g}\right) d_{j}
$$

Nusselt No.

$$
N u_{j g}=2.0+0.65 R e_{j g}^{0.5} \operatorname{Pr}_{g}^{0.333}
$$

Transpiration parameter

$$
B_{j}=\frac{r_{j} C_{p g}}{2 \pi d_{j} k_{g}}
$$

Reynolds No.

$$
R e_{j g}=\frac{d_{j}\left|\vec{v}_{g}-\vec{v}_{j}\right| \rho_{g}}{\mu_{g}}
$$

Prandtl No.

$$
\operatorname{Pr}_{g}=\frac{C_{p g} \mu_{g}}{k_{g}}
$$

Liquid enthalpy

$$
h_{w j}=h_{f w j}^{0}+\int_{298}^{T_{j}} C_{p w j} d T_{j}
$$

Raw coal enthalpy

$$
h_{c j}=h_{f c j}^{0}+\int_{298}^{T_{j}} C_{p c j} d T_{j}
$$

Char enthalpy

$$
h_{h j}=h_{f h j}^{0}+\int_{298}^{T_{j}} C_{p h j} d T_{j}
$$


Ash enthalpy

$$
h_{a j}=h_{f a j}^{o}+\int_{298}^{T_{j}} C_{p a j} d T_{j}
$$

Volatiles enthalpy

$$
h_{v j m}=\frac{h_{c j}-h_{h j}\left(1-Y_{j m}\right)}{Y_{j m}}
$$

Total particle product gas enthalpy

$$
\begin{aligned}
h_{j g}= & \frac{1}{r}\left[\sum_{m} r_{v j m} h_{v j m}+h_{h j} \sum_{l} r_{h j l}+r_{w j} h_{w j}\right. \\
& \left.+\zeta\left(\sum_{m} r_{v j m} \Delta h_{j m}^{r}+\sum_{l} r_{h j l} \Delta h_{j l}^{r}+r_{w j} \Delta h_{w j}^{\nu}\right)\right]
\end{aligned}
$$

Particle heat of formation

$$
h_{f j}^{o}=\frac{\alpha_{w j} h_{f w j}^{o}+\alpha_{h j} h_{f h j}^{o}+\alpha_{c j} h_{f c j}^{o}+\alpha_{a j} h_{f a j}^{o}}{\alpha_{j}}
$$

The equations for the vaporization reactions are shown in Table 2-6. Vaporization is controlled by either heat transfer or mass transfer. The vaporization rate for non-boiling liquid (Eqn. 2-128) is the sum of diffusive and convective components. $K_{w}$ in the diffusion term is the mass transfer coefficient uncorrected for mass transfer. $w$ is the mass transfer correction factor. $S_{f}$ is the fraction of the particle surface area over which evaporation is taking place. The contribution of oxidizer-char reactions to the total mass flux from the particle has been neglected in the correction term because char oxidation is not usually important during slurry liquid vaporization. Slurry droplets often are not predicted to boil unless the local gas phase mass fraction of slurry liquid (e.g. water) reaches a high value (Baxter, 1987). However, the vaporization rate is normally heat-transfer-limited. 
TABLE 2-6

PARTICLE REACTIONS FOR PARTICLE MODEL

Type Equation

Liquid vaporization rate (non-boiling)

$$
r_{w j}=\frac{S_{f} A_{j} k_{j w} \rho_{g}\left(X_{w j s}-X_{w b}\right)+X_{w j s} \sum_{m} S_{f} r_{v j m}}{I-X_{w j s}}
$$

$\begin{aligned} & \text { Liquid vaporization } \\ & \text { rate (boiling) }\end{aligned} r_{w j}=\frac{-Q_{j}+Q_{r j}+r_{c j}\left(h_{j g}-h_{c j}\right) \zeta+r_{h j} \zeta\left(h_{j g}-h_{h j}\right)}{\zeta\left(h_{j g}-h_{w j}\right)}$

Particle reaction rate $r_{j}=\sum_{l} r_{h j l}+\sum_{m} r_{v j m}+r_{w j}$

to gas phase

Net char reaction

$$
r_{h j}=\sum_{m} r_{h j m}-\sum_{l} r_{h j l}
$$

rate

Oxidizer-char reaction rate

$$
r_{h j l}=\frac{A_{j}^{2} M_{h j} M_{g} \phi_{l} k_{c j i} k_{j l} \zeta_{j} C_{o l g} C_{g}}{M_{g} A_{j} C_{g}\left(\zeta_{j} k_{j l}+k_{c j l}\right)+r_{j}}
$$

Kinetic char reaction rate

$$
k_{j l}=A_{j l} T_{j}^{n} \exp \left(\frac{-E_{j l}}{R T_{j}}\right)
$$

coefficient

Transpiration parameters for mass transfer

Mass transfer coefficient

$$
B_{j i}=\frac{r_{j}}{2 \pi D_{i m} \rho_{g} d_{j}}
$$

Total coal reaction rate

$$
\begin{aligned}
& k_{c j i}=\frac{S h_{j g} D_{i m} B_{j i}}{d_{j}\left(\exp B_{j i}-1\right)} \\
& r_{c j}=\sum_{m}-r_{c j m}=-\sum_{m}\left(r_{h j m}+r_{v j m}\right)
\end{aligned}
$$


Theoretical Development

TABLE 2-6 (continued)

Type

Equation

Volatile production

$r_{v j m}=k_{m j} Y_{m j} \alpha_{c j}$

rate

$\begin{aligned} & \text { Char production } \\ & \text { rate }\end{aligned} r_{h j m}=\frac{r_{v j m}\left(1-Y_{m j}\right)}{Y_{m j}}$

$\begin{aligned} & \text { Coal kinetic rate } \\ & \text { coefficient }\end{aligned} \quad k_{m j}=A_{m j} \exp \left(\frac{-E_{m j}}{R T_{j}}\right)$

The kinetic rate of production of volatiles from the $m^{\text {th }}$ reaction is given in Eqn. 2-137, where the reaction rate constant is given in Eqn. 2-138. Diffusional effects only become important when the volatiles repolymerize or condense prior to diffusion into the gas phase. Equation 2-137 is used because of uncertainties in the repolymerization rate coefficients, the nature of the volatiles diffusion process, and because techniques and models used to correlate existing pyrolysis data most often have taken forms like Eqn. 2-137.

The recommended rate constants for devolatilization are shown in Table 27. The two-step model (Ubhayakar et al., 1976) is a reasonable compromise between a simple one-step model (e.g. Godridge and Read, 1976), which would not account for the effects of particle size and pressure, and a more complex, multistep mechanism. The rates of Ubhayakar have been most often used in the past.

In accordance with Eqn. 2-95, the devolatilization of the raw coal can produce char. A simple mass balance results in the rate of formation of char from the $j^{\text {th }}$ particle type from the $m^{\text {th }}$ reaction as shown in Eqn. 2-138. Finally, the net rate of disappearance of the raw coal in the $j^{t h}$ particle class may be obtained by summing over all $M$ devolatilization reactions. The rate of raw coal disappearance is the rate of char plus volatiles formation according to Eqn. 2-95 and as shown in Eqn. 2-136. 
Char is assumed to react heterogeneously with gaseous oxidizers that diffuse to the particle surface from the bulk gas phase. Two rate-limiting steps are considered for this process: 1) gas phase diffusion and 2) heterogeneous reaction. The combined reaction rate is given in Eqn. 2-132. In this form of the equation, the surface reaction has been assumed to be first-order in the surface concentration of the oxidizer The Arrhenius expression for the rate constant is given in Eqn. 2-133.

TABLE 2-7

DEVOLATILZATION RATE EXPRESSIONS AND PARAMETERS

Single-Step Model:

raw coal $\stackrel{k}{\longrightarrow} Y$ (volatiles $)+(I-Y)$ (char)

\begin{tabular}{clccc} 
Rank & Reference & $\mathrm{Y}$ & $\mathrm{A}\left(\mathrm{s}^{-1}\right)$ & $\mathrm{E}(\mathrm{J} / \mathrm{kmol})$ \\
\hline \multirow{2}{*}{ Lignite } & Solomon et al., 1986 & 0.4 & $4.3 \times 10^{14}$ & $2.29 \times 10^{8}$ \\
& Kobayashi et al., & 0.7 & $6.6 \times 10^{4}$ & $1.05 \times 10^{8}$ \\
& 1977 & 0.4 & $2.83 \times 10^{2}$ & $4.64 \times 10^{7}$ \\
& Anthony et al., 1976 & 0.4 & $6.2 \times 10^{3}$ & $4.64 \times 10^{7}$ \\
& Truelove \& & 0.64 & \\
& Jamaluddin, 1986 & & & \\
Bituminous & Solomon et al., 1986 & 0.4 & $4.3 \times 10^{14}$ & $2.29 \times 10^{8}$ \\
& Kobayashi et al., & 0.7 & $6.6 \times 10^{4}$ & $1.05 \times 10^{8}$ \\
& 1977 & & \\
& Anthony et al., 1976 & 0.4 & $7.1 \times 10^{2}$ & $4.94 \times 10^{7}$ \\
& Truelove \& & 0.4 & $2.0 \times 10^{4}$ & $4.94 \times 10^{7}$ \\
& Jamaluddin, 1986 & 0.5 & $4.8 \times 10^{1}$ & $6.27 \times 10^{7}$ \\
& Wiser et al., 1967 & 0.5 & \\
& Badzioch \& & 0.4 & $1.14 \times 10^{5}$ & $7.45 \times 10^{7}$ \\
& Hawksley, 1970 & 0.4 & $3.12 \times 10^{5}$ & $7.45 \times 10^{7}$ \\
& Badzioch \& & & & \\
& Hawksley, 1970 & & &
\end{tabular}


Two-Step Model:

TABLE 2-7 (continued)

raw coal $\left\{\begin{array}{l}\left.\stackrel{k_{1}}{\longrightarrow} Y_{1} \text { (volatiles }\right)+\left(1-Y_{1}\right)(\text { char }) \\ \left.\stackrel{k_{2}}{\longrightarrow} Y_{2} \text { (volatiles }\right)+\left(1-Y_{2}\right)(\text { char })\end{array}\right.$

\begin{tabular}{|c|c|c|c|c|c|}
\hline Rank & Reference & $i$ & $Y_{i}$ & $A\left(\mathrm{~s}^{-1}\right)$ & $E(\mathrm{~J} / \mathrm{kmol})$ \\
\hline Lignite & Kobayashi et al., 1977 & $\begin{array}{l}1 \\
2\end{array}$ & $\begin{array}{l}0.3 \\
1.0\end{array}$ & $\begin{array}{l}2.0 \times 10^{5} \\
1.3 \times 10^{7}\end{array}$ & $\begin{array}{l}1.05 \times 10^{8} \\
1.67 \times 10^{8}\end{array}$ \\
\hline Bituminous & $\begin{array}{l}\text { Kobayashi et al., } 1977 \\
\text { Ubhayakar et al., } 19771\end{array}$ & $\begin{array}{l}1 \\
2 \\
1 \\
2\end{array}$ & $\begin{array}{l}0.3 \\
1.0 \\
0.4 \\
0.8\end{array}$ & $\begin{array}{l}2.0 \times 10^{5} \\
1.3 \times 10^{7} \\
3.7 \times 10^{5} \\
1.5 \times 10^{13}\end{array}$ & $\begin{array}{l}1.05 \times 10^{8} \\
1.67 \times 10^{8} \\
7.36 \times 10^{7} \\
2.51 \times 10^{8}\end{array}$ \\
\hline
\end{tabular}

Values of the rate parameters to use for char reaction with oxygen and carbon dioxide in PCGC-2 are shown in Table 2-8 A sample calculation for $A_{j l}$ is given in Appendix K. Given the lack of reliable data for char reaction with steam and hydrogen, the assumptions of Hobbs et al. (1992) are recommended. They assumed that the steam-char reaction rate is the same as the carbon dioxide reaction rate and that the char gasification rate by hydrogen is three orders of magnitude smaller than the gasification rate by carbon dioxide. Their assumptions were based on the data of Walker et al. (1959), Yoon (1978), Wen et al. (1982), Wen and Chaung (1979), and Blackwood (1959). The mass transfer coefficient, which is also required, must be corrected for particle blowing or high rates of mass transfer. The mass transfer coefficient without blowing may be calculated from an empirical correlation for the Sherwood Number (Sh) (Bird et al., 1960):

$$
S h_{j g}=\frac{k_{j i} d_{j}}{D_{i m}}=2.0+0.6\left(R e_{j g}\right)^{1 / 2}(S c)^{1 / 3}
$$


TABLE 2-8

COEFFICIENTS FOR CHAR REACTION WITH O $\mathrm{O}_{2} \mathrm{AND} \mathrm{CO}_{2}$

$$
k_{j l} A_{j l} T_{j}^{n} \exp \left(\frac{-E_{j l}}{R T_{j}}\right)
$$

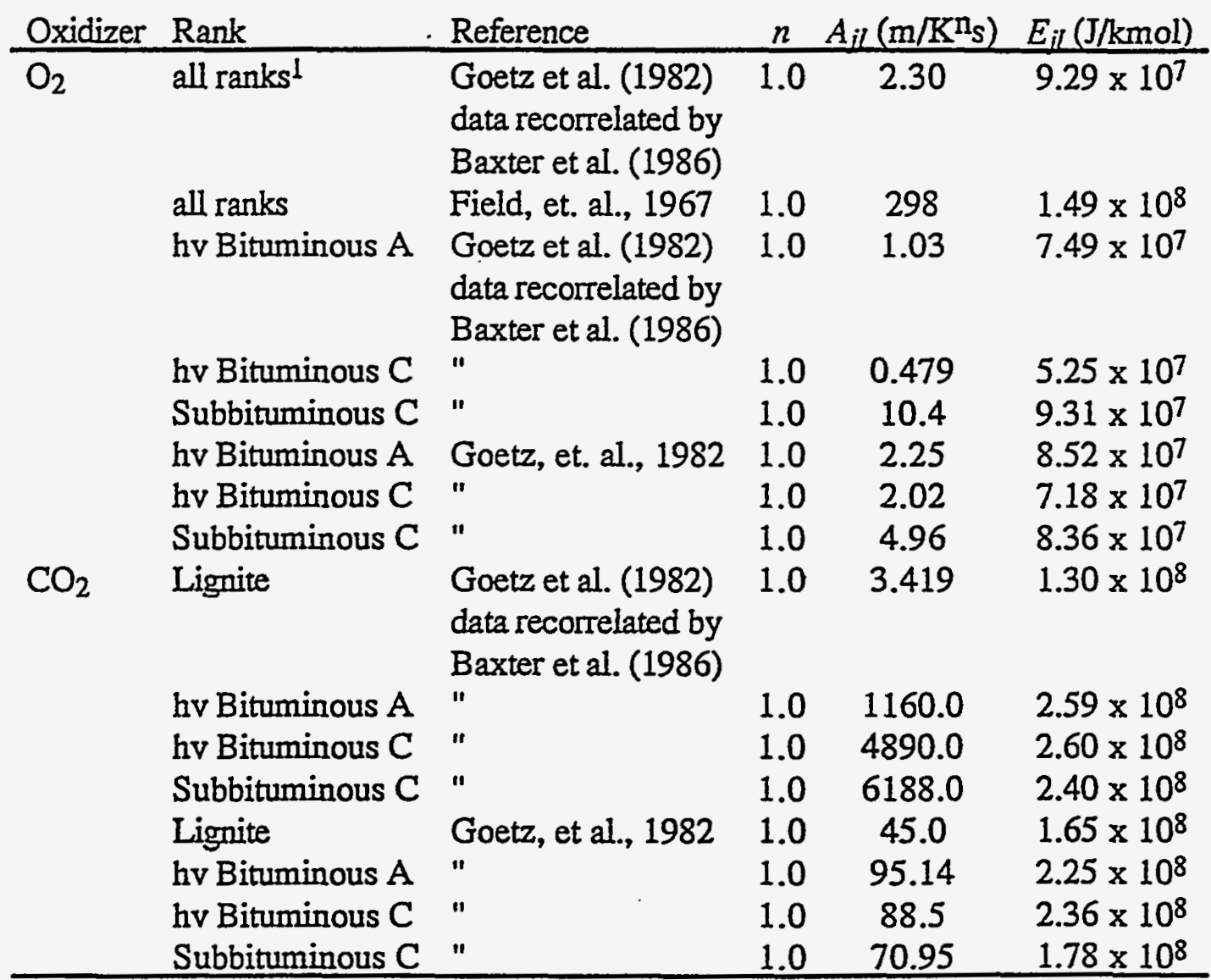

where the Schmidt Number (Sc) is $\frac{\mu_{g}}{\rho_{g} D_{i m}}$, and the Reynolds Number $(R e)$ is based on the difference in velocity between the particle and gas phases. If the Reynolds 
number is zero, this expression reduces to the theoretical value of 2.0 for molecular diffusion. The resulting expression for $k_{c o}$, corrected for particle blowing, is given in Eqn. 2-135. In this case, the blowing parameter is that for mass transfer (Eqn. 2-123).

Since char is being formed by each of the $M$ devolatilization reactions and depleted by each of the $L$ oxidation reactions, the net rate of char reaction is given in Eqn. 2-92. Further, since the heterogeneous reaction is zero order with respect to the char concentration, heterogeneous ignition is permitted. The ignition phenomenon of the coal is not limited to devolatilization. Heterogeneous surface ignition is possible even if devolatilization has not been initiated.

\section{Radiation}

\section{Background}

Radiative transfer becomes increasingly important as the furnace dimensions are increased, and in a typical coal-fired furnace environment, radiation includes contributions from both particulates (coal/char, ash and soot) and the gas phase (mainly $\mathrm{CO}_{2}$ and $\mathrm{H}_{2} \mathrm{O}$ ). The accuracy of the radiation calculation depends on a combination of the accuracy of the calculation method, and the degree of certainty with which the radiative properties of the medium and surrounding walls are known. The radiative properties of the particulates depend on their concentration, size distribution, and complex refractive indices; those of the gases depend on their temperature, partial pressures and the optical thickness of the enclosure. Considerable uncertainties exist, in particular, regarding the size and concentration of soot, and the refractive index of ash. A recent study (Jamaluddin and Smith, 1987) of the sensitivity of predicted radiation fluxes to variations in the inputs indicated that these predictions are most sensitive to the particle concentration and size distribution, while the effect of the absorption and scattering efficiencies of the particles are rather insignificant. As for the gas phase radiation, emissivity data (Sarofim and Hottel, 1978) are known with more certainty than the local variations in gas temperature and species concentrations. 
Radiative heat transfer models of gas- or oil-fired (Field et al., 1967; Bueters et al., 1974), and pulverized coal-fired furnaces (Lowe et al., 1974) have been ieported using zone and flux methods with simple empirical assumptions for flow characteristics, temperatures, heat release rates, and gas composition. Recent radiation modeling has focused on the more efficient but less fundamental flux methods.

The flux methods commonly used are the four-flux type (Gosman and Lockwood, 1973; Lowes et al., 1973; DeMarco and Lockwood, 1975; Varma, 1979). Most of these models suffer from a lack of coupling between the directional fluxes. The discrete ordinates method (an extension of the flux method) corrects this defect, and still offers an equation form which is amenable to numerical methods consistent with the fluid mechanics. The consequences of the lack of effective coupling between the directional fluxes on the predictions of the radiation models has been demonstrated earlier (Jamaluddin and Smith, 1986). PCGC-2 uses either the Varma axisymmetric six-flux method (VFM), or the discrete ordinates method (DOM).

\section{Formulation}

Radiation calculations are based on an energy balance for a beam of radiation passing through a volume element (see Fig. 2-4) containing an absorbing-emittingscattering medium:

$$
\begin{aligned}
& \left(\mu_{m} / r\right)\left[\partial\left(r I_{m}\right) / \partial r\right]-(1 / r)\left[\partial\left(n_{m} I_{m}\right) / \partial \psi\right]+\xi_{m}\left(\partial I_{m} / \partial z\right) \\
& =-\left(k_{a}+k_{s}\right) I_{m}+k_{a} I_{b}+\frac{k_{s}}{4 \pi} \int_{4 \pi} p\left(\Omega, \Omega^{I}\right) I_{m} d \Omega^{\circ}
\end{aligned}
$$



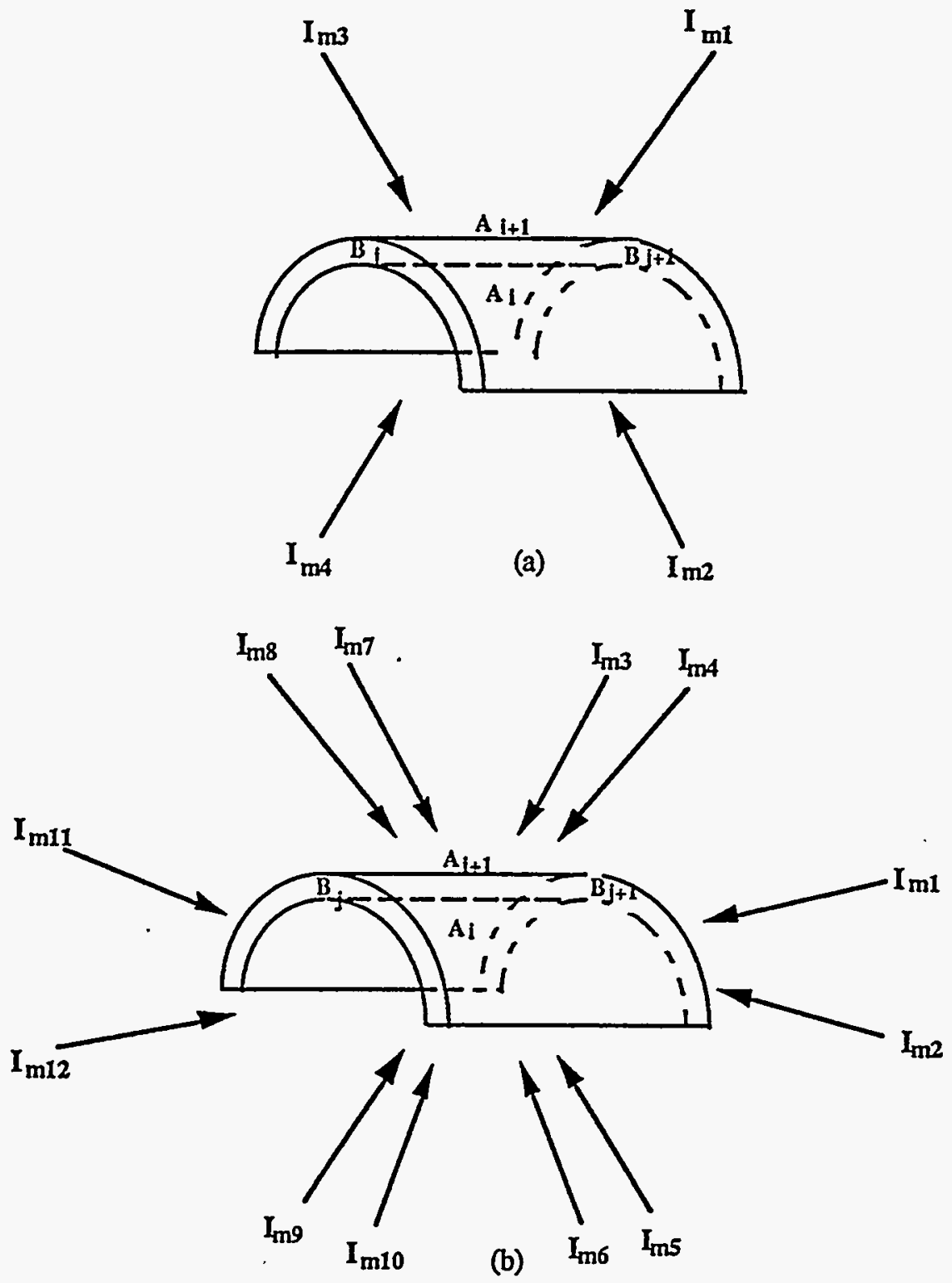

Figure 2-4. Directional intensities reaching a volumelelement. (a) $S_{2}$ (b) $S_{4}$. 
The left side of Eqn. 2-140 represents the gradients of intensity in the direction of propagation, and the right side represents, respectively, the attenuation of intensity due to absorpiion and out-scattering, and the contribution to the direction intensity due to emission by the medium, and in-scattering. $P\left(\Omega, \Omega^{\circ}\right)$ is a probability density function, formally known as the phase function, which determines the distribution of the scattered intensity. Eqn. 2-140 is better known as the radiation transport equation. The quantities $\mu_{m}, \eta_{m}$, and $\xi_{m}$ represent the direction cosines for the direction $\Omega$, and are expressed as (see Fig. 2-5)

$$
\mu_{m}=\sin \theta \cos \psi, \quad \eta_{m}=\sin \theta \sin \psi, \quad \xi_{m}=\cos \theta
$$

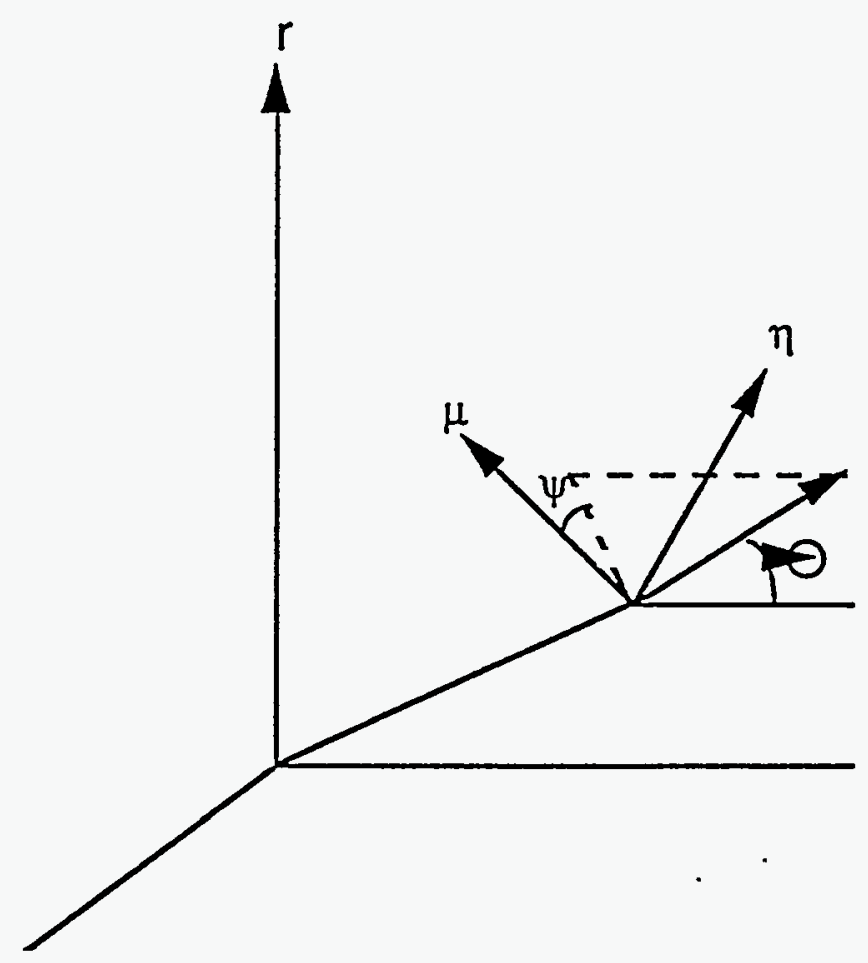

Figure 2-5. The direction cosines. 
The flux methods (including the discrete ordinates method) solve Eqn. 2140 in a number of pre-determined directions. The number of directions, $N_{m}$ depends on the order of the approximation. In discrete ordinates method, this is given by $N_{m}=n(n+2)$ (Carlson and Lathrop, 1968), where $n$ represents the order of the discrete ordinates approximation (the number of values for the direction cosines considered within the range \pm 1.0 ). The angular integral is evaluated using numerical quadrature. Each direction $\Omega_{m}$ is pictured as a point on the surface of a unit sphere with which a surface area $w_{m}$ is associated. $w_{m}$ can be viewed as the angular quadrature weight, with the requirement that

$$
\sum_{m} w_{m}=4 \pi
$$

In the conventional flux methods (e.g., VFM), the quadrature weight is assumed to be uniform for all directions. In the VFM this is assigned a value of $4 \pi / 6$. In the discrete ordinates method, the entire quadrature scheme (i.e., the direction cosines and the weights) is obtained using the "moment matching" technique of Carlson and Lathrop (1968). The quadrature schemes for the $S_{2}$ and $\mathrm{S}_{4}$ discrete ordinates, where radiation transport equation is solved in 4 and 12 directions, respectively, $\left(\mathrm{N}_{\mathrm{m}} / 2\right.$ directions required due to axial symmetry), are reported in Tables 2-9 and 2-10 (Truelove, 1976, 1978). These direction cosines establish a coupling between the directional intensities. In the orthogonal flux methods (like the VFM), the directions chosen to solve the radiative transport equation coincide with the coordinate directions, and therefore the coupling between the directional intensities is lost (for every direction, one of the direction cosines has a value of unity, while the other two are zeroes).

Considering the surrounding surfaces to be diffusely emitting-reflecting, the boundary conditions for the above equation are 


$$
\begin{array}{ll}
\text { at } r=R: & I_{m}=\varepsilon_{w} I_{b w}+\left(1-\varepsilon_{w}\right) \frac{q_{r}^{+}}{\pi} ; \mu_{m}<0 \\
\text { at } r=0: & I_{m}=I_{m^{\prime}} ; \mu_{m^{\prime}}=\mu_{m} \\
\text { at } z=0: & I_{m}=\varepsilon_{w} I_{b w}+\left(1-\varepsilon_{w}\right) \frac{q_{z}^{-}}{\pi} ; \xi_{m}>0 \\
\text { at } z=L: & I_{m}=\varepsilon_{w} I_{b w}+\left(1-\varepsilon_{w}\right) \frac{q_{z}^{+}}{\pi} ; \xi_{m}<0
\end{array}
$$

TABLE 2-9

THE $S_{2}$ QUADRATURE FOR AXISYMMETRIC CYLINDRICAL ENCLOSURES

\begin{tabular}{ccccc}
\hline$m$ & $\mu$ & $\eta$ & $\xi$ & $w_{m}$ \\
\hline 1 & -0.5 & 0.7071 & -0.5 & $\pi$ \\
2 & 0.5 & 0.7071 & -0.5 & $\pi$ \\
3 & -0.5 & 0.7071 & 0.5 & $\pi$ \\
4 & 0.5 & 0.7071 & 0.5 & $\pi$ \\
\hline
\end{tabular}

TABLE 2-10

THE $S_{4}$ QUADRATURE FOR AXISYMMETRIC CYLINDRICAL ENCLOSURES

\begin{tabular}{lrrrl}
\hline$m$ & $\mu$ & $\eta$ & $\xi$ & $w_{m}$ \\
\hline 1 & -0.2959 & 0.2959 & -0.9082 & $\pi / 3$ \\
2 & 0.2959 & 0.2959 & -0.9082 & $\pi / 3$ \\
3 & -0.9082 & 0.2959 & -0.2959 & $\pi / 3$ \\
4 & -0.2959 & 0.9082 & -0.2959 & $\pi / 3$ \\
5 & 0.2959 & 0.9082 & -0.2959 & $\pi / 3$ \\
6 & 0.9082 & 0.2959 & -0.2959 & $\pi / 3$
\end{tabular}


TABLE 2-10 (continued)

\begin{tabular}{rrrrr}
\hline$m$ & $\mu$ & $\eta$ & $\xi$ & $w_{m}$ \\
\hline 7 & -0.9082 & 0.2959 & 0.2959 & $\pi / 3$ \\
8 & 0.2959 & 0.9082 & 0.2959 & $\pi / 3$ \\
9 & 0.2959 & 0.9082 & 0.2959 & $\pi / 3$ \\
10 & 0.9082 & 0.2959 & 0.2959 & $\pi / 3$ \\
11 & -0.2959 & 0.2959 & 0.9820 & $\pi / 3$ \\
12 & 0.2959 & 0.2959 & 0.9820 & $\pi / 3$ \\
\hline
\end{tabular}

with the hemispherical fluxes given by

$$
\begin{aligned}
& q_{r}^{+}=\sum_{m} W_{m} \mu_{m} I_{m} ; \mu_{m}>0 \\
& q_{r}^{-}=\sum_{m} W_{m}\left|\mu_{m}\right|_{m} ; \mu_{m}<0 \\
& q_{z}^{+}=\sum_{m} W_{m} \xi_{m} I_{m} ; \xi_{m}>0 \\
& q_{z}^{-}=\sum_{m} W_{m}\left|\xi_{m}\right|_{m} ; \xi_{m}<0
\end{aligned}
$$

In the VFM, these fluxes are obtained as

$$
\begin{aligned}
& q^{+}=\frac{F+q}{2} \\
& q^{-}=\frac{F-q}{2}
\end{aligned}
$$

the flux-sums and the net fluxes being those from the previous iteration.

Assuming scatter to be linearly anisotropic, the phase function may be represented by 


$$
P\left(\Omega, \Omega^{\prime}\right)=1+a_{0} \cos \phi
$$

where $\phi$ is the angle between the incident and the scattered intensities, and $a_{0}$ is an asymmetry, factor with a value between +1.0 and -1.0 . In terms of the direction cosines, $P\left(\underline{\Omega}, \Omega^{\circ}\right)$ can be written as

$$
P\left(\underline{\Omega}, \Omega^{\prime}\right)=1+a_{0}\left(\mu_{m} \mu_{m^{\prime}}+\xi_{m} \xi_{m^{\prime}}\right)
$$

In the VFM, the directional contributions due to scatter are evaluated as

$$
\begin{aligned}
& \bar{f}=2 \pi \int_{0}^{\pi / 2} P\left(\Omega, \Omega^{\prime}\right) \sin \phi \cos ^{2} \phi d \phi \\
& \bar{b}=2 \pi \int_{\pi / 2}^{\pi} P\left(\Omega, \Omega^{\circ}\right) \cos ^{2} \phi \sin \phi d \phi \\
& \bar{s}=(1-\bar{f}-\bar{b}) / 4
\end{aligned}
$$

where $\bar{f}, \bar{b}$, and $\bar{s}$ are the forward-, backward-, and side-scattered components.

The angular derivative term in Eqn. 2-140 is evaluated using a directdifferencing technique (Carlson and Lathrop (1968)). Using this technique, the second term on the left hand side of Eqn. 2-140 can be re-written as

$$
(1 / r)\left[\partial\left(\eta_{m} I_{m}\right) / \partial \psi\right]=(1 / r)\left[\left(\alpha_{m+1 / 2} I_{m}+1 / 2-\alpha_{m-1 / 2} I_{m}-1 / 2\right)\right] / w_{m}
$$

The direction $m \pm 1 / 2$ define the edges of the angular range of $w_{m}$, the two terms representing, respectively, the flow out of and into the angular range. A direct relationship between $\alpha_{m}$ and $w_{m}$ can be drawn on the basis of isotropic radiation

$$
\alpha_{m+1 / 2}-\alpha_{m-1 / 2}=w_{m} \mu_{m}
$$

The $\alpha^{\prime} s$ are chosen so that the radiatign intensity is only redistributive in the azimuthal direction (i.e., no net transfer). In the Varma method this is achieved by 
letting $d I_{\psi}^{+} / d \psi=d I_{\psi}^{-} / d \psi=0.0$, which eliminates two of the six first-order equations of intensity distribution and reduces them to algebraic form.

\section{Working Equations}

The working equations for DOM and VFM are quite different, and deserve separate discussion.

Discrete Ordinates Method (DOM). Multiplying both sides of Eqn. 2-140 by $2 \pi$ rdrdz, and integrating over the volume element (Fig. 2-4), we obtain

$$
\begin{aligned}
& \mu_{m}\left(A_{i+1} I_{i+1}-A_{i} I_{i}\right)-\left(A_{i+1}-A_{i}\right)\left[\left(\alpha_{m+1 / 2} I_{m+1 / 2}-\alpha_{m-1 / 2} I_{m-1 / 2}\right) / w_{m}\right] \\
&+\xi_{m}\left(B_{j+1} I_{j-1}-B_{j} I_{j}\right)=-\left(k_{a}+k_{s}\right) V_{p} I_{m}+k_{a} V_{p} I_{b} \\
&+\frac{k_{s} V_{p}}{r \pi} \sum_{m}\left[1+a_{o}\left(\mu_{m} \mu_{m^{\prime}}+\xi \xi_{m^{\prime}}\right)\right] w_{m}
\end{aligned}
$$

The intensities $I_{i+1}, I_{j+1}$ and $I_{m+1 / 2}$ can be expressed in terms of the discrete intensities $I_{i}, I_{j}$ and $I_{m-1 / 2}$ using central differencing:

$$
I_{i}+I_{i+1}=I_{j}+I_{j+1}=I_{m-1 / 2}+I_{m+1 / 2}=2 I_{m}
$$

$I_{m}$ intensity at the center of the volume element can therefore be evaluated as

$$
I_{m}=\frac{\mu_{m} A I_{i}+\beta_{m} I_{m-1 / 2}+\xi_{m} B I_{j}+V_{p}\left(k_{a} I_{b}+k_{s} I_{s}\right)}{\mu_{m} A-\beta_{m}+\xi_{m} B+V_{p}\left(k_{a}+k_{s}\right)}
$$

where 


$$
\begin{aligned}
A & =A_{i}+A_{i+1} \\
B & =B_{j}+B_{j+1} \\
B_{m} & =-\left(\alpha_{m+1 / 2}+\alpha_{m-1 / 2}\right)\left(A_{i+1}-A_{i}\right) / w_{m} \\
I_{s} & =\sum_{m} w_{m} I_{m}\left[\frac{1+a_{o}\left(\mu_{m} \mu_{m^{\circ}}+\xi_{m} \xi_{m^{\circ}}\right)}{4 \pi}\right]
\end{aligned}
$$

Eqn. 2-151 is strictly applicable to the case where both $\mu_{m}$ and $\xi_{m}$ are positive. For other combinations of $\mu_{m}$ and $\xi_{m}$, the equation changes.

Varma Six-Flux Method. The four first-order equations for the $r-$ and $x-$ directed intensities are combined into two second-order equations following the approach of Gosman and Lockwood (1973). The radiation flux sum and the net radiation are defined as

$$
\begin{aligned}
& F=q^{+}+q^{-} \\
& q=q^{+}-q^{-}
\end{aligned}
$$

Combining each pair of radiation flux equations results in an expression of the following form (for the $x$-component):

$$
q_{x}=-\Gamma_{x}\left(d F_{x} / d x\right)
$$

where

$$
\Gamma_{x}=\left[k_{a}+k_{s}(1-\bar{b}-\bar{f})\right]^{-1}
$$

Adding each pair, and combining with Eqn. 2-156, the following equation for the $x$-component results

$$
-\frac{d}{d x}\left(\Gamma_{x} \frac{d F_{x}}{d x}\right)=\left(c_{1}+c_{2}\right) F_{x}+2 c_{3} F_{r}+2 c_{4} E_{b}
$$


The corresponding equations for the $r$ and $\psi$ components are

$$
\begin{gathered}
\frac{1}{r} \frac{d}{d x}\left(r F_{r}\right)=\left(c_{1}+c_{2}\right) F_{r}+2 c_{3} F_{x}+2 c_{4} E_{b} \\
F_{\theta}=2 c_{5}\left(F_{x}+F_{r}\right)+2 c_{6} E_{b}
\end{gathered}
$$

The necessary coefficients are defined in Table 2-11. The extinction coefficient and the albedo of scatter are given by

$$
\begin{gathered}
k_{t}=k_{a}+k_{s} \\
\omega_{o}=\frac{k_{s}}{k_{t}}
\end{gathered}
$$

TABLE 2-11

COEFFICIENTS FOR FLUX SUMS

$$
\begin{gathered}
c_{1}=-k_{t}\left(I-\omega_{o} f\right)+k_{t}\left(\frac{2 \omega_{o}^{2} s^{2}}{1-\omega_{o} f-\omega_{o} b}\right) \\
c_{2}=k_{t} \omega_{o} b+k_{t}\left(\frac{2 \omega_{o}^{2} s^{2}}{1-\omega_{o} f-\omega_{o} b}\right) \\
c_{3}=k_{t} \omega_{o} s+k_{t}\left(\frac{2 \omega_{o}^{2} s^{2}}{1-\omega_{o} f-\omega_{o} b}\right)
\end{gathered}
$$


TABLE 2-11 (continued)

$$
\begin{gathered}
c_{4}=k_{t}\left(\frac{1-\omega_{0}}{6}\right)\left(1+\frac{2 \omega_{o} s}{1-\omega_{o} f-\omega_{o} b}\right) \\
c_{5}=\frac{\omega_{o} s}{1-\omega_{o} f-\omega_{o} b} \\
c_{6}=\frac{1-\omega_{o}}{6\left(1-\omega_{o} f-\omega_{o} b\right)}
\end{gathered}
$$

\section{Radiative Properties}

To carry out the above calculations, information is needed on the absorption and scattering cross-sections for the particulates, as well as the scattering phase functions. These can be calculated using Mie theory (Mie, 1908) if the particle size, the wavelength of the radiation, and the complex refractive index of the particle are known. Sample results from such calculations, using a published program (Dave, 1968), are presented in Fig. 2-6 for size parameters $(\pi d j / \lambda)$ in the range of 0-100. In this figure, the refractive index of char is taken as $1.93(1.0-0.53 i)$ at a wavelength of $2 \mu \mathrm{m}$.

Optical properties of coal are not well characterized (Solomon et al., 1986; Brewster and Kunitomo, 1984; Baxter et al., 1988). The absorption and scattering efficiencies depend strongly on these properties. Once the absorption and scattering efficiencies are known from Mie theory, the absorption and scattering coefficients of the particulates for a cell can be evaluated as

$$
k_{a p, c e l l}=\sum_{i} \sum_{j} k_{a i j, c e l l}=(\pi / 4) \sum_{i} \sum_{j} \dot{n}_{i j} \int_{c e l l} Q_{a j} d_{j}^{2} d t
$$




$$
k_{s p, c e l l}=\sum_{i} \sum_{j} k_{s i j, c e l l}=(\pi / 4) \sum_{i} \sum_{j} \dot{n}_{i j} \int_{c e l l} Q_{s j} d_{j}^{2} d t
$$

where $t$ is the time along a Lagrangian trajectory, and $\dot{n}_{i j}$ is the particle number flowrate for the $i^{\text {th }}$ starting location and $j^{\text {th }}$ particle size. The scattering efficiency plotted in Fig. 2-6 includes a contribution of 1.0 due to diffraction which must be discounted in heat transfer calculations (Varma, 1979). This makes the value of $Q_{s}$ used in Eqn. 2-163 equal to 1.0 less than that plotted in Fig. 2-6. Soot particles absorb, but do not scatter radiation significantly due to their microscopic size. Soot radiation is considered below. data:

The absorption coefficients for the gases are evaluated from gas emissivity

$$
k_{a g}=\left(1 / L_{e}\right)\left[\ln \left(I-\varepsilon_{g}\right)\right]
$$

where

$$
\varepsilon_{g}=C_{\mathrm{CO}_{2}} \varepsilon_{\mathrm{CO}_{2}}+C_{\mathrm{H}_{2} \mathrm{O}} \varepsilon_{\mathrm{H}_{2} \mathrm{O}}-\Delta \varepsilon
$$

and the mean (effective) beam length (a single overall value is used in PCGC-2) is given by

$$
L_{e}=3.5\left(V_{F} / A_{F}\right)
$$

$C_{\mathrm{CO}_{2} \text { and }} C_{\mathrm{H}_{2} \mathrm{O}}$ represent the pressure corrections to the gas emissivities, and $\Delta \varepsilon$ represents the spectral overlap correction. The gas emissivity data are taken from Hottel and Sarofim (1967) and Siegel and Howell (1981). The overall absorption coefficient for a cell can therefore be obtained as

$$
k_{a, \text { cell }}=k_{a p, \text { cell }}+k_{a g, \text { cell }}
$$

and the total heat absorption by the cell as

$$
q_{r, \text { cell }}=k_{a, \text { cell }}\left(F_{x}+F_{r}+F \psi-E_{b}\right)_{\text {cell }}
$$




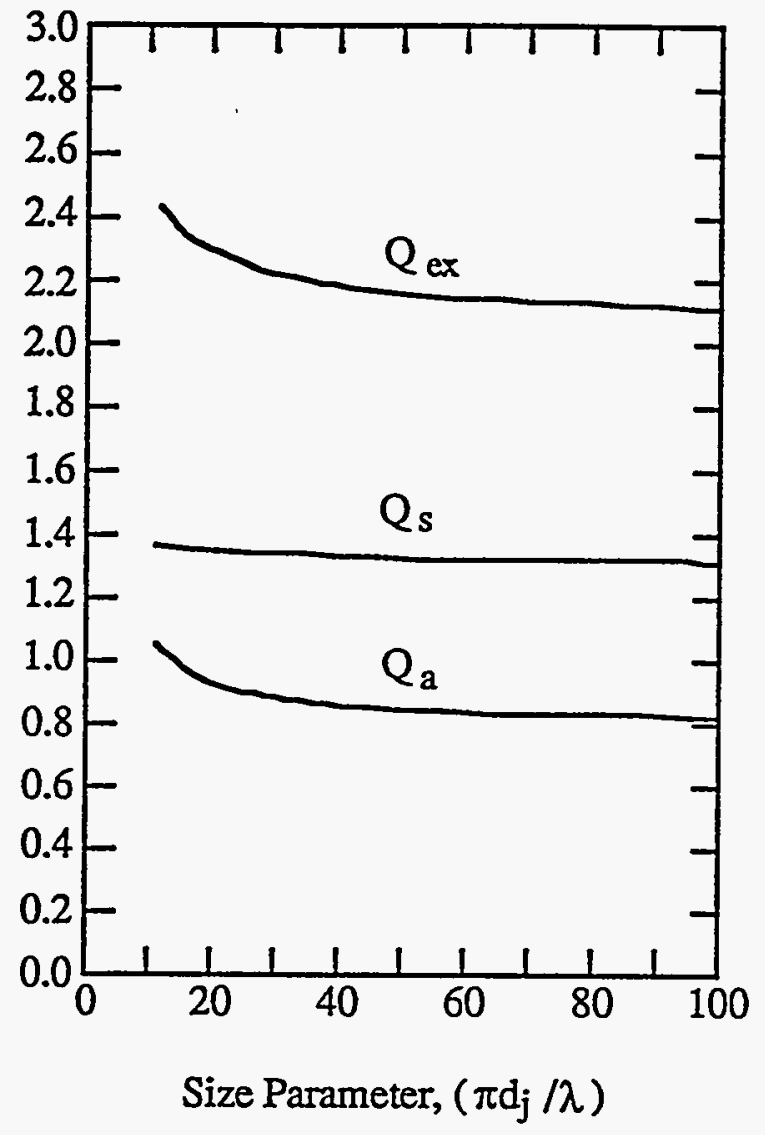

Fig. 2-6. The absorption, scattering, and extinction efficiencies of char particles at wavelength of $2 \mu \mathrm{m}$ and refractive index of 1.93 [1-i(0.53)].

out of which a fraction $q_{r g}=\left(k_{a g} / k_{a}\right) q_{r}$ is absorbed by the gas phase, and the remainder $q_{r p}=\left(k_{a p} / k_{a}\right) q_{r}$ is absorbed by the particle phase within the cell. The radiation transfer rate to the Lagrangian particles is given by 


$$
Q_{r j}=\frac{\pi d_{j}^{2}}{4} Q_{a j}\left(F_{x}+F_{r}+F_{\psi}-E_{b}\right)
$$

The blackbody emissive power, $E_{b}$, is given by (Siegel and Howell, 1981, p. 36)

$$
E_{b}=4 \sigma T_{g}^{A}
$$

where $T_{g}$ is the local gas temperature (Siegel and Howell, 1981, p.36).

\section{Soot Radiation}

When soot radiation is included, Eqn. 2-153 becomes

$$
I_{m}=\frac{\mu_{m} A I_{i}+\beta_{m} I_{m-1 / 2}+\xi_{m} B I_{j}+V_{p}\left(k_{a} I_{b}+k_{s} I_{s}+k_{\text {soot em }} I_{b}\right)}{\mu_{m} A-\beta_{m}+\xi_{m} B+V_{p}\left(k_{a}+k_{s}+k_{\text {soot abs }}\right)}
$$

The values of $k_{\text {soot } \mathrm{em}}$ and $k_{\text {soot abs }}$ are given by (Kent and Honnery, 1990)

$$
k_{\text {soot } \mathrm{cm}}=1.9 \times 10^{3} f_{v} T_{e}\left(m^{-1}\right)
$$

and

$$
k_{\text {soot abs }}=1.9 \times 10^{3} f_{v} T_{b}\left(m^{-1}\right)
$$

where $f_{\nu}$ is the volume fraction of soot, and the volume of soot (which does not include entrained gas) $=$ (moles of soot) $\times 12$ grams per mole/soot density. The Favre-averaged mole fraction of solid, condensed carbon is taken as the mean soot concentration since there is currently no kinetics-based soot formation and decay model in PCGC-2. With this assumption, the model will probably underpredict the contribution due to soot radiation, since soot formation kinetics are fairly rapid, but decay is fairly slow. These values are for small soot particles and are averaged over wavelength and temperature. $T_{e}$ is the local gas temperature in Kelvins, and $T_{b}$ is the effective blackbody temperature of the radiation incident on the local volume.

The correction due to spectral overlap of soot and gas radiation is given by (Hottel and Sarofim, 1967) 


$$
\Delta \varepsilon=\sum_{i} \varepsilon_{i}-\left[1-\Pi_{i}\left(1-\varepsilon_{i}\right)\right]
$$

With a three-component model for continuum radiation (soot, $\mathrm{H}_{2} \mathrm{O}$, and $\mathrm{CO}_{2}$ ), the correction becomes

$$
\Delta \varepsilon=\varepsilon_{\mathrm{H}_{2} \mathrm{O}} c_{\mathrm{H}_{2} \mathrm{O}}+\varepsilon_{\mathrm{CO}_{2}} c_{\mathrm{CO}_{2}}+\varepsilon_{\text {soot }}-\left[1-\left(1-\varepsilon_{\mathrm{H}_{2} \mathrm{O}} c_{\mathrm{H}_{2} \mathrm{O}}\right)\left(1-\varepsilon_{\mathrm{CO}_{2}} c_{\mathrm{CO}_{2}}\right)\left(1-\varepsilon_{\text {soot }}\right)\right]
$$

With the correction due to the overlap of $\mathrm{CO}_{2}$ and $\mathrm{H}_{2} \mathrm{O}$ lines already included, the additional correction simplifies to two pairwise corrections, for soot- $\mathrm{CO}_{2}$ and soot$\mathrm{H}_{2} \mathrm{O}$ :

$$
\Delta \varepsilon_{\text {soot, } \mathrm{CO}_{2}}=\varepsilon_{\text {soot }} \times \varepsilon_{\mathrm{CO}_{2}}
$$

and

$$
\Delta \varepsilon_{\text {soot }, H_{2} \mathrm{O}}=\varepsilon_{\text {soot }} \times \varepsilon_{\mathrm{H}_{2} \mathrm{O}}
$$

However, Eqn. 2-174 only applies if all but one of the species is a grey-body radiator; not the case for soot. The correct calculation for the correction term is shown below. Equations 2-176 and 2-177 calculate the correction to within 4 percent for all temperatures and concentrations of interest. 


\section{Background}

The development of an effective model to predict nitrogen pollutant formation during pulverized coal combustion requires an adequate description of nitrogen conversion from coal, char, and soot to volatile gaseous nitrogen species and subsequent homogeneous and heterogeneous reactions among the nitrogen, fuel, and oxidizer species. The kinetic mechanism must be limited to sufficiently few homogeneous reactions to allow for coupling with the turbulent mixing process. The approach used for calculating each reaction rate is dependent on the relative time scales of reaction and turbulent fluctuation.

\section{Reaction Mechanisms}

Two simplified fuel-NO mechanisms are available for predicting the conversion of coal-bound nitrogen to $\mathrm{HCN}, \mathrm{NH}_{3}, \mathrm{NO}$ and $\mathrm{N}_{2}$ as illustrated by the schematics in Figure 2-7. Either mechanism can be coupled with the Zel'dovich mechanism. One mechanism is an extension of the $\mathrm{NO}_{\mathrm{x}}$ model in previous versions of PCGC-2. It allows volatile coal and char nitrogen to be partitioned between $\mathrm{HCN}$ and $\mathrm{NH}_{3}$ (see Figure 2-7a). The second mechanism (see Figure 2-7b) is based on the general concept that HCN decays to $\mathrm{NH}_{3}$ which is subsequently oxidized to NO or competitively reduced to $\mathrm{N}_{2}$. The Zel'dovich mechanism is used to predict thermal NO formation. Thermal NO formation can also be predicted for gaseous fuel cases. Joint or separate prediction of fuel NO and thermal NO can be made for coalcontaining cases. 
a) Expanded mechanism

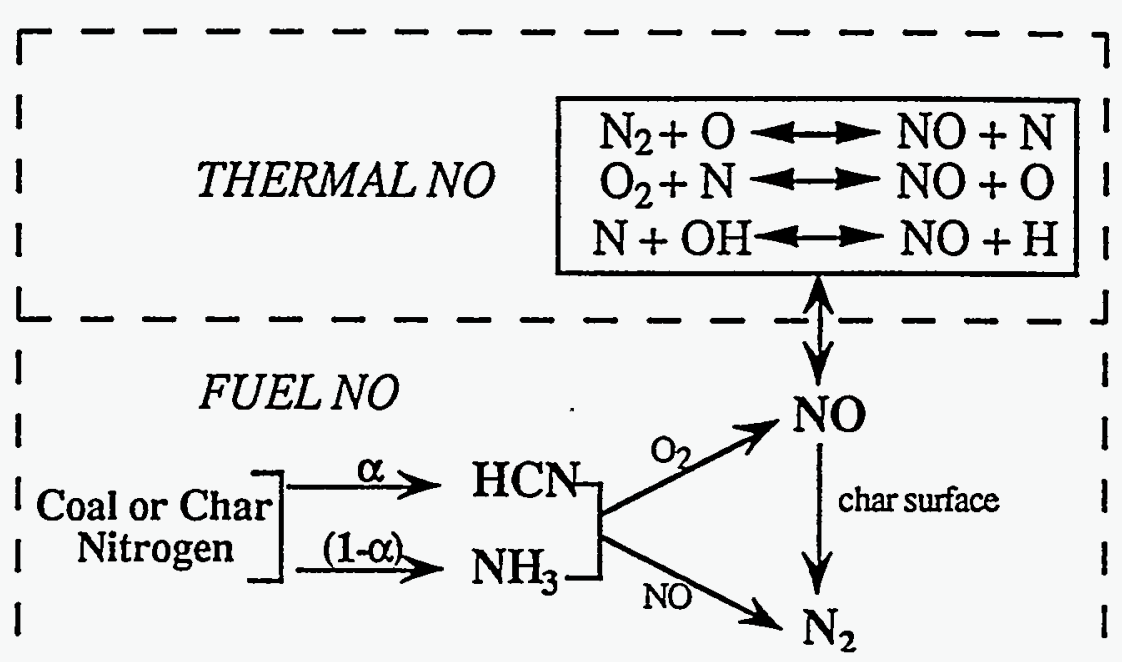

b) Alternative mechanism

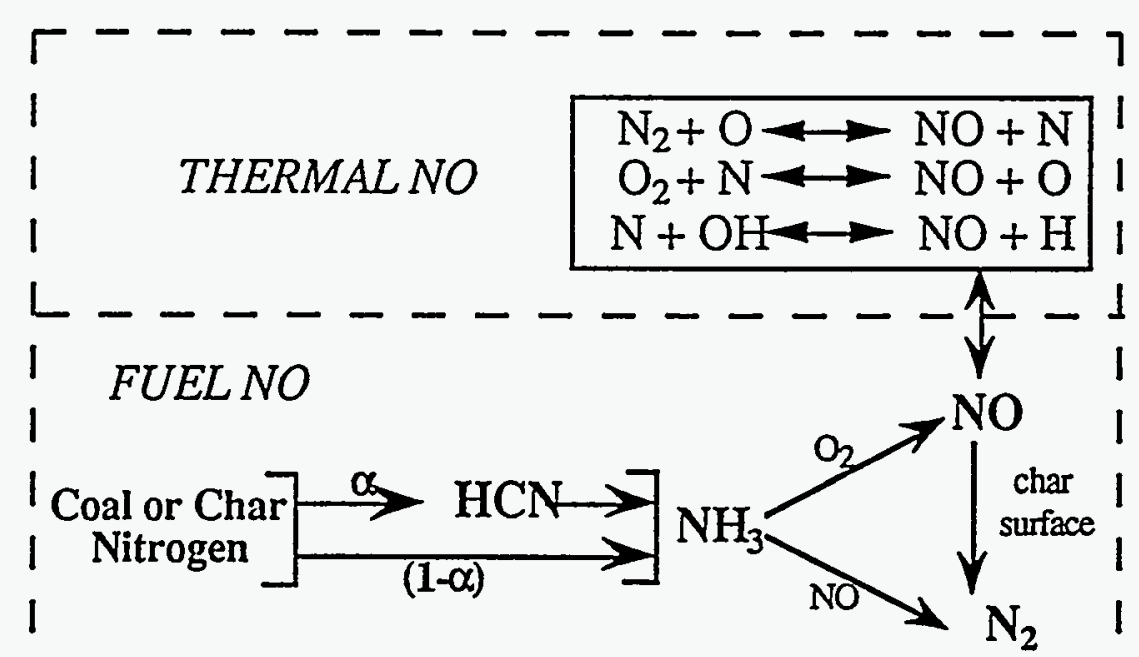

Figure 2-7. Kinetic mechanisms for prediction of fuel and thermal NO. 
Prompt NO formation occurs in coal flames, but since coal generally contains 1-2\% nitrogen, prompt NO is likely to be overshadowed by fuel NO. It is estimated that prompt NO typically accounts for less than $5 \%$ of the total NO formed. However, prompt NO formation may be significant during gaseous combustion. Reactions describing the interaction of nitrogen species with fuel fragments, called "reburning" reactions, may be important in fuel-rich flame conditions. Neither prompt NO nor reburning reactions are currently modeled in this version of the $\mathrm{NO}_{\mathrm{x}}$ submodel.

The global rate expressions reported by de Soëte (1975) and Bose et al., (1988) and the empirical rate correlations by Mitchell and Tarbell (1982) appear to be the best available rates for predicting the homogeneous fuel NO mechanism steps. The user has the flexibility of selecting the rate expressions to fit the alternative fuel NO mechanisms. The rate parameters for the thermal NO reactions were taken from Bowman (1975) and Miller and Bowman (1989). An empirical rate expression is used to predict char/NO reaction. Table 2-11 documents the rate parameters for the individual reaction steps.

It is generally agreed that the reaction network for thermal NO is correctly described by the modified Zel'dovich mechanism based on research originally carried out by Zel'dovich (1947).

$$
\begin{aligned}
& \mathrm{N}_{2}+\mathrm{O} \leftrightarrow \mathrm{NO}+\mathrm{N} \\
& \mathrm{N}+\mathrm{O}_{2} \leftrightarrow \mathrm{NO}+\mathrm{O}
\end{aligned}
$$

In fuel-rich environments (stoichiometric ratios of air to fuel $<1.0$ ), at least one additional step should be included in this mechanism (Lavoie et al., 1970).

$$
\mathrm{N}+\mathrm{OH} \leftrightarrow \mathrm{NO}+\mathrm{H}
$$



(1971)

The following thermal NO rate expression is derived by Westenberg,

$$
\frac{d[N O]}{d t}=2[O]\left\{\frac{k_{A 6}\left[N_{2}\right]-\frac{k_{-A 6} k_{-A 7}[N O]^{2}}{k_{A 7}\left[O_{2}\right]}}{1+\frac{k_{-A 6}[N O]}{k_{A 7}\left[O_{2}\right]+k_{A \delta}[O H]}}\right\} \quad \text { gmole } \mathrm{cm}^{-3} \mathrm{~s}^{-1}
$$

For most lean flames, the reaction in Eqn. 2-180 may be neglected. In early stages of the flame, NO concentrations are very low; thus, the reverse reactions for Reactions in eqns. 2-178 and 2-179 are negligible. This yields a simpler thermal NO rate expression when the "steady state assumption" for atomic nitrogen is also invoked.

$$
\frac{d[N O]}{d t}=2 k_{A 6}[O]\left[N_{2}\right] \quad \text { gmole } \mathrm{cm}^{-3} \mathrm{~s}^{-1}
$$

Both eqns. 2-181 and 2-182 have been made available in the $\mathrm{NO}_{\mathrm{x}}$ submodel. Both expressions are coupled to the combustion kinetics through competition for the oxygen atom. In fuel lean, secondary combustion zones, where $\mathrm{CO}$ is oxidized to $\mathrm{CO}_{2}, \mathrm{O}$ is often assumed to be only in equilibrium with $\mathrm{O}_{2}$.

$$
\begin{gathered}
\mathrm{O}_{2}+\mathrm{M} \leftrightarrow 2 \mathrm{O}+\mathrm{M} \\
{[\mathrm{O}]=\left\{K_{\text {eq }}^{\prime}\left[\mathrm{O}_{2}\right]\right\}^{1 / 2}}
\end{gathered}
$$

Another approach, which accounts for $[\mathrm{O}]$ and $[\mathrm{OH}]$ in excess of their equilibrium values, is assumed to be valid in regions where hydrocarbons are consumed (Iverach et al., 1973; Sarofim and Pohl, 1973; and Thompson et al., 1981). 


$$
[\mathrm{O}]=K_{e q} \frac{\left[\mathrm{O}_{2}\right][\mathrm{CO}]}{\left[\mathrm{CO}_{2}\right]}
$$
submodel.

Both options for calculating $\mathrm{O}$ concentrations have been included in the $\mathrm{NO}_{\mathrm{x}}$

When the global rate expressions of Wendt and coworkers are selected to predict fuel NO formation, $\mathrm{OH}$ concentrations must be estimated for the HCN oxidation reaction (see Table 2-12). Two options are available in the $\mathrm{NO}_{\mathrm{x}}$ submodel. The default option is to use the gaseous equilibrium $\mathrm{OH}$ concentrations calculated by PCGC-2. The second option is to predict the deviation from equilibrium using a empirical expression. Wendt et al. (1989) presented an equation for estimating the "overshoot" in radical $\mathrm{OH}$ concentration as a function of temperature for their reactor system and conditions.

$$
O H=O H_{e q}\left[1.1 \times 10^{-4} \exp \left(\frac{15469}{T}\right)\right]
$$

This expression is included in the revised $\mathrm{NO}_{\mathrm{x}}$ submodel and can be used to adjust $\mathrm{OH}$ concentrations. When experimental $\mathrm{OH}$ data are available for a particular flame condition, the temperature-dependent constants can be changed.

TABLE 2-12

REACTION EXPRESSION ALTERNATTVES FOR THE GENERALIZED NITRIC OXIDE MODEL

\begin{tabular}{lcccc}
\hline & Thermal NO Mechanism Reactions \\
& \multicolumn{4}{c}{$k=A T^{\beta}$ exp $(-E / R T)$} \\
\hline Reaction Expression & Concentration & $A$ & $\beta$ & $E(\mathrm{~J} / \mathrm{gmole})$ \\
$\mathrm{O}+\mathrm{N}_{2} \rightarrow \mathrm{NO}+\mathrm{N}$ & $\mathrm{X}_{\mathrm{N}_{2}} \mathrm{X}_{\mathrm{O}}$ & $1.36 \times 10^{14}$ & 0 & 315,900 \\
$\mathrm{~N}+\mathrm{NO} \rightarrow \mathrm{N}_{2}+\mathrm{O}$ & $\mathrm{X}_{\mathrm{NO}} \mathrm{X}_{\mathrm{N}}$ & $3.27 \times 10^{12}$ & 0.300 & - \\
$\mathrm{N}+\mathrm{O}_{2} \rightarrow \mathrm{NO}+\mathrm{O}$ & $\mathrm{X}_{\mathrm{O}_{2}} \mathrm{X}_{\mathrm{N}}$ & $6.40 \times 10^{9}$ & 1.000 & 26,300 \\
$\mathrm{O}+\mathrm{NO} \rightarrow \mathrm{O}_{2}+\mathrm{N}$ & $\mathrm{X}_{\mathrm{NO}} \mathrm{X}_{\mathrm{O}}$ & $1.50 \times 10^{9}$ & 1.000 & 162,100 \\
$\mathrm{~N}+\mathrm{OH} \rightarrow \mathrm{NO}+\mathrm{H}$ & $\mathrm{X}_{\mathrm{OH}} \mathrm{X}_{\mathrm{N}}$ & $3.80 \times 10^{13}$ & 0 & - \\
$\mathrm{H}+\mathrm{NO} \rightarrow \mathrm{OH}+\mathrm{N}$ & $\mathrm{X}_{\mathrm{NO}} \mathrm{X}_{\mathrm{H}}$ & $2.00 \times 10^{14}$ & 0 & 196,600
\end{tabular}


TABLE 2-12 (continued)

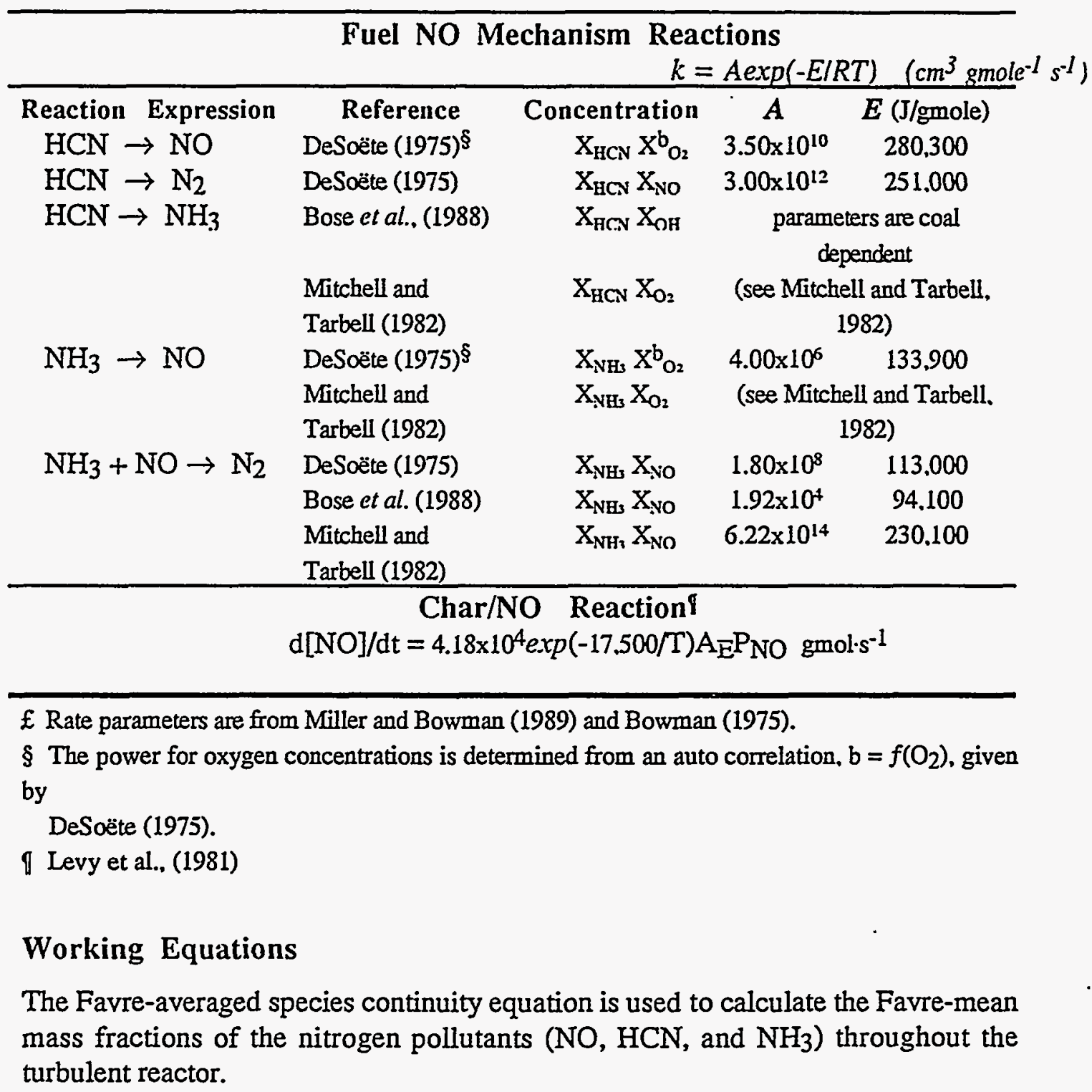




$$
\bar{W}_{i}=\bar{\rho} \bar{u}\left(\frac{\partial \bar{Y}_{i}}{\partial x}\right)+\bar{\rho} \bar{v}\left(\frac{\partial \bar{Y}_{i}}{\partial r}\right)-\left(\frac{\partial}{\partial x}\right)\left(\bar{D}_{Y} \frac{\partial \bar{Y}_{i}}{\partial x}\right)-\frac{1}{r}\left(\frac{\partial}{\partial r}\right)\left(r \bar{D}_{Y} \frac{\partial \bar{Y}_{i}}{\partial r}\right)
$$

Here, $\overline{\mathrm{W}}_{\mathrm{i}}$ is the overall mean chemical reaction source or sink term calculated by summing all individual (global and elementary-step) mean reaction rates, $\bar{\Phi}_{j}$, involving formation or destruction of species $i$ by the $j$ th reaction.

$$
\bar{W}_{i}=\sum_{j} \bar{\omega}\left(X_{i}, X_{k}, T\right)_{j}
$$

Smith et al. (1982) assumed that $\omega_{j}$ for turbulent reacting gases could be approximated by convolving the instantaneous rates over the random fluctuations of the two mixture fraction progress variables $f$ and $\eta$.

$$
\bar{\omega}_{\mathrm{i}}=\bar{\rho} \int_{f} \int_{\eta}\left(\omega_{\mathrm{i}}(f, \eta, \mathrm{h}) / \rho(f, \eta, \mathrm{h})\right) \overline{\mathrm{P}}(f) \overline{\mathrm{P}}(\eta) \mathrm{d} \eta \mathrm{d} f
$$

In order to jointly integrate the chemical kinetics and turbulent fluctuations, local instantaneous species concentrations must be known for every probable state of "mixedness". The overall fractional conversion of each species is tracked in the revised $\mathrm{NO}_{\mathrm{x}}$ submodel. Individual species are bounded between their maximum and minimum values, depending on their initial concentrations and the limiting reactants. The fractional conversion of individual species reactions between these bounds is used to calculate the desired instantaneous concentrations.

The maximum amount of $\mathrm{HCN}$ or $\mathrm{NH}_{3}$ available for reaction at a location in the reactor is determined by the local instantaneous extent of coal mass loss, tracked by the coal off-gas mixture fraction. The maximum $\mathrm{HCN}$ and $\mathrm{NH}_{3}$ concentrations are determined from the ultimate yield of fuel nitrogen at each location in the reactor. The local minimum $\mathrm{HCN}$ and $\mathrm{NH}_{3}$ concentrations are determined by the local availability of oxidizer. Thus, the local fractional conversion, $\zeta_{i}$, is bounded between 0.0 and 1.0 and is expressed as:

$$
\zeta_{i}=\left(\frac{X_{i}^{f}-X_{i}}{X_{i}^{f}-X_{i}^{\min }}\right) \quad i=H C N, N H_{3}
$$


Here $X_{i}^{f}$ represents the local maximum concentration for $\mathrm{HCN}$ or $\mathrm{NH}_{3}$ and $X_{i}^{\text {min }}$ represents the local minimum. When $\mathrm{HCN}$ or $\mathrm{NH}_{3}$ is the limiting reactant, $X_{i}^{\text {mis }}$ is 0.0 . Except in fuel-rich regions, $X_{i}^{\text {min }}$ is typically 0.0 icr coal combustion. During coal gasification, $X_{i}^{\min }$ is frequently not zero.

The maximum amount of fuel NO that can be formed locally is determined from the maximum concentrations of $\mathrm{HCN}$ and $\mathrm{NH}_{3}$ combined, since in the limit, where enough oxygen is available, all of the $\mathrm{HCN}$ and $\mathrm{NH}_{3}$ can be converted to NO. When NO does not enter the reactor with any inlet stream, the minimum amount of NO is zero and the fractional conversion of NO reduces to the following equation:

$$
\zeta_{N O}^{\text {fuel }}=\left(\frac{X_{N O}}{X_{N O}^{f}}\right)
$$

The formation of thermal NO is bounded by the local equilibrium concentrations since the extended Zel'dovich mechanism contains reversible elementary-step reactions. The reaction progress for thermal NO reactions is determined by scaling the local instantaneous NO concentration by the local equilibrium quantity. Local NO equilibrium concentrations is calculated by an equilibrium subroutine in PCGC-2. The fractional conversion for thermal NO may be viewed as an equilibrium deviation factor and is not bounded by unity.

$$
\zeta_{N O}^{\text {chemal }}=\left(\frac{X_{N O}}{X_{N O}^{\text {cq }}}\right)
$$

When $\zeta_{N O}^{\text {thermal }}$ is less than 1.0 , the thermal NO mechanism favors NO formation; conversely, when the value of $\zeta_{\mathrm{NO}}^{\text {themal }}$ is greater than unity, thermal NO shifts back to atomic and molecular oxygen and nitrogen according to the extended Zel'dovich mechanism.

When joint thermal and fuel NO predictions are made, the maximum NO concentration possible is calculated as the sum of the maximum amount of fuel NO and maximum thermal NO possible. The maximum concentration of thermal NO possible can be calculated by the sum of the forward reaction steps of the Zel'dovich mechanism, neglecting reverse mechanism reactions. The fuel NO and 
thermal NO mechanisms couple in such a manner that final NO concentrations rarely equal the sum of thermal NO and fuel NO concentrations when predicted separately. Both mechanisms compete for oxygen concentrations and NO is destroyed simultaneously by both mechanisms, regardless of the origin of NO.

It is assumed that the local instantaneous reaction progress variables are approximately equal to their time-mean values; in other words, turbulent fluctuations do not affect the local extent-of-reaction. Thus, the local extent-of-reaction is calculated using time-mean values. For example;

$$
\zeta_{i}=\tilde{\zeta}_{i}=\left(\frac{\tilde{X}_{i}^{f}-\tilde{X}_{i}}{\tilde{X}_{i}^{f}-\tilde{X}_{i}^{m i n}}\right) \quad i=H C N, N H_{3}
$$

Once the local fractional conversion of a species is known, the local instantaneous concentrations are calculated by scaling the local instantaneous maximum or equilibrium concentrations with the appropriate fractional conversion variable. For example;

$$
X_{i}=X_{i}^{f}-\left(X_{i}^{f}-X_{i}^{\min }\right) \tilde{\zeta}_{i} \quad i=H C N, N H_{3}
$$

The amount of nitrogen released from the coal is calculated directly from the source of particle mass added to the gas phase $\left(s_{p}^{\eta}\right)$. Experimental evidence shows that $\mathrm{HCN}$ and $\mathrm{NH}_{3}$ are rapidly produced and exist as relatively long lived species under all coal flame conditions. Several investigators have reported that $\mathrm{HCN}$ precedes the appearance of $\mathrm{NH}_{3}$ for both high and low rank coals over a broad range of stoichiometries (Ghani and Wendt, 1990, Haussmann and Kruger, 1989, Freihaut and Proscia, 1991). However, other investigations (Peck et al., 1991 and Chen et al., 1982) observed that $\mathrm{NH}_{3}$ may be a direct pyrolysis product in subbituminous flames and that light-gas $\mathrm{NH}_{3}$ may also be produced by heterogeneous processes. Until a comprehensive nitrogen pyrolysis model is incorporated into the $\mathrm{NO}_{\mathrm{x}}$ submodel, the fraction of coal and char nitrogen released as $\mathrm{HCN}$ or $\mathrm{NH}_{3}$ is specified by adjustable parameters. The FG/DVC model has not been adapted into the submodel in this version of the code.

Two adjustable parameters are available in the model to control nitrogen release to the gas phase. The parameter $\zeta_{\mathrm{n}}$ specifies the fractional conversion of 
coal and/or char nitrogen to gaseous nitrogen while the adjustable parameter $\alpha_{n}$ is used to partition the initial volatile nitrogen between $\mathrm{HCN}$ and $\mathrm{NH}_{3}$. In the model, the coal off-gas mixture fraction tracks the extent of coal and char conversion to the gas phase. Hence, the yield of coal-nitrogen released to the gas phase is expressed by the equations:

$$
\begin{gathered}
\left(Y_{H C N}\right)_{\max }=\eta \cdot \zeta_{n} \cdot \alpha_{n} \cdot b c n \cdot M W_{H C N} \\
\left(Y_{N H_{3}}\right)_{\max }=\eta \cdot \zeta_{n} \cdot\left(1-\alpha_{n}\right) \cdot b c n \cdot M W_{N H_{3}}
\end{gathered}
$$

Here, $b c n$, is the percentage of nitrogen in the coal and is determined by ultimate analysis of the coal. $\eta$ is the fraction of coal off-gas at a position in the reactor. The fractional nitrogen release parameters are bounded to $\left(0.0 \leq \zeta_{n} \leq 1.0\right)$ and $\left(0.0 \leq \alpha_{\mathrm{n}} \leq 1.0\right)$.

\section{Submodel Summary}

All of the equations that are solved by the $\mathrm{NO}_{\mathrm{x}}$ submodel are listed in Table 2-12. The following is a summary of the key model assumptions.

i. The formation of nitrogen pollutant species does not impact the governing flame structure.

ii. NO formation/destruction mechanisms other than thermal NO and fuel NO are neglected.

iii. Fuel NO formation is adequately described by global mechanisms explicit only in $\mathrm{HCN}, \mathrm{NH}_{3}, \mathrm{NO}$, and $\mathrm{N}_{2}$.

iv. Thermal $\mathrm{NO}$ is adequately predicted by the extended Zel'dovich mechanism. Two rate expressions are available.

v. Atomic oxygen can be predicted using quasi-equilibrium expressions dependent on major species concentrations $\left(\mathrm{O}_{2}, \mathrm{CO}_{2}\right.$, and $\left.\mathrm{CO}\right)$. Two expressions are available.

vi. Nitrogen is devolatilized or released from char at a rate proportional to the rate of total coal weight loss. The proportionality constant is an adjustable parameter. 
vii. Volatile nitrogen is instantaneously converted to $\mathrm{HCN}$ and/or $\mathrm{NH}_{3}$ once it is released to the gas. The partition between $\mathrm{HCN}$ and $\mathrm{NH}_{3}$ is specified by an adjustable parameter.

viii. Instantaneous species concentrations can be tracked using fractional conversion expressions.

ix. Instantaneous and Favre-average fractional conversions are approximately equal.

x. Mean homogeneous reaction rates are obtained by convolving over the probability of mixture fraction progress variables.

xi. Heterogeneous char/NO reactions are not affected by turbulence.

xii. Soot/NO interaction are neglected.

TABLE 2-13

EQUATION SET FOR A GENERALIZED NO MODEL

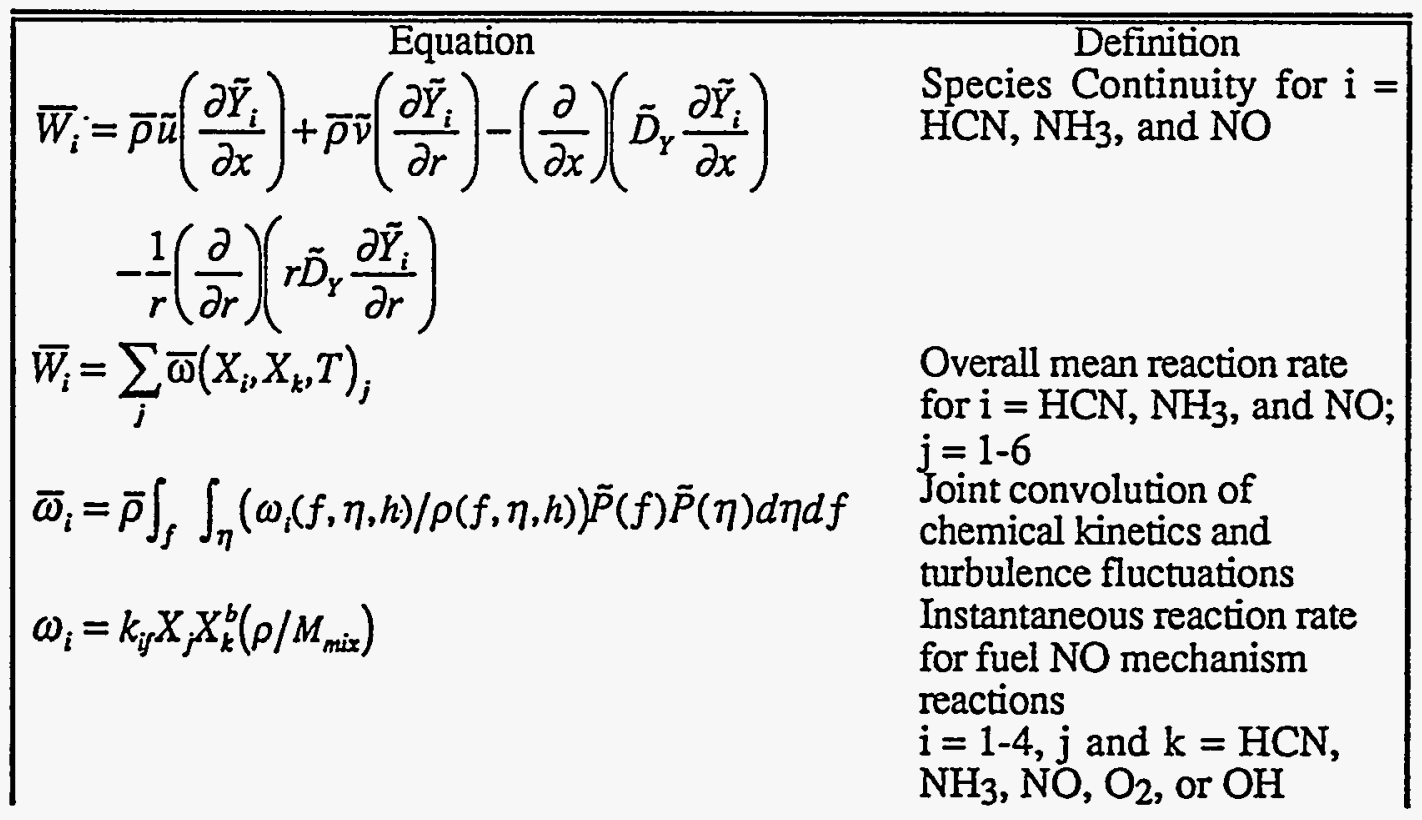


TABLE 2-13 (continued)

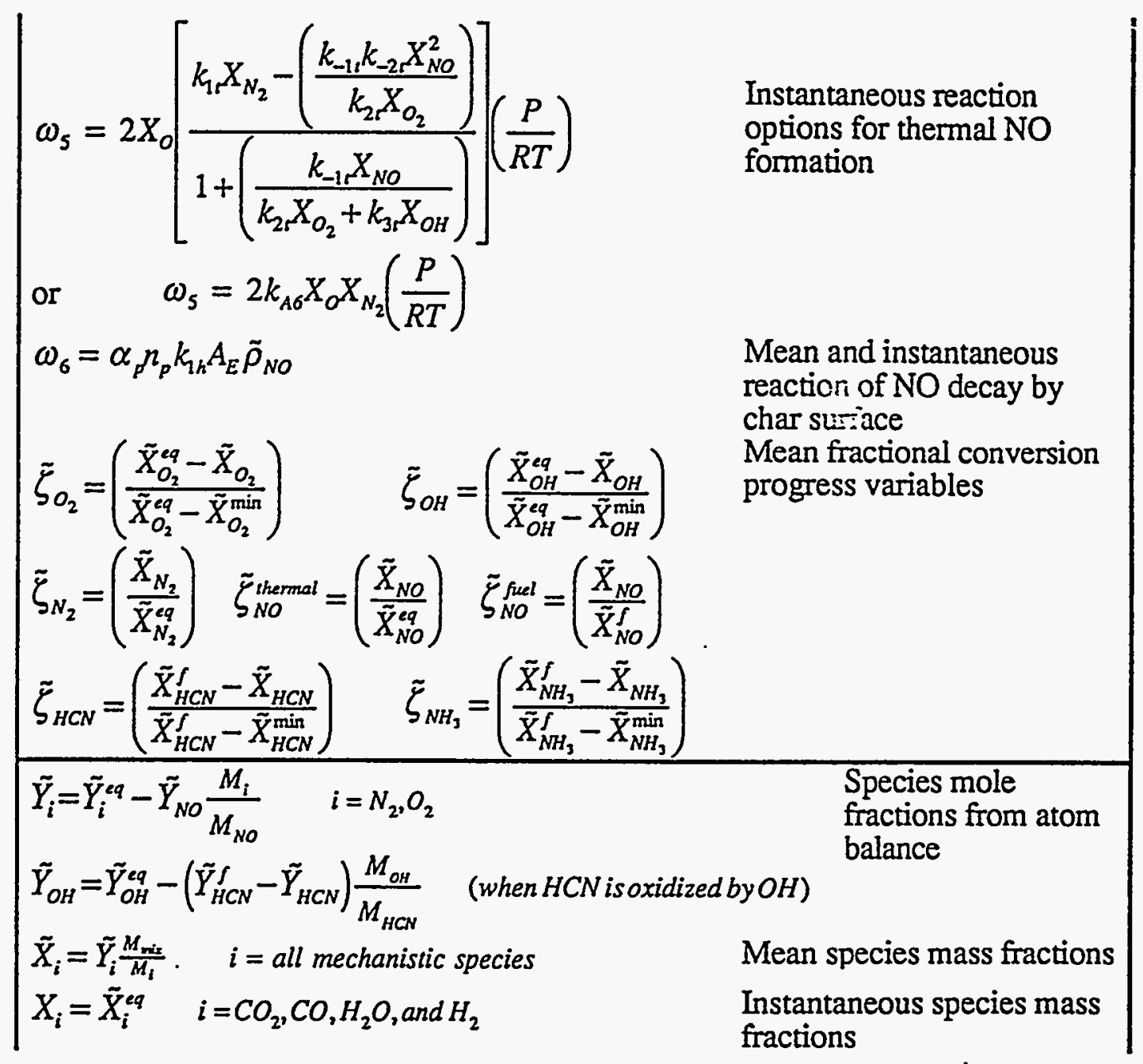


TABLE 2-13 (continued)

$$
\begin{aligned}
& X_{O_{2}}=X_{O_{2}}^{e q}-\left(X_{O_{2}}^{e q}-X_{O_{2}}^{\min }\right) \tilde{\zeta}_{O_{2}} \quad X_{O H}=X_{O H}^{e q}-\left(X_{O H}^{e q}-X_{O H}^{\min }\right) \tilde{\zeta}_{O H} \\
& X_{N_{2}}=\left(X_{N_{2}}^{c q}\right) \tilde{\zeta}_{N_{2}} \\
& X_{i}=X_{i}^{f}-\left(X_{i}^{f}-X_{i}^{\min }\right) \tilde{\zeta}_{i} \quad i=H C N, N H_{3} \\
& X_{N O}=\left(X_{N O}^{e q}\right) \tilde{\zeta}_{N O}^{\text {themal }} \text { or } X_{N O}=\left(X_{N O}^{f}\right) \tilde{\zeta}_{N O}^{\text {fuel }} \\
& X_{i}^{\min }=\max \left(0.0, X_{i}^{e q}-\left(\frac{X_{H C N}^{f}}{M_{H C N}}+\frac{X_{N H_{3}}^{f}}{M_{N H_{3}}}+\frac{X_{N O}^{e q}}{M_{N O}}\right) \frac{M_{i}}{2}\right) \quad i=O_{2}, N_{2} \\
& X_{i}^{\min }=\max \left(0.0, X_{i}^{f}-\left(X_{O_{2}}^{e q} 4 M_{i} / M_{O_{2}}\right)\right) \quad i=H C N, N H_{3}{ }^{3} \\
& X_{O H}^{\min }=\max \left(0.0, X_{O H}^{e q}-X_{H C N}^{f} \frac{M_{O H}}{M_{H C N}}\right) \\
& X_{H C N}^{f}=\alpha_{N}(b c n) \eta \quad X_{N H_{3}}^{f}=\left(1-\alpha_{N}\right)(b c n) \eta \\
& X_{N O}^{f}=X_{H C N}^{f}+X_{N H_{3}}^{f}+\left(X_{N O}^{\text {chermal }}\right)^{s} \\
& X_{O}=\left[K_{e q} X_{O_{2}}\right]^{1 / 2} \quad \text { or } \quad X_{O}=K_{e q} \frac{X_{O_{2}} X_{C O}}{X_{\mathrm{CO}_{2}}} \quad \begin{array}{l}
\begin{array}{l}
\text { Quasi-equilibrium } \\
\text { expressions for estimating } \\
\text { radical oxygen } \\
\text { concentrations }
\end{array} \\
\text { conction }
\end{array}
\end{aligned}
$$

I A factor of 4 is used since every molecule of $\mathrm{NO}$ formed by oxidation of $\mathrm{HCN}$ or $\mathrm{NH}_{3}$ can reduce another $\mathrm{NH}_{3}$ or $\mathrm{HCN}$ molecule to $\mathrm{N}_{2}$.

$\S$ Term added to the maximum concentration of NO possible when joint fuel and thermal NO calculations are made.

\section{Sulfur Pollutants and Sorbent Reactions Submodel}

\section{Background}

Sulfur-containing pollutants formed by burning fossil fuels include $\mathrm{SO}_{2}, \mathrm{SO}_{3}$, $\mathrm{H}_{2} \mathrm{~S}, \mathrm{COS}$, and $\mathrm{CS}_{2}$. Under normal boiler operating conditions, with excess 
oxygen, virtually all of the sulfur is oxidized to $\mathrm{SO}_{2}$ with small quantities of $\mathrm{SO}_{3}$. Fuel-rich species (primarily $\mathrm{H}_{2} \mathrm{~S}$ ) exist only in regions of the reactor where the coal particles are rapidly devolatilizing and oxygen is depleted. When coal is gasified under oxygen-deficient conditions, the principal sulfur-containing species is $\mathrm{H}_{2} \mathrm{~S}$, with $\mathrm{SO}_{2}$ also occurring locally in various regions of the gasifier.

Sulfur pollutants can be captured during the combustion process or in the flue gas ducts by injecting sorbents. Calcium-based sorbents are particularly attractive due to their low cost and the inertness of the calcium sulfate (in the case of $\mathrm{SO}_{2}$ capture) or calcium sulfide (in the case of $\mathrm{H}_{2} \mathrm{~S}$ capture) by-product. The calcium sorbents first undergo calcination followed by particle sulfation. The reacted sorbents are typically collected with the fly ash by the bag house or electrostatic precipitator.

\section{$\mathrm{SO}_{\mathrm{x}}$ Pollutant Species}

Local instantaneous equilibrium is assumed for the homogeneous chemistry; thus, the volatile sulfur is assumed to be locally equilibrated with the gas prior to capture by the sorbents. This information is provided by the chemical equilibrium subroutines of PCGC-2. The model does not currently allow for the conversion of $\mathrm{SO}_{2}$ to $\mathrm{H}_{2} \mathrm{~S}$ or visa versa once it is formed or after one species is preferentially captured by the sorbents.

\section{Sorbent Reactions Model}

When hydrated lime $\left[\mathrm{Ca}(\mathrm{OH})_{2}\right]$ and limestone [calcium carbonate $\left.\left(\mathrm{CaCO}_{3}\right)\right]$ sorbent particles are injected into the combustor and thermally heated, calcination occurs:

$$
\begin{aligned}
& \mathrm{CaCO}_{3}(\mathrm{~s}) \rightarrow \mathrm{CaO}(\mathrm{s})+\mathrm{CO}_{2}(\mathrm{~g}) \\
& \mathrm{Ca}(\mathrm{OH})_{2}(\mathrm{~s}) \rightarrow \mathrm{CaO}(\mathrm{s})+\mathrm{H}_{2} \mathrm{O}(\mathrm{g})
\end{aligned}
$$

Complete calcination of a $\mathrm{CaCO}_{3}$ particle yields a particle of $\mathrm{CaO}$ with about 50 percent free pore volume. Once calcined material is produced, sulfur capture can 
occur, provided the temperature is conducive for sulfation reactions. The following simplified reactions are generally accepted to occur:

$$
\begin{gathered}
\mathrm{CaO}(\mathrm{s})+\frac{1}{2} \mathrm{O}_{2}(\mathrm{~g})+\mathrm{SO}_{2}(\mathrm{~g}) \rightarrow \mathrm{CaSO}_{4}(\mathrm{~s}) \\
\mathrm{CaO}(\mathrm{s})+\mathrm{H}_{2} \mathrm{~S}(\mathrm{~g}) \rightarrow \mathrm{CaS}(\mathrm{s})+\mathrm{H}_{2} \mathrm{O}(\mathrm{g}) \\
\mathrm{CaO}(\mathrm{s})+\mathrm{SO}_{3}(\mathrm{~g}) \rightarrow \mathrm{CaSO}_{4}(\mathrm{~s})
\end{gathered}
$$

Some simplifying assumptions which have made in the current sorbent reactions submodel include: 1) The sorbent particles are assumed to be instantaneously calcined to $\mathrm{CaO} ; 2$ ) The sorbent particles are isothermal and in thermal equilibrium with the local gas; and 3) Sulfation is considered irreversible.

A shrinking-core grain model developed and evaluated by Silcox et al. $(1985,1989)$ is used to predict the capture of $\mathrm{SO}_{2}$ and $\mathrm{H}_{2} \mathrm{~S}$. This model assumes that calcined sorbent particles consist of an agglomeration of small spherical grains of $\mathrm{CaO}$. Sulfation occurs by the following sequence of physical and chemical events:

(1) diffusion of reactants from the bulk gas to the particle surface.

(2) pore diffusion of reactants to the particle internal (i.e., grain) surface.

(3) solid-state diffusion through the product layer (except at the onset of reaction).

(4) reaction of with $\mathrm{CaO}$

Figure 2-8 gives a conceptual view of the shrinking-core grain model for a sorbent particle with a radius of $R_{p}$. The enlargement of an area of grains at an internal radius of $R$ (or a subshell) illustrates the band of reaction product (e.g $\mathrm{CaSO}_{4}$ ) with product radius of $\mathrm{Ig}_{\mathrm{g}}$, extended beyond the original grain radius, $\mathrm{Ig}_{\mathrm{g}}$, and the core radius, $r$, of the unreacted calcined material. 


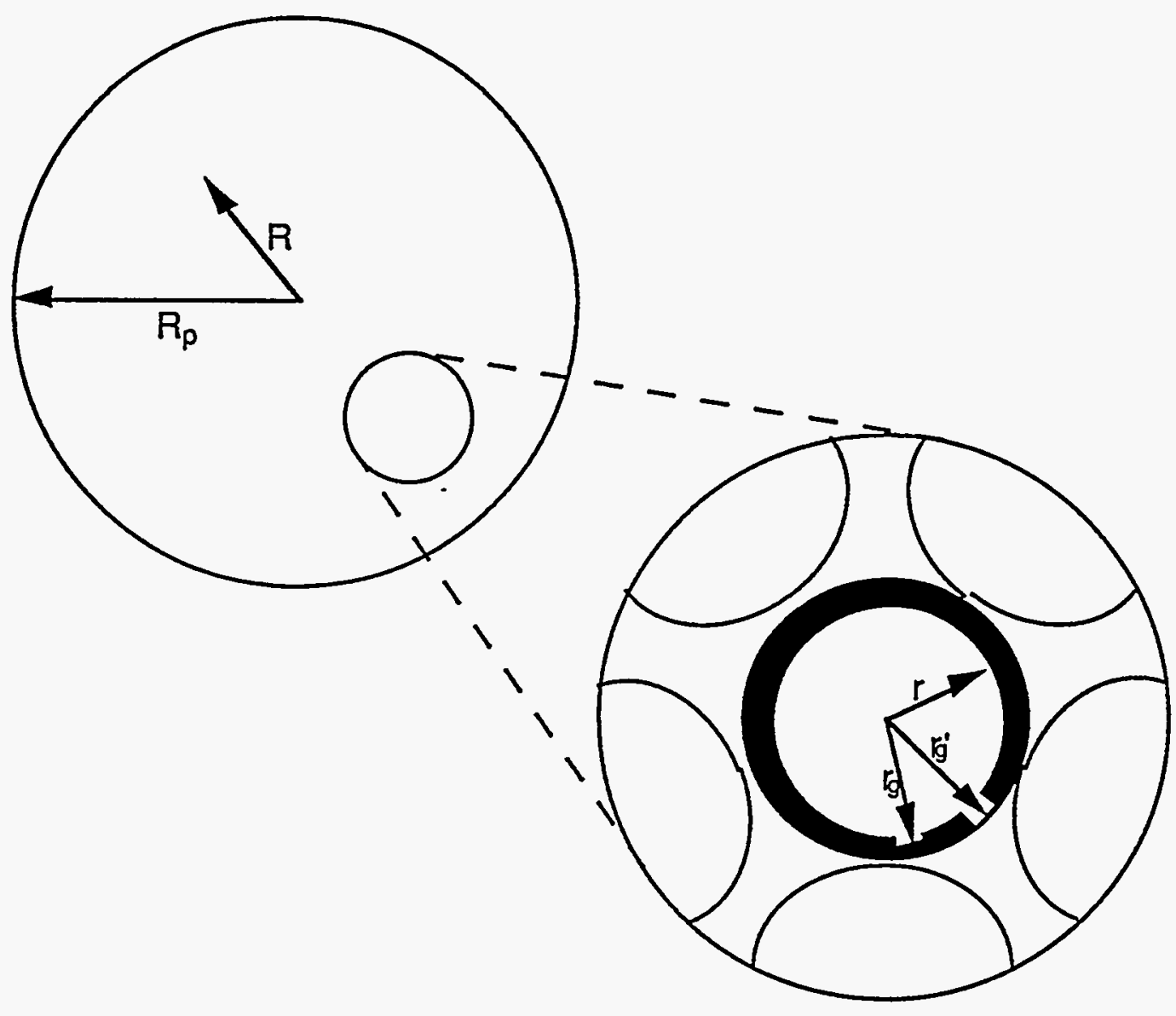

Figure 2-8. Conceptual view of the shrinking-core grain model. (Figure adapted with permission from Silcox (1985)

For sorbent particles which are spherical in shape, a material balance for the reacting gas in a concentric shell of the particle yields, 


$$
\frac{d^{2} C}{d R^{2}}+\left(\frac{2}{R}+\frac{1}{D_{e f f}} \frac{d D_{e f f}}{d R}\right) \frac{d C}{d R}-\frac{N}{D_{e f f}}=0
$$

where $C$ is the $\mathrm{SO}_{2}$ concentration, $R$ is the radial coordinate of the sorbent, $\bar{D}_{\text {eff }}$ is the effective pore diffusivity, and $N$ is the rate of reaction of $\mathrm{SO}_{2}$ per unit volume of porous solid. The boundary conditions which satisfy this equation are,

$$
\begin{gathered}
R=R_{p} \quad D_{\text {eff }} \frac{d C}{d R}=k_{m}\left(C_{b}-C\right) \\
R=0 \quad \frac{\partial C}{\partial R}=0
\end{gathered}
$$

Here $C_{b}$ is the bulk gas $\mathrm{SO}_{2}$ concentration and $k_{m}$ is a mass transfer coefficient which can be obtained from a suitable Sherwood number.

The reaction term, $N$, is given for reaction of order $n$, with respect to the sulfur-containing reactant, by,

$$
N=\frac{C^{n}}{\frac{1}{k_{f}}+\frac{r^{2}}{D_{s p}}\left(\frac{1}{r}-\frac{l}{r_{8}}\right)}(A), \quad \quad \quad \quad \operatorname{mol~cc^{-3}s^{-l}}
$$

where $k_{f}$ is the forward heterogeneous reaction expression, $D_{s p}$ is the diffusion coefficient of $\mathrm{SO}_{2}$ in the solid product layer, and $A$ is the interfacial area available for reaction per unit volume. The reaction order, $n$, has been not been generalized in the current version. First and half-order reactions can be selected for $\mathrm{SO}_{2}$ capture and only a first order reaction is allowable for $\mathrm{H}_{2} \mathrm{~S}$ capture. The first order reaction expressions have been tested and found to converge without difficulty.

The sulfation grain model is solved at discrete time steps as the particles progress through the combustor. Thus, the instantaneous sorbent particle conversion (sulfation) is dependent on the local gaseous properties (composition, temperature, etc.). 


\section{Working Equation}

The Favre-averaged species continuity equation is used to calculate the Favre-mean mass fraction of the sulfur pollutants $\left(\mathrm{SO}_{2}\right.$ and $\left.\mathrm{H}_{2} \mathrm{~S}\right)$ throughout the turbulent reactor.

$$
\bar{W}_{i}=\bar{\rho} \tilde{u}\left(\frac{\partial \tilde{Y}_{i}}{\partial x}\right)+\bar{\rho} \tilde{v}\left(\frac{\partial \tilde{Y}_{i}}{\partial r}\right)-\left(\frac{\partial}{\partial x}\right)\left(\tilde{D}_{Y} \frac{\partial \tilde{Y}_{i}}{\partial x}\right)-\frac{1}{r}\left(\frac{\partial}{\partial r}\right)\left(r \tilde{D}_{Y} \frac{\partial \tilde{Y}_{i}}{\partial r}\right)
$$

$\bar{W}_{i}$ is the mean chemical reaction source or sink term for the species mass fraction, either $\mathrm{SO}_{2}$ or $\mathrm{H}_{2} \mathrm{~S}$, depending on the dominant species formed in the gas phase. The source term for each finite-difference cell is comprised of two contributions; 1) the sink due to sorbent capture by particles passing through the cell, and 2) the contribution of sulfur released to the cell by reacting coal particles within the cell.

Lagrangian trajectories for the reacting sorbent particles, analogous to the method of tracking coal particles, are calculated simultaneously with solution of the sorbent reaction grain model. The effects of the sorbent particles on the gas velocity and radiation fluxes are neglected. The particles are also assumed to follow the motion of the gas. 


\section{Chapter Three}

\section{Solution Technique}

Approach

An information flow diagram for PCGC-2 is shown in Figure 3-1. The basic approach is to decouple the gas and particle equations using the particle source terms as "tear" variables. After initializing the flow field and geometric quantities, the gas variables are first solved for an assumed set of source terms. After converging the gas phase, the gas properties are updated, the Eulerian particle number density fields are calculated, and the radiation field is solved. The Lagrangian particle trajectories are then solved one at a time. New values of the source terms are calculated from particle mass, enthalpy, and velocity, when trajectories cross cell boundaries. Based on the previously guessed values of the source terms and the newly calculated values, an improved guess of the source terms is made, the gas phase reconverged, the particle trajectories recalculated, and so forth, until overall convergence of both phases is achieved. An option is also provided (INEACH $=\mathrm{T}$ ) for solving the gas and particle phases simultaneously, in semi-coupled form, rather than in nested form as described above. The approaches for solving the gas and particle phases are discussed in more detail below.

\section{Gas Phase}

All of the gas phase equations are Eulerian, steady-state, second order, non-linear, elliptical partial differential equations. All of the gas phase equations are conveniently cast into one finite difference form, so that only one solution technique 
is required. Table 2-1 contains a summary of this form for all of the gas phase equations.

The gas field is solved using a line-by-line technique; a tri-diagonal algorithm is used to solve the finite difference equations along a line using appropriate boundary conditions. PCGC-2 is coded to calculate the gas field lineby-line in either direction, or else in alternate directions. The tri-diagonal algorithm is solved iteratively for a specific variable until convergence is achieved. These iterations on a specific variable are termed "micro-iterations"; generally only three micro-iterations are necessary to obtain convergence for each variable. Each variable in Table $2-1$ is solved in succession, starting with $u$ and ending with $h$. A "macro-iteration" is completed after the complete set of variables has been calculated once (Steps 2 and 3 in Fig. 3-1) . Between 200 and 1000 macro-iterations are required to completely converge the gas phase (Steps 2-4 in Fig 3-1; this comprises one "gas-phase iteration").

The particles are accounted for only by appropriate particle source terms of mass $\left(S_{p}^{m}\right)$, momentum $\left(S_{p}^{u}\right.$ and $\left.S_{p}^{v}\right)$, and enthalpy $\left(S_{p}^{h}\right)$. These terms represent the addition of mass, momentum, and energy to the gas phase by the particles. The source terms are found along a particle trajectory by taking the difference in the particle properties on each side of a computational cell and dividing by the cell volume.

Roache (1976) presents a good review of techniques for solving fluid dynamics problems. The particular fluid flow problem solved by PCGC-2 consists of a recirculating fluid, and this type of problem has been heavily investigated by researchers at Imperial College. A method similar to the TEACH technique (Gosman and Pun, 1973) developed at Imperial College is used in PCGC-2 to solve the gas equations in the primitive variables. This technique is an iterative, steady-state, finite difference approach, and is widely used (Lockwood et al., 1980; Smith, 1979; Smoot and Pratt, 1979). Recently, finite element methods have been applied successfully to fluid flow problems (Gallagher et al., 1975). These methods have some strong advantages, particularly pertaining to arbitrary boundary shapes, but they are still in a developing stage and have not been applied extensively to compressible recirculating flows. 


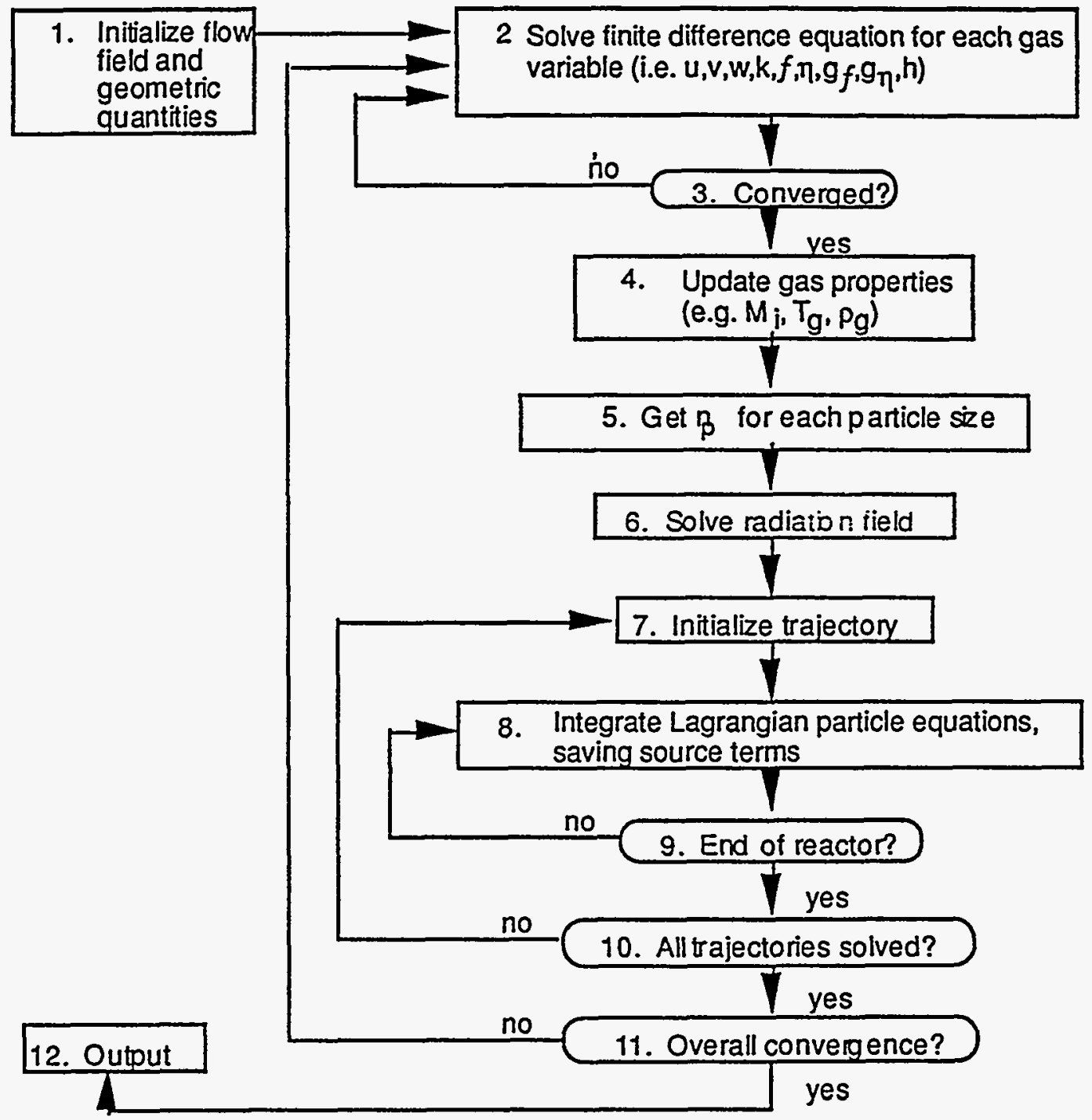

Fig. 3-1. Information flow diagram. 
Solution Technique

Each of the differential equations must be cast in finite difference form and solved over some appropriate grid spacing. A series of grid lines running orthogonally to the coordinate directions define at their intersection, node points, where the values of the dependent variables are usually identified. Roach (1976) has reviewed application of the flow equations to various possible mesh systems and show that in the $(u, v, p)$ formulation, the variables $u$ and $v$ are most conveniently and accurately evaluated with node points lying on the boundary and with $p$ and $\rho$ being placed at one-half grid spacing off the boundary. This staggered-mesh system is shown in Fig. 3-2 as it is applied in PCGC-2. The grid spacing may be non-uniform, with grid points concentrated in areas of steep gradients. This spacing increases the convergence rate and accuracy of the computations. PCGC-2 locates all faces midway between node points. Arithmetic averaging is used to obtain property values at mesh boundaries between node points. Throughout PCGC-2, the distance between nodes is stored and used in the differencing scheme. Completely arbitrary grid spacing is thus permitted.

It is has already been mentioned that special difficulties arise in the primitive variable approach in that the momentum equations contain the pressure gradient terms, yet pressure does not explicitly appear in the continuity equation. Roach (1976) used the Los Alamos Marker and Cell (MAC) method for solving this problem. Patankar $(1975,1980)$ developed a semi-implicit method for pressurelinked equations (SIMPLE). PCGC-2 has been coded to include the SIMPLE algorithm and two of its variants (SIMPLER and SIMPLEC). A brief outline of the basic technique is presented here. More complete derivations may be found in the next section.

Figure 3-2 shows a typical computational cell. Attention is focused on the node point $P$ and its nearest neighbors (N, S, E, W). The pressure, along with other variables, is calculated for these nodes. The velocity components associated with this node point are calculated at cell faces as shown. This staggered grid helps in writing the mass conservation equation for a cell since the velocities at the cell faces are needed in the convection terms. In addition, the difference of pressure between two adjacent nodes is used to derive the velocity component for the point midway between them. 


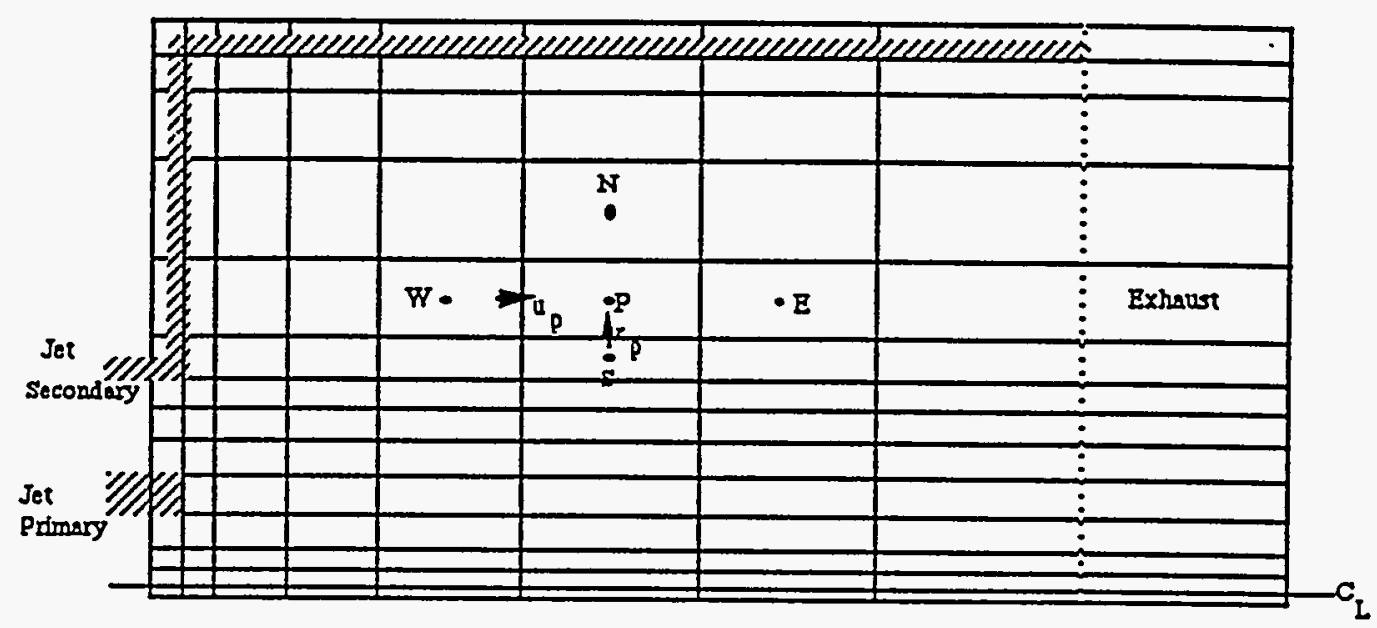

Figure 3-2. Sample grid pattern for PCGC-2.

When the momentum equations are cast in finite difference form (discussed in the next section) the unknown pressure terms will appear. At this point, the true pressure $\bar{p}$ is decomposed into a best estimate $p^{*}$ and a pressure correction term $\bar{p}^{c}$.

$$
\bar{p}=\bar{p}^{*}+\bar{p}^{c}
$$

With the help of this decomposition of the true pressure and the two momentum difference equations, expressions may be found for two velocity components in terms of starred velocity components and pressure-correction variables $\left(\bar{p}^{c}\right)$. The continuity equation permits closure of the problem. By writing it in infinite difference form and substituting the velocity equation in terms of $\bar{p}^{c}$, a finite difference equation for the pressure correction is formed. In other words, the continuity equation is written in terms of the unknown pressure corrections in Eqn. 3-1.

The overall iterative solution procedure for the SIMPLE algorithm is: 
1. Begin with the best available initial guess for all variables including pressure $\left(\bar{p}^{*}\right)$.

2. Calculate auxiliary variables such as density, temperature, etc., from the associated combustion mode.

3. Solve the momentum difference equations for the starred velocity field.

4. Solve the pressure correction form of the continuity equation for $\bar{p}^{c}$.

5. Calculate the pressure (from Eqn. 3-1) and corrected velocities.

6. Solve the difference equations for all other associated variables (i.e., $\left.k, \varepsilon, g_{f}, \eta, g_{\eta}, \bar{h}\right)$.

7. With the new updated variables, return to Step 2 and continue iteration until convergence is achieved.

The overall iterative solution procedure for the SIMPLER algorithm is similar to the procedure for the SIMPLE algorithm (as shown above). The continuity equation is used in connection with the momentum equations to formulate an equation for pressure $(\bar{p})$ instead of the pressure correction $\left(\bar{p}^{c}\right)$. The iterative scheme is discussed later in the text. The SIMPLEC method (Van Doormaal and Raithby, 1984) corrects an inconsistency in the derivation of the pressure correction equation and results in faster convergence.

\section{Particle Phase}

The particle source-in-cell (PSI-CELL) technique of Crowe and coworkers (1977) has been followed directly to account for the Lagrangian particle field in the Eulerian gas field. The procedure is outlined below:

1. The Eulerian gas field is solved without particles using the above algorithm.

2. The radiation field is solved using the flux method.

3. The Lagrangian particle field is solved with a representative number of trajectories and the particle source term field is calculated.

4. The gas is solved with the updated particle source term field.

5. Step 2 is repeated and iteration continued until overall convergence is achieved.

The PSI-CELL technique is very efficient with respect to storage and computational time required. The only significant storage requirement is for the particle source term field. Steps 2 through 4 represent one "particle iteration" (Steps 7 through 11 
in Fig. 3-1). Convergence is achieved when the gas phase does not change significantly between particle iterations.

\section{Finite Difference Scheme}

The majority of this section was taken originally from another source with the author's permission (Wormeck, 1976). Modifications to the original material were made as appropriate.

\section{Introduction}

This section presents the basic fluid flow solution procedure. This derivation brings to light various questions and possible alternative routes to take. The pressure is usually the variable giving the most trouble and can be eliminated by cross-differentiation of the momentum equations and introducing vorticity $\xi$ as a dependent variable. The two velocity components are then removed by defining the stream function $\psi$. The resultant $(\psi, \xi)$ system requires two equations to be solved for the unknown vorticity and stream function. If the flow is compressible, then the pressure must be recovered by the usual technique of integrating one of the momentum equations, after which the density can be updated by an equation of state.

The $(\psi, \xi)$ system has been developed and published elsewhere (Gosman et al., 1969), but because of difficulties with boundary conditions, failure for many compressible flows, and not being readily extendible to three-dimensional flows, the method was abandoned in favor of a new algorithm (Gosman and Pun, 1973) which solves the flow equations in the primitive variables. The latter method is discussed in this section.

\section{Differential Equations}

The gas-phase equation set was presented in Chapter 2, illustrating that the equations can be cast into one standard form. The presentation in this section will focus on this general form and on deviations from this form for the momentum equations due to the staggered grid. The continuity and momentum equations are presented here in their customary form (Bird et al., 1960) with particle source terms: 


$$
\operatorname{MASS} \text { (continuity): } \frac{\partial \rho}{\partial t}+\vec{\nabla} \bullet(\rho \bar{v})=S_{p}^{m}
$$

$$
\text { MOMENTUM: } \rho \frac{D \vec{v}}{D t}=-\vec{\nabla} p-\vec{\nabla} \bullet \overline{\bar{\tau}}+\rho \vec{g}+\vec{v}_{p g} S_{p}^{m}+S_{p}^{\bar{v}}
$$

where $D / D t$ is the substantive derivative, and $\overline{\bar{\tau}}$ is the stress tensor. The $S_{p}$ terms are the source terms due to the particle phase. After the complete derivation, an analysis will be performed to modify the finite-difference equations such that total mass is conserved.

Neglecting body forces, this system of equations can be expanded in terms of cylindrical coordinates for steady, axi-symmetric flows (Gosman et al., 1969):

$$
\text { CONTINUITY: } \frac{\partial}{\partial x}(\rho u)+\frac{1}{r} \frac{\partial}{\partial r}(r \rho v)=S_{p}^{m}
$$

U-MOMENTUM (direction $\mathrm{x}$ ):

$$
\begin{aligned}
& \frac{\partial}{\partial x}(\rho u u)+\frac{1}{r} \frac{\partial}{\partial r}(r \rho u v)-\frac{\partial}{\partial x}\left(\mu \frac{\partial u}{\partial x}\right)-\frac{1}{r} \frac{\partial}{\partial r}\left(\mu r \frac{\partial u}{\partial r}\right) \\
& =-\frac{\partial p}{\partial x}+\frac{\partial}{\partial x}\left(\mu \frac{\partial u}{\partial x}\right)+\frac{1}{r} \frac{\partial}{\partial r}\left(\mu r \frac{\partial v}{\partial x}\right)-\frac{\partial}{\partial x}\left(\frac{2}{3} \mu d i v \vec{v}\right)+u_{p g} S_{p}^{m}+S_{p}^{u}
\end{aligned}
$$

V-MOMENTUM (direction r):

$$
\begin{aligned}
& \frac{\partial}{\partial x}(\rho u v)+\frac{1}{r} \frac{\partial}{\partial r}(r \rho v v)-\frac{\partial}{\partial x}\left(\mu \frac{\partial v}{\partial x}\right)-\frac{1}{r} \frac{\partial}{\partial r}\left(\mu r \frac{\partial v}{\partial r}\right)= \\
& -\frac{\partial p}{\partial r}+\frac{\partial}{\partial x}\left(\mu \frac{\partial u}{\partial r}\right)+\frac{1}{r} \frac{\partial}{\partial r}\left(\mu r \frac{\partial v}{\partial r}\right)-\frac{2 \mu v}{r^{2}}-\frac{1}{r} \frac{\partial}{\partial r}\left(\frac{2}{3} \mu r \operatorname{div} \vec{v}\right) \\
& +\frac{2}{3} \frac{\mu d i v \vec{v}}{r}+\frac{\rho w w}{r}+v_{p z} S_{p}^{m}+r S_{p}^{v} .
\end{aligned}
$$


W-MOMENTUM (tangential direction):

$$
\begin{aligned}
& \frac{\partial}{\partial x}(\rho u w)+\frac{1}{r} \frac{\partial}{\partial r}(r \rho w)-\frac{\partial}{\partial x} \mu \frac{\partial w}{\partial x}-\frac{1}{r} \frac{\partial}{\partial r}\left(r \mu \frac{\partial w}{\partial r}\right)=-\frac{\rho w w}{r} \\
& -\frac{1}{r^{2}} \frac{\partial}{\partial r} r^{3} \mu \frac{\partial w}{\partial r}+W_{p g} S_{p}^{m}+S_{p}^{w}
\end{aligned}
$$

All of the above equations can be cast into a standard form:

$$
\operatorname{div}(\rho \bar{v} \phi)-\operatorname{div}(\Gamma \operatorname{grad} \phi)=S
$$

where $\phi$ is taken as the dependent variable, $\Gamma$ as the exchange coefficient relating the force with the flux in the transport law for $\phi$, and $S$ as the source term that includes all terms not contained in the first two terms. The first term is generally referred to as the convection term while the second term is the diffusion term. Writing Eqn. 38 in cylindrical two-dimensional coordinates,

$$
\frac{1}{r}\left[\frac{\partial}{\partial x}(r \rho u \phi)+\frac{\partial}{\partial r}(r \rho v \phi)\right]-\frac{1}{r}\left[\frac{\partial}{\partial x}\left(r \Gamma \frac{\partial \phi}{\partial x}\right)+\frac{\partial}{\partial r}\left(r \Gamma \frac{\partial \phi}{\partial r}\right)\right]=S
$$

The corresponding terms in Eqns. 3-4 to 3-7 are easily identified.

Note that the diffusion terms are simply the divergence of a gradient and that any other form of a transport law must be contained in the source term. In particular, the $(\partial / \partial r)[2 \mu r(\partial v / \partial r)]$ term in the second momentum equation has been split, with half of it becoming the second diffusion term in Eqn. 3-6 while the remainder becomes the third source term on the right. This is required for success of the method; the reasoning will follow once the two modifications for correcting mass conservation are developed below.

Therefore our equation system becomes

CONTINUITY $(\phi=1)$ :

$$
\frac{\partial}{\partial x}(\rho u)+\frac{1}{r} \frac{\partial}{\partial r}(r \rho v)=S_{p}^{m}
$$


U-MOMENTUM $(\phi=u)$ :

$$
\frac{\partial}{\partial x}(\rho u u)+\frac{1}{r} \frac{\partial}{\partial r}(r \rho u v)-\frac{\partial}{\partial x}\left(\mu \frac{\partial u}{\partial x}\right)-\frac{1}{r} \frac{\partial}{\partial r}\left(\ddot{r \mu} \frac{\partial u}{\partial r}\right)=-\frac{\partial p}{\partial x}+S^{u}
$$

V-MOMENTUM $(\phi=v)$ :

$$
\frac{\partial}{\partial x}(\rho u v)+\frac{1}{r} \frac{\partial}{\partial r}(r \rho v)-\frac{\partial}{\partial x}\left(\mu \frac{\partial v}{\partial x}\right)-\frac{1}{r} \frac{\partial}{\partial r}\left(r \mu \frac{\partial v}{\partial r}\right)=-\frac{\partial p}{\partial r}+S^{v}
$$

W-MOMENTUM $(\phi=w)$ :

$$
\frac{\partial}{\partial x}(\rho u w)+\frac{1}{r} \frac{\partial}{\partial r}(r \rho v w)-\frac{\partial}{\partial x}\left(\mu \frac{\partial w}{\partial x}\right)-\frac{1}{r} \frac{\partial}{\partial r}\left(r \mu \frac{\partial w}{\partial r}\right)=S^{w}
$$

where the three source terms are given by:

$$
\begin{gathered}
S^{u}=\frac{\partial}{\partial x}\left(\mu \frac{\partial u}{\partial x}\right)+\frac{1}{r} \frac{\partial}{\partial r}\left(r \mu \frac{\partial v}{\partial x}\right)-\frac{\partial}{\partial x}\left(\frac{2}{3} \mu d i v \vec{v}\right)+u_{p s} S_{p}^{m}+r S_{p}^{u} \\
S^{v}=\frac{\partial}{\partial x}\left(\mu \frac{\partial u}{\partial r}\right)+\frac{1}{r} \frac{\partial}{\partial r}\left(r \mu \frac{\partial v}{\partial r}\right)-\frac{2 \mu \nu}{r^{2}}-\frac{1}{r} \frac{\partial}{\partial r}\left(\frac{2}{3} r \mu d i v \vec{v}\right) \\
+\frac{2}{3} \frac{\mu d i v \vec{v}}{r}+\frac{\rho w w}{r}+v_{p g} S_{p}^{m}+S_{p}^{v} \\
S^{w}=-\frac{\rho v w}{r}-\frac{1}{r^{2}} \frac{\partial}{\partial r} r^{3} \mu \frac{\partial w}{\partial r}+w_{p g} S_{p}^{m}+S_{p}^{w}
\end{gathered}
$$

The div $\vec{v}$ term is always dropped in the TEACH program. The effect of particle mass source terms on tangential momentum is ignored in this version of the code. 


\section{Finite-Difference Equations}

There are at least four methods of deriving finite-difference equations from the differential equations. These include Taylor series expansions, polynomial fitting, integral methods, and the control volume approach. The approach taken here is to use the integral technique (Gosman et al., 1969).

Grid. Before integrating the standard equations over the flow domain, a satisfactory grid is required. The boundaries of the flow domain are made to coincide with one of the constant coordinate directions, with only the Cartesian and cylindrical geometries considered.

A series of grid lines orthogonal to the coordinate directions define (at their intersections) node points where the values of the dependent variables will usually be identified. TEACH incorporates non-uniform spacing between these grid points to obtain higher resolution (and therefore accuracy) in regions where the flow gradients are expected to be severe, but does not utilize the more numerically accurate coordinate-stretching transformations, e.g. exponential strerch.

The choice of mesh system depends on whether the node points are to lie on the flow boundary or one-half grid spacing away from it. Roache (1976) derives and compares all of the flow equations in these various mesh systems and shows that in the $(u, v, p)$ formulation, the variable $u$ and $v$ are most conveniently and accurately evaluated in the mesh system with node points laying on the boundary, while $p$ and $\rho$ are most conveniently and accurately evaluated when node values are one-half grid spacing off the walls. The use of a hybrid- or staggered-mesh system thus suggests itself.

A staggered grid is used as shown in Fig. 3-3, where a boomerang-shaped structure has been drawn to emphasize the field positions of the dependent variables $u, v, p$, and $\phi$ (a general variable) that correspond to FORTRAN indices I and J.

It should be noted that the flow domain is a continuous field which has been discretized by a mesh to perform a numerical analysis. All dependent variables and fluid properties are stored in two-dimensional FORTRAN arrays and all grid coordinates and distances are stored in one-dimensional arrays. Referencing a variable requires consideration of the field location specified by subscripts and its representation in the computer code.

The various computational cells are centered at the point of definition of the variable of interest and extend halfway to the four adjacent (in two-dimensional) neighboring node points. Thus we speak of the $p$-cell (main cell), $u$-cell and $v$-cell. The practice in TEACH is to name the node point of interest as $\mathrm{P}$ and the neighboring nodes as the four points of a compass. 


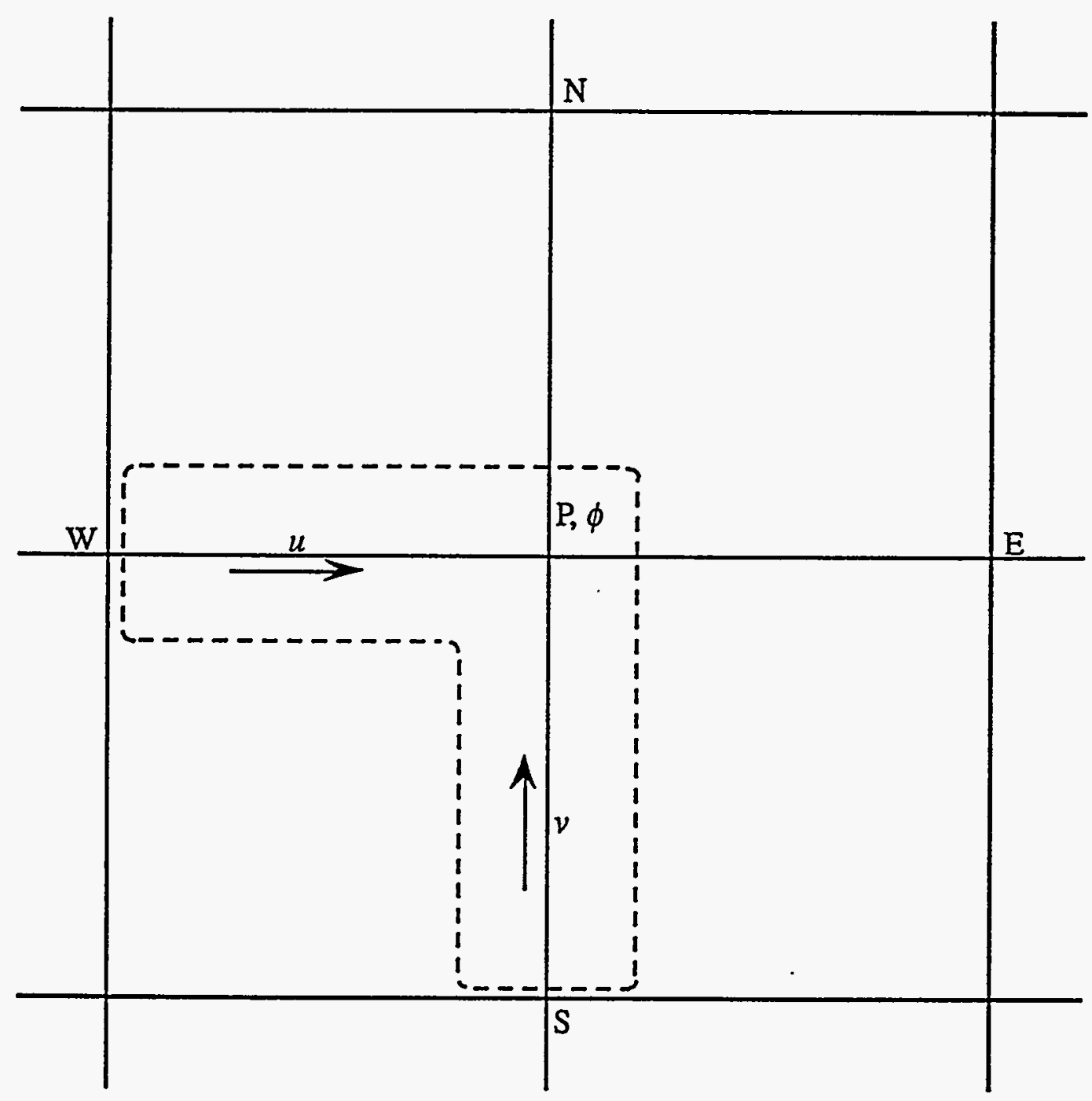

Fig. 3-3. The boomerang-shaped structure enclosing the points of definition of $u$, $v, p$, and $\phi$ corresponding to FORTRAN index I, J. 
NW

$\mathrm{N}$

NE

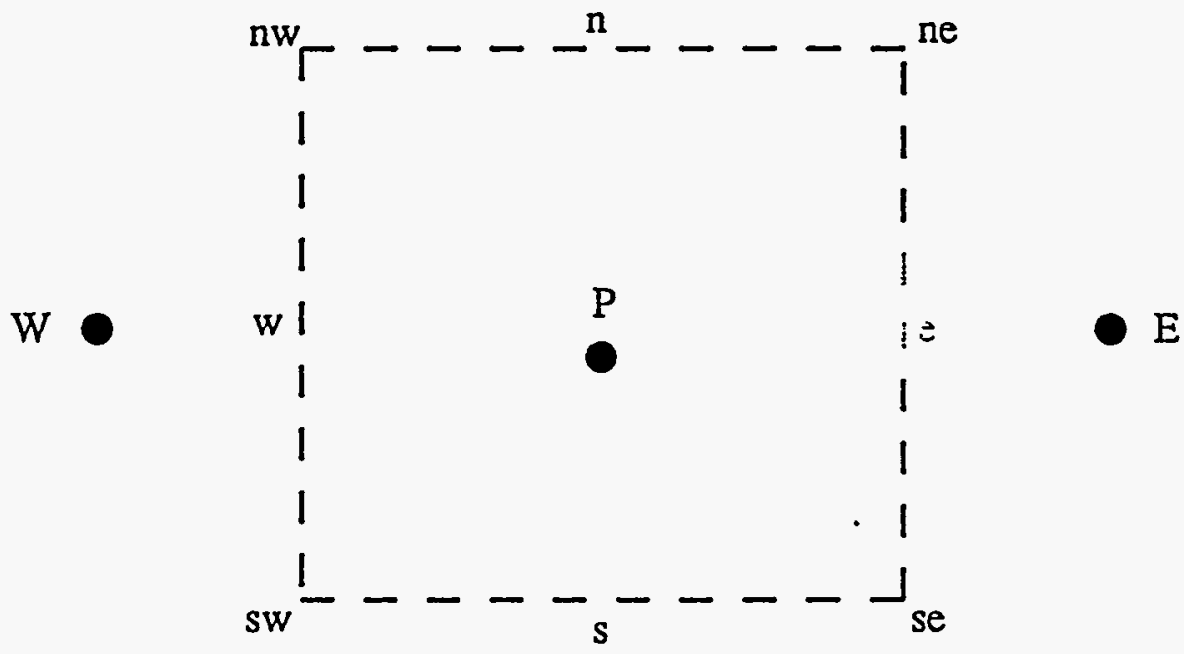

SW

S

SE

Fig. 3-4. Illustration of the grid symbols for a computational cell.

The mathematical description is illustrated in Fig. 3-4, where the absence of a subscript on these compass points signifies the main cell and addition of $u$ or $v$ superscripts implies the $u$ - or $v$-cell respectively. Figs. 3-5, 3-6, and 3-7 illustrate 
the FORTRAN description of these three computational cells. Note how the velocity cells "overlap" the grid lines when the grid spacing is non-uniform.

Three computational cells are associated with each node point. The node points are specified by the FORTRAN indices $I$ and $J$. The main cell center is denoted by subscript $p$ and is located at $\left(x_{p}, r_{p}\right)$ [FORTRAN symbols $\mathrm{X}(\mathrm{I})$ and $\mathrm{R}(J)$ ]. The $u$-cell center has the same radial location as the $p$-cell and is referenced automatically by subscript $p$ and superscript $u$. It is located at $x_{p}^{u}, r_{p}$ in the mathematical developments and in FORTRAN by coordinates [XU(I), R(J)]. With the $v$-cell, the center has the same $x$ location as the main cell, but the radial coordinate is $r_{p}^{\nu}[R V(J)]$. Thus Fig. 3-5 is a template which, when centered on grid location $X(I), R(J)$, references the main cell (no superscripts). Adding a superscript $u$ to the symbols will describe the field if node $\mathrm{P}$ is moved to the center of the $u$-cell at coordinates XU(I), R(J). Similarly, the $v$-cell notation is shown by moving Fig. 3-5 to the center of the $v$-cell $[\mathrm{X}(\mathrm{I}), \mathrm{RV}(\mathrm{J})]$ and addition of the $v$ superscript to all variables.

A location can be referenced in several ways; for example, consider the center of the west face of the main cell. A variable $\phi$ is referenced there mathematically by either $\phi_{w}$ or $\phi_{p}$ and approximately (exactly in a uniform grid) by $\phi_{n w}^{\nu}$. In the derivation of the finite-difference equations which will follow, these different notations will be utilized to reference the same position. When writing the computer code, the best available FORTRAN variables are employed.

Use of the staggered grid also has the convenient feature that velocity is defined at the locations where it is needed in the convective terms. Pressure is defined so as to make it easy to compute the pressure gradients in the two momentum equations.

\section{Integration of the Standard Equation}

The standard equation (Eqn. 3-9) will be developed first, followed by the momentum equations and the equation for pressure.

General Finite-Difference Equation ( $\phi$-Equation). Fig. 3-8 displays a typical internal main cell where the $\phi$-equation (Eqn. 3-9) can be integrated over the volume obtained by rotating the area, represented by the dotted lines, about the symmetry axis to give: 


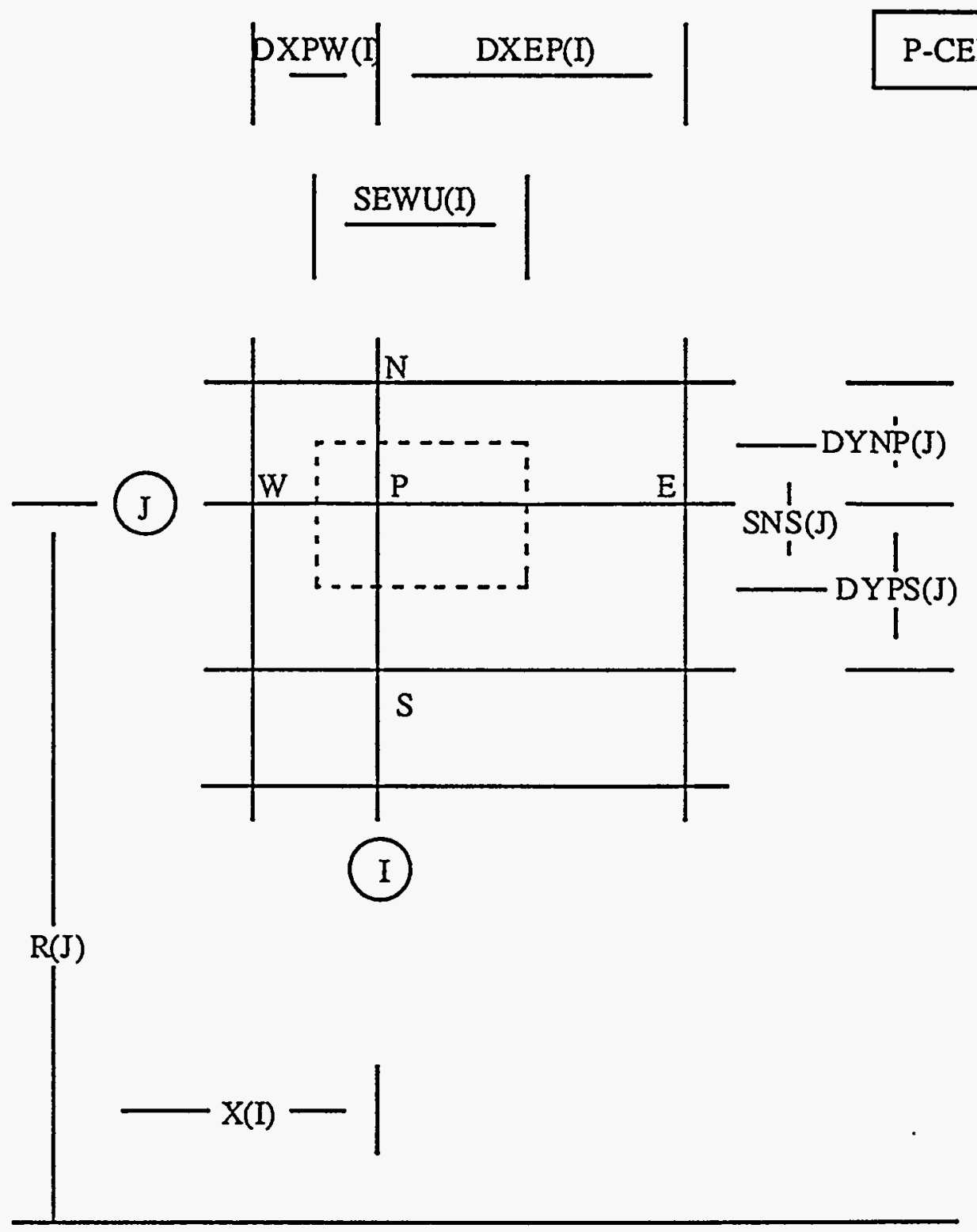

Fig. 3-5. FORTRAN symbols for p-cell. 


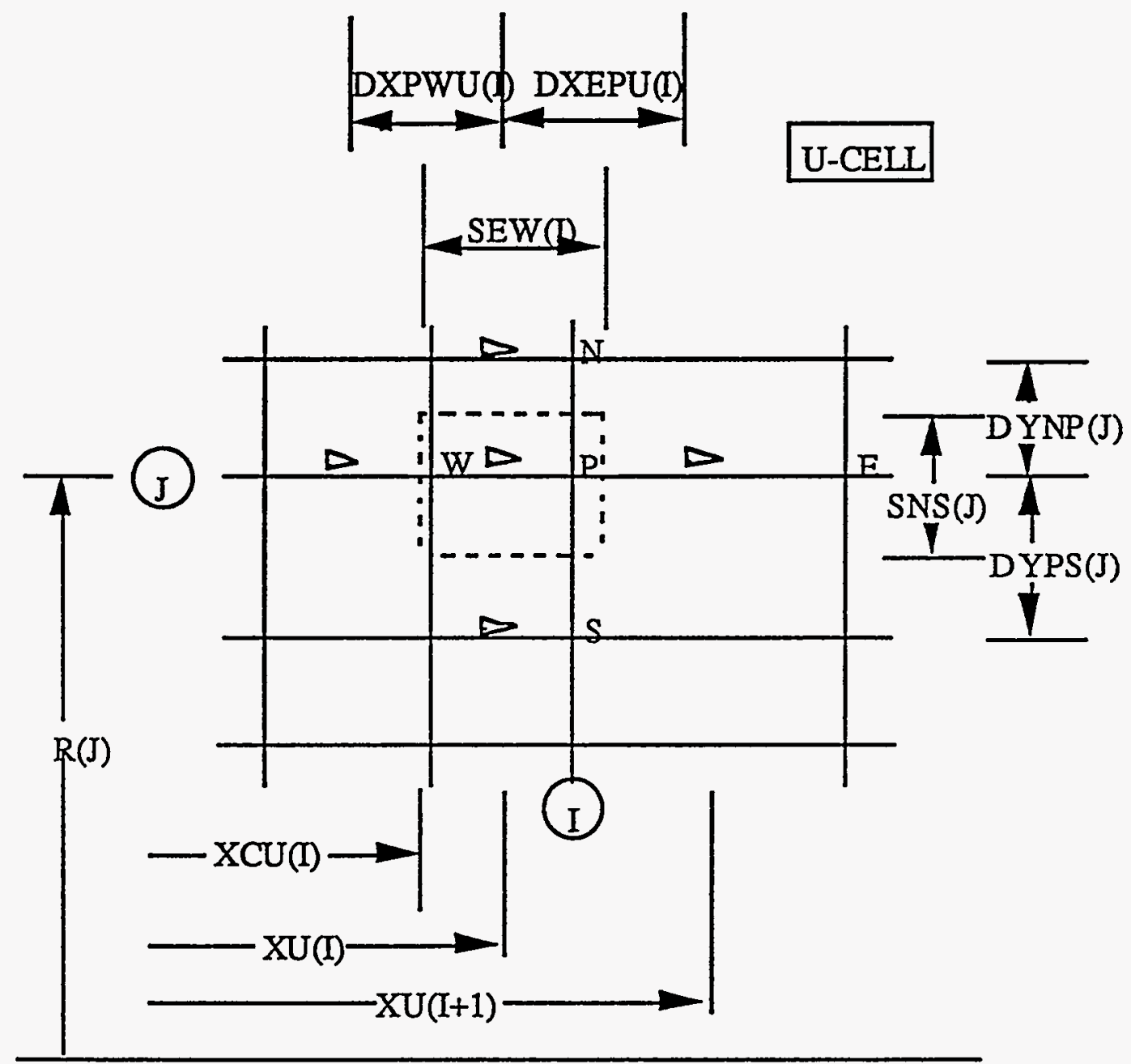

Fig. 3-6. FORTRAN symbols for $u$-cell. 


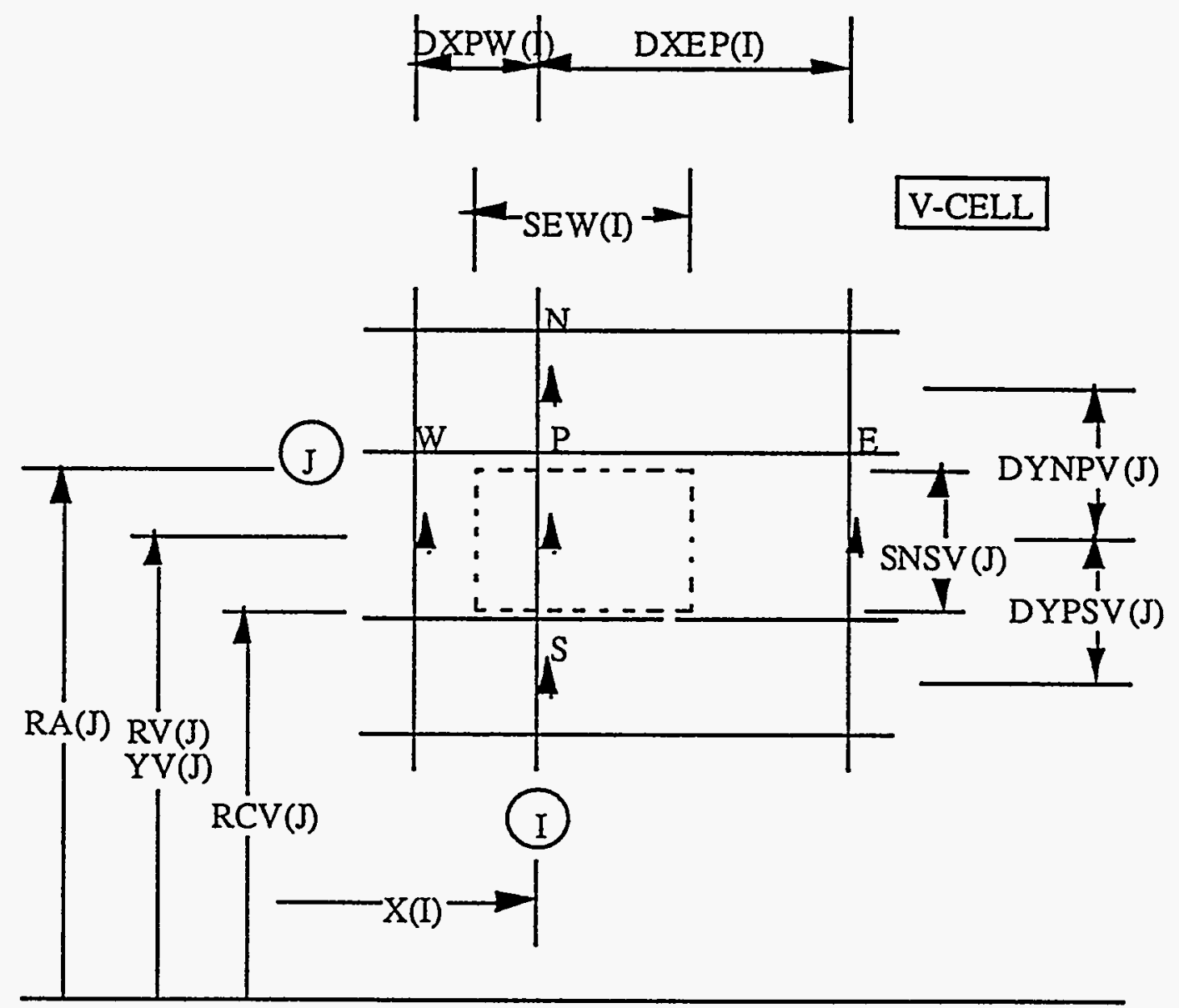

Fig. 3-7. FORTRAN symbols for $v$-cell. 
Solution Technique
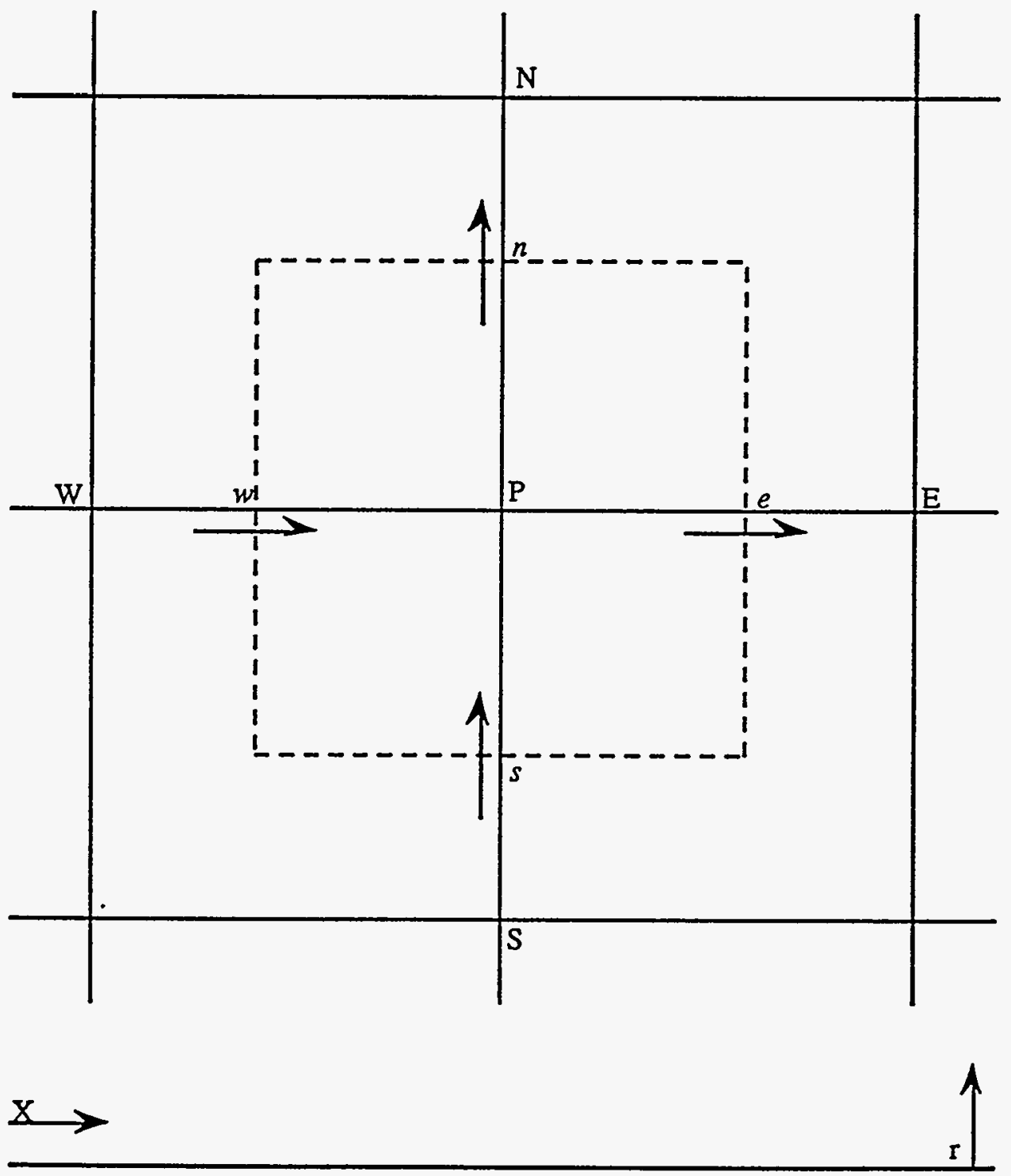

Fig. 3-8. Illustration of the main cell finite-difference grid showing the node point labeling.

$3-18$

93-PCGC-2 User' s Manual 


$$
\int_{x_{w} r_{3}}^{x_{1} r_{x}} \int_{0}^{1}\left[\frac{\partial}{\partial x}(\rho u r \phi)+\frac{\partial}{\partial r}(\rho v r \phi)-\frac{\partial}{\partial x}\left(r \Gamma \frac{\partial \phi}{\partial x}\right)-\frac{\partial}{\partial r}\left(r \Gamma \frac{\partial \phi}{\partial r}\right)-r S^{\phi}\right] d x d r d \xi_{3}=0
$$

where the third coordinate, $\xi_{3}$, has the integration limits of 0 and 1 radians for convenience instead of 0 to $2 \pi$ radians, because of assumed axial symmetry (i.e. the final overall equation is divided by $2 \pi$ ).

Considering the first convection term in Eqn. 3-17

$$
\int_{x_{w} r_{3}}^{x_{s} r_{n}} \int_{0}^{1} \frac{\partial}{\partial x}(\rho u r \phi) d x d r d \xi_{3}
$$

and noting that all properties are uniform in the third direction, we can perform two exact integrations, giving

$$
\int_{r_{s}}^{r_{n}}[\rho u r \phi]_{w}^{e} d r
$$

where $e$ and $w$ indicate that the expression is to be evaluated at the east and west faces, respectively. The derivation to this point is exact. Factoring $r$ from the integrand and applying the mean-value theorem

$$
\begin{aligned}
\int_{r_{s}}^{r_{n}}[\rho u r \phi]_{w}^{e} d r & =\int_{r_{s}}^{r_{n}}[\rho u \phi]_{w}^{e} r d r=\int_{r_{s}}^{r_{n}}\left\{[\rho u \phi]_{e}-[\rho u \phi]_{w}\right\} r d r \\
& \cong\left\{[\overline{\rho u \phi}]_{e}-[\overline{\rho u \phi}]_{w}\right\} \frac{r_{n}^{2}-r_{s}^{2}}{2}
\end{aligned}
$$

where the subscript stands for evaluation at that particular face. The right-hand side of Eqn. 3-21 may be rewritten as 


$$
\left\{[\overline{\rho u \phi}]_{e}-[\overline{\rho u \phi}]_{w}\right\} A_{e w}
$$

where $A_{\text {ew }}$, the area of the east or west face of the cell, is given by

$$
A_{c N}=\int_{r_{3}}^{r_{s}} \int_{0}^{1} r d r d \xi_{3}=\frac{1}{2}\left(r_{n}^{2}-r_{s}^{2}\right)
$$

or

$$
A_{e w}=\frac{1}{2}\left(r_{n}-r_{s}\right)\left(r_{n}+r_{s}\right)=r_{a p}\left(r_{n}-r_{s}\right)
$$

where

$$
r_{a p}=\frac{r_{n}+r_{s}}{2}
$$

For arbitrary grid spacing, $r_{a p}$ is not the same as $r_{p}$. Thus $r_{a p}$ is stored throughout the entire calculational domain.

In the staggered grid system, the $u$ velocities are defined at the cell boundaries where they are required $\left(u_{e}=u_{E}, u_{w}=u_{p}\right)$ and the first convection term now becomes

$$
\rho_{e} \phi_{e} u_{E} A_{e w}-\rho_{w} \phi_{w} u_{p} A_{e w}
$$

We can define convection coefficients as

$$
\begin{aligned}
& C_{E}=\rho_{e} u_{E} A_{e w} \\
& C_{W}=\rho_{w} u_{p} A_{e w}
\end{aligned}
$$

which gives the mass flowrate through the face corresponding to the subscript.

Both $\rho$ and $\phi$ are defined at the main grid nodes and some sort of interpolation is needed to determine their values at the faces midway between node 
points. The practice typically employed is to linearly interpolate dependent variables and use simple averaging for fluid properties. Thus,

$$
\rho_{e}=\frac{1}{2}\left(\rho_{p}+\rho_{E}\right), \rho_{w}=\frac{1}{2}\left(\rho_{w}+\rho_{p}\right)
$$

and

$$
\begin{gathered}
x_{P}<x_{\phi}<x_{E} \quad \phi_{e}=\left(1-f_{E}\right) \phi_{P}+f_{E} \phi_{E}, f_{E}=\frac{x-x_{P}}{x_{E}-x_{P}} \\
x_{W}<x_{\phi}<x_{P} \quad \phi_{w}=\left(1-f_{W}\right) \phi_{W}+f_{W} \phi_{P}, \quad f_{W}=\frac{x-x_{W}}{x_{P}-x_{W}} \\
r_{P}<r_{\phi}<r_{N} \quad \phi_{n}=\left(1-f_{N}\right) \phi_{P}+f_{N} \phi_{N}, \quad f_{N}=\frac{r-r_{P}}{r_{N}-r_{P}} \\
r_{S}<r_{\phi}<r_{P} \quad \phi_{S}=\left(1-f_{S}\right) \phi_{S}+f_{S} \phi_{P}, \quad f_{S}=\frac{r-r_{S}}{r_{P}-r_{S}}
\end{gathered}
$$

Since PCGC-2 always employs cell faces halfway between node points, this interpolation is identical to simple averaging, even for non-uniform grid spacing, since the $f$ s are always equal to 0.5 . Using these relationships, with all $f$ 's set equal to 0.5 , the convection coefficients become

$$
\begin{aligned}
& C_{E}=\frac{1}{2}\left(\rho_{P}+\rho_{E}\right) u_{E} A_{e w} \\
& C_{W}=\frac{1}{2}\left(\rho_{P}+\rho_{w}\right) u_{P} A_{e w}
\end{aligned}
$$


The first convection term in the $f$-equation (Eqn. 3-17) then becomes (upon substitution)

$$
C_{E} f_{E} \phi_{E}-C_{W}\left(1-f_{W}\right) \phi_{W}+\left[C_{E}\left(1-f_{E}\right)-C_{W} f_{W}\right] \phi_{P}
$$

Similarly, the second convection term in Eqn. 3-17 is

$$
\int_{x_{v} r_{2}}^{x_{2} r_{1}} \int_{0}^{1} \frac{\partial}{\partial r}(\rho v r \phi) d x d r d \xi_{3}
$$

Again, two exact integrations can be performed to give

$$
\int_{x_{w}}^{x_{s}}[\rho v r \phi]_{s}^{n} d x
$$

From the mean-value theorem this term becomes

$$
\left\{[\rho v \phi]_{n} r_{n}-[\rho v \phi]_{s} r_{s}\right\}\left(x_{e}-x_{w}\right)
$$

In terms of the geometric quantities

$$
\begin{aligned}
& A_{n}=r_{n}\left(x_{e}-x_{w}\right) \\
& A_{s}=r_{s}\left(x_{e}-x_{w}\right)
\end{aligned}
$$

the convection coefficients are defined as:

$$
\begin{gathered}
C_{N}=\rho_{n} v_{n} A_{n}=\frac{1}{2}\left(\rho_{N}+\rho_{P}\right) v_{N} A_{n} \\
C_{S}=\rho_{s} v_{s} A_{s}=\frac{1}{2}\left(\rho_{S}+\rho_{P}\right) v_{P} A_{s}
\end{gathered}
$$


The final form of the second convection term of the $\phi$-equation becomes:

$$
C_{N} f_{N} \phi_{N}-C_{S}\left(1-f_{S}\right) \phi_{S}+\left[C_{N}\left(1-f_{N}\right)-C_{S} f_{S}\right] \phi_{P}
$$

Considering the diffusion terms in Eqn. 3-17 separately, we have:

$$
\int_{x_{w} r_{s}}^{x_{1} r_{0}} \int_{0}^{1}\left[-\frac{\partial}{\partial x}\left(r \Gamma \frac{\partial \phi}{\partial x}\right)-\frac{\partial}{\partial r}\left(r \Gamma \frac{\partial \phi}{\partial r}\right)\right] d x d r d \xi_{3}
$$

and integrating twice gives:

$$
-\int_{r_{s}}^{r_{s}}\left[r \Gamma \frac{\partial \phi}{\partial x}\right]_{w}^{e} d r-\int_{x_{w}}^{x_{s}}\left[r \Gamma \frac{\partial \phi}{\partial r}\right]_{s}^{n} d x
$$

Using the same technique as presented for convection terms, these last integrals can be evaluated as:

$$
\begin{aligned}
& -\Gamma_{e}\left(\frac{\partial \phi}{\partial x}\right)_{e} r_{a p}\left(r_{n}-r_{s}\right)+\Gamma_{w}\left(\frac{\partial \phi}{\partial x}\right)_{w} r_{a p}\left(r_{n}-r_{s}\right) \\
& -\Gamma_{n}\left(\frac{\partial \phi}{\partial r}\right)_{n} r_{n}\left(x_{e}-x_{w}\right)+\Gamma_{s}\left(\frac{\partial \phi}{\partial r}\right)_{s} r_{s}\left(x_{e}-x_{w}\right)
\end{aligned}
$$

As expected, the same geometric quantities appear as in the convection terms; substituting Eqns. 3-26, 3-40, and 3-41 yields:

$$
-\Gamma_{e}\left(\frac{\partial \phi}{\partial x}\right)_{e} A_{c w}+\Gamma_{w}\left(\frac{\partial \phi}{\partial x}\right)_{w} A_{e w}-\Gamma_{n}\left(\frac{\partial \phi}{\partial r}\right)_{n} A_{n}+\Gamma_{s}\left(\frac{\partial \phi}{\partial r}\right)_{s} A_{s}
$$

The derivatives at the four faces must be expressed in terms of variables at the main node points. Employing central differences (which are second-order accurate) (Dave, 1968) gives: 


$$
\begin{aligned}
& -\Gamma_{e}\left(\frac{\phi_{E}-\phi_{P}}{\delta x_{P E}}\right) A_{e w}+\Gamma_{w}\left(\frac{\phi_{P}-\phi_{W}}{\delta x_{P W}}\right) A_{e w} \\
& -\Gamma_{n}\left(\frac{\phi_{N}-\phi_{P}}{\delta y_{N P}}\right) A_{n}+\Gamma_{s}\left(\frac{\phi_{P}-\phi_{S}}{\delta y_{P S}}\right) A_{s}
\end{aligned}
$$

Where the $\delta x$ and $\delta y$ stand for coordinate distance between the node points indicated by their corresponding subscripts.

Diffusion coefficients can be defined and calculated as:

$$
\begin{gathered}
D_{E}=\Gamma_{e} \frac{A_{e w}}{\delta x_{P E}}=\left(\frac{\Gamma_{P}+\Gamma_{E}}{2}\right)\left(\frac{A_{e w}}{\delta x_{P W}}\right) \\
D_{W}=\Gamma_{w} \frac{A_{e w}}{\delta x_{P W}}=\left(\frac{\Gamma_{P}+\Gamma_{W}}{2}\right)\left(\frac{A_{e w}}{\delta x_{P W}}\right) \\
D_{N}=\Gamma_{n} \frac{A_{n}}{\delta y_{N P}}=\left(\frac{\Gamma_{P}+\Gamma_{N}}{2}\right)\left(\frac{A_{n}}{\delta y_{N P}}\right) \\
D_{S}=\Gamma_{s} \frac{A_{s}}{\delta y_{P S}}=\left(\frac{\Gamma_{P}+\Gamma_{S}}{2}\right)\left(\frac{A_{s}}{\delta y_{P S}}\right)
\end{gathered}
$$

Thus the diffusion terms can be expressed as

$$
-D_{E}\left(\phi_{E}-\phi_{P}\right)+D_{W}\left(\phi_{P}-\phi_{W}\right)-D_{N}\left(\phi_{N}-\phi_{P}\right)+D_{S}\left(\phi_{P}-\phi_{S}\right)
$$

Note the similarity with a control-volume formulation: the exchange coefficients and geometric quantities are contained in the diffusion coefficients $D$, while the 
difference in $\phi$, which drives the diffusion, is explicitly shown with the correct sign ( $\phi$ enters the cell when the $\phi$-difference is negative).

Finally, we are left with the source terms in Eqn. 3-17,

$$
\int_{x_{w}}^{x_{2} r_{n}} \int_{0}^{1} \int_{0} r S^{\phi} d x d r d \xi_{3}
$$

One of the major reasons for the success of the TEACH formulation is the linearization of this source term. Thus

$$
\int_{x_{w} r_{s}}^{x_{2} r_{2}} \int_{0}^{1} \int_{0} r S^{\phi} d x d r d \xi_{3}=S_{U}^{\phi}+S_{P}^{\phi} \phi_{P}
$$

which defines two source term coefficients, $S_{U}^{\phi}$ and $S_{P}^{\phi}$. If the source term happens to be nonlinear in terms of the dependent variable, $\phi_{P}$, the technique calls for $\phi_{P}$ to be factored from the expression (if possible) and to appear with the $S_{P}^{\phi}$ coefficient in Eqn. 3-57. Thus, $\phi_{P}$ appears implicitly while the remaining factored expression involving $\phi_{P}$ will be considered as known (explicit, based on previous values) and lumped together in the $S_{U}^{\phi}$ coefficient of Eqn. 3-57. Furthermore, as shown later in this chapter, $S_{P}^{\phi}$ must be negative to guarantee stable convergence.

In general, $S^{\phi}$ will be a function of all the dependent variables and other fluid properties as well as various types of derivatives involving these quantities. When integrating this source term, the value prevailing at the cell center will be used for all quantities. Any derivatives will be evaluated by central differencing. Therefore, the source term is considered constant, and:

$$
\int_{x_{w} r_{s}}^{x_{4} r_{0}} \int_{0}^{1} r S^{\phi} d x d r d \xi_{3}=S^{\phi}(\Delta V)=S_{U}^{\phi}+S_{P}^{\phi} \phi P
$$

where $\Delta V$ is the volume of the cell.

Upon substitution of these newly defined coefficients, the general $\phi$ equation becomes 


$$
\begin{aligned}
& {\left[C_{E} f_{E}-D_{E}\right] \phi_{E}-\left[C_{W}\left(1-f_{W}\right)+D_{W}\right] \phi_{W}+\left[C_{N} f_{N}-D_{N}\right] \phi_{N}-\left[C_{S}\left(1-f_{S}\right)+D_{S}\right] \phi_{S}} \\
& +\left[C_{E}\left(1-f_{E}\right)+D_{E}-C_{W} f_{W}+D_{W}+C_{N}\left(1-f_{N}\right)+D_{N}-C_{S} f_{S}+D_{S}\right] \phi_{P}=S_{U}+S_{P} \phi_{P}
\end{aligned}
$$

Adding and subtracting $C_{E}-C_{W}+C_{N}-C_{S}$ from the bracket preceding $\phi_{P}$ and rearranging to obtain common expressions yields:

$$
\begin{aligned}
& {\left[\left(C_{E}-C_{W}+C_{N}-C_{S}\right)+D_{W}+\left(1-f_{W}\right) C_{W}\right.} \\
& \left.+D_{E}-f_{E} C_{E}+D_{S}+\left(1-f_{S}\right) C_{S}+D_{N}-f_{N} C_{N}\right] \phi_{P} \\
& =\left[D_{W}+\left(1-f_{W}\right) C_{W}\right] \phi_{W}+\left[D_{E}-f_{E} C_{E}\right] \phi_{E}+\left[D_{S}+\left(1-f_{S}\right) C_{S}\right] \phi_{S} \\
& +\left[D_{N}-f_{N} C_{N}\right] \phi_{N}+S_{U}+S_{P} \phi_{P}
\end{aligned}
$$

It is convenient to define new total coefficients to replace these common expressions:

$$
\begin{gathered}
A_{E}=D_{E}-f_{E} C_{E} \\
A_{W}=D_{W}+\left(1-f_{W}\right) C_{W} \\
A_{N}=D_{N}-f_{N} C_{N} \\
A_{S}=D_{S}+\left(1-f_{S}\right) C_{S}
\end{gathered}
$$

The $A$ 's with the single subscript are not area coefficients, but convection/diffusion coefficients. In terms of these total coefficients, the finitedifference form of the $\phi$-equation becomes 


$$
\begin{aligned}
& {\left[C_{E}-C_{W}+C_{N}-C_{S}+A_{E}+A_{W}+A_{N}+A_{S}\right] \phi_{P}} \\
& =A_{E} \phi_{E}+A_{W} \phi_{W}+A_{N} \phi_{N}+A_{S} \phi_{S}+S_{U}+S_{P} \phi_{P}
\end{aligned}
$$

\section{First Modification (Stability)}

Checking to see if mass is conserved, we can express the continuity equation in finite-difference form as follows. From Eqn. 3-10

$$
\int_{x_{w} r_{1}}^{x_{1} r_{0} r_{1}}\left[\frac{\partial}{\partial x}(r \rho u)+\frac{\partial}{\partial r}(r \rho v)-r S_{p}^{m}\right] d x d r d \xi_{3}=0
$$

or

$$
\int_{r_{s}}^{r_{n}}[r \rho u]_{w}^{e} d r+\int_{x_{w}}^{x_{e}}[r \rho v]_{s}^{n} d x=S_{p}^{m}(\Delta V)
$$

and in terms of convection coefficients,

$$
C_{E}-C_{W}+C_{N}-C_{S}-S_{p}^{m}(\Delta V)=\dot{M}_{n e t}
$$

At steady-state, $\dot{M}_{\text {net }}$ should equal zero in a converged solution. During the course of iterating to obtain a converged solution, the quantity $\left(C_{E}-C_{W}+C_{N}-C_{S}-S_{p}^{m}(\Delta V)\right)$ will not in general vanish. In addition, a situation may arise where all of the total coefficients ( $A$ 's) are zero, thus causing the finitedifference $\phi$-equation (Eqn. 3-65) to become singular. Therefore, we cannot allow this grouping of convection coefficients on the LHS of Eqn. 3-65 to become equal to zero. From the definitions of these coefficients (Eqns. 3-61 to 3-64), if $\dot{M}_{\text {net }}$ is positive, then mass is being lost in the cell and a mass flow rate of $C_{E}-C_{W}+C_{N}-C_{S}-S_{p}^{m}(\Delta V)$ is carrying the property of $\phi$ out of the cell. Under such conditions, it is reasonable to set $\dot{M}_{\text {net }}$ to zero and to include an additional term corresponding to the mass flow rate $C_{E}-C_{W}+C_{N}-C_{S}-S_{p}^{m}(\Delta V)$ carrying the (old) property $\phi_{p}^{\text {old }}$ with it. The other possibility (of $\dot{M}_{\text {net }}$ being negative) 
implies accumulation of mass in the cell, with $\phi$ being transported from the neighboring cells. Since Eqn 3-65 predicts this, no modification is necessary. Combining this modification into the source coefficients, Eqn. 3-65 becomes

$$
\left[A_{E}+A_{W}+A_{N}+A_{S}\right] \phi_{P}=A_{E} \phi_{E}+A_{W} \phi_{W}+A_{N} \phi_{N}+A_{S} \phi_{S}+S_{U}+S_{P} \phi_{P}
$$

with

$$
\begin{gathered}
S_{U}=\max \left\{C_{E}-C_{W}+C_{N}-C_{S}-S_{P}^{m}(\Delta V), 0\right\} \phi_{P}^{\text {old }}+S_{U}^{\text {rest }} \\
S_{P}=-\max \left\{C_{E}-C_{W}+C_{N}-C_{S}-S_{P}^{m}(\Delta V), 0\right\}+S_{P}^{\text {rest }}-S_{P}^{m}(\Delta V)
\end{gathered}
$$

where

$$
\phi_{P}^{\text {old }}=\text { old or known value of } \phi \text { at node } P
$$

$$
\begin{aligned}
S_{U}^{\text {rest }}, S_{P}^{\text {rest }}= & \text { source coefficients of the original equation } \\
& \text { given by Eqn. III.B }-57 \text { times the volume of the cell }
\end{aligned}
$$

This modification will ensure that the finite-difference equation will behave reasonably when the mass flow rates for the control volume do not satisfy continuity.

Equation 3-69 can be written more compactly by introducing a subscript $d$ for the four directions $(E, W, N, S)$, and combining the coefficients of the $\phi_{P}$ term:

$$
\left[\left(\sum_{d} A_{d}\right)-S_{P}\right] \phi_{P}=\sum_{d} A_{d} \phi_{d}+S_{U}
$$

where $S_{U}$ and $S_{P}$ are given by Eqns. 3-70 and 3-71. Another coefficient can be defined as

$$
A_{P}=\sum_{d} A_{d}-S_{P}
$$


and the standard equation form which TEACH solves is expressed as

$$
A_{P} \phi_{P}=\sum_{d} A_{d} \phi_{d}+S_{U}
$$

\section{Second Modification (Improved Differencing Scheme)}

Thus far the derivation resulting in Eqn. 3-76 has used central differencing for all terms. Because of non-linearities in the transport equations, central differencing is not exact. For problems in computational fluid mechanics with large convection terms (i.e. cell Reynolds numbers greater than 2), central differencing causes problems in some locations (Roache, 1976; Patankar, 1980).

A simple, one-dimensional analysis, for which a known exact solution exists, can be performed (Wormeck, 1976; Patankar, 1980). It can be concluded from such an analysis that central differencing is preferable for small cell Reynolds numbers, and upwind differencing is performed for large values, but neither is satisfactory for all Reynolds numbers. Equation 3-76 is therefore modified to conform to these non-linearities while at the same time conserving mass. This approach is discussed in more detail by Patankar (1980). Questions and modifications concerning stability and convergence rates will be discussed in the next section.

The technique employed by PCGC-2 alters the differencing scheme according to the local value of the cell Reynolds Number $(R e)$. For example, for a west face we note that:

$$
\begin{gathered}
G_{w} A_{w}=(\rho u A)_{w}=C_{W} \\
R e_{w}=\left(\frac{\rho u \delta}{\mu}\right)_{w}=\left(\frac{\rho u A}{\frac{\mu}{\delta} A}\right)_{w}=\frac{C_{W}}{D_{W}}
\end{gathered}
$$

Therefore, the local cell Reynolds number is a measure of the relative magnitude of convection and diffusion. When convection terms are very small relative to diffusion terms (small $R e$ ), then central differencing is preferred. However, when convection terms are large relative to diffusion terms (large $R e$ ) then upwind differencing is preferred. In middle regions of $R e$, some combination is preferred. PCGC-2 uses either 1) hybrid differencing or 2) power-law differencing. 
The mathematical representation of the alternate schemes is as follows. The total coefficients for each direction are rewritten as:

$$
\begin{gathered}
A_{N}=\Omega_{N}+\max \left(0,-C_{N}\right) \\
A_{S}=\Omega_{S}+\max \left(0, C_{S}\right) \\
A_{E}=\Omega_{E}+\max \left(0,-C_{E}\right) \\
A_{W}=\Omega_{E}+\max \left(0, C_{W}\right)
\end{gathered}
$$

where $\Omega$ depends on the differencing scheme used. For upwind differencing:

$$
\Omega_{d}=D_{d}
$$

For central differencing:

$$
\Omega_{d}=D_{d}-0.5\left|C_{d}\right|
$$

For hybrid differencing:

$$
\Omega_{d}=\max \left[0,\left(D_{d}-0.5\left|C_{d}\right|\right)\right]
$$

For power-law differencing:

$$
\Omega_{d}=\max \left\{0, D_{d}\left[\frac{D_{d}-0.1\left|C_{d}\right|}{D_{d}}\right]^{5}\right\}
$$

The difference between the various differencing schemes can be summarized as follows: in upwind differencing, the finite difference is taken in the direction from which the velocity vector is arriving. Central differencing has already been 
discussed. Hybrid differencing uses upwind differencing when cell Reynolds

Numbers are larger than 2 or less than -2 and central differencing when $-2<\operatorname{Re}<2$. Power-law differencing uses the fifth-order-power-law expression for $-10<\mathrm{R} .<<10$ and upwind differencing outside this range. For both power-law and hybrid differencing, the appropriate differencing is applied to convection terms. Diffusion terms are ignored for high Reynolds numbers (pure upwind differencing). These advanced differencing options allow for greater accuracy with less grid resolution than is permissible with central differencing. The power-law scheme is recommended in all cases.

\section{Convergence Test}

The finite-difference equations will now be analyzed for convergence criteria. Writing Eqn. 3-76 as

$$
\phi_{p}=\sum_{d} \dot{A_{d}} \phi_{d}+\dot{S_{U}}
$$

where $A_{d}^{\prime}=A_{d} / A_{p}$ and $S_{U}^{\prime}=S_{U} / A_{p}$, we have a system of linear algebraic equations of the form

$$
x_{i}=\sum_{j, j \neq i}\left(a_{i j} x_{j}\right)+b_{i}
$$

From the theory of linear equations (Gosman et al., 1969; Patankar and Spalding, 1970 ) if the $a_{i j}$ and $b_{i}$ are constants, the sufficient conditions for convergence are:

$$
\sum_{i, j \neq i}\left|a_{i j}\right|\left\{\begin{array}{l}
<1 \text { for all } i \\
<1 \text { for at least one } i
\end{array}\right.
$$

Since $A_{d}^{\prime}$ and $S_{U}^{\prime}$ in Eqn. 3-87 are not constant, the criteria in Eqn. 3-89 do not apply exactly. However, as an approximation, we may expect convergence if

$$
\sum_{d} \frac{A_{d}}{A_{d}-S_{p}}\left\{\begin{array}{l}
<1 \text { for all } i \\
<1 \text { for at least one } i
\end{array}\right.
$$


which implies that $S_{p}$ must be less than zero. The $S_{p}$ term is the source term involving the dependent variable implicitly (based on new value). Therefore, to insure a negative value for $S_{p}$, if any source term containing the dependent variable is positive, the old value of $\phi$ must be used and the term combined this term with the $S_{U}$ quantity (i.e., appearing explicitly).

Since the coefficients are not constant, convergence is not guaranteed by Eqn. 3-88 but is reasonably assured if $S_{p}<0$. If divergence is encountered, underrelaxation may be used as discussed later. PCGC-2 generally converges if the source terms are linear or mildly nonlinear. Highly nonlinear source terms may not converge or require very small $(0.1-0.3)$ under-relaxation factors.

\section{Solution of the Finite-Difference Equations (TDMA)}

PCGC-2 has a large system of linear algebraic finite-difference equations to solve, and vast numbers of techniques are available (Carnahan et al., 1969) to solve this system of equations. Some of the more popular methods to solve the fluid flow type of equation systems are presented elsewhere (Roache, 1976).

Matrix inversion and other direct techniques are out of the question because of the large number of equations involved, and therefore iterative methods must be employed. The formulation in terms of vorticity and stream function (Gosman, et al., 1969) utilized a Gauss-Seidel algorithm, which is a point-by-point method. The equations are solved one at a time, passing from node point to node point throughout the flow field. New values of the variables are used as soon as they become available, and the complete flow field is solved for each dependent variable before going to the next dependent variable.

The technique used in PCGC-2 is to solve simultaneously a line of node points in a so-called line-by-line method. This line-by-line technique is implemented in a very efficiently numbered scheme, the Tri-Diagonal Matrix Algorithm (TDMA). The equations are of the form:

$$
D_{i} \phi_{i}=A_{i} \phi_{i+1}+B_{i} \phi_{i-1}+C_{i}
$$

where $i=2, N-1$ and the values of the constant coefficients $A_{i}, B_{i}, C_{i}$, and $D_{i}$ are given for $i=2, N-1$ together with the boundary values $\phi_{1}$ and $\phi_{N}$. The procedure calls for rewriting Eqn. 3-91 as:

$$
\phi_{i}=\dot{A_{i}} \phi_{i+1}+\dot{C_{i}}
$$


and obtaining a solution by successive substitution, starting with $i=N-1$ and working backwards to $i=2$. The transformed coefficients $A_{i}^{\prime}$ and $C_{i}^{\prime}$ are given by the recurrence relations:

$$
\begin{aligned}
& \dot{A_{i}}=\frac{A_{i}}{D_{i}-B_{i} A_{i-1}} \\
& C_{i}=\frac{C_{i}+C_{i-1} B_{i}}{D_{i}-B_{i} \dot{A_{i-1}}}
\end{aligned}
$$

with the starting values of $\dot{A_{l}}=0$ and $C_{1}^{i}=\phi_{1}$.

The TDMA can be implemented to solve Eqn. 3-76 by considering the equations for all the control volumes along a grid line with the last or best estimates for the values of $\phi$ along the two neighboring grid lines, and hence constructing an equation set which can be solved by the TDMA. In this manner, we can first traverse along all the grid lines in, say, the $x$ direction; then, using this solution as the best estimate, go over lines in the $r$ direction.

For the r-direction sweep, Eqn. 3-76 is written as:

$$
A_{P} \phi_{P}^{\prime}=A_{N} \phi_{N}^{\prime}+A_{S} \phi_{S}^{\prime}+\left(A_{E} \phi_{E}+A_{W} \phi_{W}+S_{U}\right)
$$

where the term in the parentheses is considered known and TDMA can be applied. The superscript 'denotes the values obtained from this first phase of solution, while the second phase, the $\mathrm{x}$-direction sweep, is the solution of:

$$
A_{P} \phi_{P}^{\prime \prime}=A_{E} \phi_{E}^{\prime \prime}+A_{W} \phi^{\prime \prime}{ }_{W}+\left(A_{N} \phi_{N}^{\prime}+A_{S} \phi_{S}^{\prime}+S_{U}\right)
$$

in a similar manner.

It must be remembered that the TDMA is an iterative solution technique to solve the finite-difference equations, the coefficients of which are only tentative and require updating to account for the changes in the values of the variables. The number of sweeps needed for an accurate solution of the finite-difference equations before the coefficients are recalculated is arbitrary and problem-dependent. Convergence criteria are used to converge the equations uniformly (van Doormaal and Raithby, 1984). 


\section{Final Form}

The final form of the $f$-finite-difference equation in PCGC-2 is:

$$
A_{P} \phi_{P}=A_{E} \phi_{E}+A_{W} \phi_{W}+A_{N} \phi_{N}+A_{S} \phi_{S}+S_{U}
$$

where

$$
\begin{gathered}
A_{P}=A_{E}+A_{W}+A_{N}+A_{S}-S_{P} \\
A_{E}=\Omega_{E}+\max \left(0,-C_{E}\right) \\
A_{W}=\Omega_{W}+\max \left(0, C_{W}\right) \\
A_{N}=\Omega_{N}+\max \left(0,-C_{N}\right) \\
\Omega_{d}=\left\{\begin{array}{c}
A_{S}=\Omega_{S}+\max \left(0, C_{S}\right) \\
\max \left[0, D_{d}\left(\frac{D_{d}-0.1\left|C_{d}\right|}{D_{d}}\right)^{5}\right] \text { for power - law differencing } \\
\max \left[0, D_{d}-0.5\left|C_{d}\right|\right] \\
\text { for hybrid differencing } \\
\text { where } d=E, W, N, \text { or } S \\
D_{E}=\frac{\frac{1}{2}\left(\Gamma_{P}+\Gamma_{E}\right)\left(A_{\text {ew }}\right)}{\delta x_{P E}}
\end{array}\right.
\end{gathered}
$$




$$
\begin{aligned}
& D_{W}=\frac{\frac{1}{2}\left(\Gamma_{P}+\Gamma_{W}\right)\left(A_{e W}\right)}{\delta x_{P W}} \\
& D_{N}=\frac{\frac{1}{2}\left(\Gamma_{P}+\Gamma_{N}\right)\left(A_{n}\right)}{\delta x_{N P}} \\
& D_{S}=\frac{\frac{1}{2}\left(\Gamma_{P}+\Gamma_{S}\right)\left(A_{S}\right)}{\delta x_{P S}} \\
& C_{E}=\frac{\frac{1}{2}\left(\rho_{P}+\rho_{E}\right)\left(A_{c W}\right)}{u_{E}} \\
& C_{W}=\frac{\frac{1}{2}\left(\rho_{P}+\rho_{W}\right)\left(A_{c W}\right)}{u_{P}} \\
& C_{S}=\frac{\frac{1}{2}\left(\rho_{P}+\rho_{S}\right)\left(A_{S}\right)}{v_{P}} \\
& C_{N}=\frac{\frac{1}{2}\left(\rho_{P}+\rho_{N}\right)\left(A_{N}\right)}{v_{N}}
\end{aligned}
$$

$$
S_{U}=\max \left[C_{E}-C_{W}+C_{N}-C_{S}-S_{P}^{m}(\Delta V), 0\right] \phi_{P}^{\text {old }}+S_{U}^{\text {rest }}(\text { vol })
$$

$$
S_{P}=-\max \left[C_{E}-C_{W}+C_{N}-C_{S}-S_{P}^{m}(\Delta V), 0\right]+S_{P}^{\text {rest }}(\text { vol })-S_{P}^{m}(\Delta V)
$$




$$
S_{U}^{r e s t}+S_{P}^{r e s t} \phi_{P}=S^{\phi}
$$

and the grid definition implies that all the $f$ s are equal to 0.5 .

\section{Finite-Difference Form of the Momentum Equation}

The finite-difference form of the momentum equation closely resembles Eqn. 3-97 for the general $\phi$-equation, with two exceptions. First, as mentioned previously, a staggered-velocity grid is employed for the definition of the velocity components (see Figs. 3-9 and 3-10), and therefore control volumes centered at these velocity locations are defined which are different from those for the other dependent variables. Secondly, the pressure gradient term, which may be regarded as a source of momentum, is treated in a special manner, and the geometric coefficient which relates a velocity component to the pressure difference between the adjacent nodes is stored for use in the pressure equation.

If the pressure distribution were known, the momentum equations (Eqns. 311 to 3-13) would be uncoupled from the continuity equation (Eqn. 3-10) and could be solved in a straightforward manner. Unfortunately, the pressures are not known in advance.

PCGC-2 employs the SIMPLE (Semi-Implicit Method for Pressure-Linked Equations) algorithm and two of its variants: SIMPLER (SIMPLE-Revised) and SIMPLEC (Patankar and Spalding, 1970, 1972; Patankar, 1980; van Doormaal and Raithby, 1984). In these techniques, the pressure distribution is guessed (i.e. assumed known) and denoted by $p^{*}$. The solution of the momentum equations for the two velocity components that correspond with this guessed (or "starred") pressure field becomes the "starred" velocity field. Corrections are then made to the "starred" pressure field so as to bring the velocity field into conformity with the continuity equation.

u-Equation. From Eqn. 3-11, the u-equation is:

$$
\frac{\partial}{\partial x}(r \rho u u)+\frac{\partial}{\partial r}(r \rho u v)-\frac{\partial}{\partial x}\left(r \mu \frac{\partial u}{\partial x}\right)-\frac{\partial}{\partial r}\left(r \mu \frac{\partial u}{\partial r}\right)=-r \frac{\partial p}{\partial x}+r S^{\mu}
$$

where the source term is given by Eqn. 3-14. The convection and diffusion terms are identical to the $\phi$-equation with $\phi=u$ and $\Gamma=\mu$, and hence the derivation of the finite-difference equations is similar to the $f$-equation, but the integration is performed over the $u$-control volume, which is staggered from the main $\phi$-cell. The pressure term is handled explicitly. Figure 3-9 shows a typical $u$-cell, and the 
superscript $u$ means that reference to this $u$-cell is being made instead of to the main control volume.

Integrating Eqn. 3-115 over the $u$-cell in the same manner as the $\phi$-equation was integrated over the main or $p$-cell, the first convection term becomes:

$$
\int_{x_{m}^{*} r_{d}^{*}}^{x_{0}^{k} r_{0}^{k}} \int_{0}^{1} \frac{\partial}{\partial x}(r p u u) d \xi_{3} d r d x
$$

or

$$
(\rho u u)_{e}^{u} A_{e w}^{u}-(\rho u u)_{w}^{u} A_{e w}^{u}
$$

A problem arises here as to which way to expand the density and velocity. For example, one method would be to linearly interpolate the velocities and simply average the density. Another method would be to linearly interpolate the product $\rho u=G$ as well as the remaining $u$. The former method results in:

$$
\rho_{e}^{u}\left(u_{e}^{u}\right)^{2}=\rho_{e}^{u}\left[\left(1-f_{E}^{u}\right) u_{p}+f_{E}^{u} u_{E}\right]^{2}
$$

and, for $f_{e}^{u}=\frac{1}{2}$, the east face convection term is:

$$
\frac{1}{4} A_{c w}^{u} \rho_{P}\left(u_{P}+u_{E}\right)^{2}
$$

The latter method yields

$$
(\rho u)_{e}^{u} u_{e}^{u}=\left[\left(1-f_{E}^{u}\right)(\rho u)_{P}+f_{E}^{u}(\rho u)_{E}\right]\left[\left(1-f_{E}^{u}\right) u_{P}+f_{E}^{u} u_{E}\right]
$$

requiring the densities midway between their points of definition. By averaging them and taking $f_{c}^{u}=\frac{1}{2}$, the convection coefficient through the east face is given by:

$$
\frac{1}{2} A_{s w}^{u}\left[\left(\frac{\rho_{E}+\rho_{P}}{2}\right) u_{E}+\left(\frac{\rho_{P}+\rho_{W}}{2}\right) u_{P}\right]
$$




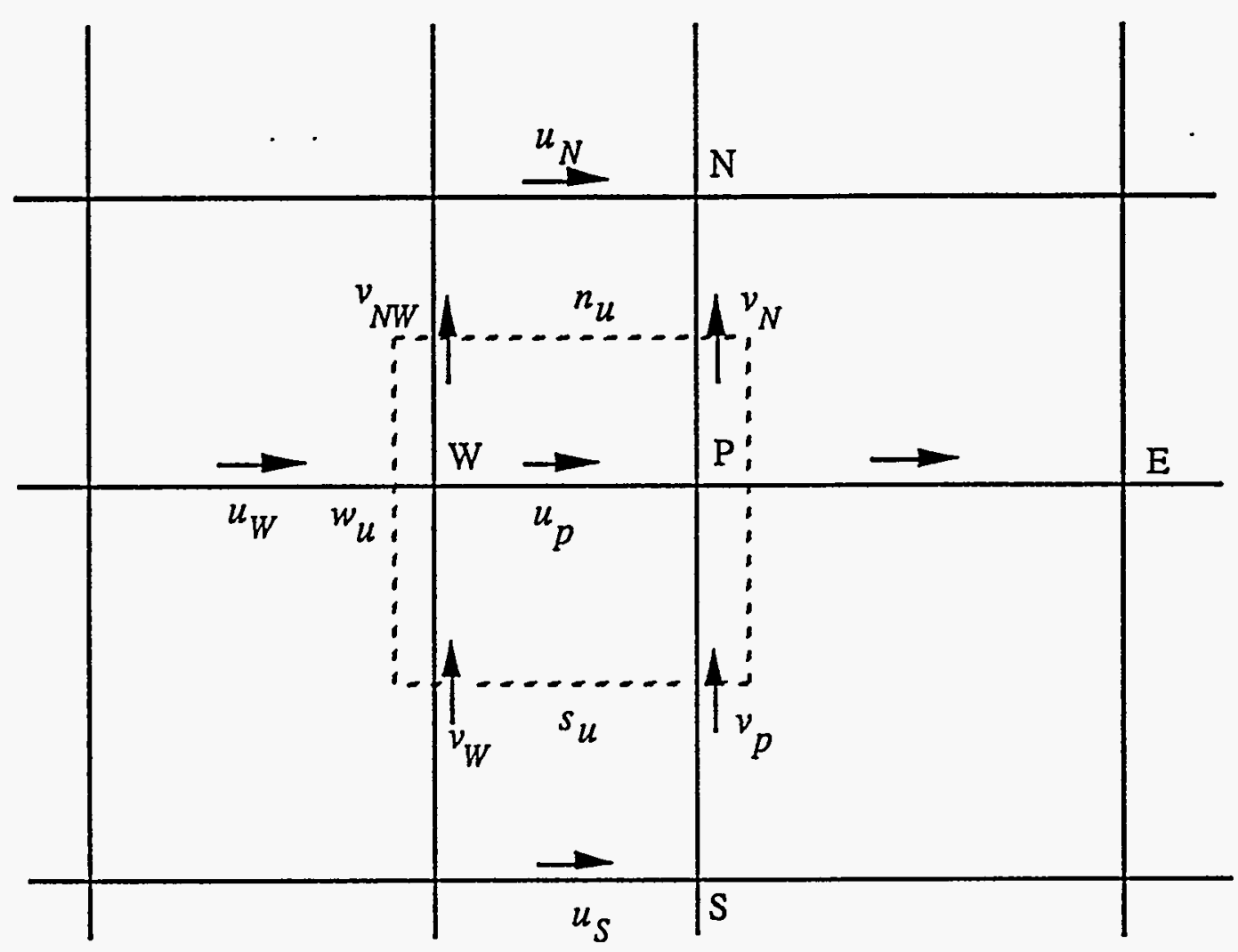

Fig. 3-9. A typical $u$-cell showing adjacent velocities. Other symbols define the location of the control volume over which the first momentum equation is integrated. 


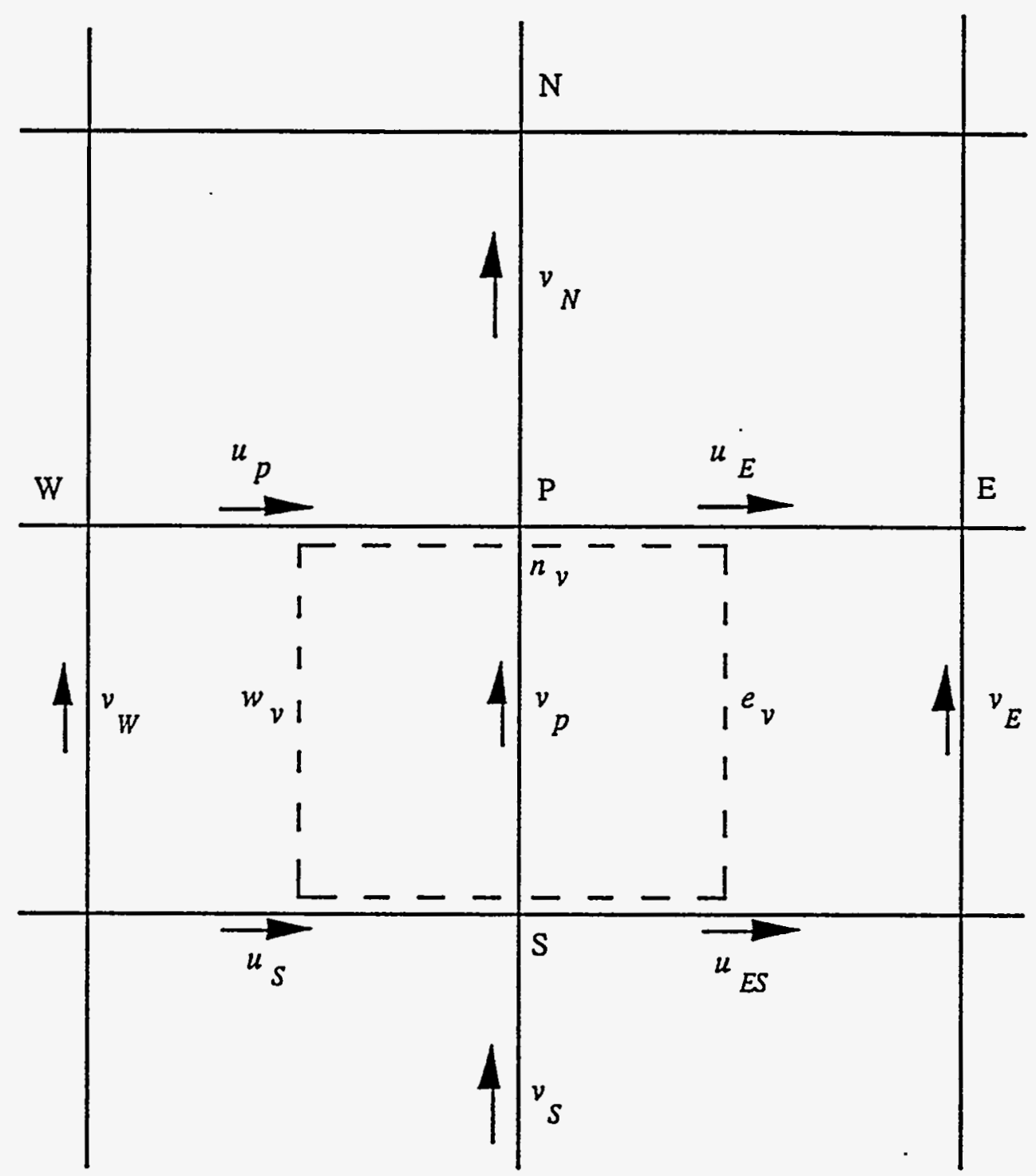

Fig. 3-10. A typical v-cell showing adjacent velocities. Other symbols define the location of the control volume over which the second momentum equation is integrated. 
$(6 Z I-\varepsilon)$

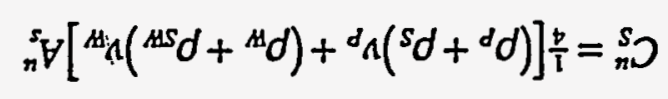

$(8 Z I-\varepsilon)$

$$
{ }_{n}^{W}\left[\left[{ }^{M N} \wedge\left({ }^{M N} d+{ }^{M} d\right)+{ }^{N} n\left({ }^{N} d+{ }^{d} d\right)\right] \frac{b}{I}={ }_{n}^{N} D\right.
$$

$(L Z I-\varepsilon)$

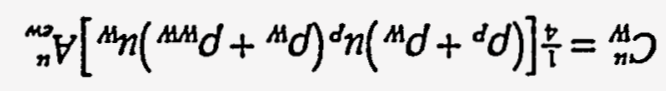

$(9 Z I-\varepsilon)$

$$
{ }_{n}^{m}\left[{ }^{d} n\left({ }^{A} d+{ }^{d} d\right)+{ }^{3} n\left({ }^{3} d+d d\right)\right] \frac{b}{1}={ }_{n}^{3} D
$$

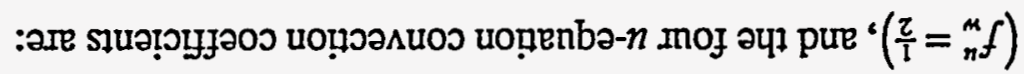

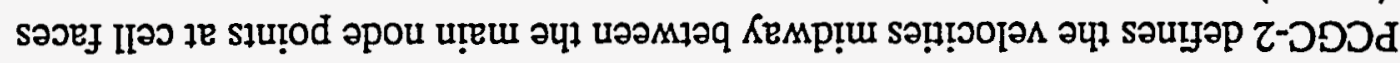
$(\varsigma Z I-\varepsilon)$

$$
\frac{M x-d x}{M_{X}-d_{n} x}=M_{n} f
$$

วรวบМ

$(\pitchfork Z I-\varepsilon)$

$$
d_{n}\left(\frac{\tau}{d d+S d}\right) M_{n} f+M_{\Lambda}\left(\frac{Z}{M S_{d}+M_{D}}\right)\left(M_{n} f-I\right)={ }_{n}^{s}(\Lambda d)
$$

$(\varepsilon \tau I-\varepsilon)$

$$
N_{\Lambda}\left(\frac{\tau}{d d+N_{d}}\right) M f+M N_{n}\left(\frac{\tau}{M N_{d}+M d}\right)\left(M_{n} f-I\right)={ }_{n}^{u}(\Lambda d)
$$

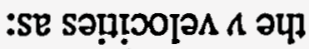

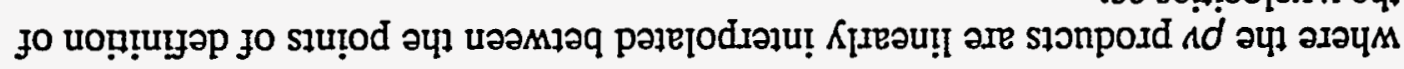
$(Z Z I-\varepsilon)$

$$
{ }_{n}^{s}{ }_{n}^{s}(n \cap d)-{ }_{n}^{u}{ }_{n}^{u}(n \cap d) \text {. }
$$

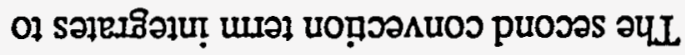


where

$$
\rho_{W W}=\rho_{i-2, j}
$$

The diffusion terms for the $u$-equation are defined as:

$$
\begin{aligned}
& D_{E}^{\mu}=\frac{\mu_{e}^{u} A_{e w}^{u}}{\delta x_{E P}^{u}} \\
& D_{W}^{u}=\frac{\mu_{w}^{u} A_{e w}^{u}}{\delta x_{P W}^{u}} \\
& D_{N}^{u}=\frac{\mu_{n}^{u} A_{n}^{u}}{\delta y_{N P}} \\
& D_{S}^{\mu}=\frac{\mu_{s}^{u} A_{s}^{u}}{\delta y_{P S}}
\end{aligned}
$$

where the viscosities are interpolated as:

$$
\begin{gathered}
\mu_{e}^{u} \approx \mu_{P} \\
\mu_{w}^{u} \approx \mu_{W} \\
\mu_{n}^{u}=\frac{1}{4}\left(\mu_{N W}+\mu_{N}+\mu_{W}+\mu_{P}\right) \\
\mu_{s}^{u}=\frac{1}{4}\left(\mu_{w}+\mu_{P}+\mu_{S W}+\mu_{s}\right)
\end{gathered}
$$


Considering the pressure term in Eqn. 3-115

$$
\int_{x_{n}^{*}=r_{0}^{0}}^{x_{0}^{*} r_{n}^{*}} \int_{0}^{1}\left(-r \frac{\partial p}{\partial x}\right) d \xi_{3} d r d x
$$

and integrating twice yields:

$$
\begin{aligned}
& -\int_{x_{n}^{*}}^{x_{s}^{u}}\left[\frac{1}{2}\left(r_{n}^{u}\right)^{2}-\left(r_{s}^{u}\right)^{2}\left(\frac{\partial p}{\partial x}\right)\right] d x \\
& =-\frac{1}{2}\left(r_{n}^{u}-r_{s}^{u}\right)\left(r_{n}^{u}+r_{s}^{u}\right)[p]_{x_{s}^{*}}^{x_{u}^{*}}
\end{aligned}
$$

and since

$$
\begin{aligned}
& r_{P}=\frac{1}{2}\left(r_{n}^{u}+r_{s}^{u}\right) \\
& A_{e w}^{u}\left(-p_{e}^{u}+p_{w}^{u}\right)
\end{aligned}
$$

where these pressures are already defined at their required locations to give:

$$
A_{e w_{*}}\left(p_{W}-p_{P}\right)
$$

Since the leading coefficient will be needed in the pressure equation, it is stored as a new area coefficient, $D^{u}=A_{e}^{u}$. The expression

$$
D^{u}\left(p_{W}-p_{P}\right)
$$

will be incorporated into the $S_{U}$ term. Also note that for turbulent flow, the normal stress (pressure) is augmented by $\frac{2}{3} \rho k$.

Evaluating the source terms the same way, the final $u$-equation is 


$$
A_{P} u_{P}=\sum_{d} A_{d} u_{d}+D^{u}\left(p_{W}-p_{P}\right)+S_{U}
$$

where ${ }^{1}$

$$
\begin{gathered}
A_{p}=\sum_{d} A_{d}-S_{p} \\
A_{d}=\Omega_{d}+\max \left(0,-C_{d}\right) \text { for } d=E \text { or } N \\
A_{d}=\Omega_{d}+\max \left(0, C_{d}\right) \text { for } d=W \text { or } S \\
\Omega_{d}=\max \left[0, D_{d}\left(\frac{D_{d}-0.1\left|C_{d}\right|}{D_{d}}\right)^{5}\right] \\
S_{U}=\max \left\{C_{E}-C_{W}+C_{N}-C_{S}-S_{p}^{m}(\Delta V), 0\right\} u_{P}+S_{U}^{\text {rest }}(\mathrm{vol}) \\
S_{p}=-\max \left\{C_{E}-C_{W}+C_{N}-C_{S}-S_{p}^{m}(\Delta V), 0\right\}+\left(S_{P}^{\text {rest }}\right)(\text { vol })-S_{p}^{m}(\Delta V)
\end{gathered}
$$

and $C_{d}$ and $D_{d}$ are given by Eqns. 3-126 to 3-134.

$y$ and $w$ Equations. Following a similar procedure, the second momentum equation, Eqn. 3-12 can be expressed as:

$$
A_{p} v_{p}=\sum_{d} A_{d} v_{d}+S_{U}
$$

${ }^{1}$ This $\Omega_{d}$ is for power-law differencing. If hybrid differencing is used, $\Omega_{d}=\max \left[0, D_{d}-0.5\left|C_{d}\right|\right]$. 
where the total coefficients are defined in the usual manner, but $S_{U}$ is given as:

$$
S_{U}=\max \left\{C_{E}-C_{W}+C_{N}-C_{s}-S_{p}^{m}(\Delta V), 0\right\} v_{p}+D_{v}\left(p_{s}-p_{p}\right)+\left(S_{U}^{\text {rest }}\right)(\mathrm{vol})
$$

The v-equation coefficients are:

$$
\begin{gathered}
C_{E}=\frac{1}{4}\left[\left(\rho_{P}+\rho_{E}\right) u_{E}+\left(\rho_{S}+\rho_{S E}\right) u_{S E}\right] \\
C_{W}=\frac{1}{4}\left[\left(\rho_{P}+\rho_{W}\right) u_{P}+\left(\rho_{S}+\rho_{S W}\right) u_{S}\right] \\
\quad: \\
C_{N}=\frac{1}{4}\left[\left(\rho_{P}+\rho_{N}\right) v_{N}+\left(\rho_{P}+\rho_{S}\right) v_{P}\right] \\
C_{S}=\frac{1}{4}\left[\left(\rho_{P}+\rho_{S}\right) v_{P}\left(\rho_{S}+\rho_{S S}\right) v_{S}\right]
\end{gathered}
$$

The diffusion coefficients are given by:

$$
\begin{aligned}
& D_{E}=\frac{\mu_{e}^{v} A_{c W}^{v}}{\delta x_{E P}} \\
& D_{W}=\frac{\mu_{w}^{v} A_{c W}^{v}}{\delta x_{P W}} \\
& D_{N}=\frac{\mu_{p} A_{n}^{v}}{\delta y_{N P}^{v}}
\end{aligned}
$$




$$
D_{s}=\frac{\mu_{s} A_{s}^{v}}{\delta y_{P S}^{v}}
$$

Figure 3-10 shows a typical $v$ control volume and notation. Similar results can be obtained for the tangential momentum equation.

\section{Pressure Correction Equation (Continuity)}

The next task in the SIMPLE/R/C algorithms is to correct the starred pressure and velocity fields such that the continuity equation is satisfied. The two finitedifference momentum equations are from Eqns 3-146 and 3-153:

$$
\begin{aligned}
& A_{p}^{u} u_{p}=\sum_{d} A_{d}^{u} u_{d}+D^{u}\left(p_{W}+p_{p}\right)+S_{U}^{u} \\
& A_{p}^{v} v_{p}=\sum_{d} A_{d}^{v} v_{d}+D^{v}\left(p_{S}-p_{p}\right)+S_{U}^{v}
\end{aligned}
$$

Lacking knowledge of the pressure distribution, the pressures in the above momentum equations are guessed (denoted by a star superscript) to allow the computation of the two velocity components $u^{*}$ and $v^{*}$ corresponding to the $p^{*}$ distribution. This true pressure is

$$
p=p^{*}+p^{\prime}
$$

where $p^{\prime}$ is the pressure correction which, when summed with the guessed pressure, will give the correct pressure.

The SIMPLE algorithm utilizes the continuity equation to determine the pressure corrections. The equations expressing the true velocities in terms of the guessed velocities and pressure corrections are developed below. Substituting the guessed pressures in Eqns. 3-163 and 3-164 yields:

$$
A_{p}^{u} u_{p}^{*}=\sum_{d} A_{d}^{u} u_{d}^{*}+D^{u}\left(p_{W}^{*}-p_{p}^{*}\right)+S_{U}^{u}
$$




$$
A_{p}^{v} v_{p}^{*}=\sum_{d} A_{d}^{v} v_{d}^{*}+D^{v}\left(p_{s}^{*}-p_{p}^{*}\right)+S_{U}^{v}
$$

These equations are solved for the "starred" velocity fields. Subtracting the corresponding Eqns. 3-166 and 3-167 from Eqns. 3-163 and 3-164 and substituting the pressure corrections, we have:

$$
\begin{aligned}
& A_{p}^{u}\left(u_{p}-u_{p}^{*}\right)=\sum_{d} \mathrm{~A}_{d}^{u}\left(u_{d}-u_{d}^{*}\right)+D^{\mu}\left(p_{w}^{\prime}-p_{P}^{\prime}\right) \\
& A_{p}^{v}\left(v_{p}-v_{p}^{*}\right)=\sum_{d} \mathrm{~A}_{\mathrm{d}}^{v}\left(v_{d}-v_{d}^{*}\right)+D^{\nu}\left(p_{s}^{\prime}-p_{P}^{\prime}\right)
\end{aligned}
$$

which gives the true velocities as:

$$
\begin{gathered}
u_{p}=u_{p}^{*}+\frac{D^{u}}{A_{p}^{u}}\left(p_{W}^{\prime}-p_{P}^{\prime}\right)+\sum_{d} \frac{A_{d}^{u}}{A_{p}^{u}}\left(u_{d}-u_{d}^{*}\right) \\
v_{p}=v_{p}^{*}+\frac{D^{v}}{A_{p}^{v}}\left(p_{S}^{\prime}-p_{p}^{\prime}\right)+\sum_{d} \frac{A_{d}^{v}}{A_{p}^{v}}\left(v_{d}-v_{c}^{*}\right)
\end{gathered}
$$

A similar derivation applied to the neighboring nodes leads to

$$
\begin{aligned}
& u_{E}=u_{E}^{*}+\left(\frac{D^{\mu}}{A_{p}^{\mu}}\right)_{E}\left(p_{P}^{\prime}-p_{E}^{\prime}\right)+\sum_{d_{E}}\left(\frac{A_{d}^{u}}{A_{p}^{\mu}}\right)_{E}\left(u_{d}-u_{d}^{*}\right) \\
& v_{N}=v_{N}^{*}+\left(\frac{D^{v}}{A_{p}^{v}}\right)_{N}\left(p_{P}^{\prime}-p_{N}^{\prime}\right)+\sum_{d_{N}}\left(\frac{A_{d}^{v}}{A_{p}^{v}}\right)_{N}\left(v_{d}-v_{d}^{*}\right)
\end{aligned}
$$

where the new subscript refers to total coefficients at the node point.

From Eqn. 3-68, the finite-difference form of the continuity equation is 


$$
\rho_{e} u_{E} A_{e}-\rho_{w} u_{p} A_{w}+\rho_{n} v_{N} A_{n}-\rho_{s} v_{P} A_{s}-S_{p}^{m}(\Delta V)=0
$$

In the so-called incompressible pressure-correction equation, the density does not depend on pressure; this derivation follows.

Equation 3-174 calls for substitution of Eqns. 3-170 to 3-173 for the velocities, but the last term in each of these equations is lengthy and complicated, so that it will be dropped to give the true velocities as:

$$
\begin{aligned}
& u_{P}=u_{P}^{*}+D_{P}^{u}\left(p_{W}^{\prime}-p_{P}^{\prime}\right) \\
& v_{P}=v_{P}^{*}+D_{P}^{v}\left(p_{S}^{\prime}-p_{P}^{\prime}\right) \\
& u_{E}=u_{E}^{*}+D_{E}^{u}\left(p_{P}^{\prime}-p_{E}^{\prime}\right) \\
& v_{N}=v_{N}^{*}+D_{N}^{\nu}\left(p_{P}^{\prime}-p_{N}^{\prime}\right)
\end{aligned}
$$

where the new pressure coefficients include the corresponding $A_{P}$ terms. Now substituting these velocities, the continuity equation becomes:

$$
\begin{aligned}
& \left(\rho_{e} A_{e} D_{E}^{u}+\rho_{w} A_{w} D_{P}^{u}+\rho_{n} A_{n} D_{N}^{v}+\rho_{s} A_{s} D_{P}^{v}\right) p_{P}^{\prime}=\left(\rho_{e} A_{e} D_{E}^{u}\right) p_{E}^{\prime}+\left(\rho_{w} A_{w} D_{P}^{u}\right) p_{W}^{\prime}+ \\
& \left(\rho_{n} A_{n} D_{N}^{v}\right) p_{N}^{\prime}+\left(\rho_{s} A_{s} D_{S}^{v}\right) p_{S}^{\prime}-\rho_{e} A_{e} u_{E}^{*}+\rho_{w} A_{w} u_{P}^{*}-\rho_{n} A_{n} v_{N}^{*}+\rho_{s} A_{s} v_{P}^{*}+S_{p}^{m}(\Delta V)
\end{aligned}
$$

This equation allows the pressure corrections to be determined from known quantities, and furthermore has a similar form to the standard finite-difference equation. Defining the following pressure-correction coefficients:

$$
A_{E}=\rho_{e} A_{e} D_{E}^{\mu}
$$




$$
\begin{gathered}
A_{W}=\rho_{w} A_{w} D_{P}^{\mu} \\
A_{N}=\rho_{n} A_{n} D_{N}^{\nu} \\
A_{S}=\rho_{s} A_{s} D_{P}^{\nu} \\
S_{U}=-\rho_{e} A_{e} u_{E}^{*}+\rho_{w} A_{w} u_{P}^{*}-\rho_{n} A_{n} v_{N}^{*}+\rho_{s} A_{s} v_{P}^{*}+S_{p}^{m}(\Delta V)
\end{gathered}
$$

the pressure-correction equation becomes simply:

$$
\left(\sum_{d} A_{d}\right) p_{P}^{\prime}=\sum_{d} A_{d} p_{d}^{\prime}+S_{U}
$$

which can be solved by the TDMA. Note that no hybrid differencing is required.

The quantity $S_{U}$ defined above is the net mass source resulting from the starred velocity field which, in general, does not satisfy continuity. The aim of the pressure correction equation is to reduce this mass source to zero; indeed, by monitoring this quantity, the extent of convergence is known, with complete convergence occurring when the sum of these pressure source terms throughout the flow domain is less than some input tolerance (typically 0.001-0.0001).

After resolving the pressure correction equation by the TDMA, the correct pressure and velocities are given by

$$
\begin{gathered}
p=p^{*}+p^{\prime} \\
u_{p}=u_{p}^{*}+D_{u}\left(p_{W}^{\prime}-p_{P}^{\prime}\right) \\
v_{p}=v_{p}^{*}+D_{\nu}\left(p_{S}^{\prime}-p_{P}^{\prime}\right)
\end{gathered}
$$


It should be pointed out that the pressure correction equation calculates just the corrections to the pressure field; and in a converged solution these corrections vanish, and therefore, the dropping of the last terms in the momentum equations, to arrive at Eqns. 3-175 to 3-179, has no effect on the accuracy of the solution. Also, it must be noted that under-relaxation is required $(\sim 0.5)$ so that only a portion of these corrections is added at a time.

The SIMPLER (SIMPLE-Revised) algorithm was developed by Patankar (1980) to allow faster convergence than the SIMPLE algorithm. Solving Equations 3-163 and 3-164 for $u_{p}$ and $v_{p}$ yields:

$$
\begin{aligned}
& u_{p}=\frac{\sum_{d} A_{d}^{u} u_{d}+D_{P}^{u}\left(p_{W}-p_{P}\right)-S_{U}^{u}}{A_{P}^{u}} \\
& v_{p}=\frac{\sum_{d} A_{d}^{v} v_{d}+D_{P}^{\nu}\left(p_{S}-p_{P}\right)-S_{U}^{\nu}}{A_{P}^{\nu}}
\end{aligned}
$$

Pseudo-velocities $\left(\hat{u}_{p}, \hat{v}_{p}\right)$ are defined to simplify Eqns. 3-188 and 3-189:

$$
\begin{gathered}
\hat{u}_{P}=\frac{\sum_{d} A_{d}^{u} u_{d}+S_{U}^{u}}{A_{P}^{u}} \\
\hat{v}_{P}=\frac{\sum_{d} A_{d}^{v} v_{d}+S_{U}^{v}}{A_{P}^{v}}
\end{gathered}
$$

Similar equations are easily derived for $\hat{u}_{E}$ and $\hat{v}_{N}$. Using Eqns. 3-191 and 3-192, Eqns. 3-188 and 3-189 are reduced to:

$$
u_{P}=\hat{u}_{P}+D_{P}^{u}\left(p_{W}-p_{P}\right)
$$




$$
v_{P}=\hat{v}_{P}+D_{P}^{\nu}\left(p_{S}-p_{P}\right)
$$

Equations 3-193 and 3-194 are very similar to Eqns. 3-175 and 3-176. Substituting the velocities from Eqns. 3-193 and 3-194 into the continuity equation yields an equation similar to Eqn. 3-185, except that the pseudo velocities are used instead of the starred velocities:

$$
\left(\sum_{d} A_{d}\right) p_{P}=\sum_{d} A_{d} p_{d}+S_{u}^{P}
$$

where the $A$ 's are given by Eqns. $3-180$ through $3-183$, and $S_{u}^{p}$ is defined by:

$$
S_{u}^{P}=-\rho_{e} A_{e} \hat{u}_{E}+\rho_{w} A_{w} \hat{u}_{P}-\rho_{n} A_{n} \hat{v}_{N}+\rho_{s} A_{s} \hat{v}_{P}+S_{p}^{m}(\Delta V)
$$

The SIMPLER algorithm is summarized below (Patankar, 1980):

1. Start with a guessed velocity field.

2. Calculate the coefficients for the axial and radial momentum equations and then calculate the pseudo-velocities from Eqns. 3-191 and 3-192 using the guessed velocity field.

3. Calculate the pressure coefficients and solve for the pressure field using Eqn. 3-195.

4. Treating this pressure field as $p^{*}$, solve for the starred velocity field using Eqns. 3-166 and 3-167.

5. Solve the pressure correction equation (Eqn. 3-185) to get $p^{\prime}$.

6. Correct the starred velocity field using Eqns. 3-175 and 3-176, but do not correct the pressure field.

7. Solve the other conservation equations, as necessary $(w, k, \varepsilon, f, \mu$, etc.).

8. Return to step 2 and repeat as necessary.

The SIMPLER algorithm has been shown to reduce the convergence time in PCGC-2 by nearly one-half of that required by the SIMPLE algorithm (Fletcher, 1983). It should be pointed out that in Eqn. 3-194, $S_{U}^{P}$ should not necessarily vanish when convergence is achieved like $S_{U}$ in Eqn. 3-185. Also, it must be noted that best results are achieved when under-relaxation is not used for either the pressure (Eqn. 3-195) or the pressure correction (Eqn. 3-185) when the SIMPLER 
algorithm is used. The SIMPLER algorithm makes it possible to use much larger values for all other under-relaxation factors in the model (i.e. less under-relaxation). A typical value is 0.7 instead of 0.5 .

Dropping the last term in Eqns. 3-170 through 3-173 is equivalent to dropping the first term on the right-hand side of Eqns. 3-168 and 3-169, which has been pointed out to be inconsistent since this term is equivalent in magnitude to a term on the left-hand side that results when Eqn. 3-147 is substituted for $A_{p}^{u}$ (van Doormaal and Raithby, 1984). A "consistent" approximation which still leads to a suitable expression for $p^{\prime}$ can be obtained by subtracting the term $\sum_{d} A_{d}^{u}\left(u_{p}-u_{p}^{*}\right)$ from both sides of Eqn. 3-168:

$$
\left(A_{p}^{u}-\sum_{d} A_{d}^{u}\right) u_{p}^{\prime}=\sum_{d} A_{d}^{u}\left(u_{d}^{\prime}-u_{p}^{\prime}\right)+D^{u}\left(p_{W}^{\prime}-p_{P}^{\prime}\right)
$$

A similar equation can be obtained for Eqn. (3-169). In the SIMPLEC approximation, the underlined term $\sum_{d} A_{d}^{u}\left(u_{d}^{\prime}-u_{p}^{\prime}\right)$ is neglected. $\mathrm{A}$ " $\mathrm{C}$ " is appended to the name SIMPLE as a reminder that this is a "consistent" approximation. Equation 3-197 then becomes equivalent to Eqn. 3-174, where

$$
D_{P}^{u}=\frac{A_{c}^{u}}{A_{p}^{u}-\sum_{d} A_{d}^{u}}
$$

$p^{\prime}$ should not be under-relaxed in SIMPLEC.

\section{Under-Relaxation}

The solution of the finite-difference equations in the form of Eqn. 3-195 generally diverges unless the iterative corrections are under-relaxed. The conventional practice in computational fluid dynamics is to under-relax the dependent variables to avoid numerical instabilities. However, based on considerable experience, underrelaxation in this code is accomplished by under-relaxing the source terms. as follows:

If $f_{U R}$ is the under-relaxation factor, then under-relaxation is accomplished 


$$
\begin{gathered}
A_{P}^{\text {new }}=\frac{A_{P}^{\text {old }}}{f_{U R}} \\
S_{U}^{\text {new }}=S_{U}^{\text {old }}+\frac{\left(1-f_{U R}\right) \phi_{P}^{\text {old }} A_{P}^{\text {new }}}{f_{U R}}
\end{gathered}
$$

where $\phi_{o l d}^{P}$ is the known value of $\phi$ computed during the previous iteration.

These modifications are performed before solution of the finite-difference equations by TDMA. All dependent variables are relaxed by this method, where the under-relaxation factor can be different for each variable. In the pressure-correction equation (Eqn. 3-185), the pressure coefficients can also be under-relaxed:

$$
\begin{aligned}
& D^{u}=\left(D^{u}\right) f_{U R}^{u} / A_{P}^{u} \\
& D^{\nu}=\left(D^{\nu}\right) f_{U R}^{v} / A_{P}^{\nu}
\end{aligned}
$$

It may also be required to under-relax the fluid properties if they change rapidly as is the case with viscosity in turbulent flow and the density in compressible flow. If $\phi^{(n)}$ is the value of the property used in iteration $n+1$, and $\phi^{(n+1)}$ is the value computed in the $n+1^{s t}$ iteration, the value used in the $n+l^{\text {st }}$ iteration is computed from

$$
\phi=f_{U R} \phi^{(n+1)}+\left(1-f_{U R}\right) \phi^{(n)}
$$

The values of the under-relaxation factors must be between zero and one, and for computational efficiency, should be the largest value allowing a stable solution. The optimum values of the under-relaxation factors are problemdependent. Should divergence occur, smaller values may be required. 


\section{Boundary Conditions}

There exist an infinite number of solutions to the governing equations, and only the boundary corditions distinguish one solution from another. Thus, the boundary conditions play an important role. An excellent presentation of many types of boundary conditions occurring in fluid dynamic calculations and a discussion of their effects on numerical stability and accuracy have been given elsewhere (Roache, 1976).

In PCGC-2, all boundaries coincide with the control volume sides as illustrated in Fig. 3-11. The implementation of the boundary conditions in the numerical procedure is accomplished by modifying the finite-difference equations for the boundary cells:

$$
\left(\sum_{d} A_{d}-S_{p}\right) \phi_{p}=A_{E} \phi_{E}+A_{W} \phi_{W}+A_{N} \phi_{N}+A_{S} \phi_{S}+S_{U}
$$

The four total coefficients ( $A^{\prime}$ s) and the two source term coefficients $S_{U}$ and $S_{p}$ in the above equation are altered in such a way that the boundary conditions are satisfied. This technique, generally referred to as "breaking the link," is illustrated by rewriting the equation as

$$
A_{E}\left(\phi_{P}-\phi_{E}\right)+A_{W}\left(\phi_{P}-\phi_{W}\right)+A_{N}\left(\phi_{P}-\phi_{N}\right)+A_{S}\left(\phi_{P}-\phi_{S}\right)=S_{U}+S_{P} \phi_{P}
$$

It may be seen that $\phi_{p}$ may be considered as being "linked" to its four neighbors. The above equation is correct for interior cells; for boundary cells, the appropriate total coefficients ( $A$ 's) must be set to zero, and the correct conditions of convection and diffusion at the boundary included by modifying the two source terms. Thus the procedure calls for specifying the boundary conditions as linear functions of the dependent variable. All three types of boundary conditions for elliptic equations are permissible, and will be discussed in tum.

Dirichlet conditions, or prescribed values of the function, are inserted by calculating the correct convection and diffusion coefficients based on the prescribed values of the dependent variable on the boundary, and using the hybrid differencing scheme to obtain a new total coefficient $A^{\text {(new). }}$

Hence: 


$$
\begin{gathered}
A_{d}^{(\text {old })}=0 \\
D_{d}=\frac{\Gamma_{B} A_{B}}{\delta_{B P}} \\
C_{d}=\rho_{B} v e l_{B} A_{B} \\
A_{d}^{(\text {new })}=H Y B R I D\left[C_{d}, D_{d}\right] \\
S_{U}^{(\text {new })}=S_{U}^{(\text {old })}+A_{d}^{(\text {new })} \phi_{B} \\
S_{P}^{(\text {new })}=S_{P}^{(\text {old })}-A_{d}^{(\text {new })}
\end{gathered}
$$

where the subscript $B$ refers to the conditions at the boundary located at a distance of $\delta_{B P}$ from the cell center in the $d$ direction, $\phi_{B}$ is the prescribed boundary value, $A_{B}$ is the area of the control volume on the boundary, and $C_{d}$ and $D_{d}$ are the convection and diffusion coefficients at the control volume face on the boundary. If the velocity at the boundary, $v e l_{B}$, is zero (as in the case of a solid wall), then Eqns. 3-206 to 3-211 reduce to:

$$
\begin{gathered}
A_{d}^{(\text {new })}=0 \\
S_{U}^{(\text {new })}=S_{U}^{(\text {old })}+\left(\frac{\Gamma_{B} A_{B}}{\delta_{B P}}\right) \phi_{B}
\end{gathered}
$$




$$
S_{P}^{(\text {new })}=S_{P}^{(\text {old })}-\left(\frac{\Gamma_{B} A_{B}}{\delta_{B P}}\right)
$$

where $A_{B}$ is the area of the bounding face.

For Neumann conditions, for which the normal derivative of the function is specified, the usual "links" to all the adjacent exterior cells are broken by setting to zero the appropriate A's and inserting the prescribed boundary fluxes, as follows:

$$
\begin{gathered}
S_{U}^{(\text {new })}=S_{U}^{(\text {old })}+\left(c \frac{\partial \phi}{\partial n}\right)_{B}(\text { area })_{B} \\
S_{P}^{(\text {new })}=S_{P}^{(\text {old })}
\end{gathered}
$$

where $\left(c \frac{\partial \phi}{\partial n}\right)_{B}$ is the specified flux at the boundary.

The third type of boundary condition is the mixed or Robbin's type with the linear combination of the function value and normal gradient specified (for example, in a convective heat transfer calculation). Consider heat conduction occurring from node W in Fig. 3-11 to the boundary, where convection takes place with a heat transfer coefficient $\alpha$. Then the required boundary condition (for steady state) is:

$$
q=\alpha A_{W}\left(T_{B}-T_{P}\right)=k_{B} A_{W} \frac{T_{W}-T_{B}}{\delta_{W B}}
$$

where $k_{B}$ is the conductivity of the conducting media, $T_{B}$ is the boundary temperature, and $\delta_{W B}$ is the distance between node $W$ and the boundary. The boundary temperature can be eliminated in the above equation to give:

$$
q=\frac{T_{W}-T_{P}}{\frac{1}{\alpha A_{W}}+\frac{k_{B} A_{W}}{\delta_{W B}}}
$$




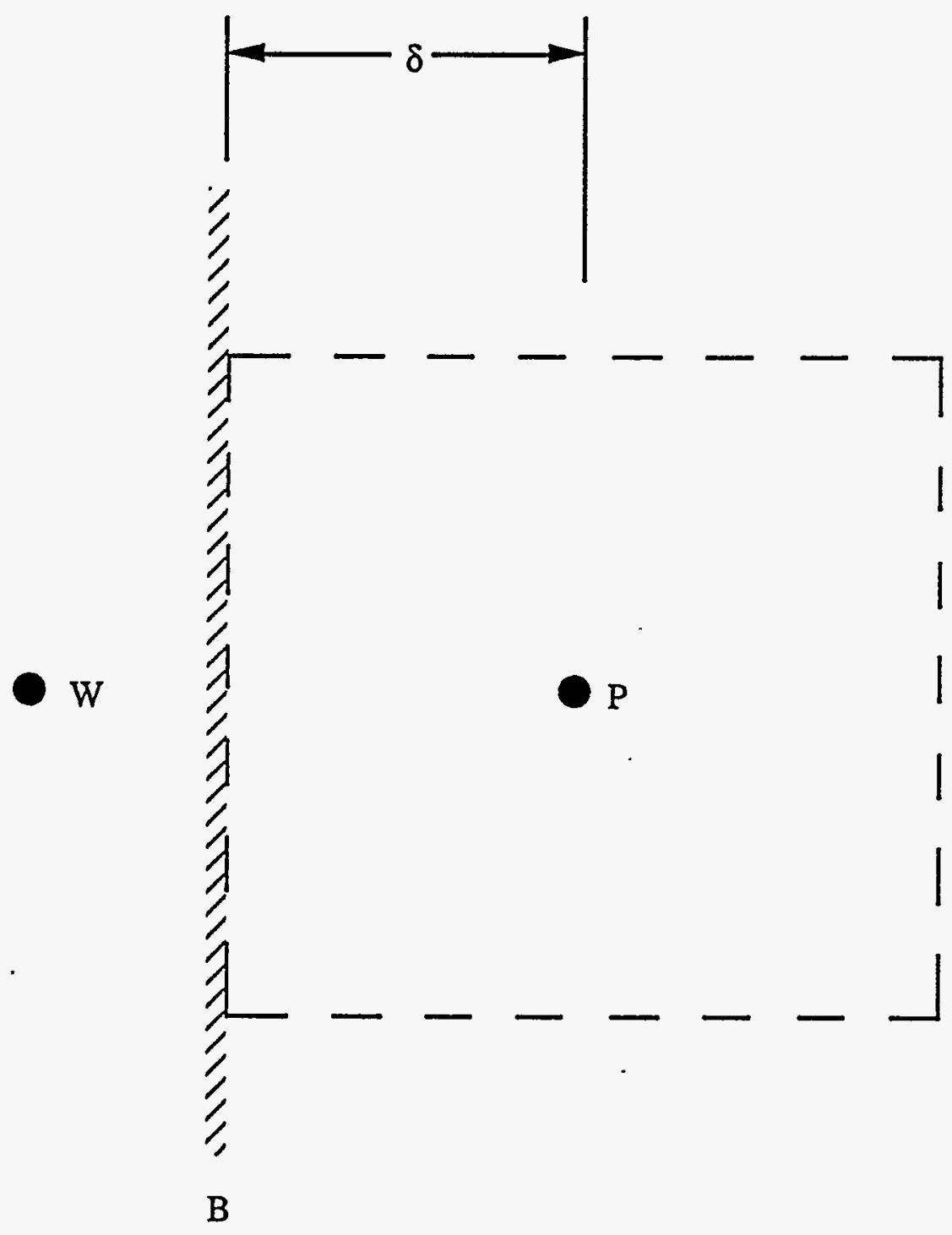


and the two source terms become:

$$
\begin{gathered}
S_{U}^{T(\text { new })}=S_{U}^{T(\text { old })}+\frac{T_{W}}{R_{T}} \\
S_{P}^{T(\text { new })}=S_{P}^{T(\text { old })}-\frac{I}{R_{T}}
\end{gathered}
$$

where

$$
R_{T}=\frac{1}{\alpha A_{w}}+\frac{k_{B} A_{w}}{\delta_{W B}}
$$

Normally, PCGC-2 iterates on a rectangular domain (being controlled by FORTRAN do-loops) and if this domain contains node points which are outside the flow field domain, either the computer code can be altered to skip these nodes or the following technique can be employed to fix the value of $\phi$ at the node:

$$
\begin{aligned}
& S_{U}=\left(\gamma \phi_{p}\right) \\
& S_{P}=(-\gamma)
\end{aligned}
$$

where $\gamma$ is a large number $\left(10^{30}\right)$ and $\phi_{p}$ is the value desired at the node point. Boundary conditions must be inserted for each boundary cell.

\section{Numerical Diffusion}

The hybrid differencing scheme introduces numerical errors in the convection terms of order $O \Delta x)$ when upwind differencing is used and $O\left(\Delta x^{2}\right)$ when central differencing is used. When upwind differencing is used, the error introduced from the finite-difference approximation can be viewed as an artificial numerical diffusion (Roache, 1976). This numerical diffusion is a function of the Courant number: 


$$
C_{x}=\frac{u \Delta t}{\Delta x}
$$

If $C_{x}=1$, the numerical diffusion terms will be identically zero (Roache, 1976), meaning that the finite-difference approximation is exact. The errors introduced by upwind differencing should be investigated by using a different finite-differencing scheme. Central-differencing, however, is thought to be unstable for elliptical partial differential equations (Roache, 1976).

Several authors (Leonard, 1979; Castro, 1978; Raithby, 1976; Argarwal, 1981; Leschiziner, 1980; Lillington, 1978) have developed methods to eliminate numerical diffusion in simple flow systems. Significant numerical diffusion has been shown to arise in predictions where convection dominates physical diffusion and where there is a streamline-to-grid skewness (Leschiziner, 1980). The abovereferenced predictions were made when the streamlines were at a 45-degree angle to the grid. Significant numerical diffusion has also been reported when gradients exist normal to the streamlines in the dependent variable and source terms (Lillington, 1978). It was also noted that higher-order differencing schemes could have poor convergence in some elliptical flow regimes. Raithby (1976) found that numerical diffusion was significant only when the source terms take a certain form and if the streamlines were skew to the grid. Leonard (1979) showed predictions where significant numerical diffusion occurred when streamlines were at an 18degree angle to the grid. Leonard and co-workers also argued that their scheme allows larger grid spacing since it was more accurate.

Fletcher (1983) incorporated the method of Leonard (1979) into PCGC-2 to determine the effect of numerical diffusion in typical PCGC-2 predictions. The basis for the differencing scheme of Leonard (1979) is a second-order interpolation scheme for the values for the variable of interest $(\phi)$ at the cell wall. The predictions made using Leonard's higher-order differencing scheme required 10 to 15 times the computational time required for the prediction made using upwind differencing. The results for the non-reacting flow system indicated that numerical diffusion does not seriously affect PCGC-2 predictions, and that the hybrid differencing prediction is sufficiently accurate for this particular flow system. However, it is important to note that this observation was made for only a single computation, and was not achieved in a reacting or particle-laden system.

The negligible effect of numerical diffusion is somewhat surprising, since the streamlines in the recirculation zone of the reactor were definitely skew to the grid. Roache (1976) states that if the grid size is small enough, the effects of numerical diffusion will not be significant. He also says that a free outflow boundary condition (such as that used in PCGC-2) tends to reduce upstream error in elliptical systems. 
In light of these results, the effects of numerical diffusion in PCGC-2 predictions for this particular geometry (namely the BYU Combustor) are thought to be of secondary importance. The higher-order differencing algorithm of Leonard (1979) was not used in PCGC-2 for any other predictions. Other higher-order differencing methods should not be used unless they are first demonstrated for reacting flows.

\section{Boundary Conditions}

A discussion of the numerical approach used in PCGC-2 is not complete without a discussion of the boundary conditions for all of the equations used. Each variable must have a boundary condition specified at each of the boundaries for elliptic equations. Symmetry conditions are imposed along the centerline, while other types of boundary conditions must be specified at the reactor walls, inlets, and exits.

\section{Inlet Streams}

The inlet mass flow rate is used to set the inlet velocities, with assumed parallel injection $\left(v_{p r i}=v_{s e c}=0\right)$. This gives a Dirichlet condition for $u$ and $v$. Turbulence intensities must be specified for the inlet streams in order to get Dirichlet boundary conditions for $k$. Turbulence intensities are defined as follows:

$$
I=\frac{\left[{\overline{\left(u^{\prime}\right)}}^{2}+{\overline{\left(v^{\prime}\right)}}^{2}\right]^{\frac{1}{2}}}{\left[\bar{u}^{2}+\bar{v}^{2}\right]^{\frac{1}{2}}}=\frac{[2 k]^{1 / 2}}{\|\bar{v}\|}
$$

where $\|\bar{v}\|$ is the magnitude of vector $\vec{v}$. Since $\bar{u}$ and $\bar{v}$ are known in the inlet streams, $k$ is specified when $I$ is set. The dissipation level $(\varepsilon)$ must be estimated at the inlet from empirical correlations (Syed and Sturgess, 1980):

$$
\varepsilon=\frac{C_{\mu} k^{1.5}}{0.03 D_{e}}
$$


where $D_{e}$ is the effective inlet diameter. The mixture fraction is known by definition in each of the inlet streams, and the mixture fraction fluctuations $\left(g_{f}\right)$ are assumed zero in the inlet streams. The coal is not allowed to react in the inlet streams, so that $\eta$ and $g_{\eta}$ are both zero at the inlet boundary.

PCGC-2 is coded to allow user-specified inlet profiles of $u, v, w, r, k$, and $\varepsilon$ in both the primary and/or secondary streams. Theoretical or experimental velocity profiles can be used. Theoretical inlet velocity profiles are calculated according to Bird, et al. (1960). The inlet velocity profile is normalized to match continuity with the specified inlet mass flowrate.

\section{Centerline}

Symmetry conditions are imposed at the centerline on all variables, meaning that the radial component of the gradient of any variable is zero $\left(\frac{\partial \beta}{\partial r}=0\right)$ at the centerline. This specifies a Neumann condition at the axis or symmetry for all variables. The only exception to this rule is the radial radiation flux $\left(F_{r}\right)$. The quantity $\left(r F_{r}\right)$ is assumed zero along the centerline.

\section{Qutlet Stream}

The radial component of the velocity $(\tilde{v})$ is set to zero at the reactor exit, while the axial component $(\bar{u})$ is adjusted to satisfy over-all continuity with the inlet gas mass flow rates. The outlet values of $f$ and $\eta$ are set to force continuity with the inlet particle mass droplet flow rate. It is assumed that all of the other variables have smooth axial profiles at each radial location near the reactor exit. The outlet condition for these variables is determined by setting the axial gradient equal to zero.

\section{Walls}

The wall boundary conditions are of special interest. Of course, it would be possible to use parabolic boundary layer equations and solve them all the way to the wall; however, to reduce computer storage and run times, it is convenient to bridge over the wall region. The Van Driest hypothesis on turbulent flow near a wall is used to derive wall functions which are consistent with the logarithmic law of the 
wall. Launder and Spalding (1972) outline the derivation and Patankar and Spalding (1970) give more details. In this way the dependent variables at the wall are linked to those in the logarithmic region (alsc see Khalil et al., 1975). For the velocity component normal to the wall in question, a Neumann condition is used and the gradient is set equal to zero (i.e., $\partial v / \partial r=0$ for the north wall). For the component of velocity parallel to the wall in question, a no-slip boundary condition is assumed. A Dirichlet condition is imposed and the velocity component is set equal to zero (i.e., $\bar{u}=0$ for the north wall; however, in this near-wall region, the fully developed turbulent exchange coefficient is not valid, and an exchange coefficient (effective turbulent viscosity) is calculated from the logarithmic law of the wall as given below and derived by Launder and Spalding (1972) (for a north wall, $u$ velocity):

$$
\Gamma_{u}=\mu_{c}=\frac{\tau_{w} \Delta r}{\Delta u}=\frac{\tau_{w} \Delta r}{u_{p}}=\frac{\rho C_{\mu}^{1 / 4} k^{1 / 2} \kappa \Delta r}{\ln \left[\frac{E C_{\mu}^{1 / 4} k^{1 / 2} \Delta r \rho}{\mu}\right]}
$$

When the reactor is non-adiabatic and convective/conductive heat losses must be considered, a Neumann condition is specified for the enthalpy $(h)$. The normal derivative is calculated from a universal temperature profile in the logarithmic region for the near-wall turbulence (for the north wall)

$$
\begin{gathered}
\Gamma \frac{\partial h}{\partial r}=-\left(\Gamma \frac{\partial h}{\partial r}\right)_{w}=-\left(\frac{C_{p} \Gamma \partial T}{\partial r}\right)_{w}=q_{w} \\
q_{w}=\left(T_{p}-T_{w}\right) \rho C_{\mu}^{1 / 4} k^{1 / 2} \sigma_{k, t} C_{p \operatorname{mix}}\left[P+\frac{1}{\kappa} \ln \left(\frac{E \Delta r C_{\mu}^{1 / 4} k^{1 / 2} \rho}{\mu}\right)\right]
\end{gathered}
$$

where $P$ is the extra resistance to heat transfer that arises due to the difference in $\sigma_{k}$ and $\sigma_{h, t}$. Jayatilleke (1969) gives the following correlation for $P$ :

$$
P=9.24\left[\left(\frac{\sigma_{h}}{\sigma_{h, 1}}\right)^{3 / 4}-1\right]
$$


where $\sigma_{h, t}=0.9$. Equation 3-230 was found to cause numerical instability in PCGC-2, apparently due to some nodes being too close to the wall. Rather than sacrifice the accuracy of the bulk flow solution by moving all nodes farther away from the wall, a constant value of unity is used for $P$. No instability is caused when $P$ is non-negative. For $\sigma_{h}=0.8$, the available data (Jayatilleke, 1969) are extremely scattered, and $P$ ranges from -8 to +23 . The value predicted by Eqn. $3-$ 230 is -0.78 , however the correlation is thought to be inaccurate at the low value of $\sigma_{h}$ due to the scatter in the data. Therefore, a constant value of unity is reasonable. The quantity $[\Gamma(\partial h / \partial r)]_{w}$ is the total turbulent flux of enthalpy to or from the wall and is the quantity needed by the numerical scheme for the Neumann condition.

At the walls, the normal derivative of the turbulent energy is zero:

$$
\frac{\partial k}{\partial x_{i}}=0
$$

However, the production of turbulent kinetic energy $(P)$ and the dissipation $(\varepsilon)$ near the walls require modification. Near a wall in axi-symmetric, polar-cylindrical coordinates,

$$
P_{W}=-\overline{u_{x}^{\prime} u_{x}^{\prime}} \frac{\partial \bar{u}_{x}}{\partial x}-\overline{u_{r}^{\prime} u_{r}^{\prime}} \frac{\partial\left(r \bar{u}_{r}\right)}{\partial r}+\frac{\tau_{w}}{\rho}\left(\frac{\partial \bar{u}_{x}}{\partial r}+\frac{\partial \bar{u}_{r}}{\partial x}\right)
$$

When the wall is parallel to the $u_{x}$ direction, $\partial \vec{u}_{r} / \partial x=0$. For a wall perpendicular to $u_{x}, \partial u_{x} / \partial x=0$. An extension of Eqn. 3-332 to include axi-symmetric, swirling flows is discussed by Lilly and Rhode (1982).

The wall shear stress $\left(\tau_{w}\right)$ can be estimated from turbulent Couette flow near a wall. The modified log-law is used:

$$
u^{+}=\frac{1}{\kappa} \ln \left(E y^{+}\right)
$$

where 


$$
y^{+}=\frac{y \rho\left(C_{\mu}^{\frac{1}{2}} k\right)^{\frac{1}{2}}}{\mu}
$$

and

$$
u^{+}=\frac{\bar{u}}{\sqrt{\frac{\tau_{w}}{\rho}}}
$$

Thus, the wall shear stress can be calculated directly:

$$
\tau_{w}=\frac{\vec{u} \kappa\left(C_{\mu}^{\frac{1}{2}} k\right)^{\frac{1}{2}} \rho}{\ln \left[\frac{E \Delta r\left(C_{\mu}^{\frac{1}{2}} k\right)^{\frac{1}{2}} \rho}{\mu}\right]}
$$

Equation 3-336 is substituted for Eqn. 3-332 for the production of turbulent energy near a wall $\left(P_{w}\right)$.

The dissipation rate at solid boundaries is harder to evaluate. In this case, it is suggested that the last node point in the flow field near a wall be set according to the following mixing length approximation.

$$
\ell_{m}=\kappa \Delta y_{i}
$$

where $x$ is a mixing length constant $(=0.42)$ and $\Delta y_{i}$ is the distance from the wall:

$$
\varepsilon=\frac{C_{\mu}^{\frac{3}{4}} k^{\frac{3}{2}}}{\kappa \Delta y_{i}}
$$

The dissipation in the $k$-equation can also be altered accordingly. The wall boundary conditions on $\tilde{f}, \tilde{g}_{f}, \bar{\eta}$, and $\bar{g}_{\eta}$ are simply Neumann conditions of zero normal derivative $(\partial \phi / \partial r=0$, or $\partial \phi / \partial x=0)$. 


\section{Summary}

A summary of the boundary conditions for each variable is found in Table 3-1.

TABLE 3-1

\section{PCGC-2 BOUNDARY CONDITIONS}

\begin{tabular}{|c|c|c|c|c|c|c|c|c|}
\hline & $\begin{array}{l}\text { Primary } \\
\text { jet }\end{array}$ & $\begin{array}{l}\text { Secondary } \\
\text { jet }\end{array}$ & $\begin{array}{l}\text { Symmetry } \\
\text { axis }\end{array}$ & $\begin{array}{l}\text { North } \\
\text { wall }\end{array}$ & South wall & West wall & East wall & Outlet \\
\hline $\bar{u}$ & $\begin{array}{l}\text { uniform } \\
\text { at } u_{p} \text {, or } \\
\text { profile }\end{array}$ & $\begin{array}{l}\text { uniform at } \\
u s \text {, or } \\
\text { profile }\end{array}$ & $\bar{u}_{i, l}=\bar{u}_{i, 2}$ & $\begin{array}{l}\tau_{w} \text { from } \\
\text { wall } \\
\text { function }\end{array}$ & $\begin{array}{l}\tau_{w} \text { from } \\
\text { wall } \\
\text { function }\end{array}$ & 0 & 0 & $\bar{u}_{i, j}=\bar{u}_{i-1, j}$ \\
\hline $\bar{v}$ & $\begin{array}{l}0, \text { or } \\
\text { profile }\end{array}$ & $\begin{array}{l}\text { uniform at } \\
v_{s,} \text { or } \\
\text { profile }\end{array}$ & 0 & 0 & 0 & $\begin{array}{l}\tau_{w} \text { from } \\
\text { wall } \\
\text { function }\end{array}$ & $\begin{array}{l}\tau_{w} \text { from } \\
\text { wall } \\
\text { function }\end{array}$ & 0 \\
\hline $\bar{w}$ & $\begin{array}{l}0, \text { or } \\
\text { profile }\end{array}$ & $\begin{array}{l}0, \text { or } \\
\text { profile }\end{array}$ & $\begin{array}{l}0, \text { or } \\
\tilde{w}_{i, I}=\bar{w}_{i, 2}\end{array}$ & $\begin{array}{l}\tau_{w} \text { from } \\
\text { wall } \\
\text { function }\end{array}$ & $\begin{array}{l}\tau_{w} \text { from } \\
\text { wall } \\
\text { function }\end{array}$ & $\begin{array}{l}\tau_{w} \text { from } \\
\text { wall } \\
\text { function }\end{array}$ & $\begin{array}{l}\tau_{w} \text { from } \\
\text { wall } \\
\text { function }\end{array}$ & $\bar{w}_{i, j}=\bar{w}_{i-1, j}$ \\
\hline$\tilde{k}$ & $\begin{array}{l}\text { from } \\
\text { primary } \\
\text { turbulence } \\
\text { intensity }\end{array}$ & $\begin{array}{l}\text { from } \\
\text { secondary } \\
\text { turbulence } \\
\text { intensity }\end{array}$ & $\bar{k}_{i, 1}=\bar{k}_{i, 2}$ & $\begin{array}{l}\text { Near-wall } \\
\text { values } \\
\text { from wall } \\
\text { function }\end{array}$ & $\begin{array}{l}\text { Near-wall } \\
\text { values } \\
\text { from wall } \\
\text { function }\end{array}$ & $\begin{array}{l}\text { Near-wall } \\
\text { values } \\
\text { from wall } \\
\text { function }\end{array}$ & $\begin{array}{l}\text { Near-wall } \\
\text { values } \\
\text { from wall } \\
\text { function }\end{array}$ & $\overline{\bar{k}_{i, j}}=\bar{k}_{i-1, j}$ \\
\hline $\bar{\varepsilon}$ & $\begin{array}{l}\text { from } \\
\text { empirical } \\
\text { correla- } \\
\text { tions }\end{array}$ & $\begin{array}{l}\text { from } \\
\text { empirical } \\
\text { correla- } \\
\text { tions }\end{array}$ & $\vec{\varepsilon}_{i, 1}=\vec{\varepsilon}_{i, 2}$ & $\begin{array}{l}\text { Near-wall } \\
\text { values } \\
\text { from } \\
\text { length } \\
\text { scale } \\
\end{array}$ & $\begin{array}{l}\text { Near-wall } \\
\text { values } \\
\text { from } \\
\text { length } \\
\text { scale } \\
\end{array}$ & $\begin{array}{l}\text { Near-wall } \\
\text { values } \\
\text { from } \\
\text { length } \\
\text { scale } \\
\end{array}$ & $\begin{array}{l}\text { Near-wall } \\
\text { values } \\
\text { from } \\
\text { length } \\
\text { scale } \\
\end{array}$ & $\varepsilon_{i, j}=\varepsilon_{i-1, j}$ \\
\hline $\begin{array}{l}g_{f}, \\
g_{n}\end{array}$ & & 0 & $g i, 1=g i, 2$ & \multicolumn{5}{|c|}{$\bar{g}_{i, l}=\bar{g}_{i, j}-1 \bar{g}_{i, j}=\bar{g}_{i, j}+1 \bar{g}_{i, j}=\bar{g}_{i+1, j} \bar{g}_{i, j}=\bar{g}_{i}-1, j \bar{g}_{i, j}=\bar{g}_{i-1, j}$} \\
\hline $\bar{h}$ & $\begin{array}{l}\text { uniform at } \\
h_{p}\end{array}$ & $\begin{array}{l}\text { uniform at } \\
h_{S}\end{array}$ & $\bar{h}_{i, l}=\bar{h}_{i, 2}$ & $\begin{array}{l}q w \text { from } \\
\text { wall } \\
\text { function }\end{array}$ & $\begin{array}{l}q_{w} \text { from } \\
\text { wall } \\
\text { function }\end{array}$ & $\begin{array}{l}q_{w} \text { from } \\
\text { wall } \\
\text { function }\end{array}$ & $\begin{array}{l}q_{w} \text { from } \\
\text { wall } \\
\text { function }\end{array}$ & $\bar{h}_{i, j}=\bar{h}_{i-1, j}$ \\
\hline
\end{tabular}


TABLE 3-1 (continued)

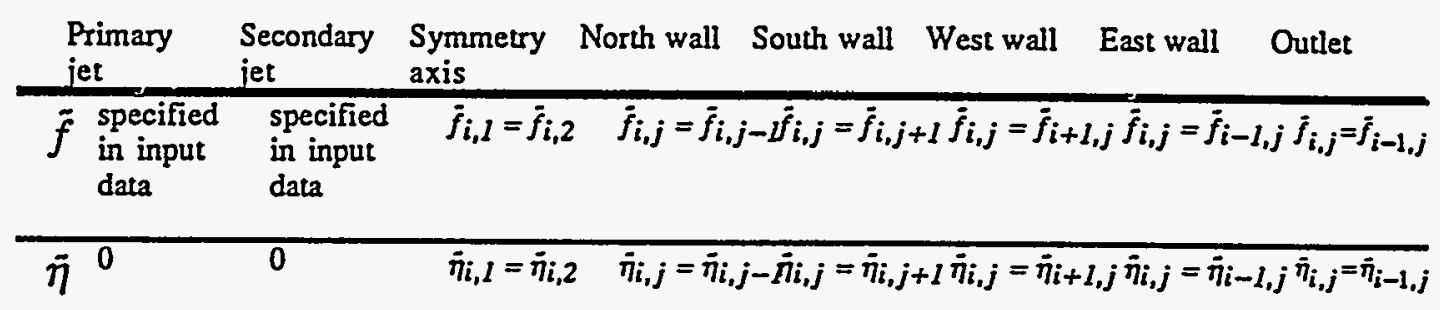

NOTE: Saying $\phi_{i, 1}=\phi_{i, 2}$, etc., is the differencing scheme for $(\partial \bar{\phi} / \partial n)=0$.

\section{Generalized Geometry}

The principal purpose of this section is to describe the treatment of axi-symmetric combustors with non-uniform cross-sections. Modern pulverized combustors and gasifiers and CWM combustors cannot always be represented in two dimensions using a cylindrical reactor vessel. Some reactors incorporate radiation baffles, "wasp waists," exit constrictions, and other extensions of the wall into the flow field. When modeling such reactors, the boundary conditions must be modified to account for these flow field "intrusions." PCGC-2 allows for generalized wall boundary conditions. Figure 3-12 shows sample reactor configurations that are possible with the code.

\section{Approach}

Eulerian gas phase equations are steady-state, yielding an elliptic system of partial differential equations. Such systems require boundary conditions at every boundary surrounding the flow domain. In a two-dimensional, axi-symmetric framework, boundary conditions are set at the inlets, along the centerline, along the exit, and along the walls. Cell faces are labeled like the directions of a compass (north, south, east, west) for ease of description. The flow chambers are generally modeled with inlets in the west wall, the centerline along the south boundary, and the exit along the east boundary, as if the reactor were horizontal. 

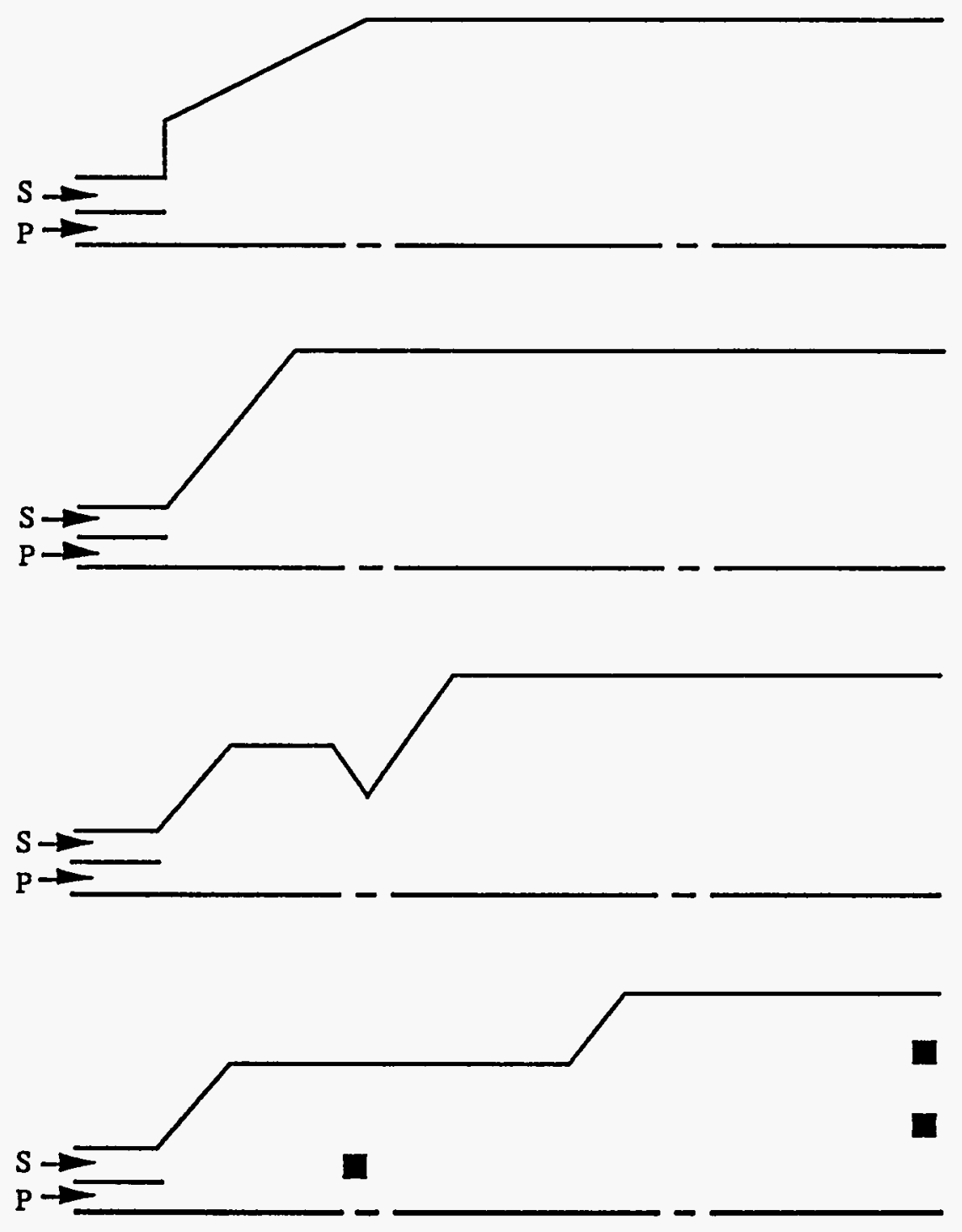

Figure. 3-12. Sample reactor configurations possible with modified PCGC-2. ( 1 are intrusions) 
Gas phase equations are transformed into algebraic form using power-law finite differencing (Patankar, 1980). A staggered grid is used in the finite difference approach, with velocities stored at cell faces and other variables (e.g. density, mixture fraction) stored at cell centers. Figure 3-2 showed a sample grid used for PCGC-2 calculations.

Table 3-1 shows typical boundary conditions used in PCGC-2 for the main gas field variables. In this formulation, any deviation from the cylinder wall is termed an "intrusion" into the flow field. All intrusions must be symmetrical and around the centerline of the reactor. For example, a triangular exit constriction is modeled as a cone when rotated around the centerline.

Intrusions are allowed in any part of the computational domain, and need not be connected to the outer wall in this formulation. The only restriction to this geometric formulation is that each grid cell is rectangular (in two dimensions), and not triangular. This means that any smooth intrusions must be modeled in a sawtooth manner, as shown in Fig. 3-13. In the limit, if grid spacing is small, smooth intrusions can be accurately modeled. However, computational time and memory requirements generally limit the fineness of the grid.

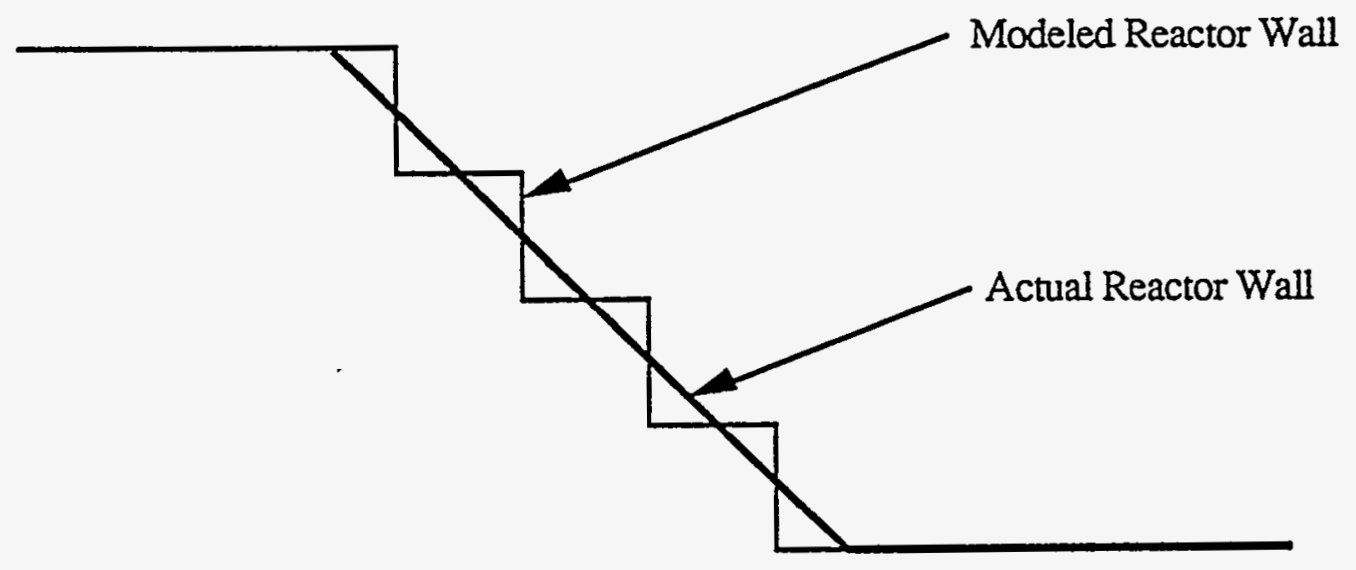

Figure 3-13. Example of saw-tooth representation of reactor wall. 


\section{Numerical Method}

An arbitrary grid cell is pictured in Fig. 3-14 along with the eight adjacent cells. The center cell has location $X(I)$ and $R(J)$, and the surrounding cells are therefore described in terms of $I+1, I-1, J+1$, and $J-1$. The array INTR $(I, J)$ is defined to be 1 if the cell is an intrusion (wail) and 0 if the cell is part of the flow field. The value of INTR $(I, N)$ is checked at each cell in the computational domain to see where the intrusions are located. When an intrusion is located, the boundary conditions of the surrounding cells are adjusted accordingly.

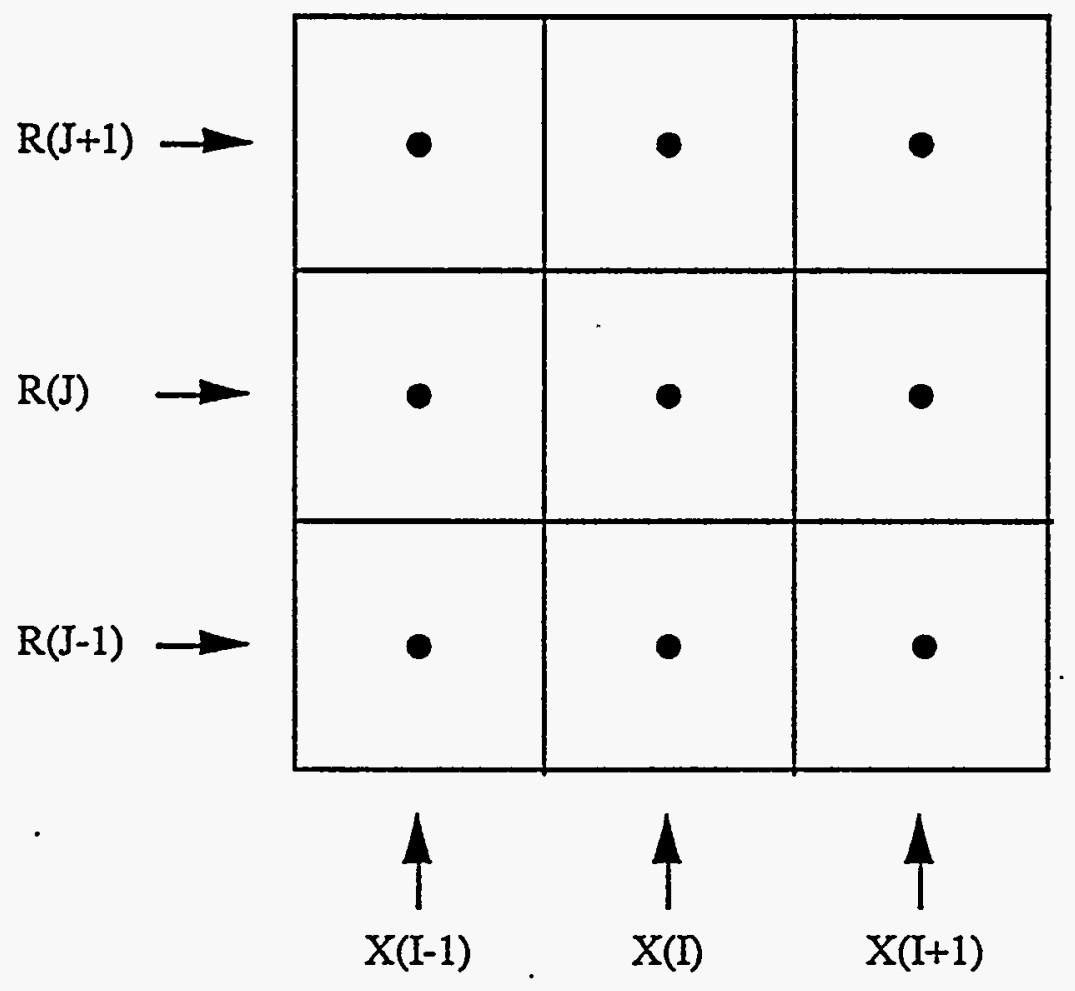

Figure 3-14. Arbitrary grid cell showing location of X, R, I and J. 
Suppose that cell $(I, J)$ is an intrusion and that we are solving the mixture fraction $(F)$ equation. The value of $F(I, N)$ is set to zero as follows:

$$
\begin{gathered}
S U(I, J)=0 \\
S P(I, J)=-1.0 \times 10^{30}
\end{gathered}
$$

Table 3-1 indicates that boundary conditions are satisfied for the $f$ equation by setting the gradient at the wall to zero. For the $f$ equation, this done by setting the appropriate " $A$ " coefficient (i.e. $A E, A W, A N$, or $A S$ ) to zero in the neighboring cell. For example, the north neighbor cell $(I, J+I)$ to an intrusion at $(I, J)$ must have the south boundary modified so that

$$
A S(I, J+1)=0
$$

Similarly, the other neighbor cells to an intrusion are modified as follows:

$$
\begin{aligned}
& A N(I, J-I)=0 \\
& A W(I+1, J)=0 \\
& A E(I-1, J)=0
\end{aligned}
$$

If a neighboring cell is also an intrusion, the boundary between the cells is not modified at all, since Eqns. 3-239 and 3-240 negate the effect of the $A$ coefficients.

Intrusions are used to describe the reactor walls as well as intrusions into the main flow field. When modifying the boundary conditions around the cells at the outer wall, care is taken not to exceed the dimensions of the arrays. For example at the north wall $(I, N J)$, the north neighbor cell is not used and hence is not modified.

All P-cell variables $\left(w, k, \varepsilon, g_{f}, g_{\eta}, h, f, \eta, p, p^{\prime}\right)$ are adjusted for intrusions as described above using the boundary conditions from Table 3-1 and the north, south, east and west neighboring cells. However, since a staggered grid is used, $u$ and $v$ are defined on the faces of the P-cells. Intrusions are also defined on 
the P-cells, which complicates the boundary conditions for $u$ and $v$ (U-cells and Vcells).

Figure 3-15 shows an intrusion with the $u$ velocity locations at the neighboring cells. Both $U(I, J)$ and $U(I+I, J)$ are zero because they are located on the edge of the intrusion. The $S_{u}$ and $S_{p}$ terms are modified as shown in Eqns. 3239 and 3-240 for these two cells. The east and west neighbor cell boundary conditions for the $u$ velocity are set as follows:

$$
A E(I-I, J)=0
$$

$$
A W(I+2, J)=0
$$

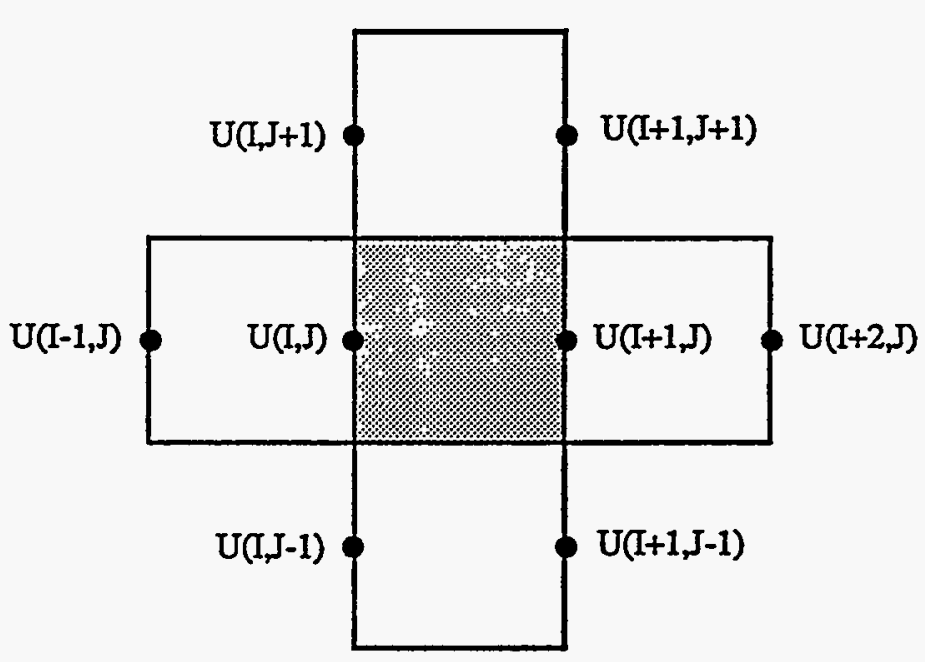

Figure 3-15. Arbitrary U-cell showing locations of cells affected by intrusion at $(\mathrm{I}, \mathrm{J})$.

For a single cell intrusion, $u$ velocity boundary conditions must also be applied to the northeast, northwest, southeast, and southwest neighboring $u$ cells. These boundaries are located at the corners of the intrusion, and hence have characteristics of both boundary cells and free flow field cells. Khalil et al. (1975) 
suggest some type of averaging for the corner cells so that "inclined" walls can be modeled. The approach here is to treat the corner cells as if they were bounded by the wall along the entire cell boundary. The northeast $(I+I, J+I)$ and northwest $(I, J+1)$ U-cells therefore have south wall boundary conditions (see Table 3-1), and the southeast $(I+I, J-1)$ and southwest $(I, J-1)$ U-cells have north wall boundary conditions. In the course of development, these corner cells were also treated as free-flow field cells, but results did not merit the use of this approach.

Figure 3-16 shows an arbitrary V-cell with the location of the six neighboring V-cells. The $v$ at the north and south edges of the intrusions are set to zero for $(I, J)$ and $(I, J+I)$. The north and south neighbor cell boundary conditions simply become:

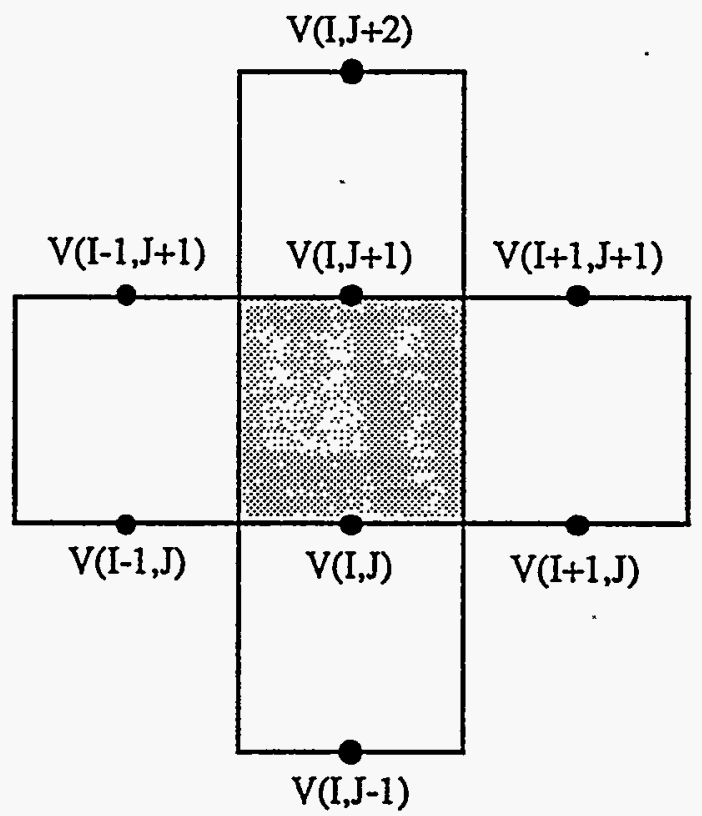

Figure 3-16. Arbitrary V-cell showing locations of cells affected by intrusion at $(\mathrm{I}, \mathrm{J})$. 


$$
\begin{aligned}
& A N(I, J-I)=0 \\
& A S(I, J+2)=0
\end{aligned}
$$

The northeast $(I+1, J+I)$ and southeast $(I+1, J)$ V-cells have west wall boundary conditions based on the logarithmic wall function (see Table 3-1) and the northwest $(I-I, J+I)$ and southwest $(I-I, I)$ V-cells have east wall boundary conditions.

\section{Input and Output for Intrusions}

The locations of the intrusions in the flow field are read from the grid data file (also used to read in the locations of grid points). A sample grid data file is shown in Appendix G. A two-dimensional array of $X^{\prime} s$ and $O$ 's is found at the bottom of the grid data file; the $X$ 's represent intrusions and walls and the $O$ 's represent the free flow field. This two-dimensional array is also used to govern the location of the additional inlets, as described in the next section. Each location in this twodimensional array represents a single computational cell, and the $x-y$ coordinates of the cells are given at the top of the grid file.

There are two logical parameters that govern the use of the intrusions in the flow field: INRDGD and INTRUS. Table 3-2 shows how these two variables affect the code operation. When starting from scratch (without a restart), setting both variables to.FALSE. will allow the code to generate a new grid data file which can then be modified to allow for intrusions. The modified grid data file will then be used with both variables.TRUE. in order to read the data file and look for intrusions.

TABLE 3-2

\begin{tabular}{|c|c|c|c|c|c|}
\hline INRDGD & INTRUS & $\begin{array}{l}\text { Reads New } \\
X^{\prime} \text { ' \& Y's }\end{array}$ & $\begin{array}{l}\text { Reads New } \\
\text { Intrusions }\end{array}$ & $\begin{array}{c}\text { Allows } \\
\text { (INORL=T) }\end{array}$ & $\begin{array}{c}\text { Writes Out } \\
\text { New Grid File } \\
\text { (GRDOUT=T }\end{array}$ \\
\hline $\mathrm{T}$ & $T$ & yes & yes & yes & yes \\
\hline $\mathrm{T}$ & F & yes & no & yes & yes \\
\hline *F & ${ }^{*} \mathrm{~T}$ & no & no & yes & yes \\
\hline F & F & no & no & I yes & yes \\
\hline
\end{tabular}

USE OF FORTRAN INPUT VARIABLES INRDGD AND INTRUS 


\section{Additional Inlets}

The principal purpose of this section is to describe the formulation which permits multiple feed streams at arbitrary locations along the combustor boundaries. A primary stream located symmetrically about the centerline, one secondary stream, and up to three additional streams in either the north or west walls of the reactor vessel are allowed. Any stream can contain particles or droplets. Figure 3-17 shows sample feed stream configurations possible with the code. Since this is a 2$\mathrm{D}$, axi-symmetric formulation, all geometries must be rotated around the centerline. A north wall inlet, therefore, is really modeled as a slit all the way around the outer reactor wall.

\section{Approach}

Table 3-1 showed boundary conditions used for the primary and secondary streams. Additional inlets along the west wall have similar boundary conditions, except for the inlet gas mixture fraction $f$. As discussed previously in the approach to gas phase chemistry, only two mixing variables are solved. This allows definition of three independent sources of mass to the gas phase. Inlet compositions and temperatures for all inlet streams must be a function of the two mixing variables. Since one mixing variable $\eta$ is generally used to account for coal off-gas, the inlets are usually specified with the remaining mixing variable $f$. For example, some inlets may contain high temperature air while others contain lowtemperature air. The three sources of mass would then be 1) coal off-gas, 2) hightemperature air, and 3) low-temperature air. The mixture fraction would be the flow ratio of high-temperature air to total inlet air, and the $f$ value for each inlet stream would determine the mixture of air entering the reactor from that stream. For CWM computations, all inlets are regarded as having the same composition and energy level, and $f$ is the mass addition of water into the gas phase from the droplets.

The staggered grid used in PCGC-2 makes the determination of inlet velocities different from $P$-cell variables. For west wall inlets, grid node $I=1$ is a false boundary used to establish velocities. Figure 3-18 shows a west wall inlet with 3 different grid nodes in the axial direction. The velocity $U(I, J)$ is specified by knowing the inlet mass flow rate, density, and cross-sectional area (in FORTRAN variables): 

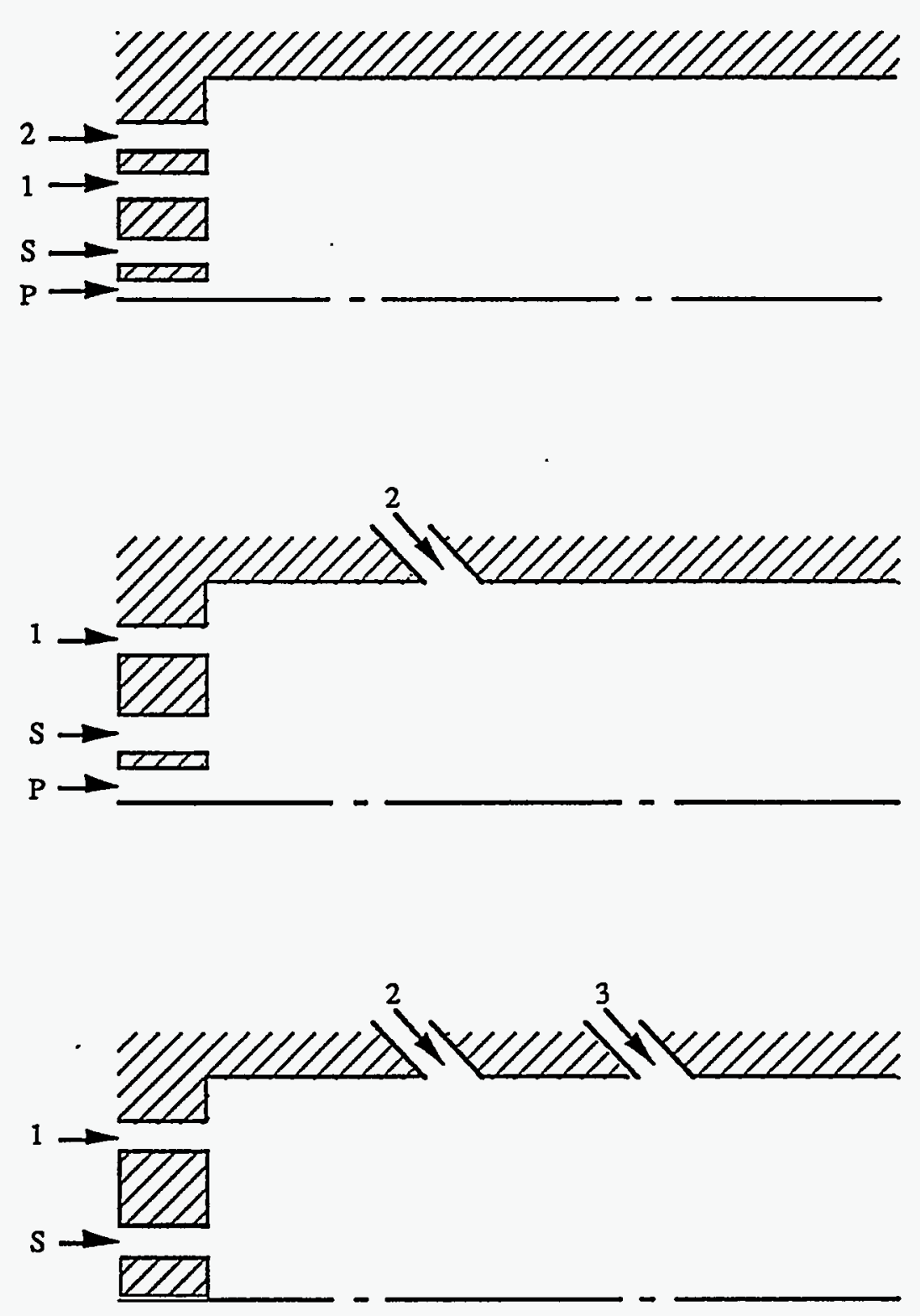

Figure 3-17. Example feed stream configurations. 


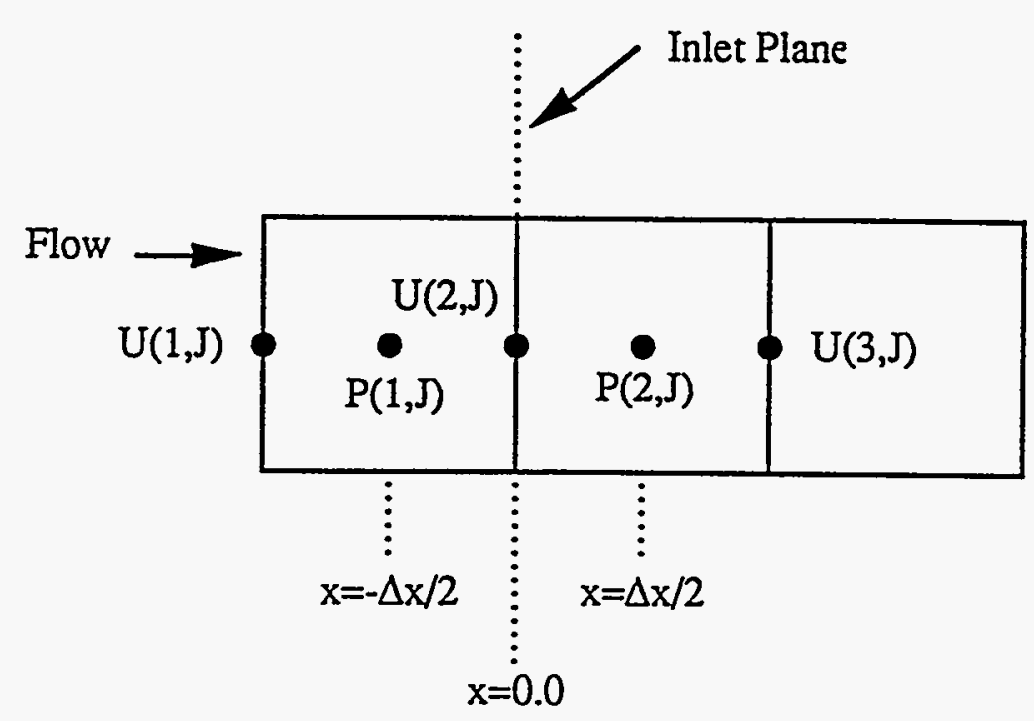

Figure 3-18. Axial grid locations for west wall inlets.

$$
U(1, J)=\dot{m}_{\text {cell }} / R H O(1, J) / A_{\text {cell }}
$$

The velocity at $U(2, J)$ is different than $U(1, J)$ due to a change in density, but continuity yields the following equation:

$$
U(1, J) * R H O(1, J)=U(2, J) *[R H O(1, J)+R H O(2, J)] / 2.0
$$

or

$$
U(2, J)=U(I, J) * \frac{R H O(I, J) * 2.0}{R H O(1, J)+R H O(2, J)}
$$
changes.

The value of $U(2, J)$ changes at every gas phase iteration because $R H O(2, J)$ 
Figure 3-19 shows a north wall inlet with 3 different grids in the radial direction. However, the maximum dimension on the $V$ array is $N J$, so the location of the $V(I, N J+I)$ is undefined. The array, VINLT(I), stores the velocities in the false boundary above the north wall. The inlet velocity at the inlet plane is then:

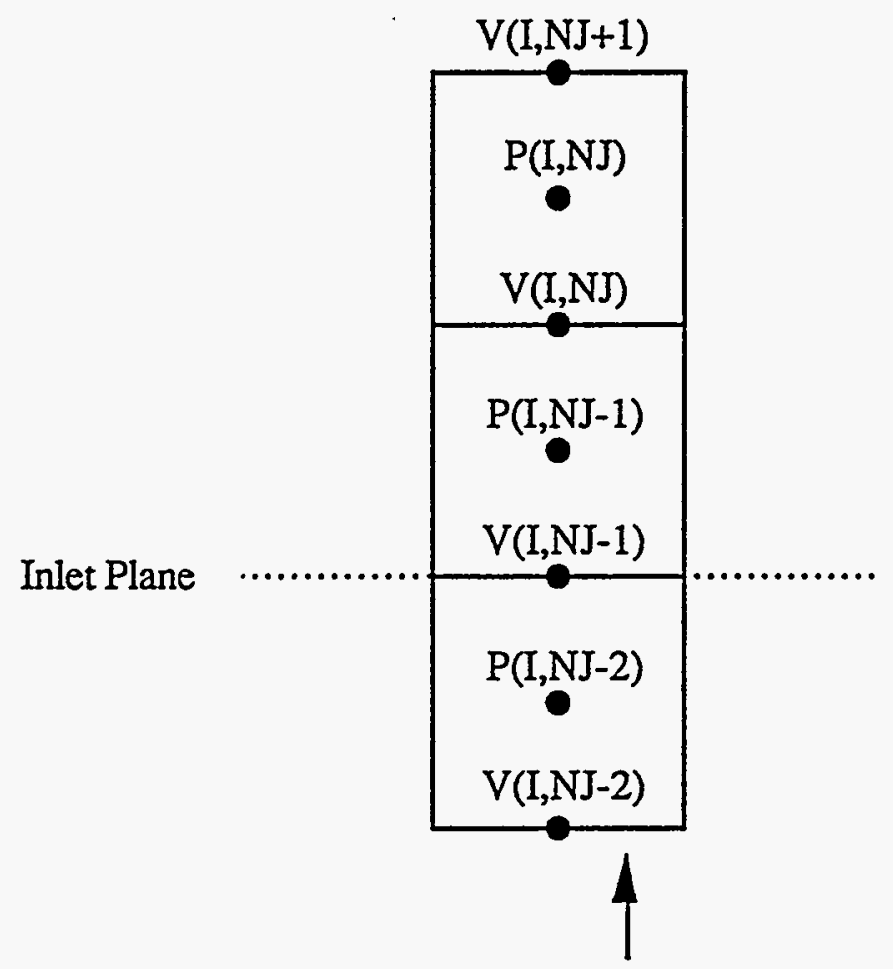

Flow

Figure 3-19. Radial grid locations for north wall inlets.

$$
V(I, N J)=V I N L T(I) * \frac{R H O(I, N J) * 2.0}{R H O(I, N J)+R H O(I, N J-I)}
$$


Provisions are made to allow for non-parallel injection in the additional inlets, but detailed velocity profiles in each additional inlet were not incorporated. The only detailed velocity profiles allowed are in the primary and secondary streams.

All inlets must be in the outer reactor shell with paths to the main flow field. Inlets are calculated only in the false boundary along the west and north walls. No inlets are permitted in the east wall (the east reactor wall is considered an exit).

\section{Input and Qutput for Additional Inlets}

A mixture fraction for each inlet stream, including primary and secondary streams is read from the main input data file. The $f$ value in the primary and secondary streams need not be 1.0 and 0.0 . Flow rates and turbulence intensities for each inlet stream are also read from the main data file.

The locations of the primary and secondary streams are read from the main data file, but the additional inlet stream locations are specified by the grid data file. $P$ represents a cell in the primary stream, the $S$ represents a cell in the secondary stream, and the numbers 1,2 , or 3 represent the first, second, or third additional inlets. Primary and secondary stream locations can be changed using either the main data file or the grid data file.

\section{Gas Phase Properties}

In Chapter 2, the theory was introduced for finding the mean turbulent properties such as species mole fraction, density, etc. The approach requires instantaneous values of the energy level $(h)$ and the weight fraction of the elements $\left(b_{k}\right)$ to be calculated from the instantaneous values of the mixture fraction and (in the case of non-adiabatic reactors) residual enthalpy. From this input, the Gibb's free energy is minimized to give the instantaneous local equilibrium properties. These properties are then convoluted over the complete probability density function with proper account for intermittency to produce the mean properties. This can be done for either Reynolds-averaging or Favre-averaging.

The largest computational portion of this algorithm is the equilibrium computations. Pratt and Wormeck (1976) have developed a code (CREK, Combustion Reaction Equilibrium and Kinetics) for either complex kinetic or equilibrium computations. This code was developed as a module for incorporation into larger fluid mechanics schemes. The approach for equilibrium computations is the same as was described in Chapter 2 of Pratt and Wormeck (1976). CREK was 
used as a base but was stripped of all statements pertaining to the kinetic computations. The input was also restructured to receive local element mass fractions $\left(b_{k}\right)$ as required by the above approach. The resulting submodel was named CREE (Chemical Reaction Equilibrium for Elements). The FORTRAN comment statements are profuse and with reference to Pratt and Wormeck (1976), the user explanation should be more than sufficient.

CREE is applicable to gaseous combustion or fuel-lean coal combustion. Because it does not treat condensed-phases, it does not give good predictions for fuel-rich coal systems (e.g. gasification) where solid carbon is a significant species at equilibrium. Therefore, another chemical equilibrium option has been provided based on the METCEC code developed by Nicoletti (1986). METCEC was based on the NASA-Lewis CEC code and properly accounts for condensed phases. It requires more time to converge than does CREE, and therefore both algorithms are provided as options in PCGC-2.

Computational efficiency is enhanced significantly by constructing table search procedures for the equilibrium properties. When the equilibrium properties are functions of only two independent variables $(f$ and $\eta)$, a table is constructed listing each property as a function of $f$ and $\eta$. The inlet and coal gas mixture fractions are physically restricted to lie between the values of 0 and 1 . A table is constructed once at the beginning of the code calculations using CREE. All subsequent calls for equilibrium properties are performed by interpolation within the table. The subroutine TABLE in PCGC-2 does all the table construction and retrieval. This subroutine uses knowledge of the stoichiometric mixture fraction for each $\eta$ even though intervals of $f$ may be different.

Chapter 2 showed how the PDF approach is altered when the system is non-adiabatic. In this case, the equilibrium properties are a function of three variables: the mixture fraction $(f)$, the coal gas mixture fraction $(\eta)$, and the residual enthalpy $\left(h_{r}\right)$. The table look-up approach is still used; however, the table is three-rather than two-dimensional. Since the three-dimensional table is quite large, it is saved from one calculation to the next.

Two types of PDF's have been used in PCGC-2; a Gaussian distribution and a top-hat or uniform distribution. The Gaussian distribution produces slightly smoother gas property profiles, but the uniform distribution requires slightly less time to obtain a converged gas-phase solution. The present version of PCGC-2 uses only the Gaussian distribution.

The convolution of gas properties with a PDF requires numerical integration (see Eqn. 2-80). This integration is time-consuming because the particle properties have to be either repeatedly calculated or interpolated from a table of values (Smith, 1979). The integration must be performed with a minimum of function evaluations. 
The method used to perform these integrations is five-point Gaussian quadrature (Abramowitz and Stegun, 1972). This method combines reasonable accuracy with a minimum number of function evaluations.

Intermittency for the Gaussian PDF is calculated as shown in Eqns. 2-47, $2-48,2-82$, and $2-83$. It is easily seen that the equations for $\alpha$ give the area under the normal curve, which is tabulated in standard math handbooks. Tabulated values of the area under the normal curve were fitted using a cubic spline routine.

\section{Radiation}

Equation 2-141 is solved in each direction, forming a set of $N$ differential equations (coupled pde's in DOM, uncoupled ode's in VFMI). Due to the different forms of the working equations, the solution techniques used in the DOM and VFM are also quite different.

\section{Discrete Ordinates Method (DOM)}

The calculation is started at the top right-hand comer of the computational domain using the boundary conditions (Eqn. 2-143). The boundary condition on the axis is based on the conservation of flux (i.e., a reflective boundary). An initial estimate of the azimuthal component of intensity, before starting with the recursive solution scheme, is obtained by solving Eqn. $2-140$ in the special direction where $\eta_{m}=0.0$. The solution thus obtained is assigned a zero weight. The directions of traverse are chosen so that the values of the direction cosines gradually increase, a change in the sign of the direction cosine signifying a reversal in the corresponding direction of integration.

The solution to the discrete ordinates equations must be obtained iteratively, as the calculated intensities enter the boundary conditions and the in-scattering term (Eqn. 2-140). Once the intensity at a cell-center is known (from Eqn. 2-154), the intensity at the downstream surface of the volume element can be obtained by extrapolation using Eqn. 2-153. However, the central differencing scheme used in Eqn. 2-153 often results in negative intensities (particularly in the presence of steep gradients, or where spatial resolution is not high enough). Such negative intensities are impossible on physical grounds, and my be avoided using a combination of central and upwind differencing (Truelove, 1978): 


$$
I_{i+1}=(I+f) I_{m}-f I_{i}, \text { etc. }, 0 \leq f \leq 1.0
$$

where $f=1.0$ yields central differencing, and $f=0.0$ yields pure upwind differencing. If negative intensities are encountered during calculations, $f$ is gradually decreased from its initial value of 1.0 till the negativity is removed. For $f$ $=0.0$, the intensity is always non-negative, and special directions are not required to initiate the recursive solution.

In the case of a scattering medium, an approximate solution is first obtained without the in- and out-scattering terms. The scattering terms are then included as a perturbation to the approximate solution to obtain the final, converged solution for the absorbing-emitting-scattering case.

\section{Varma Flux Method}

The two ordinary differential equations (Eqns. 2-157 and 2-158) are cast into finite difference form as shown in Table 3-3. The ' $\mathrm{C}$ ' coefficients are defined in Table 211. These equations are solved using a line-by-line technique as in the gas phase. The tridiagonal matrix algorithm (TDMA) gives an exact solution on each line. The radiation calculations generally converge quickly, and do not take much computational time relative to the gas-phase calculations. Axial symmetry is used for the centerline boundary condition, and the wall boundary conditions (see Eqn. 2-143) are treated in the same way as those for the gas-phase calculations.

\section{TABLE 3-3}

FINITE DIFFERENCE FOR THE RADIATION FLUX SUMS

$$
\begin{array}{ll}
\phi & \text { Equation } \\
\hline F_{x} & A_{p}^{x} \phi_{p}=A_{E} \phi_{E}+A_{W} \phi_{W}+S_{U}^{x} \\
F_{r} & A_{p}^{r} \phi_{p}=A_{N} \phi_{N}+A_{S} \phi_{S}+S_{U}^{r} \\
\hline & A_{p}^{x}=A_{E}+A_{W}-S_{p}^{x} \\
& A_{p}^{r}=A_{N}+A_{S}-S_{p}^{n}
\end{array}
$$


TABLE 3-3 (continued)

$$
\begin{gathered}
A_{E}=\frac{\Gamma_{P}+\Gamma_{E}}{2 \delta x_{P E}} \\
A_{W}=\frac{\Gamma_{W}+\Gamma_{P}}{2 \delta x_{P W}} \\
A_{N}=\frac{\Gamma_{P}+\Gamma_{N}}{2 \delta r_{N P}} \\
A_{S}=\frac{\Gamma_{S}+\Gamma_{P}}{2 \delta r_{P S}} \\
S_{u}^{x}=2\left(C_{3} F_{r}+C_{4} I_{b}\right)(S E W) \\
S_{u}^{r}=2 r\left(C_{3} F_{x}+C_{4} I_{b}\right)(S N S) \\
S_{p}^{x}=\left(C_{2}+C_{l}\right)(S E W) \\
S_{p}^{r}=\left(C_{2}+C_{l}\right)(S N S)
\end{gathered}
$$




\section{Approach}

The particle source-in-cell (PSI-CELL) technique of Crowe and coworkers (Crowe et al., 1977) has been followed directly to account for the Lagrangian particle field in the Eulerian gas field. The procedure is outlined below:

1. The Eulerian gas field is solved without particles as described earlier.

2. The radiation field is solved using the flux method, as described earlier.

3. The Lagrangian particle field is solved with a representative number of trajectories and the particle source term field is calculated.

4. The gas field is solved with the updated particle source term field.

5. Step 2 is repeated and iteration continued until overall convergence is achieved.

The PSI-CELL technique is very efficient with respect to storage and computational time required. The only significant storage requirement is for the particle source term field. Steps 2 through 4 represent one "particle iteration" (steps 7 through 11 in Fig. 3-1). Convergence is achieved when the gas field does not change between particle iterations.

The Lagrangian particle equation of motion (Eqn. 2-19) is integrated once analytically to save computational time. The $x$-component of the equation can be written as

$$
\frac{d u_{j}}{d t}=\frac{\Gamma_{d}\left(u_{g}-u_{j}\right)}{\alpha_{j}} \pm g
$$

where the gravitational force term assumes the reactor is vertical ( + for down-fired, - for up-fired). Substituting Eqn. 2-21 for $\Gamma_{d}$ and rearranging, Eqn. 3-353 becomes

$$
\frac{d u_{j}}{d t}=\frac{C_{d} R e}{24 t_{j}}\left(u_{g}-u_{j}\right) \pm g
$$


where $C_{d}$ is given by Eqns. 2-22 through 2-24, $R e$ is given by

$$
R e=\frac{\rho_{g}\left\|\vec{v}_{j}-\vec{v}_{g}\right\| d_{j}}{\mu_{g}}
$$

and $t_{j}$ is given by Eqn. 2-30. Taking $u_{g}$ and $\frac{C_{d} R e}{t_{j}}$ constant, Eqn. 3-255 may be integrated to obtain

$$
\ln \left[\frac{A\left(u_{g}-u_{j}^{\text {new }}\right) \pm g}{A\left(u_{g}-u_{j}^{\text {old }}\right) \pm g}\right]=-A(\Delta t)
$$

where $A=\frac{C_{d} R e}{24 t_{j}}$. Equation 3-257 may be solved for $u_{j}^{\text {new }}$ to obtain

$$
u_{j}^{\text {new }}=u_{g}-\left(u_{g}-u_{j}^{o l d}\right) e^{-A(\Delta t)} \pm \frac{g}{A}\left(1-e^{-A(\Delta t)}\right)
$$

A similar treatment of the $r$-component of Eqn. 2-19 leads to

$$
v_{j}^{\text {new }}=v_{g}-\left(v_{g}-v_{j}^{o l d}\right) e^{-A(\Delta t)}
$$

where the gravitational force term is missing because of the assumed vertical orientation of the reactor. Equations 3-357 and 3-358 are integrated by the simple Euler method to obtain particle position as a function of time.

The simple Euler's method was found satisfactory for calculating the particle trajectory, but unsatisfactory for integrating the particle continuity (Eqns. 290 through 2-94) and energy (Eqn. 2-97) equations; hence, the modified Euler's method is used to integrate the latter. The step-size for the integration scheme must be small enough so that the particle does not cross more than one cell boundary between function evaluations. Step sizes must be small in regions of rapid coal reaction in order to achieve numerical accuracy, but large enough in all regions to be numerically efficient. PCGC-2 uses a self-adjusting step-size control based on the difference between the predictor and corrector of the modified Euler's method.

The diffusive component of particle velocity is determined from Eqn. 2-34: 


$$
\vec{v}_{j d}=-\Gamma_{j} \frac{\vec{\nabla} \bar{n}_{j}}{\bar{n}_{j}}
$$

In practice, $\bar{\nabla} \bar{n}_{j}$ is difficult to calculate on the computer, because $\bar{n}_{j}$ is a large number. The calculation becomes inaccurate because of computer roundoff and taking the difference of two large numbers. To overcome this computer limitation, $\vec{\nabla} \bar{n}_{j}$ is calculated as follows:

$$
\vec{v} \bar{n}_{j}=\bar{n}_{j} \vec{\nabla}\left(\ln \bar{n}_{j}\right)
$$

Equation 3-359 becomes

$$
\vec{v}_{j d}=-\Gamma_{j} \vec{\nabla}\left(\ln \bar{n}_{j}\right)
$$

When a particle collides with the wall, the particle is allowed to 1) stick to the wall (in which case the particle trajectory calculation stops), or 2) bounce off the wall. The particles are arbitrarily allowed to exit from recirculation zones by setting the diffusion velocity to zero after three loops, if such a situation occurs.

\section{Stiffness}

Problems with stability occur in the integration of the energy equation when the integrand changes slowly. In such areas, oscillations in the energy level occur that generally increase in amplitude until solution is no loner feasible (these are called numerically "stiff" equations). When the integrand begins to change slowly, a numerical approximation is made in which the left-hand side and 3rd term on the right-hand side of Eqn. 2-97 are set to zero and the particle properties are solved with an algebraic equation for the temperature:

$$
T_{p j}=T_{g}+\frac{Q_{r p}}{N u_{j} \pi k_{g} d_{j}}
$$

This equation can also be viewed from a physical standpoint. In downstream regions of the reactor, temperature gradients are small and radiation is the main cause of differences between gas and particle temperatures. 
The particle energy stiffness assumption was validated by comparison with a prediction using very small step sizes. This stiffness assumption greatly decreases computational time for each trajectory without significant loss of accuracy. Care is taken to de-dimensionalize the criteria for employing the stiffness assumption in PCGC-2, in order to simplify manipulation by the user. Smith (1979) found that the errors introduced in 1-DICOG from the stiffness assumption are negligible, and that significant computational time is saved because larger integration steps can then be taken. The same conclusions are found true of the particle energy equation in PCGC-2 (Fletcher, 1980).

\section{Particle Temperature}

The particle temperature depends on its composition and enthalpy. All of the components of the particle, i.e. composition and enthalpy, are predicted as independent quantities. At each time step, these properties are used to solve for particle temperature. Because there are several options in the code for expressing the particle heat capacity and its dependence on temperature, an efficient and robust algorithm is used which is based on a combination of bisection and regula-falsi techniques.

\section{Particle Reactions}

Calculation of the particle reaction rates and heat fluxes is iterative. Oxidizer-char reaction rates (Eqn. 2-131) depend on the char mass transfer coefficient (Eqn. 2134) which, in turn, depends on the transpiration parameter for mass transfer (Eqn. 2-133) and the reaction rate. The transpiration parameter and mass transfer coefficient are calculated using assumed reaction rates, and the reactions are then iterated on until converged. Under-relaxation is used to make the calculation more stable.

\section{Particle-Gas Coupling}

\section{Mixed Eulerian-Lagrangian Particle Model}

An information flow diagram for the mixed Eulerian-Lagrangian particle model is shown in Fig. 3-20. Eulerian particle number density is needed by the radiation 


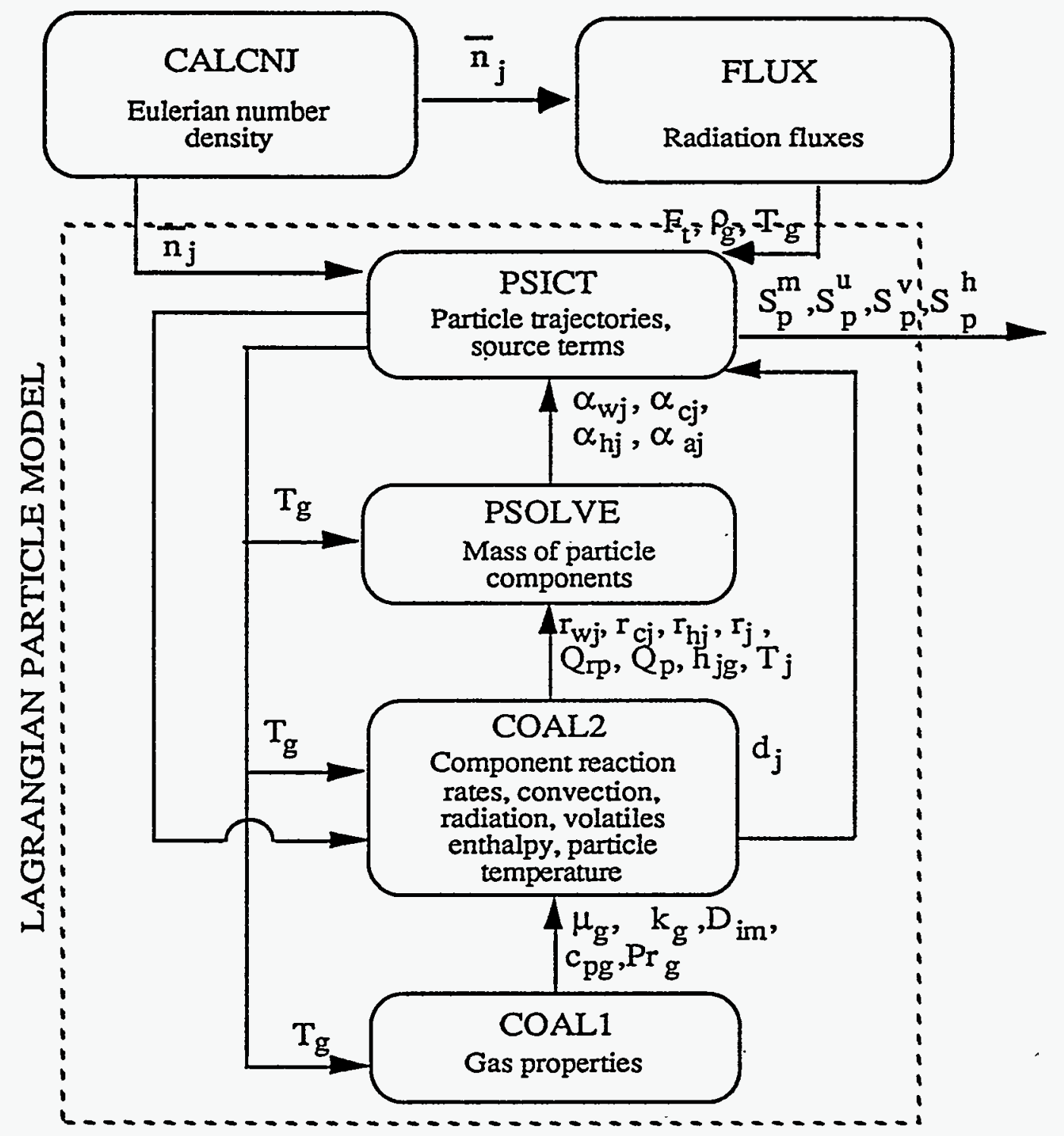

Fig. 3-20. Information flow diagram for mixed Eulerian-Lagrangian particle model. 
submodel and the Lagrangian particle submodel for calculating turbulent particle dispersion. Four routines perform most of the calculations in the Lagrangian particle model. PSICT calculates the particle trajectories and gas-phase source terms. PSOLVE integrates the particle enthalpy and continuity equations. COAL2 calculates the coal and char reaction rates, rates of heat transfer to the particle, particle temperature, and coal volatiles enthalpy. COAL1 calculates the local gas properties needed by COAL2.

\section{Particle Source Terms}

The Eulerian gas phase equations are coupled to the Lagrangian particle phase equations through particle source terms $\left(S_{p}^{m}, S_{p}^{u}, S_{p}^{v}, S_{p}^{h}\right)$. The particle field is modeled as a set of discrete trajectories, where each trajectory represents a number of particles of uniform particle size and starting location in the primary jet. It is assumed that the particle number flow rate $\left(\dot{n}_{i j}\right)$ along a trajectory is constant, so that $\dot{n}_{i j}$ may be calculated from the initial mass flow rate of particles $\left(\dot{m}_{p o}\right)$, the appropriate initial mass fractions representing particle size and location, and the initial particle mass $\left(\alpha_{j i o}\right)$. The particle number flow rate of the $i^{\text {th }}$ particle size and the $j^{\text {th }}$ starting location is calculated from the input conditions as follows:

$$
\dot{n}_{i j}=\frac{\dot{m}_{j o} X_{i o} Y_{j o}}{\alpha_{j i o}}
$$

where $X_{i o}$ is the mass fraction of particles of the $i^{t h}$ particle size and $Y_{j o}$ is the mass fraction of the $j^{\text {th }}$ starting location.

The particle mass source $\left(S_{p}^{m}\right)$ to the gas phase is the change in mass of all particles that traverse the particular cell of interest. For the $k^{\text {th }}$ cell, this change is represented by:

$$
\left(\Delta \dot{m}_{p i j}\right)_{k c e l l}=\dot{n}_{i j}\left[\left(\alpha_{p i j}\right)_{o u t}-\left(\alpha_{p i j}\right)_{i n}\right]_{k c e l l}
$$

The mass source term is taken to be negative when the particle loses mass in order to fit the gas-phase sign convention. The mass source term is calculated from: 


$$
\left(S_{p}^{m}\right)_{k c e l l}=\left[\frac{\sum_{i} \sum_{i} \Delta \dot{m}_{p i j}}{V}\right]_{k c e l l}
$$

The particle momentum source term $\left(S_{p}^{u}\right)$ for the $u$ component of momentum is similarly derived:

$$
\left(S_{p}^{u}\right)_{k c e l l}=\left(\frac{I}{V} \sum_{i} \sum_{j} \dot{n}_{i j}\left[\left(u_{p i j} \alpha_{p i j}\right)_{o u t}-\left(u_{p i j} \alpha_{p i j}\right)_{i n}\right]\right)_{k c e l l}
$$

The equation for $S_{p}^{v}$ is similar to that for $S_{p}^{u}$ :

$$
\left(S_{p}^{v}\right)_{k c e l l}=\left(\frac{1}{V} \sum_{i} \sum_{j} \dot{n}_{i j}\left[\left(v_{p i j} \alpha_{p i j}\right)_{o u t}-\left(v_{p i j} \alpha_{p i j}\right)_{i n}\right]\right)_{k c e l l}
$$

The particle energy source term $\left(S_{p}^{h}\right)$ represents the energy given to the gasphase by the particles. The enthalpy of the solid phase changes by convection, reaction, and radiation. The change in solids enthalpy due to convection and reaction affects the enthalpy of the gas in the cell, but the change due to radiation does not. Therefore, $S_{p}^{h}$ is calculated from the net change in solids enthalpy excluding the effects of radiation:

$$
\left(S_{p}^{k}\right)_{k c e l l}=\left(\frac{1}{V} \sum_{i} \sum_{j} \dot{n}_{i j}\left[\left(h_{p i j}^{\prime} \alpha_{p i j}\right)_{o u t}-\left(h_{p i j}^{\prime} \alpha_{p i j}\right)_{i n}\right]\right)_{k c e l l}
$$

where $h_{p i j}^{\prime}$ is a pseudo enthalpy for the particle that neglects the effects of radiation. It is calculated from Eqn. 2-97 with the radiation term removed:

$$
\frac{d\left(\alpha_{j} h_{j}\right)}{d t}=Q_{j}-(1-\chi) r_{j} h_{j g}
$$


It should be realized that Eqn. 3-368 includes the effects of chemical reaction.

It has been found that the particle mass source terms sometimes introduce instabilities into the program especially during the first few gas-phase iterations. Therefore, the source terms are under-relaxed according to Eqn. 3-202. A value of 0.5-0.7 is typically recommended for the under-relaxation factor.

\section{Particle Number Density}

The bulk particle number density couples the particle field with the gas field in two ways: turbulent particle dispersion, and radiation coupling between the phases. This is evident in Eqns. 2-33, 2-34, and 2-140. The equation for $n_{j}$ is found in Chapter 2 (Eqn. 2-35). This equation does not fit the general form of the gas phase equations in Table 2-1 due to the missing density $(\rho)$. As was noted in the earlier discussion of Eqn. 2-35, the $u_{j}$ and $v_{j}$ terms are replaced by $u_{g}$ and $v_{g}$ :

$$
\frac{\partial}{\partial x}\left(r \tilde{u}_{g} \bar{n}_{j}\right)+\frac{\partial}{\partial r}\left(r \bar{v}_{g} \bar{n}_{j}\right)-\frac{\partial}{\partial x}\left(r \Gamma_{j} \frac{\partial \bar{n}_{j}}{\partial x}\right)-\frac{\partial}{\partial r}\left(r \Gamma_{j} \frac{\partial \bar{n}_{j}}{\partial r}\right)=0
$$

This equation is cast into finite difference form in a manner analogous to the other gas phase variables. Differences occur in the definition of the convection coefficients ( $C$ 's) and in the two modifications. The resulting finite difference equation is shown in Table 3-4. This equation is solved using the TDMA.

In the calculation of $\Gamma_{j}$ from Eqns. 2-28 through 2-32, the ratio $\alpha_{j} / d_{j}$ is required. In PCGC-2 this ratio is evaluated at the reactor inlet $\left(\alpha_{j o} / d_{j o}\right)$ and assumed constant. The boundary conditions for Eqn. 3-370 are shown in Table 35 .

TABLE 3-4

FINITE DIFFERENCE EQUATION FOR $\theta=\bar{n}_{j}$

$$
A_{P} \theta_{P}=A_{E} \theta_{E}+A_{W} \theta_{W}+A_{N} \theta_{N}+A_{S} \theta_{S}+S_{U}
$$

where

$$
A_{P}=A_{E}+A_{W}+A_{N}+A_{S}-S_{P}
$$


TABLE 3-4 (continued)

$$
\begin{gathered}
A_{E}=D_{E}^{*}-\frac{1}{2} C_{E} \\
A_{W}=D_{W}^{*}+\frac{1}{2} C_{W} \\
A_{N}=D_{N}^{*}+\frac{1}{2} C_{N} \\
A_{S}=D_{S}^{*}+\frac{1}{2} C_{S} \\
D_{d}^{*}=\max \left(D_{d}, \frac{-C_{d}}{2}, \frac{C_{d}}{2}\right) w h e r e d=E, W, N, o r S \\
D_{E}=\frac{\left(\Gamma_{\phi P}+\Gamma_{\phi E}\right) A_{e w}}{2 \delta x_{P E}} \\
D_{W}=\frac{\left(\Gamma_{\phi P}+\Gamma_{\phi W}\right) A_{e W}}{2 \delta x_{P W}} \\
D_{N}=\frac{\left(\Gamma_{\phi P}+\Gamma_{\phi N}\right) A_{n}}{2 \delta x_{P N}} \\
D_{S}=\frac{\left(\Gamma_{\phi P}+\Gamma_{\phi S}\right) A_{s}}{2 \delta x_{P S}} \\
C_{E}=A_{e w} u_{E}
\end{gathered}
$$


TABLE 3-4 (continued)

$$
\begin{gathered}
C_{W}=A_{e w} u_{P} \\
C_{N}=A_{n} v_{N} \\
C_{S}=A_{s} v_{P} \\
S_{U}=-\min \left[\left(C_{E}-C_{W}+C_{N}-C_{S}\right), 0\right] \phi_{P}^{\text {old }} \\
S_{P}=-\max \left[\left(C_{E}-C_{W}+C_{N}-C_{S}\right), 0\right]
\end{gathered}
$$

TABLE 3-5

BOUNDARY CONDITIONS FOR $\bar{n}_{j}$

Inlet: primary: $\quad \bar{n}_{j}=\bar{n}_{j o}$ secondary: $\quad \bar{n}_{j}=0$

Side walls:

$\frac{d}{d x}\left(\bar{n}_{j}\right)=0$

Top wall:

$\frac{d}{d r}\left(\bar{n}_{j}\right)=0$

Symmetry Axis

$\frac{d}{d r}\left(n_{j}\right)=0$

Outlet:

Quadratic upstream extrapolation 
Solution Technique

Nitrogen Pollutants Submodel

\section{Algorithm}

The equations in the $\mathrm{NO}_{\mathrm{x}}$ model are similar in form to the Eulerian gas-phase equations, and use the same solution technique as described previously. The major difference in solution of the equations in the $\mathrm{NO}_{\mathbf{x}}$ model is the calculation of the overall reaction rates in the species continuity equations for $\mathrm{NO}, \mathrm{HCN}$, and $\mathrm{NH}_{3}$. The procedure and equations used to calculate these reaction rates in the $\mathrm{NO}_{x}$ model were described in Chapter 2 .

The equations for the pollutant species are decoupled from the coal combustion model since small pollutant levels will not significantly affect the velocity, density, or temperature fields. However, the equations for the pollutant species are coupled with each other since the pollutant reaction rates are solved following convergence of the fuel oxidation calculations.

The solution algorithm of the NO model involves starting with a guess for the initial pollutant concentrations throughout the reactor. Fractional conversion variables are then calculated for each nitrogen pollutant species, $\mathrm{O}_{2}$, and $\mathrm{N}_{2}$. Next, integration of the instantaneous rates over the probability of fluctuating mixture fractions is made. The local instantaneous temperature is calculated by knowing unique values of $f$ and $\eta$ associated with each point in the Gaussian quadrature. Values of the instantaneous species are calculated by scaling the local instantaneous maximum or equilibrium concentrations by the appropriate local fractional conversion variable. Local instantaneous rates are next calculated for each reaction in the kinetic mechanism. The integrated rate expressions are then summed to obtain overall mean rates for each nitrogen pollutant species. Species continuity is then solved for $\mathrm{HCN}, \mathrm{NH}_{3}$, and $\mathrm{NO}$ using the routines available in PCGC-2. $\mathrm{O}_{2}$, $\mathrm{OH}$, and $\mathrm{N}_{2}$ mass fractions are calculated by performing atom balances. The updated mass fractions are then used to re-calculate the reaction progress variables. Thus, the solution is iterative, converging on the concentration fields for $\mathrm{HCN}$, $\mathrm{NH}_{3}$, and NO. Figures 3-21 and 3-22 show a flow chart of this procedure. 


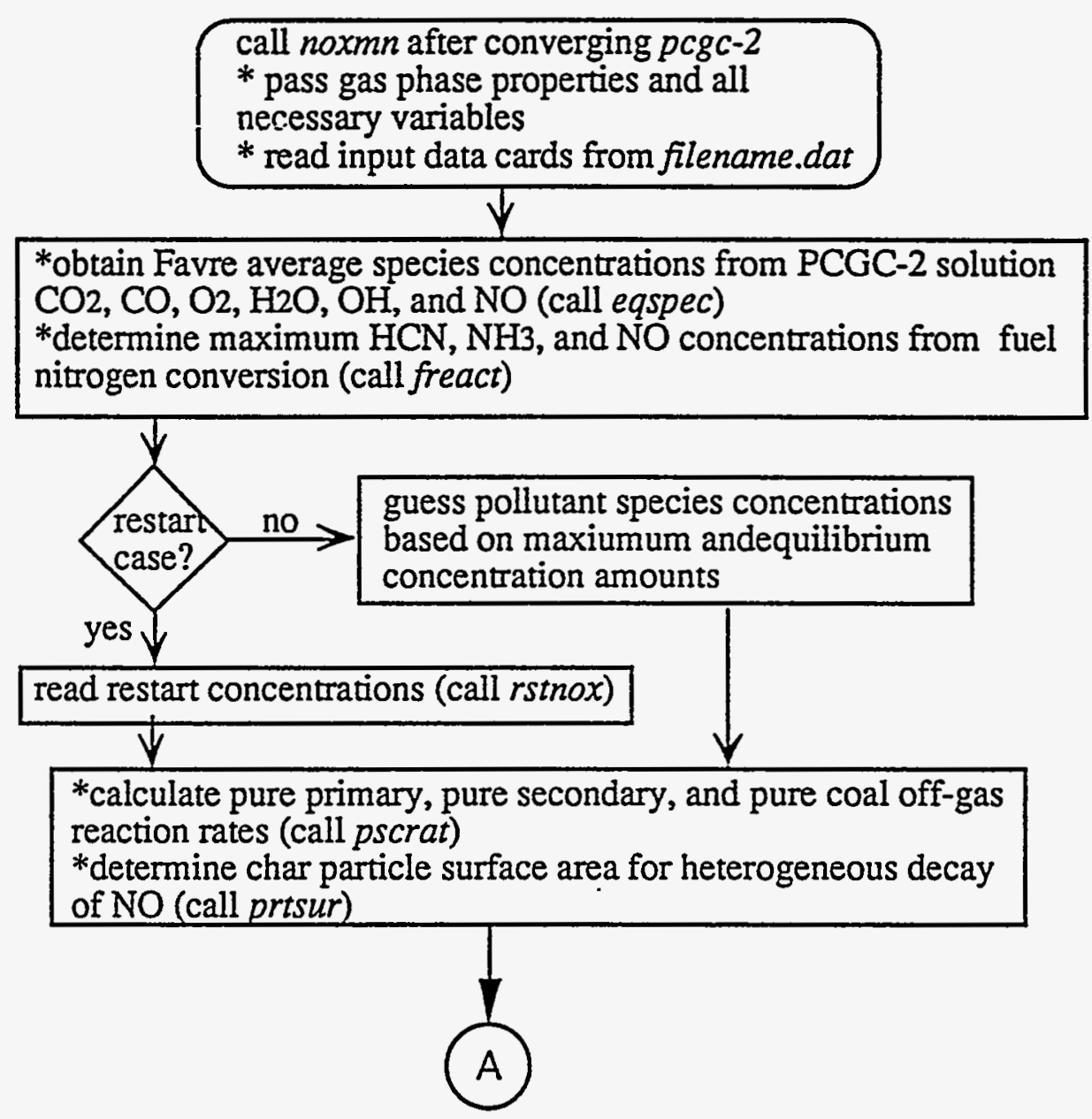




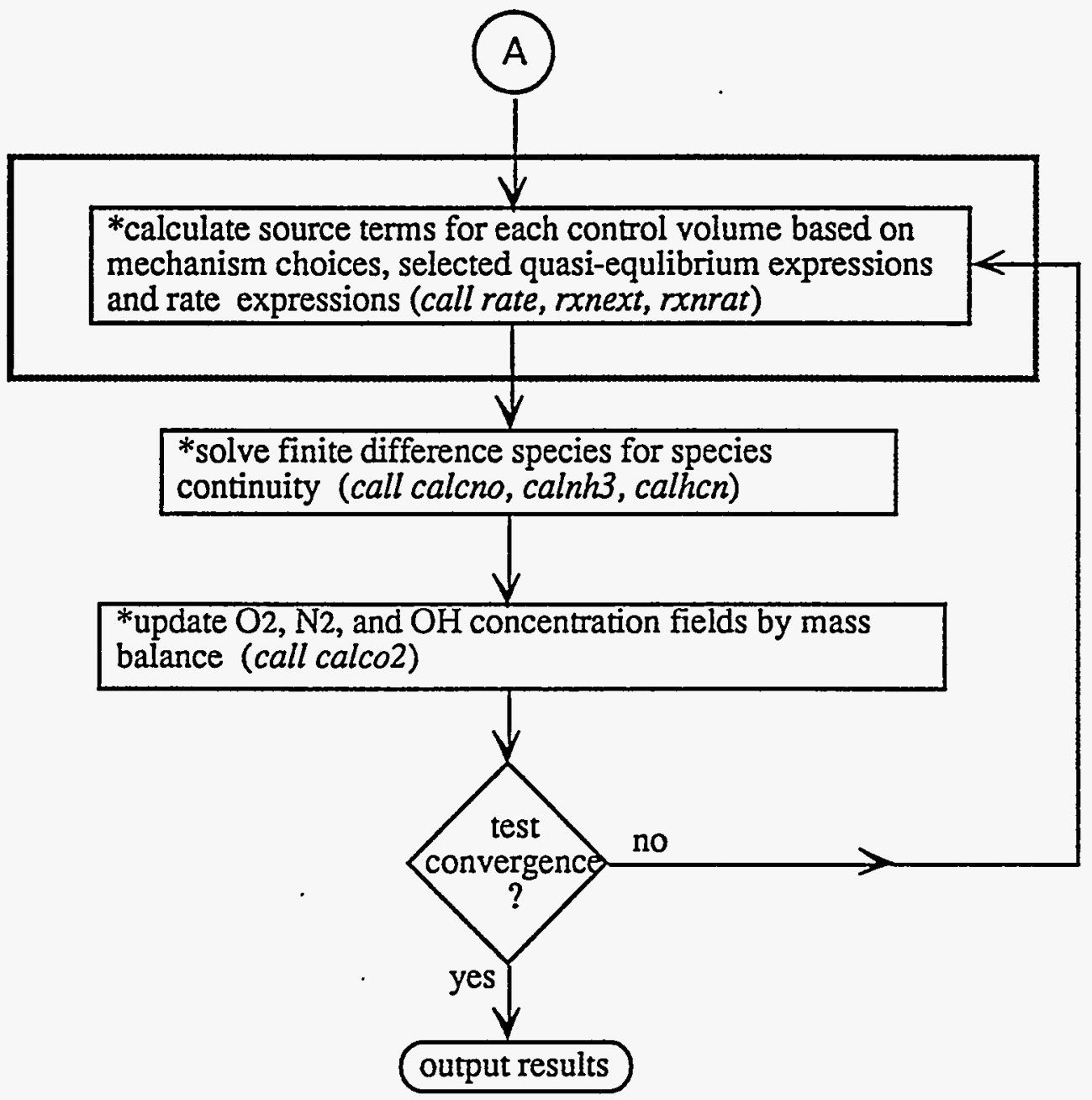

Fig. 3-21. $\mathrm{NO}_{\mathrm{x}}$ model logical sequencing. Shaded box is expanded in Figure 322. 


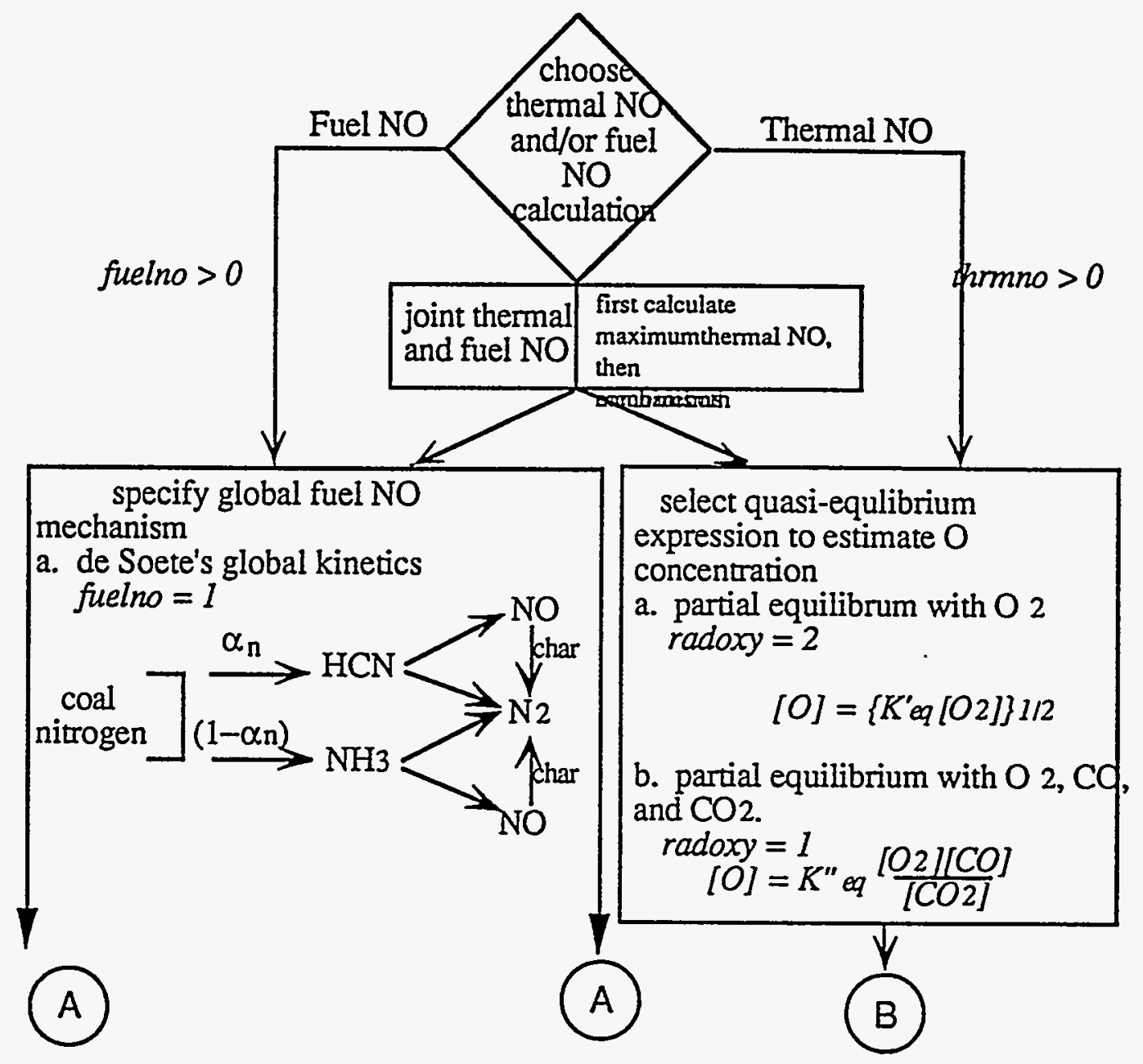




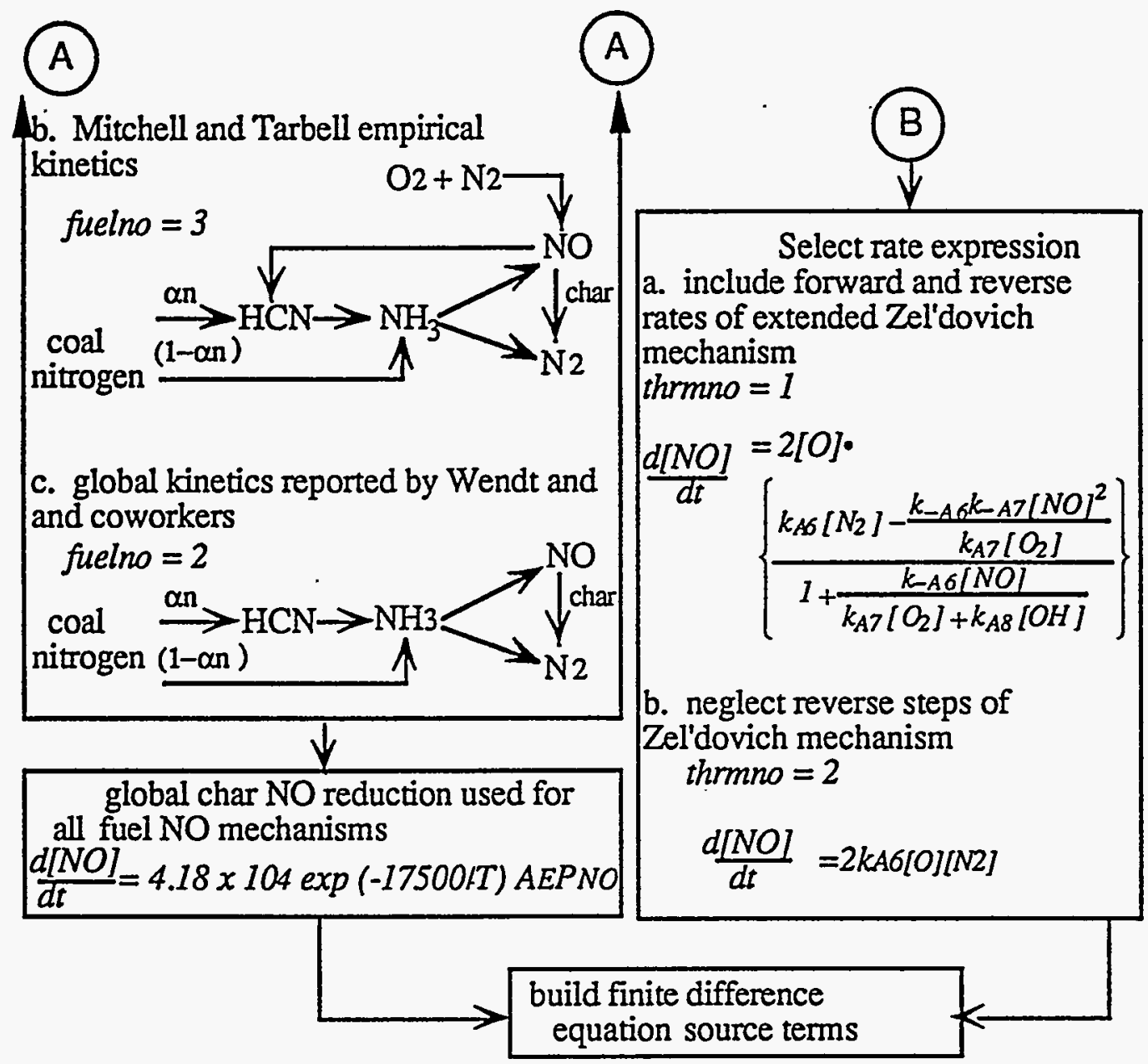

Fig. 3-22. Expansion of shaded box in Fig. 3-21. showing the alternative mechanisms and options available in the revised $\mathrm{NO}_{\mathrm{x}}$ model. Input flags and values are discussed in the Chapter 5. 
Sulfur Pollutants and Sorbent Reactions Submodel

\section{Sulfur Pollutants and Sorbent Reactions Submodel}

The sulfur reactions submodel is also implemented as a post-processor to the comprehensive particle-gas flame calculations. An information flow diagram is shown in Fig. 3-23. After converging the reacting gas and particle flowfield, the sorbent model is called, and Eulerian gas properties are passed to it. Eulerian equations are then solved for sorbent particle number density, in a manner similar to the coal particles. Lagrangian trajectories representing ensembles of sorbent particles are then calculated, also in similar manner to the coal particles. The sorbent particles are allowed to react with either $\mathrm{SO}_{2}$ or $\mathrm{H}_{2} \mathrm{~S}$, providing source (sink) terms for the selected sulfur species. After calculating ing sorbent particle trajectories and $\mathrm{SO}_{2}$ or $\mathrm{H}_{2} \mathrm{~S}$ source (sink) terms, a continuity eqLi:ion is solved for either $\mathrm{SO}_{2}$ or $\mathrm{H}_{2} \mathrm{~S}$, to distribute the effects of the capture througinout the flowfield. This provides a new concentration field for the sulfur species, and the sorbent particle trajectories and reactions are calculated again, providing new source terms, which are used to calculate a new sulfur species concentration field. This procedure is repeated until converged.

When calculating the new sulfur species concentration field, it is assumed that sulfur evolving from the coal forms $\mathrm{SO}_{2}$ and $\mathrm{H}_{2} \mathrm{~S}$ in a ratio equal to that predicted for the reactor effluent gas at the conclusion of Step 1. Since there is no homogeneous chemistry considered in the submodel, there is no interconversion between $\mathrm{SO}_{2}$ and $\mathrm{H}_{2} \mathrm{~S}$, once formed. This leads to error in the prediction of sulfur species concentration in the fuel-rich region of a fuel-lean flame and in the fuel-lean region of a fuel-rich flame, but these regions are fairly small compared to the overall size of the combustor, and most sulfur capture occurs in the post-flame region. In fact, sorbent is often injected in the post-flame region in order to avoid the high flame temperatures that might cause sintering and loss of effectiveness. 


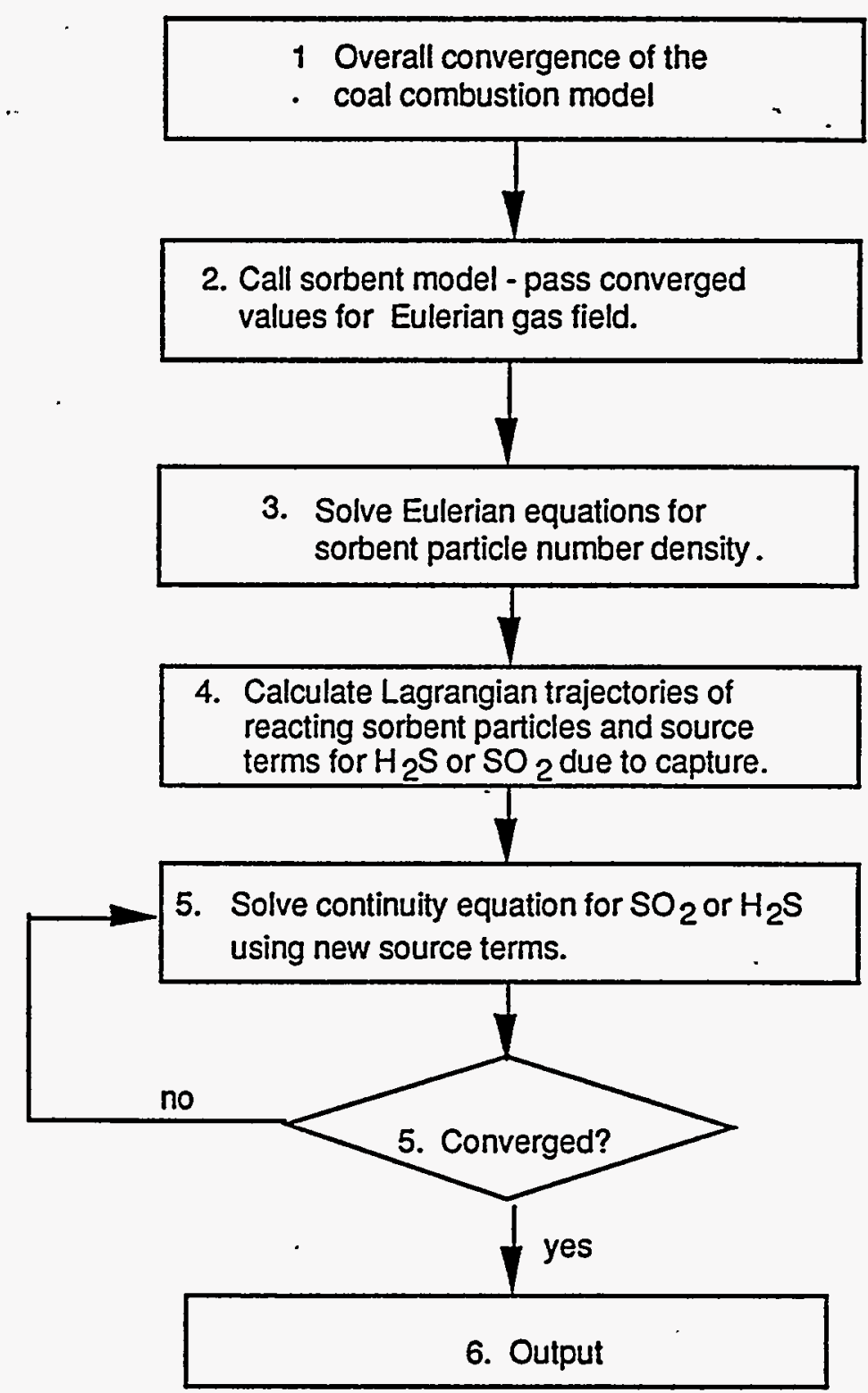

Fig. 3-23. Information flow diagram for the sorbent/sulfur capture submodel. 


\section{Chapter Four}

\section{Generalized Particle Reactions}

This chapter presents a general description of FG-DVC, the model which simulates the coal thermal decomposition in the absence of oxygen, with its three submodels: coal viscosity, coal particle swelling and sulfur and nitrogen devolatilization. The coal reactivity model is discussed in this chapter as well. The nomenclature of all the variables in the equations of this chapter is given in Table 4-1.

\section{FG-DVC Model Basis}

FG-DVC is a general model for coal pyrolysis which predicts the coal's decomposition into tar, char, and gas, given the ambient pressure and the temperature history of an homogeneous coal particle. The model predicts the amount, functional group composition, elemental composition, and molecular weight distribution of tar and char and the amount and composition of the gas. The model is described in more detail in Appendices J-O.

The letters FG stand for Functional Group, and DVC for Depolymerization, Vaporization and Crosslinking. The FG model considers certain functional groups in the coal which decompose to form the light gas 
species. At the same time, the DVC model describes the overall depolymerization of the macromolecular network which combines bridge breaking and crosslinking to produce fragments of the coal macromolecule. These fragments are then subjected to transport behavior, specifically the vaporization of the lightest fragments to form tar. The tar fragmentation process provides a second mechanism for the removal of functional groups from the coal. The model, whose parameters are determined in the laboratory at moderate temperatures and one atmosphere, can then be used to extrapolate away from the laboratory conditions to predict pyrolysis and combustion in high temperature reactions, or liquefaction at high pressure.

The model for coal thermal decomposition has six basic concepts.

- Functional Groups (decompose to produce light gases)

- Macromolecular Network (decomposes to produce tar and metaplast)

- Network Coordination Number (possible number of attachments per cluster)

- Bridge Breaking (limited by hydrogen availability)

- Crosslinking (related to gas evolution)

- Mass Transport of Tar (evaporation of light network fragments into light gases)

The first concept is that light gases are formed by the decomposition of certain functional groups in the coal. For example, methyl groups can lead to the formation of methane, carboxyl groups can lead to the formation of $\mathrm{CO}_{2}$, etc. The second concept is that coal consists of a macromolecular network. This network is made up of fused aromatic ring clusters (which are described by their molecular weight) linked by bridges, some of which are relatively weak. There are some unattached parts of the network which can be extracted. Sometimes, there is also a second component of high polymethylene content. When heated, this network decomposes to produce smaller fragments. The lightest of the fragments evaporate to produce tar and the heavier fragments form the metaplast. These heavier molecules are the primary liquid fragments in liquefaction or the fragments that make coal fluid. 
The third concept is that one of the most important properties of the network is its coordination number. The coordination number describes the geometry of the network, and specifies how many possible attachments there are per aromatic ring cluster. For example, a linear polymer chain has a coordination number of 2 , because each fused aromatic ring has two possible attachments to link it in the chain. On the other hand, a square "fish net" has a coordination number of 4 , because there are four possible attachments at each ring cluster. The coordination number controls the molecular weight distribution of the network fragments at a given extent of decomposition. The extent of decomposition is specified by the probability that the possible attachments are made. For example, for $20 \%$ of broken bridges, a linear chain is totally fragmented, while a "fish net" will have some holes but is almost totally connected. In describing the network, a crosslink is defined to occur at a branch point where there are more than two attachments on a ring cluster. The coordination number is thus related to the crosslink density. With no possible crosslinks, the coordination number is two. With increasing crosslink density the coordination number increases.

The second important property of the network is the fraction of possible attachments, $p$, which are actually present. During thermal decomposition, this fraction is determined by the rates of bridge breaking. The factors which control how many of the weak links can break are the rate constant and the amount of hydrogen that can be donated from the coal to stabilize the free radicals which form when the links break.

A competitive process with the bond breaking is the retrogressive process of crosslinking. Crosslinking reactions appear to be related to the evolution of certain gases. Specifically, for low rank coals, crosslinking at low temperature (prior to bridge breaking) seems to be related to the evolution of carbon dioxide. For coals of all rank, a higher temperature crosslinking event (following bridge breaking) seems to be related to the evolution of methane. At high temperatures, the evolution of hydrogen is also related to crosslinking.

The final concept is that the tar evolution is controlled by mass transport. Bridge breaking and crosslinking produce a set of fragments with a molecular weight distribution. The lightest fragments can leave the coal melt by evaporation 
into the light gas species. The heavier fragments remain, forming the metaplast which controls the coal's fluidity. In the following sections, the descriptions of DVC and FG, and other coal chemistry submodels: viscosity, particle swelling, sulfer and nitrogen, and reactivity, are given. More details can be found in Appendices $\mathrm{J}$ and $\mathrm{K}$ for FG-DVC.

\section{Depolymerization-Vaporization-Crosslinking (DVC) Formulation}

The DVC model predicts the yield and molecular weight distribution of the tar, and the yield and molecular weight distribution, extract yield, and the crosslink density of the char. The theory describes the combined effects of: 1) depolymerization and hydrogen consumption; 2) cross-linking; and 3) transport. These processes, which are described below, are incorporated into a computer code which employs a percolation theory method for performing the statistical analysis(see Appendix L).

Process 1. Depolymerization and Hydrogen Consumption. Bond cleavage in coal is likely to be very complicated, including homolytic cleavage, ipso substitution, and hydrogen-transfer-induced bond-scission reactions for a variety of bond types. However, it has been observed that tar evolution is consistent with a narrow distribution of activation energies which allows consideration in the model of a single representative bridge (while acknowledging that other types may be present). Also, the rate for tar formation from coal, $k_{\text {tar }}$, is in good agreement with the rate determined for the breaking of ethylene bridges between naphthalene rings, $k_{B}$. This kinetic rate, $k_{B}$, employs an activation energy which is in agreement with resonance-stabilization calculations and an overall rate which agrees with previous measurements on model compounds. In view of these observations, a single type of bond (ethylene). undergoing homolytic cleavage is assumed for coal as a simple approximation of a complex behavior.

Bond cleavage is accompanied by the consumption of donatable hydrogens, $\mathrm{H}(\mathrm{al})$, to cap free radicals, along with corresponding carbon-carbon double bond formation at the donor site. For simplicity, the DVC model assumes all the coal's 
donatable hydrogens, whether in bridges or in hydroaromatic rings, are in bridges, i.e., $\mathrm{H}(\mathrm{al})=(2 / 28) \mathrm{W}_{\mathrm{B}}$, where $\mathrm{H}(\mathrm{al})$ is the weight fraction of donatable hydrogen and $W_{B}$ is the weight fraction of labile bridges. This approximation will produce some error in tar yield since a broken bond in a hydroaromatic ring will not be as effective as a broken bond in a bridge in fragmenting the coal. But this effect will be compensated for, since $\mathrm{H}(\mathrm{al})$ is a parameter which is determined for each coal from a selected pyrolysis experiment. $\mathrm{H}$ (al) could, in principle, be determined by FT-IR or NMR, but not with sufficient accuracy.

The equation describing the disappearance of labile bridges in the char, $\mathrm{W}_{\mathrm{B}}$ (char), due to bridge breaking and hydrogen donation is,

$$
d W_{B} / d t=-2 k_{B} W_{B}
$$

The value for $k_{B}$ is the same as for tar formation, $k_{\text {tar }}$. The rate of decrease of labile bridges is twice the rate of bridge breaking since for each broken bridge, an additional labile bridge is converted to a non-labile bridge with the donation of hydrogen. By assuming that all the donatable hydrogens are in the labile bridges, the consumption of labile bridges and donatable hydrogens occurs simultaneously. The redistribution of hydrogen creates source and loss terms, $\mathrm{dW}_{\mathrm{i}}(\mathrm{DVC}) / \mathrm{dt}$, in the equations for the char functional groups $\mathrm{W}_{\mathrm{i}}$ (char), as will be discussed with the FG part of the model (see Eq. 4-13).

Equation 4-1 only describes the loss due to bridge breaking and hydrogen donation. The loss of labile bridges due to evolution with the tar is computed by using the transport equations (Eqs. 4-3 and 4-7) discussed below.

Process 2. Crosslinking. Crosslinking reactions are important in describing the rank and heating rate dependence of the tar molecular weight distributions and yields. It appears that a correlation exists between gas evolution and crosslinking, which permits the rates for crosslinking and the number of crosslink sites to be related to rates and yields for gas evolution. The model assumes the following expression for the rate of increase of the number of crosslinks, per gram of coal, $\mathrm{m}$ 


$$
\frac{d m}{d t}=N_{0}\left\lfloor\left\lfloor\frac{d W_{C O_{2}}(g a s) / d t}{44}\right\rfloor+\left\lfloor\frac{d W_{C H 4}(g a s) / d t}{16}\right\rfloor\right\rfloor
$$

where the rates, $d W_{i} / d t$, of evolution per gram of coal of $\mathrm{CO}_{2}$ and $\mathrm{CH}_{4}$ are calculated in the FG subroutine. $\mathrm{N}_{\mathrm{o}}$ is Avogadro's number.

Again, caution should be added that the reactions which have been assumed must be a gross simplification of a very complicated set of chemical reactions. This is especially true for the crosslinks occurring during methane formation, during which time there is extensive bond breaking and crosslinking accompanying tar formation.

Process 3. Transport. The external transport of tars from the particle surface to the bulk gas by vaporization and diffusion through a gas boundary layer is described with the model of Unger and Suuberg (Unger and Suuberg, 1981). The rate of evolution per gram of coal, $\left(\mathrm{dn}_{\mathrm{j}} / \mathrm{dt}\right)_{\mathrm{ET}}$, of oligomers of molecular weight $M_{\mathrm{j}}$ is given by

$$
\left(d n_{j} / d t\right)_{E T}=\left(3 / r_{0}^{3} \rho\right) r D_{j} x_{j}^{s}\left(P_{j} / R T\right)
$$

where $r$ is the particle radius assumed to shrink with the cubic root of its mass and $r_{0}$ is the initial particle radius, $\rho$ is the particle density, $\chi_{j}^{s}$ is the mole fraction of species of molecular weight $\mathrm{M}_{\mathrm{j}}$ in the metaplast at the surface of the particle, $P_{j}$ is the vapor pressure for oligomers of molecular weight $M_{j}$ (Fletcher et al., 1992),

$$
P_{j}=87,060 \exp \left(-299 M_{j}^{0.59} / T\right)
$$

$D_{j}$ is the gas-phase diffusivity of species of molecular weight $M_{j}, R$ is the gas constant and $T$ is the particle temperature. 
For softening coals, the internal transport mechanisms include: i) the transport of tar molecules through the liquid to the surface; ii) the transit of bubbles containing tar from the interior of the particle to the surface; iii) the transport of tars within the liquid to the bubbles; and iv) the stirring action of the bubble evolution. For non-softening coals, transport occurs by v) convection and diffusion within the pores.

A common feature of mechanisms iii and $v$ is that tars are transported out of the particle with the light devolatilization products which exit the coal via bubbles or pores. In the DVC model, we assume the upper limit for this process. This limit, which occurs when the tars achieve their equilibrium vapor pressure in the evolving gases, can be computed with few assumptions. In this case, the rate of transport, per gram of coal $\left(\mathrm{dn}_{\mathrm{j}} / \mathrm{dt}\right)_{\mathrm{IT}}$, for tar component $\mathrm{j}$ is proportional to the volume of gases evolved, $\mathrm{dV} / \mathrm{dt}$. That is

$$
\left(d n_{j} / d t\right)_{I T}=P_{j} x_{j}^{b}(d V / d t)(1 / R T)
$$

The volume of gases is proportional to the number of gas molecules and the temperature. It is inversely proportional to the pressure within the particle, $P_{0}$ $+\Delta P$ where $P_{0}$ is the ambient pressure and $\Delta P$ is the average pressure difference between the surface and the particle's interior. Then

$$
d V / d t=\sum_{i}\left(d n_{i} / d t\right)_{g a s}\left(\frac{R T}{P_{0}+\Delta P}\right)
$$

where $\Sigma_{\mathrm{i}}\left(\mathrm{dn}_{\mathrm{i}} / \mathrm{dt}\right)_{\text {gas }}$ is the rate of production per gram of coal of gas components i summed over all gas and light tar species. For gas molecules, $\mathrm{dn}_{\mathrm{i}} / \mathrm{dt}$ is taken as the rate of production given by the FG model. For light tar molecules $d n_{i} / d t$ is taken as the total amount transported out of the particle as tar computed in the previous time step. For computational efficiency, the sum has been limited to molecular weights less than $300 \mathrm{amu}$, since this accounts for over $90 \%$ of the volume. Combining the two equations with this approximation gives, 


$$
\left(d n_{j} / d t\right)_{I T}=P_{j} x_{j}^{b} \sum_{i<300}\left(d n_{i} / d t\right)_{g a s}\left[\frac{1}{P_{0}+\Delta P}\right]
$$

$\Delta P$ is used as an adjustable parameter which varies with the coal and experimental conditions. For the highly fluid Pittsburgh Seam bituminous coal, in cases where $P_{0}$ is one atmosphere or greater, we have considered the upper limit to this rate where $P_{0}>>\Delta$. Then all the terms in Eq. 4-7 can be determined by the combined FG-DVC model.

While $\Delta P=0$ appears to be a good approximation for fluid coals at one atmosphere or more, $\Delta P>0$ is expected for some coals and situations. $\Delta P$ is proportional to the coal's viscosity and so, will become important for less fluid coals. $\Delta P$ is also important when $P_{0}$ is small, for large particles and when the heating rates are very high. For non fluid coals, $\Delta P$ is very large due to the limited gas transport ability of these coals, while the highly fluid coals have a small $\Delta P$. In the model, $\Delta P$ is $20 \mathrm{~atm}$ for non fluid coals and $0.2 \mathrm{~atm}$ for fluid coals.

Since we do not have a good method to determine $\chi_{\mathrm{j}}^{5}$, calculations are made assuming that the external-transport term can be neglected i.e.,

$$
\left(d n_{i} / d t\right)_{t o t}=(d n / d t)_{I T}
$$

This provides an excellent fit to the data for $50 \mu \mathrm{m}$ diameter particles. It is likely that the external transport term will be increasingly important for smaller particles, but this will require better knowledge of the liquid phase diffusion coefficient, (mechanism i) and the stirring action of bubbles (mechanism iv).

The quantity needed for the FG part of the model $[\mathrm{X}]$ is given as:

$$
d X / d t=-\sum_{J}\left(d n_{j} / d t\right)_{T o t} \cdot M_{J} / M
$$


where $n_{j}$ is the number of molecules of molecular weight $M_{j}$ in the tar, and $M$ is the weight of the molecule being computed.

\section{Functional Group (FG) Model Formulation}

The Functional Group (FG) model permits the detailed prediction of the composition of volatile species (gas yield, tar yield and tar functional group and elemental composition) and of char (elemental and functional group composition). It employs coal dependent rates for the decomposition of individual assumed functional groups in the coal and char to produce gas species. The ultimate yields of each gas species are related to the coal's functional group composition. Tar evolution is a parallel process which competes for all the functional groups in the coal. There are three additional processes in the FG part.

Process 4. Gas Formation. The evolution of each gas species is assumed to be a first order reaction,

$$
d W_{i} \text { (gas) } / d t=k_{i} W_{i}(\text { char })=k_{i} X Y_{i} .
$$

where, $d W_{i}$ (gas)/dt is the rate of evolution of species $i$ into the gas phase, $k_{i}$ is a distributed rate for species $i$, and $W_{i}($ char) is the functional group source remaining in the char. $\mathrm{X}$ is an arbitrary coordinate representing the extent of pyrolysis and is defined as the weight fraction of the char. It equals 1.0 at time zero(the beginning of the pyrolysis) and will reduce to a value between 1.0 and 0 at the end of the pyrolysis. $Y_{i}$ is the weight fraction of $i^{\text {th }}$ species in the char. This representation of pyrolysis can be well explained with Figure 4 in Appendix J.

We employ the concept of the distributed rate where $k_{i}$ is given by an Arrhenius expression $k_{i}=k_{i}^{0} \exp \left(-\left(E_{i} \pm \sigma_{i}\right) / R T\right)$, where $\pm \sigma_{i}$ indicates that a Gaussian distribution is employed to describe the product sources, $\mathrm{W}_{\mathrm{i}}\left(\mathrm{E}_{\mathrm{i}}\right)$, as a function of the activation energies $E_{i}$. 


$$
W_{i}\left(E_{i}\right)=\frac{W_{i}^{0}}{(2 \pi)^{1 / 2} \sigma_{i}} \exp \left(-\frac{\left(E_{i}-E_{i}^{0}\right)^{2}}{2 \sigma_{i}^{2}}\right)
$$

$E_{i}^{0}$ is the average activation energy and $\sigma_{i}$ is the standard deviation of the Gaussian distribution.

Process 5. Tar Formation. The tar composition is tracked by summing the functional group contributions evolved with the tar. The rate of evolution of each contribution is:

$$
d W_{i}(\text { tar }) / d t=(d X / d t) Y_{i}
$$

where $\mathrm{dW}_{\mathrm{i}}(\operatorname{tar}) / \mathrm{dt}$ is the rate of evolution of each functional group component with the tar.

Process 6. Char Formation. The change in the $\mathrm{i}^{\text {th }}$ char pool, $\mathrm{W}_{\mathrm{i}}$ (char), is computed by summing the losses to the gas and tar, and the redistributions determined in the DVC subroutine,

$$
\begin{aligned}
d W_{i}(\text { char }) / d t & =-d W_{i}(\text { gas }) / d t-d W_{i}(\text { tar }) / d \\
& +d W_{i}(D V C) / d t
\end{aligned}
$$

where $d W_{i}(D V C) / d t$ includes the source and loss terms from the DVC model, given by $(30 / 28) \mathrm{k}_{B} \mathrm{~W}_{B},(2 / 28) \mathrm{k}_{B} \mathrm{~W}_{B},(24 / 28) \mathrm{k}_{\mathrm{B}} \mathrm{W}_{\mathrm{B}}$ and $-2 \mathrm{k}_{\mathrm{B}} \mathrm{W}_{\mathrm{B}}$ for methyl, aromatic $\mathrm{H}$, aromatic $\mathrm{C}$, and labile bridge functional groups, respectively.

\section{Percolation Theory}

The coal macromolecular network can be represented using several 
methods. This model uses the percolation theory, which predicts analytically the probability of a bond to be occupied given the coordination number and the starting bond occupation probability(Appendix L).

The DVC model predicts, and experiments confirm, that there are more than one kind of bond bridges and crosslinks which have different coordination numbers, and independent probabilities of being broken. To take advantage of the benefits of percolation theory, we have extended percolation theory on a Bethe lattice (one with no loops) to use two independent sub-networks, as illustrated in Figure 4-1. In the figure, double lines represent one of the bond types, while single lines represent the other. As can be seen by comparing Figure 4-1a and 4-1b, this lattice has the desired feature of modeling a transition from chain-like structures (a) to fishnet structures (b). The mathematics of this two-bond percolation theory follows closely that of the standard theory. The mathematics are presented in Appendix L.

There are four parameters describing the network: $\mathrm{p}, \mathrm{q}, \sigma_{1}$ and $\sigma_{2} . \mathrm{p}$ and $\mathrm{q}$ are the occupation probabilities of bridges and crosslinks, respectively, while $\sigma_{1}+1$ and $\sigma_{2}+1$ are the coordination numbers for bridge linkage and crosslink linkage, respectively. $\sigma_{1}$ and $\sigma_{2}$ are model constants. $\mathrm{p}$ and $\mathrm{q}$, are the state variables computed at each time step, and are set equal to the input parameters $p_{o}, q_{o}$, at time equal $0 . p_{o}, q_{o}$ vary with each coal.

\section{Coal Viscosity Model}

The coal fluidity model is based on the decomposition and condensation of the macromolecular network under the influence of bridge breaking and crosslinking reactions(Appendix $M$ ). The fluidity is dependent on the relative amounts of the liquid, (molecules detached from the network) and solid (the remaining network) and on the fluidity of the liquid component. The fluidity of the liquid component depends on the average molecular weights of the liquid and on the temperature.

The viscosity model used here has terms that depend on the coal liquid 
temperature, and the volume fraction of solids, with an inhomogeneous gel-point. It is the last two factors which are the most important. In common with previous studies of coal viscosity, a two-phase model is used. The particular equation chosen is as follows:

$$
\ln \left(\eta / \eta_{l i q)}\right)=\left[\frac{k_{E} \phi_{s}}{1-\phi_{s} / \phi_{c}}\right]
$$

where $\eta$ is the viscosity of the suspension, $\eta_{\text {liq }}$ is the viscosity of the liquid, $k_{E}$ is the Einstein coefficient, and $\phi_{\mathrm{s}}$ is the volume fraction of solids, having a critical value $\phi_{\mathrm{c}}$ at which $\eta$ goes to infinity.

The viscosity of the liquid phase is given by the Andrade equation

$$
\eta_{l i q}=C \exp \left(E_{\eta} / R T^{*}\right)
$$

which on combining with Eq. 4-14 leads to:

$$
\eta=C \exp \left(E_{\eta} / R T^{*}\right)\left[\exp \frac{k_{E} \phi_{s}}{1-\phi_{s} / \phi_{c}}\right]
$$

The constants used in the viscosity theory are as follows:

$\mathrm{k}_{\mathrm{E}}: \quad$ Einstein coefficient, $\mathrm{k}_{\mathrm{E}}=5.0$

$\phi_{\mathrm{c}}$ : Volume fraction of the solid phase at the gel-point, $\phi_{\mathrm{c}}=0.65$

$\eta_{\text {liq }}: \quad$ viscosity of the liquid phase

$\eta_{\text {liq }}=\mathrm{C}_{1} \exp \left(\mathrm{E}_{\eta 1} / \mathrm{RT}^{*}\right)=5.0 \times 10^{-30} \exp \left(100,000 / \mathrm{RT}^{*}\right)$ for $\mathrm{T} \leq 708 \mathrm{~K}$

$\eta_{\text {liq }}=\mathrm{C}_{2} \exp \left(\mathrm{E}_{\eta 2} / \mathrm{RT}^{*}\right)=1.082 \times 10^{-14} \exp \left(50,000 / \mathrm{RT}^{*}\right)$

$T^{*}$ : Absolute temperature, cut-off at $T^{c}=750 \mathrm{~K}$ for $T \geq 708 \mathrm{~K}$

$$
\text { i.e., } T^{*}=T \text { for } T \leq T^{c} K: T^{*}=T^{c} \text { for } T>T^{c} K
$$


$\mathrm{R}$ : $\quad$ Gas constant, $\mathrm{R}=1.98 \mathrm{cal} \mathrm{mole}^{-1} \mathrm{~K}^{-1}$

The value of $k_{E}=5$ was arrived at empirically, i.e., to fit the data. This value, however, matches the situation in which liquid is entrapped within large agglomerates of solid. The value of the activation energies for coal was chosen based on measured liquid viscosities (from 50 to $100 \mathrm{kcal} / \mathrm{mole}$ ).

\section{Coal Swelling Model}

Under certain conditions, coals which become fluid when heated also swell, due to the internal pressure of trapped, evolved gases. Experimental observations of the swelling are made both on single particles which have swollen in essential isolation, and on agglomerates, as in a measurement of the freeswelling index. Although the same basic phenomena are involved in both cases, only the swelling of individual particles of pulverized coal (radius $<100 \mu \mathrm{m}$, say) has been modeled to date.

When microscopic observations of swollen coal particles are made it becomes apparent that the particles do not behave in an identical fashion. At average heating rates, between $10^{2}$ and $10^{4} \mathrm{~K} \mathrm{~s}^{-1}$, the swollen particles of a typical swelling coal, Pittsburgh No. 8, can be categorized into one of three types: a small fraction $(\sim 10 \%)$ which shows virtually no sign of fluidity, and hence no swelling; the majority ( $-80 \%)$ which experience swelling of about the same magnitude; and another small fraction $(\sim 10 \%)$ which exhibits behavior that can be associated with a much higher fluidity (lower viscosity) than the majority. We have associated the three behaviors with the three main maceral types of the coal; inertinite, vitrinite, and exinite, respectively. In the model the properties only of average particles are described, and this must be recognized when comparing predictions with microscopic observations.

The range of "swelling" behaviors encompassed by our model includes the swelling which is observed for moderate heating, but also the behaviors at the extremes of heating rate. At very low heating rates $\left(\leq 10 \mathrm{~K} \mathrm{~s}^{-1}\right)$ there is 
virtually no swelling. At extremely high rates $\left(\geq 5 \times 10^{4} \mathrm{~K} \mathrm{~s}^{-1}\right)$, the liquid walls surrounding the gas-filled internal voids rupture, allowing the gas to escape. Minimal swelling is observed in this case. Microscopic observations of particles which have ruptured in this way reveal a variety of images. For some coals the shapes are not unlike fairly smooth skinned potatoes, but with many holes over the surface. For others the surfaces are still pitted with holes, but with a more angular morphology indicative of low flow.

The starting point for our model is the equation described by (Chiou and Levine, 1981), who considered the swelling rate of a pulverized coal particle of external radius $r_{2}$, with a single void of radius $r_{1}$, with spherical symmetry. The swelling they depict is due to the pressure, $\Delta \mathrm{P}_{t}$, of trapped evolved gases, doing work against viscous forces (viscosity $=\eta$ ). Their equation was further modified to take account of the pyrolysis weight loss.

For this single bubble model, the equation for the velocity of the outer wall is given by

$$
\frac{d r_{2}}{d t}=\frac{r_{1}^{3} r_{2} \Delta P_{t}}{4 \eta\left(r_{2}^{3}-r_{1}^{3}\right)}+\frac{r_{2}}{3\left(r_{2}^{3}-r_{1}^{3}\right)} \frac{d\left(r^{3}\right)}{d t}
$$

Where $r_{1}$ and $r_{2}$ are the radius of the inner bubble and the coal particle with one bubble, respectively; $4 \pi / 3 r^{3}$ is the coal volume in this particle.

In our model, the temperature-dependent viscosity is supplied by the viscosity model. The accounting of excess internal pressure begins at $t=0$, considering the gas gain from trapped, evolved gas, and loss due to out-diffusion. The number of moles of gas, $n_{\mathrm{g}}$, evolved in one second, by the particle walls of volume, $\Delta v$, is obtained from the FG-DVC model, $\Delta v$ being updated to account for pyrolysis. Of the evolved gas, a fraction $r_{1}^{2} /\left(r_{1}^{2}+r_{2}^{2}\right)$ is captured within 
the void, so that the captured gas is given by

$$
d n_{c}=n_{g} r_{1}^{2} /\left(r_{1}^{2}+r_{2}^{2}\right) \cdot d t
$$

In this same period an amount of gas diffuses out:

$$
d n_{d}=\frac{4 \pi D_{l} c_{1} d t}{\left(1 / r_{1}-1 / r_{2}\right)}
$$

where $D_{1}$ is the diffusivity of the gas in the coal liquid, $c_{1}=3 n_{t} / 4 \pi r_{1}^{3}$, and the total gas within the cenosphere, $n_{t}$, is the "sum" of Eqs. 4-18 and 4-19

$$
\frac{d n_{t}}{d t}=\frac{d n_{c}}{d t}-\frac{d n_{d}}{d t}
$$

The excess of internal over external pressure, $\Delta \mathrm{P}_{\mathrm{g}}$, due to the trapped gas is given by

$$
\Delta P_{8}=\frac{3 n_{t} R T}{4 \pi r_{1}^{3}}
$$

The calculation of the amount of the trapped ambient gas $n_{a}$ follows the similar argument in Eq. 4-19 and 4-20: 


$$
\frac{d n_{a}}{d t}=\frac{4 \pi D_{a}}{1 / r_{1}-1 / r_{2}}\left[\frac{P_{0}}{R T}-\frac{n_{a}}{4 \pi r_{1}^{3} / 3}\right]
$$

where $P_{0}$ is the ambient pressure and $D_{a}$ the diffusivity of ambient gas. The pressure difference is then

$$
\Delta P^{\prime}{ }_{t}=\frac{3\left(n_{t}+n_{a}\right) K I}{4 \pi r_{1}^{3}}-P_{0}
$$

But this is reduced by the effective negative pressure due to the surface tension, $\sigma$; so that the total outward pressure differential, $\Delta \mathrm{P}_{\imath}$, is

$$
\Delta P_{t}=\frac{3\left(n_{t}+n_{a}\right) R T}{4 \pi r_{1}^{3}}-\sigma\left(\frac{1}{r_{1}}+\frac{1}{r_{2}}\right)-P_{0}
$$

This is the pressure difference used in Eq. 4-17, to calculate swelling.

If $\Delta \mathrm{P}_{\mathrm{t}}{ }_{\mathrm{t}}$ is sufficiently large, the stress of the wall will exceed the strength of the wall $S_{c}$. Cell rupture is defined to occur when

$$
S_{c} \leq-P_{0}+\frac{1.5 r_{1}^{3} \Delta P_{t}^{\prime}}{\left(r_{2}^{3}-r_{1}^{3}\right)}
$$

The parameters of the model are: $D_{l}, D_{a}, \sigma$, and $S_{c}$, which are listed in Table 42. 
Large particles are expected to contain more than one bubble. The value of $\mathrm{I}_{2}$, the radius of a particle with only one bubble, is found to be $20 \mu \mathrm{m}$ to fit the data of most of the coals. The extrapolation of this single bubble model to large particles needs to be further investigated. The current model assumes that the number of bubbles in a particle of radius $R$ is $\left(R / r_{2}\right)^{3}$.

\section{Char Reactivity Model}

As previously mentioned in section II-G(PCGC-2 1988 version), three rate-limiting steps are considered for this process: 1) gas phase diffusion, 2) heterogeneous reaction, 3 ) heterogeneous reaction + pore diffusion. This section considers both the determination of the intrinsic rate coefficient and the incorporation of the effect of particle diffusion limitations. In order to obtain the intrinsic rate coefficient, the model uses correlations with chemical properties such as the char hydrogen content and the coal oxygen and mineral contents. In the diffusion regime, the model determines the value of the reaction rate as a function of the intrinsic reaction rate coefficient and char structural properties such as porosity, tortuosity and mean pore radius. Specifically, it calculates the effectiveness factor (i.e. the ratio of the actual rate over the rate without diffusion limitations) using the Thiele modulus. In the pore diffusion regime when high Thiele modulus values are assumed (i.e. reaction is thought to occur mostly on the external surface of the particle), the model predicts that the activation energy is approximately half the intrinsic activation energy. A first order equation is used for the intrinsic reaction. The overall reaction rate (including gas phase diffusion) $\mathrm{r}_{\mathrm{bjl}}$ is calculated following equation 114 (PCGC-2, 1988 version) :

$$
r_{h j l}=\frac{A_{j}^{\leftarrow} M_{h j} M_{g} \phi_{l} k_{c j l} k_{j l} \xi_{j} C_{o l g} C_{g}}{M_{g} A_{j} C_{g}\left(\xi_{j} k_{j l}+k_{c j l}\right)+r_{j}}
$$

If mass transfer is neglected, the equation for the particle reaction rate becomes :

$$
r_{h j l}=A_{j} M_{h j} \phi_{l} k_{j l} \xi_{j} C_{o l g} \quad \text { with } \quad \xi_{j}=\eta \text { surf } / \text { surfe }
$$


where $A_{j}$ is the external surface area, $M_{b j}$ is the molecular weight of carbon, $\phi_{1}$ is a stochiometic factor, $k_{j l}$ is the intrinsic rate coefficient, $\xi$ is a surface coefficient, $\mathrm{C}_{\text {olg }}$ is the oxidant concentration, $\eta$ is the effectiveness factor, and surf and surfe are the internal and external surface areas respectively (both in $\mathrm{m}^{2} / \mathrm{g}$ available char).

The value of the intrinsic reaction rate coefficient $k_{j l}$ has been experimentally found to be well represented by the equation :

$$
k_{j l}=A 1_{j l} T_{j} \exp \left(-\frac{E 1_{j l}}{R T_{j}}\right)
$$

where $\mathrm{T}_{\mathrm{j}}$ is the particle temperature, $\mathrm{E} 1_{\mathrm{jl}}$ is the activation energy in the kinetic regime $(32000 \mathrm{cal} / \mathrm{mol})$, and $A 1_{j l}$ is the pre-exponential factor which depends on the starting coal and the pyrolysis conditions. For reaction with oxygen, $\mathrm{Al}_{\mathrm{jl}}$ is defined, for high rank coals (oxygen content lower than $13 \%$ ), as :

$$
A I_{l l}=4.75 \times 10^{-4} \exp \left(1.49 H_{\text {char }}\right) \exp \left(0.28 O_{\text {coal }}\right)
$$

where $\mathrm{H}_{\text {char }}$ is the char hydrogen content, and $\mathrm{O}_{\text {coal }}$ the coal oxygen content. For low rank coals (oxygen content higher than $13 \%$ ) $\mathrm{Al}_{\mathrm{jl}}$ becomes :

$$
A 1_{j l}=5.33 \times 10^{-2} \exp \left(1.49 H_{\text {char }}\right) \exp (1.65 \mathrm{~min})
$$

where min represents the "effective" coal dispersed mineral content. At low 
temperature, $\min$ is equal to the measured dispersed mineral content min 0 , while at high temperature, $\mathrm{min}$ is calculated using a deactivation function as follows:

$$
\min =\min 0 \exp \left(-\frac{E m_{j l}}{R} \times\left(\frac{1}{T m}-\frac{1}{T_{j}}\right)\right)
$$

where $\mathrm{Em}_{\mathrm{jl}}$ is the deactivation energy, and $\mathrm{Tm}$ is an adjustable temperature.

The effectiveness factor $\eta$ (and as a result, the surface coefficient $\xi_{i}$ ) is calculated using the Thiele modulus $\Phi_{s}$ :

$$
\eta=\frac{3}{\Phi_{s}}\left[\frac{1}{\tanh \Phi_{s}}-\frac{1}{\Phi_{s}}\right]
$$

where $\Phi_{s}$ is given by :

$$
\Phi_{s}=\frac{d}{2}\left(\frac{M_{g} \text { surf sigma }}{D_{e f f} \Phi_{l} M_{h j}} k_{j l}\right)^{0.5}
$$

where Deff is the effective diffusivity, and sigma is the particle density. The effective diffusivity Deff is dependent on the mean pore radius radp (needed to calculate the Knudsen diffusivity), the porosity eps and the tortuosity tau, and is given by :

$$
\text { Deff }=9.7 \times 10^{1} \mathrm{radp}\left(\frac{T_{j}}{M_{g}}\right)^{0.5} \frac{\text { eps }}{\text { tau }}
$$

At this point, the model contains numerical parameters for the $\mathrm{O}_{2}$-char reaction 
only. However the same approach can be used for other gas-char reactions.

\section{Coal Sulfur and Nitrogen Devolatilization Model}

The phenomenon of coal sulfur decompositions is complicated by the various forms of sulfur existing in the coal structure on one hand and the various gas species produced by the decompositions on the other. The weak absorbance of $\mathrm{H}_{2} \mathrm{~S}$, which is a major product of sulfur pyrolysis evolution, from FTIR spectra makes the understanding of the physical and chemical nature of this process more difficult. The lack of measures to distinguish the origins of sulfur products in the spectra is the major obstacle in our investigation. Efforts have been made in analyzing the data of the post oxidized runs at AFR (Bassilakis, 1992) and those of $\mathrm{COS}$ and $\mathrm{SO}_{2}$ of the ordinary pyrolysis. A preliminary understanding of this phenomenon has been reached and forms the basis of the sulfur model in the current code.

Sulfur exists in coals in three forms: organic sulfur, pyritic sulfur, and sulfates. Organic sulfur is in the coal structure, either in aromatic rings or in the aliphatic portion. Pyrite exists in coal as dispersed particles, but interactions with the coal structure to a certain extend are expected. Sulfates are only a very small part of the total sulfur in most coals, especially coals from Argonne Coal collection (Vorres, 1989), and therefore, are ignored in the current model.

During devolatilization, sulfur in various forms decomposes into gas species, including $\mathrm{H}_{2} \mathrm{~S}, \mathrm{COS}, \mathrm{SO}_{2}$ and $\mathrm{CS}_{2}$, and a large amount of it remains in char and then decomposes in the combustion cycle. Some amount of the sulfur is entrained by tar as other gas pools, and it does not constitute a modeling problem here. Pyrite $\mathrm{FeS}_{2}$ is believed to decompose mainly as $\mathrm{FeS}_{2} \rightarrow \mathrm{FeS}+\mathrm{S}$, and the further reaction of $\mathrm{FeS}$ is slow during pyrolysis. Alternative pyrite reactions with coal gases are possible, but they may be less significant. $\mathrm{S}$ from $\mathrm{FeS}_{2}$ decomposition reacts with coal structure forming all possible sulfur gas species listed above. Among all the gases, $\mathrm{H}_{2} \mathrm{~S}$ is the major species of sulfur evolution, and other gases are just the background under the $\mathrm{H}_{2} \mathrm{~S}$ peaks. Therefore, efforts have only been made to predict the overall sulfur evolution with $\mathrm{H}_{2} \mathrm{~S}$. For all 
coals, a part of the organic sulfur is retained in char and the percentage of the retention is coal rank dependent.

As shown by Bassilakis (Bassilakis, 1992), $\mathrm{SO}_{2}$ evolution in a post oxidized run has three peaks during pyrolysis. The first peak at low temperature is attributed mainly to the aliphatic organic sulfur, but for low rank coals it has part of the sulfur from the pyrite as the product of its low temperature decomposition. The second peak is sharp and coincides with the pure pyrite decomposition temperature, so it is believed mainly due to pyrite devolatilization. The third peak at high temperature is much broader than the rwo previous peaks and is actually a shoulder of the second peak. This peak cor. sts of th: products from the high temperature evolution of organic sulfur ana pyritic sulfur. A schematic representation of above discussion is given in Figure 4-3.

In FG-DVC, the organic sulfur is distributed into two $\mathrm{H}_{2} \mathrm{~S}$ (loose and tight), one $\mathrm{SO}_{2}$, one $\mathrm{COS}$, one $\mathrm{CS}_{2}$ and one char $\mathrm{S}$ pools. The pyritic sulfur decomposes at two temperatures. The low temperature decomposition is the result of the coal+pyrite reaction, which produces $\mathrm{H}_{2} \mathrm{~S}$. The high temperature decomposition releases $\mathrm{H}_{2} \mathrm{~S}, \mathrm{SO}_{2}, \mathrm{COS}$, and $\mathrm{CS}_{2}$ gases. Some sulfur released from this reaction remains in the coal structure and evolves at higher temperature as $\mathrm{H}_{2} \mathrm{~S}$. Another part of it could remain in the char. The product distribution discussed above is depicted in Table 4-3.

Under oxidized conditions, the decompositions of both organic and pyritic sulfur are $100 \%$. The biggest change is the release of FeS and the organic char sulfur which are stable in absence of oxygen. However, the positions of these additional peaks are tentatively assigned and more work needs to be done. In FG-DVC, the FeS evolution is invoked only when the inlet gas is oxidized. The FeS peak is placed at temperature slightly high than the $\mathrm{FeS}_{2}$ intrinsic sharp peak, and the organic char sulfur oxidation peak is in between the first peak and the second peak. This overall evolution profile is consistent with the result by LaCount (LaCount, 1991), but our peak assignment(Figure 4-3) is different.

All the devolatilizations follow the standard description of gas evolutions in Eq. (4-10). 
The forms of the coal nitrogen are much simple comparing to sulfur. Most of the coal nitrogen is in pyrrole and pyridine structures, which are both aromatic. There is only a very small amount of amine in lignite. The main evolution gases are $\mathrm{HCN}$ and $\mathrm{NH}_{3}$. What makes the nitrogen evolution difficult to be modeled is the heating rate dependency (Bassilakis, 1992). At $30^{\circ} \mathrm{C} / \mathrm{min}$, $\mathrm{NH}_{3}$ was the major product of nitrogen evolution in pyrolysis, and there was only less than $5 \%$ of the volatile nitrogen in $\mathrm{HCN}$. While in an entrained flow reactor of furnace temperature at $1100^{\circ} \mathrm{C}, \mathrm{HCN}$ evolution was strongly enhanced and the amount of $\mathrm{NH}_{3}$ became too low to be detected.

The modeling scheme used in this version is outlined in Figure 4-4. HCN evolves directly from its pool precursors. $\mathrm{NH}_{3}$ gas comes from two sources: the direct evolution from coal nitrogen(pools) and the secondary conversion from evolved gaseous $\mathrm{HCN}$ to $\mathrm{NH}_{3}$. The former contributes only a small amount, while the later is the major reaction pathway. The conversion is assumed via the reaction of the gaseous HCN with the coal hydrogen in the pore structure of coals when HCN is making its way out of the coal particles. The completeness of this reaction is obviously dependant upon the comparison of the gas residence time in the pore structure to the coal reaction residence time in reactors. Since nitrogen evolves at higher temperature than other gases, the gas residence time depends on the char structure instead of the coal structure. The precise determination of the gas residence time is difficult. It can be more complicated if pyrolysis of large coal chunks is to be modeled, in which case bed conditions can affect the gas release.

This model uses a simplified single cell structure to estimate the gas residence time. Consider a spherical char particle with an original radius $r_{0}$. The radius changes to $r_{2}$ in the pyrolysis due to the weight loss and swelling. The particle has a single inner bubble of radius $r_{1}$. During pyrolysis, the evolution gas flux in this particle is always positively outward. We assume that the gas starts to transport outward immediately after it evolves, so the gas residence time is defined as the time elapse of its traveling from the sphere origin to the particle surface. It is obvious that if the coal particles are packed the residence time will be longer than we estimate here. 
With this single cell particle model, for the gas that evolves at a point inside this particle of distance $r$ from the center of the sphere, the residence time, $\Delta t_{n}$, of the evolution gas before leaving the particle is

$$
\Delta t_{n}(r)=\Delta t_{n 0} \ln \frac{r_{2}}{r}
$$

and

$$
\Delta t_{n 0}=\frac{3 P^{*} \phi_{\nu}}{\rho n_{z o t} R T}
$$

where $P^{*}$ is the gas pressure in the coal pores, $n_{\text {tot }}$ is the total gas evolution rate per unit weight coal, $\mathrm{R}$ is the gas constant, $\mathrm{T}$ is the temperature, $\rho$ is the coal solid density, and $\phi_{v}$ is the volume swelling ratio that can be calculated by swelling model. The HCN gas that is generated within the shell $4 \pi \mathrm{r}^{2} \mathrm{dr}$ has a time period of $\Delta t_{n}$ to react to $\mathrm{NH}_{3}$. The modified $\mathrm{HCN}$ evolution rate is then

$$
\left.\frac{d W^{*}{ }_{H C N}(g a s)}{d t}\right|_{r}=\frac{d W_{H C N}(g a s)}{d t} e^{\left(-k_{R} \Delta t_{n} \ln \left(\frac{r_{2}}{r}\right)\right)}
$$

where $k_{n}$ is the reaction constant of $\mathrm{HCN}$ to $\mathrm{NH}_{3}$ conversion, and $\mathrm{dW}_{\mathrm{HCN}}(\mathrm{gas}) / \mathrm{dt}$ is the HCN evolution rate given by Eq. (4-10). Averaging over the whole particle leads to

$$
\frac{d W^{*}{ }_{H C N}(g a s)}{d t}=f_{n} \frac{d W_{H C N}(g a s)}{d t}
$$


where

$$
f_{n}=\left(1-f_{n 0}\right) \frac{1-e^{\left(-k_{n} \Delta t_{x d} \ln \left(\frac{r_{2}}{r_{1}}\right)\right)}\left(r_{1} / r_{2}\right)^{3}}{\left(1-\left(r_{1} / r_{2}\right)^{3}\right)\left(1+k_{n} \Delta t_{n d} / 3\right)}+f_{n 0}
$$

where $f_{n 0}$ is a non-zero residue fraction of $H C N$ rate. $\left(1-f_{n}\right)$ is the $H C N$ to $N_{3}$ conversion factor. And

$$
\frac{d W^{*}{ }_{N H_{3}}(\text { gas })}{d t}=\frac{M_{N H_{3}}}{M_{H C N}}\left(1-f_{n}\right) \frac{d W_{H C N}(\text { gas })}{d t}
$$

where $\mathrm{M}_{\mathrm{HCN}}$ and $\mathrm{M}_{\mathrm{NH}}$ are the molecular weights of $\mathrm{HCN}$ and $\mathrm{NH}_{3}$, respectively.

To extend this single cell structure model to the generally heterogeneous coal/char structure, the residue HCN rate fraction $f_{n 0}$, the coal volume swelling ratio, $\phi_{v}$, and coal particle-bubble radius ratio, $r_{1} / r_{2}$, are the model parameters.

A non zero $\mathrm{f}_{\mathrm{n} 0}$ is to retain a small fraction of $\mathrm{HCN}$ after the most of it has been converted to $\mathrm{NH}_{3}$, even at very low heating rate (Bassilakis, 1992). $\mathrm{f}_{\mathrm{n} 0}$ is affected by the char particle size and the char structure, which in tum are affected by the heating rate. $f_{n 0}$ is also a function of reaction bed conditions that could alter $\Delta t_{n}$ evaluated above. The model suggested $f_{n 0}$ value is 0.1 . It is only important for low heating rate, while for very high heating rate cases in which the conversion has no time to complete, $f_{n 0}$ is not important.

Although the char volume swelling ratio, $\phi_{v}$, and the ratio of the inner bubble size to the char particle size, $r_{1} / r_{2}$, can be calculated directly from the swelling model, we would rather treat them as model parameters, because the single cell swelling model is too primitive to describe the heterogeneity of the char structure. For most of the combustion cases that PCGC-2 is to apply, swelling is not significant due to the very high heating rate and the presence of oxygen. Therefore, $\phi_{\mathrm{v}}$ is set to 4 and $r_{1} / r_{2}$ to 0.8 . $\mathrm{P}^{*}$ is also related to the 
sweiling computation, since it is the gas pressure in the coal pores. But because nitrogen evolves at higher temperature than most of the gases and after the swelling, at the time nitrogen starts to evolve, $\mathrm{P}$ is almost at equilibrium with the ambient gas pressure. So $\mathrm{P}^{*}$ is set to equal to the ambient pressure in nitrogen computation.

In the current version these model parameters are not adjustable by users.

\section{Solution Technique}

FG-DVC calculations are performed at each time step along the particle trajectory calculation of every particle. At each time step, the time increment, the current particle temperature and the local pressure are passed to FG-DVC subroutines. The coupled equations in FG-DVC are integrated for this time increment to get the updated particle elemental composition, the weight loss due to devolatilization, and the new particle size due to swelling for PCGC2.

The coupled Eqs. 4-10, 4-12, and 4-13 in FG part are integrated by using a basic Euler method. The size of the sub-time step is controlled by the quickest evolving species at the time so that the evolved amount of this gas pool at this time step must be less than a few percent of its original amount in the coal. This scheme is proven to be accurate enough for the current problem. Integrations start with initial gas pool composition read from FG-DVC input coal data file for the coal in the calculation. Gas evolution kinetic data are read from FG-DVC kinetic file which is also coal dependent.

In the DVC part, the network parameters $\mathrm{p}$ and $\mathrm{q}$ are updated using the bond breaking rate and crosslinking rate passed from FG part(Eq. 4-2), so that an updated network oligomer weight distribution can be obtained, and is used to calculated liquid/solid fractions of the coal. The oligomers with molecular weight less than $300 \mathrm{amu}$ are the candidates for tar formation. Tar vaporization is determined by Eq. 4-7, an internal transport limit process, where higher pressure will reduce tar formation rate. The FG-DVC predicted pressure effect on pyrolysis agrees with experimental data very well. The initial molecular 
distribution and network parameters $p_{0}$ and $q_{0}$ are read from FG-DVC coal dependent polymer file.

The coal viscosity at one time step is calculated directly from Eq. 4-16 following the FG-DVC calculation.

The particle swelling calculation proceeds by integrating Eqs. 4-17, 4-20, and 4-22. $n_{g}$ in Eq. 4-18 is the sum of all gas evolution rates per particle from FG-DVC. The viscosity $\eta$ in Eq. $4-17$ is from viscosity model. At time equal to zero,

$$
n_{t}=0
$$

and

$$
n_{a}=\frac{4 \pi r_{1}^{3} P_{0}}{3 R T}
$$

where $P_{0}$ should be the local pressure at the place the particle trajectory starts. Before integrating Eq. 4-17, it is transformed to

$$
\begin{gathered}
\frac{d\left(r_{2}-r_{1}\right)}{d t}=-\frac{\left(r_{2}-r_{1}\right)\left(r_{2}+r_{1}\right) r_{2}}{r_{1}^{2} r^{3}}\left[\frac{r_{1}^{3} \Delta P}{4 \eta}+\frac{1}{3} \frac{d\left(r^{3}\right)}{d t}\right] \\
+\frac{1}{3 r_{1}^{2}} \frac{d\left(r^{3}\right)}{d t}
\end{gathered}
$$


where $4 \pi r^{3} / 3$ is the current volume of the particle, and $4 \pi / 3 d\left(r^{3}\right) / d t$ is the rate of volume loss due to combustion and pyrolysis. The relationship

$$
r_{2}^{3}=r_{1}^{3}+r^{3}
$$

is used to derive Eq. 4-43. Defining a new variable $w=r_{2}-r_{1}$, Eq. $4-43$ is integrated instead of Eq. 4-17. Because the bubble shell thickness $r_{2}-r_{1}$ will be in the order of $10^{-3} \times \mathrm{r}_{2}$ after the particle starts to swell, the very small time step required in integrating Eq. 4-17 to insure the accuracy of $r_{2}-r_{1}$ makes the calculation very inefficient. Using Eq. 4-43 to repiace Eq. 4-17 solves this problem.

Numerical difficulties in this submodel originate from the abrupt nature of swelling at high heating rates and the subtle pressure balance to be maintained across the bubble shell. An error control Runge-Kutta-Fehlberg method is used in the integration. This variable step size method allows using large step sizes at the time the particle does not swell and small step sizes when the particle does swell.

This set of coupled equations becomes numerically stiff, when the particle heating rate is low or the particle residence time is large $(>3$ second). The diffusion equations of Eqs. 4-20 and 4-22 are the origin of the stiffness. This was resolved partially by analytically integrating Eqs. 4-20 and 4-22 in one sub time increment $\Delta t$ to yield

$$
n_{t}^{\text {now }}=\frac{-B+\left(A n_{t}+B\right)_{\Delta t=0} \exp (A \Delta t)}{A} .
$$

and

$$
n_{a}^{\text {new }}=\frac{-C+\left(A_{a} n_{t}+C\right)_{\Delta t=0} \exp \left(A_{a} \Delta t\right)}{A}
$$


where

$$
\begin{aligned}
& A=-\left[\frac{3 D_{l} r_{2}}{\left(r_{2}-r_{1}\right) r_{1}^{2}}\right]_{\Delta s=0} \\
& B=\left[\frac{r_{2}^{2}}{r_{1}^{2}+r_{2}^{2}} n_{g}\right]_{\Delta s=0} \\
& C=\left[\frac{4 \pi D_{1} P r_{1} r_{2}}{R T\left(r_{2}-r_{1}\right)}\right]_{\Delta s=0} \\
& A_{a}=-\left[\frac{3 D_{c} r_{2}}{\left(r_{2}-r_{1}\right) r_{1}^{2}}\right]_{\Delta s=0}
\end{aligned}
$$

$P$ is the local pressure; $T$ is the particle temperature; $D_{1}$ and $D_{a}$ are the apparent diffusivities of evolved gas and ambient gas, respectively. 
In the reactivity model, the calculation of the mass loss rate due to heterogeneous reactions is performed through iterations with temperature. $\mathrm{k}_{\mathrm{jl}}$ and $\xi$ are calculated at each call of the subroutine KLCALC and are passed onto COAL2.

\section{Model Parameters}

In parallel with the model development of FG-DVC, much work has been done to obtain the coal dependent pyrolysis gas evolution kinetic rates, coal functional group compositions, coal polymer network parameters, and other model parameters in the viscosity and swelling models to fit experimental data. Table 4-4 lists the elemental compositions of the eight Argonne Premium Coals and three PSOC coals, for which coal data have been derived experimentally. The kinetic data of major gas pools of the Argonne coals are listed in Table 4-5. The elemental and functional group compositions, kinetic rates, and coal polymer network parameters of each Argonne coal are collected in three FG-DVC coal data input files
composition file
coal.arN.7
kinetics file
kin.arN.7
network parameter file
polymr.arN.7

respectively. The letter $N$ will be the coal serial number defined in Table 4-4. For instance, the coal composition file for N. D. zap-lignite is 'coal.ar1.7'. Only files of Argonne coals are provide with the PCGC2 package. To select a coal for a PCGC-2 run, it is necessary to specify the correct coal data file names for this coal in PCGC-2 input file. The description of these files are given in User's Guide part of the manual.

If the coal in use is not one of the Argonne coals, a new set of the above three coal data files has to be created. It is not simple for users to do this, because getting these data requires special instruments, understandings of FGDVC, and a stand-alone FG-DVC program. A utility program COALS was therefore written to assist users in maintaining and creating the FG-DVC coal data 
Generalized Particle Reactions

files. The full functions of COALS have been incorporated into PCG2-2 preprocessing program PREC. For the usage of PREC, read Chapter 6. 


\section{References}

Fletcher, T. H., Kerstein, A. R., Pugmire, R. J., Solum, M. S., and Grant, D. M., "Chemical Percolation Model for Devolatilization. 3. Direct Use of ${ }^{13}$ C NMR Data to Predict Effects of Coal Type", Energy \& Fuels, 1992, 6, 414.

Fong, W. S., Peters, W. A., Howard, J. B.," Plastic Behaviour of Coal under Rapid-Heating High-Temperature Conditions", Fuel 1986, 65, 195

Chiou, M. J., and Levine, H. B.," Investigation of Structure Deformation of Coal Particles in Pyrolysis", Internal Report, JAYCOR, 1981.

Bassilakis, R., Serio, M. A., and Solomon, P. R., "Sulfur and Nitrogen Evolution in the Argonne Coals", Prep. Papers Am. Chem. Soc. Div. Fuel Chem., 1992, 37(4), 1712.

Vorres, K., (prepared),"Users Handbook for the Argonne Premium Coal Sample Program", October, 1989, Argonne National Laboratory.

LaCount, R. B., Kern, D. G., King, W. P., LaCount Jr., R. B., Miltz Jr., D. J., Stewart, A. L., Trulli, T. K., Walker, D. K., and Wicker, R. K., "Advances in Coal Characterization by Programmed-Temperature Oxidation", Prep. Papers Am. Chem. Soc. Div. Fuel Chem., 1991, 36(3), 1217.

Unger, P.E. and Suuberg, E.M., "Modeling the Devolatilization Behavior of a Softening Bituminous Coal", 8th Symp. (Int'l) on Combust., 1203, 1981. 


\begin{tabular}{|c|c|}
\hline Table 4-1 & Nomenclature of the Varibles in Chapter 4 \\
\hline Symbol & Definition \\
\hline$A 1_{\mathrm{jl}}$ & $\begin{array}{l}\text { Pre-exponential factor, kinetic regime, reactivity } \\
\text { model, } \mathrm{m} / \mathrm{sK}\end{array}$ \\
\hline$A_{j}$ & External surface area, $\mathrm{m}^{2}$ \\
\hline $\mathrm{C}_{\mathrm{olg}}$ & Oxidant concentration, $\mathrm{kmol} / \mathrm{m}^{3}$ \\
\hline $\mathrm{D}_{\mathrm{a}}$ & Apparent ambient gas diffusivity in coal liquid, $\mathrm{cm}^{2} / \mathrm{s}$ \\
\hline Deff & Effective diffusivity, reactivity model, $\mathrm{m}^{2} / \mathrm{s}$ \\
\hline$D_{j}$ & $\begin{array}{l}\text { Tar vapor diffusivity of species of molecular weight } \\
\mathrm{M}_{\mathrm{j}}\end{array}$ \\
\hline $\mathrm{D}_{\mathrm{l}}$ & Apparent evolution gas diffusivity in coal liquid, $\mathrm{cm}^{2} / \mathrm{s}$ \\
\hline $\mathrm{d}$ & Particle diameter, $\mathrm{m}$ \\
\hline$E_{i}$ & Activation energy for $k_{i}, K$ \\
\hline $\mathrm{E}_{\eta}$ & Activation energy for coal liquid viscosity \\
\hline$E 1_{j l}$ & $\begin{array}{l}\text { Activation energy for kinetic regime, reactivity model, } \\
\mathrm{cal} / \mathrm{mol}\end{array}$ \\
\hline $\mathrm{Em}_{\mathrm{jl}}$ & $\begin{array}{l}\text { Minerals deactivation energy, reactivity model, } \\
\mathrm{cal} / \mathrm{mol}\end{array}$ \\
\hline eps & Porosity, reactivity model \\
\hline$f_{n}$ & $1-f_{n}$ is the $\mathrm{HCN}$ to $\mathrm{NH}_{3}$ conversion fraction \\
\hline$f_{\text {no }}$ & $\mathrm{HCN}$ to $\mathrm{NH}_{3}$ residue fraction \\
\hline
\end{tabular}




\begin{tabular}{|c|c|}
\hline Table 4-1 & Nomenclature of the Varibles in Chapter 4 - cont. \\
\hline Symbol & Definition \\
\hline$h_{\text {char }}$ & Char hydrogen content, reactivity model, wt \% \\
\hline $\mathrm{k}_{\mathrm{E}}$ & Einstein coefficient, viscosity model \\
\hline$k_{i}$ & Rate constant of gas species $i, 1 / s$ \\
\hline$k_{j l}$ & Intrinsic reaction rate coefficient, reactivity model, $\mathrm{m} / \mathrm{s}$ \\
\hline$k_{\mathrm{a}}$ & $\mathrm{HCN}$ to $\mathrm{NH} 3$ reaction rate constant, $1 / \mathrm{s}$ \\
\hline$M_{\text {olg }}$ & $\begin{array}{l}\text { Molecular weight of oxidant gas, reactivity model, } \\
\text { g/mole }\end{array}$ \\
\hline $\mathrm{M}_{\mathrm{bj}}$ & Molecular weight of material, reactivity model, $\mathrm{g} / \mathrm{mol}$ \\
\hline $\mathrm{M}_{\mathrm{j}}$ & Molecular weight of $j^{\text {th }} \operatorname{tar}$ species \\
\hline $\min$ & Effective mineral content, reactivity model, wt\% \\
\hline $\min 0$ & $\begin{array}{l}\text { Measured dispersed mineral content, reactivity model, } \\
\text { wt\% }\end{array}$ \\
\hline $\mathrm{n}_{\mathrm{a}}$ & $\begin{array}{l}\text { Mole of ambient gas trapped in the coal bubble, } \\
\text { swelling model }\end{array}$ \\
\hline$n_{\mathbf{i}}$ & Mole of gas species i per gram raw coal \\
\hline$n_{j}$ & $\begin{array}{l}\text { Tar species of molecular weight } M_{j} \text {, mole per gram } \\
\text { coal }\end{array}$ \\
\hline$n_{c}$ & $\begin{array}{l}\text { Mole of evolved gas trapped in the coal bubble, } \\
\text { swelling model }\end{array}$ \\
\hline $\mathrm{dn} \mathrm{d}_{\mathrm{d}} / \mathrm{dt}$ & $\begin{array}{l}\text { Rate of gas diffusing out the bubble, swelling model, } \\
\text { mole/s }\end{array}$ \\
\hline
\end{tabular}


Table 4-1

Symbol

$\mathrm{n}_{\mathrm{g}}$

$\mathrm{n}_{\mathrm{t}}$

$\mathrm{n}_{\text {tot }}$

Ocoal

$\mathbf{P}_{\mathbf{j}}$

$\mathrm{P}_{0}$

$\Delta P$

$\Delta \mathrm{P}$

I

$r_{0}$

$r_{1}$

$\mathrm{I}_{2}$

$\mathrm{r}_{\mathrm{bjl}}$

radp

$S_{c}$

sigma
Nomenclature of the Varibles in Chapter 4 - cont.

Definition

Rate of gas evolution of the coal particle, swelling model, mole/s

Amount of evolution gas trapped in the bubble, swelling model, mole

Total gas evolution rate, moles per gram DAF coal per second

Coal oxygen content, reactivity model, wt \%

Vapor pressure of the oligomer of molecular weight $\mathrm{M}_{\mathrm{j}}$

Ambient pressure, atm

Pressure drop from coal inner pores to outside, atm

Effective pressure difference across the coal bubble wall, outward positive, atm

Particle radius

Initial particle radius, $\mathrm{cm}$

Inner bubble radius, swelling model, $\mathrm{cm}$

Particle radius, swelling model, $\mathrm{cm}$

Overall reaction rate, reactivity model, $\mathrm{kg} / \mathrm{s}$

Mean pore radius, reactivity model, $\mathrm{m}$

Strength of coal bubble wall, swelling model, atm

Particle density, reactivity model, $\mathrm{kg} / \mathrm{m}^{3}$ 
Table 4-1

\begin{tabular}{ll}
\hline Symbol & Definition \\
\hline surf & Internal surface area, reactivity model, $\mathrm{m}^{2} / \mathrm{g}$ \\
surfe & External surface area, reactivity model, $\mathrm{m}^{2} / \mathrm{g}$ \\
tau & Tortuosity, reactivity model \\
$\mathrm{T}_{\mathrm{m}}$ & Deactivation temperature $\mathrm{I}$, reactivity model, $\mathrm{K}$ \\
$\mathrm{T}_{\mathrm{j}}$ & Temperature of $\mathrm{j}^{\text {th }}$ particle classification, reactivity \\
$\Delta \mathrm{t}_{\mathrm{n}}$ & model, $\mathrm{K}$ \\
$\Delta \mathrm{t}_{\mathrm{mo}}$ & HCN residence time in coal particle \\
$\mathrm{V}$ & HCN residence time constant \\
$\mathrm{W}_{\mathrm{i}}, \mathrm{i}=$ gas species & Amount of gas species $\mathrm{i}$, gram per gram raw coal \\
$\xi_{\mathrm{j}}$ & Surface area factor, reactivity model \\
$\eta$ & Coal viscosity, pascal*second \\
$\eta$ & Effectiveness factor, reactivity model \\
$\rho$ & Coal density, g/cm ${ }^{3}$ \\
$\sigma$ & Surface tension of coal liquid, dynes/cm ${ }^{2}$ \\
$\sigma_{\mathrm{i}}$ & Standard deviation of distributed evolution rate of gas \\
$\phi_{\mathrm{l}}$ & species $\mathrm{i}$ \\
& stochiometric factor, reactivity model \\
&
\end{tabular}


Table 4-1 Nomenclature of the Varibles in Chapter 4 - cont.

\begin{tabular}{ll}
\hline Symbol & Definition \\
\hline$\phi_{\text {liq }}$ & $\begin{array}{l}\text { Volume fraction of the liquid phase in the coal } \\
\text { particle, viscosity model } \\
\text { Volume fraction of the solid phase in the coal particle } \\
\phi_{s}\end{array}$ \\
$\phi_{\mathrm{c}}$ & $\begin{array}{l}\text { viscosity model } \\
\text { Volume fraction of the solid phase at the gel-point, } \\
\text { viscosity model }\end{array}$ \\
$\phi_{\mathrm{v}}$ & $\begin{array}{l}\text { Volume swelling ratio } \\
\chi_{\mathrm{j}}^{\mathrm{b}}\end{array}$ \\
$\chi_{\mathrm{j}}^{\mathrm{s}}$ & $\begin{array}{l}\text { Mole fraction of species of molecular weight } \mathrm{M}_{\mathrm{j}} \text { in the } \\
\text { metaplast in the bulk of the particle }\end{array}$ \\
& $\begin{array}{l}\text { Mole fraction of species of molecular weight } \mathrm{M}_{\mathrm{j}} \text { in the } \\
\text { metaplast at the surface of the particle }\end{array}$
\end{tabular}


Table 4-2 Swelling model parameters.

\begin{tabular}{|l|r|}
\hline Calculation Particle Radius, cm & 0.002 \\
\hline Bubble Radius, cm & 0.0008 \\
\hline Apparent Pyrolysis Gas Diffusivity, $\mathrm{cm}^{2} / \mathrm{sec}$ & $7.5 \mathrm{e}-5(\mathrm{~T} / 773)^{1.8}$ \\
\hline Apparent Ambient Gas Diffusivity, $\mathrm{cm}^{2} / \mathrm{sec}$ & $7.5 \mathrm{e}-4$ \\
\hline Critical Bubble Wall Stress, atm & 1.0 \\
\hline Coal Liquid Surface Tension, dynes $/ \mathrm{cm}^{2}$ & 30.0 \\
\hline
\end{tabular}


Table 4-3 Sulfur devolatilization: correspondence between pools and gases.

\begin{tabular}{|c|c|c|}
\hline \multirow{5}{*}{ Peak 1} & \multirow{4}{*}{ Organic Sulfur } & $16-\mathrm{SO}_{2}$ \\
\hline & & $17-\cos$ \\
\hline & & $18-C_{2}$ \\
\hline & & $20-\mathrm{H}_{2} \mathrm{~S}$ Loose \\
\hline & Pyritic Sulfur & $58-\mathrm{H}_{2} \mathrm{~S}$ \\
\hline \multirow{5}{*}{ Peak 2} & \multirow{5}{*}{ Pyritic Sulfur } & $51-\mathrm{SO}_{2}$ \\
\hline & & $52-\cos$ \\
\hline & & $53-\mathrm{CS}_{2}$ \\
\hline & & $55-\mathrm{H}_{2} \mathrm{~S}$ Loose \\
\hline & & $57-\mathrm{H}_{2} \mathrm{~S}$ Gas \\
\hline \multirow{2}{*}{ Peak 3} & Organic Sulfur & $19-\mathrm{H}_{2} \mathrm{~S}$ Tight \\
\hline & Pyritic Sulfur & $54-\mathrm{H}_{2} \mathrm{~S}$ Tight \\
\hline
\end{tabular}


Table 4-4 Elemental Compositions of the Eight Argonne Coals and the Three PSOC Coals. Pyrite sulfur contents are given in grams per 100 grams DAF coal.

\begin{tabular}{|c|c|c|c|c|c|c|c|}
\hline \multirow[b]{2}{*}{$\begin{array}{c}\text { Coal } \\
\# \\
\end{array}$} & \multicolumn{7}{|c|}{ Elemental Composition, wt\%(DAF) } \\
\hline & Coals & $\mathrm{C}$ & $\mathrm{H}$ & 0 & $\mathbf{N}$ & $S$ & $\begin{array}{c}S \\
\text { (pyrite) }\end{array}$ \\
\hline 1 & Beulah-Zap & 72.97 & 4.83 & 20.35 & 1.15 & 0.7 & 0.155 \\
\hline 2 & $\begin{array}{l}\text { Wyodak- } \\
\text { Anderson }\end{array}$ & 75.03 & 5.34 & 18.04 & 1.12 & 0.47 & 0.186 \\
\hline 3 & Illinois \#6 & 77.72 & 5.00 & 13.53 & 1.37 & 2.38 & 3.32 \\
\hline 4 & $\begin{array}{c}\text { Blind Canyon, } \\
\text { UT }\end{array}$ & 80.71 & 5.76 & 11.59 & 1.57 & 0.37 & 0.251 \\
\hline 5 & $\begin{array}{c}\text { Lewis-Stockton } \\
\text { WV }\end{array}$ & 82.69 & 5.26 & 9.84 & 1.56 & 0.65 & 0.200 \\
\hline 6 & $\begin{array}{l}\text { Pittsburgh } \\
\text { Seam }\end{array}$ & 83.30 & 5.33 & 8.84 & 1.64 & 0.89 & 1.50 \\
\hline 7 & $\begin{array}{c}\text { Upper Freeport } \\
\text { PA }\end{array}$ & 85.50 & 4.70 & 7.51 & 1.55 & 0.74 & 2.04 \\
\hline 8 & $\begin{array}{c}\text { Pocahontas \#3 } \\
\text { VA }\end{array}$ & 91.23 & 4.45 & 2.49 & 1.33 & 0.50 & 0.158 \\
\hline 9 & PSOC 1448 & 84.08 & 6.04 & 7.52 & 1.84 & 0.52 & 0.05 \\
\hline 10 & PSOC 1521 & 87.00 & 5.13 & 5.08 & 1.87 & 0.92 & 0.52 \\
\hline 11 & PSOC 1522 & 89.09 & 4.56 & 3.47 & 1.89 & 0.90 & 0.02 \\
\hline
\end{tabular}


Table 4-5 Kinetic Data of Major Functional Group Pools. $R$ is the Gas Constant, $A$ is in $1 /$ sec., $E / R$ and $\sigma / R$ are in $K$

Rinetic Rate Parameters for Argonne Cosls

\begin{tabular}{|c|c|c|c|c|c|c|c|c|}
\hline $\mathrm{CO}_{2} \cdot \mathrm{XI}$ & $\frac{Z A P}{A=5.00 e+12}$ & wyo & III & ITA & stos & EIT & UPE & ROC \\
\hline 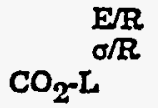 & $\begin{array}{l}18000 \\
1500.0 \\
A=5.00 \mathrm{E}+12\end{array}$ & $\begin{array}{l}18000 \\
1500.0\end{array}$ & $\begin{array}{l}20500 \\
3000.0\end{array}$ & $\begin{array}{l}21000 . \\
4000.0\end{array}$ & $\begin{array}{l}21250 . \\
3500.0\end{array}$ & $\begin{array}{l}21500 . \\
3600.0\end{array}$ & $\begin{array}{l}22000 \\
2000.0\end{array}$ & $\begin{array}{l}23000 . \\
2500.0\end{array}$ \\
\hline 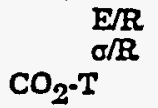 & $\begin{array}{l}23500 \\
2000.0 \\
A=7.500 \mathrm{E}+12\end{array}$ & $\begin{array}{l}24000 \\
2500.0 \\
2\end{array}$ & $\begin{array}{l}24750 . \\
1750.0\end{array}$ & $\begin{array}{l}25500 \\
2000.0\end{array}$ & $\begin{array}{l}26000 . \\
3000.0\end{array}$ & $\begin{array}{l}26500 \\
3000.0\end{array}$ & $\begin{array}{l}27000 \\
3000.0\end{array}$ & $\begin{array}{l}28000 . \\
2500.0\end{array}$ \\
\hline 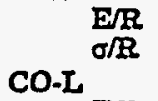 & $\begin{array}{l}31000 \\
3000.0 \\
A=5.00 \mathrm{E}+12\end{array}$ & $\begin{array}{l}32000 \\
2800.0\end{array}$ & $\begin{array}{l}32000 . \\
2750.0\end{array}$ & $\begin{array}{l}32000 \\
3000.0\end{array}$ & $\begin{array}{l}32000 . \\
3200.0\end{array}$ & $\begin{array}{l}32000 \\
3000.0\end{array}$ & $\begin{array}{l}32000 . \\
2500.0\end{array}$ & $\begin{array}{l}33500 . \\
2700.0\end{array}$ \\
\hline $\mathrm{CO}^{\frac{\mathrm{E}}{\alpha / R}}{ }^{\frac{E / R}{R}}$ & $\begin{array}{l}24500 \\
3000.0 \\
A=5.00 \mathrm{E}+12\end{array}$ & $\begin{array}{l}24750 . \\
2500.0\end{array}$ & $\begin{array}{l}25000 . \\
1000.0\end{array}$ & $\begin{array}{l}25000 \\
1250.0\end{array}$ & $\begin{array}{l}25500 . \\
1100.0\end{array}$ & $\begin{array}{l}26000 . \\
1250.0\end{array}$ & $\begin{array}{l}28000 \\
750.0\end{array}$ & $\begin{array}{l}29000 . \\
1250.0\end{array}$ \\
\hline$\underset{\substack{E / R \\
\sigma / R}}{c o-X T}$ & $\begin{array}{l}30000 \\
3000.0 \\
A=2.00 E+14\end{array}$ & $\begin{array}{l}30250.0 \\
3000.0\end{array}$ & $\begin{array}{l}30500 . \\
2000.0\end{array}$ & $\begin{array}{l}30500 . \\
2000.0\end{array}$ & $\begin{array}{l}30500 \\
1600.0\end{array}$ & $\begin{array}{l}30750 . \\
1900.0\end{array}$ & $\begin{array}{l}31500 . \\
1400.0\end{array}$ & $\begin{array}{l}32000 . \\
1500.0\end{array}$ \\
\hline $\mathrm{CE}_{4^{-\mathrm{L}}} \underset{\mathrm{G} / \mathrm{R}}{\mathrm{E} / \mathrm{R}}$ & $\begin{array}{l}39000 . \\
2500.0 \\
A=3.00 \mathrm{E}+13\end{array}$ & $\begin{array}{l}39750 . \\
2500.0\end{array}$ & $\begin{array}{l}40000 \\
3000.0\end{array}$ & $\begin{array}{l}40000 \\
2500.0\end{array}$ & $\begin{array}{l}400000 \\
3000.0\end{array}$ & $\begin{array}{l}40000 . \\
2800.0\end{array}$ & $\begin{array}{l}40000 \\
2250.0\end{array}$ & $\begin{array}{l}40000 . \\
3200.0\end{array}$ \\
\hline $\begin{array}{r}\underset{E / R}{\sigma / R} \\
\mathrm{CH}_{4}-\mathrm{T}\end{array}$ & $\begin{array}{l}28000 . \\
2500.0 \\
A=6.00 E+13\end{array}$ & $\begin{array}{l}28000 . \\
2250.0\end{array}$ & $\begin{array}{l}28000 . \\
1800.0\end{array}$ & $\begin{array}{l}28300 \\
1800.0\end{array}$ & $\begin{array}{l}28000 . \\
1200.0\end{array}$ & $\begin{array}{l}28000 \\
1300.0\end{array}$ & $\begin{array}{l}28750 \\
800.0\end{array}$ & $\begin{array}{l}29500 . \\
750.0\end{array}$ \\
\hline $\begin{array}{c}E / R \\
\frac{E}{\sigma / R} \\
\mathrm{H}_{2} \mathrm{O} \cdot \mathrm{XI}\end{array}$ & $\begin{array}{l}32000 \\
2200.0 \\
A=5.000 E+12\end{array}$ & $\begin{array}{l}32000 . \\
2500.0 \\
2\end{array}$ & $\begin{array}{l}32000 . \\
2200.0\end{array}$ & $\begin{array}{l}31500 . \\
2200.0\end{array}$ & $\begin{array}{l}32000 . \\
2200.0\end{array}$ & $\begin{array}{l}32000 . \\
2200.0\end{array}$ & $\begin{array}{l}32000 . \\
2000.0\end{array}$ & $\begin{array}{l}33000 . \\
1700.0\end{array}$ \\
\hline 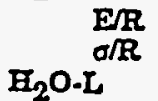 & $\begin{array}{l}16500 \\
1500.0 \\
A=5.000 \mathrm{E}+12\end{array}$ & $\begin{array}{l}17000 . \\
1500.0\end{array}$ & $\begin{array}{l}18000 . \\
1800.0\end{array}$ & $=$ & $=$ & $=$ & $=$ & 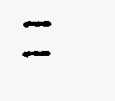 \\
\hline $\mathrm{H}_{2} \mathrm{O}-\mathrm{T} / \mathrm{T}$ & $\begin{array}{l}23000 \\
2700.0 \\
A=2.000 E+14\end{array}$ & $\begin{array}{l}24250 \\
2500.0 \\
4\end{array}$ & $\begin{array}{l}25000 . \\
1500.0\end{array}$ & $\begin{array}{l}25000 \\
1250.0\end{array}$ & $\begin{array}{l}25500 . \\
1250.0\end{array}$ & $\begin{array}{l}26000 \\
1250.0\end{array}$ & $\begin{array}{l}27500 . \\
1250.0\end{array}$ & $\begin{array}{l}28000 . \\
1250.0\end{array}$ \\
\hline $\begin{array}{r}\mathbf{E} / \mathbf{R} \\
\sigma / \mathbb{R} \\
T a x-B \mathbf{B}\end{array}$ & 31000 . & $\begin{array}{l}31000 \\
3500.0\end{array}$ & $\begin{array}{l}32000 \\
2800.0\end{array}$ & $\begin{array}{l}32000 \\
2500.0\end{array}$ & $\begin{array}{l}32000 . \\
2500.0\end{array}$ & $\begin{array}{l}32000 . \\
2500.0\end{array}$ & $\begin{array}{l}34000 . \\
2000.0\end{array}$ & $\begin{array}{l}35000 . \\
2500.0\end{array}$ \\
\hline $\begin{array}{c}\mathbf{L}-\mathbf{B} B \\
\mathbf{E} / \mathbf{R} \\
\mathbf{\alpha} / \mathbf{R}\end{array}$ & $\begin{array}{l}A=1.000 \mathrm{E}+14 \\
26000 . \\
1000.0\end{array}$ & $\begin{array}{l}4 \\
26000 \\
1000.0\end{array}$ & $\begin{array}{l}26000 . \\
750.0\end{array}$ & $\begin{array}{l}27000 . \\
1250.0\end{array}$ & $\begin{array}{l}27250 . \\
1000.0\end{array}$ & $\begin{array}{l}27500 . \\
1250.0\end{array}$ & $\begin{array}{l}28250 . \\
1250.0\end{array}$ & $\begin{array}{l}29000 . \\
750.0\end{array}$ \\
\hline
\end{tabular}




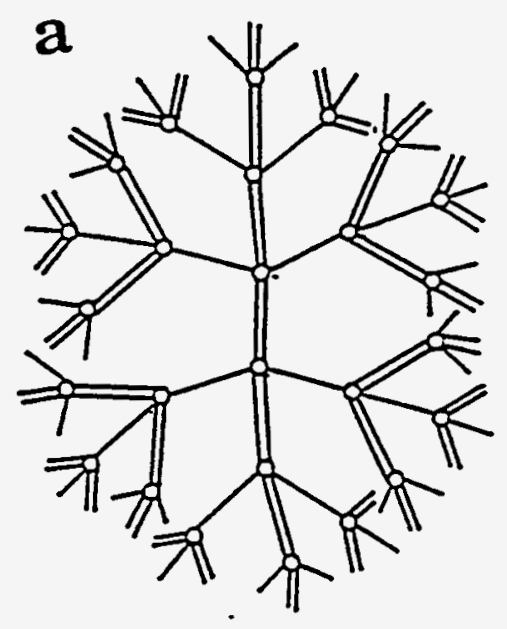

b

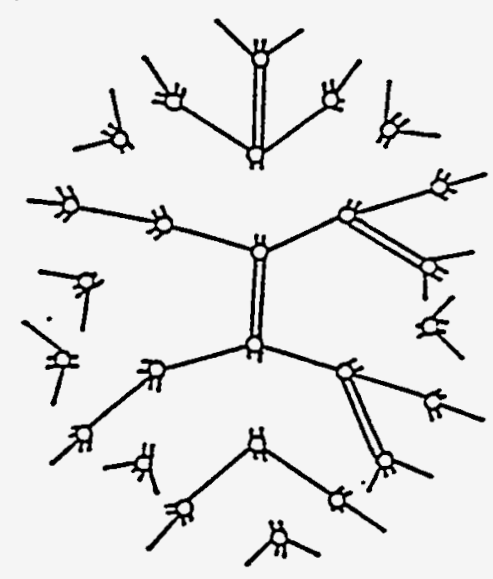

Figure 4-1 Bethe Lattice for Two- $\sigma$ Model with $\sigma_{1}=\sigma_{2}=1$ (shown as single bonds). a) Fully Linked Case $(p=q=1)$ is like One- $\sigma$ Model with $\sigma=3$. b) With Most Double Bonds Representing the Crosslinks not yet Formed to Represent the Starting Coal. The Lattice is Like One- $\sigma$ Model with $\sigma=1$, Linear Chains. 


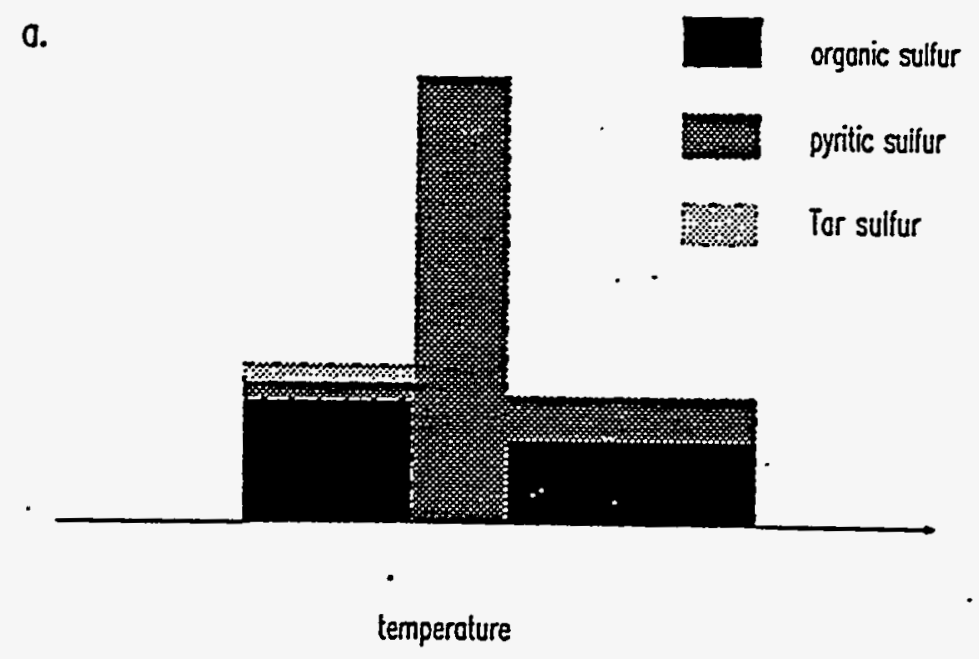

b.

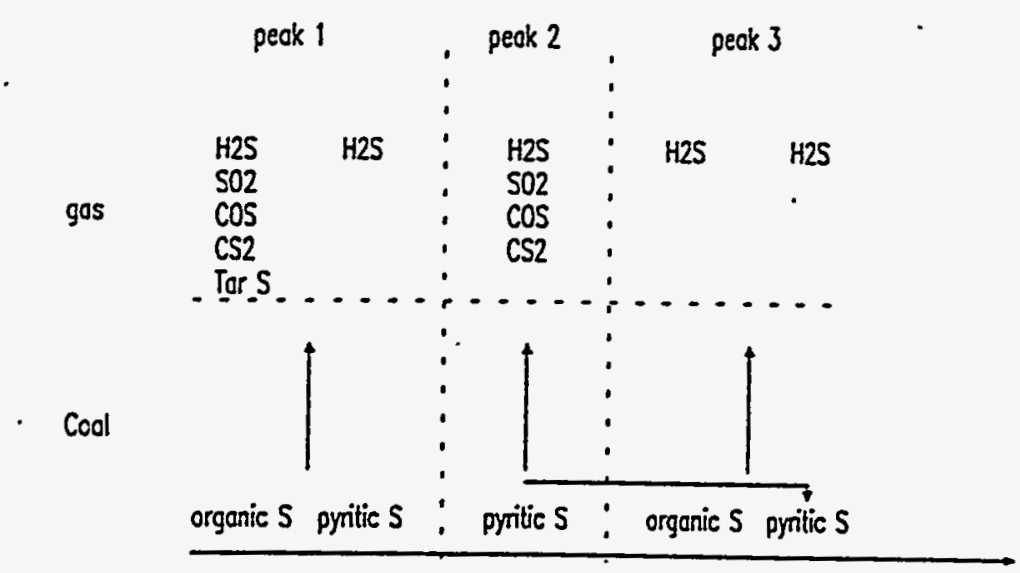

Intrecroise

Figure 4-2 Schematic diagrams of sulfur devolatilization: $a$. the three peak framework. b. sulfur gas distribution. 


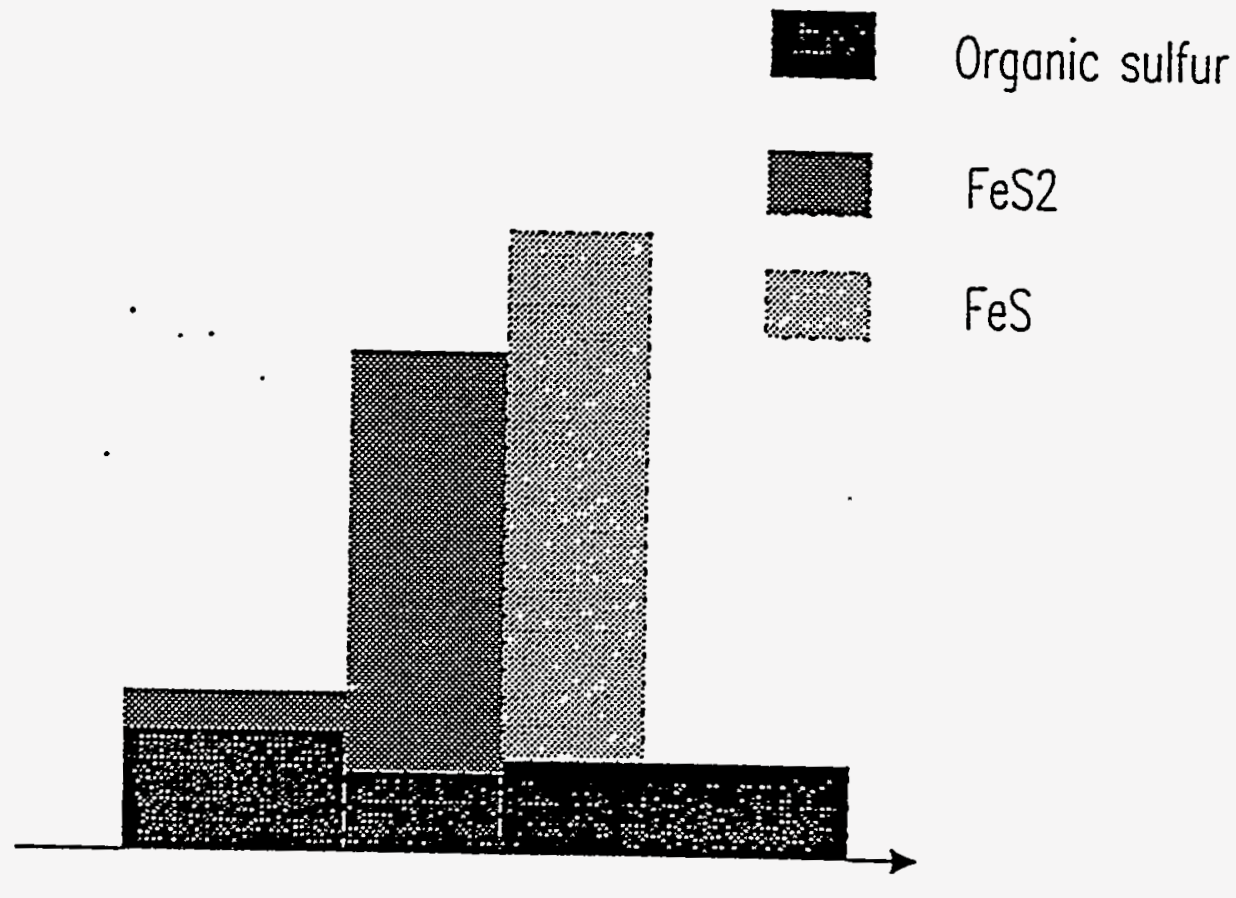

Temperature

Figure 4-3 A schematic diagram of sulfur devolatilization in oxidation conditions. 


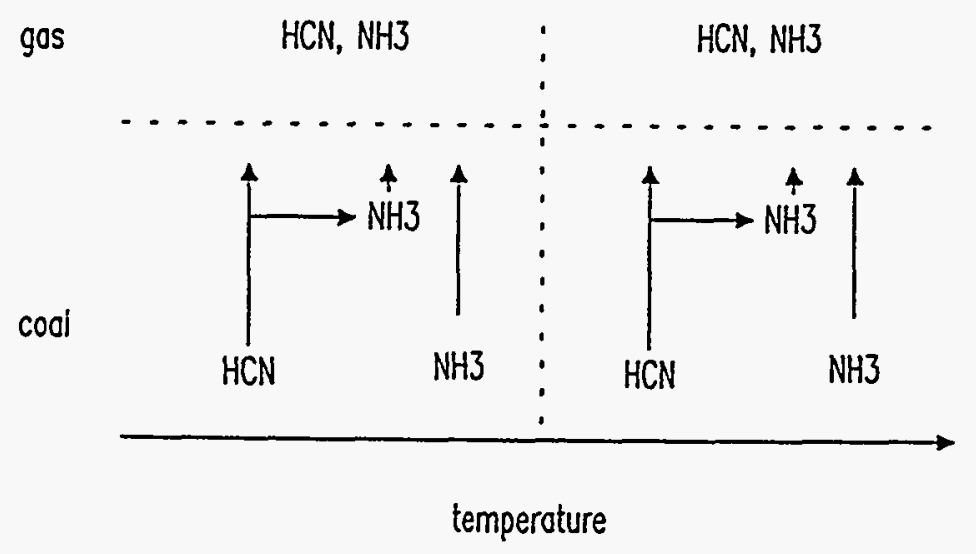

Figure 4-4 A schematic diagram of nitrogen gas distribution. 


\section{Chapter Five}

\section{User's Guide}

\section{General Description}

PCGC-2 was originally developed on a VAX/11-750 computer with VMS FORTRAN. Recent development has been done on UNIX machines, adhering to the FORTRAN 77 standard. Plotting or graphics routines are not distributed with the code, but are available separately. Graphics is very important for understanding the code's voluminous output of data, and graphics is discussed in a section below. Intermediate computational values of all variables can be saved for restarting, so that computations need not start from scratch each time. Approximately 1.5 megabytes of disk space are required for the FORTRAN listing. At least two megabytes of real memory are required for running the code.

All input data to PCGC-2 are in SI units with the exception of angles, which are in degrees. The essential input is summarized in Table 5-1. All working variables within the program are either dimensionless or in SI units. Units associated with all variables are given in the nomenclature.

CPU run time varies widely according to the particular simulation and the machine. Ten CPU-hours is typical for simulation of simple pulverized-coal combustion with a Sun SPARC II workstation. 
TABLE 5-1

Geometry

\section{SUMMARY OF INPUT FOR PCGC-2}

Primary tube diameter (m)

Secondary tube diameter $(\mathrm{m})$

Chamber diameter $(\mathrm{m})$

Chamber length (m)

Additional ${ }^{l}$ inlets, location and sizes

Feed Rates

Primary gas $(\mathrm{kg} / \mathrm{s})$

Secondary gas $(\mathrm{kg} / \mathrm{s})$

Additional inlet \#1 $(\mathrm{kg} / \mathrm{s})$

Additional inlet $\# 2(\mathrm{~kg} / \mathrm{s})$, etc.

Coal particle loading in each inlet

Sorbent particle loading in each inlet

Inlet Gas Properties

Primary swirl number

Primary turbulence intensity (\% or $\mathrm{m} / \mathrm{s}$ )

Primary temperature $(\mathrm{K})$

Primary mole fractions of all species

Secondary swirl number

Secondary turbulence intensity (\% or $\mathrm{m} / \mathrm{s})$

Secondary temperature (K)

Secondary mole fractions of all species

Turbulence intensity, temperature and composition for additional

inlets 2

Reactor Parameters

Reactor pressure (N/sq m)

Wall temperatures $(\mathrm{K})$

Particle Parameters

Particle solid density (kg/cu m)

Mass mean particle diameter and distribution of particle sizes (m)

${ }^{1}$ Up to 3 additional inlets are allowed

2 Some restrictions apply to temperature and composition of additional inlets. 
TABLE 5-1 (continued)

Coal composition ( $\neq$ proximate analysis) $(\%)$ raw coal

char

ash

Coal type

\section{Description of Subroutines}

A complete description of the routines is given in Appendix B. A brief description is given below.

The main program is PCGC-2. INFLOW reads the input data for the inlet streams. GRD generates the numerical grid. GRMAP modifies the numerical grid if a grid data file is being used and writes a new grid data file. GRDGRF writes a schematic showing the grid locations in the output file. CREEO reads the reactant composition data and thermochemical data file. INIT initializes arrays used in the code.

PSICTO, COALO, FLUXO, and NOXMNO read input data and perform initial calculations for the particle trajectory calculations, particle reaction scheme, radiation submodel, and nitrogen pollutants submodel, respectively. Other input data are read primarily by the main program and by the driver program for the nitrogen oxide submodel (NOXMN).

TABLE is the driver program for generating the table of equilibrium gas properties for lookup. TBLF, TBLFE, TBLFEH, and TBLFH generate tables of equilibrium properties as functions of $f$ only; both $f$ and $h, f, h$, and $h_{r}$; and both $f$ and $h_{r}$; respectively. These four tables correspond to adiabatic, gas only; adiabatic, gas and reacting particles; non-adiabatic, gas and reacting particles; and nonadiabatic, gas only; respectively. All four table generators call CREE to calculate the equilibrium properties. TBLFE and TBLFEH provide for table restart (TBLRSTFE and TBLRSTFEH).

PROFL reads input data and sets the boundary values for the gas inlet streams. PRINT is a routine that is called frequently to print values of field variables to the output file. RESRT reads and writes gas-phase restart files. Particle phase restart files are read and written by the main program. FLINT initializes the flow field variables and calculates the gas density field. EULINT is an entry in PSICTO that calculates the particle number flowrate for each particle trajectory. 
PROPS calculates the time-mean gas properties, incorporating the effects of turbulence. FGTABL is a routine that provides the upper and lower limits of the clipped Gaussian probability density function from a table of precalculated values. CREE may be utilized to calculate equilibrium gas properties when residual enthalpy falls outside the table limits. UPDATE is an entry point in TABLE that calls the appropriate entry point in TBLF, TBLFE, TBLFEH or TBLFH, to retrieve the needed gas properties.

STREAM calculates the velocity streamlines for plotting. PLTWRT writes plotting files.

The CALC routines calculate the flowfield variables such as velocities, turbulence properties, mixture fractions, and pressure, at each node throughout the flow field (Step 2 of Fig. 3-1). Each CALC routine calls an entry point in PROMOD or a separate "MOD" routine to modify the coefficients and source terms in the appropriate finite difference equation in order to account for the boundary conditions as described in Chapter 3. LISOLV is the solver that performs the operations of the TDMA algorithm to solve the finite difference equations.

PROG monitors the progress of the calculation toward convergence by recording the values of the variables at a specified location for each iteration.

EOLP is the driver program for the particle calculations. CALCNJ solves the Eulerian particle density equation using the TDMA (LISOLV). CALACJ, CALAHI, and PSOLVP solve the Eulerian particle calculations. Although the pure Eulerian approach for solving the particle phase has been programmed into PCGC2 , it is not operational. FLUX is the driver program for the radiation submodel. BVALIN is an entry point in BPROPS that inserts reasonable values into boundary intrusions in order to allow for 2-D interpolation of gas properties. PSICT solves the particle trajectories using the Particle-Source-In-Cell of Crowe and coworkers (1977) and calculates the particle source terms. COAL1 calculates the gas properties. COAL 2 calculates the particle reaction rates, and PSOLVE solves the particle continuity equations. PSOLVS is a special solver that is used when the particle enthalpy equation goes stiff (after burnout it is essentially complete). BVALOUT is an entry in BPROPS that substitutes the original values of properties back into the boundary intrusions after particle trajectory calculations are complete.

An information flow diagram for the combined Eulerian-Lagrangian particle model is shown in Figure 5-1. CALCNJ first calculates the Eulerian number density field for each particle size. The Eulerian number density is needed by the FLUX routines for calculating the radiation fluxes and by the PSI-CELL routine for calculating the turbulent diffusion component of particle velocity as given by Eqn. 2-34. PSICT solves the particle momentum equations for each trajectory, keeps track of particle position and properties as a function of time, and calculates the appropriate source terms for each cell. PSICT also interpolates Eulerian properties 


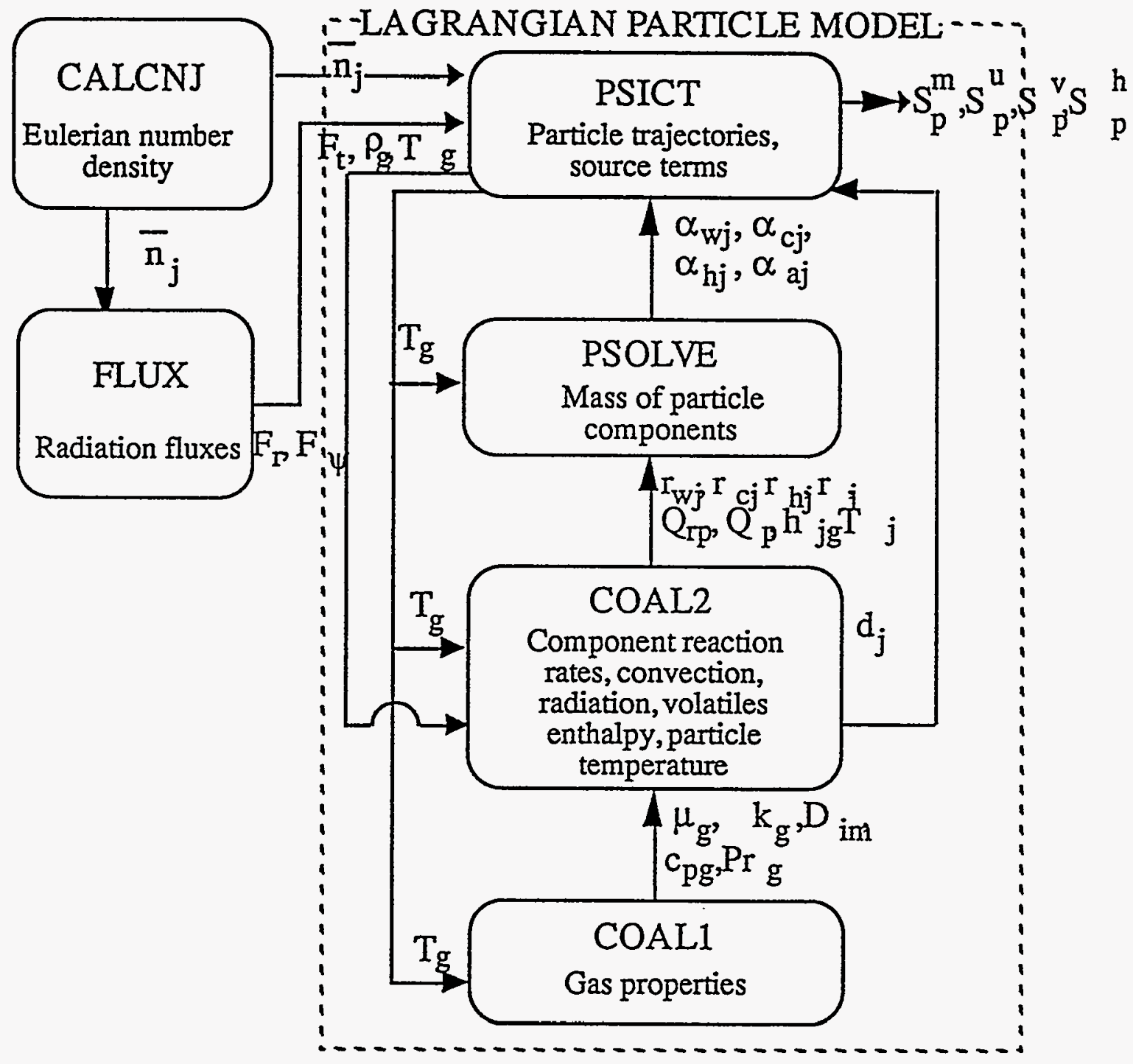

Fig. 5-1. Information flow diagram for combined Eulerian-Lagrangian particle model. 
User's Guide

(total radiation flux, gas density, and gas temperature) across cells to be used in the particle calculations. COAL1 uses the local gas temperature to calculate gas viscosity, gas thermal conductivity, species diffusivities, gas thermal heat capacity, and Prandtl number. COAL2 uses this information to calculate the component reaction rates, convection and radiation heat transfer rates, volatiles enthalpy, and particle temperature. Particle diameter is also calculated by COAL 2 and used by PSICT in solving the momentum equations. PSOLVE uses the particle reaction rates, heat transfer rates, particle temperature, and volatiles enthalpy to solve the particle continuity equations and provide the mass of each particle component at each time step. The final product of the particle model is the source terms which are returned to the gas phase.

RADCOF calculates the gas emissivity and absorption and scattering coefficients for either the VARMA six-flux (VFM) or discrete ordinates (DOM) radiation model. EICO2 and EIH20 calculate the emissivity contributions for carbon dioxide and water vapor, respectively. DISORD solves the radiation equations using the DOM model. RESULT prints a summary of the results of the DOM calculations in the output file. SETUP calculates the coefficients for the VFM model. FLUXX, FLUXR, and FLUXT solve the VFM model equations for the axial, radial, and tangential directions, respectively. The equation for the tangential direction is algebraic due to the axial symmetry and does not utilize the TDMA solver (LISOLV).

NOXMN is the driver for the $\mathrm{NO}_{\mathrm{x}}$ pollutant submodel. NOXMN reads input cards from the data file, controls the $\mathrm{NO}_{\mathrm{x}}$ model logical sequence, tests for convergence, and prints out intermediate and final results. EQSPEC calculates Favre average equilibrium concentrations for gas species used to estimate atomic oxygen $(\mathrm{O})$ and hydroxide $(\mathrm{OH})$ concentrations. FREACT calculates the maximum possible concentrations of $\mathrm{HCN}, \mathrm{NH}_{3}$ and $\mathrm{NO}$ at each grid location for fuel nitrogen conversion. PSCRAT calculates the rate of homogeneous reactions for intermittent primary, secondary and coal off-gas. Mean reaction rates and species continuity source terms are calculated in RATE which calls RXNEXT to calculate reaction progress variables and RXNRAT to calculate the local instantaneous homogeneous chemical reaction rates for all mechanisms. RATE incorporates the time-mean effects of turbulence on the homogeneous reaction rates, analogous to the manner in which PROPS incorporates the effects of turbulence on the time-mean gas properties. The subroutine B is called to determine the power for oxygen concentrations from the auto-correlation given by de Soëte (1975) for $\mathrm{HCN}$ and $\mathrm{NH}_{3}$ global oxidation reactions. PRTSUR calculates the surface area of char particles which is required for the heterogeneous NO decay reactions. CALCNO, CALHCN, and CALNH3 calculate the field values for $N O, H C N$, and $\mathrm{NH}_{3}$ respectively, using LISOLV, analogous to the other CALC routines which 
solve the field variables for the gas phase. CALCO2 adjusts the time-mean mass fractions of $\mathrm{O}_{2}, \mathrm{~N}_{2}$, and $\mathrm{OH}$ throughout the flowfield following the pollutant reactions. Finally, INTGRT computes integrated average dry mole fraction at each axial node for final output while RSTNOX reads and writes to a restart file.

SORPAR is the driver for the $\mathrm{SO}_{\mathrm{x}}$ /sorbent reactions submodel. SORPAR controls the sorbent reactions model logical sequence, tests for convergence, and prints out intermediate and final results. SORBO reads the input files while CALCSJ calculates the Eulerian number density for the sorbent particles. SPSICT calculates the Lagrangian trajectories for the sorbent particles and calls SULFAT and SULFHS which calculate the progressive sulfation of the sorbents due to $\mathrm{SO}_{2}$ and $\mathrm{H}_{2} \mathrm{~S}$ capture. Thus, SPSICT calculates the source terms for $\mathrm{SO}_{2}$ and $\mathrm{H}_{2} \mathrm{~S}$ capture in each computational cell. SULFAT and SULFHS are based on the shrinking-core grain model. CALSO2 and CALH2S calculate the field values for $\mathrm{SO}_{2}$ and $\mathrm{H}_{2} \mathrm{~S}$ respectively, using LISOLV, analogous to the other CALC routines which solve the field variables for the gas phase.

\section{Program Input}

At least three input files are required by PCGC-2: the main data file (PCGCIN), the thermodynamic properties data file (THERMO), and the inlet profile data file (INLET). Other data files may also be required. For example, if the individual grid locations are to be specified (INRDGD $=\mathrm{T}$ in the main data file) a grid data file (GRID) is needed. If the calculation is being restarted from the results of a previous calculation (INRST $=\mathrm{T}$ and/or $\mathbb{I N P R S T}=\mathrm{T}$ in the main data file), restart files are needed for the gas (INOUT) and/or particles (PARSOU). If the FG-DVC submodel for devolatilization is being used, additional data files are needed.

The data contained in the three required input files are shown in Table 5-2 and are briefly described below. The data are given in the actual order in which they are read by the program, and are divided into seventeen groups according to function. Also shown in Table 5-2 are the calling routine and the required format. A detailed description of the input data is given in Appendix $C$.

Group 1 in Table 5-2 contains comment statements that are printed out as a header at the beginning of the main output (PCGCOT) and log files. Any number of comment statements may be included in the header.

Group 2 contains program control information, including which field variables are to be calculated, and numerical parameters for degree of underrelaxation, convergence criteria, etc. The reactor geometry, temperature bounds for 
the physical properties table, reactor heat loss, wall temperatures, and gas viscosity and pressure are also specified in Group 2.

Group 3 describes the flowrate, composition (in terms of the reactant streams described below.), swirl, turbulence intensity, coal particle loading, and sorbent particle loading of each of the gas inlet streams. Up to three additional inlets (in addition to the primary and secondary) may be included, but they must all be described in terms of the reactant streams specified below.

Data in Groups 1,2, and 3 are all contained in the main input file, PCGCIN. For Groups 4 and 5, only the first data item, i.e. the keywords ELEM and THER, are contained in file PCGCIN. The remaining data in these groups are contained in the THERMO file. Group 4 gives the molecular weight and valence of each of the elements. The order in which the elements are listed is the order in which they are treated in the program calculations. Group 5 gives the elemental composition, heat of formation, temperature ranges, and heat capacity coefficients for each of the molecular species.

Group 6 describes the reactant streams that are used to specify the inlet stream compositions and temperatures. Only two reactant streams are allowed. The inlet streams are specified as a mixture of the two reactant streams. Usually, the first reactant stream is used to specify the primary gas, and the second reactant stream is used to specify the secondary and any additional inlets. However, any inlet may also be specified as any mixture of both reactant streams using the input variable FFLOW in Group 3.

TABLE 5-2

INPUT DATA

\begin{tabular}{|c|c|c|c|c|c|}
\hline Group & $\begin{array}{l}\text { Calling } \\
\text { Routine }\end{array}$ & Data File ${ }^{1}$ & Line & $\underline{\text { Data Items }}$ & Format \\
\hline 1 & MAIN & PCGCIN & $\begin{array}{r}1 \\
2 \\
\text { NSAY }\end{array}$ & 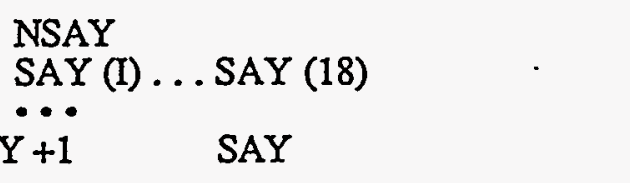 & $\begin{array}{r}* \\
18 \mathrm{A4} \\
\bullet\end{array}$ \\
\hline 2 & MAIN & PCGCIN & $\begin{array}{l}1 \\
2 \\
3\end{array}$ & $\begin{array}{l}\text { INRST, INCALF, INCREK, INCALH, } \\
\text { INCALG } \\
\text { IPSICT, INPRST, INEACH, INCLET, } \\
\text { INCLGE } \\
\text { INCALN, INCURF, LEULP, } \\
\text { INTFRZ, INSIMP }\end{array}$ & 8LF \\
\hline
\end{tabular}




\begin{tabular}{|c|c|c|c|c|c|}
\hline \multirow[t]{2}{*}{ Group } & $\begin{array}{l}\text { Calling } \\
\text { Routine }\end{array}$ & Data File ${ }^{1}$ & Line & TABLE 5-2 (continued) & Format \\
\hline & & & $\begin{array}{r}4 \\
5 \\
6 \\
7 \\
8 \\
\\
9 \\
10 \\
11 \\
12 \\
13 \\
14 \\
15 \\
16 \\
17 \\
18 \\
19 \\
20 \\
21 \\
22 \\
23\end{array}$ & $\begin{array}{l}\text { INSMPC,INCLKE,LPOST } \\
\text { INCNOX, POLLUT, INQRL, } \\
\text { INCSWP, INCSWS } \\
\text { INNOZZ, INFSOU, LTBUG, } \\
\text { GRDOUT, INPROG } \\
\text { INCFP, INRAD, INRDGD, INTRUS, } \\
\text { MAGHJER } \\
\text { INETA2,HTRACK,FGDVC,LAMIN } \\
\text { ARI IBUOY } \\
\text { UPDOWN } \\
\text { URFU, URFV, URFW, URFH } \\
\text { URFE, URFK, URFF, URFG, URFVIS } \\
\text { URFDEN, URFETA, URFGET, } \\
\text { URFNJ, URFPP,URFP } \\
\text { DIAP, DIAS, DIACH } \\
\text { NDIA(REAL), THICK } \\
\text { MAXIT, INDPRI, INDRST } \\
\text { NJINP, NJINS, NIWOQ } \\
\text { AL1, EPSX, NL, EPSI, EPSD } \\
\text { TMIN, TMAX, HLOSS } \\
\text { TLODEL, THIDEL } \\
\text { NIINQ, QHA, QLX } \\
\text { TBN, TBW, TBE } \\
\text { VISCOS, PRES, IEUCK } \\
\text { SORMAX, SORMIN }\end{array}$ & $\begin{array}{l}* \\
* \\
* \\
* \\
* \\
* \\
* \\
* \\
* \\
* \\
*\end{array}$ \\
\hline 3 & INFLOW & PCGCIN & $\begin{array}{l}1 \\
2\end{array}$ & $\begin{array}{l}\text { STRING } \\
\text { FLOW(I), FFLOW(I), SWIRIN(I), } \\
\text { TINFLO(I), PLOAD(I), PLOADS(I) } \\
\text { I=primary, secondary }[, 1, \ldots \\
\text {,NINLET] }\end{array}$ & $\begin{array}{l}\text { A5 } \\
*\end{array}$ \\
\hline 4 & CREE $\varnothing$ & $\begin{array}{l}\text { PCGCIN } \\
\text { THERMO }\end{array}$ & $\begin{array}{l}1 \\
2\end{array}$ & $\begin{array}{l}\text { ELEM } \\
\text { ATOMID(NLM), ATOM(1, NLM), } \\
\text { ATOM( } 2, N L M) \\
\text { repeat the above card for each } \\
\text { element (NLM total) } \\
\text { BLANK LINE }\end{array}$ & $\begin{array}{r}\text { A4 } \\
\text { A2, } 7 \mathrm{X} \\
2 \mathrm{~F} 10.6\end{array}$ \\
\hline 5 & CREE $\varnothing$ & $\begin{array}{l}\text { PCGCIN } \\
\text { THERMO }\end{array}$ & $\begin{array}{l}1 \\
2\end{array}$ & $\begin{array}{l}\text { THER } \\
\text { DATA(1), DATA(2), DATA(3) }\end{array}$ & $\begin{array}{r}\mathrm{A} 4 \\
3 \mathrm{~A} 4, \mathrm{XX}\end{array}$ \\
\hline
\end{tabular}


TABLE 5-2 (continued)

\begin{tabular}{|c|c|c|c|c|c|}
\hline \multirow[t]{2}{*}{ Group } & $\begin{array}{l}\text { Calling } \\
\text { Routine }\end{array}$ & Data File & Line & Data Items & Format \\
\hline & & & 5 & $\begin{array}{l}\text { DT1, DT2, AT(1), B(1) } \\
\text { AT(2), B(2), AT(3), B(3) } \\
\text { AT(4), B(4), PHASE } \\
\text { TLOW, THIGH } \\
\text { IDON(1), IDON(2), IDON(3), } \\
\text { IDON(4) } \\
\text { NCD } \\
Z(1,1, N S), Z(2,1, N S), Z(3,1, N S) \\
Z(4,1, N S), Z(5,1, N S), N C D \\
Z(6,1, N S), Z(7,1, N S), Z(1,2, N S) \\
Z(2,2, N S), Z(3,2, N S), N C D \\
Z(4,2, N S), Z(5,2, N S), Z(6,2, N S) \\
\text { repeat the above } 4 \text { cards for each } \\
\text { chemical species (NS total) } \\
\text { BLANK LINE }\end{array}$ & $\begin{array}{r}2 \mathrm{~A} 3, \mathrm{~A} 2, \\
\mathrm{~F} 3.0 \\
2(\mathrm{~A} 2, \\
\mathrm{F} 3.0) \\
\mathrm{A} 2, \mathrm{~F} 3.0, \\
\mathrm{~A} 1 \\
2 \mathrm{~F} 10.3 \\
4 \mathrm{I} 3 \\
\mathrm{I} 3 \\
3 \mathrm{E} 15.8 \\
2 \mathrm{E} 15.8, \\
\mathrm{I} \\
3 \mathrm{E} 15.8 \\
2 \mathrm{E} 15.8, \\
\mathrm{I} 5 \\
3 \mathrm{E} 15.8, \\
\mathrm{I} 5\end{array}$ \\
\hline 6 & CREE $\varnothing$ & PCGCIN & $\begin{array}{l}1 \\
2 \\
3\end{array}$ & $\begin{array}{l}\text { REAC } \\
\text { TMP } \\
\text { AT(1), B(1), . . AT(4),B(4), } \\
\text { ASUB(1), ASUB(2), PECWT, } \\
\text { MOLE, PHASE } \\
\text { Repeat the above card for each } \\
\text { species in the primary reactant } \\
\text { stream } \\
\text { BLANK LINE } \\
\text { Repeat group } 6 \text { once for the } \\
\text { secondary stream }\end{array}$ & $\begin{array}{r}\text { A4 } \\
* \\
4(\mathrm{~A} 2 \\
\mathrm{F} 7.5) \\
2 \mathrm{~A} 4,1 \mathrm{X}, \\
\mathrm{F} 7.5 \\
\mathrm{~A} 1,9 \mathrm{X}, \\
\mathrm{A} 1\end{array}$ \\
\hline 7 & PSICTळ & PCGCIN & $\begin{array}{l}1 \\
2 \\
3\end{array}$ & $\begin{array}{l}\text { NSL, NPS } \\
\text { PDEN } \\
\text { XPS(ISL), YPS(ISL), SPRANG(ISL) } \\
\text { Repeat previous line for a total of } \\
\text { NSL times }\end{array}$ & \\
\hline
\end{tabular}


TABLE 5-2 (continued)

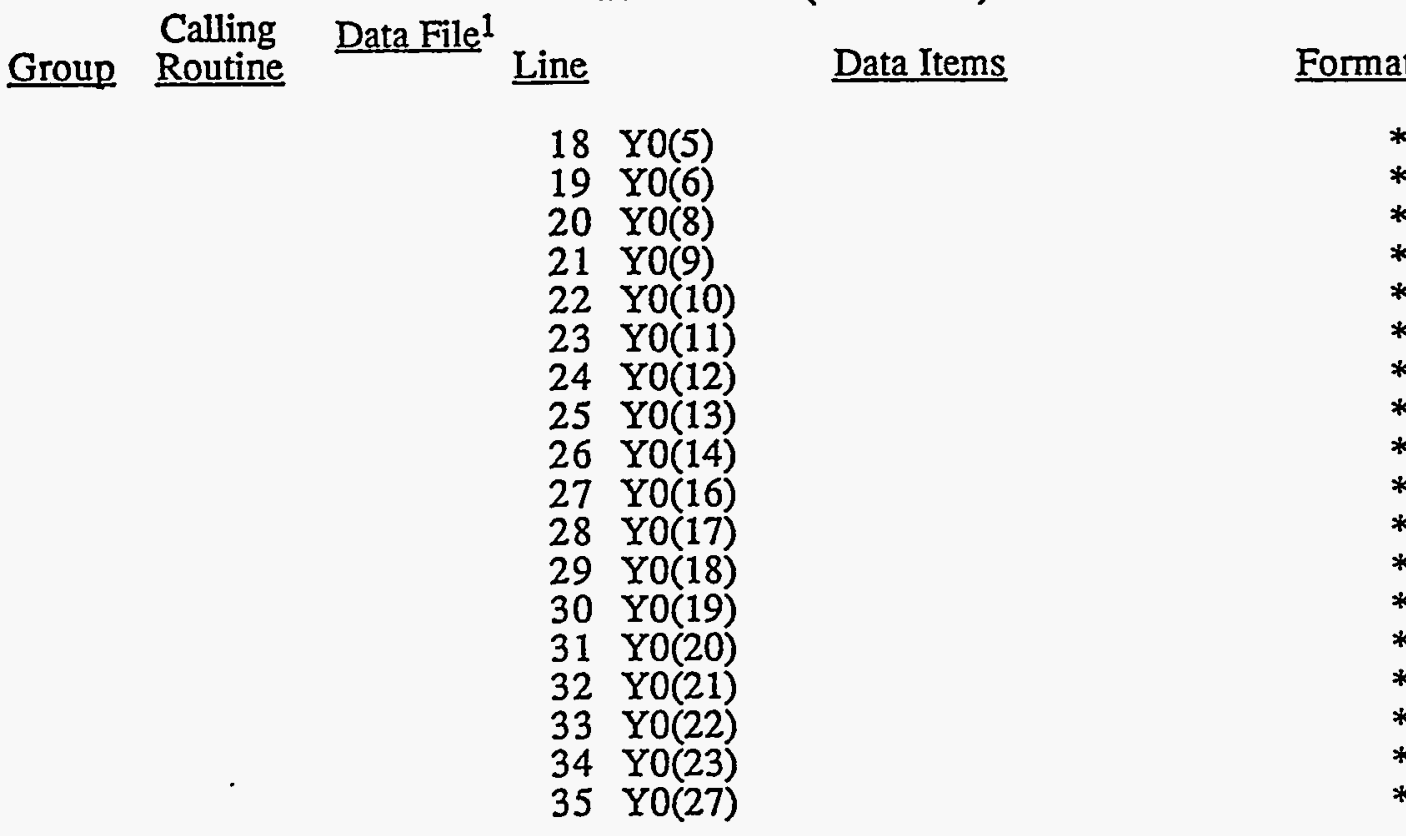

9 GETKIN PCGCIN 1 STRING (kinetics file name)

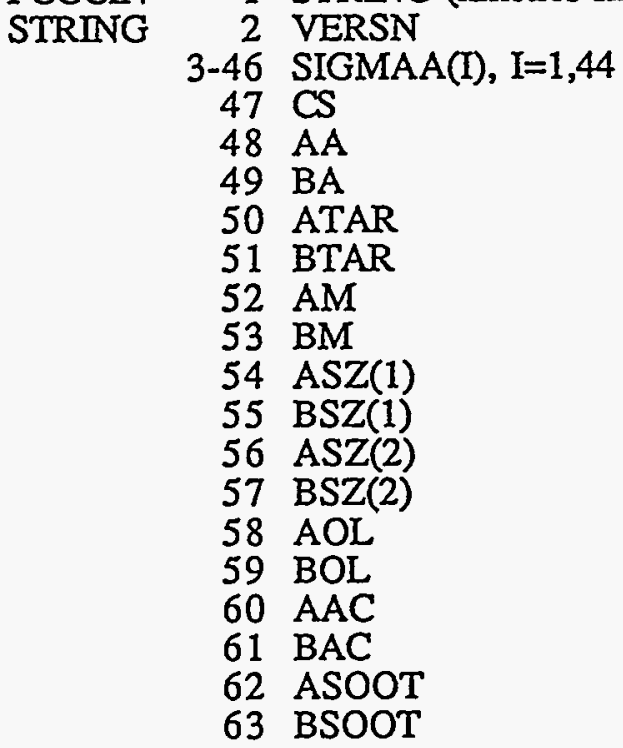

10 NETIN PCGCIN 1 STRING (polymer file name) STRING 2 VERSN 
Calling Data File ${ }^{1}$

Group Routine

TABLE 5-2 (continued)

Line Data Items

Format

3 OLIGST, MONO, FETHYL, FLINK, BEAD, XTO, CNST2, Q0, PHO

4 MMASS(1), MNUMB(1)

$\begin{array}{cl}\vdots & \vdots \\ \text { MONO+3 } & \text { MMASS(MONO) } \\ & \text { MNUMB(MONO) }\end{array}$

MONO+4 XEFF(J), $J=1,35$

MONO+5 RADIO, DENSTY, PRESS,

AMVSOL, AMWCOL,

VSRCOL, VSRMIN

MONO+6 MERS, DVG1, VISENG, DVG3, DVG4, VSTMAX, VISMAX, PHIMAX

MONO+7 MERMAS( $(J), J=1$, MERS

MONO +8 MASSLM(J), $\mathrm{J}=1,2$

MONO+10 LJUNK1

MONO+11 LJUNK2

MONO +12 S1, S2

MONO+13 SURFO

MONO+14 EPSO

11 NETIN mass_bin

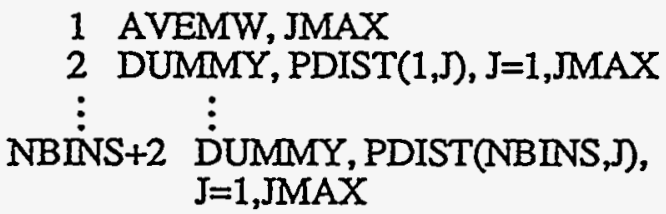

7F10.3

NBİS +2

$$
\mathrm{J}=1 \text {,JMAX }
$$

7F10.3

$12 \operatorname{COAL} \varnothing \quad$ PCGCIN

1 NCARD

*

2 COMENT(1,1), . . COMENT (1, 14) ...

$14 \mathrm{~A} 4$

$2 \operatorname{COMENT}(2,1), \ldots$,

COMENT $(2,14) \ldots$

2 COMENT(NCARD, 1$), \ldots$,

COMIMENT(NCARD, 14)

13 COAL $\varnothing$ PCGCIN 1 INCOAL

2 URFPM, URFPH

3 NCRXN, NHRXN, NPROP, KEQ, NSHRNK

4 DELTPI, DELRRJ, GAMMA 


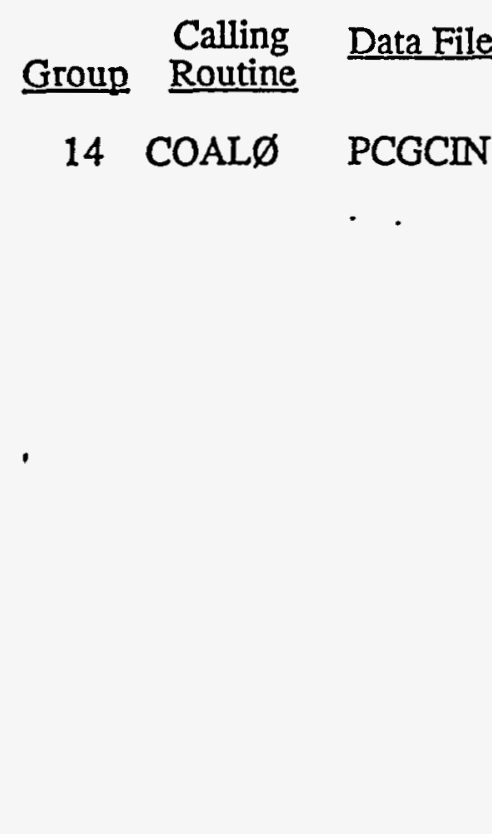

15 GETRCTO PCGCIN STRING

16 COALO PCGCIN

17 MAIN THERMO
TABLE 5-2 (continued)

Line

Data Items

Format

$1 \mathrm{XI}(1)$

2 QHC(1), HHO(1), HAO(1)

3 HWO(1), TNBP

4 OMEGAC(1), OMEGAH(1), OMEGAA(1)

5 OMEGAW(1)

$6 \operatorname{AMJ}(1,1), \operatorname{EMJ}(1,1), Y Y(1,1)$

7 HGV $(1,1)$

Repeat previous two lines for each raw coal reaction up to a total of NCRXN

$8 \operatorname{OXYD(1),~OXYD(2)\ldots .}$ OXYD(NHRXN)

9 PHIL (1), PHIL(2), . . PHIL(NHRXN)

$10 \operatorname{AL}(1,1), \operatorname{EL}(1,1), \operatorname{EMM}(1,1)$ Repeat the previous line for each char reaction up to a total of NHRXN

1 STRING (reactivity input file name)

2 SURFO, EPSO, TAUO, PSI, RADP, MINO

3 E1(JP,1), E2(JP,2), EM(JP,2)

$\vdots \vdots$

$N X N+2$ E1(JP,NXN), E2(JP,NXN), EM(JP,NXN)

NXN+3 THIELE

1 WIC(1,1), WIC $(1,2), \ldots$ WIC(1, NLM)

Repeat the previous line enough times to specify values for NLM elements, 3 on a line Group 11 parameters are used for all particles

$5 \mathrm{~A} 4$

*

*

A

*

:

*

$*$

$3 \mathrm{~A} 4$

$1 \operatorname{ASUB}(1, \mathrm{~J}), \mathrm{J}=1,3$

2 S(1), EK(1), DELTA(1),HFORM(1) Repeat the previous two lines for each species up to a total of NS 


\begin{tabular}{|c|c|c|c|c|c|}
\hline \multirow[t]{2}{*}{ Group } & $\begin{array}{l}\text { Calling } \\
\text { Routine }\end{array}$ & Data File $^{1}$ & $\underline{\text { Line }}$ & TABLE 5-2 (continued) & Format \\
\hline & & & $\begin{array}{r}7 \\
\\
8 \\
9 \\
10 \\
11 \\
12\end{array}$ & $\begin{array}{l}\text { UPLAG(IPS), IPS }=1, \text { NPS } \\
\text { Repeat previous line enough times to } \\
\text { specify values for NPS particle sizes, } \\
5 \text { on a line } \\
\text { TLAG(IPS), IPS }=1, \text { NPS } \\
\text { Repeat previous line enough times to } \\
\text { specify values for NPS particle sizes, } \\
5 \text { on a line } \\
\text { PD(IPS), IPS }=1, \text { NPS } \\
\text { Repeat previous line enough times to } \\
\text { specify values for NPS particle sizes, } \\
3 \text { on a line } \\
\text { PMF(IPS), IPS }=1 \text {, NPS } \\
\text { Repeat previous line enough times to } \\
\text { specify values for NPS particle sizes, } \\
5 \text { on a line } \\
\text { LPBUG, LYPS, LPARTP, LPARTS, } \\
\text { LPBOTH } \\
\text { LSPM, LSPU, LSPV, LSPH } \\
\text { YPSH, YPSL } \\
\text { MAXITP, MINITP, IGASV } \\
\text { PRK(IPS), IPS=1, NPS } \\
\text { Repeat previous line enough times to } \\
\text { Specify values for NPS particle sizes, } \\
5 \text { on a line }\end{array}$ & $\begin{array}{r}5 L 5 \\
5 L 5 \\
* \\
* \\
*\end{array}$ \\
\hline 8 & GETCDF & $\begin{array}{l}\text { PCGCIN } \\
\text { STRING }\end{array}$ & $\begin{array}{r}1 \\
2 \\
3-5 \\
6 \\
7 \\
8 \\
9 \\
10 \\
11 \\
12 \\
13 \\
14 \\
15 \\
16 \\
17\end{array}$ & $\begin{array}{l}\text { STRING (coal composition file } \\
\text { name) } \\
\text { TITLE } \\
\text { Lines not used } \\
\text { CA } \\
\text { CB } \\
\text { XCO0 } \\
\text { XHO0 } \\
\text { XO0 } \\
\text { XNO0 } \\
\text { XSO0 } \\
\text { XSMO } \\
\text { XOH } \\
\text { YO(1) } \\
\text { YO(2) } \\
\text { YO(3) }\end{array}$ & $\begin{array}{l}\text { A } \\
* \\
* \\
* \\
* \\
* \\
* \\
* \\
* \\
* \\
* \\
* \\
*\end{array}$ \\
\hline
\end{tabular}


TABLE 5-2 (continued)

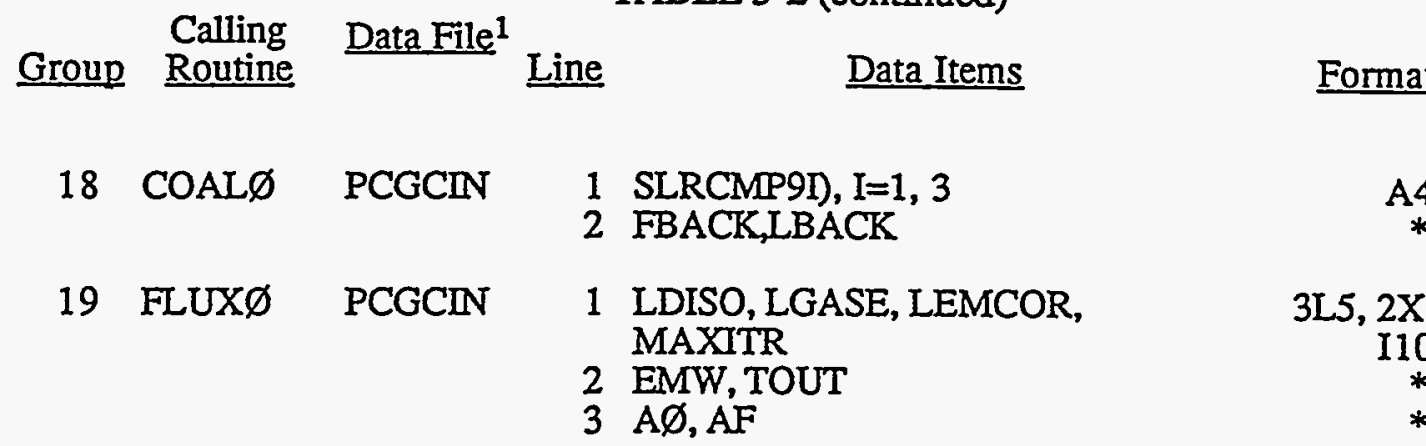

20 PROFIL INLET 1 UPDAT, KOPUP, USDAT, KOPUS 2(1X, L1,

1 UPD(N), KOPY(N), N=1, NINLET $3(1 X, 13)$ 2 VPDAT, KOPVP, VSDAT, KOPVS 2(1X, I1), IX, I3)

2 VPD(N), KOPV(N), N=1, NINLET $3(1 X, L 1$, 3 WPDAT, KOPWP, WSDAT, KOPWS 2(1X, L1,

3 WPD(N) KOPW(N) N=1, NINLET $3(1 \times, 13)$

1X, I3)

4 TIPDAT, KOPTEP, TISDAT, $\quad$ 2(1X, L1, KOPTES 1X, I3)

4 TIPD(N),KOPTE(N), N=1, NINLE $3(1 \mathrm{X}, \mathrm{L} 1$, EDSDAT,

5 EDPD(N), KOPED(N), N=1, $\quad 3(1 X, L 1$, NINLET $\quad$ IX, I3)

21 PROFIL INLET 1 PLS, SLS

2 NSFORM, ALPHAO

3 RP, RS1, RS2

4 UIN(1), VIN(1), WIN(1), TURBIN(1)

4 EDIN(1), RIN(1)

Repeat line 4 for entire profile defined by an arbitrary number of radial locations

22 NOXMN NOXIN 1 . NSAYNX

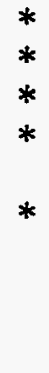


TABLE 5-2 (continued)

Calling Data File ${ }^{1}$

Line Data Items

Format

2 SAYNX(1), SAYNX(2),...

$18 \mathrm{~A} 4$

SAYNX(18)

- $\bullet$

NSAYNX+1 SAYNX(1), SAYNX(2), ... SAYNX(18)

$18 \mathrm{~A} 4$

23 NOXMN NOXIN

1 FUELNO

2 THRMNO

3 RADOXY, EQTEST, RADOH, OHADJS

4 CHARNO

5 PRNOX,PRHCN, PRNH3, FCTNO, FCTHCN,FCTNH3

6 MXITNX, ITYNX, INDPNX, ICALCN

7 XIANOX, ZEDA, FN2PRT, MAXRES

8 IRSTNX, IPLTNX, INHTNX

3L5

24 SORBO SOXIN

1 NSAYSX

2 SAYSX(1), SAYSX(2),... SAYSX(18)

$\begin{array}{clll}\vdots & \vdots & \\ \text { NSAYYXX+1 } & \text { SAYSX(1), SAYSX(2), ... } & \text { 18A4 }\end{array}$

$18 \mathrm{~A} 4$

$$
\text { SAYSX(18) }
$$

25 SORBO SOXIN

1 NSTYPE

2 NSLS, NPSS

3 SPDEN

4 BETSUR

5 XPS(ISL), YPS(ISL), SPRANG(ISL) Repeat the above line for each sorbent particle starting location.

6 PDS(IPS), IPS $=1,3$

Repeat the above line enough times to specify particle diameter for all sorbent particle sizes, 3 on a line.

7 PMFS(IPS), IPS=1,5

Repeat the above line enough times to specify mass fractions for all particle sizes, 5 on a line. 
Group Routine Data File $^{1}$ Line

TABLE 5-2 (continued)

TABLE 5-2 (continued)
8 LSPBUG, LSYPS, LPARTP, LPARTS, LPBOTH

Data Items

Format

9 LRBNDS

10 YPSH, YPSL

11 PRKS(IPS), IPS=1,5

Repeat the above line enough times

to specify values for all particle sizes,

5 on a line.

12 PRSO2

13 MXITSX, MAXRES

14 URFSO2
5L5

$5 \mathrm{LS}$

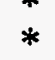

$*$

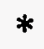

*

1Indicates data file where information is found.
2If case uses restart file for particles (INPRST = TRUE), it is read in prior to reading
data group 6.

Group 7 gives information about the particles, including the number of particle starting locations (up to NUMSTR), number of particle sizes (up to NUMPAR), particle density, starting location of the trajectories, initial particle velocity and temperature, initial particle size distribution, particle Prandtl number, and information controlling the particle phase calculations, such as maximum number of particle iterations.

Groups 8 through 18 give information about the solids composition and reactions. Groups $8,9,10$, and 11 contain input data for the FG-DVC submodel and are described in more detail in Chapter 6 . Group 12 contains any number of comment cards which are reprinted in the output file. Group 13 specifies the number of reactions and numerical control parameters. Group 14 gives kinetic parameters for each of the devolatilization and char oxidation reactions (if FGDVC $=F$ ), as well as the oxidizing species and stoichiometry. Group is contains input data for the reactivity submodel that goes along with the FG-DVC devolatilization submodel. These data are explained in more detail in Chapter 6. Group 16 gives the elemental analysis of the "raw coal" portion of the particles. Group 17 gives the Lennard-Jones parameters for predicting the transport properties of the gas species. Group 18 specifies the slurry component and fraction of enthalpy from volatiles combustion that should be fed back directly to the particle.

Group 19 gives information for the radiation submodel, including the method of solving the governing equations (Varma six-flux or discrete ordinates), 
whether to include gas emissivities sand corrections, and maximum number of iterations. Absorption and scattering cross-sections are calculated by the model, based on particle size.

Groups 20 and 21 contain data for calculating (theoretical) or reading (experimental) profile data for velocity, turbulence intensity, and eddy dissipation for the primary and secondary inlets. These data are contained in the inlet profile data file.

Groups 22 and 23 contain input data for the $\mathrm{NO}_{x}$ pollutant submodel. Group 22 contains comment statements. Group 23 contains the kinetic, numerical, and program control parameters.

Groups 24 and 25 contain input data for the sorbent reactions submodel.

As explained above, several optional data files may be included as input to PCGC-2. These include: 1) a file containing information which describes the grid geometry (assigned logical name GRIDS in the FORTRAN subroutine GRMAP), 2) files used as initial guesses based on previous calculations for the gas phase (assigned logical name INOUT in the FORTRAN subroutine RESTRT) and for the particle phase (assigned logical name PARSOU in the FORTRAN program PCGC2), and 3) a file consisting of the equilibrium table (assigned logical name TBLRST in the FORTRAN subroutines TBLRSTFE and TBLRSTFEH) used in calculating gas properties (temperature, density, etc.) as functions of the progress variables $f, h$, and $h r .^{*}$ Each of the data files are accessed in VAX/VMS FORTRAN using an OPEN statement. Some use the IOSTAT qualifier to insure proper operation. IOSTAT is assumed equal to 0 if the open command is successful.

* An option is provided (INCAIH $=F$ ) for assuming a locally adiabatic reactor, in which case $h$ is not independent of $f$ and $\eta$.

\section{Program Output}

Program output is contained in the main output (PCGCOT) and log (for batch runs) files. The quantity of output in the main output file is controlled by the relative values of INDPRI and MAXIT ash shown in Table 5-3. Three options are available: Output can be a) turned completely off, b) restricted to the beginning and end of the calculation, or c) printed periodically during program execution. Option (a) is used most commonly, since the output file can get rather large and cumbersome otherwise, and graphics are usually used to examine code results. 
Table 5-4 presents an outline of the output file produced during a PCGC-2 computation. All of the input parameters (i.e. thermodynamic properties, flowrates, particle size information, coal reaction rate information, and radiation information) and many parameters calculated from them are written to the output file early during execution (Option $b$ and $c$ ). Also, the initial values for each scalar field (i.e. gas temperature, velocities, pressure, etc.) used for initialization are written to the output file. During the iteration process, values of each critical field variable are printed for each node point in the computational grid (Options c). The y (or r) values of the grid are found at the right-hand side of the array, and the axial location $(x)$ is found along the bottom of the grid printout. Since the sample grid is too long to be printed in one block, it is "wrapped around" in three segments. The index values for the node point $(I$ and $J$ are also printed out. Other gas properties are printed only upon convergence or after other normal termination (Options b and c).

The particle trajectory information is printed out each 10 trajectory time steps as the calculations proceed. Among other things, the output shows the location of the particle (XP and YP), the time step of integration (DT), and the elapsed residence time along the particle trajectory (TIME), the particle and gas temperatures (TMP and TG), the mass of liquid, raw coal, char, ash, and total mass of the particle normalized by the original particle mass (WFRAC, CFRAC, HFRAC, AFRAC, TFRAC, respectively). This information is given for each of the particle trajectories (ISL = initial starting location, and IPS = initial particle size).

In addition to the main output file, several other files contain program results. These are summarized in Table 5-5. Standard FORTRAN 77 commands were used, where possible, to manipulate these files.

TABLE 5-3

\section{PROGRAM OUTPUT CONTROL}

INDPRI
a) $<0$ or $>$ MAXIT
b) $=$ MAXIT
c) $>0$ and $<$ MAXIT

Output

None

Input summary, initial values of field variables, final values of field variable. Same as b) plus intermediate output of field variables every INDPRI iterations. 
TABLE 5-4

I. INPUT DATA FOR COMPUTATION

a) Comment lines describing calculation

b) Elements considered

c) Thermodynamic data required to predict equilibrium properties

d) Inlet stream information

e) Particle property information

f) Particle reaction information

g) Radiation information

h) Reactor parameters

i) Computational grid information

j) Initial scalar fields (i.e. velocity, mixture fraction, etc.)

II. GAS PHIASE ITERATION INFORMATION (see note below)

a) Normalized residual source sums

III. PARTICLE ITERATION INFORMATION

a) Time/position information for Lagrangian particle trajectories

b) Axial mixing-cup coal burnout

IV. FINAL OUTPUT

a) All scalar fields

b) Summary of CPU time requirements

Note: Residual terms are printed for either INDRST iterations or until gas phase convergence is obtained. On gas phase convergence, the field variables are printed and the computation proceeds in the particle phase until convergence. After each Lagrangian particle calculation is completed, computation returns to the gas phase at which time the field variables are computed with the updated particle source terms. If the difference between and the most recent calculated field variables and those calculated at the end of the last gas phase iteration is less than SORMIN the calculation is complete. Otherwise, computation continues in the gas phase after gas phase. If the gas phase converges in fewer than MINITP iterations, the calculation is considered complete. Otherwise, computation returns to the particle phase after gas phase convergence, and the process is repeated. On overall convergence, the field variables are written to the output file along with a summary of the time required. 
TABLE 5-5

\section{PCGC-2 OUTPUT FILES}

\begin{tabular}{|c|c|c|}
\hline Filename & Type & Description \\
\hline GASPLT & Text & Eulerian gas properties and particle density for plotting \\
\hline INOUT & Binary & Eulerian gas properties for plotting \\
\hline output.fg & Text & FGDVC submodel results \\
\hline PARSOU & Binary & Particle source terms for restart (INPRST $=\mathrm{T}$ ) \\
\hline PCGCOT & Text & Main output file \\
\hline POLPLT & Binary & Pollutant data for plotting \\
\hline PPLOT1 & Text & $\begin{array}{l}\text { Number of records for each trajectory in PPLOT2 and } \\
\text { pointers to trajectories in PPLOT3 }\end{array}$ \\
\hline PPLOT2 & Text & $\begin{array}{l}\text { Particle coordinate residence time, and time increment } \\
\text { for plotting. Also contains radially integrated burnout } \\
\text { and centerline mixture fraction data }\end{array}$ \\
\hline PPLOT3 & Text & $\begin{array}{l}\text { Particle coordinates, composition, temperature, and gas } \\
\text { temperature for plotting }\end{array}$ \\
\hline PRGRSS & Text & $\begin{array}{l}\text { Residual source sums and gas properties at a selected } \\
\text { point in the reactor for monitoring progress toward } \\
\text { convergence }\end{array}$ \\
\hline $\begin{array}{l}\text { PSORB1, } \\
\text { PSORB2, } \\
\text { PSORB3 }\end{array}$ & Text & $\begin{array}{l}\text { Same as PPLOT1, PPLOT2, PPLOT3, except for } \\
\text { sorbent particles }\end{array}$ \\
\hline RSNXIO & Binary & $\mathrm{NO}_{\mathrm{x}}$ pollutant data for restart $(\mathrm{RSTNX}=\mathrm{T})$ \\
\hline SORDN1 & Text & Restart file for sorbent particle number density \\
\hline TBLRST & Binary & Gas property data \\
\hline
\end{tabular}


TABLE 5-5 (continued)

Filename Type Description

TWODDBV Binary Gas plotting file for CEQUEL post-processor

YH2S1 Text Restart file for $\mathrm{H}_{2} \mathrm{~S}$ concentration with sorbents

YSO21 Text Restart file for $\mathrm{SO}_{2}$ concentration with sorbents

\section{Code Operation}

PCGC-2 is a "user-specialist" code that requires familiarity with the theory and structure of the code in order to be used correctly and efficiently. This section gives several hints for new users. A thorough understanding of the problem physics and numerical assumptions is essential.

\section{New Cases}

When setting up a new problem for simulation, it is easiest to start by editing input files from a previous, similar, simulation, if one is available. If not, the pre-processor can be used as explained later in this chapter.

\section{Restart}

Unless the user has a converged or partially converged solution of a similar problem with identical geometry, he will probably want to start his problem from "scratch". To do so, the logical variables that will cause the code to try to "restart" from a guessed solution (INRST, INPRST, and IRSTNX) must be set equal to F in the main input file. This will not prevent the code from writing restart files for future restarts, since writing to restart files is automatic at a frequency specified by the variable INDRST. A value of 50 is recommended. Subsequent runs for the same case can be restarted from a previously converged solution by setting the above logical variables equal to $\mathrm{T}$ in the input file. The restart feature is useful 
when small changes are made for the same case and geometry. If the changes are large or the geometry changes, the restart option should no be used.

\section{Particle and Gas Iterations}

The maximum number of particle iterations is controlled by the parameter MAXITP. It typically takes 5-15 particle iterations to converge a PCGC-2 calculation from scratch. The maximum number of gas iterations is controlled by the parameter MAXIT. A typical value of MAXIT is 1000 .

\section{Gas Convergence}

The code has the capability of completely converging the gas phase for every calculation of the particle phase, which is the normal approach, or of calculating the particle phase every 5 iterations of the gas phase. The latter approach is used when INEACH is set to $T$. and may be useful in cases where the gas and particles are highly coupled. In all cases, convergence of the gas phase is controlled by the value of SORMAX. Overall convergence is controlled by SORMIN.

An efficient approach to obtaining a solution is as follows. Obtain an approximate solution by selecting large values for SORMAX and SORMIN, 1.0 and 2.5 respectively, for example. Systematically reduce both parameters, converging the case between each change. For example, after a case is converged with $S O R M A X=1.0$ and SORMIN $=2.5$, change the parameters to 0.1 and 0.5 , respectively. Then change them to 0.01 and 0.10 , respectively. This is usually sufficient for most cases. If detailed analysis of trace species is desired, both may be reduced further. It is usually desirable to solve the $\mathrm{NO}_{\mathrm{x}}$ and sorbent reactions submodels only after the main code is converged to the desired level.

\section{Gaseous Combustion (No Particles)}

For gaseous combustion (no particles), the following control variables should be turned off (set equal to F): INCLET, INCLGE, IPSICT, INPRST and INCALN. This will prevent the calculation of the coal gas mixture fraction and its variance, particle trajectories, and particle Eulerian density. In addition, the input data for the particle phase (data groups 7-13) should be removed. 
User's Guide

\section{Coal Particle Combustion}

In most combustion simulations, both the primary and secondary gas streams are composed of air and are therefore identical in composition. If the gas streams were also identical in temperature, it would be unnecessary to calculate the gas mixture fraction (controlled by INCALF) or its variance (INCALG). However, the secondary stream is usually preheated, and in this case is may be important to calculate the gas mixture fraction. However, the turbulent fluctuations can often be ignored, even though there is a difference in temperature. Of course, the coal-gas mixture fraction (controlled by INCLET) and its variance (controlled by INCLGE) must always be calculated when reacting coal particles are present.

\section{Energy Equation}

If a locally adiabatic solution is desired, the calculation of enthalpy can be turned off with the logical INCALH. Otherwise, the energy equation is explicitly solved subject to the given temperature boundary conditions (TBN, TBW, and TBE).

If the energy equation is to be solved, upper (TMAX) and lower (TMMN) bounds for temperature must be given. These bounds are for the equilibrium table for gas properties. It is recommended that the table debug flag (LTBUG) be turned on when generating a new table. This will stop execution after table generation so that the results of the table generation calculations can be reviewed before the actual simulation begins. If failures in the equilibrium algorithm occur during table generation, messages will appear on the screen and in the output file (if one is being generated).

TMIN should be set equal to the lowest expected temperature in the simulation. This is usually the temperature of the coldest inlet stream. TMAX should be set equal to the highest expected temperature. This is usually $2500 \mathrm{~K}$ for combustion and $3500 \mathrm{~K}$ for oxygen-blown gasification. Sometimes, wider limits may be required. If so, the code will print a message to that effect during execution. It is desirable to keep the limits as narrow as possible in order to confine the table to the region of interest and maximize the accuracy of the table look-up and interpolation procedure. In order to further narrow the table limits and improve accuracy, two other parameters are required. TLODEL and THIDEL specify the maximum variation in temperature on the low side and on the high side compared with the adiabatic temperature at a given stoichiometry. IF TLODEL and THIDEL are large, TMMN and TMAX will always be limiting. If TLODEL and THWDEL are not large, they may be used to confine the region of the table closer to the adiabatic temperature. Again, if the code requires properties at temperatures outside the table 
limits, it will print a message to that effect on the screen and tell the user which parameter to adjust and suggest a value to adjust it to.

Whenever table values are needed by the program outside the range of the table, the residual enthalpy is reset to the table limiting value for the particular values of $f$ and $\eta$, and a counter (NCREE) is incremented. NCREE is printed in the $\log$ and output files, and therefore represents the number of times (for a given gasphase iteration) the residual enthalpy was outside the table limits. For an unconstrained solution, NCREE should be equal to zero at the solution. The narrower the table limits, the fewer the number of table failures, but the larger the value of NCREE.

After successfully generating the table, LTBUG should be turned off to allow the combustion calculation to proceed using the table that was generated previously. A new table will then be generated only when a variable that affects the table generation is altered. If for some reason the user desires to regenerate the table, even though no variable affecting the table generation has been altered, he must delete the file containing the previous table (Runid.TBL or TBLRST).

There is a high degree of energy coupling between the particle and gas phases, and it may be necessary to reduce the under-relaxation factor for the particle source term for enthalpy (URFPH). A value of 0.5 is typically used, but values as low as 0.1 may be required. As the solution is approached, it should generally be possible to slowly increase URFPH.

\section{Radiation}

In the 1987 version of PCGC-2, the discrete ordinates method was first included as an option in the radiation submodel. This option can be chosen by setting the logical variable LDISO equal to true in the main data file. Gas-phase radiation is included if LGASE is set to true; otherwise only radiation due to the particulates (excluding soot) is considered. The gas phase radiation is calculated in two ways: if LEMCOR is set to true, the calculation includes the corrections in gas emissivities due to species partial pressures and spectral overlap of $\mathrm{CO}_{2}$ an $\mathrm{H}_{2} \mathrm{O}$ emissivities, using the Hottel chars (Hottel and Sarofim, 1967); otherwise, the overall emissivity of the gas is calculated using the Hadvig (1970) plot. 'QAB' and 'QSC' are the absorption and scattering efficiencies of the particulates based on Mie theory. These are calculated internally by the code, as a function of particle diameter.

The scattering phase function was changed to linearly anisotropic in the 1987 version of the code because it can be adjusted to account for either isotropic, or strongly forward- or backward-directed scatter. Since scatter by coal/char particles is known to be strongly forward-directed, the recommended value for $a_{0}$, 
the asymmetry factor, is 1.0. The phase function used in earlier versions of PCGC-2 was that for ash particles (Hottel and Sarofim, 1967), which represented strongly backward-directed scatter.

Finally, the user can choose a value for the differencing factor, $f$, in extrapolating intensities across volume elements. This parameter is contained in the main data file. If a value of 1.0 (i.e., central differencing scheme) is chosen, and negative intensities are encountered, the radiation submodel will automatically adjust (reduce) the value of $f$ until the negative intensities are all removed. This is expected to result in slightly more accurate predictions of the intensity field as compared with upwind differencing (i.e., $f=0.0$ ), but requires more computer time. It is therefore recommended that upwind differencing $(\mathrm{f}=0.0)$ be used.

\section{Numerical Algorithm}

Two choices of numerical algorithm for solving the gas phase are provided: SIMPLE and SIMPLER. In addition, either method may be used with the SIMPLEC approximation. SIMPLER (selected by INSIMP equals $T$ ) with SIMPLEC (INSMPC $=\mathrm{T}$ ) is recommended in all cases because it is more efficient. The use of SIMPLE requires significantly lower under-relaxation parameters, as explained below.

\section{Under-relaxation}

When using SIMPLER, values of 0.7-0.9 are recommended for all underrelaxation factors for the independent gas field variables except URFPP and URFP (which should be 1.0) and URFDEN (which should be 0.5-0.7). When using SIMPLE, all factors should be substantially lower (0.3-0.6). A value of 0.9 is recommended for URFH for both algorithms. Of course, these factors are problem-dependent, and the convergence rate can be increased by increasing the under-relaxation factor of variables that seem to be controlling the convergence rate, as determined by monitoring the terminal-directed output from the code. The most efficient mode of operation is for each under-relaxation parameter to be as high as possible without causing the program to oscillate or diverge. However, optimizing the under-relaxation parameters is a difficult process and often takes more time than converging the code with non-optimum values. In addition to being problemdependent, optimum values also change with level of convergence for a single problem, further complicating any optimum procedure. If desired, under-relaxation factors can be altered interactively every 25 iterations by setting INCURF equal to T. 
A value of $0.5-1.0$ is recommended for the particle phase under-relaxation (URFPM). This parameter is applied to all particle source terms except enthalpy. The latter uses URFPH, which often requires a much lower value (e.g. 0.1) to converge.

\section{Maximum Number of Iterations and Generation of Output}

A value of 1000 is recommended for the maximum number of iterations for the gas phase (MAXIT). The frequency of intermediate output is determined by INDPRI. A value of zero is recommended unless printed output is desired.

\section{Grid Generation}

The maximum number of nodes for both the axial and radial directions is determined by the values of NUMXPT and NUMYPT in the file PARAMETER.INC. Enough node points should be used to achieve gridindependence. In most cases, it is wise to use at least $40^{\circ}$ node points in each direction. Many cases may require more node points.

Only the number of axial node points is specified directly in the input. This is done by the variables NIINQ (number of nodes inside the quarl region) and NIWOQ (number of nodes outside the quarl). The sum of these two variables is the total number of axial nodes (must be less than or equal to NUMXPT). The spacing of the nodes is determined by AL1, EPSX, and NL.

The number of radial nodes is specified indirectly by NJINP, NJINS, EPSI, EPSD, and INNOZ. The grid generation algorithm uses these parameters to generate a grid for specified reactor dimensions that spaces the grid points closely near the reactor centerline and near the walls, where gradients are expected to be large, and less closely in regions where the gradients are expected to be small. Trial and error is often required to generate an acceptable grid for new geometry.

The program also allows the user to specify the grid locations directly. In this case, another input file for the grid must be created, and the variable INRDGD in the input must be set equal to $\mathrm{T}$. The recommended approach is to let PCGC-2 generate a grid file (GRDOUT equal to $T$ and INRDGD equal to $F$ ) and then edit the resulting grid file that is generated. INRDGD can then be set equal to T.

Either grid point or cell face locations can be specified. If specifying grid point locations, the key word at the beginning of the GRIDS (.grd) file should be "NODE". If specifying cell face locations, the key word should be "FACE". It is easier to specify node locations, since faces are located midway between nodes. However, it is easier to model complicated geometry by specifying face locations. If the grid spacing is too non-uniform when specifying face locations, it may be 
impossible to calculate the node locations such that the faces are located midway between adjacent nodes, unless the nodes are located outside of the cells. This condition is unacceptable. If it occurs, the cell face locations must be altered to achieve a more uniform grid, or more cells must be added.

The grid in the radial direction is configured based on either the secondary duct diameter (INNOZ = F) or both the primary and secondary duct diameters (INNOZ = T). The latter method was designed for modeling nozzles in slurry combustion. For dry (non-slurry) coal combustion, INNOZ is ordinarily set equal to false. In this case, NJINS represents the total number of nodes desired in the secondary duct and in the tube wall. The nodes in the secondary are all equally spaced. The equal node spacing in the secondary duct is also extended through the tube wall toward the primary duct. In the primary duct, the node spacing is decreased toward the centerline by the factor EPSD. It is possible, depending on the primary and secondary duct diameters and the values of NJINS and EPSD, to specify a condition where an infinite number of nodes would be required to reach the centerline. In this case, an error message will be printed in the log and output files. The remedy is to decrease NJINS, increase EPSD, or both. Alternatively, the nozzle configuring option (described below) may provide an acceptable grid in some cases (e.g. reactors with a large diameter relative to the primary stream).

To use the nozzle configuring option, INNOZ should be set equal to true. The grid will then be configured based on both NJINS and NJINP, the number of nodes specified in the secondary and primary ducts, respectively. In this case, the nodes are equally spaced in both the primary and secondary ducts, and the node spacing is incremented in the tube wall. This option puts the most closely spaced nodes in the primary duct.

\section{Intrusions}

The program can accommodate intrusions within the constraint of axisymmetric geometry. An intrusion is a boundary cell in the interior of the flowfield. To define intrusions, the grid structure must be read from an input file. Therefore, the easiest approach is to first generate a grid input file as described above, and then add intrusions at the appropriate locations by changing O's to X's. The logical variables INTRUS and INRDGD must both be equal to $T$ to use this option.

\section{Additional Inlets}

Additional inlets are used in a manner similar to intrusions by altering a previously generated grid file. Up to three additional inlets may be specified by 
changing X's to 1 's, 2 's or 3's at the appropriate boundary locations on the north or west walls. The flowrate, composition (in terms of the reactant streams), swirl number, and turbulence intensity must be specified in the input file for each additional inlet. In addition to modifying the grid file to show he location of the additional inlets, the inlet profile data file must be modified to give information for the velocity and turbulence properties profiles for the additional inlets. Currently, a flat profile is the only option for additional inlets.

\section{Overall Code Convergence}

Overall convergence of the code is assumed when the particle source terms do not change significantly between two consecutive particle iterations. Two criteria are used to determine this condition quantitatively, and these criteria are mutually independent. Satisfying either criterion causes the program to terminate with the assumption of overall convergence.

The convergence criteria are specified by two input parameters: SORMIN and MMNITP. If the maximum residual source sum is less than SORMIN (typically 0.1 ) on the first iteration, then overall convergence is assumed. This is the first criterion. Since the gas phase is converged to the value of SORMAX (typically 0.01 ) each time, this means that if the maximum residual source sum increases from 0.01 to no more than 0.1 following a particle iteration, overall convergence is assumed. Overall convergence is also assumed if the gas phase can be reconverged to the value of SORMIN in less than MINITP (typically 25) iterations, regardless of the value of the maximum residual source sum on the first iteration after a particle iteration. This is the second, or alternative, convergence criterion. It is recommended that users attempt to converge their cases according to the first criterion because it puts a quantitative limit on the degree of mismatch between the particle and gas solutions.

The second criterion is intended primarily as a safeguard in case the user specifies values of SORMIN and SORMAX that make it impossible to satisfy the first criterion. For example, if the gas phase is not converged tightly enough (SORMIN too large), it may be impossible to satisfy the SORMIN criterion due to variations in the gas phase solution that feed to the particle phase and then back to the gas phase. This situation is usually typified by a solution that reaches a steady state and stops improving. The gas phase converges readily each time, but the maximum residual source sum on the first gas iteration after a particle iteration stops improving (decreasing). Tightening up (decreasing) the value of SORMAX causes the gas phase to be converged more tightly and may result in being able to satisfy the SORMIN criterion for overall convergence. This adjustment has been found to be particularly useful in cases without swirl. For example, if the swirl in the coal 
User's Guide

combustion case in Appendix $G$ is turned off, the case will not converge without tightening up the value of SORMAX from 0.01 to 0.005 .

\section{Particle Reaction Rate Parameters}

The particle reaction rate parameters (AMJ, EMJ, YY, ALJ, EL, EMM, etc.) are coal-specific. Also, the code predictions are quite sensitive to these parameters, and they need to be chosen carefully, based on experimental data for the coal-type and conditions of interest (See Chapter 2).

\section{$\mathrm{NO}_{\mathrm{x}} /$ Sorbent Reactions Submodel}

The $\mathrm{NO}_{\mathrm{x}}$ and sorbent reactions submodels are decoupled from the main solution and can be executed separately, based on a previous solution of the main code. This is accomplished by setting POILUT equal to $\mathrm{T}$. The $\mathrm{NO}_{\mathrm{x}}$ submodel is executed when INCNOX is set equal to .TRUE. The sorbent reaction submodel is executed when INSORB is set equal to .TRUE .

It was found that a value for MAXRES $\leq 0.001$ was sufficient to obtain a converged solution for a natural gas flame simulated with 1600 nodes. If less nodes are used, MAXRES should be set lower since the error is summed for all nodes. In determining convergence for PCGC-2, the analogous convergence criteria for all gas phase equations (e.g. pressure, enthalpy, and mixture fractions) is typically set equal to 0.01 . This value is also suggested as a minimum for MAXRES for any natural gas or coal combustion simulation.

Achieving converged NO model solutions is often not trivial and requires appropriate selection of under-relaxation factors. Some difficulties may be encountered in obtaining converged solutions when either the empirical kinetics of Mitchell and Tarbell (1982) or the global rate expressions of Wendt and coworkers (Bose et al., 1988) are used. This difficulty is attributed to the coupling between chemical reactions. In order to dampen oscillations in the solutions from iteration to iteration, several combinations of under-relaxation factors may be required. This dampening can best be accomplished by running the code in an interactive debugger to allow manipulation of the under-relaxation factors. Despite this level of user-interaction, some cases may still be difficult to converge. In order to improve convergence, the parameter ICALCN can be used to dampen coupled interactions between the $\mathrm{HCN}$ and $\mathrm{NH}_{3}$ reactions. With ICALCN assigned a value of 10 , both the $\mathrm{NH}_{3}$ and $\mathrm{NO}$ species continuity equations are often effectively converged while at the same time slowly progressing the convergence of the $\mathrm{HCN}$ continuity equation. 


\section{Chemical Species}

The chemical species considered in the equilibrium calculations are governed by the input in the THERMO file. Data for additional species not being considered can be stored by moving them to the end of the file (after the LeonardJones parameters).

\section{Coal-Water Mixtures}

Simulation of coal-water slurries is limited to cases where all inlet gas streams have the same composition and temperature. The gas mixture fraction variable is then used to track the evaporating slurry liquid from the droplets rather than the inlet gas mixing. In such cases, INFSOU should be set equal to $T$ to include a source term in the $f$ equation. The variable OMEGAW is used to specify the initial liquid content of the slurry. For ordinary combustion of pulverized coal, OMEGAW is set equal to zero, and the inherent moisture of the coal is incorporated directly in the input composition and flowrate of the primary gas.

The variable INNOZZ allows specification of the primary nodes for grid generation rather than the secondary nodes. This option is useful when primary diameters are small, such as when slurry nozzles are being modeled. The option can also be used to generate a grid for dry coal combustion, however, if the diameter of the reactor is large compared with the primary diameter.

\section{Graphics}

\section{Pre-processor}

The pre-processor generates input files for PCGC-2. It is a separately licensable product available from ACERC as described in Chapter 1. It is based on the CQUEL program developed by the Engineering Computer Graphics Laboratory at BYU. Its purpose is to allow the experienced or inexperienced user of PCGC-2 to quickly set up the input files for a new case from scratch without having to understand all of the details about the input data, formatting, etc. The program is designed to be used with $\mathrm{X}$-windows terminals or workstations.

When the program is first started, a menu bar appears at the top of the screen as shown in Fig. 5-2. A help window is provided near the bottom of the screen (It is shown near the top in Fig. 5-2 for illustrative purposes). 


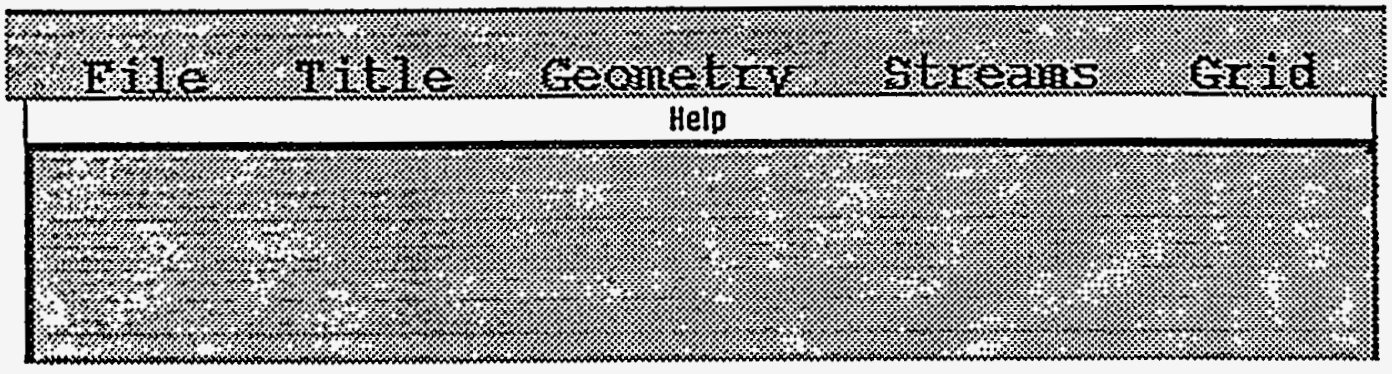

Fig. 5-2. Menu bar and help window.

Step 1: Load data files. The user first clicks on the word "File" in the main menu and selects either "New" or "Read" as shown in Fig. 5-3. "New" reads in default values from a set of files that the user can customize to his/her own particular needs. A standard set of default files are provided with the program. "Read" brings up a window where the user can type in the specific location and name of the files he/she wants to read into the pre-processor as shown in Fig. 5-4. Thus it is possible for the user to create a new case based on a previous case.

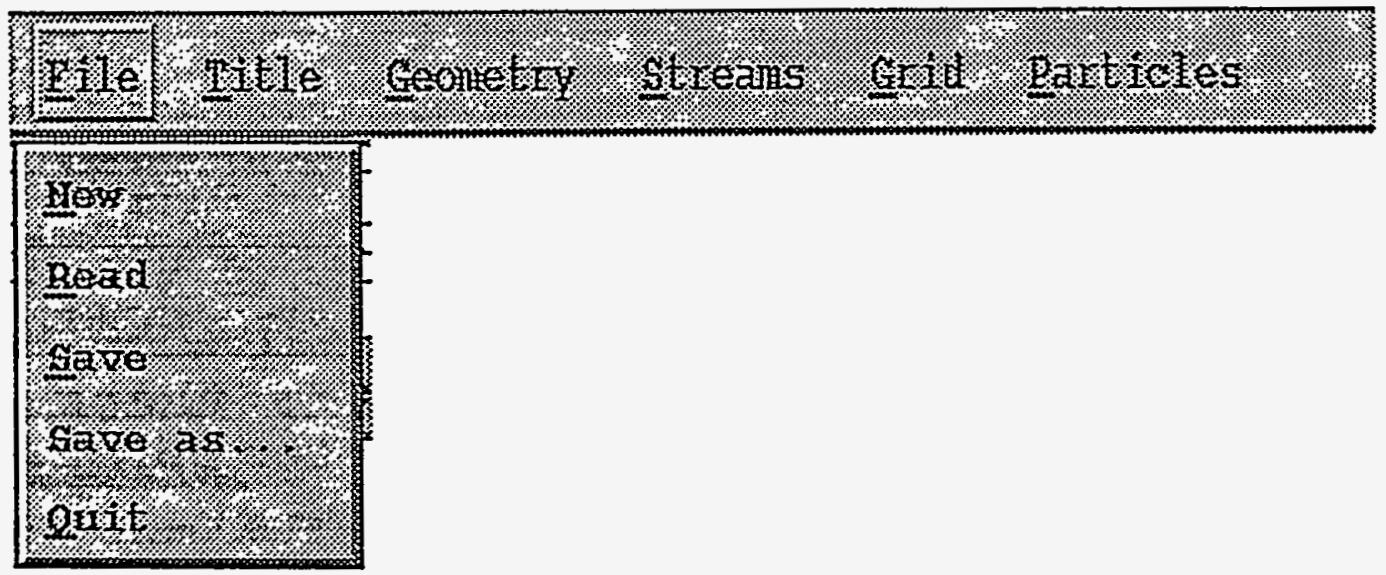


Fig. 5-3. Pull-down menu for reading and saving input files.

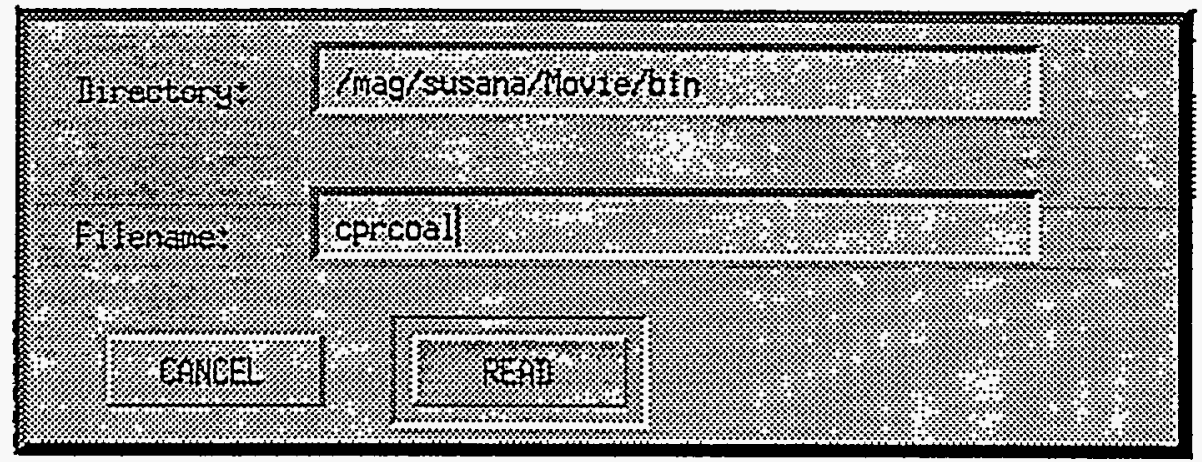

Fig. 5-4. Window for specifying filename for reading input files.

Step 2: Edit the title. The user then clicks on the word "Title" in the main menu and brings up the title window shown in Fig. 5-5. The title which appears is the one that was read in during Step 1. This can now be edited using standard mouse and keyboard entry techniques. After entering the title, the user clicks on the "SAVE" button to save his/her changes. Clicking on the "RESET" button changes the title back to what was originally read, and clicking on the "CANCEL" button gets rid of the window without saving any changes. 


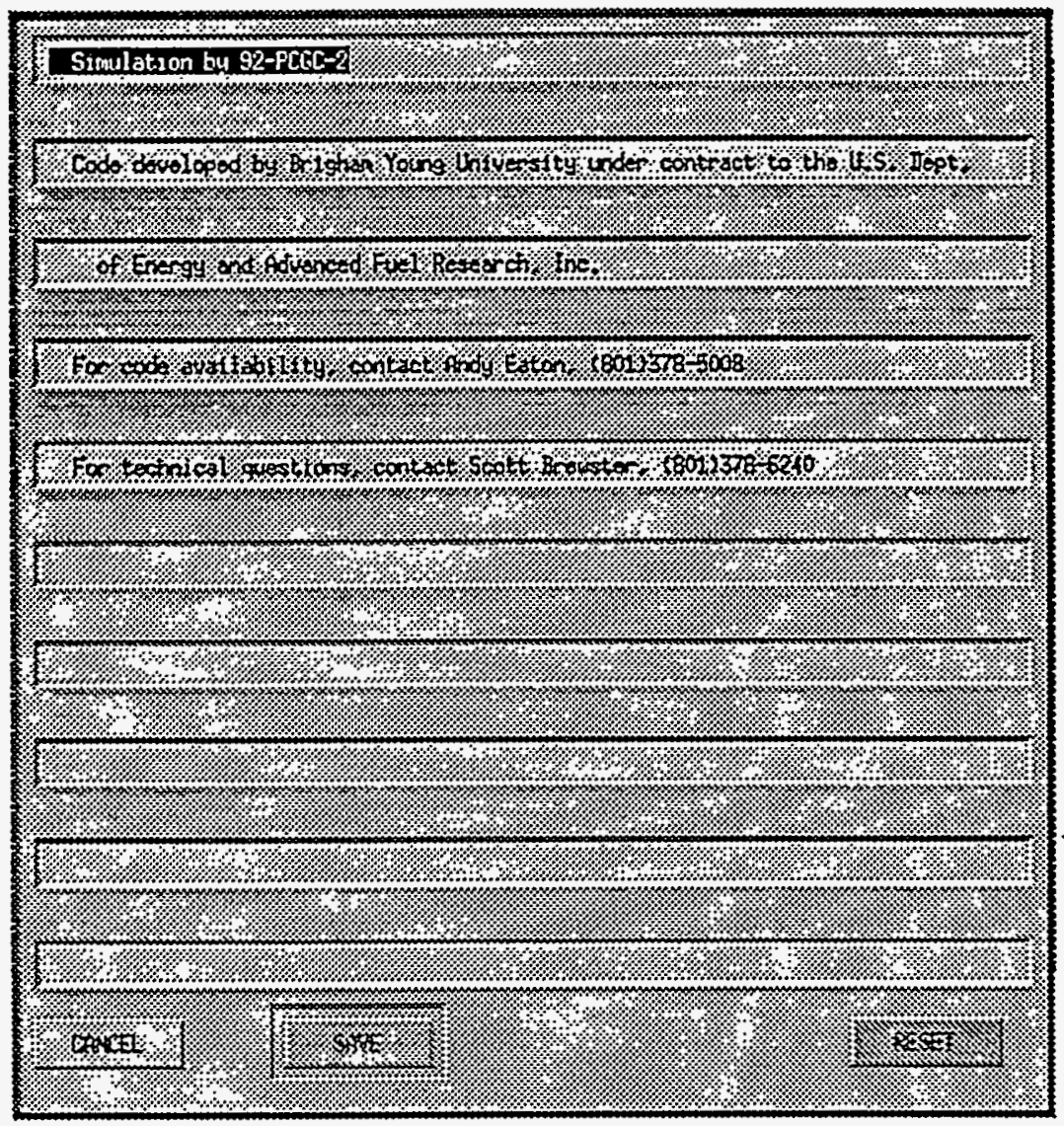

Fig. 5-5. Window for specifying simulation title.

Step 3: Enter the reactor geometry. The user then clicks on the word "Geometry" in the main menu and brings up the geometry window shown in Fig. 5-6. He/she edits the information shown, clicking on the desired units button for each data entry. He/she clicks on the desired configuration button, and then clicks on "DONE" to save the changes. 


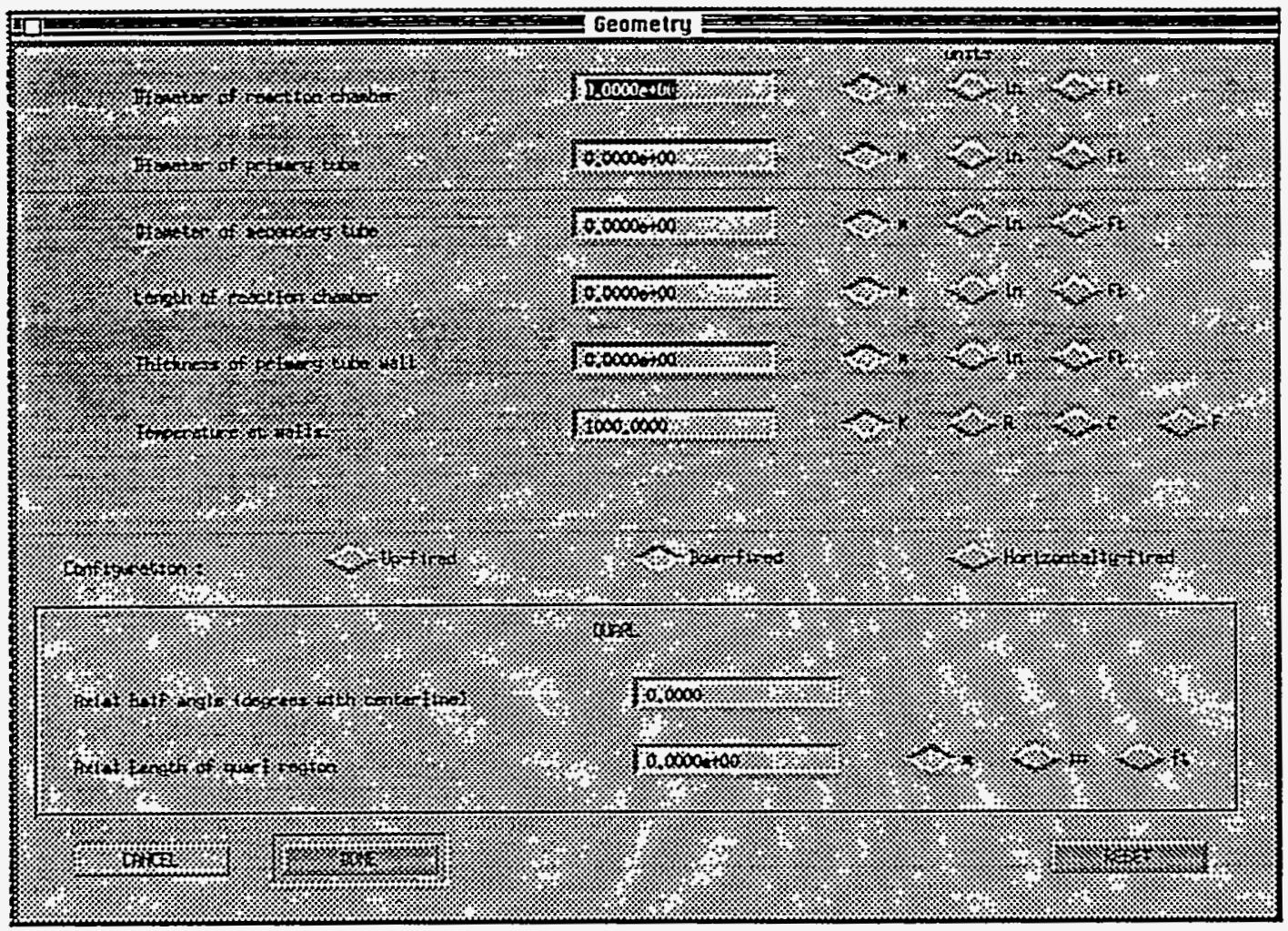

Fig. 5-6. Window for specifying reactor geometry.

Step 4: Specify the primary stream. The user then clicks on the word "S primary stream window as shown in Fig. 5-7. Again, he/she edits the information shown using the mouse and keyboard. The "Other Species" button brings up a window with a scrollable list of species as shown in Fig. 5-8. To add one of these species to the primary stream, the user need merely select it and then click "DONE." The species gets entered automatically into the lower portion of the primary stream window. If the user knows beforehand what species are available and the library name of the species, it can entered directly into the primary stream window. 


\section{User's Guide}

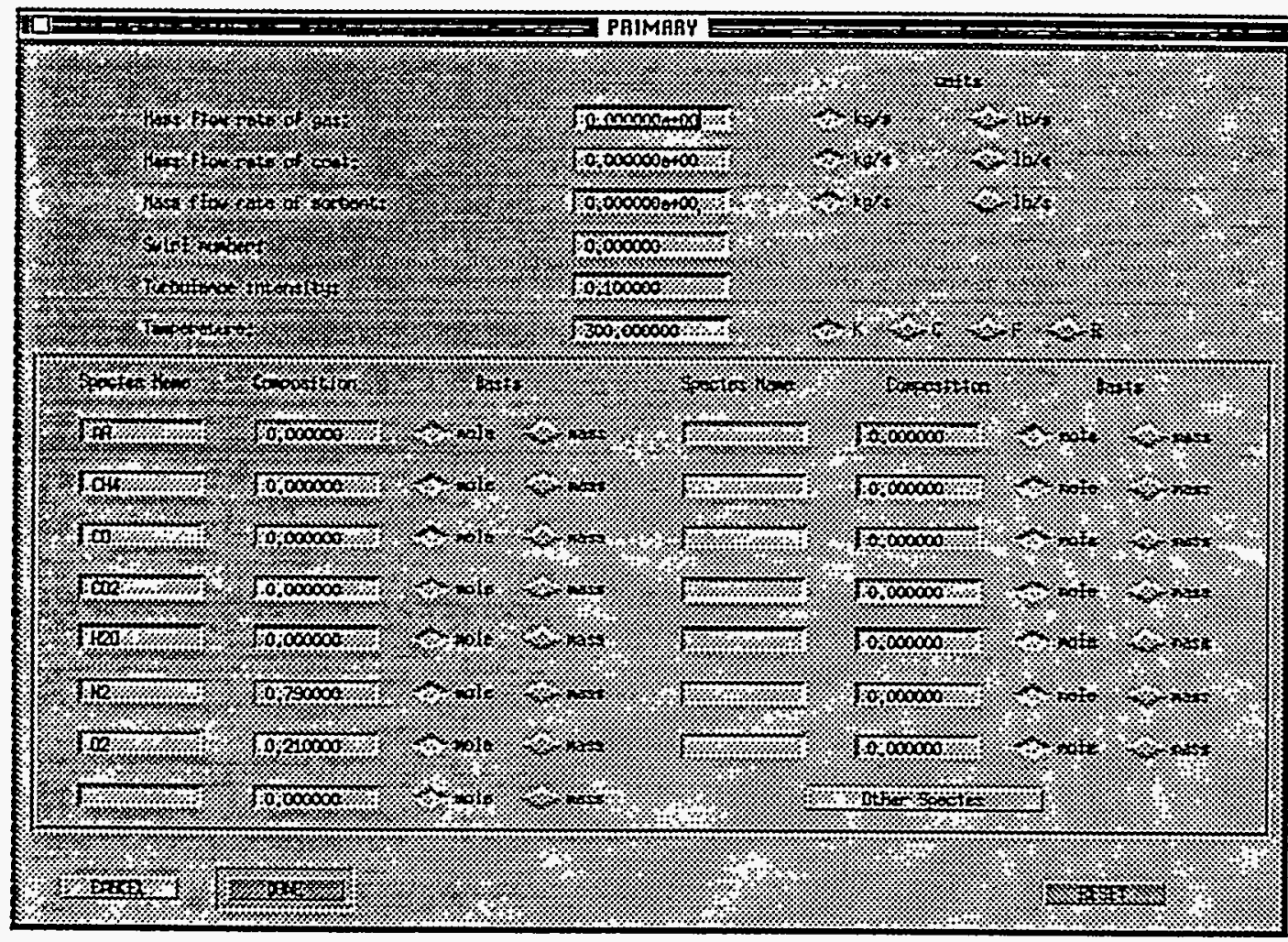

Fig. 5-7. Window for specifying primary inlet parameters. 


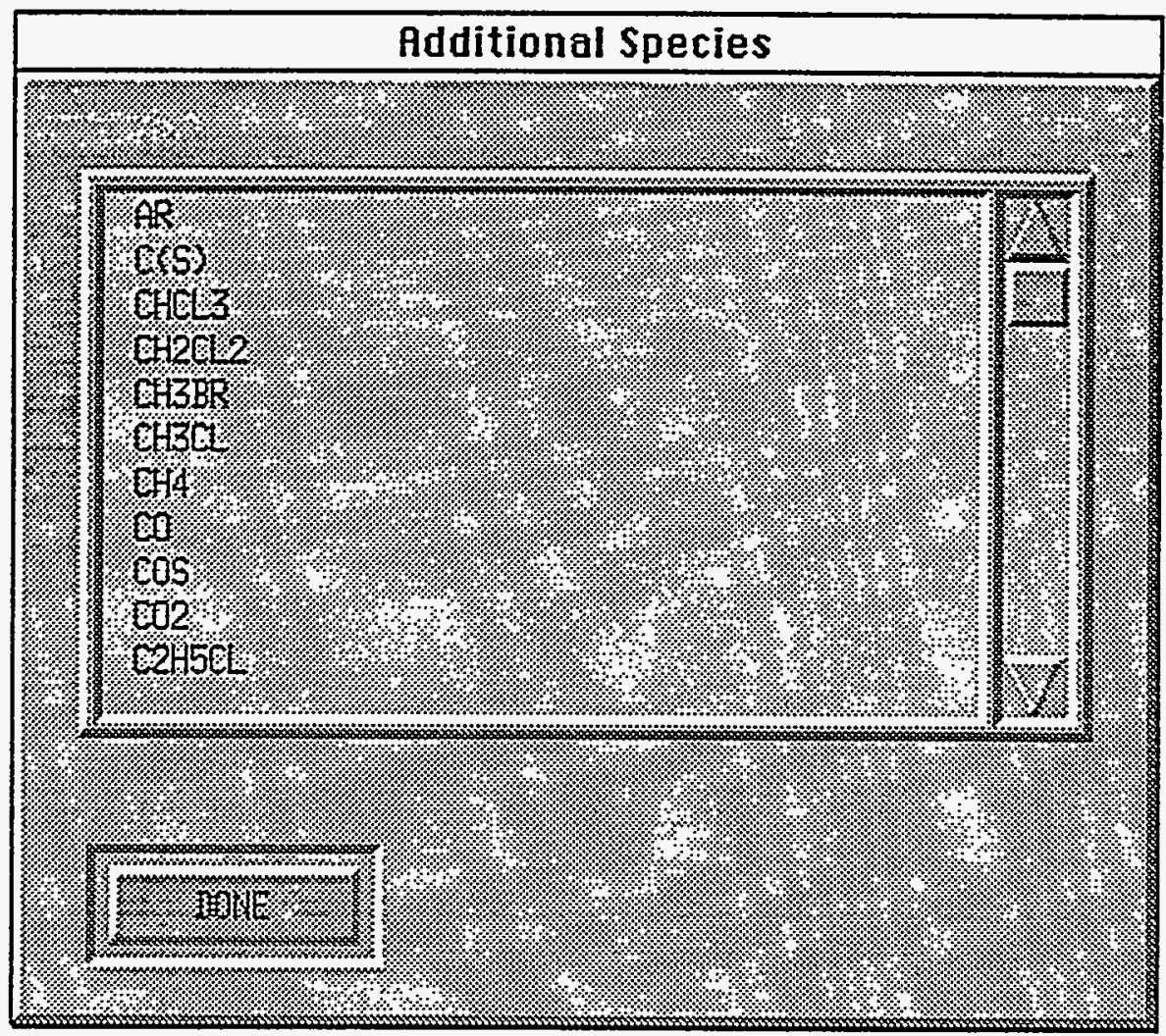

Fig. 5-8. Window for selecting chemical species to add.

Step 5: Specify the secondary stream. After specifying the primary stream, the user clicks again on the word "S "Secondary," bringing up the secondary stream window. This window is identical to the primary stream window, and the user edits the data for the secondary stream just as was done for the primary stream.

Step 6: Generate the computational grid. This step may be performed any time after Step 3. To construct the computational grid, the user clicks on the word "Grid" in the main menu, thus bringing up the grid window shown in Fig. 5-9. 


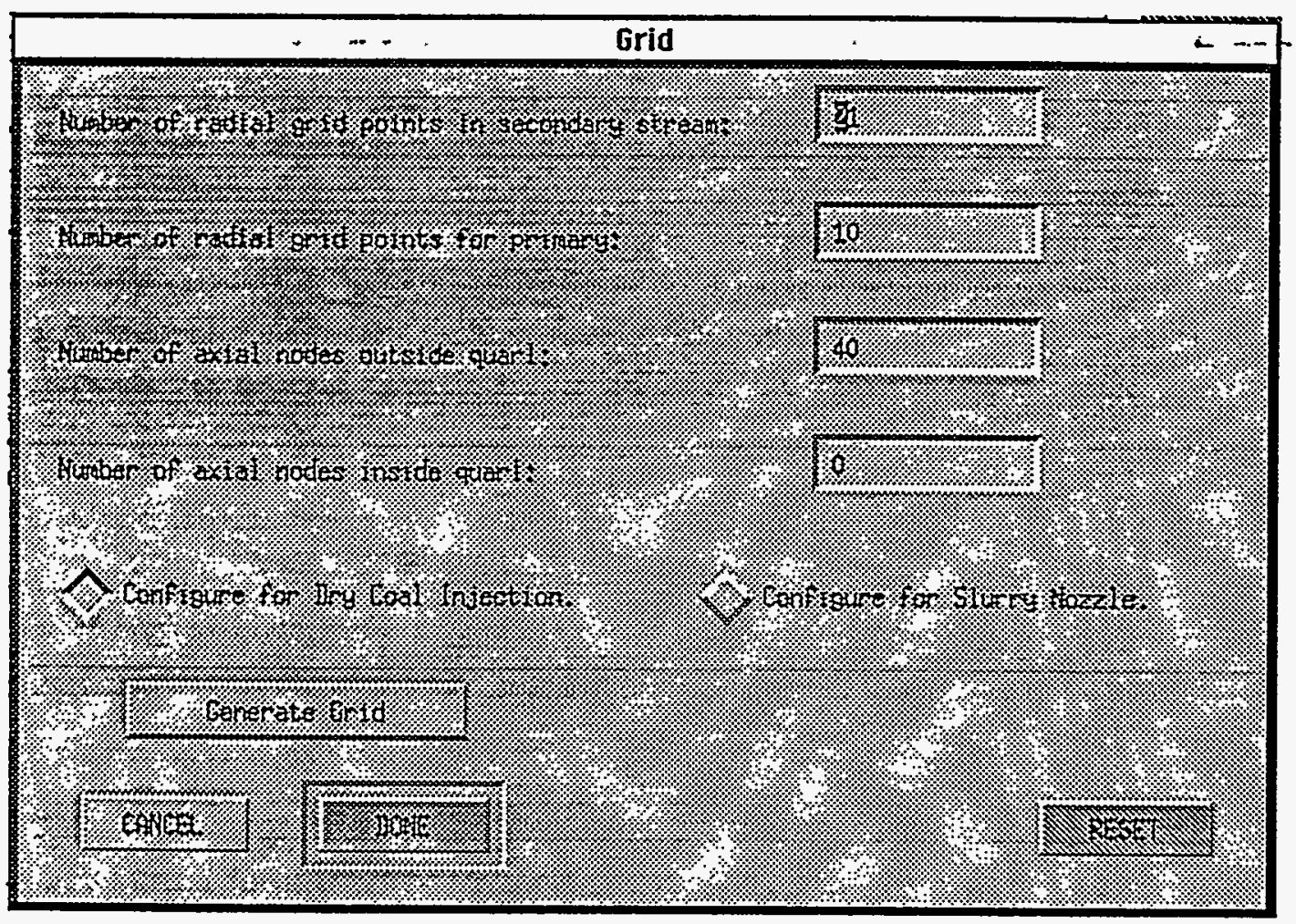

Fig. 5-9. Window for specifying grid generation parameters.

The user first enters an estimate for the number of radial grid points for the secondary duct. This is typically on the order of five. If the primary diameter is quite small relative to the secondary duct, he/she also enters an estimate for the number of grid points in the primary. The pulverized fuel combustion option is the one intended for most applications, for this case, a value of zero number for the radial grid points in the primary is used independently of the value inputted in the window. For slurry nozzles a non zero value for the radial grid points in the 
primary must be given. Next the user enters the number of axial nodes desired for the quarl and outside-of-the-quarl regions. The total of these two numbers should be less than or equal to 100 . After entering the required parameters, the grid can be generated by clicking the mouse on the "Generate Grid" button. A message will appear in the information window as shown in Fig. 5-10 giving the results. If the grid generation is successful, the number of radial grid points (NJ) will be printed. NJ must be less than or equal to 100 . If the grid generation is unsuccessful, a diagnostic message will be printed.

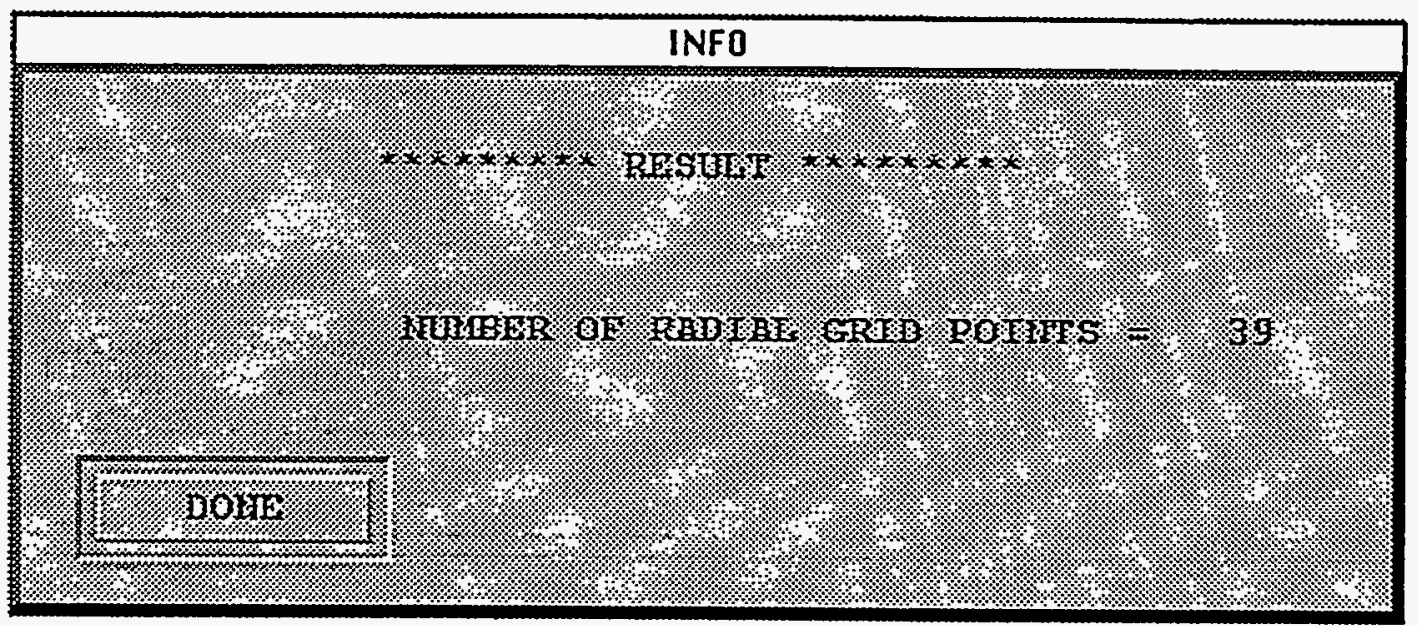

Fig. 5-10. Message window for generating the calculational grid. 
Step 7: Enter the coal particle data. If coal particles are entrained in either the primary or secondary stream, the user can click on the word "Particles" in the main menu as shown in Fig. 5-11 and select the word "Coal."

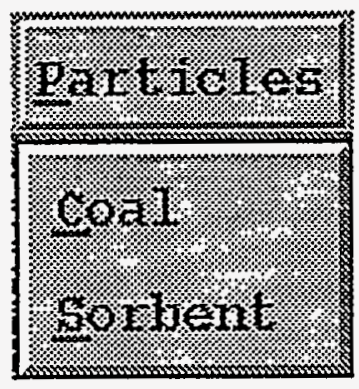

Fig. 5-11. Pull-down menu for selecting coal or sorbent particle properties.

The user will then enter the coal particle data in the coal window shown in Fig. 5-12. The pre-processor calculates the required input data for a flat particle size distribution based on the minimum and maximum particle sizes entered in this window. Any of the Argonne data bank coals can be selected by clicking on the appropriate box. The pre-processor assumes the user desires to use the FG-DVC devolatilization submodel. The desired oxidizers can also be selected by clicking with the mouse. 


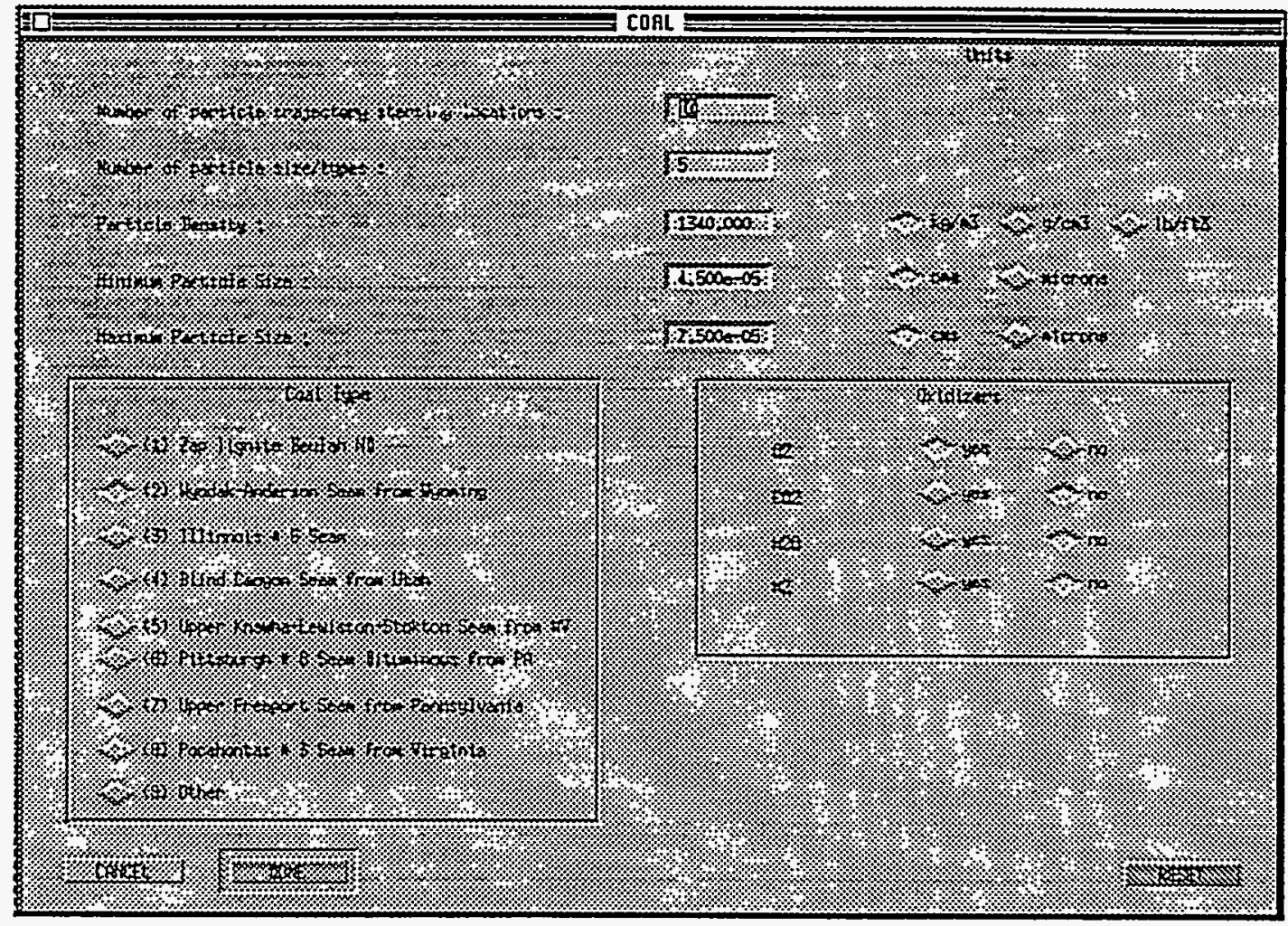

Fig. 5-12. Window for specifying coal particle input data.

If the user has a coal different from those of the Argonne data bank, the preprocessor can interpolate the Argonne data base to generate required data. This is done by clicking on the "other" button. The coal elemental composition is specified in the window shown in Fig. 5-13. After entering the required parameters, the coal data can be generated by clicking the mouse on the "Generate Coal Data File" button. A message will appear in the information window shown in Fig. 5-14 giving the results of the coal generation program. The user can also make changes to existing coal data files or the ones he/she just generated. To read existing data 
files, the user must click on the "Data from Files" button. The Program will pop up a window so the user can specify the name of the files to be read.

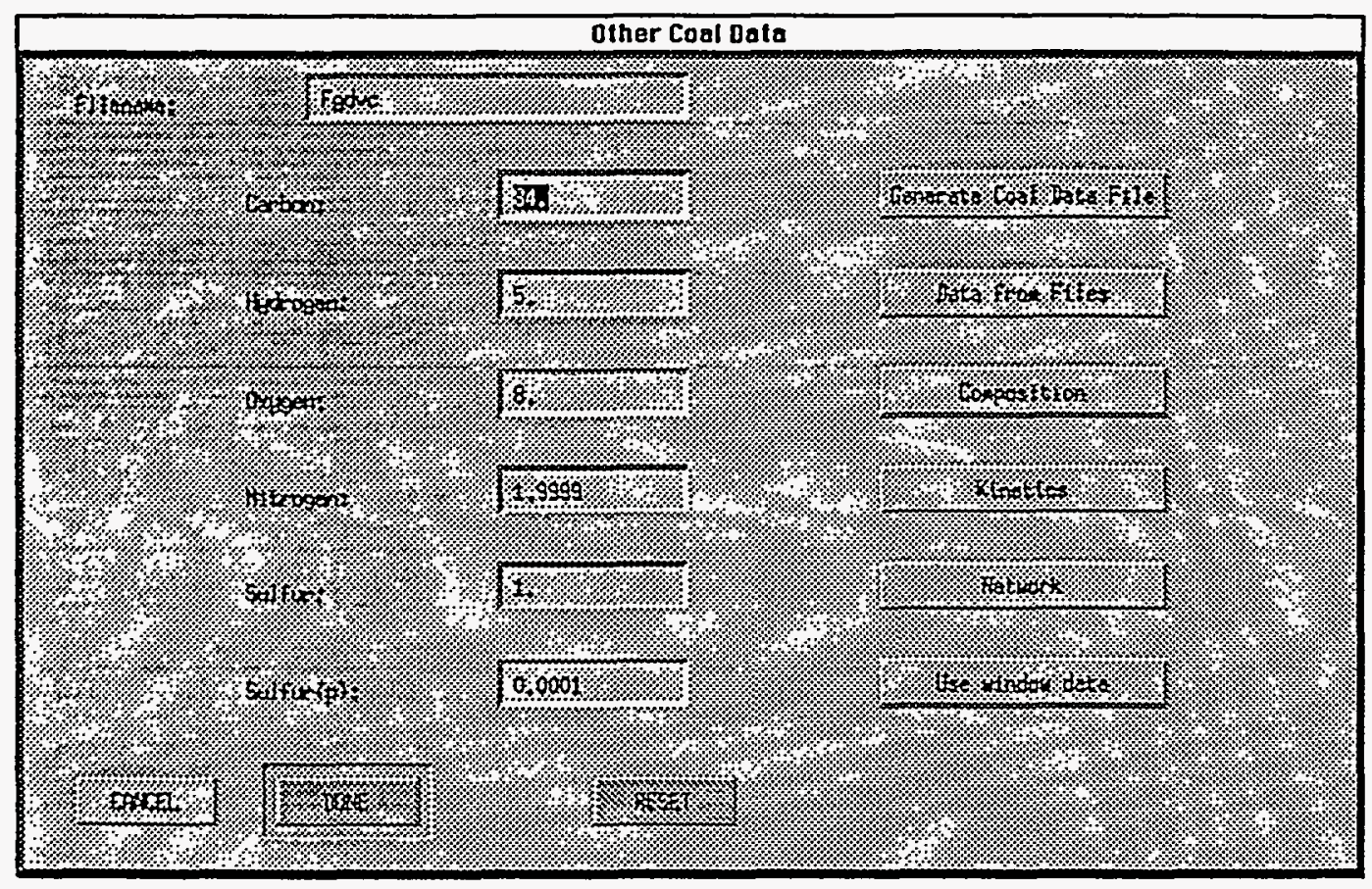

Fig. 5-13. Window for specifying elemental coal composition for coals not included in the Argonne data bank. 


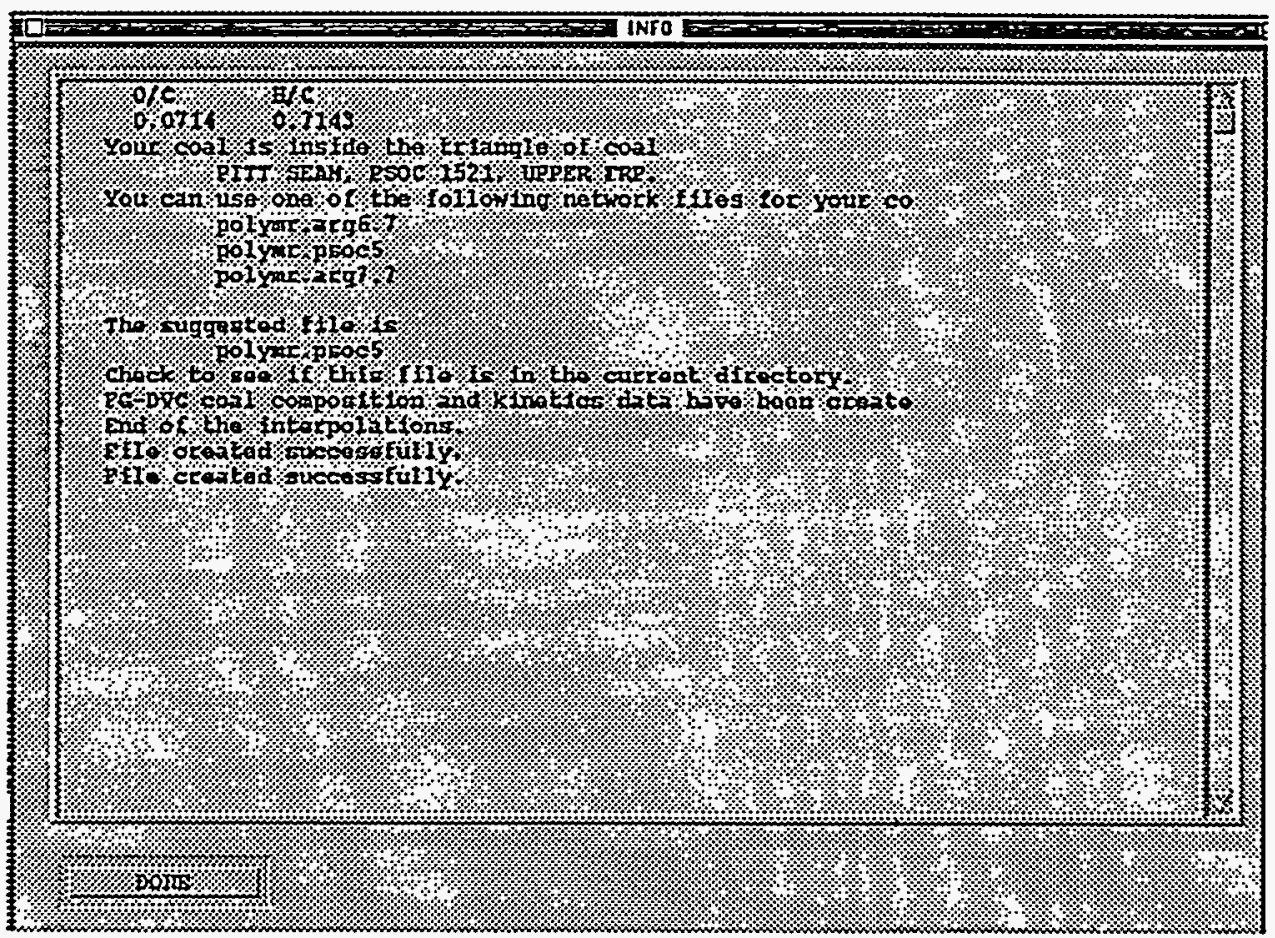

Fig. 5-14. Information window displaying results of coal data file generation program.

To make changes to the composition file, the user must click on the "Composition" button. This button will write the functional group composition of the coal in the information window, and will pop up a window, as shown in Fig. 515, where the user can make changes. Similarly, the kinetics data file can be modified as shown in Fig. 5-16. 


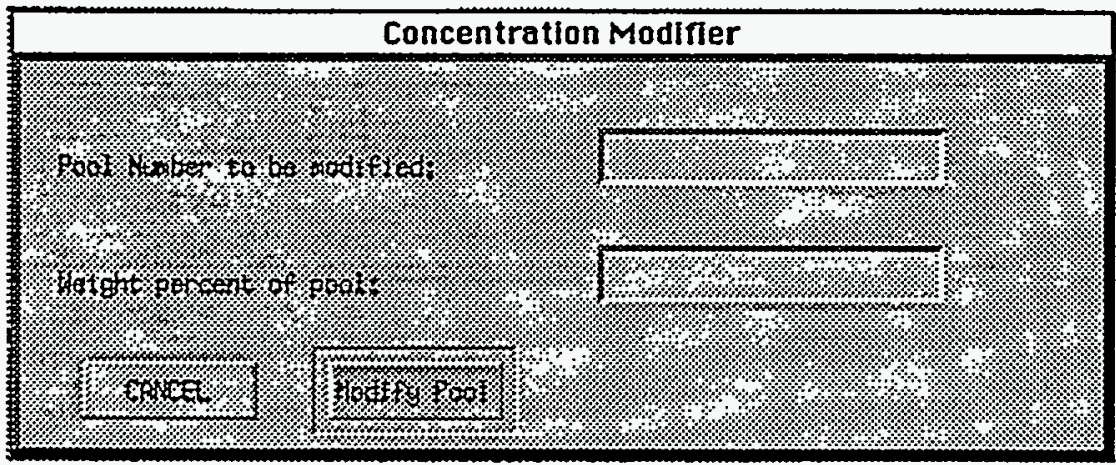

Fig. 5-15. Window for modifying coal composition data files.

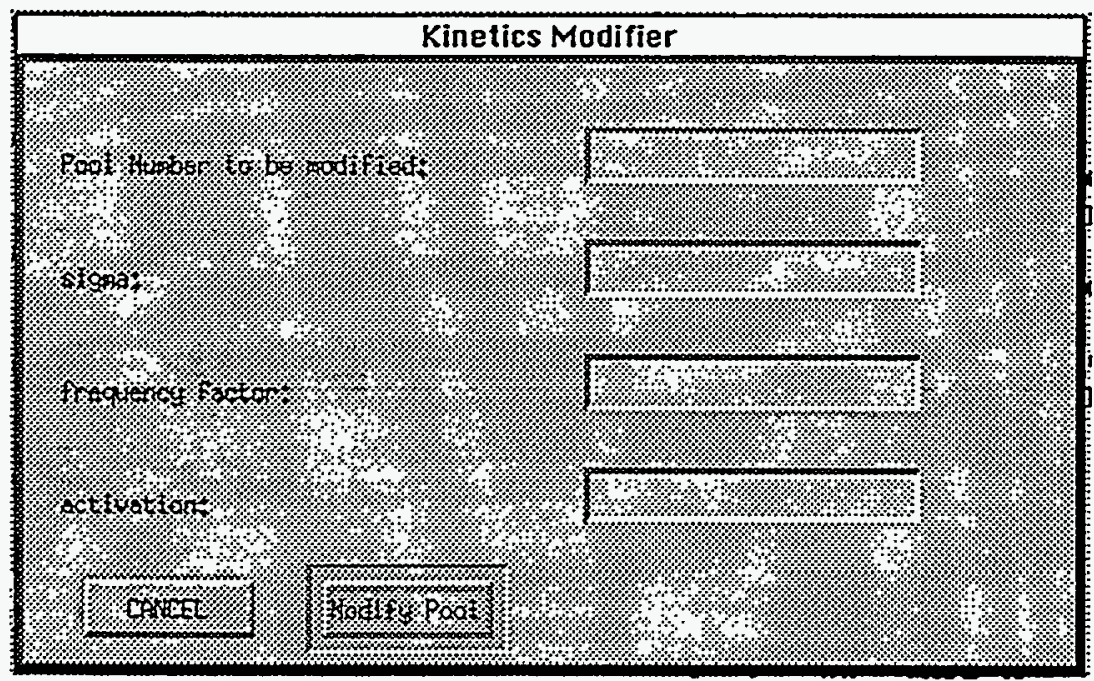

Fig. 5-16. Window for modifying coal kinetics data files. 
The user can go back to the data in the window with the "Use window data" button. If an error occurs during any step, the program will pop up a different information window to help the user make the needed changes. The user can save the files by clicking on the "DONE" button. The program will save the files using the name indicated. If the files already exist, the program will ask for a different name to save the resulting data. For more information on the specifics of the coal data file generation program, the user can refer to Chapter 7.

Step 8: Enter the sorbent particle data. If sorbent particles are entrained in either the primary or secondary gas streams, the user clicks again on the word "Particles" in the main menu and selects the word "Sorbent." The sorbents window is shown in Fig. 5-17. As with the coal, a flat particle size distribution is assumed, based on the specified minimum and maximum particle sizes.

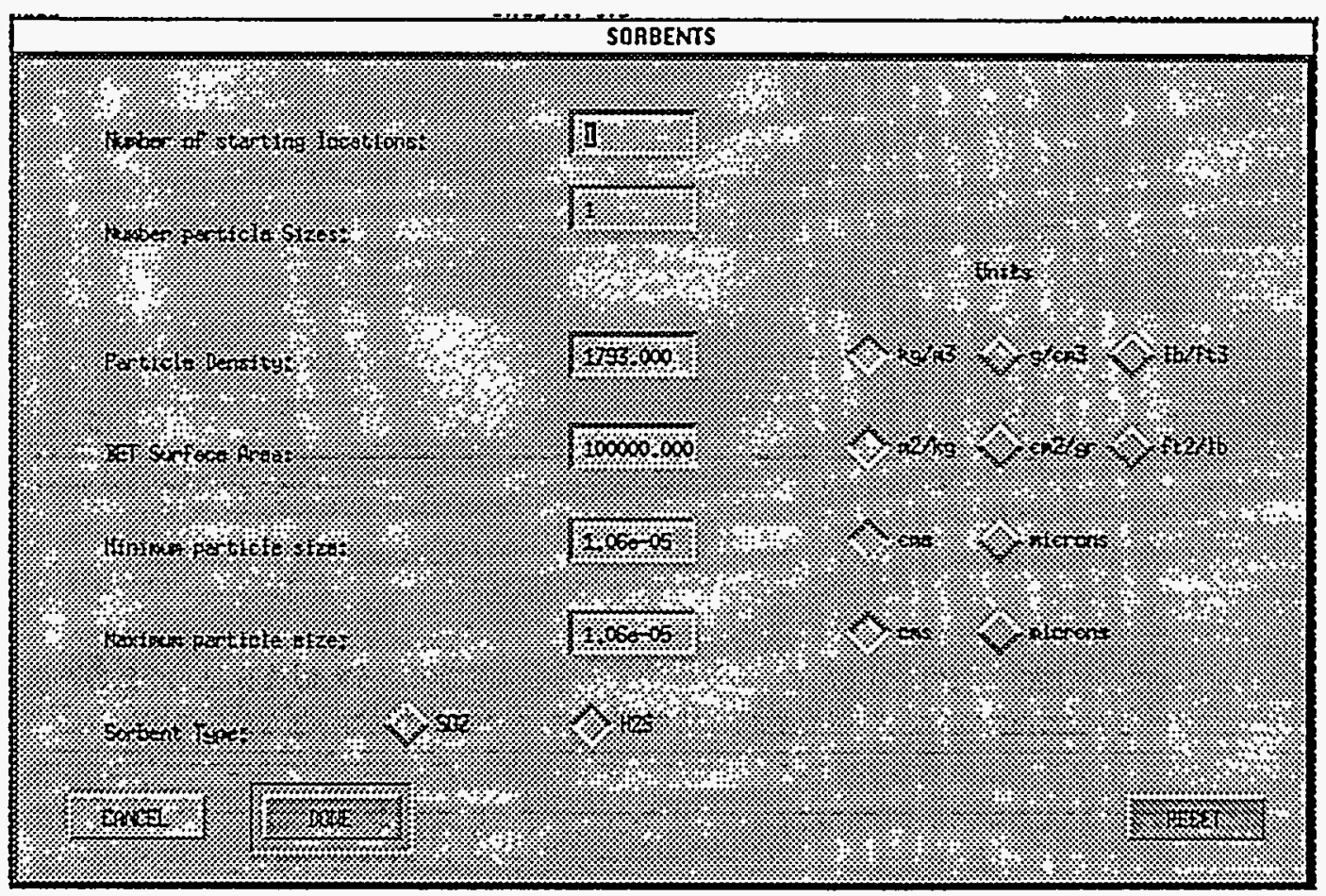

Fig. 5-17. Window for specifying sorbent particle input data. 
Step 9: Save the new input files. After all of the data entries are completed and the grid has been successfully generated, the user clicks again on the word "File" in the main menu and selects the word "Save" or "Save As." "Save" saves the files under the same name they were opened under. "Save A $s$ " saves them under a new name which the user can specify in the window shown in Fig. 5-18. If the user tries to quit the program without saving the new data files, he/she is prompted with the window shown in Fig. 5-19.

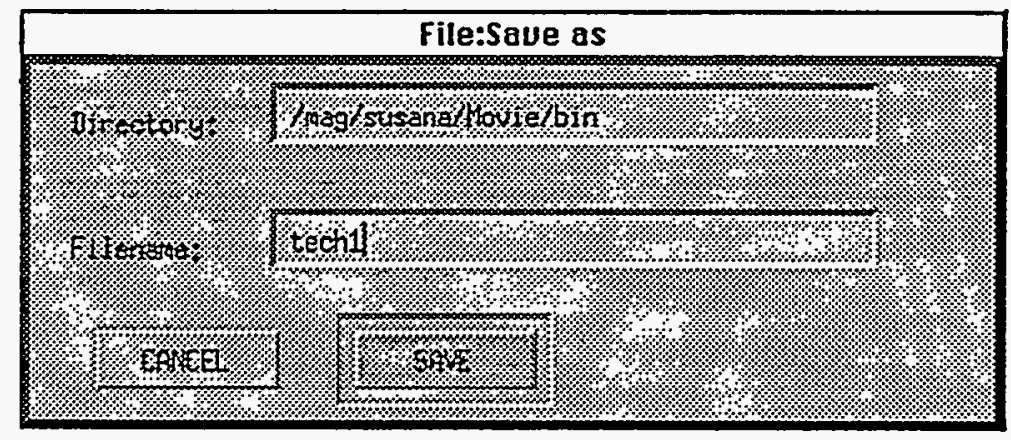

Fig. 5-18. Window for specifying filename to save input data.

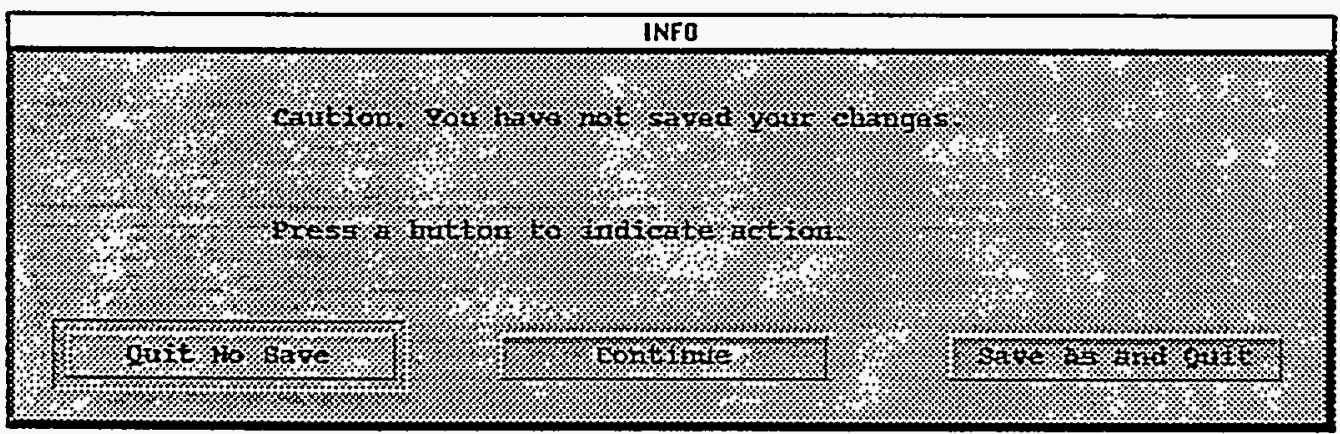

Fig. 5-19. Prompting window for saving data files. 


\title{
DISSPLA Post-processor
}

PCPLOT is a menu-driven driver program for PCGC-2, used in conjunction with the DISSPLA ${ }^{3}$ graphics package. It produces specially formatted meta-files that can be displayed on the screen or printed in hard-copy, depending on the user's system. PCPLOT reads the following plotting files from PCGC-2:

\author{
filename.gsp: gas properties \\ filename.grd: grid configuration \\ filename.pp 1: coal particle \\ filename.pp2: coal particle \\ filename.pp 3: coal particle \\ filename.ps 1: sorbent particle \\ filename.ps2: sorbent particle \\ filename.ps 3: sorbent particle \\ filename.nxp: $\mathrm{NO}_{x}$ \\ filename.sxp: $\mathrm{SO}_{x}$
}

The initial menu displays the types of plots that can be produced with PCPLOT and the program options for modifying these plots :

1. Gas Properties

2. Coal Particle Properties

3. Sorbent Particle Properties

4. Experimental Comparisons

5. Sox Pollutants

6. NOx Pollutants

7. Spreadsheet Format

8. Velocity Vector Plots

9. Zoom-in Plots

10. Plot Grid Setup

${ }^{3}$ Available from : Computer Associates International, Inc.

711 Stewart Avenue

Garden City, NY 11530-4787

tel 1-800-645-3042 
11. Read New File Name

12. Omit Title

13. Normalize

14. Black and White

15. STOP

Gas Properties. Option 1 from the main menu, brings up a new menu . This menu shows the properties included in the .gsp file. For example:

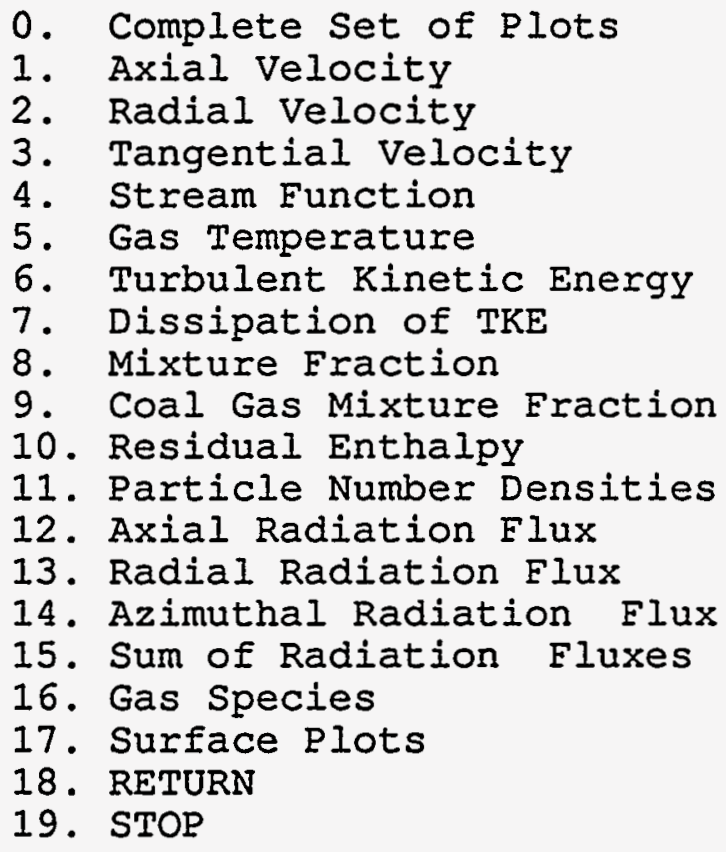

All gas properties can be plotted either as surface plots or as contour plots. Option 17 produces four different rotations of the surface plots in addition to the contour plot. The user can choose to have one plot on each page or all four rotations on the same page. The first choice plots all the gaseous variables that PCGC-2 wrote in the .gsp file. Plotting all of these properties, however, will take a lot of time and a lot of space for the resulting meta-files. Option 11 will produce a menu telling the user how many particle diameters there are in the file and will ask him/her how many of these he/she wants to plot. If the user chooses Option 16, PCPLOT will list the names of all the species in the .gsp file, so he/she can choose 
which ones to plot. Option 18 is used to go back to the main menu. Option 19 is used to clean-up and finish the resulting plots.

As an example, the following sequence was used to generate Fig. 5-20:

ENTER FILENAME

CPR

cpr.pop IF FILE EXISTS, DO YOU WANT TO WRITE OVER ? ( $\mathrm{y} / \mathrm{n})$

$\mathbf{Y}$

SELECT :

1. Gas Properties

2. Coal Particle Properties

3. Sorbent Particle Properties

4. Experimental Comparisons

5. Sox Pollutants

6. NOx Pollutants

7. Spreadsheet Format

8. Velocity Vectors plots

9. Zoom-in plots

10. Plot Grid Setup

11. Read New File Name

12. Omit Titles

13. Normalize

14. Black and White

15. STOP

1

SELECT VARIABLE:

0 . Complete set of Plots

1. Axial Velocity

2. Radial Velocity 
3. Tangential Velocity

4. Stream Function

5. Gas Temperature

6. Turbulent Kinetic Energy

7. Dissipation of TKE

8. Mixture Fraction

9. Coal Gas Mixture Eraction

10. Residual Enthalpy

11 - 25. Particle Number Densities

26. Axial Radiation Flux

27. Radial Radiation Flux

28. Azimuthal Radiation Flux

29. Sum of Radiation Fluxes

30 - 50. Gas Species

51. Surface Plots

52. RETURN

53. STOP

51

SURFACES: 4 PER PAGE OR 1 PER PAGE? $(4,1)$

4

SELECT VARIABLE:

0 . Complete set of Plots

1. Axial Velocity

2. Radial Velocity

3. Tangential Velocity

4. Stream Function

5. Gas Temperature

6. Turbulent Kinetic Energy

7. Dissipation of TKE

8. Mixture Fraction

9. Coal Gas Mixture Fraction

10. Residual Enthalpy 
11 - 25. Particle Number Densities

26. Axial Radiation Flux

27. Radial Radiation Flux

28. Azimuthal Radiation Flux

29. Sum of Radiation Fluxes

30 - 50. Gas Species

51. Surface Plots

52. RETURN

53. STOP

5

PLOTTING TEMPERATURE (K)

SELECT VARIABLE:

0 . Complete set of plots

1. Axial Velocity

2. Radial Velocity

3. Tangential Velocity

4. Stream Function

5. Gas Temperature

6. Turbulent Kinetic Energy

7. Dissipation of TKE

8. Mixture Eraction

9. Coal Gas Mixture Fraction

10. Residual Enthalpy

11 - 25. Particle Number Densities

26. Axial Radiation Flux

27. Radial Radiation Flux

28. Azimuthal Radiation Flux

29. Sum of Radiation Fluxes

30 - 50. Gas Species

51. Surface Plots

52. RETURN

53. STOP

\section{3}



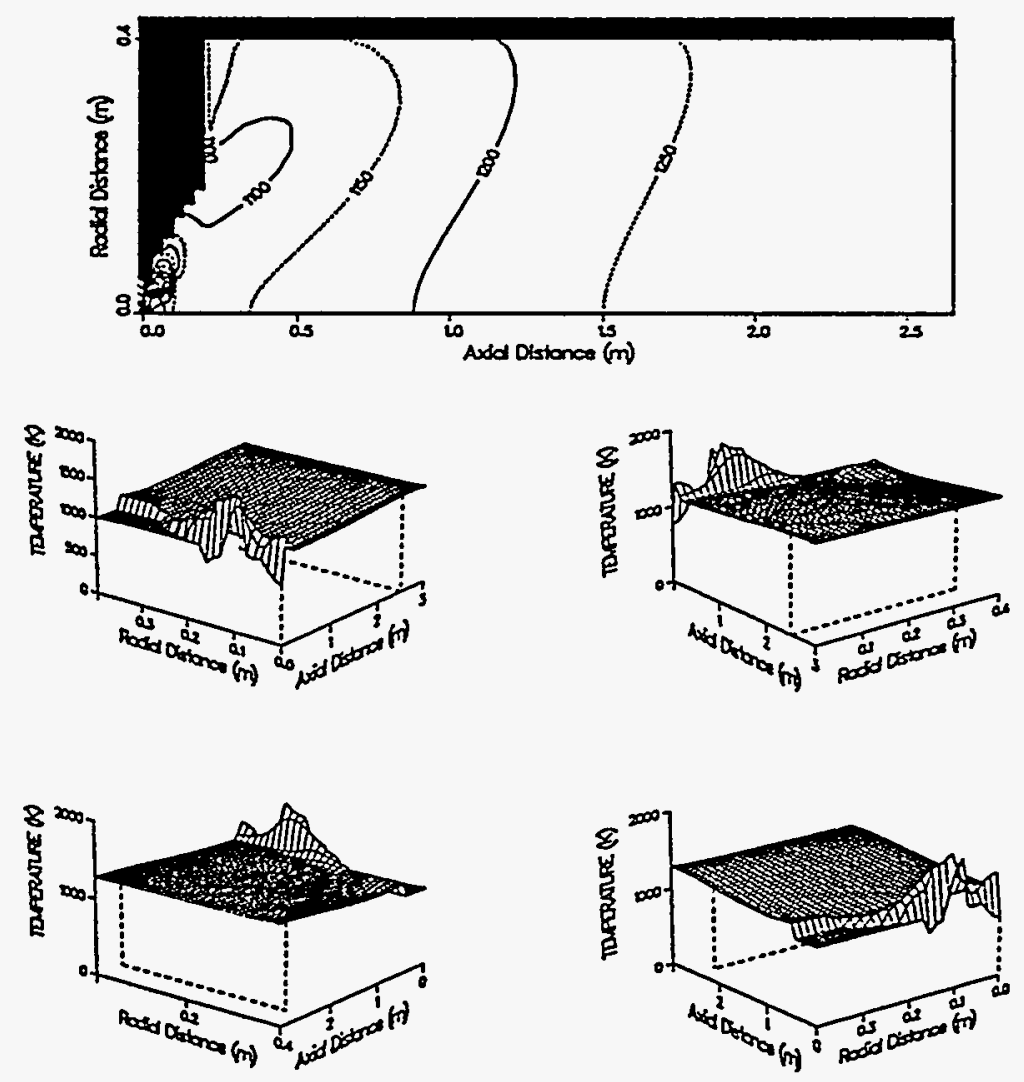

Fig. 5-20. Sample plot of gas temperature. 
Coal and Sorbent Particle Properties. When the user chooses either Option 2 or Option 3 from the main menu, a new menu will appear. This menu shows the properties included in either the .pp1, .pp2, and .pp3 files for coal properties or the .ps 1, .ps2, and .ps 3 files for sorbent particles. For the coal, the user can choose from the following menu:

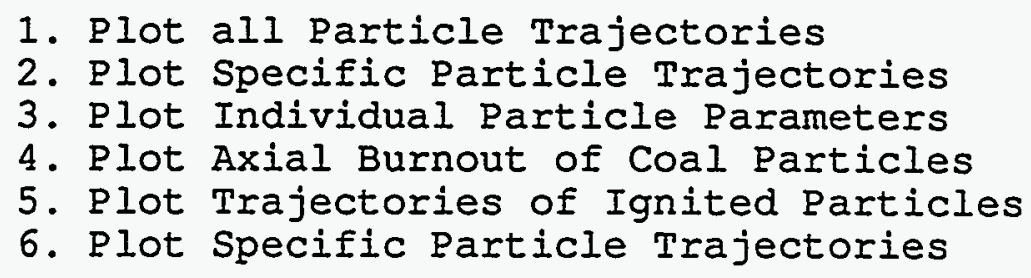

Option 3 will provide a menu telling the user how many particle diameters there are in the file, and will ask how many and which ones to plot. Similarly, when the user chooses Option 2, he/she will be able to choose which particle trajectories he/she wants to plot from the limits given. A similar menu appears when the user wants to plot sorbent particle properties.

Experimental Comparisons. PCPLOT compares data from the .gsp file with experimental data in the .exp file with the special format:

number of points (integer) radial position (real)

list of axial positions (real)

data values (real)

a final value of 0.0 that tells the program to stop reading data.

For example:

90.19

$0.00 .05 \quad 0.1 \quad 0.150 .20 .250 .3 \quad 0.350 .4$

.0274 .0346 .0376 .0173 .013 .012 .0086 .012

00.

Usually the locations of the experimental data do not coincide with the grid used by PCGC-2. To obtain data in the same locations as the experimental data, PCPLOT performs a linear interpolation of the PCGC-2 data, in both the axial and radial directions. PCPLOT calculates the limits of the plots as the minimum and 
maximum $x$ positions and the smallest and biggest values of the variable to be plotted, from all the experimental and interpolated data. If the user wants to look only at the portion of the interpolated data close to his/her experimental points, he can change the initial and final value of the $x$ positions.

$\underline{S O}_{\underline{X}}$ and NO $\mathrm{NO}_{\mathrm{X}}$ Pollutants. These plots are specific for the cases when $\mathrm{SO}_{\mathrm{X}}$ and $\mathrm{NO}_{\mathrm{x}}$ calculations have been performed. They use the . nxp and . sxp files. As with the other options, the user will get a menu showing the possible plots he/she can generate. For the $\mathrm{NO}_{\mathrm{x}}$ data files, the menu is :

$$
\begin{aligned}
& \text { 1. Mole fraction of } \mathrm{NO}_{x} \text { (ppm) } \\
& \text { 2. Mole fraction of } \mathrm{HCN}^{(\mathrm{ppm})} \\
& \text { 3. Mole fraction of } \mathrm{NH}_{3}(\mathrm{ppm}) \\
& \text { 4. Mole fraction of } \mathrm{O}_{2} \text { (ppm) } \\
& \text { 5. } \mathrm{NO}_{x} \text { rate } \\
& \text { 6. } \mathrm{HCN} \text { rate } \\
& \text { 7. } \mathrm{NH}_{3} \text { rate } \\
& \text { 8. RETURN } \\
& \text { 9. STOP }
\end{aligned}
$$

For the $\mathrm{SO}_{\mathrm{X}}$ data files, the menu is :

$$
\begin{aligned}
& \text { 1. Equilibrium } \mathrm{SO}_{2} \\
& \text { 2. } \mathrm{SO}_{2} \\
& \text { 3. Equilibrium } \mathrm{H}_{2} \mathrm{~S} \\
& \text { 4. } \mathrm{H}_{2} \mathrm{~S} \\
& \text { 5. RETURN } \\
& \text { 6. STOP }
\end{aligned}
$$

Spreadsheet Format. PCPLOT allows the user to generate a .sp1 file in a text format that can be read by standard spreadsheet programs. The user can choose any variable he/she wants to write in this file. The main spreadsheet dialog is:

1. Gas Properties

2. Coal Particle Properties

2. Sorbent Particle Properties

4. Experimental comparisons 
5. Sox Pollutants

6 NOx Pollutants

7. Close-up

8. Normalize

9. RETURN

10. STOP

Each of the menus works in exactly the same way as the corresponding menu of the DISSPLA graphics. For example, to generate a coal particle spreadsheet file with all the properties for a given particle, as shown in Table 5-5, the following sequence was used:

ENTER FILENAME

CPR

cpr.pop IF FILE EXISTS, DO YOU WANT TO WRITE OVER ? $(\mathrm{y} / \mathrm{n})$

$Y$

SEIECT :

1. Gas Properties

2. Coal Particle Properties

3. Sorbent Particle Properties

4. Experimental Comparisons

5. SOx Pollutants

6. NOx Pollutants

7. Spreadsheet Format

8. Velocity Vectors plots

9. Zoom-in plots

10. Plot Grid Setup

11. Read New File Name

12. Omit Titles

13. Normalize

14. Black and White

13. STOP

7

SELECT : 
1. Gas Properties

2. Coal Particle Properties

3. Sorbent Particle Properties

4. Experimental Comparisons

5. Sox Pollutants

6. NOx Pollutants

7 Close-up

8. Read New File Name

9. Normalize

10. $* * *$ RETURN $* * \star$

11. $* * *$ STOP $* * *$

2

READING CPR INPUT FIIE (S)

READING PARTICLE SIZE : 1 LOCATION : 1

...

$\cdots$

READING PARTICLE SIZE : 5 LOCATION : 5

READING OF PARTICLE INPUT IS COMPLETE

1. Plot All Particle Trajectories

2. Plot Specific Size Class Trajectories

3. Plot Individual Particle Trajectories

4. Plot Axial Burnout of Coal Particles

5. Plot Trajectories of Ignited Coal Particles

6. Plot Specific Starting Locations

7. RETURN

8. STOP

CHOOSE A NUMBER (1-8)

3 
HOW MANY PARTICLES DO YOU WANT PLOTTED? (1-25)

1

INPUT THE STARTING IOC. AND SIZE CLASS DESIRED FOR PLOT NUMBER 1 (i.e. ISL $=1$, IPS $=2$ ):

1,3

1. Plot All Particle Trajectories

2. Plot Specific Size Class Trajectories

3. Plot Individual Particle Trajectories

4. Plot Axial Burnout of Coal particles

5. Plot Trajectories of Ignited Coal Particles

6. Plot Specific Starting Locations

7. RETURN

8. STOP

CHOOSE A NUMBER (1-8)

8

TABLE 5-6

SPREADSHEET OUTPUT FROM PCPLOT

Starting Location $1 \quad$ Particle Size 3

Axial location (m) Radial location (m) Total Fraction $\quad$ Water Fract ...

$\begin{array}{llll}9.010 \mathrm{E}-07 & 2.660 \mathrm{E}-04 & 1.000 \mathrm{E}+00 & 0.000 \mathrm{E}+00 \ldots \\ 1.390 \mathrm{E}-02 & 2.750 \mathrm{E}-04 & 1.000 \mathrm{E}+00 & 0.000 \mathrm{E}+00 \ldots \\ 4.390 \mathrm{E}-02 & 3.250 \mathrm{E}-04 & 1.000 \mathrm{E}+00 & 0.000 \mathrm{E}+00 \ldots \\ 7.370 \mathrm{E}-02 & 3.770 \mathrm{E}-04 & 1.000 \mathrm{E}+00 & 0.000 \mathrm{E}+00 \ldots \\ 1.040 \mathrm{E}-01 & 4.430 \mathrm{E}-04 & 1.000 \mathrm{E}+00 & 0.000 \mathrm{E}+00 \ldots \\ 1.330 \mathrm{E}-01 & 5.760 \mathrm{E}-04 & 9.990 \mathrm{E}-01 & 0.000 \mathrm{E}+00 \ldots \\ 1.410 \mathrm{E}-01 & 6.140 \mathrm{E}-04 & 9.910 \mathrm{E}-01 & 0.000 \mathrm{E}+00 \ldots \\ 1.480 \mathrm{E}-01 & 6.520 \mathrm{E}-04 & 9.800 \mathrm{E}-01 & 0.000 \mathrm{E}+00 \ldots \\ 1.630 \mathrm{E}-01 & 7.220 \mathrm{E}-04 & 9.250 \mathrm{E}-01 & 0.000 \mathrm{E}+00 \ldots \\ 1.780 \mathrm{E}-01 & 7.860 \mathrm{E}-04 & 8.250 \mathrm{E}-01 & 0.000 \mathrm{E}+00 \ldots\end{array}$


TABLE 5-6 (continued)

$\begin{array}{llll}1.930 \mathrm{E}-01 & 8.550 \mathrm{E}-04 & 7.800 \mathrm{E}-01 & 0.000 \mathrm{E}+00 \ldots \\ 2.080 \mathrm{E}-01 & 9.580 \mathrm{E}-04 & 7.650 \mathrm{E}-01 & 0.000 \mathrm{E}+00 \ldots \\ 2.360 \mathrm{E}-01 & 1.450 \mathrm{E}-03 & 7.590 \mathrm{E}-01 & 0.000 \mathrm{E}+00 \ldots \\ 2.490 \mathrm{E}-01 & 2.100 \mathrm{E}-03 & 7.480 \mathrm{E}-01 & 0.000 \mathrm{E}+00 \ldots \\ 2.540 \mathrm{E}-01 & 2.680 \mathrm{E}-03 & 6.780 \mathrm{E}-01 & 0.000 \mathrm{E}+00 \ldots \\ 2.570 \mathrm{E}-01 & 3.310 \mathrm{E}-03 & 6.350 \mathrm{E}-01 & 0.000 \mathrm{E}+00 \ldots \\ 2.580 \mathrm{E}-01 & 3.490 \mathrm{E}-03 & 6.300 \mathrm{E}-01 & 0.000 \mathrm{E}+00 \ldots \\ 2.590 \mathrm{E}-01 & 3.770 \mathrm{E}-03 & 6.210 \mathrm{E}-01 & 0.000 \mathrm{E}+00 \ldots \\ 2.600 \mathrm{E}-01 & 4.040 \mathrm{E}-03 & 6.150 \mathrm{E}-01 & 0.000 \mathrm{E}+00 \ldots \\ 2.600 \mathrm{E}-01 & 4.350 \mathrm{E}-03 & 6.110 \mathrm{E}-01 & 0.000 \mathrm{E}+00 \ldots \\ 2.610 \mathrm{E}-01 & 4.960 \mathrm{E}-03 & 6.040 \mathrm{E}-01 & 0.000 \mathrm{E}+00 \ldots \\ 2.620 \mathrm{E}-01 & 5.570 \mathrm{E}-03 & 6.000 \mathrm{E}-01 & 0.000 \mathrm{E}+00 \ldots \\ 2.630 \mathrm{E}-01 & 6.690 \mathrm{E}-03 & 5.960 \mathrm{E}-01 & 0.000 \mathrm{E}+00 \ldots \\ 2.630 \mathrm{E}-01 & 7.940 \mathrm{E}-03 & 5.930 \mathrm{E}-01 & 0.000 \mathrm{E}+00 \ldots \\ 2.630 \mathrm{E}-01 & 1.050 \mathrm{E}-02 & 5.890 \mathrm{E}-01 & 0.000 \mathrm{E}+00 \ldots \\ 2.630 \mathrm{E}-01 & 1.560 \mathrm{E}-02 & 5.850 \mathrm{E}-01 & 0.000 \mathrm{E}+00 \ldots \\ 2.600 \mathrm{E}-01 & 2.790 \mathrm{E}-02 & 5.760 \mathrm{E}-01 & 0.000 \mathrm{E}+00 \ldots \\ 2.550 \mathrm{E}-01 & 4.000 \mathrm{E}-02 & 5.710 \mathrm{E}-01 & 0.000 \mathrm{E}+00 \ldots \\ 2.470 \mathrm{E}-01 & 5.210 \mathrm{E}-02 & 5.650 \mathrm{E}-01 & 0.000 \mathrm{E}+00 \ldots \\ 2.350 \mathrm{E}-01 & 6.660 \mathrm{E}-02 & 5.590 \mathrm{E}-01 & 0.000 \mathrm{E}+00 \ldots \\ 2.120 \mathrm{E}-01 & 8.560 \mathrm{E}-02 & 5.480 \mathrm{E}-01 & 0.000 \mathrm{E}+00 \ldots \\ 2.120 \mathrm{E}-01 & 1.120 \mathrm{E}-01 & 5.420 \mathrm{E}-01 & 0.000 \mathrm{E}+00 \ldots \\ 2.520 \mathrm{E}-01 & 1.380 \mathrm{E}-01 & 5.330 \mathrm{E}-01 & 0.000 \mathrm{E}+00 \ldots \\ 3.040 \mathrm{E}-01 & 1.630 \mathrm{E}-01 & 5.200 \mathrm{E}-01 & 0.000 \mathrm{E}+00 \ldots \\ 3.730 \mathrm{E}-01 & 1.730 \mathrm{E}-01 & 5.140 \mathrm{E}-01 & 0.000 \mathrm{E}+00 \ldots \\ 4.640 \mathrm{E}-01 & 1.600 \mathrm{E}-01 & 5.100 \mathrm{E}-01 & 0.000 \mathrm{E}+00 \ldots \\ 5.850 \mathrm{E}-01 & 1.350 \mathrm{E}-01 & 5.060 \mathrm{E}-01 & 0.000 \mathrm{E}+00 \ldots \\ 7.460 \mathrm{E}-01 & 1.390 \mathrm{E}-01 & 5.030 \mathrm{E}-01 & 0.000 \mathrm{E}+00 \ldots \\ 9.610 \mathrm{E}-01 & 1.710 \mathrm{E}-01 & 4.990 \mathrm{E}-01 & 0.000 \mathrm{E}+00 \ldots \\ 1.240 \mathrm{E}+00 & 1.960 \mathrm{E}-01 & 4.940 \mathrm{E}-01 & 0.000 \mathrm{E}+00 \ldots \\ 1.620 \mathrm{E}+00 & 1.930 \mathrm{E}-01 & 4.870 \mathrm{E}-01 & 0.000 \mathrm{E}+00 \ldots \\ 2.120 \mathrm{E}+00 & 1.870 \mathrm{E}-01 & 4.790 \mathrm{E}-01 & 0.000 \mathrm{E}+00 \ldots \\ 2.710 \mathrm{E}+00 & 1.870 \mathrm{E}-01 & 4.730 \mathrm{E}-01 & 0.000 \mathrm{E}+00 \ldots\end{array}$

Velocity Vector Plots. With Option 8 the user can generate both velocity vector plots and superimposed plots of particle trajectories and velocity vectors. It plots the U-V velocities as vectors with a magnitude proportional to the velocity at a grid point. For this case, the menu is : 
1. U-V Velocity Vectors

2. U-V Velocity Vectors and Coal Part. Trajs

3. U-V Velocity Vectors and Sorbent Part. Trajs.

4. Renormalize

5. Plot all vectors

6. Reduce number of vectors

7. RETURN

8. STOP

CHOOSE A NUMBER $(1-8)$

Note that the grid point corresponds to the originating end of the arrow. This does not mean that there is flow going into the wall but that the velocity at the grid point has the magnitude and direction shown in the piot. PCPLOT will automatically reduce the number of vectors plotted to eiiminate confusing page blackening from overlapping vectors. The user can plot the vectors at all grid points with Option 5. The user can further reduce the number of plotted vectors with Option 6.

For example, to generate Fig. 5-21, the following sequence was used:

ENTER FILENAME

CPR

cpr.pop IF FLLE EXISTS, DO YOU WANT TO WRITE OVER ? $(\mathrm{y} / \mathrm{n})$

$Y$

SELECT:

1. Gas Properties

2. Coal Particle Properties

3. Sorbent Particle Properties

4. Experimental Comparisons

5. Sox Pollutants

6. NOx Pollutants

7. Spreadsheet Format

8. Velocity Vectors plots

9. Zoom-in plots

10. Plot Grid Setup

11. Read New File Name 
12. Omit Titles

13. Normalize

14. Black and White

13. STOP

8

1. U-V Velocity Vectors

2. U-V Velocity Vectors and Coal Part. Trajs

3. U-V Velocity Vectors and Sorbent Part. Trajs.

4. Renormalize

5. Plot all vectors

6. Reduce number of vectors

7. RETURN

8. STOP

CHOOSE A NUMBER $(1-8)$

1

DOING VECTOR PLOTS

1. U-V Velocity Vectors

2. U-V Velocity Vectors and Coal Part. Trajs

3. U-V Velocity Vectors and Sorbent Part. Trajs.

4. Renormalize

5. Plot all vectors

6. Reduce number of vectors

7. RETURN

8. STOP

CHOOSE A NUMBER $(1-8)$

8 


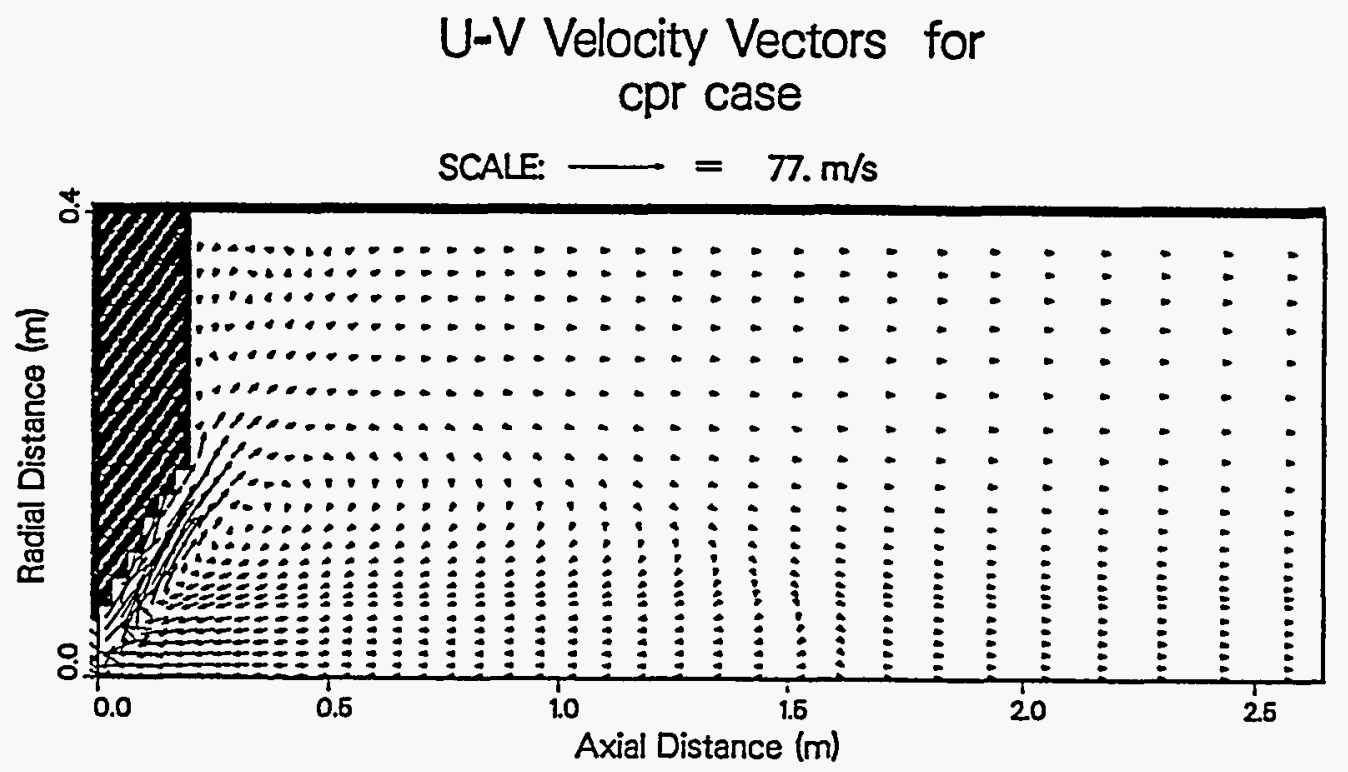

4 Velocty Ranges $-024 \varepsilon+02$ to $0.84 E+02 \mathrm{ln} / \mathrm{d}$ $\checkmark$ Volocity Punge: $-0.214 E+01$ to $0.202 E+02 \mathrm{ln} / \mathrm{l}$

Fig. 5-21. Sample U-V velocity vector plot.

If the user selects combined particle and trajectories plots, PCPLOT will prompt the user to specify whether he/she wants all the particle trajectories or several specific ones.

There are special cases when the resulting vectors are too large, and the plot is unclear. The user can renormalize the vectors using Option 4 . The user can do one or two normalizations. When the user selects this option, PCPLOT will display the values used in previous plots and will ask if the user will use one or two normalizations. For one normalization the user will need to give only one normalization value. 
For example, to generate both Figs. 5-22 and Fig. 5-23, the following sequence was used:

ENTER FILENAME

CPRCOAI

cprcoal.pop IE FILE EXISTS, DO YOU WANT TO WRITE OVER ? $(y / n)$

$\Psi$

SELECT :

1. Gas Properties

2. Coal Particle Properties

3. Sorbent Particle Properties

4. Experimental Comparisons

5. Sox Pollutants

6. NOx Pollutants

7. Spreadsheet Format

8. Velocity Vectors plots

9. Zoom-in plots

10. Plot Grid Setup

11. Read New File Name

12. Omit Titles

13. Normalize

14. Black and White

15. STOP

9

DO YOU WANT ONLY ZOOM-IN PLOTS? $(Y, N)$

$\mathbf{Y}$

SELECT :

1. Gas Properties

2. Coal Particle Properties 


\author{
3. Sorbent Particle Properties \\ 4. Experimental Comparisons \\ 5. Sox Pollutants \\ 6. NOx Pollutants \\ 7. Spreadsheet Format \\ 8. Velocity Vectors plots \\ 9. Zoom-in plots \\ 10. Plot Grid Setup \\ 11. Read New File Name \\ 12. Omit Titles \\ 13. Normalize \\ 14. Black and White \\ 15. STOP
}

8

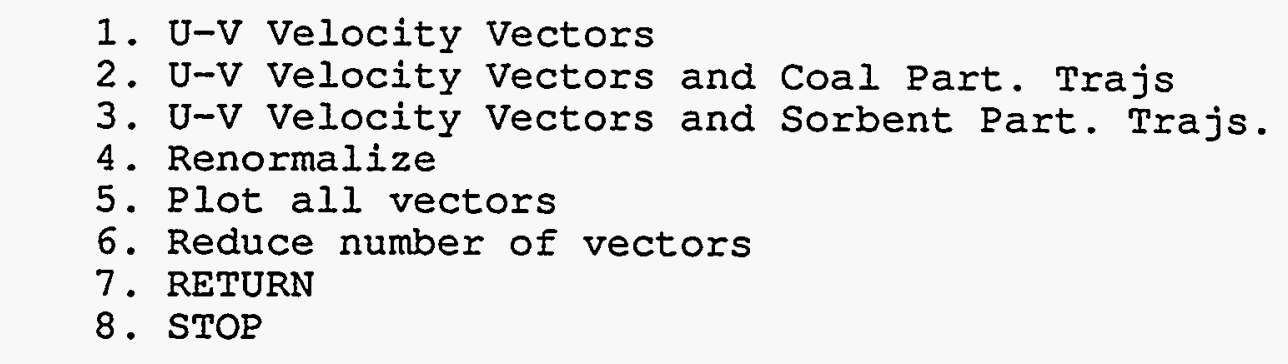

CHOOSE A NUMBER $(1-8)$

1

Initial $x$ value $(0,2.6)$

0 .

Einal $x$ value $(0,2.6)$

0.5

Initial $y$ value $(0,0.4)$

0 .

Final $y$ value $(0,0.4)$ 
0.2

DOING VELOCITY VECTORS PLOT

1. U-V Velocity Vectors

2. U-V Velocity Vectors and Coal Part. Trajs

3. U-V Velocity Vectors and Sorbent Part. Trajs.

4. Renormalize

5. Plot all vectors

6 . Reduce number of vectors

7. RETURN

8. STOP

CHOOSE A NUMBER $(1-8)$

4

VHNORM AND VLNORM FOR IAST RUN $47.2743 \quad 0$.

ONE OR TWO VECTOR NORMALIZATIONS ?

2

GIVE VECTOR IOW AND HIGH NORMAIIZATIONS

$47.3 \quad 94.6$

SMALI-TO-IARGE VECTOR DIVISION POINT

8

1. U-V Velocity Vectors

2. U-V Velocity Vectors and Coal Part. Trajs

3. U-V Velocity Vectors and Sorbent Part. Trajs.

4. Renormalize

5. Plot all vectors

6 . Reduce number of vectors

7. RETURN

8. STOP

CHOOSE A NUMBER $(1-8)$ 
DOING VECTOR PLOTS

1. U-V Velocity Vectors

2. U-V Velocity Vectors and Coal Part. Trajs

3. U-V Velocity Vectors and Sorbing Part. Trajs.

4. Renormalize

5. Plot all vectors

6. Reduce number of vectors

7. RETURN

8. STOP

CHOOSE A NUMBER $(1-8)$

8

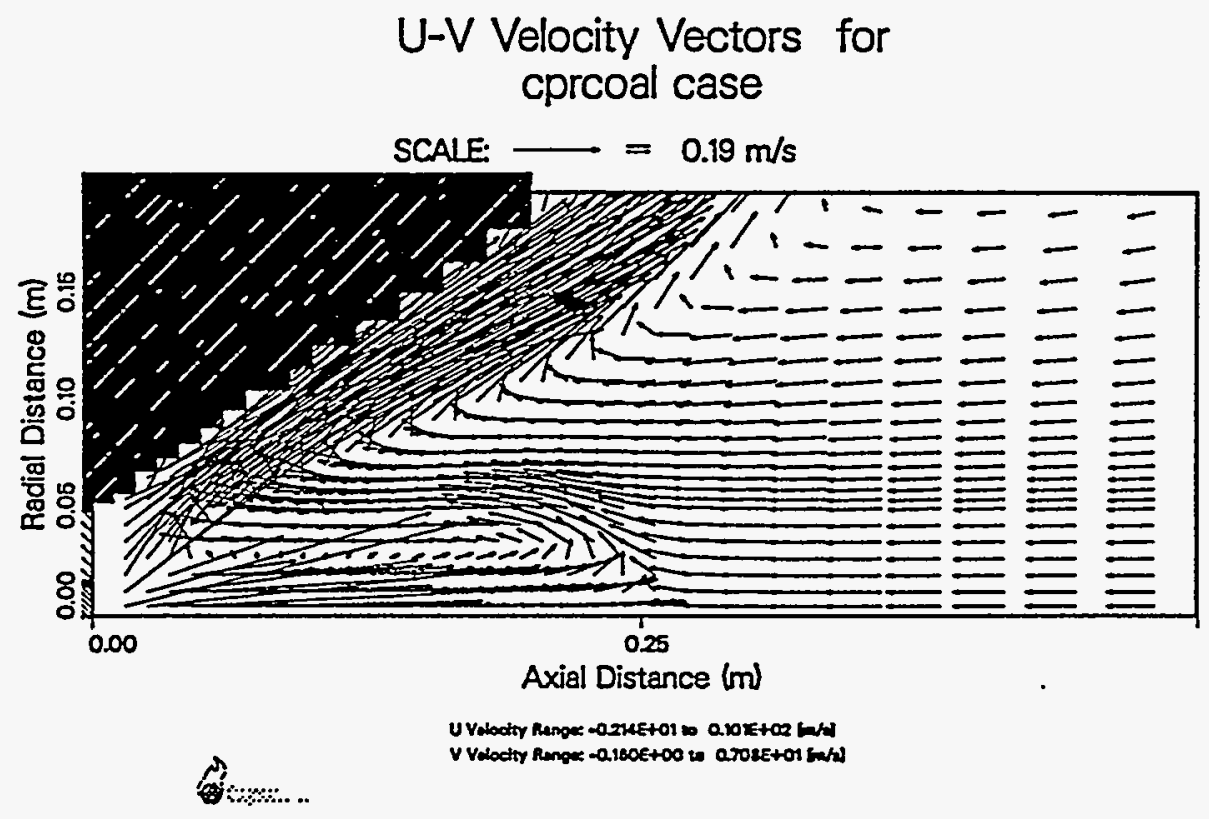

Fig. 5-22. Near-Burner U-V velocity vector plot. 
The velocity plot in Fig. 5-22 is a zoom-in of the near-burner region of the reactor and has a normalization value was 47.3. If two normalizations are used, the resulting plot will have two type of vectors. In this case, the user will have to give values for both high and low normalization as well as small-to-large vector division point. The vector division point decides at what length large instead of small vectors will be plotted. It is usually set to approximately 6.0 .

As shown, two normalization values were used: a high normalization value of 94.6 (twice the original normalization value) and low normalization value of 47.3. The division point value of $8(\sim=47.3 / 5.9)$ was used for normalizing only the vectors over $5.9 \mathrm{~m} / \mathrm{s}$ with the higher normalization value, a different type of vector, and half their original size.

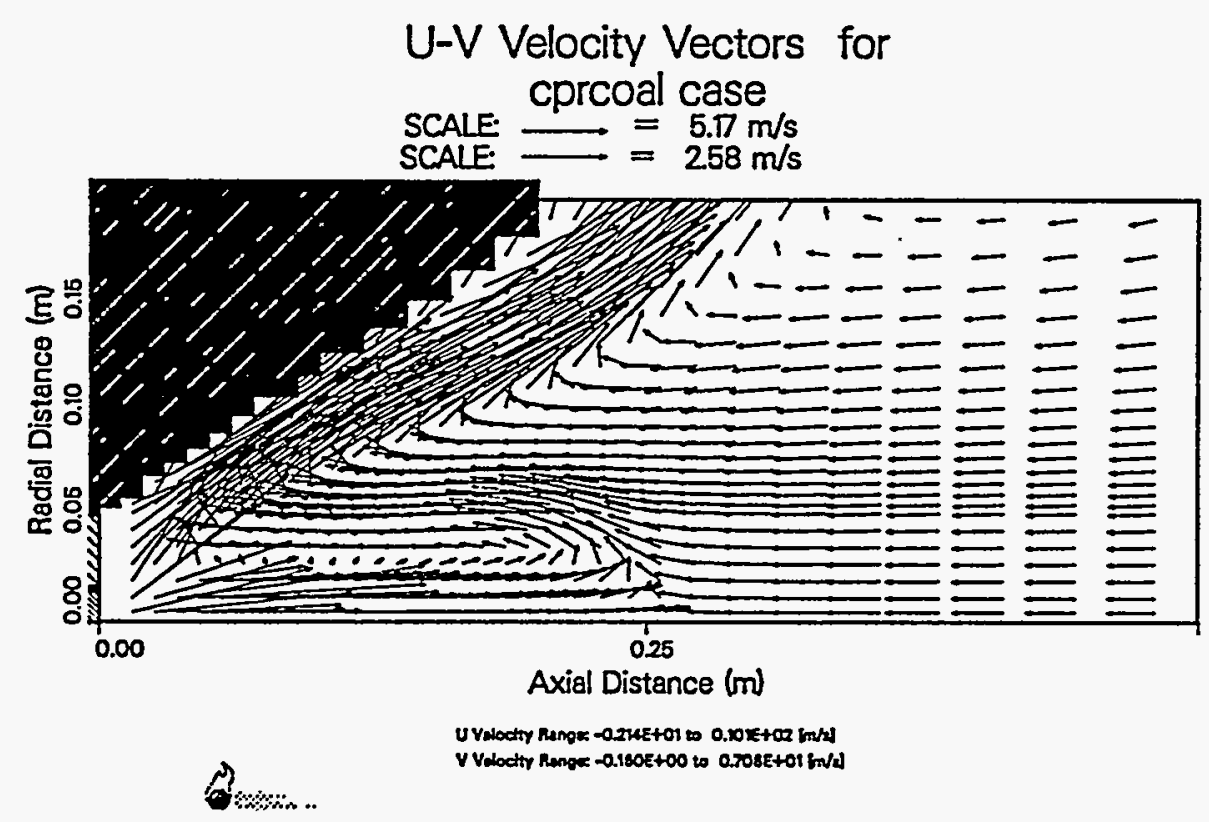

Fig. 5-23. Near-burner U-V velocity vector plot with two types of arrows representing two velocity scales. 
Zoom-in Plots. Option 9 in the main menu can be used to zoom in at the details of one or all variables in a given region. If the user wants zoom-in plots, he/she must specify whether he/she wants only these plots, or both complete data plots and zoom-in plots. Once the user turns on this option, all the following plots will be generated using this option. The first time a zoom-in plot is produced, the user must specify the initial and final $x$ and $y$ positions for the plots. PCPLOT will ask him/her for the values:

$$
\begin{array}{ll}
\text { initial } x \text { value } & \left(x_{0}, x_{n j}\right) \\
\text { final } x \text { value } & \left(x, x_{n j}\right) \\
\text { initial } y \text { value } & \left(y_{0}, y_{n j}\right) \\
\text { final } y \text { value } & \left(y, y_{n j}\right)
\end{array}
$$

where the allowed range for the input value is given in parentheses. $X_{0}$ is the original value at the origin of the $x$ axis, $y_{0}$ is the original value of the origin of the $y$ axis, $x_{n i}$ is the original value at the extreme of the $x$ axis, $y_{n j}$ is the original value of the extreme of the $y$ axis, and $x, y$ are the values typed by the user. If the user types a value outside the range given in the parentheses, the program will indicate the value is outside the range and will ask for new values.

Plot Grid Setup. The grid setup used by PCGC-2 can be plotted with Option 10. The location and type of cells is read from the .grd file. The resulting plot shows the grid points as well as the cell boundaries.

Read New File Name. A new group of data files can be read without leaving PCPLOT using Option 11. Once the name of a data file is read, PCPLOT checks to see if a . pop metafile exists. If the metafile exists, PCPLOT will advise the user of its existence. He/she can now write over this file or give a new name for the resulting metafile.

Omit Titles. The user can omit the titles on the plots by using Option 12. $\mathrm{He} /$ she can toggle back to typing titles by using Option 12 which will now read Print Titles. Option 12 is useful when preparing figures for reports, publications, etc.

Normalize. If the user wants to normalize the axis values, he/she can do so with Option 13. This option toggles between using normalized scales and actual scales. Option 13 is useful when preparing figures for presentation when the exact dimensions of the reactor are proprietary and cannot be divulged. 
Black and White. PCPLOT produces plots in black and white, or in color, according to the user's choice and his/her particular hardware. Initially the color is turned on. If the user selects Option 14 in the main menu, the color is turned off and the option will now read: Colors. The user can then toggle back to color plots again with this option. 


\section{Chapter Six}

\section{FG-DVC Submodel Descriptions}

The coal dependent capability of PCGC-2 is provided by FG-DVC submodel. The physical background of this submodel is given in Chapter Four. FG-DVC has three coal description files, i. e., a coal composition file, a kinetic file, and a polymer file, to form a set of descriptions of a coal's chemical and physical properties needed in its computation. The reactivity submodel has its own input file. This section discusses the FG-DVC subroutines and explains the structures of these input files. A list of subroutines is given in Figure 6-1.

Eight sets of coal input files for the eight Argonne coals are provided with the PCGC-2 package. Three additional sets of input files for three PSOC coals are provided for the interpolation of FG-DVC input data. The elemental compositions(DAF) of these coals and three PSOC coals are listed in Table 4-4. When running PCGC-2 with an Argonne coal on this list, a user only needs to specify the file names of the files of this coal. When using a coal not-on this list, the user needs to create a new set of files for the coal with PCGC-2 preprocessor PREC. All the FORTRAN variables of the input data are listed in Figure 6-2.

\section{FG-DVC Subroutines}

FG-DVC subroutines are called by PCGC-2 to compute the coal particle 
devolatilization, if the FORTRAN variable FGDVC in the PCGC-2 data input file is set to be TRUE. FG-DVC calculations are done at each time-temperature step. The main PCGC-2/FG-DVC interface is in COAL2. A logical flow diagram of this part of the program is given in Figure 6-3. In each step THER15P is called first and the FG part of the calculations is executed for each of the function groups there, and later PERCVAP is called for the DVC part. TBLRTS calculates the kinetic rates of all functional group pools as well as the bondbreaking rate at a given temperature. In PERCVAP, the bond breaking and cross linking rates, which are being calculated in parallel with FG calculations, are used to update the macromolecular network parameters $\mathrm{p}$ and $\mathrm{q}$. These two parameters are then used to calculated the new mass bin distribution and coal liquid fraction. The results are submitted to subroutine TARVAP to calculate the tar evaporation rate, in combination with coal dependent network properties such as the molecular weight distribution of ring clusters of coals. After returning to THER15P, appropriate function group redistributions are necessary due to the network change to further update the function group composition. COAL2 calls the other two subroutines VISCOSP and DWGOUT. VISCOSP calculates the coal viscosity, while DWGOUT calculates the total evolution rate and individual rates of all gas pools and calls SWESUB to invoke the coal particle swelling calculation. RK is called in SWESUB to perform Runge-Kutta integration at this time increment. SWEFUN, NGFUN, and WGFUN are the three functions used in the integration. FINFL1 is called to prepare the output.

In parallel to the FG-DVC calculations of the coal material, the pyrite decomposition is calculated by calling PYRITE1, PYDIST, and PYRITE2 at appropriate points in THER15P.

PER12 calculates the elemental compositions of char, tar, and gas phase. PER12P is the counterpart of PER12 for pyrite distribution.

GETCDF, GETKIN, and NETIN are three input subroutines to input the coal related data for FG-DVC routines. INITYO and REINIT initialize the functional group compositions and the coal network parameters at the beginning of the particle trajectory calculations. 
NTRGN2 is called from inside THER15P to compute the secondary nitrogenous gas reaction that converts $\mathrm{HCN}$ to $\mathrm{Nin}_{3}$.

\section{The Coal Composition File}

The coal composition file contains the elemental and functional group composition information of a coal. The structure of this file can be explained by an example in Figure 6-4. The first line of this file is the version number line. It must start with a version number in REAL format. Lines 2 through 6 are comment lines in which users can write anything they want. In this example, the second line gives the name of the coal. Line 7 is the fraction of the volatile pyrite sulfur that decomposes at low temperatures. Lines 8 through 12 contain the coal's elemental composition, while line 13 is the pyrite sulfur content, which should be given in grams per 100 grams DAF coal. Line 14 is not active. Lines 15 through 46 list the compositions of all the functional groups. Lines 47 through 52 are the fractions that determine how the pyrite decomposes into gases during the high temperature decomposition. Line 53 is the fraction of $-\mathrm{H}_{2} \mathrm{C}-\mathrm{CH}_{2}-$ type bridges among total bridges, which affects the ultimate yield of $\mathrm{CH}_{4}$.

Any data line users only the first value. Anything after one datum and a comma is considered as a comment. Merger of any two lines will cause severe problems.

\section{The Kinetics File}

The kinetics file contains kinetics that control the evolutions of all functional groups. An example is given in Figure 6-5. As in coal data files, the first line is the version line starting with a version number. Lines 2 through 40 are the $\sigma$ values of all the functional groups. Line 41 is the $\sigma$ for pyrite low temperature decomposition. After two inactive lines, lines 44 and 45 are the sigma values for pyrite $\mathrm{FeS}_{2}$ and $\mathrm{FeS}$. Lines 49 and 50 are the pre-exponential factor and activation energy for bond breaking. Lines 63 through 97 are the preexponential factors of all the functional groups, line 102 is for pyrite low 
temperature decomposition, and lines 103 and 104 of pyrite $\mathrm{FeS}_{2}$ and $\mathrm{FeS}$. Lines 105 through 139 are the activation energies of all the functional groups. Line 144 is for pyrite low temperature decomposition, and lines 145 and 146 of pyrite $\mathrm{FeS}_{2}$ and $\mathrm{FeS}$. All the other lines are inactive but should not be modified.

Any data line uses only the first value. Anything after one datum and a comma is considered as comment. Merger of any two lines will cause severe problems.

\section{The Polymer File}

The polymer file contains coal macromolecular network parameters. Its structure is displayed in Figure 6-6.

Among the few adjustable parameters in polymer files are the last two numbers at the second line of the files, $q_{0}$ and $p_{\mathrm{k} 0}$. $q_{0}$ is the initial value of the occupation probability of crosslinks, $\mathrm{q}$, and $\mathrm{p}_{\mathrm{b} 0}$ the initial value of the unbreakable bond fraction, $\mathrm{p}_{\mathrm{h}}$, (see Chapter 4 for the description of FG-DVC model). An increase of any of these two values will decrease the tar yield and the coal fluidity, with $\mathrm{p}_{\mathrm{b} 0}$ most effective for tar yield and $\mathrm{q}_{0}$ for fluidity. Table 6-1 lists the $\mathrm{q}_{0}$ and $\mathrm{p}_{\mathrm{b} 0}$ of the eight Argonne coals and they should be used as a reference in selecting these two values. In most of the cases users can use the polymer file of a coal on the list with $\mathrm{H} / \mathrm{C}$ and $\mathrm{O} / \mathrm{C}$ closest to those of the coal in use without any modification.

Another adjustable parameter in polymer files is $x t 0$, which is the pyridine extractable yield in weight fraction of a coal measured at room temperature. In FG-DVC, it is a measure of the initial looseness of a coal material and is used to calculate the initial breakable bond fraction, $\mathrm{p}_{0}$, with $\mathrm{p}_{\mathrm{b}}$.

mbin is the option number for mass bin file selection, and should be 1 for Pocahontas \#3 and 0 for all another Argonne coals. See Section Mass Bin Files. 
The Mass Bin File

\section{The Mass Bin File}

It is found that the weight distributions of oligomers in coals are invariant and independent of coal rank for almost all rank coals except very high rank coals like Pocahontas \#3 of the Argonne coals. So only two mass bin files are supplied, mass_bin and mass_bil. The former is for all Argonne coals except Pocahontas \#3, and contains the oligomer weight distributions up to 6-mers. The latter is for Pocahontas \#3 only.

\section{The Reactivity Input File}

Input parameters for the reactivity submodel are of two types : coal (and char) dependent parameters, and model dependent parameters. Parameters from the first type include : eps0, min0, psi, radp, surfo, while the parameters $E 1_{\mathrm{jl}}$, $\mathrm{E}_{\mathrm{jl}}$, Em and tau0 are considered as model parameters, and are kept constant. The parameter "thiele" is a logical variable which, when set to "true", corresponds to the use of the Thiele modulus to calculate the rate in the pore diffusion regime.

The internal surface area surfo is obtained by $\mathrm{N}_{2}$ or $\mathrm{CO}_{2}$ adsorption (with the char having at least $5 \%$ burnoff), and is typically found in the range 50 to $1000 \mathrm{~m}^{2} / \mathrm{g}$. An average value which can be taken is $300 \mathrm{~m}^{2} / \mathrm{g}$. The porosity eps0 varies with the pyrolysis conditions (the higher the heating rate, the more porous the resulting char) and the starting coal type (chars from high rank coals have, in general, lower porosities then the ones from low rank coals). Chars from high rank coals usually give porosities between 0.1 and 0.3 , while those from low rank coals show porosities in the range 0.5 to 0.8 . The mean pore radius radp can be obtained experimentally from pore size distribution measurements using gas adsorption. For high heating rate chars (which is typical for pulverized coal conditions), a useful range for this parameter is $100-200 \AA$. The parameter $\min 0$ is coal dependent, is used in the case of low rank coals only, and represents the amount of dispersed minerals (in particular calcium), as measured by FTIR and SEM. As an example, in the case of a Zap lignite, a 
typical value for this parameter is about $1 \%$, while for a Wyodak sub-bituminous coal the value is about $0.9 \%$. The parameter psi is used to represent the variations of internal surface area (in the kinetic regime, for high rank coals) as a function of burnoff, and is char dependent. Its value generally falls between 5 and 10.

Concerning the model parameters, tau 0 represents the tortuosity of the char pore network, cannot be measured directly and is consequently kept to a constant value of 2. E1 is the intrinsic activation energy, which has been found to be relatively constant for different chars, and is equal to $30 \mathrm{kcal} / \mathrm{mol}$. E2 represents the activation energy observed in the pore diffusion regime. This parameter is generally not used since, following the Thiele model, half the intrinsic activation energy is usually taken for the pore diffusion regime. Em represents the energy of "deactivation" (to account for sintering, etc..) of the minerals, and is in effect in the pore diffusion regime (i.e. at high temperature). "Thiele" is a flag which, when set to "true", allows the calculation to follow the Thiele model. In the case where "thiele" is set to "false", E2 is used for the activation energy in the pore diffusion regime.

\section{FG-DVC Input Data Creation and Maintenance}

The stand-along FG-DVC has a utility program COALS for creating coal input data files for a new coal and maintaining the existing coal data files. The full capability of COALS has been incorporated into PCGC-2's preprocessor PREC. When users click on a designated button in PREC's pull down menu, the appropriate functions of COALS will be invoked. If users elect to enter a new coal, the program enters the creation mode. If users type in some valid file names of existing FG-DVC coal data files, it is in the maintenance mode. This section discusses the basic concepts of COALS. For operational instructions of PREC, read appropriate sections in this manual.

In the creation mode, COALS interpolates the functional group composition and gas evolution kinetics of a new coal from the data of six of the 
Argonne coals and three additional PSOC coals. The interpolation is based on a triangular finite element construction and uses $\mathrm{H} / \mathrm{C}$ and $\mathrm{O} / \mathrm{C}$ as two basic coordinates. The triangular finite element mesh in the $\mathrm{H} / \mathrm{C}-\mathrm{O} / \mathrm{C}$ two dimensional plane is plotted in Figure 6-7. The nine nodal points correspond to the nine coals, while the two points not connected are Stockton and Pocahontas \# 3 which are not selected for interpolations. Interpolations can be performed for coals with $\mathrm{H} / \mathrm{C}$ an $\mathrm{O} / \mathrm{C}$ inside this range only. Any coal with its elemental composition out of this range is not covered. In this mode, users need to give the elemental composition(DAF) of the new coal with the content of pyrite sulfur given in grams of sulfur in pyrite per 100 grams of DAF coal. Upon requests, COALS will display the functional group compositions and the pool kinetics created by the interpolation and enter the maintenance mode in which manual adjustments can be done.

COALS does not create FG-DVC coal polymer data, since the rank dependence of the coal network is more complicated and has not been fully studied. Based on the elemental compositions users entered, COALS recommends users to use one of the polymer files of the eight Argonne coals and the three PSOC coals. Modifications of the polymer files have to be done with a text editor, like UNIX vi.

In the maintenance mode, the interpolation step is skipped and users have choices to enter the composition or kinetics branch of the code.

\section{Composition Branch}

In the composition branch, the functional group composition of the coal is displayed and users have three options: 1) creating a new coal composition file, 2) modifying the composition manually, 3) exit to main selection. By choosing option 2, users can modify the FG composition according to their own knowledge of the coals. It is very important for users to understand that most of the pools cannot be adjusted without changing other pools because of the mass conservation. Oxygen is distributed in $\mathrm{H}_{2} \mathrm{O}, \mathrm{CO}, \mathrm{CO}_{2}$ and, in relatively small amount, $\mathrm{SO}_{2}$ and COS. Altering any of these pools will cause other pool sizes to be changed. The same situation exists for the sulfur and nitrogen pools. In 
other words, changes in one pool will cause reshuffling of other pools based on the mass conservation of $\mathrm{O}, \mathrm{S}$, and $\mathrm{N}$. Internal rules in COALS in this respect are:

1) Total amount of $\mathrm{H}_{2} \mathrm{O}, \mathrm{CO}$, or $\mathrm{CO}_{2}$

Adjusting the size of any one of the above will cause one of the others to change size, according to the following rule:

$$
\mathrm{H}_{2} \mathrm{O} \rightarrow \quad \mathrm{CO} \rightarrow \mathrm{CO}_{2} \rightarrow \mathrm{H}_{2} \mathrm{O}
$$

while maintaining the total oxygen content in these three pool groups unchanged.

2) Individual pool sizes of $\mathrm{H}_{2} \mathrm{O}, \mathrm{CO}$ and $\mathrm{CO}_{2}$ pools

Adjusting the size of any one of the individual pools of one of the above pool groups will cause one of the other pools in the same group to change size, according to the following rule:

\section{Extra Tight $\rightarrow$ Tight $\rightarrow$ Loose $\rightarrow$ Extra Loose $\rightarrow$ Extra Tight}

while maintaining the total amount of this pool group unchanged. For instance. A reduction of $\mathrm{CO}$ loose pool size will lead to an increase in the $\mathrm{CO}$ extra loose pool size, and so on.

3) Individual pool sizes of the organic sulfur pools

Adjusting the size of any one pool in the sulfur pool group except $\mathrm{H}_{2} \mathrm{~S}$ tight will cause $\mathrm{H}_{2} \mathrm{~S}$ tight to change size to maintain the sulfur content. Changing the $\mathrm{H}_{2} \mathrm{~S}$ tight pool size can only be done indirectly by changing the other sulfur pool sizes. 
Notice that the changes in $\mathrm{SO}_{2}$ and $\mathrm{COS}$ pool sizes will alter oxygen content in $\mathrm{H}_{2} \mathrm{O}, \mathrm{CO}$ and $\mathrm{CO}_{2}$ pools, and therefore modify these pool sizes.

4) Individual pool sizes of the organic nitrogen pools

Adjusting the size of any one pool in the nitrogen pool group except HCN tight will cause $\mathrm{HCN}$ tight to change size to maintain the nitrogen content. Changing the HCN tight pool size can only be done indirectly by changing the other nitrogen pool sizes.

5) Individual pool sizes of $\mathrm{CH}_{4}$, Olefin and Paraffin, and the fraction of $\mathrm{CH}_{2} \mathrm{CH}_{2}$ - type bond, $\mathrm{X}_{-} \mathrm{CH}_{4}$.

These pools can be modified independently. The $\mathrm{CH}_{4}$ pool amounts will be altered in the FG-DVC subroutine REINIT according to source term redistribution rules. The additional $\mathrm{CH}_{4}$ contribution from $-\mathrm{CH}_{2}-\mathrm{CH}_{2}$ - bridge breaking, termed terminal $\mathrm{CH}_{4}$, changes the ultimate $\mathrm{CH}_{4}$ yield. Modifying $\mathrm{X}_{-} \mathrm{CH}_{4}$ in coal data files changes the amount of this contribution.

$\mathrm{X}_{-} \mathrm{CH}_{4}$ is assigned to pool number 61 in COALS only. It is not a gas pool in PCGC2/FG-DVC. It is the fraction of $-\mathrm{CH}_{2} \mathrm{CH}_{2}$ - type bond that creates two terminal $\mathrm{CH}_{3}$ 's when it breaks.

6) Pyrite pools

Pyrite in coals will decomposes into various sulfur gases and FeS during the pyrolysis. In FG-DVC, FeS is assumed stable in pyrolysis. The other half of the sulfur from the pyrite evolves into $\mathrm{H}_{2} \mathrm{~S}, \mathrm{SO}_{2}, \mathrm{COS}$ and $\mathrm{CS}_{2}$. Part of it could stay in the coal structure as char sulfur. Users are allowed to specify the fractions of evolving pyritic sulfur into the above pools. Note that it is the fraction of evolving pyritic sulfur(half of the total pyritic sulfur) that is used.

Pyrite decomposes at two temperatures. The low temperature 
decomposition produces $\mathrm{H}_{2} \mathrm{~S}$ only. Changing the size of this pool will change the pools in the high temperature decomposition group proportionally.

In the higher temperature group, all the pools except $\mathrm{H}_{2} \mathrm{~S}-\mathrm{GAS}$ can be modified directly, but the $\mathrm{H}_{2} \mathrm{~S}$-GAS pool size changes indirectly if any of those pools gets changed.

These pool numbers are valid in COALS only.

7) Other pools

All the other pools are not manually adjustable, but aliphatic $\mathrm{C}$ and $\mathrm{H}$ pools act like two reservoirs for $\mathrm{C}$ and $\mathrm{H}$, respectively. They save all the remaining $\mathrm{C}$ and $\mathrm{H}$ after they are distributed into the other pools.

Increasing or reducing any pool too much will cause some component pool sizes to be negative, suggesting further adjustments.

\section{Kinetics Branch}

In kinetics branch, users can select a number of pools to modify their devolatilization kinetics, which include the sigma and the mean activation energy for the distributed activation energy model, and the pre exponential factor. The modifications are straight forward.

\section{Coals not in the range}

There will be cases in which users have coals with elemental compositions outside the $\mathrm{H} / \mathrm{C}-\mathrm{O} / \mathrm{C}$ range covered by COALS. If that happens, follow the suggestions here to create the coal composition files:

Step 1. Take the coal composition file of a coal with the elemental composition closest to the new coal, and use a text editor to modify the file so that the composition of the new coal 
is specified, referring to the section of Coal Composition File and Figure 6-4. Save the file.

Step 2. Use COALS to modify this file further.

To create a new kinetics file for this coal, compare the coal elemental composition with those of the eleven coals provided, select the kinetics file of one of these coals, which has its composition closest to that of the new coal, and modify it with PREC based on your own knowledge of the coal. After the kinetics of file an Argonne coal has been selected, the polymer file of the same coal should be used for the new coal. 


\section{FG-DVC Subroutines}

AMNT

DECOD

DWGOUT

ELBACK

FINFL1

FINFL2

FKSU

FNPQ

FPQ

Figure 6-1 FG-DVC Subroutines files. oligmers. FPQ.
Real function for calculating the amount of change in a given functional group pool.

Subroutine for reading the value of a variable from input

Subroutine to calculate the time derivatives of gas species and to call the swelling subroutine.

Subroutine calculating at the end of particle trajectory the amount of coal species that reacted with pyrite.

Subroutine for preparing an output file (Part I).

Calculates updated amounts and elemental compositions of char, tar, gases and their elemental compositions.

Subroutine for preparing an output file (Part II).

Calculates the distribution of individual functional groups in char, tar, gases, and missing.

Real Function to calculate the probability of a site belonging to an n-mer from a given combination of two

Subroutine to obtain tar, extract, and liquid fractions using percolation theory. It uses or calls FKSU and

Subroutine to calculate the liquid fraction and the mean cluster size for a given Bethe lattice. 
GETCDF

GETKIN

INITYO

KBAR

MAKNAM

NETIN

NETOUT

NFCN

NGFUN

NTRGN2
Subroutine to read a coal composition file (elemental composition and functional group composition of a specified coal).

Subroutine to read a kinetic file, including frequency factors, activation energies and their distribution parameters for all functional groups.

Subroutine to initialize all functional groups in terms of weight fraction (daf basis).

Real function for averaging kinetic rates for a given FG pool based on distributed activation energies.

Subroutine for making a name in output for a specified time step.

Subroutine to read the coal network/polymer file and to calculate the related network parameters.

Subroutine to write the computational results in DVC part into an output file.

Real function for calculating new distribution functions for kinetic rates.

Real funtion for evaluating ambient gas diffusion function in swelling integration.

Subroutine to compute the nitrogen gas evolutions.

Figure 6-1 FG-DVC Subroutines - continued 
PER12 Subroutine to calculate the elemental composition in char, tar, and gas phase, etc.

PER12P Subroutine to calculate the elemental contents in char, tar, and gas phase, contributed by pyrite.

PERCVAP Driver routine to perform the DVC computations using percolation theory.

PYDIST Subroutine to distribute pyrite decomposition products into gas pools.

PYRITE1 Subroutine to calculate pyrite thermo-decomposition, part 1.

PYRITE2 Subroutine to calculate pyrite thermodecomposition, part 2.

REINIT Subroutine to reinitialize variables to zero or their starting values.

RK Subroutine to perform Runge-Kutta integration of swelling equations.

SWEFUN Real function to evaluate the swelling function in swelling integration.

SWELLDATA Block data subroutine for swelling parameters.

SWESUB Driver subroutine to simulate swelling process based on the given viscosity values computed in subroutine VISCOS and total gas evolution rate.

Figure 6-1 FG-DVC Subroutines - continued 
TARVAP

TBLRTS

THER13P

THER15P

VISCOSP

VOLSWL

WGFUN

WRTOUT
Subroutine to calculate tar vaporization and incorporate the results into the percolation computations.

Subroutine for setting up kinetic rates for functional groups, performed at each time step.

Driver routine to simulate pyrolysis using the FG-DVC model. Uses theory 13 and assumes that evolved tars are removed immediately or stay for a short residence time to further produce gases.

Driver routine to simulate pyrolysis using the FG-DVC model. Uses theory 15 and assumes that evolved tar further reacts to produce gases the same way as char does.

Real function for calculating viscosity of coal at a given temperature.

Subroutine for calculating the volumetric swelling ratio from crosslink density.

Real funtion for evaluating devolati-lized gas diffusion function in swelling integration.

Subroutine for writing the computational results of the FG part into an output file.

Figure 6-1 FG-DVC Subroutines - continued 


\begin{tabular}{|c|c|c|c|}
\hline Variables & Type & Unit & Description and Usual Symbol \\
\hline A1 to A44 & Real & Kelvin & $\begin{array}{l}\text { Activation energy of pool } \\
\text { devolatilizations, } E_{i} \text { for } k_{i} \text { in Eq. } \\
(4-10) \text {. }\end{array}$ \\
\hline amvsol & Real & -- & $\begin{array}{l}\text { Molar volume of solvent (pyridine), } \\
\text { not used in PCGC- } 2 \text {. }\end{array}$ \\
\hline amwcol & Real & -- & $\begin{array}{l}\text { Start crosslinking density, not used } \\
\text { in PCGC- } 2 \text {. }\end{array}$ \\
\hline atar & Real & Kelvin & $\begin{array}{l}\text { Activation energy of bond } \\
\text { breaking, for } k_{B} \text { in Eq. (4-1). }\end{array}$ \\
\hline $\mathrm{B} 1$ to $\mathrm{B} 44$ & Real & $\mathrm{s}^{-1}$ & $\begin{array}{l}\text { Pre-exponential factor of pool } \\
\text { devolitilization } k_{i}^{0} \text { for } k_{i} \text { in Eq. (4-. } \\
\text { 10). }\end{array}$ \\
\hline btar & Real & $\mathrm{s}^{-1}$ & $\begin{array}{l}\text { Pre-exponential factor of bond } \\
\text { breaking, for } k_{B} \text { in Eq. }(4-10) \text {. }\end{array}$ \\
\hline bead & Integer & -- & $\begin{array}{l}\text { Total number of monomers in } \\
\text { network sample, not used. }\end{array}$ \\
\hline cnst2 & Real & atm & $\begin{array}{l}\text { Gas pressure drop from outside to } \\
\text { inner coal pores, } \triangle \mathrm{P} \text { in Eq. (4-6) } \\
\text { AND (4-7). }\end{array}$ \\
\hline
\end{tabular}

Figure 6-2 A list of FORTRAN variables of the input data for FG-DVC. 


$\begin{array}{llll}\text { Variables } & \text { Type } & \text { Unit } & \text { Description and Usual Symbol } \\ \text { density } & \text { Real } & \text { g/cm3 } & \text { Coal density, not used PCGC-2. } \\ \text { dvg3 } & \text { Real } & - & \text { Not used. } \\ \text { dvg4 } & \text { Real } & - & \text { Not used. } \\ \text { dummy } & \text { Real } & - & \text { Dummy variable. } \\ \text { fethyl } & \text { Real } & -- & \begin{array}{l}\text { Initial number of hard (double) } \\ \text { bonds, not used in PCGC-2. }\end{array} \\ \text { flink } & \text { Real } & - & \begin{array}{l}\text { Initial number of crosslinks in } \\ \text { starting coal, not used in PCGC-2. }\end{array} \\ \text { Idummy } & \text { Logical } & - & \begin{array}{l}\text { Dummy variable. } \\ \text { massim(i) }\end{array} \\ \text { Real } & - & \text { Char bin parameters, not used. } \\ \text { mers } & \text { Integer } & - & \begin{array}{l}\text { Number of mass bins for tar } \\ \text { vaporization. }\end{array} \\ \text { mermas(i) } & \text { Real } & \text { Dalton } & \begin{array}{l}\text { Binned mass of oligmers for tar } \\ \text { vaporization. }\end{array} \\ \text { oligst } & \text { Real } & - & \begin{array}{l}\text { Starting number of oligomers in the } \\ \text { network, not used. }\end{array}\end{array}$

Figure 6-2 A list of FORTRAN variables of the input data for FG-DVC, (continued). 


\begin{tabular}{|c|c|c|c|}
\hline Variables & Type & Unit & Description and Usual Symbol \\
\hline ph0 & Real & -- & $\begin{array}{l}\text { Initial hard bond fraction of the } \\
\text { coal network, used with xt0 to } \\
\text { initialize } p \text {. }\end{array}$ \\
\hline phimax & Real & - & $\begin{array}{l}\text { Volume fraction of solid in coal at } \\
\text { gel point for coal viscosity model, } \\
\phi_{c} \text { in Eq. }(4-13) \text { and }(4-15) \text {. }\end{array}$ \\
\hline q0 & Real & -- & $\begin{array}{l}\text { Initial occupation probability of } \\
\text { crosslinks, } \mathrm{q}_{0} \text {. }\end{array}$ \\
\hline radis0 & Real & -- & Coal particle radius, not used. \\
\hline $\mathrm{s} 1, \mathrm{~s} 2$ & Integer & -- & $\begin{array}{l}\text { Coal network coordination } \\
\text { numbers, } \sigma_{1} \text { and } \sigma_{2} \text {. }\end{array}$ \\
\hline sigma(i) & Real & Kelvin & $\begin{array}{l}\text { Standard deviation of Gaussion, for } \\
\text { distributed activation energies of } \\
\text { pool deviations, } \sigma_{i} \text { for } k_{i} \text { in Eq. (4- } \\
\text { 10). }\end{array}$ \\
\hline viseng & Real & Kelvin & $\begin{array}{l}\text { Activation energy for coal } \\
\text { viscosity, } k_{E} \text { in Eq. (4-13) and (4- } \\
\text { 15). }\end{array}$ \\
\hline vismax & Real & pacal*sec & Maximum viscosity. \\
\hline vsrcol & Real & -- & $\begin{array}{l}\text { Volumetric swelling ratio of raw } \\
\text { coal, not used. }\end{array}$ \\
\hline
\end{tabular}

Figure 6-2 A list of FORTRAN variables of the input data for FG-DVC, (continued). 


\begin{tabular}{|c|c|c|c|}
\hline Variables & Type & Unit & Description and Usual Symbol \\
\hline vsrmin & Real & - & $\begin{array}{l}\text { Minimum volumetric swelling ratio } \\
\text { of char, not used. }\end{array}$ \\
\hline $\operatorname{xeff(i)}$ & Real & - & Crosslink coefficients of gas pools. \\
\hline $\mathrm{xt0}$ & Real & -- & $\begin{array}{l}\text { Weight fraction of solvent } \\
\text { extractables of starting coal at room } \\
\text { temperature, use with ph0 to } \\
\text { initialize p. }\end{array}$ \\
\hline $\mathrm{E} 1(\mathrm{j}, \mathrm{I})$ & Real & - & $\begin{array}{l}\text { Intrinsic activation energy for } \\
\text { reactivity model, for particle size } \\
\text { index } \mathrm{j} \text { and reaction } \mathrm{l}, \mathrm{E} 1_{\mathrm{jl}} \text {. }\end{array}$ \\
\hline $\mathrm{E} 2(\mathrm{j}, \mathrm{I})$ & Real & - & $\begin{array}{l}\text { Activation energy in the pore } \\
\text { diffusion regime, for particle size } \\
\text { index } j \text { and reaction } 1, E 2_{j l} \text {, } \\
\text { reactivity model. }\end{array}$ \\
\hline $\operatorname{Em}(J, \mathrm{I})$ & Real & - & $\begin{array}{l}\text { Activation energy for } \\
\text { "deactivation" of the minerals (to } \\
\text { account for sintering), for particle } \\
\text { size index } j \text { and reaction } 1, \mathrm{Em}_{\mathrm{jl}} \text {, } \\
\text { reactivity model. }\end{array}$ \\
\hline eps0 & Real & -- & $\begin{array}{l}\text { Tortuosity of the char network. } \\
\text { Since it cannot be measured, it is } \\
\text { kept at a constant value of } 2 \text {, eps } 0 \text {, } \\
\text { reactivity model. }\end{array}$ \\
\hline
\end{tabular}

Figure 6-2 A list of FORTRAN variables of the input data for FG-DVC, (continued). 


\begin{tabular}{|c|c|c|c|}
\hline Variables & Type & Unit & Description and Usual Symbol \\
\hline $\min 0$ & Real & - & $\begin{array}{l}\text { Dispersed minerals concentration } \\
\text { (in \%). Represents the amount of } \\
\text { calcium as measured by FTIR and } \\
\text { SEM, min0, reactivity model. }\end{array}$ \\
\hline psi & Real & -- & $\begin{array}{l}\text { Structure parameter, to represent } \\
\text { variations in internal surface area } \\
\text { for the kinetic regime, for high } \\
\text { rank coal. It's value usually falls } \\
\text { between } 5 \text { and } 10, \Psi \text {, reactivity } \\
\text { model. }\end{array}$ \\
\hline radp & Real & - & $\begin{array}{l}\text { Mean pore radius. Obtained from } \\
\text { gas adsorption measurements, radp, } \\
\text { reactivity model. }\end{array}$ \\
\hline thiele & Logical & - & $\begin{array}{l}\text { true. means that the Theile model } \\
\text { is used, i.e. that } \mathrm{E} 1_{\mathrm{jl}} / 2 \text { is the } \\
\text { activation energy used in the pore } \\
\text { diffusion regime. If. false., then } \\
\mathrm{E} 2_{\mathrm{jl}} \text { is used, reactivity model. }\end{array}$ \\
\hline surfo & Real & -- & $\begin{array}{l}\text { Internal surface area in } \mathrm{m}^{2} / \mathrm{g} \text {, } \\
\text { obtained from gas adsorption, } \\
\text { surfo, reactivity model. }\end{array}$ \\
\hline $\operatorname{tau} 0$ & Real & - & $\begin{array}{l}\text { Tortuosity factor, taken as a value } \\
\text { of } 2 \text {, tau0, reactivity model. }\end{array}$ \\
\hline
\end{tabular}

Figure 6-2 A list of FORTRAN variables of the input data for FG-DVC, (continued). 


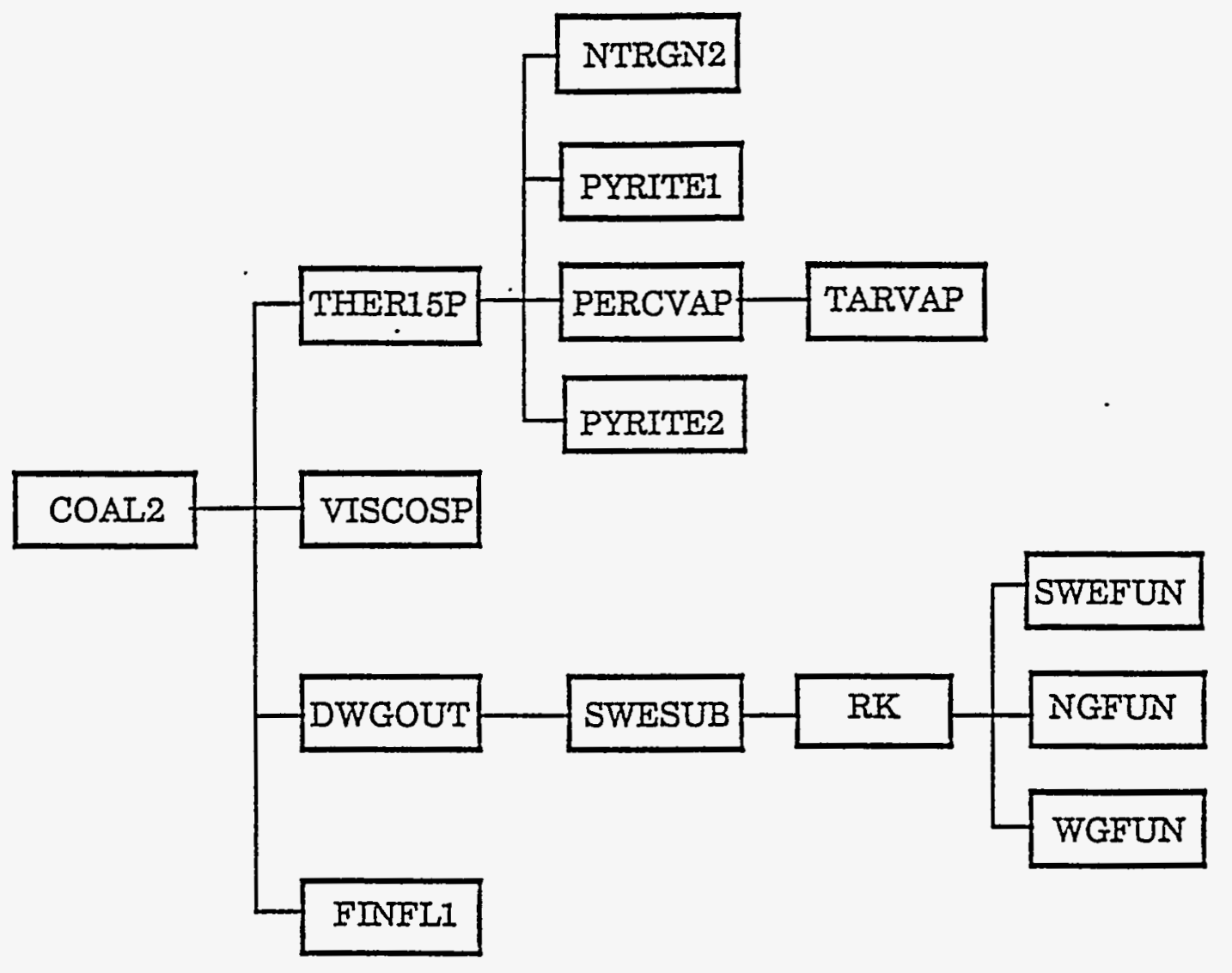

Figure 6-3 FG-DVC Subroutine Called from COAL2. 
7.0 , created by "coals"

Illnois \#6, Argonne Coal

comment line

comment line

comment line

0.000000 , inactive

0.1860 , Fraction of low temp. FeS2

77.7200, XCO

$5.0000, \mathrm{XHO}$

13.5300, XO0

$1.3700, \mathrm{XNO}$

2.3800, XSO

3.320000 , XSM0

0.000000 , inactive

0.109500 , XOH

$0.011627,1-\mathrm{CO} 2$

$0.001500,2-\mathrm{NH} 3-$ loose

0.530000 , 3-Fraction of $\mathrm{CO} 2$ tight

$0.015711,5-\mathrm{H}$-aromatic

$0.009762,6-\mathrm{HCN}$ tight

$0.000000,7-\mathrm{HCN}$ loose

0.600000 , 8-Fraction of $\mathrm{H} 2 \mathrm{O}$ tight

0.080000 , 9-Fraction of $\mathrm{H} 2 \mathrm{O}$ extra-loose

$0.034401,10-\mathrm{CO}$-extra-tight

0.931507 , 11-Fraction of Water in $\mathrm{OH}$

0.119996 , 12-Fraction of $\mathrm{CO} 2$ extra-loose

0.003225 , 13-CO-loose

$0.000000,14-T e r m i n a l ~ C H 4$, not an input

$0.000000,15-\mathrm{CO}-\mathrm{XX}$-tight

$0.001000,16-\mathrm{SO} 2$

$0.001000,17-\mathrm{COS}$

$0.000000,18-\mathrm{CS} 2$

0.004300 , 19-H2S-tight

Figure 6-4 An example of the coal composition file. 

$0.011400,21-$ Char $S$
$0.000000,22$-Empty
$0.000000,24-\mathrm{NH} 3$-tight
$0.000000,25$-Empty
$0.004000,26-\mathrm{C} 2 \mathrm{H} 4$
$0.000000,27-\mathrm{C} 3 \mathrm{H} 6$
$0.027000,28-$ Olefins-C $6 \mathrm{H} 12$
$0.004000,29-\mathrm{C} 2 \mathrm{H} 6$
$0.000000,30-\mathrm{C} 3 \mathrm{H} 8$
$0.025000,31$-Paraffins-c $6 \mathrm{~h} 14$
$0.030500,32-\mathrm{CH} 3$
0.622951 , 33-Fraction of $\mathrm{CH} 4$ tight
0.550000 , 37-Carbon
0.050000 , 1-SO2-pyrite
$0.050000,2$-COS-pyrite
$0.000000,3-C S 2$-pyrite
$0.400000,4-H 2 S-L O O S E-p y r i t e$
$0.000000,5-\mathrm{H} 2 \mathrm{~S}$-TIGHT-pyrite
$0.000000,6$-CHAR-S
$1.000000, \quad-X \_C H 4-C-C-B R I D G E$ FRACTION

Figure 6-4 An example of the coal composition file (continued). 
FG-DVC Submodel Descriptions

7.0, VERION \# /OCT., 1991/

1750., $\rightarrow$ 1200., SIGMA1-CO2-LOOSE *** / ILLIN6 / ARGONNE /

OCTOBER $90 /$

1500.,2000., SIGMA2-NH3

2750., --> 4500., SIGMA3-CO2-TIGHT

2000., SIGMA4-CO-TIGHT

6000., SIGMA5-HYDROGEN

3000.,4750., SIGMA6-HCN-TIGHT

3000 ., 1500., SIGMA7-HCN-LOOSE

2800., SIGMA8-H20-TIGHT

1800., SIGMA9-H2O-EX-LOOSE

3000., SIGMA10-CO-EX-T

1500., SIGMA11-H20-LOOSE

3000., SIGMA12-CO2-EXTRA-LOOSE

1000., SIGMA13-CO-LOOSE

2500., 2000., SIGMA14-TERMINAL METHYL

1000.0, SIGMA15-CO-XXT

1000.0, SIGMA16-SO2

1000.0, SIGMA17-COS

1000.0, SIGMA18-CS2

2500.0, SIGMA19-H2S-TIGHT

1000.0, SIGMA20-H2S-LOOSE

2000.0, SIGMA21-CHAR-S

1000.0, SIGMA22-EMPTY

2000.0, SIGMA23-CHAR-N

2500., 2000.0, SIGMA24-NH3-TIGHT

3000.0, SIGMA25-HCN-to-NH3

1500., SIGMA26-C2H4

1500., SIGMA27-C3H6

1500., SIGMA28-OLEFINS

1500., SIGMA29-C2H6

1500., SIGMA30-C3H8

1500., SIGMA31-PARAFFINS

Figure 6-5 An example of the kinetic file. 


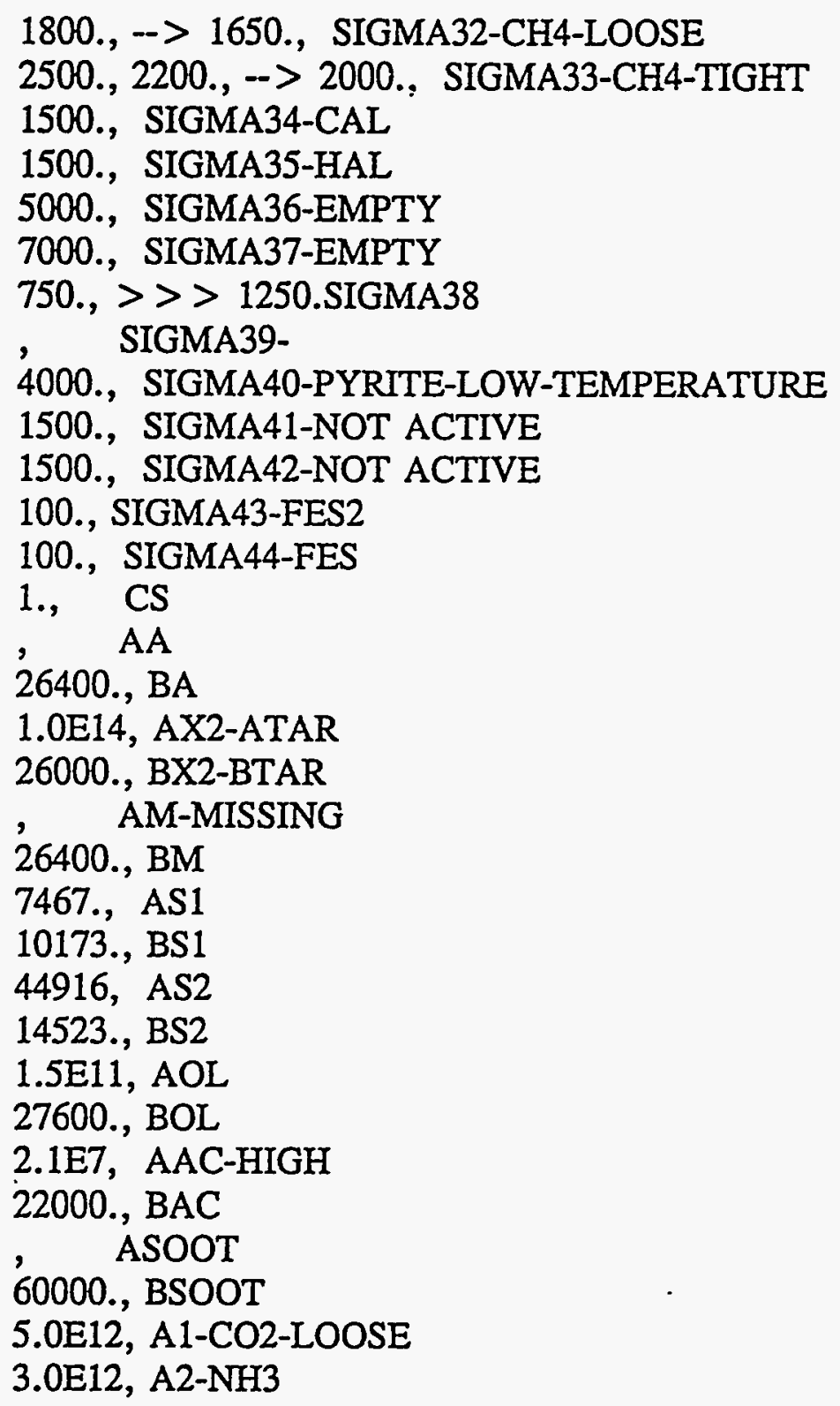

Figure 6-5 An example of the kinetic file (continued). 
FGDVC Submodel Descriptions

7.5E12, A3-CO2-TIGHT

5.0E12, A4-CO-TIGHT

.25E15, A5-HYDROGEN

5E12, 17.3E12,A6-HCN-TIGHT

5E12, 4.2E13, A7-HCN-LOOSE

2.0E14, A8-WATER-TIGHT

5.0E12, A9-H20-EX-LOOSE

2.0E14, A10-CO-EX-TIGHT

5.0E12, A11-WATER-LOOSE

5.0E12, A12-CO2-EXTRA-LOOSE

5.0E12, A13-CO-LOOSE

6.0E13, A14-TERMINAL METHYL

0.00, A15-CO-XXT

5.0E12, A16-SO2

5.0E12, A17-COS

$0.0, \quad$ A18-CS2

5.0E12, A19-H2S-TIGHT

5.0E12, A20-H2S-LOOSE

0.00, A21-CHAR-S

$0.0, \quad$ A22-EMPTY

0.00, A23-CHAR-N

5.0E12, A24-NH3-TIGHT

5.0E13, A25-HCN-to-NH3

5.0E12, A26-C2H4

5.0E12, A27-C3H6

5.0E12, A28-OLEFINS

5.0E12, A29-C2H6

5.0E12, A30-C3H8

5.0E12, A31-PARAFFINS

3.0E13, A32-CH4-LOOSE

6.0E13, A33-CH4-TIGHT

$0 ., \quad$ A34-CAL

0., A35-HAL

Figure 6-5 An example of the kinetic file (continued). 
5E34,A36-EMPTY

5E17, 5E31,A37-EMPTY

2.5E11, A38-AAC-LOW

10, A39,TEMP CROSSOVER ACET

5.0E12, 368.5, A40-PYRITE-LOW-TEMPERATURE

5.0E12, A43-FES2-PYRITE

5.0E12, A44-FES

24750., --> 24500., B1-CO2-LOOSE

26000., 25500., 27300., B2-NH3

32000., B3-CO2-TIGHT

30500., B4-CO-TIGHT

40500., 40500., B5-HYDROGEN

34000., B6-HCN-TIGHT

33000., 23500., B7-HCN-LOOSE

32000., B8-H20-TIGHT

18000., B9-H20-EX-LOOSE

40000., B10-CO-EXTRA-TIGHT

25000., B11-WATER-LOOSE

20500., --> 20750., B12-CO2-EXTRA-LOOSE

25000., B13-CO-LOOSE

31500., 32000., B14-TERMINAL METHYL

$0.00, \mathrm{~B} 15-\mathrm{CO}-\mathrm{XXT}$

24000., 31000., B16-SO2

24000., B17-COS

24000., 0.00, B18-CS2

29500., B19-H2S-TIGHT

24300., B20-H2S-LOOSE

0.00, B21-CHAR-S

25500.,B22-NOT ACTIVE

0.00, B23-CHAR-N

33000., B24-NH3-TIGHT

15000., B25-HCN-to-NH3

25000., B26-C2H4

Figure 6-5 An example of the kinetic file, (continued). 
25000., B27-C3H6

25000., B28-OLEFINS

25000., B29-C2H6

25000., B30-C $3 \mathrm{H} 8$

25000., B31-PARAFFINS

28000., B32-CH4-LOOSE

32000., B33-CH4-TIGHT

30000., B34-CAL

30000., B35-HAL

75000., B36-NOT ACTIVE

100000., B37-NOT ACTIVE

32500., B38-NOT ACTIVE

8408., B39-NOT ACTIVE

23500., B40-PYRITE-LOW-TEMPERATURE

28500., B43-FES2-PYRITE

30000 ., B44-FES

Figure 6-5 An example of the kinetic file, (continued). 


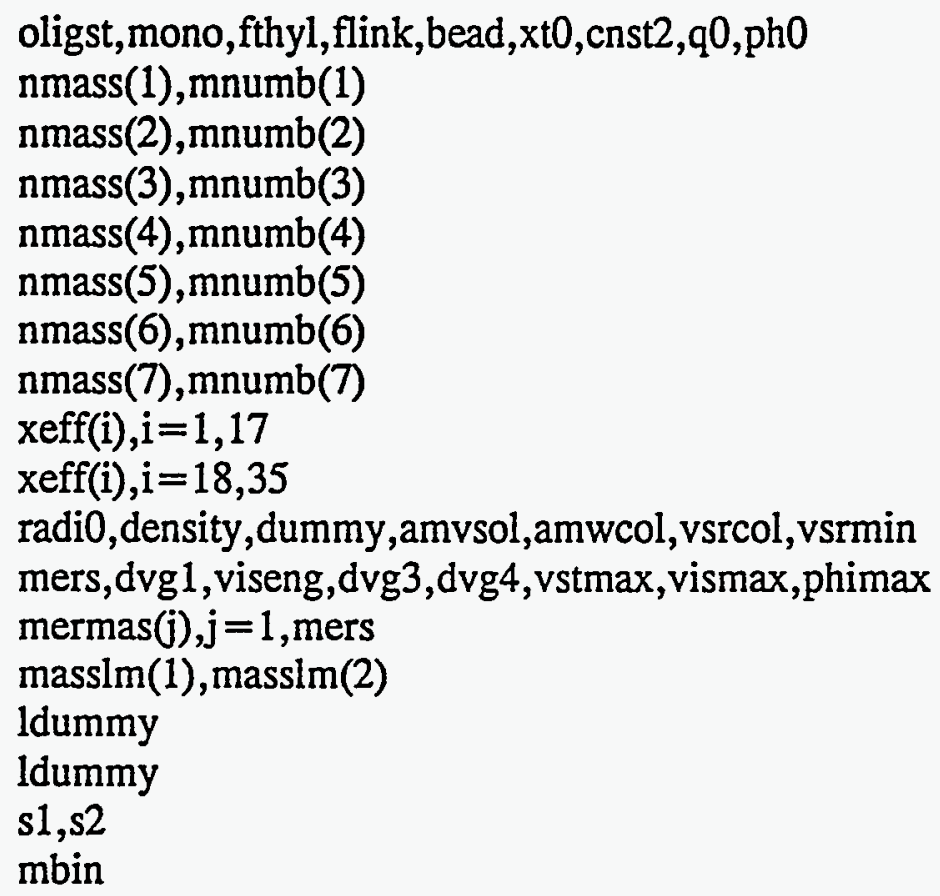

Figure 6-6 The data structure of the polymer file. 


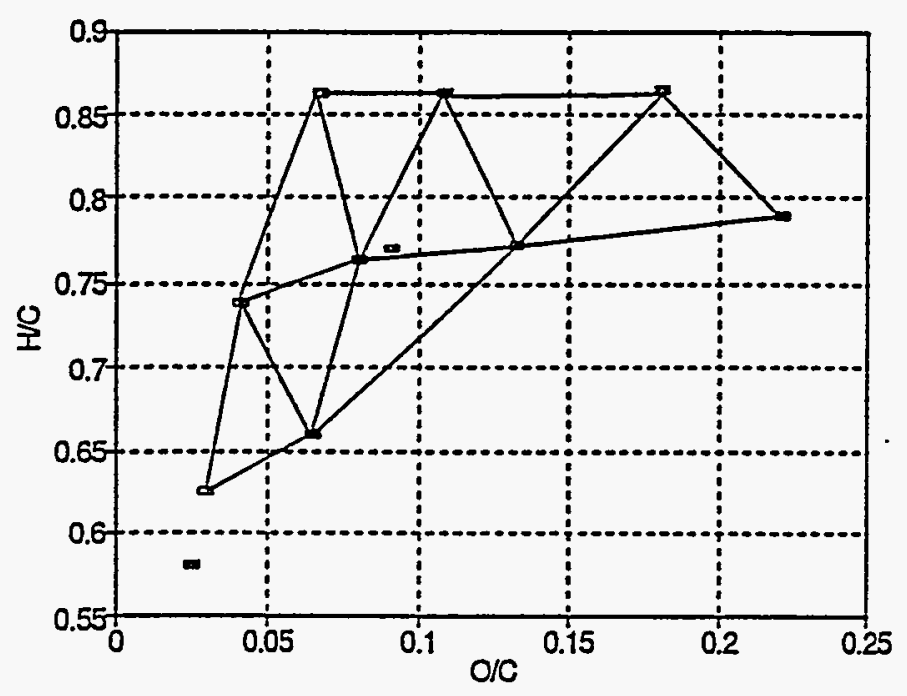

Figure 6-7. The mesh and the nodal points used for interpolations. 
Table 6-1 $\mathrm{q}_{0}$ 's and $\mathrm{p}_{\mathrm{t} 0}$ 's of the Eight Argonne Coals.

\begin{tabular}{||l|l|l||}
\hline Argonne Coals & $\mathrm{q}_{0}$ & $\mathrm{p}_{\mathrm{b}}$ \\
\hline 1 & 0.35 & 0.06 \\
\hline 2 & 0.2 & 0.045 \\
\hline 3 & 0.06 & 0.1 \\
\hline 4 & 0.22 & 0.06 \\
\hline 5 & 0 & 0.06 \\
\hline 6 & 0 & 0.037 \\
\hline 7 & 0.1 & 0.03 \\
\hline 8 & 0.05 & 0.036 \\
\hline
\end{tabular}




\section{Chapter Seven}

\section{Implementation Guide}

\section{Code Files}

This chapter discusses the implementation of PCGC-2 on UNIX operating systems. The concepts that are illustrated are applicable to other computer systems as well.

A typical simulation requires approximately 30 input and output files, and programmed procedures, such as script (command) files, are recommended to perform the file management task. Script files can also be used to automatically recompile subroutines that have been modified, relink the program, submit jobs to a batch queue, and prompt interactively for names of files or batch queues.

Typical script files are included in Appendix D. The operation of these files is as follows: First, filenames are specified. These filenames include the main data file (geometry and general flow conditions), inlet data file (inlet velocity flow conditions), thermo data file (thermodynamic parameters), output data files (formatted output file and files created specifically for graphics display), and restart data files (for both gas and particle phases). The script (command) file prompts interactively for the names of these five sets of files if they are not given on the command line. If only a single parameter is specified on the command line, all filenames are assumed identical except for the extensions.

After specifying the filenames for the particular run, links are created (logical variables are assigned) that correspond with the FORTRAN filenames used in PCGC-2. For example, the FORTRAN name for the main data file is PCGCIN. Creating a link called PCGCIN for the filename that was specified above for the main data file allows the data file to be opened by the program. The FORTRAN 
Implementation Guide

filenames that are opened by PCGC-2 and a brief description of each are given in Table 7-1.

Filenames for the coal, kinetics, polymer, and char reactivity data files are specified in the main data file. The file name for the file containing the probability distribution of the mass bins (either MASS_BNN or MASS_BIN8) is determined by the program. After creating links for each of the filenames where required, PCGC2 is compiled and linked using the most recent versions of each subroutine. This step is especially important if code development is being performed. The MAKE utility automates this step on UNIX systems. An example of a "Make" file is shown in Appendix E. After compiling and linking the program (if necessary), the program is executed. The script file may prompt for the name of the batch queue at this point. After the program has finished executing, the links are deleted.

TABLE 7-1

FILES OPENED BY PCGC-2

\begin{tabular}{|c|c|c|c|c|}
\hline$\frac{\text { FORTRAN }}{\text { Filename }}$ & $\begin{array}{l}\text { Filename } \\
\text { Extension }\end{array}$ & Subroutine & $\frac{\text { Unit }}{\text { No. }}$ & Description \\
\hline $\begin{array}{c}\text { Character } \\
\text { string }\end{array}$ & NA & GETCDF & 10 & Coal data file \\
\hline $\begin{array}{l}\text { Character } \\
\text { string }\end{array}$ & NA & GETKIN & 10 & Kinetics data file \\
\hline $\begin{array}{l}\text { Character } \\
\text { string }\end{array}$ & NA & GETRCTO & 25 & Char reactivity data file \\
\hline $\begin{array}{l}\text { Character } \\
\text { string }\end{array}$ & NA & NETIN & 10 & Polymer data file \\
\hline GASPLT & .gsp & PLTWRT & 23 & Gas plotting file \\
\hline GRIDS & .grd & GRMAP & 13 & Grid data file \\
\hline INLET &. inl & PROFIL & 20 & Inlet data file \\
\hline INOUT & .rst & RESTRT & 21 & Gàs restart file \\
\hline
\end{tabular}


TABLE 7-1 (continued

\begin{tabular}{|c|c|c|c|c|}
\hline $\begin{array}{c}\text { Fortran } \\
\text { Filename }\end{array}$ & $\begin{array}{l}\text { Filename } \\
\text { Extension }\end{array}$ & Subroutine & $\frac{\text { Unit }}{\text { No. }}$ & Description \\
\hline $\begin{array}{l}\text { nass_bin8 or } \\
\text { mass_bin }\end{array}$ & NA & NETIN & 11 & $\begin{array}{l}\text { Probability distribution of } \\
\text { mass bins }\end{array}$ \\
\hline NOXIN & nox & NOXMN & 7 & $\mathrm{NO}_{x}$ data file \\
\hline PARSOU & .pso & $\begin{array}{l}\text { EOLP, Main } \\
\text { program }\end{array}$ & 22 & Coal particle restart file \\
\hline PCGCIN & dat & Main program & 1 & Main data file \\
\hline PCGCOT & .out & Main program & 3 & Output file \\
\hline POLPLT &. $\mathrm{nxp}$ & NOXMN & 24 & $\mathrm{NO}_{x}$ plotting file \\
\hline PPLOT1 & .pp1 & PSICT & 23 & Coal particle plotting file \\
\hline PPLOT2 &.$p p 2$ & EOLP & 15 & Coal particle plotting file \\
\hline PPLOT3 & .pp3 & PSICT & 20 & Coal particle plotting file \\
\hline PRGRSS & .prg & PROG & 14 & Progress monitoring file \\
\hline PSORB1 & .ps1 & SPSICT & 23 & Sorbent particle plotting file \\
\hline PSORB2 & .ps2 & SPSICT & 27 & Sorbent particle plotting file \\
\hline PSORB3 & .ps3 & SPSICT & 20 & Sorbent particle plotting file \\
\hline RSNXIO & $\operatorname{sn} x$ & RSTNOX & 22 & $\mathrm{NO}_{\mathrm{x}}$ restart file \\
\hline SORDN1 &.$s d n$ & SDNRST & 27 & $\begin{array}{l}\text { Sorbent particle no. density } \\
\text { restart file }\end{array}$ \\
\hline SOXIN & .sox & SORBO & 8 & Sorbent reactions data file \\
\hline
\end{tabular}




\section{TABLE 7-1 (continued}

\begin{tabular}{|c|c|c|c|c|}
\hline $\begin{array}{l}\text { Fortran } \\
\text { Filename }\end{array}$ & $\begin{array}{l}\text { Filename } \\
\text { Extension }\end{array}$ & Subroutine & $\frac{\text { Unit }}{\mathrm{No}}$ & Description \\
\hline SOXPOL &. $\operatorname{sxp}$ & SORPAR & 24 & $\begin{array}{l}\text { Sorbent reactions gas } \\
\text { plotting file }\end{array}$ \\
\hline TBLRST & .tbl & $\begin{array}{l}\text { TBLRSFEH, } \\
\text { TBLRSTFE }\end{array}$ & 28 & Table restart file \\
\hline THERMO & thm & Main program & 21 & Thermodynamic data file \\
\hline TWODDBV &.$d b v$ & OVENDB & 110 & $\begin{array}{l}\text { CEQUII post-processor } \\
\text { plotting file }\end{array}$ \\
\hline YH2S1 & .h2s & SLFRST & 27 & $\mathrm{H}_{2} \mathrm{~S}$ restart file \\
\hline YSO21 & .so2 & SLFRST & 27 & $\mathrm{SO}_{2}$ restart file \\
\hline
\end{tabular}

FORTRAN and Machine Incompatibilities

PCGC-2 was originally developed on a VAX/VMS computer. Development and maintenance during the past six years has been on UNIX machines. The programming conforms to FORTRAN-77 standards. Therefore, the code should be easily portable to other systems with the following considerations:

1. Scripts for running the code have been developed for the $\mathrm{C}$-shell under UNIX and will need to be converted to run under other UNIX shells or other operation systems.

2. Some variables may not be initialized. PCGC-2 assumes that all memory registers allocated for its use are initialized prior to execution. Most compilers do this automatically or have an option to do it. If the compiler being used cannot perform this initialization, modifications may be required in the code.

3 Direct-access files are used in some instances. The direct-access feature of these files is useful for post-processing of data for plotting. All of these files can be changed to sequential without changing the performance of the code, with the exception of the PRGRSS file. The purpose of this file is to 
be accessible and provide information on the progress of the simulation during execution. This file is unnecessary for UNIX systems as all input and output files are accessible for viewing while the program is rinning. Machine-specific modifications of this file may be needed on other systems.

4. Before closing the restart, plotting, and other sequential files, it may be necessary to rewind them so they will be written from the beginning the next time they are opened. Otherwise, they may be appended, defeating their purpose and consuming large amounts of disk space.

5. PCGC-2 frequently calls a subroutine called CPUTIM, which uses a system-dependent utility to monitor the amount of CPU time used in various sections of the code. The CPUTIM and GETCPU subroutines may need to be modified for this function. Alternatively, the calls to CPUTIM may be commented out, or dummy variables may be passed back from CPUTIM to PCGC-2 .

6. PCGC-2 sometimes uses calls to FLUSH, a UNIX utility, for emptying the contents of output buffers in a timely fashion. These calls need to be replaced with calls to a similar utility applicable on the current machine. Alternatively, they may be removed, but removing these calls would make the progress of the calculation more difficult to monitor and increase the difficulty of debugging. 
Implementation Guide 


\section{Chapter Eight}

\section{Sample Problems}

Three sample problems are presented in this chapter: gaseous combustion, coal combustion, and coal gasification. Input data files for all cases are included in the appendices and are available on magnetic tape with the licensing of the program as explained in Chapter 1. The output files are too lengthy to include here, but are included on the tape.

\section{Gaseous Combustion}

This sample problem is the combustion of natural gas in air. Natural gas is fed at room temperature in the primary stream, and air is fed at room temperature in the secondary stream. Primary stream is not swirled. The secondary stream is swirled and enters the reactor at a swirl number of 1.45 . The reactor is at a pressure slightly below atmospheric pressure. Important parameters for the problem are shown in Table 8-1, and the inlet files are contained in Appendix E.

\section{Coal Combustion with $N O_{x}$ Formation}

This sample problem is the combustion of coal in air. Pulverized coal entrained in air is fed at room temperature in the primary stream. The secondary air is preheated and swirled. The reactor is at atmospheric pressure. The coal is a bituminous coal (Utah Blind Canyon). Other important parameters are shown in Table 8-2. The case uses ten chemical species and fifty particle trajectories. Input files are included in Appendix F. The inlet and thermo files are not included 
TABLE 8-1

KEY INPUT DATA FOR NATURAL GAS COMBUSTION CASE

Geometry

Primary tube diameter (m)

Secondary tube diameter $(m)$

Chamber diameter $(\mathrm{m})$

0.027

Chamber length $(\mathrm{m})$

0.098

0.800

2.650

Feed Rates

Primary gas $(\mathrm{kg} / \mathrm{s})$

2.611E-03

Secondary gas $(\mathrm{kg} / \mathrm{s})$

0.150

Inlet Gas Properties

Primary swirl number

Primary turbulent intensity

0.000

Primary temperature $(\mathrm{K})$

0.10

Primary mole fractions:
$\mathrm{CH}_{4}$
$\mathrm{CO}_{2}$
$\mathrm{C}_{2} \mathrm{H}_{6}$
$\mathrm{C}_{3} \mathrm{H}_{8}$
$\mathrm{N}_{2}$

Secondary swirl number

Secondary turbulent intensity

Secondary temperature (K)

Secondary mole fractions:

$\mathrm{O}_{2}$

$\mathrm{N}_{2}$

Reactor Parameters

Reactor pressure (N/sq m)

Side wall temperature (K)
298.0

0.801

0.016

0.120

0.054

0.009

1.450

0.10

298.0

0.233

0.767

$8.6 \mathrm{E}+04$

1000.0 
TABLE 8-2

\section{KEY INPUT DATA FOR PIRVERIZED COAL COMBUSTION CASE}

Geometry

Primary tube diameter $(\mathrm{m})$

Secondary tube diameter $(\mathrm{m})$

0.027

Chamber diameter $(\mathrm{m})$

0.098

Chamber length (m)

0.800

2.650

Feed Rates

Primary gas $(\mathrm{kg} / \mathrm{s})$

Secondary gas $(\mathrm{kg} / \mathrm{s})$

4.170E-03

Coal in primary $(\mathrm{kg} / \mathrm{s})$

0.035

3.169E-03

Inlet Gas Properties

Primary swirl number

Primary turbulent intensity

Primary temperature (K)

Primary mole fractions:

$\mathrm{H}_{2} \mathrm{O}$

$\mathrm{N}_{2}$

$\mathrm{O}_{2}$

0.000

0.10

298.0

0.062

0.720

0.219

Secondary swirl number

1.400

Secondary turbulent intensity

0.10

Secondary temperature $(\mathrm{K})$

423.0

Secondary mole fractions:

$\mathrm{N}_{2}$

0.767

$\mathrm{O}_{2}$

0.233

Reactor Parameters

Reactor pressure (N/sq m)

Side wall temperature $(\mathrm{K})$

$1.013 \mathrm{E}+05$

1250.0

Particle Parameters

Particle solid density $(\mathrm{kg} / \mathrm{cu} \mathrm{m})$

1340.0

Heat of formation, daf $(\mathrm{J} / \mathrm{kg})$

Mass mean particle diameter (m)*

4.50E-05 
Initial analysis:

TABLE 8-2 (continued) raw coal

0.861

ash

Elemental analysis (daf):

C

0.696

$\mathrm{H}$

0.053

O

0.094

$N$

0.013

$\mathrm{S}$

0.005

*Continuous distribution simulated with 5 discrete sizes.

because they are essentially the same as the ones shown in Appendix E. A grid file is shown, which can be used to alter the node locations, add nodes (up to a total of 100 in each direction), add intrusions, and/or add additional inlets (up to 3) as discussed in Chapters 3 and 5. The right-hand side of the bottom portion of the grid file is truncated because of space limitations on the printed page.

The log file for this case is also included in Appendix F. Information identifying the computation was first printed out, followed by a figure showing the important node locations. As shown, this problem used a $55 \times 49$ grid. After the figure, the names of the FG-DVC input files are printed out followed by a set of parametric values calculated when using the FG-DVC submodel. Next, a header was printed out for the gas phase solution. Next, the physical properties table was generated. Because the energy equation was solved in this case (INCALH is set to $T$ in the main data files), the table is a function of three independent variables: gas mixture fraction $(f)$, coal gas mixture fraction $(\eta)$, and residual enthalpy $\left(h_{r}\right)$.

After generating the table, the first gas phase iteration began. As shown, information was printed out for the first iteration and every 5 gas macro-iterations thereafter. This corresponds to Steps 2 and 3 in Fig. 3a-1. Residual source sums were printed out for the $u$-velocity, $v$-velocity, $w$-velocity, mass source term, pressure, enthalpy, mixture fraction, and coal-gas mixture fraction. The maximum of these source sums was printed to the left. At the far right, is shown the number of potential calls to CREE, or the number of times equilibrium properties were needed for a value of residual enthalpy that was outside the table limits. For this particular case there was no potential call to CREE. 
After the gas phase was calculated, the gas properties were updated (Step 4, Fig. 3a-1) and then the Eulerian particle number density field was calculated (Step 5, Fig. 3a-1). The bulk velocity of the particles is assumed equal to that of the gas, with the particles diffusing according to the concentration gradient. This calculation is indicated in the log file by the brief header starting with "NJITER" shown following the gas phase. Residual source sums for each of the allowable five particle sizes are printed out in a manner similar to the source sums for the gas phase every five iterations. For this case, the number density calculation converged at between 5 and 10 iterations, after the first gas phase iteration.

After the particle density fields were calculated, the radiation field was solved (Step 6, Fig. 3a-1). In this case, the discrete ordinates method was used (LDISO $=\mathrm{T}$ in the main input file of Appendix F). As shown in the log file, the radiation model is solved alternately without (SCATTER ITER: 0) and with (SCATTER ITER: 1) radiation scattering by particles included. A total of six iterations were required the first time without scattering followed by four iterations to converge with scattering. Iteration continued until the change in total net wall flux fell below a prescribed level.

After solving the gas phase, particle density, and radiation fields, the Lagrangian particle calculation was performed (Steps 7-10, Fig. 3a-1). The assumption for this case was that all particles stick when they hit the wall. For each particle size and starting location, the terminating location, transit time, and number of iteration steps are shown. If the trajectory recirculated, it was so noted. After the particle trajectory information, a summary of the solid phase coupling was printed. This is the sum of the particle source terms for all of the gas cells for $\eta, f$, mass $u$-velocity, $v$-velocity, and enthalpy. Since this is a coal combustion case, the values for SSPE $(\eta)$ and SSPM (mass) are identical, since the only source of mass to the gas phase is from the coal. For coal-water mixture combustion, the variable $f$ is used to track the mixing of the moisture from the coal-water droplets, and both SSPE and SSPF would be non-zero, with SSPM being the sum of both. Finally, the cumulative particle burnout was printed for the outlet location of the reactor. After the particle phase was calculated, the gas phase was recalculated, using the new particle source terms.

This case was converged from restart in four particle iterations. The convergence criterion which finally terminated the calculation was the SORMIN criterion. After the third particle iteration, the maximum residual source sum for the gas phase during the first iteration was 0.09512 , which is less than the value of 0.1 specified for SORMIN in the main data file. The gas phase was converged one more time, followed by one more particle iteration. For the final gas phase calculation, less than 15 macro-iterations were required. 
After converging the particle-gas combustion model, the $\mathrm{NO}_{\mathrm{x}}$ calculations are performed. In this case, only fuel $\mathrm{NO}_{\mathrm{x}}$ was calculated. The $\mathrm{NO}_{\mathrm{x}}$ iterations can be seen at the end of the log file.

\section{Coal Gasification with Sidewall Sorbent Injection and Sulfur Capture}

This sample problem is the gasification of Illinois No. 6 bituminous coal at $2 \mathrm{~atm}$ in the BYU gasifier (Huber, 1985). The primary stream consisted of coal transported by Argon. The secondary stream was oxygen. Sorbent was injected through the sidewall. Important parameters are shown in Table 8-3 and the input files are included in Appendix G.

TABLE 8-3

\section{PARAMETERS FOR COAL GASIFICATION CASE}

\section{Geometry}

Primary tube diameter $(\mathrm{m}) \quad 0.013$

Secondary tube diameter $(\mathrm{m}) \quad 0.029$

Chamber diameter $(\mathrm{m}) \quad 0.200$

Chamber length (m) $\quad 2.000$

Feed Rates

Primary gas $(\mathrm{kg} / \mathrm{s}) \quad 0.0011$

Secondary gas $(\mathrm{kg} / \mathrm{s}) \quad 0.0052$

Tertiary (sidewall) gas (kg/s) 0.0013

Coal in primary $(\mathrm{kg} / \mathrm{s}) \quad 0.0083$

Inlet Gas Properties

Primary swirl number $\quad 0.000$

Primary turbulence intensity $\quad 0.010$

Primary temperature (K) $\quad 356.0$

Primary mole fractions: Ar

1.000

$\begin{array}{ll}\text { Secondary swirl number } & 0.000\end{array}$ 
TABLE 8-3 (continued)

Secondary turbulence intensity

Secondary temperature $(\mathrm{K})$

0.100

Secondary mole fractions:

589.0

$\mathrm{O}_{2}$

1.000

Tertiary swirl number

Tertiary turbulence intensity

0.000

Tertiary temperature (K)

0.010

Tertiary mole fractions

$$
\mathrm{Ar}
$$

356.0

1.000

Reactor Parameters

Reactor pressure (N/sq m)

$1.84 \mathrm{E}+05$

Side wall temperature $(\mathrm{K})$

1100.0

Coal Particle Parameters

Particle solid density $(\mathrm{kg} / \mathrm{cu} \mathrm{m})$

1340

Particle mass fractions

$5 \mu \mathrm{m}$

$13 \mu \mathrm{m}$

$32 \mu \mathrm{m}$

$50 \mu \mathrm{m}$

$100 \mu \mathrm{m}$

Initial analysis:

raw coal

ash

Elemental analysis (daf):

$\mathrm{C}$
$\mathrm{H}$
$\mathrm{O}$
$\mathrm{N}$
$\mathrm{S}$

0.200

0.200

0.200

0.200

0.200

0.941

0.059

0.777

0.050

0.137

0.014

0.022

Sorbent Particle Parameters

Particle solid density ( $\mathrm{kg} / \mathrm{cu} \mathrm{m}$ )

Particle mass fractions

$10.6 \mu \mathrm{m}$

BET surface area (sq $\mathrm{m} / \mathrm{g}$ )

1.000

$1 E+5$ 


\section{Nomenclature}

Nomenclature for Chapters 4 and 6 are found at the end of those chapters. Nomenclature for all other chapters is found below.

\begin{tabular}{|c|c|c|}
\hline Symbol & $\underline{\text { Units }}$ & Definition \\
\hline$\dot{m}, \dot{M}$ & $\mathrm{~kg} \mathrm{~s}-1$ & Mass flow rate \\
\hline $\bar{b}, \bar{f}, \bar{s}$ & - & $\begin{array}{l}\text { Backward-, forward- and side-scattered } \\
\text { components }\end{array}$ \\
\hline$A$ & $\mathrm{~kg} \mathrm{~s}^{-1}$ & Total coefficient (convection plus diffusion) \\
\hline$A$ & varies & Pre-exponential factor \\
\hline$a$ & - & Size parameter $\pi d / \gamma$ \\
\hline$A, B$ & $\mathrm{~m}^{2}$ & Area \\
\hline$A_{E}$ & $\mathrm{~m}^{2}$ & Char surface area in $\mathrm{NO}_{\mathrm{x}}$ submodel \\
\hline$a_{0}$ & - & Asymmetry factor \\
\hline
\end{tabular}




\begin{tabular}{|c|c|c|}
\hline Symbel & Units & Definition \\
\hline$B$ & -- & Blowing parameter \\
\hline$b$ & -- & $\begin{array}{l}\text { Coefficient on oxygen concentrations in } \\
\mathrm{NO}_{\mathrm{x}} \text { submodel }\end{array}$ \\
\hline$b$ & -- & Element mass fraction, back scatter \\
\hline$b c n$ & -- & $\begin{array}{l}\text { Weight fraction of nitrogen in coal in } \mathrm{NO}_{\mathrm{x}} \\
\text { submodel }\end{array}$ \\
\hline$C$ & (VFM) & Pressure-correction factor, coefficients \\
\hline$C$ & $\mathrm{~kg} \mathrm{~m}^{-3}$ & Concentration \\
\hline$C$ & $\mathrm{~kg} \mathrm{~s}^{-1}$ & Convection coefficient \\
\hline$C$ & -- & "Universal" constant \\
\hline$c$ & varies & Variable constant \\
\hline$C_{d}$ & -- & Drag coefficient \\
\hline$C_{p}$ & $\mathrm{~J} \mathrm{~kg}^{-1} \mathrm{~K}^{-1}$ & Heat capacity \\
\hline$D$ & $\mathrm{~kg} \mathrm{~s}^{-1}$ & Numerical coefficient for diffusion \\
\hline$D$ & $\mathrm{~m}^{2} \mathrm{~s}^{-1}$ & Diffusivity \\
\hline$D, d$ & $\mathrm{~m}$ & Diameter \\
\hline$E$ & 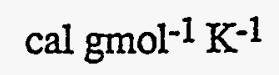 & Activation energy in $\mathrm{NO}_{\mathrm{x}}$ submodel \\
\hline$E$ & $\mathrm{~J} \mathrm{kmol-1}$ & Activation energy \\
\hline$E$ & $\mathrm{~kW} / \mathrm{m}^{2}$ & Emissive power \\
\hline$E$ & - & Constant in the law of the wall \\
\hline
\end{tabular}




\begin{tabular}{|c|c|c|}
\hline Symbol & Units & Definition \\
\hline$F$ & $\mathrm{~J} \mathrm{~m}^{-2} \mathrm{~s}^{-1}$ & Radiation flux \\
\hline$F$ & $\mathrm{~kW} / \mathrm{m}^{2}$ & Radiative flux-sum \\
\hline$F$ & -- & Adjusted mixture fraction (in PDF) \\
\hline$f$ & $\mathrm{~N} \mathrm{~m}^{-2}$ & Drag force \\
\hline$f$ & -- & Differencing factor \\
\hline$f$ & -- & $\begin{array}{l}\text { Mixture fraction, interpolation factor, } \\
\text { forward scatter }\end{array}$ \\
\hline$f_{p}$ & - & Mixture fraction corrected for $h$ \\
\hline$G$ & $\mathrm{~kg} \mathrm{~m}^{-2} \mathrm{~s}^{-1}$ & Mass flux . \\
\hline$G$ & - & Adjusted variance \\
\hline$g$ & $\mathrm{~m} \mathrm{~s}^{-2}$ & Gravitational acceleration \\
\hline$g$ & - & Mean square fluctuation (variance) \\
\hline$h$ & $\mathrm{~J} \mathrm{~kg}^{-1}$ & Enthalpy \\
\hline hg & $\mathrm{J} \mathrm{kg}^{-1}$ & Enthalpy reaction \\
\hline$I$ & varies & Turbulence intensity, radiation intensity \\
\hline$i$ & -- & Imaginary $\left(\mathrm{i}^{2}=-1\right)$ \\
\hline$j$ & $\mathrm{~kg} \mathrm{~m}^{-2} \mathrm{~s}^{-1}$ & Mass flux \\
\hline$K$ & - & Absorption coefficient \\
\hline$K$ & - & $\begin{array}{l}\text { Chemical equilibrium constant in } \mathrm{NO}_{\mathrm{x}} \\
\text { submodel }\end{array}$ \\
\hline
\end{tabular}




\begin{tabular}{|c|c|c|}
\hline Symbol & Units & Definition \\
\hline$K$ & - & Scattering coefficient \\
\hline$k$ & $\mathrm{~J} \mathrm{~m}^{-1} \mathrm{~K}^{-1} \mathrm{~s}^{-1}$ & Thermal conductivity \\
\hline$k$ & $\mathrm{~m}^{2} \mathrm{~s}^{-2}$ & Kinetic energy of turbulence \\
\hline$k$ & $\mathrm{~m}^{3} \mathrm{kmol}^{-1} \mathrm{~s}^{-1}$ & Arrhenius rate constant in $\mathrm{NO}_{\mathrm{x}}$ submodel \\
\hline$k$ & varies & Constant, kinetic rate coefficient \\
\hline$k_{a}, k_{s}, k_{t}$ & $\mathrm{~m}$ & $\begin{array}{l}\text { Absorption, scattering and extinction } \\
\text { coefficients }\end{array}$ \\
\hline$L$ & $\mathrm{~m}$ & Length \\
\hline$L$ & -- & Lower limit \\
\hline$L_{e}$ & $\mathrm{~m}$ & Mean (effective) beam length \\
\hline$M$ & $\mathrm{~kg}$ & Mass of fluid atoms \\
\hline$M$ & $\mathrm{~kg} \mathrm{kmol}^{-1}$ & Molecular weight \\
\hline$M, m$ & -- & Mass fraction of species \\
\hline$M W$ & $\mathrm{~kg} \mathrm{kmol}^{-1}$ & Molecular weight in $\mathrm{NO}_{\mathrm{x}}$ submodel \\
\hline$N$ & - & Total number of directions used \\
\hline$n$ & $\mathrm{~m}^{-3}$ & Particle number density \\
\hline$n$ & $s^{-1}$ & Particle number flow rate \\
\hline$n$ & - & Direction normal to boundary \\
\hline$n$ & -- & Order of discrete ordinates approximation \\
\hline$N u$ & .- & Nusselt number \\
\hline
\end{tabular}




\begin{tabular}{|c|c|c|}
\hline Symbol & Units & Definition \\
\hline$P$ & $\mathrm{~Pa}$ & Pressure in $\mathrm{NO}_{\mathrm{x}}$ submodel \\
\hline$P$ & - & $\begin{array}{l}\text { Probability density function (PDF), phase } \\
\text { function, } P \text { cell }\end{array}$ \\
\hline$P$ & - & Scattering phase function \\
\hline$p, P$ & $\mathrm{Nm}^{-2}$ & Pressure \\
\hline $\operatorname{Pr}$ & -- & Prandtl number \\
\hline$Q$ & $\mathrm{~J} \mathrm{~s}^{-1}$ & Heat flow \\
\hline$q$ & $\mathrm{~J} \mathrm{~m}^{-2} \mathrm{~s}^{-1}$ & Heat flux \\
\hline$q$ & $\mathrm{~kW} / \mathrm{m}^{2}$ & Net flux \\
\hline$Q_{a}, Q_{s}$ & -- & Absorption and scattering efficiency \\
\hline$Q_{R}$ & $\mathrm{~kW}$ & Net radiative exchange for a single particle \\
\hline$q_{R}$ & $\mathrm{~kW} / \mathrm{m}^{3}$ & $\begin{array}{l}\text { Net radiative exchange for a volume } \\
\text { element }\end{array}$ \\
\hline$q^{ \pm}$ & $\mathrm{kW} / \mathrm{m}^{2}$ & Hemispherical flux \\
\hline$R$ & $\mathrm{~J} \mathrm{kmol}^{-1} \mathrm{~K}^{-1}$ & Universal gas constant \\
\hline$R$ & $\underset{\mathrm{K}^{-1}}{\mathrm{~m}^{3} \mathrm{~Pa} \mathrm{kmol}^{-1}}$ & Universal gas constant in $\mathrm{NO}_{\mathrm{x}}$ submodel \\
\hline$r$ & $\mathrm{~kg} \mathrm{~s}-1$ & Reaction rate \\
\hline$r, R$ & M & Radius, radial direction \\
\hline$r, x$ & $\mathrm{~m}$ & Distance along radial and axial directions \\
\hline$R e$ & -- & Reynolds number \\
\hline
\end{tabular}




\begin{tabular}{|c|c|c|}
\hline Symbol & Units & Definition \\
\hline$S$ & varies & Source term \\
\hline$s$ & - & Scattering factor, conserved scalar \\
\hline Sc & - & Schmidt number \\
\hline$S h$ & - & Sherwood number \\
\hline$T$ & $\mathrm{~K}$ & Temperature \\
\hline$T, t$ & s & Time, time period \\
\hline$U$ & -- & Upper limit \\
\hline$u$ & $\mathrm{~m} \mathrm{~s}^{-1}$ & Axial velocity \\
\hline$V$ & $\mathrm{~m}^{3}$ & Volume \\
\hline$v$ & $\mathrm{~m} \mathrm{~s}^{-1}$ & Radial velocity, velocity vector \\
\hline$W$ & $\mathrm{~kg} \mathrm{~m}^{-3} \mathrm{~s}^{-1}$ & $\begin{array}{l}\text { Overall mean reaction rate or source term in } \\
\mathrm{NO}_{\mathrm{x}} \text { submodel }\end{array}$ \\
\hline$W$ & $\mathrm{~kg} \mathrm{~m}^{-3} \mathrm{~s}^{-1}$ & Overall species reaction rate \\
\hline$w$ & $\mathrm{kmol} \mathrm{m}^{-3} \mathrm{~s}^{-1}$ & Individual reaction rate \\
\hline$w$ & $\mathrm{~m} \mathrm{~s}^{-1}$ & Tangential velocity \\
\hline$w_{m}$ & sr & Angular quadrature weight \\
\hline$X$ & -- & Mole mass fraction \\
\hline$X$ & -- & Particle size function \\
\hline$x$ & $\mathrm{~m}$ & Axial position \\
\hline
\end{tabular}




$\begin{array}{ccl}\text { Symbol } & \text { Units } & \begin{array}{l}\text { Definition } \\ \text { Volatiles coefficient, particle starting } \\ \text { location fraction }\end{array} \\ y & - & \begin{array}{l}\text { Species mole fraction, fluctuating mass } \\ \text { fraction }\end{array} \\ Z & -- & \begin{array}{l}\text { Arbitrary variable } \\ \text { Concentration of enclosed species symbol } \\ \left(\mathrm{NO}_{\mathrm{x}} \text { submodel) }\right.\end{array}\end{array}$

Greek Symbols

$\begin{array}{ccl}\text { Svmbol } & \text { Units } & \text { Definition } \\ \Omega, \Omega^{\prime} & -- & \text { Outward and inward directions of radiation } \\ \alpha & \text { kg } & \text { Mass } \\ \alpha & - & \text { Intermittency factor } \\ \beta & \text { varies } & \text { Arbitrary property } \\ \beta & -- & \text { Intermittency } \\ \chi & \mathrm{m} & \text { Wavelength of radiation } \\ \delta, \Delta & -- & \text { Difference operator } \\ \varepsilon & \mathrm{m}^{2} \mathrm{~s}^{-1} & \text { Dissipation rate of turbulence energy } \\ \varepsilon & -- & \text { Emissivity }\end{array}$




\begin{tabular}{|c|c|c|}
\hline Symbol & $\underline{\text { Units }}$ & Definition \\
\hline$\Phi$ & $\mathrm{kg} \mathrm{m}^{-1} \mathrm{~s}^{-1}$ & Dissipation function \\
\hline$\phi$ & varies & General variable \\
\hline$\phi$ & -- & $\begin{array}{l}\text { Angle between incident and scattered } \\
\text { intensities }\end{array}$ \\
\hline$\Gamma$ & varies & Exchange coefficient \\
\hline$\gamma$ & -- & Heat loss factor \\
\hline$\gamma$ & -- & Particle swelling coefficient \\
\hline$H$ & radians & Azimuthal direction \\
\hline$\eta$ & -- & Coal gas mixture fraction \\
\hline$\kappa$ & -- & Constant \\
\hline$\lambda$ & -- & $\begin{array}{l}\text { Under-relaxation factor, wavelength of } \\
\text { radiation }\end{array}$ \\
\hline$\mu$ & $\mathrm{kg} \mathrm{m}^{-1} \mathrm{~s}^{-1}$ & Dynamic viscosity \\
\hline$\mu, \eta, \xi$ & -- & Direction cosines \\
\hline$v$ & $\mathrm{~m}^{2} \mathrm{~s}^{-1}$ & Kinematic viscosity \\
\hline$\pi$ & -- & Percentage perturbation \\
\hline$\theta, \psi$ & - & Polar and azimuthal angles/directions \\
\hline$\rho$ & $\mathrm{kg} \mathrm{m}^{-3}$ & Density \\
\hline
\end{tabular}




\begin{tabular}{|c|c|c|}
\hline Symbol & Units & Definition \\
\hline$\sigma$ & - & Schmidt or Prandtl number \\
\hline$\tau$ & $\mathrm{m}^{2} \mathrm{~s}^{-2}$ & Shear stress \\
\hline$\Omega$ & -- & Collision integral \\
\hline$\Omega$ & -- & Differencing scheme coefficient \\
\hline$\omega$ & $\mathrm{kmol} \mathrm{m}^{-3} \mathrm{~s}^{-1}$ & Reaction rate $\left(\mathrm{NO}_{\mathrm{x}}\right.$ submodel) \\
\hline$\omega_{0}$ & -- & Albedo for forward radiation scattering \\
\hline$\xi$ & $s^{-1}$ & Vorticity \\
\hline$\xi$ & - & Dimensionless coordinate of integration \\
\hline$\Psi$ & $\mathrm{m}^{2} \mathrm{~s}^{-1}$ & Stream function \\
\hline$\zeta$ & -- & Constant in $\mathrm{NO}_{\mathrm{x}}$ model \\
\hline$\zeta$ & -- & $\begin{array}{l}\text { Fraction of heat of reaction given to particle } \\
\text { phase }\end{array}$ \\
\hline$\zeta$ & -- & Pore diffusion adjustment factor \\
\hline$\zeta$ & -- & $\begin{array}{l}\text { Reaction progress (extent of reaction or } \\
\text { deviation from equilibrium factor) or } \\
\text { fraction of nitrogen released from coal } \\
\left(\mathrm{NO}_{\mathrm{x}} \text { submodel) }\right.\end{array}$ \\
\hline$\zeta$ & -- & Surface area factor \\
\hline
\end{tabular}




\section{Subscripts}

Symbol Definition

a Absorptive, ash, arbitrary

b Black-body, bulk, boundary, backward scattering

B Boundary

c Cell, pure coal off-gas, convective, raw coal

$d \quad$ Direction, diffusive, drag

$E, e \quad$ East direction, effective

$e q \quad$ Equilibrium $\left(\mathrm{NO}_{\mathrm{x}}\right.$ submodel)

$f \quad$ Mixture fraction, formation

$f \quad$ Fuel $\mathrm{NO}$ mechanism reaction $\left(\mathrm{NO}_{\mathrm{x}}\right.$ submodel $)$

F $\quad$ Radiation flux, furnace

$g \quad$ Mean square fluctuations (variance), gas phase

$g_{1}, g_{2} \quad$ Universal (found empirically)

$h \quad$ Enthalpy, char

$h \quad$ Heterogeneous reaction $\left(\mathrm{NO}_{\mathrm{x}}\right.$ submodel $)$

$i \quad$ Gas species index, summation index

$i$, Species or reaction index $\left(\mathrm{NO}_{\mathrm{x}}\right.$ submodel)

$i, j \quad$ Area indices in the radial and axial directions, $\mathrm{j}^{\text {th }}$ particle size 


\begin{tabular}{|c|c|}
\hline Symbol & Definition \\
\hline$I$ & Pure inlet gas \\
\hline$j$ & Particle size, gas species index \\
\hline$k$ & Turbulent kinetic energy, element \\
\hline$k$ & Species or reaction index $\left(\mathrm{NO}_{\mathrm{x}}\right.$ submodel $)$ \\
\hline$l$ & Char reaction index \\
\hline$m$ & Devolatilization reaction index, mass, mixture \\
\hline$m, m^{\prime}$ & Directions of the discrete ordinates \\
\hline$n$ & Number density, nitrogen $\left(\mathrm{NO}_{\mathrm{x}}\right.$ submodel) \\
\hline$N, n$ & North direction \\
\hline$N$ & Nitrogen \\
\hline 0 & Initial, oxidizer \\
\hline$P, p$ & Primary stream, particle, P-cell \\
\hline$r e f$ & Reference \\
\hline$r$ & Reactor, radial, radiation \\
\hline$r$ & Non-chemical sources ( $\mathrm{NO}_{\mathrm{x}}$ submodel) \\
\hline$s$ & Secondary stream, scattering, south direction \\
\hline$S$ & South direction, source \\
\hline$t$ & Turbulence \\
\hline$t$ & Thermal NO mechanism reaction constant $\left(\mathrm{NO}_{\mathrm{x}}\right.$ submodel $)$ \\
\hline$T$ & Temperature \\
\hline
\end{tabular}




$\begin{array}{cll}\text { Symbol } & & \text { Definition } \\ u & & \text { Total }\left(\mathrm{NO}_{\mathrm{x}} \text { submodel }\right) \\ u & & \text { Axial velocity, u cell } \\ \nu & \text { Non-proportional part of source term } \\ w & \text { Radial velocity, v cell, volatiles, devolatilization } \\ W, w & \text { Wall } \\ x, z & \text { Axial direction } \\ Y & \text { Gas phase species }\left(\mathrm{NO}_{\mathrm{x}} \text { submodel }\right) \\ \varepsilon & \text { Dissipation rate } \\ \eta & \text { Coal gas mixture fraction } \\ \theta & \text { Azimuthal direction } \\ \mu & \text { Viscosity } \\ \sigma & \text { Solid } \\ \phi & \text { General variable } \\ \lambda & \text { Coal component } \\ 0 & \text { Initial value }\end{array}$


Superscripts

Superscripts Definition

$\quad$ Favre mean

- Fluctuation, flux

: ", "' Used to distinguish different reaction rate constants $\left(\mathrm{NO}_{\mathrm{x}}\right.$ submodel)

* $\quad$ Guessed

$=\quad$ Tensor

$\rightarrow \quad$ Vector

c $\quad$ Corrected

$\varepsilon \quad$ Dissipation of turbulent kinetic energy

eq equilibrium species value $\left(\mathrm{NO}_{x}\right.$ submodel)

$\phi \quad$ Arbitrary variable

$f \quad$ f mixture fraction

$f \quad$ Frozen

$f \quad$ Full conversion of volatile fuel nitrogen to species $\left(\mathrm{NO}_{\mathrm{x}}\right.$ submodel)

fuel Related to fuel $\mathrm{NO}$ mechanism $\left(\mathrm{NO}_{\mathrm{x}}\right.$ submodel)

$\eta \quad$ Coal gas mixture fraction 


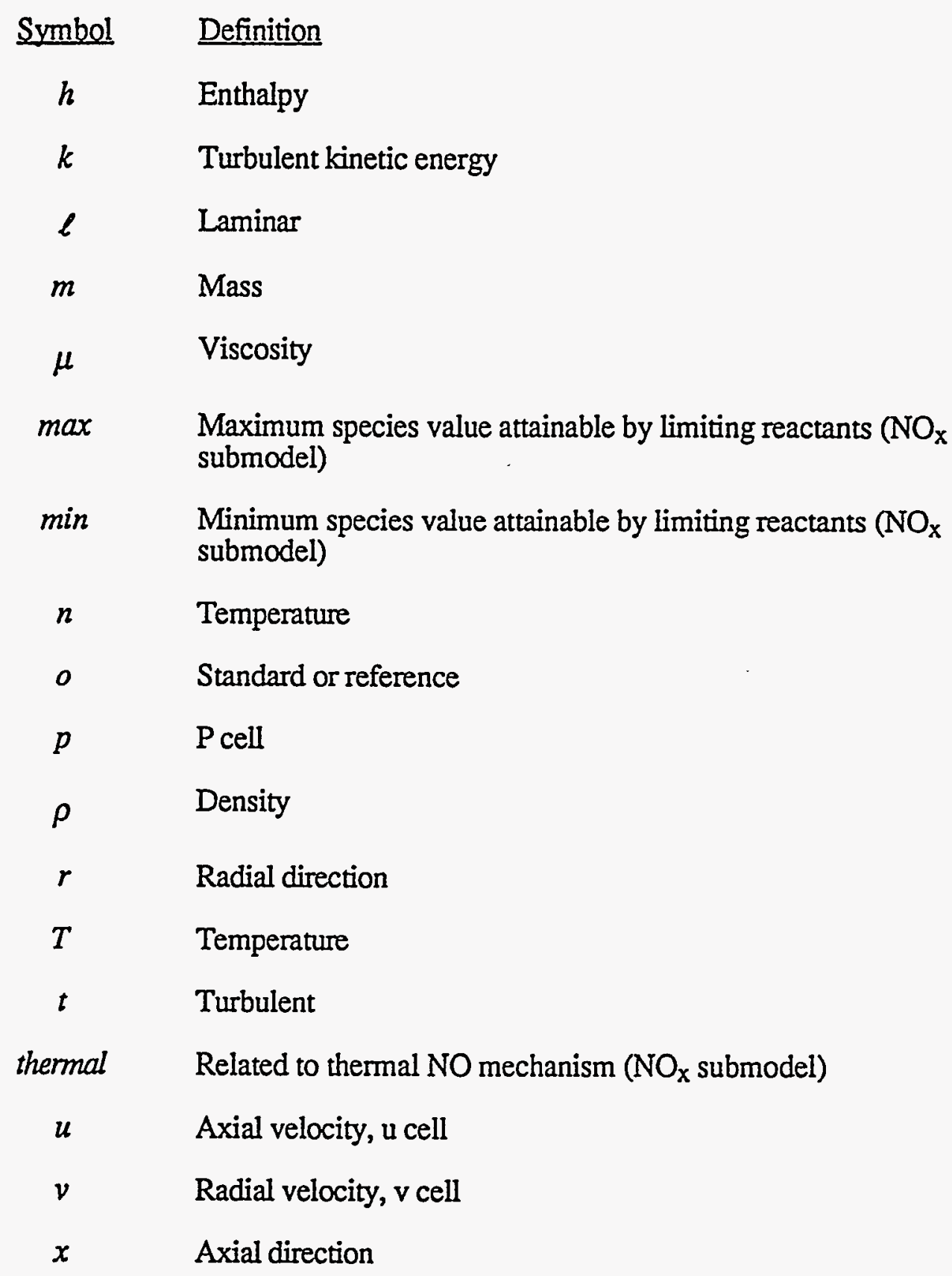




\begin{tabular}{cl} 
Symbol & Definition \\
\hline & Initial $\left(\mathrm{NO}_{\mathrm{x}}\right.$ submodel) \\
\pm & Positive and negative directions of propagation \\
- & Reynolds mean
\end{tabular}


Nomenclature 


\section{References}

References for Chapters 4 and 6 are found at the end of those chapters. References for all other chapters are found below.

Abramowitz, M. and Stegun, A. S., Handbook of mathematical functions, Dover Publications, New York, NY (1972).

Anson, D., Moles, F. D. and Street, P. J., "Structure and surface area of pulverized coal during combustion," Combust. Flame, 16, 265 (1971).

Anthony, D. B., Howard, J. B., Hottel, H. C. and Meissner, H. P., "Rapid devolatilization and hydrogasification of bituminous coal," Fuel, 55, 121 (1976).

Argarwal, R. K., A third-order-accurate upwind scheme for Navier-Stokes solutions at high Reynolds numbers, St. Louis, MO, AIAA 19th Aerospace Sciences Meeting, January, St. Louis, MO (1981).

Badzioch, S. and Hawksley, P. G. W., "Kinetics of thermal decomposition of pulverized coal particles," IEC Proc. Des. Dev., 9, 521-30 (1970).

Bailey, G. H., Slater, I. W. and Eisenklam, P., "Dynamic equations and solutions for particles undergoing mass transfer," Brit. Chem. Eng., 15, 912-16 (1970).

Baxter, L. L., Fletcher, T. H. and Ottesen, D. K., "Spectral emittance measurements of coal particles," Energy \& Fuels, 2, 423-430 (1988). 


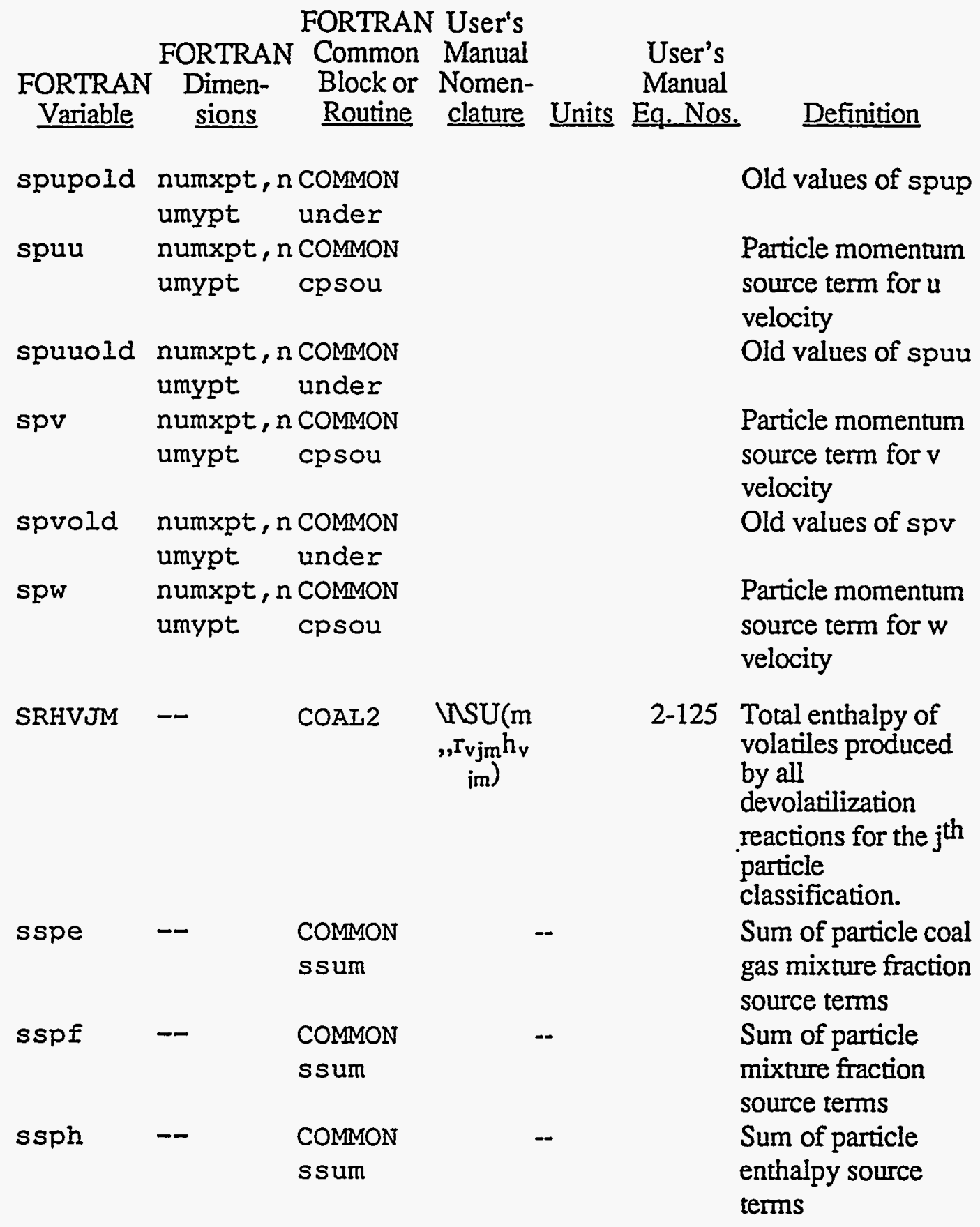




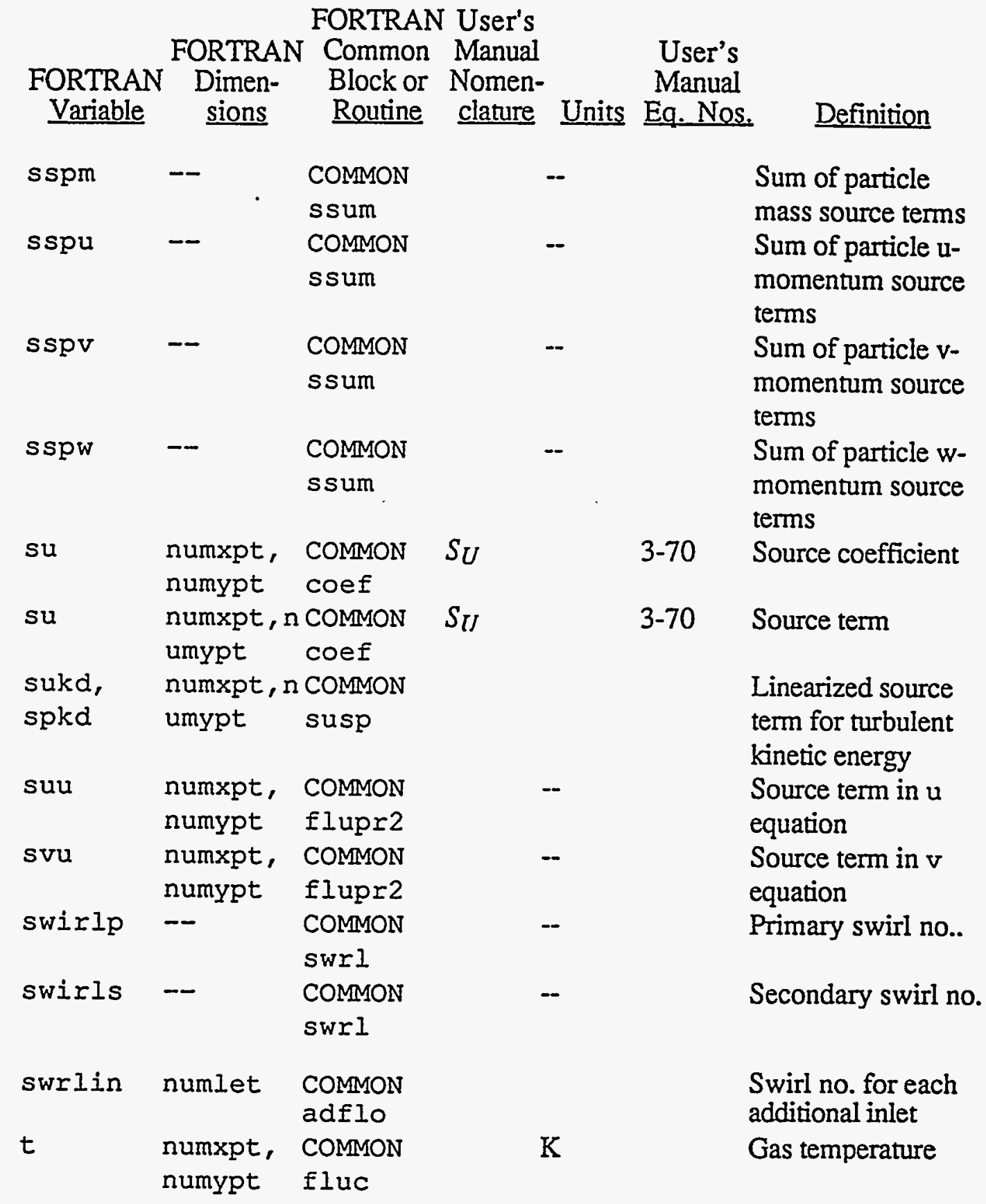


FORTRAN User's

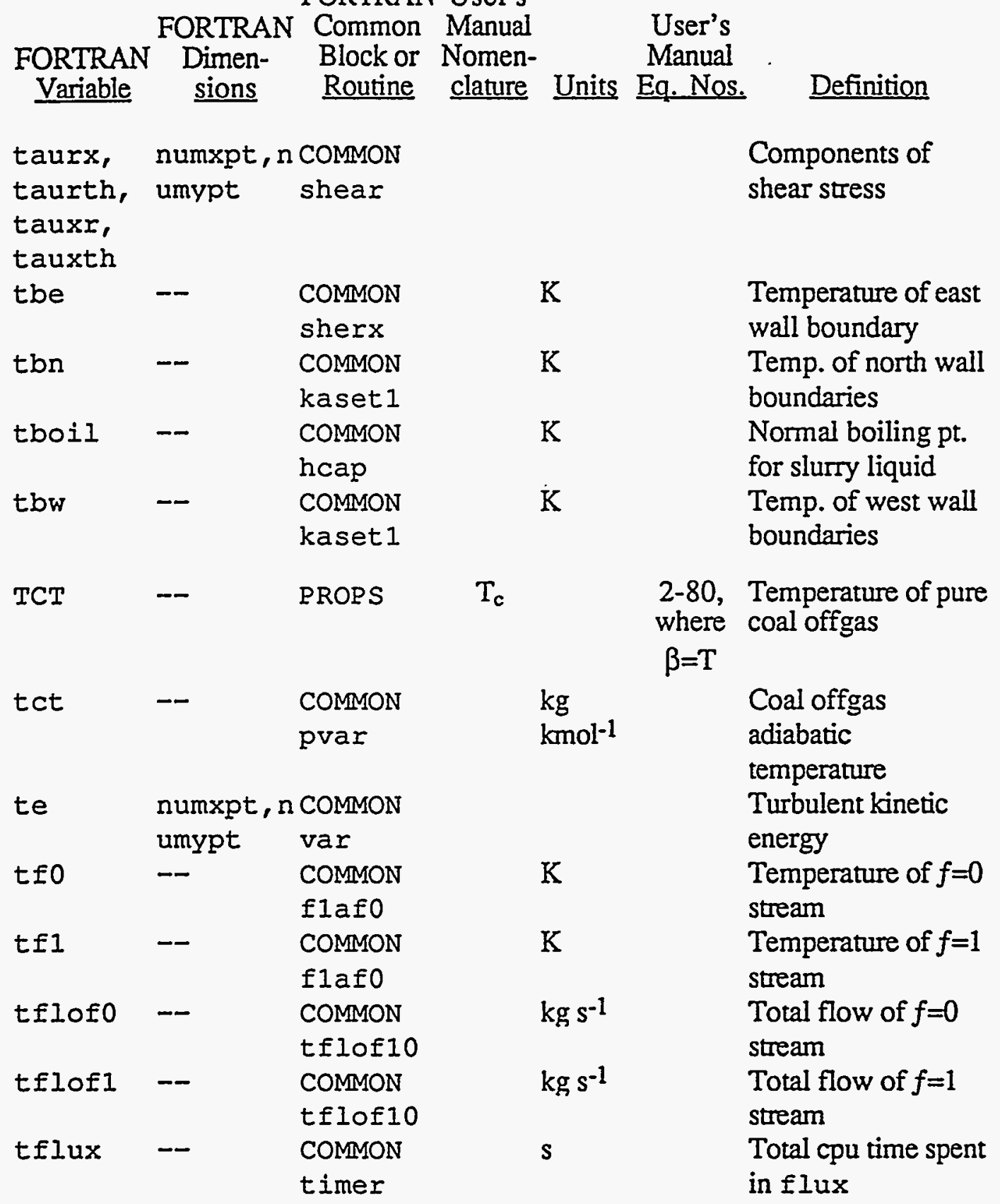




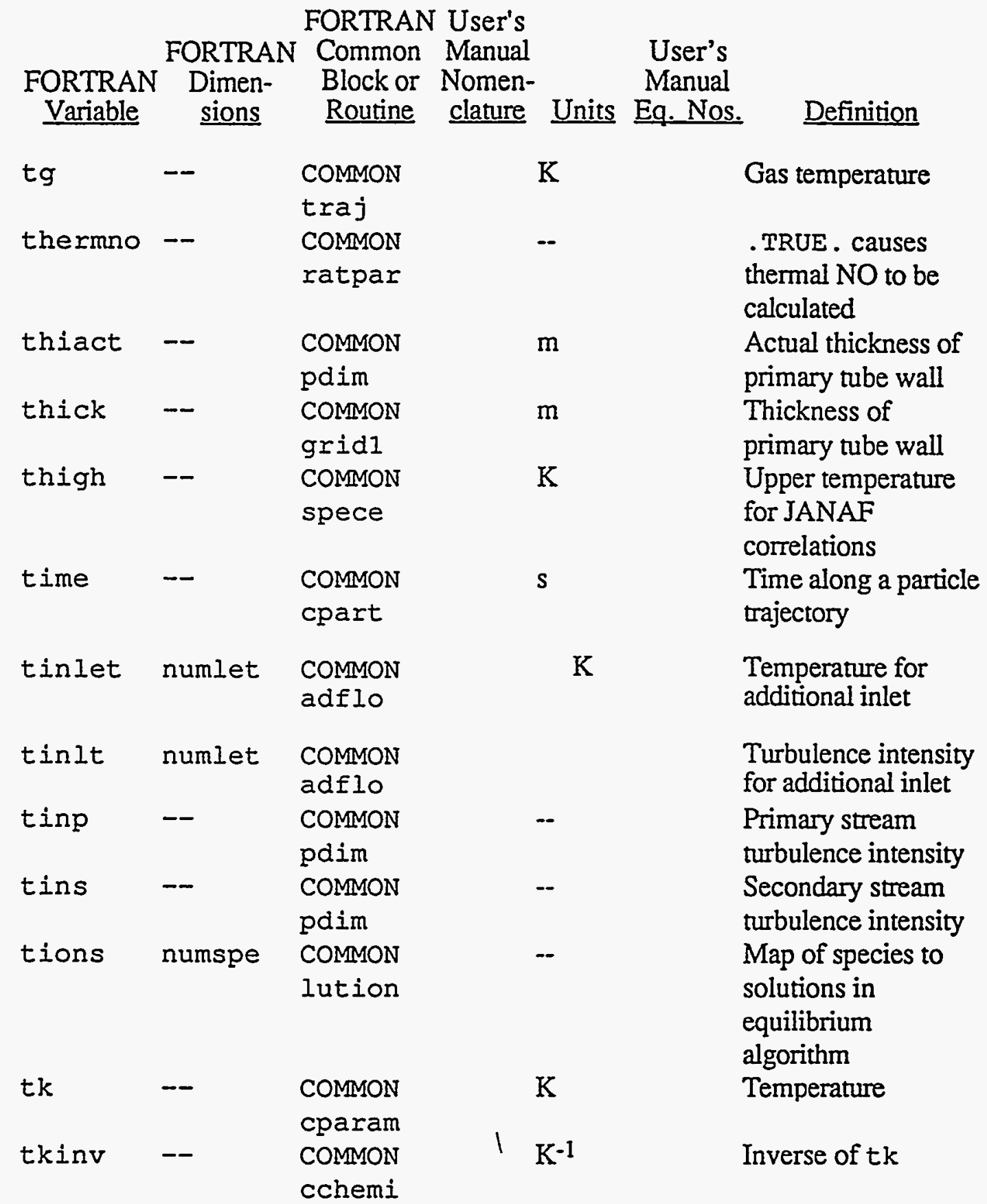


FORTRAN User's

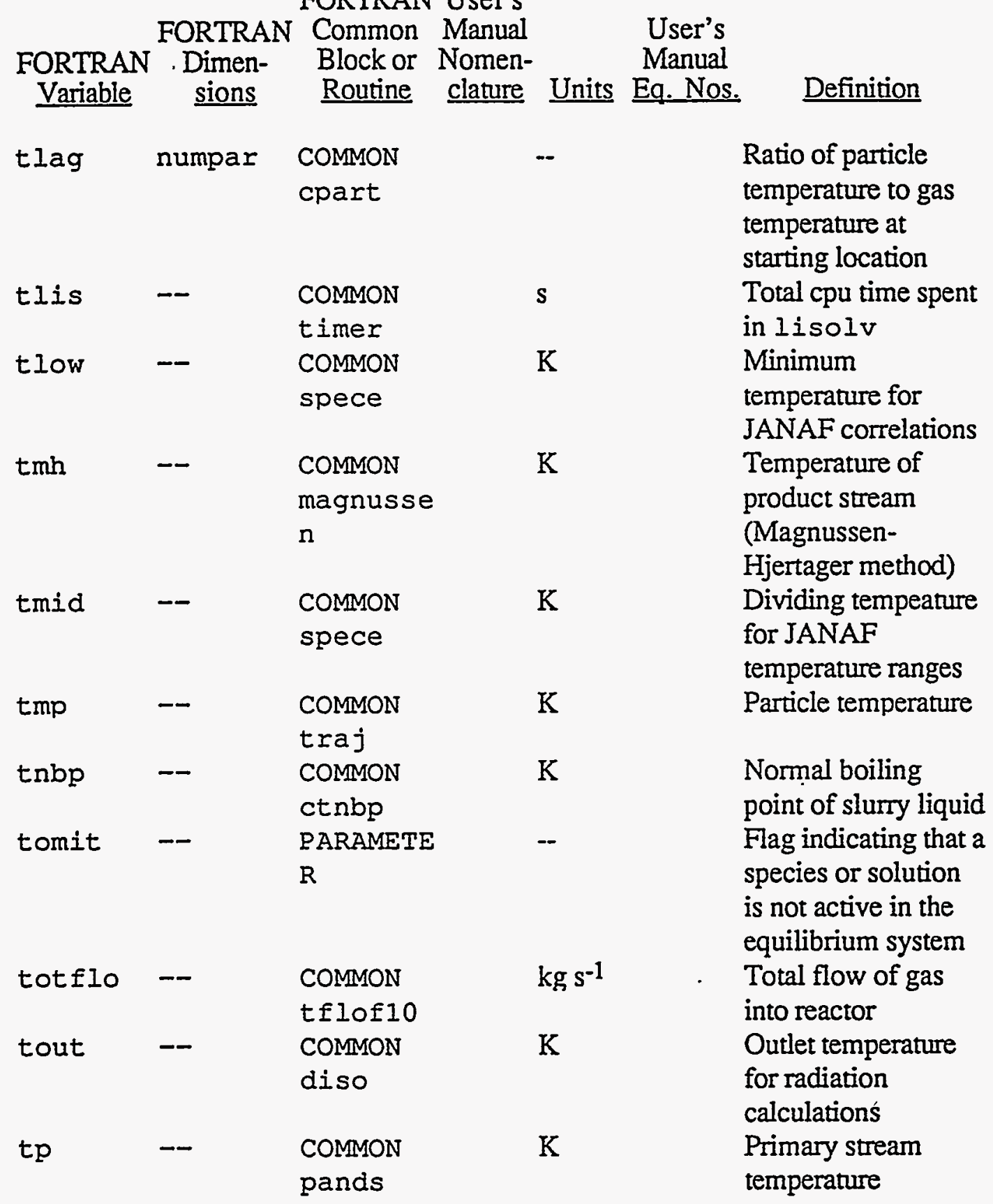




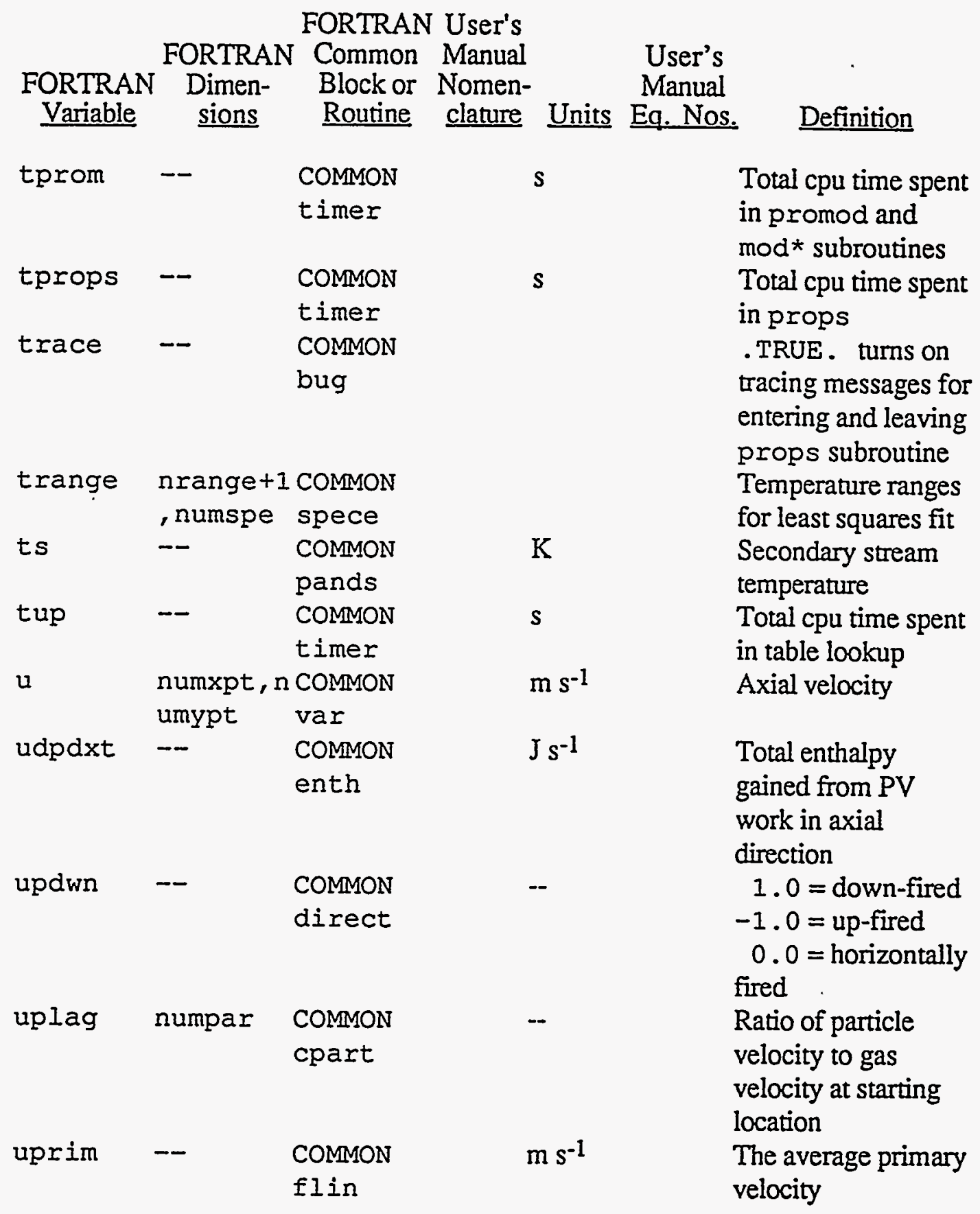




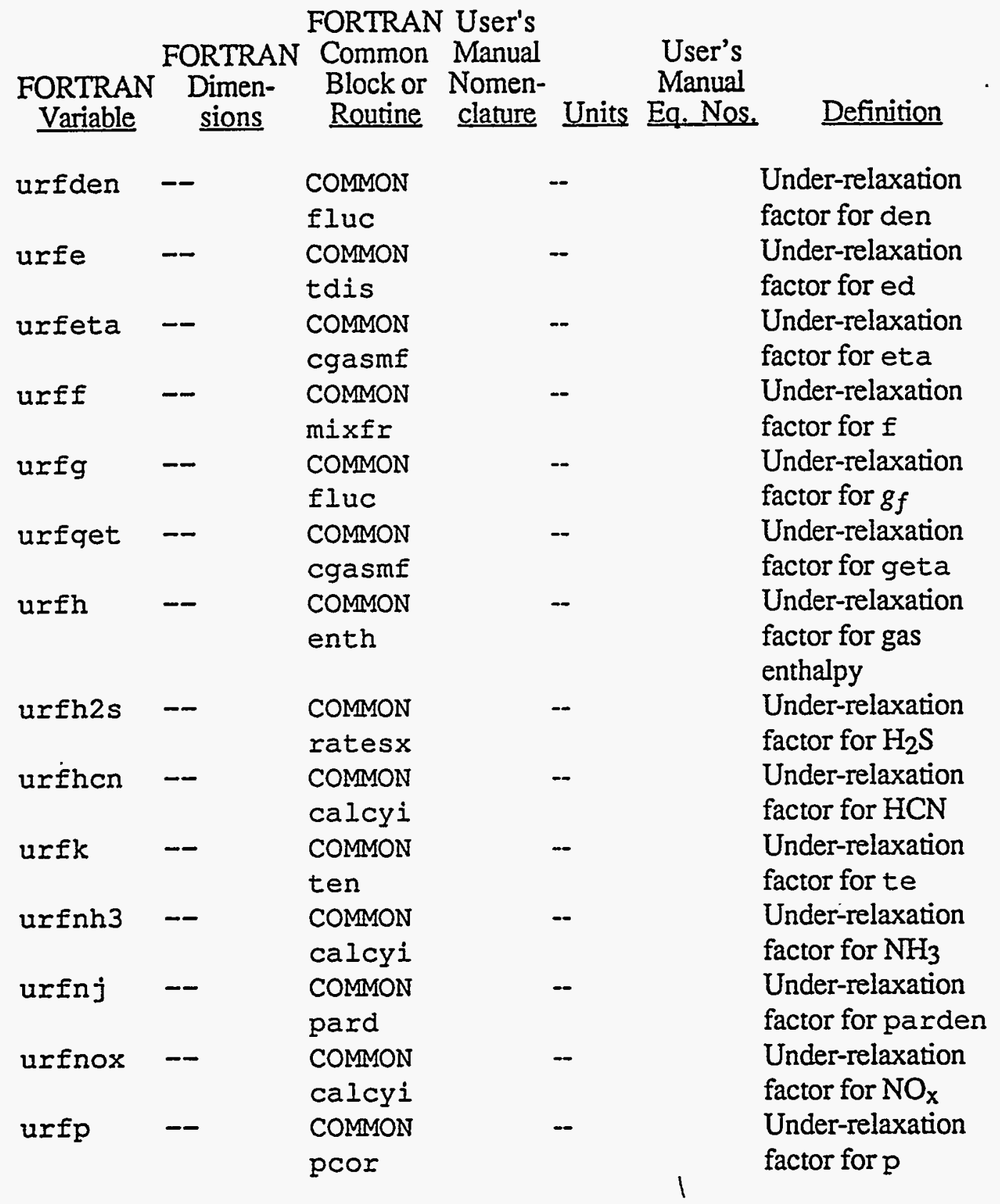




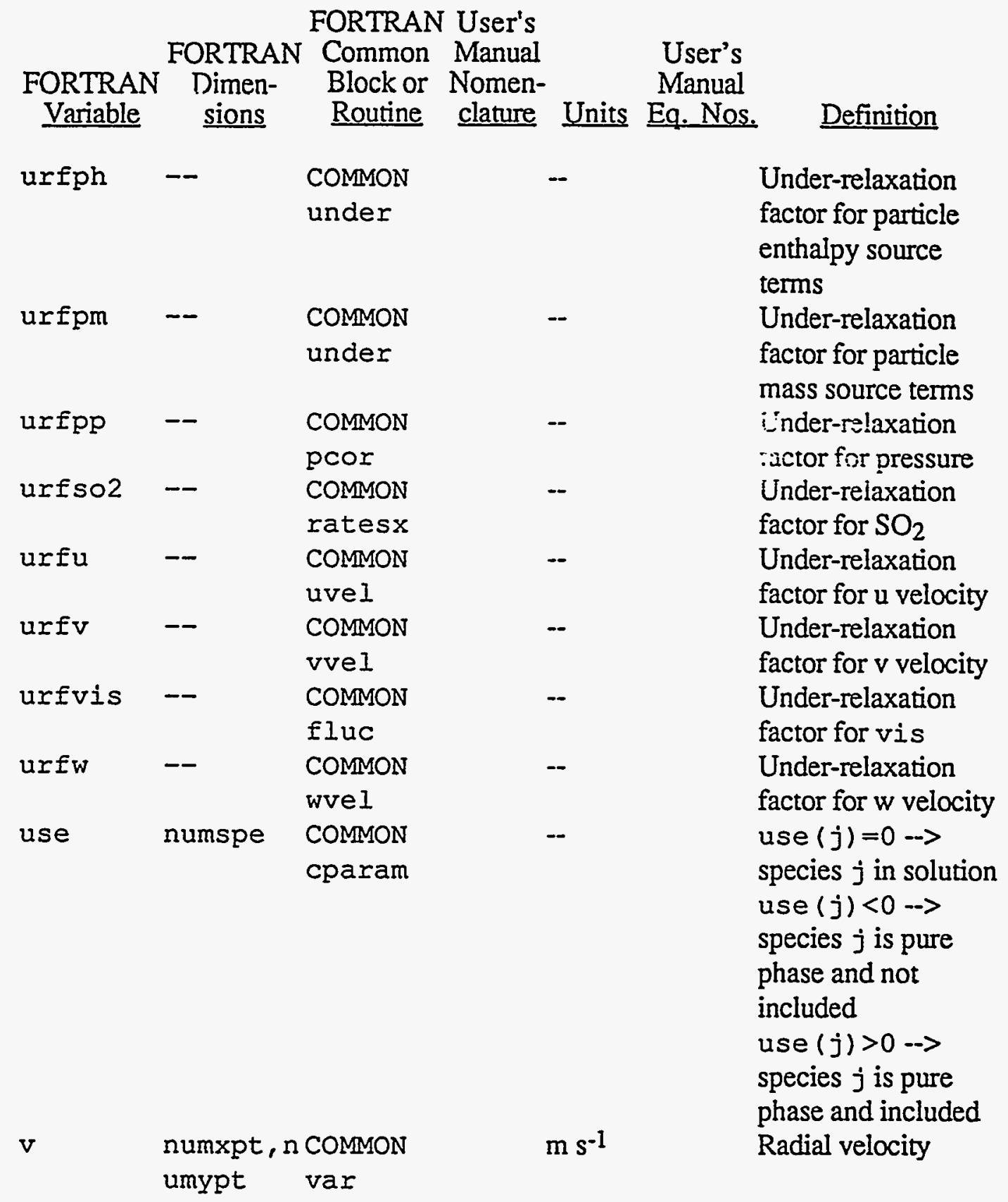


FORTRAN User's

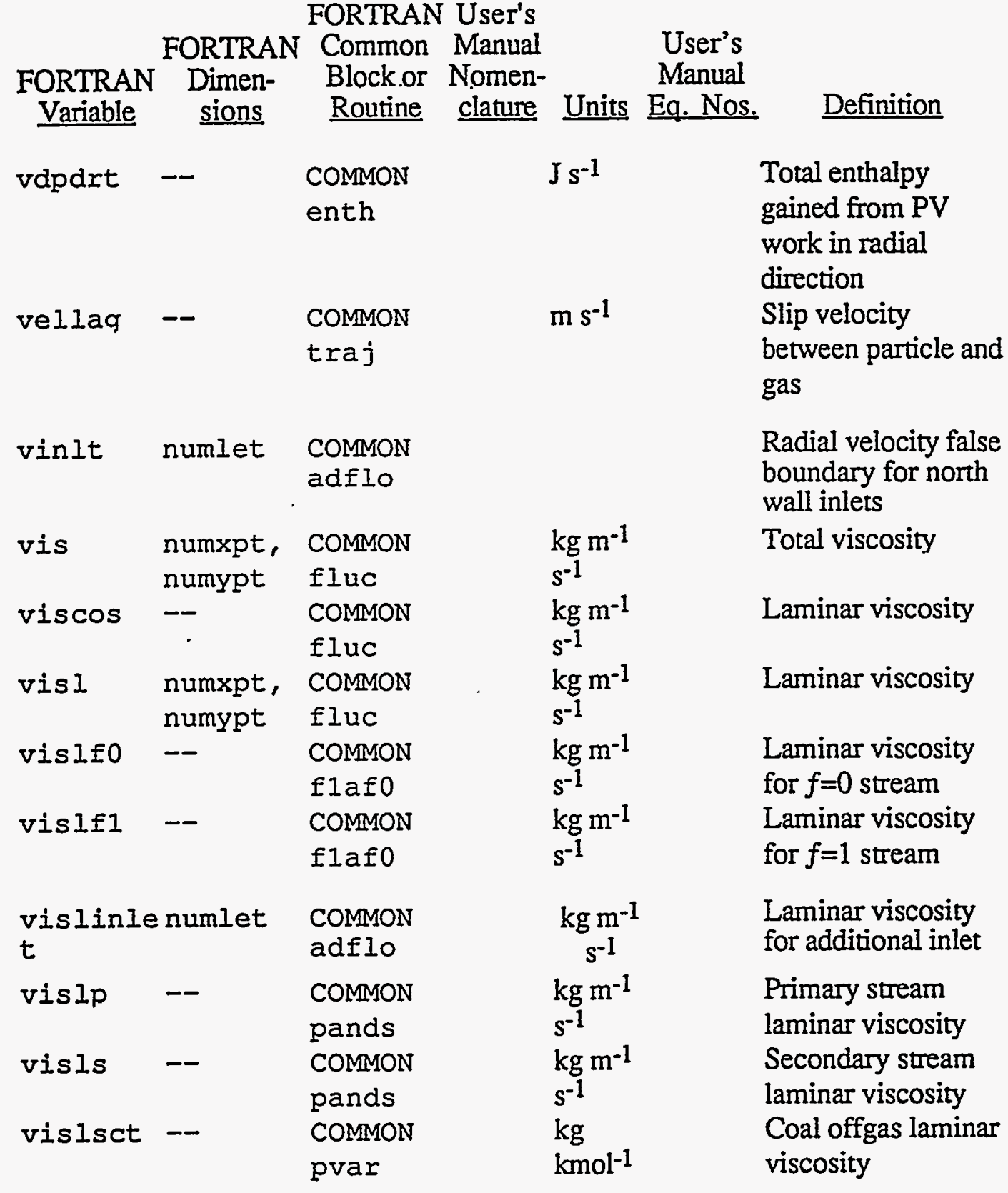




\begin{tabular}{|c|c|c|c|c|c|c|}
\hline $\begin{array}{l}\text { FORTRAN } \\
\text { Variable }\end{array}$ & $\begin{array}{l}\text { FORTRAN } \\
\text { Dimen- } \\
\text { sions }\end{array}$ & $\begin{array}{l}\text { FORTRAN } \\
\text { Common } \\
\text { Block or } \\
\text { Routine } \\
\end{array}$ & $\begin{array}{l}\text { User's } \\
\text { Manual } \\
\text { Nomen- } \\
\text { clature }\end{array}$ & Units & $\begin{array}{c}\text { User's } \\
\text { Manual } \\
\text { Eq. Nos. }\end{array}$ & Definition \\
\hline W & numxpt, $n$ & COMMON & & $\mathrm{m} \mathrm{s}^{-1}$ & & Tangential velocity \\
\hline & umypt & $\operatorname{var}$ & & & & \\
\hline wfac & numxpt & $\begin{array}{l}\text { COMMON } \\
\text { geom }\end{array}$ & & - & & Geometric factor \\
\hline wfinit & -- & $\begin{array}{l}\text { COMMON } \\
\text { cwater }\end{array}$ & & -- & & $\begin{array}{l}\text { Initial overall } \\
\text { fraction of water in } \\
\text { particles }\end{array}$ \\
\hline wmin & -- & $\begin{array}{l}\text { COMMON } \\
\text { cwater }\end{array}$ & & $\mathrm{kg} \mathrm{s}^{-1}$ & & $\begin{array}{l}\text { Total mass flow rate } \\
\text { of water into reactor }\end{array}$ \\
\hline wq & 4,5 & $\begin{array}{l}\text { COMMON } \\
\text { diso }\end{array}$ & $w_{m}$ & -- & $2-142$ & $\begin{array}{l}\text { Angular quadrature } \\
\text { weight }\end{array}$ \\
\hline wtm & $\begin{array}{l}\text { numxpt, } \\
\text { numypt }\end{array}$ & $\begin{array}{l}\text { COMMON } \\
\text { molwt }\end{array}$ & & $\begin{array}{l}\mathrm{kmol}^{2} \\
\mathrm{~kg}^{-1}\end{array}$ & & $\begin{array}{l}\text { Inverse of mixture } \\
\text { mol. wt. }\end{array}$ \\
\hline $\mathrm{x}$ & numspe+2 & $\begin{array}{l}\text { COMMON } \\
\text { cmatri }\end{array}$ & & & & $\begin{array}{l}\text { Solution to Gauss } \\
\text { matrix }\end{array}$ \\
\hline $\mathrm{x}$ & numspe+2 & $\begin{array}{l}\text { COMMON } \\
\text { cmatri }\end{array}$ & & & & $\begin{array}{l}\text { Solution to Gauss } \\
\text { matrix }\end{array}$ \\
\hline$x$ & numxpt & $\begin{array}{l}\text { COMMON } \\
\text { geom }\end{array}$ & & $\mathrm{m}$ & & $\begin{array}{l}\text { Location of axial } \\
\text { nodes }\end{array}$ \\
\hline XERAC & NUMSPE & $\begin{array}{l}\text { Common } \\
\text { TRAJ }\end{array}$ & & $\underset{\mathrm{kg}^{-1}}{\mathrm{kmoli}}$ & & $\begin{array}{l}\text { Species mole } \\
\text { number }\end{array}$ \\
\hline xfrac & numspe & $\begin{array}{l}\text { COMMON } \\
\text { traj }\end{array}$ & & -- & & Gas mole fractions \\
\hline $\mathrm{xh} 2 \mathrm{~s}$ & $\begin{array}{l}\text { numxpt, } \\
\text { numypt }\end{array}$ & $\begin{array}{l}\text { COMMON } \\
\text { ratesx }\end{array}$ & & -- & & $\mathrm{H}_{2} \mathrm{~S}$ mole fraction \\
\hline$X I$ & NUMP AR & $\begin{array}{l}\text { Common } \\
\text { PEAC }\end{array}$ & $\zeta$ & -- & $2-131$ & Surface area factor. \\
\hline
\end{tabular}




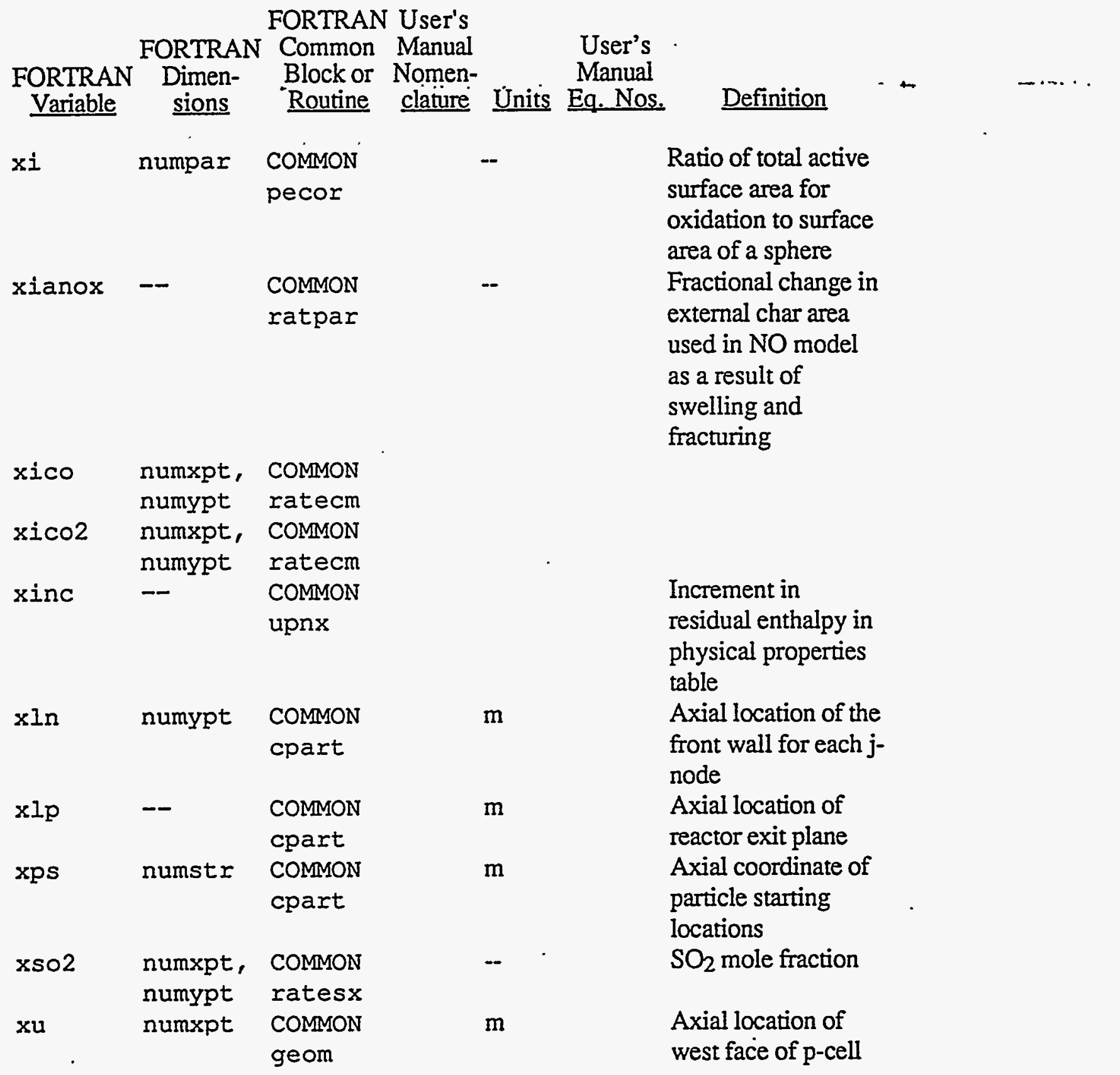




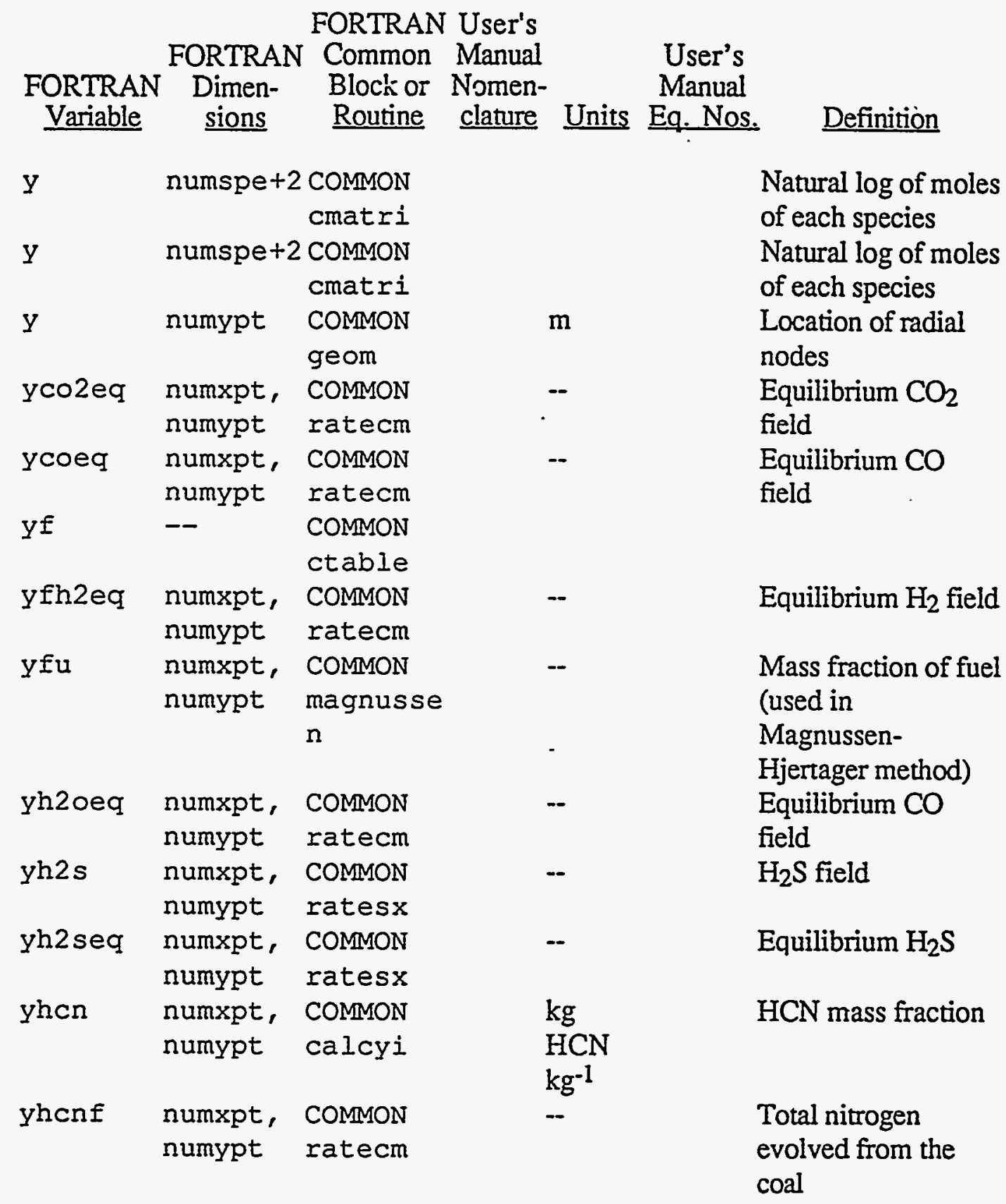




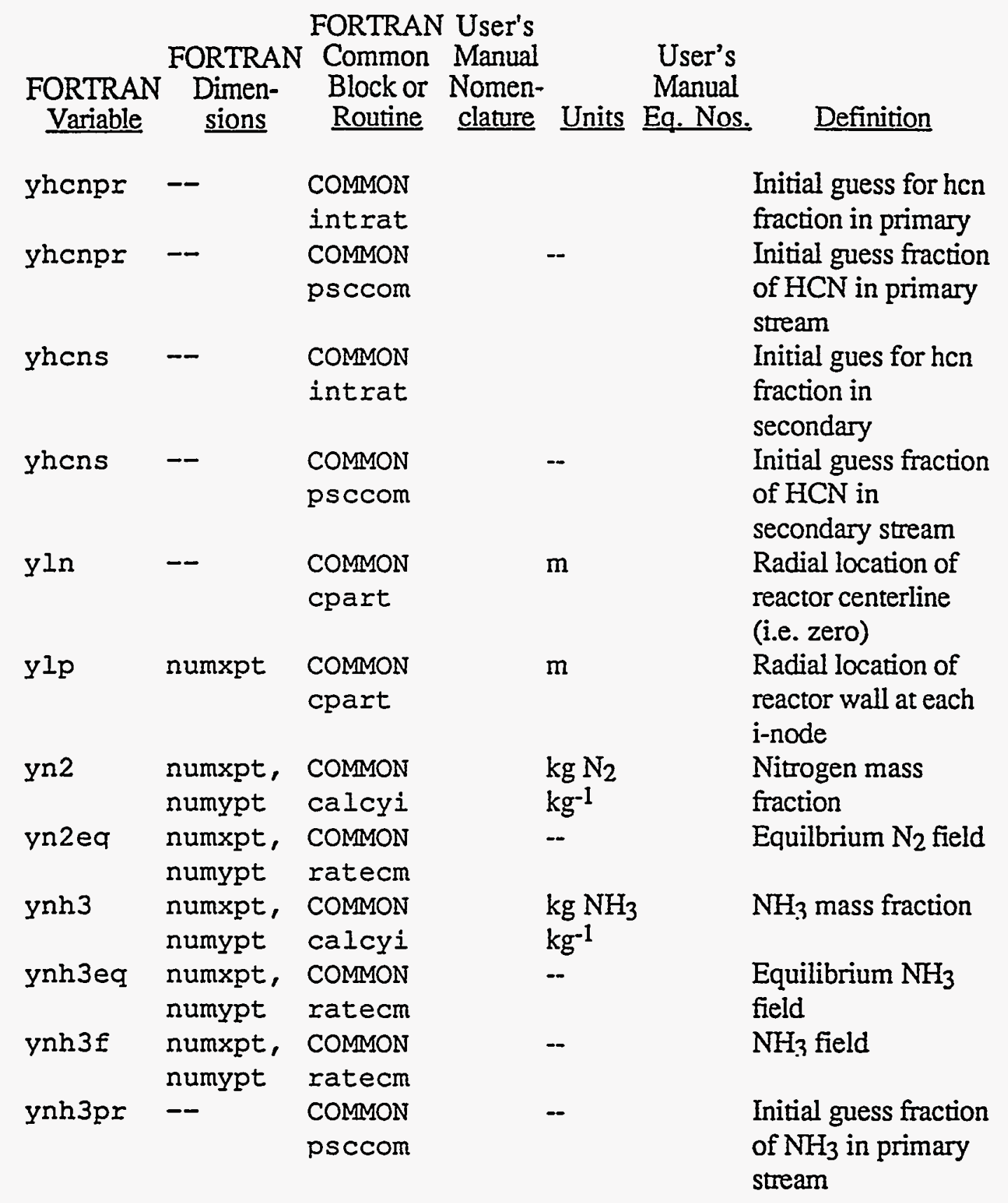




\begin{tabular}{|c|c|c|c|c|c|}
\hline $\begin{array}{l}\text { FORTRAN } \\
\text { Variable }\end{array}$ & $\begin{array}{l}\text { FORTRAN } \\
\text { Dimen- } \\
\text { sions }\end{array}$ & $\begin{array}{l}\text { FORTRAN } \\
\text { Common } \\
\text { Block or } \\
\text { Routine }\end{array}$ & $\begin{array}{l}\text { User's } \\
\text { Manual } \\
\text { Nomen- } \\
\text { clature }\end{array}$ & $\begin{array}{r}\text { User's } \\
\text { Manual } \\
\text { Units Eq. Nos. }\end{array}$ & Definition \\
\hline ynh3s & - & $\begin{array}{l}\text { COMMON } \\
\text { psccom }\end{array}$ & & -- & $\begin{array}{l}\text { Initial guess fraction } \\
\text { of } \mathrm{NH}_{3} \text { in } \\
\text { secondary stream }\end{array}$ \\
\hline ynoeq & $\begin{array}{l}\text { numxpt, } \\
\text { numypt }\end{array}$ & $\begin{array}{l}\text { COMMON } \\
\text { ratecm }\end{array}$ & & -- & $\begin{array}{l}\text { Equilibrium NO } \\
\text { field }\end{array}$ \\
\hline ynox & $\begin{array}{l}\text { numxpt, } \\
\text { numypt }\end{array}$ & $\begin{array}{l}\text { COMMON } \\
\text { calcyi }\end{array}$ & & $\begin{array}{l}\mathrm{kg} \mathrm{NO} \mathrm{NO}_{\mathrm{x}} \\
\mathrm{kg}^{-1}\end{array}$ & $\mathrm{NO}_{\mathrm{x}}$ mass fraction \\
\hline ynoxf & $\begin{array}{l}\text { numxpt, } \\
\text { numypt }\end{array}$ & $\begin{array}{l}\text { COMMON } \\
\text { ratecm }\end{array}$ & & . & $\begin{array}{l}\text { The amount of } \\
\text { nitrogen available } \\
\text { from the coal plus } \\
\text { the maximum } \\
\text { amount of thermal } \\
\text { NO that can be } \\
\text { formed when joint } \\
\text { thermal NO and fuel } \\
\text { NO calculations are } \\
\text { made }\end{array}$ \\
\hline yo2 & $\begin{array}{l}\text { numxpt, } \\
\text { numypt }\end{array}$ & $\begin{array}{l}\text { COMMON } \\
\text { calcyi }\end{array}$ & & $\begin{array}{l}\mathrm{kg} \mathrm{O}_{2} \\
\mathrm{~kg}^{-1}\end{array}$ & $\begin{array}{l}\text { Oxygen mass } \\
\text { fraction }\end{array}$ \\
\hline yo2eq & $\begin{array}{l}\text { numxpt, } \\
\text { numypt }\end{array}$ & $\begin{array}{l}\text { COMMON } \\
\text { ratecm }\end{array}$ & & -- & Equilibrium $\mathrm{O}_{2}$ field \\
\hline yoh & $\begin{array}{l}\text { numxpt, } \\
\text { numypt }\end{array}$ & $\begin{array}{l}\text { COMMON } \\
\text { ratecm }\end{array}$ & & -- & OH field \\
\hline yoheq & $\begin{array}{l}\text { numxpt, } \\
\text { numypt }\end{array}$ & $\begin{array}{l}\text { COMMON } \\
\text { ratecm }\end{array}$ & & -- & $\begin{array}{l}\text { Equilibrium } \mathrm{OH} \\
\text { field }\end{array}$ \\
\hline yox & $\begin{array}{l}\text { numxpt, } \\
\text { numypt }\end{array}$ & $\begin{array}{l}\text { COMMON } \\
\text { magnusse } \\
\text { n }\end{array}$ & & -- & $\begin{array}{l}\text { Mass fraction of } \\
\text { oxygen } \\
\text { (Magnussen- } \\
\text { Hjertager method) }\end{array}$ \\
\hline
\end{tabular}


FORTRAN User's

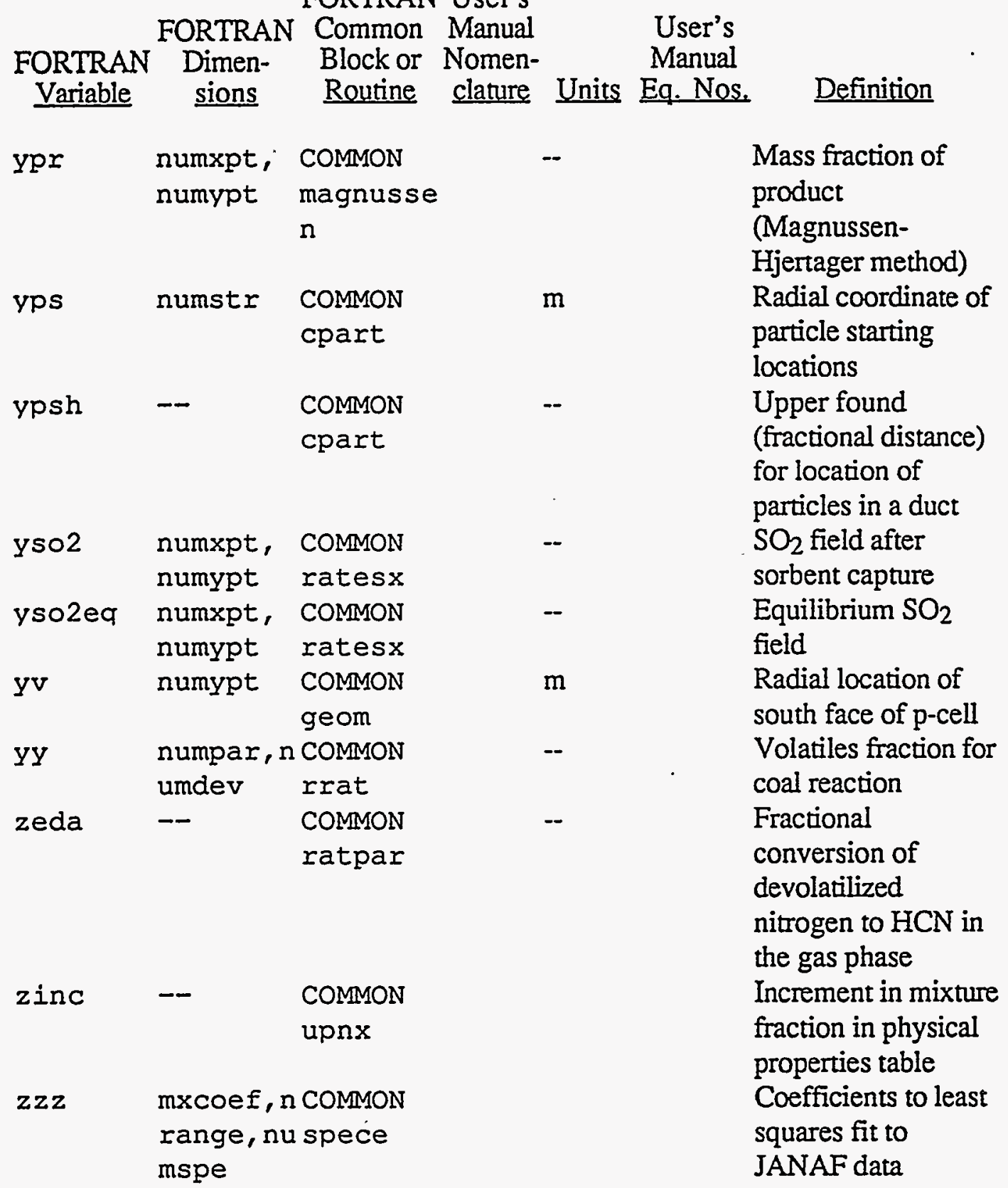




\section{Appendix I}

\section{Sample Calculations}

\section{Char Oxidation Parameters}

Table 2-8 reports a value of $300 \frac{\mathrm{m}}{\mathrm{s} \mathrm{K}}$ for $\mathrm{A}_{\mathrm{jl}}$ for the data of Field et al. (1967) for char $/ \mathrm{O}_{2}$ reaction. This value is derived from the value reported by Field et al. $\left(8710 \frac{\mathrm{g}}{\mathrm{cm}^{2} \mathrm{~s} \mathrm{~atm}}\right)$ as follows:

The oxidizer-char reaction rate is given by Eqn. 2-131, which takes into account the effect of mass transfer on the reaction rate. If mass transfer is neglected, Eqn. 2-131 becomes

$$
r_{h j l}=A_{j} M_{k j} \phi_{l} k_{j l} \zeta_{j} C_{o l g}
$$

where $r_{h j l}$ is $\mathrm{kg}$ char/s reacting for the $j^{\text {th }}$ particle classification and the $l^{\text {th }}$ char reaction. $A_{j}$ is the particle surface area $\left(\mathrm{m}^{2}\right), M_{h j}$ is the char molecular weight, $\phi_{l}$ is the stoichiometric factor (moles char required per mole of oxidizer), $\zeta_{j}$ is a particle surface area factor, and $C_{\text {olg }}$ is bulk concentration of oxidizer $\left(\mathrm{kmol}\right.$ oxidizer $\left./ \mathrm{m}^{3}\right)$. The rate coefficient $k_{j l}(\mathrm{~m} / \mathrm{s})$ is given by Eqn. $2-132$, using the parameters in Table 2-8. The units of Eqn. (I-1) are thus 
Sample Calculations

$\left(\frac{\mathrm{kg} \mathrm{char}}{\mathrm{s}}\right)=\left(\mathrm{m}^{2}\right)\left(\frac{\mathrm{kg} \mathrm{char}}{\mathrm{kmol} \mathrm{char}}\right)\left(\frac{\mathrm{kmol} \mathrm{char}}{\mathrm{kmol} \mathrm{oxidizer}}\right)\left(\frac{\mathrm{m}}{\mathrm{s}}\right)\left(\frac{\mathrm{m}^{2}}{\mathrm{~m}^{2}}\right)\left(\frac{\mathrm{kmol} \mathrm{oxidizer}}{\mathrm{m}^{3}}\right)$

Using the reaction rate of Field et al. (1967), $r_{h j l}$ is given by:

$$
r_{h j l}=A_{j} k_{j l} \zeta_{j} P_{\text {olg }}
$$

where the subscript " $\rho$ " has been added to $k_{j l}$ to indicate that this is the rate constant for Field et al. The rate coefficient $k_{j l}$ is given by

$$
k_{j l}=A_{j l} \exp \left(\frac{-E_{j l}}{R T_{j}}\right)
$$

where the subscript " $f$ " on $A_{j l f}$ indicates the Field et al. value. The units of Eqn. (I3) are given by

$$
\left(\frac{\mathrm{kg} \mathrm{char}}{\mathrm{s}}\right)=\left(\mathrm{m}^{2}\right)\left(\frac{\mathrm{kg} \mathrm{char}}{\mathrm{m}^{2} \mathrm{~s} \mathrm{~Pa}}\right)\left(\frac{\mathrm{m}^{2}}{\mathrm{~m}^{2}}\right)(\mathrm{Pa})
$$

Equating (I-1) and (I-3), one obtains

$$
k_{j l}=\frac{k_{j l g} P_{o l g}}{M_{h j} \phi_{l} C_{o l g}}
$$

The relationship between $A_{j l}$ and $A_{j l f}$ can be obtained by substituting Eqn. 2-132 with $n$ equal to unity and (I-4) into $(1-6)$, and by using the ideal gas law to replace the ratio of $P_{\text {olg }}$ and $C_{\text {olg }}$ with $R T_{j}$ to obtain

$$
A_{j l}=A_{j l}\left(\frac{R}{M_{h j} \phi_{l}}\right)
$$

The value of $A_{j l}$ reported in Table 2-8 for Field et al. is thus obtained as follows: 


$$
A_{j l}=\left(8710 \frac{\mathrm{g}-\mathrm{char}}{\mathrm{cm}^{2} \mathrm{~s} \mathrm{~atm}}\right)\left[\frac{82.05 \frac{\mathrm{cm}^{3} \mathrm{~atm}}{\mathrm{~mol}-\mathrm{O}_{2} \mathrm{~K}}}{\left(\frac{12 \mathrm{~g}-\mathrm{char}}{\mathrm{mol}-\mathrm{char}}\right)\left(\frac{2 \mathrm{~mol}-\mathrm{char}}{\mathrm{mol}-\mathrm{O}_{2}}\right)}\right]\left(\frac{\mathrm{m}}{100 \mathrm{~cm}}\right)=300 \frac{\mathrm{m}}{\mathrm{s} \mathrm{K}}
$$


Appendix $J$

$$
\begin{gathered}
\text { General Model of Coal } \\
\text { Devolatilization }
\end{gathered}
$$


General Model of Coal Devolatilization 
Reprinted from Joural of ENERGY \& FUELS. 1988, 2, 405.

Copyright ( $) 1988$ by the American Chemical Society and reprinted by permission of the copyright owner.

\title{
General Model of Coal Devolatilization ${ }^{\dagger}$
}

\author{
P. R. Solomon,* D. G. Hamblen, R. M. Carangelo, M. A. Serio, and \\ G. V. Deshpande \\ Advanced Fuel Research, Inc., 87 Church Street, East Hartford, Connecticut 06108 \\ Received December 21, 1987. Revised Manuscript Received April 21, 1988
}

\begin{abstract}
A general model for coal devolatilization, which combines a functional group model for gas evolution and a statistical model for tar formation, has been presented. The tar formation model includes depolymerization, cross-linking, external transport, and internal transport. The cross-linking is related to the evolutions of $\mathrm{CO}_{2}$ and $\mathrm{CH}_{4}$, with one cross-link formed per molecule evisived. The model predictions compare favorably with a variety of data for the devolatilization of F:trtsburgh Seam coal and North Dakota (Beulah) lignite, including volatile yields, extract yields, cross-link densities, and tar molecular weight distributions. The variations with pressure, devolatilization temperature, rank, and heating rate were accurately predicted. Comparison of the model with several sets of data employing alternative assumptions on transport suggests that assuming that the particle is well mixed (i.e. the surface concentration of tar molecules is the same as the bulk) overpredicts the transport rate. For $50-\mu \mathrm{m}$ particles, assuming that the internal-transport limitation dominates (i.e. neglecting the external transport) provides a good fit to the data. The rank dependence of tar formation, extract yields, cross-linking, and viscosity appears to be explained by the rank dependence of $\mathrm{CO}_{2}$ yields and its associated cross-linking. High $\mathrm{CO}_{2}$ yields in low-rank coals produce rapid cross-linking at low temperatures and hence thermosetting behavior, low tar yields, low extract yields, loss of solventswelling properties, and high viscosities. The relative importance of cross-linking compared to bond breaking is, however, sensitive to heating rate, and this effect is predicted by the model. Areas for improving the model include (1) refinement of the internal and external transport assumptions, (2) accounting for hydroaromatic structures and bridge structures besides ethylene, and (3) including polymethylene "guest". molecules.
\end{abstract}

\section{Introduction}

Coal devolatilization is a process in which cool is transformed at elevated temperatures to produce gases, tar, and char. (Tar is defined as the room-temperature condensibles formed during coal devolatilization.) The combined chemical and physical processes in devolatilization have been reviewed by a number of investigators. ${ }^{1-6}$ Gas formation can often be related to the thermal decomposition of specific functional groups in the coal and can be predicted with reasonable accuracy by models employing first-order reactions with ultimate yields. ${ }^{5-15}$ On the other hand, tar and char formation are more complicated, and success in mechanistic modeling of tar formation has been more limited.

Predicting tar formation is, however, important for several reasons. Tar is a major volatile product (up to $40 \%$

* To whom correspondence is to be addressed

t Presented at the Symposium on Coal Pyrolysis: Mechanisms and Modeling, 194th National Meeting of the American Chemical Society, New Orleans. LA. August 31-September 4, 1987. of the DAF coal weight for some bituminous coals). Tar yields vary substantially depending on reactor conditions

(1) Howard. J. B.; Peters, W. A.: Serio, M. A. -Coal Devolatilization Information for Resctor Modeling"; Final Roport on EPRI Projoct No. 986-5, 1981.

(2) Howard, J. B. In Chemistry of Coal Utilization; Elliott, M. A., Ed; Wilay: New York, 1981: Chapter 12, p 665.

(3) Gavalas, G. R. Coal Pyrolysis; Finevier: Amsterdem, The Neth. erlands, 1981.

(4) Suuberz. E. M. In Chemistry of Coal Conversion; Schloubers, R H.. Ed. Plenum: Now York, 1985; Chapter 4.

(5) Solomon, P. R; Hamblen, D. G. In Chemiztry of Coal Converrion; Schloubers, R. H. Ed; Plenum: Now York, 1985; Chaptes 5, p 121. (6) Serio, M. A; Hemblon, D. G.; Mrrkhem, J. R.; Solomon, P. R. Energy Fuel 1987, 1, 138

(7) Suuberg, E. M. Poters, W. A.; Howard, J. B. Sevenseenth Symposium (International) on Comburtion; The Comburtion Institute: Pittsburgh, PA, 1979; p 117.

(8) Juntgen. H; van Heek, K. H. Fuel Process. Technol. 1979, 2, 261. (9) Weimer, R. F.; Ngan, D. Y. Prepr. Pap.-Am. Chem. Soc., Div. Fuel Chem. 1979, 24(3), 129.

(10) Campbell J. H. Fuel 1978, 57, 217.

(11) Solomon. P. R.; Colket, M. B. Seventeenth Symposium IInternational) on Combustion: The Comburtion Institute: Pittuburgh, PA, 1979; p 131. 
(pressure, heating rate, final temperature, bed geometry, particle size, etc.). In combustion or gasification, tar is often the volatile product of highest initial yield and thus controls ignition and flame stability. It is a precursor to soot, which is important to radiative heat transfer. The process of tar formation is linked to the char viscosity ${ }^{16-19}$ and the subsequent physical and chemical structure of the char and so is important to char swelling and reactivity. Also, because tar molecules are sometimes minimally disturbed coal molecular fragments, primary tars provide important clues to the structure of the parent coal. $5,6,20$

It is generally agreed that the tar formation includes the following steps: (1) depolymerization by rupture of weaker bridges in the coal macromolecule to release smaller fragments that make up the "metaplast";,5,7.16,21-33 (2) repolymerization (cross-linking) of metaplast molecules; $35,7,16,21-33$ (3) transport of lighter molecules away from the surface of the coal particles by combined vaporization and gas phase diffusion;, 2332 (4) internal transport of lighter molecules to the surface of the coal particles by convection and diffusion in the pores of nonsoftening coals $24,27,34,35$ and liquid-phase or bubble transport in softening coals. $17,36-38$ Char is formed from the unreleased or recondensed fragments. Varying amounts of loosely bound "guest" molecules, usually associated with the extractable material, are also released in devolatilization.

(12) Solomon, P. R.; Hamblen, D. G.; Carangelo, R. M.; Krause, J. L Nineteenth Symposium (International) on Combustion; The Combus tion Institute: Pittsburgh, PA, 1982; p 1139.

(13) Solomon, P. R.; Serio, M. A.; Carangelo, R. M.; Markham, J. R. Fuel 1986, 65, 182 .

(14) Xu, W.-C.; Tomita, A. Fuel 1987, 66, 627.

(15) Juntgen, H. Fuel 1984, 63, 731.

(16) Fong, W. S.; Peters, W. A.; Howard, J. B. Fuel 1986, 65, 251.

(17) Oh, M. S.: Peters, W. A.; Howard, J. B. Proceedings of the 1983 Intermational Conference on Coal Science; Center for Conference Management: Pittsburgh, PA, 1983; p 483.

(18) Fong, W. S.; Khalil, Y. F.; Peters, W. A.; Howard, J. B. Fuel 1986, $65,195$.

(19) Van Krevelen, D. W. Properties of Polymers; Elsevier: Amsterdam, 1976.

(20) Solomon, P. R. New Approaches in Coal Chemistry; ACS Symposium Series 169; American Chemical Society: Weshington, DC, 1981; pp 61-71.

(21) van Krovelon, D. W.; Schuyer, J. Coal Science; Elsevier: Amsterdam, 1957.

(22) Anthony, D. B.; Howard, J. B.; Hottel, H. C.; Meissner, H. P. Fifteenth Symposium (International) on Combustion: The Combustion Institute: Pittsburgh, PA, 1974; p 1303.

(23) Unger, P. E.; Suuberg, E. M. Eighteenth Symposium (International) on Combustion; The Combustion Institurte: Pittsburgh, PA, 1981; p 1203.

(24) Russel, W. B.; Saville, D. A.; Greene, M. I. AIChE J. 1979, 25, 65.

(25) James, R. K.; Mills, A. F. Lett. Heat Mass Transfer 1976, 3, 1.

(26) Lewellen, P. C. S.M. Thesis, Department of Chemical Engineering, MIT, 1975.

(27) Chen, I. W; Wen, C. Y. Prepr. Pap.-Am. Chem. Soc., Div. Fuel Chem. 1979, 24(3), p 141 .

(28) Nikes, S.; Keratein, A. R. Combust. Flame 1986, 66, 95.

(29) Niksa, S. Combust. Flame 1986, 66, 111.

(30) Solomon, P. R; Squire, K. R.; Carangelo, R. M. Proceedings of the International Conference on Coal Science; Pergamon: Sydney, Australia, 1985; p 945.

(31) Solomon, P. R. Squire, K. R. Prepr. Pap.-Am. Chem. Soc o, Div. Fuel Chem. 1985, 30(4), 347.

(32) Suuberg, E. M; Unger, P. E; Lilly, W. D. Fuel 1985, 64, 956.

(33) Solomon, P. R.; Hamblen, D. G.; Carangelo, R. M.; Serio, M. A. Deshpande, G. V. Prepr. Pap.-Am. Chem. Soc., Div. Fuel Chem. 1987, 32(3), 83.

(34) Gavales, G. R.; Wilke, K. A. AIChE J. 1980, 26, 201.

(35) Simons, G. A. Prog. Energy Combust. Sci. 1983, 9, 269.

(36) Suuberg, E. M. Sezen, Y. Proceedings of the International Conference on Coal Science; Pergamon: Sydney, Australia, 1985; p 913.

(37) Melie, P. F.; Bowman, C. T. Combust. Sci. Technol. 1983, 31, 195; Paper presented at the Western States Section of the Combustion Institute, Salt Lake City, UT, 1982.

(38) Oh. M. S. Sc.D. Thesis, Department of Chemical Engineering, MIT, Cambridge, MA, 1985.
The complexity of proposed devolatilization models varies substantially. They can be divided into four categories. The simplest are the "weight loss models" employing a single rate, ${ }^{622,30-42}$ two rates, ${ }^{39,43}$ multiple parallel rates, or distributed rates. ${ }^{9.22}$ These models do not account for the variations in tar yield with reaction conditions, and a number of "tar formation models" incorporating retrogressive char-forming reactions and mass transport have been proposed that account for such variations. $16.21-33,33,4-19$ A recent innovation has been the description of the decomposition and repolymerization of the macromolecular network by using statistical methods. ${ }^{28,29,44-66,50.51}$

Most of the above models do not consider the evolution of gas species, which have been treated in a number of "species evolution/functional group models" as parallel first-order reactions. ${ }^{5-13}$ More complicated "comprehensive chemical models" also describe the composition of the char and $\operatorname{tar} .3,5,6,11-13,33,48,49.51$

The level of detail required in a model depends on its application. In the modeling of combustion and gasification, the simple "weight loss models" have often been employed. However, to predict the variations in yield with reactor conditions, the more complicated "tar formation modelg" must be used. A case can also be made for employing "species evolution/functional group models" or "comprehensive chemical models". For example, in predicting the energy released from combustion of the volatiles, it is important to know that for low-rank coals a high percentage of the volatiles may be noncombustible $\mathrm{H}_{2} \mathrm{O}$ and $\mathrm{CO}_{2}$. For a North Dakota lignite, the total of these two components can be as high as $35 \%$ of the rapidly released volatiles which are important for ignition. ${ }^{6}$ In addition, the swelling, particle agglomeration properties, char reactivity, and char fragmentation are functions of the char composition. Soot formation (which can dominate radiative energy transport) is controlled by the tar amount.

In the modeling of liquefaction and mild gasification, knowledge of the chemical makeup and molecular weight distribution of the soluble and volatile products is essential, requiring the more complete "comprehensive chemical models".

This paper presents a "comprehensive chemical model" for coal devolatilization that considers the evolution of gas, tar, char, and guest molecules. The model is general in its applicability to bituminous coals, subbituminous coals,

(39) Kobayashi, H.; Howard, J. B.; Sarofim, A. F. Sixteenth Symposium (International) on Combustion: The Combustion Institute: Pittsburgh, PA, 1977; p 411. Kobayashi, H. Ph.D. Thesis, Department of Mechanical Engineering, MIT, Cambridge, MA. 1976.

(40) Nikss, S.; Heyd, L E; Russel, W. B.; Saville, D. A. Twentieth Symposium (Intermational) on Combustion; The Combustion Institute: Pittsburgh, PA, 1984; p 1445.

(41) Badzioch, S.; Hawksley, P. G. W. Ind. Eng. Chem. Process Des. Dev. 1970, 9, 521 .

(42) Maloney, D. J; Jenkins, R. G. Twentieth Symposium (Internetional) on Combustion; The Combustion Institute: Pittsburgh, PA, 1984; p 1435.

(43) Witte, A B. Gat N. Presented at the DOE Direct Utilization AR\&TD Contractor's Meeting, Pittsburgh, PA, 1983.

(44) Solomon, P. R; King, H. H. Fuel 1984, 63, 1302.

(45) Solomon, P. R. Squire. K. R. Carangelo, R. M. Prepr. Pop.-Am. Chem. Soc., Div. Fuel Chem. 1984, 29(1), 10.

(46) Squire, K. R.; Solomon, P. R.; Carangelo, R. M.; DiTaranto, M. B. Fuel 1986, 65, 833 .

(47) Squire, K. R.; Solomon, P. R.; DiTaranto, M. B.; Carangelo, R. M. Prepr. Pap.-Am. Chem. Soc., Div. Fuel Chem. 1985, 30(1), 386. (48) Gavalas, G. R. Cheong, P. H.: Jain. R. Ind. Eng. Chem. Fundam. 1981, $20,113$.

(49) Gavalas, G. R.; Cheong, P. H.; Jain, R. Ind. Eng. Chem. Fundam. 1981, 20, 122.

(50) Solomon, P. R; Hamblen, D. G.; Carangelo, R. M.; Serio, M. A.; Deshpande, G. V. Combust. Flame 1988, 71, 137.

(51) Solomon, P. R.; Hamblen, D. G.; Deshpande, G. V.; Serio, M. A. Coal Sci. Technol. 1987, 11, 601. 
and lignites (employing rank-independent kinetic parameters), in its application to reactors of widely differing heating rates $\left(0.05\right.$ to $\left.20000^{\circ} \mathrm{C} / \mathrm{s}\right)$, and in its ability to predict the variations in tar yield with reactor conditions.

Two previously developed models, a functional group (FG) model 5,6,11-13 (a "species evolution/functional group model $l^{n}$ ) and a depolymerization-vaporization-cross-linking (DVC) model ${ }^{30,31,4 \text { Hin }^{-1}}$ ( $\mathrm{a}$ "tar formation model") have been combined as subroutines of what is now called the "FGDVC' model. $.33,50,51$ The DVC subroutine is employed to determine the yield of tar and the molecular weight distribution of the tar and char. The FG subroutine is used to describe the gas evolution and the elemental and functional group compositions of the tar and char. Cross-linking in the DVC subroutine is computed by assuming that this event is correlated with $\mathrm{CO}_{2}$ and $\mathrm{CH}_{4}$ evolutions predicted in the FG subroutine. The dependence of the yield of rapidly released $\mathrm{CO}_{2}$ (which is related to coal rank or weathering) is the factor that controls the thermosetting or thermoplastic behavior of coals.

The combined FG-DVC model was described in two previous publications, ${ }^{50,51}$ and comparisons were made to a limited set of data. In this paper, a description of internal transport has been added to the model. The model equations are presented, and comparisons are made to a wider set of data. The paper also includes a discussion of the assumptions, approximations, and exceptions to the model and a sensitivity analysis for the parameters of the DVC subroutine. The model describes the processes of (1) depolymerization and hydrogen consumption, (2) cross-linking, (3) external transport, (4) internal transport, (5) gas formation for all principal species, (6) tar composition, and (7) char composition.

The work presented here is limited to dilute phase reactions of small coal particles where internal temperature gradients can be neglected. Secondary gas phase reactions have been discussed elsewhere, ${ }^{6}$ and reactions of pyrolysis products with a char bed and large particle effects have not yet been included. Only reactions involving $\mathrm{C}, \mathrm{H}$, and $O$ are discussed here.

A number of coal composition parameters and reactor parameters (pressure, particle time-temperature profile) are required to predict the pyrolysis behavior. A substantial reduction in the number of parameters that must be measured for each coal is made by the use of rank-independent kinetic rates. These parameters have already been determined for a wide variety of coals and reactors. This simplification is a good first approximation to describe the kinetics of individual evolved species and the functional group decompositions. $5,5,9,52-55$ The properties predicted as a function of time, include the following: for tar, molecular weight distribution, elemental and functional group composition, and yield; for char, molecular weight distribution, elemental and functional group composition, yield, cross-link density, and extract yield; for gas, yields of individual light gas species. Results are presented for a Pittsburgh Seam bituminous coal and a North Dakota lignite.

\section{Experimental Section}

Coals Examined. The two coals described in this paper are a Pittsburgh Seam bituminous coal and a North Dakota (Beulah, Zap) lignite. Samples of the Pittsburgh Seam coal were obtained

(52) Solomon, P. R; Hamblen, D. G. Prog. Energy Combust. Sci. 1983, 9, 323.

(53) Xu, W. C.; Tomita. A. Fuel 1987, 66, 632

(54) Agarwal, P. K. Fuel 1985, 64, 870.

(55) Agarwal, P. K.; Agnew, J. B.; Ravindran, N.; Weimann, R. Fuel 1987, 66, 1097. from the Pittsburgh Energy Technology Center and the Argonne National Laboratory premium coal sample collection. Samples of the North Dakota (Beulah, Zap) lignite were obtained from the University of North Dakota Energy Research Center and the Argonne National Laboratory premium coal sample collection. Data on the premium samples are presented in ref 56 , and data on the other two samples, in ref. 6. The FG-DVC model was also compared to data on Pittsburgh coal samples from ref 7,16 , and 22, and characterizations of these samples are presented therein.

Coal Characterization. The cross-link density was estimated by using the volumetric swelling technique developed by Larsen and co-workers. . $^{7-69}$ Pyridine extract yields were obtained by using a Soxhlet apparatus. Molecular weight distributions of tars were obtained at SRI International on the field-ionization mass spectrometry (FIMS) apparatus described by St. John et al. ${ }^{\infty}$ Tar samples were collected from the pyrolysis apparatus and vaporized from a heated probe into the FIMS apparatus. In addition, coal samples were pyrolyzed directly in the FIMS apparatus.

Apparatus. Pyrolysis experiments were performed in several apparatuses that have been described previously including a heated-grid pyrolyzer, ${ }^{5,12}$ a heated-tube reactor, ${ }^{6,13}$ and a thermogravimetric analyzer with analysis of evolved products by Fourier transform infrared (FT-IR) spectroscopy (TG-FTIR).6.61

\section{General Model}

Any general model of a process as complicated as coal devolatilization must of course be a gross approximation. However, there are many general trends that have been observed in devolatilization. The trick in developing a model is to pick a set or cirst approximations that best match the majority of these trends. There will of course be exceptions to the trends. These exceptions can be treated as perturbations to the first-order approximation. Differences in models occur because of the subjective choice of what is a general trend and what is an exception. The following discussion presents the authors' view of the general trends and the exceptions.

General Trends in Devolatilization. The general model of coal pyrolysis is based on a number of observations that have been previously made concerning coal pyrolysis. These are as follows: (i) Pyrolysis species kinetics are insensitive to rank. $5,6,11-13,52-65$ (ii) Species amounts vary with coal rank and can be correlated with the coal's functional group compositions. $5,6,14,15,48,49,52$ The evolution of each species can be correlated with the change in the corresponding functional group composition in the char. 5,6,52 (iii) The primary tar composition is similar (except for a higher concentration of methyl groups) to that of the parent coal for bituminous coals and rapidly heated low-rank coal. $5,20,45,62-84$ (iv) Tar yields are controlled by the amount of donatable hydrogen and how efficiently it is used. $5,6,20,46$ (v) Cross-linking correlates with $\mathrm{CO}_{2}$ and $\mathrm{CH}_{4}$ evolution. ${ }^{.0,51}$

The general outline of devolatilization based on these observations was presented by Solomon and Hamblen ${ }^{5}$ and Serio et al. ${ }^{6}$ Figure 1 (adapted from ref 6) presents a hypothetical picture of the coal's or char's organic structure at successive stages of devolatilization. The figure represents (a) the raw coal, (b) the formation of tar and light

(56) Vorres, IC. S. Prepr. Pap.-Am. Chem. Soc, Div. Fuel Chem. 1987, 32(4), 221

(57) Green. T. K.; Kovac, J.; Larsen. J. W. Fuel 1984, 63, 935.

(58) Green, T. K.; Kovac, J.; Larsen, J. W. In Cocl Structure; Meyers,

R. A., Ed.; Academice New York, 1982.

(59) Suuberg, E. M.; Lee, D.; Larsen, J. W. Fuel 1985, 64, 1668.

(60) St. John, G. A.; Butrill, S. E., Jr.; Aabar, M. ACS Symp. Ser. 1978, No. 71, 223.

(61) Carangelo, R. M; Solomon, P. R.; Gerson. D. J. Fuel 1987, 66, 960.

(62) Solomon, P. R.; Colket, M. B. Fuel 1978, 57, 748.

(63) Brorrn, J. K.; Dryden. I. G. C.; Dunevein, D. H.; Joy, W. K.; Pankhurst, K. S. J. Inst. Fuel 1958, 31, 259.

(64) Orning, A. A.; Greifer, B. Fuel 1956, 35, 318. 

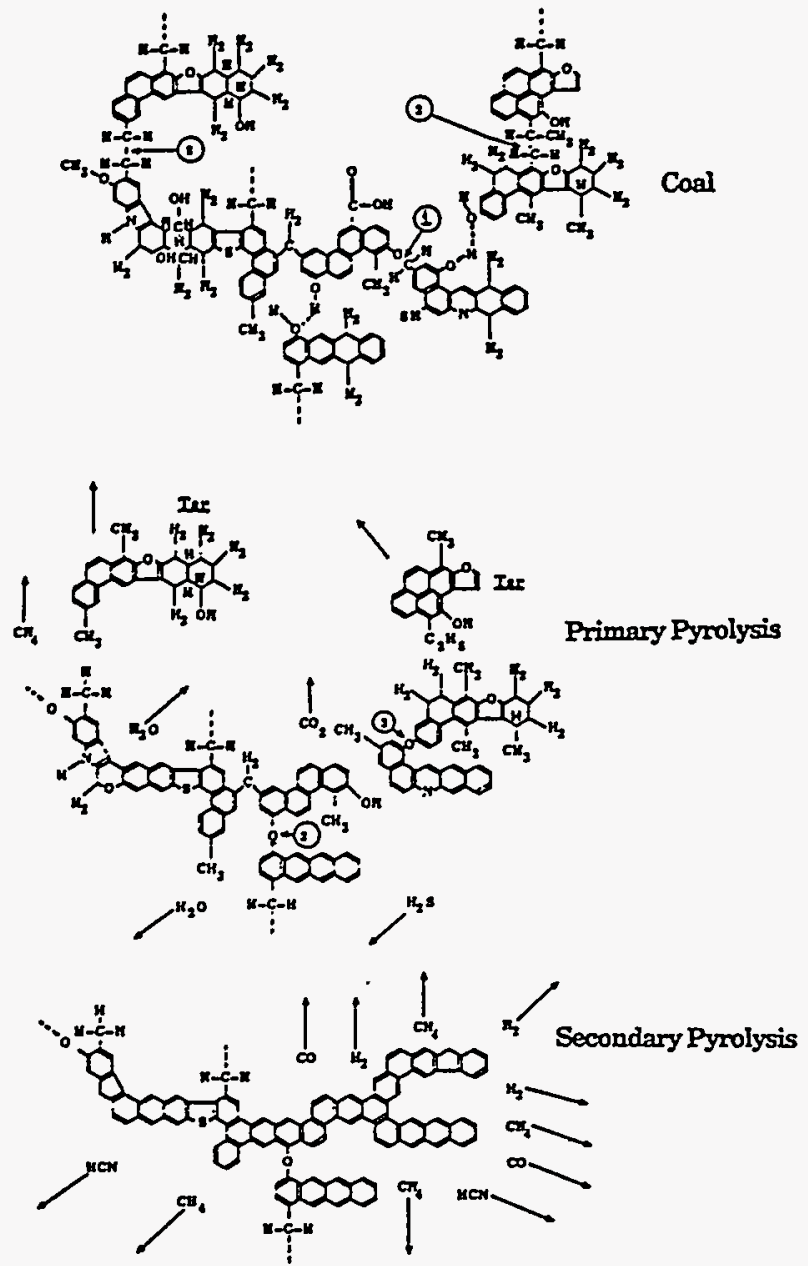

Figure 1. Hypothetical caal molecule during stages of pyrolysis. Adapted from ref 6 .

hydrocarbons during primary pyrolysis, and (c) char condensation and cross-linking during secondary pyrolysis. The hypothetical structure in Figure 1a represents the chemical and functional group compositions for a Pittsburgh Seam bituminous coal as discussed by Solomon. ${ }^{20}$ It consists of aromatic and hydroaromatic clusters linked by aliphatic bridges. During pyrolysis, the weakest bridges, labeled 1 and 2 in Figure 1a, can break producing molecular fragments (depolymerization). The fragments abstract hydrogen from the hydroaromatics or aliphatics, thus increasing the aromatic hydrogen concentration. These fragments will be released as tar if they are small enough to vaporize under typical pyrolysis conditions and do not undergo retrograde reactions before escaping from the particle. The two lightest fragments are labeled tar. The other two fragments are shown to have repolymerized, producing a molecule that is too large to vaporize.

The other events during primary pyrolysis are the decomposition of functional groups to release $\mathrm{CO}_{2}$, light aliphatic gases, and some $\mathrm{CH}_{4}$ and $\mathrm{H}_{2} \mathrm{O}$. The release of $\mathrm{CH}_{4}, \mathrm{CO}_{2}$, and $\mathrm{H}_{2} \mathrm{O}$ may produce cross-linking, $\mathrm{CH}_{4}$ by a substitution reaction in which the attachment of a larger molecule releases the methyl group, $\mathrm{CO}_{2}$ by condensation after a radical is formed on the ring when a carboxyl is removed, and $\mathrm{H}_{2} \mathrm{O}$ by the condensation of two $\mathrm{OH}$ groups to produce an ether link (labeled 3 in Figure 1b). The cross-linking is important to determine the release of tar and the viscoelastic properties of the char.

The end of primary pyrolysis occurs when the donatable hydrogens from hydroaromatic or aliphatic portion of the coal are depleted. During secondary pyrolysis (Figure 1c) there is additional methane evolution (from methyl groups), HCN from ring nitrogen compounds, $\mathrm{CO}$ from ether links, and $\mathrm{H}_{2}$ from ring condensation. These general concepts are incorporated into the combined FG-DVC model.

Exceptions to the General Trends. a. Polymethylene. The major exception to the trends described above is the presence of varying amounts (typically $0-9 \%$, but in some cases as high as $18 \%$ ) of long-chain aliphatics (polymethylenes). These have recently been reported in pyrolysis products by Nelson ${ }^{65}$ and by Calkins and coworkers ${ }^{66-69}$ and references quoted therein. The chains appear alone and attached to aromatic nucleii. ${ }^{65}$ During devolatilization, the smaller molecules may be released without bond breaking and the heavier molecules with bond breaking to contribute to the tar. The presence of these polymethylenes makes the tar more aliphatic than the parent coal. Further cracking of this material under more severe devolatilization conditions produces ethylene, propylene, and butadiene from which the concentration of polymethylenes may be determined.68 Presently, the polymethylenes are included in the FG model as part of the aliphatic functional group pool, which is assumed to decompose to produce gas products, not tar. If the amount of heavy polymethylenes is determined, these can be computed as a separate functional group pool with an appropriate release rate and added to the tar. The modeling of polymethylene evolution will be the subject of a subsequent publication.

b. Tar/Coal Similarities. The general model assumed, as a first approximation, that tar is derived from material of the same average composition as that of the parent coal. The model predicts that the tar is richer than the parent coal in methyl groups (due to hydrogen stabilization) and poorer in the rapidly removed functional groups. Evidence for this assumption is the similarities in elemental composition, infrared spectra and NMR spectra $^{5,20,45,62-64}$ between the primary tar and parent coal observed for bituminous coals. It was, however, noted $d^{5,45,70}$ that the infrared spectrum for a lignite tar was significantly different from that of the parent coal. The tar is much richer in aliphatic groups and poorer in oxygen functional groups. Freihaut et al have recently reported a systematic increase in the tar hydrogen concentration with decreasing rank that suggests a similar trend.11

There are at least two reasons for this variation with rank. One reason is the influence of the polymethylene groups. As noted by Calkins, ${ }^{68}$ the concentration of polymethylenes increases with decreasing rank ( $\sim 4 \%$ for high volatile bituminous coals compared to $\sim 8 \%$ for lignites). In addition, the tar yield decreases with decreasing rank ( $\sim 6 \%$ for the North Dakota lignite compared to $30 \%$ for the Pittsburgh Seam bituminous coal). The relative contribution of the polymethylenes to the tar is therefore increased with decreasing rank. This will lead to a higher aliphatic content and lower oxygen content for the lowrank coal tar. This effect can be treated in the FG-DVC model by the addition of polymethylenes to the tar as discussed above.

(65) Nelson, P. F. Fuel 1987, 66, 1264.

(66) Calkins, W. H.; Hagaman, E; Zeldes, H. Fuel 1984, 63, 1113

(67) Calkins, W. H.; Tyler, R. J. Fuel 1984, 63, 1119.

(68) Calkins, W. H. Fuel 1985, 63, 1125.

(69) Calkins, W. H.; Hovsepian, B. K.; Drykacz, G. R.; Bloomquist, C. A. A.; Ruscic, I. Fuel 1984, 63, 1226.

(70) Solomon, P.R Cool Structure, Advances in Chemistry Series 192. American Chemical Society: Washington, DC, 1981; p 7.

(71) Freibaut, J. D.; Proscia, W. M.; Seery, D. J. Presented at the 194th National Meeting of the American Chemical Society, New Orleans, LA. Aug 31-Sept 4, 1987. 
A second reason for differences in structure between the tar and parent coal is that the extensive cross-linking in low-rank coals is related to the carboxyl group concentration, which increases with decreasing rank. This cross-linking will thus selectively repolymerize the fragmonts rich in oxygen, while those poorer in oxygen are more likely to be released as tar. This effect has not as yet been included in the model.

It is interesting to note an exception to the above observations. At very high heating rates, the North Dakota (Beulah, Zap) lignite is observed to melt and swell and produce a higher yield of tar that resembles the parent c0al. ${ }^{13,30,31}$ The high heating rate appears to reduce the effect of cross-linking, leading to higher oxygen concentrations in the tar and to increase yields. Both effects enhance the resemblance to the parent coal.

c. Variations of Kinetic Rates with Rank. While the model assumes rank-independent kinetic rates, there is a systematic variation of rate with rank. As reported by Solomon and Hamblen, ${ }^{52}$ the variation between a lignite and bituminous coal results in a $50-75^{\circ} \mathrm{C}$ difference in the peak evolution temperature for most species (at a heating rate of $30 \mathrm{~K} / \mathrm{min}$ ). Systematic rank variations in the rate constants can be added to the model if increased accuracy is desired.

d. Macerals. Individual macerals are not considered in this model. The influence of the maceral concentration is assumed to occur through its effect on the average elemental and functional group composition. If details on macerals are desired, then each maceral must be treated as a distinct molecular population with its own functional group composition and molecular weight distribution.

e. Physical Properties of Molecular Fragments. The general model has assumed that the vaporization and solubility of the molecular fragments are functions of molecular weight alone. Both properties are expected to depend on functional group composition. Such effects can be included as corrections to the vaporization law and solubility assumptions.

Depolymerization-Vaporization-Cross-Linking (DVC) Subroutine Formulation. The DVC model has been described in a number of publications. 30,31,44-7750,51 It predicts the tar yield, the tar molecular weight distribution, the char yield, the char molecular weight distribution, the extract yield, and the cross-link density. The model had its beginning in a study of polymers representative of structural features found in coal.44 The objective of that study was to develop an understanding of coal pyrolysis by studying a simpler, more easily interpretable system. The polymers were studied in a series of pyrolysis experiments in which tar amounts and molecular weights were measured. The theory which was developed describes the combined effects of (1) depolymerization and hydrogen consumption, (2) cross-linking, and (3) external transport. Recently, an expression to describe (4) internal transport has been added to the model. ${ }^{33}$ These processes, which are described below, are incorporated into a computer code that employs a Monte Carlo method for performing the statistical analysis.

Process 1. Depolymerization and Hydrogen Consumption. Bond cleavage in coal is likely to be very complicated, including homolytic cleavage, ipso substitution, ${ }^{16}$ and hydrogen-transfer-induced bond-scission reactions ${ }^{32}$ for a variety of bond types. However, it has been observed that tar evolution is consistent with a narrow distribution of activation energies, ${ }^{5,5,12}$ which allows con-

(72) McMillen, D. F.; Malhotra, R.; Hum, G. P.; Chang, S. J. Energy Fuels 1987, 1, 193. sideration in the model of a single type of bridge (while acknowledging that other types may be present). Also, the rate for tar formation from coal, $k_{\mathrm{tan}}{ }^{6.13}$ is in good agreement with the rate determined for the breaking of ethylene bridges between naphthalene rings, $k_{\mathrm{B}}$. This kinetic rate, $k_{\mathrm{B}},{ }^{46}$ employs an activation energy that is in agreement with resonance-stabilization calculations ${ }^{7374}$ and an overall rate that agrees with previous measurements on model compounds.75 In view of these observations, a single type of bond (ethylene) undergoing homolytic cleavage is assumed for coal as a simple approximation of the complicated behavior.

Bond cleavage is accompanied by the consumption of donatable hydrogens, $H($ al), to cap free radicals, along with corresponding carbon-carbon double-bond formation at the donor site. In the polymers that were studied, the ethylene bridges were identified as a source of donatable hydrogen with the formation of a double bond between the bridge carbons. ${ }^{46,47}$ The double-bond formation was assumed to remove a breakable bond. It should be noted that hydroaromatic groups are also a source of donatable hydrogen with aromatization of the ring. However, for simplicity, the DVC model assumes all the coal's donatable hydrogens, whether in bridges or in hydroaromatic rings, are in bridges, i.e., $H(\mathrm{al})=(2 / 28) W_{\mathrm{B}}$. This approximation will produce some error in tar yield since a broken bond in a hydroaromatic ring wiil not be as effective as a broken bond in a bridge in fragmentating the coal. But this effect will be compensated for, since $H(a)$ is a parameter that is determined for each coal from a selected pyrolysis experiment. $H$ (al) couid, in principle, be determined by FT-IR or NMR, but not with sufficient accuracy.

The equation describing the disappearance of labile bridges in the char, $W_{B}$ (char), due to bond breaking and hydrogen donation is

$$
\mathrm{d} W_{\mathrm{B}} / \mathrm{d} t=-2 k_{\mathrm{B}} W_{\mathrm{B}}
$$

The value for $k_{\mathrm{B}}$ is taken as the previousiy determined $k_{\mathrm{tar}}{ }^{6}$ The rate of decrease of labile bridges is twice the rate of bond breaking since for each broken bond, an additional labile bridge is converted to a nonlabile bridge with the donation of hydrogen. By assuming that all the donatable hydrogens are in the labile bridges, the consumption of labile bridges and donatable hydrogens occur simultaneously. The redistribution of hydrogen creates source and loss terms, $d W_{\mathrm{i}}(\mathrm{DVC}) / \mathrm{d} t$, in the equations for the char functional groups $W_{i}$ (char), as will be discussed with the FG part of the model (see eq 7).

Equation 1 only describes the loss due to bond breaking and hydrogen donation. The loss of labile bridges due to evolution with the tar is computed in the Monte Carlo calculation by using the transport equations (eq 3 and 4). discussed below.

Process 2. Cross-Linking. Cross-linking reactions are important in describing the rank and heating rate dependence of the tar molecular weight distributions and yields. While cross-linking reactions were originally included in the DVC model, using adjustable parameters for the rate and amount, ${ }^{30,31,46}$ work has recently been performed to define the reactions that cause cross-linking. $33,50,51$ Under the assumption that the cross-linking reactions may also

(73) Stein, S. E. New Approceches is Coal Chemistry; Blaustein, B. D. Bockrath, B. C., Friedman, S., Eds.; ACS Symposium Series 169; American Chemical Sociaty: Washingtin. DC, 1981; p 208.

(74) Stoin. S. E "Multistop Bond Breaking and Making Processes of Relevanco to Thermal Coal Chemistry"; Annual Report for GRI Contract No. 5081-261-0556; Accession No. GRT-81/0147, 1983.

(75) Stein, S. E. Robauch, D. A.; Alfieri, A. D.; Miller, R E. J. Am. Chem. Soc. 1982, 104, 6567. 

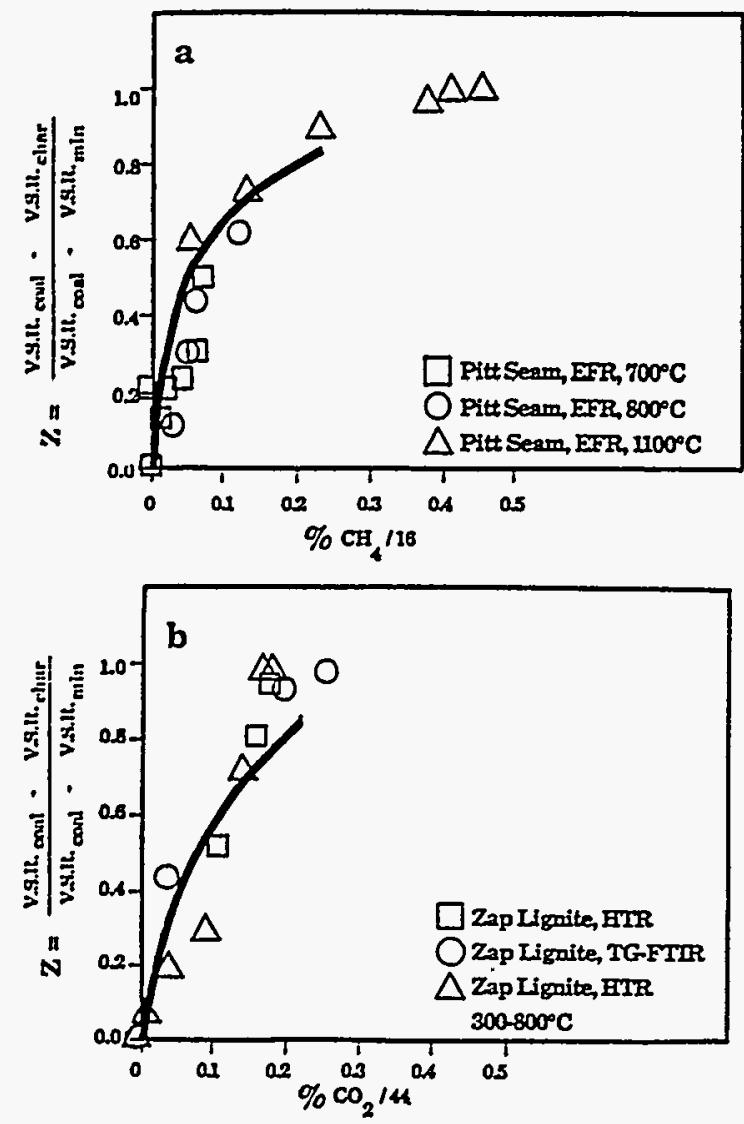

Figure 2. Measured and caiculated normalized volumetric swelling ratio (VSR) for coal and chars: (a) Pittsburgh Seam bituminous coal plotted against the methane yield; (b) Zap North Dakota lignite plotted against the $\mathrm{CO}_{2}$ yield. $\mathrm{VSR}_{\min }$ is the value achieved when crosslinking is complete. The chars were prepared in an entrained-flow reactor (EFR), a heated-tube reactor (HTR), and a thermogravimetric analyzer with evolved product analysis by FT-IR (TG-FTIR) as described in ref 61 .

release gas species, the molecular weight between crosslinks (or cross-link density) measured by solvent swelling was correlated with the observed evolution of all the major gas species during pyrolysis. Likely candidates were $\mathrm{CO}_{2}$ formation from carboxyl groups or methane formation from methyl groups. Suuberg et al. ${ }^{69}$ also noted that cross-linking in low-rank coals is correlated with $\mathrm{CO}_{2}$ evolution. Both $\mathrm{CO}_{2}$ and $\mathrm{CH}_{4}$-forming reactions may leave behind free radicals that can be stabilized by cross-linking. Condensation of hydroxyl groups to form water and an ether link is also a possible reaction.

For a series of chars, the reduction in the volumetric swelling ratio in pyridine was compared with $\mathrm{CO}_{2}$ evolution for a North Dakota (Beulah) lignite and $\mathrm{CH}_{4}$ evolution for a Pittsburgh Seam bituminous coal.50 The results are presented in Figure 2. The abscissa (parameter $Z$ ), which is the change in the volumetric swelling ratio (VSR) between coal and char divided by the maximum change, is given by

$$
Z=\left(V_{S R} R_{\text {codl }}-\text { VSR }_{\text {char }}\right) /\left(V_{S R} \text { coll }-V_{S R} R_{\text {min }}\right)
$$

$Z$ is 0 for coal and $I$ for fully cross-linked char. Since the lignite reaches marimum cross-linking before the start of methane evolution and the Pittsburgh Seam bituminous coal evolves little $\mathrm{CO}_{2}$, correlations can be made separately between cross-linking and $\mathrm{CO}_{2}$ evolution in the lignite and cross-linking and $\mathrm{CH}_{4}$ evolution in the Pittsburgh seam bituminous coal. On a molar basis, the evolution of $\mathrm{CO}_{2}$ from the lignite and the evolution of $\mathrm{CH}_{4}$ from the bitu- minous coal appear to have similar effects on the volumetric swelling ratio. The results suggest that one cross-link is formed for each $\mathrm{CO}_{2}$ or $\mathrm{CH}_{4}$ molecule evolved. No correlation was observed between the volumetric swelling ratio and tar yield for either coal. A correlation with water yield appears valid for the North Dakota (Beulah) lignite but not for the Pittsburgh Seam bituminous coal.

It therefore appears that a correlation exists between gas evolution and cross-linking which permits the rates for cross-linking and the number of cross-link sites to be related to rates and yields for gas evolution. The model assumes the following expression for the rate of increase of the number of cross-links per gram of coal, $m$

$$
\frac{\mathrm{d} m}{\mathrm{~d} t}=N_{0}\left[\frac{\mathrm{d} W_{\mathrm{CO}_{2}} \text { (gas)/dt}}{44}+\frac{\mathrm{d} W_{\mathrm{CH}_{4}} \text { (gas)/d } t}{16}\right]
$$

where the rates, $\mathrm{d} W_{\mathrm{i}} / \mathrm{d} t$, of evolution per gram of coal of $\mathrm{CO}_{2}$ and $\mathrm{CH}_{4}$ are calculated in the FG subroutine. $N_{0}$ is Avogadro's number.

Again, a caution should be added that the reactions which have been assumed must be a gross simplification of a very complicated set of chemical reactions. This is especially true for the cross-links occurring during methane formation, during which time there is extensive bond breaking and cross-linking accompanying tar formation. The inaccuracy in the description of this higher temperature cross-linking event is one of the present weaknesses in the model.

Process 3. External Transport. The external transport of tars from the particle surface to the bulk gas by vaporization and diffusion through a gas boundary layer as in the original DVC model $4-47,50,51$ is described with the model of Unger and Suuberg. ${ }^{23}$ However, in the current paper, the modified expression for the vapor pressure law of Suuberg et al. ${ }^{32}$ is now used to replace that in the model of Unger and Suuberg. The rate of evolution per gram of cosl, $\left(\mathrm{d} n_{\mathrm{j}} / \mathrm{d} t\right)_{\mathrm{ET}}$, of oligomers of molecular weight $M_{\mathrm{j}}$ is given by

$$
\left(\mathrm{d} n_{\mathrm{j}} / \mathrm{d} t\right)_{\mathrm{ET}}=\left(3 / r_{0}^{3} \rho\right) r D_{\mathrm{j}} \chi^{\mathrm{j}_{\mathrm{j}}}\left(P_{\mathrm{j}} / R T\right)
$$

where $r$ is the particle radius assumed to shrink with the cubic root of its mass $r_{0}$ is the initial particle radius, $\rho$ is the particle density, $\chi_{j}$ is the mole fraction of species of molecular weight $M_{\mathrm{j}}$ in the metaplast at the surface of the particle, $P_{j}$ is the vapor pressure for oligomers of molecular weight $M_{\mathrm{j}}$ (given by Suuberg et al. ${ }^{32}$ ), $D_{\mathrm{j}}$ is the gas-phase diffusivity of species of molecular weight $M_{\mathrm{j}},{ }^{38} R$ is the gas constant, and $T$ is the particle temperature.

In the previous work, it was assumed that the surface mole fraction, $X_{j}$, was the same as that in the bulk, $x^{b}{ }_{j}$. That is, mass transport to the surface was not a limiting factor.

Process 4. Internal Transport. When comparing the predictions of the model to available data assuming $\mathcal{X}_{j}$ $=X^{b}$ it was found that tar yields were overpredicted when devolatilization occurred at low temperatures. This was observed for either low-heating-rate experiments ${ }^{6}$ or experiments with rapid heating to relatively low temperatures. ${ }^{16}$ As discussed in the Results, it appears that the lower yields were the result of the additional transport limitations within the particle.

For softening coals, the internal transport mechanisms include (i) the transport of tar molecules through the liquid to the surface, (ii) the transit of bubbles containing tar from the interior of the particle to the surface, (iii) the transport of tars within the liquid to the bubbles, and (iv) 
the stirring action of the bubble evolution. For nonsoftening coals, transport occurs by (v) convection and diffusion within the pores.

Mechanism $i$ was treated by Suuberg and Sezen. ${ }^{36}$ The unknown factor is the diffusion coefficient of the tar molecules in the liquid. The detailed modeling of mechanisms ii and iii has been undertaken by several investigators. ${ }^{42637,38}$ Calculations for mechanism $v$ have also been published. 212534.35 The models are complicated and require many assumptions. A common feature of mechanisms iii and $\mathrm{v}$ is that tars are transported out of the particle with the light devolatilization products that exit the coal via bubbles or pores. In ref 33, the upper limit for this process was calculated. This limit, which occurs when the tars achieve their equilibrium vapor pressure in the evolving gases, can be computed with few assumptions. In this case, the rate of transport per gram of coal, $\left(\mathrm{d} n_{\mathrm{j}} / \mathrm{d} t\right)_{\mathrm{IT}}$, for tar component $j$ is proportional to the volume of gases evolved, $\mathrm{d} V / \mathrm{d} t$. That is

$$
\left(\mathrm{d} n_{\mathrm{j}} / \mathrm{d} t\right)=P_{\mathrm{j}} X_{\mathrm{j}}^{\mathrm{b}}(\mathrm{d} V / \mathrm{d} t)(1 / R T)
$$

The volume of gases is proportional to the number of gas molecules and the temperature. It is inversely proportional to the pressure within the particle, $P_{0}+\Delta P$ where $P_{0}$ is the ambient pressure and $\Delta P$ is the average pressure difference between the surface and the particle's interior. Then

$$
\mathrm{d} V / \mathrm{d} t=\sum_{\mathbf{i}}\left(\mathrm{d} n_{\mathrm{i}} / \mathrm{d} t\right)_{\operatorname{gas}}\left(\frac{R T}{P_{0}+\Delta P}\right)
$$

where $\sum_{i}\left(\mathrm{~d} n_{i} / \mathrm{d} t\right)_{\text {ges }}$ is the rate of production per gram of coal of gas components $i$ summed over all gas and light tar species. For gas molecules, $\mathrm{d} n_{\mathrm{j}} / \mathrm{d} t$ is taken as the rate of production given by the FG model. For light tar molecules, $\mathrm{d} n_{\mathrm{j}} / \mathrm{d} t$ is taken as the total amount transported out of the particle as tar computed in the previous time step. For computational efficiency, the sum has been limited to molecular weights less than $300 \mathrm{amu}$, since this accounts for over $90 \%$ of the volume. Combining the two equations with this approximation gives

$$
\left(\mathrm{d} n_{\mathrm{j}} / \mathrm{d} t\right)_{\mathrm{TF}}=P_{\mathrm{j}} \chi^{\mathrm{b}}{ }_{\mathrm{i}<300} \sum_{1}\left(\mathrm{~d} n_{\mathrm{i}} / \mathrm{d} t\right)_{\mathrm{gas}}\left[\frac{1}{P_{0}+\Delta P}\right]
$$

$\Delta P$ is used as an adjustable parameter that varies with the coal and experimental conditions. For the highly fluid Pittsburgh Seam bituminous coal, in cases where $P_{0}$ is 1 atm or greater, we have considered the upper limit to this rate where $P_{0} \gg \Delta P$. Then all the terms in eq 4 can be determined by the combined FG-DVC model. This limit coincides with assumptions recently used by Niksa in his FLASHKIN model for Pittsburgh Seam bituminous coal ${ }^{76}$

While $\Delta P=0$ appears to be a good approximation for fluid coals at one atmosphere or more, $\Delta P>0$ is expected for some coals and situations. $\Delta P$ is proportional to the coal's viscosity and so will become important for less fluid coals. $\Delta P$ is also important when $P_{0}$ is small, when particles are large, and when the heating rates are very high.

Two possibilities have been considered for combining the internal and external transportation. In an earlier publication, ${ }^{33}$ the internal-transport term and externaltransport term (with $X_{j}=X_{j}^{b}$ ) were assumed to be in series. Then the transport was controlled by the smaller term. The internal-transport term was the smaller for all

(76) Niksa, S. Presented at the Western States Section/The Combustion Institute, Spring Meeting, Salt Lake City, UT, March 1988; Paper $88-4$. pyrolysis cases that were considered and so it dominated. In fact, calculations performed by neglecting the external transport limitation where almost identical with those made by assuming the two terms to be in series.

Alternatively, a case can be made that the total transport should be the sum of eq 3 and 4 . The reasoning is that internal transport assumes the tars to be in equilibrium with the escaping light gases. It is more likely that this mechanism will transport the tars to the ambient gas than to the surface. In this case, the mechanism considered in eq 4 transports the tars away from the surface in parallel with the surface vaporization and gas diffusion considered in eq 3.

If the two terms are taken in parallel, it is again obvious that $\chi_{j}^{s_{j}}=\chi^{b_{j}}$ is a bad assumption. Since we did not have a good method to determine $X_{j}$, calculations were made by assuming that the external-transport term can be neglected, i.e.

$$
\left(\mathrm{d} n_{\mathrm{i}} / \mathrm{d} t\right)_{\text {tot }}=\left(\mathrm{d} n_{\mathrm{i}} / \mathrm{d} t\right)_{\mathrm{TT}}
$$

This provides an excellent fit to the data for $50-\mu \mathrm{m}$-diameter particles.

Therefore, for either parallel or series combinations of the transport terms, it appears best to neglect the external transport. It is likely that the external transport term will be increasingly important for smaller particles, but this will require better knowledge of the liquid-phase diffusion coefficient (mecrianism i), and the stirring action of bubbles (mechanism iv). The relative importance of the various internal-and external-transport mechanisms is the subject of ongoing research.

Schematic Representation of DVC Model. In the current DVC model, the parent coal is represented as a two-dimensional network of monomers (condensed ring clusters) linked by strong and weak bridges as shown in Figure $3 \mathrm{a}$. The monomers are linked to form unbranched oligomers of length " $l^{n}$ by breakable and nonbreakable bridges (shown as horizontal single or double lines, respectively, in Figure 3a). The monomers are represented by circles with molecular weights shown in each circle. The molecular weight distribution of the monomers is assumed to be Gaussian and is described by two parameters, $M_{\mathrm{av}}$ (mean) and $\sigma$ (standard deviation). The breakable bridges (assumed to be ethylene) are represented by single lines, the unbreakable bridges by double lines. " $m_{0}$ " cross-links per monomer are added (as vertical double lines in Figure 3a) to connect the oligomers of length $l$ so that the molecular weight between cross-links, $M_{c}$ corresponds to the value reported in the literature ${ }^{77}$ for coals of similar rank. The cross-links form the branch points in the macromolecule. Unconnected "guest" molecules (the extract yield) are obtained by choosing the value of $l$. A large value of $l$ will mean that a completely connected macromolecule. will be formed when even a small number of cross-links are added, leaving no extractable material. For smaller values of $l$ some of the oligomers will be unattached after the cross-links are added, and these are the guest molecules. The number of ethylene bridges, $W_{B}$, (two donatable hydrogens per bridge) is chosen to obtain the appropriate value for total donatable hydrogen (i.e., to fit a selected laboratory pyrolysis experiment). The remainder are nonbreakable bridges whose carbons are counted with the aromatic carbons.

The parameters $M_{\mathrm{c}}, l, M_{\mathrm{av}}$ and $\sigma$ determine the molecular weight distribution of oligomers in the starting coal molecule. A histogram showing the distribution created

(77) Nelson, J. R. Fuel 1983, 62, 112 
Table I. Coal Structure Parameters for DVC Subrontine

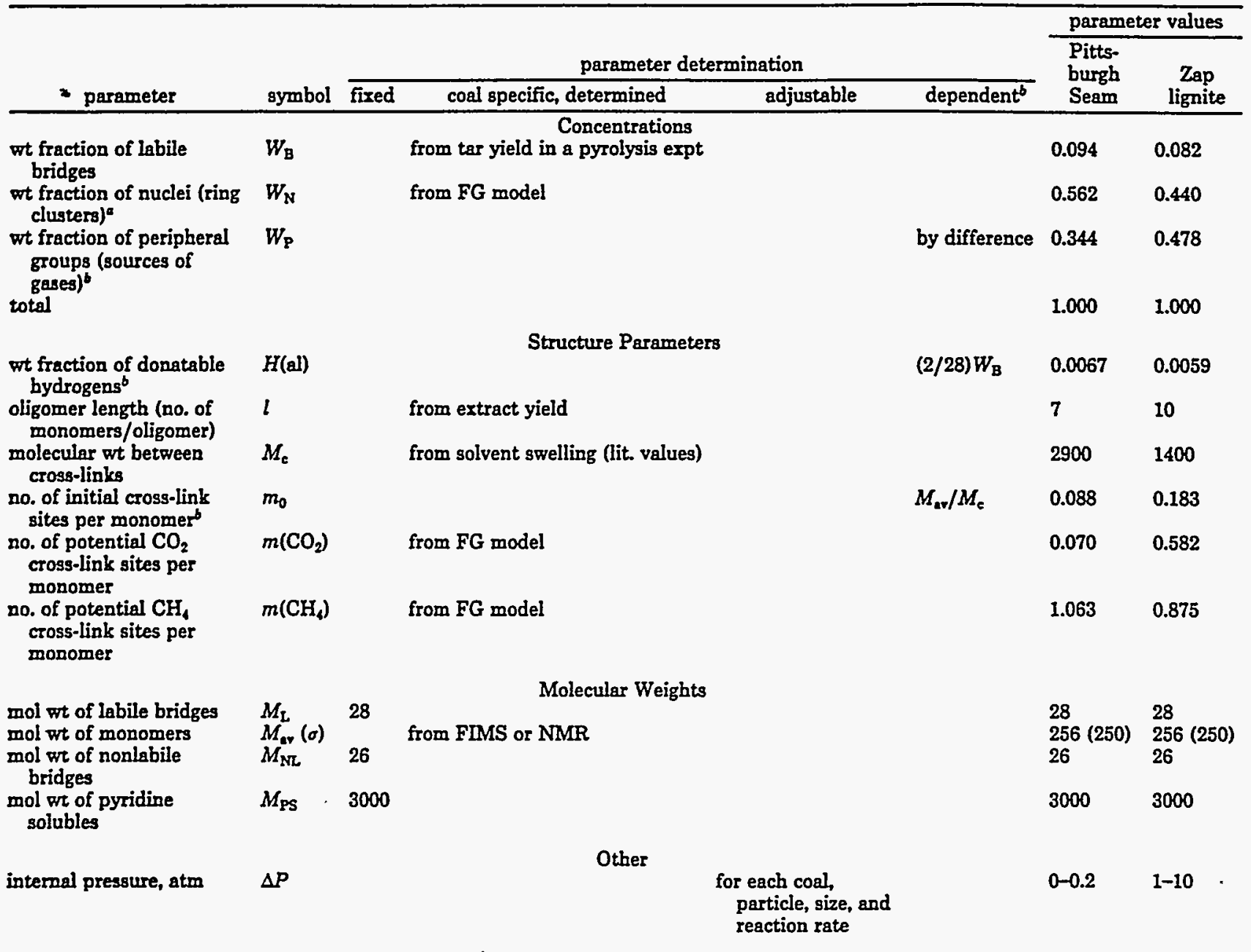

a Carbon in aromatic rings plus nonlabile bridges. 'Dependent parameters are calculated from the independent parameters.

by randomly picking monomers to form oligomers of length $l$ and randomly cross-linking them to achieve an average molecular weight between cross-links, $M_{c}$, is presented at the right of Figure 3a. The distribution is divided into a pyridine-soluble portion below 3000 amu (light shading) and a pyridine-insoluble portion above $3000 \mathrm{amu}$ (dark shading).

Figure $3 \mathrm{~b}$ shows the molecule during pyrolysis. The rates for bond breaking and cross-linking are from the FG model and are the same for all coals and all experiments. Some bonds have broken, other bonds have been converted to unbreakable bonds by the abstraction of hydrogen to stabilize the free radicals, and new cross-links have been formed. To determine the change of state of the computer molecules during a time step, the number of cross-links formed is determined by using the FG subroutine and passed to the DVC subroutine. These cross-links are distributed randomly throughout the char, assuming that the cross-linking probability is proportional to the molecular weight of the monomer. Then the DVC subroutine breaks the appropriate number of bridging bonds and calculates the quantity of tar evolved for this time step by using the internal and external transport equations. The result is the coal molecule representation and the molecular weight distributions shown in Figure $3 \mathrm{~b}$. The lighter "tar molecules", which leave the particle according to the transport equations, are shown as crosshatched. A fraction of the donatable hydrogen is used to stabilize the free radicals formed by bridge breaking, creating two new methyl groups per bridge and the same fraction of breakable bridges is converted into (unbreakable) double bonds.

Figure 3c shows the final char, which is highly crosslinked with unbreakable bonds and has no remaining donatable hydrogen. The histogram now shows only tar and pyridine-insoluble fractions. The extractables have been eliminated by tar formation and cross-linking.

The output of the DVC subroutine is the molecular weight distribution in the coal, its time-dependent transformation during devolatilization, and the evolution of tar determined by the transport of the lighter components.

Selection of DVC Parameters. The DVC composition parameters employed for a Pittsburgh Seam coal and North Dakota lignite are summarized in Table I. The FG composition parameters and the kinetic parameters, which are fixed for all coals and experiments, are presented in Table II. In Table I, there are 11 independent composition parameters. Three parameters are fixed, the molecular weight of the labile bridges, $M_{\mathrm{L}}$, the nonlabile bridges, $M_{\mathrm{NL}}$, and the pyridine-extractable limit, $M_{\mathrm{PS}}$.

Eight parameters are coal specific'(i.e., fixed for each coal, for all conditions) and must be determined by some measurement. $M_{\mathrm{c}}$ and $l$ are determined experimentally for each coal by the measured molecular weight between cross-links and the pyridine extract yield, respectively. The weight fraction of carbon in nucleii and nonbreakable bridges, $W_{\mathrm{N}}$, is obtained from the FG model and is equal to the amount of nonvolatile carbon. This value is, in 
a. Starting Molecule
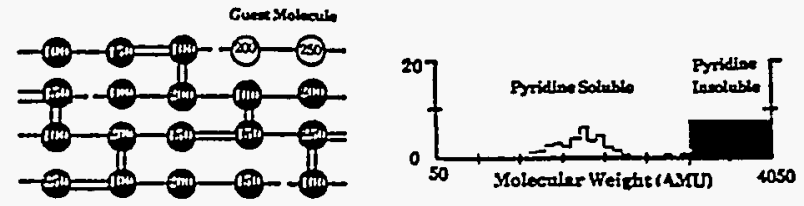

b. During Tar Formation

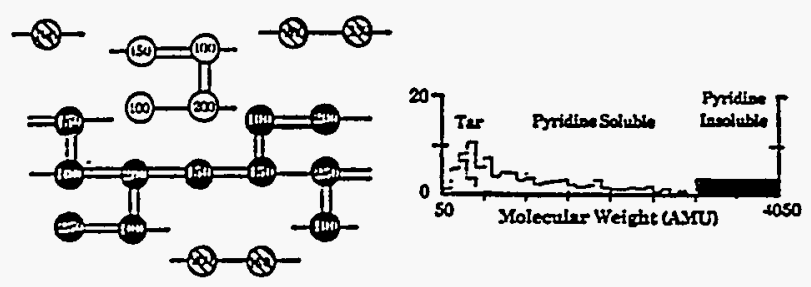

c. Char Formed

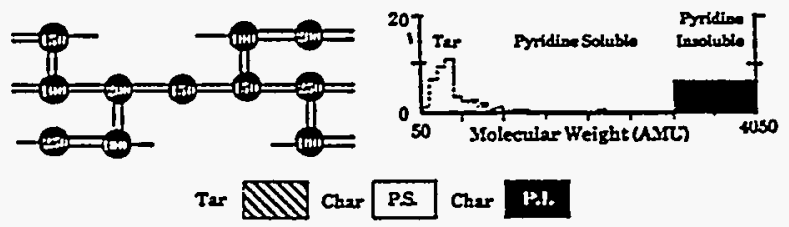

Figure 3. Representation of coal molecule in the DVC simulation and corresponding molecular weight distribution. In the molecule, the circles represent monomers (ring clusters and peripheral groups). The molecular weight shown by the numbers is the molecular weight of the monomer including the attached bridges. The single-line bridges are breakable and can donate hydrogen. The Double-line bridges are unbreakable and do not donate hydrogen. The molecular weight distribution of the coal. tar, and chars are shown as a histogram at the right. The histogram is divided into tar and char with pyridine-soluble and pyridine-insoluble fractions. The area under the histogram corresponds to the weight percent of the oligomers.

principle, determined for each coal for a single pyrolysis experiment. In practice, several experiments are performed. The number of potential cross-link sites, $m\left(\mathrm{CO}_{2}\right)$ and $m\left(\mathrm{CH}_{4}\right)$, are proportional to the total yield of $\mathrm{CO}_{2}$ and the total yield of $\mathrm{CH}_{4}$, respectively. $W_{\mathrm{B}}, M_{\mathrm{av}}$, and $\sigma$ are determined by using the model to fit selected pyrolysis experiments. The value of $W_{B}$ is adjustable to fit the tar yield or total volatile yield from one or two selected experiments. In principle, $W_{B}$ could be measured by FT-IR or NMR but not with sufficient accuracy for this highly sensitive parameter. The values of $M_{\mathrm{av}}$ and $\sigma$ are chosen based on FIMS analysis of the coal. $M_{\mathrm{av}}$ can be determined from the average cluster size determined by NMR. ${ }^{78,79}$ The value of 256 chosen for both the lignite and bituminous coal is in reasonable agreement with these reported by Solum, et al., 290 for Zap and 300 for the Pittsburgh Seam coal.

One parameter, $\Delta P$, is adjustable and can vary with each type of experiment. For fluid coals at pressures above one atmosphere, $\Delta P \approx 0$. For low external pressures, less fluid coals, large particles, or high heating rates, $\Delta P>0$.

There are three dependent parameters that are computed from the other parameters: the weight fraction of peripheral groups, $W_{p}$; the donatable hydrogen, $H$ (al); and the number of initial cross-link sites per monomer, $m_{0}$.

Functional Group (FG) Model Formulation. The Functional Group (FG) model has been described in a

(78) Gerstein, B. C.; Murphy, P. D.; Ryan, L. M. Coal Structure; Meyers, R A., Ed.; Academic Press: New York, 1982; Chapter 4.

(79) Solum, M. A.; Pugmire, R. J.; Grant, D. M.; Wolfenden, W. R. submitted for publication in Energy Fuels. a
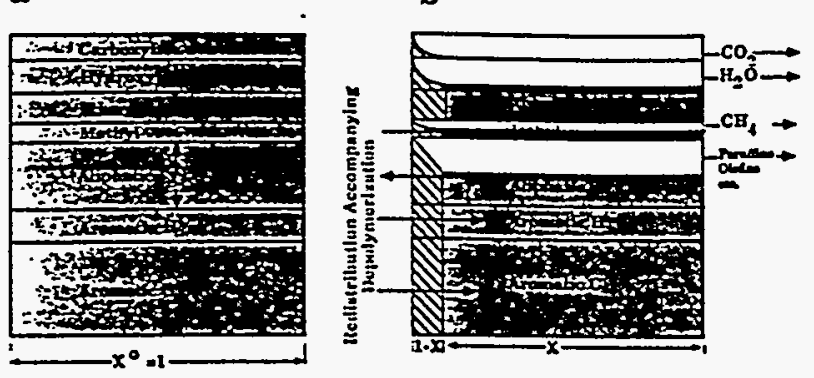

c

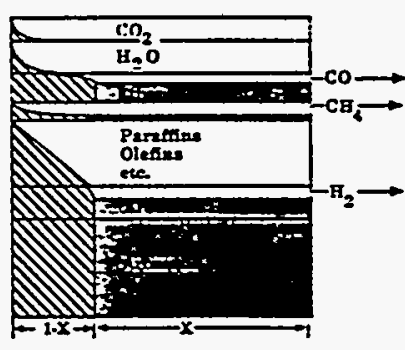

d

$$
\text { Cᄄ- }
$$

Figure 4. Schematic representation of functional group (FG) model: (a) Initial Coal Composition; (b) composition during tar formation; (c) composition trier completion of tar formation; (d) composition after complerion of devolatilization.

number of publications..$^{3.6,11-13}$ It permits the detailed prediction of the composition of volatile species (gas yield, tar yield, and tar functional group and elemental composition) and of char (elemental and functional group composition). It employs coal-independent rates for the decomposition of individual assumed functional groups in the coal and char to produce gas species. The ultimate yields of each gas species are related to the coal's functional group composition. Tar evolution is a parallel process which competes for all the functional groups in the coal. In the original FG model, the potential tar forming fraction of the coal, $X^{0}$, was an input parameter that was adjusted for each coal and type of experiment. In the combined FG-DVC model, the DVC subroutine provides this parameter.

Schematic Representation of FG Model. The mathematical description of the functional group pyrolysis model has been presented previously.,6,11-13 The evolution of tar and light-gas species provides two competing mechanisms for removal of a functional group from the coal: evolution as a part of a tar molecule and evolution as a distinct gas species. This process is shown schematically in Figure 4. To model these two paths, with one path yielding a product that is similar in composition to the parent coal, the coal is represented as a rectangular area with $X$ and $Y$ dimensions. As shown in Figure 4a, the $Y$ dimension is divided into fractions according to the chemical composition of the coal. $Y_{i}^{0}$ represents the initial fraction of a particular component (carboxyl, aromatic hydrogen, etc.), and the sum of the $Y_{i}^{0}$ 's equal 1 . The evolution of each component into the gas (carboxyl into $\mathrm{CO}_{2}$, aromatic hydrogen into $\mathrm{H}_{2}$, etc.) is represented by the first-order diminishing of the $Y_{\mathrm{i}}$ dimension, $\mathrm{d} Y_{\mathrm{i}} / \mathrm{d} t=$ $-k_{\mathrm{i}} Y_{\mathrm{i}}$.

The $X$ dimension is divided into char, $X$, and tar, (1 $X)$; initially $X=1$. The evolution of the tar is represented by the decreasing of the $X$ dimension, $\mathrm{d} X / \mathrm{d} t$, computed in the DVC subroutine as

$$
\mathrm{dX} / \mathrm{d} t=-\sum_{j}\left(\mathrm{~d} n_{\mathrm{j}} / \mathrm{d} t\right)_{c_{0}} M_{\mathrm{j}}
$$


Table II. Kinetic Rate Coefficients and Species Composition Parameters for FG Subroutine

\begin{tabular}{|c|c|c|c|c|c|}
\hline $\begin{array}{c}\text { composition } \\
\text { params }\end{array}$ & ges & $\begin{array}{c}\text { primary } \\
\text { functional } \\
\text { group source }\end{array}$ & rate eq ${ }^{\alpha}$ & $\begin{array}{c}\text { Pittsburgh No. } \\
8 \text { bituminous } \\
\text { coal }\end{array}$ & $\begin{array}{c}\text { North } \\
\text { Dakots } \\
\text { Zap } \\
\text { lignite } \\
\end{array}$ \\
\hline $\begin{array}{l}\text { C } \\
H \\
N \\
\text { S(org) } \\
0\end{array}$ & & & & $\begin{array}{l}0.821 \\
0.056 \\
0.017 \\
0.024 \\
0.082\end{array}$ & $\begin{array}{l}0.665 \\
0.048 \\
0.011 \\
0.011 \\
0.265\end{array}$ \\
\hline total & & & & 1.000 & 1.000 \\
\hline 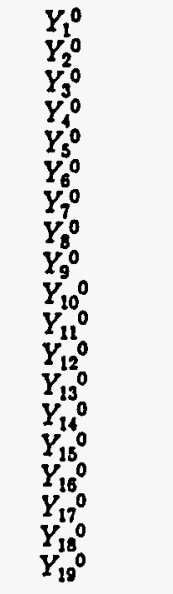 & $\begin{array}{l}\mathrm{CO}_{2} \text { extra loose } \\
\mathrm{CO}_{2} \text { loose } \\
\mathrm{CO}_{2} \text { tight } \\
\mathrm{H}_{2} \mathrm{O} \text { loose } \\
\mathrm{H}_{2} \mathrm{O} \text { tight } \\
\mathrm{CO} \text { ether loose } \\
\mathrm{CO} \text { ether tight } \\
\mathrm{HCN} \text { loose } \\
\mathrm{HCN} \text { tight } \\
\mathrm{NH}_{3} \\
\mathrm{CH}_{s} \text { aliphatic } \\
\text { methane extra loose } \\
\text { methane loose } \\
\text { methane tight } \\
\mathrm{H} \text { aromatic } \\
\text { methanol } \\
\mathrm{CO} \text { extra tight } \\
\mathrm{C} \text { nonvolatile } \\
\mathrm{S} \text { organic }\end{array}$ & $\begin{array}{l}\text { carboryl } \\
\text { carboryl } \\
\text { hydroryl } \\
\text { hydroxyl } \\
\text { ether } 0\end{array}$ & $\begin{array}{l}k_{1}=0.81 \mathrm{E}+13 \exp (-(22500 \pm 1500) / T) \\
k_{2}=0.65 \mathrm{E}+17 \exp (-(33850 \pm 1500) / T) \\
k_{3}=0.11 \mathrm{E}+16 \exp (-(38315 \pm 2000) / T) \\
k_{4}=0.22 \mathrm{E}+19 \exp (-(30000 \pm 1500) / T) \\
k_{5}=0.17 \mathrm{E}+14 \exp (-(32700 \pm 1500) / T) \\
k_{6}=0.14 \mathrm{E}+19 \exp (-(40000 \pm 6000) / T) \\
k_{7}=0.15 \mathrm{E}+16 \exp (-(40500 \pm 1500) / T) \\
k_{8}=0.17 \mathrm{E}+14 \exp (-(30000 \pm 1500) / T) \\
k_{9}=0.69 \mathrm{E}+13 \exp (-(42500 \pm 4750) / T) \\
k_{10}=0.12 \mathrm{E}+13 \exp (-(27300 \pm 3000) / T) \\
k_{11}=0.84 \mathrm{E}+15 \exp (-(30000 \pm 1500) / T) \\
k_{12}=0.84 \mathrm{E}+15 \exp (-(30000 \pm 1500) / T) \\
k_{13}=0.75 \mathrm{E}+14 \exp (-(30000 \pm 2000) / T) \\
k_{11}=0.34 \mathrm{E}+12 \exp (-(30000 \pm 2000) / T) \\
k_{15}=0.10 \mathrm{E}+15 \exp (-(40500 \pm 6000) / T) \\
k_{16}=0 \\
k_{17}=0.20 \mathrm{E}+14 \exp (-(45500 \pm 1500) / T) \\
k_{18}=0\end{array}$ & $\begin{array}{l}0.000 \\
0.007 \\
0.005 \\
0.012 \\
0.012 \\
0.050 \\
0.021 \\
0.009 \\
0.023 \\
0.000 \\
0.207 \\
0.000 \\
0.020 \\
0.015 \\
0.013 \\
0.000 \\
0.020 \\
0.562 \\
0.024\end{array}$ & $\begin{array}{l}0.065 \\
0.030 \\
0.005 \\
0.062 \\
0.033 \\
0.060 \\
0.038 \\
0.007 \\
0.013 \\
0.001 \\
0.102 \\
0.000 \\
0.017 \\
0.009 \\
0.017 \\
0.000 \\
0.090 \\
0.440 \\
0.011\end{array}$ \\
\hline total & & & & 1.000 & 1.000 \\
\hline$X^{0}$ & $\operatorname{tar}$ & & $k_{\mathrm{B}}=k_{\mathrm{T}}=0.86 \mathrm{E}+15 \exp (-(27700 \pm 1500) / T)$ & & \\
\hline
\end{tabular}

a The rate equation is of the form $k_{n}=k_{0} \exp (-(E / R \pm \sigma / R) / T)$, with $k_{0}$ in $\mathrm{s}^{-1}, E / R$ in $\mathrm{K}$, and $\sigma / R$ in $\mathrm{K}$. $\sigma$ designates the spread in activation energies in a Gaussian distribution. The notation for $k_{0}$ is defined as follows: $0.81 E+13$ is equivalent to $0.81 \times 10^{13}$ etc.

The fractional amount of a particular functional group component in the char is

$$
W_{\mathrm{i}}(\mathrm{char})=X Y_{\mathrm{i}}
$$

and the amounts in the gas and tar may be obtained by integration with respect to time starting from $t=0$.

Secondary reactions such as further decomposition of aliphatic species to form olefins, acetylene, and soot modify the basic equations. Some of these have been described elsewhere. ${ }^{6}$ These types of secondary reactions are not considered in the current paper.

Figure $4 \mathrm{a}$ shows the initial state of the coal. Values for $Y_{i}^{0}$ are obtained from elemental analysis and FT-IR analysis of the raw coal or from analysis of the products of one or two selected pyrolysis experiments. Figure $4 \mathrm{~b}$ shows the initial stage of devolatilization, during which the most volatile components, $\mathrm{H}_{2} \mathrm{O}, \mathrm{CO}$ (loose), and $\mathrm{CO}_{2}$ evolve from the hydroxyl, ether-loose, and carboxyl groups, respectively, along with aliphatics and tar. At a later stage (Figure $4 \mathrm{c}$ ) $\mathrm{CO}$ (tight), $\mathrm{HCN}$ and $\mathrm{H}_{2}$ are evolved from the ether-tight, ring nitrogen, and aromatic hydrogen groups. Figure $4 \mathrm{~d}$ shows the final state of the char, tar, and gas.

The evolution of gas and the composition of the char and tar are then described mathematically as follows.

Process 5. Gas Formation. The evolution of each gas species is assumed to be a first-order reaction

$$
\mathrm{d} W_{\mathrm{i}} \text { (gas) } / \mathrm{d} t=k_{\mathrm{i}} W_{\mathrm{i}} \text { (char) }=k_{\mathrm{i}} X Y_{\mathrm{i}}
$$

where, $\mathrm{d} W_{1}$ (gas)/d $t$ is the rate of evolution of speices $i$ into the gas phase, $k_{1}$ is a distributed rate for species $i$ and $W_{\mathrm{l}}$ (char) is the functional group source remaining in the char. The concept of the distributed rate was introduced by Pitt $^{B 0}$ and subsequently employed by Rennhack ${ }^{81}$ and
Anthony et al. ${ }^{22}$ to describe weight loss. Hanbaba et al., ${ }^{82}$ van Heek et al., ${ }^{83}$ Weimer and Ngan, ${ }^{9}$ and Solomon et al. ${ }^{12}$ employed distributed rates for individual species. In the FG subroutine, $k_{i}$ is given by an Arrhenius expression $k_{i}$ $=k_{i}^{0} \exp \left(-\left(E_{i} \pm \sigma_{i}\right) / R T\right)$, where $\pm \sigma_{i}$ indicates that a Gaussian distribution is employed to describe the product sources, $W_{\mathrm{i}}\left(E_{\mathrm{i}}\right)$, as a function of the activation energies $E_{\mathrm{i}}{ }^{5,9,12,22} W_{\mathrm{i}}\left(E_{\mathrm{i}}\right)=\left(W_{\mathrm{i}}^{0} / \sigma_{\mathrm{i}}(2 \pi)^{1 / 2}\right) \exp \left(-\left(E_{\mathrm{i}}-E_{\mathrm{i}}^{0}\right)^{2} / 2 \sigma_{\mathrm{i}}{ }^{2}\right)$. $E_{i}^{0}$ is the average activation energy, and $\sigma_{i}$ is the width of the Gaussian distribution.

Note that $W_{i}$ (char) also is decreased by its evolution with the tar.

Process 6. Tar Formation. The tar composition is tracked by summing the functional group contributions evolved with the tar. The rate of evolution of each contribution is

$$
\mathrm{d} W_{\mathrm{i}}(\operatorname{tar}) / \mathrm{d} t=-(\mathrm{d} X / \mathrm{d} t) Y_{\mathrm{i}}
$$

where $\mathrm{d} W_{\mathrm{i}}(\mathrm{tar}) / \mathrm{d} t$ is the rate of evolution of each functional group component with the tar.

Process 7 Char Formation. The change in the ith char pool, $W_{\mathrm{i}}$ (char), is computed by summing the losses to the gas and tar and the redistributions determined in the DVC subroutine

$$
\begin{aligned}
& \mathrm{d} W_{\mathrm{i}}(\text { char }) / \mathrm{d} t= \\
& \quad-\mathrm{d} W_{\mathrm{i}}(\text { gas }) / \mathrm{d} t-\mathrm{d} W_{\mathrm{i}}(\text { tar }) / \mathrm{d} t+\mathrm{d} W_{\mathrm{i}}(\mathrm{DVC}) / \mathrm{d} t
\end{aligned}
$$

where $\mathrm{d} W_{\mathrm{i}}(\mathrm{DVC}) / \mathrm{d} t$ includes the source and loss terms from the DVC model, given by $(30 / 28) k_{\mathrm{B}} W_{\mathrm{B}},(2 / 28) k_{\mathrm{B}} W_{\mathrm{B}}$,

(81) Rennhack, R. Brennst.-Chem. 1964, 45, 300.

(82) Hanbaba, P.; Juntgen, H.; Peters, W. Brennst.-Chem. 1968, 49, 368.

(83) Van Heel, K. H.; Juntgen, H, Peters, W. Ber. Bunsen-Ges. Phys. Chem. 1967, 71, 113 . 


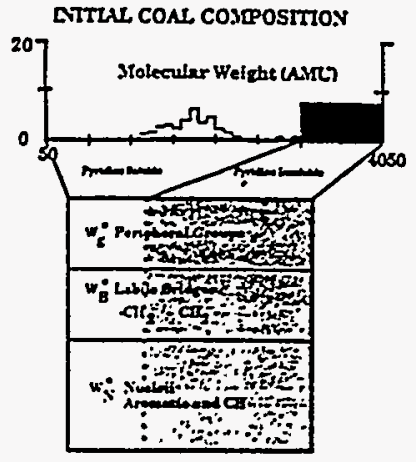

PRDARY DEVOLATRIZATION
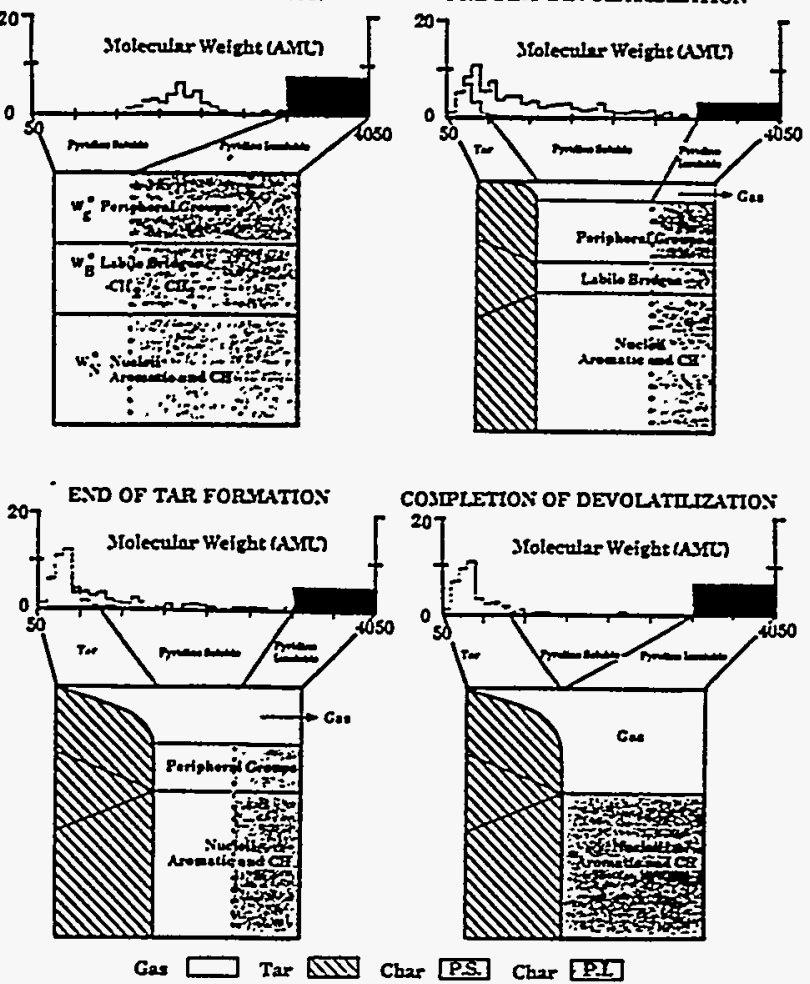

Figure 5. Schematic representation of the FG-DVC model combining the DVC and FG subroutines. The FG subroutine is illustrated for a single gas species only. The area under the histogram corresponds to the weight percent of the oligomers.

$(24 / 28) k_{\mathrm{B}} W_{\mathrm{B}}$, and $-2 k_{\mathrm{B}} W_{\mathrm{B}}$ for methyl, aromatic $\mathrm{H}$, aromatic $C$, and labile bridge functional groups, respectively.

The general rates and specific composition parameters for Pittsburgh Seam coal and North Dakota lignite are presented in Table II.

Schematic and Execution of FG-DVC Model. Figure 5 presents a schematic of the linked model for a simple case of only one gas species. The combined model connects the upper (DVC portion) and lower (FG portion) parts of Figure 5a-d. The model is initiated by specifying the functional group composition parameters $\left(W_{\mathrm{B}}, W_{\mathrm{N}}\right.$, and, in this case, only one gas species parameter, $W_{p}$ ) and the coal structure parameters (starting oligomer length, $l$, number of added cross-links per monomer, $m_{0}$, and the monomer molecular weight distribution parameters, $M_{\mathrm{av}}$ and $\sigma$ ). The starting molecular weight distribution of oligomers is presented at the top of Figure 5a. The monomers are assumed to have the average elemental and functional group composition given by the FG parameters. The functional groups are divided into pyridine-soluble and pyridine-insoluble parts. Each computer simulation considers coal to consist of a network made from $2100-2400$ monomers.

Once the starting distribution of oligomers in the coal is established, it is then subjected to a time-temperature profile made up of a series of isothermal time steps. Each time step is chosen so the temperature rise in each step does not exceed a fixed maximum. During each step, the gas yields, elemental composition, and functional group composition are computed by using the FG subroutine. The $\mathrm{CO}_{2}$ and $\mathrm{CH}_{4}$ yields are used to determine the number of new cross-links to be randomly added to the molecule. The molecular weight distribution, the escape of tar molecules, and the redistribution of hydrogens and carbons from the labile groups is computed with the DVC subroutine. Figure $5 \mathrm{~b}$ illustrates tar formation simultaneous with gas formation. The labile bridges are either evolved with the tar, converted to methyl groups (and thus added to the peripheral groups), or converted to unbreakable bridges (and thus added to aromatic $\mathrm{C}$ and $\mathrm{H}$ groups). Tar formation is complete (Figure $5 \mathrm{c}$ ) when all the labile bridges are consumed. Devolatilization is completed (Figure 5d) when all volatile functional groups (in this case the single gas species represented as peripheral groups) are removed from the char.

The model has been programmed in Fortran 77 and runs on the Sun Microsystems $3 / 260$ and 3/50 computers. Run times on a Sun 3/260 are between 83 and $550 \mathrm{~s} /$ simulation for 2100-2400 monomers. A streamlined version of the code designed to run as a subroutine in a comprehensive combustion or gasification reactor simulation employs from 400 to 800 monomers and requires approximately $10 \mathrm{~s}$ / simulation for the pyrolysis of a single particle.

Summary of FG Subroutine Assumptions. (a) Light-gas species are formed from the decomposition of specific functional groups with rate coefficients that depend on the functional group but are independent of coal rank. The evolution rate is first order in the remaining functional group concentration in the char. The rates follow an Arrhenius expression with a Gaussian distribution of activation energies. ${ }^{5,12,22}$

(b) Simultaneous with the production of light-gas species is the thermal cleavage of bridge structures in the coal to release molecular iragments of the coal, which consist of a representative sampling of the functional group ensem. ble. These fragments may be transported out of the coal particle to form tar. The instantaneous tar yield is given by the DVC subroutine.

(c) Under conditions where pyrolysis products remain hot (such as an entrained-flow reactor), pyrolysis of the functional groups in the tar continues at the same rates used for functional groups in the char (e.g., the rate for methone formation from methyl groups in tar is the same as from methyl groups in the char).

Summary of DVC Subroutine Assumptions. (d) The oligomer length, $l$, the number of cross-links per monomer, $m_{0}$, and the fraction of labile bridges, $W_{B}$, are parameters of the model, chosen to be consistent with the coal's measured extract yield, cross-link density, and volatile yield in selected calibration experiments.

(e) The molecular weight distribution is adjusted so that the model predictions fit the observed molecular weight distribution for that coal, measured by pyrolysis of the coal (in vacuum at $3^{\circ} \mathrm{C} / \min$ to $500^{\circ} \mathrm{C}$ ) in a FIMS apparatus. ${ }^{60}$ Molecular weights 106, 156, 206, 256,306, 356, and 406 (which are aromatic one-ring through seven-ring compounds with two methyl substituents) are considered as representative of typical monomer molecular weights. .

(f) During pyrolysis, the breakable bonds are assumed to rupture randomly at a rate $k_{\mathrm{B}}=k_{\mathrm{tar}}$, described by an Arrhenius expression with a Gaussian distribution of sources as a function of activation energies. Each rupture creates two free radicals that consume two donatable hydrogens to form two new methyl groups and convert two more donatable hydrogens to two aromatic $\mathrm{CH}$ groups. Oxymethylene bridges, which may be important for lowrank coals, have not been modeled although a second class of labile bridges could easily be added.

(g) All the donatable hydrogens are assumed to be located in the labile bridges. Two donatable hydrogens are available at each bridge. The consumption of the donatable hydrogen converts the bridge into an unbreakable bridge by the formation of a double bond. The unbreakable bridges are included in the aromatic hydrogen and 
aromatic carbon functional groups.

(h) Tar formation continues until all the donatable hydrogens are consumed.

(i) During pyrolysis, additional unbreakable cross-links are added at a rate determined by the evolution of $\mathrm{CH}_{\text {, }}$ and $\mathrm{CO}_{2}$. One cross-link is created for each evolved molecule. The rates of $\mathrm{CH}_{4}$ and $\mathrm{CO}_{2}$ evolution are given by the FG subroutine.

(j) The cross-links are distributed randomly, with the probability of attachment on any one monomer being proportional to the molecular weight of the monomer.

(k) Tar molecules are assumed to vaporize from the surface of the coal particle (or into bubbles) with a molecular weight and temperature dependence based on the vapor pressure correlation of Suuberg et al. ${ }^{32}$ The external-transport model is based on the surface-evaporation model of Unger and Suuberg. ${ }^{23}$

(l) To describe internal transport, a simple empirical expression (eq 4) is used to describe both bubble transport in softening coals and convective transport through pores in nonsoftening coals. The tar is assumed to be transported at its equilibrium vapor pressure in the light-gas species. The pressure increase that drives the transport within the particle, $\Delta P$, is between 0 and $0.2 \mathrm{~atm}$ for the bituminous coal and between 0 and $10 \mathrm{~atm}$ for the lignite, depending on the experimental conditions.

(m) Extractable material (in boiling pyridine) in the char is assumed to consist of all molecules less than $3000 \mathrm{amu}$. This limit can be adjusted depending on the solvent and extraction conditions.

(n) The molecular weight between cross-links, $M_{\mathrm{c}}$, is computed to be the total molecular weight of the computer molecule divided by the total number of cross-links. This assumption will underestimate $M_{\mathrm{c}}$ since broken bridges are not considered.

\section{Results}

The model predictions have been compared to the results obtained from a number of experiments on the pyrolysis of a Pittsburgh Seam coal 6,7,16,22 and a North Dakota (Beulah, Zap) lignite.651 The coal composition and kinetic parameters are presented in Tables I and II. It should be noted that different samples of Pittsburgh seam coal from different sources were employed. While the elemental compositions were similar, extract yields varied depending on the sample source. The oligomer length in Table I was chosen to fit an extract yield of $30 \%$ for the Pittsburgh Seam coal and $1 \%$ for the lignite. Comparisons are considered for gas yields, tar yields, tar molecular weight distributions, extract yields, and volumetric swelling ratios.

Volatile Yields. Extensive comparisons of the FG model with gas yields have been presented previously for high- and low-heating-rate devolatilization experiments. 5,6,11-13 The evolution of gases for the combined model is similar to results of the FG model and will not be repeated here. There is good agreement between the measured and predicted results. The functional group parameters and the linetic rates used for this work for the Pittsburgh Seam coal and North Dakota (Zap) lignite are principally those determined previously and published in ref 6. The methane parameters for the Pittsburgh Seam coal were, however, adjusted (methane $X-L=0.0$, methane- $\mathrm{L}=0.02$, methane- $\mathrm{T}=0.015$, unchanged) to better match yields of refs. 5-7 (see Figure 20c in ref 6). Also note that the $\mathrm{CH}_{2}$ - aliphatic rate in ref 6 applies to the observed gas species (paraffins, olefins, $\mathrm{C}_{2} \mathrm{H}_{6}, \mathrm{C}_{2} \mathrm{H}_{4}$ ) only. The aliphatic material in the labile bridge part of the aliphatic groups is assumed to be made up of bridges that volatilize only when attached to a tar molecule (i.e., $k_{i}=0$ ). Also,

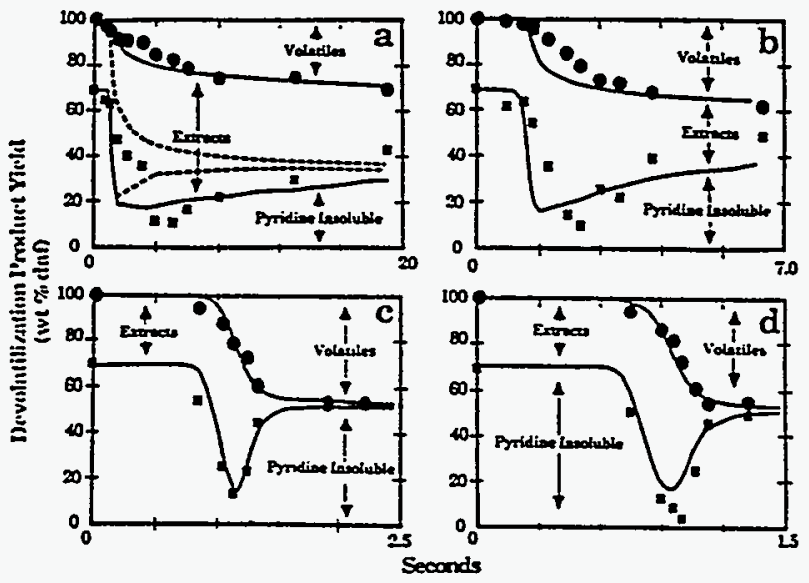

Figure 6. Comparison of FG-DVC model predictions with the data of Fong et al. ${ }^{16}$ (symbols) for Pittsburgh Seam coal: (a) 813 $\mathrm{K}$ at $470 \mathrm{~K} / \mathrm{s}$; (b) $858 \mathrm{~K}$ at $446 \mathrm{~K} / \mathrm{s}$; (c) $992 \mathrm{~K}$ at $514 \mathrm{~K} / \mathrm{s}$; (d) $1018 \mathrm{~K}$ at $640 \mathrm{~K} / \mathrm{s}$. $P=0.85 \mathrm{~atm}$. The solid line assumes transport by eq $4(\Delta P=0 \mathrm{~atm})$ and no external transport. The dashed line in part a shows the predicted yield assuming $X_{\mathrm{j}}^{\mathrm{s}}=$ $x^{b}{ }_{j}$ in eq 3 and no internal-transport limitations.

the rate for $\mathrm{CO}_{2}$ (loose) has been adjusted to improve the predictions of the change in tar molecular weight distributions and yield with heating rate. The predictions of gas yield due to this change have not been changed noticeably. The predicted values of $X^{0}$ from the DVC subroutine vary with heating rate and final temperature and are in good agreement with the values of $X^{0}$ used in the original FG model.

Extract Yields. Figure 6 compares the FG-DVC predictions to the data of Fong et al. ${ }^{16}$ on total volatile yield and extract yield as a function of temperature in pyrolysis at $0.85 \mathrm{~atm}$. The experiments were performed in a heated-grid apparatus at heating rates of approximately 500 ${ }^{\circ} \mathrm{C} / \mathrm{s}$, with variable holding times and rapid cooldown. The predictions at the two higher temperatures (Figures 6c,d) are in excellent agreement with the data.

The initial predictions for the two lower temperature cases, which neglected interal-transport limitations, were not good. The dashed line in Figure $6 \mathrm{a}$ shows the predicted yield in the absence of internal transport limitations (i.e., $\left(d n_{j} / d t\right)_{I T}=0$ and with $\chi_{j}^{s}=\chi_{b}^{b}$ in eq 3 ). The predicted ultimate yield is clearly too high. The data suggest that the low yields are not a result of unbroken bonds (which would result from a lower bond breaking rate, $k_{\mathrm{B}}$ ), since the extract yields at low temperatures are equivalent to those at the higher temperatures. The coal molecule thus appears to be well decomposed, the low yields resulting from poor transport out of the coal. This suggested an additional transport limitation in getting molecules to the surface, so $X_{j}^{b}=X_{j}^{b}$ appears to be a bad assumption.

Equation 4 was employed for the internal-transport rate, and surface evaporation by eq 3 was assumed to be unimportant $\left(X_{j}^{*}=0\right)$. Then, $W_{B}$ had to be slightly readjusted from 0.096 in ref 50 to 0.094 to match the $1018 \mathrm{~K}$ case. This new value of $W_{B}$ was used for subsequent cases. The predictions with this assumption are the solid lines in Figure 6. The internal-transport limitation is most important when pyrolysis occurs at low temperatures and $\sum_{\text {light }} \mathrm{d} n_{\mathrm{i}} / \mathrm{d} t$ in eq 4 is small.

There still is a discrepancy between the prediction and the data at early times for the two lower temperature cases (Figure 6a,b). While it is possible that the rate $k_{\mathrm{b}}$ for bond breaking is too high, adjustment of this rate alone would significantly lower the extractable yield, since the lower depolymerization rate is closer to the methane cross-linking 

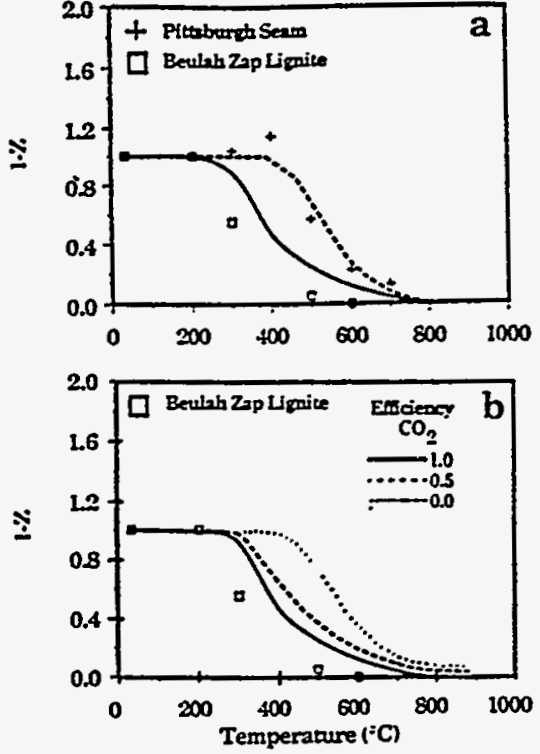

Figure 7. (a) Comparison of measured and predicted normalized volumetric swelling ratio as a function of temperature. The solid line is the prediction of Beulah lignite; the dashed line is for Pittsburgh Seam coal. The cross-link efficiency of $\mathrm{CO}_{2}$ is 1.0. (b) Effect of cross-link efficiency of $\mathrm{CO}_{2}$ on the normalized volumetric swelling ratio profile with temperature. For parts $a$ and $b$ the heating rate is $0.5^{\circ} \mathrm{C} / \mathrm{s} . \Delta P=0$ atm.

rate. In addition, both the methane and depolymerization rates appear to be in good agreement with the data at even lower temperatures. 6 Another possibility is that the coal particles heat more slowly than the nominal temperatures given by Fong et al. ${ }^{16}$ Such an effect could be caused by having some clumps of particle that would heat more slowly than isolated particles, by reduction in the convective heat transfer due to the volatile evolution (blowing effect) or by endothermic tar forming reactions. A firm conclusion as to the source of this remaining discrepancy cannot be drawn without further investigation.

It is also seen in Figure 6a,b that the cross-linking rate is higher than predicted. This can be due to other crosslinking events not considered. These possibilities are currently under investigation.

Cross-Link Density. To examine the effect of coal rank on cross-linking, the volumetric swelling ratios (VSR) for North Dakota (Beulah, Zap) lignite and Pittsburgh Seam bituminous coal were measured as a function of temperature at $0.5^{\circ} \mathrm{C} / \mathrm{s}$. The VSR can be related to the cross-link density. 77 The swelling data are plotted in Figure 7a as $1-Z$, where $Z$ is the change in VSR between coal and char normalized by the maximum change. For coal, $Z$ is 0 and for completely cross-linked char, $Z$ is 1 . While the weight loss profiles of the two samples look similar at $0.5^{\circ} \mathrm{C} / \mathrm{s}$, the swelling behaviors in Figure 7a are quite different. The Pittsburgh Seam coal starts to cross-link during tar evolution, and the Beulah lignite cross-links well before tar evolution. Similar results have been reported by Suuberg et al. ${ }^{59}$ who also suggested a correlation between cross-linking in lignites and $\mathrm{CO}_{2} \mathrm{ev}$ olution. The coals that undergo early cross-linking are less fluid, produce less tar, and produce lower molecular weight tar compared with coals that do not experience early cross-linking. $30,31,4$

As discussed previously, under the assumption that the cross-linking reactions may also release gas species, the VSR was correlated with the observed evolution of gas species during pyrolysis. Correlations presented in Figure 2 show that on a molar basis, the evolution of $\mathrm{CO}_{2}$ from the lignite and $\mathrm{CH}_{4}$ from the bituminous coal appear to have similar effects on the VSR. Reactions that form these gases, leave behind free radicals that can be stabilized by cross-linking.

Assuming that one cross-link is formed for each $\mathrm{CO}_{2}$ or $\mathrm{CH}_{4}$ evolved from the char, the FG-DVC model predictions are presented as the lines in Figures 2 and $7 \mathrm{a}$. The agreement between theory and experiment is good except that the increase in $1-Z$ for the Pittsburgh Seam coal in Figure $7 \mathrm{a}$ is not predicted. This may be related to the restrictions of assumption n (see summary of DVC subroutine assumptions). The predictions in Figure $2 a$ are different from those originally presented in ref 50 . In ref 50 , the value used for $\mathrm{VSR}_{\min }$ was not appropriate for the fully cross-linked molecule. This error has now been corrected.

In Figure $7 \mathrm{~b}$, the effect of varying the $\mathrm{CO}_{2}$ cross-linking efficiency is considered. The figure shows cases calculated for the lignite assuming $0,0.5$, and 1.0 cross-links are formed per $\mathrm{CO}_{2}$ evolved. Varying this assumption has a major effect on the early cross-linking of the lignite. Assuming that the cross-linking efficiency per $\mathrm{CO}_{2}$ is 1.0 gives the best agreement with the data.

The difference in crosslinking behavior between the two coals is manifested in several areas. At low heating rates, the Pittsburgh Seam chars soften; the Beulah, Zap chars do not. This is in agreement with the high predicted maximum extract yields in the Pittsburgh char (70\%) compared to the low extract yields in the Beulah, Zap lignite (7\%). The measured values are $71 \%{ }^{16}$ and $\sim 6 \%$, respectively. The predicted yield of tar plus aliphatic gases at I atm, $0.5^{\circ} \mathrm{C} / \mathrm{s}$ to $900^{\circ} \mathrm{C}$, of $26 \%$ is in good agreement with the measured value of $28 \%$ for the Pittsburgh Seam coal. The predicted value of $11 \%$ (for $\Delta P=10 \mathrm{~atm}$ ) is in good agreement with the measured value of $10 \%$ for the Beulah, Zap lignite.

Molecular Weight Distribution. A sensitive test of the general model is the ability to predict the tar molecular weight distribution and its variations with rank, pressure, and heating rate. The input to the model is the distribution of monomer molecular weights. The tar, which consists of oligomers, has a different distribution from the monomer distribution and is controlled by the relative effects of bond breaking, cross-linking, and transport. The tar molecular weight distribution is not highly sensitive to the choice of $M_{\mathrm{av}}$ and $\sigma$. For Pittsburgh Seam coal, the average monomer was assumed to be a three-ring compound $\left(M_{\mathrm{av}}=256\right)$ and a fairiy broad distribution $(\sigma=250)$ was chosen. The same values appeared to work for the lignite. These are in reasonable agreement with the measured values of $\sim 300$ reported by Solum et al. ${ }^{79}$ for. both coals.

Figure 8c,d show results for the Pittsburgh Seam bituminous coal and the Beulah, Zap lignite pyrolyzed in the FIMS apparatus. The data have been summed over 50 amu intervals. While the Pittsburgh bituminous coal shows a peak intensity at about $400 \mathrm{amu}$, the lignite peak is at $100 \mathrm{amu}$. The predicted average tar molecular weight distributions are in good agreement with FIMS data as shown in Figure $8 \mathrm{a}, \mathrm{b}$. Since both tar distributions are from the same monomer distribution, the enhanced drop off in amplitude with increased molecular weight for the lignite compared to the bituminous coal must be due to early cross-linking and transport effects in the lignite.

Pressure Effects. The predicted effect of pressure on the tar molecular weight distribution is illustrated in Figure $9 \mathrm{a}, \mathrm{b}$. Pressure enters the model through the transport eq 3 and 4. The internal-transport rate (eq 4), which is assumed to dominate, is inversely proportional to the am- 
Table III. Comparison of Measured and Predicted Yields for Pittsburgh Seam Bituminous Coal $(\Delta P=0$ atm)

\begin{tabular}{|c|c|c|c|c|c|c|c|}
\hline \multirow[b]{2}{*}{ experiment } & \multirow[b]{2}{*}{ beating rate, ${ }^{\circ} \mathrm{C} / \mathrm{s}$} & \multirow[b]{2}{*}{$P_{0}$, atm } & \multirow[b]{2}{*}{ final temp, ${ }^{\circ} \mathrm{C}$} & \multicolumn{2}{|c|}{$\begin{array}{l}\text { max yield of tar }+ \\
\text { aliphatic gases, \% }\end{array}$} & \multicolumn{2}{|c|}{ total max volatiles, wt \% } \\
\hline & & & & measd & predicted & measd & predicted \\
\hline $\begin{array}{l}\text { TG-FTIR } \\
\text { entrained flow } \\
\text { heated grid }\end{array}$ & $\begin{array}{c}0.5 \\
5000 \\
640\end{array}$ & $\begin{array}{l}1.0 \\
1.0 \\
0.85\end{array}$ & $\begin{array}{l}600 \\
700 \\
745\end{array}$ & $\begin{array}{l}25 \\
36\end{array}$ & $\begin{array}{l}29 \\
37 \\
40\end{array}$ & $\begin{array}{l}35 \\
43 \\
47\end{array}$ & $\begin{array}{l}37 \\
43 \\
47\end{array}$ \\
\hline
\end{tabular}

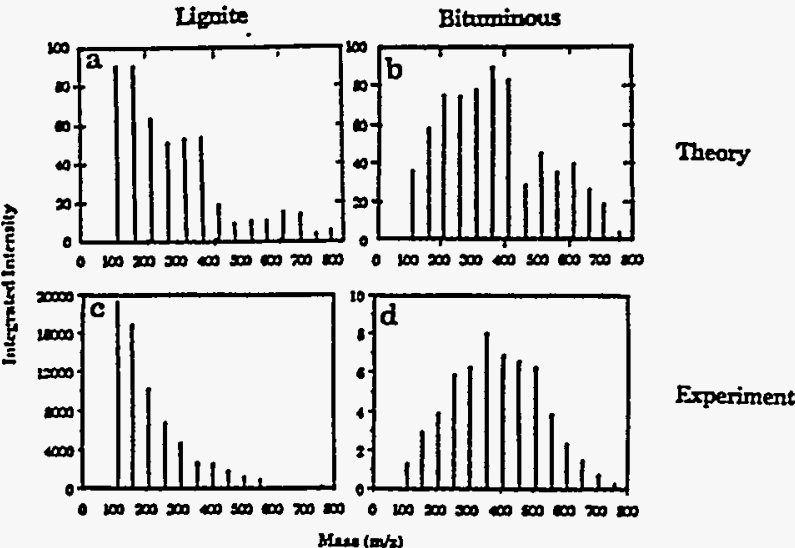

Figure 8. Comparison of measured and predicted tar molecular weight distributions for lignite and bituminous coals. The experiments are performed by pyrolysis of coal samples in a FIMS apparatus. Intensities have been summed over 50 amu intervals. For part a $\Delta P=10 \mathrm{~atm}$, and for part b $\Delta P=0.0 \mathrm{~atm}$.

bient pressure $P_{0}$. The reduced transport rate reduces the evolution rate of the heavier molecules. Therefore, the average molecular weight and vaporization "cutoff" decrease with increasing pressure. The trends are in agreement with observed tar molecular weight distributions shown in Figure 9c,d. The spectra are for previously formed tar that has been collected and analyzed in a FIMS apparatus. ${ }^{60}$ The low values of intensity between 100 and 200 mass units are believed to be due to loss of these components due to their higher volatility.

Pressure effects on yields have also been examined. Figure 10 compares the predicted and measured pressure dependence on yield for a Pittsburgh Seam coal. Figure 10 a compares the prediction to the total volatile yield data of Anthony et al. ${ }^{22}$ while Figure $10 \mathrm{~b}$ compares the prediction to the tar plus liquids data of Suuberg et al. ${ }^{7}$ The agreement between theory and experiment is good at $1 \mathrm{~atm}$ and above, but the theory with $\Delta P=0$ (solid line) overpredicts the yields at low pressure. Below 1 atm, it is expected that $\Delta P$ within the particle will become important compared to the ambient pressure, $P_{0}$. The dashed lines, which agree with the data, were obtained by assuming $\Delta P$ $=0.2 \mathrm{~atm}$, which is physically reasonable.

Heating-Rate Effects. It is well-known that the heating rate can affect the amount of volatiles produced. $20,76,84-86$ Heating rate can also affect the melting and swelling behavior of low-rank coals. ${ }^{13}$ Considering the mechanisms proposed for pyrolysis (including those in this paper), it is the relative rates of competing processes for tar formation (e.g., bond breaking, cross-linking, and mass transport) that provide the heating-rate effects. The relative rates of these processes change with temperature, and it is the heating rate that determines the temperature at which the controlling reactions occur. So it is really the

(84) Gibbins-Matham, J; Kandiyoti, R. Prepr. Pap.-Am. Chem. Soc., Div. Fuel Chem. 1987. 32(4), 318.

(85) Freihaut, J. D. Seery, D. J. Prepr. Pap.-Am. Chem. Soc., Div. Fuel Chem. 1983, 28(4), 265.

(86) Serio, M. A.; Peters, W. A.; Sawada, K.; Howard, J. B. Prepr. Pap.-Am. Chem. Soc. Div. Fuel Chem. 1984, 29(2), 65.
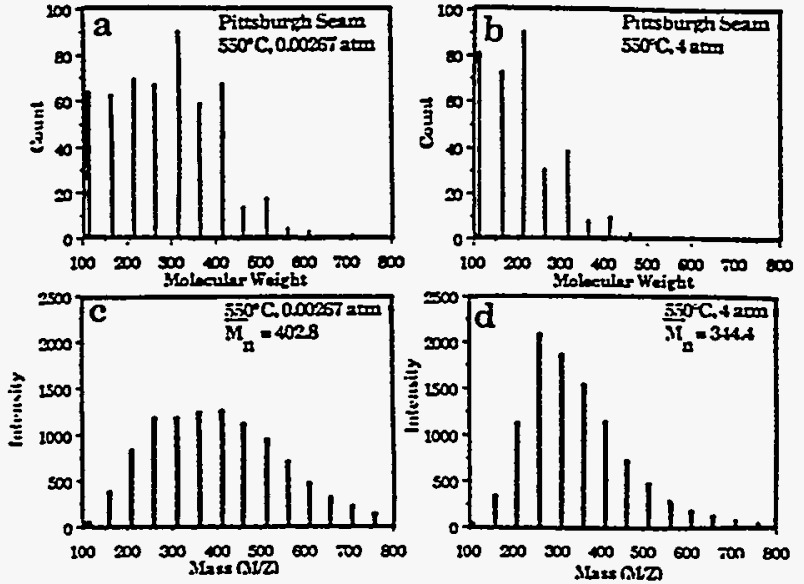

Figure 9. Comparison of predicted ( $a$ and $b$ ) and measured ( $c$ and d) tar molecular weight distribution for pyrolysis of a Pittsburgh Seam coal in a heat-grid apparatus at a heating rate of $500^{\circ} \mathrm{C} / \mathrm{s}$ to $550^{\circ} \mathrm{C}$. Parts a and c compare the prediction and the measurement at $0.00267 \mathrm{~atm}$. Parts b and d compare the prediction and measurement at $4.0 \mathrm{~atm} . \Delta P=0.2 \mathrm{~atm}$.

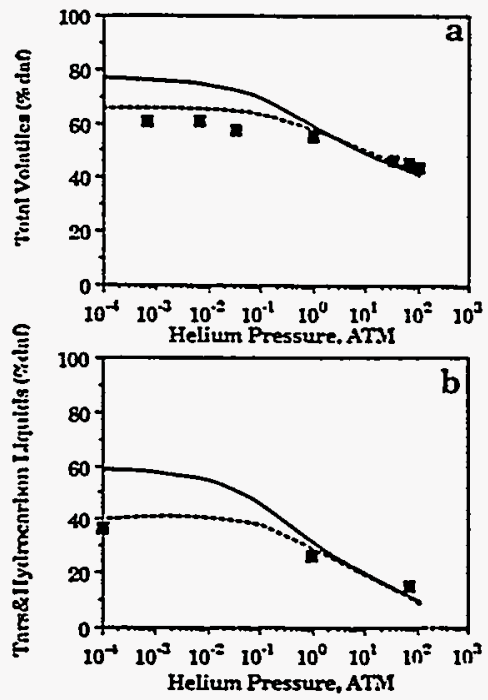

Figure 10. Comparison of measured and predicted volatile yield for a Pittsburgh Seam bituminous coal; (a) total volatiles, data of Anthony et al.;2 (b) tars and hydrocarbon liquids, data of Suuberg et al. ${ }^{7}$ The solid line assumes $\Delta P=0 \mathrm{~atm}$; the dashed line assumes $\Delta P=0.2 \mathrm{~atm}$.

temperature of tar formation and not the heating rate per se which is important.

Consider first the effects of heating rate on the yields of a Pittsburgh Seam bituminous coal. Table III summarizes the results for three experiments ${ }^{16,87,88}$ in which the heating rate varied from 0.5 to $5000^{\circ} \mathrm{C} / \mathrm{s}$ and in which the final temperature reached is sufficiently high for tar

(87) Serio, M. A.; Solomon, P. R.; Carangelo, R. M. Prepr. Pap.-Am. Chem. Soc., Div. Fuel Chem. 1988, 33(2), 295.

(88) Solomon, P. R.; Hamblen, D. G.; Serio, M. A.; Smoot, L. D.; Brewster, S. "Measurement and Modeling of Advanced Coal Conversion"; First Annual Report for U.S. METC Contract No. DE-AC21-86MC23075, 1987. 

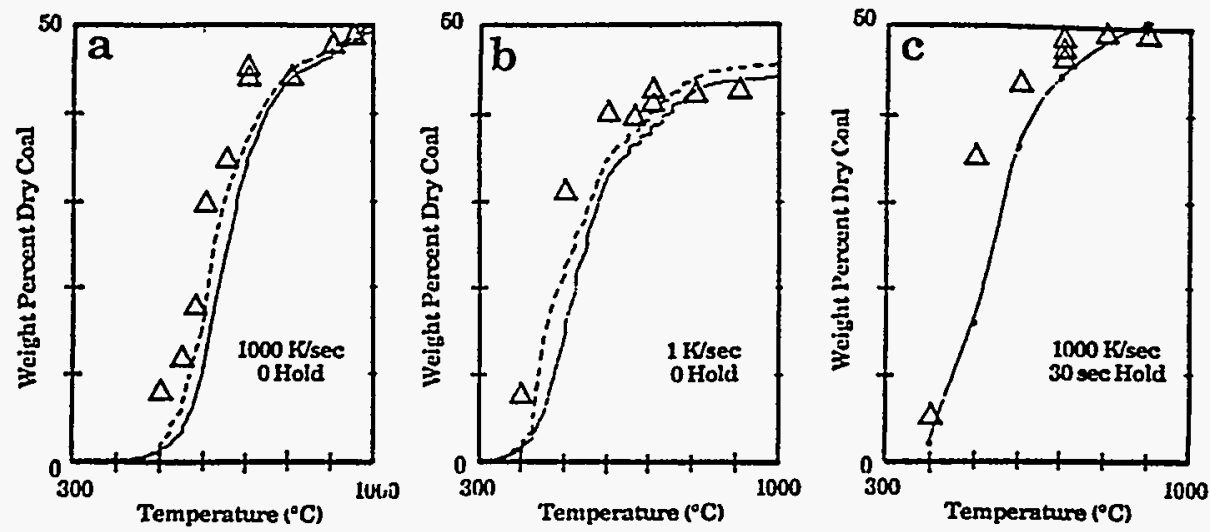

Figure 11. Comparison of FG-DVC model predictions with the data of Gibbins-Matham and Kandiyoti ${ }^{3}$ (symbols) for Pittsburgh Seam coal: (a) $1000 \mathrm{~K} / \mathrm{s}$, zero hold; (b) $1 \mathrm{~K} / \mathrm{s}$, zero hold; (c) $1000 \mathrm{~K} / \mathrm{s}, 30-\mathrm{s}$ hold. $P=1.18$ atm. Transport is by eq $4(\Delta P=0)$ and no external-transport limitation. The dashed line assumes no transport limitations for molecules whose vapor pressure exceeds $P_{0}$ $+\perp P$.

formation to be completed during the heating period. As can be seen, the predicted and measured volatile yields increased by about $10 \%$ from low to high heating rates. As can also be seen, the increase in yield results from the increase in tar plus aliphatic gases. Examination of the rates in the model shows that the major contribution to the variation in yield is the internal-transport rate relative to the bond-breaking rate. At low temperatures, internal transport severely limits the evolution of the heavier molecules, resulting in smaller tar molecules and inefficient use of the donatable hydrogens.

A set of data showing the effect of heating rate on yield for the Argonne Pittsburgh Seam coal was recently reported by Gibbins-Matham and Kandiyoti. ${ }^{84}$ Data were obtained in a wire-grid apparatus at 1 and $1000^{\circ} \mathrm{C} / \mathrm{s}$ with no holding time and at $1000^{\circ} \mathrm{C} / \mathrm{s}$ with a $30-\mathrm{s}$ hold. These data (triangles) are compared to predictions of the model in Figure 11. For all three cases, the theory predicts the correct pyrolysis final yields, the correct yield variation with heating rate, and the correct temperature shift with heating rate.

The predicted yields, however, occur at temperatures from 20 to $80^{\circ} \mathrm{C}$ higher than the comparable experimental yields. At this time, the reason for the discrepancy is not clear. One possible reason is the assumptions used for the internal transport limitations. Calculations were made assuming that molecules for which $P_{\mathrm{j}} \geq P_{0}+\Delta P$ evolve as they are produced, while only heavier molecules evolve as described in eq 4. The predicted curves (dashed lines in Figure 11) are $20-40^{\circ} \mathrm{C}$ lower than in the original calculation. Alternatively, the vapor pressure may not be accurately described by the expression of Suuberg et al. ${ }^{32}$ $\mathrm{Oh}^{38}$ compared a number of correlations for the tar vapor pressure. At $1000^{\circ} \mathrm{C}$, the expression of Suuberg et al.$^{32}$ gave vapor pressures from 1-2 orders of magnitude lower than other published expression.89,90 Calculations using the expression for aliphatic molecules of Maiorella ${ }^{89}$ gave predictions at about $40^{\circ} \mathrm{C}$ lower temperatures, in better agreement with the data of Gibbons-Matham and Kandiyoti. The simulation, however, required a lower value of $W_{\mathrm{B}}(0.060)$ to compensate for the higher volatility. Predictions using the same assumptions failed to match those of Fong et al..$^{26}$ in Figure 6 with regard to the temperature of evolution and the amount of extract produced. Possible refinements of the internal-transport model are

(89) Maiorella, B. L. B.S. Thesis, Department of Chemical Engineering, MT, Cambridge, MA, 1975.

(90) Grey, J. A.; Brady, A. J.; Cunningham, J. R.; Freeman, J. R.; Wilson, G. M. Ind. Eng. Chem. Process Des. Dev. 1983, 22, 410.
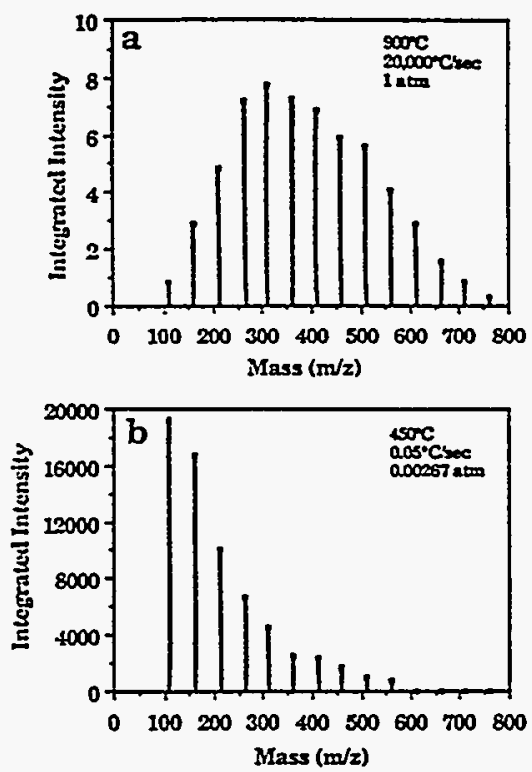

Figure 12. Comparison of FIMS spectra of tars of Beulah Zap lignite formed at (a) high heating rate $\left(20000^{\circ} \mathrm{C} / \mathrm{s}\right)$ and (b) low heating rate $\left(0.05^{\circ} \mathrm{C} / \mathrm{s}\right)$.

being considered.

Another possible explanation for the discrepancy is the accuracy of the reported pyrolysis temperature, which has been notoriously variable among investigators. Other Pittsburgh Seam coal data (not shown) for Niksa et al. ${ }^{10}$ under the same conditions as Figure $11 \mathrm{c}\left(1000^{\circ} \mathrm{C} / \mathrm{s}, 30-\mathrm{s}\right.$ hold) and from $\mathrm{Oh}^{38}$ and Suuberg et al. ${ }^{7}$ for the same conditions as Figure 11a $\left(1000^{\circ} \mathrm{C} / \mathrm{s}\right.$, zero hold) show. substantial variations in temperature compared to the results of Gibbons-Matham and Kandiyoti.84 The theoretical predictions would lie within the scatter of the several data sets. Work is in progress to resolve this question.

Low-rank coals also exhibit heating-rates effects. It has been found that Beulah lignite chars soften and exhibit bubble formation at high heating rates $\left(\sim 20000^{\circ} \mathrm{C} / \mathrm{s}\right) .^{13}$ Under these conditions, molecular weight distribution of tars of Beulah lignite look like that of a bituminous coal ${ }^{30,31}$ The infrared spectrum of the tar is also closer in appearance to that of the parent coal. ${ }^{31}$ The mass spectra of the tars formed at high heating rate $\left(20000^{\circ} \mathrm{C} / \mathrm{s}\right)$ and low heating rate $\left(0.05^{\circ} \mathrm{C} / \mathrm{s}\right)$ are shown in parts $a$ and $b$ of Figure 12, respectively. The low values of intensity between 100 and 200 mass units in Figure 12b are believed 


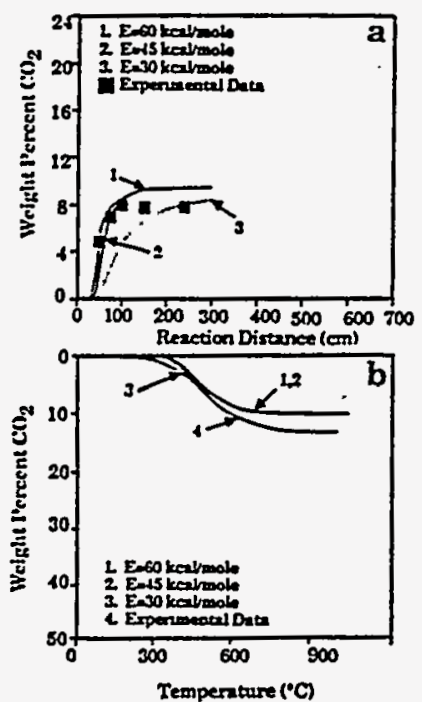

Figure 13. Comparison of $\mathrm{CO}_{2}$ evolution data from North Dakota lignite for low-heating-rate $\left(0.5^{\circ} \mathrm{C} / \mathrm{s}\right)$ and high-heating-rate $(20000$ ${ }^{\circ} \mathrm{C} / \mathrm{s}$ ) experiments with model predictions for different values of activation energy for $\mathrm{CO}_{2}$ (extra loose) in the FG-DVC Model: (a) heated-tube-reactor experiments; ${ }^{6}$ (b) TG-FTIR experiments. ${ }^{6}$

to be due to loss of these components due to their high volatility. The molecular weight distribution of the tars is very sensitive to the heating rate. The effect is attributed to the higher rate of depolymerization reactions relative to cross-linking reactions at high temperatures, as discussed in the sensitivity section.

The FG-DVC model, assuming the internal-masstransport limitations, was used to simulate the low heating rate $\left(0.05^{\circ} \mathrm{C} / \mathrm{s}\right)$ and high heating rate $\left(20000^{\circ} \mathrm{C} / \mathrm{s}\right) \mathrm{py}-$ rolysis of Beulah lignite. The activation energy for $\mathrm{CO}_{2}$ (extra loose) in the FG subroutine was reduced from 60 to $45 \mathrm{kcal} / \mathrm{mol}$ in order to make it lower than the activation energy for bond breaking ( $55 \mathrm{kcal} / \mathrm{mol})$. This was done since measurements of the rate of cross-linking at high heating rates suggested that the relative rate of bondbreaking and cross-linking reactions associated with $\mathrm{CO}_{2}$ evolution is increased with increasing temperature. ${ }^{91}$ This change in the activation energy makes only a slight change in the $\mathrm{CO}_{2}$ evolution profiles for high-heating-rate (20000 $\left.{ }^{\circ} \mathrm{C} / \mathrm{s}\right)$ and low-heating rate $\left(0.5^{\circ} \mathrm{C} / \mathrm{s}\right)$ predictions. The $\mathrm{CO}_{2}$ gas evolution profiles are compared to the data in Figure 13a,b for high-heating-rate $\left(20000^{\circ} \mathrm{C} / \mathrm{s}\right)$ and lowheating-rate $\left(0.5^{\circ} \mathrm{C} / 8\right)$ experiments with Beulah lignite using activation energies of 60,45 , and $30 \mathrm{kcal} / \mathrm{mol}$ When the activation energy for $\mathrm{CO}_{2}$ (extra loose) evolution was reduced to $45 \mathrm{kcal} / \mathrm{mol}$, acceptable fits to the gas evoluation data were still obtained. However, at $30 \mathrm{kcal} / \mathrm{mol}$, the high-heating-rate $\mathrm{CO}_{2}$ evolution profile was quite different and did not agree with the experimental data.

The model, with internal-mass-transport limitations included, was used to simulate the tar molecular weight distributions with $\Delta P=0$ atm for Beulah lignite for high heating rate $\left(20000^{\circ} \mathrm{C} / \mathrm{s}\right)$ in Figure $14 \mathrm{a}, \mathrm{b}$. The simulations were done for both the original activation energy ( 60 $\mathrm{kcal} / \mathrm{mol})$ and altered activation energy $(45 \mathrm{kcal} / \mathrm{mol})$ for $\mathrm{CO}_{2}$ (extra loose) evolution. The tar molecular weight distributions (for $\Delta P=0 \mathrm{~atm}$ ) at high heating rates (Figure $14 a, b)$ show the observed high values of the tar molecular weight at high heating rate (Figure 12a). The lower activation energy case (Figure 14a) exhibits more high molecular weight molecules and gives a higher tar yield (10\%)

(91) Deahpande, G. V.; Solomon, P. R.; Serio, M. A Prepr. Pap.-Am. Chem. Soc., Div. Fuel Chem. 1988, 33(2), 310.
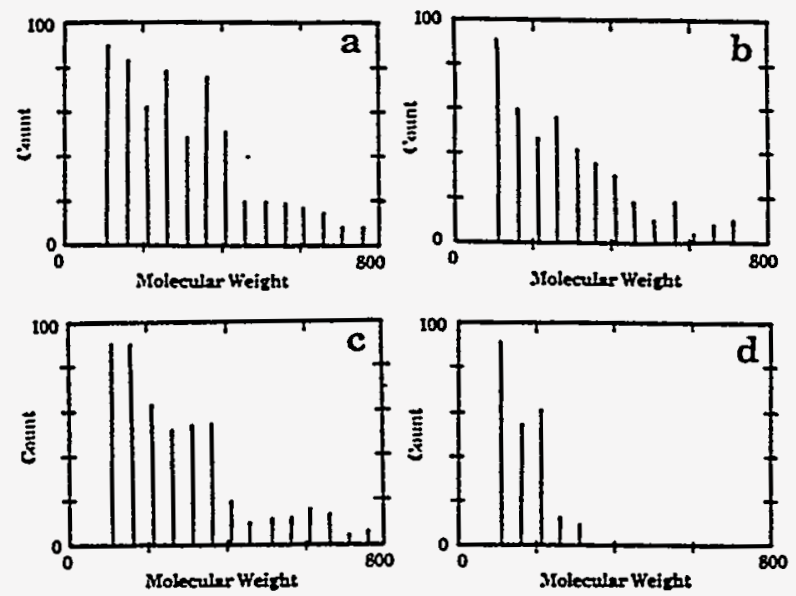

Figure 14. Comparison of predicted molecular weight distribution of tars of Beulah lignite for ( $a$ and $b$ ) high heating rate $\left(20000^{\circ} \mathrm{C} / \mathrm{s}\right)$ and $(\mathrm{c}$ and $\mathrm{d})$ low heating rate $\left(0.05^{\circ} \mathrm{C} / \mathrm{s}\right)$. In parts $\mathrm{a}, \mathrm{c}$, and d the $\mathrm{CO}_{2}$ activation energy is $45 \mathrm{kcal} / \mathrm{mol}$ and in part $\mathrm{b}$ it is $60 \mathrm{kcal} / \mathrm{mol}$. In parts $\mathrm{a}-\mathrm{c} \perp P=0 \mathrm{~atm}$, and in part $\mathrm{d} \Delta P$ $=10$ atm.
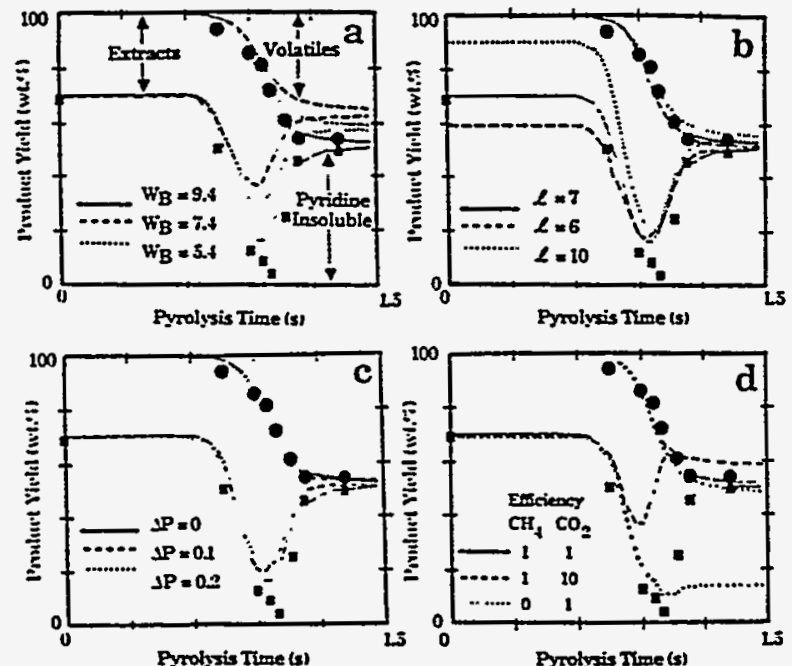

Figure 15. Effect on product yields of (a) fraction of labile bridges, $W_{B}$, (b) oligomer length, $l$, (c) internal pressure difference, $\triangle P$, and (d) cross-linking efficiency. Data were taken from Fong et al ${ }^{16}$ for Pittsburgh Seam bituminous coal (1018 K at $640 \mathrm{~K} / \mathrm{s}$, $P=0.85$ atm); $\triangle P=0$ atm.

than the high activation energy case (8\%) (Figure 14b). The low-heating-rate $\left(0.05^{\circ} \mathrm{C} / \mathrm{s}\right)$ case $(\Delta P=0)$ (Figure $\left.14 \mathrm{c}\right)$ exhibits lower molecular weights consistent with Figure 12b. At high heating rates, where cross-linking reactions are curbed and the lignite melts, $\Delta P$ is likely to be low. At low heating rate, due to the higher extent of cross-linking before tar evolution, the coal is less fluid, and hence $\Delta P$ (which is related to viscosity of the solid/liquid mixture) is likely to be higher. A simulation for the slow-heatingrate case with $\triangle P=10$ atm is shown in Figure 14d. The measured molecular weight distribution in Figure I2b appears to be intermediate between the $\Delta P=0$ and $\Delta P$ $=10 \mathrm{~atm}$ cases.

Sensitivity Analysis. This section considers the sensitivity of the FG-DVC model to variations in the DVC parameters. The FG parameter sensitivities have been considered elsewhere. ${ }^{52}$

(a) Variations in $W_{\mathrm{B}}$. The number of labile bridges is the most important parameter in determining tar yield. The value of $W_{B}$ for the Pittsburgh Seam coal was reduced from its value of 9.4 to 7.4 and 5.4. The results in Figure 15 a were calculated for the case considered in Figure $6 \mathrm{~d}$. 

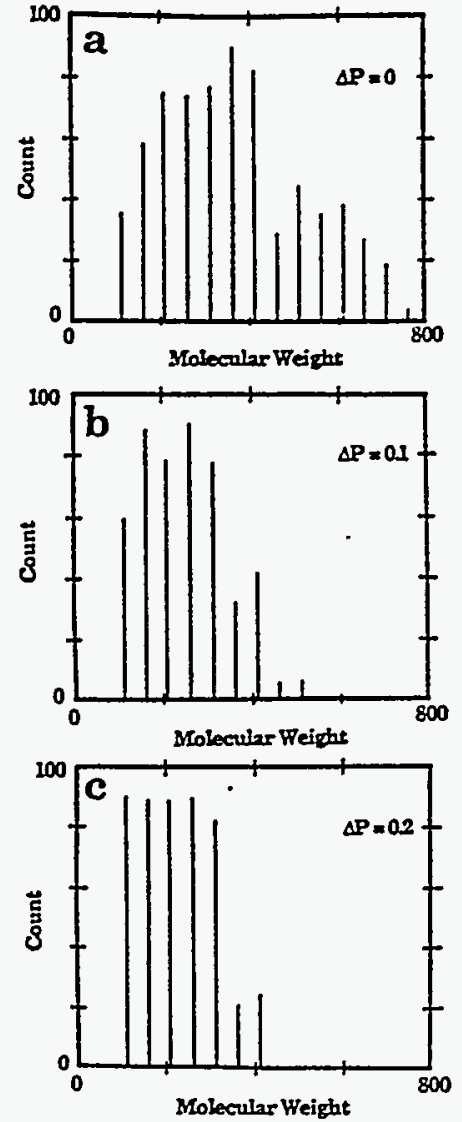

Figure 16. Effect of $\Delta P$ on tar molecular weight distribution for Pittsburgh Seam bituminous coal heated to $723 \mathrm{~K}$ at $0.05 \mathrm{~K} / \mathrm{s}$. $P=0.00267$ atm.

The reduction in $W_{\mathrm{B}}$ reduces the tar yield, the total volatile yield, and the extract yield. Higher values of $W_{B}$ could not be considered because the molecule already contained the maximum number of labile bridges. This is a limitation in the model as it is currently formulated since all the donatable hydrogens are assumed to be in bridges.

(b) Variations in 1 . The parameter $l$ affects mainly the extract yield in the raw coal. Figure $15 \mathrm{~b}$ demonstrates variations in $l$ from 6 to 10 around the base value of 7 . The initial extract yield varies substantially while there is only a minor effect on the tar yield, total volatile yield, and extract yield at elevated temperature.

(c) Variations in $\Delta P$. The effect of variations in $\Delta P$ on the overall yield are considered in Figure 10. There is no effect at 1 atm pressure and above but a strong effect at lower ambient pressures. Figure $15 \mathrm{c}$ confirms that $\Delta P$ has little effect on the tar yield or the total volatile yield for pyrolysis at 1 atm pressure. Only the extract yield is slightly affected.

Figure 16 illustrates the effect on the molecular weight distribution for three values of $\Delta P$ for pyrolysis in vacuum $\left(P_{0}=0\right)$. The yield of higher molecular weight tars present for $\Delta P=0$ is lower for $\Delta P=0.1 \mathrm{~atm}$ and eliminated for $\Delta P=0.2 \mathrm{~atm}$. The total tar yields are $39 \%, 21 \%$, and $17 \%$ for $\Delta P=0,0.1$, and $0.2 \mathrm{~atm}$ respectively. The tar molecular weight distribution for $\Delta \bar{P}=0$ atm gives the best match to Figure $9 \mathrm{c}$, but $\Delta P=0.1-0.2 \mathrm{~atm}$ provides the best match to the yield.

The variation of $\Delta P$ in the tar molecular weight distribution for lignite is discussed with reference to Figure 13.

(d) Variations in $m\left(\mathrm{CO}_{2}\right)$ and $m\left(\mathrm{CH}_{4}\right)$. Variations in $m\left(\mathrm{CO}_{2}\right)$ were considered for the lignite in the discussion accompanying Figure 7. Variations in both $m\left(\mathrm{CO}_{2}\right)$ and
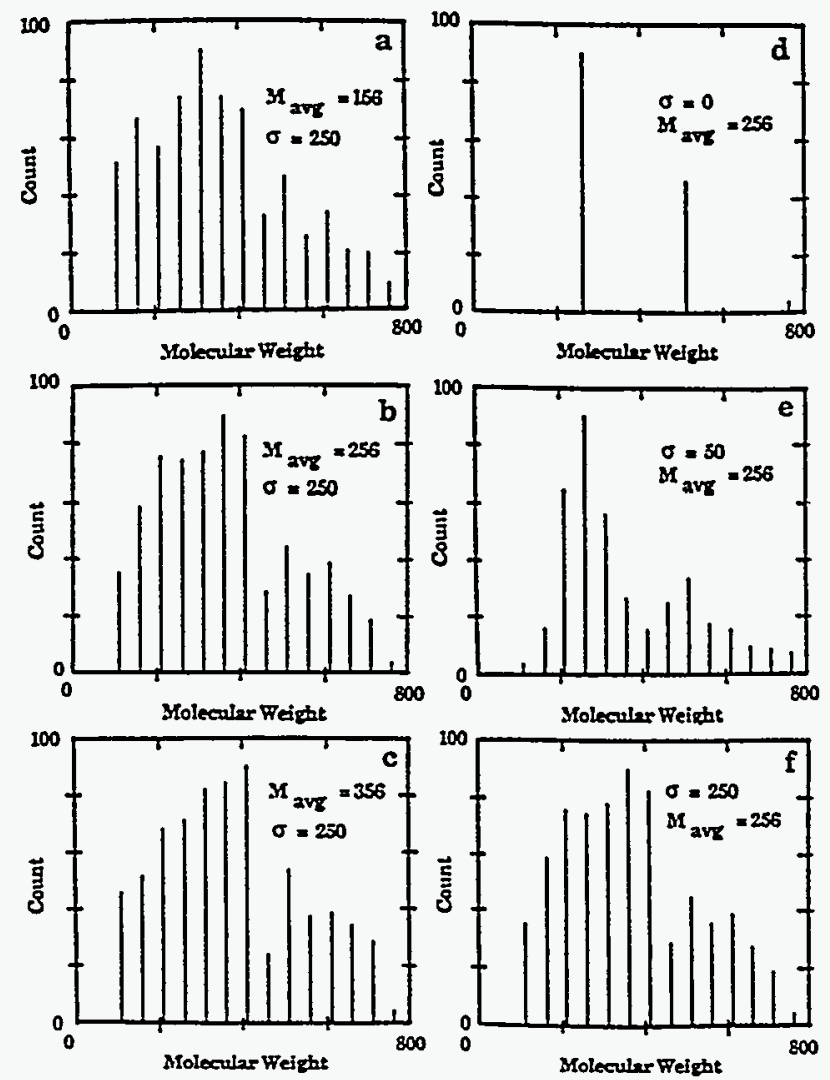

Figure 17. (a-c) Effect of $M_{a v}$ on the shape of the tar spectrum and $(d-f)$ effect of $\sigma$ on the shape of the tar spectrum. The heating rate is $0.05^{\circ} \mathrm{C} / \mathrm{s}$ to $450^{\circ} \mathrm{C}, P=0.00267 \mathrm{~atm}$, and $\triangle P=0 \mathrm{~atm}$.

$m\left(\mathrm{CH}_{4}\right)$ are considered in Figure 15d. These have a major effect on the yields. Increasing $m\left(\mathrm{CO}_{2}\right)$ from 1 to 10 reduces the extract and volatile yields while reducing $m\left(\mathrm{CH}_{4}\right)$ from 1 to 0 prevents the repolymerization of the extract.

(e) Variations in $M_{\mathrm{c}}$ Variations in the $M_{\mathrm{c}}$ values were made. These chiefly affect the extract yield, requiring an adjustment in $l$. They have little effect on the subsequent cross-linking in the coal. The reason for this can be seen in Table I. The initial value of $M_{\mathrm{c}}$ consistent with the literature required only 0.09 and 0.18 cross-link/monomer for the bituminous coal and lignite, respectively. The total number of cross-links added during pyrolysis are 0.49 and 0.89 , respectively. The added number of cross-links is thus much larger than that in the raw coal and, consequently, dominates the char's behavior.

(f) Variations in $M_{\mathrm{av}}$ and $\sigma$. Figure 17a-c illustrates the effects of variations in $M_{a v}$. Varying $M_{\text {av }}$ changes the shape of the tar spectrum, but not drastically. The shape is still dominated by the transport properties (e.g., see Figure 16). The effect on the tar yield is also modest, giving values of $45 \%, 44 \%$, and $42 \%$ for $M_{\mathrm{av}}$ values of 156 , 256 , and 356 , respectively.

A similar lack of sensitivity of the molecular weight distribution to $M_{\mathrm{av}}$ was exhibited for the lignite for both high-heating-rate $\left(\sim 2000{ }^{\circ} \mathrm{C} / \mathrm{s}\right)$ and low-heating-rate $\left(0.05^{\circ} \mathrm{C} / \mathrm{s}\right)$ cases (not shown).

The effect of variations in $\sigma$ is illustrated in Figure 17d-f. $\sigma=250$ fills in the spectrum in a more realistic fashion and is more aesthetically pleasing than the two smaller values of $\sigma$. The effect on the total tar yield is minor with yields of $41 \%, 46 \%$, and $45 \%$ for $\sigma=0,50$, and 250 , respectively.

(g) Variations in $W_{\mathrm{N}}$. This parameter, which is taken from the FG model, controls the split between tar, char, and gas. 
Table IV. Summary of Sensitivity Analysis ${ }^{a}$

\begin{tabular}{|c|c|c|c|c|c|c|c|c|c|}
\hline & $W_{N}$ & $W_{\mathrm{B}}$ &.$l$ & $M_{\mathrm{c}}$ & $m\left(\mathrm{CO}_{2}\right)$ & $m\left(\mathrm{CH}_{4}\right)$ & $M_{\text {av }}$ & $\sigma$ & $\Delta P$ \\
\hline $\begin{array}{l}\text { tar molecular weight } \\
\text { tar yield } \\
\text { char extract yield } \\
\text { coal extract yield } \\
\text { char solvent-swelling ratio } \\
\text { coal solvent-swelling ratio }\end{array}$ & $\begin{array}{l}W \\
\mathbf{S} \\
W \\
W \\
W \\
W\end{array}$ & $\begin{array}{l}W \\
S \\
S \\
W \\
W \\
W\end{array}$ & $\begin{array}{l}W \\
W \\
W \\
S \\
W \\
W\end{array}$ & $\begin{array}{l}\mathbf{W} \\
\mathbf{W} \\
\mathbf{W} \\
\mathbf{M} \\
\mathbf{S} \\
\mathbf{W}\end{array}$ & $\begin{array}{l}\mathbf{S} \\
\mathbf{S} \\
\mathbf{S} \\
\mathbf{W} \\
\mathbf{S} \\
\mathbf{W}\end{array}$ & $\begin{array}{l}\mathbf{W} \\
\mathbf{M} \\
\mathbf{S} \\
\mathbf{W} \\
\mathbf{S} \\
\mathbf{W}\end{array}$ & $\begin{array}{l}M \\
W \\
W \\
W \\
W \\
W\end{array}$ & $\begin{array}{l}M \\
W \\
W \\
W \\
W \\
W\end{array}$ & $\begin{array}{l}\mathbf{S} \\
\mathbf{S} \\
\mathbf{M} \\
\mathbf{W} \\
\mathbf{W} \\
\mathbf{W}\end{array}$ \\
\hline
\end{tabular}

a Key: $W=$ weak or none; $M=$ moderate; $S=$ strong.

(h) Vaporization Law. The resuits are sensitive to the choice of the tar vapor pressure correlation. Higher vapor pressures result in faster tar evolution and higher yields as discussed in reference to Figure 11.

A summary of the sensitivity analysis is presented in Table IV. The concentration of labile bridges $W_{B}$ and the $\mathrm{CO}_{2}$ cross-linking parameter $m\left(\mathrm{CO}_{2}\right)$ are the most important parameters in determining yields.

\section{Conclusions}

A general FG-DVC model for coal devolatilization, which combines a functional group model for gas evolution and a statistical model for tar formation, has been presented. The tar formation model includes depolymerization, cross-linking, external transport and internal transport. The cross-linking is related to the evolutions of $\mathrm{CO}_{2}$ and $\mathrm{CH}_{4}$, with one cross-link formed per molecule evolved. The predictions of the tar formation model are made by using Monte Carlo calculation methods. Predictions take between $10 \mathrm{~s}$ and $10 \mathrm{~min}$ (depending on coal rank, experimental conditions, and accuracy required) on a Sun $3 / 260$ computer.

The FG-DVC model predictions compare favorably with a variety of data for the devolatilization of Pittsburgh Seam coal and North Dakota (Beulah) lignite, including volatile yields, extract yields, cross-link densities, and tar molecular weight distributions. The variations with pressure, devolatilization temperature, rank, and heating rate were accurately predicted. Comparison of the model with several sets of data employing alternative assumptions on transport suggests assuming that the particle is well mixed (i.e. the surface concentration of tar molecules is the same as the bulk) overpredicts the transport rate. For 50- $\mu \mathrm{m}$ particles, assuming that the internal-transport limitation dominates (i.e. neglecting the external transport) provides a good fit to the data. This is consistent with assuming (a) that the internal- and external-transport mechanisms act in series or (b) that they act in parallel but liquid-phase diffusion of tar molecules to the surface is very small and so the external transport term can be neglected.

The rank dependence of tar formation, extract yields, cross-linking, and viscosity appears to be explained by the rank dependence of $\mathrm{CO}_{2}$ yields. The high $\mathrm{CO}_{2}$ yields in low-ranle coals produce rapid cross-linking at low temperatures and hence low tar yields, low extract yields, loss of solvent-swelling properties, and high viscosities. The relative importance of cross-linking compared to bond breaking is, however, sensitive to heating rate, and this effect is predicted by the FG-DVC model. The predicted cross-linking associated with methane evolution appears to match the observed cross-linking in high-rank coals (which evolve little $\mathrm{CO}_{2}$ ).

The model has eight coal structure parameters that must be determined for each coal from selected laboratory experiments. Once determined, these remain fixed for all experiments. The model also contains one adjustable parameter, $\Delta P$, the internal pressure difference that drives the volatiles out of the particle. A sensitivity analysis shows that the volatile yield is most sensitive to the fraction of labile bridges, $W_{\mathrm{B}}$, the cross-linking parameters $m\left(\mathrm{CO}_{2}\right)$ and $m\left(\mathrm{CH}_{4}\right)$, and, in some cases (low-rank coals, low pressure), to $\Delta P$. The monomer molecular weight distribution parameters, $M_{\mathrm{av}}$ and $\sigma$, have only a weak effect on yields and tar molecular weight distributions. The initial molecular weight between cross-links, $M_{c}$, and the initial oligomer length, $l$, affect the coal's solvent-swelling ratio and extract yield but have little effect on the subsequent pyrolysis behavior.

The model currently has several deficiencies. There is no model for estimating liquid-phase diffusion of tar molecules, which may be important for very small particles. The calculation of the average molecular weight between cross-links neglects the effect of labile bridge rupture. The assumption that all the donatable hydrogen is in bridges may be restrictive for some high hydrogen coals. The model presented here has neglected polymethylene in coal and the effect of other types of weak bonds besides ethylene bridges. There are some discrepancies between the predictions and reported temperatures of pyrolysis experiments. It is unclear at this time whether this is due to errors in the reported temperatures or in the transport predictions. Many of these deficiencies require only minor modifications to the model and are currently being addressed.

Acknowledgment. This work was supported under DOE Contracts DE-AC21-85MC22050, DE-AC2184MC21004, DE-AC21-86MC23075, and DE-FG2285PC80910. We express our thanks to Professor Eric Suuberg for many helpful discussions on transport properties and to Dr. Zhen Zhong Yu for assistance with the model calculations. 
Appendix $K$

Can Coal Science Be
Predictive 
Can Coal Science Be Predictive

3 


\section{CAN COAL SCIENCE BE PREDICTIVE}

Peter R. Solomon, David G. Hamblen, Michael A. Serio, Zhen-Zhong Yu, and Sylvie Charpenay Advanced Fuel Research Inc., 87 Church Street, East Hartford, CT 06108

KEY WORDS: Coal, Pyrolysis, Network

\section{ABSTRACT}

This paper considers the development of a predictive macromolecular network decomposition model for coal conversion which is based on experimental results from a variety of modern analytical techniques. Six concepts which are the foundation of the Functional Group, Depolymerization, Vaporization, Crosslinking (FG-DVC) model are considered: 1) The decomposition of functional group sources in the coal yield the light gas species in thermal decomposition. The amount and evolution kinetics can be measured by TG-FTIR, the functional group changes by FT-IR and NMR. 2) The decomposition of a macromolecular network yields tar and metaplast. The amount and kinetics of the tar evolution can be measured by TG-FTIR and the molecular weight by FIMS. The kinetics of metaplast formation and destruction can be measured by solvent extraction, by Geissler plastometer and by proton magnetic resonance thermal analysis (PMRTA). 3) The molecular weight distribution of the metaplast depends on the network coordination number (average number of attachments on aromatic ring clusters). The coordination number can be determined by solvent swelling and NMR. 4) The network decomposition is controlled by bridge breaking. The number of bridges broken is limited by the available donatable hydrogen. 5) The network solidification is controlled by crosslinking. The changing crosslink density can be measured by solvent swelling and NMR. Crosslinking appears to occur with evolution of both $\mathrm{CO}_{2}$ (prior to bridge breaking) and $\mathrm{CH}_{4}$ after bridge breaking. Thus, low rank coals (which form a lot of $\mathrm{CO}_{2}$ ) crosslink prior to bridge breaking and are thus thermosetting. High volatile bituminous coals (which form little $\mathrm{CO}_{2}$ ) undergo significant bridge breaking prior to crosslinking and become highly fluid. Weathering, which increases the $\mathrm{CO}_{2}$ yield, causes increased crosslinking and lowers fluidity. 6) The evolution of tar is controlled by mass transport in which the tar molecules evaporate into the light gas species and are carried out of the coal at rates proportion to their vapor pressure and the volume of light gases. High pressures reduce the volume of light gases and hence reduces the yield of heavy molecules with low vapor pressures. These changes can be studied with FIMS.

The paper describes how the coal kinetic and composition parameters are obtained by TG-FTIR, solvent swelling, solvent extraction, and Geissler plastometer data. The model is compared to a variety of experimental data in which heating rate, temperature, and pressure are all varied. There is good agreement with theory for most of the data available from our laboratory and in the literature.

\section{INTRODUCTION}

The question addressed by this paper is, can coal science be predictive? More specifically, is it possible, to accurately predict the way a coal behaves in a coal conversion process, given coal characteristics which can be measured in the laboratory. For example, Fig. 1 illustrates the behavior of coal in combustion. The left hand side of the figure shows a picture of a coal burning in a reactor where the coal is injected into the center of a hot air stream. The processes that occur are illustrated on the right hand side. Starting from the bottom, the figure represents the heating of the coal, coal softening, devolatilization, swelling, the ignition of the volatiles, the formation of soot, the burning of the volatiles, the ignition of the char, the combustion of the char, and finally the fragmentation of the char which determine the ultimate distribution of the ash particles. Can one qualitatively predict pyrolysis yields, swelling, soot formation, char reactivity, etc.?

As a second example, consider coal in a liquefaction process. The important step is the fragmentation of the coal macromolecule into small pieces. As shown in Fig. 2 , that fragmentation takes place very quickly for a bituminous coal. The coal dissolves into the solvent, and the subsequent reactions between the solvent and the coal are all liquid-liquid phase interactions, which can occur very rapidly. In a lignite, this fragmentation process is prevented by low temperature crosslinking. The result is that 
there is no quick solubilization of the coal, and most of the reaction takes place between the solvent and a solid crosslinked residue. Can one predict macromolecular fragmentation and crosslinking?

The research conducted during the last ten years suggests that many of the steps discussed above can be accurately predicted. Figure 3 shows the concept employed in our laboratory for developing predictive capabilities. We start with a set of laboratory characterization procedures that allow the appropriate kinetic and composition parameters for coal to be determined. Five kinds of experiments allow us to define the parameters for our model. The most important is the TG-FTIR, a thermogravimetric (TG) analyzer with the analysis of the evolved product by Fourier Transform Infrared (FT-IR) spectroscopy (1). This instrument allows us to determine the amount of the volatiles, their composition, the kinetics for their evolution, the reactivity of the char, and also the moisture and ash content of the coal. We also measure the solvent swelling ratio $(2,3)$, the extract yield, and the fluidity in a Geissler plastometer (4), and employ nuclear magnetic resonance (NMR) (5), and Field lonization Mass spectrometry (FIMS) data (6). These experiments determine the macromolecular network parameters for the model.

The model is the FG-DVC model 7,8$)$. The letters FG stand for Functional Group, and DVC for Depolymerization, Vaporization and Crosslinking. The FG model considers certain functional groups in the coal which decompose to form the light gas species (9-12). At the same time, the DVC.model describes the overall depolymerization of the macromolecular network which combines bridge breaking and crosslinking to produce fragments of the coal macromolecular (13-15). These fragments are then subjected to transport behavior, specifically the vaporization of the lightest fragments to form tar. The fragmentation process provides a second mechanism for the removal of functional groups from the coal. The model, whose parameters are determined in the laboratory at moderate temperatures and one atmosphere, can then be used to extrapolate away from the laboratory conditions to predict pyrolysis and combustion in high temperature reactions, or liquefaction at high pressure. Recently, we have explored extrapolation of the kinetics and reactions to low temperature geological transformations in coal beds (16).

The model for coal thermal decomposition has six basic concepts:

- Functional Groups (decompose to produce light gases)
Macromolecular Network (decomposes to produce tar and metaplast)
Network Coordination Number (determines fragment molecular weights)
Bridge Breaking (limited by hydrogen availability)
- Crosslinking (related to gas evolution)
Mass Transport of Tar (evaporation of light network fragments into light gases)

The first concept is that light gases are formed by the decomposition of certain functional groups in the coal. For example, methyl groups can decompose to form methane, carboxyl groups can decompose to form $\mathrm{CO}_{2}$, etc. $(9-12,17-20)$. The second concept is that coal consists of a macromolecular network $(2,3,7,13-15,21-36)$. This network is made up of fused aromatic ring clusters (which are described by their molecular weight) linked by bridges, some of which are relatively weak. There are some unattached parts of the network which can be extracted. Sometimes, there is also a second polymethylene component (37-41). When heated, this network decomposes to produce smaller fragments. The lightest of the fragments evaporate to produce tar $(7,42)$, and the heavier fragments form the metaplast. These heavier molecules are the primary liquid fragments in liquefaction or the fragments that make coal fluid $(8,43)$.

The third concept is that one of the most important properties of the network is its coordination number. The coordination number describes the geometry of the network by specifying how many possible attachments there are per aromatic ring cluster (node) (31-36). For example, a linear polymer chain has a coordination number of 2 , because each fused aromatic ring has two possible attachments to link it in the chain. On the other hand, a "fish net" has a coordination number of 4 , because there are four possible attachments at each node. The coordination number controls the molecular weight distribution of the network fragments at a given extent of decomposition. The extent of decomposition is specified by the probability that the possible attachments are made. For example, for $20 \%$ of broken 
bridges, a linear chain is totally fragmented, while a "fish net" will have some holes but is almost totally connected. In describing the network, a crosslink is defined to occur at a node where there are more than two attachments. The coordination number is thus, related to the crosslink density. With no possible crosslinks, the coordination number is two. With increasing crosslink density the coordination number increases.

The second important property of the network is the fraction of possible attachments which are actually made. During thermal decomposition, this fraction is determined by the rates of bond breaking and crosslinking $(7,15,44-47)$. The factors which control how many of the weak links can break are the rate constant and the amount of hydrogen that can be donated from the coal to stabilize the free radicals which form when the links break (10).

A competitive process with the bond breaking is the retrogressive process of crosslinking. Crosslinking reactions appear to be related to the evolution of certain gases $(7,15,44,47)$. Specifically, for low rank coals, crosslinking at low temperature (prior to bridge breaking) seems to be related to the evolution of carbon dioxide (or possibly water). For coals of all rank, a higher temperature crosslinking event (following bridge breaking) seems to be related to the evolution of methane. At high temperatures, the evolution of hydrogen is also related to crosslinking.

The final concept is that the tar evolution is controlled by mass transport. Bridge breaking and crosslinking produce fragments with a molecular weight distribution. The lightest fragments can leave the coal melt by evaporation into the light gas species $(7,42)$. The heavier fragments remain, forming the metaplast which controls the coal's fluidity.

The remainder of the paper describes how these concepts are incorporated into a practical predictive model. Section II considers the FG-DVC model in detail. It discusses each of the six concepts and the evidence for each assumption. Section III considers the experiments employed to obtain the model parameters, and Section IV compares predictions of the model with a variety of experimental data. Section $V$ is the summary.

COAL PYROLYSIS MODEL

\section{Functional Group Decomposition Model}

Figure 4 illustrates the phenomena in coal thermal decomposition considered in the functional group model. The figure is not meant to describe the exact structure of coal or the exact chemistry which occurs in pyrolysis. It is meant to illustrate the kinds of structures that are being considered and the classes of phenomena that can occur. The important processes are the decomposition of the individual functional group to form the light gases and the competitive decomposition of the macromolecular network to form fragments, the lightest of which can evaporate as tar.

Figure 4a shows a representative piece of a Pittsburgh Seam coal macromolecule. The structure is based on measurements of the aromatic ring cluster size, the functional group composition and the elemental composition (48). The molecule consists of several fused aromatic ring clusters linked by labile bridges. The ring clusters have various functional groups attached to them. When the coal is heated, two things happen to the functional groups. The first is that certain functional groups can decompose to form light gases. The second is that fragmentation of the network, and removal of light fragments as tar, can cause the same type of functional group to be removed as part of the tar. So, there are two parallel processes for the volatilization of the functional groups.

The way the coal behaves during pyrolysis is illustrated in Fig. 4b. The carbon-carbon aliphatic bridge in the upper left hand corner of the molecule (labeled 2) broke and picked up hydrogen to form two methyl groups. This process creates a fragment which is light enough to evolve as tar. There is also independent decomposition of functional groups to form light gases. The carboxyl group that was shown in the middle of Fig. $4 a$ is shown as a carbon dioxide evolving in Fig. 4b. Methyl groups have decomposed to form methane, there has been a condensation of hydroxyl groups to form water and an ether link (labeled 3 ), mercaptans decompose to form $\mathrm{H}_{2} \mathrm{~S}$, etc. 
The evidence for this description is as follows: 1) for bituminous coals and low rank coals heated rapidly, the tar is strikingly similar in elemental and functional group composition to the parent coal $(9,10,48-50)$. The tar appears to consist of representative fragments of the parent coal macromolecule; 2) there is a correlation between the decrease in the functional group sources in the char and the evolution of specific gases $(9-12) ; 3)$ there is a systematic variation in functional group composition with rank, and this variation is correlated with the evolved gas composition.

While there is good evidence for the above description, the details of the chemistry are not yet well understood. Also, tar produced from low rank coals at low heating rates appears to be significantly different in composition from the coal (51) and is probably dominated by polymethylenes.

\section{Macromolecular Network Decomposition Model}

The concept of a macromolecular network decomposition model, is illustrated in Fig. 5, which recently appeared in a paper by Grant and coworkers (36). The figure represents aromatic ring clusters with four possible attachments to their neighbors, arranged in a "fish net" type network, (a network with a coordination number of 4). Figure $5 a$ illustrates what happens when $20 \%$ of the possible attachments are broken. As can be seen, there are only three fragments which are created, shown by the clusters with boxes around them. The breaking of $20 \%$ of the bridges produces very little fragmentation of the network. On the other hand, consider in Fig. $5 \mathrm{~b}$ what happens when $45 \%$ of the bridges are broken. Now, there is a much higher concentration of fragments and the fragments have a molecular weight distribution from monomers up to 7 -mers (consisting of 7 fused ring clusters linked together). The lightest of these fragments, monomers, dimers, and trimers can evaporate into the light gas species and are removed from the coal particles as tar. The heavier fragments make up the metaplast. The lightest of these can be extracted using solvent, while others are too heavy to be extracted. The presence of a sufficiently large fraction of these fragments are what makes these materials fluid.

\section{Network Coordination Number}

The importance of the network coordination number is illustrated in Figs. 5 and 6 . In Fig. 5 a with $20 \%$ of the bridges broken in a "fish net", only a small number of fragments are produced, and they are all monomers. On the other hand, if $20 \%$ of the bridges in the linear chain are broken, $100 \%$ of the material becomes fragments and there will be many dimers, trimers, etc. Thus, the molecular weight distribution of the fragments depends very strongly on the coordination number.

In Fig. 5, the molecular distribution was computed using Monte Carlo calculations in which a representative network is set up in computer memory and the fragment molecular weight distribution is calculated after the broken bridges are randomly distributed. Alternatively, a technique called percolation theory allows a closed form analytical solution of the molecular weight distribution as a function of the number of actual attachments per ring cluster.

Figure 6 shows percolation theory calculations for networks with two different coordination numbers: Fig. $6 a$ is for a coordination number of 2.2 and Fig. $6 \mathrm{~b}$ is for 4.6. The variable $\sigma$ is one less than the coordination number. The figure shows the calculated distributions of: i) monomers, ii) up to trimers (i.e., monomer, dimer, trimer) representative of what might be evolved as tar for a ring cluster size of 300 Daltons, iii) the yields of all n-mer up to ten representative of extractable material, and 4) the yield of all $n$-mers. These are plotted as a function of $\alpha$, which is the average number of bridges per fused aromatic rings. This term $\alpha$ is equal to the probability, $p$, that a bridge is occupied times the coordination number of the network divided by two, $\alpha=p(\sigma+1) / 2$. As can be seen, there is a very different distribution of fragments depending on the coordination number. For example, at a value of $\alpha=0.9$, the network with coordination number 4.6 has most of the fragments in the tar, with only a small number of $n$-mers between 3 and 10 and almost no n-mers above 10 . On the other hand, for a network with a coordination number of 2.2 at $\alpha=0.9$, there is a smaller number of monomers, a somewhat smaller concentration of tar, but a much higher concentration of n-mers up to 10 and a $100 \%$ yield of all $n$-mers. In other words, for 0.9 bridges per cluster, most of the molecules had decomposed to produce fragments of one size or another. 
The DVC model was originally implemented using a Monte Carlo solution method, which allows an arbitrary network geometry. Percolation theory, however, offers significant benefits in computational speed and reproducibility, at the cost of restricting the network geometries.

As we shall see below, in the Monte Carlo version of the model, the starting network is represented by linear chains of monomers (6-12 aromatic clusters) with some amount of crosslinking which tie the chains together. Thus the starting network has a coordination number between 2 (straight chains) and three or more (fully cross linked). As pyrolysis proceeds, the linear chain bonds (bridges) are broken, and crosslinks (the side bonds) are formed. Thus, the coordination number, or degree of branching, increases with extent of pyrolysis. The conventional percolation theory models of coal decomposition do not model this feature. With conventional percolation theory, one can make any identification of the various chemical bonds with the percolation lattice bonds, so that the probability of a bond being occupied tracks the chemistry; but the occupied bonds must be randomly distributed within the lattice. The structure cannot be converted from "chain-like" to "fishnet-like".

The DVC model predicts, and experiments confirm, that there are more than one kind of bond (bridges and crosslinks) which have different coordination numbers, and independent probabilities of being broken. To take advantage of the benefits of percolation theory, we have extended percolation theory on a Bethe lattice (one with no loops) to use two independent sub-networks, as illustrated in Figure 7 (32). In the Figure, double lines represent one of the bond types, while single lines represent the other. As can be seen by comparing Fig $7 a$ and $7 b$, this lattice has the desired feature of modeling a transition from chain-like structures (a) to fishnet structures (b). The mathematics of this 2-bond percolation theory follows closely that of the standard theory (32). A comparison of the results obtained from the 2-bond percolation theory agree well with those obtained from the original Monte Carlo calculations, as will be discussed in the Results Section.

\section{Bridge Breaking and Hydrogen Utilization}

There are two questions with respect to bridge breaking: what is the bridge breaking rate and how many bridges break. Pyrolysis rates have been reviewed by a number of authors (10,52-54). One of the problems in pyrolysis over the last two decades is a very wide variation in the reported rates for either weight loss or tar evolution in pyrolysis.

Figure 8 a presents several of the extremes in rates reported prior to 1985 for high heating rate experiments (10,55-58). At $800^{\circ} \mathrm{C}$ there is almost a four order of magnitude variation in the rate constant which has been reported. An analysis of the data shows that one can not ascribe this sort of variation in kinetics to variations in the coal type, because investigators who measured more than one coal type found that the variations in kinetics rates among coals are typically within a factor of 10. So there has to be another explanation for why there is such a wide variety of reported rates. The answer appears to be the knowledge of the coal particle temperatures $(53,54,59-62)$. Almost none of the experiments are done with direct measurements of the coal particle temperature. For entrained flow reactor experiments, the temperature is usually calculated and the calculations depend critically on the rate of mixing of the preheated gases with the coal stream. A factor of two error in the heating rate can lead to errors of hundreds of degrees celsius in the particle temperature during pyrolysis. For heated grid experiments, temperature measurements are made with a thermocouple and the inference is made that the thermocouple temperature is the same as the coal particle temperature. Recent reviews of experiments for Pittsburgh Seam coal heated at $1000^{\circ} \mathrm{C} / \mathrm{sec}$ show a wide variation in pyrolysis temperatures, suggesting that this is just notla good assumption $(60,63)$.

Since 1985, several experiments have been performed in which coal particle temperatures were measured during pyrolysis $(12,53,61,62,64)$. Careful experiments have also been performed at several low heating rates where the thermocouple temperature is a good measure of the coal particle temperature $(65,66)$. As can be seen from Fig. $8 b$, the data are much more tightly grouped. There is a systematic variation with the rank of the coal and the kinetic rate constants appear to have an activation energy between 45 and $55 \mathrm{Kcal}$. This is the magnitude one would expect for the kind of labile bridges depicted in Fig. $4(14,67)$. 
Besides the kinetic rate for bridge breaking, one needs to know the number of bridges that can be broken. The number depends upon the amount of hydrogen that is available to stabilize the free radicals formed when bridges break. How the hydrogen utilization controls the amount of tar and its hydrogen concentration is illustrated in Fig. 9. The figure is based on the following consideration: every time a bridge is broken, the available hydrogen is used to stabilize the free radicals. Two radicals are assumed to be stabilized per tar molecule. If the tar is made up of large fragments, the utilization s of hydrogen per unit weight of tar is very efficient. On the other hand, if the tar consists of small molecules, the utilization of hydrogen is much less efficient. The figure shows the results for $0.3 \%$ hydrogen in the coal available for donation to the tar. The figure presents the yield of tar and the percent of additional hydrogen in the tar as a function of the average molecular weight in the tar. For an average molecular weight of 100 Daltons, $15 \%$ tar is produced. The amount of additional hydrogen in the tar is $2 \%$. On the other hand, at an average molecular weight of 300 Daltons the yield is up to $45 \%$, while the weight percent of additional hydrogen per unit mass is only $0.7 \%$. The average molecular weight of the tar is affected by crosslinking, pressure, heating rate, and bed geometry.

\section{Crosslinking}

During pyrolysis, another important process occurs besides bridge breaking. It is the process of crosslinking, where new bonds are formed between the fused aromatic ring clusters. One of the ways of measuring the crosslink density is through solvent swelling $(2,3)$, in which a solvent (e.g., pyridine) is used to swell the char or coal $(7,15,44-47)$. To understand how solvent swelling indicates the crosslink density, consider the analogy of an air mattress. An air mattress is stitched in long rows along the length of the mattress. When the mattress is inflated there are several connected small tubes, instead of one big round tube. The small tubes have a smaller volume than one large tube and the volume can be used to infer the limiting circumference of the tubes. In a similar manner, the addition of the solvent to a coal indicates the circumference of linked molecules that make a loop to limit the swelling. Since it is crosslinks (more than two attachments per cluster) that allow loops to be formed, the amount of swelling indicates the average molecular weight between crosslinks.

Figure 10 shows the behavior of the solvent swelling ratio as a function of the char temperature for coals of several ranks. Chars are produced by heating up to the indicated temperatures at $30^{\circ} \mathrm{C} / \mathrm{min}$ in an inert atmosphere and then cooling. These chars are subjected to solvent swelling experiments which determines the volume of swollen coal divided by the unswollen volume. Coals have solvent swelling ratios as high as 2.7. As char is formed, new crosslinks reduce the swelling ratio to unity. The solvent swelling ratio in Fig. 10 is normalized. The parameter $x$ is the difference in solvent swelling ratio between the coal and the char divided by the maximum differences that can be achieved. This normalization allows us to compare different coals with different starting solvent swelling ratios in a convenient manner. Figure 10 presents 1 - $x$ as a function of the char temperature. If $x=0$, we have a material that swells the same as coal and if $x=1$ we have a fully crosslinked char.

There is a wide variation in behavior depending upon rank. This rank dependence of the crosslinking behavior was first noted by Suuberg and coworkers (44) who measured a lignite and a bituminous coal and found the same sort of difference that has been exhibited here. The lowest rank coal, Zap lignite, is shown by the open squares. At temperatures as low as $200^{\circ} \mathrm{C}$, the char starts to undergo crosslinking, loosing most of its solvent swelling properties by a temperature of $400^{\circ} \mathrm{C}$. It is between : 400 and $500^{\circ} \mathrm{C}$ that most of the pyrolysis weight loss is occurring for this material. Thus, for a low rank coal, the crosslinking occurs well in advance of the bridge breaking.

For higher rank coals, the crosslinking event is delayed relative to bridge breaking. For a highly sottening bituminous coal like Pittsburgh Seam coal, or Kentucky No. 9, we find the material swells even more as it is heated into the region of pyrolysis, and only looses its solvent swelling properties after most of the weight loss has occurred in pyrolysis. There is, thus, a very strong rank dependence of the crosslinking behavior. Low rank coals crosslink early, prior to bridge breaking, while high rank coals undergo crosslinking after most of the bridge breaking has taken place.

The results of solvent swelling experiments are not unambiguous because the solvent swelling ratio depends on two things: 1) the crosslink density and 2) the solvent interaction parameter. This 
parameter can change with the functional group composition of the coal. Since the functional group composition will change as the coal pyrolyzes, the change in solvent swelling ratio could be due to the change in the functional group composition, not crosslinking. However, an analysis of how much the solvent swelling ratio may change with the functional group composition indicates that the kind of drastic change from a solvent swelling ratio of 2.7, typical for coal, down to 1 for char is not likely to occur for the small changes in the functional group composition with low temperature pyrolysis (47).

Another way of investigating the crosslink density is by experiments done using nuclear magnetic resonance (NMR). The work was performed at the University of Utah in collaboration with Solum and coworkers (68). Results of the NMR experiments are shown in Fig. 11. The NMR experiments employ cross polarization with magic angle spinning and dipolar dephasing (5). Dipolar dephasing allows the determination of the functional group form of the carbons that are being studied. When all the different kinds of bonds are considered, it is possible to determine an average molecular weight for the ring clusters and also the average number of attachments per ring cluster. In Fig. 11, the average number of attachments are compared to the solvent swelling data for Pittsburgh Seam bituminous coal and a North Dakota lignite. The average number of attachments determined by the NMR are also normalized to determine an NMR index which can be compared to $1-x$. Figure 11 compares the results for the lignite. The change in crosslink density determined by NMR is in reasonable agreement with that determined by the solvent swelling ratio. As can be seen, the material starts to crosslink at a reasonably low temperature and is almost completely crosslinked by a temperature of about $700 \mathrm{~K}$ $\left(427^{\circ} \mathrm{C}\right)$, prior to significant bond breaking. For the Pittsburgh Seam coal shown in Fig. $11 \mathrm{~b}$ the NMR index is in reasonable agreement with the solvent swelling ratio, and in this case both indices show that the char has not undergone appreciable crosslinking by a temperature of 700 .

To develop an understanding of the chemistry of crosslinking, we attempted to determine whether the addition of crosslinks could be correlated with any other observation, specifically, the evolution of gases. In the initial work of Suuberg and coworkers (44), they noted that the one gas species which correlated with the early creation of crosslinking in the lignite was carbon dioxide.

Figure 12 shows the results obtained in our laboratory. Figure $12 a$ presents the parameter $x$ as a function of the carbon dioxide yield divided by 44 so that it is on a molecular basis. In Fig. 12 we have plotted $x$ rather than $1-x$ which was plotted in Figs. 10 and 11 . For a wide variety of experiments (some at high heating rates and some at low heating rates) there is an very reasonable correlation between the loss of swelling and the appearance of carbon dioxide in pyrolysis. For all the low rank coals studied, there appears to be a good correlation between the appearance of crosslinks and the appearance of carbon dioxide. The line shown in Fig. 12a is from our FG-DVC model, where one crosslink is assumed for each carbon dioxide evolved.

For a higher rank coal, which does not produce significant yields of $\mathrm{CO}_{2}$, a different correlation is observed. Figure $12 \mathrm{~b}$ compares the normalized solvent swelling ratio for a Pittsburgh Seam coal with the evolution of methane divided by 16. There is a good correlation between these two parameters for chars created at a number of different temperatures at high heating rates. The line in Fig. $12 \mathrm{~b}$ is from the FG-DVC model, where it is assumed that one crosslink is formed for each methane evolved.

We examined the correlation of the loss of swelling with other parameters and found the correlation between carbon dioxide and methane to be the best. There is a correlation for low rank coals between the formation of crosslinks and the water evolution, but not quite as good as for $\mathrm{CO}_{2}$. There was no good correlation for high rank coals between crosslinking and tar evolution.

Three experiments which exhibit the phenomena of bridge breaking and crosslinking are presented in Fig. 13. Figure 13 a presents the proton magnetic resonance thermal analysis (PMRTA) experiment done at CSIRO by Lynch, Sakurovs and coworkers (69-71). This experiment, which measures the relaxation time for protons, can distinguish between protons attached to mobile molecules (which are free to rotate) and those attached to a ridged lattice. The higher the concentration of mobile protons, the lower the values of the parameter $M_{2 T^{*}}$. The data taken at $4^{\circ} \mathrm{C} / \mathrm{sec}$ was provided by Dr. Sakurovs (72). The decrease in $M_{2 T}$ at low temperatures appears to be associated with melting, the sharp drop 
in $M_{2 T}$ above $400^{\circ} \mathrm{C}$ is due to bridge breaking and the sharp increase of $M_{2 T}$ above $440^{\circ} \mathrm{C}$ is due to the crosslinking.

Figure 13b shows fluidity data measured with a Geissler plastometer for the same coal at a similar heating rate $\left(3^{\circ} \mathrm{C} / \mathrm{sec}\right)(4)$. While the fluidity below $400^{\circ} \mathrm{C}$ is probably due to melting, above $400^{\circ} \mathrm{C}$ bond breaking becomes important, while above $440^{\circ} \mathrm{C}$ crosslinking resolidifies the network.

Figure $13 \mathrm{c}$ presents data of Fong (73) on the extract yield in chars produced at a high heating rate of $640^{\circ} \mathrm{C} / \mathrm{sec}$. The maximum extract yield occurs at a much higher temperature than for the other two experiments due to the high heating rate. The increase in extract yield is due to bridge breaking and the decrease to crosslinking.

\section{Transport}

The above discussion shows how bridge breaking and crosslinking, can fragment the macromolecular network and allow small pieces to be formed. The evolution of tar is controlled by the formation of these small fragments and their transport out of the metaplast. In our FG-DVC model, we've assumed a very simple transport process. The assumption is that the fragments reach their equilibrium vapor pressure in the light gas species and are removed from the metaplast by convective transport in the light gas species (7). In a highly fluid coal, the expulsion of the light gases occurs by bubble transport. In a low rank thermosetting coal the transport of the light gas species is through the pores. In either case, the degree to which the tar molecules are transported depends upon the volume of light species that evolve and the vapor pressure of the molecule. The low macromolecular weight species that have high vapor pressure are therefore easily transported while heavy molecular weight species are not. The result is that the tar for a bituminous coal pyrolyzed at 1 atmosphere or below consists of molecules up to about 800 Daltons. As the pressure is increased the volume of the light gases is reduced and those marginal heavy products, which were previously transported at one atmosphere, can no longer be transported. Thus, as pressure is increased, the average molecular weight of the tar is reduced. The amount of tar is also reduced because of the reduced efficiency of hydrogen utilization.

For low rank coals, low temperature crosslinking increases the effective coordination number of the network and only small molecules are produced. The yields are low and pressure has little influence on the yield or molecular weight distribution.

\section{Summary of FG-DVC Model}

Figure 14 summarizes the FG-DVC model. In Fig. 14a we start with an assumed macromolecular network. In the Monte Carlo version of the model, each piece of this network is actually described in the computer memory. The description of the network contains the molecular weight of the aromatic ring clusters (shown as the number in the circles), and the crosslinking density (shown by the vertical double line). The potential number of labile bridges (related to the donatable hydrogen) are indicated by the single horizontal lines. The starting molecule is.constructed from linear chains of a certain length (typically between 6 and 12 aromatic ring clusters) connected by the appropriate number of crosslinks. When this is done a certain number of the chains may be unattached to the rest of the macromolecular network. These are the guest molecules whose molecular weight is less than 3000 Daltons and would be pyridine soluble. The length of the chains is adjusted to obtain the proper pyridine solubles. The number of crosslinks is picked to get a coordination number which yields the right ratio of tar to heavier fragments (e.g., extracts) in the metaplast. The number of labile bridges (amount of donatable hydrogen) is picked to get the proper tar yield in the TG-FTIR experiments.

Figure $14 \mathrm{~b}$ considers what happens during pyrolysis. As the temperature increases, some of the weak bridges (which are the single horizontal lines), can break according to the bridge breaking rate. The hydrogen limitation is accomplished by requiring that for each bridge that is broken, another one of the labile bridges becomes an unbreakable bridge as its hydrogen is used to stabilize the free radicals caused by the broken bridges. Thus, for each broken bridge, two of the labile bridges are consumed. The broken bridges and new unbreakable bridges are distributed randomly. 
In the model, the crosslinking is assumed to correlate with the $\mathrm{CO}_{2}$ and methane evolution. The evolution of these species is determined from the functional group part of the model and one crosslink is inserted randomly for each carbon dioxide and each methane group which is evolved. If bridge breaking dominates over crosslinking, the macromolecular network is broken up into smaller fragments. On the right hand side of the figure, the molecular weight distribution which results from the bridge breaking and crosslinking events is shown. Molecules below 3000 Daltons are increased, the lightest molecules escape as tar, and the rest of the network is described as pyridine insoluble.

Figure $14 \mathrm{c}$ shows the network at the conclusion of the pyrolysis process. When all the labile bridges are consumed, the decomposition of the network is complete. All of the network is completely connected by unbreakable bridges, and is highly crosslinking. All the previously loose fragments have been incorporated into the network by crosslinking or have escaped as tar.

\section{ANALYSIS OF COMPOSITION AND KINETIC PARAMETERS}

In this section, the laboratory characterization to determine the model parameters is considered. An analysis by TG-FTIR is employed to determine kinetic rates and functional group compositions. Solvent swelling, solvent extraction, and fluidity measurements in a Geissler plastometer are employed to obtain information on the molecular weight distribution of the metaplast and hence determine the network parameters. NMR and FIMS are used to determine the molecular weight of the ring clusters. These measurements are considered below.

\section{TG-FTIR}

Apparatus - As indicated in Fig. 3, TG-FTIR analysis of coal is employed to obtain the composition and kinetic parameter for the model. A schematic of the instrument is presented in Fig. 15 . Its components are as follows: a DuPont ${ }^{\mathrm{n}} 951$ TGA; a hardware interface (including a furnace power supply); an Infrared Analysis 16 pass gas cell with transfer optics; a MICHELSON MB Series FT-IR; (Resolution: $4 \mathrm{~cm}^{-1}$, Detector: $\mathrm{MCT}$ ); and a PC-AT compatible computer. The cell is connected without restrictions to the sample area and a helium sweep gas is employed to bring evolved products from the TGA directly into the gas cell. This instrument is now available as the TG/plus from Bomem, inc.

The most difficult volatiles to analyze are the tars which condense at room temperature. In the $T G / p l u s$, the rapid cooling from the high thermal conductivity helium sweep gas causes these products to form an aerosol which is fine enough to follow the gas through the analysis cell. The aerosol is also fine enough that there is little scattering of the infrared beam and it thus appears as though the tar was in the gas phase.

As an example of the analysis procedure, the pyrolysis and oxidation of a bituminous coal is described. More detail can be found in Ref. 1. Figure 16a illustrates the weight loss from this sample and the temperature history. A $25 \mathrm{mg}$ sample of Pittsburgh Seam coal, loaded in the sample basket of the DuPont ${ }^{\mathrm{N}} 951$, is taken on a $30^{\circ} \mathrm{C} / \mathrm{min}$ temperature excursion in the helium sweep gas, first to $150^{\circ} \mathrm{C}$ to dry, then to $900^{\circ} \mathrm{C}$ for pyrolysis. After cooling, a small flow of $\mathrm{O}_{2}$ is added to the furnace at the 57 minute mark and the temperature is ramped to $900^{\circ} \mathrm{C}$ for oxidation.

During this excursion, infrared spectra are obtained once every forty seconds. As discussed previously (1) the spectra show absorption bands for $\mathrm{CO}, \mathrm{CO}_{2}, \mathrm{CH}_{4}, \mathrm{H}_{2} \mathrm{O}, \mathrm{SO}_{2}, \mathrm{COS}, \mathrm{C}_{2} \mathrm{H}_{4}, \mathrm{HCl}$, and $\mathrm{NH}_{3}$. The spectra above $400^{\circ} \mathrm{C}$ also show aliphatic, aromatic, hydroxyl, carbonyl and ether bands from tar. The evolution of gases derived from the IR absorbance spectra are obtained by a quantitative analysis program which employs a database of calibration spectra for different compounds. The routine decides which regions of each calibration spectrum to use for best quantitation with the least interferences. A correlation between the sample spectrum and the reference spectrum is performed to determine gas amounts. A database of integration windows is also available for tracking functional groups absorptions. Tar quantitation is discussed in Ref. 1 . The routine is fast enough so that the product analysis can be performed and displayed every 40 seconds during the actual experiment. 
Figure $16 \mathrm{~b}$ illustrates the integral of the evolution curves to obtain cumulative evolved product amounts. Because the data are quantitative, the sum of these curves match the weight loss as determined by the TGA balance. Discrepancies occur because of components such as $\mathrm{H}_{2}$ which cannot be seen by IR. When $\mathrm{O}_{2}$ is introduced, the balance shows a net gain in weight due to $\mathrm{O}_{2}$ chemisorption.

Determination of FG-DVC Model Parameters - The kinetic and composition parameters for the FGDVC model are obtained from the TG/plus pyrolysis cycle. The pyrolysis cycle for lllinois No. 6 coal (Argonne premium sample) is presented in Fig. 17. Figure 17a presents the weight loss and temperature profile. Also presented (dashed line) is the sum of species (tar, $\mathrm{CH}_{4}, \mathrm{H}_{2} \mathrm{O}, \mathrm{CO}, \mathrm{CO}, \mathrm{SO}_{2}$ $\mathrm{NH}_{3}, \mathrm{C}_{2} \mathrm{H}_{4}$, and COS). The sum of species is within a few percent of the weight loss.

The water evolution (Fig. 17b) consists of a low temperature moisture peak followed by a pyrolysis. peak. To fit the wide pyrolysis peak by the FG submodel, three sources are used for $\mathrm{H}_{2} \mathrm{O}$. Each source evolves according to

$$
d W_{1} / d t=k_{1} W_{1} \text { (char) }
$$

were $W_{1}$ is the gas species and $W_{i}$ (char) is the amount of the functional group source remaining in the char. The rate constant, $k_{1}$ is given by an Arrhenius expression of the form

$$
k_{1}=A_{1} \exp \left(\left(-E_{1} \pm \sigma_{1}\right) / R T\right)
$$

where $A_{1}$ is the frequency factor, $E_{1}$ the activation energy and $\sigma_{1}$ the distribution in activation energies. Two sources are employed for $\mathrm{CH}_{4}$, and three sources for $\mathrm{CO}$ and $\mathrm{CO}_{2}$. Note the elimination of the calcite $\mathrm{CO}_{2}$ evolution peak (Fig. 17d) and the increase in tar (Fig. 17c) for the demineralized coal.

To obtain the model parameters, the model is fit to the TG/plus data at three heating rates $(3,30$, and $100^{\circ} \mathrm{C} / \mathrm{min}$ ). When there are multiple sources for a given species and the sources have overlapping peaks, the determination of parameters is not unique and some rules must be assumed. Based on chemical arguments, $A$ is restricted between $10^{12}$ and $10^{15} \mathrm{sec}^{-1}$. Also, the preexponential for a given species pool is assumed rank invariant based on the observed rank variation of the evolution curves. As the coal is increased in rank, the leading edges and the early peaks (Extra Loose or Loose pools) shift to higher temperatures while the trailing edge (Tight or Extra Tight pools) remain at the same temperature. An example of this is shown for water for five coals in Fig. 18a (16). From this figure it appears that the shift in the evolution curve with rank can be explained by the geological aging process. With increasing aging temperature and time, the maturation process gradually evolve the loosely bounded functional groups and leaves the tightly bounded groups intact.

The shift can be simulated by pyrolyzing a species described by a distribution of activation energies (Eq. 2) up to different bed temperatures. An example is shown in Fig. 18b. Starting with the evolution profile for Zap lignite, the coal is assumed to pyrolyze at $10^{\circ} \mathrm{C} /$ million years up to temperatures of $(60$, 120,150 , and $180^{\circ} \mathrm{C}$. The resulting geologically aged samples is simulated using the TG/plus temperature profile and the predicted results are plotted. The curves for geological aging at 120,150, and $180^{\circ} \mathrm{C}$ are similar to the actual TG/plus evolution curves shown in Fig. 18a for Illinois No. 6 , Pittsburgh, No. 8, and Upper Freeport, respectively. Thus, the frequency factor is assumed to be constant as the rank increases, and the activation energy of the pool increased with increasing coal rank to fit the data. We find that the activation energy of tight pools generally change with rank much less than do the loose pools.

A typical comparison of theory and TG-FTIR experiments is shown in Fig. 19 for the Pittsburgh Seam coal for one heating rate. The amounts of the functional group pools are obtained from this procedure. The resolution of the hydrocarbon evolution into paraffins, olefins, ethane, ethylene, propane, and propylene is done in other experiments if required. Figure 20 compares the theory and experiment for three heating rates for weight loss, tar evolution, and methane evolution. The kinetic parameters are derived from these experiments. The agreement between the theory and experiment is quite good. 
We have applied these curve fitting procedures for the eight Argonne coals according to the rules cited above (i.e., frequency factor between $10^{12}$ and $10^{15} / \mathrm{sec}$ and constant for a given gas species pool independent of coal rank). Results for the rates for bond breaking, the evolution of methane (two pools), $\mathrm{CO}$ (three pools), $\mathrm{CO}_{2}$ (three pools) and $\mathrm{H}_{2} \mathrm{O}$ (three pools) are presented as a function of the coal's oxygen concentration in Fig. 21. As can be seen, there is a systematic increase in activation energy with increasing rank. The variation in activation energy is maximum for the loose pool and reduces as the activation energy increases. The amounts for these pools are presented in Fig. 22. There is a systematic variation in the amounts with rank.

\section{Solvent Swelling, Extraction and NMR}

Solvent swelling and extraction data for the Argonne coals are presented in Table 1. As discussed above, the extract yield is employed to determine the length of the chains (Monte Carlo) or the starting bond probabilities (percolation) used in the model. There appears to be some problem in employing this approach for the highest rank coals (Pocahontas and Upper Freeport). The swelling and extract yields for these coals in pyridine appears to be limited by weak crosslinking (other. than hydrogen bonding) forces which are not eliminated by pyridine.

The solvent swelling ratio has been employed to determine the crosslink density $(2,3,24-29)$. The various theories and values for the solvent interaction parameter (24-29) suggest that there are between 4 and 8 ring clusters between crosslinks, indicating a value of $\sigma+1$ between 2.13 and 2.25 (32). NMR results of Solum et al. (5) for the number of bridges and loops suggest a value of $\sigma+1$ of between 2 and 3 , so a value in the neighborhood of 2.5 seems reasonable. However, the uncertainty of these determinations is too large to employ them in the model. The crosslink density is instead considered an adjustable parameter employed to fit the fluidity data.

\section{Geissler Fluidity}

As discussed above, the crosslink density controls the effective coordination number of the network, and hence the molecular weight distribution and amount of the fragments. For bituminous coals, it is the initial crosslink density which is important, since few new crosslinks are formed prior to pyrolysis. A recent theory for fluidity was developed based on the liquid fraction in the coal computed by the FGDVC model $(8,43)$. Measurements of the tar and the fluidity thus provide a constraint on the molecular distribution of the fragments and hence on the crosslink density.

Figure 23 presents a comparison of theory and experiment for four of the Argonne coals with the kinetic parameters fit from TG-FTIR data. The fitting procedure for fluidity and tar determines a unique combination of the crosslink density and donatable hydrogen.

\section{Monomer Molecular Weight Distribution}

The molecular weight distribution of the monomers is chosen based on the ring cluster size determined by NMR (5) and the results of the model checked with FIMS data (6).

\section{RESULTS}

\section{Volatiles Evolution}

A good test of the validity of using the TG-FTIR method over a range of low heating rates to obtain kinetic parameters is the ability to use the kinetic parameters to extrapolate to high heating rate conditions. Figure 24 presents results for Illinois No. 6 coal (obtained from Combustion Engineering) using the complete FG-DVC model and the most recent kinetic and composition parameters derived from the TG/plus (65). The predicted rates of evolution for each species are in good agreement with the observed rates except for water where moisture sometimes creates measurement errors. The data were obtained in the heated tube reactor where FT-IR emission and transmission measurements of coal particle temperatures determined the heating rate to be over $20,000 \mathrm{~K} / \mathrm{sec}$. 


\section{Char}

A number of char characteristics can be measured and compared with the model. These include fluidity (already discussed in Section III), functional group composition, crosslink density, PMRTA, and extract yield. These are discussed below.

- Functional Group Composition - The functional group composition can be determined by FT-IR (74-77) or NMA CP-MS with dipolar dephasing (5). A set of chars was prepared by heating Pittsburgh Seam coal up to temperatures of $200,300,400,500$, and $600^{\circ} \mathrm{C}$ at $30^{\circ} \mathrm{C} / \mathrm{sec}$ and the chars were characterized (68). Figure 25 compares the theory with NMR and FT-IR measurements. The fractions of aliphatic and aromatic carbons are compared in Fig. 25a, and aliphatic, aromatic, methyl, and hydroxyl hydrogens in Fig. 25b. Figures 25c and 25d compare the theory and FT-IR measurements for the same quantities (except methyl and aliphatic hydrogen are lumped together). The tar yield (Fig. 26a), and methane yield (Fig. 26b) are presented for comparison. The model predictions are in excellent agreement with the data.

Crosslink Density - The application of the volumetric swelling ratio to obtain the changing crosslink density in the char was discussed in Section II. Comparison with theory was discussed in Ref. 7. Figure 26c compares the theory and experiment for the set of chars in Figs. 25 and 26. The agreement is good. Figure 27 compares the theory and experiment for two coals (Zap lignite and Pittsburgh Seam). The theory predicts the early crosslinking in Zap lignite (related to $\mathrm{CO}_{2}$ evolution) not seen for the bituminous coal. The agreement between theory and experiment is good except that the increase in $1-x$ for the Pittsburgh Seam coal in Fig. 27 is not predicted.

The NMR data also provide a direct measurement of the number of attachments per cluster (5). Figure 28 presents data (68) for total number of attachments (which includes peripheral groups, bridges, and loops), and just bridges and loops ( $B \& L$ ) as a function of final temperature for Pittsburgh Seam coal heated at $30^{\circ} \mathrm{C} / \mathrm{min}$ to the indicated temperature. The FG-DVC model predicts the number of $B$ \& $L$. This quantity (near 2 for coal) is the coordination number, suggesting that coal is quite chain-like. There is little change in $B$ \& $L$ up to $400^{\circ} \mathrm{C}$. B\&L increased at $500^{\circ} \mathrm{C}$ and above where crosslinking related to methane evolution is believed to occur.

Notice that the total number of attachments changes very little. This would be reasonable if the methane peripheral groups were replaced by bridges in substitution reactions. This is believed to be the reason for the correlation between methane evolution and crosslinking.

PMRTA - Proton magnetic resonance thermal analysis (PMRTA) is employed at CSIRO as an alternative to fluidity measurements. The measurement of proton mobility can distinguish protons on molecules free to rotate from protons on a rigid lattice. The molecules ability to rotate depends on its freedom from the network (i.e., it must be unattached or attached at only one place) and on the mobility of free molecules to rotate (which depends on the temperature). From the measured $M_{2 T}$ values, a "mobile" liquid fraction can be defined by the expression (72)

$$
\text { Liquid Fraction }=\frac{M_{2 T} \text { (room temperature) }-M_{2 T}(T)}{M_{2 T} \text { (room temperature) }}
$$

At sufficiently high temperature, when the free molecules have sufficient energy to rotate, this quantity should be equal to the FG-DVC liquid fraction. Figures $29 \mathrm{a}$ and $29 \mathrm{~d}$, compare the measured and predicted liquid fraction using both the Monte Carlo and percolation models. As expected, the theory and experiment do not agree at low temperature, but there is good agreement on the softening temperature, peak fluidity temperature, and solidification temperature. The liquid amounts in the two theories are defined differently and so the absolute amounts do not agree. Also shown for comparison are the fluidity and tar evolution curves for the same coal at a similar heating rate. The agreement between the data and both theories is good. 
Extract Ylelds - Figure 30 compares the FG-DVC predictions to the data of Fong et al. (73) on total volatile yield and extract yield as a function of temperature in pyrolysis at $0.85 \mathrm{~atm}$. The experiment was performed in a heated grid apparatus at heating rates of $640^{\circ} \mathrm{C}$ to $1018 \mathrm{~K}$, with variable holding times and rapid cooldown. The predictions in Fig. 30 are in reasonable agreement with the data. The predicted extract yields are not as high as the measured yields. However, such high yields of extracts have not been duplicated by others, and there is some possibility that the extracted fraction also contains some colloidal material.

Weathering - Oxidation of a Pittsburgh Seam coal in our laboratory was performed at $80^{\circ} \mathrm{C}$ for 10 , 20 , and 62 days. In our model, the loss of fluidity with increasing oxygen concentration is related to the increase in $\mathrm{CO}_{2}$ evolution and hence increases in low temperature crosslinking. To determine the $\mathrm{CO}_{2}$ evolution, measurements were made in the TG-FTIR (78). The data in Fig. 31 a shows that the low temperature $\mathrm{CO}_{2}$ evolution was significantly increased after 10 days of oxidation, becoming comparable to that for llinois No. 6. After 20 days, the early $\mathrm{CO}_{2}$ evolution was larger than that for the Utah bituminous coal. After three months at $110^{\circ} \mathrm{C}$ the $\mathrm{CO}_{2}$ evolution was comparable to that of a lignite. When these increased $\mathrm{CO}_{2}$ yields were incorporated in the simulation for the oxidized Pittsburgh Seam coal's fluidity, the maximum fluidity was reduced. We compare our predicted maximum fluidity with the measurement of Wu et al. (79) for comparable coal and oxidation treatment in Fig. $31 \mathrm{~b}$. The agreement is quite reasonable.

Predicted Molecular Weight Distribution in Char - The dominant event in determining the char's properties is the starting and low temperature crosslinking behavior. Figures $32 \mathrm{a}$ and $32 \mathrm{~b}$ compare the predicted molecular weight distributions in the char for Zap lignite and a Pittsburgh Seam bituminous coal. The bituminous coal (Fig. 32a) exhibits substantial fragmentation of tar precursors ( $n$-mers 1-3), extracts (n-mers 4-10), and liquids (n-mers 11-100). On the other hand, the initial crosslink density in the lignite, and the subsequent increase due to $\mathrm{CO}_{2}$ related crosslinking, allows almost no n-mers except monomers, dimers, and trimers to be formed (Fig. 32b). These predictions of the model are related to the extract yields, PMRTA analysis fluidity, and tar yields.

\section{Tar}

Molecular Weight Distribution - The tar is evolved from the lightest fractions of the metaplast and depends on the metaplast distribution and the transport. This is illustrated in Fig. $32 \mathrm{c}$ and $32 \mathrm{~d}$, which are the predicted tar distribution for a bituminous coal and a lignite (two cases discussed above). For the bituminous coal, the upper molecular weight is limited only by the vapor pressure for the large molecules. For the lignite, the metaplast distribution limits the amount and molecular weight distribution.

Figures 33c, and 33d show measurements for the Pittsburgh Seam bituminous coal and the Beulah, Zap lignite pyrolyzed in the FIMS apparatus. The data have been summed over 50 amu intervals. While the Pittsburgh bituminous coal shows a peak intensity at about 400 Daltons, the lignite peak is at 100 Daltons. The predicted average tar molecular weight distributions are in good agreement with FIMS data as shown in Figs. 33a and 33b. Since both tar distributions are from the same monomer distribution, the enhanced drop off in amplitude with increased molecular weight for the lignite compared to the bituminous coal must be due to early crosslinking and transport effects in the lignite.

Pressure Effects - The prediction effect of pressure on the tar molecular weight distribution is illustrated in Figs. $34 \mathrm{a}$ and $34 \mathrm{~b}$. Pressure enters the model through the transport assumption. The internal transport rate is inversely proportional to the ambient pressure. The reduced transport rate reduces the evolution rate of the heavier molecules. Therefore, the average molecular weight and vaporization "cutoff" decrease with increasing pressure. The trends are in agreement with observed tar molecular weight distributions shown in Figs. $34 \mathrm{C}$ and $34 \mathrm{~d}$. The spectra are for previously formed tars that have been collected and analyzed in a FIMS apparatus (6). The low values of intensity between 100 and 200 Daltons are believed to be due to loss of these components due to their higher volatility.

Yield - The tar yields are measured in the TG-FTIR. Figure 35 compares the measured and predicted yield as a function of temperature. The agreement is good except for the low temperature evolution 
of guest molecules, which is not well predicted in the standard model. Improvements to predict this early peak have been made (8).

\section{Network Parameters}

Figure 36 presents the adjustable network parameters which have been chosen to fit the tar evolution and fluidity data as functions of the oxygen concentration. The oligomer length and the molecular weight between crosslinks increase smoothly with rank. The concentration of available hydrogen for ring stabilization has a maximum for the high volatile bituminous coals.

In Fig. 37, we compare the predicted and measured extract yield and the predicted and estimated molecular weight between crosslinks. The crosslink density for the bituminous coals is within the range of measured values. The model, however, requires a high molecular weight between crosslinks, $M_{c}$, for Pocahontas and Upper Freeport, while the solvent swelling ratios would indicate a low $M_{c}$ value. The model also requires a high extract yield for Upper Freeport while the measured yields are low. There may be an additional kind of weak crosslink for high rank coals, possibly associated with the aromatic-aromatic interactions suggested by Larsen (80). When the Upper Freeport coal is heated to $300^{\circ} \mathrm{C}$ and then cooled the solvent swelling ratio increases from 1.32 to 2.13 and the extract yield from 10.4 to $21 \%$, suggesting that this treatment may loosen some of these weak bonds non-reversibly.

\section{SUMMARY AND CONCLUSIONS}

The paper poses the question, can coal science be predictive? The answer is yes for coal thermal decomposition in particles small enough to be isothermal. It is possible to construct a model based on reasonable assumptions to predict almost all of the observed behavior. The model has only one parameter which is adjusted for the process conditions. This is the internal pressure in the transport submodel. All other model parameters of the coal are fixed for each coal. The model has composition and kinetic parameters to describe the evolution of each individual gas species. These can be determined in TG-FTIR experiments and exhibit a systematic variation with rank. There are three network parameters in the Monte Carlo version of the model, the chain length, the crosslink density, and the available donatable hydrogen. A similar set of network parameters is used in the percolation theory. These are adjusted to fit the TG-FTIR, tar yields, extract yields, and fluidity. These also exhibit a systematic variation with rank.

The paper explores the six concepts which are the foundation of the FG-DVC model:

1) The decomposition of functional group sources in the coal yield the light gas species in thermal decomposition. The amount and evolution kinetics can be measured by TG-FTIR, the functional group changes by FT-IR and NMR. There is good agreement between the model and NMR, FT-IR, and TG-FTIR measurements on a Pittsburgh Seam coal heated at $30^{\circ} \mathrm{C} / \mathrm{min}$ and for gas evolution for a lignite and an llinois No. 6 bituminous coal at $30^{\circ} \mathrm{C} / \mathrm{min}$ and $20,000^{\circ} \mathrm{C} / \mathrm{sec}$.

2) The decomposition of a macromolecular network yields tar and metaplast. The amount and kinetics of the tar evolution can be measured by TG-FTIR and the molecular weight by FIMS. The kinetics of metaplast formation and destruction can be measured by solvent extraction, by Geissler plastometer and proton magnetic resonance thermal analysis (PMRTA). Reasonable agreement has been demonstrated for solvent extract of a Pittsburgh Seam coal pyrolyzed at $30^{\circ} \mathrm{C} / \mathrm{min}$ and $640^{\circ} \mathrm{C} / \mathrm{sec}$. Good agreement was shown for four of the Argonne coal samples for fluidity by Geissler plastometer, and for one coal by PMTRA.

3) The molecular weight distribution of the metaplast depend on the network coordination number. The coordination number can be determined by solvent swelling and NMR.

4) The network decomposition is controlled by bridge breaking and the amount of bridge breaking is limited by the available donatable hydrogen. 
5) The network solidification is controlled by crosslinking. The changing crosslink density can be measured by solvent swelling and NMR. Crosslinking appears to occur with evolution of both $\mathrm{CO}_{2}$ (prior to bridge breaking) and $\mathrm{CH}_{4}$ after bridge breaking. Thus, low rank coals (which form a lot of $\mathrm{CO}_{2}$ ) crosslink prior to bridge breaking and are thus thermosetting. High volatile bituminous coals (which form little $\mathrm{CO}_{2}$ ) undergo significant bridge breaking prior to crosslinking and become highly fluid. Weathering, which increases the $\mathrm{CO}_{2}$ yield, causes increased crosslinking and lowers fluidity. There is good agreement between the predicted and measured crosslink densities and fluidities in the FG-DVC model in which crosslinks are correlated with $\mathrm{CO}_{2}$ and $\mathrm{CH}_{4}$ gas evolution.

6) The evolution of tar is controlled by mass transport in which the tar molecules evaporate into the light gas species and are carried out of the coal at rates proportion to their vapor pressure and the volume of light gases. High pressures reduces the volume of light gases and hence reduces the yield of heavy molecules with low vapor pressure. These changes can be studied with FIMS. The changes in tar yield and molecular weight distribution with pressure have been accurately predicted using the vapor pressure law, of Suuberg and coworkers.

The paper describes how the coal kinetics and composition parameters are obtained by TG-FTIR, solvent swelling, solvent extraction, Geissler plastometer data, NMR data and FIMS data. The model is compared to a variety of experimental data in which heating rate $\left(0.05\right.$ to $\left.20,000^{\circ} \mathrm{C} / \mathrm{sec}\right)$, temperature $\left(100\right.$ to $1600^{\circ} \mathrm{C}$ ), and pressure (vacuum to $100 \mathrm{~atm}$ ) are all varied. There is good agreement with theory (both Monte Carlo and percolation) and most of the data available from our laboratory and in the literature.

The network parameters employed in the model have been presented. The results suggest that there is some form of weak crosslinks for Pocahontas and Upper Freeport coal.

While the experimental results and the model are consistent with the suggested processes, the chemical reactions for bridge breaking, crossiinking, and functional group decomposition are not defined in detail. Also, there is only sparse data to validate the transport assumption and the internal pressure in the particle is an adjustable parameter of the model.

\section{ACKNOWLEDGEMENTS}

The authors wish to acknowledge the following organizations for their support over the last ten years: U.S. Department of Energy, Morgantown Energy Technology Center, U.S. Department of Energy, Pittsburgh Energy Technology Center, National Science Foundation, and Gas Research Institute. The authors also wish to acknowledge the extensive contribution from many coauthors who have collaborated on the various aspects of this work: Robert Carangelo, James Markham, Philip Best, Erik Kroo, Yan Ping Zhang, Rosemary Bassilakis, Marie DiTaranto, Girish Deshpande, Po-Liang Chien, H.H. King, Tom Squires, and Kevin Squire who worked at Advanced Fuel Research, Inc., Meredith Colket from United Technologies Research Center, Douglas Smoot and Scott Brewster from Brigham Young University, Eric Suuberg from Brown University, Ronald Pugmire, David Grant, and Mark Solum from the University of Utah, Donald McMillen and Ripudaman Malhotra from SRI international, Rashid Khan from Texaco, Tom Fletcher from Șandia National Laboratory, Leo Lynch and Richard Sakurovs from CSIRO, Bernie Gerstein from lowa State University, Jean Whelan from Woods Hole: Oceanographic Institute, Harold Schobert and Caroline Burgess at Penn State University, Garry Vail, Fred Baudais, Michel Baillargeon, Daniel Gravel from Bomem, Inc. and Dennis Gerson from IBM Instruments.

\section{REFERENCES}

1. Solomon, P.R., Serio, M.A., Carangelo, R.M., Bassilakis, R., Gravel, D., Baillargeon, M., Baudais, F., and Vail, G., Energy and Fuels, 1990, 4(3), 319.

2. Green, T.K., Kovac, J., and Larsen, J.W., Fuel, 1984, 63, 935.

3. Green, T.K., Kovac, J., and Larsen, J.W., Coal Structure, R.A. Meyers, Ed., Academic, NY, 1982, p. 199.

4. The Geissler Plastometer data was supplied by Mr. George Engelke from Commercial Testing 
and Engineering Company.

5. Solum, M.A., Pugmire, R.J., and Grant, D.M., Energy \& Fuels, 1989, 3, 187.

6. St. John, G.A., Butrill, S.E., Jr., and Anbar, M., ACS Symposium Series 71, American Chemical Society, Washington, DC, 1978, p. 223.

7. Solomon, P.R., Hamblen, D.G., Carangelo, R.M.'., Serio, M.A., and Deshpande, G.V., Energy and Fuel, 1988, 2, 405.

8. Solomon, P.R., Serio, M.A., Hamblen, D.G., Yu, Z.Z, and Charpenay, S., ACS Div. of Fuel Chem. Preprints, 1990, 35(2), 479.

9. Solomon, P.R., in Coal Structure, Advances in Chemistry Series, 1981, 192, 95.

10. Solomon, P.R. and Hamblen, D.G., "Pyrolysis", in Chemistry of Coal Conversion, (R.H. Schlosberg, Editor), Plenum, New York, NY, 1985, pg. 121-251.

11. Solomon, P.R., Hamblen, D.G., Carangelo, R.M., and Krause, J.L., Nineteenth Symposium (Int) on Combustion, 1139, The Combustion Institute, Pittsburgh, PA, 1982, 1139.

12. Serio, M.A., Hamblen, D.G., Markham, J.R., Solomon, P.R., Energy and Fuel, 1987, 1, 138.

13. Solomon, P.R. and King, H.H., Fuel, 1984, 63, 1302.

14. Squire, K.R., Carangelo, R.M., DiTaranto, M.B., and Solomon, P.R., Fuel, 1986, 65, 833.

15. Solomon, P.R., Hamblen, D.G., Carangelo, R.M., Serio, M.A. and Deshpande, G.V., Combustion and Flame, 1988, 71, 137.

16. Solomon, P.R., Serio, M.A., Carangelo, R.M., Bassilakis, R., Yu, Z.Z., Charpenay, S., and Whelan, J., "Analysis of Coal by TG-FTIR and Pyrolysis Modeling", presented at the Pyrolysis '90 Meeting in Holland, June 1990, to be published in Journal of Analytical and Applied Pyrolysis.

17. Suuberg, E.M., Peters, W.A., and Howard, J.B., Seventeenth Symposium (Int) on Combustion, The Combustion Institute, Pittsburgh, PA 1979, 117.

18. Gavalas, G.R., Cheong, P.H., and Jain, R., Ind. Eng. Chem. Fundam., 1981, 20, 113.

19. Gavalas, G.R., Cheong, P.H., and Jain, R., Ind. Eng. Chem. Fundam., 1981, 20, 122.

20. Xu, W.C. and Tomita, A., Fuel, 1987, 66, 627.

21. van Krevelen, D.W., Coal, Elsevier, Amsterdam, 1961.

22. Solomon, P.R., Squire, K.R., and Carangelo, R.M., Int. Conf. on Coal Science, Pergamon, Sydney, Australia, 1985, p. 945.

23. Brenner, D., Fuel, 1985, 64, 167.

24. Lucht, L.M. and Peppas, N.A., Fuel, 1987, 66, 803.

25. Lucht, L.M., Larsen, J.M., and Peppas, N.A., Energy \& Fuels, 1987, 1, 56.

26. Larsen, J.W., ACS Fuel Chem. Div. Preprints, 1985, 30(4), 444.

27. Green, T., Kovac, J., Brenner, D., and Larsen, J., Coal Structure, (R.A. Meyers, Ed.), Academic, NY, 1982, p 199.

28. Hall, P.J., Marsh, H., and Thomas, K.M., Fuel, 1988, 67, 863.

29. Sanada, Y. and Honda, H., Fuel, 1966, 45, 295.

30. Suuberg, E.M., Yoshi, O., and Deevo. S., ACS Div. Fuel Chem. Prepr., 1988, 33(1), 387.

31. Gavalas, G.R., Coal Pyrolysis, Elsevier, NY, 1982 p. 51

32. Solomon, P.R., Hamblen, D.G., Yu, Z.Z., and Serio, M.A., Fuel, 1990, 69, 754.

33. Niksa, S., and Kerstein, A.R., Combustion and Flame, 1986, 66, 95.

34. Niksa, S., Combustion and Flame, 1986, 66111.

35. Niksa, S. and Kerstein, A.R., Fuel, 1987, 66, 1389.

36. Grant, D.M., Pugmire, R.J., Fletcher, T.H., and Kerstein, A.R., Energy \& Fuels, 1989, 3, 175.

37. Nelson, P.F., Fuel, 1987, 66, 1264.

38. Calkins, W.H., Hagaman, E., and Zeldes, H., Fuel, 1984, 63, 1113.

39. Calkins, W.H., and Tyler, R.J., Fuel, 1984, 63, 1119.

40. Calkins, W.H., Fuel, 1985, 64, 1125.

41. Calkins, W.H., Hovsepian, B.K., Drykacz, G.R., Bloomquist, C.A.A., and Ruscic, L., Fuel, 1984, $63,1226$.

42. Niksa, S., AlChE J., 1988, 34, 790.

43. Solomon, P.R., Best, P.E., Yu, Z.Z., and Deshpande, G.V., ACS Div. of Fuel Chem. Preprints, 1989, 34(3), 895.

44. Suuberg, E.M., Lee, D., and Larsen, J.W., Fuel, 1985, 64, 1668.

45. Suuberg, E.M., Unger, P.E., and Larsen, J.W., Energy and Fuels, 1987, 1, 305.

46. Bockrath, B.C., Illig, E.G., and Eassell-Bridger, W.D., Energy \& Fuels, 1987, 1, 227.

47. Solomon, P.R., Serio, M.A., Deshpande, G.V., and Kroo, E., Energy \& Fuels, 1990, 4(1), 42. 
48. Solomon, P.R., "Coal Structure and Thermal Decomposition", in New Approaches in Coal Chemistry, ACS Symposium Series 169, 1981, 61.

49. Brown, J.K., Dryden, I.G.C., Dunevein, D.H., Joy, W.K, and Pankhurst, K.S., Inst. Fuel, 1958, 31, 259.

50. Orning, A.A. and Greifer, B., Fuel, 1956, 35, 318.

51. Freihaut, J.D., Proscia, W.M., and Seery, D.J., Energy \& Fuels, 1989, 3, 692.

52. Anthony, D.B. and Howard, J.B., AlChE J., 1976, 22, 625.

53. Solomon, P.R., Serio, M.A., Carangelo, R.M., and Markham, J.R., Fuel, 1986, 65, 182.

54. Solomon, P.R. and Serio, M.A., "Evaluation of Coal Pyrolysis Kinetics", in Fundamentals of the Physical-Chemistry of Pulverized Coal Combustion, J. Lahaye and G. Prado, Eds.), Martinus Nijhoff, 1987, 126.

55. Kobayashi, H., Howard, J.B., and Sarofim, A.F., Sixteenth Symposium (Int) on Combustion, The Combustion Institute, Pittsburgh, PA, 1977, 411.

56. Solomon, P.R., and Colket, M.B., Seventeenth Symposium (Int) on Combustion, The Combustion Institute, Pittsburgh, PA, 1979, 131.

57. Anthony, D.B., Howard, J.B., Hottel, H.C., and Meissner, H.P., Fifteenth Symposium (Int) on Combustion, The Combustion Institute, Pittsburgh, PA, 1974, 1303.

58. Badzioch, S., and Hawksley, P.G., Ind. Eng. Chem. Proc. Des., Dev., 1970, 9, 521.

59. Solomon, P.R., Fletcher, T.H., and Pugmire, R.J., Progress in Coal Pyrolysis, Pittsburgh Coal Conference, (Sept. 10-14, 1990).

60. Solomon, P.R., Serio, M.A., and Suuberg, E.M., Review of Coal Pyrolysis: Experiments, Kinetic Rates, and Mechanisms, Progress in Energy and Combustion Science, submitted (1990).

61. Fletcher, T.H., Combustion and Flame, 1989, 78, 223.

62. Fletcher, T.H., Combust. Sci. and Tech., 1989, 63, 89.

63. Freihaut, J.D. and Proscia, W.M., Energy \& Fuels, 1989, 3(5), 625.

64. Solomon, P.R., Serio, M.A., and Markham, J.R., Kinetics of Coal Pyrolysis, Int. Conference on Coal Science Proceedings, IEA, Tokyo, Japan, p. 575, (October 23-27, 1989).

65. Serio, M.A., Solomon, P.R., Charpenay, S., Yu, Z.Z., and Bassilakis, R., ACS Div of Fuel Chem. Preprints, 1990, 35(3), 808.

66. Burnham, A.K., Oh, M.S., Crawford, R.W., and Samoun, A.M., Energy \& Fuel, 1989, 3, 42.

67. Stein, S.E., Robauch, D.A., Alfieri, A.D., and Miller, R.E., J. Am. Chem. Soc., 1982, 104, 6567.

68. Solum, M.S., Pugmire, R.J., Grant, D.M., Fletcher, T.H. and Solomon, P.R., Studies of Coal Char Structure Evolution: 1. Solid State ${ }^{13} \mathrm{C}$ NMR, to be submitted, (1989).

69. Lynch, LJ., Sakurovs, R., Webster, D.S., and Redlich, P.J., Fuel, 1988, 67, 1036.

70. Lynch, L.J., Webster, D.S., Sakurovs, R., Barton, W.A., and Maher, T.P., Fuel, 1988, 67, 579.

71. Barton, W.A. and Lynch, L.J., Energy \& Fuels, 1989, 3, 402.

72. Dr. Richard Sakurovs, personal communications.

73. Fong, W.S., Peters, W.A., and Howard, J.B., Fuel, 1986, 65, 251.

74. Sobkowwiak, M., Riesser, B., Given, R., and Painter, P., Fuel, 1984, 63, 1245.

75. Riesser, B., Starsinic, M., Squires, E., Davis, A., and Painter, P., Fuel, 1984, 63, 1253.

76. Solomon, P.R. and Carangelo, R.M., Fuel, 1988, 67, 949.

77. Solomon, P.R., and Carangelo, R.M., Fuel, 1982, 61, 663.

78. Solomon, P.R., Best, P.E., Yu, Z.Z., and Charpenay, S., "A Macromolecular Network Model for Coal Fluidity", to be submitted to Fuel, (1990).

79. Wu, M.M., Robbins, G.A., Winscheil, R.A., and Burke, F.P., Energy \& Fuels, 1988, 2, 150.

80. Larsen, J.W., ACS Div. of Fuel Chem. Preprints, 1988, 33(1), 400. 
Appendix $L$

Network Models of Coal

Thermal Decomposition 
Network Models of Coal Thermal Decomposition 


\title{
Network models of coal thermal decomposition
}

\author{
Peter R. Solomon, David G. Hamblen, Zhen-Zhong $Y u$ and \\ Michael A. Serio \\ Advanced Fuel Research, Inc., 87 Church Street, East Hartford, CT 06108. USA \\ (Received 24 July 1989; revised 2 February 1990)
}

\begin{abstract}
Several groups have considered statistical network fragmentation models to describe coal thermal decomposition. In these models. the coal macromolecule is viewed as a collection of fused aromatic rings (monomers) linked by bridges. During thermal decomposition, existing bridges break and new bridges are formed. The parameters of the models are the geometry of the network. which is expressed as the number of attachments per monomer (the coordination number. $\sigma+1$ ), and the chemistry of bridge breaking and formation. Given $\sigma+1$ and the instantaneous number of unbroken and formed bridges, the molecular weight distribution can be predicted. The different groups have employed both Monte Carlo methods and percolation theory to describe the network statistics. The former approach has advantages in terms of describing both the depolymerization and crosslinking processes in coal decomposition. since it does not require a constant coordination number. The latter method provides closed form solutions and is computationally less demanding. The models differ in the geometry of the network, the chemistry of bridge breaking and bridge formation (crosslinking) and the mass transport assumptions. This paper considers for three such models: the mathematical schemes; the assumed network geometries; the assumed bond breaking and bond formation chemistries: and the mass transport assumptions. The predictions of three models were compared by comparing the oligomer populations as a function of the number of unbroken bridges per ring cluster. This paper also presents results from a new model which combines the geometry, chemistry and mass transport assumptions of the FG-DVC model with the mathematics of a modified percolation theory.
\end{abstract}

(Keywords: thermal decomposition: coal: modelling)

Many recent studies have proposed that coal can be thought of as having a macromolecular network structure to which concepts of crosslinked polymers can be applied $^{1-10}$. These concepts have been employed to understand and model such properties of coal as: the insolubility; the equilibrium swelling and penetration of solvents; the viscoelastic properties: similarities between the parent coal and products of hydrogenolysis. or mild oxidation: crosslinking during char formation ${ }^{11.12}$; and the formation of coal tar in pyrolysis ${ }^{13-17}$. With the success of these concepts in describing coal properties, it appears logical to extend macromolecular network concepts to completely describe the thermal decomposition behaviour of coal.

A number of investigators have used statistical methods to predict how the network behaves when subjected to thermally induced bridge breaking, crosslinking, and mass transport processes ${ }^{17-30}$. Gavalas et al. ${ }^{20}$ employed statistical methods to predict the release of monomers from a randomly connected network. The model of Niksa and Kerstein employed percolation theory in a model called DISARAY ${ }^{28}$, which extended their previous model built on chain statistics ${ }^{24.25}$. Grant et al. $^{30}$ employed percolation theory in a model called chemical percolation devolatilization (CPD). Solomon et al. ${ }^{21-23,27}$ used Monte Carlo methods in a network model called the depolymerization, vaporization. and crosslinking (DVC) model. This was an extension of their previous model for linear polymers ${ }^{17,20}$. The DVC model was recently combined with their functional group (FG) model 27.29 to produce the general FG-DVC pyrolvsis model. This model is currently being applied to model the devolatilization behaviour of Argonne premium coals $s^{31}$ and to predict the fluidity of coals ${ }^{32}$. Other statistical methods for network behaviour have been employed in the polymer literature 33-38. $^{\text {. }}$

In applying network models to coal thermal decomposition, one considers the coal to consist of aromatic ring clusters linked together by bridges in some geometry. The geometry is described by the degree of branching in the network. An unbranched linear network will have one bridge per ring cluster attaching it to the next cluster. Thus each cluster has two attachments and is said to have a coordination number $(\sigma+1)$ of two. A highly branched 'fish net' would have two bridges per cluster, attaching it to the neighbouring four clusters and thus a coordination number of four. A branch point is considered to occur at any cluster connected to more than two neighbours (i.e. having more than two attachments).

When the coal is heated, the bridges can break and new bridges can form. Various statistical methods can be employed to predict the concentration of individual aromatic ring clusters (monomers) and linked clusters (oligomers of $n$ clusters. ' $n$-mers') up to a totally linked network. By assigning an average or distribution of molecular weights to the monomers. the amounts of tar, extractables. liquids or char can then be defined from the distribution of oligomer sizes. The models vary in the assumed chemistry of bridge breaking and crosslinking, 
in the definition of tar, extracts. liquids, and char and in the statistical methods used.

In view of the importance of macromolecular network models to the accurate predictions of coal processing behaviour. this paper assesses the assumptions and limitations of the proposed models. It appears that the way one performs the statistics (Monte Carlo, percolation theory, or other statistical methods) makes little difference. For example, percolation theory methods were substituted for Monte Carlo calculations in the FG-DVC model. and comparable predictions were obtained for appropriately restricted cases. The important differences among models are in the assumptions for: the network geometry (i.e. the degree of branching or coordination number); the chemistry of bridge breaking; the chemistry of crosslink formation; hydrogen utilization; and mass transport. This paper compares the three most recent models (DISARAY, CPD, and FG-DVC) and considers how the assumed network properties relate to behaviours observed for coal.

\section{MACROMOLECULAR NETWORKS}

\section{General properties of networks}

Figures 1 and 2 present the networks employed in the FG-DVC Monte Carlo calculations and percolation theory, respectively. For the. FG-DVC Monte Carlo calculation. linear oligomers of $l$ clusters (shown as the horizontal chains of clusters) of a molecular weight distribution defined by $M_{\text {avg }}$ and deviation. $\Delta M$. are linked by $m_{0}$ "crosslinks' per monomer ${ }^{26.29}$. These 'crosslinks' are indicated by the vertical double lines as shown in Figure 1. The branch points in the network are defined to occur at those clusters where more than two attachments connect a cluster to another cluster. Unless the crosslink occurs at the end of the oligomer. it forms at least one branch point. The term 'crosslink' has previously been used to mean the extra bridges that can form a branch point. and this is the definition employed here ${ }^{27.29}$. During thermal decomposition. bridges break. new crosslinks are added and the molecular weight of the oligomers is calculated by randomly distributing these changes.

For the percolation theory, a Bethe lattice is employed ${ }^{28,30.39}$. Lattices are characterized by the coordination number, $(\sigma+1)$, which is the number of attachment sites for bridges per cluster and the probability, $p$, that an unbroken bridge occupies the site. Figure 2 shows lattices at $p=1$ (i.e. all possible bridges

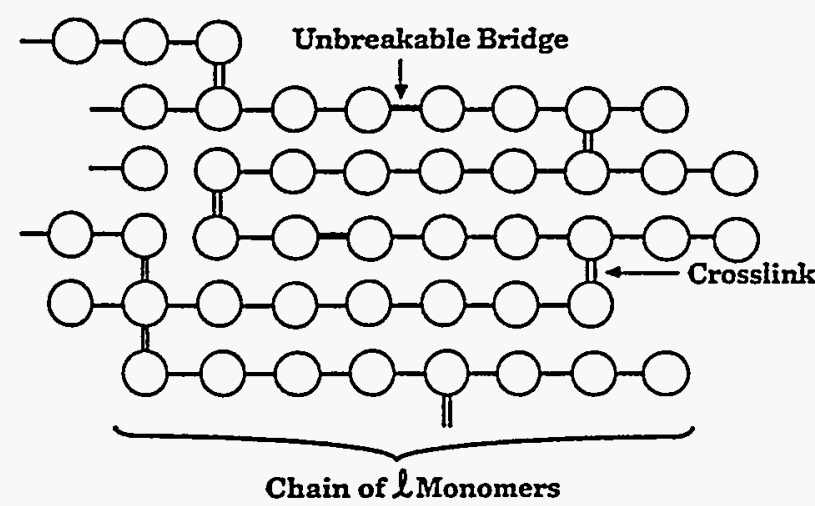

Figure 1 Macromolecular network used in Monte Carlo simulation a

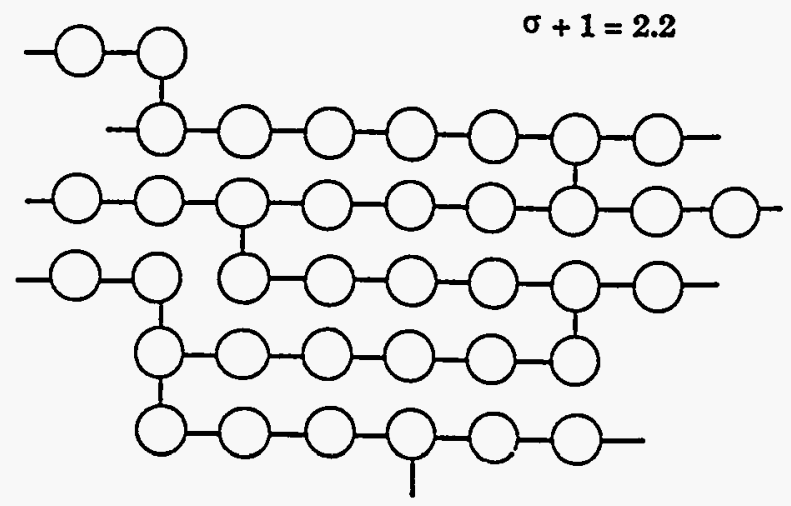

b

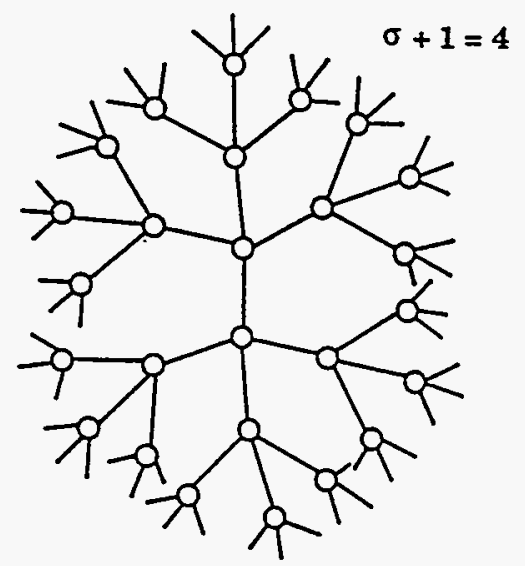

Figure 2 Bethe lattice for a, coordination number 2.2. $p=1: b$. coordination number $4 . p=1$

occupied) for $\sigma+1=2.2$ and $\sigma+1=4$. The Bethe lattice has no loops, but it has been demonstrated that this lattice is a good approximation to a lattice of equivalent coordination number containing loops ${ }^{39}$. As shown in Figure 2, the $\sigma+1=2.2$ lattice has branch points every 5th cluster. This network is close to a linear polymer $\sigma+1=2$ which has no branch points. The $\sigma+1=4$ lattice is much more highly branched. having double branch points on each cluster (this would be the Bethe lattice analogue of the 'fish net'). For values of $p$ less than 1 . the bridges are randomly removed from the network. It is important to realize that the statistical distribution of unbroken bridges means that even when the average number of unbranched bridges per cluster is identical for the two networks in Figure 2. the $\sigma+1=4$ network can never look like the $\sigma+1=2.2$ network (except for. the case $p=0$ ).

The loop free geometry of the Bethe lattice allows for the number of free oligomers to be analytically expressed as a function of $\sigma+1$ and the probability $p$ of bonds being unbroken. This is the feature which makes the percolation theory so attractive from the standpoint of computer efficiency and for understanding the behaviour of networks under conditions of varying bridge populations. Figure 3 shows calculations using percolation theory (for three values of $\sigma+1$ ) for the monomer, the sum of oligomers up to 3 , up to 10 , and the sum of all free oligomers as a function of the number of unbroken bridges per ring cluster $\alpha$, where $\alpha=1 / 2 p(\sigma+1)$. The $1 / 2$ enters because $\sigma+1$ is the number of attachments per cluster, which is twice the number of bridges (i.e. each 

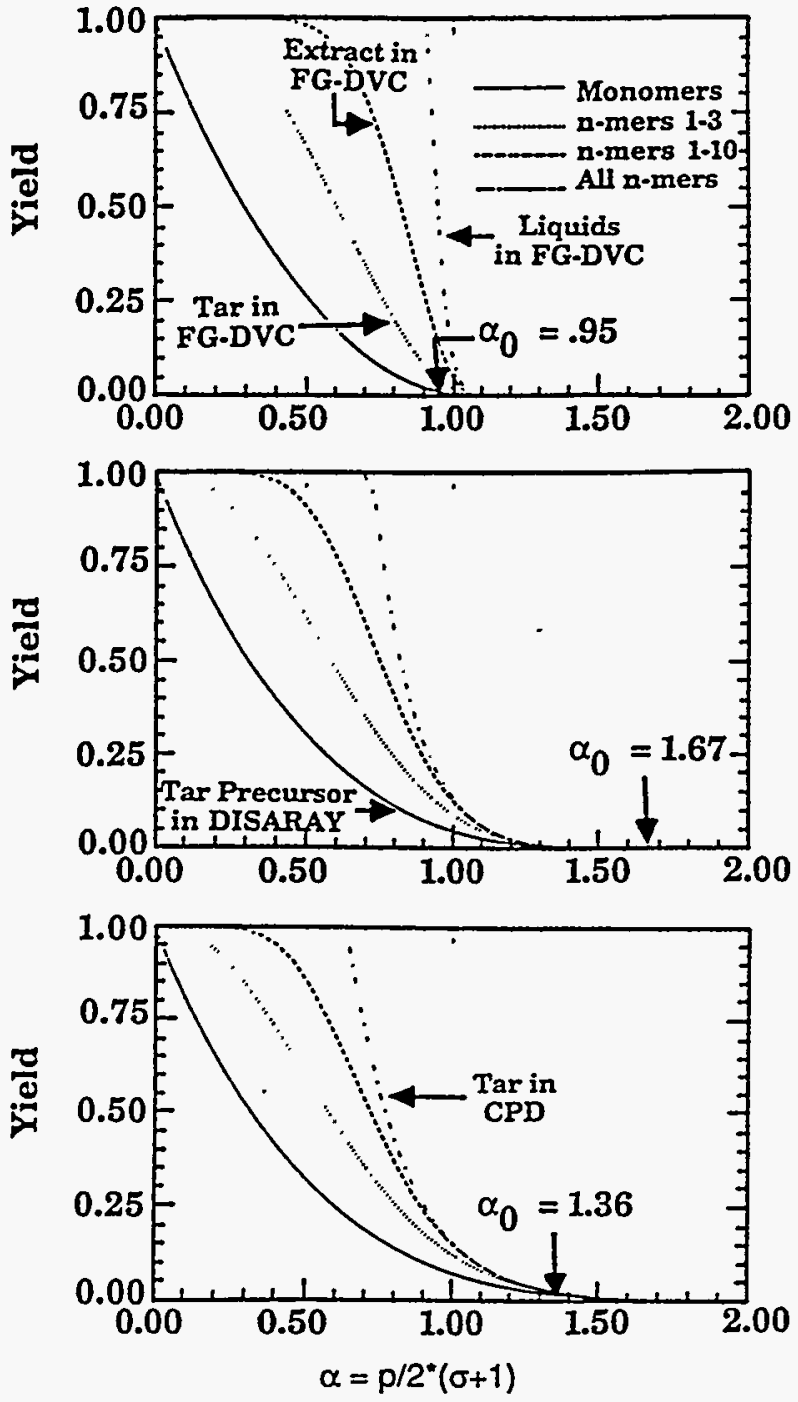

Figure 3 Percolation theory predictions for pyrolysis products (monomers. tar. extracts and total liquids) for three values of the coordination number $(\sigma+1): a, \sigma+1=2.2: b, \sigma+l=3.25: c . \sigma+l=4.6$

bridge forms two attachments). If $\sigma+1$ remains constant during pyrolysis, the molecular weight distribution is a single valued function of $\alpha$. For ring clusters of molecular weight $300 \mathrm{amu}$, the sum of $1-3 n$-mers corresponds roughly to the potential tar fraction (up to $900 \mathrm{amu}$ ), the sum of $1-10 n$-mers corresponds to the extractable fraction (up to $3000 \mathrm{amu}$ ), and the sum of all oligomers corresponds to the liquids fraction (all free oligomers). It can be seen that, with increasing $\sigma+1$, more broken bridges are required to achieve equivalent fractions of free oligomers. Also, at a fixed value of $\alpha$, the relative amounts of tar, extracts, and liquids vary with $\sigma+1$.

\section{Network geometries representative of coal}

The three important parameters of the network are the average ring cluster size $M_{\text {avg }}$, the coordination number $(\sigma+1)$, and the starting probability of bridges being unbroken, $p_{0}$. To compare networks of different coordination numbers, it is convenient to use $\alpha$ and $\alpha_{0}$ rather than $p$ and $p_{0}$. The assumptions of DISARAY, $C P D$, and FG-DVC are considered below.

Ring cluster size. Ring cluster sizes have been estimated from n.m.r. alone ${ }^{40}$, n.m.r. and FT-i.r. ${ }^{41}$, mild degradation $^{42}$, and the molecular weight distribution of $\operatorname{tar}^{15.16 .29}$. Based on these results, the average ring cluster size for coals with less than $90 \%$ carbon is expected to be between 2 and 3 aromatic rings or a total molecular weight per cluster. including peripheral groups, of 200-400 amu. DISARAY assumes a value of $1400 \mathrm{amu}$ for the monomer which can split into two $700 \mathrm{amu}$ tar fragments. CPD does not specify the monomer molecular weight. For coals with less than $90 \%$ carbon. FG-DVC employs a distribution of monomers with an $M_{\text {avg }}$ of 256 amu.

Coordination number. Information on the coordination number comes from solvent swelling measurements and recent estimates made using n.m.r. of the number of non-peripheral group attachments to the cluster ${ }^{+0}$. There is some controversy about the meaning of the measurements of the solvent swelling ratio. Network theories have. however, been applied ${ }^{2-9}$ to relate the solvent swelling ratio. $Q$, and the average molecular weight of a cluster, $M_{\text {avg }}$, to the average molecular weight between branch points, $M_{\mathrm{c}}$. The $M_{\mathrm{c}}$ determinations ${ }^{2-9}$ suggest that there are between 4 and 8 repeating units (ring clusters) between branch points for coals with less than $90 \%$ carbon. (In this work the term crosslink has been used to indicate th: bridge which makes a ring cluster into a branch point: : This indicates a value for $\sigma+1$ between 2.13 and 2.25, since $\sigma+1$ is the average number of attachments per cluster (1 bridge makes two attachments). The n.m.r. data suggest that there are between 2 and 3 bridge or loop attachments per cluster (see Figure 8 of Ref. 40). This suggests $\sigma+1$ is between 2 and 3. Based on these two measurements, the coordination number for the starting coal for describing the break up of the network by bridge cleavage should be less than 3. and probably between 2.2 and 2.5. A different value of $\sigma+1$ might be appropriate for describing crosslinking (branch point formation at higher temperature). To model a high volatile bituminous coal. the different models used networks with $(\sigma+1)=3.25$ (DISARAY), 4.6 (CPD), and $\approx 2.1$ (FG-DVC).

Initial bridge population. $\mathrm{p}_{0}$. The starting macromolecular network for FG-DVC is chosen to match the measured extract yield and molecular weight between crosslinks by picking two parameters: the length of the linear oligomer chain. $l$; and the number of initial crosslinks (branch points) per monomer, $m_{0}$. First $m_{0}$ is picked such that $m_{0}=M_{\text {avg }} / M_{\mathrm{e}}$, where $M_{\mathrm{avg}}$ is the average ring cluster (monomer) molecular weight and $M_{c}$ is the molecular weight between crosslinks determined from solvent swelling ${ }^{2-9}$. Then $l$ is chosen so that when the molecule is randomly constructed, the weight per cent of oligomers less than $3000 \mathrm{amu}$ matches the measured extract yield. There is the implicit assumption that the extract yield is due to the unpolymerized fraction of a homogeneous network. Polymethylenes or highly fluid macerals (e.g. exinites), which can be a significant portion of the extracts in coal. should really be treated as separate components but were not in the first version of the FG-DVC model. The initial value of $x$ is approximately $\left((l-1) / l+m_{0}\right)$, which for the Pittsburgh seam coal modelled in Ref. 29 is $\alpha_{0} \approx 0.95$. This initial value is indicated in Figure 3a. In DISARAY, $p_{0}$ is set equal to $1\left(\alpha_{0}=1.63\right)$. This is illustrated in Figure $3 b$. 
The starting macromolecular network in the CPD model is chosen by picking two parameters: the coordination number $\sigma+1$, picked to match the average number of all connections (including peripheral groups in addition to bridges and loop attachments) per ring determined by n.m.r. ${ }^{30.40}$; and $p_{0}$, the starting probability of unbroken bonds. For the high volatile bituminous coal simulated in Ref. 30, $z_{0}=1 / 2 p_{0}$ $(\sigma+1)=1.36$. This initial value is indicated in Figure 3 .

\section{PROCESSES CONTROLLING THE NETWORK DECOMPOSITION}

This section considers the important processes in pyrolysis: bridge breaking, and hydrogen utilization; crosslinking (branch point formation): and the mass transport processes which control the distribution of oligomers into tar. extracts, liquids. and solids. The processes are summarized in Figure 4.

\section{Bridge breaking and hydrogen utilization}

Figure $4 a$ summarizes the bridge breaking assumptions of the three models. Both the FG-DVC and CPD models assume similar (within a factor of 3 ) bridge breaking rates, $0.86 \times 10^{15} \exp -(55400 / R T) s^{-1}$ for FG-DVC* and $2.6 \times 10^{15} \exp -(55400 / R T) s^{-1}$ for CPD. Both models employ rank independent kinetics. The FG-DVC model rate was determined in experiments in which particle temperatures were directly measured ${ }^{43}$. The rate was recently confirmed within a factor of 2 by Fletcher et $a l^{44}$ in a second experiment to directly measure particle temperatures. The DISARAY model* assumes a bridge dissociation rate which can produce monomers of $6 \times 10^{8} \exp -(30000 / \mathrm{RT}) \mathrm{s}^{-1}$. The monomers subsequently decompose at $1.4 \times 10^{7} \mathrm{exp}-(31000 / \mathrm{RT}) \mathrm{s}^{-1}$ to form tar.

In DISARAY, all the initial bridges can break. In FG-DVC and CPD, there is a process for creating unbreakable bridges associated with the bridge breaking process. The FG-DVC model includes three kinds of bonds: labile bridges, 'unbreakable bridges' (which do not form branch points), and crosslinks (which do form branch points). The unbreakable bridges are represented by the heavy horizontal lines in Figure 1. For each broken labile bridge, FG-DVC requires that hydrogen be available to stabilize the free radicals. It is assumed that all the donatable hydrogen (aliphatic plus hydroaromatic) is located in the labile bridges, so that only half the labile bridges can break with the other half becoming unbreakable with the donation of their hydrogen (i.e. there is a 1:1 ratio between the occurrence of bridge breaking and the formation of additional 'unbreakable bridges'). In the FG-DVC model, crosslinks are also considered to be bridges which cannot be broken. but are not in the 'unbreakable bridges' pool. The weight fraction of the initial bridges in the chain of length $/$ which are labile is given by the parameter $W_{B}$; the rest are assumed to be unbreakable bridges. $W_{B}$ is a fitting parameter chosen to make the model fit the pyrolysis data.

In a similar manner, in CPD there are both unbreakable bridges with probability $c_{0}$ and labile bridges with probability $L_{0}\left(L_{0}+c_{0}=p_{0}\right)$. As pyrolysis

- Both FG-DVC and DISARAY employ distributed activation energy expressions. The rates quoted above are for the centre of the distribution proceeds. the labile bridges can break and react by two possible routes to form unbreakable 'char' bridges or broken bridges. CPD assumes a 0.9:1.0 ratio of broken bridge to char bridge formation. That assumption is almost identical to the 1:1 ratio used in FG-DVC. In

\section{a Bridge Breaking}

DISARAY
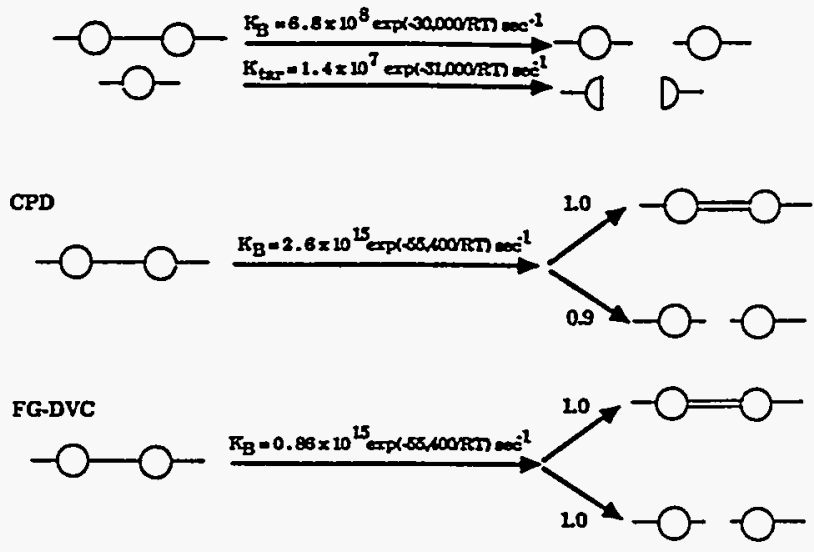

b Crosslinking

DISARAY

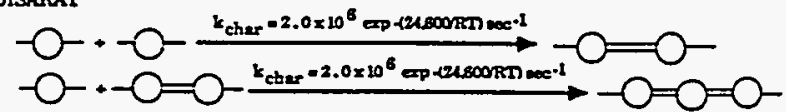

CPD

No Independers Croedinking Procem

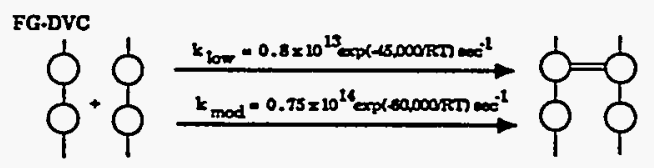

c Broduct Distribution

DISARAY

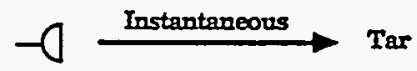

CPD
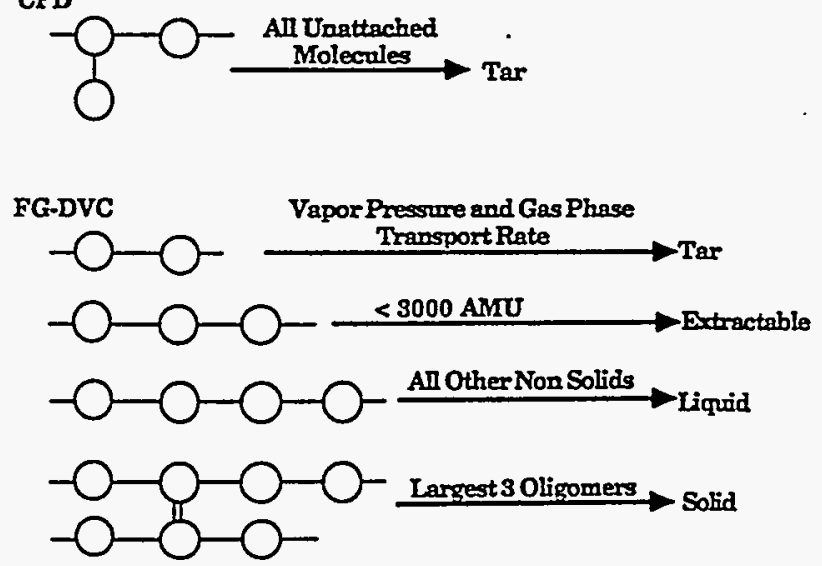

Figure 4 Summary of model assumptions for a, bridge breaking: $b$. crosslinking: c. product distribution 
CPD there is no distinction between unbreakable bridges and crosslinks. A branch point occurs any place there are three or more attachments per cluster.

\section{Crosslinking}

The crosslinking (branch point forming) reactions are summarized in Figure $4 b$. CPD does not define any distinct crosslinking processes. Char forming reactions produce unbreakable bridges as a consequence of bridge breaking. These reactions form branch points (three or more attachments per cluster) randomly depending on the coordination number. $\sigma+1$ and probability, $p$.

DISARAY assumes char formation occurs at a rate $2 \times 10^{6} \mathrm{exp}-(24600 / \mathrm{RT}) \mathrm{s}^{-1}$. Char formation is assumed to occur by monomers attaching to the original lattice or to each other.

FG-DVC assumes two independent crosslinking reactions. These are the only reactions which can form branch points in the Monte Carlo formulation. One process occurs at low temperature (below that for bridge breaking) for low rank coals and is associated with $\mathrm{CO}_{2}$ evolution ${ }^{11.12,45}$. Crosslinking also occurs at moderate temperatures, slightly higher than bridge breaking, and is associated with the evolution of $\mathrm{CH}_{4}$. The model assumes one crosslink is formed for each $\mathrm{CH}_{4}$ or $\mathrm{CO}_{2}$ evolved $^{29}$. The mechanistic basis for these assumptions has been discussed elsewhere ${ }^{25}$.

\section{Product distribution}

The product distribution assumptions are summarized in Figure $4 c$. The identification of different size oligomers with tar, extracts. liquids. and solids is related to their molecular weight. The oligomers which can form tar are the lightest fraction. Tar formation is controlled in part by the vapour pressure of the components. This idea is supported by the observation that tar yields are strongly influenced by external pressure 29.46 .47 .

Only oligomers with molecular weights less than 1000 amu have sufficient vapour pressure to become gas at typical pyrolysis tempratures, so tar is roughly limited to $<1000 \mathrm{amu}^{+7-51}$. The extract yield is controlled by the solubility of the oligomers. For coal fragments in pyridine this limit is roughly $3000 \mathrm{amu}^{47-49.52}$. Larger fragments appear to be important to the fluid properties of $\mathrm{coal}^{32}$.

In FG-DVC, the Monte Carlo calculation is employed to determine the molecular weight distribution in the decomposing char. Then. a mass transport equation is applied to determine the probability of the light $n$-mers evolving as tar. The transport equation assumes that a molecular weight dependent vapour pressure controls the appearance of these molecules in the gas phase and that they escape the coal particles by convective transport of the gas $^{29}$. Tar is thus the light end of the molecular weight spectrum, i.e. those with sufficiently high vapour pressures. This produces tar with number average molecular weights of $300-400 \mathrm{amu}$ and maximum weights of $800-1000 \mathrm{amu}$. Thus, in FG-DVC, tar is approximately the sum of 1-3 n-mers in Figure $3 a$. Extractable material is defined as all molecules up to $3000 \mathrm{amu}$ (sum of 1-10 $n$-mers) and liquids are defined as all molecules not attached to the starting network. An alternative definition. which has been employed here. is to assume the largest three molecules constitute the solid, and all the rest are liquid.

In DISARAY, tar is defined as half the monomer. and the monomer is taken as $1400 \mathrm{amu}$. Consequently, the tar would be defined as some fraction of the monomer curve in Figure $3 b$. No transport equations are employed in CPD. Tar is defined as all molecules not attached to the infinite lattice. Thus tar is represented by the highest line in Figure $3 c$.

One advantage of the Monte Carlo method over the percolation theory is that, when tar is produced. molecules can be removed from the network. In percolation theory, there is no consistent way to remove molecules from the network and to allow the formation of new bridges such as the crosslinking events in FG-DVC. CPD avoids this problem by excluding any independent crosslinking which would reconnect oligomers. This presents the limitation that independent crosslinking and mass transport cannot be treated with the exact percolation theory expressions.

\section{EXAMPLES OF MODEL CALCULATIONS}

\section{Formation of pyrolysis products}

The evolution of the macromolecular network in the CPD model is illustrated in Figure 5. Figure 5a shows the percolation theory predictions for the total of
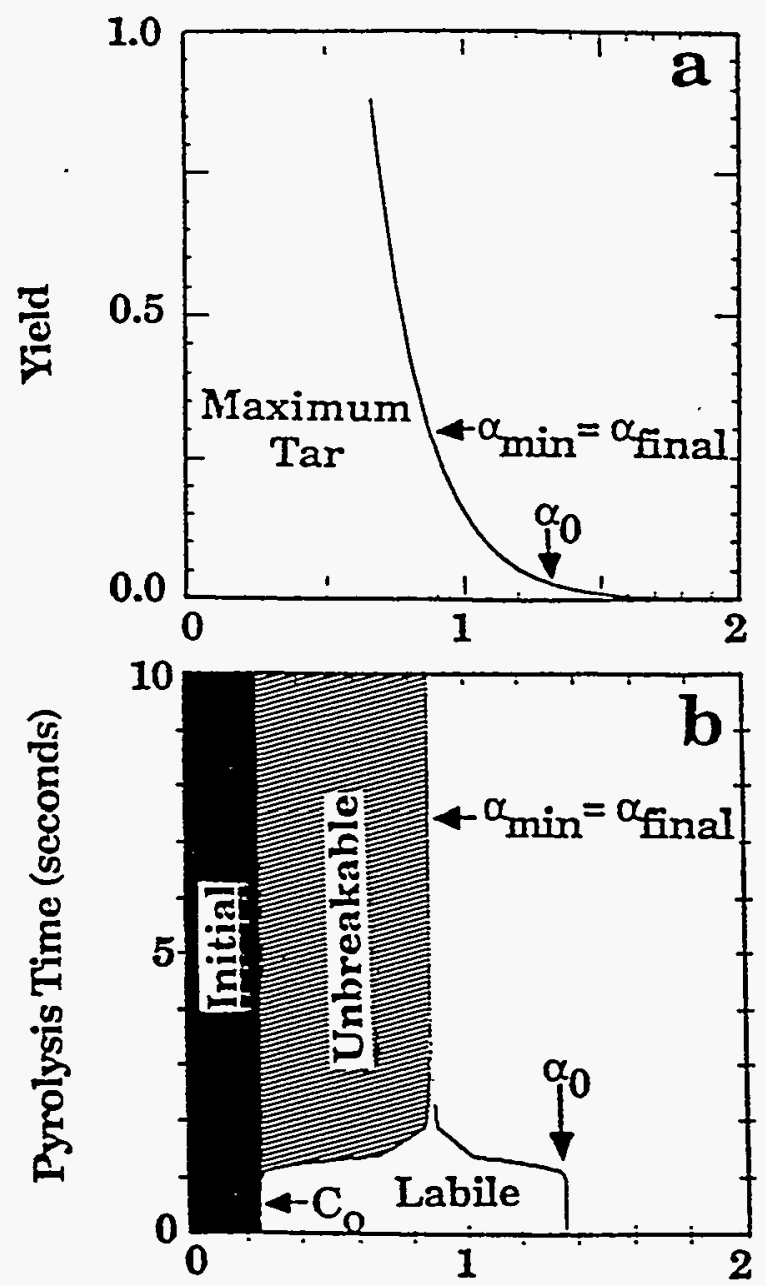

No. of UnbrokenBridges per Bead, $\alpha$

Figure 5 Tar yield for a bituminous coal predicted by the CPD model: a. tar yield versus $x ; b$, variation in $x$ with time, heating at $450^{\circ} \mathrm{Cs}^{-i}$ to $936 \mathrm{~K}$. The shaded areas show the relative amounts of the two types of crosslinks (initial: unbreakable bridge formation) 
Network models of coal thermal decomposition: P. R. Solomon et al.
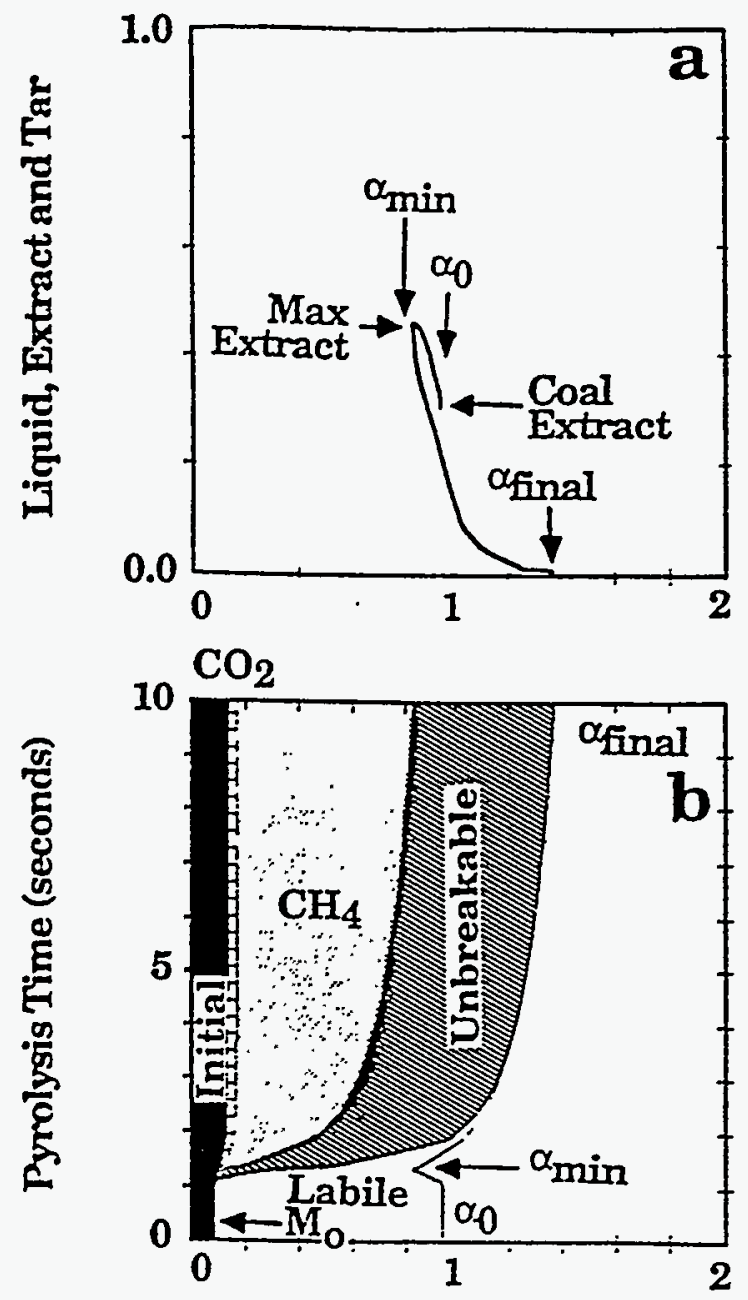

No. of UnbrokenBridges per Bead, $\alpha$

Figure 6 Extract yield for a bituminous coal predicted by the FG-DVC model: a. extract yield versus $\alpha: b$. variation in $z$ with time. heating at $450^{\circ} \mathrm{C} \mathrm{s}^{-1}$ to $936 \mathrm{~K}$. The shaded areas show the relative amounts of the three types of crosslinks (initial: crosslinks related to gas evolution: unbreakable bridge formation)

unattached oligomers (defined to be the tar) as a function of $\alpha$. The coal is represented at $p_{0}=0.59$ or $\alpha_{0}=1 / 2 p_{0}$ $(\sigma+1)=1.36$. During pyrolysis, the labile bridges form either broken bridges or unbreakable char bridges in the ratio 0.9 to 1.0 . Figure $5 b$ shows how $\alpha$ changes during pyrolysis. Pyrolysis proceeds until $\alpha_{\min }$ is reached. and all the labile bridges are either broken or have formed unbreakable bridges. Thus

$$
\alpha_{\min }=1 / 2(\sigma+1)\left(c_{0}+(1.0 / 1.9) L_{0}\right)=0.83
$$

and the change in $\alpha$ during pyrolysis was 0.53 . Note that $\alpha$ can only decrease in the CPD model.

The evolution of the macromolecular network for FG-DVC computed using the Monte Carlo method for a bituminous coal is illustrated in Figure 6. Figure $6 a$ shows the calculated extract yield as a function of $\alpha$. The initial probability of unbroken bridges, $\alpha_{0}$, starts out at close to 1.0 to produce the measured extract yield $(30 \%)$. Figure $6 b$ shows the computed value of $\alpha$ with its contributions from the initial crosslinks $m_{0}$, the conversion of labile bridges to broken bridges and unbreakable bridges and the added crosslinks related to gas evolution. For the bituminous coal. the added crosslinks are almost all due to $\mathrm{CH}_{4}$ related processes. $\alpha$ goes back up in the FG-DVC model to resolidify the lattice. This is necessary to model fluidity effects ${ }^{32}$.

Results of the FG-DVC model applied to a lignite are presented in Figure 7. For the lignite, the formation of low temperature crosslinks from $\mathrm{CO}_{2}$ evolution prevents $\approx$ from being reduced due to bridge breaking. Thus pyrolysis produces no additional extract yield. The thermosetting behaviour of the low rank coal, and the release of little tar or extracts is related to this low temperature crosslinking process.

\section{Utilization of donatable hydrogen}

As discussed above, $W_{B}$, the initial fraction of labile bridges, is a parameter of the FG-DVC model. This parameter is related to the fraction of donatable hydrogen by $H(d)=2 / 28 W_{B}$, i.e. there are two donatable hydrogens per labile bridge. This parameter has a strong effect on $x_{\min }$ and hence the yield of tar, extracts, and liquids.

There are two ways to estimate the amount of hydrogen donated. During pyrolysis the donation of hydrogen converts two aliphatic or hydroaromatic
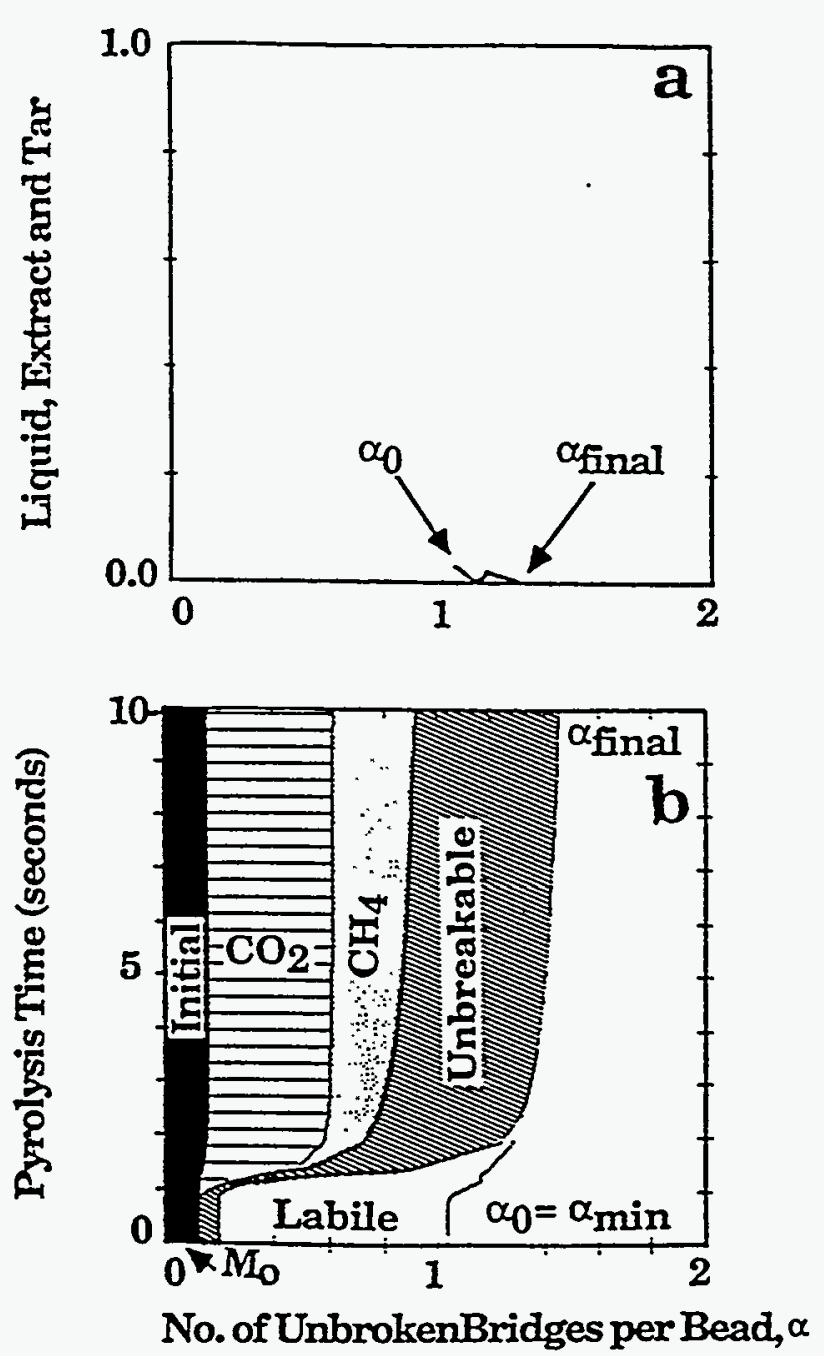

Figure 7 Extract yield for a lignite predicted by the FG-DVC model: a. extract yield versus $\alpha$; b. variation in $\alpha$ with time, heaing at $450^{\circ} \mathrm{C} \mathrm{s}^{-i}$ to $936 \mathrm{~K}$. The shaded areas show the relative amounts of the three types of crosslinks (initial: crosslinks related to gas evolution: unbreakable bridge formation) 


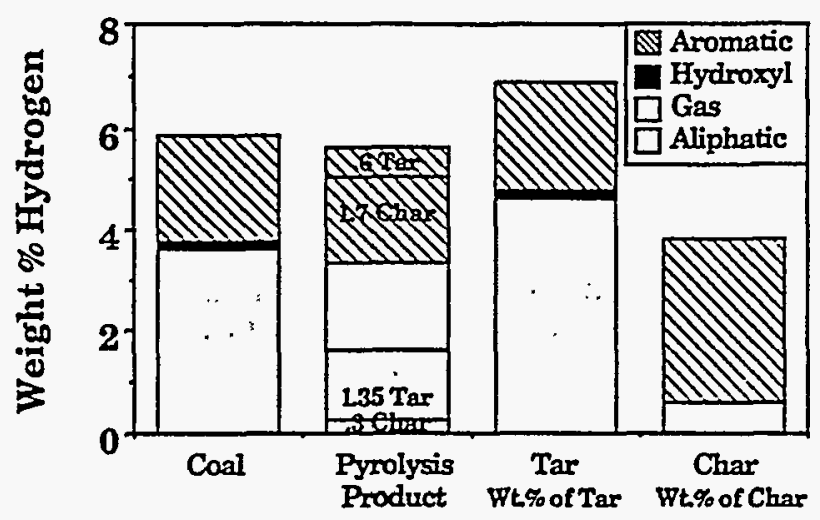

Figure 8 Distribution of hydrogen in coal and pyrolysis products. Pyrolysis produced approximately $53 \%$ char, $30 \%$ tar and $21 \%$ gas

hydrogens into a donated aliphatic hydrogen plus a newly formed aromatic hydrogen. The increase in aromatic hydrogen in the pyrolysis products, and the increase in aliphatic hydrogen in the tar, can both be measured using quantitative FT-i.r. analysis ${ }^{33.54}$. The results for a Pittsburgh seam coal are summarized in Figure 8. They show that the aromatic hydrogen in the total pyrolysis products increased from 2.1 to $2.4 \%$, or an increase of $0.3 \%$ on a starting coal basis. This increased aromatic content is all in the char. The aromatic content in the tar remains about the same. The tar, which is approximately $30 \%$ of the starting coal. increases its aliphatic hydrogen content by about $1 \%$ or $0.3 \%$ on the starting coal basis. The two numbers are thus consistent: $0.6 \%$ aliphatic or hydroaromatic hydrogens in the coal are converted to $0.3 \%$ new aromatics plus $0.3 \%$ donated. aliphatics. If it is assumed that a monomer has a molecular weight of $300 \mathrm{amu}$, then one breakable bridge per monomer with four aliphatic hydrogens is $1.33 \%$. Half the bridges can break $(0.67 \%)$ and the other half $(0.67 \%)$ can donate half its hydrogen $(0.34 \%)$, in reasonable agreement with the experimentally estimated value of $0.3 \%$ hydrogens actually donated. The value assumed ${ }^{29}$ in FG-DVC for $H(d)$ for the Pittsburgh seam coal is $0.67 \%$.

The value of $H(d)$ has implications for the CPD model. If there is only one labile bridge per monomer, then

$$
\begin{aligned}
\alpha_{\min } & =1 / 2(\sigma+1)\left(c_{0}+(1.0 / 1.9) L_{0}\right) \\
& =1 / 2(4.6)(0.37+(1.0 / 1.9) 0.22)=1.11
\end{aligned}
$$

rather than 0.83 . In this case, the value of $\sigma+1$ would have to be reduced to match the data. Also, the average molecular weight for the unattached molecules is too high to be identified as tar. If a more reasonable definition of tar is used (e.g. the sum of oligomers up to 3 ), then $\sigma+1$ has to be reduced still further.

\section{Comparison of Monte Carlo calculation with percolation theory}

To further illustrate some of the differences between the FG-DVC Monte Carlo model and percolation theory calculations, the extract yield calculated for a case similar to that in Figure $6 a$, but with tar evolution not permitted. is plotted in Figure 9 along with the predictions of percolation theory for several values of $\sigma+1$. The FG-DVC Monte Carlo prediction is not a single valued function of $\alpha$. As pyrolysis proceeds, the increase in extract yield follows $\sigma+1 \approx 2.2$ while the decrease in extract yield follows $\sigma+1 \approx 4$.

It is important to know whether this result is an artefact of the Monte Carlo calculation or a real feature of pyrolysis. Based on what is known to happen in pyrolysis, the result does make sense. For a bituminous coal. the initial process occurring in pyrolysis is bridge breaking. This occurs by breaking bridges in the network described by $\sigma+1$ between 2.1 and 2.5 . No crosslinking occurs initially as the solvent swelling ratio is observed to increase during this period ${ }^{45}$. Thus. the coordination number used in CPD, which includes all connections to the ring cluster (bridges, loops, and peripheral groups $)^{30.40}$. may not be appropriate to this phase of pyrolysis. Eventually crosslinks start forming, resulting in an increase in the coordination number and in $\alpha$. There is evidence that crosslinks form at peripheral group sites so that the coordination number used in CPD (the sum of branches and peripheral groups) is appropriate for this phase of the process. Consequently, the network cannot adequately be described by one type of bridge site with a single coordination number. There are bridge sites for labile bridges and for crosslinks, each with their own coordination number. This observation motivated the development of a more general percolation theory model.

\section{LATTICE MODEL WITL TWO BRIDGE BOND TYPES}

Two- $\sigma$ model

To deal with a structure with a time dependent coordination number. a Bethe lattice with two types of bridging bonds is considered, with coordination numbers and probabilities of occupation given by $\sigma_{1}+1, p$, and $\sigma_{2}+1, q$, for the two types. respectively. Such a lattice for $\sigma_{1}+1=\sigma_{2}+1=2$ is illustrated in Figure 10. The analysis can be carried through using the procedures described previously ${ }^{30.39}$, but with extensions to deal with the extra variables. The equations are presented in Appendix A.

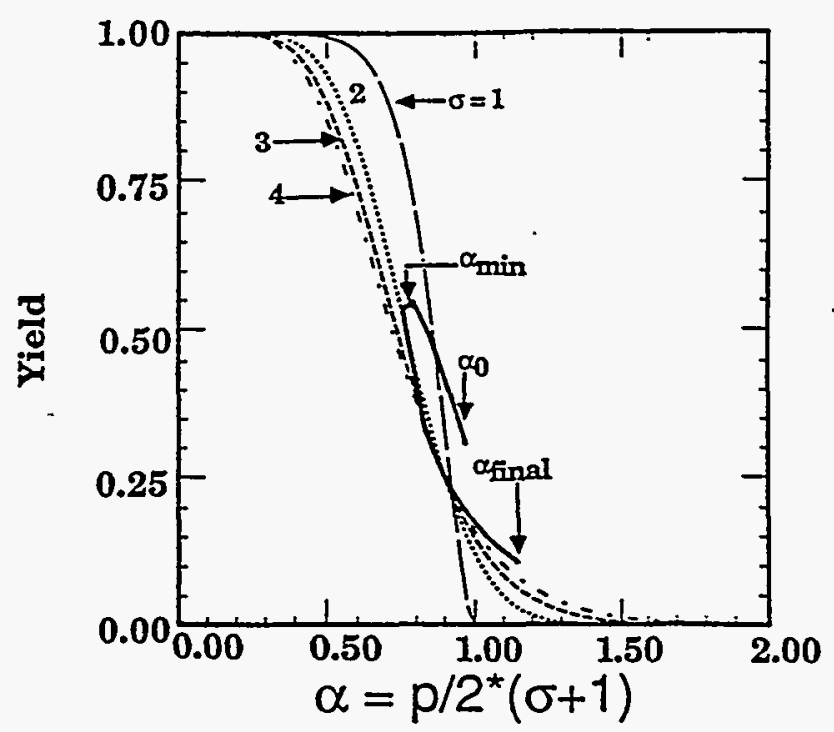

Figure 9 Comparison of extract yield predictions from FG.DVC model with percolation theory for $\sigma=1,2.3$ and 4. FG-DVC predictions are for Pittsburgh seam coal heated at $450^{\circ} \mathrm{C} \mathrm{s}^{-1}$ to $936 \mathrm{~K}$ with no tar evolved 


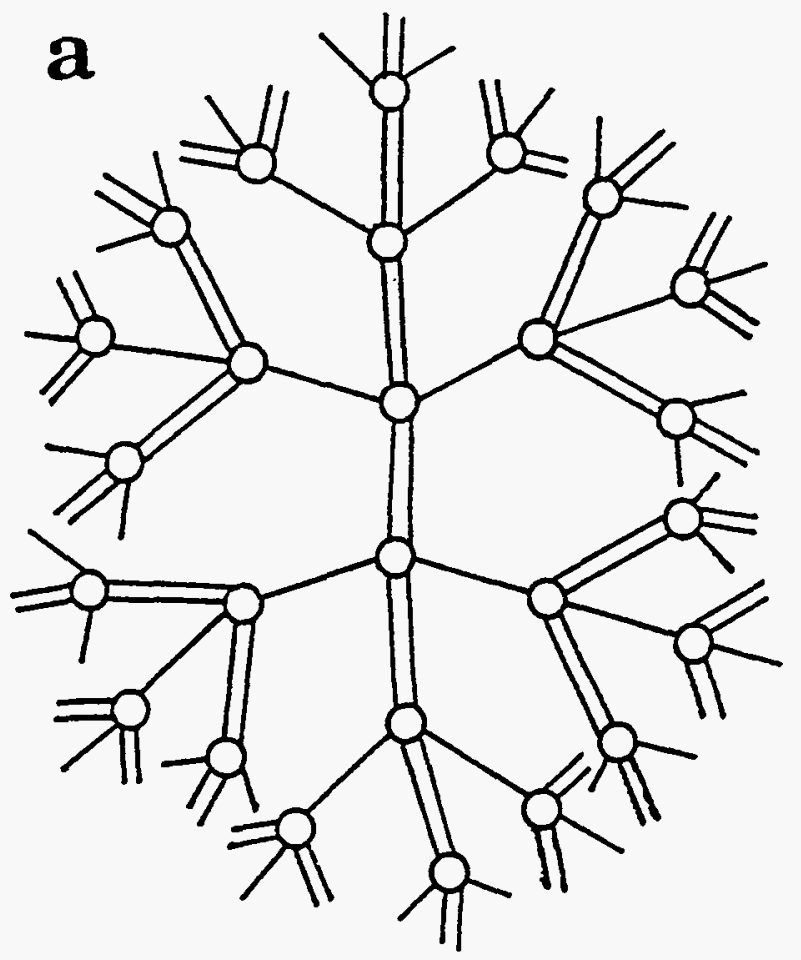

b

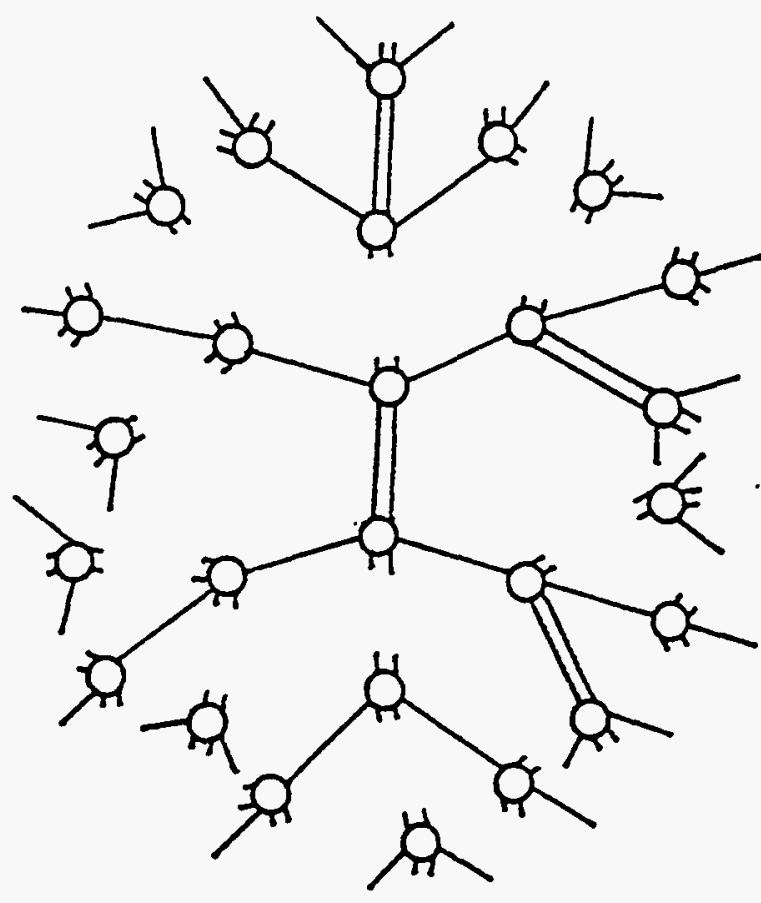

Figure 10 Bethe lattice for two- $\sigma$ model with $\sigma_{1}=1$ (shown as single bonds) and $\sigma_{2}=1$ (shown as double bonds): a. fully linked case $(p=q=1)$ is like one- $\sigma$ model with $\sigma=3 ; b$, shown with most double bonds (representing the crosslinks) not yet formed to represent the starting coal. This lattice is like a one $\sigma$ model with $\sigma=1$, linear chains

\section{Application of two- $\sigma$ model}

Figure 11 presents a comparison of the predictions for pyrolysis assuming the FG-DVC chemistry, using: the Monte Carlo calculation; the two- $\sigma$ percolation calculations $\left(\sigma_{1}+1=2, \sigma_{2}+1=2\right)$; and two cases of the one- $\sigma$ percolation calculation $(\sigma+1=2.2$ and $\sigma+1=3.2)$. The calculations are made under the assumption that no tar is evolved. The tar values in Figure 11 are the sum of 1-3n-mers remaining in the char. The Monte Carlo calculation in Figure $11 a$ is matched best by the two- $\sigma$ model if liquids are assumed to be the sum of the first $100 n$-mers (i.e. up to $300000 \mathrm{amu}$ ). The two- $\sigma$ model has a reasonable value for the initial extract yield but predicts slightly more initial tar. Neither of the one- $\sigma$ cases is a good match. Use of $\sigma+1=2.2$ is good at low temperature, but overpredicts the maximum values of extracts and liquids and resolidifies the network very abruptly at too low a temperature. Use of $\sigma+1=3.2$ does a much better job at predicting the maximum values of tar, extract and liquids, and resolidifies the network more slowly at a higher temperature, but the initial ratio of tar to extract is not consistent with that observed for coal and the rate of increase of $n$-mers is too slow. It thus appears that the two- $\sigma$ model can be used instead of the Monte Carlo calculations when no tar is evolved. while one- $\sigma$ calculations are less accurate.

The real test, however, is how well the models fit the data for coal. A comparison of tar yield is not a sufficient test since $x_{0}$ and $\Delta \alpha$ can always be selected in conjunction with the network geometry to fit the data. A critical test requires a careful comparison of how $\alpha_{0}$ and $\alpha(t)$ match with measurement of functional group changes in the char (e.g. the transformation of hydrogen functional groups and bridges), solvent swelling behaviour (i.e. crosslink density), and the complete molecular weight distribution as reflected in the amounts of tar, extracts. and fluidity.

\section{COMPARISON OF NETWORK MODELS}

A summary of the processes predicted by the three recent network models. CPD. DISARAY and FG-DVC is presented in Table 1 . All the models predict their primary objective. i.e. the variations in tar and gas yield with time and temperature. All three are capable of predicting
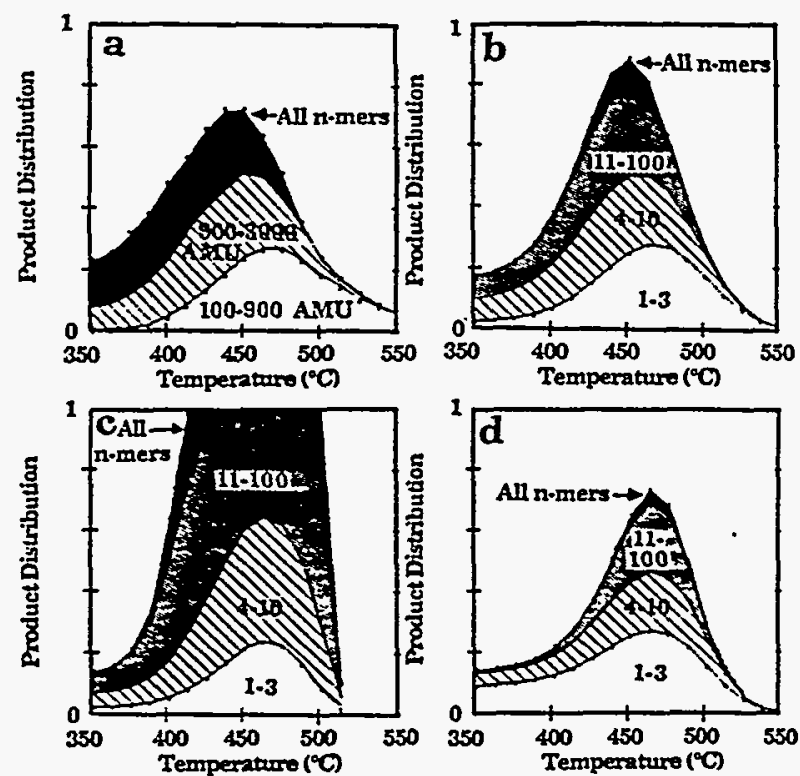

Figure 11 Comparison of distribution of $n$-mers for pyrolysis of upper Freeport coal at $3^{\circ} \mathrm{C}$ min $^{-1}:$ a, Monte Carlo calculation; b. two- $\sigma$ model $\left(\sigma_{2}=1, \sigma_{2}=1\right) ; c$, one- $\sigma$ model $(\sigma=1.2)$; d, one- $\sigma$ model $(\sigma=2.2)$ 
Table 1 Comparison of network models

\begin{tabular}{lllll}
\hline & & & FG-DVC \\
& CPD & DISARAY & or $2 \sigma$ & Relevant model process \\
\hline Tar yield versus time & Yes & Yes & Yes & Bond breaking \\
Extract yield versus time & No & No & Yes & Bond breaking \\
Gas yield versus time & Yes & Yes & Yes & From peripheral groups \\
Tar yield versus heating rate & Not yet & Yes & Yes & Relative rates of bond breaking and crosslinking \\
Variation of tar molecular weight with heating rate & No & No & Yes & Relative rates of bond breaking and crosslinking \\
Molecular weight of tar & No & No & Yes & Mass transport limitation \\
Tar yields versus pressure & No & No & Yes & Mass transport limitation \\
Molecular weight versus pressure & No & No & Yes & Mass transport limitation \\
Solvent swelling of char & No & No & Yes & Crosslinking \\
\hline
\end{tabular}

- All oligomers are defined as tar

variations of tar yield with heating rate, but $C P D$ has not yet done this. All three models are capable of predicting the complete molecular weight distributions of fragments. but only FG-DVC uses this information to predict the extract yield. the tar yield and the tar molecular weight distribution. DISARAY uses only the prediction for monomers (defined as tar precursor) and CPD uses only the prediction for all oligomers (defined as tarl. In a recent paper ${ }^{32}$, the total oligomer population computed by the FG-DVC model was used to predict coal nuidity behaviour. Only FG-DVC employs a mass transport equation which is necessary to predict tar molecular weights and the variations of yield and molecular weights with pressure. Only FG-DVC predicts the solvent swelling ratio, which is determined by the crosslink density in the char.

\section{CONCLUSIONS}

This paper examines the extension of macromolecular network concepts to describe coal thermal decomposition. The statistical methods (Monte Carlo calculations and percolation theories) and the classes of chemical reactions (labile bridge breaking, hydrogen utilization, crosslinking) and mass transport (vaporization and convection) employed by different researchers have been compared. The conclusion are as follows:

1. The application of macromolecular network concepts appears to be a very promising and versatile approach.

2. Monte Carlo methods for computing the network statistics are the most versatile, but are computationally demanding.

3. The use of percolation theory is computationally eflicient and helps provide insight into network behaviour, but the use of a fixed coordination number may be inadequate to accurately describe coal thermal decomposition. The network appears to require a coordination number between 2.2 and 2.5 during labile bridge breaking and greater than 3 during crosslinking.

4. Alternatively, a more general percolation theory model for a network with two types of bridging bonds was developed, each with their own $\sigma+1$.

5. When the two- $\sigma$ percolation model is applied using the FG-DVC chemistry to cases in which tar is not removed, it is much more flexible in matching the Monte Carlo calculations. The one- $\sigma$ models either decompose at too low a temperature for large values of $\sigma+1$, or decompose too much and resolidify at too low a temperature for small values of $\sigma+1$. Applying percolation theory to cases where tar is removed requires additional approximations.

6. Of the three models compared (CPD, DISARAY, and FG-DVC), FG-DVC is the most complete in treating the molecular weight distribution of network fragments and the processes of vaporization and mass transport to define tar. the tar molecular weight distribution and the extract

7. Of the three models. FG-DVC is the most closely related with the previous concepts of coal as a macromolecular network by requiring that the model predict the coal solvent swelling ratios and measured extract yields. The assumptions which define the parameters of the starting network are open to question and must be explored.

8. Future efforts should focus on identifying the chemistry for the processes of bridge breaking, low temperature crosslinking, moderate temperature crosslinking, and hydrogen utilization.

\section{ACKNOWLEDGEMENTS}

The authors acknowiedge support for the work provided by the Morgantown Energy Technology Center of the Department of Energy under Contract No. DE-AC21$86 \mathrm{MC} 23075$.

\section{REFERENCES}

1 van Krevelen. D. W. in 'Coal', Elsevier, Amsterdam. The Netherlands. 1961

2 Green. T. K.. Kovac, J. and Larsen. J. W. Fuel 1984. 63. 935

3 Brenner. D. Fuel 1985. 64, 167

4 Lucht. L. M. and Peppas. N. A. Fuel 1987, 66, 803

5 Lucht. L. M.. Larsen. J. M. and Peppas, N. A. Energy \& Fuels 1987. 1.56

$6)$ Larsen. J. W. Am. Chem. Soc. Div. Fuel Chem. Prepr. 1985, 30(4). 444

7 Green. T., Kovac. J.. Brenner. D. and Larsen. J. in Coal Structure (Ed. R. A. Meyers). Academic Press. NY. USA. 1982. p. 199

8 Hall. P. J.. Marsh. H. and Thomas. K. M. Fuel 1988, 67, 863

9 Sanada. Y. and Honda. H. Fuel 1966, 45, 295

10 Suuberg. E. M.. Yoshi. O. and Deevo. S. Am. Chem. Soc. Div. Fuel Chem. Prepr. 1988. 33(1). 387

11 Suuberg, E.M., Lee. D. and Larsen.J. W. Fuel 1985,64, 1668

12 Suuberg. E. M.. Unger, P. E. and Larsen. J. W. Energy \& Fuels 1987, 1, 305

13 Brown. J. K.. Dryden. I. G. C.. Dunevein. D. H. et al. J. Inst. Fuel 1958. 31, 259 
14 Oming, A. A. and Greifer, B. Fuel 1956, 35, 318

15 Solomon. P. R. and Hamblen, D. G. in 'Chemistry in Coal Conversion' (Ed. R. H. Schlosberg), Plenum Press, New York, USA, chapter 5, p. 121

16 Solomon. P. R. 'New Approaches in Coal Chemistry'. ACS Symposium Series 169.ACS, Washington. DC. USA. 1981, p.61

17 Solomon, P. R. and King, H. H. Fuel 1984. 63. 1302

18 Gavalas, G. R. in 'Coal Pyrolysis' Elsevier, NY, USA, 1982. p. 51

19 Gavalas. G. R., Cheong, P. H. and Jain. R. Ind. Eng. Chem. Fundam. 1981, 20, 122

20 Squire. K. R.. Solomon, P. R., Carangelo. R. M. and DiTaranto, M. B. Fuel 1986. 65. 833

21 Squire, K. R., Solomon P. R.. DiTaranto. M. B. and Carangelo, R. M. Am. Chem. Soc. Div. Fuel Chem. Prepr. 1985. 30(1), 386

22 Solomon, P. R.. Squire, K. R. and Carangelo. R. M. 'Proc. Int. Conf. on Coal Science', Pergamon. Sydney, Australia. 1985. p. 945

23 Solomon, P. R. and Squire. K. R. Am. Chem. Soc. Div. Fuel Chem. Prepr. 1985. 30(4). 347

24 Niksa. S. and Kerstein, A. R. Comb. and Flame 1986, 66, 95

25 Niksa. S. Comb. and Flame 1986. 66. 111

26 Solomon. P. R.. Hamblen. D. G., Deshpande. G. V. and Serio. M. A. 'Proc. Int. Coal Science Conference', Elsevier. NY, USA. 1987, p. 601

27 Solomon. P. R., Hamblen. D. G., Carangelo. R. M. et al. Comb. and Flame 1988, 71, 137

28 Niksa. S. and Kerstein. A. R. Fuel 1987, 66, 1389

29 Solomon. P. R., Hamblen, D. G., Carangelo, R. M. et al. Energy \& Fuels 1988, 2, 405

30 Grant, D. M., Pugmire, R. J., Fletcher, T. H. and Kerstein, A. R. Energy \& Fuels $1989,3,175$

31 Serio. M. A., Solomon. P. R., Yu. Z. Z. et al. Am. Chem. Soc. Div. Fuel Chem. Prepr. 1988, 33(3), 91

32 Solomon. P. R., Best. P. E.. Yu. Z. Z. and Deshpande. G. V. Am. Chem. Soc. Div. Fuel Chem. Prepr. 1989. 34(3). 895

33 Nielsen. L. E. in 'Mechanical Properties of Polymers and Composites', Volume 2. Marcel Dekker. Inc.. NY. USA. 1974

34 Bartels. C. R.. Crist. B.. Felters. L. J. and Graessley. W. W. . Wacromolecules $1986,19,785$

Nazem. F. F. Fuel $1980,59,851$

Macosko. C. W. Brit. Polymer Journ. 1985, 17, 239

Flory, P. J. J. Am. Chem. Soc. 1941, 63. 3083, 3097; 'Principles of Polymer Chemistry', Cornell University Press. Ithaca. NY. USA. 1953, chapter 9

38 Stockmayer, W. H. J. Chem. Phys. 1943. 11, 45: 1944. 12. 125

39 Fisher. M. E. and Essam. J. W. J. Math. Phvs. 1961. 2. 609

40 Solum. M.. Pugmire, R. J. and Grant. D. M. Energy \& Fuels $1989,3,40$

41 Gerstein. B. C. Murphy, D. P. and Ryan, L. M. in Coal Structure' (Ed. R. A. Meyers), Academic Press, NY, USA, 1982. chapter 4, p. 87

42 Hooker, D. T. II, Lucht. L. M. and Peppas, N. A. Ind. Eng. Chem. Fundam. 1986. 25. 103

43 Solomon, P. R., Serio, M. A.. Carangelo, R. M. and Markham. J. R. Fuel 1986, 65. 182

44 Fletcher, T. H. Comb. Sci. and Tech. 1989, 63, 87

45 Solomon. P. R., Serio, M. A., Deshpande. G. V. and Kroo, E. Energy \& Fuels submitted for publication; Deshpande. G. V. Solomon. P. R. and Serio, M. A. Am. Chem. Soc. Dic. Fuel Chem. Prepr. 1988, 33(2), 310

46 Unger, P. E. and Suuberg, E. M. '18th Symposium (Int) on Combustion', The Combustion Institute, Pittsburgh, PA, USA, 1981. p. 1203

47 Suuberg, E.M., Unger, P. E. and Lilly, W.D. Fuel 1985.64.956

48 Unger, P. E. and Suuberg. S. E. Fuel 1984, 63, 606

49 Oh. M. S., Peters, W. A. and Howard, J. B. AIChE J. 1989. 35(5), 775

50 Serio, M. A. Ph.D. Thesis Massachusetts Institute of Technology, Cambridge, MA. USA. 1984
51 Khan. M. R.. Serio, M. A.. Malhotra. R. and Solomon. P. R. Am. Chem. Soc. Div. Fuel Chem. Prepr. 1989, 34(4), 1054 52 Fong, W.S. Peters, W. A. and Howard, J. B. Fuel 1986. 65.251

53 Solomon. P. R. and Carangelo, R. M. Fuel 1982, 61, 663

54 Solomon. P. R. and Carangelo. R. M. Fuel 1988, 67, 949

\section{APPENDIX A}

The probability $F_{\text {s.u }}(p, q)$, that a site is a member of a cluster of $n$ sites with $s$ type 1 bridges and $u$ type 2 bridges is given by

$$
F_{\mathrm{s}, u}(p, q)=a_{\mathrm{s}, u} p^{\mathrm{s}}(1-p)^{\mathrm{t}} q^{\mathrm{u}}(1-q)^{\mathrm{v}}
$$

where

$$
\begin{aligned}
& n=u+s+1 \\
& \tau=\left(\sigma_{1}+1\right) n-2 s \\
& v=\left(\sigma_{2}+1\right) n-2 u
\end{aligned}
$$

$\tau$ and $v$ are the number of broken bridges of type 1 and 2 , respectively, on the perimeter of the cluster, and $a_{5,4}$ is the number of different ways to form such a cluster. Following the procedure used by Fisher and Essam. an expression for the configuration coefincient can be derived

$$
a_{s, u}=\frac{\left(\sigma_{1}+1\right)\left(\sigma_{2}+1\right)}{(s+\tau)(u+v)} \Gamma(s+\tau+1) \Gamma(u+v+1)(u+s+1)
$$

where $\Gamma$ is a gamma function. Note that for $u=0$ (no type 2 bonds), this reduces to the quantity $n b_{n}$ in Ref. 30 . To determine the probability, $F_{\mathrm{n}}(p, q)$ that a given site is a member of a cluster of $n$ sites. i.e. the fraction of $n$-mers, Equation (1) must be summed over all possible values of $s$ and $u$ that give an $n$-site cluster:

$$
F_{\mathrm{n}}(p, q)=\sum_{s=0}^{n-1} a_{\mathrm{s}, \mathrm{u}} p^{\mathrm{s}}(1-p)^{\mathrm{z}} q^{\mathrm{u}}(1-q)^{\nu} \quad u=n-s-1
$$

The total fraction of sites, $F(p, q)$ in finite clusters is the sum over all $s$ and $u$

$$
F(p, q)=\sum_{s=0}^{x} \sum_{u=0}^{\infty} F_{s, u}(p, q)=\left(\frac{1-p}{1-p^{*}}\right)^{\sigma_{1}+1}\left(\frac{1-q}{1-q^{*}}\right)^{\sigma_{2}+1}
$$

where $p^{*}$ and $q^{*}$ are obtained by finding the least roots of

$$
\begin{aligned}
& p^{*}\left(1-p^{*}\right)^{\sigma_{1}-1}\left(1-q^{*}\right)^{\sigma_{2}+1}-p(1-\dot{p})^{\sigma_{1}-1}(1-q)^{\sigma_{2}+1}=0 \\
& q^{*}\left(1-q^{*}\right)^{\sigma_{2}-1}\left(1-p^{*}\right)^{\sigma_{1}+1}-q(1-q)^{\sigma_{2}-1}(1-p)^{\sigma_{1}+1}=0 .
\end{aligned}
$$

The critical point at which an infinite lattice begins to form (i.e. $F(p, q)$ begins to decrease) becomes a critical curve which divides the $p-q$ plane into two regions. Note that for $q=0$, the equations all reduce to the single $\sigma$ case given in Ref. 30 . 
Appendix $M$

An Empirical Model for Coal Fluidity Based on a Macromolecular Network

Pyrolysis Model 
An Empirical Model for Coal Fluidity Based on a Macromolecular Network Pyrolysis Model 
References

Baxter, L. L., "Turbulent transport of particles," Ph.D. dissertation, Brigham Young University, Provo, UT, (1989).

Baxter, L. L., Smith, P. J. and Smoot, L. D., "Optimization of coal devolatilization model parameters for comprehensive gasifiction and combustion models," Western States Section/The Combustion Institute, Banff, Alberta, Canada, April 27-30, (1986).

Bilger, R. W., "Chemical reaction calculations in turbulent flows: application to a CO-containing turbulent plume," Adv. in Geophysics, 188, 349 (1974).

Bilger, R. W., "Turbulent jet diffusion flames," Prog. Energy Comb. Sci., 1, 87 (1976).

Bird, R. B., Stewart, W. E. and Lightfoot, E. N., Transport phenomena, Wiley, New York (1960).

Borghi, R., "Chemical reaction calculations in turbulent flows: application to a cocontaining turbulent plume," Adv. Geophysics, 188, 349 (1974).

Bose, A. C., Dannecker, K. M. and Wendt, J. O. L., "Coal domposition effects on mechamisms governing the destruction of NO and other nitrogenous species during fuel-rich combustion," Energy \& Fuels, 2, 301 (1988).

Boussinesq, J., "Theorie de l'ecoulement tourbillant," Mem. Acac. Sci., 23(No. 46), (1877).

Bowman, C. T., "Kinetics of pollutant formation and destruction in combustion," Prog. in Energy Comb. Sci., 1, 1 (1975).

Brewster, B. S., Baxter, L. L. and Smoot, L. D., "Treatment of coal devolatilization in comprehensive combustion modeling," Energy \& Fuels, 2, 362-370 (1988).

Brewster, M. Q. and Kunitomo, T., "The optical constants of coal, char, and limestone," J. Heat Trans./Trans. ASME, 106, 678-83 (1984).

Bueters, K. A., Cogoli, J. G. and Habelt, W. E., "Performance prediction of tangentially fired utility furnaces by computer módel," Fifteenth Symposium (International) on Combustion, The Combustion Institute, Pittsburgh, PA, 1245-1260 (1974). 
Caretto, L. S., "Mathematical modeling pollutant formation," Prog. Energy Comb. Sci., 1, (1976).

Carlson, B. G. and Lathrop, K. D., "Computing methods in reactor physics," Transport theory - the method of discrete ordinates, Eds, Gordon Breach Science Publishers, New York, (1968).

Carnahan, B., Luther, H. A. and Wilkes, J. O., Applied numerical methods, John Wiley and Sons, New York, NY (1969).

Castro, I. P., The numerical prediction of recirculating flows in numerical methods in laminar and turbulent flow, Pentech Press, London (1978).

Chen, S. L., Pershing, D. W. and Martin, G. B., "Influence of coal composition on the fate of volatile and char nitrogen during combustion," Nineteenth Symposium (International) on Combustion, The Combustion Institute, Pittsburgh, PA, 1271 (1982).

Crowe, C. T., Sharma, M. P. and Stock, D. E., "The particle-source-in-cell (PSICELL) model for gas-droplet flows, "J. Fluids Eng., Trans. of the ASME, 99, 325-332 (1977).

DeMarco, A. G. and Lockwood, F. C., "A new flux model for the calculation of radiation in furnaces," LaRivista dei Combustibili (Italian Flame Day), 29, 184 (1975).

DeSoete, G. G., Overall reaction rates of NO and N2 formation from fuel nitrogen, 15th Symposium (International) on Combustion, The Combustion Institute, Pittsburgh, PA, 1093-1102 (1975).

Field, M. A., Gill, D. W., Morgan, B. B. and Hawksley, P. G. W., Combustion of pulverized coal, The British Coal Utilisation Research Association, Leatherhead, Surrey, England (1967).

Fletcher, T. H., "Theoretical modeling of reacting coal particles in pulverized coal combustion and gasification," M.S. thesis, Brigham Young University, Provo, UT, (1980).

Fletcher, T. H., "A two-dimensional model for coal gasification and combustion," Ph.D. dissertation, Brigham Young University, Provo, UT, (1983). 
Freihaut, J. D. and Proscia, W. M., First International Conference on Combustion Technologies for a Clean Environment, Vilamoura (Algarve), Portugal, (1991).

Gallagher, R. H., Oden, J. T., Taylor, C. and Zienkiewicz, O. C., Finite elements in fluids volume 1 and 2, John Wiley and Sons, London (1975).

Ghani, M. U. and Wendt, J. O. L., "Early evolution of coal nitrogen in opposed flow combustion configurations," Twenty-Third Symposium ( International) on Combustion, The Combustion Institute, Pittsburgh, Orléans, France, July 22-27, 1281-1288 (1990).

Godridge, A. M. and Read, A. W., "Combustion and heat transfer in large boiler furnaces," Prog. Energy Combust. Sci., 2, 83-95 (1976).

Goetz, G. J., Nsakala, N. Y., Patel, K. L. and Lao, T. C., "Combustion and gasification kinetics of chars from four commercially significant coals of varying rank," Second Annual Conference on Coal Gasification, EPRI, Palo Alto, CA, October, (1982).

Gosman, A. D. and Lockwood, F. C., "Incorporation of a flux method for radiation into a finite-difference procedure for furnace calculations," in Fourteenth Symposium (International) on Combustion, The Combustion Institute, Pittsburgh, PA, University Park, PA, August 20-25, 1972, 661671 (1973).

Gosman, A. D., Lockwood, F. C. and Salooja, A. P., The prediction of cylindrical furnaces gaseous fueled with premixed and diffusion burners, $17 \mathrm{th}$ Symposium (International) on Combustion, The Combustion Institute, (1978).

Gosman, A. D., Pun, W. M., Ruchal, A. K., Spalding, D. B. and Wolfshstein, $\mathrm{R}$., Heat and mass transfer in recirculating flows, Academic Press, London (1969).

Gosman, A. D. and Pun, W. M., Picture notes for course entitled calculation of recirculating Flows, Imperial College, London (December 1973).

Hadvig, S., "Gas emissivity and absorptivity: a thermodynamic study," J. Inst. Fuel, 129-135 (April 1970). 
Haussmann, G. J. and Kruger, C. H., "Evolution and reaction of fuel nitrogen during rapid coal pyrolysis and combustion," Western States Section/The Combustion Institute, Livermore, CA, (1989).

Hobbs, M. L., Radulovic, P. T. and Smoot, L. D., "Modeling fixed-bed coal gasifiers," AIChE Journal, 38, 681-702 (1992).

Hottel, H. C. and Sarofim, A. F., Radiative transfer, New York, McGraw-Hill (1967).

Huber, A. M., "Effect of sorbent on sulfur pollutant species in an entrained-flow coal gasifier," M.S. thesis, Brigham Young University, Provo, Utah, (1989).

Iverach, D., Basden, K. S. and Kirov, N. Y., "Formation of nitric oxide in fuellean and fuel-rich flames," Fourteenth Symposium (International) on Combustion/The Combustion Institute, Pittsburgh, PA, 767 (1973).

Jamaluddin, A. S. and Smith, P. J., Prediction of radiative heat transfer in cylindrical furnaces, Western States and Canadian Sections, The Combustion Institute, Banff, Canada, April, (1986).

Jamaluddin, A. S. and Smith, P. J., "Predicting radiative transfer in axisymmetric cylindrical enclosures using the discrete ordinates method," Comb. Flame, (1987).

Jayatilleke, C. L. V., "The influence of Prandtl number and surface roughness on the resistance of the laminar sub-layer to momentum and heat transfer," Prog. Heat Mass Trans., 1, 193-237 (1969).

Jones, W. P. and Launder, B. E., "The calculation of low-Reynolds-number phenomena with a two-equation model of turbulence," Int. J. Heat Mass Transfer, 16, 1119-1130 (1973).

Jones, W. P. and Launder, B. E., "The prediction of laminarization with a twoequation model of turbulence," Int. J. Heat Mass Transfer, 15, 301-314 (1972).

Kent, J. H. and Bilger, R. W., "The prediction of turbulent diffusion flame fields and nitric oxide formation," 16th Symp. (International) on Combustion, The Combustion Institute, Pittsburgh, PA, 1643-1656 (1977). 
References

Kent, J. H. and Honnery, D. R., "A soot formation rate map for a laminar ethylene diffusion flame," Comb. Flame, 79, 289-298 (1990).

Khalil, E. E., Spalding, D. B. and Whitelaw, J. H., "The calculation of local flow properties in two-dimensional furnaces," Int. J. Heat and Mass Trans., 18, 775-791 (1975).

Kobayashi, H., "Devolatilization of coal at high temperatures," Ph.D. dissertation, M.I.T., Cambridge, MA, (1976).

Kuo, K. K., Principles of combustion, John Wiley, New York (1986).

Launder, B. E. and Spalding, D. B., Mathematical models of turbulence, Academic Press, London (1972).

Lavoie, G. A., Heywood, J. B. and Keck, J. C., "Experimental and theoretical study of nitric oxide formation in internal combustion engines," Comb. Sci. Tech., 1, 313 (1970).

Leonard, B. P., "A stable and accurate convective modeling procedure based on quadratic upstream interpolation," Computer Methods in Applied Mechanics and Engineering, 19, 59-98 (1979).

Leschiziner, M. A., "Practical evaluation of three finite difference schemes for the computation of steady-State recirculating flows," Computer Methods in Applied Mechanics and Engineering, 23, 293-312 (1980).

Levy, J. M., Chen, L. K., Sarofim, A. F. and Béer, J. M., NO/char reactions at pulverized coal flame conditions, 18th Symposium (International) on Combustion, The Combustion Institute, Pittsburgh, PA, 111-120 (1981).

Libby, P. A., "On turbulent flows with fast chemical reactions part III: twodimensional mixing with highly dilute reactants," Combust. Sci. Tech., 13, 79-98 (1976).

Lillington, J. N., "Numerical methods in laminar and trubulent flow," A comparison of finite difference methods for the prediction of temperature in recirculating flows in rod cluster geometry, Pentech Press, London, 515525 (1978). 
Lilly, D. G. and Rhode, D. L., A computer code for swirling turbulent axisymmetric recirculating flows in practical isothermal combustor geometries, NASA Contractor Report 3442, prepared for Lewis Research Center under Grant NAG 3-74, (1982).

Lilly, G. P., "Effect of particle size on particle eddy diffusivity," Ind. Eng. Chem. Fund., 12, 268-275 (1973).

Lockwood, F. C. and Naguib, A. S., "The prediction of the fluctuations in the properties of free, round-Jet, turbulent, diffusion flame," Comb. Flame, 24, 109-124 (1975).

Lockwood, F. C., Salooja, A. P. and Syed, S. A., "A prediction method for coalfired furnaces," Comb. Flame, 38, 1-15 (1980).

Longwell, J. P. and Weiss, M. A., "Mixing and distribution of liquids in highvelocity air streams," Ind. Eng. Chem., 45, 1-15 (1980).

Lowe, A., Wall, T. F. and Stewart, I., "A zoned heat transfer model of large tangentially fired pulverized coal boilers," Fifteenth Symposium (International) on Combustion, The Combustion Institute, Pittsburgh, PA, 1261-1270 (1974).

Lowes, T. M., Bartelds, H., Heap, M. P., Michelfelder, S. and Pai, B. R., "The prediction of radient heat transfer in axisymmetrical systems," Report No. GO2/a/25, International Flame Research Foundation, IJmuiden, The Netherlands, (1973).

Melville, E. K. and Bray, N. C., "A Model of the two-phase turbulent jet," Int. J. Heat and Mass Trans., 22, 647-656 (1979).

Merrick, D., "Mathematical models of the thermal decomposition of coal. 2. Specific heats and heats of reaction," Fuel, 62, 540-546 (1983).

Mie, G., "Optics of turbid media," Ann. Phys., 25, 377-445 (1908).

Miller, J. A. and Bowman, C. T., "Mechanism and modeling of nitrogen chemistry in combustion," Prog. Energy Combust. Sci., 15, 287 (1989).

Mitchell, J. W. and Tarbell, J. M., "A kinetic model of nitric oxide formation during pulverized coal combustion," AIChE J., 28, 302-311 (1982). 
References

Mitchell, R. E., "Determination of the intrinsic reactivities of pulverized-coal chars at high temperature," (1988).

Mitchell, R. E., "The influence of the mineral matter content of coal on the temperatures and burning rates of char particles during pulverized coal combustion," Sixth Annual International Pittsburgh Coal Conference Proceedings, Pittsburgh, PA, September 25-29, 32-52 (1989).

Nicoletti, P. A., METCEC - USER'S MANUAL, Final Report, DOE-METC Contract No. DE-AC21-85MC21353, EG\&G WASC, Inc., Morgantown, WV, (1986).

Nicoletti, P. A., METCEC - PROGRAM LOGIC MANUAL, Final Report, DOEMETC Contract No. DE-AC21-85MC21353, EG\&G WASC, Inc., Morgantown, WV, (1986).

Patankar, S. V. and Spalding, D. B., "A calculation procedure for heat, mass and momentum transfer in three-dimensional parabolic flows," International Journal of Heat and Mass Transfer, (Oct. 1972).

Patankar, S. V. and Spalding, D. B., Heat and mass transfer in boundary layers, Intertext Books, London (1970).

Patankar, S. V., Numerical heat transfer and fluid flow, Hemisphere Publishing, Washington (1980).

Patanker, S. V., "Studies in converction," in Chapter 1, Numerical prediction of three - d i m e n s i o n a l f l o w s , Ed, Academic Press, London, (1975).

Peck, R. E., Glarborg, P. and Johnsson, J. E., Comb. Sci. Tech., 76, 81 (1991).

Pratt, D. T., "Mixing and chemical reaction in continuous combustion," Prog. Energy Comb. Sci, 73-86 (1976).

Pratt, D. T. and Wormeck, J. J., CREK A computer program for calculation of combustion reaction equilibrium and kinetics in laminar or turbulent flows, WSU-COE-B-76-341, Washington State Univ., Pullman, WA, (1976). 
Raithby, G. D., "Skew upstream differencing schemes for problems involving fluid flow," Computer Methods in Applied Mechanics and Engineering, 9, 153-164 (1976).

Roache, P. J., Computational fluid dynamics, Hermosa Publishers, Albuquerque, NM (1976).

Sarofim, A. F. and Hottel, H. C., "Radiative transfer in combustion chambers: Influence of alternative fuels," Sixth International Heat Transfer Conference, Toronto, Canada, (1978).

Sarofim, A. F. and Pohl, J. H., "Kinetics of nitric oxide formation in premixed laminar flames," Fourteenth Symposium (International) on Combustion/The Combustion Institute, Pittsburgh, PA, 739 (1973).

Siegel, R. and Howell, J. R., Thermal radiation heat transfer, Hemisphere Publishing, New York (1981).

Silcox, G. D., "Analysis of the SO2-lime reaction system: Mathematical modeling and experimental studies emphasis on stoker applications," Ph.D. dissertation, The University of Utah, (1985).

Sloan, D. G., Smith, P. J. and Smoot, L. D., "Modeling of swirl in turbulent flow systems," Prog. Energy Comb. Sci., 12, 63-250 (1986).

Smith, P. J., "Theoretical modeling of coal and gas-fired turbulent combustion and gasification process," Ph.D. dissertation, Brigham Young University, Provo, UT, (1979).

Smith, P. J., Hill, S. C. and Smoot, L. D., "Theory for NO formation in turbulent coal flames," 19th Symposium (International) on Combustion, The Combustion Institute, Pittsburgh, PA, 1263 (1982).

Smith, P. J., Fletcher, T. H. and Smoot, L. D., "Model for pulverized coal-fired reactors," Eighteenth Symposium (International) on Combustion, The Combustion Institute, Pittsburgh, PA, Waterloo, Canada, Aug 17-22 1980, 1285-1293 (1981).

Smoot, L. D. and Horton, M. D., "Exploratory studies of flame and explosion quenching," Final Report, Bureau of Mines Contract 101220052, Brigham Young University, Provo, UT, (1978). 
Smoot, L. D. and Pratt, D. T., Pulverized coal combustion and gasification, Plenum, New York (1979).

Smoot, L. D. and Smith, P. J., Coal combustion and gasification, Plenum, New York (1985).

Smoot, L. D. and Smith, P. J., "Comprehensive modeling of combustion systems," Proceedings of the 1987 ASME/JSME Thermal Engineering Joint Conference, eds. P. J. Marto and I. Tanasawa, American Society of Mechanical Engineers, 1, Honolulu, HI, (1987).

Solomon, P. R., Hamblen, D. G., Carangelo, R. M., Serio, M. A. and Deshpande, G. V., "General model of coal devolatilization," Energy \& Fuels, 2, 405-422 (1988).

Solomon, P. R., Serio, M. A., Carangelo, R. M. and Markham, J. R., "Very rapid coal pyrolysis," Fuel, 65, 182-194 (1986).

Spalding, D. B., "Concentration fluctuations in a round turbulent free jet," Chem. Engr. Sci., 26, 95-107 (1971).

Spalding, D. B., "A general theory of turbulent combustion," J. Energy, 2, 16-23 (1978).

Spalding, D. B., Turbulence modeling: solved and unsolved problems, turbulent mixing in nonreactive and reactive flows, Ed, Plenum Press, New York, NY, (1975).

Dave, F. V., "Subroutine for computing the parameters of the electromagnetic radiation scattered by a sphere, 360 D-17, 4.00Z," IBM Corporation, (1968).

Tennekes, H. and Lumley, J. L., A first course in turbulence, The MIT Press, Cambridge, MA (1972).

Thompson, D., Brown, T. D. and Beér, J. M., "Formation of NO in a methane-air flame," Eighteenth Symposium (International) on Combustion/The Combustion Institute, Pittsburgh, PA, 787 (1981).

Truelove, J. S., Differential equation models of radiative heat transfer, AERE R8364, AERE Harwell, U.K., (1976). 
Truelove, J. S., Evaluation of a multi-flux model for radiative heat transfer in cylindrical furnaces, AERE R-9100, AERE Harwell, U.K., (1978).

Truelove, J. S. and Jamaluddin, A. S., "Models for rapid devolatilization of pulverized coal," Combustion and Flame, 64, 369-372 (1986).

Ubhayakar, S. K., Stickler, D. B., von Rosenberg, C. W. and Gannon, R. E., "Rapid devolatilization of pulverized coal in hot combustion gases," 16 th Symposium (International) on Combustion, The Combustion Institute, Pittsburgh, PA, 427-436 (1976).

Van Doormaal, J. P. and Raithby, G. D., "Enhancements of the SIMPLE method for predicting incompressible fluid flows," Num. Heat Transf., 7, 147-163 (1984).

Varma, S. A., "Radiative heat transfer in a pulverized-coal flame," Chapter 5 in Pulverized coal combustion and gasification, Eds., Plenum Press, New York, NY, 83-106 (1979).

Varma, S. A. and Pratt, D. T., Anisotropic and multiple scattering of thermal radiation in pulverized coal combustors, 17 th Symposium (International) on Combustion, Pittsburgh, PA, (1978).

Walker, P. L. J., Rusinko, F. J. and Austin, L. G., "Gas reactions of carbon," in Eley, D. D., Selwood, P. W. and Weisz, P. B., ed., Advances in catalysis, Volume XI, ed., Academic Press, New York, 133 (1959).

Wallis, G. B., One-dimensional two-phase flow, McGraw-Hill, New York (1969).

Wen, C. Y., Chen, H. and Onozaki, M., User's manual for computer simulation and design of the moving bed coal gasifier, Final report DOE/MC/164741390, U.S. Dept. of Energy, Morgantown, WV, (1982).

Wendt, J. O. L., Bose, A. C. and Hein, K. R. G., "Fuel nitrogen mechanisms governing NOx abatement for low and high rank coals," 1988 Joint Symposium on Stationary Combustion NOx Control, San Francisco, CA, March 609, (1989).

Westenberg, A. A., "Kinetics of NO and $\mathrm{CO}$ in lean, premixed hydrocarbons air flows," Comb. Sci. Tech., 9, 59 (1971). 
References

Williams, F. A., Combustion theory, Addison-Wesley, Reading, MA (1965).

Wiser, W. H., Hill, G. R. and Kertamus, N. J., Ind. Eng. Chem. Process Des. Develop., 6, 133 (1967).

Wormeck, J. J., "Computer modeling of turbulent combustion in a longwell jetstirred Reactor," Ph.D. dissertation, Washington State University, (1976).

Yoon, H., "Modeling and analysis of moving bed coal gasifiers," Ph.D. dissertation, University of Delaware, Newark, DE, (1978).

Zeldovich, Y. B., Sadovnikov, P. Y. and Frank-Kamentskii, D. A., "Oxidation of nitrogen in combustion," translated by Academy of Sciences of USSR, (1947). 


\section{Appendix A}

\section{Description of Subroutines}

A description of the routines used in PCGC-2 is given $:: ?$ this appendix. The subroutines are grouped according to function. Entry points are shown with the name of the subroutine to which they belong in parentheses. Descriptions of routines contained in the FG-DVC submodel are found in Chapter 6.

\section{Main Program}

PCGC-2 Main driver routine for PCGC-2. Reads basic input data for grid site, inlet conditions, etc. Calls all CALC subroutines for calculating gas variables, as well as PSICT and FLIVY. Determines convergence criterion and stops program when converged.

\section{Auxiliary Subroutines}

BLOCKDATA

CPUTM
Blockdata subprogram.

Returns the elapsed CPU time. 
Description of Subroutines

FLINT

GRDGRF

GRID

GRMAP

HEATBAL

INFLOW

INIT

PRINT

PROFIL

PROG

RESTRT

VECTOR
Initializes flow field so calculations can begin. Also initializes inlet densities.

Makes visual output of grid for interactive program users.

Sets up numerical grid used to solve the equations.

Reads location of user-specified grid points from data file. Also writes calculated grid points into data file.

Calculates wall radiative heat transfer and overall heat and material balances.

Reads flowrates and properties of incoming streams.

Initializes FORTRAN arrays for all field variables.

Prints output according to specified format.

Reads in parameters governing inlet profiles of $U$ (axial velocity), V (radial velocity), W (tangential velocity), TE (turbulence kinetic energy), and ED (eddy dissipation rate), and then initializes the inlet boundary.

Writes progress file.

This subroutine does all the work necessary for reading and writing information necessary to restart a combustion calculation from a partially converged solution.

Initializes a vector (2-dimensional array) to a uniform value. Can be called on the fly in a symbolic debugger. Useful for program debugging. 
AIM

CAIACJ

CALAHJ

CALCED

CALCET

CALCF

CAICG

CALCGE
Matrix-solving routine. LISOLV is nearly always used instead of AIM. AIM should be considered in cases where convergence is slow or unattainable with LISOLV. AIM takes full-directional coupling into account (5-diagonal matrix). LISOLVE is faster when it works.

Calculates coefficients for Eulerian finite difference equation for $\mathrm{ACJ}$ at each grid point.

Calculates coefficients for Eulerian finite difference equation for $\mathrm{AHJ}$ at each grid point.

Calculates coefficients for finite difference equation for eddy dissipation and solves for the field values of eddy dissipation at each grid point.

Calculates coefficients for finite difference equation for eta (coal gas mixture fraction) and solves for the field values of eta at each grid point.

Calculates coefficients for finite difference equation for $f$ (inlet gas mixture fraction) and solves for the field values of $f$ at each grid point.

Calculates coefficients for finite difference equation for $G$ (variance of the coal gas mixture fraction) and solves for the field values of $G$ at each grid point.

Calculates coefficients for finite difference equation GETA (variance of the coal gas mixture fraction) and solves for the field values of GETA at each grid point. 
$\mathrm{CALCH}$

CALCNJ

CALCP

CALCPP

CALCTE

CALCU

CALCV

CALCW

CALCYFU

LISOLV

MODED (PROMOD)
Calculates coefficients for finite difference equation for $\mathrm{H}$ (gas enthalpy) and solves for the field values of $\mathrm{H}$ at each grid point.

Calculates coefficients for finite difference equation for NJ (bulk particle number density for jth particle type) and solves for the field values of NJ at each grid point.

Calculates coefficients for finite difference equation for $P$ (pressure) and solves for the field values of $P$ at each grid point (SIMPLER algorithm).

Calculates coefficients for finite difference equation for PP (pressure correction) and solves for the values of PP at each grid point (SIMPLE algorithm).

Calculates coefficients for finite difference equation for $\mathrm{K}$ (turbulence kinetic energy) and solves for the field values of $\mathrm{K}$ at each grid point.

Solves for the field values of $U$ (axial velocity) at each grid point.

Solves for the field values of $V$ (radial velocity) at each grid point.

Calculates coefficients for finite difference equation for $\mathrm{W}$ (tangential velocity) and solves for the field values of $W$ at each grid point.

Calculates coefficients for finite difference equation for YFU (fuel mass fraction) and solves for the field values of YFU at each grid point.

The tri-diagonal matrix solving routine (Based on Thomas Algorithm). See description of AIM.

Modifies coefficients and source terms for boundary nodes so as to take boundary conditions into account for eddy dissipation. 
MODETA

MODF (PROMOD)

MODG (PROMOD)

MODGET (PROMOD)

MODH

MODP (PROMOD)

MODPP (PROMOD)

MODTE

MODU

MODV (PROMOD)
Modifies coefficients and source terms for boundary nodes so as to take boundary conditions in to account.

Modifies coefficients and source terms for boundary nodes so as to take boundary conditions into account.

Modifies coefficients and source terms for boundary nodes so as to take boundary conditions into account.

Modifies coefficients and source terms for boundary nodes so as to take boundary conditions into account for GETA.

Modifies coefficients and source terms for boundary nodes so as to take boundary conditions into account for $\mathrm{H}$ (gas enthalpy).

Modifies coefficients and source terms for boundary nodes so as to take boundary conditions into account for $P$ (pressure).

Modifies coefficients and source terms for boundary nodes so as to take boundary conditions into account for PP (pressure correction).

Modifies coefficients and source terms for boundary nodes so as to take boundary conditions into account for $\mathrm{K}$ (turbulence kinetic energy).

Modifies coefficients and source terms for boundary nodes so as to take boundary conditions.into account for $U$ (axial velocity).

Modifies coefficients and source terms for boundary nodes so as to take boundary conditions into account for $V$ (radial velocity). 
MODW (PROMOD)

MODYFU (PROMOD)

MOSOLV

UCOEF

VCOEF

WALL
Modifies coefficients and source terms for boundary nodes so as to take boundary conditions into account for $\mathrm{W}$ (tangential velocity).

Modifies coefficients and source terms for boundary nodes so as to take boundary conditions into account for YFU (fuel mass fraction).

Modified Operator Strongly Implicit (MOSI) Scheme, a fast elliptic solver. Alternative to LISOLV.

Calculates coefficients for finite difference equation for $U$ (axial velocity).

Calculates coefficients for finite difference equation for $V$ (radial velocity).

Handles intrusion boundary conditions for $f$ (inlet gas mixture fraction), $\eta$ (coal gas mixture fraction, $g_{f}$ (variance in inlet gas mixture fraction), $g_{\eta}$ (variance in coal gas mixture fraction), $y_{f u}, p$ (pressure), and $p^{\prime}$ (pressure correction).

\section{Gas-Phase. Chemistry}

ADCOND Function which adds (if possible) a pure condensed species or an ideal solution based on lowering Gibbs free energy.

API

Auxiliary function used in thermochemical equilibrium computations.

CALC

Auxiliary subroutine for the CREE thermochemical equilibrium computations. 
CHGCON

CREE

CREEO

DGELG

DIF

ERATIO (SPECE)

ERF

ETAST (TBLFE)

ETASTH (TBLFEH)

ETASTO (TABLE)
Replaces the solid phase of a species with the liquid phase of the same species (or vise versa), depending on the current temperature. CHGCON returns .TRUE. if a change was made.

This is the main controlling routine for the thermochemical equilibrium calculations (Chemically Reacting Equilibrium, from Elemental balances).

This is strictly an initializing routine, the sole function of which is to read, store, and process data from input files. The initial statements in CREE0 cause branching to appropriate chapters, depending on which of the key words ELEM, THER, REAC, or a blank field are encountered. Data fields must be in the order given, as element data are needed in order to catalog thermodynamic data.

Gaussian elimination subroutine.

Function that compares two numbers and returns their positive fractional difference.

Calculates fuel/air equivalence ratio given atom numbers using positive and negative oxidation states (valences).

Calculates the area under the normal curve from a spline fit. This is used for values of intermittency in the turbulent combustion model.

Calculates stoichiometric $\eta$ (coal gas mixture fraction) for adiabatic reactor.

Calculates stoichiometric $\eta$ (coal gas mixture fraction) for non-adiabatic reactor.

Calls either ETASTH or ETAST. 
FAZCHK

FGTABL

FRZFE

FRZFEH

FSTE

FSTF

FSTFE

FSTFEH

FSTFH

GASFIX
Determine whether the current active list of pure condensed phase species violates the phase rule.

Calculates limits of normalized Gaussian PDF.

Calculates gas properties as a function of $f$ (inlet gas mixture fraction) and $\eta$ (coal gas mixture fraction) using frozen equilibrium scheme when the local equivalence ratio is greater than 2.0 (adiabatic reactor).

Calculates gas properties as function of $f$ (inlet gas mixture fraction), $\eta$ (coal gas mixture fraction), and $h_{r}$ (residual gas enthalpy, using frozen equilibrium scheme when the local equivalence ratio is greater than 2.0.

Calculates stoichiometric mixture fraction (adiabatic reactor with no inlet gas mixing).

Calculates stoichiometric mixture fraction (gas only and adiabatic reactor).

Calculates stoichiometric mixture fraction (adiabatic reactor with $f$ and $\eta$ ).

Calculates stoichiometric mixture fraction (using $f$, $\eta$, and $h_{r}$ ).

Calculates stoichiometric mixture fraction using $f$ (inlet gas mixture fraction) and $h_{r}$ (residual gas enthalpy) (gas only).

Called if a singular matrix is encountered and the latest condensed phase to be added has already been removed. GASFIX changes the moles of all gas phase species whose current moles equals zero to $1.0 \mathrm{E}-6$. 
GAUSS

GIBCHK

GTDELN

GTETA

HCALC

HCPS

MATRIX

MIXFE

MU

PROPS

RANGOK

REMPUR
Driver routine for DGELG, the Gaussian elimination subroutine.

Removes the condensed phase species that have the least contribution to lowering the system's Gibbs free energy. This subroutine is called only if the phase rule is being violated.

Calculates the updates to the species mole numbers for gas and solution species. The update for the pure condensed species comes directly from the solving of the iteration matrix. GTDELN also calls GTETA which calculates the control factor.

Calculates the control factor.

Calculates HSUBO from the reactant cards.

Calculates specific heat, enthalpy, and entropy for a species at a specified temperature.

Sets up the iteration matrix.

For given mixture fraction ( $\mathrm{F}$ and ETA), calculates fully mixed product without chemical reaction (adiabatic reactor).

Calculates Gibbs free energy for each species.

Calculates gas-phase properties from the cardinal variables, including turbulence effects when appropriate.

Returns .TRUE. if the current temperature is within the temperature range for the specified species.

Removes a pure condensed phase species from the equilibrium calculation. The species status, moles, the number of pure condensed species, and the SOLELE array are also updated. 
Description of Subroutines

SOLPOS

Determines the position of a specified solution in the active solution list.

SORTG

Sorts the pure condensed phase species in the active list in order of their contribution to the minimization of Gibbs free energy (from the species with the least contribution to the species with the largest contribution).

SPECE

The chemical equilibrium subroutine.

SYSERR

Called if a fatal error occurs in the equilibrium computations. It prints out a diagnostic message and stops program execution.

TABLE

Sets up table of equilibrium gas properties $\left(\rho_{g}, M_{i}\right.$, $T_{g}$ ) as a function of $f$ (inlet gas mixture fraction), $\eta$ (coal gas mixture fraction), and/or $h_{r}$ (residual gas enthalpy).

TBLE

Sets up gas equilibrium properties table as function of $\eta$ (coal gas mixture fraction) (adiabatic reactor and no inlet gas mixing).

TBLF

Sets up gas equilibrium properties table as function of $f$ (inlet gas mixture fraction) (gas only and adiabatic reactor).

TBLFE

Sets up table of equilibrium gas properties as function of $f$ (inlet gas mixture fraction) and $\eta$ (coal gas mixture fraction).

TBLFEH

Sets up table of equilibrium gas properties as a function of $f$ (inlet gas mixture fraction), $\eta$ (coal gas mixture fraction), and $h_{r}$ (residual gas enthalpy).

TBLFH

Sets up table of equilibrium gas properties as function of $f$ (inlet has mixture fraction) and $h_{r}$ (residual gas enthalpy) (gas only). 
TBLRSTFE

TBLRSFEH

TUBFEH

UPDATE (TABLE)

UPF (TBLF)

UPFE

UPFEH

UPFH
Reads and writes restart file for table of equilibrium gas properties.

Reads and writes restart file for table of equilibrium gas properties as function of $f$ (inlet gas mixture fraction), $\eta$ (coal gas mixture fraction), and $h_{r}$ (residual gas enthalpy).

Calculates the required change in THIDEL or TMAX for table generation if the upper temperature limit is being exceeded.

Interpolates table values for gas properties.

Interpolates one-dimensional $(f)$ gas properties table.

Interpolates two-dimensional ( $f$ and $\eta$ ) gas properties table.

Interpolates three-dimensional $\left(f, \eta\right.$, and $\left.h_{r}\right)$ gas properties table.

Interpolates two-dimensional ( $f$ and $h_{r}$ ) gas properties table.
B

CALCNO
Uses a cubic spline to find the reaction order for the homogeneous NO formation reaction based on the local oxygen concentration.

Calculates coefficients for finite difference equation for YNO (NO mass fraction), and solves for the field values of $Y N O$ at each grid point. 
Description of Subroutines

CALCO2

CALHCN

CALNH3

EQSPEC

FREACT

INTGRT

NOXMN

NOXDATA

RATE

RSTNOX

RXNEXT

RXNRAT
Calculates the mean $\mathrm{O}_{2}, \mathrm{~N}_{2}$, and $\mathrm{OH}$ concentrations based on species or atomic mass balances.

Computes and assembles finite difference equations for the $\mathrm{HCN}$ species continuity equation; solves for the field of predicted HCN concentrations.

Computes and assembles finite difference equations for the $\mathrm{NH}_{3}$ species continuity equation; solves for the field of predicted $\mathrm{NH}_{3}$ concentrations.

Calculates the equilibrium concentrations of nitrogen and oxygen by convolving over the probability density functions of $f$ and eta. This is necessary since there is a possibility that their storage arrays were replaced by other information if "AIM" was used in PCGC2.

Calculates the maximum possible concentrations of $\mathrm{HCN}, \mathrm{NH}_{3}$, and $\mathrm{NO}$ from fuel nitrogen conversion.

Computes integrated average dry mole fraction at each axial node for final writeout.

Reads input cards for the NO model; controls NO model logical sequence; tests for convergence; prints out intermediate and final results.

Blockdata subprogram for data initialization.

Calculates the mean reaction rates and species continuity source terms.

Reads and writes to restart file.

Calculates reaction progress variables.

Manages homogeneous chemical reaction rates for all mechanisms. 


\section{Plotting}

CLOSDB

INTP

LOADI

LOADSC

OVENDB

OVTPUT

PLTWRT

REGRID

SPREAD

STREAM

VELMAG
Closes a database for the CEQUIL post-processor.

Interpolates to find vertices from nodes for CEQUIL post-processor.

Copies integer elements into their proper place in the output array for the CEQUIL post-processor.

Copies real (scalar) elements into their proper place in the output array for the CEQULL post-processor.

Opens up a database for either read or write for CEQUIL post-processor.

Writes out in binary format the essential properties for the CEQUIL post-processor.

Writes Eulerian output into appropriate output files. The output files can be used later for plotting.

Takes irregularly spaced grid values and converts them to equal-spaced, square grid values. Linear interpolation is used.

Subroutine for writing out gas variables for spreadsheet plotting. Can be called on the fly from a symbolic debugger. Useful for program debugging.

Calculates stream lines from values of $U$ (axial velocity) and $\mathrm{V}$ (radial velocity) for plotting purposes.

Calculates the magnitude of the total velocity vector at each node for plotting. 
WRITCO

WRITGE

WRITSC

WRITVE
Writes the control variables for the CEQUIL postprocessor.

Writes the $x, y$, and $z$ values of each of the nodes for the CEQUIL post-processor.

Writes a scalar function for the CEQUIL postprocessor.

Writes a vector function for the CEQUIL postprocessor.

$S O_{x} /$ Sorbents Submodel

CALCSJ

CALH2S

CALSO2

SDNRST

SLFRST

SORB0

SORPAR

SOXDATA
Calculates sorbent particle number density.

Solves finite difference equation for $\mathrm{H}_{2} \mathrm{~S}$ species continuity.

Solves finite difference equation for $\mathrm{SO}_{2}$ species continuity.

Reads and writes to sorbent particle number density restart file.

Reads and write to sulfur species restart file.

Reads in input data and initializes sorbent particle number density.

Main submodel driver. Calculates source terms for $\mathrm{SO}_{2}$ and $\mathrm{H}_{2} \mathrm{~S}$ (sulfur entering with the coal or gas inlet streams). Determines if convergence is obtained. Prints out final results.

Block data subprogram foł variable initialization. 
$\mathrm{SO}_{\mathrm{x}} /$ Sorbents Submodel

SBVALIN (SPROPS)

SBVALOT (SPROPS)

SPSICT

SULFAT

SULFHS

Particle Phase

BVALIN (BPROPS)

BVALOUT (BPROPS)

COALO

COAL1

COAL2
Writes reasonable values into intrusions which border the flowfield in order to allow for 2-D interpolation of gas properties to sorbent particle position.

Replaces boundary values with original values.

Tracks sorbent particles through the reactor. Calculates source terms for sulfur capture.

Calculates the change in conversion of calcined $\mathrm{CaCO}_{3}$ particles to $\mathrm{CaSO}_{4}$ according to the shrinking-core model of Silcox (1985).

Calculates the change in conversion of calcined $\mathrm{CaCO}_{3}$ particles to $\mathrm{CaS}$ according to the shrinkingcore model of Silcox (1985).

Inserts reasonable properties into intrusions which border the flow field in order to allow for particle phase 2-D property interpolation.

Replaces boundary values with original properties.

Reads input coal parameters and sets up initial coal properties.

Calculates gas phase properties at a point in the reactor, such as thermal conductivity; diffusivity, and viscosity. These properties are needed in COAL2.

Calculates coal reaction rates and other auxiliary parameters from coal and gas properties at a point. 
COAL2S (COAL2)

EOLP

EULINT (PSICTO)

FIND

GRAD

PEJ

PRTSUR

PSCRAT

PSICT

PSICTO

PSOLVE
Calculates coal reaction rates and other auxiliary parameters form coal and gas properties at a point. Used when particle energy equation goẹs stiff. Pseudo steady-state assumption is used.

Driver routine for solving Eulerian or Lagrangian particle equations. Eulerian particles are not debugged in this version of PCGC-2. The Lagrangian option is currently the only viable option.

Initializes Eulerian particle number density fields.

This is a short extrapolation subroutine used by SIGMAM.

Calculates gradient of particle number density for particle dispersion submodel.

Function for calculating vapor pressure and enthalpy of vaporization for liquid component of slurry and fuel oils.

Calculates the surface area of char particles required for heterogeneous-NO decay reactions.

Calculates rate of homogeneous reactions for intermittent primary, secondary and coal off-gas.

Interpolates gas property information for each point of the Lagrangian particle information. Integrates particle velocity to get particle trajectory, and calls PSOLVE to integrate particle continuity and energy equations. Saves particle source terms for gas phase.

Reads initial particle information and initialized particle trajectory parameters.

Solves particle energy and continuity equations for reacting particles. 
PSOLVP (PSOLVE)

PSOLVS (PSOLVE)

SIGMAD

SIGMAM

Radiation Submodel
This subroutine is used when using an Eulerian particle solution (not currently implemented). Prepares the Eulerian data so that an appropriate call to each of the coal subroutines can be made. Takes the resulting coal computations and stores them in Eulerian arrays. Computes the associated source terms for the gas-particle coupling.

Solves particle energy and continuity equations for reacting particles when the particle energy equation is stiff.

Used by COAL1 for determining the value of the potential function needed for mass diffusivity as a function of the Lennard-Jones parameters and temperature.

Used by COAL1 for determining the value of the potential function needed for viscosity and thermal conductivity as a function of the Stockmayer parameters and temperature.
DISORD

EICO2

EIH2O

FLUX
Calculates radiant wall heat fluxes and flux-sums to the volume elements using the discrete ordinates approximation.

Calculates the emissivity of carbon dioxide from the Hottel charts.

Calculates the emissivity of water vapor from the Hottel charts.

Main driver routine to calculate all of the radiation flux sums. 
FLUX0

FLUXR

FLUXT

FLUXX

RADCOF

RESULT

SETUP
Reads input data for radiation flux subroutines and initializes radiation parameters.

Calculates coefficients for finite difference equation for FR (radial flux sum) and solves for the field values for $F R$ at each grid point.

Calculates FETA (azimuthal flux sum) at each grid point.

Calculates coefficients for finite difference equation for FX (axial flux sum) and solves for field values for $F X$ at each grid point.

Calculates radiation coefficients.

Writes the results of the discrete ordinates radiative heat transfer calculation to the output file.

Sets up radiation coefficients for FLUX subroutines. 


\section{Appendix B}

\section{Description of Input Data}

FORTRAN

Variable

$\mathrm{AF}$

AL1

real

m
Recommended

Description and Usual or Typical

Units

Symbol

Value

A value of 0.0 will

0.0

use upwind

differencing to solve

radiation equations in

discrete ordinates

model. A value of 1.0

will use central

differencing. An

intermediate value will

use a hybrid of

upwind and central.

Axial length where Greater than

axial dimensions of the length of

the computational cells the reactor

begin to shrink. unless it is

desired to have cells shrink at

the outlet 
FORTRAN

Variable

$\operatorname{ALJ}(J, L)^{1} \quad$ real

$\operatorname{AMJ}(J, M) \quad$ real

$\mathrm{AO}$

ASUB (I, II) character -

AT (II)

$\operatorname{ATOM}(1, \mathrm{~K}) \quad$ real

$\operatorname{ATOM}(2, K)$

ATOMID (K) character -

B (II)

BETSUR character

Type

Units

$\mathrm{m} \mathrm{s}^{-1} \mathrm{~K}^{-\mathrm{n}}$

$s^{-1}$

real

real

real

$\mathrm{m}^{2} / \mathrm{kg}$
Recommended

Description and Usual or Typical

Symbol

Value

Pre-exponential factor Depends on for the $L^{\text {th }}$ char the coal type; reaction for the $\mathrm{J}^{\text {th }}$ not needed if particle size/type, $A_{j p}$. use FG-DVC Pre-exponential factor Depends on for the $\mathrm{M}^{\text {th }}$ coal the coal type; reaction for the $\mathrm{J}^{\text {th }}$ not needed if particle size/type, $A_{j m}$. use FG-DVC Degree of 1.0 anisotropicity of scattering in discrete ordinates radiation model.

Name of $I^{\text {th }}$ species, Depends on $\mathrm{II}=1,3$. species; must correspond to name used in JANAF database

Element symbols in a Depends on species name (e.g., $\quad$ species $\mathrm{CO}_{2}$ has $\mathrm{C}$ and $\mathrm{O}$ ).

$\mathrm{kg} \mathrm{kmol}^{-1} \quad$ Atomic mass of $\mathrm{K}^{\text {th }}$ element.

Valence of $\mathrm{K}^{\text {th }}$

element.

Depends on

element

Depends on element

Name of $K^{\text {th }}$ element. Depends on element

Number of molecules Depends on of element 'AT in species species name (e.g., $\mathrm{CO}_{2}$ has 1 and 2).

Sorbent particle BET 1.E6 surface area.

${ }^{1}$ Definitions for array subscripts are at end of table. 
FORTRAN

Variable

$\begin{array}{lll}\text { BLANK } & \text { ASCII } & \mathrm{sec}^{-1} \\ \text { CHARNO } & \text { integer } & --\end{array}$

COMENT(II) character -

$\operatorname{DATA}(I=1,3)$ character

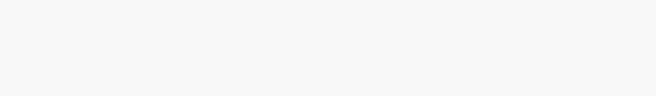

$\begin{array}{lll}\text { DELRRJ } & \text { real } \\ \text { DELTA(I) } & \text { real }\end{array}$

DELTPJ real $K$

DIACH real m

DIAP real m

Description and Usual or Typical Symbol

Value
A blank card.
Flag for selecting char-NO decay rate expression:
1=Equation by Levy output of particle information.
Molecular symbol or name of species.

Comment words for Arbitrary

Depends on species; must match names in JANAF

Fractional change in 1.E-6 total reaction rate that is acceptable for a converged reaction rate.

Stockmayer polar Depends on parameter of species I, species

$\delta_{\mathrm{i}}$.

Temperature

0.01

difference acceptable for convergence when calculating particle temperature from particle enthalpy. Diameter of reaction Depends on chamber. reactor Diameter of primary Depends on duct reactor

\footnotetext{
${ }^{2}$ Only option available for now.
} 
FORTRAN

Variable

DIAS .

DT1, DT2 character -

EDIN(II)

real

m s

EDPD

EDPDAT

Type

Units

m

real $\mathrm{m}$

$\mathrm{ms}$

$\begin{array}{lll}\text { EDPD } & \text { logical } \\ & \\ \text { EDPDAT } & \text { logical }\end{array}$

Recommended

Description and Usual or Typical

Symbol Value

Diameter of secondary Depends on duct. reactor

Date species was put Depends on in JANAF database species Experimental inlet Depends on turbulent kinetic experimental energy dissipation rate data at radial position RIN(II).

.TRUE. will use F turbulent kinetic energy dissipation rate profile data from an inlet data file for additional inlets (option not presently functional in code).

.TRUE. will use $\mathrm{F}$

turbulent kinetic energy dissipation rate profile data from an inlet data file for the primary tube, and calculates adjusted profiles at the inlet node point. FALSE.

will use theoretical calculations for the profile 
FORTRAN

Variable

EDSDAT
Type

$\underline{\text { Units }}$

logical

real

$\mathrm{K}$

$\mathrm{EL}(\mathrm{J}, \mathrm{L}) \quad$ real $\quad \mathrm{J} \mathrm{kmol}^{-1}$

ELEM

$\operatorname{EMJ}(J, M) \quad$ real

character

$\operatorname{EMM}(\mathrm{J}, \mathrm{L}) \quad$ real

$\mathrm{J} \mathrm{kmol}^{-1}$
Description and Usual or Typical

Symbol

Value

.TRUE. will use $\mathrm{F}$

turbulent kinetic

energy dissipation rate

profile data from an inlet data file for the secondary annulus, and calculates adjusted profiles at the inlet node points.

.FALSE. will use theoretical calculations for the profile.

Stockmayer parameter Depends on for the $\mathrm{I}^{\text {th }}$ species, species; must $\varepsilon / \mathrm{k}$. be paired with $S(I)$

Activation energy for Depends on the $L^{\text {th }}$ char oxidation coal type and reaction for the $J^{\text {th }}$ oxidizer particle size/type.

Formatted line must ELEM contain the letters 'ELEM'.

Activation energy for the $\mathrm{M}^{\text {th }}$

Depends on devolatilization reaction for the $\mathrm{J}^{\text {th }}$ coal type; not needed if use particle size/type.

Exponent on FǴ-DVC temperature in kinetic coal type char rate expression, 
FORTRAN

Variable

EMW

EPSD

EPSI

EPSX

EQTEST

EQTEST

FBACK

real

FCTHCN rea

real

一

FCTNH3

Type

Units

real

real

real

-

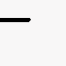

$--$

$-$

real

\section{Recommended \\ Description and Usual or Typical \\ Symbol Value}

Wall emissivity, $\varepsilon_{\mathrm{w}} \quad 0.8$

Ratio of cell size $\quad 0.9$

decrease in radial

direction.

Ratio of cell size $\quad 1.1$

increase in radial

direction.

Ratio of cell sites in $\quad 1.1$

axial direction (cf

AL1).

Local equivalence $\quad 0.5^{3}$

ratio test criterion used

to determine the

method of estimating

atomic oxygen

concentration

Fraction of volatiles 0.5

enthalpy which is fed

directly back to the

particle.

Fraction of frozen $\quad 0.8$

HCN concentrations

used to initialize $\mathrm{HCN}$

field in $\mathrm{NO}_{\mathbf{x}}$ model.

Fraction of maximum 0.1

$\mathrm{NH}_{3}$ used to estimate

initial $\mathrm{NH}_{3}$

concentration in $\mathrm{NO}_{\mathrm{x}}$

model.

${ }^{3}$ The value of EQTEST doesn't matter when the recommended option of RADOXY $=1$ is used. For RADOXY $=0$ or 2, the recommended value of EQTEST is 0.5 . 


\begin{tabular}{|c|c|c|c|c|}
\hline FORTRAN & & & Description and Usual & $\frac{\text { Recommended }}{\text { or Typical }}$ \\
\hline Variable & Type & Units & Symbol & Value \\
\hline FCTNO & real & 一 & \multicolumn{2}{|c|}{$\begin{array}{l}\text { Fraction of frozen NO } 0.1 \\
\text { concentrations used to } \\
\text { initialize NO field in } \\
\mathrm{NO}_{\mathrm{x}} \text { model. }\end{array}$} \\
\hline FFLOW & real & - & $\begin{array}{l}\text { Fraction of reactant } \\
\text { stream } 1 \text { in NINLET } \\
\text { stream. }\end{array}$ & $\begin{array}{l}1.0 \text { for } \\
\text { primary and } \\
0.0 \text { for } \\
\text { secondary }\end{array}$ \\
\hline FGDVC & logical & - & $\begin{array}{l}\text {.TRUE. uses the } \\
\text { FGDVC submodel for } \\
\text { coal devolatilization. }\end{array}$ & $T$ \\
\hline FLOW & real & $\mathrm{kg} \mathrm{s}^{-1}$ & $\begin{array}{l}\text { Mass flow rate of gas } \\
\text { in NINLET stream. }\end{array}$ & $\begin{array}{l}\text { Depends on } \\
\text { flowrate }\end{array}$ \\
\hline FN2PRT & real & - & $\begin{array}{l}\text { Partitioning factor of } \\
\text { coal or char nitrogen } \\
\text { between gaseous } \\
\mathrm{HCN} \text { and } \mathrm{NH}_{3} \text { in } \\
\mathrm{NO}_{\pi} \text { submodel. } \\
0=\text { all nitrogen evolves } \\
\text { as } \mathrm{NH}_{3} \\
1=\text { all nitrogen evolves } \\
\text { as } \mathrm{HCN}\end{array}$ & $\begin{array}{l}1.0 \text { for high- } \\
\text { rank coals; } \\
\text { something less } \\
(0.5 ?) \text { for low- } \\
\text { rank coals }\end{array}$ \\
\hline
\end{tabular}


FORTRAN

Variable

FUELNO
Type

integer
Units

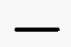

$\begin{array}{ll}\text { GAMMA } & \text { real } \\ \text { GRDOUT } & \text { logical }\end{array}$

$\mathrm{HA \emptyset (J)} \quad$ real $\quad \mathrm{J} \mathrm{kg}^{-1}$

$\operatorname{HGV}(J, M) \quad$ real

$\mathrm{HH} \emptyset$ real

\section{Recommended \\ Description and Usual or Typical \\ Symbol \\ Value}

Flag for choosing fuel 1

NO mechanism:

$0=$ fuel $N O$ not

calculated

$1=$ deSoëte kinetics ${ }^{4}$

$2=$ Wendt kinetics 5

3=Mitchell and Tarbell

kinetics

Particle swelling $\quad 1.1$

parameter, $\gamma$.

.TRUE. prints a grid $\mathrm{T}$

(.grd) file.

Heat of formation of $0.0^{6}$

the ash in the Jth

particle, $h_{a j}^{\circ}$.

Heat of reaction for $\quad 0.0^{7}$

$\mathrm{M}^{\text {th }}$ devolatilization

reaction for $\mathrm{J}^{\text {th }}$

particle.

Heat of formation of $0.0^{8}$

the char in the $\mathrm{J}^{\text {th }}$

particle, $h_{h_{j}}^{\circ}$.

${ }^{4}$ Kinetic parameters have been adjusted at BYU from what deSoëte originally reported.

5 Has a more theoretical basis than Option 1, but may cause numerical instability.

${ }^{6}$ Since ash is inert, anything could be used here, so using 0.0 is the simplest option.

${ }^{7}$ This is used to calculate volatiles enthalpy when FG:DVC is not used. FG-DVC predicts the volatiles composition and, therefore, the volatiles enthalpy.

${ }^{8}$ This value is based on assuming pure carbon for the char. 
FORTRAN

Variable

HLOSS

HTRACK logical

HWQ

ICALCN

$\operatorname{IDON}(\mathrm{I}=1,4)$ integer integer

\section{Type Units}

real

real

$\mathrm{J} \mathrm{kg}^{-1}$

一

Recommended

Description and Usual or Typical

Symbol Value

Fraction of enthalpy in 0.0 excess of ambient conditions to be removed from each computational cell. Should be 0.0 if INCALH is set TRUE.

.TRUE. causes $\quad$ F $^{9}$ hydrogen to be tracked independently from all other elements in the coal offgas when INETA2 is equal to .TRUE. Heat of formation of $-1.5866 \mathrm{E} 7^{10}$ the slurry liquid in the $J^{\text {th }}$ particle, $h_{w j}^{0}$. Iteration frequency that $\mathrm{HCN}$ species continuity is solved in $\mathrm{NO}_{\mathrm{x}}$ submodel when . fuelno $=2$ or 3 . Not used.

${ }^{9}$ This option has not yet been proven and only works when INETA2 $=\mathrm{T}$.

${ }^{10}$ This is the value for water.

${ }^{11}$ Try a value greater than 1 if have convergence problems and FUELNO $=2$ or 3 . 
FORTRAN

Variable

IEUCK

IGASV

INCALF

INCALG
Type Units

integer -

integer

logical

logical
Recommended

Description and Usual or Typical

Symbol

Value

Gas thermal

\section{1}

conductivity calculated

from the Eucken

equation if IEUCK =

1, otherwise Sage-

Galloway equation is

used.

Index for gas property 3

interpolation during

Lagrangian particle

trajectory integration.

If IGASV = 1 no

interpolation gas

properties are

assumed constant in

the cell; IGASV = 2,

gas properties are

interpolated in the

axial direction;

IGASV $=3$, gas

properties are

interpolated in both

axial and radial

directions.

.TRUE. will solve the T

f mixture fraction

equation.

.TRUE. will solve $g_{f} T$ equation. 
FORTRAN

Variable

INCALH

INCALN

INCFP

INCLET

INCLGE

logical

logical

INCLKE logical -

INCNOX logical -

INCOAL logical -

$\begin{array}{lll}\text { INCREK } & \text { logical } \\ \text { INCSWP } & \text { logical }\end{array}$

INCSWS logical -
Recommended

Description and Usual or Typical

Symbol

Value

.TRUE. will solve gas T

energy equation

(presently under

development for

particle-laden flows).

.TRUE. will solve $n_{j} T$ equation.

.TRUE. will $\mathrm{T}$ incorporate correction for particles into gas phase turbulent diffusion coefficients

(Eq. 14).

.TRUE. will solve $\eta \mathrm{T}$ equation.

.TRUE. will solve $g_{\eta} \mathrm{T}$ equations.

.TRUE. solves the $k-\varepsilon T$ equations.

.TRUE. will solve $T$ $\mathrm{NO}_{x}$ equations.

.TRUE. will solve $T$ coal reaction equations.

.TRUE. will set up $T$ gas property table. .TRUE. allows for $F$ swirl in the primary stream.

.TRUE. allows for $T$ swirl in the secondary stream. 


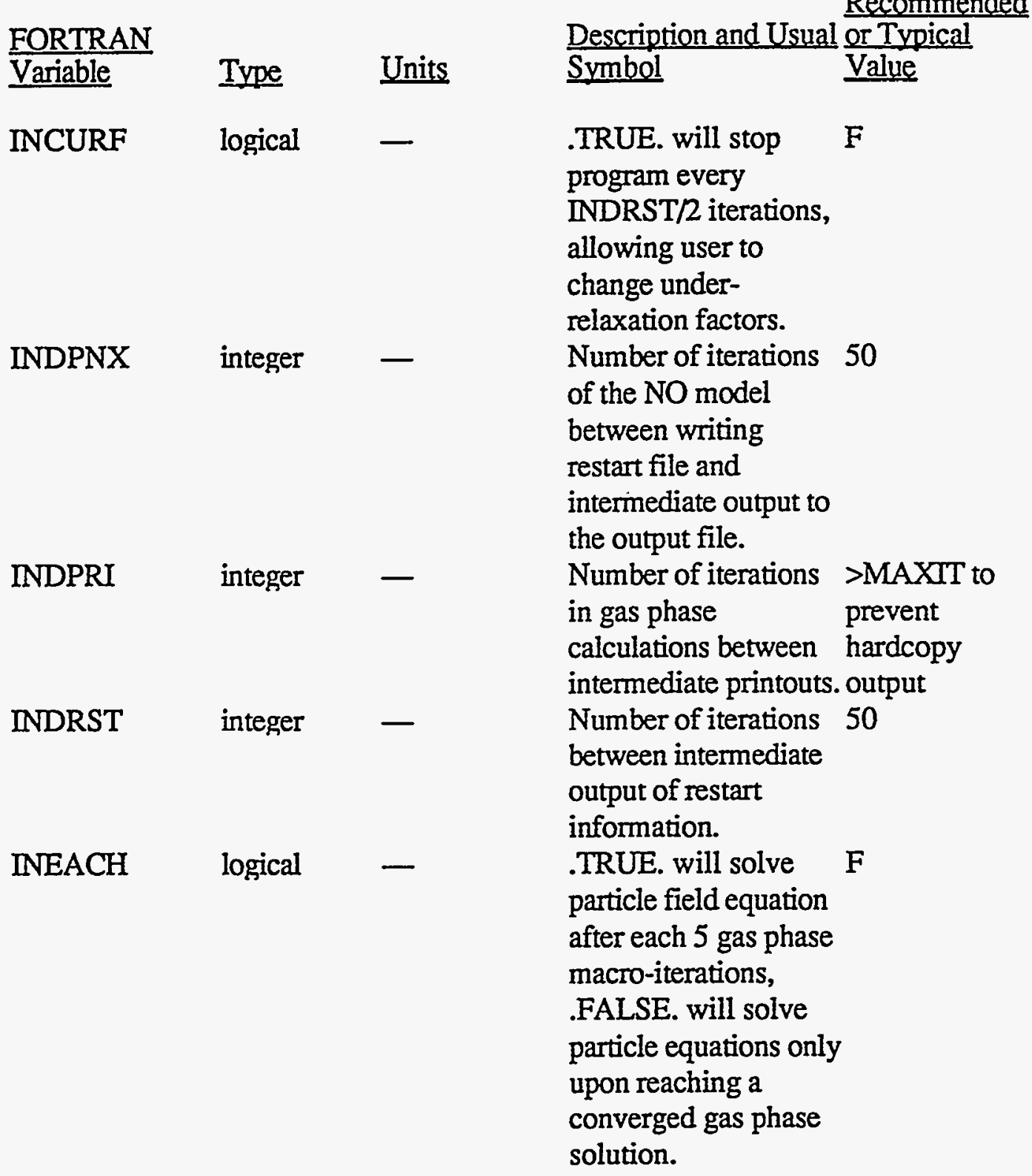




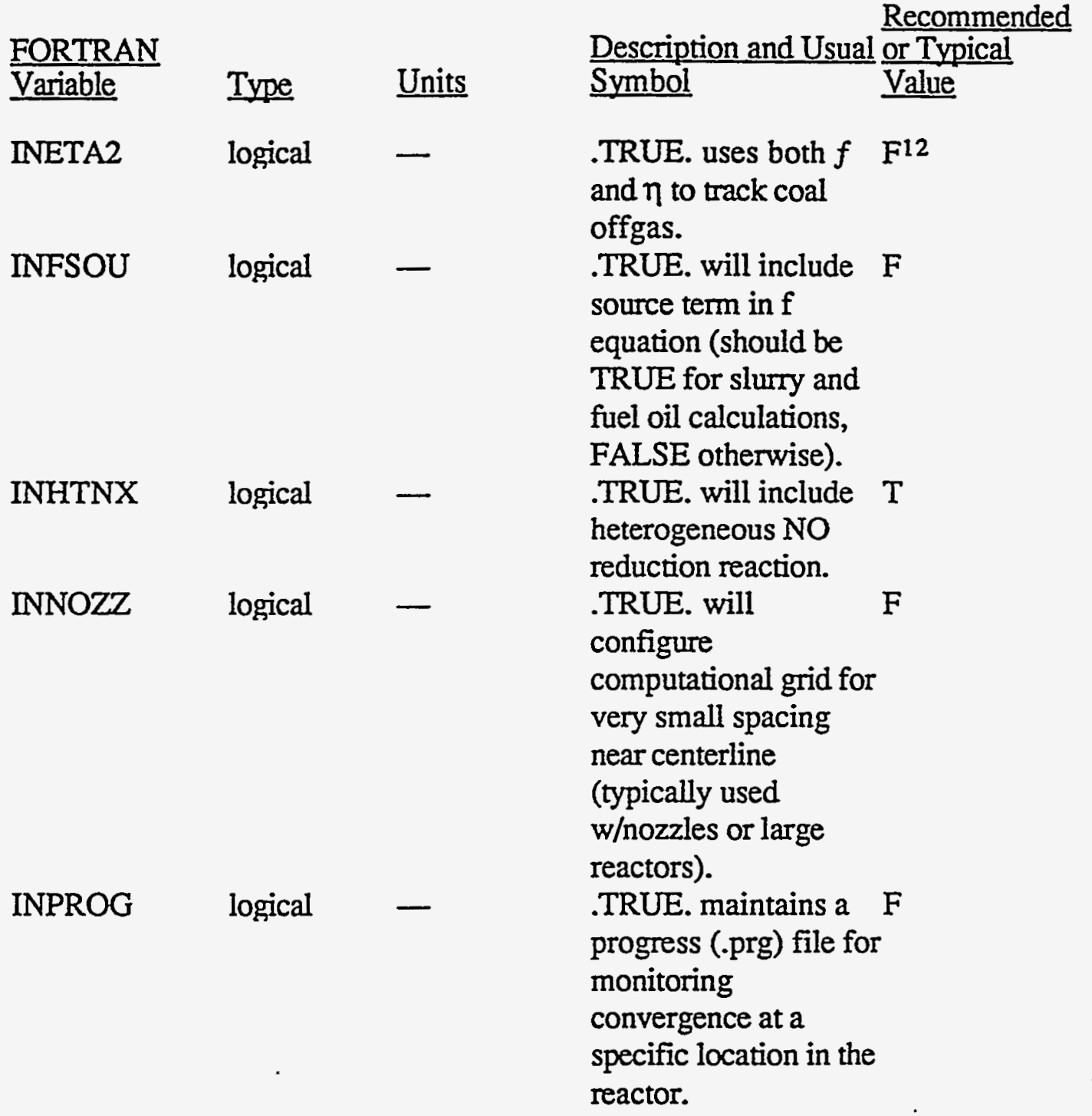

${ }^{12}$ This option is not fully debugged and is not recommended for the ordinary user. 
FORTRAN

Variable

INPRST

Type

Units

logical

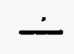

INQRL

logical

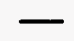

$\begin{array}{lll}\text { INRAD } & \text { logical } \\ \text { INRDGD } & \text { logical }\end{array}$

INRST

logical

INSIMP

logical

INSMPC

logical
Description and Usual or Typical

Symbol

Value.

.TRUE. will use a $\mathrm{T}$

particle restart file to

begin computations

and output

intermediate particle

restart files.

.TRUE. allows for a $T$

quarl at the secondary

inlet.

.TRUE. will solve $\mathrm{T}$ radiation equations.

.TRUE. reads location $T$

of user specified grid

points from data file.

.TRUE. will use a gas $T$

restart file to begin

computations and

output intermediate

gas phase restart files.

.TRUE. uses the $T$

SIMPLER algorithm

to solve

velocity/pressure

field. .FALSE. uses

the SIMPLE

algorithm.

.TRUE. uses the F

SIMPLEC algorithm 


\section{FORTRAN}

Variable

INSORB

INTFRZ

logical

Type

$\underline{\text { Units }}$

logical

INTFRZ

INTRUS logical -

IPLTNX

$$
\text { - logical }
$$$$
-
$$

IPSICT

IRSTNO logical -
Recommended

Description and Usual or Typical

Symbol

Value

.TRUE. calls the

$\mathrm{T}$ sorbent reactions

submodel after

converging the main

flowfield.

.TRUE. uses frozen $F$ equilibrium scheme for gas properties when the local equivalence ratio is greater than 2.0. .TRUE. will allow $\mathrm{T}$ intrusions in reactor. .TRUE. will write $\mathrm{T}$ files for plots from NO model calculations.

.TRUE. will solve $T$ particle equations (should be TRUE for all calculations involving particles). .TRUE. will use a $\mathrm{T}$ restart file to begin NO model computations and output intermediate NO model restart files. 
FORTRAN Variable

ITYNX

KEQ

KOPED

\section{Type}

Units

integer

integer

integer
Recommended

Description and Usual or Typical

Symbol Value

Number of iterations 5

of the NO model

between intermediate

typing of the residuals

to the screen or log

file.

IF $\mathrm{KEQ}=1$ the linear 0

form of the Field

(1967) char oxidation

rate expression is

used; if $\mathrm{KEQ} \neq 1$

normal Arrhenius

form used (low

temperature reactors

$(<1400 \mathrm{~K}$ ) should not

use KEQ = 1).

Selection parameter 1

which designates one

of several optional

equations to be used

in calculating a

theoretical or

experimental turbulent

kinetic energy

dissipation rate profile

for additional inlets. 
FORTRAN

Variable

KOPEDP

KOPEDS

KOPTE
Type

$\underline{\text { Units }}$

integer

integer

integer
Recommended

Description and Usual or Typical

Symbol

Value

Selection parameter 1

which designates one

of several optional

equations to be used

in calculating a

theoretical or

experimental turbulent

kinetic energy

dissipation rate profile

for the primary.

Selection parameter 1

which designates one

of several optional

equations to be used

in calculating a

theoretical or

experimental turbulent

kinetic energy

dissipation rate profile

for the secondary.

Selection parameter 1

which designates one

of several optional

equations to be used

in calculating a

theoretical or

experimental turbulent

kinetic energy profile

for the additional

inlets (option not

functional at present). 


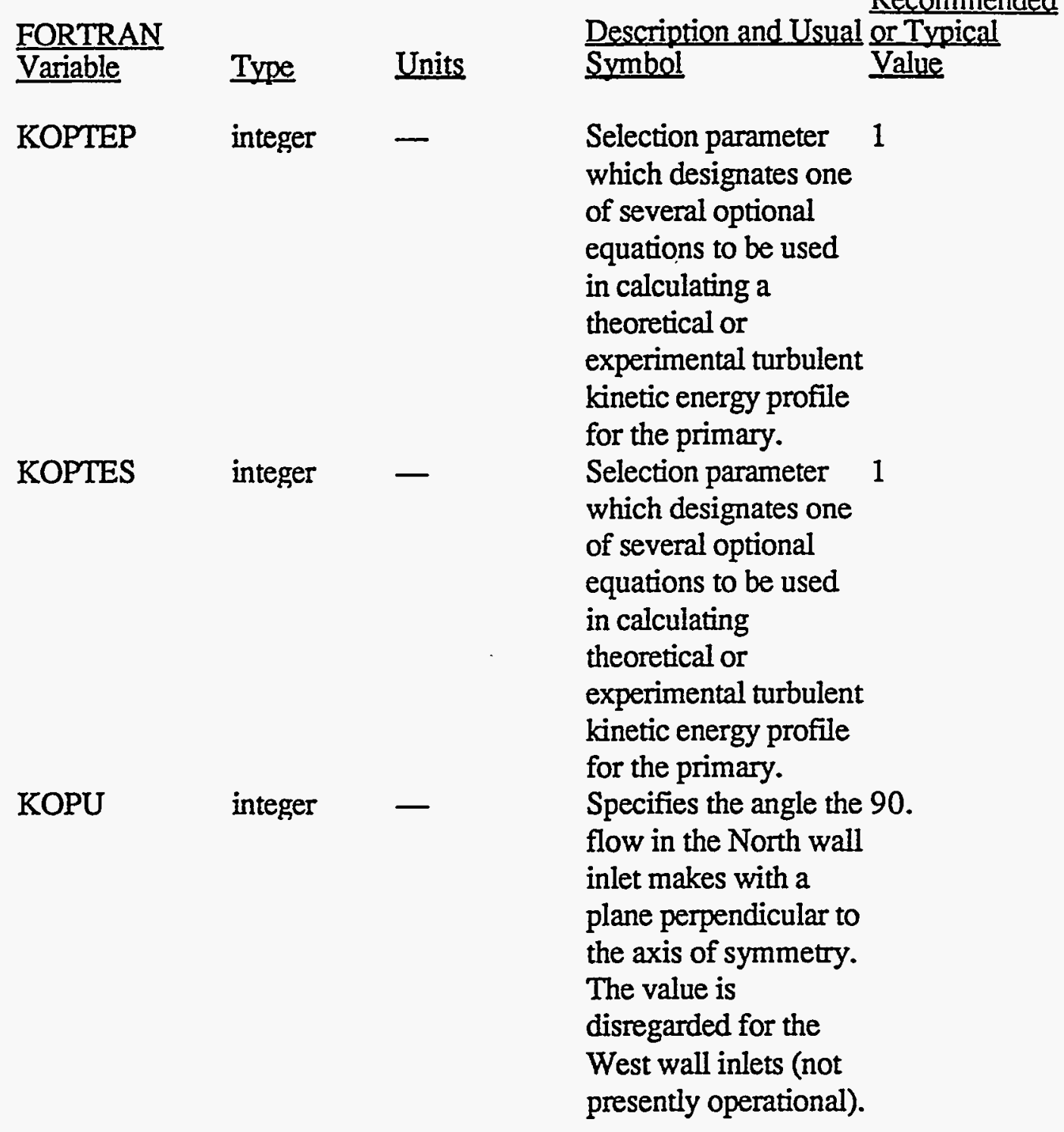


FORTRAN

Variable

KOPUP

KOPUS

KOPV
Type Units

integer

integer

integer
Recommended

Description and Usual or Typical

Symbol

Value

Selection parameter 1

which designates one

of several optional

equations to be used

in calculating a

theoretical or

experimental axial

velocity profile for the

primary.

Selection parame:er 1

which designates one

of several optional

equations to be used

in calculating a

theoretical or

experimental axial

velocity profile for the

secondary.

Specifies the angle the 0 .

flow in the West wall

inlet makes with the

axis of symmetry.

$\mathrm{KOPV}(\mathrm{N})$ is

disregarded for North

Wall inlets (not

presently operational). 
FORTRAN

Variable

KOPVP

KOPVS

integer

\section{Type}

Units

integer

一

KOPW
Recommended

Description and Usual or Typical

Symbol Value

Selection parameter 1

which designates one

of several optional

equations to be used

in calculating a

theoretical or

experimental radial

velocity profile for the

primary.

Selection parameter 1

which designates one

of several optional

equations to be used

in calculating a

theoretical or

experimental radial

velocity profile for the

secondary.

Tangential component 1

of velocity is zero for

all additional inlets

(not presently

operational). 


\begin{tabular}{|c|c|c|c|c|}
\hline FORTRAN & & & Description and Usual & $\begin{array}{l}\text { Recommended } \\
\text { or Typical }\end{array}$ \\
\hline Variable & Type & Units & Symbol & Value \\
\hline KOPWP & integer & 一 & $\begin{array}{l}\text { Selection parameter } \\
\text { which designates one } \\
\text { of several optional } \\
\text { equations to be used } \\
\text { in calculating a } \\
\text { theoretical or } \\
\text { experimental } \\
\text { tangential velocity } \\
\text { profile for the } \\
\text { primary. }\end{array}$ & 1 \\
\hline KOPWS & integer & 一 & $\begin{array}{l}\text { Selection parameter } \\
\text { which designates one } \\
\text { of several optional } \\
\text { equations to be used } \\
\text { in calculating a } \\
\text { theoretical or } \\
\text { experimental or } \\
\text { tangential velocity } \\
\text { profile for the } \\
\text { secondary. }\end{array}$ & 1 \\
\hline LAMINAR & logical & 一 & $\begin{array}{l}\text { TRUE. causes } \\
\text { molecular transport } \\
\text { properties to be } \\
\text { calculated locally, as } \\
\text { functions of } \\
\text { composition, etc., and } \\
\text { invokes the } \\
\text { laminarization } \\
\text { extension to the k- } \varepsilon \\
\text { model, if INCLKE = } \\
\text {.TRUE. }\end{array}$ & F \\
\hline
\end{tabular}




\begin{tabular}{|c|c|c|c|c|}
\hline$\frac{\text { FORTRAN }}{\text { Variable }}$ & Type & Units & $\begin{array}{l}\text { Description and Usual } \\
\text { Symbol }\end{array}$ & $\begin{array}{l}\text { Recommended } \\
\text { or Typical } \\
\text { Value }\end{array}$ \\
\hline LBACK & logical & - & $\begin{array}{l}\text { TRUE. causes a } \\
\text { portion (specified by } \\
\text { FBACK) of the } \\
\text { volatiles enthalpy to } \\
\text { be fed back directly to } \\
\text { the particle. }\end{array}$ & $\mathrm{T}$ \\
\hline LBUOY & logical & - & $\begin{array}{l}\text { TRUE. causes the } \\
\text { buoyancy term to be } \\
\text { included in the axial } \\
\text { gas momentum } \\
\text { equation. }\end{array}$ & $\mathrm{F}$ \\
\hline LDISO & logical & - & $\begin{array}{l}\text { TRUE. will solve } \\
\text { radiation equations by } \\
\text { discrete ordinates } \\
\text { method. }\end{array}$ & $\mathrm{T}$ \\
\hline LEMCOR & logical & - & $\begin{array}{l}\text { TRUE. will } \\
\text { incorporate } \\
\text { corrections for gas } \\
\text { emissivity due to } \\
\text { spectral overlap. }\end{array}$ & $\mathrm{T}$ \\
\hline LEULP & logical & - & $\begin{array}{l}\text { TRUE. solves } \\
\text { Eulerian particle } \\
\text { equations instead of } \\
\text { Lagrangian equations } \\
\text { only, .FALSE. is the } \\
\text { only currently viable } \\
\text { option. }\end{array}$ & $\mathrm{F}$ \\
\hline LGASE & & - & $\begin{array}{l}\text { TRUE. will solve for } \\
\text { gas phase emissivity. }\end{array}$ & $\mathrm{T}$ \\
\hline LPARTP & logical & - & $\begin{array}{l}\text { TRUE. allows for } \\
\text { particles in the } \\
\text { primary stream. }\end{array}$ & $\mathrm{T}$ \\
\hline
\end{tabular}


FORTRAN

Variable

Type

Units

LPARTS

logical

LPBOTH

logical

LPBUG

logical

LPOST

logical

LRBNDS

logical

LSPBUG

logical

LSPH

logical

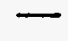

LSPM

logical

LSPU logical -
Description and Usual or Typical

Symbol

Value

.TRUE. allows for F

particles in the

secondary stream.

.TRUE. allows for $F$

particles in both the

primary and

secondary streams.

.TRUE. will print out $F$

debug information for

Lagrangian particle

calculations

.TRUE. writes a file $T$

for the CQUEL post-

processor for plotting.

.TRUE. allows

sorbent particle to

rebound from walls;

otherwise they stick

.TRUE. will print out $F$

debug information for

Lagrangian sorbent

particle calculations

.TRUE. allows for $T$

particle energy source

term.

.TRUE. allows for $T$. particle mass source

term.
.TRUE. allows for $T$ particle axial velocity source term. 
FORTRAN

Variable

LSPV

LSYPS

LTBUG

logical

Type Units

logical

logical

MAGHJER logical

MAXIT integer -

MAXITP integer -

MAXITR integer -
Recommended

Description and Usual or Typical

Symbol $\quad$ Value

.TRUE. allows for $T$ particle radial velocity source term.

.TRUE. allows the F user to specify sorbent particle trajectory starting locations

.TRUE. allows debug $\mathrm{F}$ of chemical equilibrium table.

.TRUE. allows user $F$ to specify coal particle trajectory starting location, .FALSE. assumes uniform mass flux to calculate starting locations. .TRUE. allows use of $\mathrm{F}$ the MagnussenHjertauger (coal reaction) model. Maximum number of 200 gas phase macroiterations.

Maximum number of 10 particle phase iterations.

Maximum number of 10 radiation flux iterations. 
FORTRAN

Variable

MAXRES

MINITP

MOLE

$\begin{array}{lll}\text { MXITNX } & \text { integer } & - \\ \text { MXITSX } & \text { integer } & - \\ & & \\ \text { NCARD } & \text { integer } & - \\ \text { NCD } & \text { integer } & - \\ \text { NCRXN } & \text { integer } & -\end{array}$

Recommended

Description and Usual or Typical

Symbol

Value

Maximum normalized 1.0E-04

value of residual

source for

convergence of

$\mathrm{NO}_{\mathrm{x}} / \mathrm{SO}_{\mathrm{x}}$ submodels.

If gas phase

15

reconverges within

MINTTP iterations

after a particle phase

iteration, overall

convergence is

assumed.

Gas stream relative Either blank or amount flag. When ' $M$ '

MOLE is ' $\mathrm{M}$ ', relative amounts of reactant stream are in mole percent. Otherwise, relative amounts are taken to be mass percent.

Maximum iterations 200

for $\mathrm{NO}_{\mathrm{x}}$ submodel

Maximum iterations

1000

for sorbent reactions

submodel

Number of comment Variable

cards to follow.

Card number. Variable

Number of 1 or 2

devolatilization

reactions, $M$. 
FORTRAN Variable

NDIA

NHRXN

NIINQ

NIWOQ

NJINP

integer

NJINS

NL

real

integer

integer

P

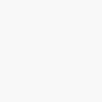

\section{Units}

$$
\text { integer }
$$

一

integer -

Description and Usual or Typical

Symbol

Value

Length of reaction Depends on

chamber divided by reactor, this is

diameter of reaction how the

chamber, L/D.

reactor length

is specified

Number of char $\quad 1$ for fuel-lean

oxidation reactions, L. and 3 for fuel-

rich

Number of axial grid 5-10

points in the quarl

region.

Number of grid points 30-100

in the axial direction

without quarl.

Number of grid points 3-5

in radial direction in

primary stream, only

used if INNOZZ is

.TRUE.

Number of grid points 3-5

in radial direction in

the secondary stream.

Cell number

Depends on

associated with AL1. AL1 and

reactor length;

doesn't matter

of AL1 is

greater than

reactor length 
FORTRAN

Variable

NPROP
Type

integer

Units

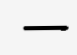

$\begin{array}{lll}\text { NPS } & \text { integer } & - \\ \text { NPSS } & \text { integer } & - \\ \text { NSAY } & \text { integer } & - \\ & & \\ \text { NSAYNX } & \text { integer } & -\end{array}$

NSAYNX(II), character $\mathrm{II}=1, \mathrm{NSAYNX}$

NSAYSX integer

NSFORM integer

NSL

integer -

Description and Usual $\frac{\text { Recommen }}{\text { or Typical }}$

Symbol

Value

Number of particle 5

integration steps

before updating gas

phase transport

properties for particle

reaction scheme.

Number of fuel 5

particle sizes/types.

Number of sorbent 1

particle sizes/types.

Number of comment $\quad 1-10$

cards to follow in

main input file.

Number of comment $\quad 1-10$

cards to follow in

$\mathrm{NO}_{x}$ input file.

Comment words for Arbitrary

output from $\mathrm{NO}_{\mathrm{x}}$

submodel.

Number of comment $\quad 1-10$

cards to follow in

sorbent reactions input

file.

Form of swirl number 1

equation to be used.

Number of particle 10

trajectory starting

locations for fuel

particles. 
FORTRAN

Variable

NSLS

NSTYPE

OHADJS

OHADS

$\begin{array}{lll}\text { OMEGAA(J) real } & - \\ \text { OMEGAC(J) real } & -\end{array}$
Recommended

Description and Usual or Typical

Symbol

Value

Number of particle 1-10

trajectory starting

locations for sorbent

particles.

$1=\mathrm{SO}_{2}$ capture by 1 for fuel-lean

sorbent systems and 2

$2=\mathrm{H}_{2} \mathrm{~S}$ capture by

sorbent

for fuel-rich

systems

Flag for adjusting $\quad \neq 1$

equilibrium

concentration:

$1=$ multiply.

equilibrium $\mathrm{OH}$

concentration by a

non-linear temperature

expression ${ }^{13}$

$\neq 1=$ no adjustment

made

Initial mass fraction of Depends on ash in Jth particle, $\omega_{a j}$.coal type Initial mass fraction of Depends on raw coal in Jth particle, $\omega_{\mathrm{ci}}$. coal type; not

needed for

FG-DVC

${ }^{13}$ This expression was developed by Wendt et al. (1989) to account for "overshoot" of $\mathrm{OH}$ and is specific to their reactor system and fuel-rich coal combustion. It is not appropriate to use this expression unless case-specific fitting parameters are substituted into this expression which appears in subroutines EQSPEC, PSCRAT, and RXNRAT. Therefore, it is recommended that OHADJS $\neq 1$. 
FORTRAN

Variable

OMEGAH(J)

real

OMEGAW(J) real

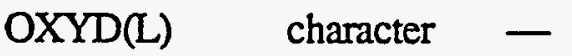

$\mathrm{PD}(\mathrm{J})$

PDEN

PDS(IPS)

PECWT

PHASE real

real

real

ASCII m

m

\section{$\underline{\text { Units }}$}
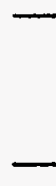

$\mathrm{kg} \mathrm{m}^{-3}$

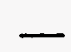

Description and Usual or Typical Symbol

Value

Initial mass fraction of 0 . unless coal char in Jth particle, is partially

$\omega_{\text {hj. }}$ devolatilized

Initial mass fraction of 0 . unless liquid in the $\mathrm{J}^{\text {th }}$ feeding a particle, $\omega_{w j}$ Species identification symbol for the oxidizer for the $L^{\text {th }}$ char oxidation reaction. Initial diameter of $J^{\text {th }}$ fuel particle, $d_{i}$. slurry 14

Always O2; sometimes $\mathrm{CO} 2, \mathrm{H} 2 \mathrm{O}$, and even $\mathrm{H} 2$ as well Depends on particle size distribution

Particle/droplet 1300. density Initial diameters of $\quad 10 . \mathrm{E}-6$ sorbent particles.

Relative amounts of each species in each input stream.

Phase of inlet stream G

Depends on stream composition species; only gases are allowed except for slurry calculations, which require one stream of liquid.

${ }^{14}$ Note that OMEGAW is not used for coal moisture. Coal moisture can only be accounted for adding it to the transporting gas stream. This is equivalent to assuming the moisture evaporates from the coal during transport into the fumace. 
FORTRAN

$\underline{\text { Variable }}$

PHIL(L)

PLOD(1)

real

Type

Units

real

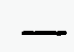

PLOD(1)

PLODS(I) real -

PLS real

PMF(J)

real

PMFS(J)

real

POLLUT

logical

$\mathrm{m}$
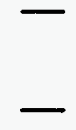

$-$

PRES

real

$\mathrm{N} \mathrm{m}^{-2}$

Recommended

Description and Usual or Typical

Symbol

Value

Ratio of moles of fuel Depends on

(C) to moles of oxidizer in the $\mathrm{L}^{\text {th }}$ char oxidation oxidizer, see discussion in Chapter 2

reaction, $\phi_{\ell}$.

Coal particle loading Whatever it is (mass of coal particles/mass of primary gas) in the $\mathrm{i}^{\text {th }}$ stream.

Sorbent particle Whatever it is loading (mass of sorbent particles/mass of primary gas) in the $i^{\text {th }}$ stream.

Local length scale in Not used primary stream. unless KOPEDP $=3$

Mass fraction of Jth Must add up to particle type.

1.0

Mass fraction of Jh Must add up to

sorbent particle type $\quad 1.0$

.TRUE. solves only $F$

$\mathrm{NO}_{\mathrm{x}}$ equations without recalculating other Eulerian variables.

Reactor outlet Whatever it is pressure. hatever it is 
FORTRAN

Variable

PRHCN

PRK(J)

PRKS(J)

PRNOX

PRSO2

QHA

QHC(J)
Type

$\underline{\text { Units }}$

real
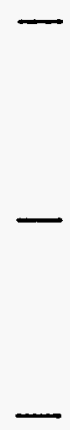

real

real

real

real

-

degrees

real

real

$\mathrm{J} / \mathrm{kg}$
Description and Usual or Typical

Symbol

Value

Turbulent

0.9

Prandtl/Schmidt

number for $\mathrm{HCN}$

concentrations.

Turbulent

0.35

Prandtl/Schmidt

number for Jth particle

type, $\sigma_{j}^{t}$.

Turbulent

0.35

Prandtl/Schmidt

number for $J^{\text {th }}$

sorbent particle type

Turbulent

0.9

Prandtl/Schmidt number for $\mathrm{NH}_{3}$.

Turbulent

Prandt/Schmidt number for NO.

Turbulent

0.9

Prandtl/Schmidt

number for $\mathrm{SO}_{2}$.

Quarl half angle (with Depends on centerline).

reactor

geometry

Gross heating value $0.0^{15}$

(constant volume) for $\mathrm{j}^{\text {th }}$ fuel particle type.

15 If the heating value is known, its value can be given here. The value of " 0.0 " causes the gross heating value to be estimated from the IGT correlation, based on ultimate analysis. 


\begin{tabular}{|c|c|c|c|c|}
\hline FORTRAN & & & Description and Usua & $\begin{array}{l}\text { Recommend } \\
\text { or Typical }\end{array}$ \\
\hline Variable & Type & Units & Symbol & Value \\
\hline QLX & real & $\mathrm{m}$ & $\begin{array}{l}\text { Axial length of quarl } \\
\text { region. }\end{array}$ & $\begin{array}{l}\text { Depends on } \\
\text { reactor } \\
\text { geometry }\end{array}$ \\
\hline $\mathrm{RADOH}$ & integer & -- & $\begin{array}{l}\text { Flag for choosing } \\
\text { quasi-equilibrium } \\
\text { expression for } \\
\text { estimating radical } \\
\text { hydroxide }(\mathrm{OH}) \\
\text { concentration: } \\
2=\text { assume equilibrium } \\
\text { with } \mathrm{H}_{2} \mathrm{O} \text { and } \mathrm{H}_{2} \\
\neq 2=\text { assume } \\
\text { equilibrium among all } \\
\text { gas species }\end{array}$ & $\neq 2$ \\
\hline
\end{tabular}




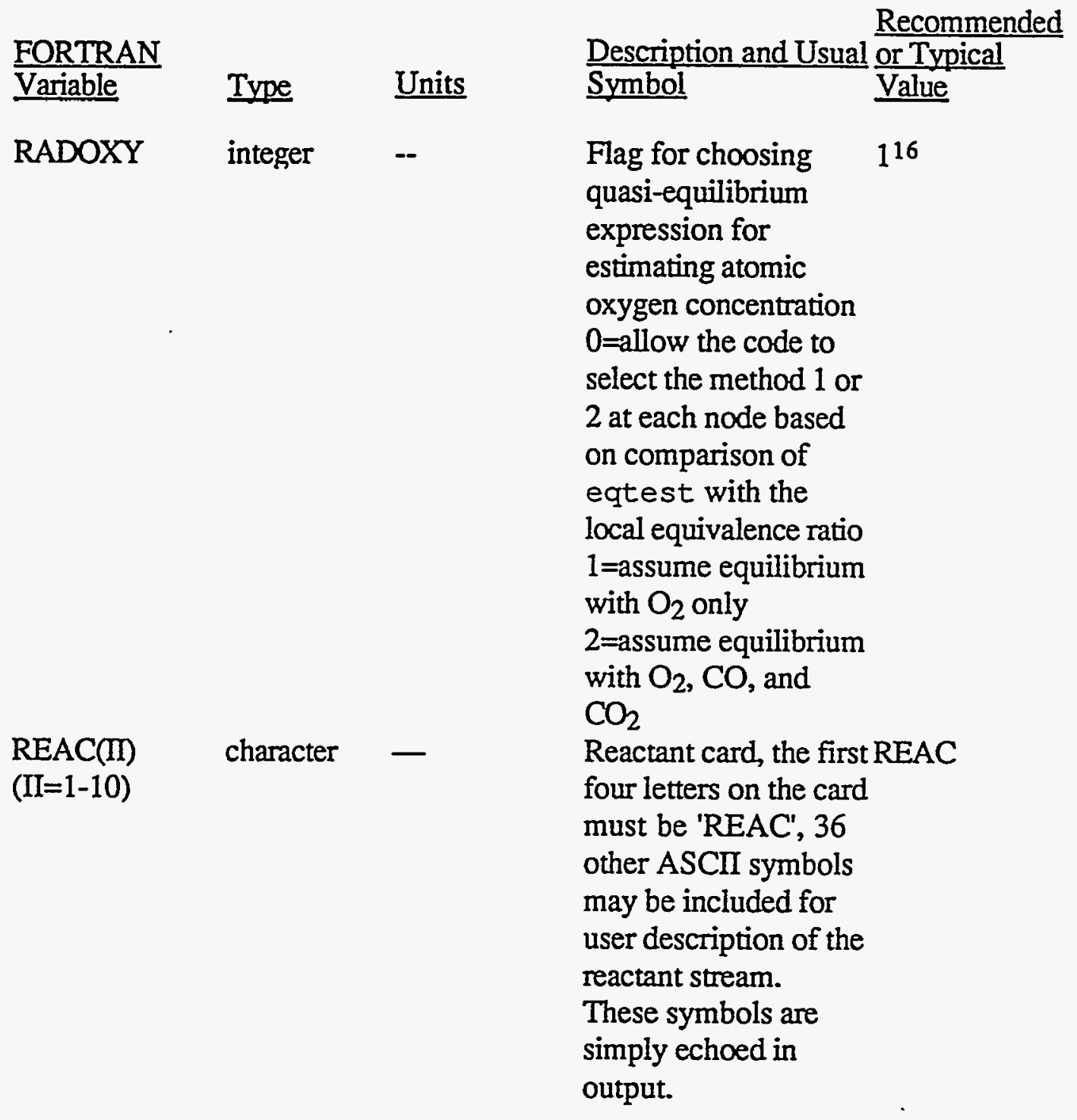

${ }^{16}$ Options 0 and 2 have a better theoretical basis, but the expression for Option 2 is not sufficiently developed. If the code included effects of non-equilibrium $\mathrm{CO} / \mathrm{CO}_{2}$, Option 0 might be the recommended choice. 


\begin{tabular}{|c|c|c|c|c|}
\hline FORTRAN & & & Description and Usual & $\begin{array}{l}\text { Recommended } \\
\text { or Typical }\end{array}$ \\
\hline Variable & Type & Units & Symbol & Value \\
\hline RIN(I) & real & $\mathrm{m}$ & $\begin{array}{l}\text { Radial location of } \\
\text { experimental inlet data } \\
\text { point. }\end{array}$ & $\begin{array}{l}\text { Depends on } \\
\text { data }\end{array}$ \\
\hline RP & real & $\mathrm{m}$ & $\begin{array}{l}\text { Primary tube inside } \\
\text { radius for } \\
\text { experimental inlet } \\
\text { data. }\end{array}$ & $\begin{array}{l}\text { Depends on } \\
\text { data }\end{array}$ \\
\hline RS1 & real & $\mathrm{m}$ & $\begin{array}{l}\text { Primary tube outside } \\
\text { radius for } \\
\text { experimental inlet } \\
\text { data. }\end{array}$ & $\begin{array}{l}\text { Depends on } \\
\text { data }\end{array}$ \\
\hline RS2 & real & $\mathrm{m}$ & $\begin{array}{l}\text { Secondary tube radius } \\
\text { for experimental inlet } \\
\text { data. }\end{array}$ & $\begin{array}{l}\text { Depends on } \\
\text { data }\end{array}$ \\
\hline $\mathrm{S}(\mathrm{I})$ & real & $\AA$ & $\begin{array}{l}\text { Stockmayer collision } \\
\text { diameter, } \sigma_{i} \text {. }\end{array}$ & $\begin{array}{l}\text { Depends on } \\
\text { species; must } \\
\text { be paired with } \\
\text { EK(I) }\end{array}$ \\
\hline $\begin{array}{l}\operatorname{SAY}(\mathrm{I}) \\
(\Pi=1-10)\end{array}$ & character & - & $\begin{array}{l}\text { Comment words for } \\
\text { beginning of output. }\end{array}$ & Variable \\
\hline $\begin{array}{l}\text { SAYSX(II), } \\
\Pi=1, N S A Y S X\end{array}$ & character & - & $\begin{array}{l}\text { Comment words for } \\
\text { output from sorbent } \\
\text { reactions submodel. }\end{array}$ & Variable \\
\hline SLRCMP (I) & character & - & $\begin{array}{l}\text { Name of slurry } \\
\text { component found in } \\
\left.\text { gas (e.g. } \mathrm{H}_{2} \mathrm{O}\right)(\mathrm{I}=1 \text {, } \\
2 \text {, and } 3 \text { ). }\end{array}$ & $\mathrm{H} 2 \mathrm{O}$ \\
\hline SLS & real & $\mathrm{m}$ & $\begin{array}{l}\text { Local length scale in } \\
\text { secondary stream. }\end{array}$ & $\begin{array}{l}\text { Not used unles } \\
\text { KOPEDS }=3\end{array}$ \\
\hline
\end{tabular}




\begin{tabular}{|c|c|c|c|c|}
\hline FORTRAN & & & Description and Usual & $\frac{\text { Recommende }}{\text { or Typical }}$ \\
\hline Variable & Type & $\underline{\text { Units }}$ & Symbol & Value \\
\hline SORMAX & real & 一 & $\begin{array}{l}\text { Maximum normalized } \\
\text { value of residual } \\
\text { source sums for } \\
\text { convergence of gas } \\
\text { phase computations. }\end{array}$ & 0.01 \\
\hline SORMIN & real & 一 & $\begin{array}{l}\text { Minimum normalized } \\
\text { value of residual } \\
\text { source sums between } \\
\text { particle phase } \\
\text { iterations for overall } \\
\text { convergence. }\end{array}$ & 0.1 \\
\hline SPDEN & real & $\mathrm{kg} / \mathrm{m}^{3}$ & $\begin{array}{l}\text { Sorbent particle } \\
\text { density. }\end{array}$ & 1800. \\
\hline SPRANG(I) & real & 一 & $\begin{array}{l}\text { Spray angle for initial } \\
\text { starting location for } \\
\text { particles. }\end{array}$ & $\begin{array}{l}\text { Depends on } \\
\text { injection } \\
\text { geometry }\end{array}$ \\
\hline STRING & character & - & $\begin{array}{l}\text { Inlet card. Must } \\
\text { contain the string } \\
\text { 'INLET'. }\end{array}$ & INLET \\
\hline SWIRLN(I) & real & 一 & $\begin{array}{l}\text { Swirl no. for the } I^{\text {th }} \\
\text { inlet. }\end{array}$ & Variable \\
\hline SWIRLS & real & - & $\begin{array}{l}\text { Swirl no. for the } \\
\text { secondary stream, } \\
\text { defined as flux of } \\
\text { angular momentum } \\
\text { divided by product of } \\
\text { axial momentum flux } \\
\text { and secondary duct } \\
\text { radius. }\end{array}$ & Variable \\
\hline TBE & real & $\mathrm{K}$ & $\begin{array}{l}\text { Temperature boundary } \\
\text { condition for the east } \\
\text { face of intrusions. }\end{array}$ & Variable \\
\hline
\end{tabular}


FORTRAN

Variable

TBN(I)

TBW

THER

THICK

THIDEL real

$\mathrm{m}$

real

real

\section{Units}

$\mathrm{K}$

real $\quad K$

Character -

$\mathrm{K}$

$\mathrm{K}$
Recommended

Description and Usual or Typical

Symbol Value

Temperature boundary Variable

condition for the north

(side) wall and north

face of intrusions.

Temperature boundary Variable

condition for the west

(front) wall and west

face of intrusions.

Thermo card. Must THER

contain the string

'THER'.

Thickness of tube wallDepends on between primary and the geometry secondary streams.

Relative (to adiabatic 500 . or greater temperature)

maximum temperature

for table generation.

Upper limit of 5000 .

temperature range for

thermodynamic

correlations for gas

species. 
FORTRAN Variable

THRMNO
Type

integer

$-$

$\begin{array}{lll}\text { TINFLO(I) } & \text { real } & - \\ \text { TIPD } & \text { logical } & -\end{array}$
Recommended

Description and Usual or Typical

Symbol Value

Flag for choosing thermal NO

mechanism:

$0=$ thermal NO not

calculated

$1=$ include forward and reverse rates of extended Zel'dovich

mechanism

2 =include only

forward rates of

Zel'dovich mechanism

Turbulence intensity 0.10

of $I^{\text {th }}$ inlet stream.

.TRUE. will use $F$

turbulence intensity

profile data from

INLET file for the additional inlet tube, and calculates adjusted profiles at the inlet node points (not functional at present). .FALSE. will use theoretical calculations for the profile. 
FORTRAN

Variable

TIPDAT

TISDAT

TLAG(J)

TLODEL logical

real

-

Type

Units

logical

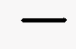

一

$\mathrm{K}$
Description and Usual Recommen

Symbol

Value

.TRUE. will use

turbulence intensity

profile data from

INLET file for the

primary tube, and

calculate adjusted

profiles at the inlet node points. .FALSE

will use theoretical calculations for the profile.

.TRUE. will use $F$

turbulence intensity

profile data from

INLET file for the

secondary annulus,

and calculate adjusted

profiles at the inlet

node points.

.FALSE. will use

theoretical calculations

for the profile.

Ratio of inlet particle 1.0

temperature $(\mathrm{K})$ to

inlet gas temperature

(K).

Relative (to adiabatic 1500 . or more

temperature) minimum

temperature for table

generation. 
FORTRAN

Variable

TLOW

TMAX

TMIN

TMP

TNBP

TOUT
Type Units

real

$-$

K

real

real

$\mathrm{K}$

$\mathrm{K}$

real

real

K

real
Description and Usual or Typical

Symbol

$\underline{\text { Value }}$

Lower limit for 300 .

temperature range of

thermodynamic

correlations for gas

species.

Absolute maximum 2500. or

temperature for table greater for generation. combustion, 3000. or greater for oxygen-blown gasification Absolute minimum Lowest of inlet temperature for table generation.

gas temperatures, often $298 \mathrm{~K}$

Temperature of inlet Often room stream NSTRM if temperature NSTRM $=1$, TMP is for primary, temperature of higher for primary gas stream; = secondary 2 , temperature of secondary gas stream.

Normal boiling point $373 \mathrm{~K}$ for of slurry liquid. $\mathrm{H}_{2} \mathrm{O}$ at $1 \mathrm{~atm}$ Outlet gas temperature 0 . for radiation $(0.0$ yields adiabatic condition). 


\section{FORTRAN}

Variable

TURBIN(I) real

UIN(I)

UPD

\section{Type Units}

real

$\mathrm{m} / \mathrm{s}^{-1}$

\section{Recommended \\ Description and Usual or Typical \\ Symbol \\ Value}

Experimental Depends on

normalized turbulent data

intensity at radial

position RIN(I). (The

unnormalized

turbulent intensity will

be utilized if the

selection parameter

KOPTE* so

specifies).

Experimental axial Depends on

velocity at radial data

position RIN(I).

.TRUE. will use axial $\mathrm{T}$

velocity profile data

from an inlet data file

for the additional inlet

tube, and calculates

adjusted profiles at the

inlet node points.

.FALSE. will use

theoretical calculations

for the profile (option

not functional at

present). 
FORTRAN

Variable

UPDAT

UPDWN

real

Type

$\underline{\text { Units }}$

logical

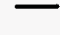

$\begin{array}{ll}\text { UPLAG(ISL) } & \text { real } \\ \text { URFDEN } & \text { real } \\ \text { URFE } & \text { real } \\ \text { URFETA } & \text { real } \\ \text { URFF } & \text { real }\end{array}$

Recommended Description and Usual or Typical Symbol

Value

.TRUE. will use axial T

velocity profile data

from an inlet data file

for the primary tube, and calculates adjusted profiles at the inlet node points.

.FALSE. will use theoretical calculations for the profile.

$1.0=$ co-gravity flow. $1.0,-1.0$, or $-1.0=$ counter-gravity 0.0

flow.

$0.0=$ horizontal flow (gravity effects neglected).

Ratio of inlet particle $\quad 1.0$ velocity to inlet gas velocity.

Under-relaxation 0.3 for factor for density, $\lambda_{n}$. SIMPLE, 0.5 for SIMPLER

Under-relaxation 0.5 for factor for eddy SIMPLE, 0.7 dissipation rate, $\lambda_{\varepsilon}$. for SIMPLER Under-relaxation 0.5 for factor for $\eta, \lambda_{n}$. SIMPLE, 0.7 for SIMPLER

Under-relaxation $\quad 0.5$ for factor for $f, \lambda_{\mathrm{f}}$.
SIMPLE, 0.7

for SIMPLER 
FORTRAN

Variable

URFG

URFGET

URFH

URFHCN

real

Type

Units

real
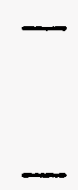

real

real

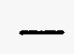

URFK real -

URFNH3 rea

$\begin{array}{lll}\text { URFNJ } & \text { real } \\ \text { URFNOX } & \text { real }\end{array}$

URFP real -

URFPH

real
Description and Usual or Typical

Symbol

Under-relaxation $\quad 0.5$ for

factor for $g_{f}, \lambda_{\mathrm{gf} .} \quad$ SIMPLE, 0.7 for SIMPLER

Under-relaxation $\quad 0.5$ for factor for $g_{\mathfrak{n}}, \lambda_{g_{n}}$. SIMPLE, 0.7 for SIMPLER

Under-relaxation $\quad 0.8$ for factor for $h$, gh. $\quad$ SIMPLE, 0.9 for SIMPLER

Under-relaxation $\quad 0.9$ factor for $\mathrm{HCN}$ in $\mathrm{NO}_{\mathrm{x}}$ submodel. Under-relaxation $\quad 0.5$ for factor for $k, \lambda_{k}$. SIMPLE, 0.7 for SIMPLER

Under-relaxation $\quad 0.9$ factor for $\mathrm{NH}_{3}$ in $\mathrm{NO}_{x}$ submodel Under-relaxation $\quad 1.0$ factor for $n_{j}, \lambda_{n_{j}}$. Under-relaxation $\quad 0.9$ factor for $\mathrm{NO}$ in $\mathrm{NO}_{\mathrm{x}}$ submodel.

Under-relaxation 1.0 for factor for $p, \lambda_{D}$. SIMPLER, not used for SIMPLE

Under-relaxation 0.5 or less, factor for $S_{p}^{h} \quad$ sometimes 0.1 
FORTRAN

Variable

URFPM

URFPP

URFSO2

URFU

URFV

URFVIS

URFW

$\begin{array}{ll}\text { Type } & \text { Units } \\ \text { real } & -\end{array}$

real

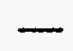

$$
\text { real }
$$

$$
\text { real }
$$

real

real

real

Recommended

Description and Usual or Typical

Symbol

Value

Under-relaxation 0.5 or less, factor for $S_{n}^{u}, S_{n}^{v}, S_{n}^{m}$. but usually not as small as URFPH

Under-relaxation $\quad 0.8$ for factor for $p^{\prime}, \lambda_{0}{ }^{\prime}$. SIMPLER, not used for SIMPLE

Under-relaxation $\quad 0.9$ factor for $\mathrm{SO}_{2}$ or $\mathrm{H}_{2} \mathrm{~S}$ mole fraction after sorbent reactions. Under-relaxation 0.5 for factor for $u, \lambda_{\mathrm{u}}$. SIMPLE, 0.7 for SIMPLER

Under-relaxation $\quad 0.5$ for factor for $v, \lambda_{v}$. SIMPLE, 0.7 for SIMPLER

Under-relaxation $\quad 0.3$ for factor for $v^{t}, \lambda_{v} \tau$. SIMPLE, 0.5 for SIMPLER

Under-relaxation for 0.5 for $w, \lambda_{w}$. SIMPLE, 0.7 for SIMPLER 
FORTRAN

Variable

USDAT

USDAT

VIN(I)

VISCOS

VPD

\section{Type}

Units

logical

real

$\mathrm{m} \mathrm{s}^{-1}$

real

logical

\section{Recommended \\ Description and Usual or Typical \\ Symbol \\ Value}

.TRUE. will use axial $\mathrm{F}$

velocity profile data

from an inlet data file

for secondary

annulus, and

calculates adjusted

profiles at the inlet

node points.

.FALSE. will use

theoretical calculations

for the profile.

Experimental radial velocity at radial

Depends on data position RIN(I).

Approximate laminar $1.79 \mathrm{E}-5$ for 1

viscosity for entire atm

reaction chamber.

.TRUE. will use $T$

radial velocity profile

data from an inlet data

file for the additional

inlet tube, and

calculates adjusted

profiles at the inlet node points.

.FALSE. will use theoretical calculations

for the profile (option

not presently

functional). 
FORTRAN

Variable

VPDAT

WIC(J, K) real

WIN (I)

$\begin{array}{ll}\text { Type } & \text { Units } \\ \text { logical } & -\end{array}$

logical

real

$\mathrm{m} \mathrm{s}^{-1}$
Description and Usual or Typical

Symbol

Value

$\mathrm{F}$

TRUE. will use
radial velocity profile

data from an inlet data

file or the primary tube, and calculates adjusted profiles at the inlet node points.

.FALSE. will use theoretical calculations for the profile. .TRUE. will use $F$ radial velocity profile data from an inlet data file for the secondary annulus, and calculates adjusted profiles at the inlet node points.,

.FALSE. will use theoretical calculations for the profile.

$\mathrm{K}^{\text {th }}$ elemental mass Depends on fraction of Jth particle coal type, not size, $\omega_{\mathrm{jk}}$, specified on needed for a daf basis in order of FG-DVC elements listed in thermodynamic file.

Experimental Depends on tangential velocity at data radial position RIN(I). 
FORTRAN

Variable

WPD

WPDAT
Type

Units

logical

-

logical -

Recommended

Description and Usual òr Typical

Symbol Value

.TRUE. will use $\mathrm{T}$

tangential velocity

profile data from an

inlet data file for the

additional inlet tube, and calculates adjusted

profiles at the inlet node points.

.FALSE. will use theoretical calculations

for the profile (option

not presently

operational).

.TRUE. will use $\mathrm{F}$

tangential velocity

profile data from an

inlet data file for the

primary tube, and

calculates adjusted

profiles at the inlet

node points.

.FALSE. will use

theoretical calculations

for the profile. 
FORTRAN Variable

WSDAT
Type

Units

logical

real

$\mathrm{XI}(\mathrm{J})$

XIANOX

real

XPS(ISL) real

m

YPS (ISL) real m

YPSH

real

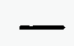

YPSL real
Description and Usual or Typical

Symbol

Value

.TRUE. will use

F

tangential velocity

profile data from an

inlet data file for the secondary annulus, and calculates adjusted

profiles at the inlet node points. .FALSE.

will use theoretical

calculations for the profile.

Surface area factor for 1.0

$J^{\text {th }}$ particle type, $\zeta_{\mathrm{j}}$.

Fractional change in $\approx 1.0$

external char area used

in NO model as a

result of swelling and

fracturing.

Axial position of the 0.0 for front

ISL starting location. wall

Radial position of the Variable

ISL starting location.

Normalized upper

0.95

bound for particle

starting locations

(r/R).

Normalized lower

bound for particle

0.05

( $\mathrm{r} / \mathrm{R})$. 
FORTRAN

Variable

YY $(\mathrm{J}, \mathrm{M})$

$\mathrm{Z}(\mathrm{N} 1, \mathrm{~N}, \mathrm{I}) \quad$ real

ZEDA

real
Recommended

Description and Usual or Typical

Units . Symbol Value

Volatiles fraction for Depends on

$J^{\text {th }}$ particle with

coal type

regards to $M^{\text {th }}$

devolatilization

reaction $Y_{j m}$.

Coefficients for $\mathrm{I}^{\text {th }}$ Depends on species heat capacity species

equation. For $N=1$, the temperature range

of the heat capacity is

generally $300 \mathrm{~K}$ -

$1000 \mathrm{~K}$ and for $\mathrm{N}=$

2 , the temperature

range is generally

$1000 \mathrm{~K}-5000 \mathrm{~K}$ (see

TLOW and THIGH).

In both cases:

$$
\begin{aligned}
& \frac{h}{R T}=\left(\sum_{i i=1}^{5} z_{i i} \frac{T^{(i i-I)}}{i i}\right) \\
& \frac{S^{o}}{R}=z_{1} \ln T+\left(\sum_{n=2}^{5} z_{i i}\right.
\end{aligned}
$$

Fractional conversion 1.0

of devolatilized

nitrogen to $\mathrm{HCN}$ in

the gas phase in $\mathrm{NO}_{x}$

submodel.

Index Identification

I Species

ISL Particle starting location

J Particle size/type 
FORTRAN

Variable
Type $\underline{\text { Units }}$

$\mathrm{K}$

L

M

$\mathrm{N}$

II
Recommended

Description and Usual or Typical Symbol

Value

Elements

Char reaction

Devolatilization reaction

Particle component

Arbitrary 
Description of Subroutines 


\section{Appendix C}

\section{Sample Script Files for UNIX Operation Systems}

Two C-shell scripts are used to run PCGC-2 in the UNIX environment at BYU. The first script (pcf) interacts with the user and assigns the filenames to environment variables which are then passed to the second script (pcgc2.com). The second script performs the file manipulation and runs the program.

Of course, these two scripts are just examples and must be adapted to each individual computer environment. Three environment variables must be defined in the C-shell before these scripts are used. \$PCSRC is the pathname of the second script (pcgc2.com). \$PCEXE is the pathname of the executable. \$PCBUG is the name of the host symbolic debugger. At BYU, these variables are defined in each user's .cshrc file. A partial listing of a sample .cshrc file is also shown to indicate how this can be done. script, type

The first script (pcf) provides several options to the user. To execute this

$$
\text { pcf - } d b \text {-batch -test filename }
$$

where filename is the specific name of the case that you are running. The above command assumes that the script or a link to it exists in the same directory as the input files for the case that is being simulated. Everything after pcf is optional. The modifier $-\mathrm{db}$ runs the program in the symbolic debugger \$PCBUG. The modifier -batch causes the script pcgc2.com to be submitted to a batch queue 
Sample Script Files for UNIX Operation Systems

rather than being submitted as an interactive process. The modifier - test causes a version of the executable in \$PCEXE/test to be used rather than \$PCEXE. This feature is useful for code development. If filename is absent, the script prompts interactively for the input, thermo, inlet profile, output, and restart filenames separately. Alternatively, they may all be given on the command line. If only a single filename is given as in the example above, all files for the simulation are assumed to have the same root filename with only the filename extension (e.g. .dat, .thm, .inl) being different.

Script File No. 1 - pcf

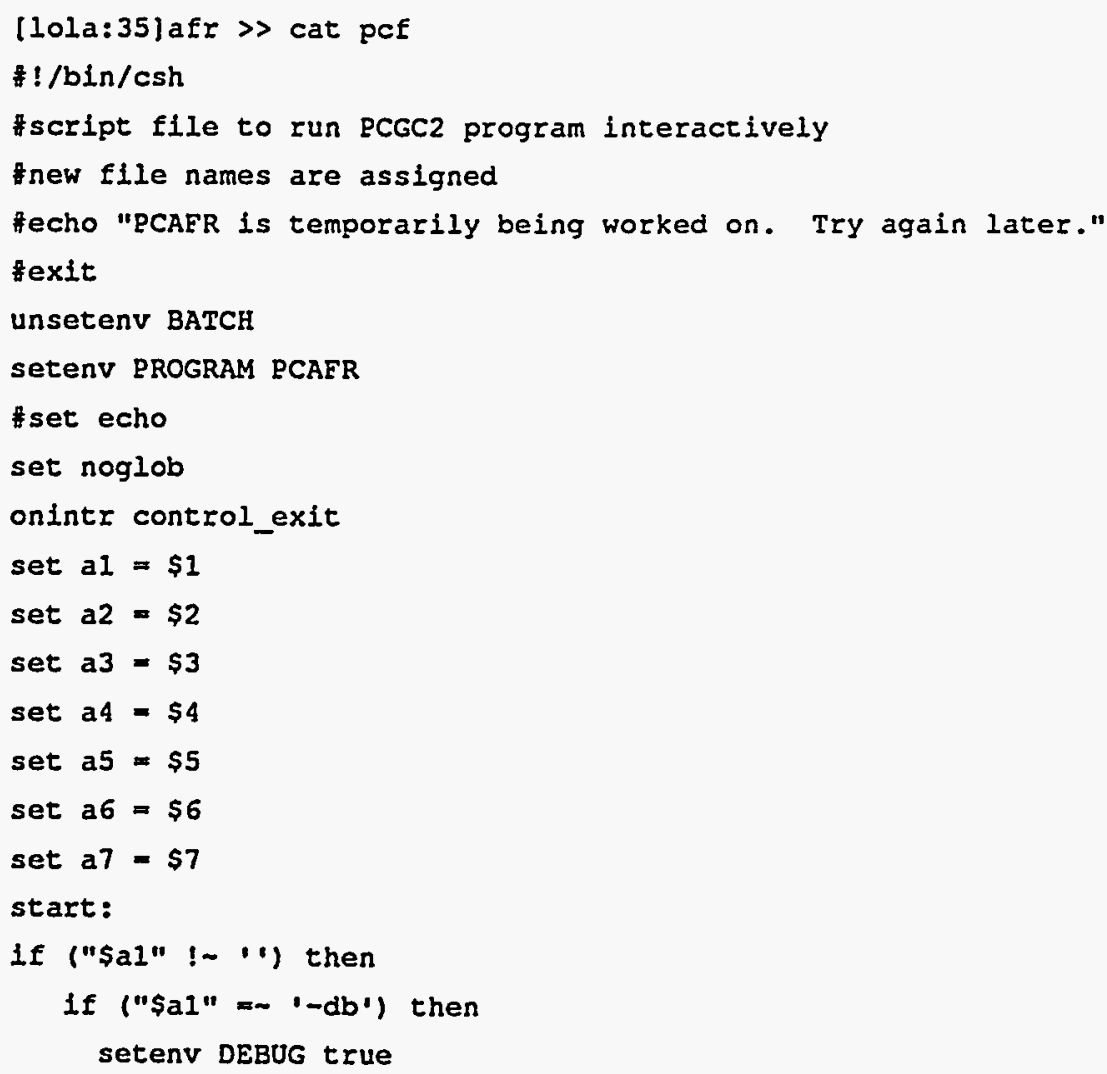




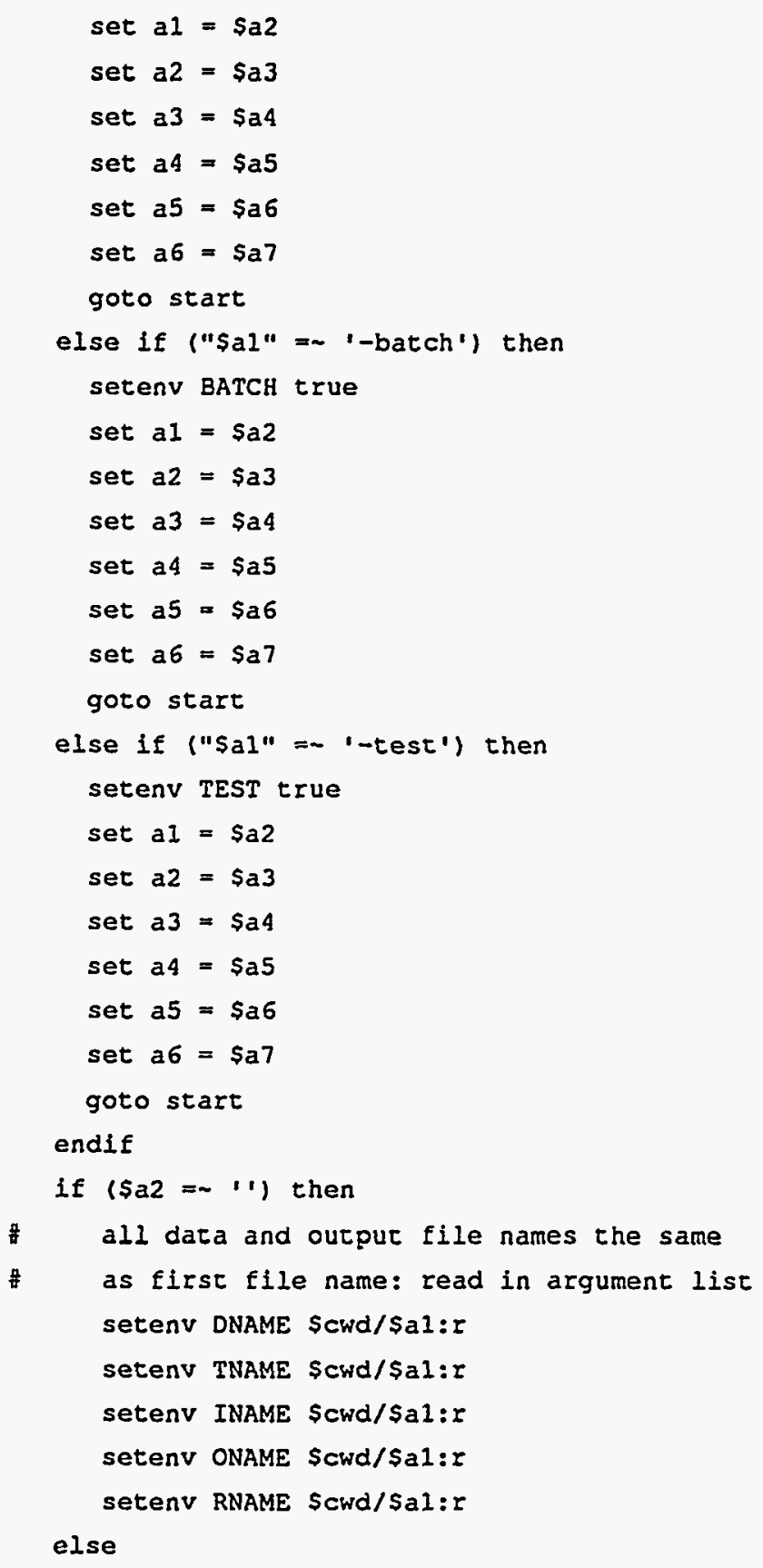


Sample Script Files for UNIX Operation Systems

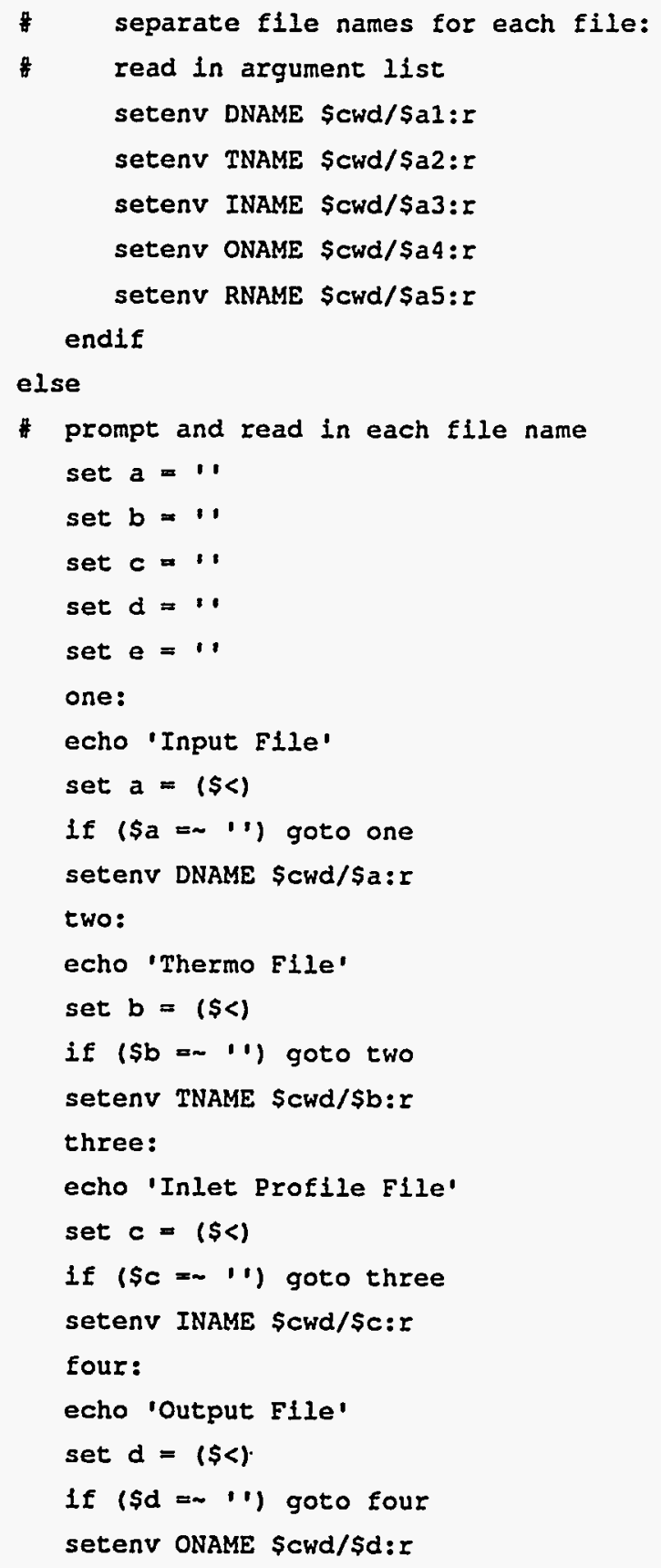


five:

echo 'Restart File'

set $e=(\$<)$

if ( $\$ \mathrm{e}=\sim$ ' ) goto five

setenv RNAME $\$ c w d / \$ e: r$

endif

endif

if (\$?BATCH) then

set oname = \$ONAME

set jobname = Soname: $r$

if ("/bin/hostname $=-$ "opus") then

echo "Which queue? $s$ = short queue (for jobs shorter than 10 minutes)"

echo" 1 = long queue (for long jobs)"

echo " $v$ = verylong queue (for very long jobs)"

echo " $a=$ acerc queue (rouces to acerc queue on lola)"

echo " $c=$ caedm queue (rouces to caedm queue on lola)"

echo "bl bestl queue (chooses the best queue from among"

echo "

long, acerc, caedm)"

set queue $=(\$<)$

goto submit

else if ("/bin/hostname" =- "lola") then

echo "Which queue? $s=$ short queue (for jobs shorter than 5 minutes)"

echo " $a$ = acerc queue (for jobs submitted by acerc group)"

echo " $\quad c=$ caedm queue (for jobs submitted by caedm group)"

echo " $\quad v=$ verylong queue (for very long jobs)"

set queue $=(\$<)$

goto submit

else

echo "Can't run batch jobs on" \$hostname". Job terminated." .

goto control_exit

endif

submit :

if (squeue $=\sim$ ' ') set queue $=$ 'bl'

if (\$?TEST) then

qsub -q \$queue -r \$PROGRAMI:\$jobname:t -o \$cwd/\$jobname:t. $\log 11$ 
Sample Script Files for UNIX Operation Systems

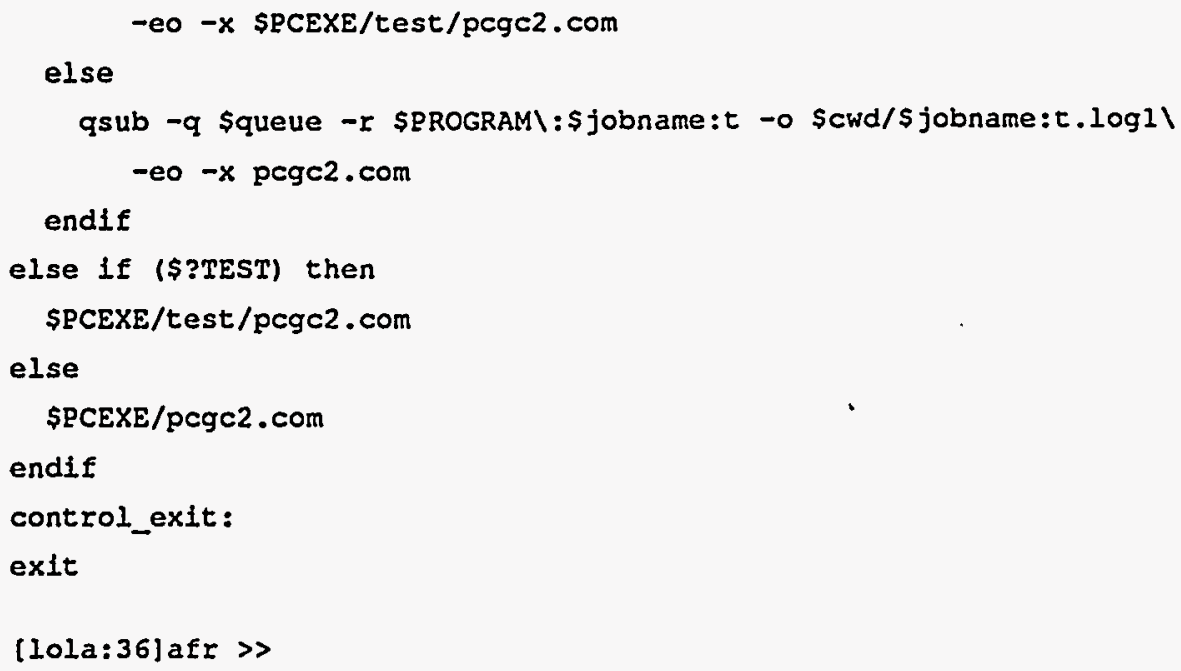

Script File No. 2 - pcgc2.com

[101a:37]c2 > cat pcgc2.com

\#/bin/csh

*script file to run PCGC2 program interactively

fset echo

\# Copy restart files to output, if different from output

if (\$RNAME ! \$ONAME) cP \$RNAME.rst SONAME.rst

if (\$RNAME ! \$ONAME) CP \$RNAME.pSO SONAME.pSO

if (\$RNAME ! SONAME) CP \$RNAME. InX SONAME.rnX

if (\$RNAME ! \$ONAME) cp \$RNAME.tbl \$ONAME.tbl

* If generic files exist, remove them

if (-e PCGCIN) rm PCGCIN

if ( $-e$ THERMO) $\mathrm{rm}$ THERMO

if ( $\mathrm{e}$ INLET) Im INLET

if ( $-e$ GRIDS) $\mathrm{rm}$ GRIDS

if (-e INOUT) $\mathrm{rm}$ INOUT

if ( -e RSNXIO) $\mathrm{cm}$ RSNXIO

if ( -e TBLRST) rm TBLRST 


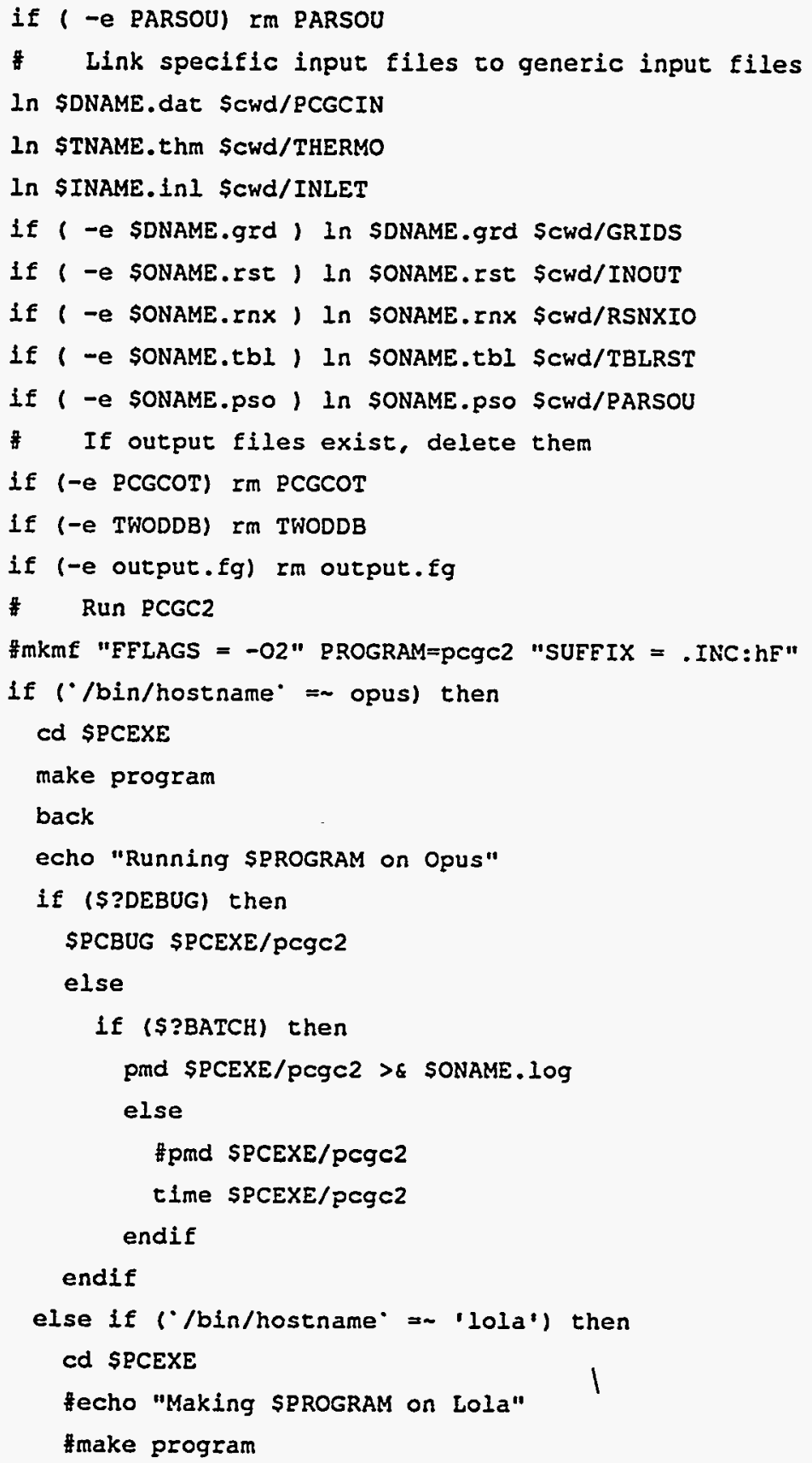




\section{Sample Script Files for UNIX Operation Systems}

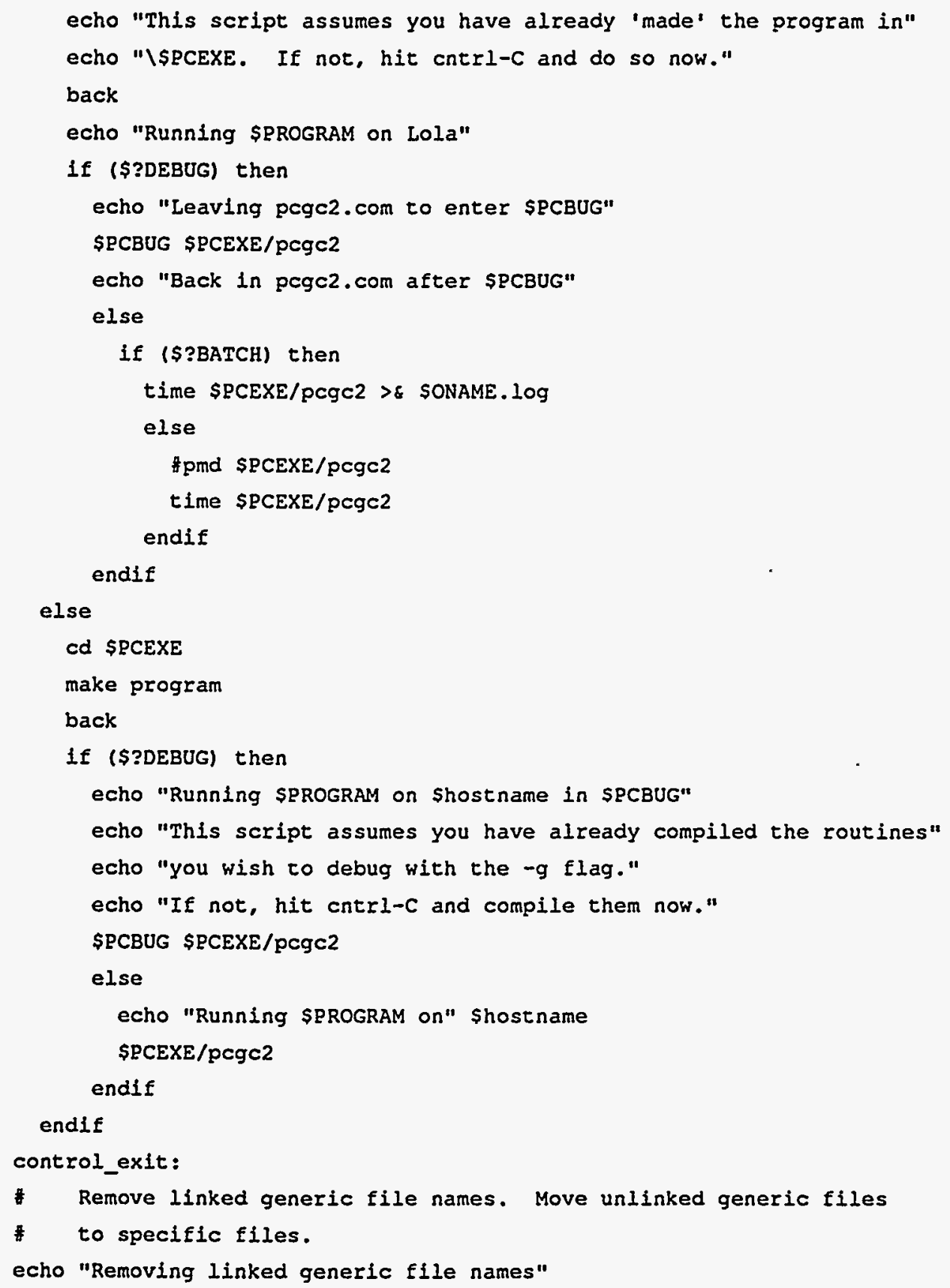

C-8 


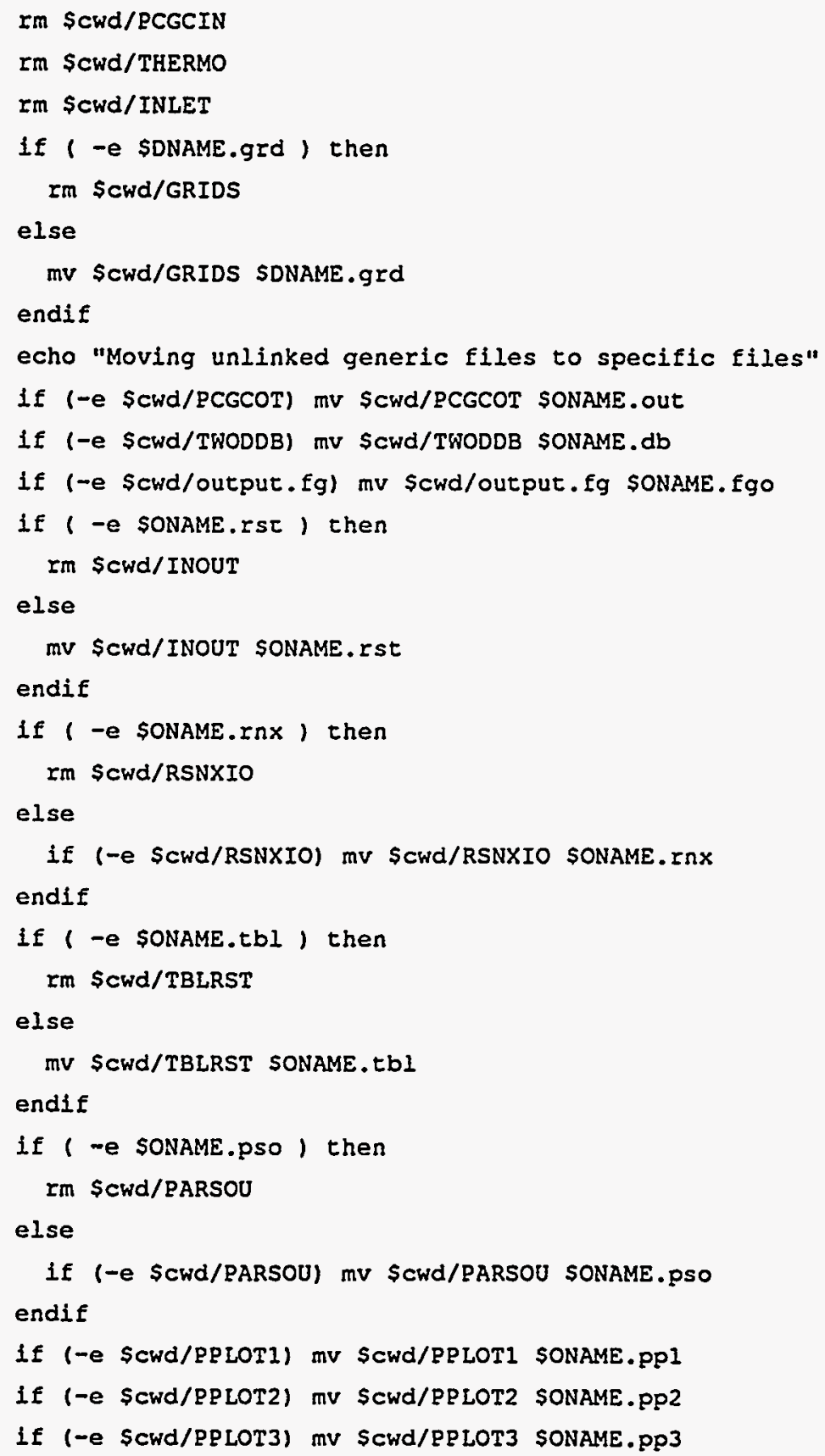


Sample Script Files for UNIX Operation Systems

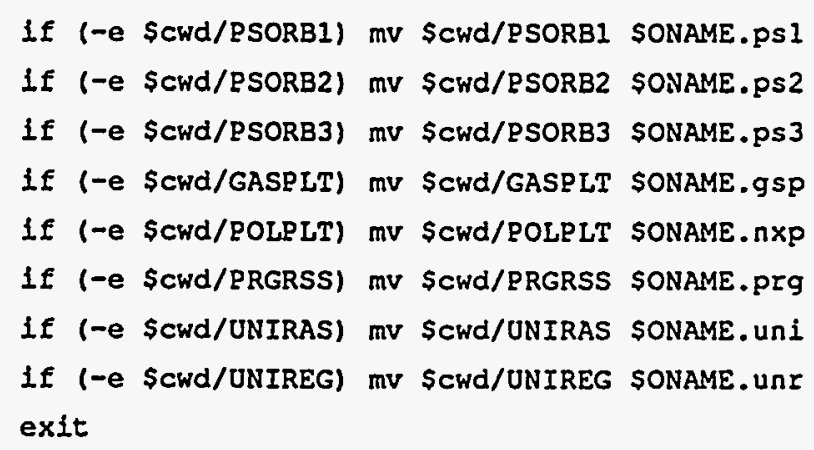

[1ola:38]c2 \>

Script File No. 3 - .cshrc (partial listing)

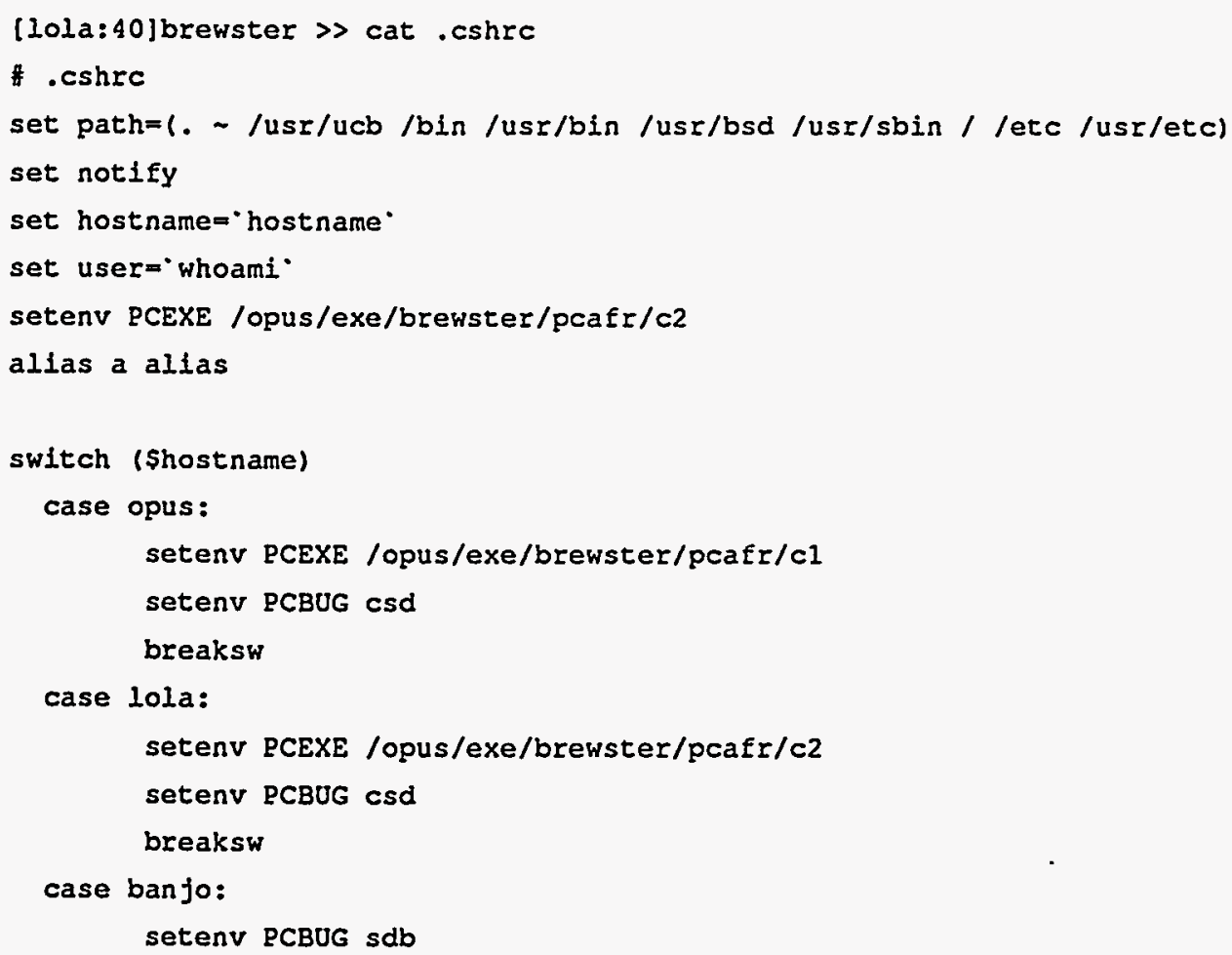




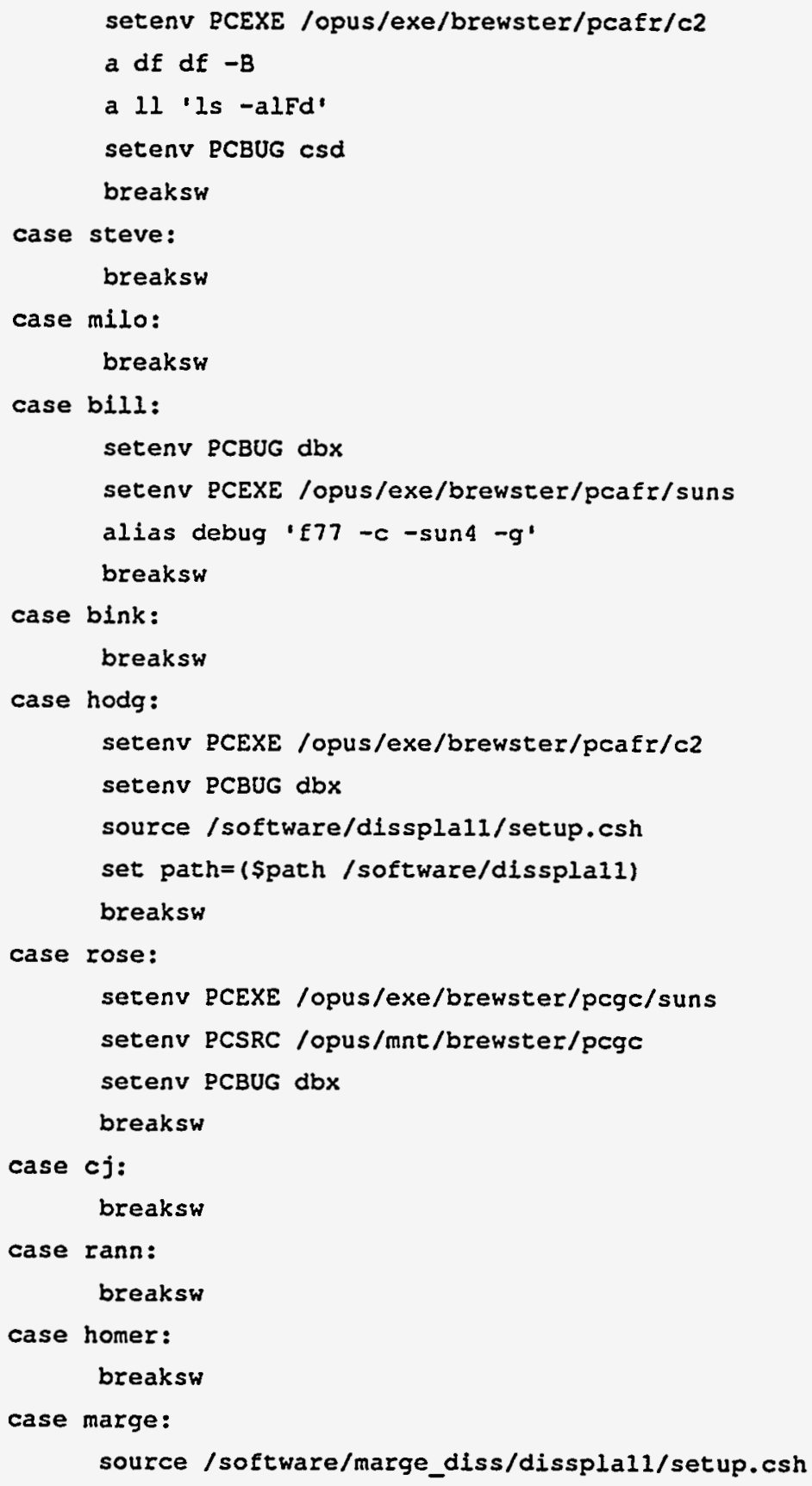


Sample Script Files for UNIX Operation Systems

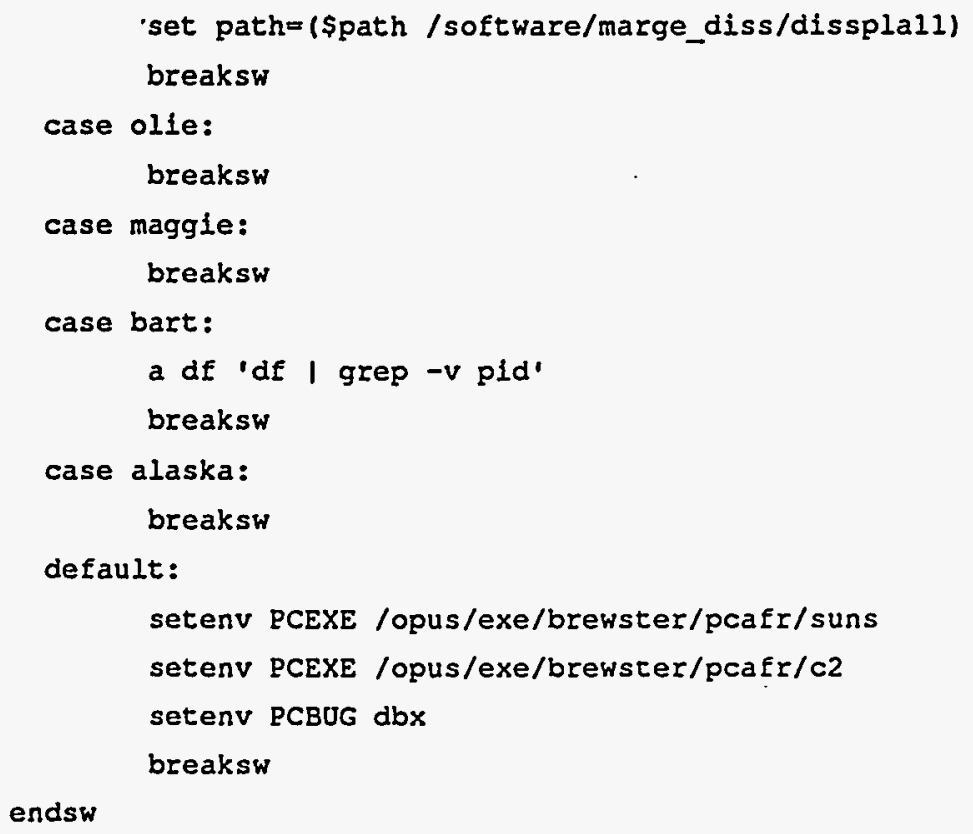




\section{Appendix D}

\section{Sample MAKE File for Program Maintenance on UNIX Systems}

Program maintenance can be simplified by using a Makefile on UNIX systems. Makefiles contain a description of all of the file dependencies in a program. Then, if one file is updated, all of the files which depend on that file will be updated automatically by issuing the make command. For example, if a FORTRAN source file is updated, it will be recompiled automatically by issuing the make command. If an INCLUDE file is updated, then all of the FORTRAN source files which incorporate that INCLUDE file will be recompiled automatically. It is possible to include the make command in the pcgc2. com script file and have the make command issued every time the program is run, as shown in Appendix C. A listing of a sample Makefile is shown below:

$\begin{array}{ll}\begin{array}{l}\text { [lola:30]test } \\ \text { DEST }\end{array} & = \\ \text { VPATH } & = \\ \text { EXTHDRS } & = \\ \text { HDRS } & = \\ \text { FFLAGS } & =-01-\mathrm{fi}-\mathrm{db}\end{array}$


Sample MAKE File for Program Maintenance on UNIX Systems

b

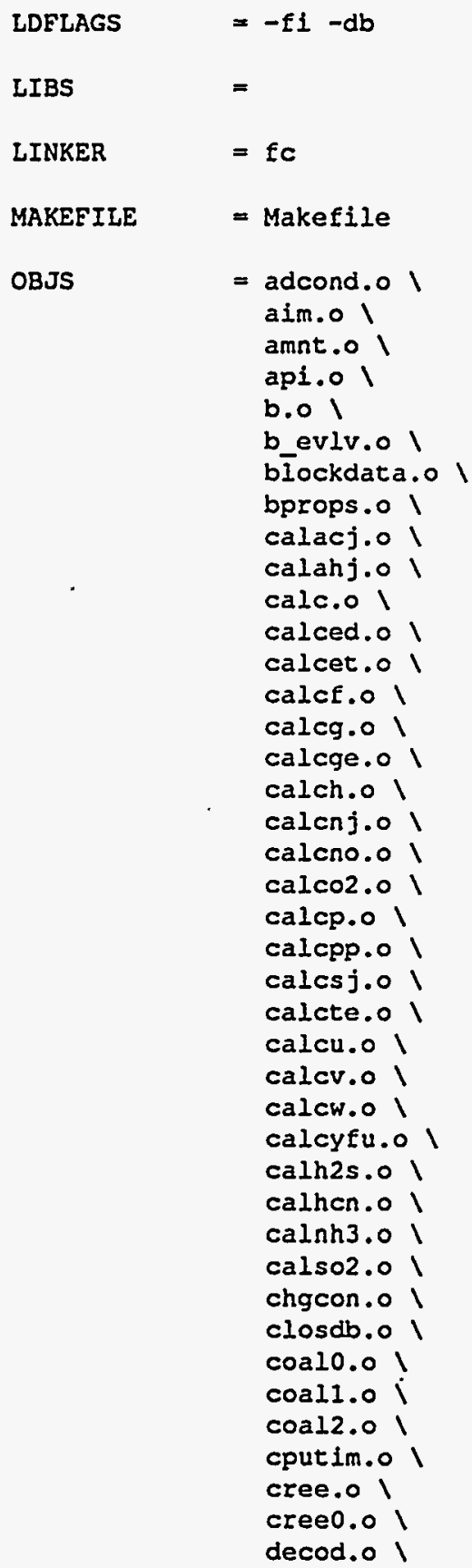




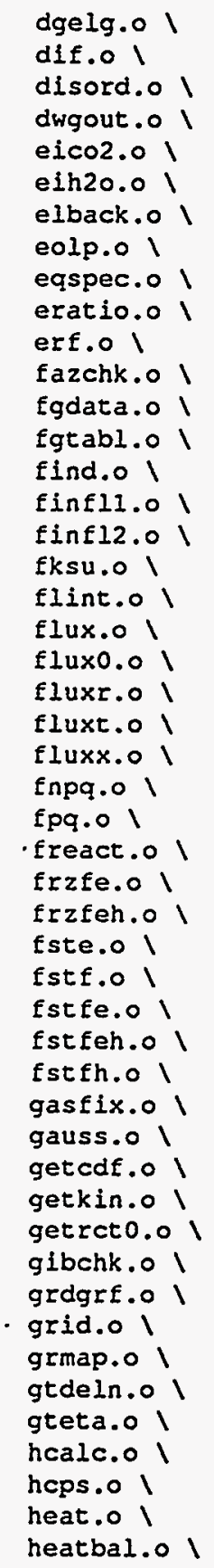




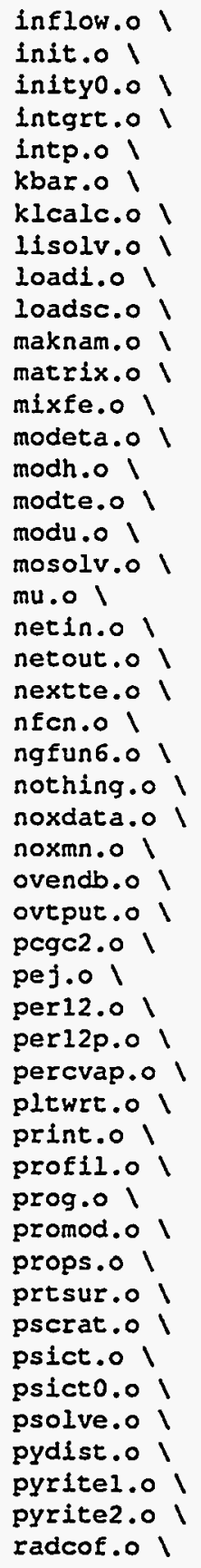




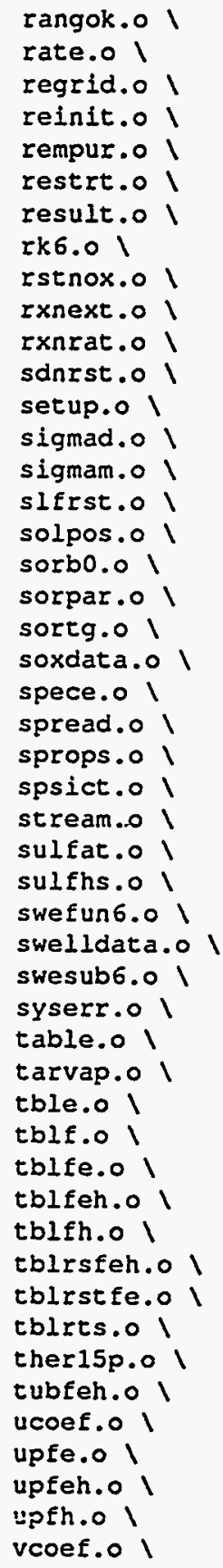




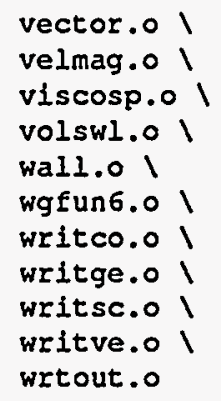

PROGRAM

$=. / \mathrm{pcgc} 2$

SRCS

$=$ adcond. $f \mid$ aim. $f$ amnt.f I api.f I

b.f 1

b_eviv.f ।

blockdata.f I

bprops.f I calacj.f I calahj.f I calc.f । calced.f I calcet.f I calcf.f । calcg.f I calcge.f I calch.f । calcnj.f I calcno.f I calco2.f I calcp.f I calcpp.f 1 calcsj.f I calcte.f I calcu.f I calcv.f । calcw.f I calcyfu.f I calh2s.f । calhon.f । calnh3.f I calso2.E I chgcon.E । 


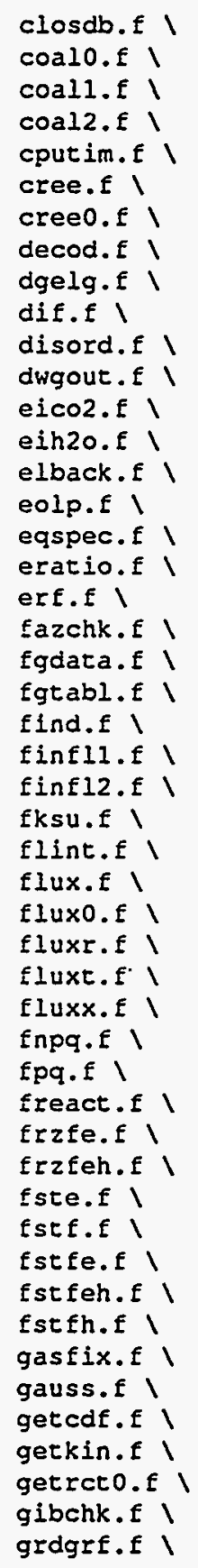


grid.f 1

grmap.f 1

gtdeln.f

gteta.f I

hcalc.f 1

hops.f

heat. I I

heatbal.f I

inflow.f ।

init.f 1

inityo.f 1

intgrt. $f 1$

intp.t 1

kbar.f I

klcalc.f ।

Iisolv.f ।

loadi.E ।

loadsc. $\mid$

maknam. I ।

matrix.f 1

mixfe.f 1

modeta.f 1

modh.f I

modte.f I

modu.f I

mosolv.f I

mu.f I

netin.f 1

netout. \& 1

nextte.f 1

nfen.f ।

ngfun6.f ।

nothing. 1

noxdata.f

noxmn.f ।

ovendb.f ।

ovtput.f I

pcgc2.f I

pej.f I

per12.f ।

per12p.f 1

percvap.f I

pltwrt.f ।

print.f ।

profil.f I

prog.f I

promod.f I

props.t I

prtsur.f 1 


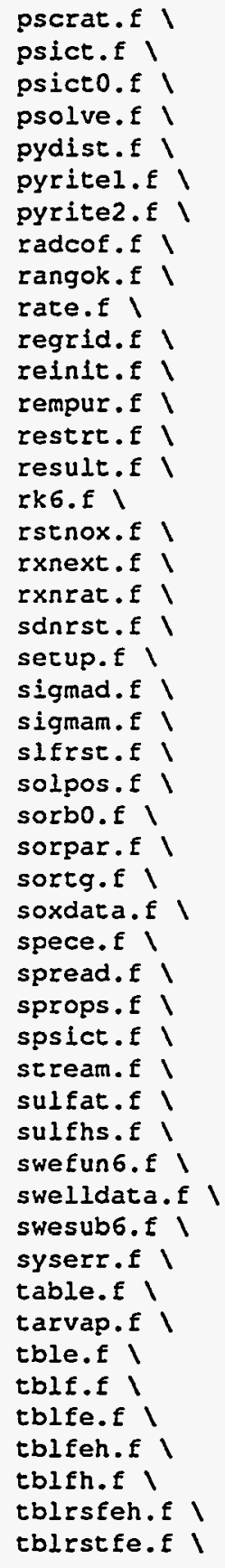




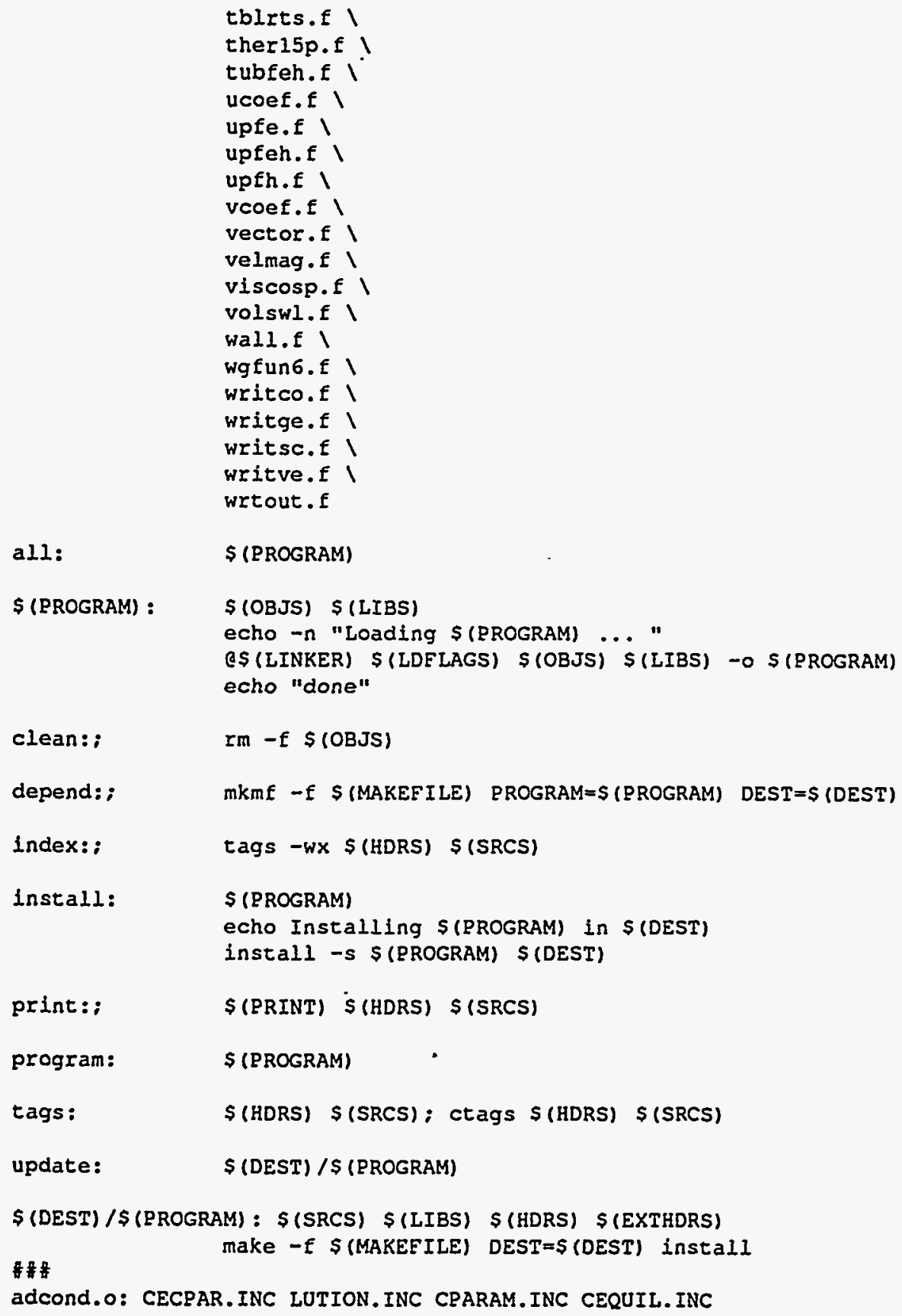


aIm.O: PARAMETER. INC COEF. INC FLURR. INC

api.O: CECPAR. INC CPARAM. INC CEQUIL.INC

b_evlv.o: $t$ ch4.inc

blockdata. O: PARAMETER. INC ADFLO.INC CSSELO.INC CPART.INC CTABLE. INC I CTNBP. INC GEOM.INC HCAP.INC KASETI.INC KRAD.INC LUTION.INC PCOR.INC I PROPIN.INC PSCRTI.INC RADCON.INC RCON.INC UVEL.INC difeq.inC

bprops.0: PARAMETER. INC ALL.INC CPARAM.INC CPART.INC CPEUL.INC FLUPR.INC I INTRUZ.INC KASETI.INC SAVBP.INC VAR. INC VARR. INC

calacj.0: PARAMETER. INC ADFLO.INC INTRUZ.INC ALL.INC CPART.INC CPSOU.INC I VAR. INC GEOM.INC FLUPR. INC COEF.INC TURB.INC PANDS.INC PARD.INC 1 TUBWAL.INC KASETI.INC CPEUL.INC SWPDIR.INC BOUND.INC CFLAG.INC I IPQAR. INC EULP. INC

calahj.0: PARAMETER. INC INTRUZ.INC ALL.INC VAR.INC GEOM.INC ELUPR.INC I COEF.INC TURB. INC PARD.INC TUBWAL.INC KASETI.INC CPEUL.INC SWPOIR.INC I BOUND.INC CFLAG.INC IRPAR. INC EULP. INC CPART.INC CPSOU.INC PANDS.INC

CaIC.O: PARAMETER. INC CCHEMI.INC CEQUIL.INC CINDEX. INC CMATRI.INC I CPARAM.INC CSPECE. INC PRIN.INC

calced.O: PARAMETER. INC AFR.INC ALL.INC TDIS.INC GEOM.INC FLUPR. INC । COEF.INC TURB.INC KASETI.INC CPSOU.INC INTRUZ.INC SWPDIR.INC SUSP.INC I VAR. INC

calcet.0: PARAMETER. INC AFR.INC CPART. INC ALL.INC CGASMF. INC VAR. INC I GEOM.INC FLUPR. INC COEF. INC TURB. INC MIXER. INC KASET1.INC CPSEE.INC । SWPDIR. INC CPSOU.INC INTRUZ.INC

CaICf.O: PARAMETER. INC AFR.INC INTRUZ.INC ALL.INC VAR.INC GEOM.INC I FLUPR.INC COEF.INC TURB.INC MIXER.INC CRSOU.INC SWPDIR.INC KASETI.INC I TABRIT.INC CGASMF.INC CPSEF.INC

Calcg.0: PARAMETER. INC ALL.INC VAR.INC GEOM.INC FLUPR. INC COEF. INC TURB. INC I FLUC.INC KASETI.INC CPSOU.INC SWPDIR.INC INTRUZ.INC

calcge.0: PARAMETER. INC ALL.INC CGASME.INC VAR. INC GEOM.INC FLUPR.INC I COEF. INC TURB. INC FLUC.INC KASETI.INC CPSOU. INC SWPDIR. INC INTRUZ.INC

calch.0: PARAMETER. INC CPART.INC AFR.INC PRIN.INC INTRUZ.INC ALL.INC I ENTH.INC VAR. INC GEOM.INC FLUPR.INC COEF.INC TURB.INC KASET1.INC I CPSOU.INC.SWPDIR. INC RADCON.INC TYPE.INC VARR. INC KRAD.INC

calcnj.0: PARAMETER. INC AFR. INC ALL.INC INTRUZ.INC VAR.INC GEOM.INC । ELUPR.INC COEF. INC TURB.INC PARD.INC TUBWAL.INC KASETI.INC CPSOU.INC I CPART.INC CPEUL.INC SWPDIR.INC CFLAG.INC PANDS.INC IPPAR. INC EULP.INC

calcno.0: PARAMETER. INC ALL.INC INTRUZ.INC VAR. INC GEOM.INC FLUPR.INC I COEF. INC TURB. INC FLUC.INC CALCYI. INC NOXMN1. INC NOXRTE. INC NSWPS.INC I KASETI.INC RATECM.INC RATPAR.INC SWPDIR.INC TUBWAL.INC CPSOU.INC

CalCO2.0: PARAMETER. INC ALL.INC CALCYI.INC CGASMF.INC CINDEX. INC INTRUZ.INC I PSCCOM.INC RATECM. INC RATPAR. INC VAR.INC

CaICP.O: PARAMETER. INC PRIN.INC ALL.INC INTRUZ.INC PCOR.INC VAR.INC I GEOM.INC COEF. INC CPSOU. INC SWPDIR. INC SIMPLE. INC FLUPR. INC I FLUPR2 . INC

CaICPP.0: PARAMETER.INC PRIN.INC ALI.INC INTRUZ.INC PCOR.INC VAR.INC I GEOM. INC COEF. INC CPSOU. INC SWPDIR.INC SIMPLE. INC SWPALT.INC I ELUPR. INC FLUPR2. INC

calcsj.0: PARAMETER. INC ALL. INC EULP.INC INTRUZ.INC VAR.INC GEOM.INC I FLUPR. INC COEF. INC TURB. INC PARD. INC TUBWAL.INC KASETI.INC CPSOU.INC I 
Sample MAKE File for Program Maintenance on UNIX Systems

CPART. INC CPEUL.INC SWPDIR. INC CELAG.INC PANDS. INC IPPAR. INC

calcte.0: PARAMETER.INC AFR.INC ALL.INC INTRUZ.INC GRIDI.INC TEN.INC I VAR. INC GEOM.INC FLUPR.INC COEF.INC TURB.INC KASET1.INC SUSP. INC I CPSOU. INC SWPDIR. INC SWRL.INC

CaLCU.0: PARAMETER. INC AFR.INC ALL.INC INTRUZ.INC UVEL.INC VAR.INC GEOM.INC I COEF.INC KASET1.INC CPSOU.INC SWPDIR.INC SIMPLE.INC FLUPR.INC । FLUPR2.INC PARD. INC PCOR.INC

CalCV.0: PARAMETER. INC AFR.INC ALL.INC INTRUZ.INC VVEL.INC VAR.INC GEOM.INC I COEF. INC KASETI.INC CPSOU.INC SWPDIR.INC SWRL.INC FLUPR.INC I FLUPR2. INC PCOR. INC SIMPLE. INC

CaICW.O: PARAMETER.INC ALL.INC INTRUZ. INC WVEL.INC VAR.INC GEOM.INC I FLUPR. INC COEF. INC KASETI. INC CPSOU.INC SWPDIR.INC

CalCY fu.O: PARAMETER. INC ALL.INC CGASMF. INC COEF. INC CPSEF. INC CPSOU.INC I FLUPR. INC GEOM.INC INTRUZ. INC KASET1.INC MAGHJ.INC MIXFR.INC । SWPDIR. INC SSUM. INC TURB. INC VAR. INC

Calh2s.0: PARAMETER. INC ALL.INC COEF. INC CPSOU.INC FLUC.INC FLUPR.INC I GEOM. INC INTRUZ. INC KASET1.INC NOXMN1. INC NSWPS.INC RATESX. INC I SOXRTE.INC SWPDIR. INC TUBWAL.INC TURB. INC VAR. INC

calhen.0: PARAMETER.INC ALL.INC INTRUZ.INC VAR.INC GEOM.INC FLUPR.INC । COEF. INC TURB. INC FLUC.INC CALCYI.INC NOXMNI.INC NOXRTE. INC NSWPS.INC I KASET1.INC RATECM.INC RATPAR.INC SWPDIR.INC TUBWAL.INC CPSOU.INC

calnh3.0: PARAMETER. INC ALL. INC INTRUZ.INC VAR.INC GEOM.INC FLUPR.INC । COEF. INC TURB. INC FLUC.INC CALCYI.INC NOXMNI.INC NOXRTE. INC NSWPS.INC I KASET1.INC RATECM.INC RATPAR.INC SWPDIR.INC TUBWAL.INC CPSOU.INC

calso2.0: PARAMETER. INC ALL. INC COEF.INC CPSOU.INC FLUC.INC FLUPR.INC I GEOM.INC INTRUZ. INC KASET1.INC NOXMNI. INC NSWPS.INC RATESX. INC I SOXRTE. INC SWPDIR. INC TUBWAL.INC TURB. INC VAR.INC

chgCON.O: CECPAR. INC LUTION. INC CSPECE. INC CPARAM.INC

COa10.0: PARAMETER. INC TFLOF10.INC MAGHJ.INC AFR.INC ALL.INC BUG.INC । CCHEMI.INC CEQUIL.INC CELAG.INC CGSELO.INC CHETRX. INC CINDEX. INC I CNDXCG.INC CPARAM.INC CPART.INC CPLOT.INC CPSEF.INC CPSOU.INC I CSPECE.INC CTNBP. INC CWATER.INC FLUPR. INC GEOM. INC GPARAM. INC I HCAP.INC HCAPFG.INC HREF.INC ICON.INC IPQAR.INC KASETI.INC MRCK.INC I PANDS.INC PFAC.INC PRIN.INC PVAR.INC RCON.INC RCONT.INC FIAFO.INC I RRAT.INC TABRIT.INC TRAJ.INC TUBWAL.INC UNDER. INC VAR.INC difeq.inC I elem.inc ffids.inc order.inc reactk. inc

cOal1.0: PARAMETER. INC PRIN. INC CSPECE. INC CEQUIL.INC CCHEMI.INC CINDEX. INC I CPARAM.INC DIFUS. INC RCON. INC RCONT.INC TRAJ.INC ICON. INC GPARAM.INC

coal2.0: PARAMETER. INC AFR. INC elem. InC CPARAM. INC CCHEMI. INC CEQUIL. INC I CINDEX.INC CHETRX.INC CSPECE.INC CPART.INC difEq.inC RCONT.INC । GEOM.INC IPPAR. INC HCAP.INC HREF.INC RRAT.INC DIFEQN.INC VARR. INC I RADCON.INC OMEGA. INC PECOR.INC PFAC.INC PRIN.INC DIFUS.INC TRAJ.INC । ICON.INC RCON.INC GPARAM.INC MRCK.INC dvccomp.inc order.inc pools.inc $I$ rates.inc finish.inc tempcm. inc xeffcm.inc CNDXCG. INC

cree.0: PARAMETER. INC CCHEMI.INC CEQUIL. INC CINDEX. INC CPARAM.INC I CSPECE. INC LUTION. INC PRIN. INC

Cree0.0: PARAMETER. INC CCHEMI.INC CEQUIL.INC CINDEX.INC CMATRI.INC । CPARAM. INC CSPECE. INC LUTION. INC PANDS. INC PRIN. INC CNDXCG. INC

decod.o: defs.inc config.inc 
disord.0: PARAMETER. INC ALL.INC BCRAD.INC CPART.INC CSET.INC DISO.INC I FLUPR.INC GEOM.INC GRIDI.INC INTRUZ.INC KASETI.INC KRAD.INC । NOXMN1.INC PARD.INC PRIN. INC RADCON.INC SHERX.INC SWPDIR. INC VARR.INC dwgout.o: defs.inc config.inc dwgswe.inc order.ınc pyrite.inc difeq.inc elback.0: pyrite.inc

EOIP.O: PARAMETER. INC AER.INC TELOF1O.INC TYPE.INC PRIN.INC ALL.INC I INTRU2.INC VAR.INC GEOM.INC FLUPR.INC COEF. INC TURB.INC RARD.INC । TUBWAL.INC KASETI.INC CPSOU.INC CPART.INC CPEUL.INC SWPDIR.INC I CFLAG.INC IPPAR. INC EULP. INC VARR. INC CGASME. INC CGSFLO.INC CPSEF.INC I UNDER. INC TIMER. INC PANDS. INC

eqspec.0: PARAMETER. INC AFR. INC ALL.INC AREXP. INC BOUND.INC BUG.INC । CALCYI. INC CCHEMI. INC CELAG.INC CGASMF. INC CGAUSS. INC CINDEX. INC । CPARAM.INC CPART.INC CPEUL.INC CPSOU.INC CSPECE. INC CTABLE.INC I DENS.INC FLUC.INC FLUPR. INC GEOM.INC GPARAM.INC INTRUZ.INC IPPAR. INC I KASET1.INC MOLWT.INC NOXMN1.INC NOXRTE. INC PANDS.INC PRORIN.INC I PRIN.INC PSCCOM.INC PVAR.INC RATECM.INC RATEST.INC RATPAR.INC I FIAEO.INC RESID.INC RMSCOM.INC TIMER.INC TABRIT.INC TRAJ.INC । TUBWAL.INC TURB. INC UPNX. INC VAR. INC XRATE. INC

erar10.0: PARAMETER. INC CCHEMI. INC CEQUIL.INC CINDEX. INC CMATRI.INC I CPARAM. INC CSPECE. INC PRIN. INC

fgdata.0: PARAMETER. INC HCAPFG.INC

fgtabl.0: CGAUSS. INC

finfl1.0: PARAMETER. INC order.inc finish.inc difeq.inc elem.inc pyrite.inc finf12.0: order.inc finish.inc pyrite.inc

flint.0: PARAMETER. INC TFLOF10. INC CTABLE. INC ADFLO. INC AFR. INC ALL.INC I CPART.INC CWATER.INC FLIN.INC GRIDI.INC INTRUZ.INC VAR.INC VVEL.INC । GEOM.INC FLUPR.INC PANDS.INC FIAEO.INC PVAR.INC KASETI.INC TUBWAL.INC I STRFNC.INC CGSELO.INC NOXMNI.INC PDIM.INC SHERX.INC GMAP.INC I CGASMF. INC CPSEF. INC TURB. INC

flUX.0: PARAMETER. INC ALL.INC INTRUZ. INC PRIN.INC VARR.INC GEOM.INC I RADCON.INC CPART.INC KRAD. INC DISO. INC COEE. INC CSET.INC RADRES. INC

flUX0.0: PARAMETER. INC TYPE. INC KASETI.INC SHERX. INC ALI.INC CPART.INC I CINDEX.INC PRIN. INC GEOM.INC DISO.INC RADCON. INC BCRAD.INC

fluXY.O: PARAMETER. INC PRIN.INC BCRAD.INC COEF. INC GEOM.INC VARR.INC I KRAD.INC CPART.INC SWPDIR. INC CSET.INC RADRES.INC ALL.INC INTRUZ.INC । RADCON. INC

FLUXT.O: PARAMETER. INC ALL.INC INTRUZ. INC COEE. INC GEOM.INC VARR.INC I KRAD. INC CPART.INC SWPDIR. INC CSET.INC RADRES. INC RADCON. INC

FlUXX.0: PARAMETER. INC PRIN.INC ALL.INC BCRAD.INC INTRUZ.INC COEF.INC I GEOM.INC VARR.INC KRAD.INC CPART.INC SWPDIR.INC CSET.INC RADRES.INC I KASETI.INC RADCON.INC

freact.O: PARAMETER. INC ALL.INC CGASMF.INC CGAUSS.INC CINDEX.INC INTRUZ.INC । NOXMNI.INC NOXRTE.INC PSCCOM.INC RATECM.INC RATEST.INC RATPAR.INC I VAR. INC

frzfe.O: PARAMETER.INC PRIN.INC CPARAM.INC UPNX.INC PVAR.INC DENS.INC I TABRIT.INC CTABLE. INC FLUPR. INC RTMTAB.INC

frzfeh.o: PARAMETER. INC PRIN.INC RTMTAB INC CPARAM.INC CINDEX.INC URNX.INC I PVAR. INC DENS.INC TABRIT.INC BUG.INC CCHEMI.INC CTABLE.INC FLUPR.INC I CSPECE. INC 
Sample MAKE File for Program Maintenance on UNIX Systems

fste.0: PARAMETER.INC FIAFO.INC PVAR.INC PRIN.INC CRARAM.INC FLUPR.INC I CTABLE. INC

fstf.o: PARAMETER.INC FIAFO.INC PRIN.INC CPARAM.INC FLUPR. INC CTABLE.INC fstfe.0: PARAMETER.INC F1AFO.INC PRIN.INC CPARAM.INC PVAR.INC BUG.INC I CTABLE. INC FLUPR. INC

fstfeh.o: PARAMETER.INC F1AFO.INC CPARAM.INC PVAR.INC PRIN.INC BUG.INC I CTABLE.INC FLUPR. INC

fstfh.o: PARAMETER. INC FIAFO.INC PRIN.INC CPARAM.INC FLURR.INC CTABLE.INC gasfix.0: CECPAR. INC CPARAM. INC LUTION.INC

getcdf.o: config.inc order.inc ffids.inc difeq.inc input.inc t_ch4.inc 1 pyrite.inc

getkin.o: order.inc config.inc ffids.inc input.ine

getrct0.0: reactk.inc

gibchk. o: CECPAR. INC

grid.0: PARAMETER. INC BOUND. INC ALL.INC INTRUZ.INC GRIDI.INC GEOM.INC I KASET1.INC TUBWAL.INC STRENC.INC CGSFLO.INC NOXMN1.INC PDIM.INC I RRIN. INC

grmap.0: PARAMETER. INC ADFLO.INC ALL.INC INTRU2.INC GRIDI.INC GEOM.INC I TUBWAL.INC NOXMN1. INC TIMER. INC PDIM.INC STRFNC.INC GMAP.INC I KASET1.INC PRIN. INC

gtdeln.O: CECPAR. INC CEQUIL.INC CPARAM.INC LUTION.INC CSPECE. INC

gteta.o: CECPAR. INC LUTION. INC CPARAM.INC

hCalc.o: CECPAR. INC RCARDS.INC CPARAM.INC COF.INC LUTION.INC CSPECE.INC I CEQUIL.INC CCHEMI.INC

hCPS.0: CECPAR. INC CPARAM. INC CCHEMI.INC CSPECE. INC CMATRI.INC LUTION.INC

heat.0: PARAMETER. INC HCAPFG. INC CEQUIL.INC order.inc elem.inc DIFEQN.INC I difeq.inc dwgswe.inc IPPAR. INC PECOR.INC

heatbal.o: PARAMETER.INC ADELO.INC ALL.INC BOUND.INC CGSFLO.INC CPART.INC I CTABLE.INC DISO.INC ENTH.INC FIAFO.INC ELUPR.INC GEOM.INC HEATCP.INC I IPPAR. INC NOXMN1. INC TUBWAL. INC VAR. INC

infIOW.O: PARAMETER. INC TELOF10.INC PRIN.INC ADELO.INC CGSELO.INC CPSEF. INC I FLIN.INC KASET1. INC PANDS.INC PDIM.INC SWRL.INC

init.o: PARAMETER. INC DISO.INC FIAFO.INC TELOE10.INC HEATCP.INC CTABLE.INC । AFR.INC TURB.INC ALL.INC INTRUZ. INC WVEL.INC VVEL.INC ENTH.INC I MAGHJ.INC MIXFR. INC VAR.INC GEOM.INC FLUPR.INC CELAG.INC KASETI.INC I COEF. INC CPSOU.INC CGASMF.INC STRFNC.INC KRAD.INC VARR. INC CGSFLO.INC I PANDS. INC CPSEF. INC UVEL.INC PCOR. INC SSUM.INC UNDER. INC

inity0.o: order.inc pyrite.ine

intgrt.0: PARAMETER. INC ALL.INC INTRUZ.INC

IntP.o: PARAMETER. INC ALL.INC GEOM. INC

kbar.o: rates.inc pools.inc

klcalc.o: reactk.inc

IISOIV.0: PARAMETER. INC COEF.INC SWPDIR. INC TIMER.INC SWPALT.INC

maknam.0: config.ine ffids.ine

matIix.o: CECPAR. INC CCHEMI.INC CEQUIL.INC CMATRI.INC CPARAM.INC CSPECE.INC I LOTION. INC

mIXfE.O: PARAMETER. INC PRIN.INC CPARAM.INC PVAR.INC DENS.INC CCHEMI.INC I BUG.INC CGSFLO.INC PANDS.INC FIAFO.INC TABRIT.INC CTABLE. INC I ELUPR. INC 
modeta.0: PARAMETER. INC ADFLO.INC INTRUZ.INC ALL. INC MAGHJ.INC VVEL.INC I WVEL.INC PCOR.INC VAR. INC GEOM.INC FIUPR. INC SHERX. INC KASETI.INC I SUSP.INC COEF. INC TURB. INC MIXER. INC SHEAR. INC TUBWAL.INC CGASMF. INC I SSUM. INC SWRL. INC TIMER. INC CPSEF. INC UVEL. INC

modh.O: PARAMETER. INC ENTH.INC HEATCP.INC CCHEMI.INC CSPECE.INC CPARAM.INC । F1AFO.INC PVAR.INC ADFLO.INC AFR.INC INTRUZ.INC ALL.INC MAGHJ.INC । VVEL.INC WVEL.INC PCOR.INC VAR.INC GEOM.INC FLUPR.INC SHERX.INC । KASETI.INC SUSP.INC COEF.INC TURB.INC MIXER. INC SHEAR.INC TUBWAL.INC I CGASME.INC SSUM.INC SWRL.INC TIMER. INC CPSEF. INC UVEL.INC

modte.O: PARAMETER. INC ADELO.INC AER. INC INTRUZ.INC ALL.INC MAGHJ.INC I WVEL.INC WVEL.INC PCOR.INC VAR.INC GEOM.INC FLUPR.INC SHERX.INC । KASETI.INC SUSP. INC COEF.INC TURB. INC MIXER. INC SHEAR.INC TUBWAL.INC I CGASMF.INC SSUM.INC SWRL.INC TIMER.INC CPSEF.INC UVEL.INC

modu.0: PARAMETER.INC ADFLO.INC AFR.INC INTRUZ.INC ALL.INC MAGHJ.INC I VVEL.INC WVEL.INC PCOR. INC VAR.INC GEOM.INC FLUPR. INC SHERX. INC I KASETI.INC SUSP. INC COEF. INC TURB. INC MIXFR. INC SHEAR.INC TUBWAL.INC I CGASMF.INC SSUM.INC SWRL.INC TIMER. INC CPSEF. INC UVEL.INC

mOSOLV.O: PARAMETER. INC COEF.INC SWPDIR. INC TIMER. INC SWPALT.INC

MU.O: CECPAR. INC LUTION. INC CPARAM.INC CSPECE. INC

netin.0: dvccomp.inc xeffcm.inc difeq.inc percol.inc input.inc

netout.o: dvccomp.inc xeffcm.ine percol.inc

nextte. 0 : defs.inc tempcm.inc config.inc

nfen.o: rates.inc pools.inc

noxdata.0: PSCCOM. INC RMSCOM. INC

nOXmn.0: PARAMETER. INC AFR. INC ALL.INC AREXP.INC BOUND. INC BUG.INC I CALCYI.INC CCHEMI. INC CEQUIL.INC CFLAG. INC CGASMF. INC CGAUSS. INC I CINDEX. INC CPARAM. INC CPART. INC CPEUL. INC CPSOU. INC COEE. INC I CSPECE.INC CTABLE. INC DENS.INC FLUC.INC ELURR. INC GEOM.INC GPARAM.INC I INTRUZ.INC IPPAR.INC KASET1.INC MOLWT.INC NOXMN1.INC NOXRTE.INC I NSWPS.INC PANDS.INC PROPIN.INC PRIN.INC PSCCOM.INC PFAC.INC PVAR.INC I RATECM.INC RATEST.INC RATPAR.INC FIAFO.INC RESID.INC RMSCOM.INC I SSUM.INC SWPDIR.INC TIMER. INC TABRIT.INC TRAJ.INC TUBWAL.INC TURB.INC I UPNX. INC VAR. INC XRATE. INC

OVtPUT.O: PARAMETER.INC ADELO. INC ALL.INC COEE. INC FLUPR.INC GEOM.INC I GMAP.INC VAR.INC CPEUL.INC INTRUZ.INC VARR.INC STRFNC.INC CPARAM.INC I CGASMF. INC

pCgC2.0: PARAMETER. INC ADFLO.INC AFR.INC ALL.INC BOUND.INC BUG.INC 1 CGASME. INC CGSFLO.INC CINDEX. INC CNDXCG.INC COEF.INC CPARAM.INC I CPART.INC CPEUL.INC CPLOT.INC CPSEF. INC CPSOU.INC CSPECE.INC I CTABLE.INC CWATER.INC DIRECT.INC DISO.INC ENTH.INC FIAFO.INC FLIN.INC FLUC.INC ELUPR.INC GEOM.INC GPARAM.INC GRIDI.INC HEATCR.INC. ICON.INC I INTRUZ.INC KASETI. INC MAGHJ.INC MIXER.INC MOLWT.INC PANDS.INC I PARD.INC PCOR.INC PDIM.INC PRIN.INC RRAT.INC SHEAR.INC SWRL.INC I TDIS.INC TEN.INC TELOEIO.INC TRAJ.INC TURB.INC TYPE.INC UVEL.INC I VAR.INC VVEL.INC WVEL.INC TUBWAL.INC PROPIN.INC VARR.INC STRENC.INC I SSUM.INC SWPDIR.INC UNDER. INC DENS. INC SIMPLE.INC NOXMN1.INC I TABRIT.INC TIMER.INC PVAR.INC CFLAG.INC PLTVAR.INC RESID.INC I CCHEMI.INC SHERX. INC EULP. INC IPPAR. INC GMAP.INC RCON.INC

pej.o: PARAMETER. INC CINDEX.INC CSPECE. INC CTNBP. INC TRAJ.INC HCAP.INC । 
Sample MAKE File for Program Maintenance on UNIX Systems

HREF. INC RCON. INC

percvap.o: dvccomp.inc xeffcm.inc percol.inc pools.inc input.inc $t$ ch4. inc

pltwrt.0: PARAMETER. INC CINDEX.INC FORMAT.INC MOLWT.INC RESID.INC AFR.INC 1

all. INC CGASMF. INC CGSFLo. INC COEF. INC CPARAM.INC CPART.INNC CPEUL.INC 1 * FLUPR.INC GEOM.INC INTRUZ.INC NOXMN1.INC STRFNC.INC VAR.INC VARR. INC

prof11.0: PARAMETER. INC ADFLO. INC ALL.INC VAR.INC GEOM.INC FLUPR.INC I KASET1.INC STRENC.INC CGSFLO.INC TURB.INC NOXMN1.INC TUBWAL.INC । PDIM.INC PANDS. INC FIAFO.INC SWRL.INC VVEL.INC

prog.0: PARAMETER. INC ALL.INC ELUC.INC VAR.INC CGASMF.INC FLUPR.INC I UVEL.INC VVEL.INC WVEL.INC PCOR.INC ENTH.INC TEN.INC TDIS.INC I MIXFR. INC PARD. INC

promod.0: PARAMETER. INC ADFLO.INC AFR.INC INTRUZ.INC ALL.INC MAGHJ.INC I VVEL.INC WVEL. INC PCOR. INC VAR.INC GEOM.INC FLURR.INC SHERX.INC I KASETI.INC SUSP. INC COEF. INC TURB.INC MIXFR. INC SHEAR.INC TUBWAL.INC I CGASMF. INC SSUM. INC SWRL.INC TIMER. INC CPSEF.INC UVEL.INC

props.0: PARAMETER. INC HEATCP. INC FIAFO.INC TRAJ.INC AFR.INC GPARAM.INC I ALL.INC INTRUZ.INC PRIN. INC MAGHJ.INC CPARAM.INC CPART.INC FLURR.INC I PANDS.INC CTABLE.INC BUG.INC VAR.INC GEOM.INC TURB.INC FLUC.INC I

- CGASMF.INC PVAR.INC MOLWT.INC PRORIN.INC DENS.INC TIMER.INC I TABRIT.INC BOUND.INC RESID.INC CFLAG.INC CPEUL.INC IPPAR.INC I CCHEMI. INC CGAUSS. INC

presur.0: PARAMETER. INC ALL.INC CPART.INC INTRUZ.INC CPEUL.INC PFAC.INC I AREXP.INC BOUND. INC GEOM.INC

pscrat.0: PARAMETER. INC CINDEX. INC CPARAM.INC CPART.INC CPSOU.INC । PSCCOM.INC PVAR.INC PANDS.INC RATPAR.INC RMSCOM.INC TABRIT.INC

psict.0: PARAMETER. INC DIRECT.INC difeq.inc FORMAT.INC PANDS.INC I

TELOF10. INC HCAP. INC AFR.INC ALL.INC CEQUIL.INC CELAG.INC CGASMF.INC I CGSFLO.INC CINDEX.INC CNDXCG.INC CPARAM.INC CPART.INC CPEUL.INC I CPLOT.INC CPSEF. INC CPSOU. INC CWATER. INC DIFEQN.INC FLUPR.INC I GEOM.INC GPARAM.INC ICON.INC INTRUZ.INC IPPAR.INC KASET1.INC 1 MOLWT.INC PECOR.INC PRIN.INC RCONT.INC RRAT.INC SSUM.INC SWRL.INC I TRAJ.INC TURB.INC TUBWAL.INC VAR.INC VARR.INC config.inc def́s.inc 1 elem.inc input.inc order.inc pools.inc tempcm.inc xeffem.inc

psict0.0: PARAMETER. INC TFLOF10. INC ADFLO. INC ALL.INC AFR. INC CFLAG.INC I CGASME. INC CGSFLO.INC CPART. INC CPEUL. INC CPLOT.INC CPSEF. INC I CPSOU.INC GRID1.INC EULP.INC FLIN.INC FLUPR.INC GEOM.INC INTRUZ.INC I IPPAR.INC KASETI.INC PANDS.INC PRIN.INC FIAFO.INC TUBWAL.INC VAR.INC

psolve.o: PARAMETER.INC difeq.inC AFR. INC ALL.INC CPARAM.INC CPART.INC I CPSOU. INC TRAJ.INC VAR. INC ELUPR. INC KASETI. INC CGSFLO.INC CPLOT.INC I CPEUL.INC MOLWT.INC IPPAR. INC CELAG.INC RCONT.INC OMEGA.INC EULP.INC I PRIN.INC VARR.INC DIFEQN.INC PECOR.INC GEOM.INC

pydist.o: pyrite.inc order.inc

pyritel.o: pyrite.inc order.inc pools.inc difeq.inc

pyrite2.0: pyrite.inc

radCOF.0: PARAMETER. INC ALL.INC CEQUIL. INC CINDEX.INC CPEUL.INC CPART.INC I DISO.INC GEOM.INC MOLWT.INC RADCON.INC KRAD.INC FLUPR.INC

rangok.O: CECPAR. INC CPARAM. INC CSPECE. INC

rate.0: BARAMETER. INC AFR.INC ALL.INC AREXR.INC BOUND.INC BUG.INC I

CALCYI.INC CCHEMI.INC CEQUIL.INC CFLAG.INC CGASMF. INC CGAUSS.INC I 
CINDEX. INC CPARAM. INC CPART.INC CPEUL.INC CPSOU.INC CSPECE.INC I CTABLE.INC DENS.INC :ZUC.INC FLUPR.INC GEOM.INC GPARAM.INC INTRUZ.INC I IPPAR.INC KASETI.INC MOLWT.INC NOXMNI. INC NOXRTE.INC PANDS.INC I PROPIN.INC PRIN.INC PSCCOM.INC PVAR.INC RATECH.INC RATEST.INC I RATPAR.INC FIAFO.INC RESID.INC RMSCOM.INC TIMER.INC TABRIT.INC I TRAJ.INC TUBWAL.INC TURB.INC UPNX.INC VAR.INC XRATE.INC

regrid.o: PARAMETER. INC

reinit.o: pools.inc order.inc pyrite.inc xeffcm.inc percol.inc plotw.inc I input, ine

rempur.O: CECPAR.INC CPARAM.INC CEQUIL.INC

restrt.0: PARAMETER.INC TURB.INC AFR. INC ALL.INC VAR.INC FLUPR.INC I MAGHJ.INC KASETI.INC CGASMF.INC KRAD.INC VARR.INC TABRIT.INC FLUC.INC result.0: PARAMETER. INC ALL.INC FLUPR. INC BCRAD.INC KRAD.INC GEOM.INC I DISO. INC VARR. INC PRIN.INC

rstnox.0: PARAMETER. INC ALL.INC CALCYI.INC CGASMF.INC KASETI.INC RATECM.INC

rXnEXT.0: PARAMETER. INC AFR. INC ALL.INC AREXP. INC BOUND.INC BUG.INC I CALCYI.INC CCHEMI.INC CEQUIL.INC CFLAG.INC CGASMF. INC CGAUSS.INC I CINDEX.INC CPARAM. INC CPART.INC CPEUL.INC CPSOU.INC ESPECE.INC I CTABLE.INC DENS.INC FLUC.INC FLUPR. INC GEOM.INC GPARAM. INC INTRUZ.INC I IPPAR.INC KASETI.INC MOLWT.INC NOXMNI.INC NOXRTE.INC PANDS.INC I PROPIN.INC PRIN.INC PSCCOM.INC PVAR.INC RATECM.INC RATEST.INC I RATPAR.INC FIAFO.INC RESID.INC RMSCOM.INC TIMER.INC TABRIT.INC I TRAJ.INC TUBWAL. INC TURB. INC UPNX. INC VAR.INC XRATE.INC

rXnrat.0: PARAMETER. INC AFR. INC ALL.INC AREXP.INC BOUND.INC BUG.INC I CALCYI.INC CCHEMI.INC CEQUIL.INC CFLAG.INC CGASMF.INC CGAUSS. INC I CINDEX.INC CPARAM. INC CPART.INC CPEUL.INC CPSOU.INC CSPECE.INC I CTABLE.INC DENS.INC FLUC.INC FLUPR. INC GEOM. INC GPARAM. INC INTRUZ.INC I IPPAR.INC KASET1.INC MOLWT.INC NOXMNI.INC NOXRTE.INC PANDS.INC I PROPIN.INC PRIN.INC PSCCOM.INC PVAR.INC RATECM.INC RATEST.INC I RATPAR.INC FIAFO.INC RESID.INC RMSCOM.INC TIMER.INC TABRIT.INC I TRAJ.INC TUBWAL.INC TURB.INC UPNX.INC VAR. INC XRATE.INC

sdnrst.0: PARAMETER. INC ALL.INC CPART. INC CPEUL.INC

setup.0: PARAMETER.INC ALL.INC CPART.INC COEF.INC GEOM.INC KRAD.INC I CSET. INC VARR. INC IAZCON.INC

SIfISt.O: PARAMETER. INC ALL.INC RATESX.INC SOXRTE.INC

SOlPOS.O: CECPAR. INC LUTION. INC

SOIbO.0: PARAMETER. INC ADFLO.INC ALL.INC CFLAG.INC CGASMF.INC CGSFLO.INC I CPART.INC CPEUL.INC CPLOT. INC CPSOU.INC EULP.INC FIAFO.INC FLIN.INC I FLUPR.INC GEOM.INC INTRUZ.INC IPPAR.INC KASET1.INC PANDS.INC PRIN.INC I RATESX.INC SOXRTE. INC SXMISC.INC TELOF1O.INC TUBWAL.INC VAR. INC

sorpar.0: PARAMETER. INC ALL. INC CEQUIL.INC CELAG.INC CGASMF.INC CGSFLO.INC I COEF. INC CINDEX. INC CNOXCG. INC CPARAM.INC CPART.INC CPEUL.INC I CPSOU.INC EULP.INC FLUC.INC FLUPR.INC GEOM.INC INTRUZ.INC IPPAR.INC I KASET1.INC MOLWT.INC NOXMN1.INC NSWPS.INC PANDS.INC PARD.INC PRIN.INC I PVAR. INC RATESX. INC SOXRTE. INC SAVBP. INC SSUM.INC SWPDIR.INC I SXMISC.INC TIMER. INC TYPE.INC TUBWAL.INC TURB.INC UNDER. INC VAR.INC I VARR, INC

SOXdata.O: PARAMETER.INC RATESX.INC

spece.O: CECPAR. INC CCHEMI.INC CEQUIL.INC CMATRI.INC CSPECE.INC CPARAM.INC I 
LUTION. INC

spread.0: PARAMETER. INC ALL. INC GEOM.INC

sprops.0: PARAMETER. INC ALL.INC CPARAM.INC CPART.INC CPEUL.INC FLUPR.INC I INTRUZ.INC KASETI. INC SAVBP. INC VAR. INC VARR. INC

spsict.o: PARAMETER. INC ALL.INC CEQUIL.INC CFLAG.INC CGASMF.INC CGSELO.INC 1 CINDEX.INC CPARAM.INC CPART.INC CPEUL.INC CPLOT.INC CPSOU. INC I FLUPR.INC FORMAT.INC GEOM.INC GPARAM.INC INTRUZ.INC KASET1.INC I MOLWT.INC PECOR.INC PRIN.INC RATESX.INC RCONT.INC SOXRTE.INC SSUM.INC I TRAJ. INC TURB. INC TUBWAL.INC VAR. INC

stream.0: PARAMETER.INC ALL.INC INTRUZ.INC VAR.INC GEOM.INC FLURR.INC I KASET1.INC TUBWAL.INC STRENC.INC CGSFLO.INC

sulfat.0: PARAMETER. INC CPART.INC GEOM.INC RATESX. INC TRAJ.INC SOXRTE.INC sulfhs.0: PARAMETER. INC CPART.INC GEOM.INC RATESX.INC TRAJ.INC SOXRTE.INC swelldata.o: tempcm.inc

swesub6.0: order.inc tempcm.inc xeffcm.inc dwgswe.inc input.inc dvecomp.inc I saveinc.inc

table.o: PARAMETER. INC CPARAM. INC TABRIT. INC TIMER. INC CTABLE. INC

tarvap.o: dvccomp.inc xeff $\mathrm{cm}$.inc percol.ine

tble.0: PARAMETER. INC TFLOF10. INC PVAR.INC PRIN.INC CPARAM.INC ELUPR.INC 1 CCHEMI.INC TABRIT. INC BUG. INC CGSFLO.INC PANDS.INC EIAFO.INC I CTABLE. INC

Tblf.0: PARAMETER. INC TFLOF10.INC AFR. INC GPARAM.INC TRAJ.INC PRIN.INC I CPARAM.INC FLUPR. INC CCHEMI.INC TABRIT.INC BUG.INC CGSFLO.INC I PANDS. INC FIAFO. INC CTABLE. INC

tblfe.0: PARAMETER. INC TFLOF1O.INC AFR.INC TRAJ.INC PRIN.INC CRARAM.INC I UPNX.INC PVAR.INC DENS.INC TABRIT.INC CCHEMI.INC BUG.INC CGSELO.INC । PANDS.INC FIAFO.INC CTABLE.INC FLUPR.INC RTMTAB.INC GPARAM.INC

tblfeh.0: PARAMETER. INC TFLOEIO.INC CINDEX. INC CSPECE.INC GPARAM.INC I AFR. INC TRAJ.INC PRIN.INC CRARAM.INC RTMTAB.INC PVAR.INC DENS.INC I TABRIT.INC CCHEMI.INC BUG.INC CGSFLO.INC PANDS.INC FIAFO.INC I CTABLE. INC FLUPR. INC

tblfh.0: PARAMETER. INC CINDEX. INC TFLOF10. INC FIAEO.INC AFR. INC TRAJ.INC I GPARAM.INC PRIN.INC CPARAM.INC FLUPR.INC CCHEMI.INC DENS.INC BUG.INC I CGSELO.INC TABRIT. INC CTABLE. INC RTMTAB. INC

tblisfeh.0: PARAMETER. INC FIAFO.INC AFR.INC PRIN.INC CRARAM.INC PVAR.INC $\backslash$ DENS.INC TABRIT. INC CCHEMI.INC BUG. INC CTABLE. INC FLUPR. INC I RTMTAB. INC

Iblistfe.O: PARAMETER. INC FIAEO.INC PRIN.INC CPARAM.INC URNX.INC EVAR.INC I DENS. INC CCHEMI.INC BUG.INC TABRIT.INC CTABLE.INC FLUPR.INC । RTMTAB. INC

tblrts.o: pools.inc order.inc finish.inc rates.inc

ther15p.0: order.inc pools.inc rates.inc xeff $\mathrm{cm}$.inc difeq.inc percol.inc 1 input.inc t_ch4.inc pyrite.inc defs.inc

tubfeh.o: PARAMETER. INC CPARAM.INC CTABLE. INC FIAEO.INC PVAR. INC RTMTAB.INC

UCOE .0: PARAMETER. INC AER.INC ALL.INC CPART.INC INTRUZ.INC OIREC:. INC I OVEL.INC VAR.INC GEOM.INC COEF.INC KASET1.INC CPSOU.INC SWPDIR.INC I SIMPLE. INC FLURR. INC FLUPR2. INC PCOR.INC

UPfe.0: PARAMETER. INC TFLOF10.INC AFR. INC TRAJ.INC PRIN.INC CPARAM.INC I URNX. INC PVAR. INC DENS.INC TABRIT. INC CCHEMI.INC BUG.INC CGSFLO.INC I 
PANDS.INC EIAFO.INC CTABLE.INC FLUPR. INC RTMTAB.INC GPARAM.INC upfeh.0: PARAMETER. INC CINOEX. INC CSPECE. INC GPARAM.INC AER. INC TRAJ.INC I PRIN.INC CPARAM.INC RTMTAB.INC PVAR.INC DENS.INC TABRIT.INC I CCHEMI.INC BUG.INC CGSFLO. INC PANDS. INC FIAFO.INC CTABLE.INC I FLURR. INC

Upth.O: PARAMETER. INC TELOEIO.INC FIAFO.INC AER.INC TRAJ.INC GPARAM.INC । PRIN.INC CPARAM.INC FLUPR. INC CCHEMI.INC DENS.INC BUG.INC CGSFLO.INC I TABRIT.INC CTABLE. INC RTMTAB. INC

vCoE. .O: PARAMETER. INC AER.INC ALL.INC CPART.INC INTRUZ.INC VVEL.INC I VAR. INC GEOM.INC COEF.INC KASETI.INC CPSOU.INC SWPDIR.INC SWRL.INC I FLUPR. INC FLUPR2. INC PCOR. INC SIMPLE. INC

vector.0: PARAMETER. INC

Velmag.0: PARAMETER.INC ALL.INC COEF.INC GEOM.INC GMAR.INC VAR.INC

viscosp.o: xeffcm.inc

volswl.o: dvccomp.inc

Wall.0: PARAMETER. INC ALL.INC INTRUZ.INC COEF.INC

wrtout.0: finish.inc ffids.inc config.inc order.inc xeffcm.inc difeq.inc। pyrite.inc 
Sample MAKE File for Program Maintenance on UNIX Systems 


\section{Sample Computer Files for Gaseous Combustion}

This sample problem is the combustion of natural gas in the BYU controlled-profile reactor (CPR).

\section{Main Data File (cpr.dat, PCGCIN)}

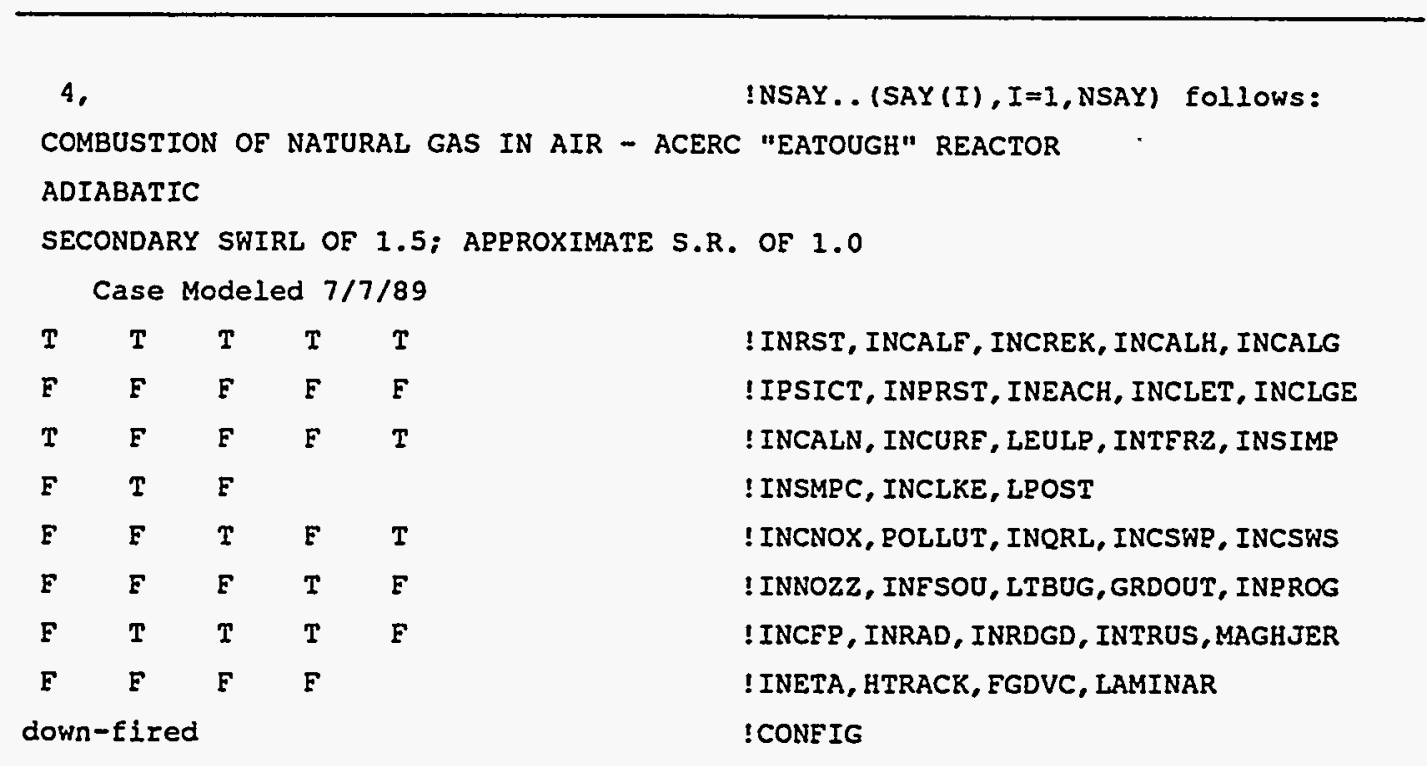




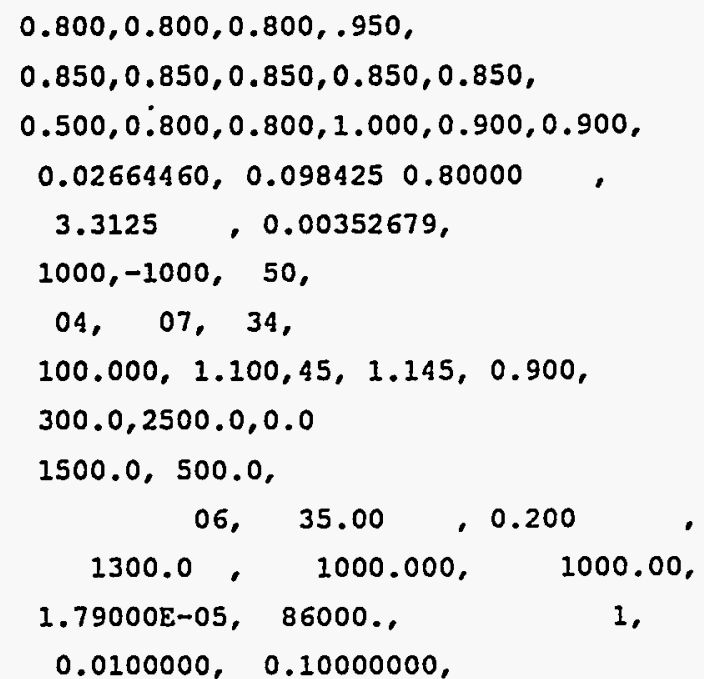
INLET 1

! URFU, URFV, URFW, URFH

! URFE, UREK, URFF, UREG, URFVIS

! URFDEN, URFETA, URFGET, URFNJ, URFPP, URFP

! DIAP, DIAS, DIACH

!NDIA(reaI), THICK

!MAXIT, INDPRI, INDRST

! NJINP, NJINS, NIWOQ

! ALI, EPSX, NL, EPSI, EPSD

! TMIN, TMAX, HLOSS

! TLODEL, THIDEL

!NIINQ, QHA, QLX

! TBN, TBW, TSE

!VISCOS, PRES, IEUCK

! SORMAX, SORMIN

! (Primary)

$2.6110 \mathrm{E}-03,1.000,0.000,0.100$,

!FLOW, FFLOW, SWIRLN, TINELO

! (Secondary)

INLET 2

!FLOW, FFLOW, SWIRLN, TINELO

(Blank line)

\section{ELEMENTS}

THERMO

tThe react. sect. is formatted

REACTANTS 1

298.000

!TMP (unformatted)

$\begin{array}{ccccc}\text { C 1. } & 02 . & 0 . & 0 . & \mathrm{CO}\end{array}$

C 1. H 4.0 0.

$\begin{array}{lllll}c \text { 2. H } 6 . & 0 . & \text { C. } & \text { H6 }\end{array}$

$\begin{array}{lllll}\text { C 3. H } 8 . & 0 . & 0 . & \text { C } 3 \mathrm{H} 8\end{array}$

$0.016252 \quad G$

$0.800761 \quad G$

$0.120040 \quad G$

N 2. 0 .

o.

o. N2

$0.054172 \quad G$

$0.008775 \quad G$

$!$

(Blank Iine).

REACTANTS 2

298.000

o 2. 0.02

N 2. $\quad$ o. $\quad$ o. $\quad$ o. N2

!TMP (unformatted)

$\begin{array}{llll}0.23300 & G & \\ 0.76700 & G & \\ \text { ! LDISO, LGASE, LEMCOR, MAXITR } & \text { (Blank line) }\end{array}$

T T T , 10 !LDISO, LGASE, LEMCOR, MAXITR 

$0.8000,1200.00$
! EMW, TOUT
$1.0000,0.0000$
!AO,AF

\section{Inlet Profile Data File (cpr.inl, INLET)}

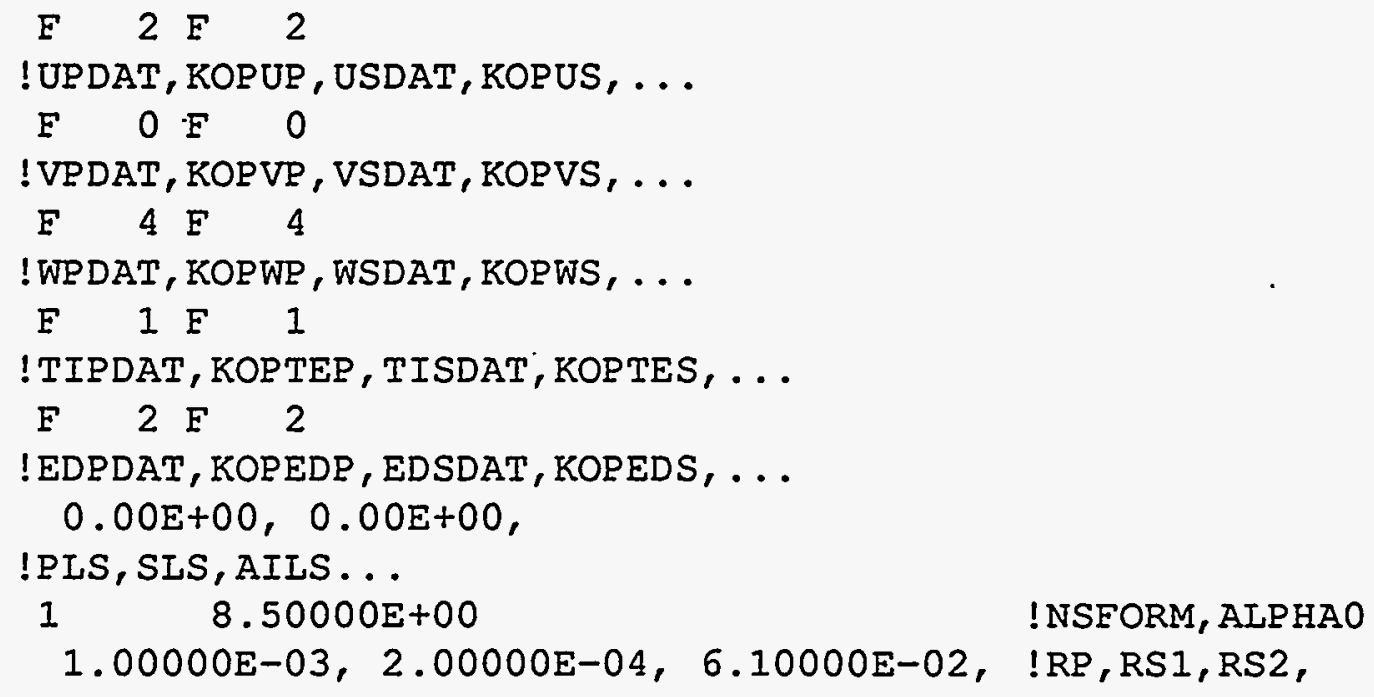

Thermo Data File (cpr.thm, THERMO)

$\begin{array}{llr}\text { C } & 12.01115 & 4.0 \\ \text { H } & 1.00797 & 1.0 \\ \text { O } & 15.9994 & -2.0 \\ \text { N } & 14.0067 & 0.0\end{array}$


Sample Computer Files for Gaseous Combustion

$0.27167616 E$, $010.29451370 E-02-0.80224368 E-06 \quad 0.10226681 E-09-0.48472104 E-14$

-0.29905820 E 05 0.66305666E $010.40701275 E$ 01-0.11084499E-02 $0.41521180 E-05$ $-0.29637404 E-08 \quad 0.80702101 E-12-0.30279719 E$ 05-0.32270038E 00

$\begin{array}{llllllll}\text { N2 } & \mathrm{J} 9 / 65 \mathrm{~N} & 2.0 & 0.0 & 0.0 & 0.6 & 300.000 & 5000.000\end{array}$

$0.28963194 E$ E $010.15154863 E-02-0.57235275 E-06 \quad 0.99807385 E-10-0.65223536 E-14$

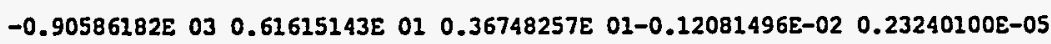
$-0.632175208-09-0.22577253 E-12-0.10611587 \varepsilon$ O4 $0.23580418 E 01$

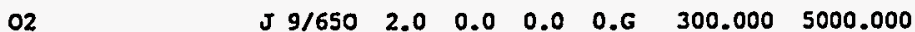

$0.36219521 E$ E $010.73618256 E-03-0.19652219 E-06 \quad 0.36201556 E-10-0.28945623 E-14$

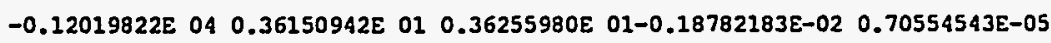
$-0.67635071 E-08 \quad 0.21555977 E-11-0.10475225 E \quad 04 \quad 0.43052769 E \quad 01$

No $\quad J 6 / 63 \mathrm{~N} \quad 1.0 \quad 1.00 \quad 0.00 \quad 0.6 \quad 300.000 \quad 5000.000$

$0.31889992 E 010.13382279 \varepsilon-02-0.52899316 \varepsilon-060.95919314 E-10-0.64847928 E-14$

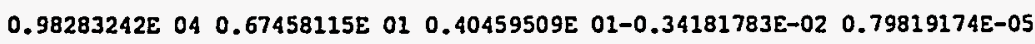

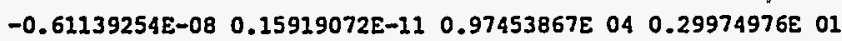

$\begin{array}{llllllll}\text { H2 } & \mathrm{J} 3 / 61 \mathrm{H} & 2.0 & 0.0 & 0.0 & 0.6 & 300.000 & 5000.000\end{array}$

$0.31001883 E$ E $01 \quad 0.51119458 E-03 \quad 0.52644204 E-07-0.34909964 E-10 \quad 0.36945341 E-14$ $-0.87738013 E$ E $03-0.19629412 E$ ol $0.30574446 E$ ol $0.26765198 E-02-0.58099149 E-05$ $0.55210343 E-08-0.18122726 E-11-0.98890430 E$ 03-0.22997046E 01

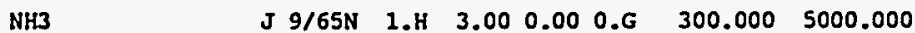

$0.24165173 E$ O1 $0.61871186 E-02-0.21785136 E-05 \quad 0.37599057 E-09-0.24448854 E-13$

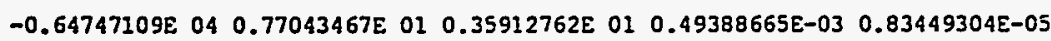
$-0.83833385 E-08 \quad 0.27299092 E-11-0.66717070 E$ OA $0.22520962 E$ OI

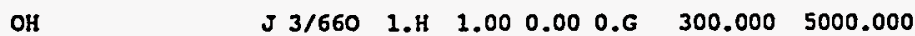

$0.29106417 E$ O1 $0.95931627 E-03-0.19441700 E-06 \quad 0.13756646 E-10 \quad 0.14224542 E-15$ $0.39353811 E$ O4 $0.54423428 E$ O $0.38375931 E$ 01-0.10778855E-02 $0.96830354 E-06$ 0.18713971E-09-0.22571089E-12 0.36412820E $04 \quad 0.49370009 E$ E 00

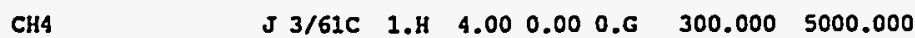

$0.15027056 \mathrm{E}$ o1 $0.10416795 \mathrm{E}-01-0.39181514 \mathrm{E}-05 \quad 0.67777872 \mathrm{E}-09-0.44283706 \mathrm{E}-13$

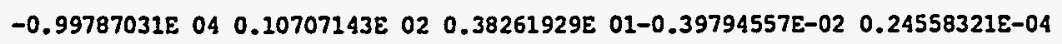
$-0.22732920 E-07 \quad 0.69626952 E-11-0.10144945 E$ O5 $0.86690062 E$ OO

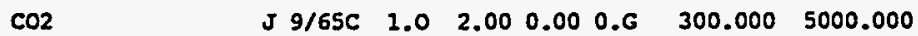

$0.44608040 \mathrm{E}$ o1 $0.30981717 E-02-0.12392566 \mathrm{E}-05 \quad 0.22741323 E-09-0.15525948 E-13$ $-0.48961438 E$ E $05-0.98635978 E$ E $0.24007788 E \quad 02 \quad 0.87350905 E-02-0.66070861 E-05$ $0.20021860 E-08 \quad 0.63274039 E-15-0.48377520 \varepsilon \quad 05 \quad 0.96951447 E \quad 01$ 


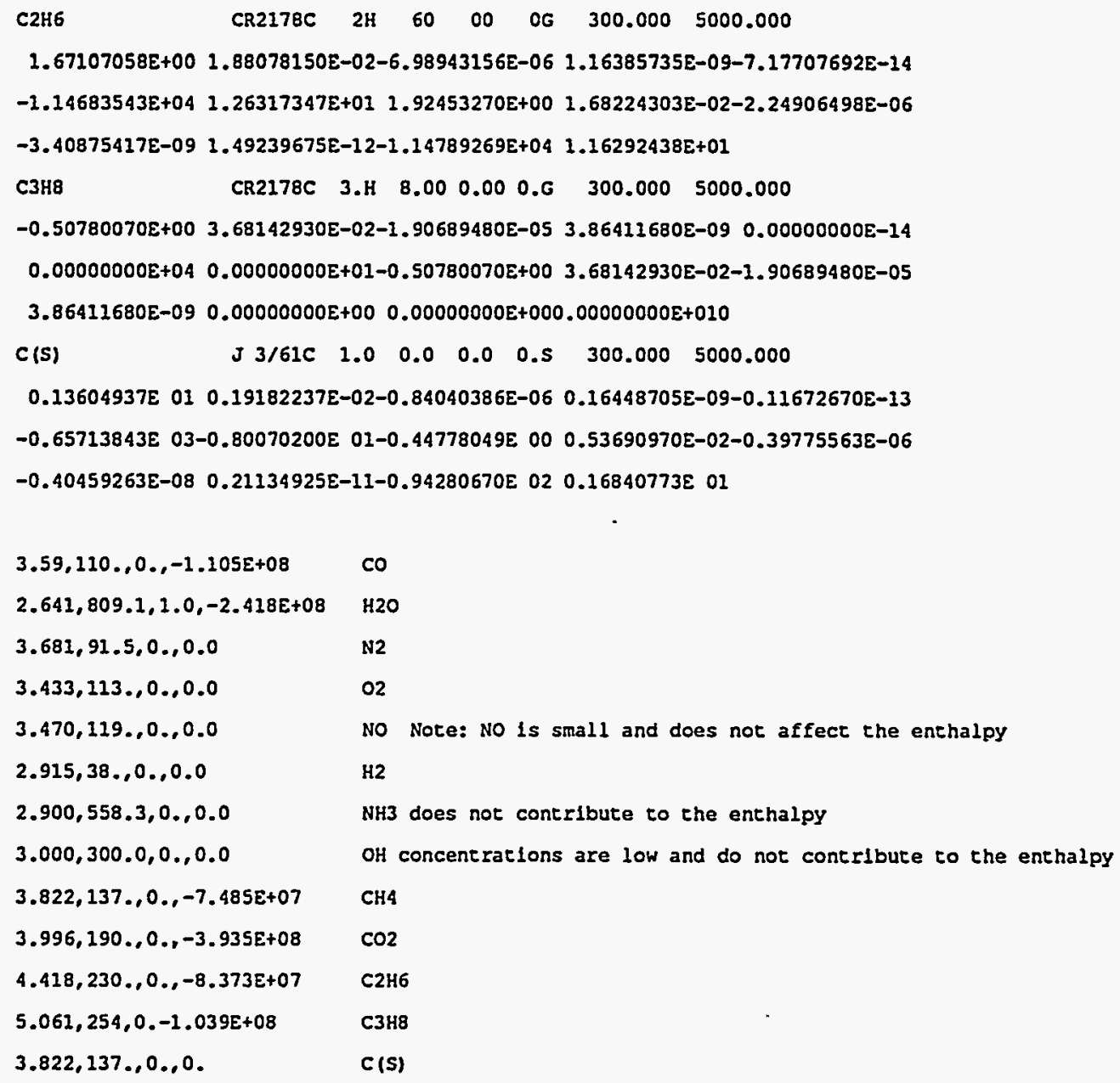


Sample Computer Files for Gaseous Combustion 


\section{Appendix $F$}

\section{Sample Computer Files for Coal Combustion with $\mathrm{NO}_{\mathrm{x}}$ Formation}

This sample problem is the combustion of coal in the BYU controlled-profile reactor (CPR) with $\mathrm{NO}_{\mathrm{x}}$ formation.

\section{Main Data File (cprcoal.dat, PCGCIN)}

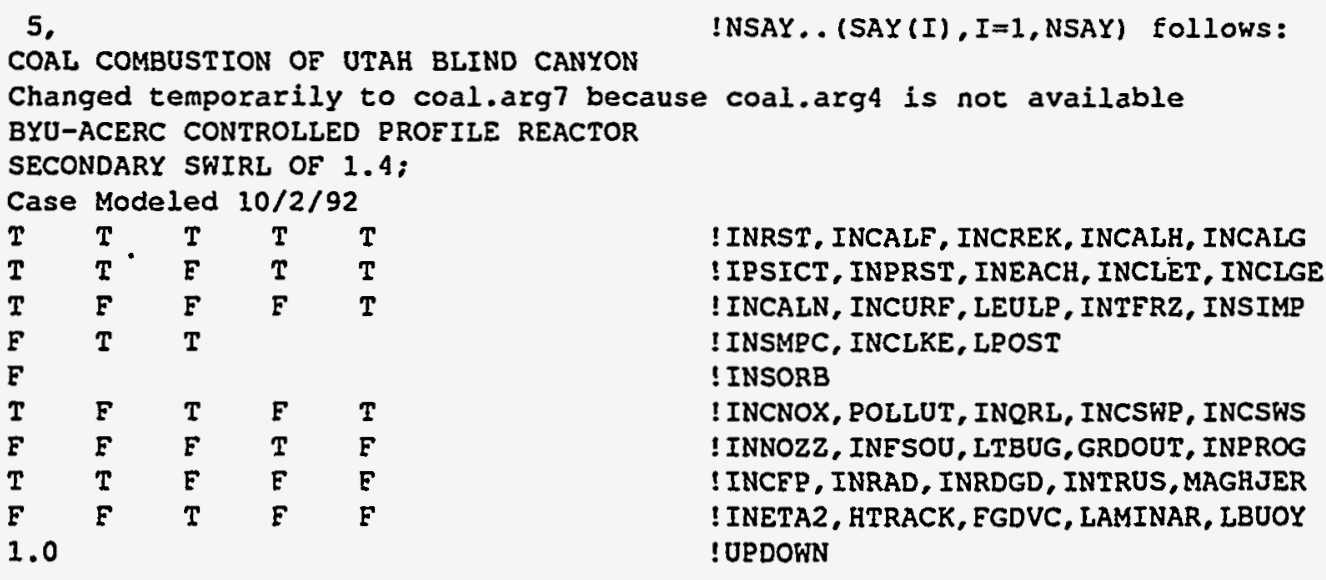




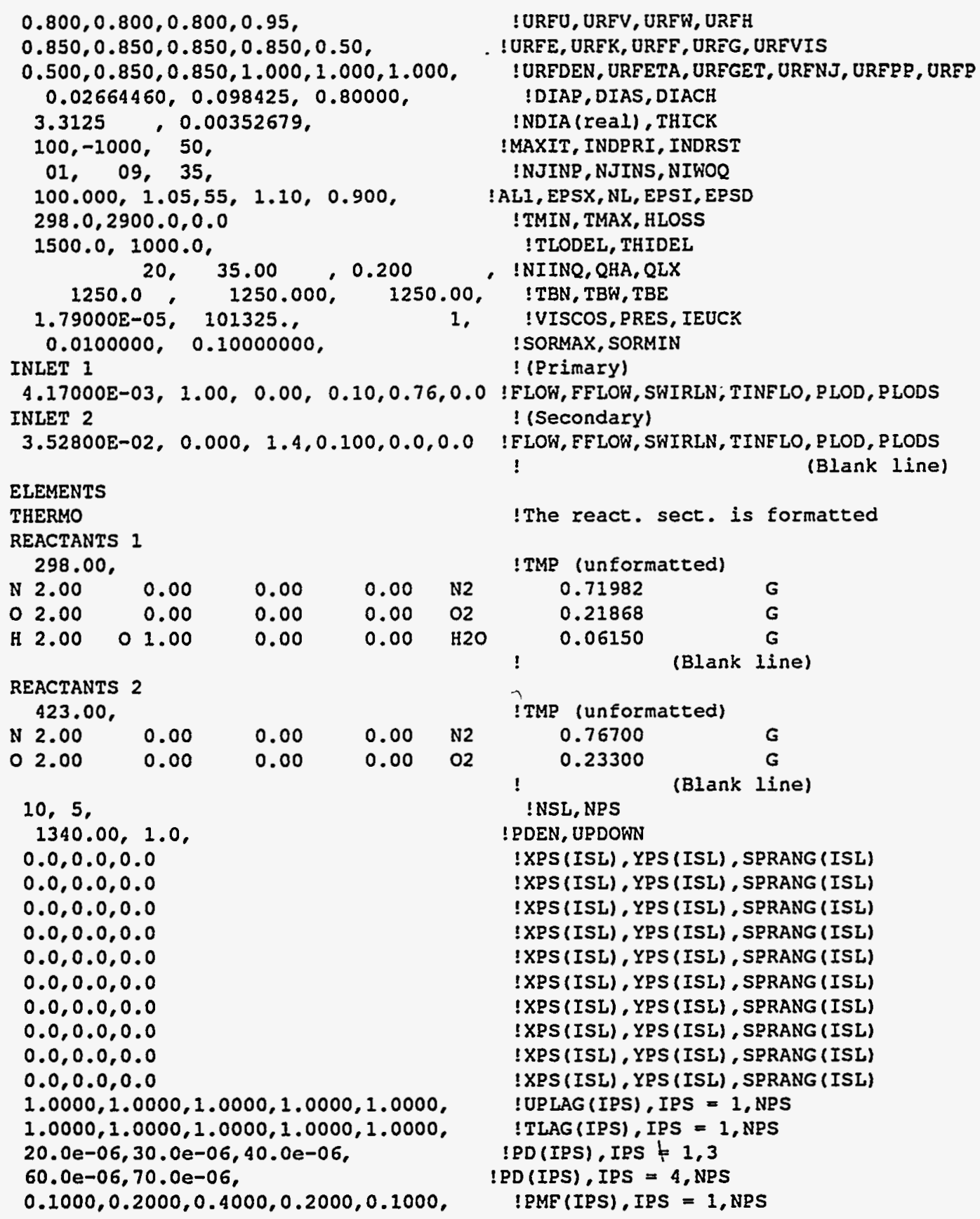

02.00

H 2.00

01.00

0.00

0.00

02

REACTANTS 2

423.00,

$\begin{array}{lllll}N 2.00 & 0.00 & 0.00 & 0.00 & \text { N2 } \\ 02.00 & 0.00 & 0.00 & 0.00 & 02\end{array}$

10,5 , $1340.00,1.0$,

$0.0,0.0,0.0$

$0.0,0.0,0.0$

$0.0,0.0,0.0$

$0.0,0.0,0.0$

$0.0,0.0,0.0$

$0.0,0.0,0.0$

$0.0,0.0,0.0$

$0.0,0.0,0.0$

$0.0,0.0,0.0$

$0.0,0.0,0.0$

$1.0000,1.0000,1.0000,1.0000,1.0000$, $1.0000,1.0000,1.0000,1.0000,1.0000$, $20.0 e-06,30.0 e-06,40.0 e-06$, $60.0 e-06,70.0 e-06$,

$0.1000,0.2000,0.4000,0.2000,0.1000$,

! UREU, URFV, URFW, URFH

. ! URFE, URFK, URFE, UREG, UREVIS

! URFDEN, UREETA, URFGET, URFNJ, URFPR, UREP

!DIAP, DIAS, DIACH

!NDIA(real), THICK

!MAXIT, INDPRI, INDRST

!NJINP, NJINS, NIWOQ

!AL1, EPSX, NL, EPSI, EPSD ! TMIN, TMAX, HLOSS ITLODEL, THIDEL

!NIINQ, QHA, QLX !TBN, TBW, TBE

1. !VISCOS, PRES, IEUCK

!SORMAX, SORMIN

! (Primary)

!FLOW, FFLOW, SWIRLN; TINFLO, PLOD, PLODS ! (Secondary)

! FLOW, FFLOW, SWIRLN, TINELO, PLOD, PLODS !

(Blank line)

tThe react. sect. is formatted

!TMP (unformatted)

$\begin{array}{ll}0.71982 & \text { G } \\ 0.21868 & \text { G } \\ 0.06150 & \text { G }\end{array}$

! (Blank Iine)

ITMP (unformatted)

$\begin{array}{ccc} & 0.76700 & G \\ 0.23300 & G \\ ! \quad & \text { (Blank line) } \\ \text { !NSL, NPS } & \end{array}$

! PDEN, UPDOWN

!XPS (ISL), YPS (ISL), SPRANG (ISL) !XPS (ISL), YPS (ISL) , SPRANG (ISL) !XPS (ISL), YPS (ISL), SPRANG (ISL) !XPS (ISL), YPS (ISL) , SPRANG (ISL) !XPS (ISL), YPS (ISL), SPRANG (ISL) !XPS (ISL), YPS (ISL), SPRANG (ISL) !XPS (ISL), YPS (ISL) , SPRANG (ISL) !XPS (ISL), YPS (ISL), SPRANG (ISL) !XPS (ISL), YPS (ISL), SPRANG (ISL) !XPS (ISL), YPS (ISL) , SPRANG (ISL) ! UPLAG (IPS), IPS $=1$, NPS 1 TLAG (IPS), IPS $=1$, NPS !PD(IPS), IPS $\neq 1,3$ IPD (IPS), IPS $=4, \mathrm{NPS}$ !PMF (IPS), IPS $=1$, NPS 


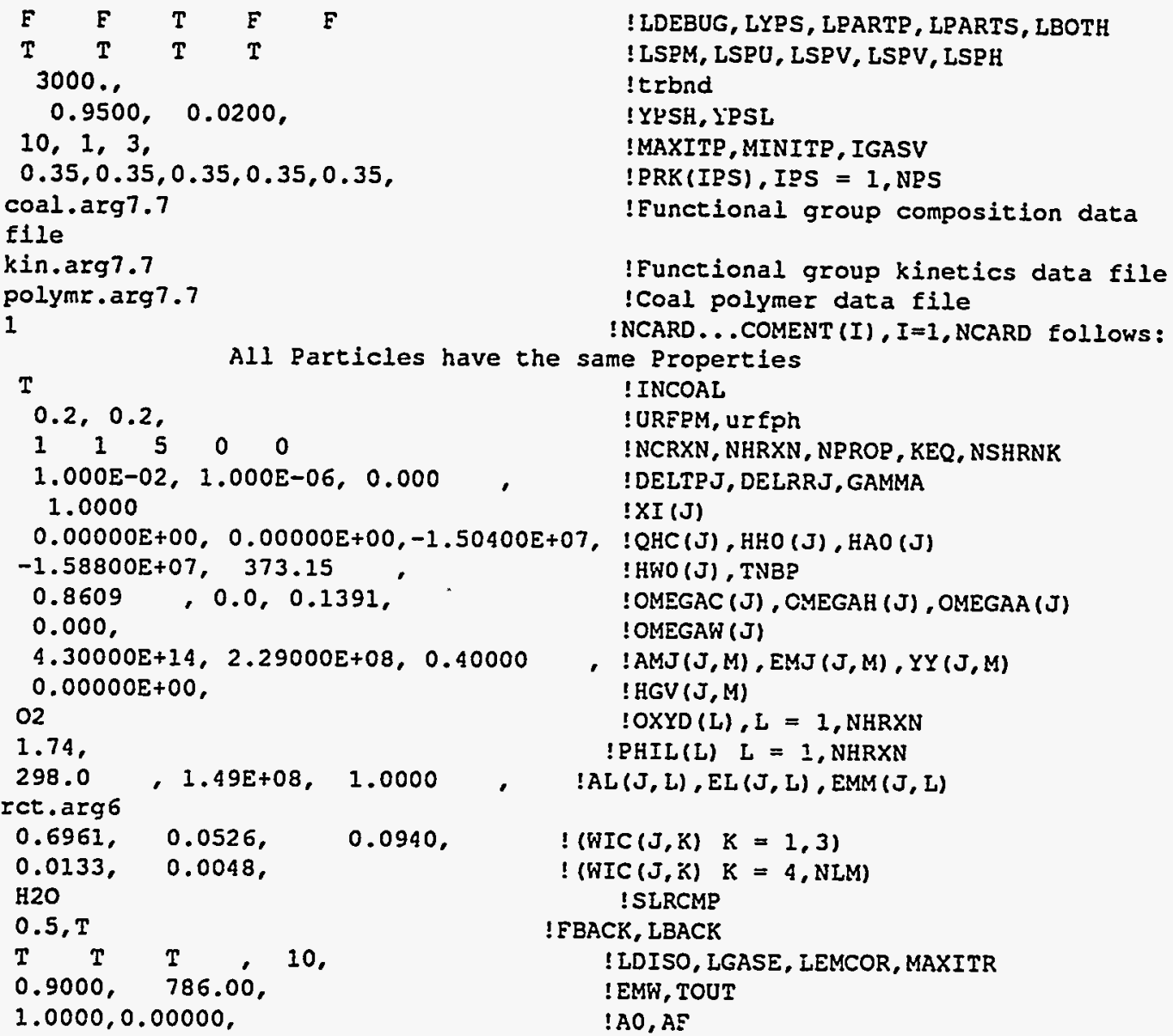

\section{Grid Data File (cprcoal.grd, GRIDS)}

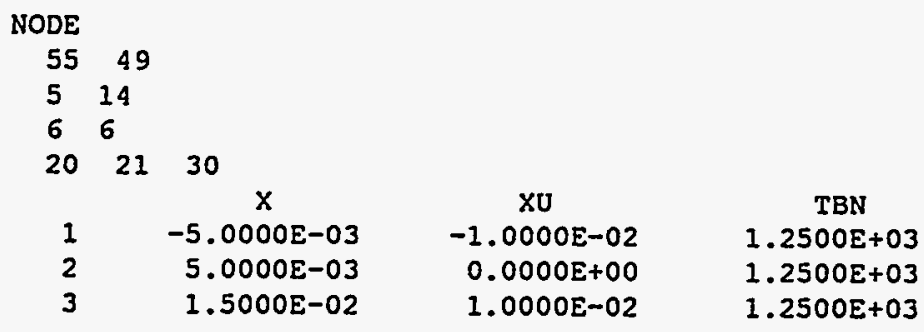




\begin{tabular}{|c|c|c|c|}
\hline 4 & $2.5000 E-02$ & $2.0000 E-02$ & $1.2500 E+03$ \\
\hline 5 & $3.5000 E-02$ & $3.0000 \mathrm{E}-02$ & $1.2500 \mathrm{E}+03$ \\
\hline 6 & $4.5000 E-02$ & $4.0000 E-02$ & $1.2500 \mathrm{E}+03$ \\
\hline 7 & $5.5000 E-02$ & $5.0000 \mathrm{E}-02$ & $1.2500 E+03$ \\
\hline 8 & $6.5000 E-02$ & $6.0000 E-02$ & $1.2500 E+03$ \\
\hline 9 & $7.5000 \mathrm{E}-02$ & $7.0000 E-02$ & $1.2500 E+03$ \\
\hline 10 & $8.5000 \mathrm{E}-02$ & $8.0000 \mathrm{E}-02$ & $1.2500 E+03$ \\
\hline 11 & $9.5000 \mathrm{E}-02$ & $9.0000 \mathrm{E}-02$ & $1.2500 E+03$ \\
\hline 12 & $1.0500 \mathrm{E}-01$ & $1.0000 \mathrm{E}-01$ & $1.2500 E+03$ \\
\hline 13 & $1.1500 \mathrm{E}-01$ & $1.1000 \mathrm{E}-01$ & $1.2500 E+03$ \\
\hline 14 & $1.2500 \mathrm{E}-01$ & $1.2000 \mathrm{E}-01$ & $1.2500 \varepsilon+03$ \\
\hline 15 & $1.3500 \mathrm{E}-01$ & $1.3000 \mathrm{E}-01$ & $1.2500 \mathrm{E}+03$ \\
\hline 16 & $1.4500 \mathrm{E}-01$ & $1.4000 E-01$ & $1.2500 \mathrm{E}+03$ \\
\hline 17 & $1.5500 \mathrm{E}-01$ & $1.5000 \mathrm{E}-01$ & $1.2500 \mathrm{E}+03$ \\
\hline 18 & $1.6500 \mathrm{E}-01$ & $1.6000 \mathrm{E}-01$ & $1.2500 \mathrm{E}+03$ \\
\hline 19 & $1.7500 \mathrm{E}-01$ & $1.7000 \mathrm{E}-01$ & $1.2500 E+03$ \\
\hline 20 & $1.8500 E-01$ & $1.8000 \mathrm{E}-01$ & $1.2500 E+03$ \\
\hline 21 & $1.9500 \mathrm{E}-01$ & $1.9000 \mathrm{E}-01$ & $1.2500 E+03$ \\
\hline 22 & $2.0500 E-01$ & $2.0000 E-01$ & $1.2500 \mathrm{E}+03$ \\
\hline 23 & $2.1602 \mathrm{E}-01$ & $2.1051 E-01$ & $1.2500 \mathrm{E}+03$ \\
\hline 24 & $2.2818 \mathrm{E}-01$ & $2.2210 \mathrm{E}-01$ & $1.2500 E+03$ \\
\hline 25 & $2.4158 \mathrm{E}-01$ & $2.3488 E-01$ & $1.2500 E+03$ \\
\hline 26 & $2.5635 \mathrm{E}-01$ & $2.4896 E-01$ & $1.2500 \mathrm{E}+03$ \\
\hline 27 & $2.7263 \mathrm{E}-01$ & $2.6449 \mathrm{E}-01$ & $1.2500 E+03$ \\
\hline 28 & $2.9059 \mathrm{E}-01$ & $2.8161 E-01$ & $1.2500 \mathrm{E}+03$ \\
\hline 29 & $3.1038 \mathrm{E}-01$ & $3.0048 E-01$ & $1.2500 E+03$ \\
\hline 30 & $3.3220 \mathrm{E}-01$ & $3.2129 \mathrm{E}-01$ & $1.2500 E+03$ \\
\hline 31 & $3.5625 \mathrm{E}-01$ & $3.4423 E-01$ & $1.2500 E+03$ \\
\hline 32 & $3.8277 \mathrm{E}-01$ & $3.6951 E-01$ & $1.2500 E+03$ \\
\hline 33 & $4.1201 E-01$ & $3.9739 E-01$ & $1.2500 E+03$ \\
\hline 34 & 4. $4424 \mathrm{E}-01$ & $4.2812 E-01$ & $1.2500 \mathrm{E}+03$ \\
\hline 35 & $4.7977 \mathrm{E}-01$ & $4.6200 E-01$ & $1.2500 \mathrm{E}+03$ \\
\hline 36 & $5.1894 \mathrm{E}-01$ & $4.9936 \mathrm{E}-0 \mathrm{I}$ & $1.2500 E+03$ \\
\hline 37 & $5.6213 E-01$ & $5.4054 \mathrm{E}-01$ & $1.2500 E+03$ \\
\hline 38 & $6.0974 E-01$ & $5.8593 E-01$ & $1.2500 E+03$ \\
\hline 39 & $6.6222 E-01$ & $6.3598 E-01$ & $1.2500 E+03$ \\
\hline 40 & $7.2009 E-01$ & $6.9116 \mathrm{E}-01$ & $1.2500 E+03$ \\
\hline 41 & $7.8388 \mathrm{E}-01$ & $7.5198 \mathrm{E}-01$ & $1.2500 E+03$ \\
\hline 42 & $8.5420 E-01$ & $8.1904 \mathrm{E}-01$ & $1.2500 E+03$ \\
\hline 43 & $9.3173 E-01$ & $8.9297 E-01$ & $1.2500 E+03$ \\
\hline 44 & $1.0172 E+00$ & $9.7447 E-01$ & $1.2500 \mathrm{E}+03$ \\
\hline 45 & $1.1114 \mathrm{E}+00$ & $1.0643 \mathrm{E}+00$ & $1.2500 \mathrm{E}+03$ \\
\hline 46 & $1.2153 E \div 00$ & $1.1634 E+00$ & $1.2500 E+03$ \\
\hline 47 & $1.3298 E+00$ & $1.2726 \mathrm{E}+00$ & $1.2500 E+03$ \\
\hline 48 & $1.4561 \mathrm{E}+00$ & $1.3930 \mathrm{E}+00$ & $1.2500 E+03$ \\
\hline 49 & $1.5953 \mathrm{E}+00$ & $1.5257 \mathrm{E}+00$ & $1.2500 \mathrm{E}+03$ \\
\hline 50 & $1.7487 \mathrm{E}+00$ & $1.6720 \mathrm{E}+00$ & $1.2500 \mathrm{E}+03$ \\
\hline 51 & $1.9179 E+00$ & $1.8333 E+00$ & $1.2500 \mathrm{E}+03$ \\
\hline 52 & $2.1044 \mathrm{E}+00$ & $2.0111 E+00$ & $1.2500 \mathrm{E}+03$ \\
\hline
\end{tabular}




\begin{tabular}{|c|c|c|c|}
\hline 53 & $2.3100 E+00$ & $2.2072 \mathrm{E}+00$ & $1.2500 E+03$ \\
\hline 54 & $2.5367 \mathrm{E}+00$ & $2.4233 E+00$ & $1.2500 E+03$ \\
\hline 55 & $2.7633 E+00$ & $2.6500 \mathrm{E}+00$ & $1.2500 E+03$ \\
\hline & $Y$ & YV & \\
\hline 1 & $-1.4815 \mathrm{E}-03$ & $-2.9630 E-03$ & \\
\hline 2 & $1.4815 \mathrm{E}-03$ & $0.0000 E+00$ & \\
\hline 3 & $4.4448 \mathrm{E}-03$ & $2.9632 \mathrm{E}-03$ & \\
\hline 4 & $7.7164 E-03$ & $6.0806 \mathrm{E}-03$ & \\
\hline 5 & $1.1328 \mathrm{E}-02$ & $9.5224 E-03$ & \\
\hline 6 & $1.5316 \mathrm{E}-02$ & $1.3322 \varepsilon-02$ & \\
\hline 7 & $1.9304 \mathrm{E}-02$ & $1.7310 \mathrm{E}-02$ & \\
\hline 8 & $2.3292 \mathrm{E}-02$ & $2.1298 \mathrm{E}-02$ & \\
\hline 9 & $2.7280 E-02$ & $2.5286 \mathrm{E}-02$ & \\
\hline 10 & $3.1267 \mathrm{E}-02$ & $2.9274 \mathrm{E}-02$ & \\
\hline 11 & $3.5255 E-02$ & $3.3261 \mathrm{E}-02$ & \\
\hline 12 & $3.9243 E-02$ & $3.7249 \mathrm{E}-02$ & \\
\hline 13 & 4. $3231 \mathrm{E}-02$ & $4.1237 \mathrm{E}-02$ & \\
\hline 14 & $4.7219 E-02$ & $4.5225 E-02$ & \\
\hline 15 & $5.1206 \mathrm{E}-02$ & $4.9213 E-02$ & \\
\hline 16 & $5.5593 \mathrm{E}-02$ & $5.3400 \mathrm{E}-02$ & \\
\hline 17 & $6.0418 E-02$ & $5.8006 E-02$ & \\
\hline 18 & $6.5726 \mathrm{E}-02$ & $6.3072 E-02$ & \\
\hline 19 & $7.1565 E-02$ & $6.8645 \mathrm{E}-02$ & \\
\hline 20 & $7.7987 \mathrm{E}-02$ & $7.4776 \mathrm{E}-02$ & \\
\hline 21 & $8.5052 \mathrm{E}-02$ & $8.1519 \mathrm{E}-02$ & \\
\hline 22 & $9.2823 E-02$ & $8.8937 E-02$ & \\
\hline 23 & $1.0137 E-01$ & $9.7097 \mathrm{E}-02$ & \\
\hline 24 & $1.1077 \mathrm{E}-01$ & $1.0607 \mathrm{E}-01$ & \\
\hline 25 & $1.2112 \mathrm{E}-01$ & $1.1595 \mathrm{E}-01$ & \\
\hline 26 & $1.3249 \mathrm{E}-01$ & $1.2681 E-01$ & \\
\hline 27 & $1.4501 E-01$ & $1.3875 \mathrm{E}-01$ & \\
\hline 28 & $1.5878 \mathrm{E}-01$ & $1.5189 \mathrm{E}-01$ & \\
\hline 29 & $1.7392 \mathrm{E}-01$ & $1.6635 \mathrm{E}-01$ & \\
\hline 30 & $1.9058 \mathrm{E}-01$ & $1.8225 E-01$ & \\
\hline 31 & $2.0890 \mathrm{E}-01$ & $1.9974 \mathrm{E}-01$ & \\
\hline 32 & $2.2906 \mathrm{E}-01$ & $2.1898 \mathrm{E}-01$ & \\
\hline 33 & $2.4922 \mathrm{E}-01$ & $2.3914 \mathrm{E}-01$ & \\
\hline 34 & $2.6736 \mathrm{E}-01$ & $2.5829 \mathrm{E}-01$ & \\
\hline 35 & $2.8368 E-01$ & $2.7552 \mathrm{E}-01$ & \\
\hline 36 & $2.9838 E-01$ & $2.9103 E-01$ & \\
\hline 37 & $3.1160 \mathrm{E}-01$ & $3.0499 \mathrm{E}-01$ & \\
\hline 38 & $3.2350 E-01$ & $3.1755 \mathrm{E}-01$ & \\
\hline 39 & $3.3421 E-01$ & $3.2886 \mathrm{E}-01$ & \\
\hline 40 & $3.4386 \mathrm{E}-01$ & $3.3904 \mathrm{E}-01$ & \\
\hline 41 & $3.5253 \mathrm{E}-01$ & $3.4819 \mathrm{E}-01$ & \\
\hline 42 & $3.6034 \mathrm{E}-01$ & $3.5644 \mathrm{E}-01$ & 1 \\
\hline 43 & $3.6737 \mathrm{E}-01$ & $3.6385 \mathrm{E}-01$ & 1 \\
\hline 44 & $3.7369 E-01$ & $3.7053 \mathrm{E}-01$ & \\
\hline 45 & $3.7939 E-01$ & $3.7654 \mathrm{E}-01$ & \\
\hline
\end{tabular}


$\begin{array}{llllllllllllllllllllllllll}\cdots & 0 & 0 & 0 & 0 & 0 & 0 & 0 & 0 & 0 & 0 & 0 & 0 & 0 & 0 & 0 & 0 & 0 & 0 & 0 & 0 & 0 & 0 & 0 & S & L\end{array}$

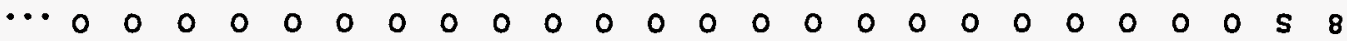

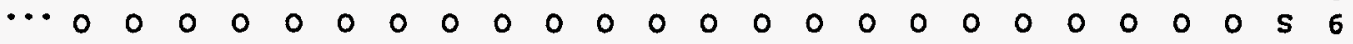

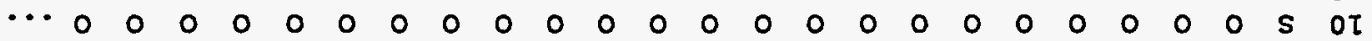

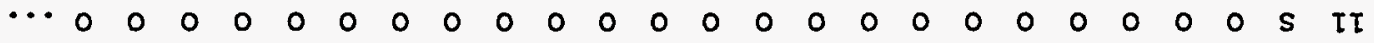

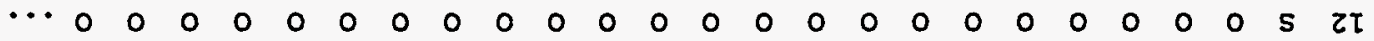

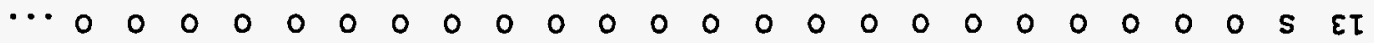

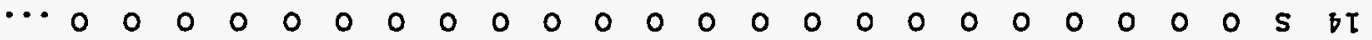

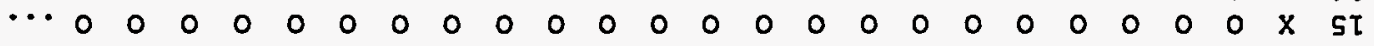

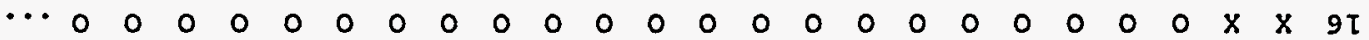

$\begin{array}{lllllllllllllllllllllllllllll}\cdots & 0 & 0 & 0 & 0 & 0 & 0 & 0 & 0 & 0 & 0 & 0 & 0 & 0 & 0 & 0 & 0 & 0 & 0 & 0 & 0 & 0 & x & x & x & L \tau\end{array}$

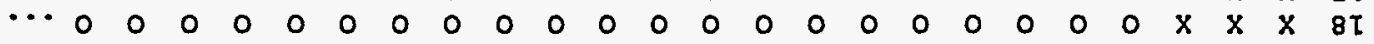

$\begin{array}{lllllllllllllllllllllllllllllll}\cdots & 0 & 0 & 0 & 0 & 0 & 0 & 0 & 0 & 0 & 0 & 0 & 0 & 0 & 0 & 0 & 0 & 0 & 0 & 0 & 0 & x & x & x & x & 6 I\end{array}$

$\begin{array}{lllllllllllllllllllllllllllllllll}\cdots & 0 & 0 & 0 & 0 & 0 & 0 & 0 & 0 & 0 & 0 & 0 & 0 & 0 & 0 & 0 & 0 & 0 & 0 & 0 & x & x & x & x & x & 0 z\end{array}$

$\begin{array}{llllllllllllllllllllllllllllllll}\cdots & 0 & 0 & 0 & 0 & 0 & 0 & 0 & 0 & 0 & 0 & 0 & 0 & 0 & 0 & 0 & 0 & 0 & 0 & x & x & x & x & x & x & \tau z\end{array}$

$\begin{array}{lllllllllllllllllllllllllllllllllll}\cdots & 0 & 0 & 0 & 0 & 0 & 0 & 0 & 0 & 0 & 0 & 0 & 0 & 0 & 0 & 0 & 0 & 0 & x & x & x & x & x & x & x & z z\end{array}$

$\begin{array}{llllllllllllllllllllllllllllllllllll}\cdots & 0 & 0 & 0 & 0 & 0 & 0 & 0 & 0 & 0 & 0 & 0 & 0 & 0 & 0 & 0 & 0 & x & x & x & x & x & x & x & x & \varepsilon z\end{array}$

$\begin{array}{lllllllllllllllllllllllllllllllllllllllllll}\cdots & 0 & 0 & 0 & 0 & 0 & 0 & 0 & 0 & 0 & 0 & 0 & 0 & 0 & 0 & x & x & x & x & x & x & x & x & x & x & b z\end{array}$

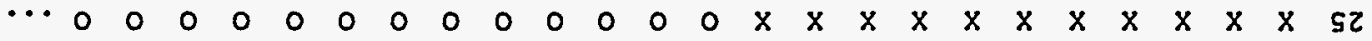

$\begin{array}{llllllllllllllllllllllllllllllllllllll}\cdots & 0 & 0 & 0 & 0 & 0 & 0 & 0 & 0 & 0 & 0 & 0 & x & x & x & x & x & x & x & x & x & x & x & x & x & 9 z\end{array}$

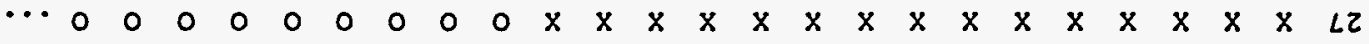

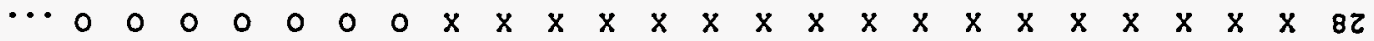

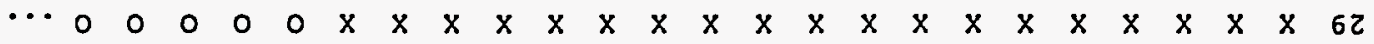

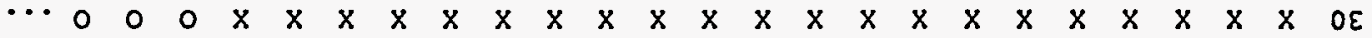

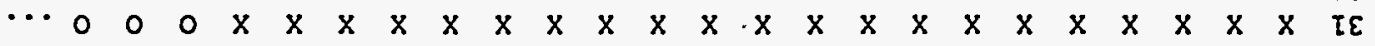

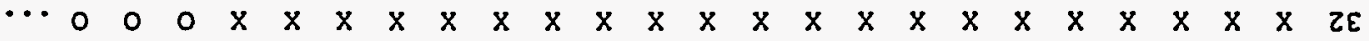

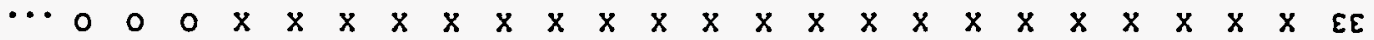

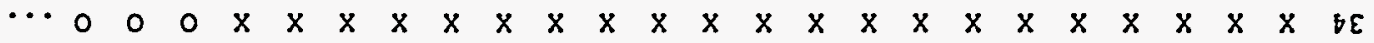

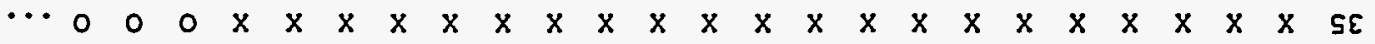

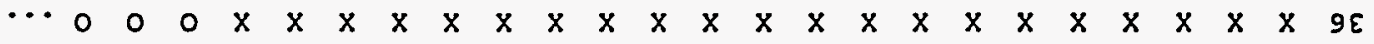

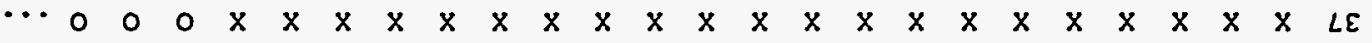

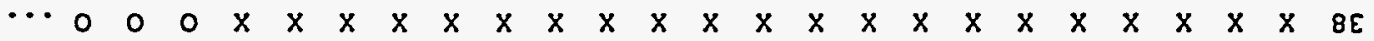

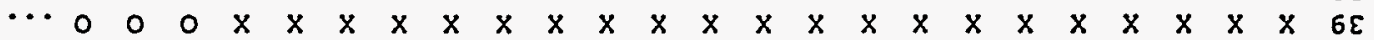

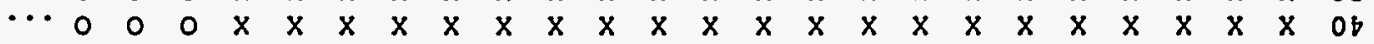

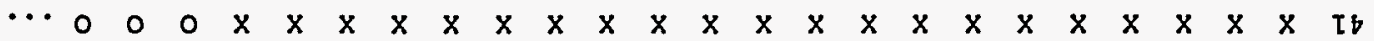

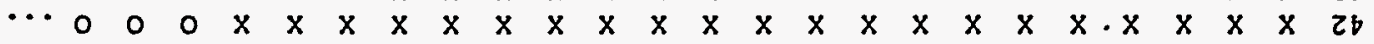

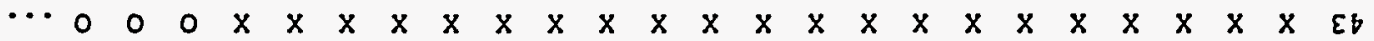

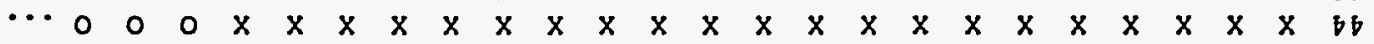

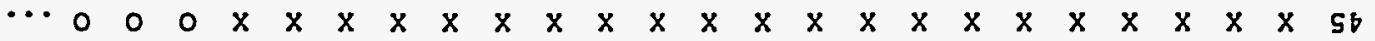

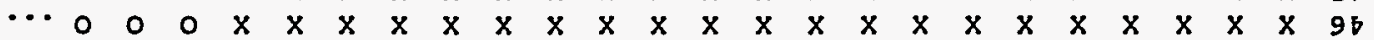

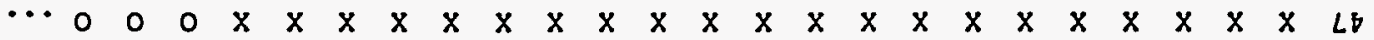

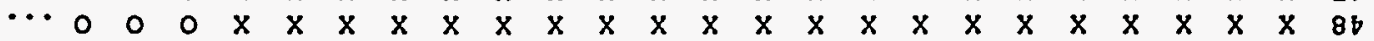

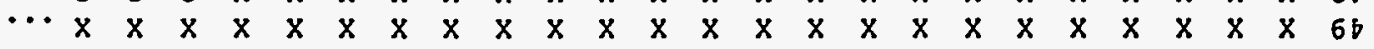

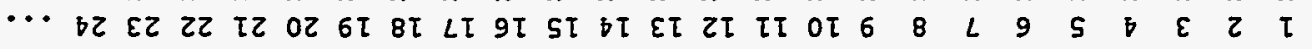
***NIYWOO MOTJ aNY SaIYYaNDOg YOIJY3Y***

TO- $30000^{\circ} \mathrm{B}$

โ0-30Zโ6 $\varepsilon$

TO-32898. $\varepsilon$

TO- $3 E \angle 90^{\circ} \mathrm{B}$

โO- $3 \angle Z E \sigma^{\circ} \varepsilon$

โO-تحโ $68^{\circ} \varepsilon$

$\tau 0-356 \tau 8^{\circ} \varepsilon$

โO- $\operatorname{TST\& 8^{\circ }\varepsilon }$

$$
\begin{aligned}
& 6 b \\
& 8 b \\
& 2 b \\
& 96
\end{aligned}
$$




$\begin{array}{llllllllllllllllllllllllll}6 & \mathrm{X} & 0 & 0 & 0 & 0 & 0 & 0 & 0 & 0 & 0 & 0 & 0 & 0 & 0 & 0 & 0 & 0 & 0 & 0 & 0 & 0 & 0 & 0 & 0 & \ldots \\ 5 & \mathrm{P} & 0 & 0 & 0 & 0 & 0 & 0 & 0 & 0 & 0 & 0 & 0 & 0 & 0 & 0 & 0 & 0 & 0 & 0 & 0 & 0 & 0 & 0 & 0 & \ldots \\ 4 & \mathrm{P} & 0 & 0 & 0 & 0 & 0 & 0 & 0 & 0 & 0 & 0 & 0 & 0 & 0 & 0 & 0 & 0 & 0 & 0 & 0 & 0 & 0 & 0 & 0 & \ldots \\ 3 & \mathrm{P} & 0 & 0 & 0 & 0 & 0 & 0 & 0 & 0 & 0 & 0 & 0 & 0 & 0 & 0 & 0 & 0 & 0 & 0 & 0 & 0 & 0 & 0 & 0 & \ldots \\ 2 & \mathrm{P} & 0 & 0 & 0 & 0 & 0 & 0 & 0 & 0 & 0 & 0 & 0 & 0 & 0 & 0 & 0 & 0 & 0 & 0 & 0 & 0 & 0 & 0 & 0 & \ldots \\ 1 & \mathrm{P} & 0 & 0 & 0 & 0 & 0 & 0 & 0 & 0 & 0 & 0 & 0 & 0 & 0 & 0 & 0 & 0 & 0 & 0 & 0 & 0 & 0 & 0 & 0 & \ldots\end{array}$

0 NOTE: THE JPRIM, JSEC, JTUBID, JTUBOD, NJINS, JQUARL

AND IQUARL ARE SUPERSEDED BY THE $X$ AND $P$ AND $S$ IN THE MAP

WHEN INTRUS=.TRUE. $N I$ and $N J$ are not superseded.

\section{NO ${ }_{x}$ Data File (cprcoal.nox, NOXIN)}

4 ,

! NSAYNX. . (SAYNX (I), I=1, NSAYNX)

follows:

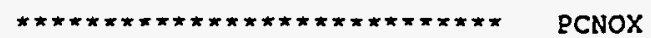

$\pi \star \star \pi \star$ PHASE AND PARTICLE PROPERTIES THROUGHOUT THE FIOW FIELD $\star \pi \pi \star \pi$

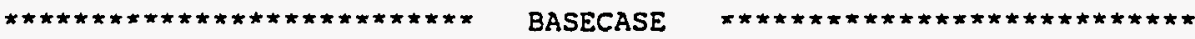

2.0

!FUELNO FLG $=1$ HSS; $=2 \mathrm{MT} ;=0$ no fuelno

0.0

$1.0,0.5,0,0$,

THRMNO FLG $=1 \mathrm{faI} ;=2 \mathrm{f}$ only; $=0$ no calc

1.0 !RADOXY, eqres $\tau$, radoh, ohadj

$0.90,0.90,0.90,0.05,0.80,0.000$, ICHARNO

20, 2, 100, 1.

!PRNOX, PRHCN, PRNH 3 , FCTNO, FCTHCN, FCTNH3

$1.0000,1.0000$

$0.95,0.95,0.75$

!MXITNX, ITYNX, INDENX, icalcn

F T T

0.5.1.00000E-04, !XIANOX, 2EDA, fn2prt, MAXRES

! urfnox, ur fher, ut fnh3

! IRSTNO, IPLTNX, INHTNX

\section{Log File (cprcoal.log)}
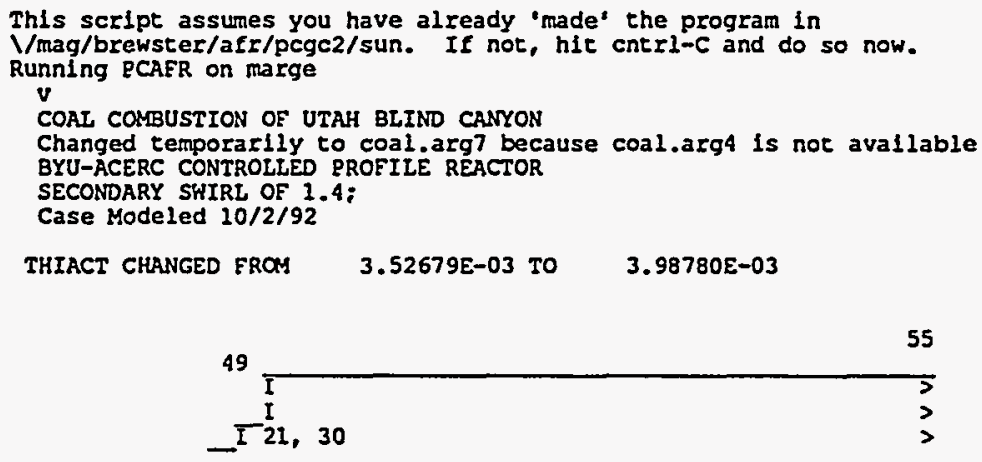


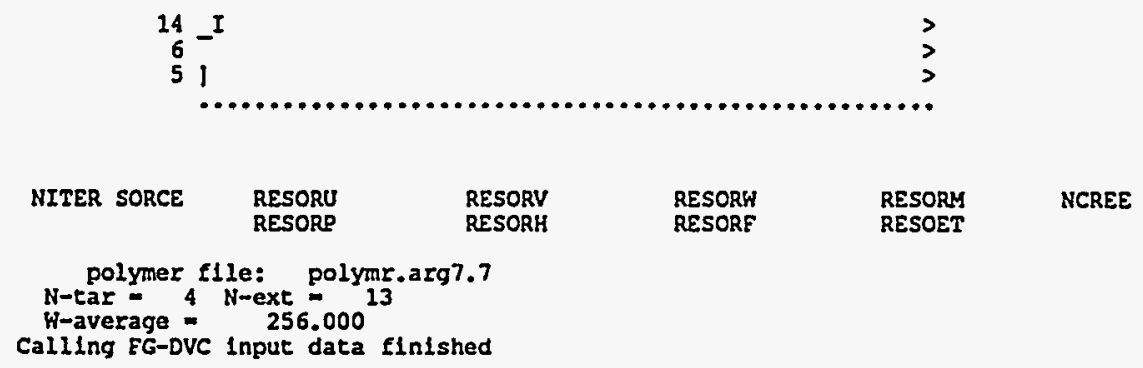

HARNING: Elemental coal composition is taken from coal data flle rather than main data file when

using FG-DVC devolatilization opelon.

Values calculated........

$\begin{array}{lll}1 & 0.829815 & 0.306192 \\ 2 & 0.808327 & 0.295413\end{array}$

$\begin{array}{lll}2 & 0.808327 & 0.295413 \\ 3 & 0.796922 & 0.288745\end{array}$

$4 \quad 0.7846490 .280553$

$5 \quad 0.780928 \quad 0.277786$

1 SIMPLER ALCORITHM INITIATED ***

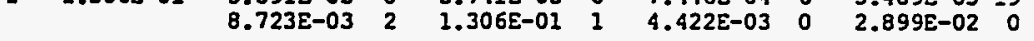

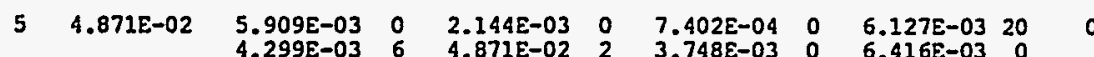

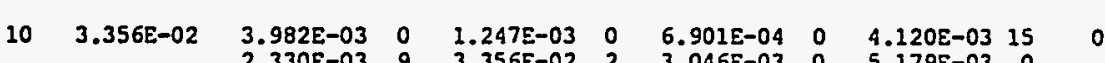

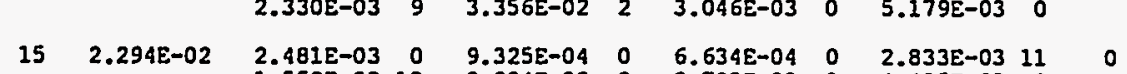

$\begin{array}{rrrrrrrrrrr}15 & 2.294 E-02 & 2.481 E-03 & 0 & 9.325 E-04 & 0 & 6.634 E-04 & 0 & 2.833 E-03 & 11 & 0 \\ & & 1.558 E-03 & 13 & 2.294 E-02 & 2 & 2.783 E-03 & 0 & 4.405 E-03 & 0\end{array}$

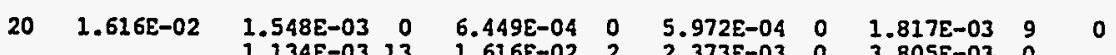

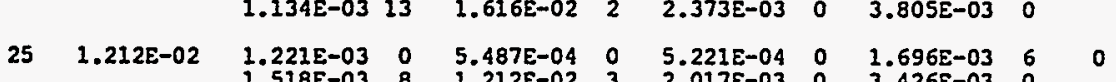

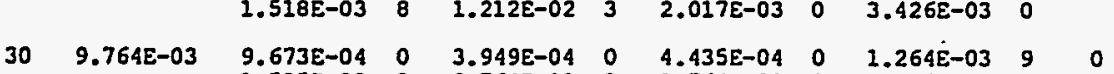

$\begin{array}{llllllll}1.535 E-03 & 3 & 9.764 E-03 & 3 & 1.764 E-03 & 0 & 3.195 E-03 & 0\end{array}$

$\begin{array}{lllllll}\text { ONJITER } & \text { SORCE } & \text { RESNJ'S } & & \\ 1.342 \mathrm{E}-03 & 1.153 \mathrm{E}-03 & 1.183 \mathrm{E}-03 & 1.238 \mathrm{E}-03 & 1.324 \mathrm{E}-03 & 1.342 \mathrm{E}-03\end{array}$

SCATTER ITER: 0 ITERATION: I ERAC CHANGE IN TOT NET WALI FLUX: 1.000E+00 SCATTER ITER: 0 ITERATION: 2 FRAC CHANGE IN TOT NET WALL FIUX: $\mathbf{7 . 5 7 0 E - 0 2}$ SCATTER ITER: 0 ITERATION: 3 FRAC CHANGE IN TOT NET HALL FLUX: $\mathbf{5 . 3 1 9 E - 0 3}$ SCATTER ITER: 0 ITERATION: 3 FRAC CHANGE IN TOT NET HALL FLUX: $5.319 E-03$ SCATTER ITER: 0 ITERATION: 4 FRAC CHANGE IN TOT NET WALI FLUX: $3.640 E-04$
SCATTER ITER: 0 ITERATION: 5 FRAC CHANGE IN TOT NET WALL ELUX: $2.380 E-05$ $\begin{array}{lll}\text { SCATTER ITER: } 0 \text { ITERATION: } 5 \text { FRAC CHANGE IN TOT NET WALI FLUX: } & 2.380 E-05 \\ \text { SCATTER ITER: } 0 \text { ITERATION: } 6 \text { FRAC CHANGE IN TOT NET WALL FLUX: } & 1.602 E-06\end{array}$ SCATTER ITER: 1 ITERATION: 1 FRAC CHANGE IN TOT NET WALL FLUX: $2.192 E-02$ SCATTER IIER: 1 ITERATION: 2 FRAC CHANGE IN TOT NET WALL ELUX: 5.6665-04 SCATTER ITER: 1 ITERATION: 3 FRAC CHANGE IN TOT NET WALI FLUX: 2.464E-05 SCATTER ITER: 1 ITERATION: 4 FRAC CHANGE IN TOT NET WALT FUUX: REBOUNDING COLLISIONS ALTOHED FOR WALLS WHEN WALT TEMPERATURE IS BEIOS $3000.0 \mathrm{~K}$ TRAJECTORY ISL= I. IPS= 1 RECIRCUIATES TRAJECTORY ISL= I, IPS= 1 RECIRCULATES
ISI= 1, IPS= 1, TERMINATED AT: IV= 54, JU-31 TIME= 8.377E+00 NPOTNT= 714 TRAJECTORY ISL $=1$, IPS= 2 RECIRCULATES

ISI - 1, IPS - 2, TERMINATED AT: IV- 54, JU-31 TIME- 8.219E+00 NPOINT- 705 TRAJECTORY ISL $=1$, IPS $=3$ RECIRCULATES

ISL $=1$, IPS $=3$, TERMINATED AT: IV $=54, \pi J=31$ TIME $=8.125 E+00$ NPOINT $=701$ TRAJECTORY ISL $=1$, IPS $=4$ RECIRCULATES

ISI- 1, IPS $=4$, TERMINATED AT: IV $=54, \pi J=32$ TIME= $7.758 E+00$ NPOINT $=736$ TRAJECTORY ISL $=1$, IPS 5 RECIRCULATES

ISI 1, IRS- 5, TERMINATED AT: IV- 54, JU-32 TIME= $7.429 E+00$ NPOINT= 731 TRAJECTORY ISL= 2, IPS- 1 RECIRCULATES 
ISL= 2, IPS- 1, TERMINATED AT: IV- 54, JU-31 TIME- $8.234 E+00$ NPOINT- 520 TRAJECTORY ISL $=2$, IPS -2 RECIRCULATES ISL- 2, IPS= 2, TERMINATED AT: IV- 54, JU-31 TIME- 8.019E+00 NPOINT= 563 TRAJECTORY ISL= 2, IPS- 3 RECIRCULATES ISL- 2, IPS= 3, TERHISATED AT: IV= 54, JU=31 TIME= 7.957E+00 NPOINT= 615 TRAJECTORY ISL $=2$, IPS $=4$ RECIRCULATES ISL= 2, IPS $=4$, TERMINATED AT: IV $=54$, JU=32 TIME= $7.844 E+00$ NPOINT= 957 TRAJECTORY ISL $=2$, IPS $=5$ RECIRCULATES ISL- 2, IPS= 5, TERHINATED AT: IV= 54, JU-30 TIME= 9.384E+00 NPOINT $=1289$ TRAJECTORY ISL $=3$, IPS- 1 RECIRCULATES ISL- 3, IPS= 1, TERMTNATED AT: IV- 54, $J=32$ TIME- 7.609E+00 NPOINT= 513 TRAJECTORY ISL $=3$, IPS- 2 RECIRCULATES ISL- 3, IPS- 2, TERMINATED AT: IV $=54, \pi J-32$ TIME- $7.675 E+00$ NPOINT= 498 TRAJECTORY ISL -3 , IPS- 3 PECIRCUTATES ISI- 3, IRS- 3, TERMINATED AT: IV- 54, JU=31 TIME- $7.789 E+00$ NPOINT $=513$ TRAJECTORY ISL $=3$, IPS 4 RECIRCULATES ISL= 3, IPS - 4, TERMINATED AT: IV= 54, JU-29 TIME- 1.001E+01 NPOINT= 874 TRAJECTORY ISL $=3$, IPS $=5$ RECIRCULATES ISL= 3, IPS $=5$, IERMINATED AT: IV $=54, \pi J=32$ TIME- $7.282 E+00$ NPOINT $=535$ TRAJECTORY ISL 4 , IPS= 1 RECIRCULATES ISL= 4, IPS= 1, TERMINATED AT: IV $=54, \pi J=33$ TIME= $7.046 E+00$ NEOINT- 462 TRA JECTORY ISL 4 TERYTSE 2 RECIRCULATES ISL 4, IPS- 2, TERMINATED AT: IV- S4, JU-33 TIME- $6.974 E+00$ NPOINT= 471 ISL 4, TRAJECTORY ISL= 4, IRS- 3 RECIRCULATES TRAJECTORY ISL $=4$, IPS $=4$ RECIRCULATES ISI 4, IPS $=4$, TERMINATED AT: IV $=54$, JU-29 TIME= 9.455E+00 NPOINT= 788 TRAJECTORY ISL- 4, IPS- 5 RECIRCULATES ISL- 4, IPS- 5, IERMINATED AT: IV- 54, JU-30 TIME- 9.122E+00 NPOINT- 804 TRAJECTORY ISL $=5$, IPS= 1 RECIRCULATES ISL $=5$, IPS $=1$, TERMINATED AT: IV $=54, \pi U=33$ TIME- $6.721 E+00$ NPOINT $=483$ TRAJECTORY ISL 5 , IPS 2 RECIRCULATES ISL - 5, IPS- 2, TERMINATED AT: IV $=54, \pi U=33$ TIME $=6.612 E+00$ NPOINT= 416 TRAJECTORY ISL $=5$, IPS $=3$ RECIRCULATES ISL= 5, IPS= 3, TERMINATED AT: IV= 54, JU=33 TIME= $6.701 E+00$ NPOINT= 611
TRAJECTORY ISL= 5, IPS= 4 RECIRCULAIES ISL= S, IPS = 4, IERMINATED AT: IV- $54, \pi J=33$ TIME- $6.685 E+00$ NPOINT- 565 TRAJECTORY ISL- 5 , IPS= 5 RECIRCULATES

ISL= 5, IPS $=5$, TERMINATED AT: IV $=54, \pi J=31$ TIME= $8.439 E+00$ NPOINT $=763$ TRAJECTORY ISL 6 TERHINATED AT: IVUTATS

ISL= 6, IPS= 1, TERMINATED AT: IV = 54, JU=34 TIME= 6.269E+00 NPOINT= 491 ISL= 6, IRS- 1, TERMINATED AT: IV= 54, JU=34 TIME= 6.269E+00 NPOINT= 491 ISL- 6, IPS- 2, TERMINATED AT: IV- 54, JU=34 TIME- 6.329E+00 NPOINT= 441 TRAJECTORY ISL $=6$, IPS $=3$ RECIRCULATES ISL= 6, IPS= 3, TERMINATED AT: IV- 54, JU-34 TIME- 6.251E+00 NPOINT- 442 TRAJECTORY ISL $=6$, IPS- 4 RECIRCULATES

ISL= 6, IPS $=4$, TERMINATED AT: IV $=54, \pi J-35$ TIME- $6.142 E+00$ NPOINT= 555 TRA EECTORY ISL $=6$, IPS $=5$ RECIRCUTATES

ISL $=6$, IPS $=5$, TERMINATED AT: IV $=54, J J=33$ TIME= $6.7558+00$ NPOINT $=771$ ISL- 7, IPS- 1, TERMINATED AT: IV $=54, \pi J=34$ TIME $=6.182 E+00$ NPOINT $=510$ TRAJECTORY ISL $=7$, IPS $=2$ RECIRCULATES ISL - 7, IPS= 2, TERMINATED AT: IV- 54, JU-34 TIME- 6.156E+00 NPOINT- 497 TRA JECTORY ISL -7 , IPS- 3 RECTRCULATES

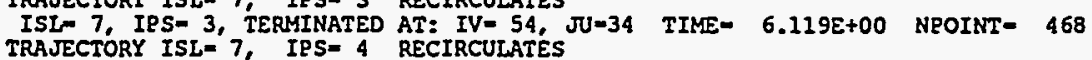

ISL $=7$, IPS $=4$, TERMINATED AT: IV $=54, \pi J-34$ TIME- $6.205 E+00$ NPOINT $=760$ TPAJECTORY ISL $=7$, IPS $=5$ PECIRCULATES

ISL- 7, IPS- 5, TERMINATED AT: IV= 54, JU-34 TIME- 6.256E+00 NPOINT= 744 ISL- 8, IPS- 1, TERMINATED AT: IV= 54, JU-35 TIME- $5.2568+00$ NPOINT= 744 TRA JECTORY ISL $=8$, IPS -2 RECIRCULATES

ISL 8 , IRS $=2$, TERMINATED AT: IV- 54, JU $=35$ TIME- $5.861 E+00$ NPOINT= 579 TRAJECTORY ISL= 8 , IPS -3 RECIRCULATES

ISL= 8, IPS- 3, TERMINATED AT: IV- 54, JU=35 TIME- 5.8948+00 NPOINT- 593 TRAJECTORY ISL $=8$, IPS- 4 RECIRCULATES

ISL $=8$, IPS $=4$, TERMINATED AT: IV $=54, J U=36$ TIME $=5.556 E+00$ NPOINT $=566$ TRAJECTÓRY ISL = 8 , IPS $=5$ RECIRCULATES

TSL ISL= 8, IPS- 5, TERMINATED AT: IV- 54, JU=37 TIME- $5.405 E+00$ NPOINT- 467

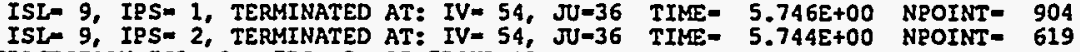
TRAJECTORY ISL $=9$, IPS- 3 RECIRCULATES 
Sample Computer Files for Coal Combustion with $\mathrm{NO}_{x}$ Formation

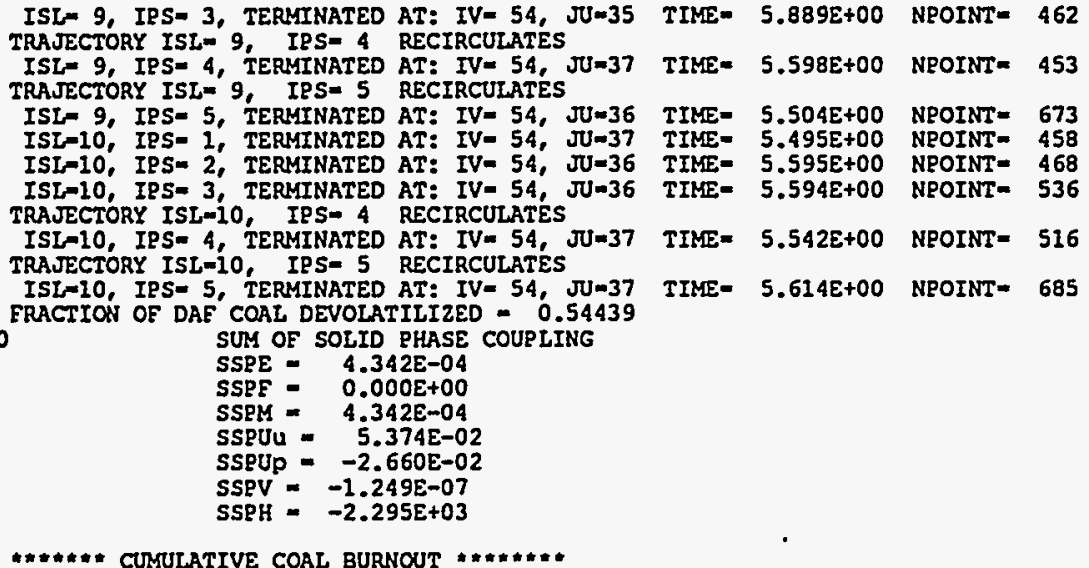

\begin{tabular}{|c|c|c|c|c|}
\hline$\left\{\begin{array}{l}x \\
x \\
x \\
x \\
x\end{array}\right.$ & $\begin{array}{l}0.00 \\
0.01 \\
0.02 \\
0.03 \\
0.04 \\
0.05 \\
0.06 \\
0.07 \\
0.08 \\
0.09 \\
0.10 \\
0.11 \\
0.12 \\
0.13 \\
0.14 \\
0.15 \\
0.16 \\
0.17 \\
0.19 \\
0.20 \\
0.21 \\
0.22 \\
0.23 \\
0.24 \\
0.26 \\
0.27 \\
0.29 \\
0.31 \\
0.33 \\
0.36 \\
0.38 \\
0.41 \\
0.44 \\
0.48 \\
0.52 \\
0.56 \\
0.61 \\
0.66 \\
0.72 \\
0.78 \\
0.85 \\
0.93 \\
1.02 \\
1.11 \\
1.22\end{array}$ & $\begin{array}{l}\text { COAL } \\
\text { COAL } \\
\text { COAL } \\
\text { COAL } \\
\text { COAL } \\
\text { COAL } \\
\text { COAL } \\
\text { COAL } \\
\text { COAL } \\
\text { COAL } \\
\text { COAL } \\
\text { COAL } \\
\text { COAL } \\
\text { COAL } \\
\text { COAL } \\
\text { COAL } \\
\text { COAL } \\
\text { COAL } \\
\text { COAL } \\
\text { COAL } \\
\text { COAL } \\
\text { COAL } \\
\text { COAL } \\
\text { COAL } \\
\text { COAL } \\
\text { COAL } \\
\text { COAL } \\
\text { COAL } \\
\text { COAL } \\
\text { COAL } \\
\text { COAL } \\
\text { COAL } \\
\text { : COAL } \\
\text { COAL } \\
\text { COAL } \\
\text { COAL } \\
\text { COAL } \\
\text { COAL } \\
\text { COAL } \\
\text { COAL } \\
\text { COAL } \\
\text { COAL } \\
\text { COAL } \\
\text { COAL } \\
\text { COAL }\end{array}$ & 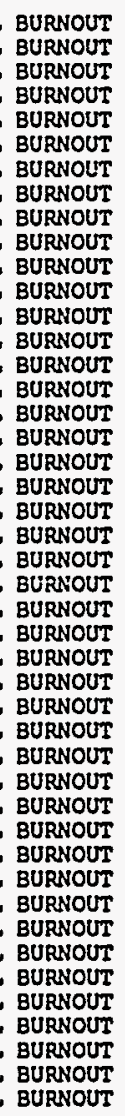 & $\begin{array}{l}=0.0000 \\
=0.1956 \\
=0.3842 \\
=0.3969 \\
=0.4262 \\
=0.4323 \\
=0.4675 \\
=0.4785 \\
=0.5106 \\
=0.5405 \\
=0.5521 \\
=0.5732 \\
=0.5950 \\
=0.6142 \\
=0.6287 \\
=0.6432 \\
=0.6564 \\
=0.6681 \\
=0.6858 \\
=0.6980 \\
=0.7110 \\
=0.7257 \\
=0.7433 \\
=0.7651 \\
=0.7916 \\
=0.8183 \\
=0.8456 \\
=0.8699 \\
=0.8917 \\
=0.9108 \\
=0.9275 \\
=0.9438 \\
=0.9565 \\
=0.9634 \\
=0.9691 \\
=0.9742 \\
=0.9792 \\
=0.9839 \\
=0.9887 \\
=0.9922 \\
=0.9962 \\
=0.9982 \\
=0.9989 \\
=0.9992\end{array}$ \\
\hline
\end{tabular}




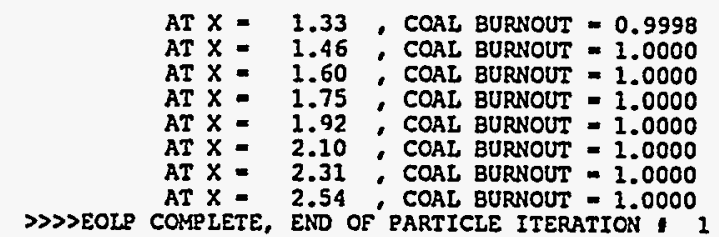

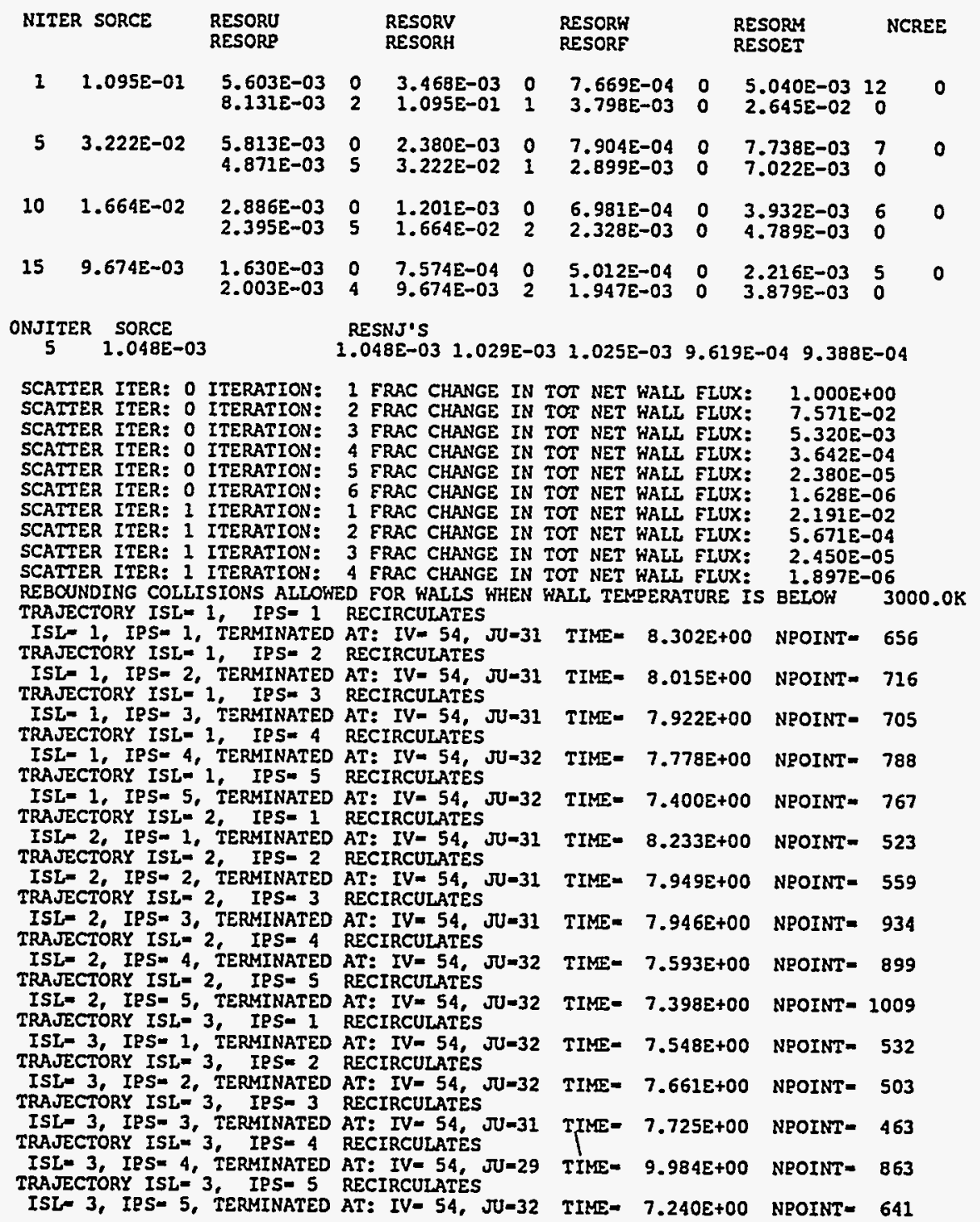


Sample Computer Files for Coal Combustion with $\mathrm{NO}_{x}$ Formation

TRAJECTORY ISL $=4$, IPS- 1 RECTRCULATES

ISI $=4$, IPS- 1 , TERMINATED AT: IV $=54, \quad J U=33$ TIME= $7.039 E+00$ NPOTNT $=445$ TRAJECTORY ISL $=4$, IPS $=2$ RECIRCULATES

ISL- 4, IPS- 2, TERMINATED AT: IV- 54, JU-33 TIME= 7.087E+00 NPOINT- 467

TRA JECTORY ISL $=4$, IPS $=3$ RECIRCULATES

ISL 4 , IPS $=3$, IERMINATED AT: IV $=54, J U=32$ TIME= $7.161 E+00$ NPOINT= 430

TRA JECTORY ISL $=4$, IPS- 4 RECIRCULATES

ISL 4, IPS= 4, TERMINATED AT: IV- 54, JU-32 TIME= 7.292E+00 NROINT- 525

TRAJECTORY ISL $=4$, IPS- 5 RECIRCULATES

ISL 4 , IPS= 5, IERMINATED AT: IV- 54 , JU-3I TIME- $7.815 E+00$ NPOINT- 599

TRAJECTORY ISL- 5 , IPS- I RECIRCULATES

ISL- 5, IPS 1, TERMINATED AT: IV- 54, JU-33 TIME- 6.694E+00 NPOINT- 486

IRAJECTORY ISL $=5$, IRS- 2 RECIRCULATES

ISL- 5, IPS = 2, TERMINATED AT: IV $=54$, JU-33 TIME- 6.742E+00 NPOINT- 416

TRAJECTORY ISL - 5, IPS -3 RECIRCULATES

ISI= 5, IPS -3 , TERMINATED AT: IV $=54,5 J=33$ TIME= 6.649E+00 NPOINT= 615

TRAJECTORY ISL $=5$, IPS $=4$ RECIRCULATES

ISL- 5, IPS - 4, TERMINATED AT: IV $=54, \pi J=33$ TIME- 6.553E+00 NPOINT= 573

TRAJECTORY ISL $=5$, IPS $=5$ RECIRCULATES

ISL= 5, IPS= 5, TERMINATED AT: IV- 54 , JU-31 TIME- $8.156 E+00$ NPOINT- 694

TRAJECTORY ISL $=6$, IPS $=1$ RECIRCULATES

ISL- 6, IPS- 1, TERMINATED AT: IV- 54, JU-34 TIME- 6.216E+00 NPOINT- 459

TRAJECTORY ISL $=6$, IPS $=2$ RECIRCULATES

ISL= 6, IPS $=2$, TERMINATED AT: IV= 54, JU=34 TIME- 6.321E+00 NPOINT= 434

TRAJECTORY ISL $=6$, IPS -3 RECIRCULATES

ISL -6 , IPS= 3, TERMINATED AT: IV- 54, JJ-34 TIME= 6.445E+00 NPOINT- 438

TRAJECTORY ISL- 6 , IPS - 4 RECIRCULATES

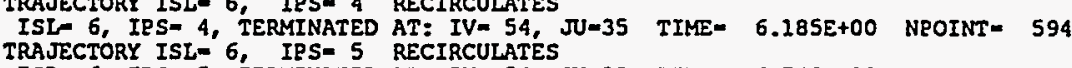

ISL $=6$, IPS $=5$, TERMINATED AT: IV $=54, J U=33$ TIME= 6.742E +00 NPOINT $=756$

ISL- 7, IRS- 1, TERMINATED AT: IV- 54, JU $=34$ TIME= $5.999 E+00$ NPOINT $=518$

TRAJECTORY ISL- 7 , IPS- 2 RECIRCULATES

ISI $=7$, IPS- 2, TERMINATED AT: IV- 54, JU-34 TIME- 6.232E+00 NPOINT = 441

TRAJECTORY ISL $=7$, IPS- 3 RECIRCULATES

ISI= 7, IPS $=3$, TERMINATED AT: IV $=54$, JU-33 TIME $=6.932 E+00$ NPOINT $=506$

TRAJECTORY ISL $=7$, IPS= 4 RECIRCULATES

ISL $=7$, IPS = 4, TERMINATED AT: IV $=54, \quad J=34$ TIME $=6.313 E+00$ NPOINT $=772$

TRAJECTORY ISL -7 , IPS- 5 RECIRCULATES

ISL- 7, IPS $=5$, TERMINATED AT: IV $=54, \pi U=36$ TIME- $5.841 E+00$ NPOINT= 547

ISI - 8, IPS= 1, TERMINATED AT: IV- 54, JU-35 TIME- 5.886E+00 NPOINT= 723

TRAJECTORY ISL- 8 , IPS- 2 RECIRCULATES

ISI - 8, IPS $=2$, TERMINATED AT: IV $=54, J U=35$ TIME $=5.944 E+00$ NPOINT $=581$

TRAJECTORY ISL= 8 , IPS- 3 RECIRCULATES

ISI = 8, IPS $=3$, TERMINATED AT: IV $=54$, JU-35 TIME- $5.809 E+00$ NPOINT- 587

TRAJECTORY ISL 8 , IPS= 4 RECIRCULATES

ISI - 8, IPS = 4, TERMINATED AT: IV $=54, \pi J=36$ TIME- 5.691E+00 NPOINT= 581

TRAJECTORY ISL $=B$, IPS $=5$ RECIRCULATES

ISI= 8, IPS $=5$, TERMINATED AT: IV= 54, JU-37 TIME= 5.422E+00 NPOINT= 438

ISL $=9$, IPS $=1$, TERMINATED AT: IV $=54, J U=36$ TIME $=5.842 E+00$ NPOINT $=460$

ISI- 9, IPS= 2, TERMINATED AT: IV- 54, JU-36 TIME- 5.692E+00 NPOTNT- 598

ISI- 9; IPS- 3;, TERMINATED AT: IV- 54, JU-35 TIME- 5.748E+00 NPOINT- 483

TRAJECTORY ISL- 9, IPS= 4 RECIRCULATES

ISL- 9, IPS= 4, TERMINATED AT: IV- 54, JU-37 TIME- $5.594 E+00$ NPOINT- 474

TRAUECTORY ISL- 9, IPS- 5 RECIRCULATES

ISL- 9, IPS - 5, TERMINATED AT: IV= 54, JU=37 TIME- 5.651E+00 NPOINT= 681

ISI-10, IPS= 1, TERMINATED AT: IV- 54, JU-37 TIME- 5.445E+00 NPOINT= 530

ISI $=10$, IPS= 2, TERMINATED AT: IV- 54, $J U=36$ TIME- $5.616 E+00$ NPOINT- 439

ISIN10, IPS= 3, TERMINATED AT: IV= 54, JU=36 TIME- 5.646E+00 NPOINT= 469

TRAJECTORY ISL-10, IPS= 4 RECIRCULATES

ISL 10 , IPS= 4, TERMINATED AT: IV $=54$, JU 36 TIME- $5.855 \varepsilon+00$ NPOINT= 694

TRAJECTORY ISL-10, IPS- 5 RECIRCULATES

ISL 10 , IRS= 5, TERMINATED AT: IV $-54, J U-37$ TIME- 5.561E+00 NPOINT= 699

FRACTION OE DAF COAL DEVOLATILIZED - 0.54595

- SUM OE SOLID PHASE COUPLING

SSPE $=4.342 E-04$

SSPF = $0.000 E+00$

SSPH = 4.342E-04

SSPH = $4.342 E-04$

SSPUu $=\quad 5.218 E-02$
SSPUP $=-2.575 E-02$

$S S P V=-1.222 E-07$ 
SSPH $=-2.291 E+03$

"*"*** CUMULATIVE COAL BURNOUT *******

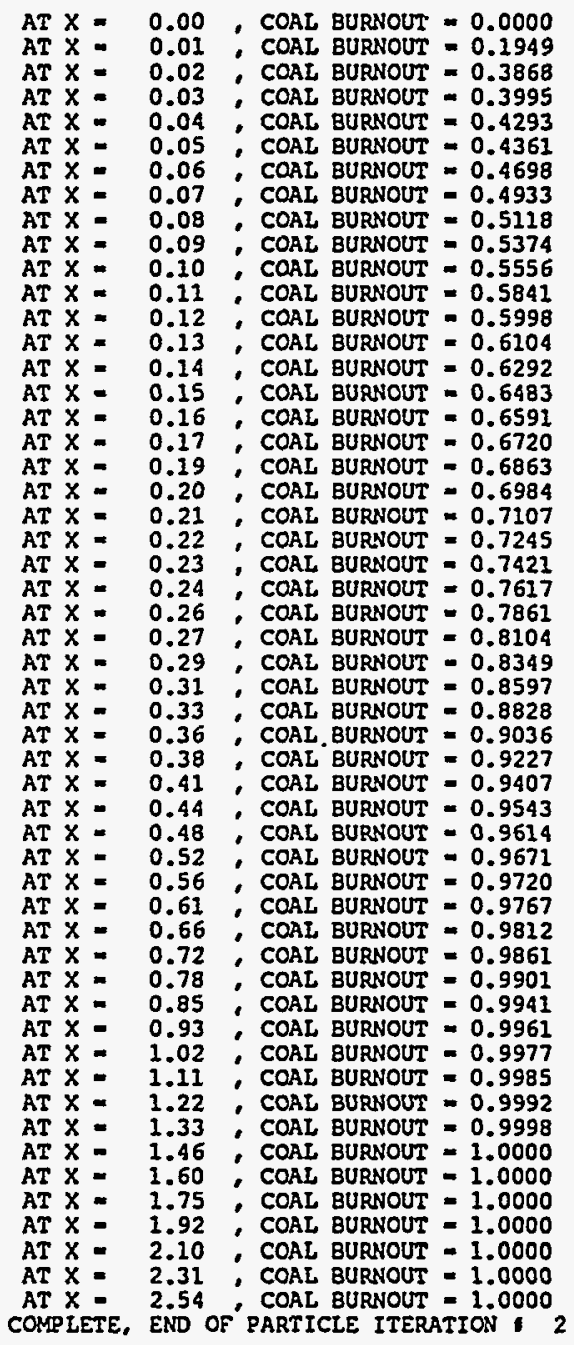

\\SOLP COMPLETE, END OF PARTICLE ITERATION 2

25

\begin{tabular}{|c|c|c|c|c|c|c|c|c|c|c|}
\hline \multicolumn{2}{|c|}{ NITER SORCE } & \multicolumn{2}{|l|}{$\begin{array}{l}\text { RESORU } \\
\text { RESORP }\end{array}$} & $\begin{array}{l}\text { RESORV } \\
\text { RESORH }\end{array}$ & \multicolumn{2}{|r|}{$\begin{array}{l}\text { RESORH } \\
\text { RESORE }\end{array}$} & \multicolumn{2}{|c|}{$\begin{array}{l}\text { RESORH } \\
\text { RESOET }\end{array}$} & \multicolumn{2}{|c|}{ NCREE } \\
\hline 1 & $1.067 E-01$ & $\begin{array}{l}5.827 E-03 \\
9.710 \mathrm{E}-03\end{array}$ & $\begin{array}{l}0 \\
2\end{array}$ & $\begin{array}{l}3.612 E-03 \\
1.067 \mathrm{E}-01\end{array}$ & $\begin{array}{l}0 \\
1\end{array}$ & $\begin{array}{l}8.049 \mathrm{E}-04 \\
4.504 \mathrm{E}-03\end{array}$ & $\begin{array}{l}0 \\
0\end{array}$ & $\begin{array}{l}5.555 E-03 \\
2.925 E-02\end{array}$ & $\begin{array}{l}6 \\
0\end{array}$ & \\
\hline 5 & $2.090 E-02$ & $\begin{array}{l}4.458 E-03 \\
3.473 E-03\end{array}$ & $\begin{array}{r}0 \\
17\end{array}$ & $\begin{array}{l}2.401 \varepsilon-03 \\
2.090 \Sigma-02\end{array}$ & $\begin{array}{l}0 \\
2\end{array}$ & $\begin{array}{l}1.035 E-03 \\
3.129 E-03\end{array}$ & $\begin{array}{l}0 \\
0\end{array}$ & $\begin{array}{l}6.050 E-03 \\
7.288 E-03\end{array}$ & $\begin{array}{r}11 \\
0\end{array}$ & \\
\hline
\end{tabular}


Sample Computer Files for Coal Combustion with $\mathrm{NO}_{x}$ Formation

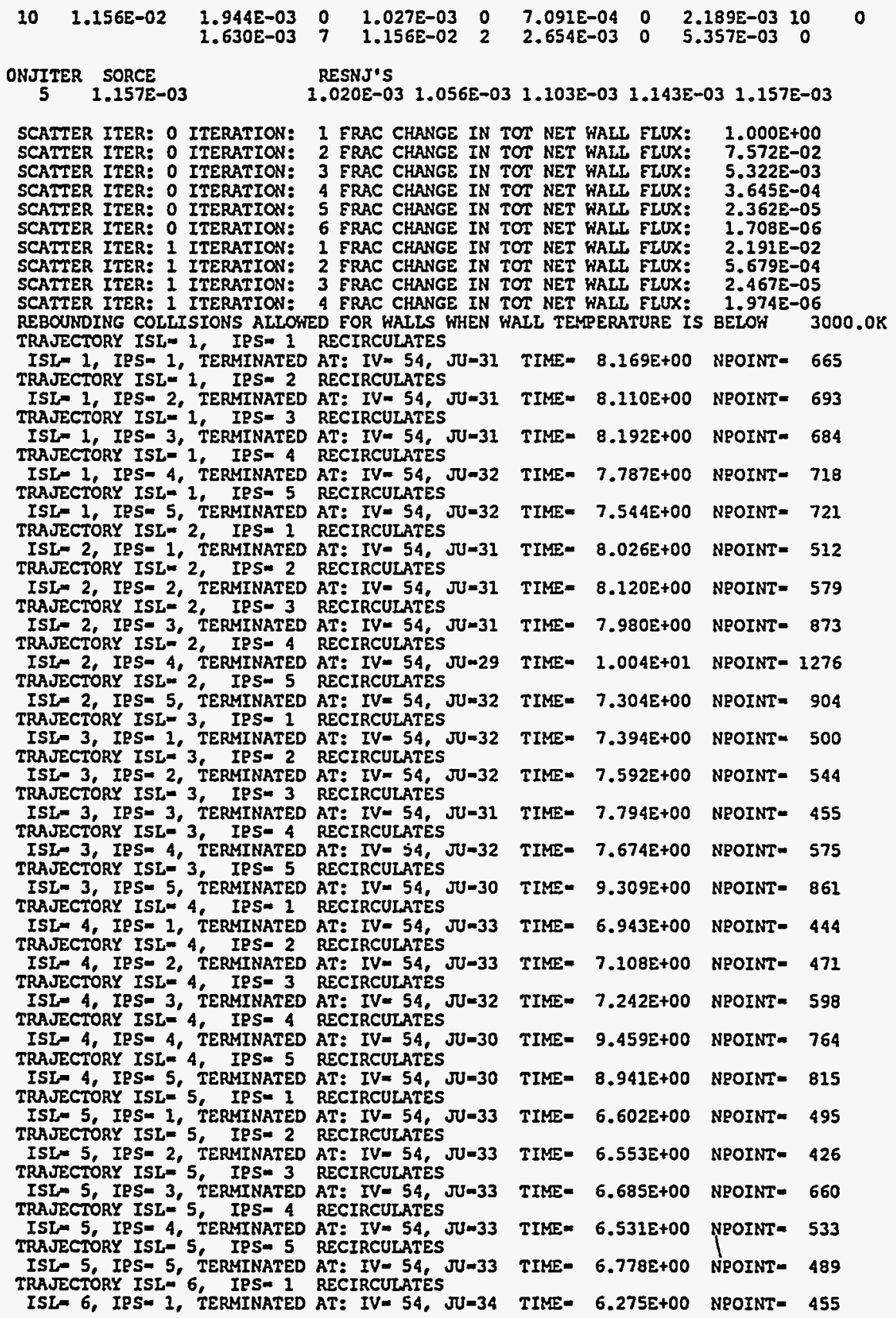

SCATTER ITER: O ITERATION: I FRAC CHANGE IN TOT NET HALL FLUX: $1.000 E+00$ SCATTER ITER: O ITERATION: 2 FRAC CHANGE IN TOT NET WALL ELUX: $7.572 E-02$ SCATTER ITER: O ITERATION: 3 FRAC CHANGE IN TOT NET HALL ELUX: $5.322 E-03$ SCATTER ITER: O ITERATION: 4 ERAC CHANGE IN TOT NET HALL FLUX: 3.645E-04 SCATTER ITER: O ITERATION: 5 ERAC CHANGE IN TOT NET WALL FLUX: $2.362 E-05$ SCATTER ITER: O ITERATION: 6 ERAC CHANGE IN TOT NET WALL FLUX: 1.708E-06 SCATTER ITER: 1 ITERATION: 1 FRAC CHANGE IN TOT NET WALL FLUX: $2.191 E-02$ SCATTER ITER: 1 ITERATION: 2 ERAC CHANGE IN TOT NET WALL FLUX: $5.679 E-04$ SCATTER ITER: I ITERATION: 3 ERAC CHANGE IN TOT NET WALL FLUX: $2.467 E-05$ SCATTER ITER: I ITERATION: 3 ERAC CHANGE IN TOT NET WALL FLUX: $2.467 E-05$ SCATTER ITER: I ITERATION: 4 FRAC CHANGE IN TOT NET WALL FUUX: $1.974 E-06$
REBOUNDING COLLISIONS ALLOHED FOR HALS WHEN WALL TEMPERATURE IS BELOW $3000.0 \mathrm{~K}$ TRAJECTORY ISL $=1$, IPS - 1 RECIRCULATES

ISL- 1, IPS- 1, TERMINATED AT: IV = 54, JU-31 TIME- $8.169 E+00$ NPOINT= 665 TRAJECTORY ISL $=1$, IPS- 2 RECIRCULATES 
TRAJECTORY ISL- 6, IPS- 2 RECIRCULATES

ISL $=6$, IPS= 2, TERMINATED AT: IV $=54, \quad J 3=34$ TIME- 6.431E+00 NPOINT= 420 TRAJECTORY ISL $=6$, IPS $=3$ RECIRCULATES ISL - 6, IPS= 3, TERMINATED AT: IV- 54 , JU-34 TIME= $6.249 E+00$ NPOINT- 438 TRAJECTORY ISL- 6 , IPS- 4 RECIRCULATES

ISI = 6, IPS- 4, TERMINATED AT: IV- 54, JU-33 TIME- 6.003E+00 NPOINT= 755 TRAJECTORY ISL= 6 , IPS- 5 RECIRCULATES

ISI= 6, IPS- 5, TERMINATED AT: IV- 54, JJ-33 TIME- 6.724E+00 NPOINT- 786 ISL- 7, IPS- 1, TERMINATED AT: IV- 54, JU-34 TIME- 6.032E+00 NPOINT- 455 TRAJECTORY ISL $=7$, IPS- 2 RECIRCULATES ISL= 7, IPS= 2, TERMINATED AT: IV= 54, JU-34 TIME- $6.130 E+00$ NPOINT- 489 TRAJECTORY ISL -7 , IRS- 3 RECIRCULATES ISI $=7$, IRS $=3$, TERMINATED AT: IV $=54, J U=35$ TIME= $6.104 E+00$ NPOINT- 528 TRAJECTORY ISL $=7$, IPS $=4$ RECIRCULATES ISL $=7$, IPS -4 , TERMINATED AT: IV $=54$, JU $=34$ TIME- $6.388 E+00$ NPOINT= 724 TRA JECTORY ISL $=7$, IPS -5 RECIRCULATES

ISL= 7, IPS $=5$, TERMINATED AT: IV $=54, \pi J=34$ TIME= $6.246 E+00$ NPOINT= 750 ISL= 8, IPS= 1, TERMINATED AT: IV- 54, JU-35 TIME- $5.808 E+00$ NPOINT= 726 TRAJECTORY ISL $=8$, IPS $=2$ RECIRCUIATES

ISL= 8, IPS= 2, TERMINATED AT: IV- 54, JJ=35 TIME= $5.817 E+00$ NPOINT= 583 TRAJECTORY ISL $=8$, IPS $=3$ RECIRCULATES

ISI $=8$, IPS $=3$, TERMINATED AT: IV- 54, JU-35 TIME- 5.892E+00 NPOINT= 543 TRAJECTORY ISL $=8$, IPS $=4$ RECIRCULATES

ISL $=8$, IPS $=4$, TERMINATED AT: IV $=54, \pi U=36$ TIME $=5.684 E+00$ NPOINT- 587 TRAJECTORY ISL- 8 , IPS- 5 RECIRCULATÉS

ISL= 8, IPS= 5, TERMINATED AT: IV- 54, JU-37 TIME- 5.422E+00 NPOINT= 440 ISL - 9, IPS= 1, TERMINATED AT: IV= 54, JJ-36 TIME= 5.844E+00 NPOINT= 455 ISL- 9, IPS $=2$, TERMINATED AT: IV= 54, JJ=36 TIME= 5.815E+00 NPOINT $=613$ ISL- 9, IPS= 3, TERMINATED AT: IV= 54, JU=35 TIME- 5.743E+00 NPOINT= 479 TRAJECTORY ISL $=9$, IPS $=4$ RECIRCULATES

ISL $=9$, IPS $=4$, TERMINATED AT: IV $=54, \pi U=35$ TRAJECTORY ISL $=9$, IPSE 5 RECIRCULATES

AT: IV $=54, \pi U=36$ TIME $=5.619 E+00$ NPOINT $=690$

ISL=10, IPS= 1, TERMINATED AT: IV= 54, JU-37 TIME- 5.455E+00 NPOINT- 894

ISL 10 , IPS- 2, TERMINATED AT: IV $=54, J U=36$ TIME $=5.590 E+00$ NPOINT $=441$

ISL-10, IPS= 3, TERMINATED AT: IV- 54, JU-36 TIME= 5.644E+00 NPOINT= 465

TRAJECTORY ISL-10, IPS= 4 RECIRCULATES

ISL-10, IPS- 4, TERMINATED AT: IV- 54, JU-36 TIME- 5.678E+00 NPOINT= 679 IRAJECTORY ISL=10, IPS- 5 RECIRCULATES

ISL-10, IPS -5 , TERMINATED AT: IV $=54, \pi U=37$ TIME= 5.563E+00 NPOINT $=682$

FRACTION OF DAF COAL DEVOLATILIZED = 0.54653

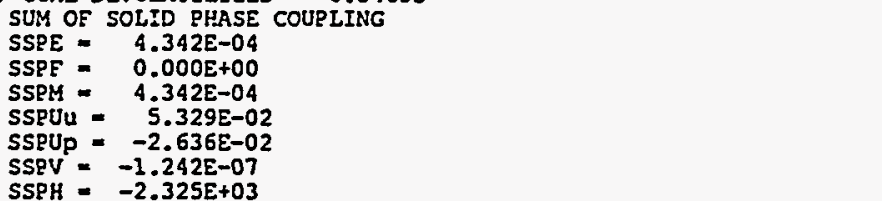

"****** CUMULATIVE COAL BURNOUT *******

\begin{tabular}{|c|c|c|}
\hline $\begin{array}{l}\text { AT } x= \\
\text { AT } x= \\
\text { AT } x= \\
\text { AT } x= \\
\text { AT } x= \\
\text { AT } x= \\
\text { AT } x= \\
\text { AT } x= \\
\text { AT } x= \\
\text { AT } x= \\
\text { AT } x= \\
\text { AT } x= \\
\text { AT } x= \\
\text { AT } x= \\
\text { AT } x=\end{array}$ & $\begin{array}{l}0.00 \\
0.01 \\
0.02 \\
0.03 \\
0.04 \\
0.05 \\
0.06 \\
0.07 \\
0.08 \\
0.09 \\
0.10 \\
0.11 \\
0.12 \\
0.13 \\
0.14 \\
0.15 \\
0.16\end{array}$ & $\begin{array}{l}\text { COAL BURNOUT } \\
\text { COAL BURNOUT } \\
\text { COAL BURNOUT } \\
\text { COAL BURNOUT } \\
\text { : COAL BURNOUT } \\
\text { COAL BURNOUT } \\
\text { COAL BURNOUT } \\
\text { COAL BURNOUT } \\
\text { COAL BURNOUT } \\
\text { COAL BURNOUT } \\
\text { COAL BURNOUT } \\
\text { COAL BURNOUT } \\
\text { COAL BURNOUT } \\
\text { COAL BURNOUT } \\
\text { COAL BURNOUT } \\
\text { COAL BURNOUT } \\
\text { COAL BURNOUT }\end{array}$ \\
\hline
\end{tabular}




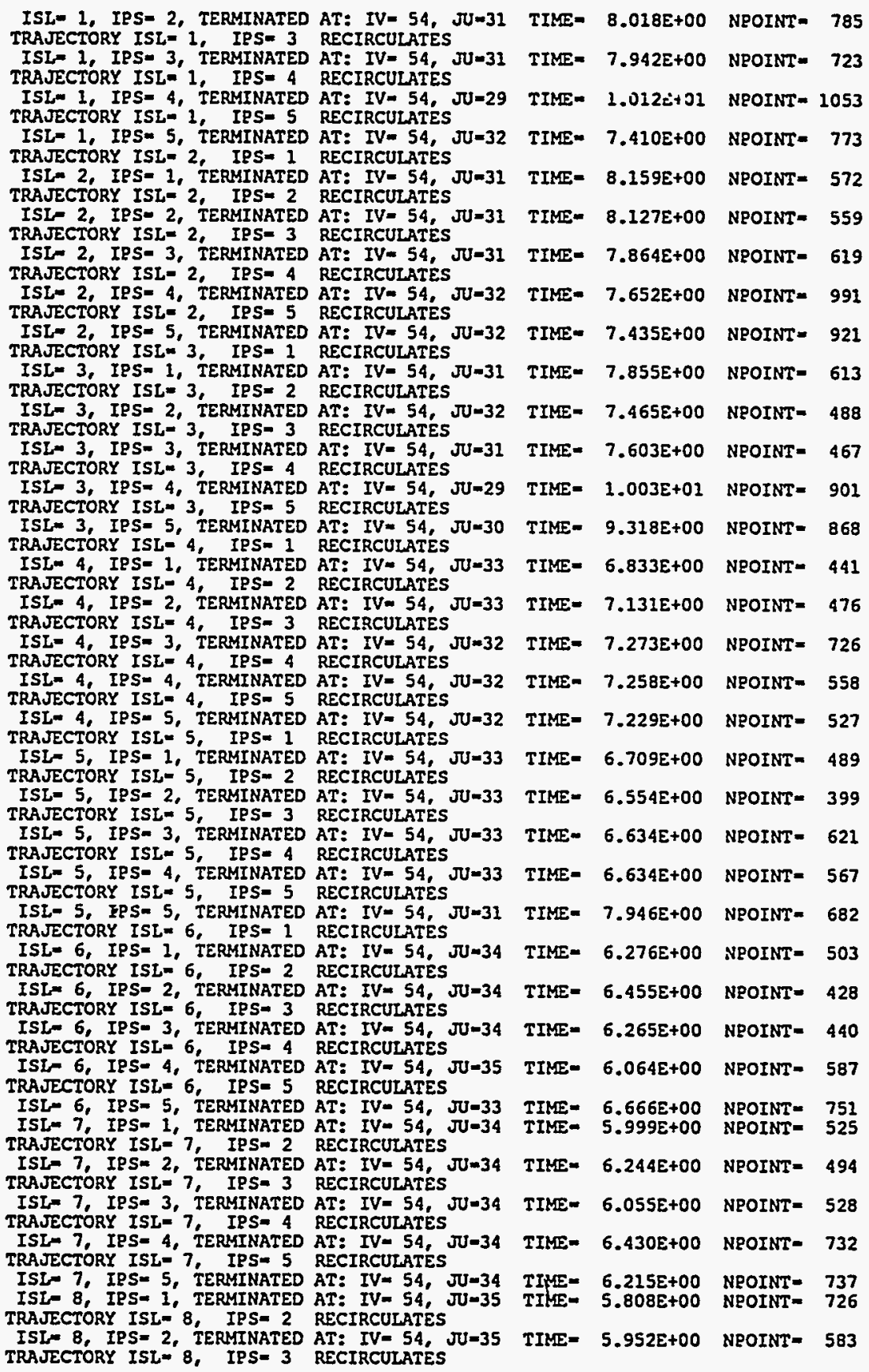


Sample Computer Files for Coal Combustion with $\mathrm{NO}_{x}$ Formation

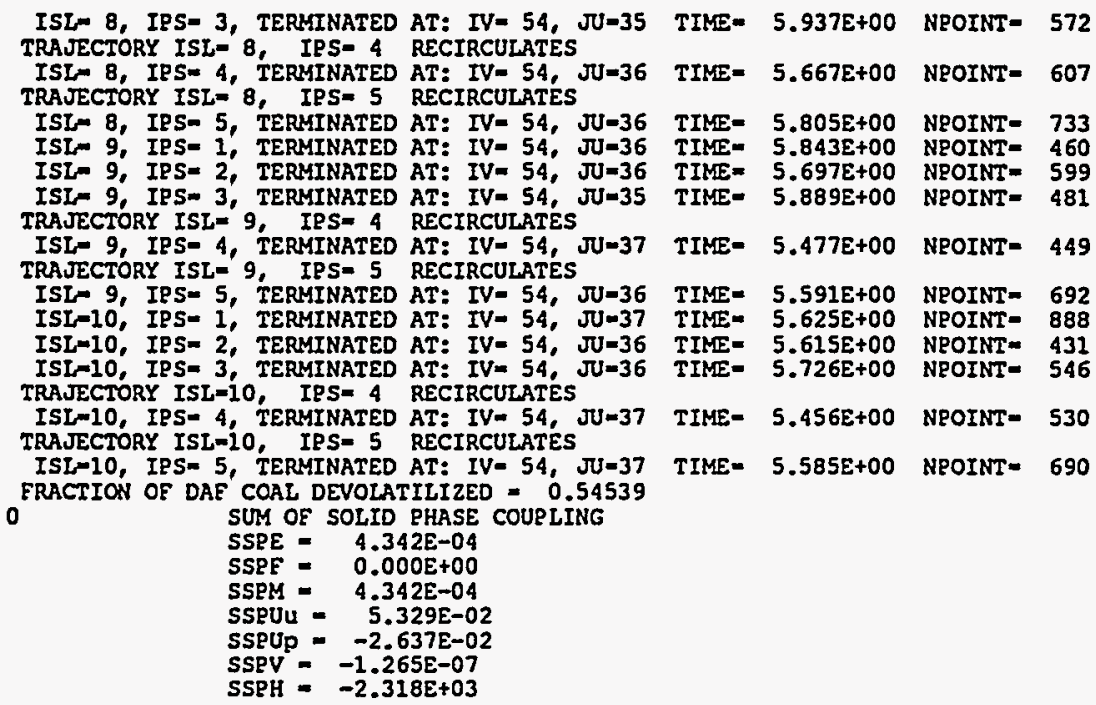

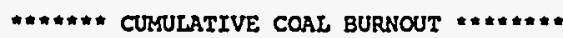

\begin{tabular}{|c|c|c|c|}
\hline $\begin{array}{l}0.01 \\
0.02 \\
0.03 \\
0.04 \\
0.05 \\
0.06 \\
0.07 \\
0.08 \\
0.09 \\
0.10 \\
0.11 \\
0.12 \\
0.13 \\
0.14 \\
0.15 \\
0.16 \\
0.17 \\
0.19 \\
0.20 \\
0.21 \\
0.22 \\
0.23 \\
0.24 \\
0.26 \\
0.27 \\
0.29 \\
0.31 \\
0.33 \\
0.36 \\
0.38 \\
0.41 \\
0.44 \\
0.48 \\
0.52 \\
0.56\end{array}$ & \multicolumn{2}{|c|}{ 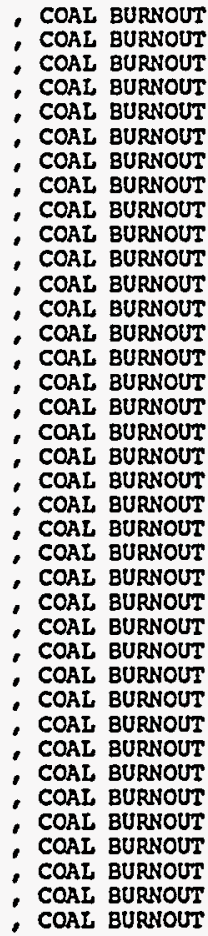 } & $\begin{array}{l}=0.0000 \\
=0.1948 \\
=0.3712 \\
=0.3850 \\
=0.4146 \\
=0.4211 \\
=0.4559 \\
=0.5002 \\
=0.5318 \\
=0.5442 \\
=0.5772 \\
=0.5989 \\
=0.6110 \\
=0.6303 \\
=0.6451 \\
=0.6642 \\
=0.6748 \\
=0.6904 \\
=0.7027 \\
=0.7153 \\
=0.7302 \\
=0.7477 \\
=0.7690 \\
=0.7934 \\
=0.8196 \\
=0.8449 \\
=0.8701 \\
=0.8934 \\
=0.9118 \\
=0.9282 \\
=0.9446 \\
=0.9567 \\
=0.9634 \\
=0.9692 \\
=0.9747 \\
=0.9800 \\
=0.9852\end{array}$ \\
\hline
\end{tabular}




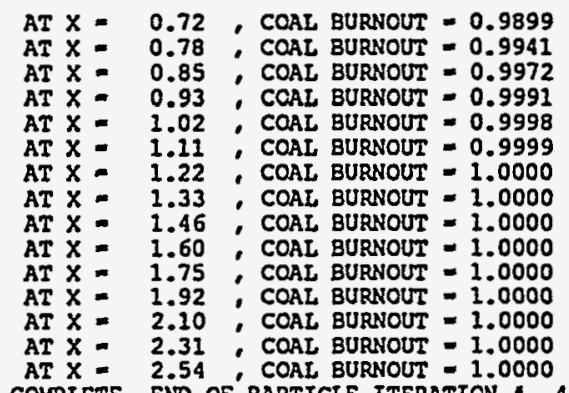

$\gg \gg$ SOLP COMPLETE, END OF' PARTICLE ITERATION 4

TOTAL MASS IN $(\mathrm{kg} / \mathrm{s})=4.26192 \mathrm{E}-02$

TOTAL MASS OUT $(\mathrm{kg} / \mathrm{s})$. $4.32478 \mathrm{E}-02$

TOTAL MASS OUT - TOTAL MASS IN $(\mathrm{kg} / \mathrm{s})=6.28643 \mathrm{E}-04$

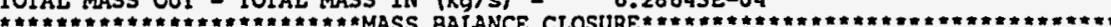

PERCENT OF TOTAL MASS IN 1 1.47502

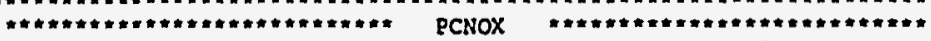

"** NOX POLIUTANT CALCULATION USING OUTPUT FROM PCGC-2 FOR THE *"

***** PHASE AND PARTICLE PROPERTIES THROUGHOUT THE FLOW FIELD **"

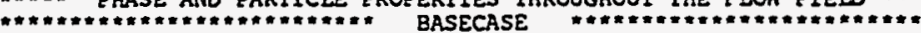

nox pollutane calculations called following convergence of pcgc2

nitnox

maxsor

reshc

resnh3

ncree

$\begin{array}{rr}2 & 3.776 E+01 \\ 4 & 3.718 \mathrm{E}+01 \\ 6 & 3.718 \mathrm{E}+01 \\ 8 & 3.718 \mathrm{E}+01 \\ 10 & 4.090 \mathrm{E}-01 \\ 12 & 3.892 \mathrm{E}-01 \\ 14 & 3.748 \mathrm{E}-01 \\ 16 & 3.683 \mathrm{E}-01 \\ 18 & 3.675 \mathrm{E}-01 \\ 20 & 3.667 \mathrm{E}-01 \\ 0 \mathrm{Tt} & =2.53667\end{array}$

$5.164 \mathrm{E}-01 \quad 3.721 E+01 \quad 3.776 \mathrm{E}+01$ $5.732 E-01 \quad 3.718 E+01$ 1.356E+00 4.814E-01 3.718E+01 $4.816 E-01$

$4.322 \varepsilon-01 \quad 3.718 \varepsilon+01 \quad 2.865 \varepsilon-01$

$4.090 \mathrm{E}-01$ 1.066E-01 2.141E-01

$3.892 E-01 \quad 6.261 E-02 \quad 1.737 E-01$

$3.748 \mathrm{E}-01$ 6.232E-02 $1.468 \mathrm{E}-01$

$3.683 \mathrm{E}-01 \quad 6.178 \mathrm{E}-02 \quad 1.263 \mathrm{E}-01$

3.675E-01 $6.093 \mathrm{E}-02$ 1.098E-01

$3.667 \varepsilon-01 \quad 5.972 E-02 \quad 9.620 E-02$

20
20
20
20
20
20
20
20
20
20
430

THIS IS A CONVERGED CASE

Removing linked generic flle names

$\mathrm{rm}$ : /mag/brewster/afr/cpr/soxIN: No such flle or dfrectory

Moving unlinked generic flles to spectfic files

mv: /mag/brewster/afr/cpr/YSO21: Cannot access: No such file or directory

mV: /mag/brewster/afr/cpr/YH2si: Cannot access: No such tile or directory

mu: /mag/brewster/afr/cpr/SORDNl: Cannot access: No such flle or dlrectory 
Sample Computer Files for Coal Combustion with $\mathrm{NO}_{x}$ Formation 


\section{Sample Computer Files for Coal Gasification with \\ Sidewall Sorbent Injection and Sulfur Capture}

This sample problem is the gasification of coal in the BYU gasifier with sidewall injection of sorbent.

Main Data File (ill.dat, PCGCIN)

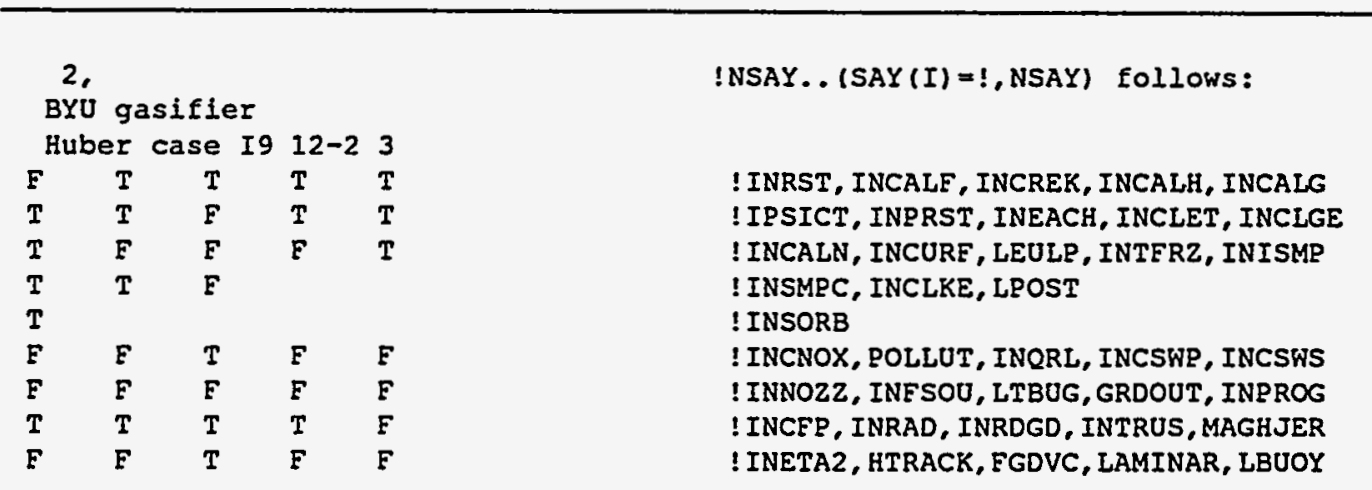




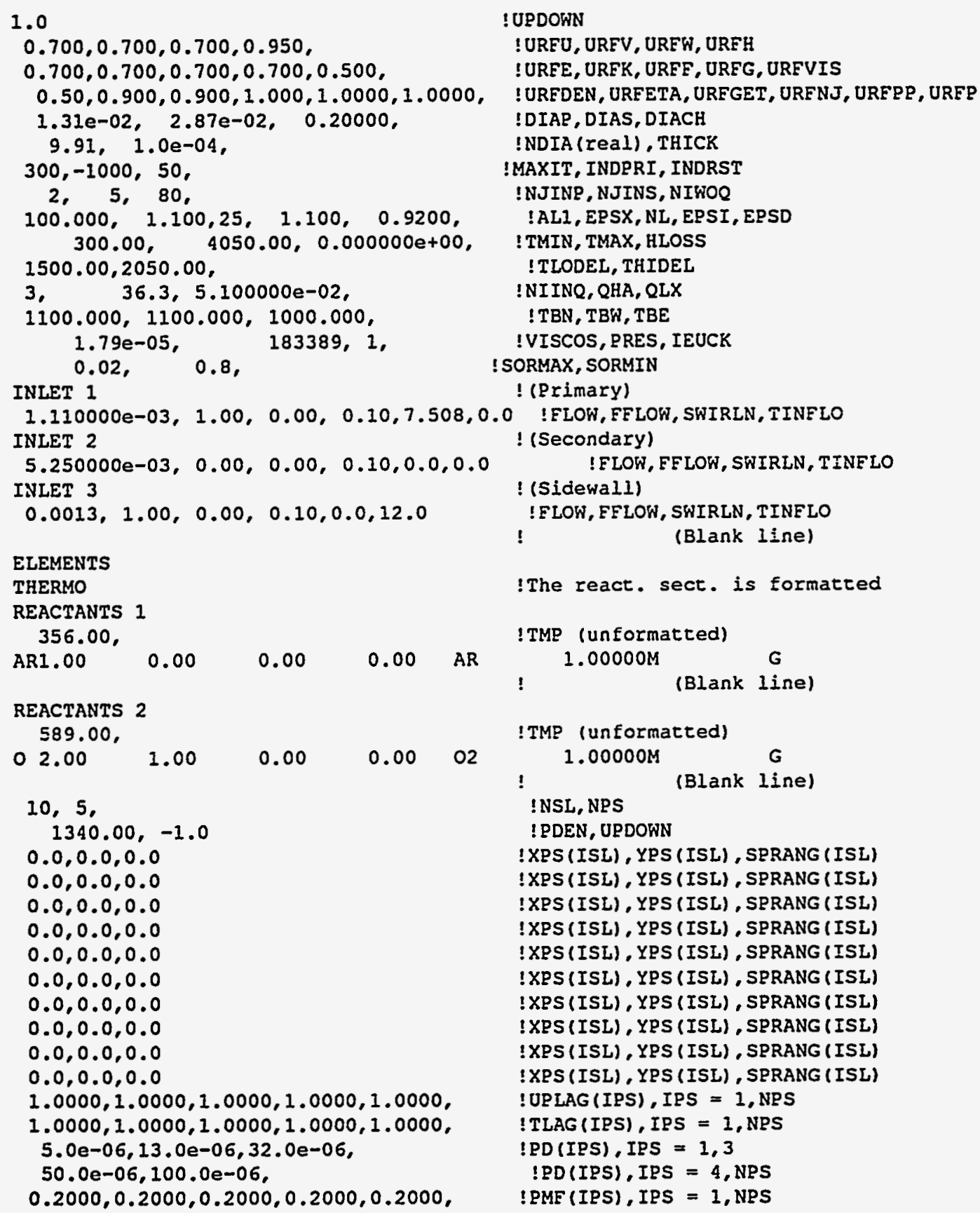




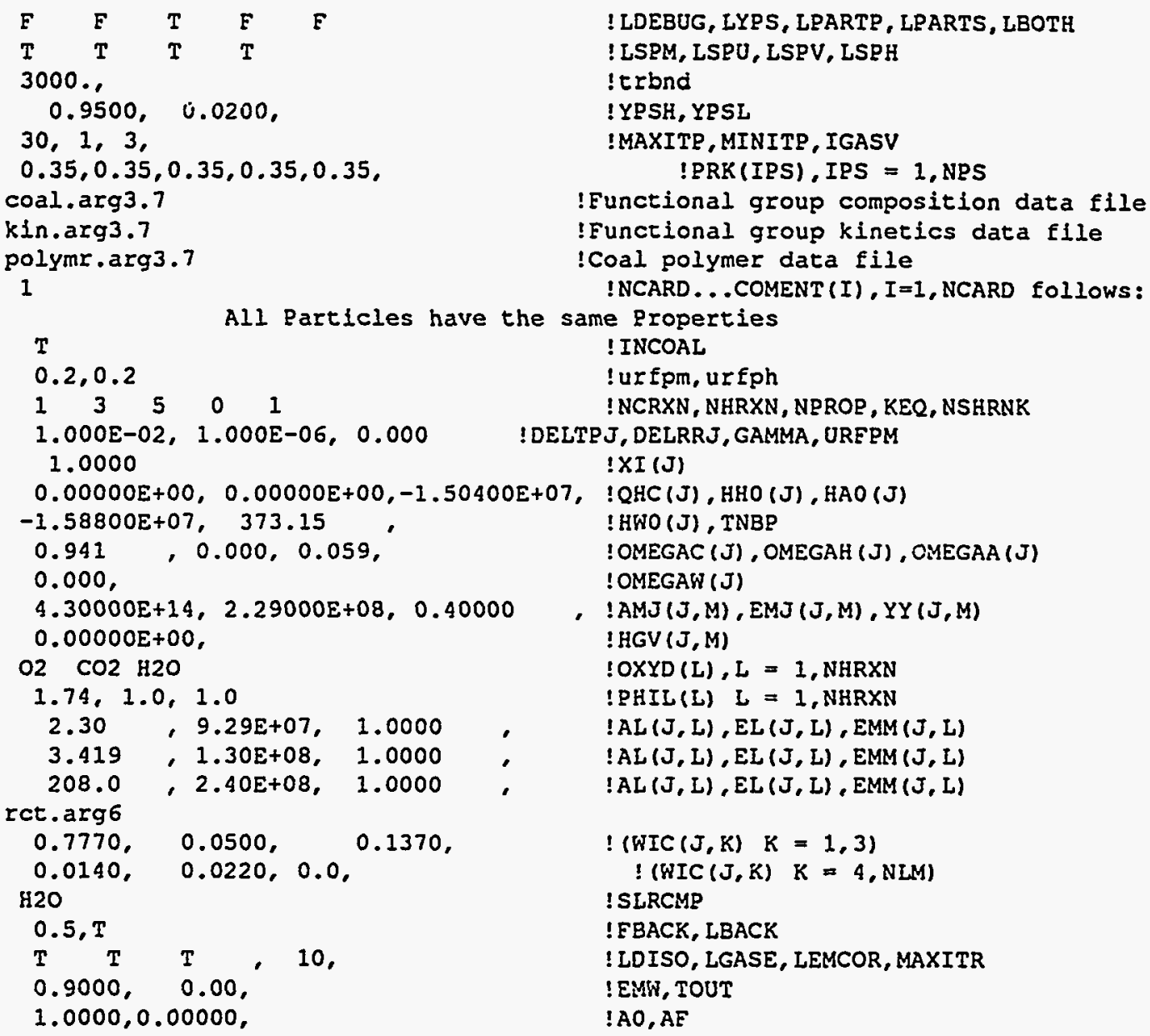

\section{Inlet Profile Data File (ill.inl, INLET)}

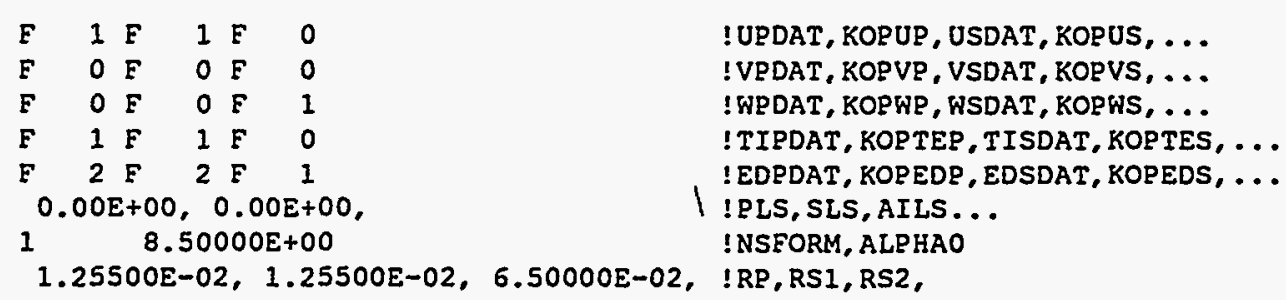


Sample Computer Files for Coal Gasification with Sidewall Sorbent Injection and Sulfur Capture

\section{Thermo Data File (ill.thm, THERMO)}

$\begin{array}{llr}\mathrm{C} & 12.01115 & 4.0 \\ \mathrm{H} & 1.00797 & 1.0 \\ \mathrm{O} & 15.9994 & -2.0 \\ \mathrm{~N} & 14.0067 & 0.0 \\ \mathrm{~S} & 32.06 & 4.0 \\ \text { AR } & 39.94800 & 0.0\end{array}$

\begin{tabular}{|c|c|c|c|c|}
\hline $\begin{array}{l}\text { AR } \\
1\end{array}$ & L 5/66AR 1.00 & $0.00 \quad 0.00 \quad 0.6$ & 300.000 & \\
\hline $2^{0.25000000 E 01}$ & 0.00000000 & 0.00000000 & 0.00000000 & 0.00000000 \\
\hline$-0.74537502 E \quad 03$ & $0.43660006 \mathrm{E} \mathrm{OI}$ & $0.25000000 \mathrm{E}$ OI & 0.00000000 & 0.00000000 \\
\hline
\end{tabular}

3
0.00000000
$0.00000000-0.74537498 E 03 \quad 0.43660006 \mathrm{E} 01$

$\mathrm{CO} 2$

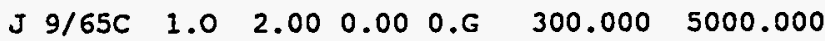

$0.44608040 E$ E $010.30981717 E-02-0.12392566 E-05 \quad 0.22741323 E-09-0.15525948 E-13$

$-0.48961438 \mathrm{E} \quad 05-0.98635978 \mathrm{E} \quad 00 \quad 0.24007788 \mathrm{E}$ 01 $0.87350905 \mathrm{E}-02-0.66070861 \mathrm{E}-05$

$0.20021860 \mathrm{E}-08 \quad 0.63274039 \mathrm{E}-15-0.48377520 \mathrm{E} \quad 05 \quad 0.96951447 \mathrm{E} \quad 01$ 120

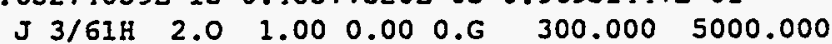

$0.27167616 \mathrm{E} \quad 01 \quad 0.29451370 \mathrm{E}-02-0.80224368 \mathrm{E}-06 \quad 0.10226681 \mathrm{E}-09-0.48472104 \mathrm{E}-14$

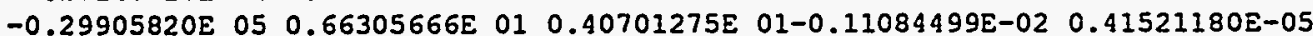
$-0.29637404 \mathrm{E}-08 \quad 0.80702101 \mathrm{E}-12-0.30279719 \mathrm{E} \quad 05-0.32270038 \mathrm{E} 00$ N2

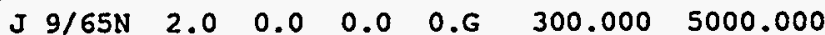

$0.28963194 \mathrm{E} \quad 01 \quad 0.15154863 \mathrm{E}-02-0.57235275 \mathrm{E}-06 \quad 0.99807385 \mathrm{E}-10-0.65223536 \mathrm{E}-14$ $\begin{array}{lllllll}-0.90586182 E & 03 & 0.61615143 E & 01 & 0.36748257 E & 01-0.12081496 E-02 & 0.23240100 E-05\end{array}$ $-0.63217520 E-09-0.22577253 E-12-0.10611587 E$ O4 $0.23580418 E \quad 01$ 02

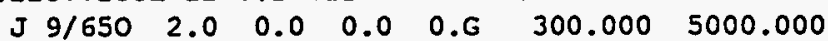

$0.36219521 \mathrm{E} 01 \quad 0.73618256 \mathrm{E}-03-0.19652219 \mathrm{E}-06 \quad 0.36201556 \mathrm{E}-10-0.28945623 \mathrm{E}-14$

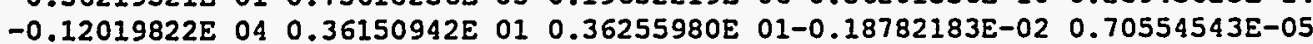
$-0.67635071 E-08 \quad 0.21555977 E-11-0.10475225 E \quad 04 \quad 0.43052769 \mathrm{E} \quad 01$ co

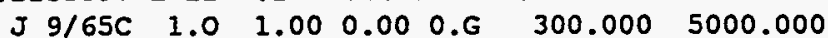

$0.29840689 \mathrm{E}$ 01 $0.14891387 \mathrm{E}-02-0.57899678 \mathrm{E}-06 \quad 0.10364576 \mathrm{E}-09-0.69353499 \mathrm{E}-14$ $-0.14245227 \mathrm{E}$ o5 $0.63479147 \mathrm{E}$ 01 $0.37100916 \mathrm{E} \quad 01-0.16190964 \mathrm{E}-02 \quad 0.36923584 \mathrm{E}-05$ $-0.20319673 \mathrm{E}-08 \quad 0.23953344 \mathrm{E}-12-0.14356309 \mathrm{E}$ 05 $0.29555340 \mathrm{E} \quad 01$ H2 $\begin{array}{llllllll}\mathrm{J} & 3 / 61 \mathrm{H} & 2.0 & 0.0 & 0.0 & 0 . \mathrm{G} & 300.000 & 5000.000\end{array}$

$0.31001883 \mathrm{E}$ o1 $0.51119458 \mathrm{E}-03 \quad 0.52644204 \mathrm{E}-07-0.34909964 \mathrm{E}-10 \quad 0.36945341 \mathrm{E}-14$ $-0.87738013 E \quad 03-0.19629412 E \quad 01 \quad 0.30574446 E$ O1 $0.26765198 E-02-0.58099149 E-05$ $0.55210343 \mathrm{E}-08-0.18122726 \mathrm{E}-11-0.98890430 \mathrm{E}$ 03-0.22997046E 01

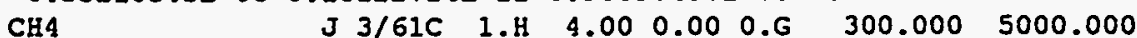

$0.15027056 \mathrm{E}$ 01 $0.10416795 \mathrm{E}-01-0.39181514 \mathrm{E}-05 \quad 0.67777872 \mathrm{E}-09-0.44283706 \mathrm{E}-13$

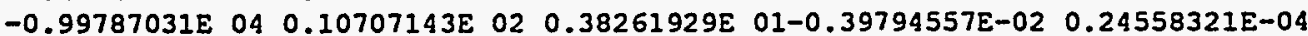
$-0.22732920 E-07 \quad 0.69626952 E-11-0.10144945 E$ OS $0.86690062 E \quad 00$

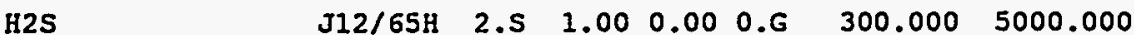


$0.28479090 E$ E $01 \quad 0.38415990 E-02-0.14099360 E-05 \quad 0.24278735 E-09-0.15783280 E-13$

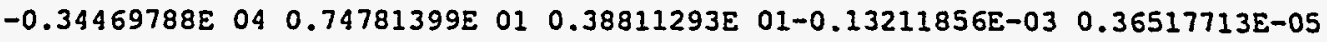

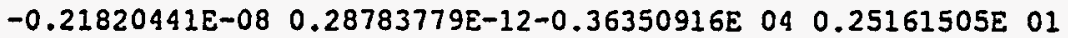
$\mathrm{SO} 2$ $\begin{array}{llllll}\mathrm{J} & 6 / 61 \mathrm{~S} \quad 1.0 & 2.00 & 0.00 & 0.6 & 300.000 \quad 5000.000\end{array}$

$0.52451363 E$ E $010.19704204 E-02-0.80375759 E-06 \quad 0.15149969 E-09-0.10557998 E-13$

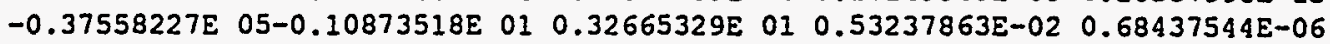
$-0.52809987 \mathrm{E}-08 \quad 0.25590450 \mathrm{E}-11-0.36908145 \mathrm{E}$ 05 $0.96513472 \mathrm{E} 01$ $C(s)$ J $3 / 61 \mathrm{C} \quad 1.0 \quad 0.0 \quad 0.0 \quad 0.5 \quad 300.000 \quad 5000.000$

$0.13604937 \mathrm{E} \quad 01 \quad 0.19182237 \mathrm{E}-02-0.84040386 \mathrm{E}-06 \quad 0.16448705 \mathrm{E}-09-0.11672670 \mathrm{E}-13$ $-0.65713843 \mathrm{E} \quad 03-0.80070200 \mathrm{E}$ 01-0.44778049E $00 \quad 0.53690970 \mathrm{E}-02-0.39775563 \mathrm{E}-06$ $-0.40459263 E-08 \quad 0.21134925 E-11-0.94280670 E \quad 02 \quad 0.16840773 E \quad 01$

AR

$3.542,93.3,0 ., 0 ., \mathrm{AR}$

$\mathrm{CO} 2$

$3.996,190 ., 0 .,-3.935 E+08, \mathrm{CO} 2$

H2O

$2.641,809.1,1.0,-2.418 E+08, \mathrm{H} 20$

N2

$3.681,91.5,0 ., 0 ., \mathrm{N} 2$

02

$3.433,113, .0 ., 0 ., 02$

CO

$3.59,110,0,0-1.105 E+08, C 0$

H2

$2.915,38 \ldots, 0,0 ., \mathrm{H} 2$

CH4

$3.758,148.6,0.0,-7.485 \mathrm{E}+07, \mathrm{CH} 4$

H2S

$3.49,343.0,0.21,-2.017 \mathrm{E}+07, \mathrm{H} 2 \mathrm{~S}$

502

$4.04,347.0,0.42,-2.969 \mathrm{E}+08, \mathrm{SO} 2$

$C(S)$

$3.822,137, .0 ., 0 ., C(5)$

\section{SO $O_{x}$ Data File (ill.sox, SOXIN)}

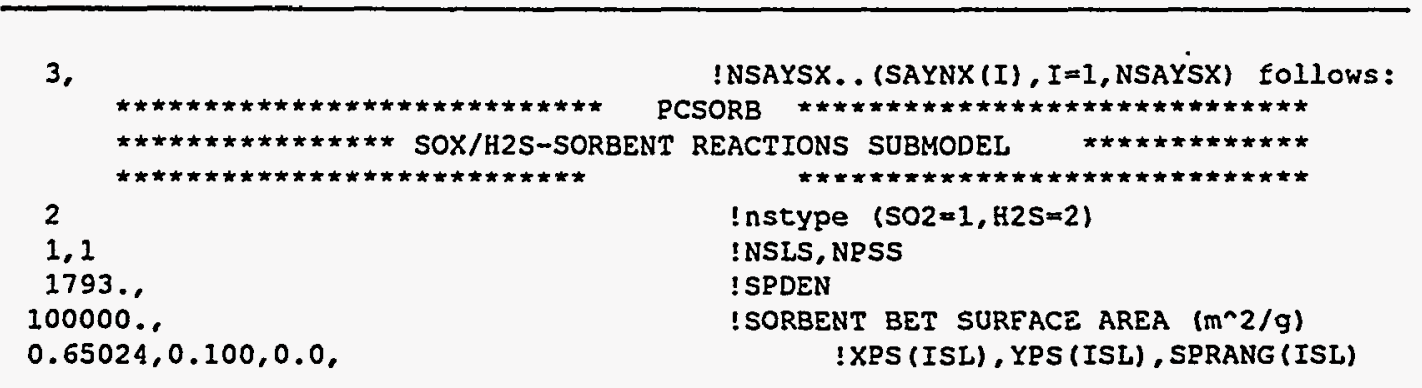



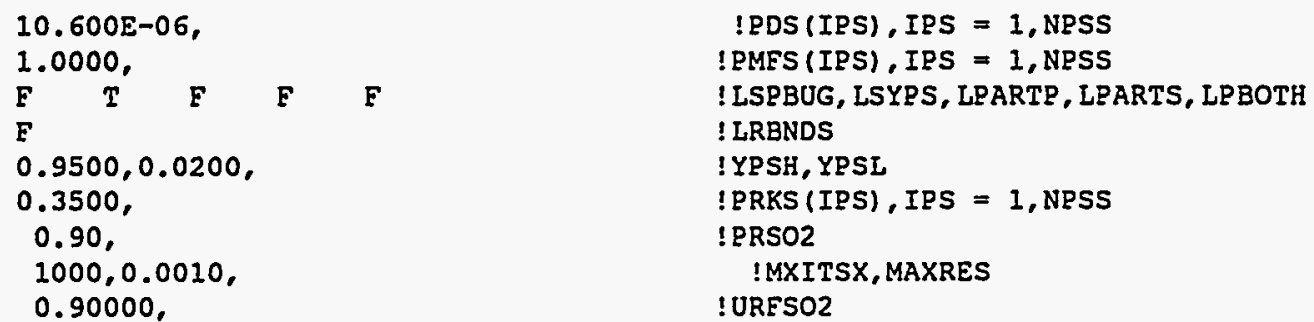

\section{Grid Data File (ill.grd, GRIDS)}

\begin{tabular}{|c|c|c|c|}
\hline \multicolumn{4}{|l|}{ NODE } \\
\hline & 100 & 37 & \\
\hline & 6 & 11 & \\
\hline & 7 & 6 & \\
\hline & 3 & 23 & \\
\hline & $\mathrm{x}$ & xu & TBN \\
\hline 1 & $-8.5000 E-03$ & $-1.7000 \mathrm{E}-02$ & 1100 . \\
\hline 2 & $8.5000 E-03$ & $0.0000 E+00$ & 1100. \\
\hline 3 & $2.5500 E-02$ & $1.7000 \mathrm{E}-02$ & 1100 . \\
\hline 4 & $4.2500 \mathrm{E}-02$ & $3.4000 E-02$ & 1100 . \\
\hline 5 & $5.9500 \mathrm{E}-02$ & $5.1000 \mathrm{E}-02$ & 1100 . \\
\hline 6 & $7.6500 \mathrm{E}-02$ & $6.8000 E-02$ & 1100 . \\
\hline 7 & $9.3500 \mathrm{E}-02$ & $8.5000 E-02$ & 1100. \\
\hline 8 & $1.1050 E-01$ & $1.0200 \mathrm{E}-01$ & 1100 . \\
\hline 9 & $1.2750 \mathrm{E}-01$ & $1.1900 E-01$ & 1100 . \\
\hline 10 & $1.4450 \mathrm{E}-01$ & $1.3600 \mathrm{E}-01$ & 1100. \\
\hline 11 & $1.6150 \mathrm{E}-01$ & $1.5300 \mathrm{E}-01$ & 1100 . \\
\hline 12 & $1.7850 E-01$ & $1.7000 \mathrm{E}-01$ & 1100 . \\
\hline 13 & $1.9550 \mathrm{E}-01$ & $1.8700 \mathrm{E}-01$ & 1100. \\
\hline 14 & $2.1250 \mathrm{E}-0 \mathrm{I}$ & $2.0400 E-01$ & 1100 . \\
\hline 15 & $2.2950 \mathrm{E}-01$ & $2.2100 E-01$ & 1100. \\
\hline 16 & $2.4650 \mathrm{E}-01$ & $2.3800 E-01$ & 1100 . \\
\hline 17 & $2.6350 \mathrm{E}-01$ & $2.5500 \mathrm{E}-01$ & 1100 . \\
\hline 18 & $2.8050 \mathrm{E}-01$ & $2.7200 \mathrm{E}-01$ & 1100 . \\
\hline 19 & $2.9750 \mathrm{E}-01$ & $2.8900 \mathrm{E}-01$ & 1100 . \\
\hline 20 & $3.1450 E-01$ & $3.0600 \mathrm{E}-01$ & 1100 . \\
\hline 21 & $3.3150 E-01$ & $3.2300 \mathrm{E}-01$ & 1100 . \\
\hline 22 & $3.4850 E-0 I$ & $3.4000 \mathrm{E}-01$ & 1100 . \\
\hline 23 & $3.6550 E-01$ & $3.5700 \mathrm{E}-01$ & 1100 . \\
\hline 24 & $3.8250 E-01$ & $3.7400 \mathrm{E}-01$ & 1100 . \\
\hline 25 & $3.9950 \mathrm{E}-01$ & $3.9100 \mathrm{E}-01$ & 1100 . \\
\hline 26 & $4.1650 \mathrm{E}-01$ & $4.0800 \mathrm{E}-01$ & 1100 . \\
\hline 27 & $4.3350 E-01$ & $4.2500 \mathrm{E}-01$ & 1100. \\
\hline 28 & $4.5050 \mathrm{E}-01$ & $4.4200 \mathrm{E}-01$ & 1100 . \\
\hline
\end{tabular}




\begin{tabular}{|c|c|c|c|}
\hline 29 & $4.6750 \mathrm{E}-01$ & $4.5900 E-01$ & 1200. \\
\hline 30 & $4.8450 E-01$ & $4.7600 \mathrm{E}-01$ & 1100. \\
\hline 31 & $5.0150 E-01$ & $4.9300 \mathrm{E}-01$ & 1100. \\
\hline 32 & $5.1680 \mathrm{E}-01$ & $5.0915 \mathrm{E}-0 \mathrm{i}$ & 1100. \\
\hline 33 & $5.3057 E-01$ & $5.2368 E-01$ & 1100. \\
\hline 34 & $5.4296 \mathrm{E}-01$ & $5.3677 E-01$ & 1100 . \\
\hline 35 & $5.5412 \mathrm{E}-01$ & $5.4854 \mathrm{E}-01$ & 1100. \\
\hline 36 & $5.6416 E-01$ & $5.5914 \mathrm{E}-01$ & 1100. \\
\hline 37 & $5.7319 E-01$ & $5.6867 E-01$ & 1100. \\
\hline 38 & $5.8132 \mathrm{E}-01$ & $5.7726 \mathrm{E}-01$ & 1100. \\
\hline 39 & $5.8864 \mathrm{E}-01$ & $5.8498 \mathrm{E}-01$ & 1100. \\
\hline 40 & $5.9522 \mathrm{E}-01$ & $5.9193 E-01$ & 1100. \\
\hline 41 & $6.0115 E-01$ & $5.9819 E-01$ & 1100. \\
\hline 42 & $6.0649 E-01$ & $6.0382 E-01$ & 1100. \\
\hline 43 & $6.1129 \mathrm{E}-01$ & $6.0889 \mathrm{E}-01$ & 1100. \\
\hline 44 & $6.1561 \mathrm{E}-01$ & $6.1345 \mathrm{E}-01$ & 1100. \\
\hline 45 & $6.1950 \mathrm{E}-01$ & $6.1755 E-01$ & 1100. \\
\hline 46 & $6.2300 \mathrm{E}-01$ & $6.2125 E-01$ & 1100. \\
\hline 47 & $6.2615 E-01$ & $6.2457 E-01$ & 1100. \\
\hline 48 & $6.2898 E-01$ & $6.2757 \mathrm{E}-01$ & 1100. \\
\hline 49 & $6.3154 E-01$ & $6.3026 \mathrm{E}-01$ & 1100. \\
\hline 50 & $6.3383 E-01$ & $6.3268 \mathrm{E}-01$ & 1100. \\
\hline 51 & $6.3590 E-01$ & $6.3487 E-01$ & 1100. \\
\hline 52 & $6.3776 E-01$ & $6.3683 E-01$ & 1100. \\
\hline 53 & $6.3943 E-01$ & $6.3860 E-01$ & 1100. \\
\hline 54 & $6.4094 E-01$ & $6.4019 E-01$ & 1100. \\
\hline 55 & $6.4230 \mathrm{E}-01$ & $6.4162 E-01$ & 1100. \\
\hline 56. & $6.4352 \mathrm{E}-01$ & $6.4291 E-01$ & 1100 . \\
\hline $57^{\circ}$ & $6.4461 E-01$ & $6.4407 E-01$ & 1100 . \\
\hline 58 & $6.4560 E-01$ & $6.4511 E-01$ & 1100. \\
\hline 59 & $6.4649 \mathrm{E}-01$ & $6.4605 E-01$ & 1100. \\
\hline 60 & $6.4729 \mathrm{E}-01$ & $6.4689 \mathrm{E}-01$ & 1100. \\
\hline 61 & $6.4801 E-01$ & $6.4765 E-01$ & 1100 . \\
\hline 62 & $6.4866 \mathrm{E}-01$ & $6.4834 \mathrm{E}-01$ & 1100. \\
\hline 63 & $6.4925 \mathrm{E}-01$ & $6.4895 E-01$ & 1100. \\
\hline 64 & $6.4977 \mathrm{E}-01$ & $6.4951 \mathrm{E}-01$ & 1100. \\
\hline 65 & $6.5024 E-01$ & $6.5001 E-01$ & 1100 . \\
\hline 66 & $6.5067 E-01$ & $6.5046 \mathrm{E}-01$ & 1100 . \\
\hline 67 & $6.5105 \mathrm{E}-01$ & $6.5086 \mathrm{E}-01$ & 1100. \\
\hline 68 & $6.5152 \mathrm{E}-01$ & $6.5129 \mathrm{E}-01$ & 1100. \\
\hline 69 & $6.5209 \mathrm{E}-01$ & $6.5181 \mathrm{E}-01$ & 1100 . \\
\hline 70 & $6.5279 \mathrm{E}-01$ & $6.5244 E-01$ & 1100 . \\
\hline 71 & $6.5363 E-01$ & $6.5321 \varepsilon-01$ & 1100 . \\
\hline 72 & $6.5467 E-01$ & $6.5415 \mathrm{E}-01$ & 1100. \\
\hline 73 & $6.5593 E-01$ & $6.5530 \mathrm{E}-01$ & 1100. \\
\hline 74 & $6.5747 E-01$ & $6.5670 \mathrm{E}-01$ & 1100. \\
\hline 75 & $6.5935 E-01$ & $6.5841 \mathrm{E}-01$ & 1100. \\
\hline 76 & $6.6165 E-01$ & $6.6050 E-01$ & 1100. \\
\hline 77 & $6.6444 E-01$ & $6.6304 E-01$ & 1100. \\
\hline
\end{tabular}


Sample Computer Files for Coal Gasification with Sidewall Sorbent Injection and Sulfur Capture

\begin{tabular}{|c|c|c|c|}
\hline 78 & $6.6786 \mathrm{E}-01$ & $6.6615 \mathrm{E}-01$ & 1100. \\
\hline 79 & $6.7202 \mathrm{E}-01$ & $6.6994 \mathrm{E}-01$ & 1100. \\
\hline 80 & $6.7710 E-01$ & $6.7456 \mathrm{E}-01$ & 1100. \\
\hline 81 & $6.8330 E-01$ & $6.8020 \mathrm{E}-01$ & 1100 . \\
\hline 82 & $6.9086 \mathrm{E}-01$ & $6.8708 \mathrm{E}-01$ & 1100. \\
\hline 83 & $7.0008 \mathrm{E}-01$ & $6.9547 \mathrm{E}-01$ & 1100 . \\
\hline 84 & $7.1134 \mathrm{E}-01$ & $7.0571 \mathrm{E}-01$ & 1100 . \\
\hline 85 & $7.2507 \mathrm{E}-01$ & $7.1820 \mathrm{E}-01$ & 1100. \\
\hline 86 & $7.4182 \mathrm{E}-01$ & $7.3344 \mathrm{E}-01$ & 1100 . \\
\hline 87 & $7.6225 \mathrm{E}-01$ & $7.5203 \mathrm{E}-01$ & 1100. \\
\hline 88 & $7.8718 E-01$ & $7.7472 \mathrm{E}-01$ & 1100. \\
\hline 89 & $8.1760 E-01$ & $8.0239 \mathrm{E}-01$ & 1100. \\
\hline 90 & 8.5470E-01 & $8.3615 \mathrm{E}-01$ & 1100 . \\
\hline 91 & $8.9997 \mathrm{E}-01$ & $8.7734 \mathrm{E}-01$ & 1100. \\
\hline 92 & $9.5520 \mathrm{E}-01$ & $9.2759 \mathrm{E}-01$ & 1100 . \\
\hline 93 & $1.0226 \mathrm{E}+00$ & $9.8890 \mathrm{E}-01$ & 1100. \\
\hline 94 & $1.1048 \mathrm{E}+00$ & $1.0637 \mathrm{E}+00$ & 1100 . \\
\hline 95 & $1.2051 E+00$ & $1.1549 \mathrm{E}+00$ & 1100. \\
\hline 96 & $1.3274 \mathrm{E}+00$ & $1.2663 E+00$ & 1100. \\
\hline 97 & $1.4767 \mathrm{E}+00$ & $1.4021 E+00$ & 1100 . \\
\hline 98 & $1.6588 \mathrm{E}+00$ & $1.5678 \mathrm{E}+00$ & 1100. \\
\hline 99 & $1.8810 \mathrm{E}+00$ & $1.7699 E+00$ & 1100. \\
\hline 100 & $2.1520 E+00$ & $2.0165 E+00$ & 1100 . \\
\hline & $Y$ & YV & \\
\hline 1 & $-5.7790 \mathrm{E}-04$ & $-1.1558 E-03$ & \\
\hline 2 & $5.7790 \mathrm{E}-04$ & $0.0000 \mathrm{E}+00$ & \\
\hline 3 & $1.7339 \mathrm{E}-03$ & $1.1559 \varepsilon-03$ & \\
\hline 4 & $2.9798 E-03$ & $2.35698-03$ & \\
\hline 5 & $4.3226 E-03$ & $3.6512 \mathrm{E}-03$ & \\
\hline 6 & $5.7700 E-03$ & $5.0463 E-03$ & \\
\hline 7 & $7.3300 \mathrm{E}-03$ & $6.5500 \mathrm{E}-03$ & \\
\hline 8 & $8.8900 \mathrm{E}-03$ & $8.1100 E-03$ & \\
\hline 9 & $1.0450 E-02$ & $9.6700 \mathrm{E}-03$ & \\
\hline 10 & $1.2010 \mathrm{E}-02$ & $1.1230 \mathrm{E}-02$ & \\
\hline 11 & $1.3570 E-02$ & $1.2790 \mathrm{E}-02$ & \\
\hline 12 & $1.5130 \mathrm{E}-02$ & $1.4350 \mathrm{E}-02$ & \\
\hline 13 & $1.6846 \mathrm{E}-02$ & $1.5988 E-02$ & \\
\hline 14 & $1.8734 \mathrm{E}-02$ & $1.77 \dot{90 E}-02$ & \\
\hline 15 & $2.0810 E-02$ & $1.9772 E-02$ & \\
\hline 16 & $2.3094 E-02$ & $2.1952 \mathrm{E}-02$ & \\
\hline 17 & $2.5606 E-02$ & $2.4350 \varepsilon-02$ & \\
\hline 18 & $2.8370 \mathrm{E}-02$ & $2.6988 \mathrm{E}-02$ & \\
\hline 19 & $3.1410 \mathrm{E}-02$ & $2.9890 \mathrm{E}-02$ & \\
\hline 20 & $3.4754 E-02$ & $3.3082 \mathrm{E}-02$ & \\
\hline 21 & $3.8432 E-02$ & 3. $6593 \mathrm{E}-02$ & \\
\hline 22 & $4.2479 \mathrm{E}-02$ & $4.0455 E-02$ & \\
\hline 23 & 4. $6929 \mathrm{E}-02$ & $4.4704 \mathrm{E}-02$ & \\
\hline 24 & $5.1825 E-02$ & $4.9377 E-02$ & \\
\hline 25 & $5.7211 E-02$ & $5.4518 \mathrm{E}-02$ & \\
\hline
\end{tabular}


Grid Data File (ill.grd, GRIDS)

$\begin{array}{lll}26 & 6.2597 \mathrm{E}-02 & 5.9904 \mathrm{E}-02 \\ 27 & 6.7551 \mathrm{E}-02 & 6.5074 \mathrm{E}-02 \\ 28 & 7.2110 \mathrm{E}-02 & 6.9830 \mathrm{E}-02 \\ 29 & 7.6303 \mathrm{E}-02 & 7.4207 \mathrm{E}-02 \\ 30 & 8.0161 \mathrm{E}-02 & 7.8232 \mathrm{E}-02 \\ 31 & 8.3711 \mathrm{E}-02 & 8.1936 \mathrm{E}-02 \\ 32 & 8.6976 \mathrm{E}-02 & 8.5343 \mathrm{E}-02 \\ 33 & 8.9981 \mathrm{E}-02 & 8.8478 \mathrm{E}-02 \\ 34 & 9.2745 \mathrm{E}-02 & 9.1363 \mathrm{E}-02 \\ 35 & 9.5288 \mathrm{E}-02 & 9.4016 \mathrm{E}-02 \\ 36 & 9.7627 \mathrm{E}-02 & 9.6457 \mathrm{E}-02 \\ 37 & 1.0237 \mathrm{E}-01 & 9.9999 \mathrm{E}-02\end{array}$

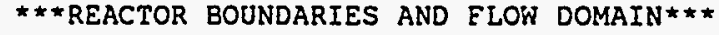

\begin{tabular}{|c|c|c|c|c|c|c|c|c|c|c|c|c|c|c|c|c|c|c|c|c|c|c|}
\hline & 1 & 2 & 3 & 4 & J & $\ldots 60$ & 61 & 62 & 63 & 64 & 65 & 66 & 67 & 68 & 69 & 70 & ...95 & 96 & 97 & 98 & 99 & 100 \\
\hline 37 & $x$ & $x$ & $x$ & $x$ & $x$ & $\ldots x$ & $x$ & $x$ & $x$ & 1 & $x$ & $x$ & $x$ & $x$ & $x$ & $x$ & $\ldots x$ & $x$ & $x$ & $x$ & $x$ & $x$ \\
\hline 36 & $x$ & 0 & 0 & 0 & 0 & $\ldots 0$ & 0 & 0 & 0 & 0 & 0 & 0 & 0 & 0 & 0 & 0 & $\ldots 0$ & 0 & 0 & 0 & 0 & 0 \\
\hline 35 & $x$ & 0 & 0 & 0 & 0 & $\ldots 0$ & 0 & 0 & 0 & 0 & 0 & 0 & 0 & 0 & 0 & 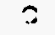 & $\ldots=$ & 2 & 0 & 0 & 0 & 0 \\
\hline 34 & $x$ & 0 & 0 & 0 & 0 & $\ldots 0$ & 0 & 0 & 0 & 0 & 0 & 0 & 0 & 0 & 0 & $=$ & $\ldots 0$ & 0 & 0 & 0 & 0 & 0 \\
\hline 33 & $x$ & 0 & 0 & 0 & 0 & ... 0 & 0 & 0 & 0 & 0 & 0 & 0 & 0 & C & 0 & $\therefore$ & $\ldots=$ & 3 & 0 & 0 & 0 & 0 \\
\hline 32 & $x$ & 0 & 0 & 0 & 0 & $\ldots 0$ & 0 & 0 & 0 & 0 & 0 & 0 & 0 & 0 & 0 & 0 & $\ldots 0$ & 0 & 0 & 0 & 0 & 0 \\
\hline 31 & $x$ & 0 & 0 & 0 & 0 & $\ldots 0$ & 0 & 0 & 0 & 0 & 0 & 0 & 0 & 0 & 0 & 0 & $\ldots 0$ & 0 & 0 & 0 & 0 & 0 \\
\hline 30 & $x$ & 0 & 0 & 0 & 0 & $\ldots o$ & 0 & 0 & 0 & 0 & 0 & 0 & 0 & 0 & 0 & 0 & $\ldots 0$ & 0 & 0 & 0 & 0 & 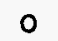 \\
\hline 29 & $x$ & 0 & 0 & 0 & 0 & ... 0 & 0 & 0 & 0 & 0 & 0 & 0 & 0 & 0 & 0 & 0 & ...o & 0 & 0 & 0 & 0 & 0 \\
\hline 28 & $x$ & 0 & 0 & 0 & 0 & $\ldots 0$ & 0 & 0 & 0 & 0 & 0 & 0 & 0 & 0 & 0 & 0 & $\ldots 0$ & 0 & 0 & 0 & 0 & 0 \\
\hline 27 & $x$ & 0 & 0 & 0 & 0 & $\ldots o$ & 0 & 0 & 0 & 0 & 0 & 0 & 0 & 0 & 0 & 0 & $\ldots 0$ & 0 & 0 & 0 & 0 & 0 \\
\hline 26 & $x$ & 0 & 0 & 0 & 0 & $\ldots 0$ & 0 & 0 & 0 & 0 & 0 & 0 & 0 & 0 & 0 & 0 & $\ldots 0$ & 0 & 0 & 0 & 0 & 0 \\
\hline 25 & $x$ & 0 & 0 & 0 & 0 & ... & 0 & 0 & 0 & 0 & 0 & 0 & 0 & 0 & 0 & 0 & $\ldots 0$ & 0 & 0 & 0 & 0 & 0 \\
\hline 24 & $x$ & 0 & 0 & 0 & 0 & ... & 0 & 0 & 0 & 0 & 0 & 0 & 0 & 0 & 0 & 0 & $\ldots 0$ & 0 & 0 & 0 & 0 & 0 \\
\hline 23 & $x$ & 0 & 0 & 0 & 0 & ... & 0 & 0 & 0 & 0 & 0 & 0 & 0 & 0 & 0 & 0 & $\ldots 0$ & 0 & 0 & 0 & 0 & 0 \\
\hline 22 & $x$ & 0 & 0 & 0 & 0 & 0 & 0 & 0 & 0 & 0 & 0 & 0 & 0 & 0 & 0 & 0 & $\ldots 0$ & 0 & 0 & 0 & 0 & 0 \\
\hline 21 & $x$ & 0 & 0 & 0 & 0 & $\ldots$ & 0 & 0 & 0 & 0 & 0 & 0 & 0 & 0 & 0 & 0 & $\ldots 0$ & 0 & 0 & 0 & 0 & 0 \\
\hline 20 & $x$ & 0 & 0 & 0 & 0 & $\ldots 0$ & 0 & 0 & 0 & 0 & 0 & 0 & 0 & 0 & 0 & 0 & $\ldots 0$ & 0 & 0 & 0 & 0 & 0 \\
\hline 19 & $x$ & 0 & 0 & 0 & 0 & ... 0 & 0 & 0 & 0 & 0 & 0 & 0 & 0 & 0 & 0 & 0 & $\ldots 0$ & 0 & 0 & 0 & 0 & 0 \\
\hline 18 & $x$ & 0 & 0 & 0 & 0 & $\ldots o$ & 0 & 0 & 0 & 0 & 0 & 0 & 0 & 0 & 0 & 0 & $\ldots 0$ & 0 & 0 & 0 & 0 & 0 \\
\hline 17 & $x$ & 0 & 0 & 0 & 0 & $\cdots$ & 0 & 0 & 0 & 0 & 0 & 0 & 0 & 0 & 0 & 0 & $\ldots 0$ & 0 & 0 & 0 & 0 & 0 \\
\hline 16 & $x$ & 0 & 0 & 0 & 0 & $\ldots$ & 0 & 0 & 0 & 0 & 0 & 0 & 0 & 0 & 0 & 0 & $\ldots 0$ & 0 & 0 & 0 & 0 & 0 \\
\hline 15 & $x$ & 0 & 0 & 0 & 0 & $\ldots 0$ & 0 & 0 & 0 & 0 & 0 & 0 & 0 & 0 & 0 & 0 & $\ldots \circ$ & 0 & 0 & 0 & 0 & 0 \\
\hline 14 & $x$ & 0 & 0 & 0 & 0 & 0 & 0 & 0 & 0 & 0 & 0 & 0 & 0 & 0 & 0 & 0 & $\ldots 0$ & 0 & 0 & 0 & 0 & 0 \\
\hline 13 & $x$ & 0 & 0 & 0 & 0 & 0 & 0 & 0 & 0 & 0 & 0 & 0 & 0 & 0 & 0 & 0 & $\ldots 0$ & 0 & 0 & 0 & 0 & 0 \\
\hline 12 & $x$ & 0 & 0 & 0 & 0 & $\ldots o$ & 0 & 0 & 0 & 0 & 0 & 0 & 0 & 0 & 0 & 0 & ... & 0 & 0 & 0 & 0 & 0 \\
\hline 11 & $\mathbf{s}$ & 0 & 0 & 0 & 0 & $\ldots o$ & 0 & 0 & 0 & 0 & 0 & 0 & 0 & 0 & 0 & 0 & $\ldots 0$ & 0 & 0 & 0 & 0 & 0 \\
\hline 10 & $\mathbf{s}$ & 0 & 0 & 0 & 0 & $\ldots o$ & 0 & 0 & 0 & 0 & 0 & 0 & 0 & 0 & 0 & 0 & $\ldots 0$ & 0 & 0 & 0 & 0 & 0 \\
\hline 9 & $s$ & 0 & 0 & 0 & 0 & $\ldots o$ & 0 & 0 & 0 & 0 & 0 & 0 & 0 & 0 & 0 & 0 & $\ldots 0$ & 0 & 0 & 0 & 0 & 0 \\
\hline 8 & $\mathbf{s}$ & 0 & 0 & 0 & 0 & 0 & 0 & 0 & 0 & 0 & 0 & 0 & 0 & 0 & 0 & 0 & $\ldots 0$ & 0 & 0 & 0 & 0 & 0 \\
\hline 7 & $\mathbf{S}$ & 0 & 0 & 0 & 0 & & 0 & 0 & 0 & 0 & 0 & 0 & 0 & 0 & 0 & 0 & $\ldots 0$ & 0 & 0 & 0 & 0 & 0 \\
\hline 6 & P & 0 & 0 & 0 & 0 & & 0 & 0 & 0 & 0 & 0 & 0 & 0 & 0 & 0 & 0 & $\ldots 0$ & 0 & 0 & 0 & 0 & 0 \\
\hline 5 & P & 0 & 0 & 0 & 0 & & 0 & 0 & 0 & 0 & $\rho$ & 0 & 0 & 0 & 0 & 0 & $\ldots 0$ & 0 & 0 & 0 & 0 & 0 \\
\hline 4 & $\mathbf{P}$ & 0 & 0 & 0 & 0 & & 0 & 0 & 0 & 0 & 0 & 0 & 0 & 0 & 0 & 0 & $\ldots 0$ & 0 & 0 & 0 & 0 & 0 \\
\hline 3 & P & 0 & 0 & 0 & 0 & . 0 & 0 & 0 & 0 & 0 & 0 & 0 & 0 & 0 & 0 & 0 & $\ldots \circ$ & 0 & 0 & 0 & 0 & 0 \\
\hline
\end{tabular}


Sample Computer Files for Coal Gasification with Sidewall Sorbent Injection and Sulfur Capture

2 P $10 \begin{array}{lllllllllllllllllllllll} & 0 & 0 & 0 & \ldots & 0 & 0 & 0 & 0 & 0 & 0 & 0 & 0 & 0 & 0 & 0 & \ldots & 0 & 0 & 0 & 0 & 0 & 0\end{array}$

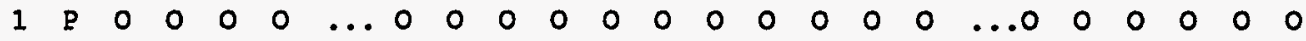

0 NOTE: THE JPRIM, JSEC, JTUBID, JTUBOD, NJINS, JQUARL

AND IQUARL ARE SUPERSEDED BY THE $X$ AND $P$ AND $S$ IN THE MAP

WHEN INTRUS $=$.TRUE. NI and NJ are not superseded. 


\section{Major FORTRAN Variables}

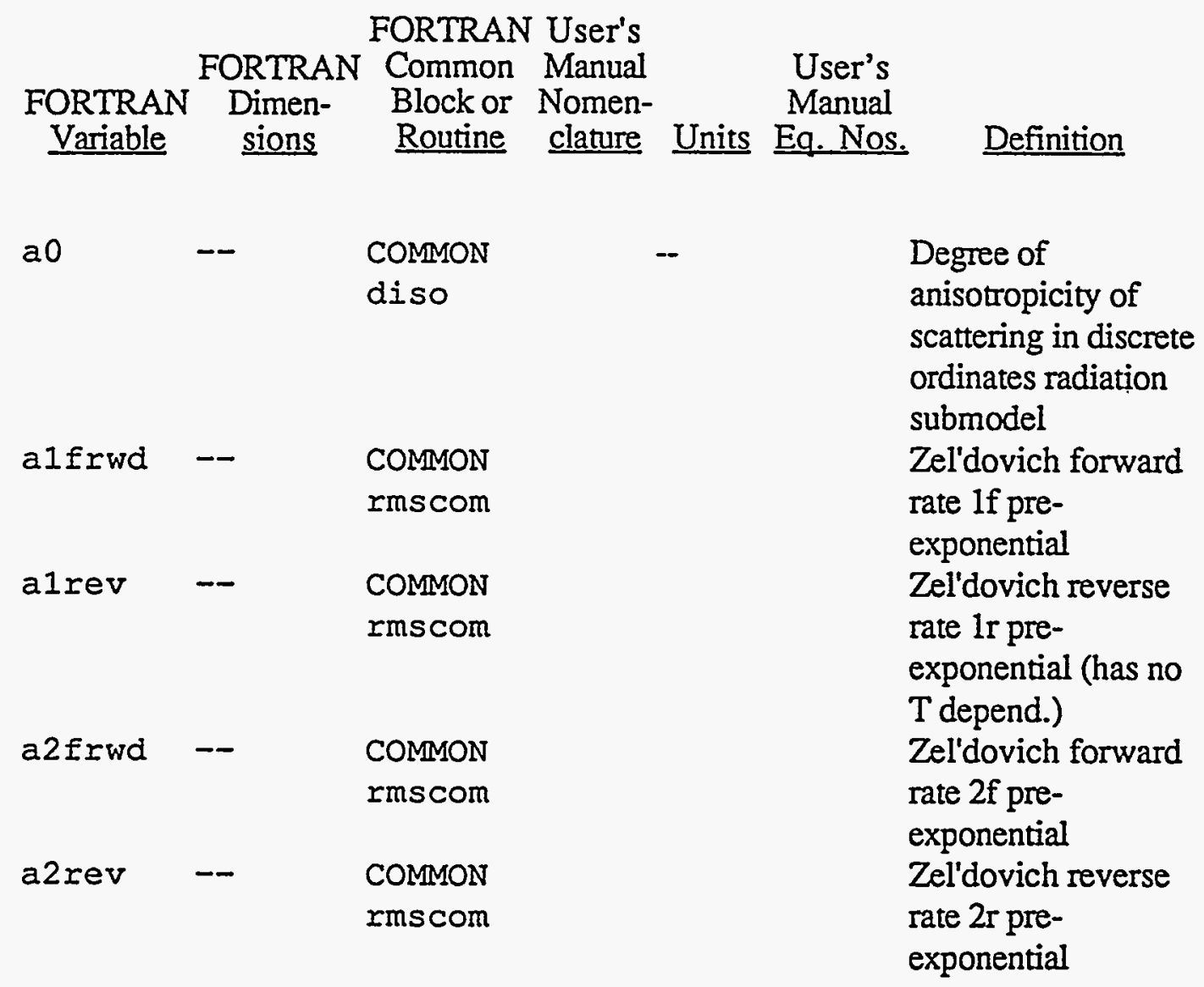




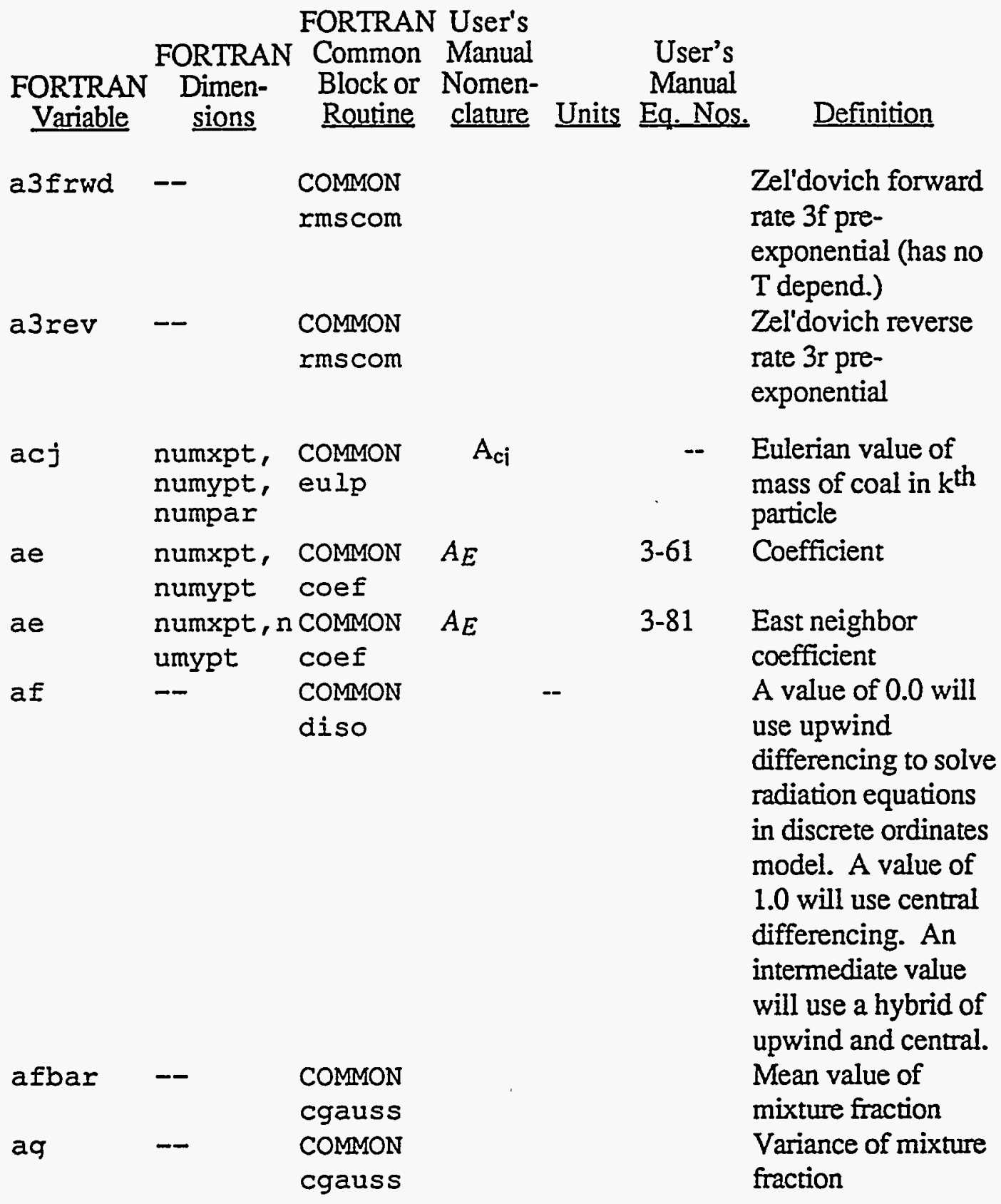




\begin{tabular}{|c|c|c|c|c|c|c|}
\hline \multicolumn{7}{|c|}{ FORTRAN User's } \\
\hline & FORTRAI & Common & Manual & & User's & \\
\hline FORTRAN & Dimen- & Block or & Nomen- & & Manual & \\
\hline Variable & sions & Routine & clature & Units & Eq. Nos. & Definition \\
\hline agsic & $\begin{array}{l}\text { numxpt, } \\
\text { numypt }\end{array}$ & $\begin{array}{l}\text { COMMON } \\
\text { krad }\end{array}$ & & & & $\begin{array}{l}\text { Absorption } \\
\text { coefficient for gases }\end{array}$ \\
\hline $\operatorname{ahj}$ & $\begin{array}{l}\text { numxpt, } \\
\text { numypt, } \\
\text { numpar }\end{array}$ & $\begin{array}{l}\text { COMMON } \\
\text { eulp }\end{array}$ & $A_{\text {hi }}$ & & -- & $\begin{array}{l}\text { Eulerian value of } \\
\text { mass of char in } k^{\text {th }} \\
\text { particle }\end{array}$ \\
\hline ahss 1 & -- & $\begin{array}{l}\text { COMMON } \\
\text { rmscom }\end{array}$ & & & & $\begin{array}{l}\text { DeSoete rate } 1 \text { pre- } \\
\text { exponential }\end{array}$ \\
\hline ahss 2 & -- & $\begin{array}{l}\text { COMMON } \\
\text { rmscom }\end{array}$ & & & & $\begin{array}{l}\text { DeSoete rate } 2 \text { pre- } \\
\text { exponential }\end{array}$ \\
\hline ahss 3 & -- & $\begin{array}{l}\text { COMMON } \\
\text { rmscom }\end{array}$ & & & & $\begin{array}{l}\text { DeScete rate } 3 \text { pre- } \\
\text { exponential }\end{array}$ \\
\hline ahss 4 & -- & $\begin{array}{l}\text { COMMON } \\
\text { rmscom }\end{array}$ & & & & $\begin{array}{l}\text { DeSoete rate } 4 \text { pre- } \\
\text { exponential }\end{array}$ \\
\hline AJ & -- & COAL2 & $A_{\mathbf{i}}$ & $\mathrm{m}^{2}$ & $2-131$ & $\begin{array}{l}\text { Particle external } \\
\text { surface area. }\end{array}$ \\
\hline al & $\begin{array}{l}\text { numele, } \\
\text { numspe }\end{array}$ & $\begin{array}{l}\text { COMMON } \\
\text { cequil }\end{array}$ & & -- & & $\begin{array}{l}\text { al }(i, j) \text { is the } \\
\text { stoichiometric } \\
\text { coefficient of the } i^{\text {th }} \\
\text { element in the } j^{\text {th }} \\
\text { species }\end{array}$ \\
\hline a.1 & -- & $\begin{array}{l}\text { COMMON } \\
\text { grid1 }\end{array}$ & & $\mathrm{m}$ & & $\begin{array}{l}\text { Axial location where } \\
\text { cell spacing begins } \\
\text { to shrink }\end{array}$ \\
\hline alfaa0 & numpar & $\begin{array}{l}\text { COMMON } \\
\text { ippar }\end{array}$ & & $\mathrm{kg}$ & & $\begin{array}{l}\text { Initial mass of ash in } \\
\text { a particle }\end{array}$ \\
\hline alfaco & numpar & $\begin{array}{l}\text { COMMON } \\
\text { ippar }\end{array}$ & & $\mathrm{kg}$ & & $\begin{array}{l}\text { Initial mass of raw } \\
\text { coal in a particle }\end{array}$ \\
\hline alfaho & numpar & $\begin{array}{l}\text { COMMON } \\
\text { ippar }\end{array}$ & & $\mathrm{kg}$ & & $\begin{array}{l}\text { Initial mass of char } \\
\text { in a particle }\end{array}$ \\
\hline
\end{tabular}




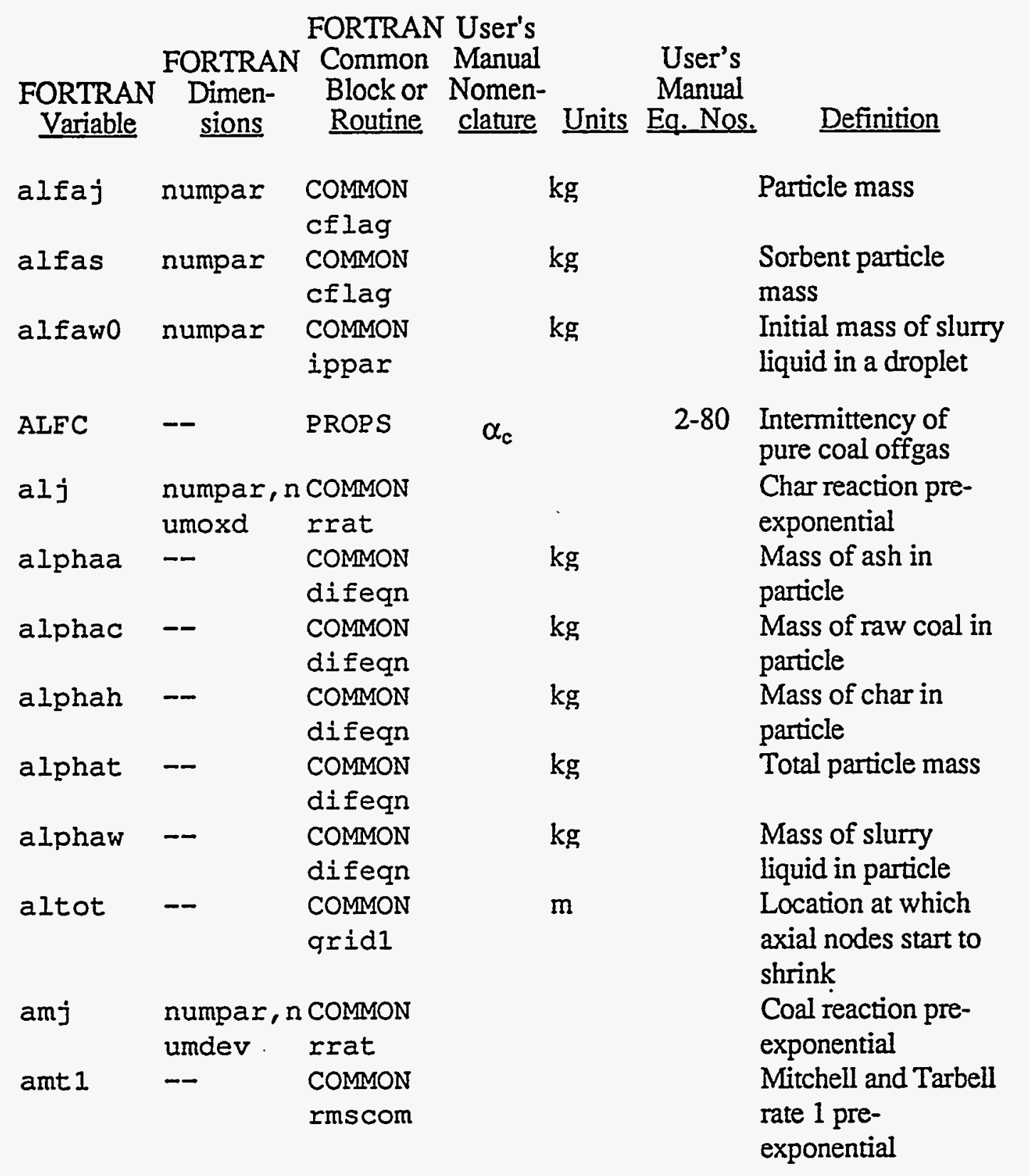




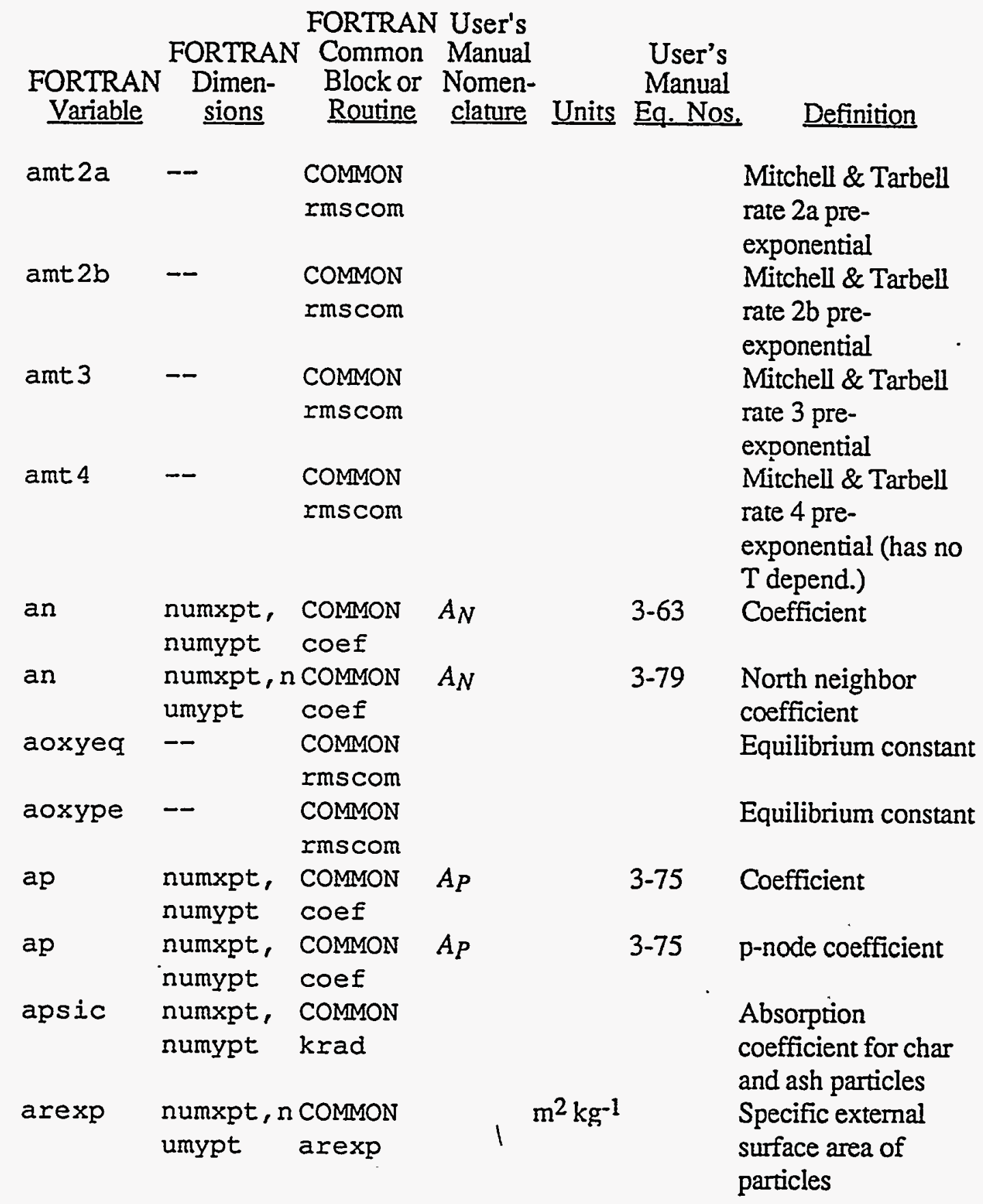




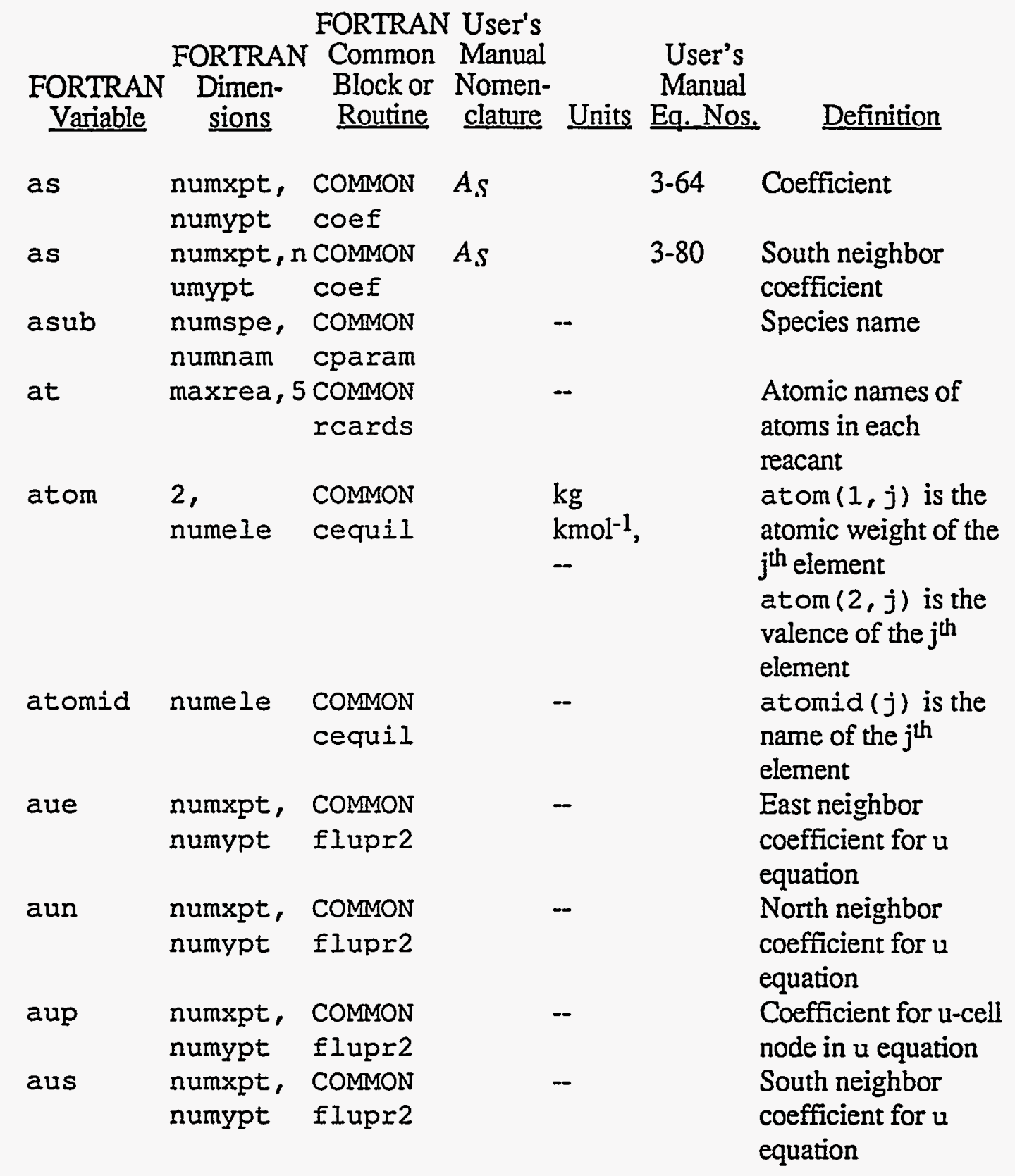




\begin{tabular}{|c|c|c|c|c|c|c|}
\hline \multirow{4}{*}{$\begin{array}{l}\text { FORTRAN } \\
\text { Variable }\end{array}$} & \multicolumn{5}{|c|}{ FORTRAN User's } & \multirow[b]{4}{*}{ Definition } \\
\hline & FORTRAN & Common & Manual & \multirow{2}{*}{\multicolumn{2}{|c|}{$\begin{array}{l}\text { User's } \\
\text { Manual }\end{array}$}} & \\
\hline & Dimen- & Block or & Nomen- & & & \\
\hline & sions & Routine & clature & Units & Eq. Nos. & \\
\hline \multirow[t]{2}{*}{ auw } & numxpt, & COMMON & & \multirow[t]{2}{*}{--} & & \multirow{2}{*}{$\begin{array}{l}\text { West neighbor } \\
\text { coefficient for } \mathrm{u} \\
\text { equation }\end{array}$} \\
\hline & numypt & flupr2 & & & & \\
\hline \multirow[t]{2}{*}{ ave } & numxpt, & COMMON & & \multirow[t]{2}{*}{--} & & \multirow{2}{*}{$\begin{array}{l}\text { East neighbor } \\
\text { coefficient in } \mathrm{v} \\
\text { equation }\end{array}$} \\
\hline & numypt & flupr2 & & & & \\
\hline \multirow[t]{2}{*}{ avgawt } & numpar & COMMON & & \multirow{2}{*}{\multicolumn{2}{|c|}{$\begin{array}{l}\mathrm{kg} \\
\mathrm{kmol}^{-1}\end{array}$}} & \multirow{2}{*}{$\begin{array}{l}\text { Average atomic } \\
\text { veight for raw coal }\end{array}$} \\
\hline & & hcap & & & & \\
\hline \multirow[t]{2}{*}{ avn } & numxpt, & COMMON & & \multirow[t]{2}{*}{--} & & \multirow{2}{*}{$\begin{array}{l}\text { North neighbor } \\
\text { coefficient in } \mathrm{v} \\
\text { equation }\end{array}$} \\
\hline & numypt & Elupr2 & & & & \\
\hline \multirow[t]{2}{*}{ avp } & numxpt, & COMMON & & \multirow[t]{2}{*}{--} & & \multirow{2}{*}{$\begin{array}{l}v \text {-cell coefficient in } \\
v \text { equation }\end{array}$} \\
\hline & numypt & flupr2 & & & & \\
\hline \multirow[t]{2}{*}{ avs } & numxpt, & COMMON & & \multirow[t]{2}{*}{-} & & \multirow{2}{*}{$\begin{array}{l}\text { South neighbor } \\
\text { coefficient in } v \\
\text { equation }\end{array}$} \\
\hline & numypt & flupr2 & & & & \\
\hline \multirow[t]{2}{*}{ avw } & numxpt, & COMMON & & \multirow[t]{2}{*}{--} & & \multirow{2}{*}{$\begin{array}{l}\text { West neighbor } \\
\text { coefficient in } \mathrm{v} \\
\text { equation }\end{array}$} \\
\hline & numypt & flupr2 & & & & \\
\hline aw & $\begin{array}{l}\text { numxpt, } \\
\text { numypt }\end{array}$ & $\begin{array}{l}\text { COMMON } \\
\text { coef }\end{array}$ & $A_{W}$ & & $3-62$ & Coefficient \\
\hline \multirow[t]{2}{*}{ aw } & numxpt, $n$ & COMMON & \multirow[t]{5}{*}{$A_{W}$} & & $3-82$ & \multirow{2}{*}{$\begin{array}{l}\text { West neighbor } \\
\text { coefficient }\end{array}$} \\
\hline & umypt & coef & & & & \\
\hline awdt 1 & $\rightarrow$ & $\begin{array}{l}\text { COMMON } \\
\text { rmscom }\end{array}$ & & & & $\begin{array}{l}\text { Wendt rate } 1 \text { pre- } \\
\text { exponential }\end{array}$ \\
\hline awdt 2 & -- & $\begin{array}{l}\text { COMMON } \\
\text { rmscom }\end{array}$ & & & & $\begin{array}{l}\text { Wendt rate } 2 \text { pre- } \\
\text { exponential }\end{array}$ \\
\hline awj & $\begin{array}{l}\text { numxpt, } \\
\text { numypt, } \\
\text { numpar }\end{array}$ & $\begin{array}{l}\text { COMMON } \\
\text { eulp }\end{array}$ & & & & $\begin{array}{l}\text { Eulerian value of } \\
\text { mass of slurry liquid } \\
\text { in } \mathrm{k}^{\text {th }} \text { particle }\end{array}$ \\
\hline
\end{tabular}


FORTRAN User's

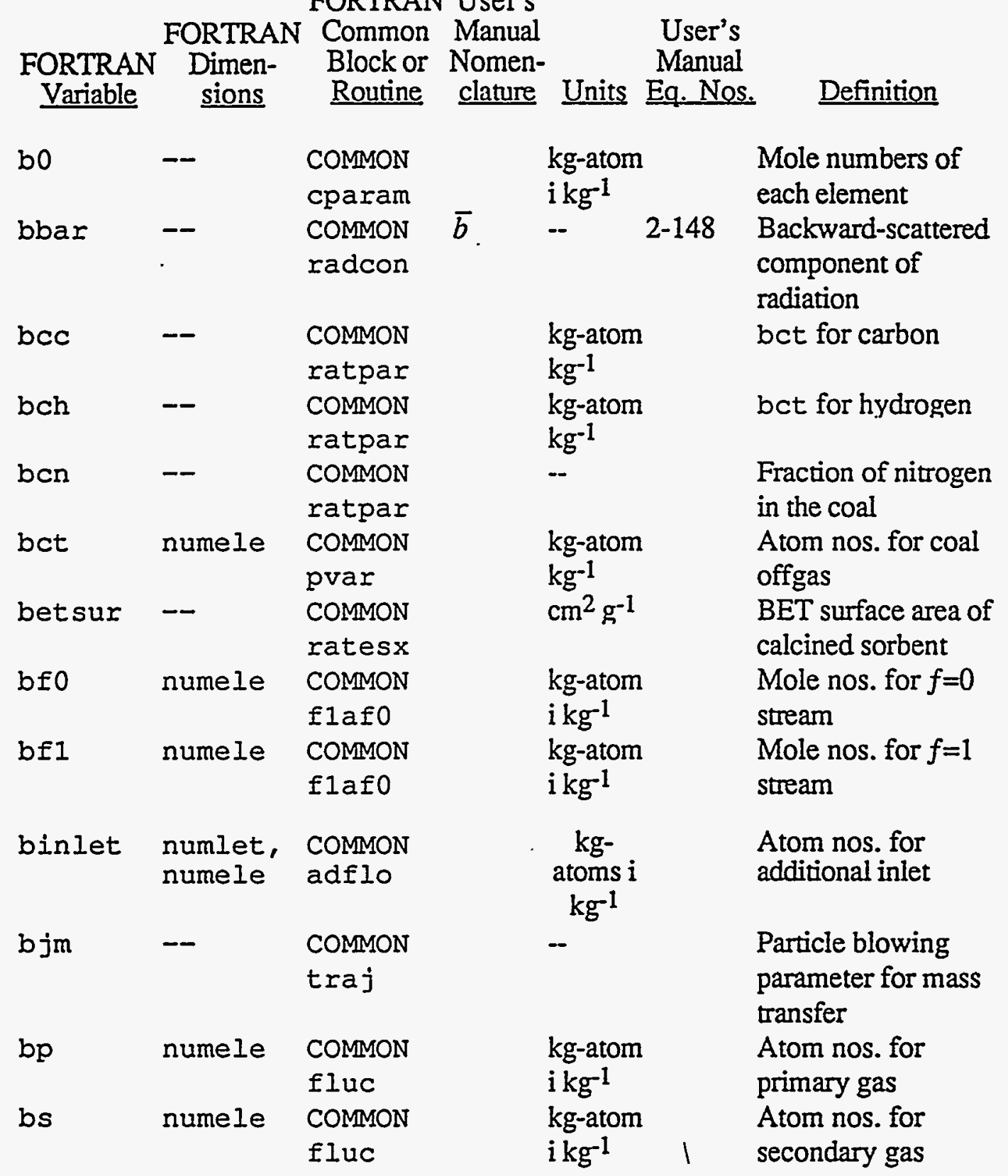




\begin{tabular}{|c|c|c|c|c|c|c|}
\hline $\begin{array}{l}\text { FORTRAN } \\
\text { Variable }\end{array}$ & $\begin{array}{l}\text { FORTRAN } \\
\text { Dimen- } \\
\text { sions }\end{array}$ & $\begin{array}{l}\text { FORTRAI } \\
\text { Common } \\
\text { Block or } \\
\text { Routine }\end{array}$ & $\begin{array}{l}\text { User's } \\
\text { Manual } \\
\text { Nomen- } \\
\text { clature }\end{array}$ & Units & $\begin{array}{r}\text { User's } \\
\text { Manual } \\
\text { Eq. Nos. }\end{array}$ & Definition \\
\hline burnot & numxpt & $\begin{array}{l}\text { COMMON } \\
\text { CElag }\end{array}$ & & -- & & $\begin{array}{l}\text { Fractional burnout } \\
\text { of coal and char } \\
\text { particles }\end{array}$ \\
\hline$c 1, c 2$, & numxpt, $n$ & COMMON & & & $2-157$ & Radiation \\
\hline$c 3, c 4$ & umypt & cset & & & & coefficients \\
\hline$c 1, c 2$ & -- & $\begin{array}{l}\text { COMMON } \\
\text { turb }\end{array}$ & $C_{1.2} C_{2}$ & -- & $2-11$ & $\begin{array}{l}\text { Turbulence model } \\
\text { constants }\end{array}$ \\
\hline cappa & -- & $\begin{array}{l}\text { COMMON } \\
\text { turb }\end{array}$ & & & & $\begin{array}{l}\text { von Karmann } \\
\text { constant }\end{array}$ \\
\hline cсpa & $\begin{array}{l}\text { numpar, } \\
\text { numcoe }\end{array}$ & $\begin{array}{l}\text { COMMON } \\
\text { hcap }\end{array}$ & & & & $\begin{array}{l}\text { Heat capacity } \\
\text { coefficients for ash }\end{array}$ \\
\hline ссрс & $\begin{array}{l}\text { numpar, } \\
\text { numcoe }\end{array}$ & $\begin{array}{l}\text { COMMON } \\
\text { hcap }\end{array}$ & & & & $\begin{array}{l}\text { Heat capacity } \\
\text { coefficients for raw } \\
\text { coal }\end{array}$ \\
\hline ccph & $\begin{array}{l}\text { numpar, } \\
\text { numcoe }\end{array}$ & $\begin{array}{l}\text { COMMON } \\
\text { hcap }\end{array}$ & & & & $\begin{array}{l}\text { Heat capacity } \\
\text { coefficients for char }\end{array}$ \\
\hline$c d$ & -- & $\begin{array}{l}\text { COMMON } \\
\text { turb }\end{array}$ & & & & \\
\hline ceiu & - & $\begin{array}{l}\text { COMMON } \\
\text { bcrad }\end{array}$ & & & & $\varepsilon_{w} /\left(2-\varepsilon_{w}\right)$ \\
\hline ceo & - & $\begin{array}{l}\text { COMMON } \\
\text { bcrad }\end{array}$ & & & & $\begin{array}{l}\varepsilon_{w} /\left(2-\varepsilon_{w}\right) \text { for } \\
\text { exit plane }\end{array}$ \\
\hline cfpabg & -- & $\begin{array}{l}\text { COMMON } \\
\text { dens } 1\end{array}$ & & -- & & $\begin{array}{l}\text { Debug flag for } \\
\text { correction factor for } \\
\text { turbulence due to } \\
\text { particles }\end{array}$ \\
\hline $\mathrm{CG}$ & - & COAI2 & & $\begin{array}{c}\mathrm{kmol} \\
\mathrm{m}^{3}\end{array}$ & & $\begin{array}{l}\text { Total gas } \\
\text { concentration }\end{array}$ \\
\hline
\end{tabular}




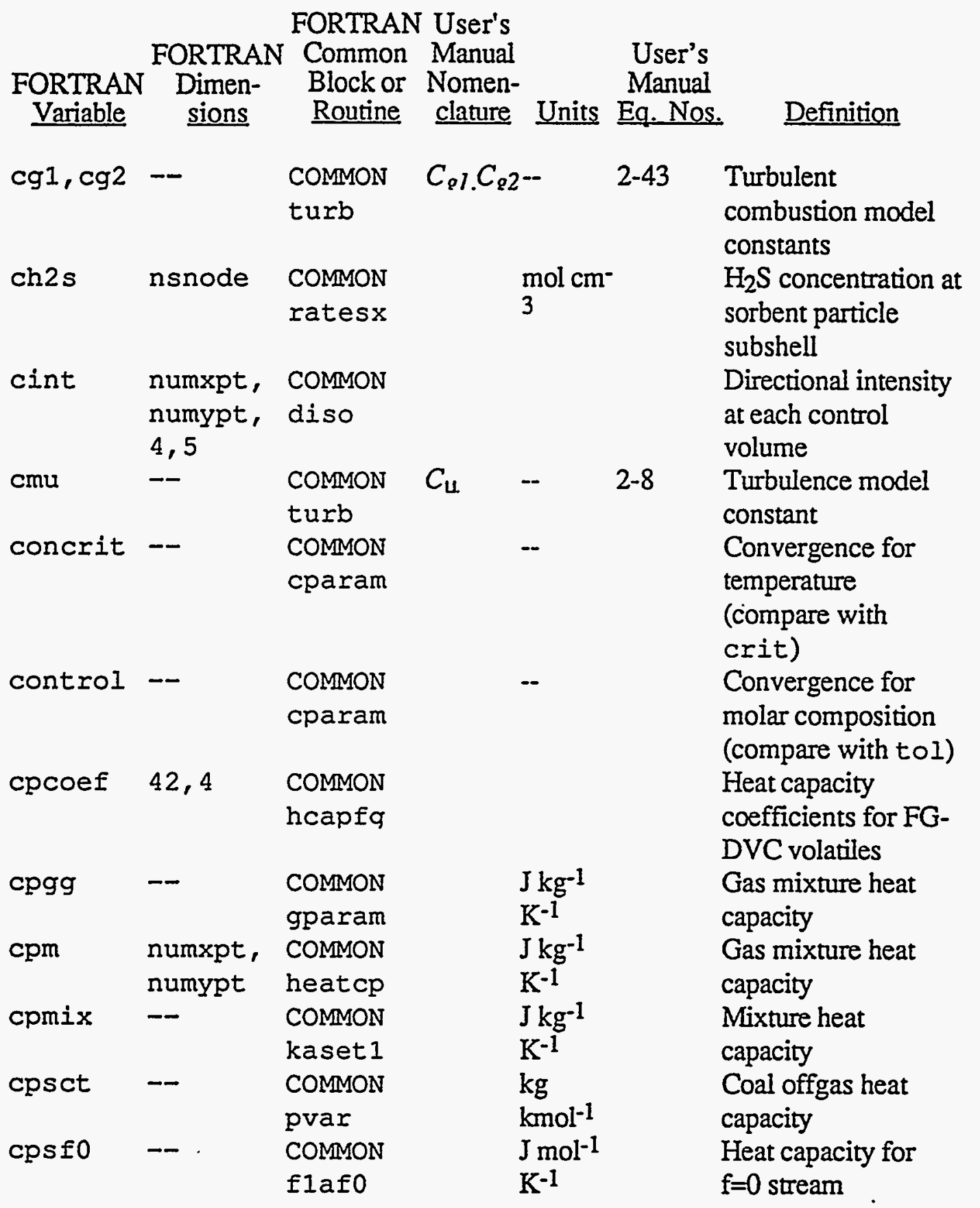


FORTRAN User's

\begin{tabular}{|c|c|c|c|c|c|c|}
\hline $\begin{array}{l}\text { FORTRAN } \\
\text { Variable }\end{array}$ & $\begin{array}{l}\text { FORTRAI } \\
\text { Dimen- } \\
\text { sions }\end{array}$ & $\begin{array}{l}\text { Common } \\
\text { Block or } \\
\text { Routine }\end{array}$ & $\begin{array}{l}\text { Manual } \\
\text { Nomen- } \\
\text { clature }\end{array}$ & Units & $\begin{array}{c}\text { User's } \\
\text { Manual } \\
\text { Eq.Nos. }\end{array}$ & Definition \\
\hline cpsf1 & -- & $\begin{array}{l}\text { COMMON } \\
\text { flafo }\end{array}$ & & $\begin{array}{l}\mathrm{J} \mathrm{mol}^{-1} \\
\mathrm{~K}^{-1}\end{array}$ & & $\begin{array}{l}\text { Heat capacity for } \\
f=1 \text { stream }\end{array}$ \\
\hline cpsinlet & numlet & $\begin{array}{l}\text { COMMON } \\
\text { adflo }\end{array}$ & & $\underset{\mathrm{K}^{-1}}{\mathrm{~J} \mathrm{~kg}^{-1}}$ & & $\begin{array}{l}\text { Heat capacity for } \\
\text { additional inlet }\end{array}$ \\
\hline cpsmh & -- & $\begin{array}{l}\text { COMMON } \\
\text { magnusse } \\
\text { n }\end{array}$ & & $\begin{array}{l}\mathrm{J} \mathrm{kg}^{-1} \\
\mathrm{~K}^{-1}\end{array}$ & & $\begin{array}{l}\text { Product stream heat } \\
\text { capacity } \\
\text { (M!agnussen- } \\
\text { Hjertager method) }\end{array}$ \\
\hline cpsp & - & $\begin{array}{l}\text { COMMON } \\
\text { pands }\end{array}$ & & $\mathrm{J} \mathrm{kg}^{-1}$ & & $\begin{array}{l}\text { Primary stream heat } \\
\text { capacity }\end{array}$ \\
\hline cpss & - & $\begin{array}{l}\text { COMMON } \\
\text { pands }\end{array}$ & & $\mathrm{J} \mathrm{kg}^{-1}$ & & $\begin{array}{l}\text { Secondary stream } \\
\text { heat capacity }\end{array}$ \\
\hline cpsum & -- & $\begin{array}{l}\text { COMMON } \\
\text { cchemi }\end{array}$ & & $\begin{array}{l}\mathrm{J} \mathrm{kg}^{-1} \\
\mathrm{~K}^{-1}\end{array}$ & & Heat capacity of gas \\
\hline $\operatorname{cso} 2$ & nsnode & $\begin{array}{l}\text { COMMON } \\
\text { ratesx }\end{array}$ & & $\mathrm{mol} \mathrm{cm}_{3}^{-}$ & & $\begin{array}{l}\mathrm{SO}_{2} \text { concentration at } \\
\text { sorbent particle } \\
\text { subshell }\end{array}$ \\
\hline $\operatorname{delrrj}$ & -- & $\begin{array}{l}\text { COMMON } \\
\text { rcont }\end{array}$ & & & & $\begin{array}{l}\text { Tolerance for } \\
\text { reaction rate }\end{array}$ \\
\hline delta & numspe & $\begin{array}{l}\text { COMMON } \\
\text { gparam }\end{array}$ & & -- & & $\begin{array}{l}\text { Stockmayer } \\
\text { parameter }\end{array}$ \\
\hline deltpj & - & $\begin{array}{l}\text { COMMON } \\
\text { rcont }\end{array}$ & & $\mathrm{K}$ & & $\begin{array}{l}\text { Tolerance for } \\
\text { particle temperature }\end{array}$ \\
\hline den & $\begin{array}{l}\text { numxpt, } \\
\text { numypt }\end{array}$ & $\begin{array}{l}\text { COMMON } \\
\text { flupr }\end{array}$ & $\rho$ & $\mathrm{kg} \mathrm{m}^{-3}$ & $2-55$ & Gas mixture density \\
\hline densct & -- & $\begin{array}{l}\text { COMMON } \\
\text { pvar }\end{array}$ & & $\begin{array}{l}\mathrm{kg} \\
\mathrm{kmol}^{-1}\end{array}$ & & Coal offgas density \\
\hline densfo & - & $\begin{array}{l}\text { COMMON } \\
\text { flafo }\end{array}$ & 1 & $\mathrm{~kg} \mathrm{~m}^{-3}$ & & $\begin{array}{l}\text { Density of } f=0 \\
\text { stream }\end{array}$ \\
\hline
\end{tabular}




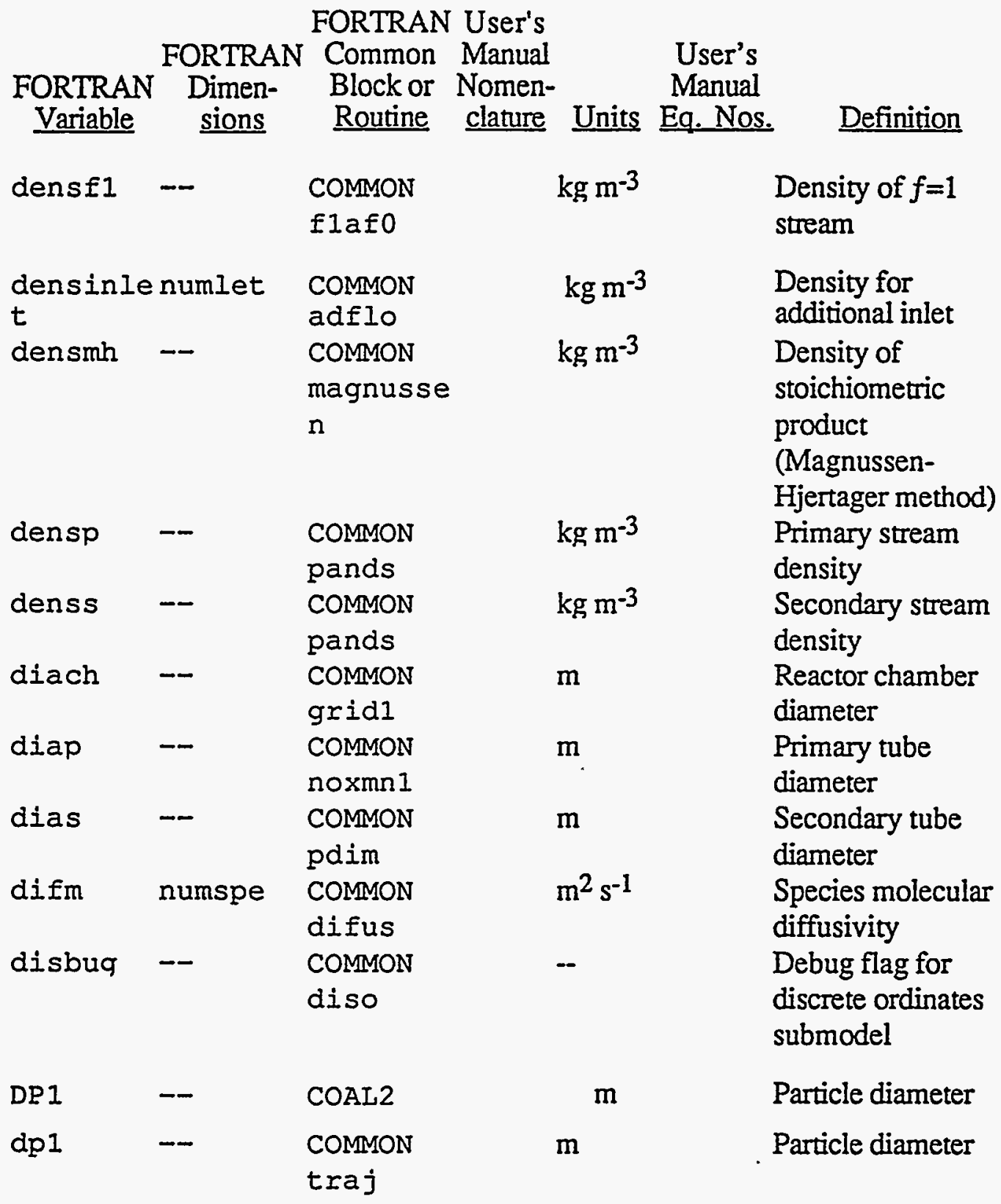




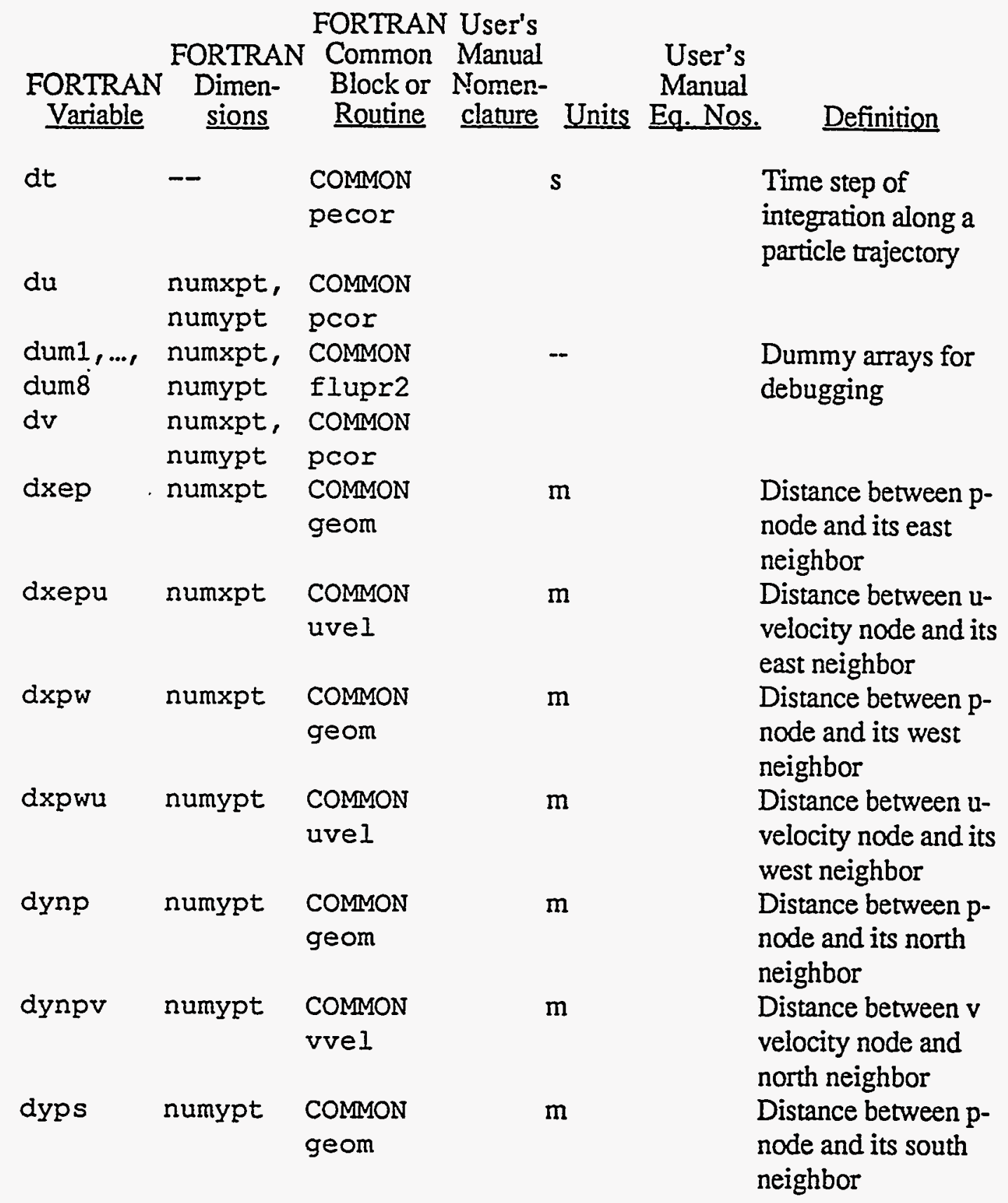




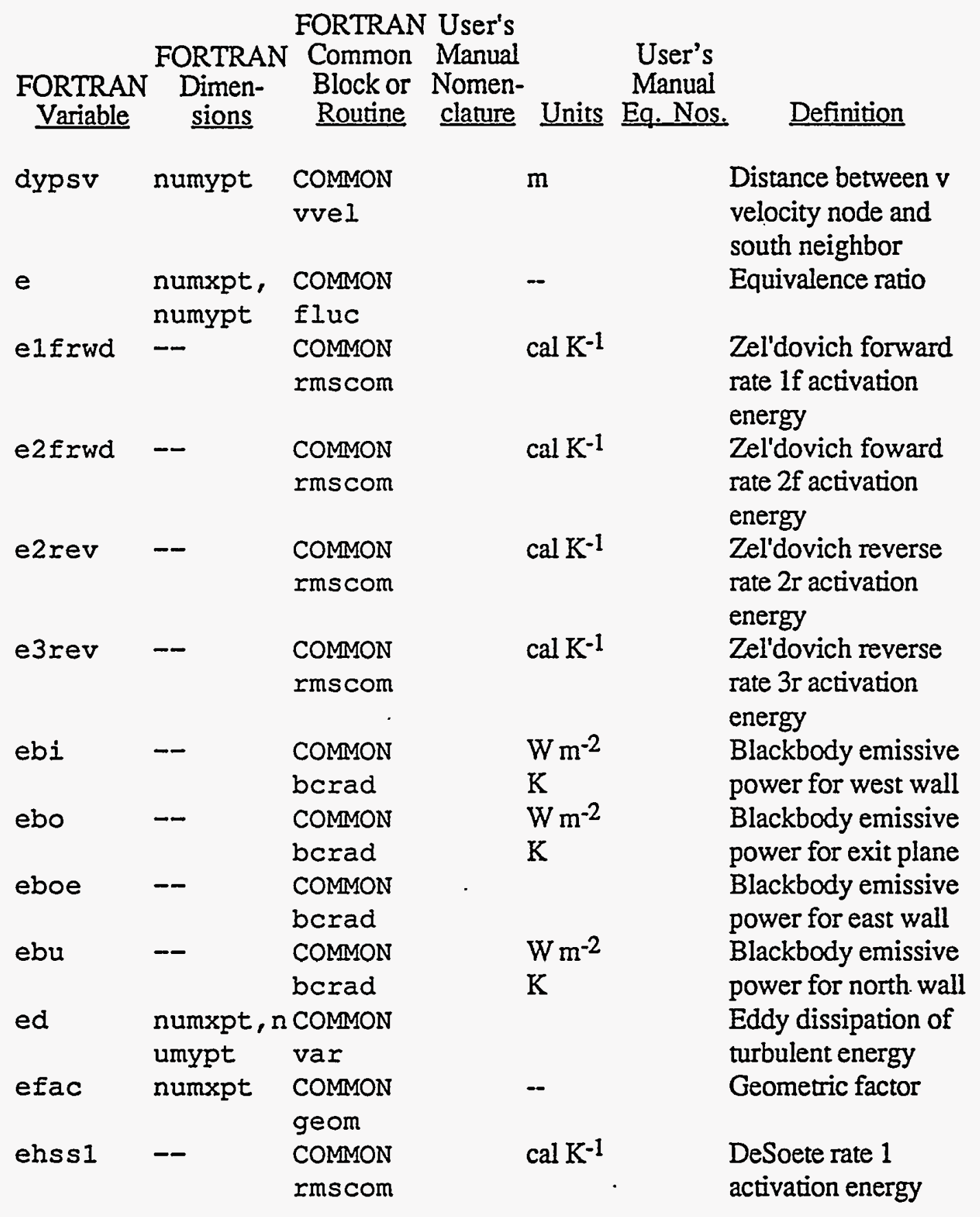




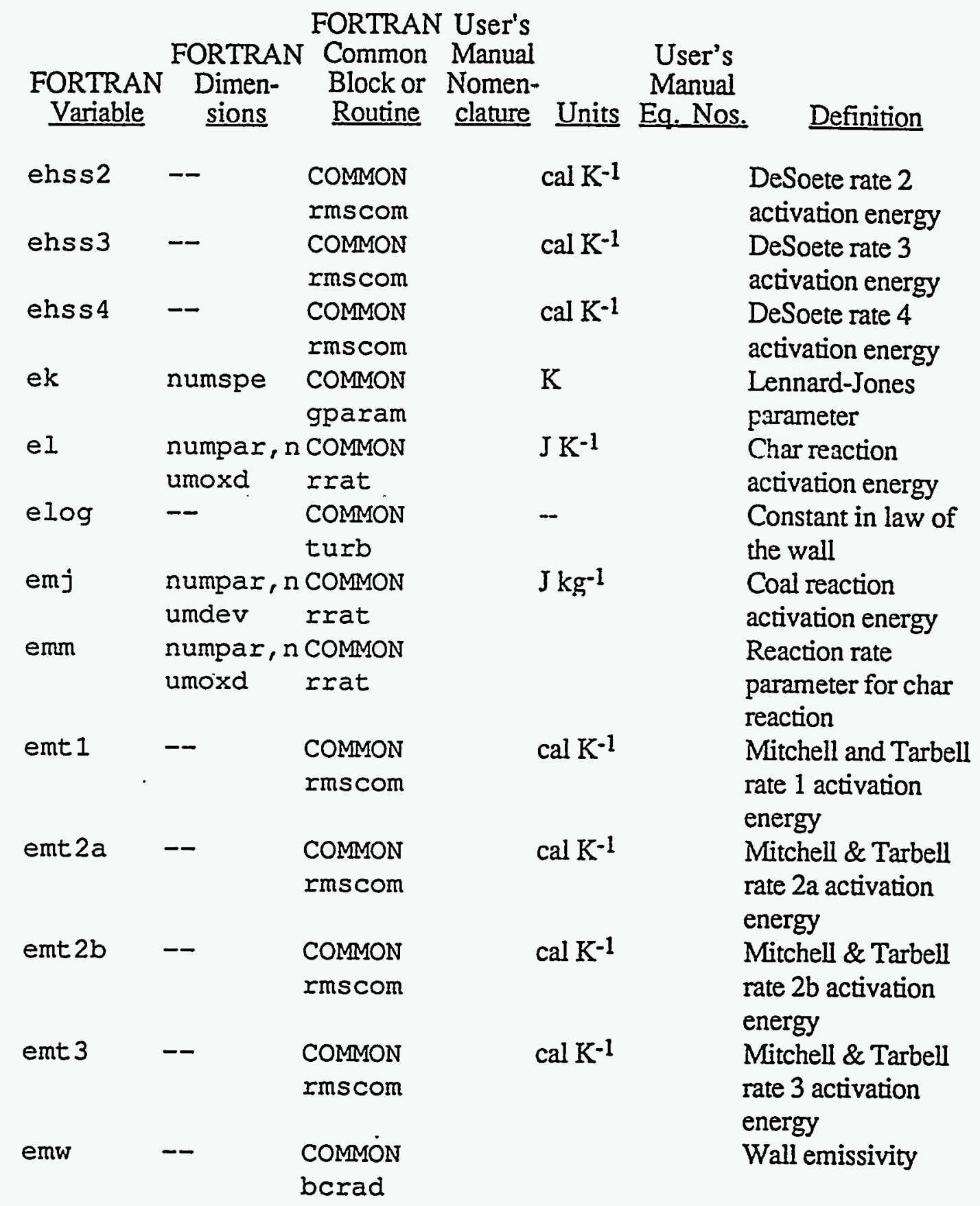




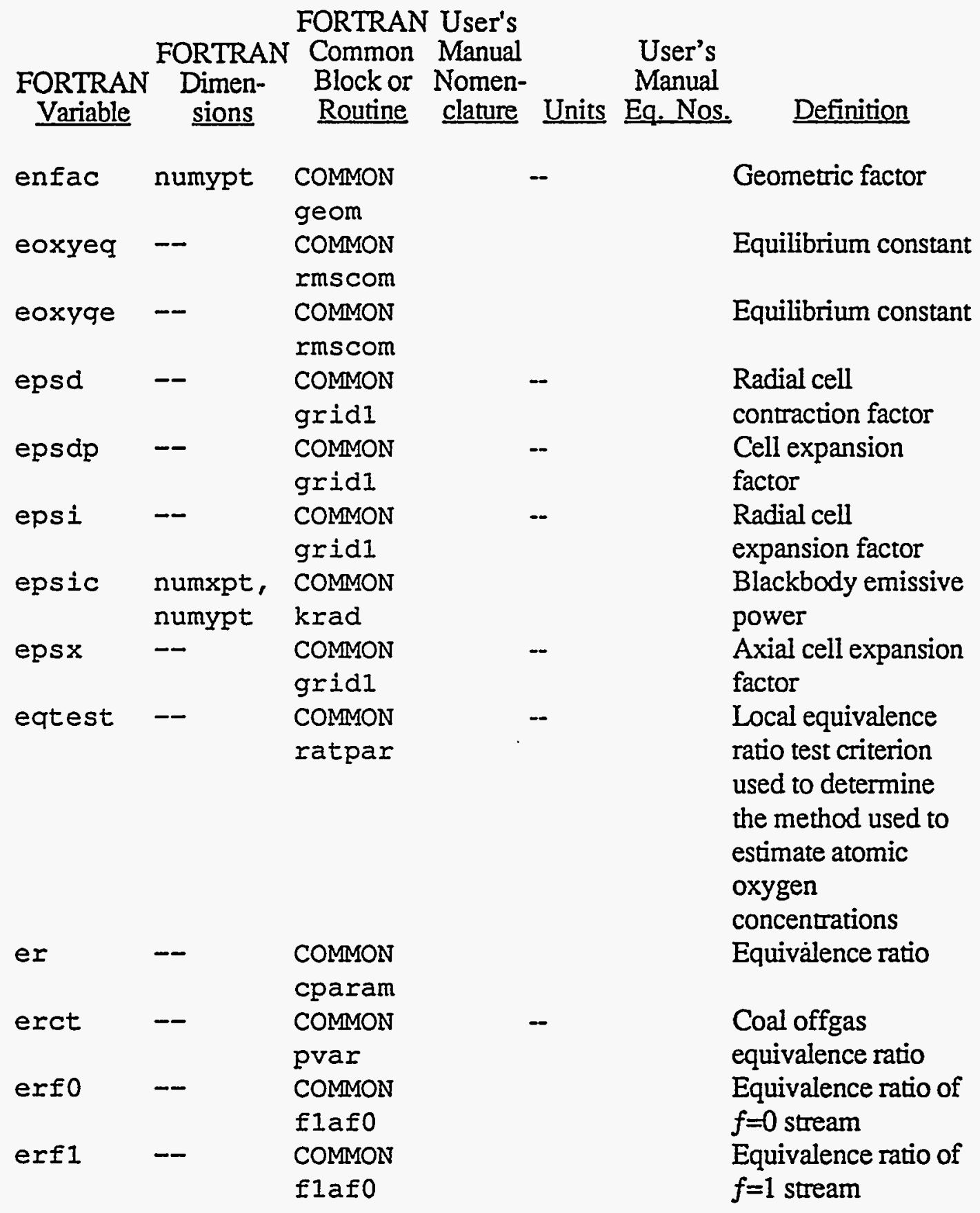


FORTRAN User's

\begin{tabular}{|c|c|c|c|c|c|}
\hline $\begin{array}{c}\text { FORTRAN } \\
\text { Variable }\end{array}$ & $\begin{array}{c}\text { FORTRAN } \\
\begin{array}{c}\text { Dimen- } \\
\text { sions }\end{array}\end{array}$ & $\begin{array}{l}\text { Common } \\
\text { Block or } \\
\text { Routine }\end{array}$ & $\begin{array}{l}\text { Manual } \\
\text { Nomen- } \\
\text { clature }\end{array}$ & 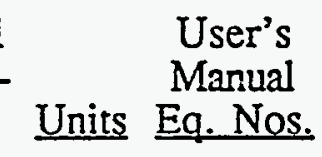 & Definition \\
\hline ermh & -- & $\begin{array}{l}\text { COMMON } \\
\text { magnusse } \\
\mathrm{n}\end{array}$ & & -- & $\begin{array}{l}\text { Equivalence ratio of } \\
\text { product stream } \\
\text { (Magnussen- } \\
\text { Hjertager method) }\end{array}$ \\
\hline erp & - & $\begin{array}{l}\text { COMMON } \\
\text { pands }\end{array}$ & & -- & $\begin{array}{l}\text { Primary stream } \\
\text { equivalence ratio }\end{array}$ \\
\hline ers & -- & $\begin{array}{l}\text { COMMON } \\
\text { pands }\end{array}$ & & -- & $\begin{array}{l}\text { Secondary stream } \\
\text { equivalence ratio }\end{array}$ \\
\hline eta & -- & $\begin{array}{l}\text { COMMON } \\
\text { cmatri }\end{array}$ & & -- & $\begin{array}{l}\text { Under-relaxation } \\
\text { factor for Newton- } \\
\text { Raphson correction } \\
\text { variables }\end{array}$ \\
\hline eta & -- & $\begin{array}{l}\text { COMMON } \\
\text { cmatri }\end{array}$ & & -- & $\begin{array}{l}\text { Under-relaxation for } \\
\text { Newton-Raphson } \\
\text { correction variables }\end{array}$ \\
\hline eta & $\begin{array}{l}\text { numxpt, } \\
\text { numypt }\end{array}$ & $\begin{array}{l}\text { COMMON } \\
\text { cgasmf }\end{array}$ & & & $\begin{array}{l}\text { Coal gas mixture } \\
\text { fraction }\end{array}$ \\
\hline etastm & -- & $\begin{array}{l}\text { COMMON } \\
\text { magnusse } \\
\mathrm{n}\end{array}$ & & -- & $\begin{array}{l}\text { Stoichiometric value } \\
\text { of } \eta\end{array}$ \\
\hline ewdt I & - & $\begin{array}{l}\text { COMMON } \\
\text { rmscom }\end{array}$ & & $\mathrm{cal} \mathrm{K}^{-1}$ & $\begin{array}{l}\text { Wendt rate } 1 \\
\text { activation energy }\end{array}$ \\
\hline ewdt2 & - & $\begin{array}{l}\text { COMMON } \\
\text { rmscom }\end{array}$ & & $\mathrm{cal} \mathrm{K}^{-1}$ & $\begin{array}{l}\text { Wendt rate } 2 \\
\text { activation energy }\end{array}$ \\
\hline exvoid & - & $\begin{array}{l}\text { COMMON } \\
\text { rates }\end{array}$ & & -- & $\begin{array}{l}\text { Sorbent-particle void } \\
\text { fraction }\end{array}$ \\
\hline f & $\begin{array}{l}\text { numxpt, } \mathrm{n} \\
\text { umypt }\end{array}$ & $\begin{array}{l}\text { COMMON } \\
\text { var }\end{array}$ & & -- & Mixture fraction \\
\hline
\end{tabular}




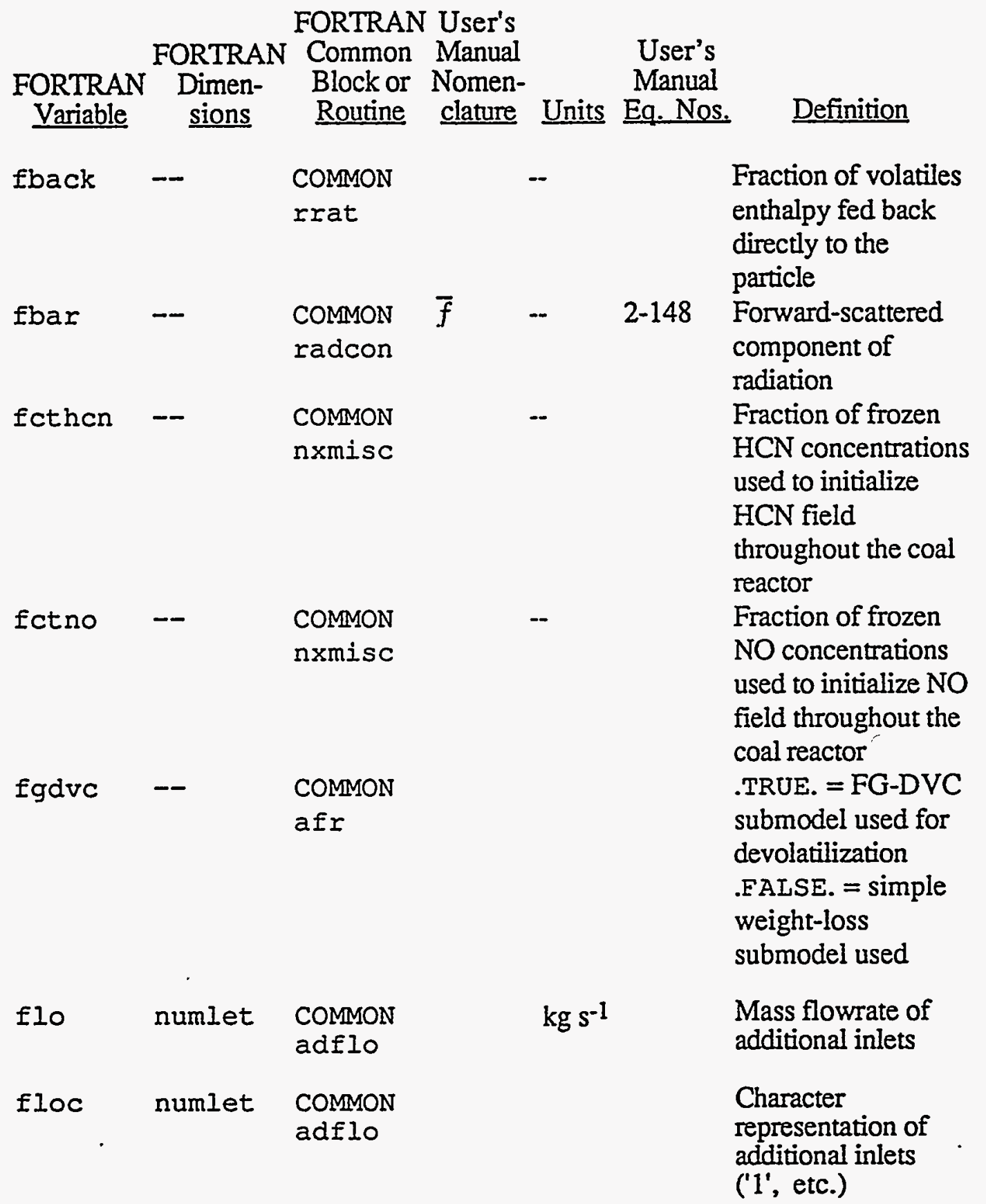




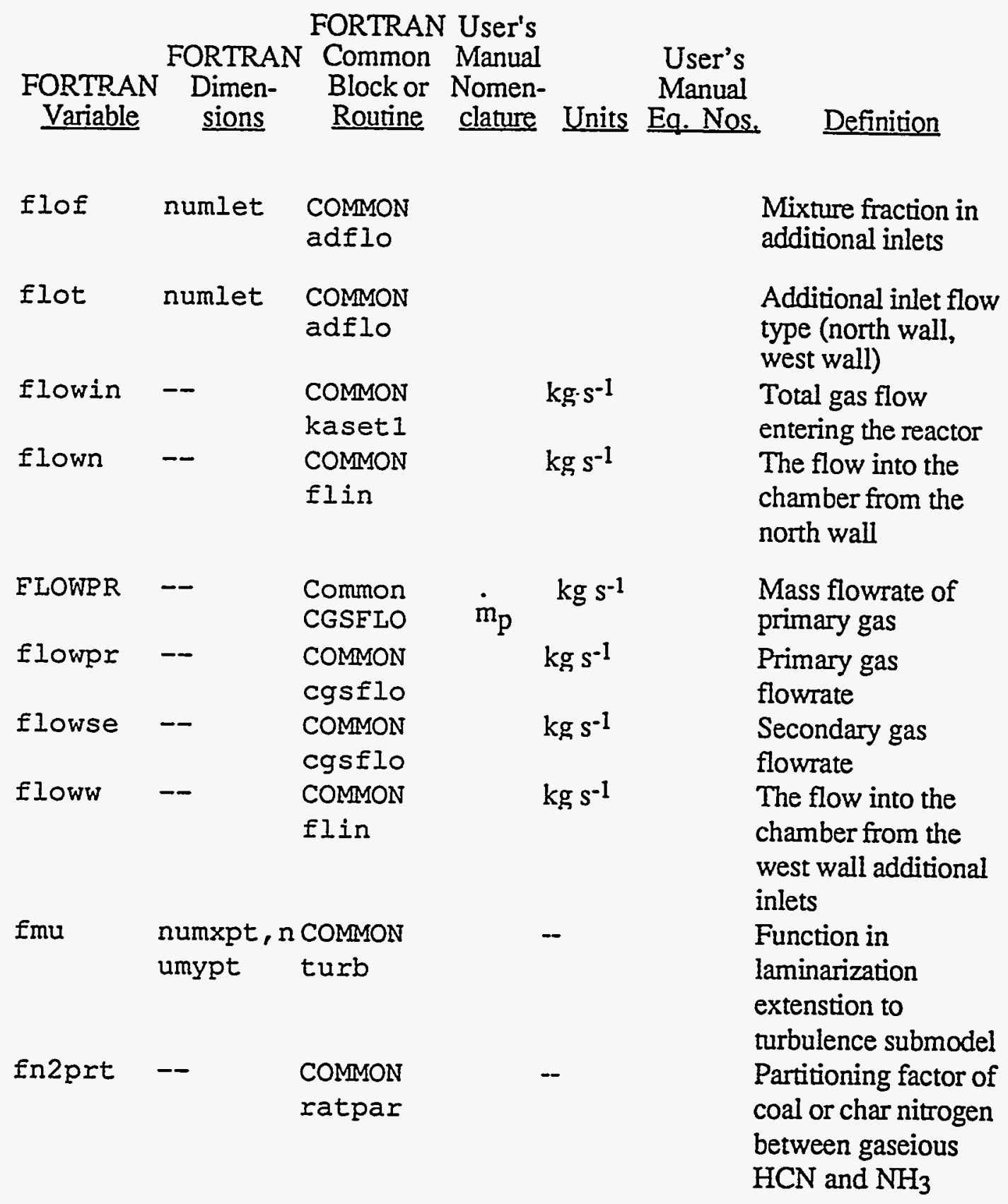




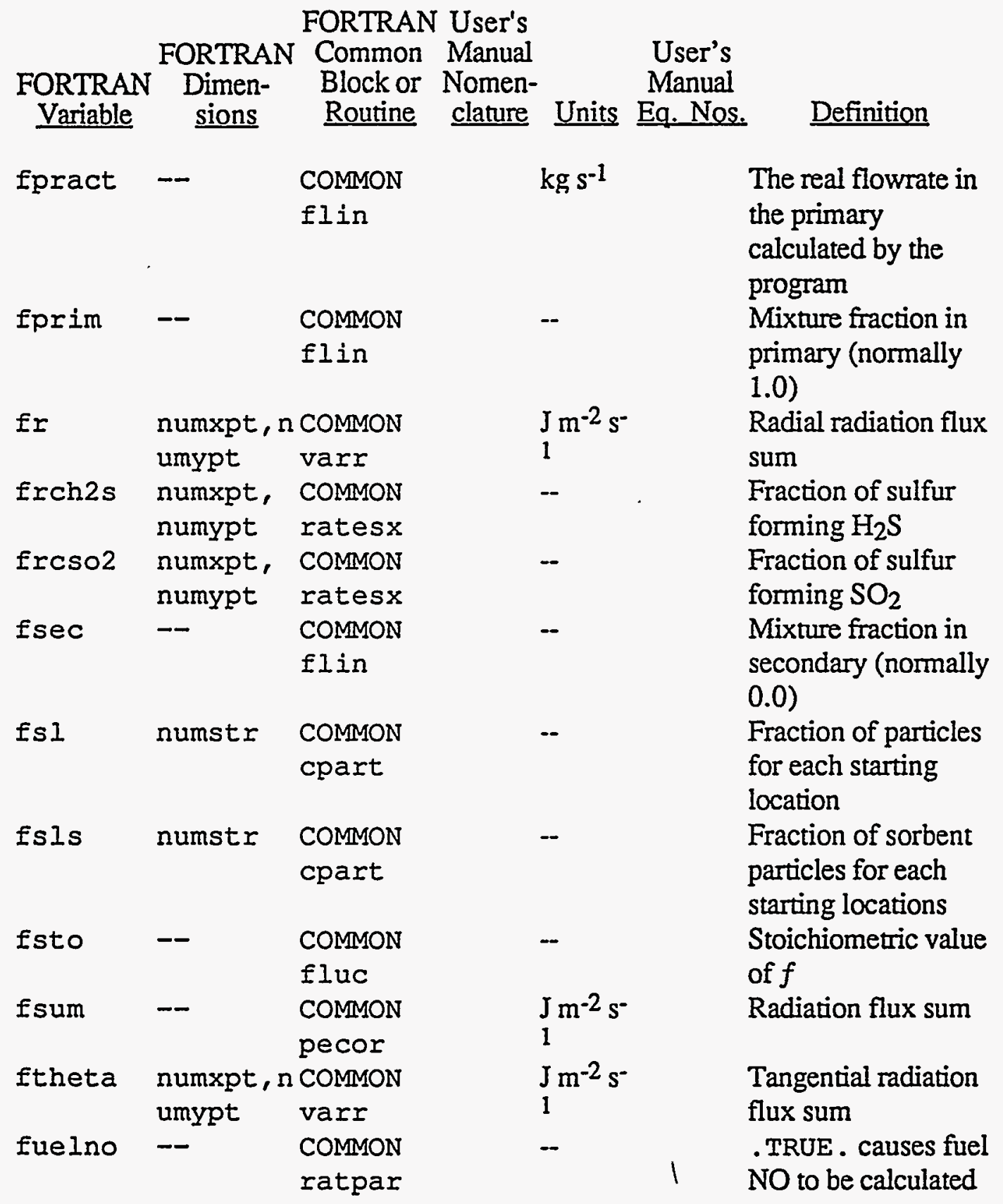


FORTRAN User's

\begin{tabular}{|c|c|c|c|c|c|c|}
\hline $\begin{array}{l}\text { FORTRAN } \\
\text { Variable }\end{array}$ & $\begin{array}{c}\text { FORTRAN } \\
\text { Dimen. } \\
\text { sions }\end{array}$ & $\begin{array}{l}\text { Common } \\
\text { Block or } \\
\text { Routine }\end{array}$ & $\begin{array}{l}\text { Manual } \\
\text { Nomen- } \\
\text { clature }\end{array}$ & Units & $\begin{array}{c}\text { User's } \\
\text { Manual } \\
\text { Eq. Nos. }\end{array}$ & Definition \\
\hline fupper & - & $\begin{array}{l}\text { COMMON } \\
\text { cgauss }\end{array}$ & & & & $\begin{array}{l}\text { Adjusted value of } \\
\text { a fbar }\end{array}$ \\
\hline $\mathrm{fx}$ & $\begin{array}{l}\text { numxpt, } n \\
\text { umypt }\end{array}$ & $\begin{array}{l}\text { COMMON } \\
\text { varr }\end{array}$ & & $\begin{array}{l}\mathrm{J}_{1} \mathrm{~m}^{-2} \mathrm{~s}^{-} \\
\end{array}$ & & $\begin{array}{l}\text { Axial radiation flux } \\
\text { sum }\end{array}$ \\
\hline$g$ & $\begin{array}{l}\text { numxpt, } n \\
\text { umypt }\end{array}$ & $\begin{array}{l}\text { COMMON } \\
\text { var }\end{array}$ & & -- & & $\begin{array}{l}\text { Variance in mixture } \\
\text { fraction }\end{array}$ \\
\hline gam & $\begin{array}{l}\text { numxpt, } n \\
\text { umypt }\end{array}$ & $\begin{array}{l}\text { COMMON } \\
\text { cset }\end{array}$ & & & & \\
\hline gamma & -- & $\begin{array}{l}\text { COMMON } \\
\text { pecor }\end{array}$ & & -- & & $\begin{array}{l}\text { Particle swelling } \\
\text { coefficient }\end{array}$ \\
\hline gasmw & -- & $\begin{array}{l}\text { COMMON } \\
\text { traj }\end{array}$ & & $\begin{array}{l}\mathrm{kg} \\
\mathrm{kmol}^{-1}\end{array}$ & & Gas mol. wt. \\
\hline gen & $\begin{array}{l}\text { numxpt, } \mathrm{n} \\
\text { umypt }\end{array}$ & $\begin{array}{l}\text { COMMON } \\
\text { turb }\end{array}$ & & & & $\begin{array}{l}\text { Generation of } \\
\text { turbulent kinetic } \\
\text { energy }\end{array}$ \\
\hline geta & $\begin{array}{l}\text { numxpt, } \\
\text { numypt }\end{array}$ & $\begin{array}{l}\text { COMMON } \\
\text { cgasmf }\end{array}$ & & & & $\begin{array}{l}\text { Variance in coal gas } \\
\text { mixture fraction }\end{array}$ \\
\hline GMW & -- & $\begin{array}{l}\text { Common } \\
\text { GPARAM }\end{array}$ & $\mathrm{M}_{\mathrm{g}}$ & $\underset{\mathrm{kmol}^{-1}}{\mathrm{~kg}}$ & $2-131$ & $\begin{array}{l}\text { Gas mixture mol. } \\
\text { wt. }\end{array}$ \\
\hline gmw & - & $\begin{array}{l}\text { COMMON } \\
\text { gparam }\end{array}$ & & $\begin{array}{l}\mathrm{kg} \\
\mathrm{kmol}^{-1}\end{array}$ & & $\begin{array}{l}\text { Gas mixture } \\
\text { molecular weight }\end{array}$ \\
\hline grdout & -- & $\begin{array}{l}\text { COMMON } \\
\text { gridl }\end{array}$ & & - & & $\begin{array}{l}\text { TRUE. causes a } \\
\text { grid file to be } \\
\text { written. }\end{array}$ \\
\hline qreat & -- & $\begin{array}{l}\text { COMMON } \\
\text { all }\end{array}$ & & & & A large number \\
\hline gupper & - & $\begin{array}{l}\text { COMMON } \\
\text { cgauss }\end{array}$ & & & & $\begin{array}{l}\text { Adjusted value of } \\
\text { ag }\end{array}$ \\
\hline 1 & $\begin{array}{l}\text { numxpt, } n \\
\text { umypt }\end{array}$ & $\begin{array}{l}\text { COMMON } \\
\text { var }\end{array}$ & $h$ & $\mathrm{~J} \mathrm{~kg}^{-1}$ & $2-89$ & Gas enthalpy \\
\hline
\end{tabular}




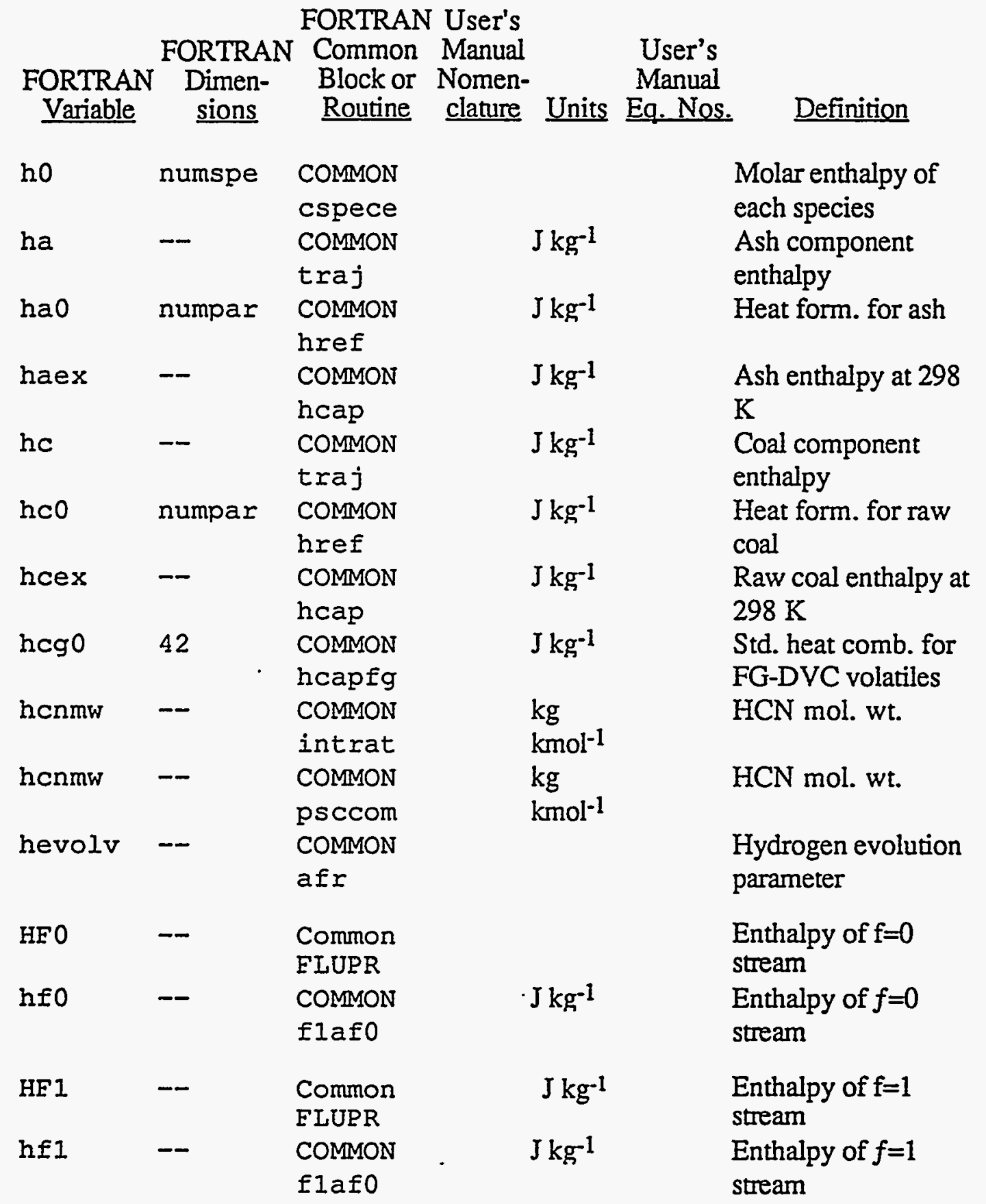




\begin{tabular}{|c|c|c|c|c|c|c|}
\hline \multicolumn{7}{|c|}{ FORTRAN User's } \\
\hline & FORTRAI & Common & Manual & \multirow{2}{*}{\multicolumn{3}{|c|}{$\begin{array}{l}\text { User's } \\
\text { Manual }\end{array}$}} \\
\hline \multirow{2}{*}{$\begin{array}{l}\text { FORTRAN } \\
\text { Variable }\end{array}$} & Dimen- & Block or & Nomen- & & & \\
\hline & & Routine & clature & $\underline{\text { Units }}$ & Eq. Nos. & Definition \\
\hline hfgo & 42 & $\begin{array}{l}\text { COMMON } \\
\text { hcapfg }\end{array}$ & & $\mathrm{J} \mathrm{kg}^{-1}$ & & $\begin{array}{l}\text { Std. heat form. for } \\
\text { FGDVC volatiles }\end{array}$ \\
\hline hform & numspe & COMMON & & J & & Species standard \\
\hline & & gparam & & $\mathrm{kmol}^{-1}$ & & heat of formation \\
\hline hg & numpar & COMMON & & $\mathrm{J} \mathrm{kg}^{-1}$ & & Coal offgas enthalpy \\
\hline & & difeqn & & & & \\
\hline hgv & $\begin{array}{l}\text { numpar, } \\
\text { numdev }\end{array}$ & $\begin{array}{l}\text { COMMON } \\
\text { chetrx }\end{array}$ & & $\mathrm{J} \mathrm{kg}^{-1}$ & & $\begin{array}{l}h g v(j, i) \text { is the } \\
\text { heat of reaction of } \\
\text { the } i^{\text {th }} \\
\text { devolatilization } \\
\text { reaction for the } j^{\text {th }} \\
\text { particle }\end{array}$ \\
\hline $\mathrm{HH}$ & -- & $\begin{array}{l}\text { Common } \\
\text { TRAJ }\end{array}$ & $h_{h i}$ & & $2-125$ & $\begin{array}{l}\text { Char enthaipy for } \\
\text { the } \mathrm{j}_{\text {th }} \text { particle } \\
\text { classification. }\end{array}$ \\
\hline $\mathrm{hh}$ & -- & $\begin{array}{l}\text { COMMON } \\
\text { traj }\end{array}$ & & $\mathrm{J} \mathrm{kg}^{-1}$ & & $\begin{array}{l}\text { Char component } \\
\text { enthalpy }\end{array}$ \\
\hline hho & numpar & $\begin{array}{l}\text { COMMON } \\
\text { href }\end{array}$ & & $\mathrm{J} \mathrm{kg}^{-1}$ & & Heat form. for char \\
\hline hhex & -- & $\begin{array}{l}\text { COMMON } \\
\text { hcap }\end{array}$ & & $\mathrm{J} \mathrm{kg}^{-1}$ & & $\begin{array}{l}\text { Char enthalpy at } 298 \\
\mathrm{~K}\end{array}$ \\
\hline hinc & numxpt & $\begin{array}{l}\text { COMMON } \\
\text { diso }\end{array}$ & & $\mathrm{kw} \mathrm{m} \mathrm{m}^{-2}$ & & $\begin{array}{l}\text { Incident radiation } \\
\text { heat flux on north } \\
\text { wall }\end{array}$ \\
\hline hinlet & numlet & $\begin{array}{l}\text { COMMON } \\
\text { adflo }\end{array}$ & & $\mathrm{J} \mathrm{kg}^{-1}$ & & $\begin{array}{l}\text { Enthalpy for } \\
\text { additional inlet }\end{array}$ \\
\hline hloss & $-\cdots$ & $\begin{array}{l}\text { COMMON } \\
\text { bug }\end{array}$ & 1 & - & & $\begin{array}{l}\text { Specified heat loss } \\
\text { factor for } \\
\text { ihloss }=\text {. TRUE. }\end{array}$ \\
\hline
\end{tabular}


FORTRAN User's

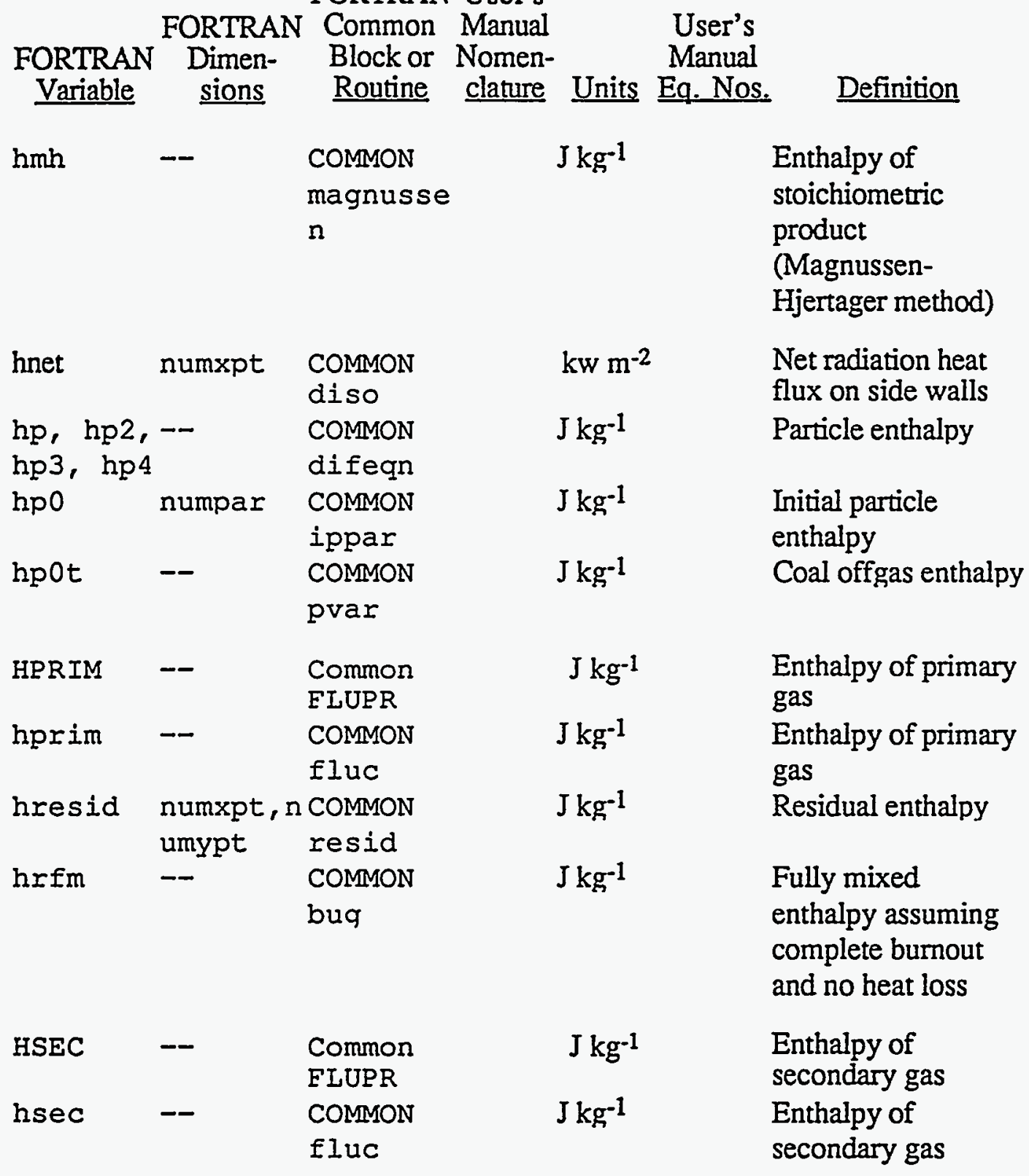




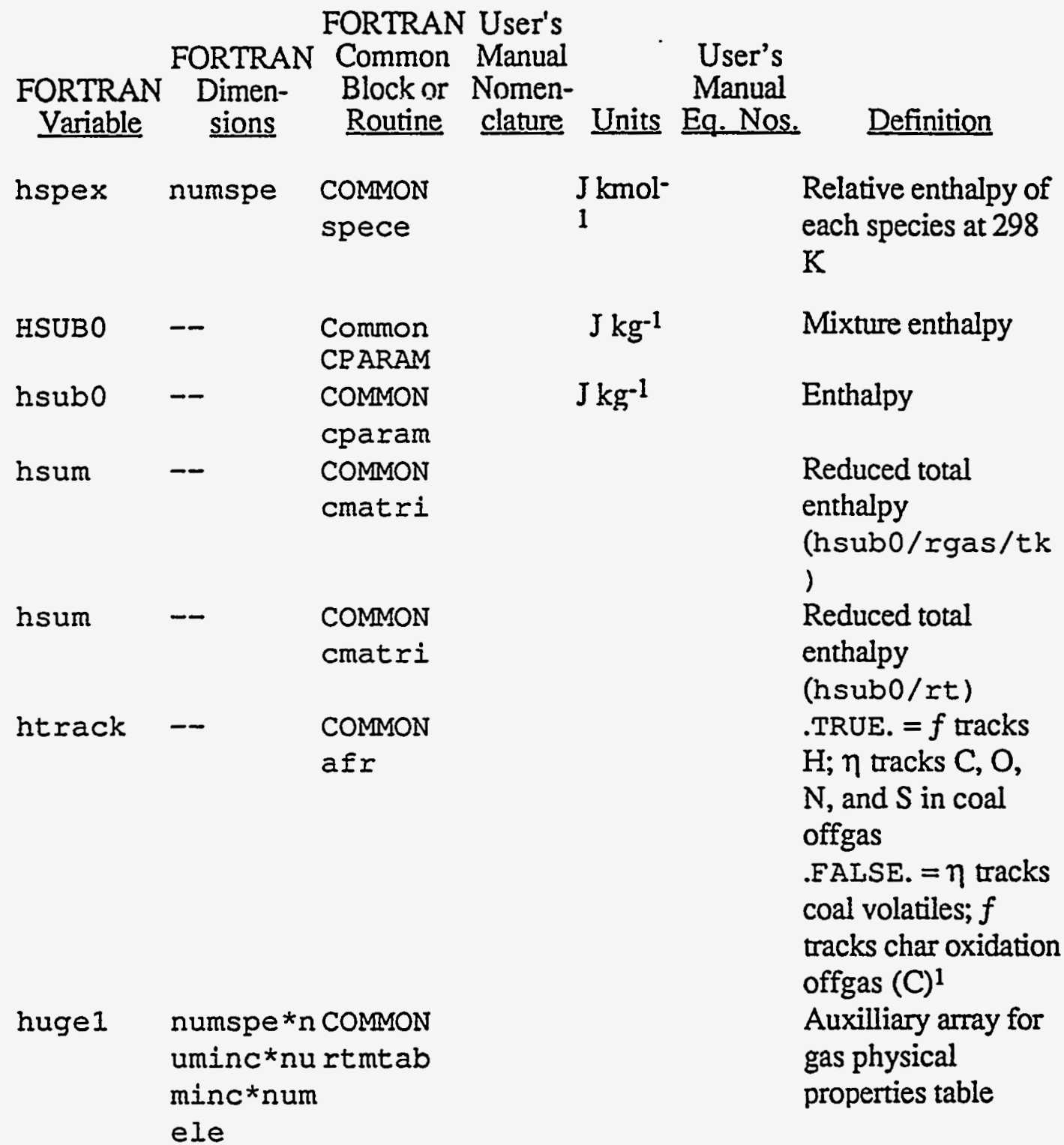

$l_{\text {ineta2 must be .TRUE. for either option of } h t \text { rack. }}$ 


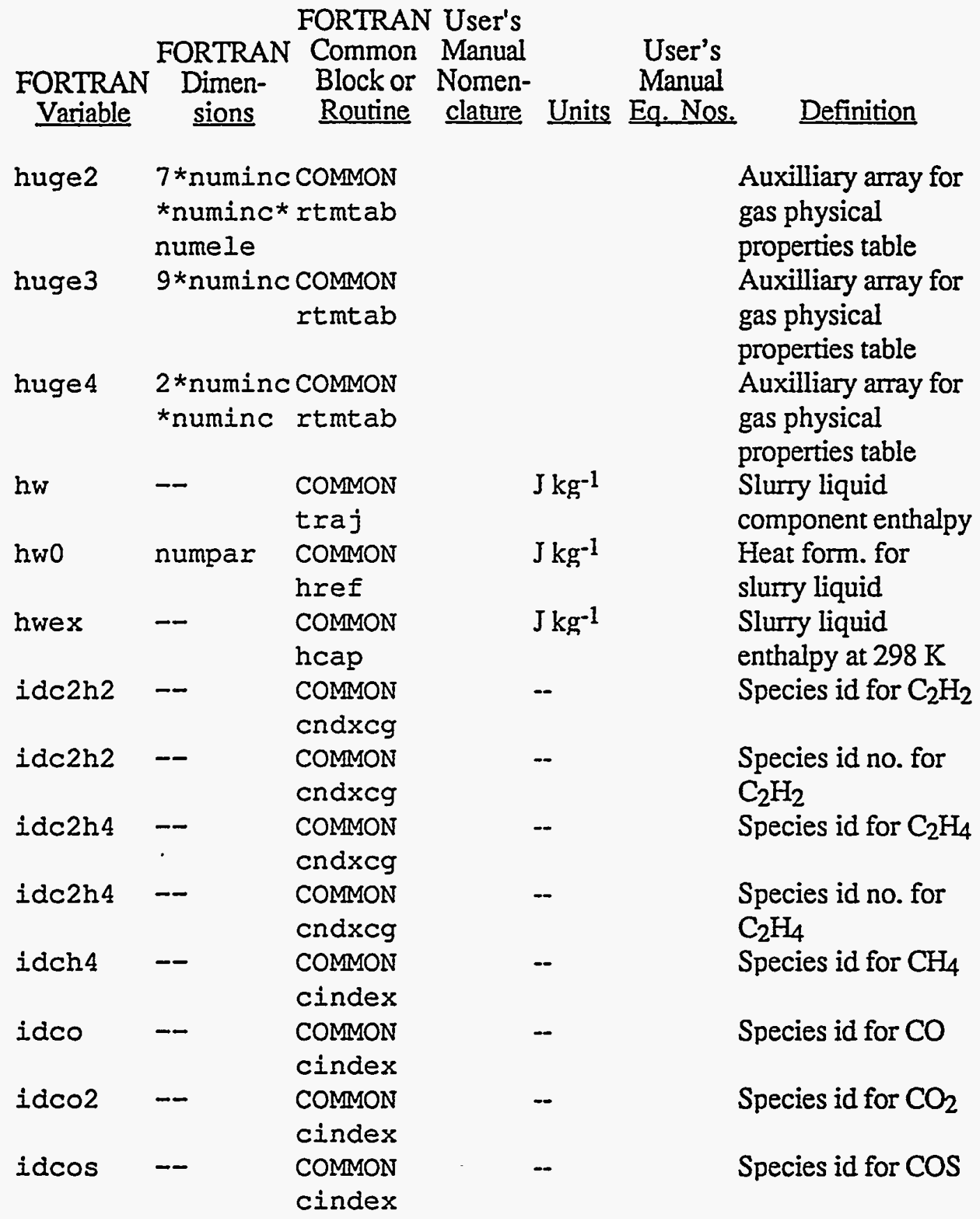




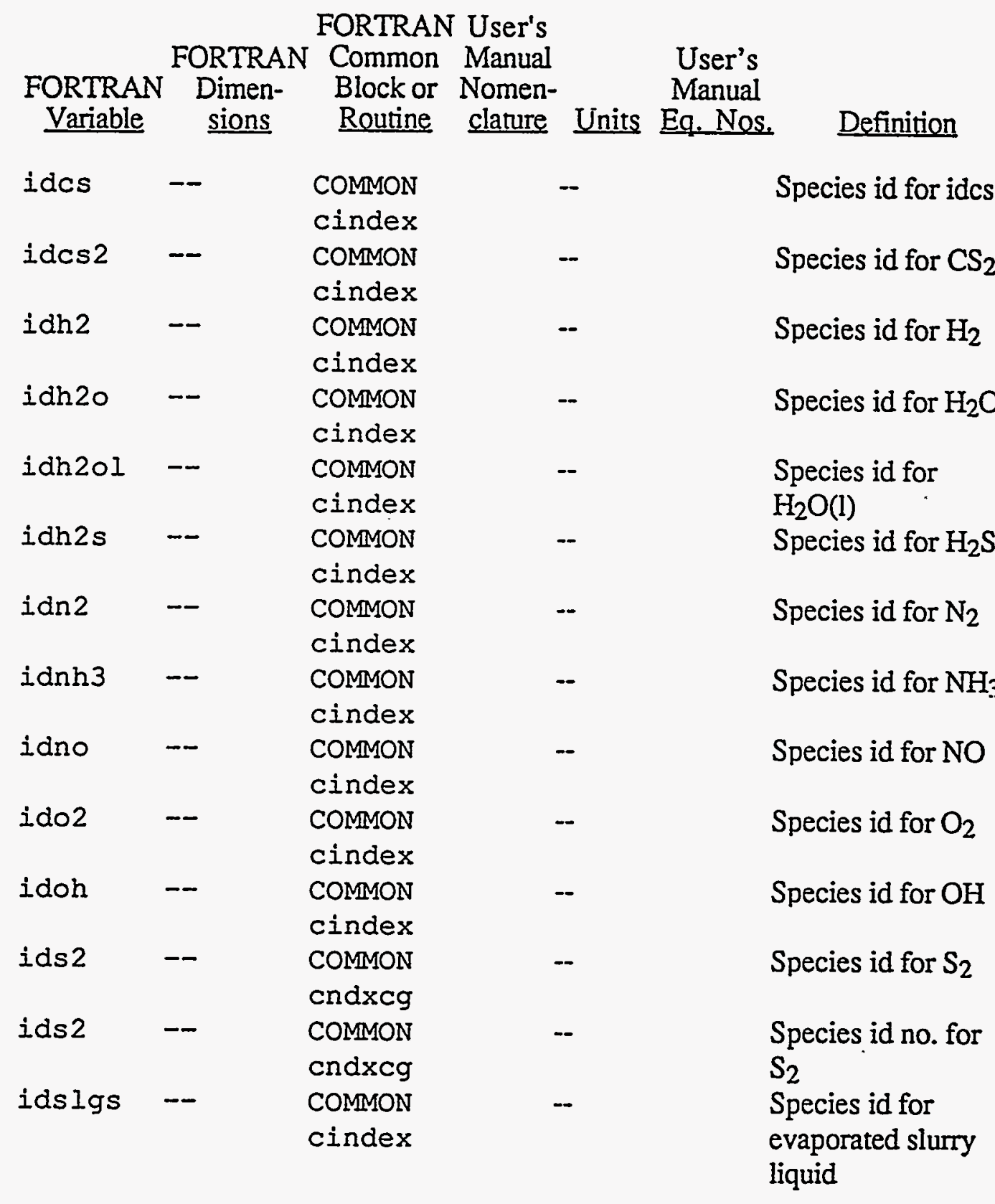




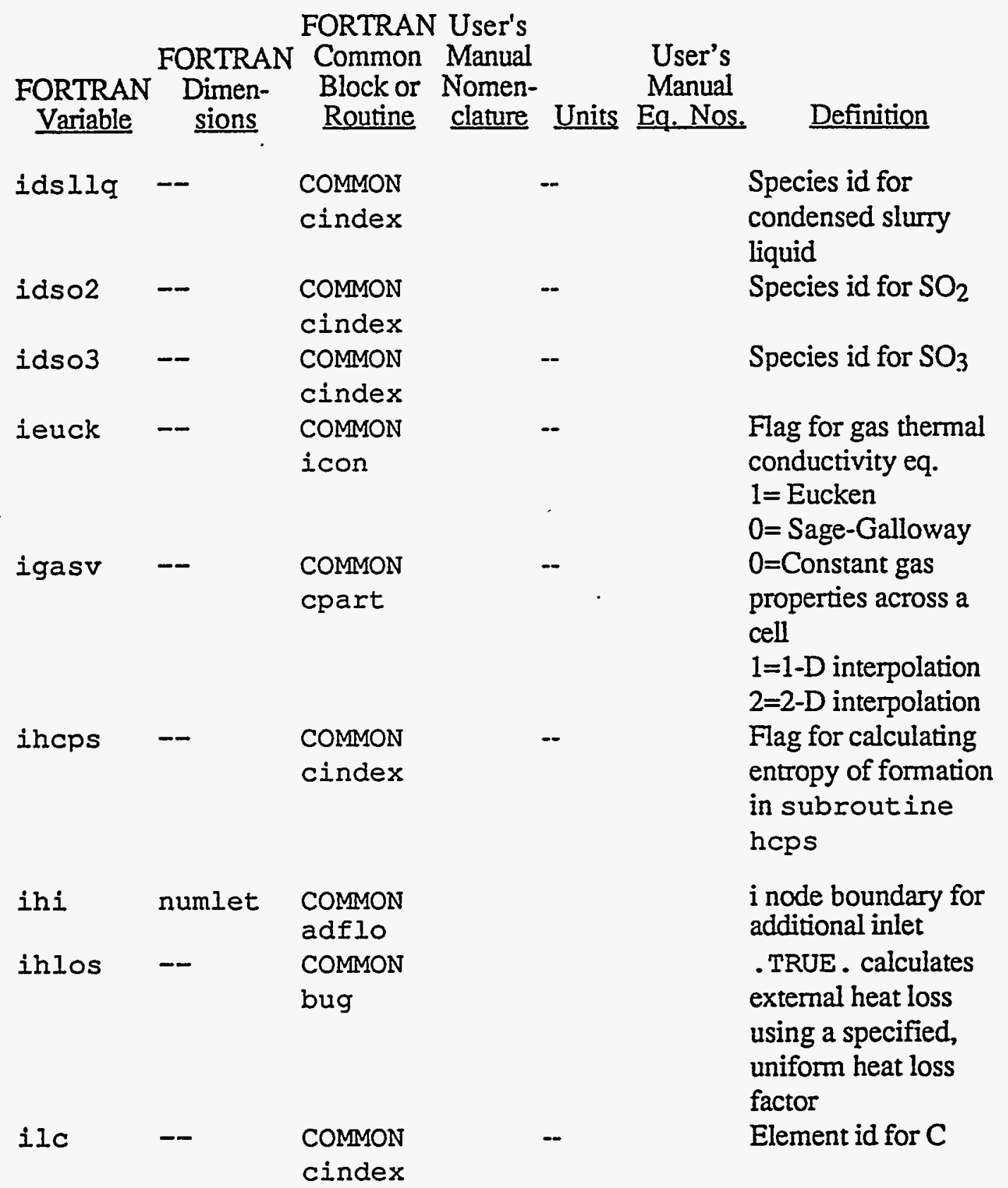




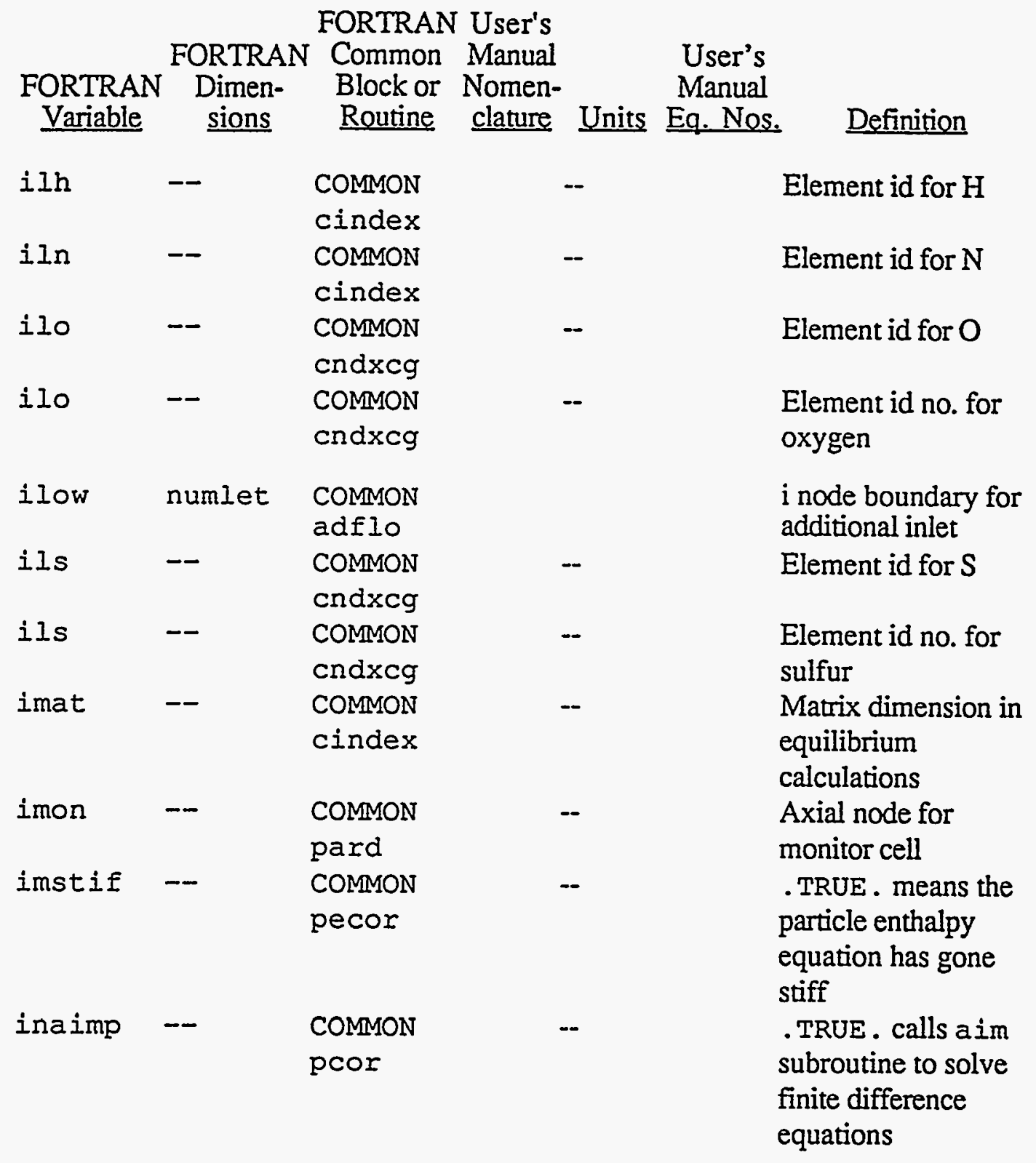


FORTRAN User's

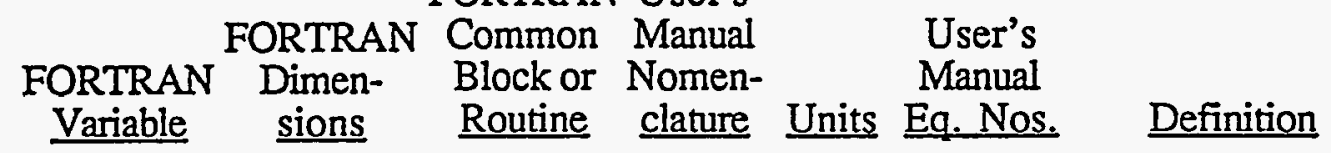

COMMON

uvel

\begin{tabular}{|c|c|c|}
\hline incald & -- & $\begin{array}{l}\text { COMMON } \\
\text { turb }\end{array}$ \\
\hline incalf & -- & $\begin{array}{l}\text { COMMON } \\
\text { tabrit }\end{array}$ \\
\hline incalg & - & $\begin{array}{l}\text { COMMON } \\
\text { fluc }\end{array}$ \\
\hline incalk & -- & $\begin{array}{l}\text { COMMON } \\
\text { turb }\end{array}$ \\
\hline incaln & -- & $\begin{array}{l}\text { COMMON } \\
\text { eulp }\end{array}$ \\
\hline incalp & -- & $\begin{array}{l}\text { COMMON } \\
\text { simple }\end{array}$ \\
\hline incalv & -- & $\begin{array}{l}\text { COMMON } \\
\text { simple }\end{array}$ \\
\hline incalyfu & $2--$ & $\begin{array}{l}\text { COMMON } \\
\text { magnusse } \\
\mathrm{n}\end{array}$ \\
\hline incfp & -- & $\begin{array}{l}\text { COMMON } \\
\text { propin }\end{array}$ \\
\hline inclet & -- & COMMON \\
\hline
\end{tabular}

.TRUE . uses a im routine to solve uvelocity finite difference equations . TRUE . causes ed to be calculated .TRUE. includes $f$ calculations .TRUE . results in $g_{f}$ being calculated .TRUE . causes te to be calculated Flag for performing particle no. density calculations .TRUE . for calculating pressure equation .TRUE . for calculating radial velocity . TRUE . causes yfu to be calculated (MagnussenHjertager method) . TRUE . causes gas phase turbulence to be corrected for particles . TRUE . includes eta calculations 


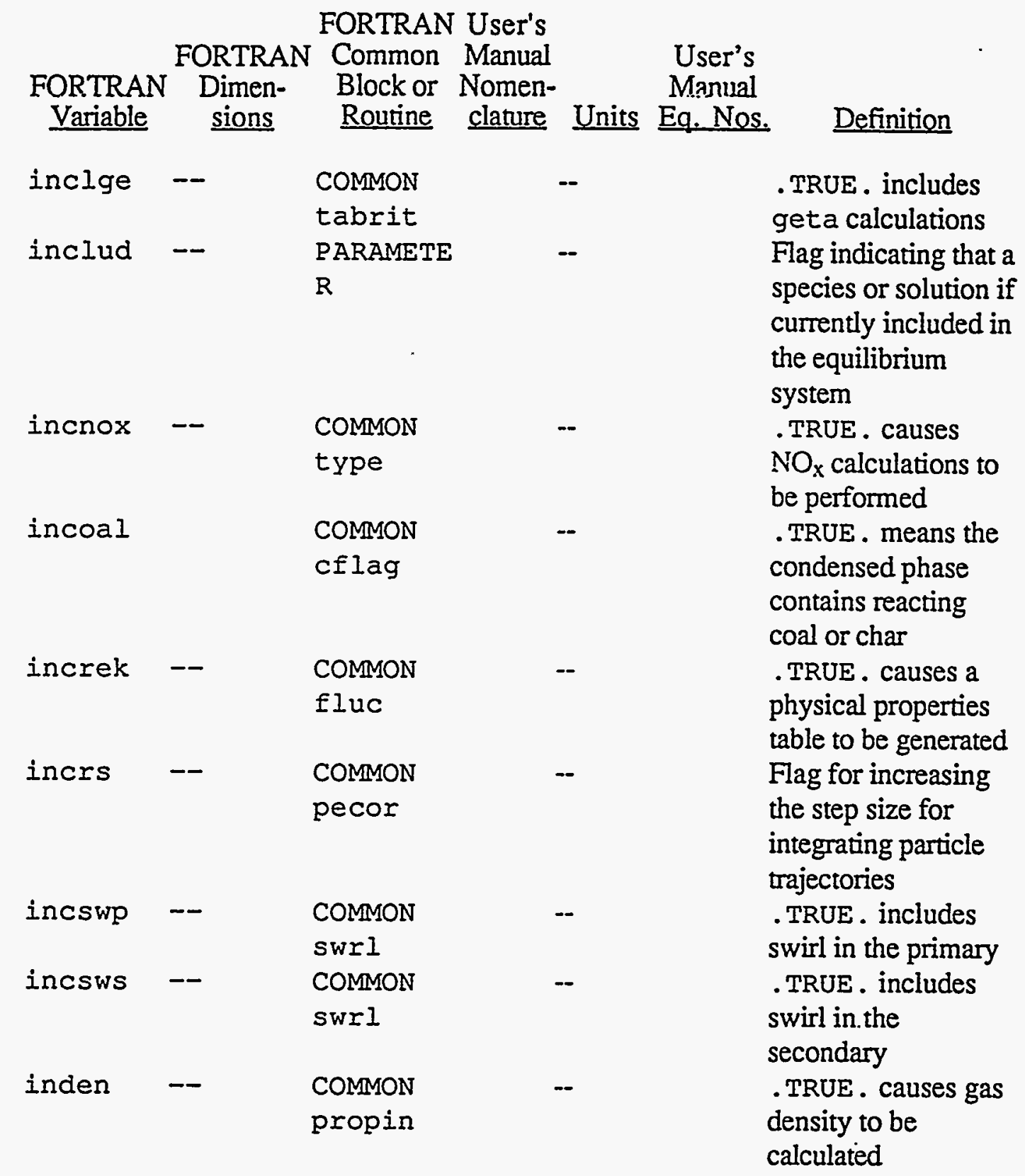




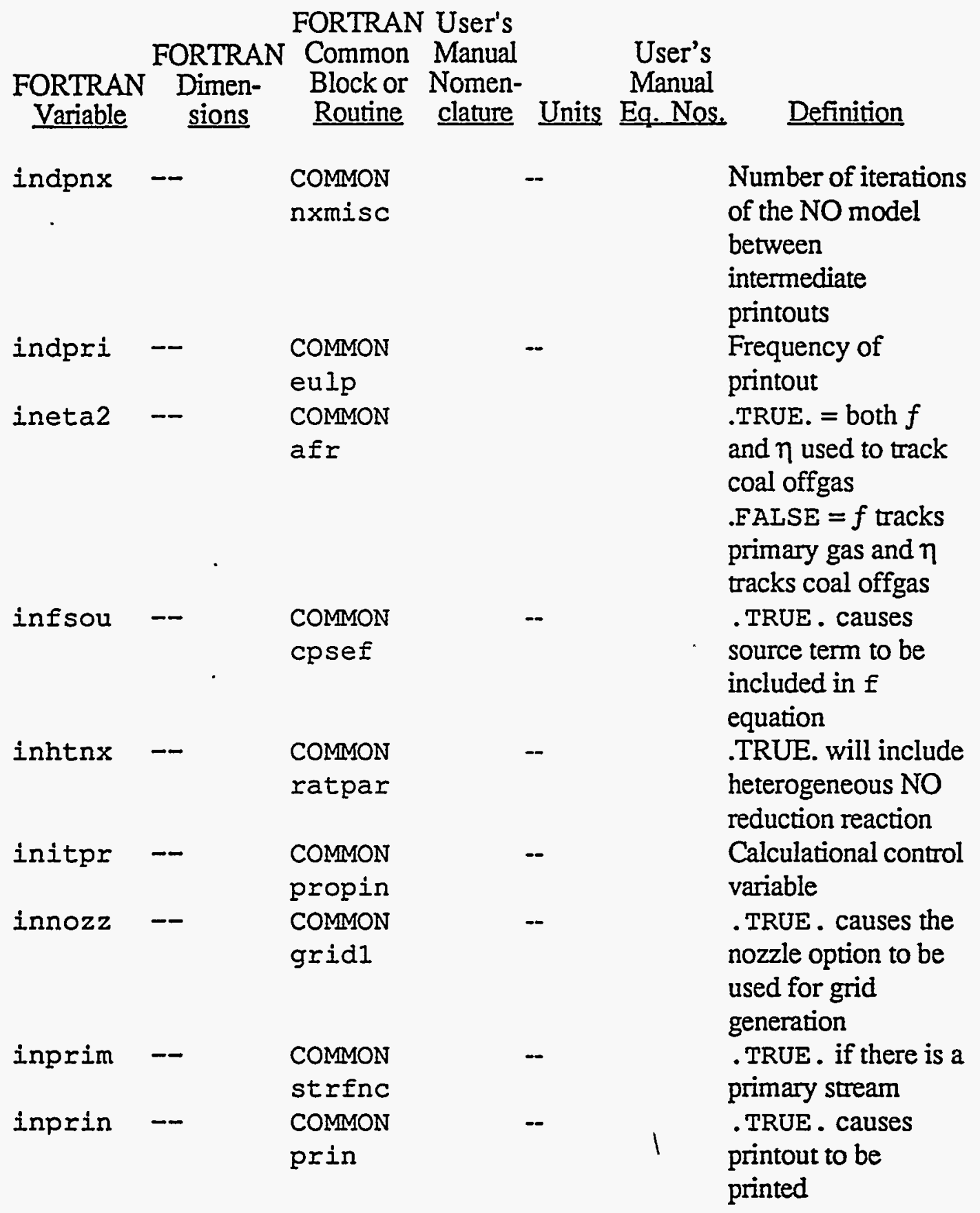




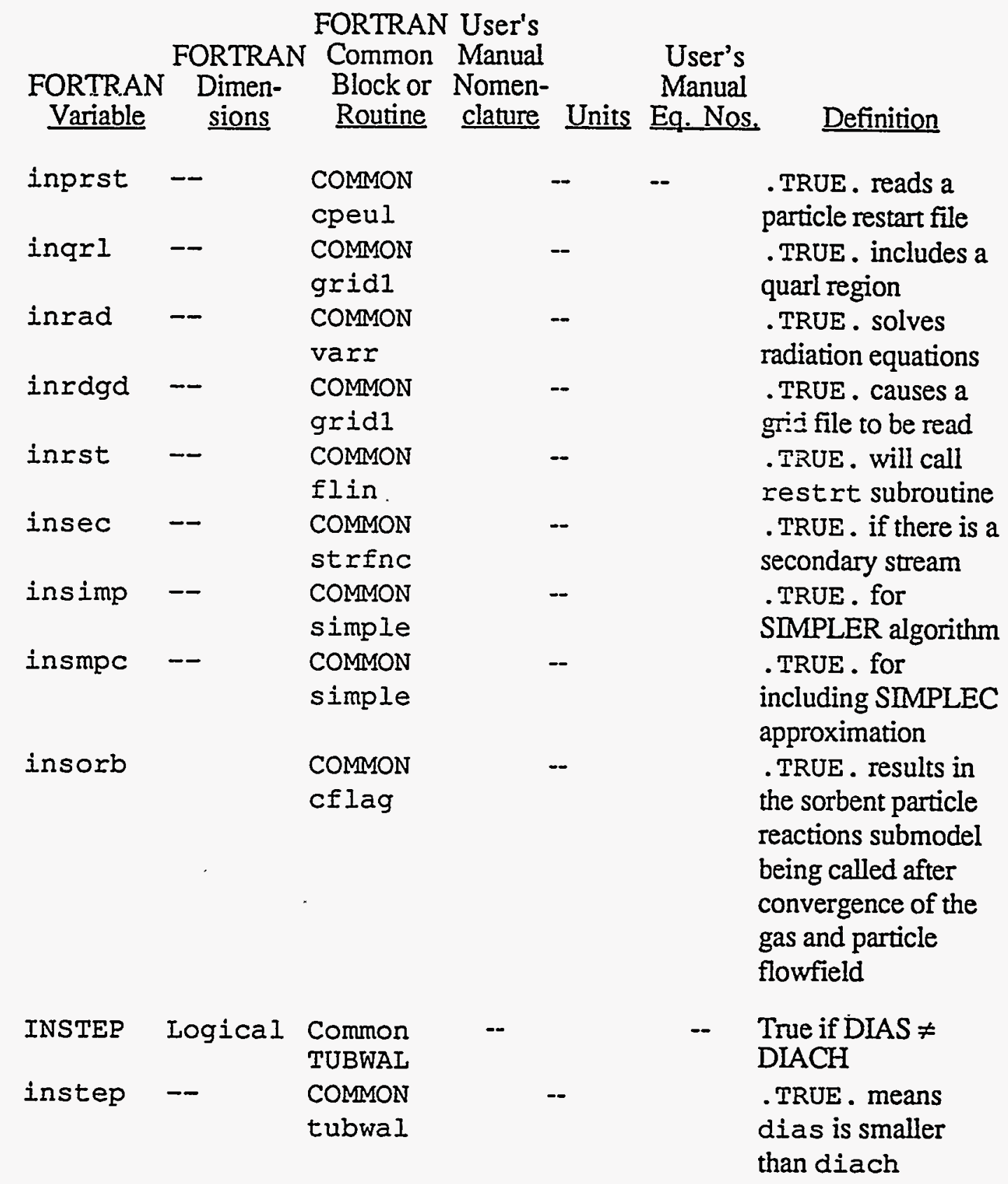




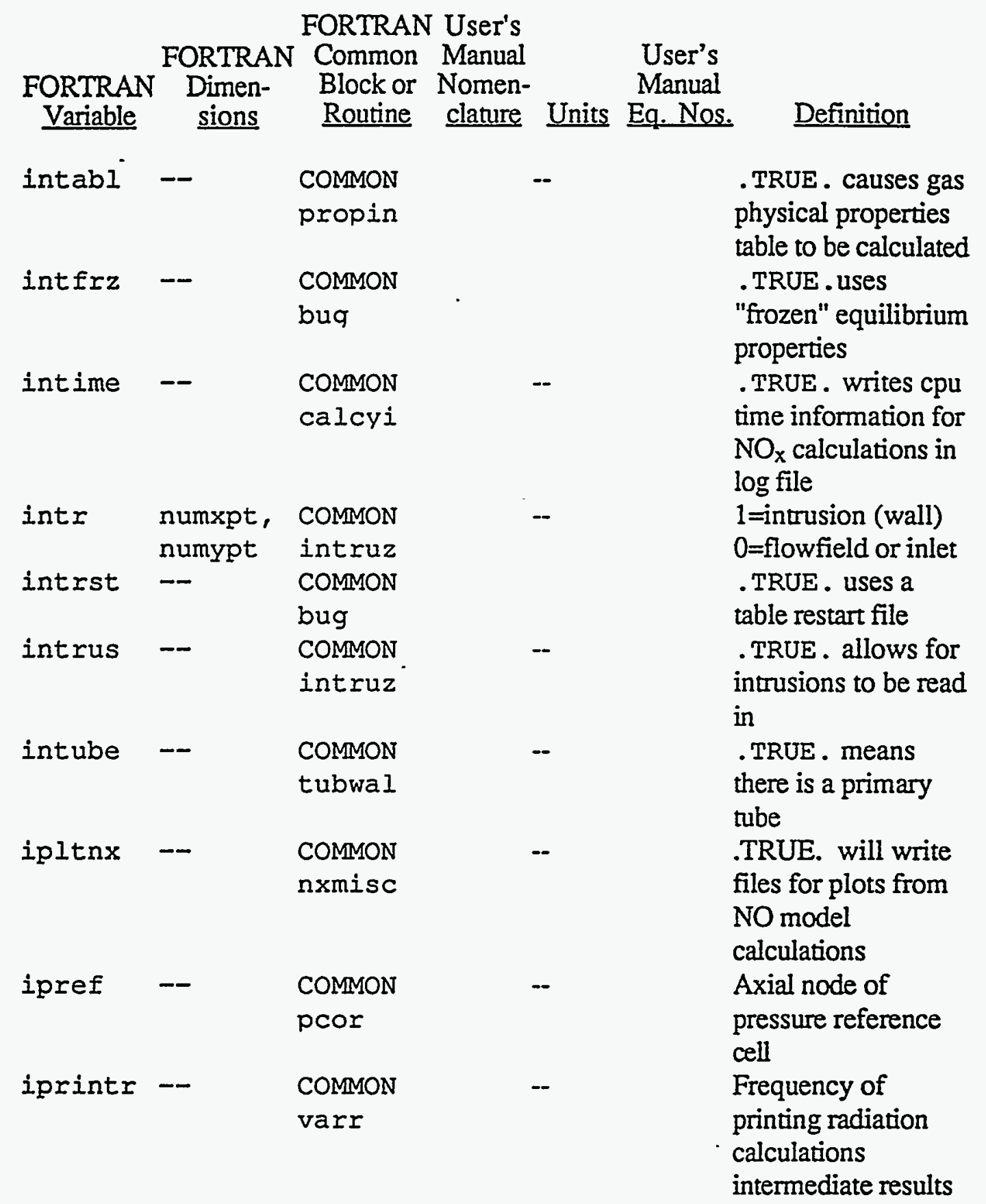




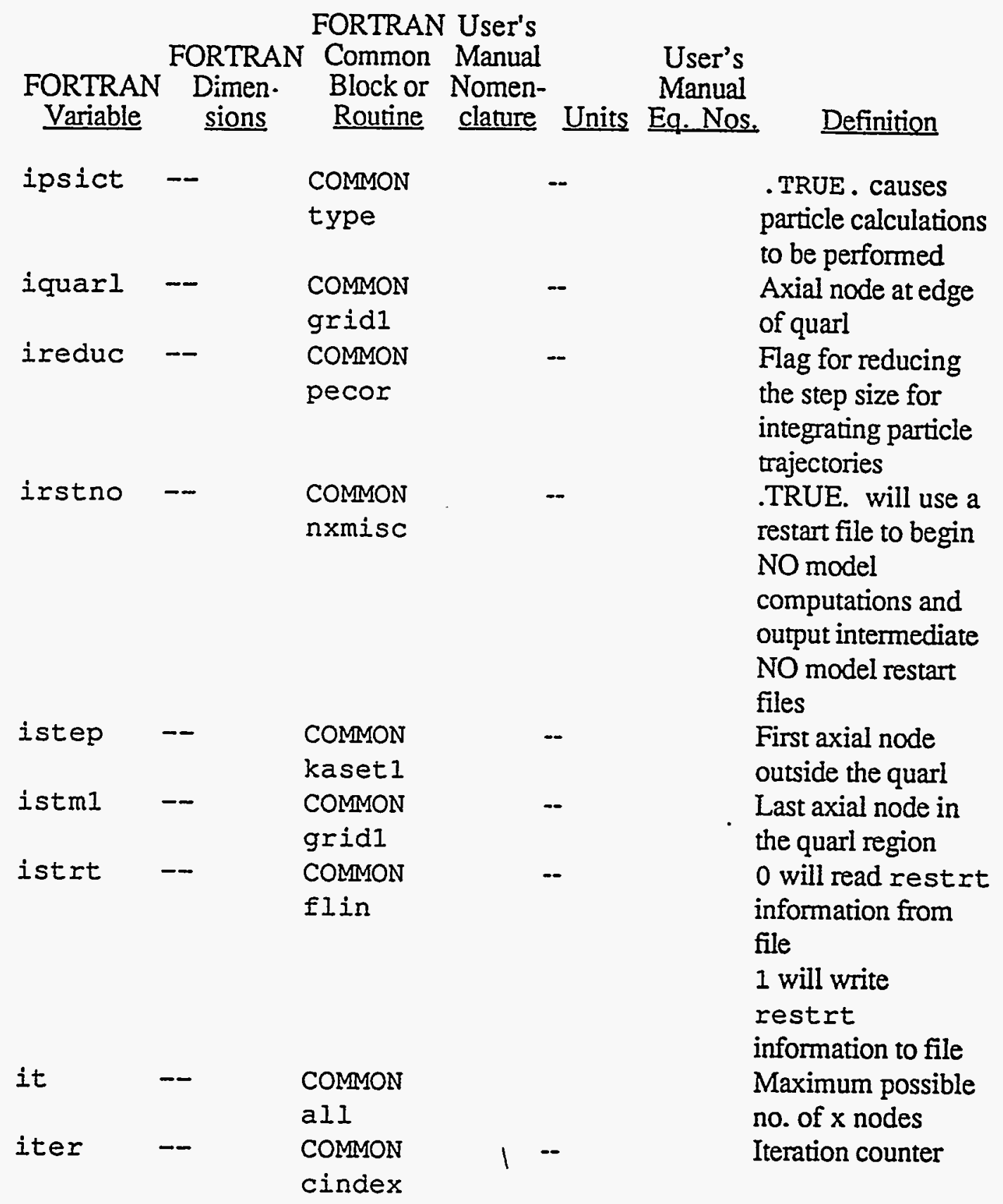




\begin{tabular}{|c|c|c|c|c|}
\hline $\begin{array}{l}\text { FORTRAN } \\
\text { Variable }\end{array}$ & $\begin{array}{l}\text { FORTRAN } \\
\text { Dimen- } \\
\text { sions }\end{array}$ & $\begin{array}{l}\text { FORTRAN User's } \\
\text { Common Manual } \\
\text { Block or Nomen- } \\
\text { Routine clature }\end{array}$ & $\begin{array}{c}\text { User's } \\
\text { Manual } \\
\text { Units Eq. Nos. }\end{array}$ & Definition \\
\hline itynx & -- & $\begin{array}{l}\text { COMMON } \\
\text { nxmisc }\end{array}$ & $\begin{array}{l}1 \\
c \\
\mathrm{~b} \\
\mathrm{i} \\
\mathrm{c}\end{array}$ & $\begin{array}{l}\text { Number of iterations } \\
\text { of the NO model } \\
\text { between } \\
\text { intermediate typing } \\
\text { of the residuals }\end{array}$ \\
\hline itype & -- & $\begin{array}{l}\text { COMMON } \\
\text { pard }\end{array}$ & -- & $\begin{array}{l}\text { Frequency of } \\
\text { printout }\end{array}$ \\
\hline ius & numstr & $\begin{array}{l}\text { COMMON } \\
\text { cpart }\end{array}$ & -- & $\begin{array}{l}\text { U-cell no. for } \\
\text { particle trajectory } \\
\text { starting locations }\end{array}$ \\
\hline ivs & numstr & $\begin{array}{l}\text { COMMON } \\
\text { cpart }\end{array}$ & I & $\begin{array}{l}\text { V-cell no. for } \\
\text { particle trajectory } \\
\text { starting locations }\end{array}$ \\
\hline iwal1 & numypt & $\begin{array}{l}\text { COMMON } \\
\text { bound }\end{array}$ & & $\begin{array}{l}\text { Axial node location } \\
\text { of the wall for each } \\
\text { radial node }\end{array}$ \\
\hline jhi & numlet & $\begin{array}{l}\text { COMMON } \\
\text { adflo }\end{array}$ & & $\begin{array}{l}\text { j node boundary for } \\
\text { additional inlet }\end{array}$ \\
\hline jlow & numlet & $\begin{array}{l}\text { COMMON } \\
\text { adflo }\end{array}$ & & $\begin{array}{l}\text { j node boundary for } \\
\text { additional inlet }\end{array}$ \\
\hline jmon & -- & $\begin{array}{l}\text { COMMON } \\
\text { pard }\end{array}$ & -- & $\begin{array}{l}\text { Radial node for } \\
\text { monitor cell }\end{array}$ \\
\hline jpref & -- & $\begin{array}{l}\text { COMMON } \\
\text { pcor }\end{array}$ & -- & $\begin{array}{l}\text { Radial node of } \\
\text { pressure reference } \\
\text { cell }\end{array}$ \\
\hline jprim & -- & $\begin{array}{l}\text { COMMON } \\
\text { noxmn } 1\end{array}$ & - & $\begin{array}{l}\text { Outermost radial } \\
\text { node in primary duct }\end{array}$ \\
\hline jquarl & -- & $\begin{array}{l}\text { COMMON } \\
\text { gridl }\end{array}$ & -- & $\begin{array}{l}\text { Radial node at edge } \\
\text { of quarl }\end{array}$ \\
\hline
\end{tabular}




\begin{tabular}{|c|c|c|c|c|c|c|}
\hline & & FORTRAI & User's & & & \\
\hline & FORTRAI & Common & Manual & & User's & \\
\hline FORTRAN & Dimen- & Block or & Nomen- & & Manual & \\
\hline Variable & $\underline{\text { sions }}$ & Routine & clature & Units & Eq. Nos. & Definition \\
\hline jsec & -- & COMMON & & - & & Outermost radial \\
\hline & & noxmn 1 & & & & $\begin{array}{l}\text { node in secondary } \\
\text { duct }\end{array}$ \\
\hline jstep & - & COMMON & & - & & First radial node in \\
\hline & & kasetI & & & & $\begin{array}{l}\text { the wall outside the } \\
\text { primary and } \\
\text { secondary }\end{array}$ \\
\hline jstpl & -- & COMMON & & - & & jstep+1 \\
\hline & & kaset 1 & & & & \\
\hline jt & -- & COMMON & & & & Maximum possible \\
\hline & & all & & & & no. of y nodes \\
\hline jtubid & -- & COMMON & & -- & & No. of the \\
\hline & & tubwal & & & & $\begin{array}{l}\text { innermost radial } \\
\text { node in the primary } \\
\text { tube wall }\end{array}$ \\
\hline jtubod & -- & COMMON & & -- & & No. of the \\
\hline & & tubwal & & & & $\begin{array}{l}\text { outermost radial } \\
\text { node in the primary } \\
\text { tube wall }\end{array}$ \\
\hline jus & numstr & COMMON & & -- & & $\mathrm{j}$-nodes for u-cells \\
\hline & & cpart & & & & $\begin{array}{l}\text { for particle starting } \\
\text { locations }\end{array}$ \\
\hline jvs & numstr & COMMON & & -- & & $\mathrm{j}$-nodes for $v$-cells \\
\hline & & cpart & & & & $\begin{array}{l}\text { for particle starting } \\
\text { locations }\end{array}$ \\
\hline jwall & numxpt & COMMON & & & & Radial node location \\
\hline & & bound & & & & $\begin{array}{l}\text { of the wall for each } \\
\text { axial node }\end{array}$ \\
\hline $\mathrm{KCJ}$ & NUMOXD & COAL2 & $k_{\mathrm{cil}}$ & $\mathrm{m} \mathrm{s}^{-1}$ & $2-134$ & $\begin{array}{l}\text { Mass transfer } \\
\text { coefficient. }\end{array}$ \\
\hline
\end{tabular}




\begin{tabular}{|c|c|c|c|c|c|c|}
\hline $\begin{array}{c}\text { FORTRAN } \\
\text { Variable }\end{array}$ & $\begin{array}{c}\text { FORTRAN } \\
\begin{array}{c}\text { Dimen- } \\
\text { sions }\end{array}\end{array}$ & $\begin{array}{l}\text { FORTRAI } \\
\text { Common } \\
\text { Block or } \\
\text { Routine } \\
\end{array}$ & $\begin{array}{l}\text { User's } \\
\text { Manual } \\
\text { Nomen- } \\
\text { clature }\end{array}$ & $\underline{\text { Units }}$ & $\begin{array}{c}\text { User's } \\
\text { Manual } \\
\text { Eq. Nos }\end{array}$ & Definition \\
\hline keq & -- & $\begin{array}{l}\text { COMMON } \\
\text { icon }\end{array}$ & & - & & $\begin{array}{l}1=\text { linear form of } \\
\text { Field eq. used for } \\
\text { char oxidation when } \\
T>1400 \mathrm{~K}\end{array}$ \\
\hline $\mathrm{kq}$ & -- & $\begin{array}{l}\text { COMMON } \\
\text { gparam }\end{array}$ & & $\begin{array}{l}\mathrm{J} \mathrm{m}^{-1} \\
\mathrm{~K}^{-1} \mathrm{~s}^{-1}\end{array}$ & & $\begin{array}{l}\text { Gas mixture thermal } \\
\text { conductivity }\end{array}$ \\
\hline$k i$ & numspe & $\begin{array}{l}\text { COMMON } \\
\text { gparam }\end{array}$ & & $\begin{array}{l}\mathrm{J} \mathrm{m}^{-1} \\
\mathrm{~K}^{-1} \mathrm{~s}^{-1}\end{array}$ & & $\begin{array}{l}\text { Species thermal } \\
\text { conductivity }\end{array}$ \\
\hline $\mathrm{KL}$ & -- & COAL2 & $k_{i l}$ & $\mathrm{~m} \mathrm{~s}^{-1}$ & $2-132$ & $\begin{array}{l}\text { Char reaction kinetic } \\
\text { rate coefficient. }\end{array}$ \\
\hline ksootab & $\begin{array}{l}\text { numxpt, } \\
\text { numypt }\end{array}$ & $\begin{array}{l}\text { COMMON } \\
\text { krad }\end{array}$ & & & & $\begin{array}{l}\text { Radiation coefficient } \\
\text { for soot }\end{array}$ \\
\hline ksootem & $\begin{array}{l}\text { numxpt, } \\
\text { numypt }\end{array}$ & $\begin{array}{l}\text { COMMON } \\
\text { krad }\end{array}$ & & & & $\begin{array}{l}\text { Radiation coefficient } \\
\text { for soot }\end{array}$ \\
\hline laminar & -- & $\begin{array}{l}\text { COMMON } \\
\text { afr }\end{array}$ & & & & $\begin{array}{l}\text {.TRUE. = laminar } \\
\text { transport properties } \\
\text { calculated locally } \\
\text {.FALSE. = laminar } \\
\text { transport properties } \\
\text { approximated } \\
\text { globally or neglected }\end{array}$ \\
\hline Ibuoy & -- & $\begin{array}{l}\text { COMMON } \\
\text { afr }\end{array}$ & & & & $\begin{array}{l}\text { TRUE } .=\text { gas } \\
\text { buoyancy included } \\
\text { in axial direction } \\
\text {.FALSE. = gas } \\
\text { buoyancy neglected }\end{array}$ \\
\hline
\end{tabular}




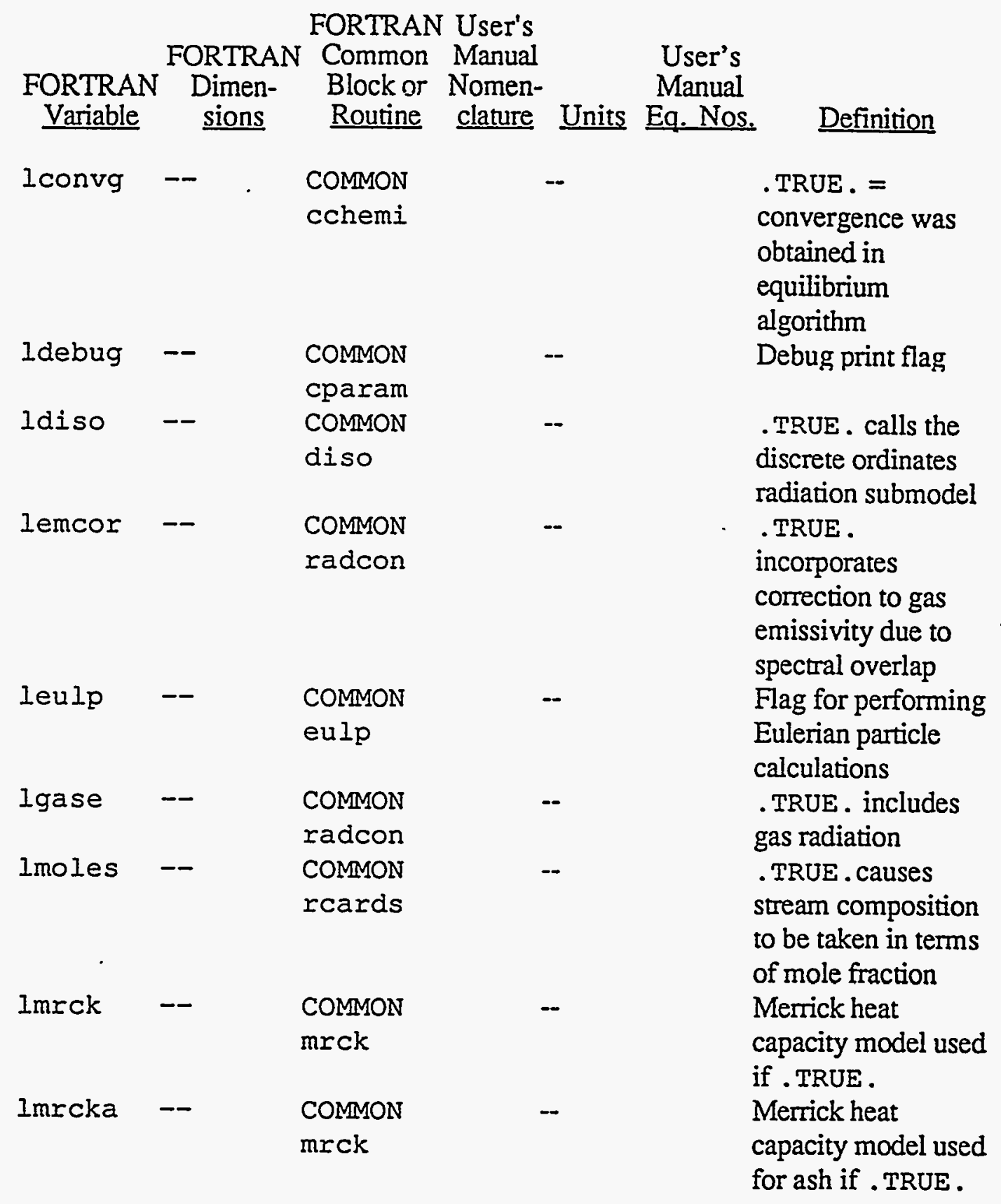




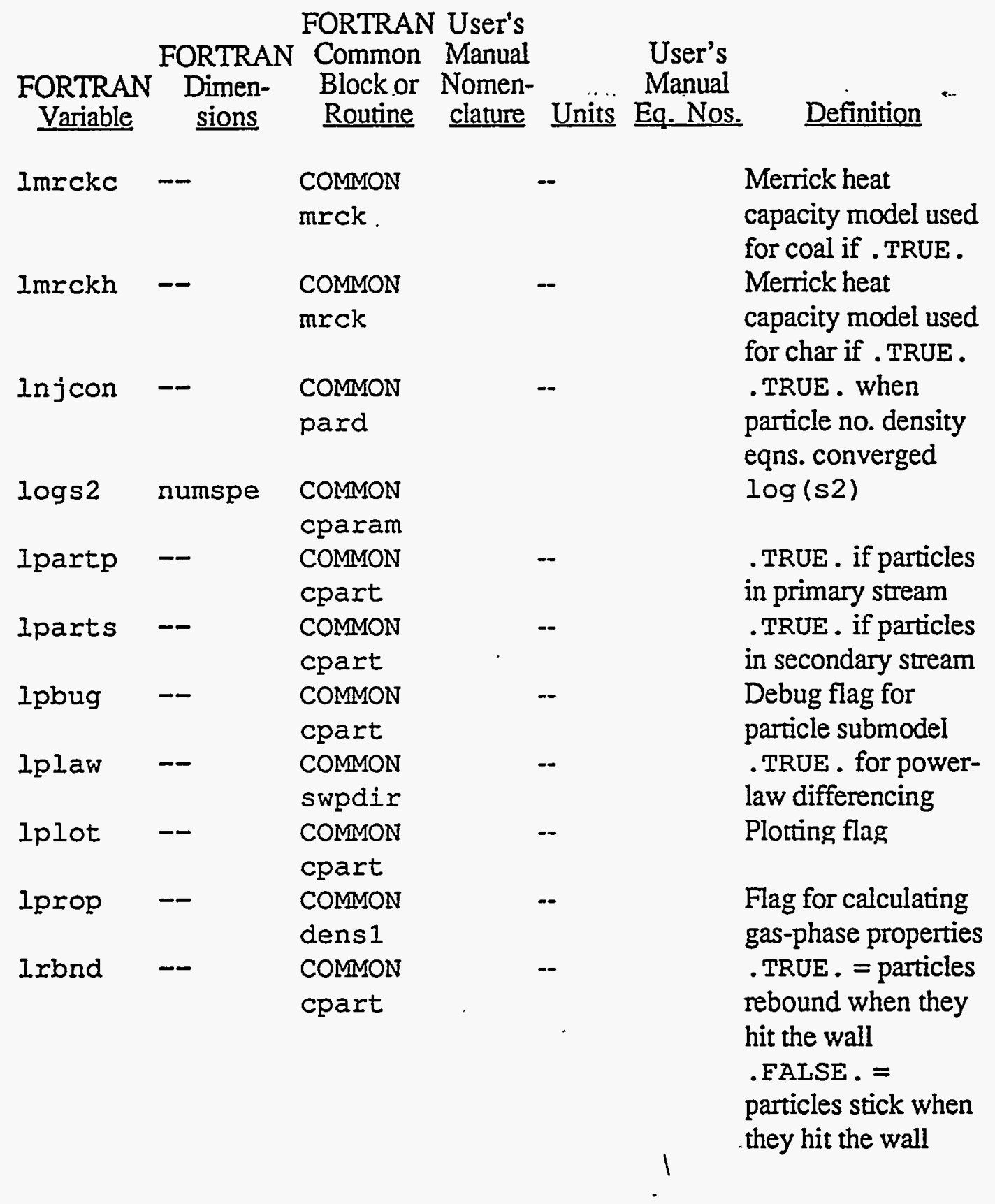




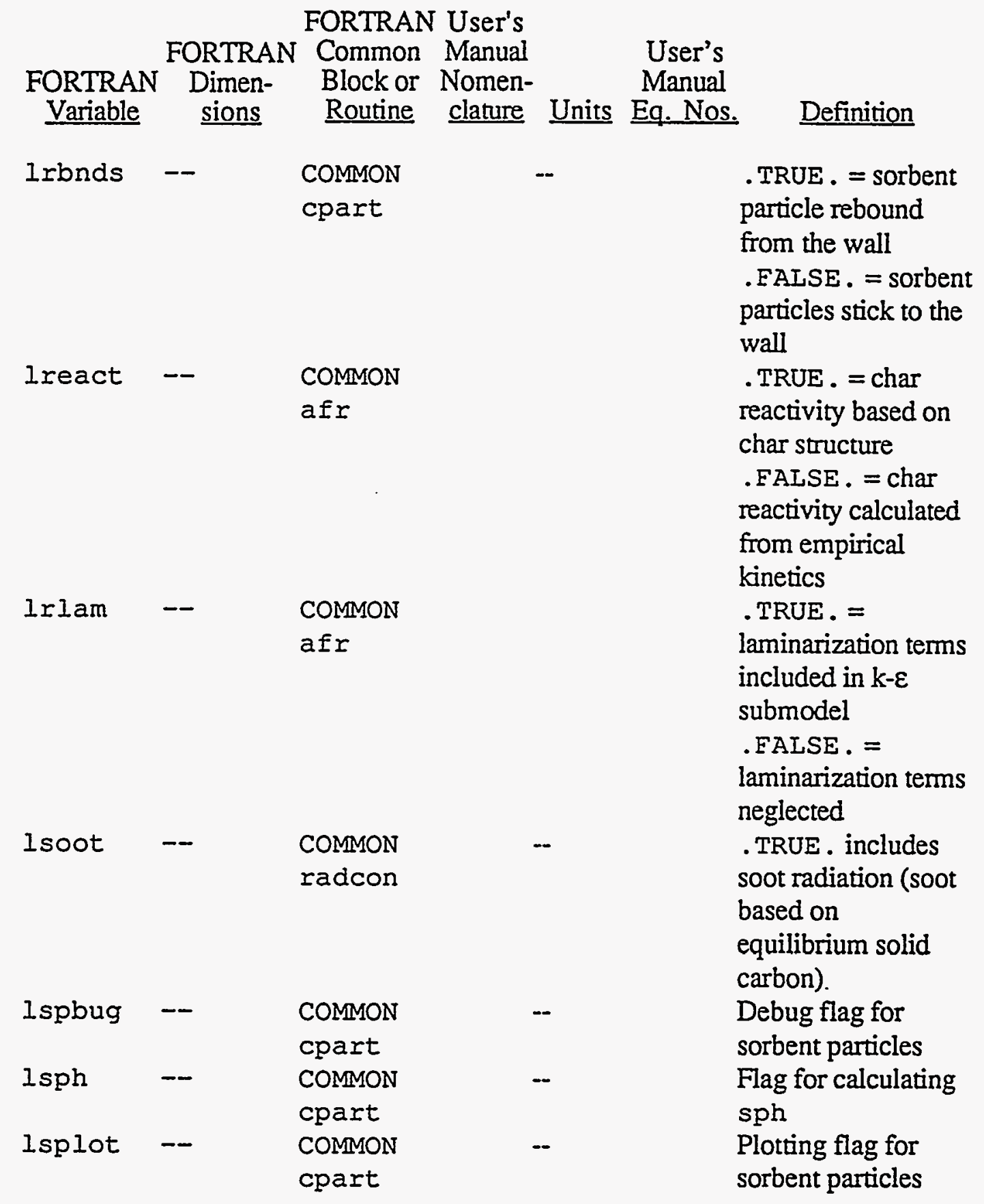




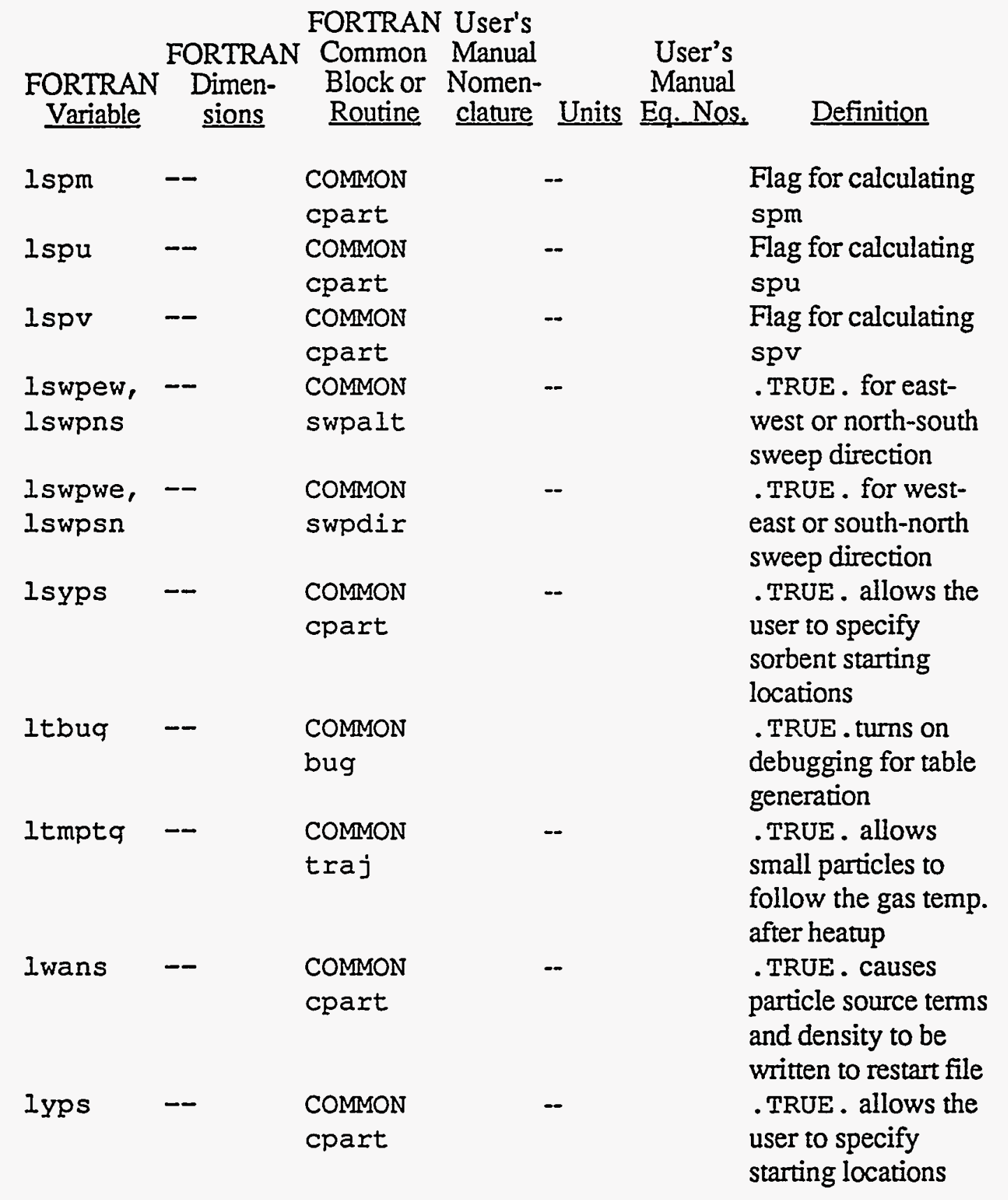




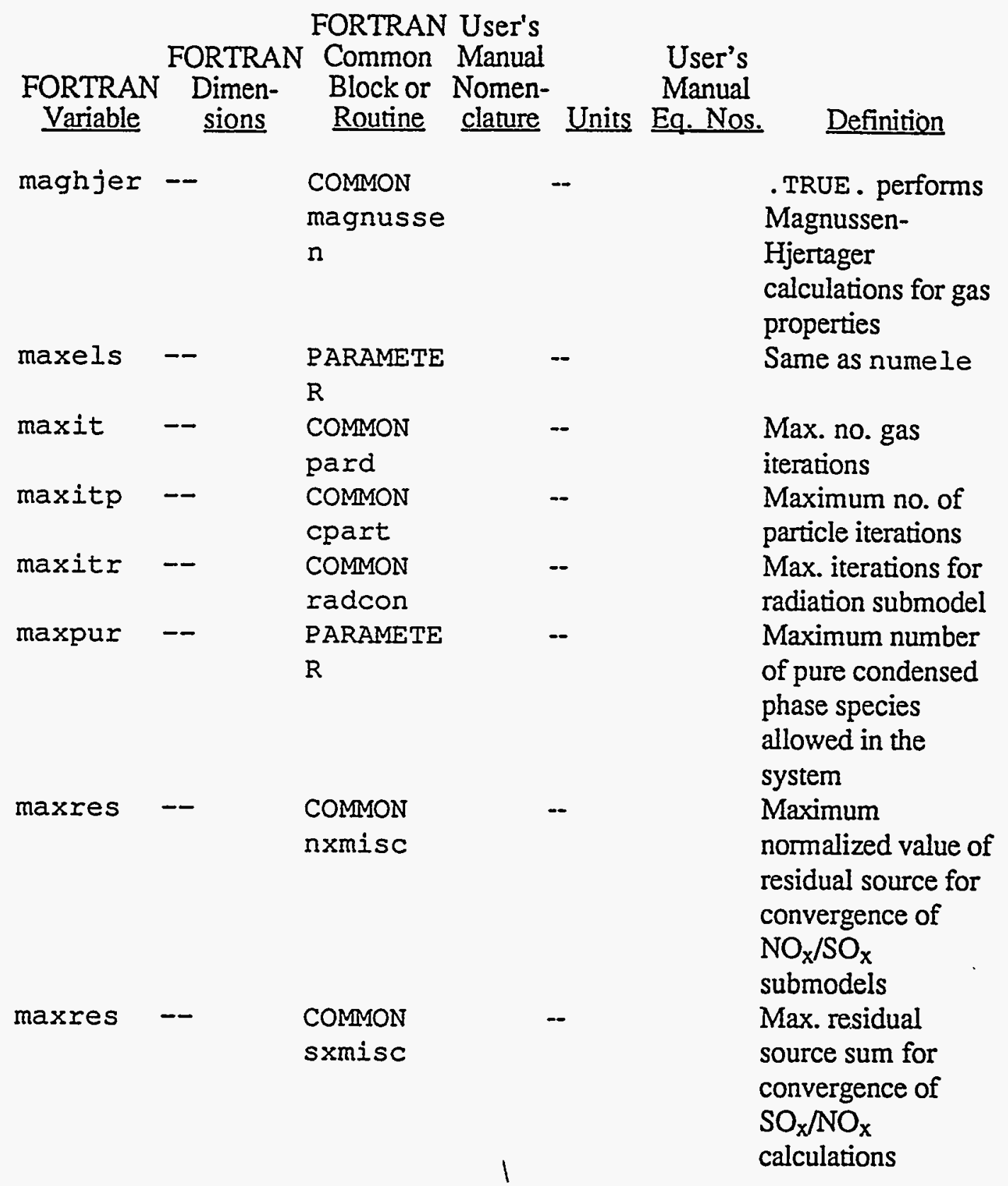




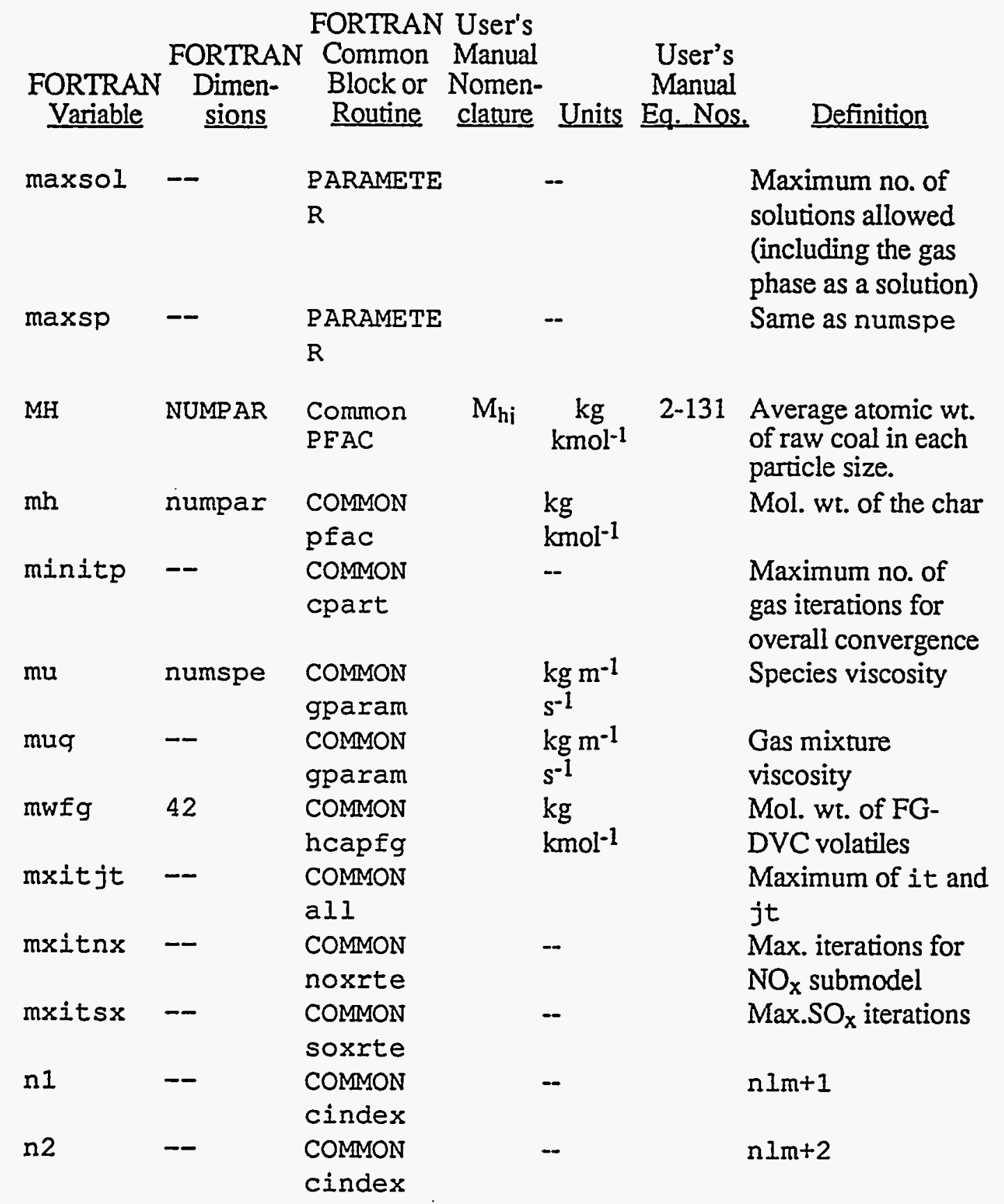




\begin{tabular}{|c|c|c|c|c|c|}
\hline \multicolumn{5}{|c|}{ FORTRAN User's } & \multirow[b]{3}{*}{ Definition } \\
\hline & FORTRAI & S Common & Manual & User's & \\
\hline $\begin{array}{l}\text { FORTRAN } \\
\text { Variable }\end{array}$ & $\begin{array}{l}\text { Dimen- } \\
\text { sions }\end{array}$ & $\begin{array}{l}\text { Block or } \\
\text { Routine }\end{array}$ & $\begin{array}{l}\text { Nomen- } \\
\text { clature }\end{array}$ & $\begin{array}{c}\text { Manual } \\
\text { Units Eq. Nos. }\end{array}$ & \\
\hline n3 & -- & $\begin{array}{l}\text { COMMON } \\
\text { cindex }\end{array}$ & & -- & $n \operatorname{lm}+3$ \\
\hline na & -- & $\begin{array}{l}\text { COMMON } \\
\text { cindex }\end{array}$ & & -- & $n s+3$ \\
\hline nc & -- & $\begin{array}{l}\text { COMMON } \\
\text { ctable }\end{array}$ & & - & $\begin{array}{l}\text { No. of components } \\
\text { (gas species) }\end{array}$ \\
\hline ncncal & -- & $\begin{array}{l}\text { COMMON } \\
\text { cparam }\end{array}$ & & -- & $\begin{array}{l}\text { No. of pure species } \\
\text { in the calculation }\end{array}$ \\
\hline ncond & -- & $\begin{array}{l}\text { COMMON } \\
\text { cparam }\end{array}$ & & -- & No. of pure species \\
\hline ncrxn & -- & $\begin{array}{l}\text { COMMON } \\
\text { icon }\end{array}$ & & -- & $\begin{array}{l}\text { No. of coal } \\
\text { reactions }\end{array}$ \\
\hline ndebug & -- & $\begin{array}{l}\text { COMMON } \\
\text { cparam }\end{array}$ & & -- & $\begin{array}{l}\text { Debug print: Higher } \\
\text { the number, the } \\
\text { more the print }\end{array}$ \\
\hline ndia & -- & $\begin{array}{l}\text { COMMON } \\
\text { gridl }\end{array}$ & & -- & $\begin{array}{l}\text { Length of reactor in } \\
\text { no. of chamber } \\
\text { diameters }\end{array}$ \\
\hline nhrxn & $-\infty$ & $\begin{array}{l}\text { COMMON } \\
\text { icon }\end{array}$ & & -- & $\begin{array}{l}\text { No. of char } \\
\text { reactions }\end{array}$ \\
\hline ni & -- & $\begin{array}{l}\text { COMMON } \\
\text { all }\end{array}$ & & & No. of $x$ nodes \\
\hline niting & -- & $\begin{array}{l}\text { COMMON } \\
\text { gridI }\end{array}$ & & -- & $\begin{array}{l}\text { No. of axial nodes } \\
\text { in quarl region }\end{array}$ \\
\hline niml & -- & $\begin{array}{l}\text { COMMON } \\
\text { aI1 }\end{array}$ & & & $\begin{array}{l}\text { No. of } x \text { nodes } \\
\text { minus } 1\end{array}$ \\
\hline ninlet & -- & $\begin{array}{l}\text { COMMON } \\
\text { adflo }\end{array}$ & & & $\begin{array}{l}\text { No. of additional } \\
\text { inlets (beyond the } \\
\text { primary and } \\
\text { secondary) }\end{array}$ \\
\hline
\end{tabular}




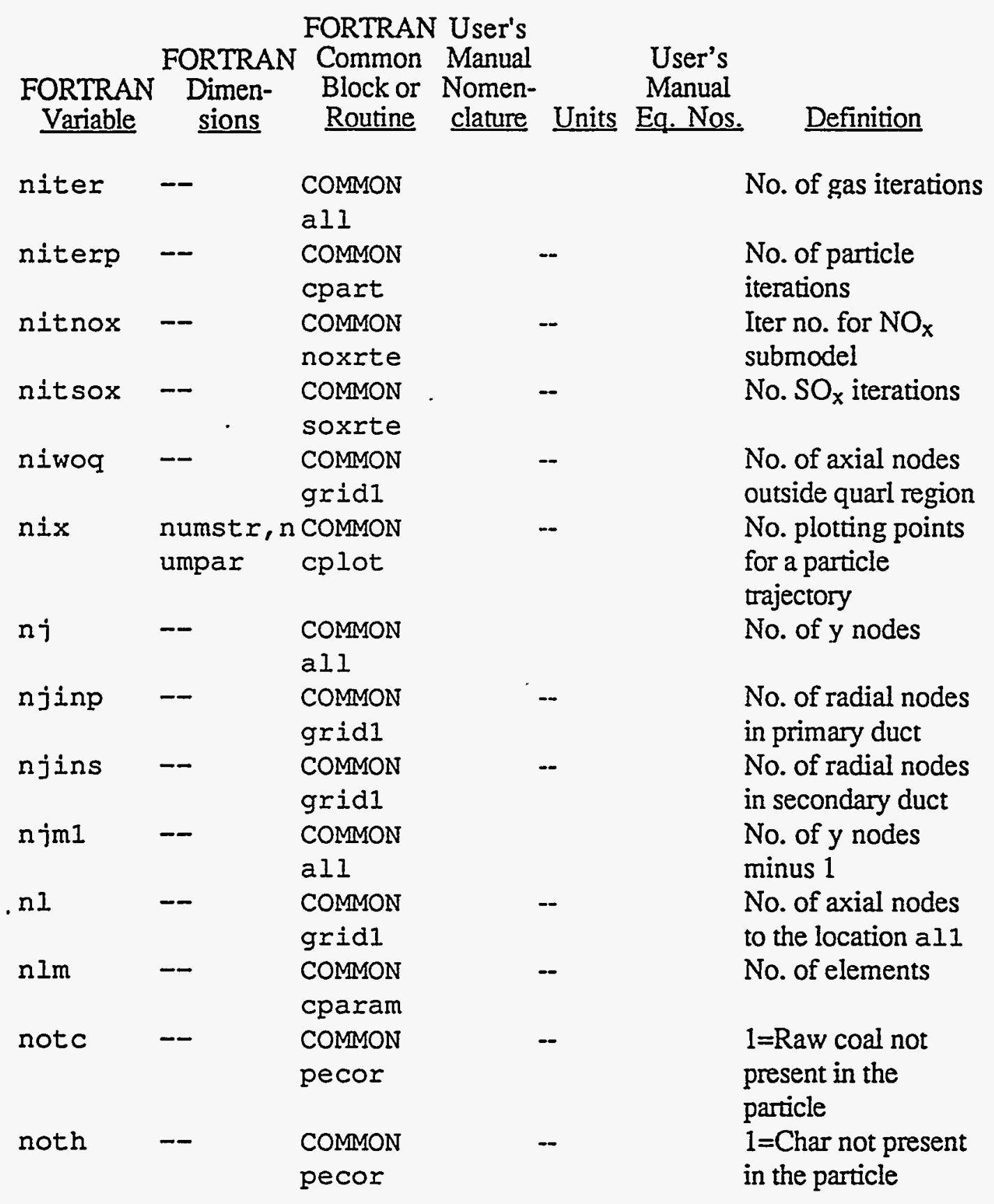




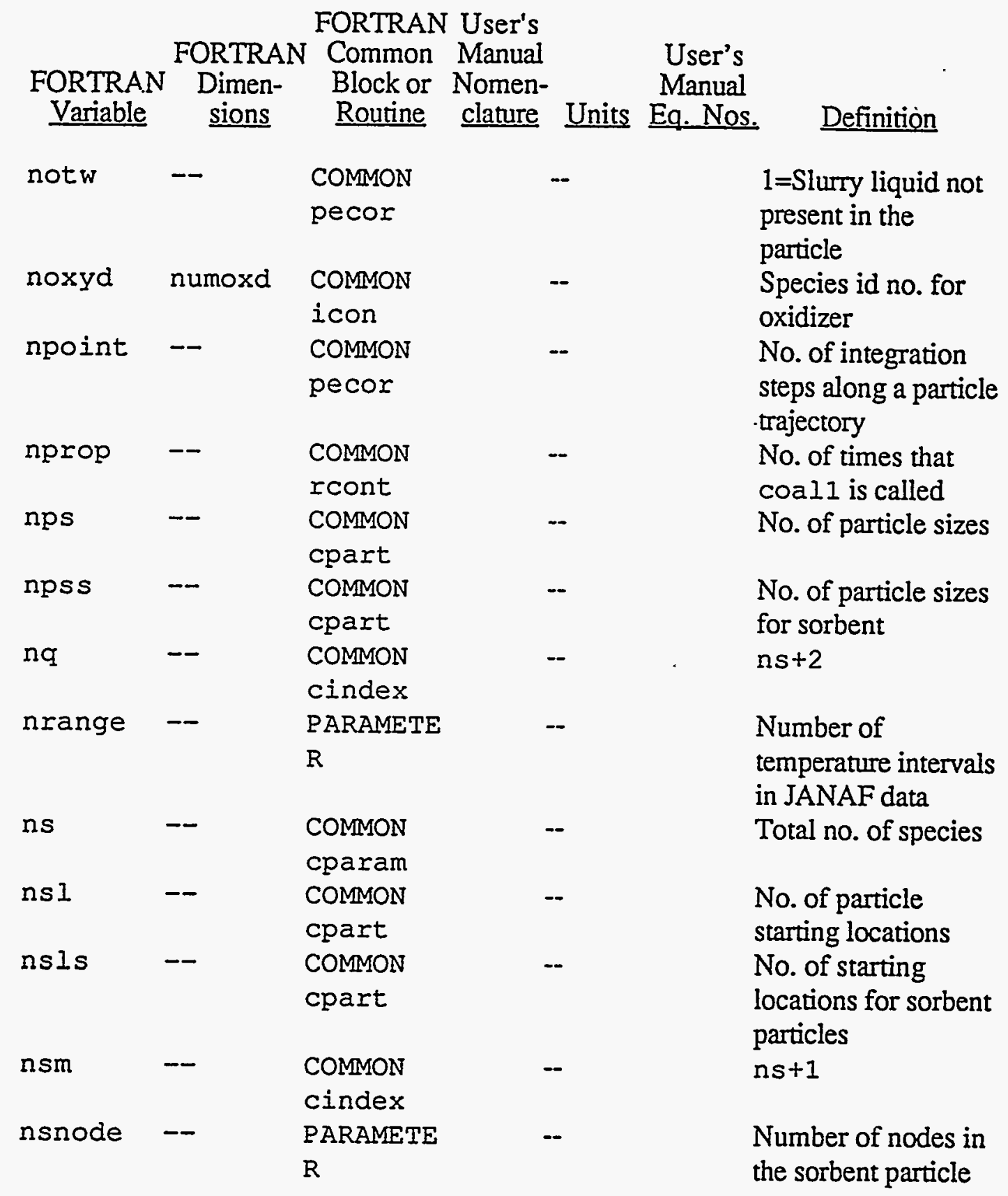




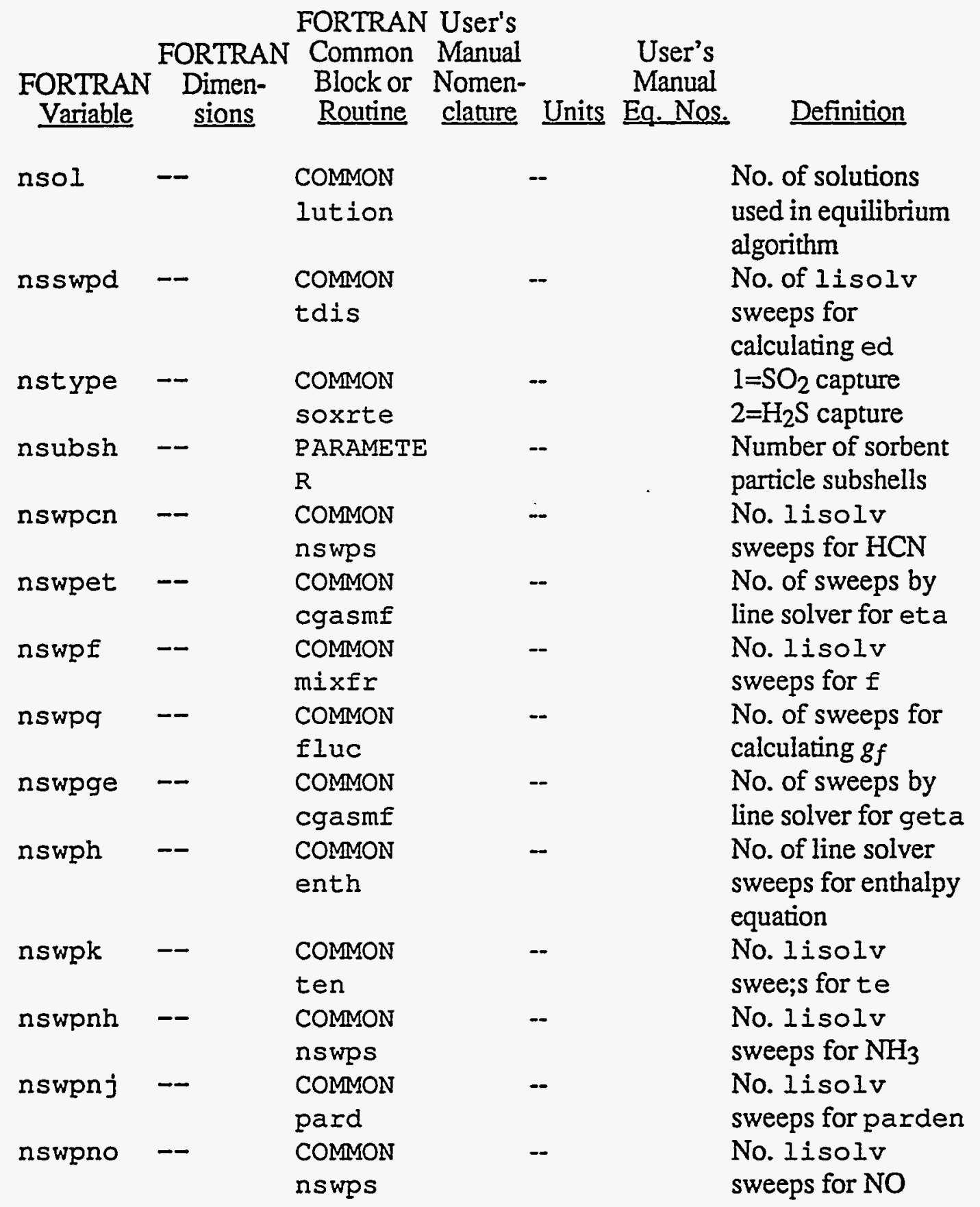




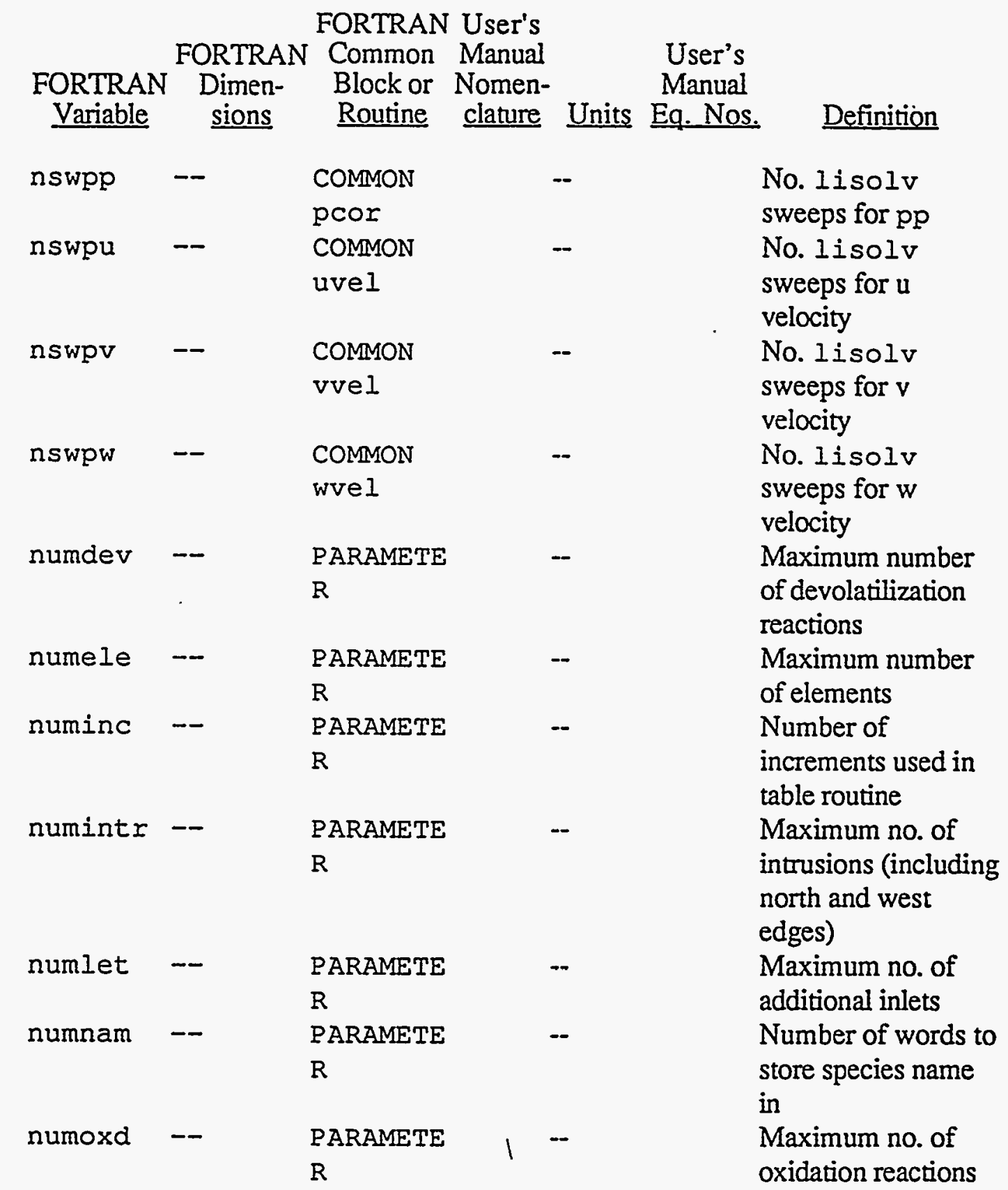


FORTRAN User's

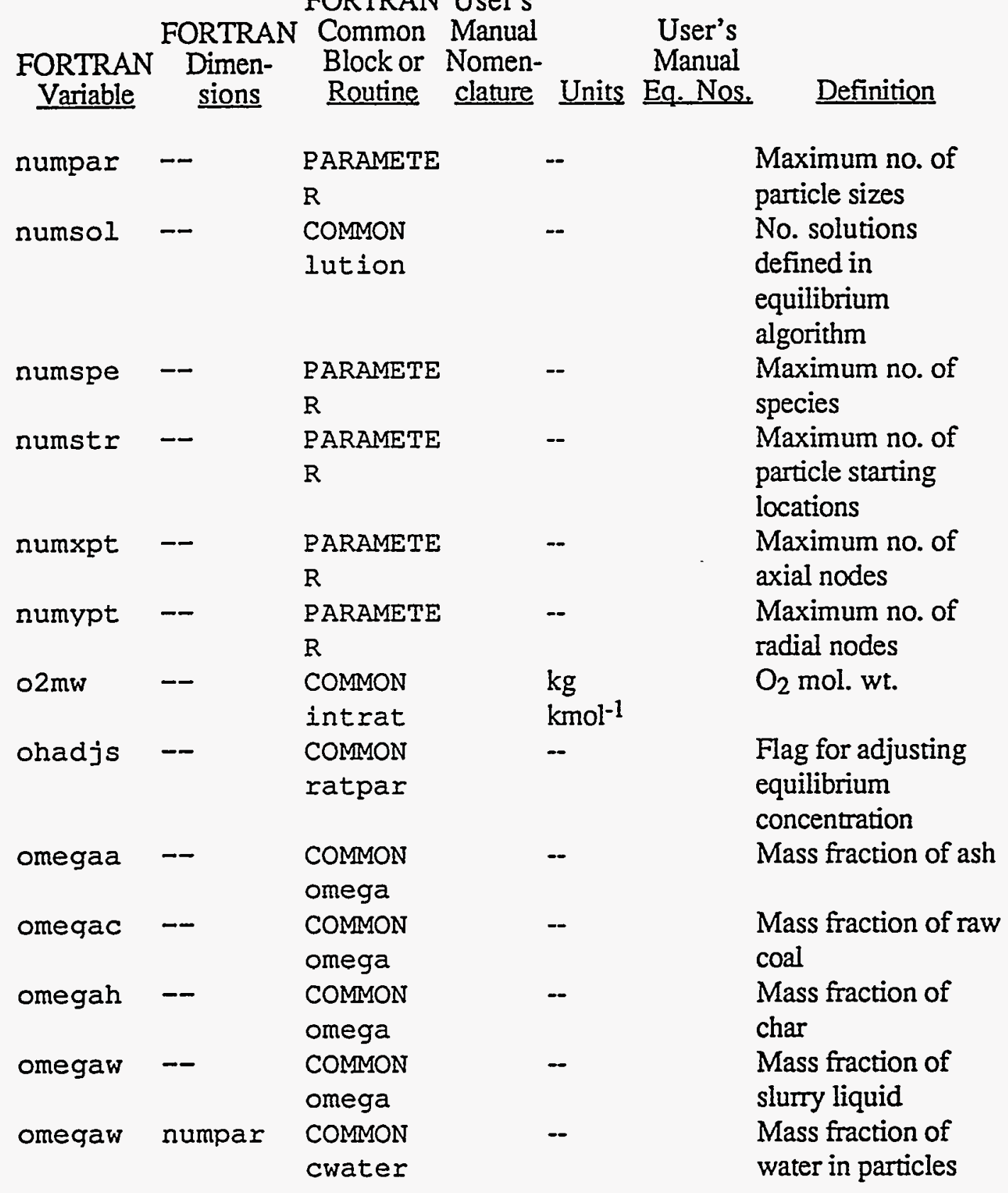




\begin{tabular}{|c|c|c|c|c|c|c|}
\hline $\begin{array}{l}\text { FORTRAN } \\
\text { Variable }\end{array}$ & $\begin{array}{c}\text { FORTRAN } \\
\text { Dimen- } \\
\text { sions }\end{array}$ & $\begin{array}{l}\text { FORTRAI } \\
\text { Common } \\
\text { Block or } \\
\text { Routine }\end{array}$ & $\begin{array}{l}\text { User's } \\
\text { Manual } \\
\text { Nomen- } \\
\text { clature }\end{array}$ & Units & $\begin{array}{c}\text { User's } \\
\text { Manual } \\
\text { Eq. Nos. }\end{array}$ & Definition \\
\hline opl & -- & $\begin{array}{l}\text { COMMON } \\
\text { radcon }\end{array}$ & & m & & Optical length \\
\hline$p$ & $\begin{array}{l}\text { numxpt, n } \\
\text { umypt }\end{array}$ & $\begin{array}{l}\text { COMMON } \\
\text { var }\end{array}$ & & $\mathrm{Pa}$ & & Pressure \\
\hline pa & -- & COMMON & & $\mathrm{Pa}$ & & Pressure \\
\hline parden & $\begin{array}{l}\text { numxpt, n } \\
\text { umypt, nu } \\
\text { mpar }\end{array}$ & $\begin{array}{l}\text { cparam } \\
\text { COMMON } \\
\text { cpeul }\end{array}$ & $\bar{n}_{\mathbf{j}}$ & & $2-35$ & Particle no. density \\
\hline pcell & $\begin{array}{l}\text { numxpt, } \\
\text { numypt }\end{array}$ & $\begin{array}{l}\text { COMMON } \\
\text { gmap }\end{array}$ & & -- & & $\begin{array}{l}X=\text { wall cell } \\
O=\text { flowfield cell } \\
P=\text { primary cell } \\
S=\text { secondary cell } \\
1,2,3=\text { additional } \\
\text { inlets }\end{array}$ \\
\hline pd & numpar & $\begin{array}{l}\text { COMMON } \\
\text { cpart }\end{array}$ & & $\mathrm{m}$ & & Particle sizes \\
\hline pden & -- & $\begin{array}{l}\text { COMMON } \\
\text { cpart }\end{array}$ & & $\mathrm{kg} \mathrm{m}^{3}$ & & Particle density \\
\hline pds & numpar & $\begin{array}{l}\text { COMMON } \\
\text { cpart }\end{array}$ & & $\mathrm{m}$ & & $\begin{array}{l}\text { Sorbent particle } \\
\text { sizes }\end{array}$ \\
\hline pecwt & maxrea & $\begin{array}{l}\text { COMMON } \\
\text { rcards }\end{array}$ & & -- & & $\begin{array}{l}\text { Percent of each } \\
\text { reactant in each } \\
\text { stream }\end{array}$ \\
\hline pfunc & -- & $\begin{array}{l}\text { COMMON } \\
\text { turb }\end{array}$ & & & & $\begin{array}{l}\text { Extra resistance to } \\
\text { heat transfer at the } \\
\text { wall that arises due } \\
\text { to the difference in } \\
\sigma_{h} \text { and } \sigma_{h, t}\end{array}$ \\
\hline
\end{tabular}




\begin{tabular}{|c|c|c|c|c|c|c|}
\hline $\begin{array}{l}\text { FORTRAN } \\
\text { Variable }\end{array}$ & $\begin{array}{c}\text { FORTRAN } \\
\begin{array}{c}\text { Dimen- } \\
\text { sions }\end{array}\end{array}$ & $\begin{array}{l}\text { FORTRAN } \\
\text { Common } \\
\text { Block or } \\
\text { Routine } \\
\end{array}$ & $\begin{array}{l}\text { User's } \\
\text { Manual } \\
\text { Nomen- } \\
\text { clature }\end{array}$ & Units & $\begin{array}{c}\text { User's } \\
\text { Manual } \\
\text { Eq. Nos. }\end{array}$ & Definition \\
\hline PHII & NUMOXD & $\begin{array}{l}\text { Common } \\
\text { RRAT }\end{array}$ & $\phi_{1}$ & -- & $2-96$ & $\begin{array}{l}\text { Moles of carbon } \\
\text { required per mole of } \\
\text { oxidizer. }\end{array}$ \\
\hline phil & numoxd & $\begin{array}{l}\text { COMMON } \\
\text { rrat }\end{array}$ & & - & & $\begin{array}{l}\text { Moles of fuel per } \\
\text { mole of oxidizer for } \\
\text { heterogeneous } \\
\text { reaction }\end{array}$ \\
\hline pi & -- & $\begin{array}{l}\text { COMMON } \\
\text { geom }\end{array}$ & & -- & & The number pi \\
\hline PIE & & & & & & 3.14159 \\
\hline piehcn & $\begin{array}{l}\text { numxpt, } \\
\text { numypt }\end{array}$ & $\begin{array}{l}\text { COMMON } \\
\text { ratecm }\end{array}$ & & -- & & $\begin{array}{l}\text { Deviation from } \\
\text { equilibrium for } \\
\text { HCN }\end{array}$ \\
\hline pien2 & $\begin{array}{l}\text { numxpt, } \\
\text { numypt }\end{array}$ & $\begin{array}{l}\text { COMMON } \\
\text { ratecm }\end{array}$ & & -- & & $\begin{array}{l}\text { Deviation from } \\
\text { equilibrium for } \mathrm{N}_{2}\end{array}$ \\
\hline pienh3 & $\begin{array}{l}\text { numxpt, } \\
\text { numypt }\end{array}$ & $\begin{array}{l}\text { COMMON } \\
\text { ratecm }\end{array}$ & & -- & & $\begin{array}{l}\text { Deviation from } \\
\text { equilibrium for } \mathrm{NH}_{3}\end{array}$ \\
\hline pienof & $\begin{array}{l}\text { numxpt, } \\
\text { numypt }\end{array}$ & $\begin{array}{l}\text { COMMON } \\
\text { ratecm }\end{array}$ & & -- & & $\begin{array}{l}\text { Deviation from } \\
\text { equilibrium for } \mathrm{NO}\end{array}$ \\
\hline pienot & $\begin{array}{l}\text { numxpt, } \\
\text { numypt }\end{array}$ & $\begin{array}{l}\text { COMMON } \\
\text { ratecm }\end{array}$ & & -- & & $\begin{array}{l}\text { Deviation from } \\
\text { equilibrium for NO }\end{array}$ \\
\hline pieo2 & $\begin{array}{l}\text { numxpt, } \\
\text { numypt }\end{array}$ & $\begin{array}{l}\text { COMMON } \\
\text { ratecm }\end{array}$ & & - & & $\begin{array}{l}\text { Deviation from } \\
\text { equilibrium for } \mathrm{O}_{2}\end{array}$ \\
\hline pieoh & $\begin{array}{l}\text { numxpt, } \\
\text { numypt }\end{array}$ & $\begin{array}{l}\text { COMMON } \\
\text { ratecm }\end{array}$ & & - & & $\begin{array}{l}\text { Deviation from } \\
\text { equilibrium for } \mathrm{OH}\end{array}$ \\
\hline pload & -- & $\begin{array}{l}\text { COMMON } \\
\text { cpart }\end{array}$ & & $\mathrm{kg} \mathrm{kg}^{-1}$ & & $\begin{array}{l}\text { Total particle } \\
\text { loading }\end{array}$ \\
\hline ploada & numlet & $\begin{array}{l}\text { COMMON } \\
\text { Cgsflo }\end{array}$ & & $\mathrm{kg} \mathrm{kg}^{-1}$ & 1 & $\begin{array}{l}\text { Coal particle loading } \\
\text { in additional inlet }\end{array}$ \\
\hline
\end{tabular}




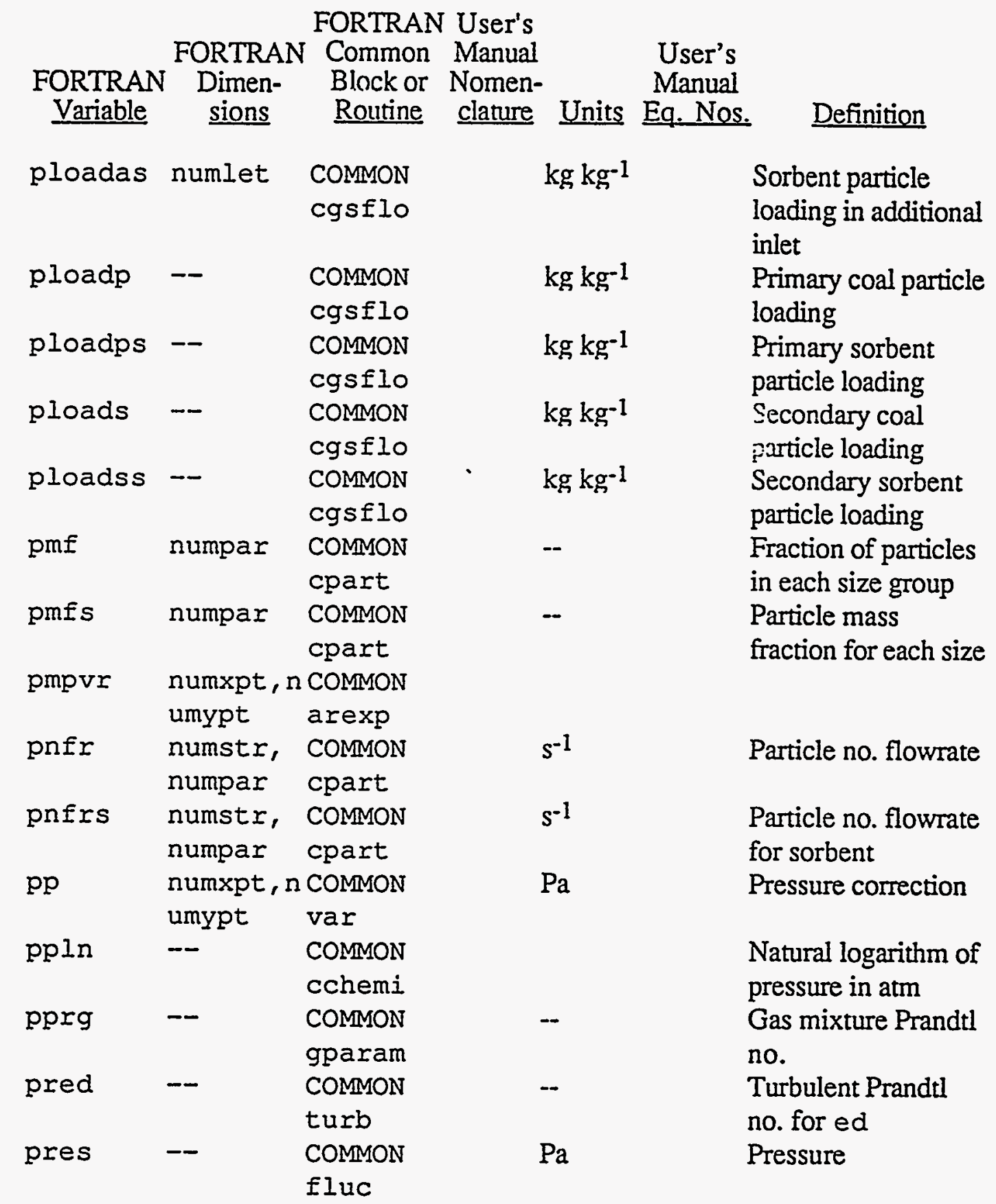




\begin{tabular}{|c|c|c|c|c|c|c|}
\hline \multicolumn{6}{|c|}{ FORTRAN User's } & \\
\hline & FORTRAN & Common & Manual & & User's & \\
\hline FORTRAN & Dimen- & Block or & Nomen- & Inits & Manual & Definition \\
\hline Vanable & & Kounne & clanure & Units & Eq. INos. & Derininon \\
\hline
\end{tabular}

\begin{tabular}{|c|c|c|c|c|}
\hline PRESP & $-\infty$ & $\begin{array}{l}\text { Common } \\
\text { TRAJ }\end{array}$ & $\mathrm{Pa}$ & $\begin{array}{l}\text { Local gas pressure } \\
\text { (assumed constant } \\
\text { across a cell) }\end{array}$ \\
\hline presp & -- & $\begin{array}{l}\text { COMMON } \\
\text { traj }\end{array}$ & $\mathrm{Pa}$ & Gas pressure \\
\hline preta & -- & $\begin{array}{l}\text { COMMON } \\
\text { cgasmf }\end{array}$ & -- & Prandtl no. for eta \\
\hline prf & -- & $\begin{array}{l}\text { COMMON } \\
\text { turb }\end{array}$ & -- & $\begin{array}{l}\text { Turbulent Prandtl } \\
\text { no. for } f\end{array}$ \\
\hline prg & -- & $\begin{array}{l}\text { COMMON } \\
\text { turb }\end{array}$ & -- & $\begin{array}{l}\text { Turbulent Prandtl } \\
\text { no. for } g\end{array}$ \\
\hline prgeta & -- & $\begin{array}{l}\text { COMMON } \\
\text { cgasmf }\end{array}$ & -- & $\begin{array}{l}\text { Prandtl no. for } \\
\text { geța }\end{array}$ \\
\hline prh2s & -- & $\begin{array}{l}\text { COMMON } \\
\text { ratesx }\end{array}$ & -- & Prandtl no. for $\mathrm{H}_{2} \mathrm{~S}$ \\
\hline prhen & -- & $\begin{array}{l}\text { COMMON } \\
\text { calcyi }\end{array}$ & -- & $\begin{array}{l}\text { Prandtl no. for } \\
\text { HCN transport }\end{array}$ \\
\hline prht & -- & $\begin{array}{l}\text { COMMON } \\
\text { turb }\end{array}$ & -- & $\begin{array}{l}\text { Turbulent Prandtl } \\
\text { no. for } h\end{array}$ \\
\hline prhtl & $\begin{array}{l}\text { numxpt, } \\
\text { numypt }\end{array}$ & $\begin{array}{l}\text { COMMON } \\
\text { fluc }\end{array}$ & -- & $\begin{array}{l}\text { Laminar Prandtl no. } \\
\text { for heat transfer }\end{array}$ \\
\hline prhtIf0 & -- & $\begin{array}{l}\text { COMMON } \\
\text { flafo }\end{array}$ & -- & $\begin{array}{l}\text { Prandtl no. for } f=0 \\
\text { stream }\end{array}$ \\
\hline prhtlf1 & $-\infty$ & $\begin{array}{l}\text { COMMON } \\
\text { flaf0 }\end{array}$ & -- & $\begin{array}{l}\text { Prandtl no. for } f=1 \\
\text { stream }\end{array}$ \\
\hline $\begin{array}{l}\text { prhtlinl } \\
\text { et }\end{array}$ & numlet & $\begin{array}{l}\text { COMMON } \\
\text { adflo }\end{array}$ & & $\begin{array}{l}\text { Laminar Prandtl no. } \\
\text { for additional inlet }\end{array}$ \\
\hline prhtlp & -- & $\begin{array}{l}\text { COMMON } \\
\text { pands }\end{array}$ & -- & $\begin{array}{l}\text { Primary stream } \\
\text { laminar Prandtl no. }\end{array}$ \\
\hline
\end{tabular}


FORTRAN User's

\begin{tabular}{|c|c|c|c|c|c|c|}
\hline $\begin{array}{l}\text { FORTRAN } \\
\text { Variable }\end{array}$ & $\begin{array}{c}\text { FORTRAI } \\
\begin{array}{c}\text { Dimen- } \\
\text { sions }\end{array}\end{array}$ & $\begin{array}{l}\text { Common } \\
\text { Block or } \\
\text { Routine }\end{array}$ & $\begin{array}{l}\text { Manual } \\
\text { Nomen- } \\
\text { clature }\end{array}$ & Units & $\begin{array}{l}\text { User's } \\
\text { Manual } \\
\text { Eq. Nos. }\end{array}$ & Definition \\
\hline prhtls & - & $\begin{array}{l}\text { COMMON } \\
\text { pands }\end{array}$ & & -- & & $\begin{array}{l}\text { Secondary stream } \\
\text { laminar Prandtl no. }\end{array}$ \\
\hline prhtlsct & $=-$ & $\begin{array}{l}\text { COMMON } \\
\text { pvar }\end{array}$ & & $\begin{array}{l}\mathrm{kg} \\
\mathrm{kmol}^{-1}\end{array}$ & & $\begin{array}{l}\text { Coal offgas laminar } \\
\text { Prandtl no. }\end{array}$ \\
\hline prk & numpar & $\begin{array}{l}\text { COMMON } \\
\text { cpeul }\end{array}$ & $\sigma_{i}^{l}$ & -- & $2-28$ & $\begin{array}{l}\text { Turbulent Prandtl } \\
\text { (Schmidt) no. for } \\
\text { coal particle } \\
\text { diffusion }\end{array}$ \\
\hline prks & - & $\begin{array}{l}\text { COMMON } \\
\text { cpeul }\end{array}$ & & -- & & $\begin{array}{l}\text { Turbulent Prandtl } \\
\text { (Schmidt) no. for } \\
\text { sorbent particle } \\
\text { diffusion }\end{array}$ \\
\hline prnh3 & - & $\begin{array}{l}\text { COMMON } \\
\text { calcyi }\end{array}$ & & -- & & Prandtl no. for $\mathrm{NH}_{3}$ \\
\hline prnox & - & $\begin{array}{l}\text { COMMON } \\
\text { calcyi }\end{array}$ & & -- & & $\begin{array}{l}\text { Prandtl no. for } \mathrm{NO}_{\mathrm{x}} \\
\text { transport }\end{array}$ \\
\hline prso2 & -- & $\begin{array}{l}\text { COMMON } \\
\text { ratesx }\end{array}$ & & -- & & Prandtl no. for $\mathrm{SO}_{2}$ \\
\hline prte & -- & $\begin{array}{l}\text { COMMON } \\
\text { turb }\end{array}$ & & -- & & $\begin{array}{l}\text { Turbulent Prandtl } \\
\text { no. for te }\end{array}$ \\
\hline pw & numstr & $\begin{array}{l}\text { COMMON } \\
\text { cpart }\end{array}$ & & $\mathrm{kg} \mathrm{s}^{-1}$ & & $\begin{array}{l}\text { Total particle mass } \\
\text { flowrate for a } \\
\text { starting location }\end{array}$ \\
\hline qab & numpar & $\begin{array}{l}\text { COMMON } \\
\text { radcon }\end{array}$ & & -- & & $\begin{array}{l}\text { Particle absorption } \\
\text { coefficient }\end{array}$ \\
\hline qcflxe & $\begin{array}{l}\text { numxpt, } \\
\text { numypt }\end{array}$ & $\begin{array}{l}\text { COMMON } \\
\text { heatcp }\end{array}$ & & $\mathrm{J} \mathrm{m}_{1}^{-2} \mathrm{~s}^{-}$ & & $\begin{array}{l}\text { Convective heat flux } \\
\text { to east faces of } \\
\text { intrusions }\end{array}$ \\
\hline geflxs & $\begin{array}{l}\text { numxpt, } \\
\text { numypt }\end{array}$ & $\begin{array}{l}\text { COMMON } \\
\text { heatcp }\end{array}$ & 1 & $\mathrm{~J}_{1}^{\mathrm{J} \mathrm{m}^{-2} \mathrm{~s}^{-}}$ & & $\begin{array}{l}\text { Convective heat flux } \\
\text { to south faces of } \\
\text { intrusions }\end{array}$ \\
\hline
\end{tabular}




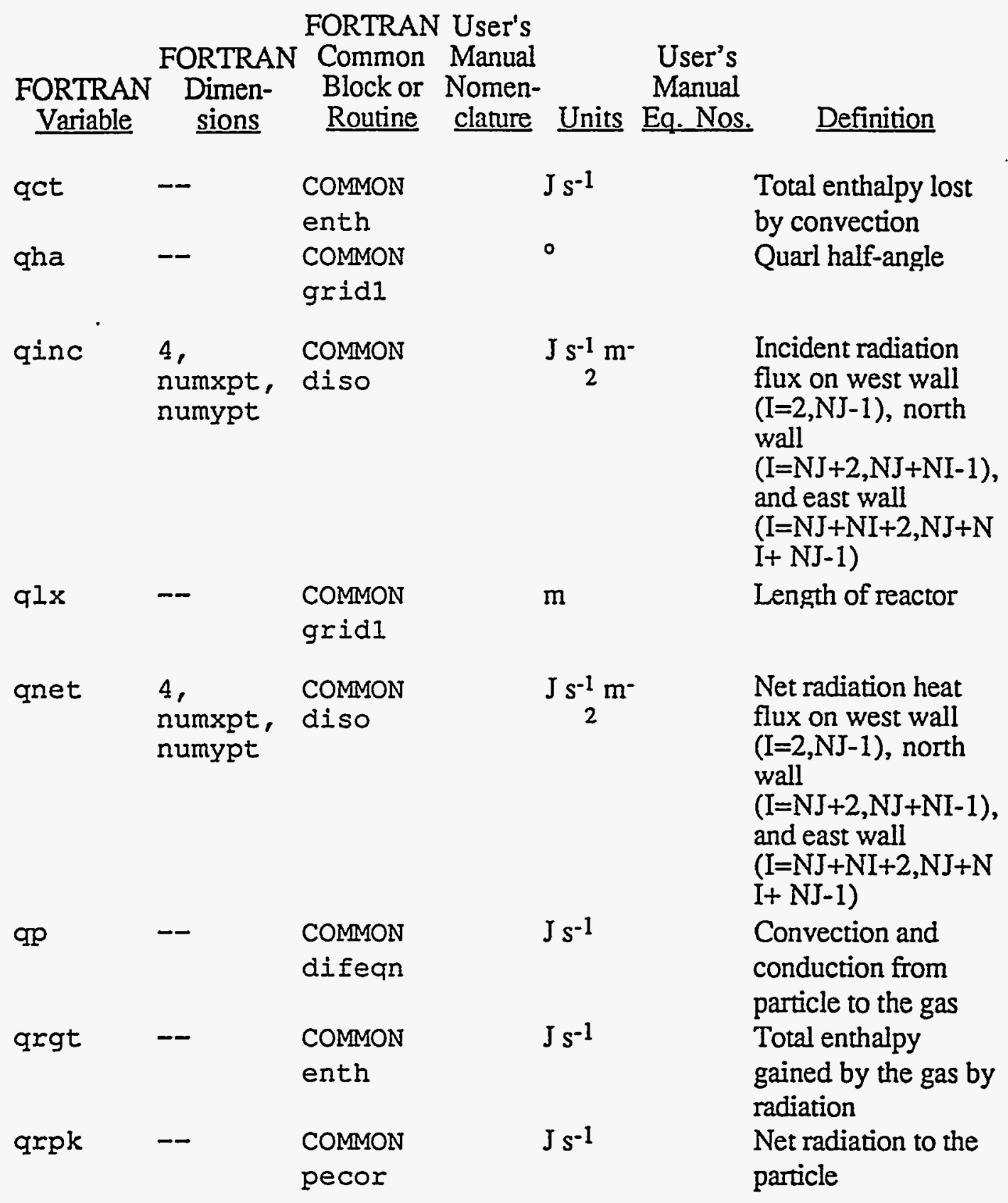




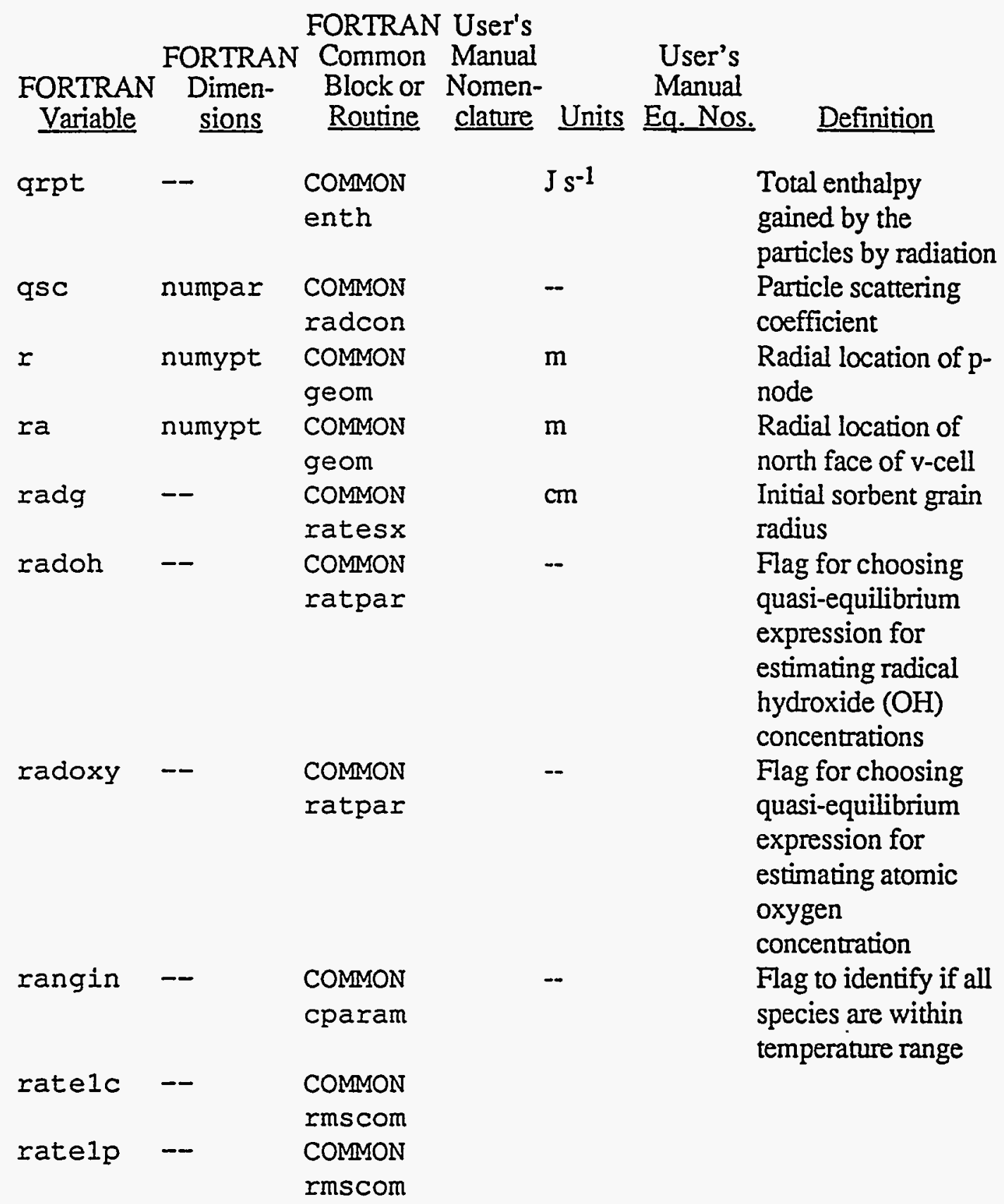




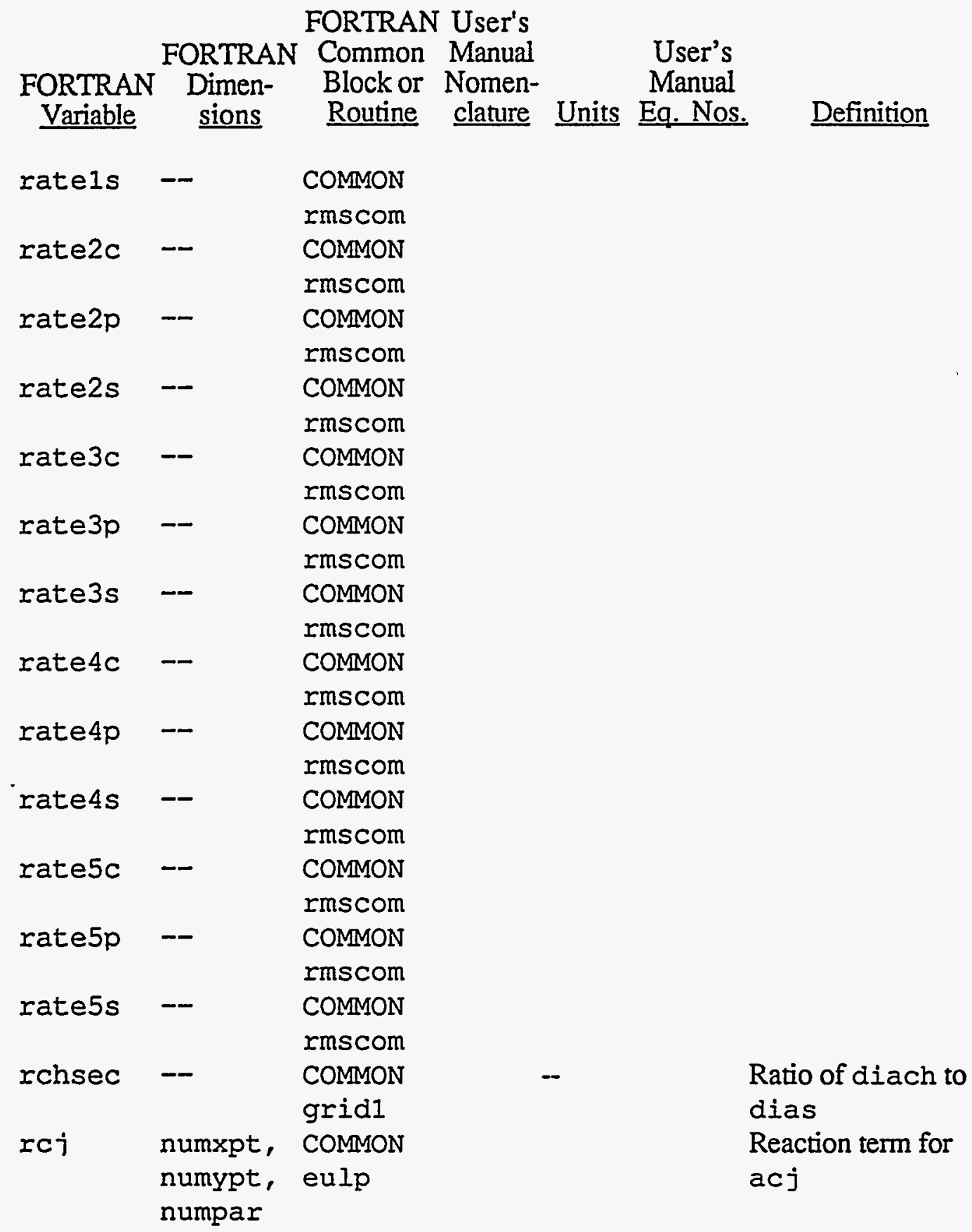




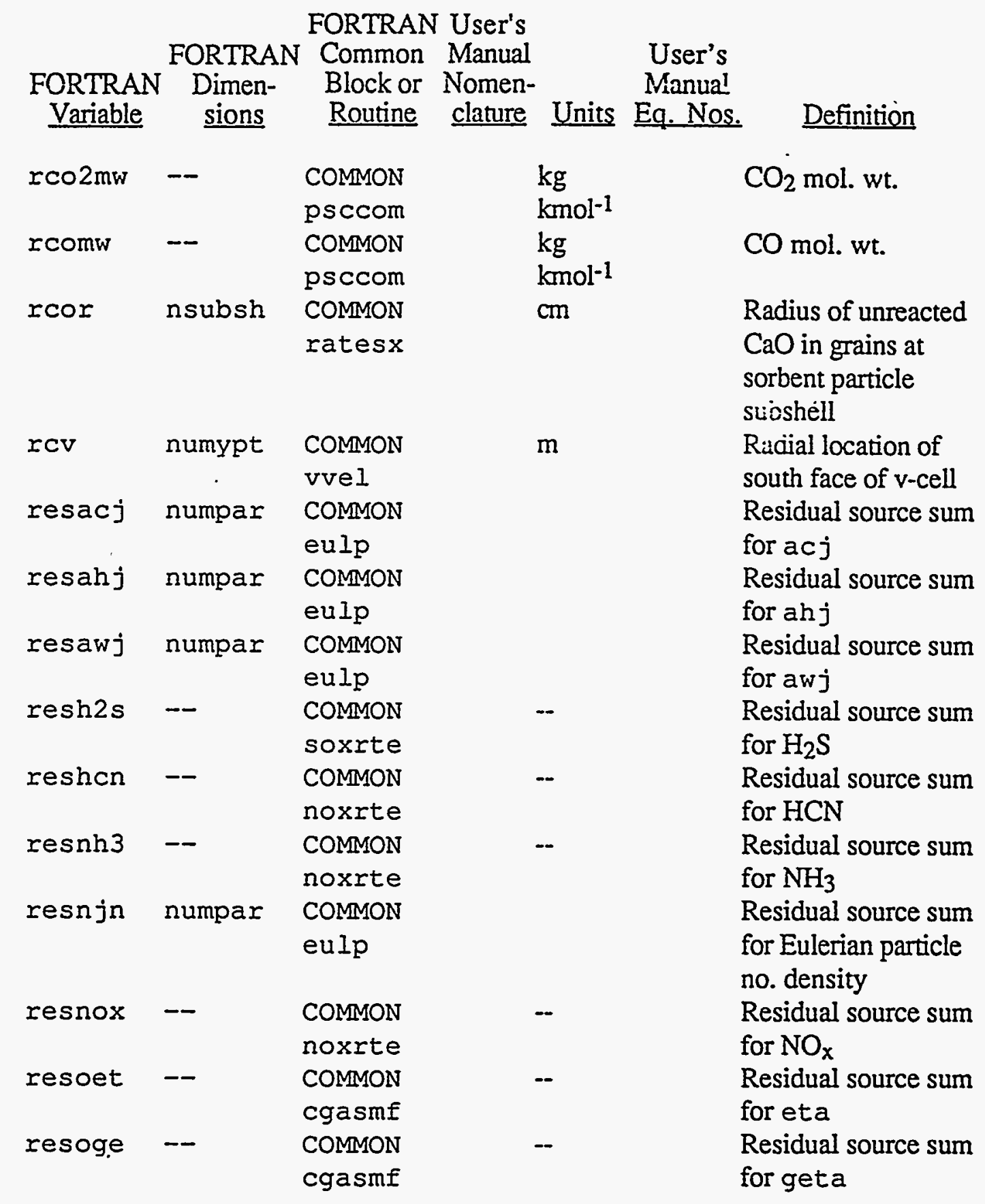




\begin{tabular}{|c|c|c|c|c|c|}
\hline & & FORTRAI & U User's & & \\
\hline & FORTRAN & Common & Manual & User's & \\
\hline FORTRAN & Dimen- & Block or & Nomen- & Manual & \\
\hline Variable & sions & Routine & clature & Units Eq. Nos. & Definition \\
\hline resore & - & $\begin{array}{l}\text { COMMON } \\
\text { tdis }\end{array}$ & & -- & $\begin{array}{l}\text { Max. residual } \\
\text { source sum for ed }\end{array}$ \\
\hline resorf & -- & $\begin{array}{l}\text { COMMON } \\
\text { mixfr }\end{array}$ & & -- & $\begin{array}{l}\text { Residual source sum } \\
\text { for } f\end{array}$ \\
\hline resorg & -- & $\begin{array}{l}\text { COMMON } \\
\text { fluc }\end{array}$ & $g_{f}$ & -- & $\begin{array}{l}\text { Residual source sum } \\
\text { for variance in } f\end{array}$ \\
\hline resorh & - & $\begin{array}{l}\text { COMMON } \\
\text { enth }\end{array}$ & & & $\begin{array}{l}\text { Residual sorce sum } \\
\text { for gas enthalpy }\end{array}$ \\
\hline resork & - & $\begin{array}{l}\text { COMMON } \\
\text { ten }\end{array}$ & & -- & $\begin{array}{l}\text { Max. residual } \\
\text { source sum for te }\end{array}$ \\
\hline resorm & - & $\begin{array}{l}\text { COMMON } \\
\text { pcor }\end{array}$ & & $\therefore$ & $\begin{array}{l}\text { Residual source sum } \\
\text { for mass } \\
\text { (continuity) }\end{array}$ \\
\hline resorn & - & $\begin{array}{l}\text { COMMON } \\
\text { pard }\end{array}$ & & -- & Residual source sum \\
\hline resorp & -- & $\begin{array}{l}\text { COMMON } \\
\text { pcor }\end{array}$ & & -- & $\begin{array}{l}\text { Pressure residual } \\
\text { source sum }\end{array}$ \\
\hline resorr & -- & $\begin{array}{l}\text { COMMON } \\
\text { radres }\end{array}$ & & - & $\begin{array}{l}\text { Residual source sum } \\
\text { for radiation in } \\
\text { radial direction }\end{array}$ \\
\hline resoru & $-\infty$ & $\begin{array}{l}\text { COMMON } \\
\text { uvel }\end{array}$ & & & $\begin{array}{l}\text { Residual source sum } \\
\text { for u velocity }\end{array}$ \\
\hline resorv & -- & $\begin{array}{l}\text { COMMON } \\
\text { vveI }\end{array}$ & & & $\begin{array}{l}\text { Residual source sum } \\
\text { for } v \text { velocity }\end{array}$ \\
\hline resorw & $\rightarrow$ & $\begin{array}{l}\text { COMMON } \\
\text { wvel }\end{array}$ & & & $\begin{array}{l}\text { Residual source sum } \\
\text { for w velocity }\end{array}$ \\
\hline resorx & -- & $\begin{array}{l}\text { COMMON } \\
\text { radres }\end{array}$ & & -- & $\begin{array}{l}\text { Residual source sum } \\
\text { for radiation in axial } \\
\text { direction }\end{array}$ \\
\hline
\end{tabular}




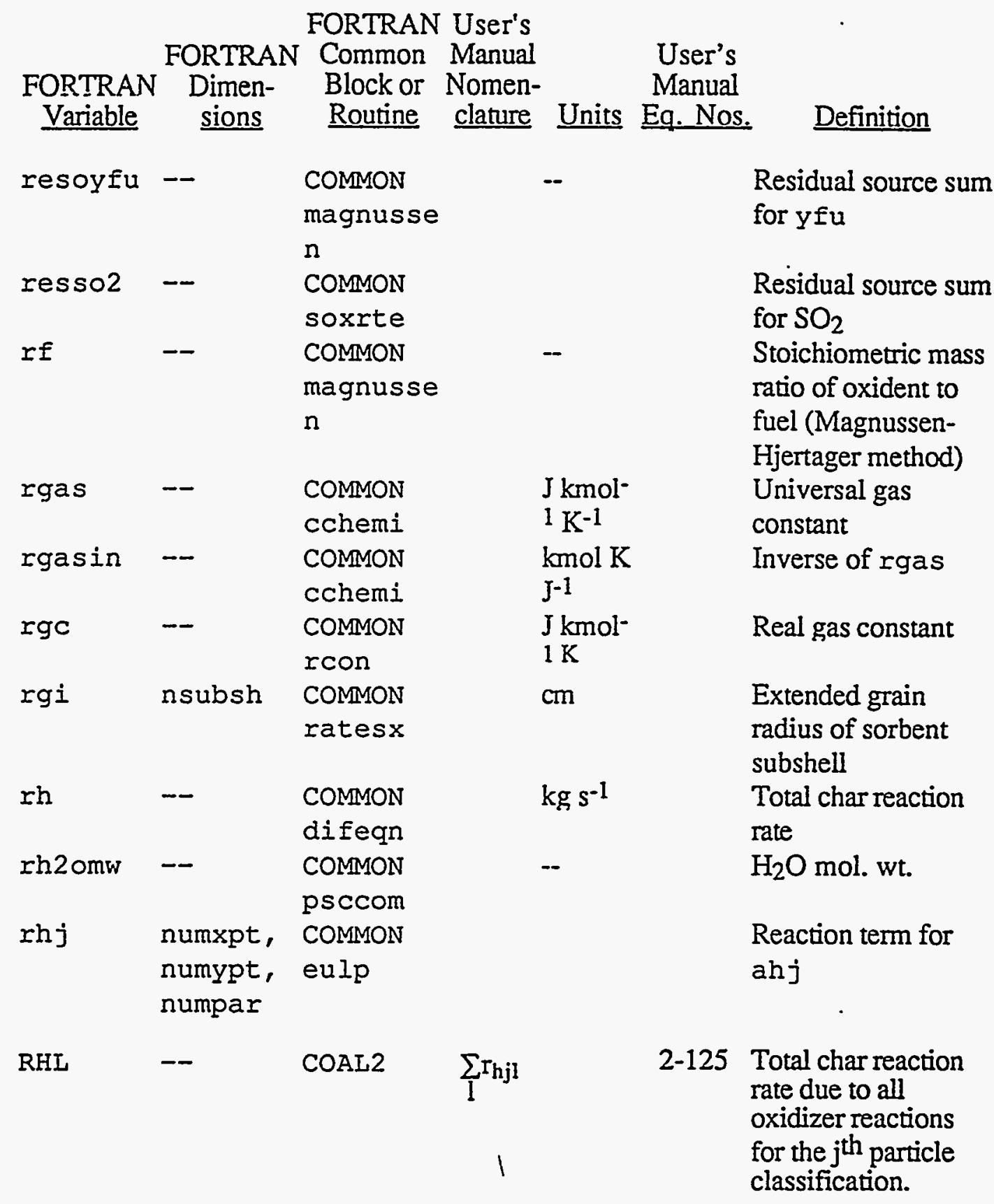




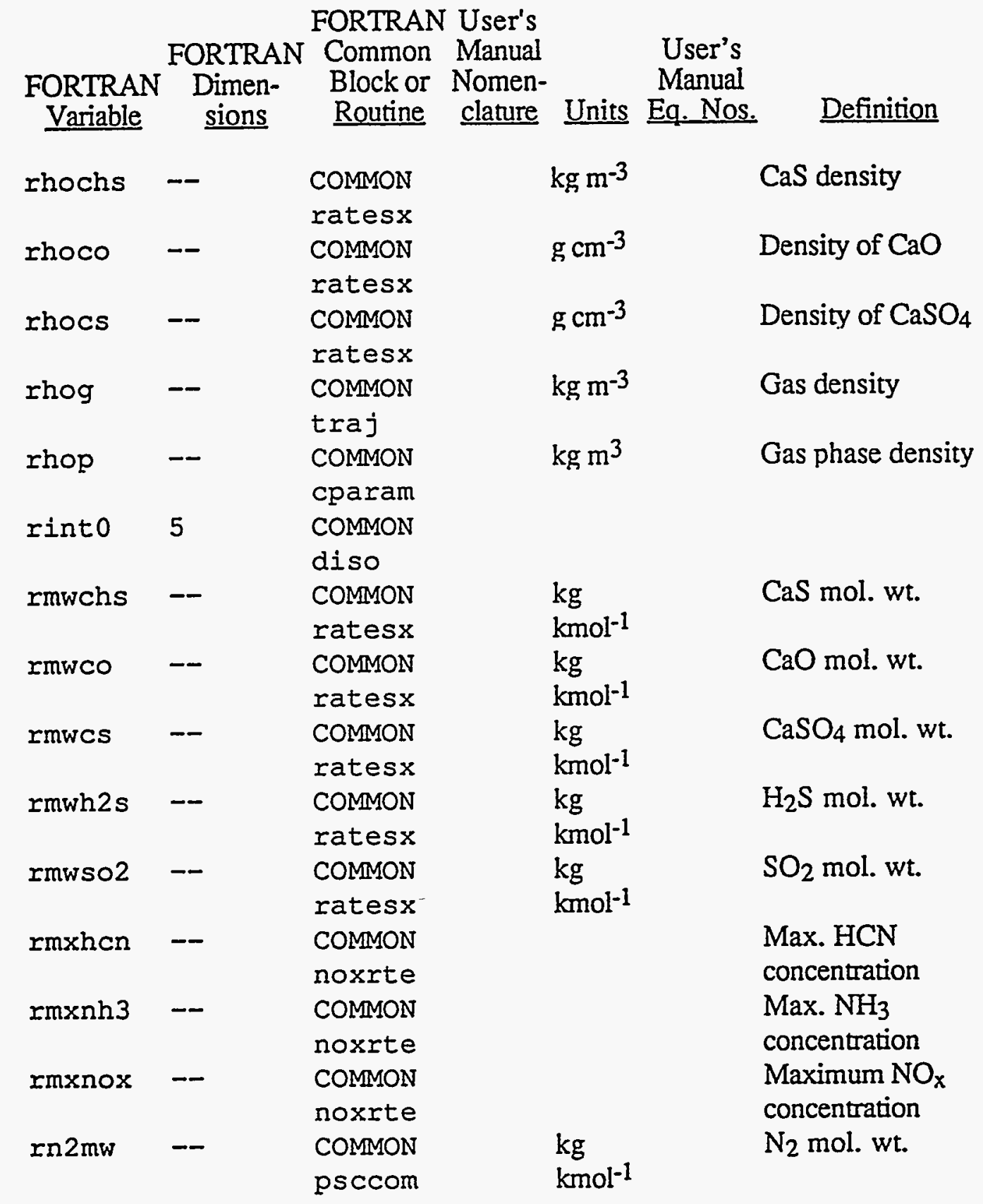




\begin{tabular}{|c|c|c|c|c|c|c|}
\hline $\begin{array}{l}\text { FORTRAN } \\
\text { Variable }\end{array}$ & $\begin{array}{c}\text { FORTRAN } \\
\text { Dimen- } \\
\text { sions }\end{array}$ & $\begin{array}{l}\text { FORTRAN } \\
\text { Common } \\
\text { Block or } \\
\text { Routine }\end{array}$ & $\begin{array}{l}\text { User's } \\
\text { Manual } \\
\text { Nomen- } \\
\text { clature }\end{array}$ & Units & $\begin{array}{c}\text { User's } \\
\text { Manual } \\
\text { Eq. Nos. }\end{array}$ & Definition \\
\hline $\mathrm{Inh} 3 \mathrm{mw}$ & -- & $\begin{array}{l}\text { COMMON } \\
\text { psccom }\end{array}$ & & $\begin{array}{l}\mathrm{kg} \\
\mathrm{kmol}^{-1}\end{array}$ & & $\mathrm{NH}_{3}$ mol. wt. \\
\hline rnomw & -- & $\begin{array}{l}\text { COMMON } \\
\text { intrat }\end{array}$ & & $\begin{array}{l}\mathrm{kg} \\
\mathrm{kmol}^{-1}\end{array}$ & & NO mol. wt. \\
\hline rnomw & -- & $\begin{array}{l}\text { COMMON } \\
\text { psccom }\end{array}$ & & $\begin{array}{l}\mathrm{kg} \\
\mathrm{kmol}^{-1}\end{array}$ & & NO mol. wt. \\
\hline ro2 $2 \mathrm{mw}$ & -- & $\begin{array}{l}\text { COMMON } \\
\text { psccom }\end{array}$ & & $\begin{array}{l}\mathrm{kg} \\
\mathrm{kmol}^{-1}\end{array}$ & & $\mathrm{O}_{2}$ mol. wt. \\
\hline rohmw & -- & $\begin{array}{l}\text { COMMON } \\
\text { psccom }\end{array}$ & & $\begin{array}{l}\mathrm{kg} \\
\mathrm{kmol}^{-1}\end{array}$ & & OH mol. wt. \\
\hline rprim & -- & $\begin{array}{l}\text { COMMON } \\
\text { grid1 }\end{array}$ & & $\mathrm{m}$ & & $\begin{array}{l}\text { Radius of primary } \\
\text { tube }\end{array}$ \\
\hline rps & -- & $\begin{array}{l}\text { COMMON } \\
\text { ratesx }\end{array}$ & & $\mathrm{cm}$ & & $\begin{array}{l}\text { Sorbent particle } \\
\text { radius }\end{array}$ \\
\hline $\operatorname{rrc}$ & -- & $\begin{array}{l}\text { COMMON } \\
\text { difeqn }\end{array}$ & & $\mathrm{kg} \mathrm{s}^{-1}$ & & $\begin{array}{l}\text { Total coal reaction } \\
\text { rate }\end{array}$ \\
\hline RRJ & -- & $\begin{array}{l}\text { Common } \\
\text { DIFEQN }\end{array}$ & $r_{\mathbf{j}}$ & $\mathrm{kg} / \mathrm{s}$ & $2-129$ & $\begin{array}{l}\text { Particle reaction rate } \\
\text { to gas phase. }\end{array}$ \\
\hline rrj & -- & $\begin{array}{l}\text { COMMON } \\
\text { difeqn }\end{array}$ & & $\mathrm{kg} \mathrm{s}^{-1}$ & & $\begin{array}{l}\text { Particle reaction rate } \\
\text { to the gas phase }\end{array}$ \\
\hline rsec & -- & $\begin{array}{l}\text { COMMON } \\
\text { gridl }\end{array}$ & & $\mathrm{m}$ & & $\begin{array}{l}\text { Radius of secondary } \\
\text { tube }\end{array}$ \\
\hline rtcnsp & $\begin{array}{l}\text { numxpt, } \\
\text { numypt }\end{array}$ & $\begin{array}{l}\text { COMMON } \\
\text { calcyi }\end{array}$ & & $\begin{array}{l}\mathrm{kg} \mathrm{m}^{-1} \\
\mathrm{~s}^{-3}\end{array}$ & & $\begin{array}{l}\text { "S } \mathrm{S}_{\mathrm{p}} \text { " source term for } \\
\mathrm{HCN}\end{array}$ \\
\hline rtcnsu & $\begin{array}{l}\text { numxpt, } \\
\text { numypt }\end{array}$ & $\begin{array}{l}\text { COMMON } \\
\text { calcyi }\end{array}$ & & $\begin{array}{l}\mathrm{kg} \\
\mathrm{HCN} \\
\mathrm{m}^{-3} \mathrm{~s}^{-1}\end{array}$ & & $\begin{array}{l}\text { "S } \mathrm{S}_{\mathrm{u}} \text { " source term for } \\
\mathrm{HCN}\end{array}$ \\
\hline rthssp & $\begin{array}{l}\text { numxpt, } \\
\text { numypt }\end{array}$ & $\begin{array}{l}\text { COMMON } \\
\text { ratesx }\end{array}$ & & -- & & $\begin{array}{l}\text { Linearized source } \\
\text { term for } \mathrm{H}_{2} \mathrm{~S}\end{array}$ \\
\hline
\end{tabular}




\begin{tabular}{|c|c|c|c|c|c|c|}
\hline & & FORTRAI & User's & & & \\
\hline & FORTRAN & Common & Manual & & User's & \\
\hline FORTRAN & Dimen- & Block or & Nomen- & & Manual & \\
\hline Variable & $\underline{\text { sions }}$ & & clature & $\underline{\text { Units }}$ & Eq. Nos. & Definition \\
\hline rthssu & numxpt, & COMMON & & -- & & Linearized source \\
\hline & numypt & ratesx & & & & term for $\mathrm{H}_{2} \mathrm{~S}$ \\
\hline rtnhsp & numxpt, & COMMON & & $\mathrm{kg} \mathrm{m}^{-3}$ & & "S $S_{p}$ " source term for \\
\hline & numypt & calcyi & & & & $\mathrm{NH}_{3}$ \\
\hline rtnhsu & numxpt, & COMMON & & $\mathrm{kg} \mathrm{NH} 3$ & & "S $S_{u}$ " source term for \\
\hline & numypt & calcyi & & $m^{-3} s^{-1}$ & & $\mathrm{NH}_{3}$ \\
\hline rtnosp & numxpt, & COMMON & & $\mathrm{kg} \mathrm{m}^{-3}$ & & "S $S_{p} "$ source term for \\
\hline & numypt & calcyi & & & & NÓ \\
\hline rtnosu & numxpt, & COMMON & & $\mathrm{kg} \mathrm{NO}$ & & "S $\mathrm{S}_{\mathrm{u}}$ " source term for \\
\hline & numypt & calcyi & & $m^{-3} \cdot s^{-1}$ & & $\mathrm{NO}$ \\
\hline rtsosp & numxpt, & COMMON & & -- & & Linearized source \\
\hline & numypt & ratesx & & & & term for $\mathrm{SO}_{2}$ \\
\hline rtsosu & numxpt, & COMMON & & -- & & Linearized source \\
\hline & numypt & ratesx & & & & term for $\mathrm{SO}_{2}$ \\
\hline rv & numypt & COMMON & & $\mathrm{m}$ & & Same as $y v$ \\
\hline & & geom & & & & \\
\hline Iw & -- & COMMON & & $\mathrm{kg} \mathrm{s}^{-1}$ & & Change of slurry \\
\hline & & difeqn & & & & $\begin{array}{l}\text { liquid mass per unit } \\
\text { time }\end{array}$ \\
\hline $\mathbf{s}$ & numspe & COMMON & & $\AA$ & & Lennard-Jones \\
\hline & & gparam & & & & parameter \\
\hline so & numspe & COMMON & & & & Molar entropy of \\
\hline & & spece & & & & each species \\
\hline$s 2$ & numspe & $\begin{array}{l}\text { COMMON } \\
\text { cparam }\end{array}$ & & $\begin{array}{l}\mathrm{kmol} \mathrm{i} \\
\mathrm{kg}^{-1}\end{array}$ & & $\begin{array}{l}\text { Mole numbers of } \\
\text { each species }\end{array}$ \\
\hline sbar & $-\infty$ & $\begin{array}{l}\text { COMMON } \\
\text { radcon }\end{array}$ & $\bar{s}$ & -- & $2-148$ & $\begin{array}{l}\text { Side-scattered } \\
\text { component of } \\
\text { radiation }\end{array}$ \\
\hline sconv & nsubsh & $\begin{array}{l}\text { COMMON } \\
\text { ratesx }\end{array}$ & & -- & 1 & $\begin{array}{l}\text { Sorbent subshell } \\
\text { conversion }\end{array}$ \\
\hline
\end{tabular}




\begin{tabular}{|c|c|c|c|c|c|c|}
\hline $\begin{array}{l}\text { FORTRAN } \\
\text { Variable }\end{array}$ & $\begin{array}{c}\text { FORTRAN } \\
\text { Dimen- } \\
\text { sions }\end{array}$ & $\begin{array}{c}\text { FORTRAI } \\
\text { Common } \\
\text { Block or } \\
\text { Routine } \\
\end{array}$ & $\begin{array}{l}\text { User's } \\
\text { Manual } \\
\text { Nomen- } \\
\text { clature }\end{array}$ & $\underline{\text { Units }}$ & $\begin{array}{l}\text { User's } \\
\text { Manual } \\
\text { Eq. Nos. }\end{array}$ & Definition \\
\hline sew & numxpt & $\begin{array}{l}\text { COMMON } \\
\text { geom }\end{array}$ & & $\mathrm{m}$ & & $\begin{array}{l}\text { East-west width of } \\
\text { p-cell }\end{array}$ \\
\hline sewu & numxpt & $\begin{array}{l}\text { COMMON } \\
\text { uvel }\end{array}$ & & -- & & Width of u-cell \\
\hline sf & numxpt, $n$ & COMMON & & -- & & Stream function \\
\hline sfac & $\begin{array}{l}\text { umypt } \\
\text { numypt }\end{array}$ & $\begin{array}{l}\text { strinc } \\
\text { COMMON } \\
\text { geom }\end{array}$ & & -- & & Geometric factor \\
\hline SHHJ & -- & COAL2 & $\frac{\sum_{l} r_{h j l} h g_{h}}{\sum_{l} r_{h j l}}$ & & $2-125$ & $\begin{array}{l}\text { Total heat of } \\
\text { reaction for all } \\
\text { oxidation reactions } \\
\text { for the } \mathrm{j} \text { th particle } \\
\text { classification } \\
\text { normalized to the } \\
\text { total rate of mass } \\
\text { loss due to all } \\
\text { oxidizer reactions. }\end{array}$ \\
\hline SHVJ & -- & COAL2 & $\begin{array}{l}\text { MSU(m } \\
\text {,r } \text { r vjm }_{\text {vim }} \text { v } \\
\text { vim }\end{array}$ & & $2-125$ & $\begin{array}{l}\text { Total heat of } \\
\text { devolatilization for } \\
\text { all devolatilization } \\
\text { reactions for the } \mathrm{j}^{\text {th }} \\
\text { particle } \\
\text { classification. }\end{array}$ \\
\hline sigma & -- & $\begin{array}{l}\text { COMMON } \\
\text { radcon }\end{array}$ & & $\begin{array}{l}\mathrm{J} \mathrm{m}^{-2} \mathrm{~s}^{-} \\
{ }^{1} \mathrm{~K}^{-4}\end{array}$ & & $\begin{array}{l}\text { Stefan-Boltzmann } \\
\text { constant }\end{array}$ \\
\hline SM & -- & $\begin{array}{l}\text { Common } \\
\text { CPARAM }\end{array}$ & & $\begin{array}{c}\mathrm{kmol} \\
\mathrm{kg}^{-1}\end{array}$ & & $\begin{array}{l}\text { Inverse of molecular } \\
\text { weight }\end{array}$ \\
\hline smet & -- & $\begin{array}{l}\text { COMMON } \\
\text { pvar }\end{array}$ & & $\begin{array}{l}\mathrm{kmol}^{-1} \\
\mathrm{~kg}^{-1}\end{array}$ & & $\begin{array}{l}\text { Coal offgas inverse } \\
\text { mol. wt. }\end{array}$ \\
\hline
\end{tabular}


FORTRAN User's

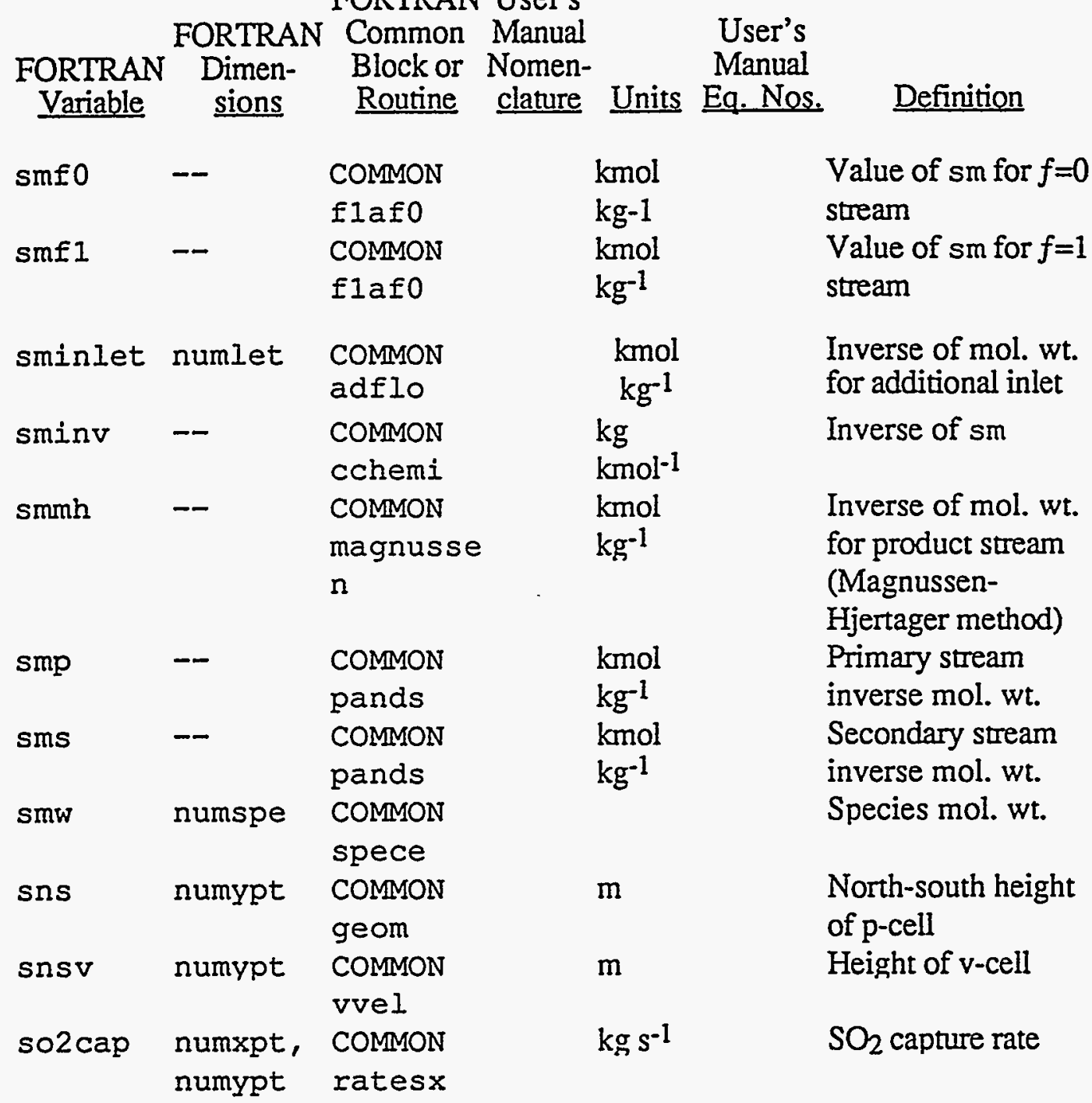




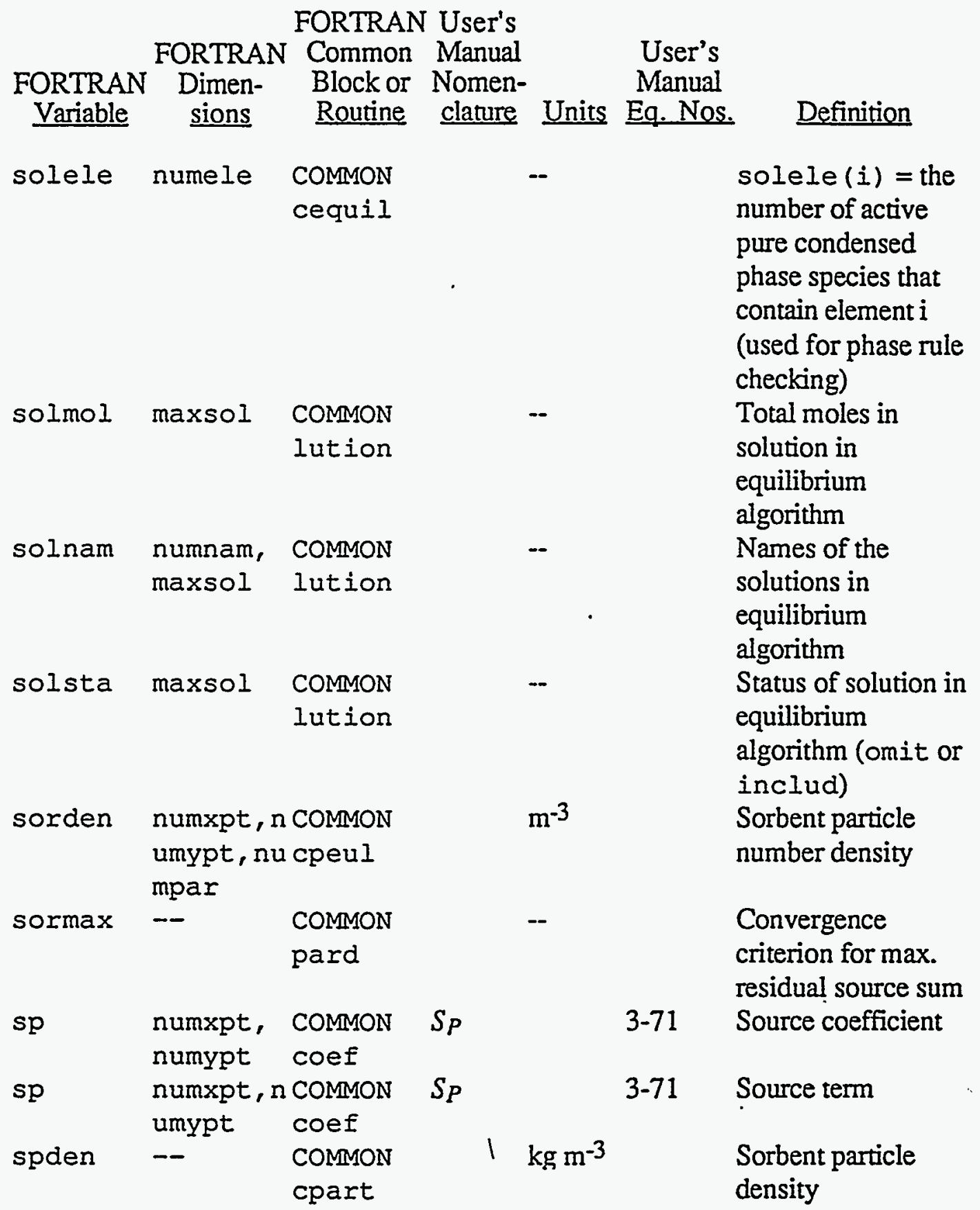




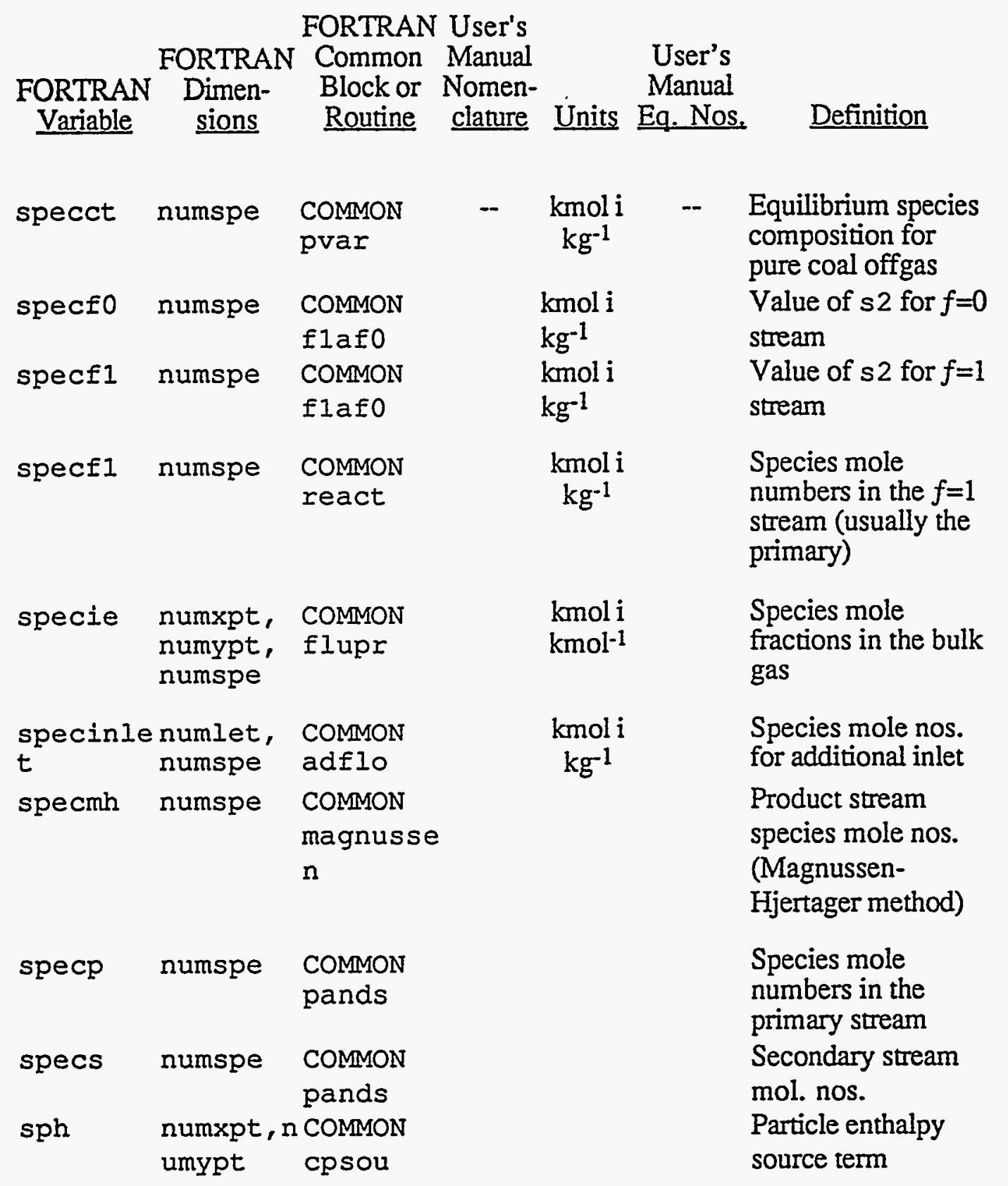




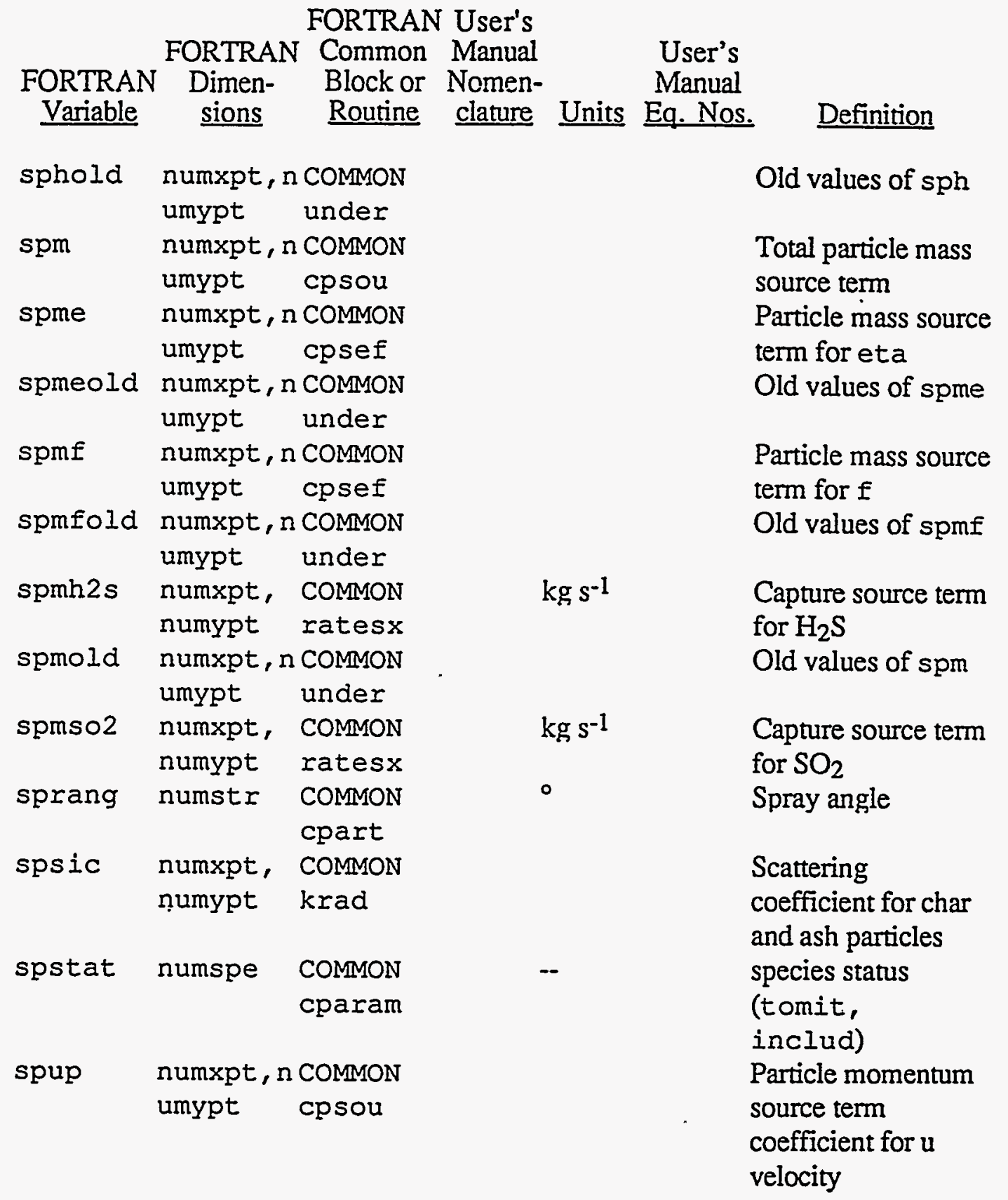




\title{
An Empirical Model for Coal Fluidity Based on a Macromolecular Network Pyrolysis Model
}

\author{
P. R. Solomon,* P. E. Best, † Z. Z. Yu, and S. Charpenay \\ Advanced Fuel Research, Inc., 87 Church Street, East Hartford, Connecticut 06108 \\ Received May 22, 1991. Revised Manuscript Received November 26, 1991
}

\begin{abstract}
We have developed a phenomenological model for coal fluidity based on a macromolecular network model for the decomposition and condensation of the network under the influence of bond breaking and cross-linking reactions. The macromolecular network model is the previously published FG-DVC model of coal pyrolysis. It employs a network consisting of aromatic ring clusters linked by bridges. The bond scissions are described by a single first-order reaction with a distribution of activation energies. Cross-linking is related to $\mathrm{CO}_{2}$ and $\mathrm{CH}_{4}$ formation which are described in multiple first-order reactions with distributions of activation energies. The fluidity is described by an empirical equation which depends on the relative amounts of the liquid (molecules detached from the network) and solid (the remaining network) and on the fluidity of the liquid component. The FG-DVC model predicts the yield of liquids. The fluidity of the liquid component is described by a second phenomenological equation which depends only on the temperature. The advantage of this model is that it is based on a previously demonstrated methodology which allows the incorporation of rank-dependent kinetics, cross-linking, weathering, and extraction phenomena into the fluidity predictions. Excellent agreement has been obtained between the model predictions and low-temperature fluidity measurements of Oxley and Pitt, van Krevelen, and Gieseler plastometer measurements for the Argonne premium coal samples. The trends for changes in the fluidity with weathering or extraction are predicted as well. Good agreement has been obtained at high temperatures between the model predictions and measurements of Fong for the onset of the fluidity. The loss of fluidity, however, is predicted to occur sooner than is indicated by the data and the maximum value of fluidity is overpredicted. The data cover over 5 orders of magnitude in fluidity, and eight coals with carbon concentration between 80 and $90 \%$. This agreement is obtained using coal-independent equations for the dependence of the fluidity on the liquid fraction and the liquid fluidity. The coal-dependent variables are the kinetic rates for bond breaking and cross-linking and the extent of cross-linking as determined from laboratory pyrolysis measurements using a TG-FTR (thermogravimetric analyzer with Fourier transform infrared analysis of evolved products). There are only two adjustable parameters in the model.
\end{abstract}

\section{Introduction}

When bituminous coals are heated, they become liquid. Those in the range of $82-89 \%$ carbon achieve the highest fluidities, ${ }^{12}$ but even lignites, if heated rapidly enough, can exhibit some fluidity. ${ }^{3}$ The understanding and ability to predict a coal's fluid properties are important in many processes. In liquefaction, highly fluid coals dissolve quickly in the process solvent, so that further chemistry occurs by liquid-liquid interactions, while nonfluid coals must undergo slower solid-liquid interactions. In combustion or gasification, fluidity controls particle swelling, ${ }^{4}$ agglomeration of particles, intrinsic char reactivity, ${ }^{5}$ and subsequent fragmentation ${ }^{6}$ of char. In coke making, fluidity controls the coke properties.78 Fluidity also affects the growth of carbon products made from coal tars.

There are a number of factors which contribute to the fluidity of coal liquid. They include (i) the fluidity of the liquid fraction, with and without molecular entanglements; (ii) the dependence of this fluidity on temperature; (iii) the contributions of suspended solids in the liquid, both "chunks" of char and mineral particles; and (iv) the formation of bubbles due to trapped gases.

Several models for coal liquid viscosity have been proposed which consider all of the influences except the bubbles. The models were based on the two step process described by van Krevelen and co-workers, ${ }_{10}^{1,9}$ which assume the following reactions to occur on heating.

'Physics Department and Institute of Material Science, University of Connecticut, Storss, CT 06268.

$$
\text { coal } \stackrel{k_{2}}{\longrightarrow} \text { metaplast } \stackrel{k_{2}}{\longrightarrow} \text { coke }
$$

where $k_{1}$ and $k_{2}$ are reaction rate constants. In the viscosity models, the change of fluidity is assumed to result from the change in solids mass fraction, $\phi_{3}$ (coal and coke), in the melt. The metaplast is the liquid fraction. Thus Bronowski et al. ${ }^{10}$ used an expression in which fluidity was directly proportional to the liquid fraction $\left(1-\phi_{2}\right)$. Expanding on this, Fitzgerald ${ }^{11,12}$ used an equation which described the relative fluidity as depending on liquid fraction raised to a power $n$ (where $n$ was chosen to be 2.5). This power law expression was based on earlier work by Roscoe ${ }^{13}$ and Brinkman. ${ }^{14}$ Frankel and Acrivos ${ }^{15}$ used an

(1) van Krevelen, D. W. Coal; Elsevier: Amsterdam, 1961.

(2) Sanada, Y.; Honda, H. Fuel 1966, 45, 295.

(3) Solomon. P. R; Serio, M. A.; Carangelo, R. M;; Markham, J. R Fuel 1986, 65, 182 .

(4) Solomon, P. R.; Hamblen, D. G. Chemistry of Coal Conversion; Schlosberg, R. H., Ed.; Plenum Publisbing. New York, 1985; Chapter 5, pp 121-251.

(5) Serio, M. A. Solomon, P. R; Bassilabis, R; Suuberg, E. M. Prepr. Pap.-Am. Chem. Soc., Div. Fuel Chem. 1989, 34 (1), 9.

(6) Helble J. J.; Sarotim, A. F. Combust. Flame 1989, 76, 183.

(7) Marsh, H. Fuel 1973, 52, 205.

(8) Marsh. H.; Neavel. R. C. Fuel 1980, 59, 511.

(9) Chermin, H. A. G.; van Krevelen, D. W. Fuel 1957, 36, 85.

(10) Bronowski, J.; Fitzgerald, D.; Gillings, D. W.; Rhys-Jones, D. C. Nature 1953, 171, 389.

(11) Fitzgerald, D. Fuel 1956, 35, 178.

(12) Fitzgerald, D. Trans. Faraday Soc. 1956, 52, 362

(13) Roscoe, Brit. J. Appl. Phys. 1952, 3, 267.

(14) Brinkman, J. J. Chem. Phys. 1952, 20, 571.

(15) Frankel, N. A.; Acrivos, A. Chem. Eng., Sci. 1976, 22, 847. 
expression in which the fluidity also depends on a power of the liquid fraction. Their model has the extra feature of a critical solids volume fraction at which fluidity disappears. This critical value occurs at the maximum volume fraction that the solids can occupy as limited by particle-particle interaction. At this critical value, the liquid fraction is insufficient to separate the solid particles. Its value is 0.64 for randomly close-packed spheres, while it ranges from 0.5 to 0.9 for other systems, depending on particle shape and state of agglomeration. ${ }^{16}$ Viscosity models predict that the fluidity vanishes for solid volume fractions equal to or greater than the critical value, and we refer to this critical value as the inhomogeneous gel point.

Fong et al. ${ }^{17}$ employed the power law of Frankel and Acrivos; 15 however, the chosen critical solids volume fraction value in Fong's model was unity, somewhat higher than usual ${ }^{16} \mathrm{~A}$ similar model was used by $\mathrm{Oh}^{18}$ The above models gave good fits to data, although in each case, four model parameters $\left(k_{1}, k_{2}, n\right.$, and the critical solids volume fraction) were chosen to fit the data for a particular coal studied in a limited number of experiments covering a nerrow range of heating rates, or holding temperatures.

On the besis of the observation that coal can be considered as a macromolecular network to which theories of cross-linked polymers may be applied, 1,219-25 we have examined the polymer literature of viscosity in polymer melts to determine the range of assumptions which have been employed.17,27-32 For nonreacting melts of branched polymers at molecular sizes below those sufficiently large for entanglements, there is experimental and theoretical support for viscosities which depend exponentially on side-arm molecular mass. ${ }^{27}$ These same authors determined temperature-dependent activation energies for viscosity, for temperatures below $200^{\circ} \mathrm{C}$. For inhomogeneous polymer melts, theories with an inhomogeneous gel point have been employed. 17,32

In reacting melts, the manner in which average molecular weight varies with extent of reaction in a homogeneous melt has been considered in the branching theory described by Macosko and co-workers. ${ }^{29}$ Insofar as it describes molecular weight distributions, this theory duplicates and ertends the results of older combinatorial methods developed by Flory 30 and by Stockmayer. ${ }^{31}$ A particular result of Macosko's work is that measured viscosity correlates well with the weight average molecular weight of the longest linear path through the molecules. This theory

(16) Nielsen, L. E Mechanical Properties of Polymers and Composites; Marcel Dekcker: New York, 1974; Vol 2.

65,195

(17) Fong, W. S.; Khalil, Y. F.; Peters, W. A.; Howard, J. B. Fuel 1986,

(18) Oh. M. S. Peter, W. A.; Howard, J. B. AIChE J. 1989, 35 (5), 775.

(19) Green, T. K.; Kovac, J.; Larsen, J. W. Fuel 1984, 63, 935.

(20) Green, T. K. Kovac, J; Larsen, J. W. Coal Structure; Meyers, R.

A. Ed.; Academic Press: New York, 1982.

(21) Nelson, J. R. Fuel 1983, 62, 112.

(22) Lucht, L. M.; Peppas, N. A. Fuel 1987, 66, 803. 56.

(23) Lucht, L. M; Larsen, J. M, Peppas, N. A. Energy Fuels 1987, 1,

(24) Green, T.; Kovac, J.; Brenner, D.; Larsen, J. Coal Structure;

Meyers, R. A., Ed; Academice New York, 1982; p 199.

(25) Hall, P. J.; Marah, H.; Thomas, K. M. Fuel 1988, 67, 863.

(26) Hirsch, P. B. Proc. R. Soc. 1954, A226, 143.

(27) Bartels, C. R. Crist, B.; Feltern, L. J.; Graessley, W. W. Macramolecules 1986, 19,785 .

(28) Nazem, F. F. Fuel 1980, 59, 851

(29) Macosko, C. W. Brit. Polymer J. 1985, 17, 239; and references therein.

(30) Flory, P. J. J. Am. Chem. Soc. 1941, 63, 3083, 3097. See also: Principles of Polymer Chemistry; Comell University Press: Ithaca, NY, 1953; Chapter 9.

(31) Stockomayer, W. H. J. Chem. Phys. 1943, 11, 45; 1944, 12, 125. (32) Mooney, M. J. Colloid Sci. 1951, 6, 162. predicts that the homogeneous gel-point (the point at which the viscosity goes to infinity) appears at the first occurrence of a solid phase (i.e., the gel point is at $\phi_{\mathrm{s}}=0$ ).

While Macosko's approach appears to be good for homogeneous polymer melts, it does not appear to be appropriate for coal, since coal is typically an inhomogeneous reacting melt. The inhomogeneities result from several factors including starting with a powdered solid (which would be sufficient to insure inhomogeneity) and having a material consisting of diverse maceral types and mineral grains. Because of these inhomogeneities, it is thought that theories which describe the viscosity of a suspension of a solid in a liquid are the most appropriate for coal, and in this work we have pursued the two phase approach with a gel point at a critical $\phi_{\mathrm{s}}$ value where $\phi_{\mathrm{s}}>0$.

To improve upon the prediction of the liquid fraction, we have employed polymer concepts to describe the molecular weight distribution during pyrolysis, from which a liquid and solid fraction can be determined. This work is based on a theory initially employing linear chain statistic ${ }^{33,34}$ and subsequently employing network statistics. ${ }^{35-38}$ Our DVC macromolecular network decomposition model includes the processes of depolymerization (bond breaking), vaporization (mass transport), and cross-linking. This model was combined with our functional group (FG) model for gas evolution ${ }^{4,39,40}$ to provide the general FG-DVC coal pyrolysis model. ${ }^{41-63}$ In the combined model, the cross-linking process in the DVC model is related to the evolution of $\mathrm{CO}_{2}$ and $\mathrm{CH}_{4}$ in the FG model. The FG-DVC model employs Monte Carlo methods to compute the network properties. More recently, network models of thermal decomposition have been proposed employing percolation theory. 4,45 We have also employed a modified percolation theory for the statistical calculations in the FG-DVC model. ${ }^{43,66,47}$

The fluidity model presented here employs the FG-DVC model to predict the molecular weight distribution of the decomposing macromolecular network. From this distribution, a solid fraction $\phi_{s}$ and a liquid fraction $\left(1-\phi_{g}\right)$ are defined. These parameters are employed using the concepts of inhomogeneous mixtures to predict the fluidity from the solid fraction, the liquid viscosity, and the tem-

(33) Solomon, P. R.; King, H. H. Fuel 1984, 63, 1302.

(34) Squire, K. R. Carangelo, R. M.; DiTaranto, M. B.; Solomon, P. R. Fuel 1986, 65, 833 .

(35) Squire, K. R.; Solomon, P. R.; DiTeranto, M. B.; Carangelo, R. M. Prepr. Pap.-Am. Chem. Soc., Div. Fuel Chem. 1985, 30 (1), 386. (36) Solomon, P. R.; Squire, K. R; Carangelo, R. M. Int. Conf. Coal Sci., Proc., Sydrey, Australia 1985, 945.

(37) Solomon, P. R. Squire, K. R. Prepr. Pap.-Am Chem. Soc, Div. Fuel Chem. 1985, 30 (4), 347.

(38) Solomon, P. R.; Hamblen, D. G.; Carangelo, R. M; Serio, M. A; Deshpande, G. V. Combust. Flame 1988, 71, 137.

(39) Solomon, P. R. Hamblen, D. G.; Carangelo, R. M.; Krause, J. L. Ninteenth Symposium (International) on Combustion; The Combustion Institute: Pittsburgh, PA, 1982; p 1139.

(40) Serio, M. A.; Hamblen, D. G.; Markham, J. R.; Solomon, P. R. Energy Fuels 1987, 1, 138.

(41) Solomon, P. R.; Hamblen, D. G.; Deshpande, G. V.; Serio, M. A. A General Model of Coal Devolatilization, In International Conference on Coal Science Proceedings; Elsevier: Amsterdam, The Netherlands, $1987 ; \mathrm{p} 601$.

(42) Solomon, P. R.; Hamblen, D. G.; Carangelo, R. M.; Serio, M. A.; Desippande, G. V. Energy Fuels 1988, 2, 405.

(43) Solomon, P. R.; Hamblen, D. G.; Serio, M. A.; Yu, Z Z; Charpenay, S., Storch Afrard Sympoiium Lecture, Prepr. Pap.-Am. Chem. Soc., Div. Fuel Chem. 1991, 36 (1), 267.

(44) Nikss, S.; Kerstein, A. R. Fuel 1987, 66, 1389

(45) Grant D. M.; Pugmire, R. J.; Fletcher, T. H.; Kerstein, A. R. Energy Fuels 1989, 3, 175.

(46) Solomon, P. R; Hamblen, D. G.; Yu, Z Z; Serio, M. A. Network Models of Coal Thermal Decomposition Fuel 1990, 69, 754.

(47) Solomon, P. R.; Serio, M. A.; Hamblen, D. G.; Yu, Z Z; Charpeney, S. Prepr. Pop.-Am. Chem. Soc., Div. Fuel Chem. 1990, 35 (2), 479. 
Table I

\begin{tabular}{|c|c|c|c|c|c|c|c|}
\hline & IIL & UTAH & UPK & $\mathrm{PIT}$ & UPF & POC & PSOC815 \\
\hline $\begin{array}{l}\text { volatile matter (daf), \% } \\
C \% \text { (daf) } \\
0 \% \text { (daf) } \\
\text { bond breaking }\end{array}$ & $\begin{array}{l}47.4 \\
77.7 \\
13.5\end{array}$ & $\begin{array}{l}48.1 \\
80.7 \\
11.6\end{array}$ & $\begin{array}{l}37.6 \\
82.6 \\
9.8\end{array}$ & $\begin{array}{l}41.7 \\
83.2 \\
8.8\end{array}$ & $\begin{array}{l}31.6 \\
85.6 \\
7.5\end{array}$ & $\begin{array}{l}19.5 \\
91.1 \\
2.5\end{array}$ & $\begin{array}{l}31.5 \\
85.6 \\
7.8\end{array}$ \\
\hline $\begin{array}{l}E_{\mathrm{BB}} / R \\
A_{\mathrm{BB}} \\
\sigma_{\mathrm{BB}} / R \\
\mathrm{BB} \text { rate at } 650^{\circ} \mathrm{C} \\
\text { methane cross-linking }\end{array}$ & $\begin{array}{l}26000 \\
1.0 \times 10^{14} \\
750 \\
2.4 \times 10^{-2}\end{array}$ & $\begin{array}{l}27000 \\
1.0 \times 10^{14} \\
1250 \\
6.0 \times 10^{-3}\end{array}$ & $\begin{array}{l}27250 \\
1.0 \times 10^{14} \\
1000 \\
4.3 \times 10^{-3}\end{array}$ & $\begin{array}{l}27500 \\
1.0 \times 10^{14} \\
1250 \\
3.0 \times 10^{-3}\end{array}$ & $\begin{array}{l}28250 \\
1.0 \times 10^{14} \\
1250 \\
1.1 \times 10^{-3}\end{array}$ & $\begin{array}{l}29000 \\
1.0 \times 10^{14} \\
750 \\
3.8 \times 10^{-4}\end{array}$ & $\begin{array}{l}28250 \\
1.0 \times 10^{14} \\
1250 \\
1.1 \times 10^{-3}\end{array}$ \\
\hline $\begin{array}{l}E_{\mathrm{CH}_{1}-\mathrm{L}} / R \\
A_{\mathrm{CO}_{2} \mathrm{~L}} \\
\sigma_{\mathrm{CO}_{2} \mathrm{~L}} / R \\
\mathrm{CH}_{1} \mathrm{~L} \text { Rate at } 650^{\circ} \mathrm{C} \\
\mathrm{CO}_{2} \text { cross-linking }\end{array}$ & $\begin{array}{l}28000 \\
3.0 \times 10^{13} \\
1800 \\
4.5 \times 10^{-4}\end{array}$ & $\begin{array}{l}28000 \\
3.0 \times 10^{13} \\
1500 \\
4.5 \times 10^{-4}\end{array}$ & $\begin{array}{l}28000 \\
3.0 \times 10^{13} \\
1200 \\
4.5 \times 10^{-4}\end{array}$ & $\begin{array}{l}28000 \\
3.0 \times 10^{13} \\
1300 \\
4.5 \times 10^{-4}\end{array}$ & $\begin{array}{l}28750 \\
3.0 \times 10^{13} \\
800 \\
1.6 \times 10^{-4}\end{array}$ & $\begin{array}{l}29500 \\
3.0 \times 10^{13} \\
750 \\
5.7 \times 10^{-5}\end{array}$ & $\begin{array}{l}28500 \\
3.0 \times 10^{13} \\
1000 \\
2.3 \times 10^{-4}\end{array}$ \\
\hline $\begin{array}{l}E_{\mathrm{CO}_{2} \mathrm{~L}} / R \\
A_{\mathrm{CO}_{z} \mathrm{~L}} \\
\sigma_{\mathrm{CO}} / R \\
\mathrm{CO}_{2} \mathrm{~L} \text { rate at } 650^{\circ} \mathrm{C} \\
M_{0} \text { (cross-link/ring cluster) } \\
l \text { (oligomer length) } \\
H(\text { al), \% }\end{array}$ & $\begin{array}{l}24750 \\
5.0 \times 10^{12} \\
1750 \\
6.8 \times 10^{-3} \\
0.21 \\
4 \\
0.51\end{array}$ & $\begin{array}{l}25000 \\
5.0 \times 10^{12} \\
1250 \\
4.8 \times 10^{-3} \\
0.21 \\
4 \\
0.55\end{array}$ & $\begin{array}{l}26000 \\
5.0 \times 10^{12} \\
3000 \\
1.2 \times 10^{-3} \\
0.13 \\
8 \\
0.67\end{array}$ & $\begin{array}{l}26500 \\
5.0 \times 10^{12} \\
3000 \\
6.0 \times 10^{-4} \\
0.08 \\
8 \\
0.62\end{array}$ & $\begin{array}{l}27000 \\
5.0 \times 10^{12} \\
3000 \\
3.0 \times 10^{-4} \\
0.08 \\
10 \\
0.55\end{array}$ & $\begin{array}{l}28000 \\
5.0 \times 10^{12} \\
2500 \\
7.6 \times 10^{-5} \\
0.128 \\
10 \\
0.35\end{array}$ & $\begin{array}{l}26750 \\
5.0 \times 10^{12} \\
3000 \\
4.3 \times 10^{-4} \\
0.08 \\
8 \\
0.51\end{array}$ \\
\hline
\end{tabular}

perature. ${ }^{17,28,32}$ The effect of bubbles has not been specifically considered but are included implicitly since bubbling occurs under conditions of the experiments whose fitting was used to devise the empirical equations. The model predictions are compared with measurements made with a Gieseler plastometer ${ }^{1,48-53}$ and with the high-temperature fluidity measurements of Fong et al. ${ }^{17}$

\section{Experimental Section}

The low-temperature fluidity data employed in this paper were obtained with a Gieseler plastometer. Data were obtained from the literature $e^{1,48-62}$ and from Commercial Testing and Engineering. ${ }^{33}$ Descriptions of the apparatus and operation may be found in the literature. ${ }^{56,55}$ The viscosity is determined by measuring the rotation rate of a stirrer in the sample when constant torque is applied.

The high-temperature data were obtained by Fong et al. ${ }^{17}$ in a device designed for rapid heating. Coal is contained in a pancake-shaped cavity in which a disk is driven at constant rotation. The torque was related to viscosity in poise using standards of known viscosity.

There are many problems of translating stirrer rotation speed to viscosity in poise, and most researchers simply report data in degrees/min or dial divisions/min (DDPM) where one DDPM $=3.6^{\circ} \mathrm{min}$. However, the Gieseler plastometer was calibrated in absolute units of viscosity by Steveng ${ }^{56}$ and by Soth and Russe ${ }^{57}$ who obtained the calibration DDPM/min $=10^{-7}$ rhe's, where 1 rhe $=1 /$ poise. We have employed this relationship as a convenience so that data of van Krevelen (at constant temperature) and Fong ${ }^{17}$ (both of whom related the experimental observations on rotation speed and torque to viscosity and thus reported data in viscosity units) could be directly compared with the other data reported in DDPM. To the extent that the coal melt is a New-

(48) Oxley, G. R.; Pitt, G. J. Fuel 1958, 37, 19.

(49) Fitzgerald, D. Fuel 1956, 35, 178.

(50) Waters. P. L. Fuel 1962, 41, 3

(51) Seki, H.; Kumagai, J.; Matsuda, M.; Ito, O.; Iino, M. Fuel 1989, 68,978 .

(52) Wu, M. M.; Robbins, G. A.; Winschel, R. A.; Burke, F. P. Energy Fuels $1988,2,150$

(53) The Gieseler Plastometer data was supplied by Mr. George Engelke from Commercial Testing and Engineering Co.

(54) Lowry, H. H. Chemistry of Coal Utilization; Wiley: New York, 1963.

(55) ASTM D 2639-74 (reapproved, 1980)

(56) Stevens, J. N. CSIRO Division of Coal Research, Investigation Report No. 12, 1957.

(57) Soth, G. C.; Russell, C. C. Proc. Am. Soc. Test. Mater. 1943, 43, 1176.

(58) Solomon, P. R.; Serio, M. A.; Deshpande, G. V.; Kroo, E. Energy Fuels $1990,4,42$

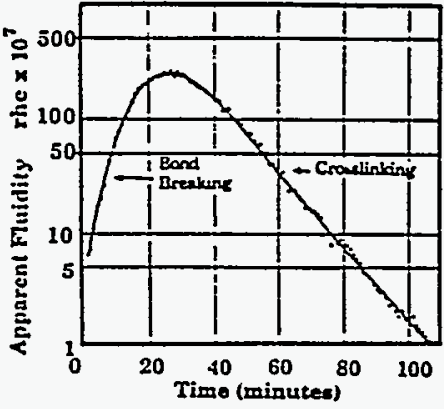

Figure 1. Apparent fluidity as a function of time at $407^{\circ} \mathrm{C}$ for a typical coling coal ( $34.4 \%$ dry ash free volatile matter, coal rank code no. $401 \mathrm{~b})$. From ref 49 .

Table II

\begin{tabular}{llll}
\hline experiments & Fong & Oxley and Pit & van Krevelen \\
volatile marter (daf), \% & 45.2 & 30.3 & 24 \\
C \% (dar) & 79 & N/A & N/A \\
coal used for modeling & Pitts 8 & Upper Kanawha & Upper Freeport \\
volatile matrer (daf), \% & 41.7 & 37.6 & 31.6 \\
C \% (daf) & 83.2 & 82.6 & 85.6 \\
parameters modified from & & & \\
Argonne caal & & & \\
$E_{\text {BB } / R}$ & & 0.17 & 29000 \\
$M_{0}$ (cross-links/bead) & & 6 & 0.07 \\
l(oligomer length) & & 0.59 & 0.19 \\
$\quad H($ al), \% & & &
\end{tabular}

tonian fluid, and the presence of bubbles and ash can be ignored, the same proportionality factor should also be applicable to the viscosity data obtained at both constant torque and constant rotation. We calibrated our model parameters using Gieseler. plastometer data. The agreement for the other sets of data suggest that the conversion factors agree to within an order of magnitude. If the absolute magnitude of the van Krevelen data is ignored, the conversion factor is unimportant.

In making fluidity measurements on coal, it has been found that there is an initial softening of coal on heating which is reversible, and this has been associated with melting and hydrogen bond breaking. This is followed by a sharp rise in fluidity due to the decomposition of the macromolecular network by covalent bond breaking. This sharp rise is illustrated in Figure 1 which presents the measured fluidity of a bituminous coal as a function of time as the coal was heated from $300^{\circ} \mathrm{C}$ at $3^{\circ} \mathrm{C} / \min$ to 407 ${ }^{\circ} \mathrm{C}$ and beld at constant temperature. 19 The slower fall in fluidity with time is due to cross-linking which resolidifies the network.

The model we present employs both the coal composition parameters and the kinetic rates for bond breaking and gas evolution. For the Argonne coals, the rates and composition pa. rameters were determined using a thermogravimetric analyzer with analysis of evolved products by Fourier transform Infrared 
spectroscopy (TG-FTIR). The TG-FTIR analysis of the Argonne coals and the determination of kinetic rates are presented in detail elsewhere. $11-43,59,50$ Table I presents the parameters for the Argonne oals and PSOC 815 used by Seki et al. ${ }^{51}$ for which fluidity data and samples were available. Since it was not possible to obtain samples for the other coals used for the fluidity measurements reported in the literature $e^{1,18.50}$ we have selected from the Argonne coal samples those which best match the reported coals in carbon concentration and volatile matter. Table II presents the available data on the coals whose fluidities have been reported in the literature and the Argonne coals chosen to represent them. More complete composition data for the Argonne coals were presented by Vorres ${ }^{61}$ and for PSOC 815 by Seki et al. 51

\section{Model}

The model of coal fluidity consists of two parts: (i) a macromolecular network model (FG-DVC) to predict the liquid fraction as a function of time and temperature (this prediction includes all the rank-dependent bond breaking and cross-linking behavior and effects of weathering or extraction); and (ii) rank-independent empirical expressions to predict fluidity from the liquid fraction, and the temperature.

The Macromolecular Network Decomposition Model. To predict the liquid fraction and its average molecular weight, we employ the FG-DVC model which describes the decomposition or condensation of the macromolecular network under the influence of bond breaking and cross-linking reactions. ${ }^{38,41-43,58}$ The model employs a sample macromolecular network in the computer, consisting of aromatic ring clusters (monomers) linked by bridges. The molecular weights of the ring clusters is estimated from FIMS data ${ }^{42}$ and ${ }^{13} \mathrm{C}$ NMR. ${ }^{62}$ The bridges are either stable and remain unbroken or are broken by bond scission reactions. New bridges are formed by cross-linking. As discussed previously, ${ }^{38,43,58}$ cross-linking occurs at low temperatures for low-rants coals by a process apparently associated with $\mathrm{CO}_{2}$ evolution. Cross-linking at moderate temperatures occurs by a process associated with $\mathrm{CH}_{4}$ evolution. This is based on an experimentally observed correlation between the evolution of $\mathrm{CH}_{4}$ and the appearance of new cross-links in the macromolecular network (as determined by the solvent swelling ratio). A possible explanation for this observation is that the methane is formed by an ipso substitution reaction in which a methyl group is replaced by a free radical attached to the network or to a network fragment. It is the latter process which leads to the reduction of fluidity shown in Figure 1.

An example of the model is shown in Figure 2. Figure $2 \mathrm{a}$ shows the starting molecule. The network is formed from linear oligomer chains of length $l$ (horizontal) joined by random cross-links (vertical). The network is characterized by the molecular weight of the ring clusters and the average number of attachments per cluster. When more than two attachments are present per cluster a cross-link or branch point occurs. During the initial development of the model, the number of initial branch points (verticle doubled lines) per monomer, $m_{0}$, was chosen to match values of molecular weight between cross-links reported in the literature. ${ }^{38,41-43}$ This parameter,

(59) Solomon, P. R.; Serio, M. A.; Carangelo, R. M.; Bassilakis, R. Gravel, D.; Baillargeon, M.; Baudais, F.; Vail, G. Energy Fuels 1990, 4 (3), 319.

(60) Serio, ML A; Solomon, P. R; Charpenay, S.; Yu, Z. Z; Bassilakis, R. Prepr. Pap.-Am. Chem. Soc., Div. Fuel Chem. 1990, 35 (3), 808. (61) Vorres, K. Prepr. Pap.-Am. Chem. Soc., Div. Fuel Chem. 1987, $32(4), 221$.

(62) Solum, M. S; Pugmire, R. J.; Grant, D. M. Energy Fuels 1989, 3.187.
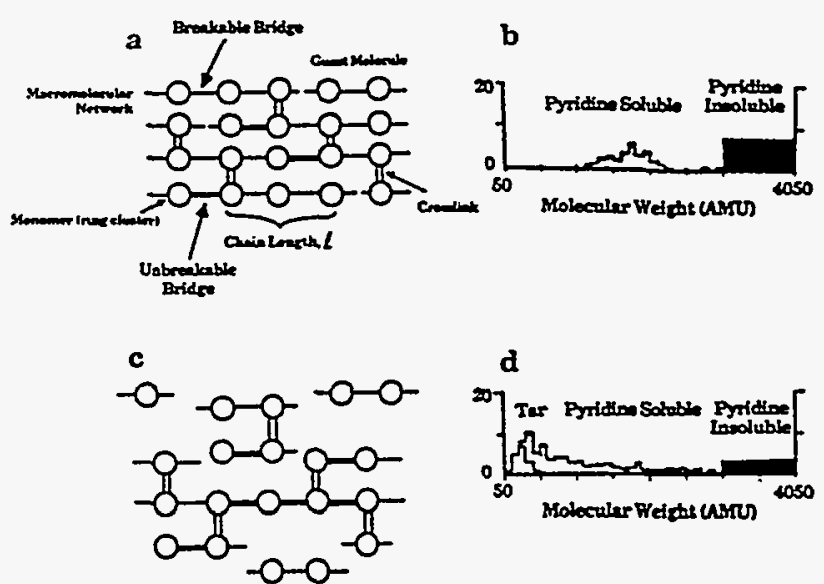

Figure 2. Representation of a coal molecular network in a Monte Carlo simulation ( $\mathrm{a}$ and $\mathrm{c}$ ) and corresponding molecular weight distribution (b and d). In the molecule, the circles represent monomers (ring clusters with their peripheral groups). The molecular weight distributions of the network are shown as histograms in $b$ and $d$. The histogram is divided into tar, pyridine-soluble and pyridine-insoluble fractions. The area under the histogram corresponds to the weight percent of the oligomers.

which is derived from solvent swelling, is, however, not well known and so instead of fixing it from swelling data, it became one of the two adjustable parameters used to fit the fluidity data. The parameter is within the range of reported values. The second parameter is the length of the linear oligomer chains $l$, which are cross-linked to form the network. This parameter is selected so that the fraction of unattached small monomer cluster matches the measured fraction of pyridine solubles. With a fixed number of cross-links, $m_{0}$, the smaller the vale of $l$, the lower the probability that a given oligomer will be attached to the network and thus the higher the value of unattached "extractables". This parameter $l$ is thus fixed for a fixed value of $m_{0}$, but varies with the choice of $m_{0}$. The final parameter in the model is the fraction of donatable hydrogens $H(\mathrm{al})$ in the network. It is assumed that all the donatable hydrogens are in the labile ethylene $\left(\mathrm{C}_{2} \mathrm{H}_{4}\right)$ bridges, so $H$ (al) determines the number of breakable bridges. These are randomly distributed in the linear oligomer chains. All other bridges in the chains are assumed to be unbreakable. The unbreakable bridges are indicated by heavy horizontal lines and the breakable bonds by thin horizontal lines. Cross-links once formed are also assumed to be unbreakable. As in the case of $m_{0}$ $H(a l)$, is not easily measured and has become the second adjustable parameter.

With the parameters $m_{0}, l$, and $H(a l)$ determining the number and type of bonds, the position of the bonds is randomly chosen and the molecular weight distribution of the initial coal molecule is computed. The result is shown in Figure 2b.

To simulate pyrolysis, the rates of bond breaking and cross-linking are determined at each time step and the appropriate changes in bridge concentration are randomly distributed in the molecule. When bonds are broken, more small molecules are formed as shown in Figure 2, $c$ and d. For each labile bridge broken, a second labile bridge is converted to an unbreakable bride $\left(\mathrm{C}_{2} \mathrm{H}_{2}\right)$ as its hydrogen is used to stabilize the free radicals. The light molecules evaporate and become tar. The value of $H(a l)$ thus determines the total number of bridges which can be broken and, thus, the extent to which the network comes apart. The parameter $m_{0}$ determines the efficiency with which the network is fragmented. The larger the value of $m_{0}$, the more bonds must be broken to produce fragments, and the 

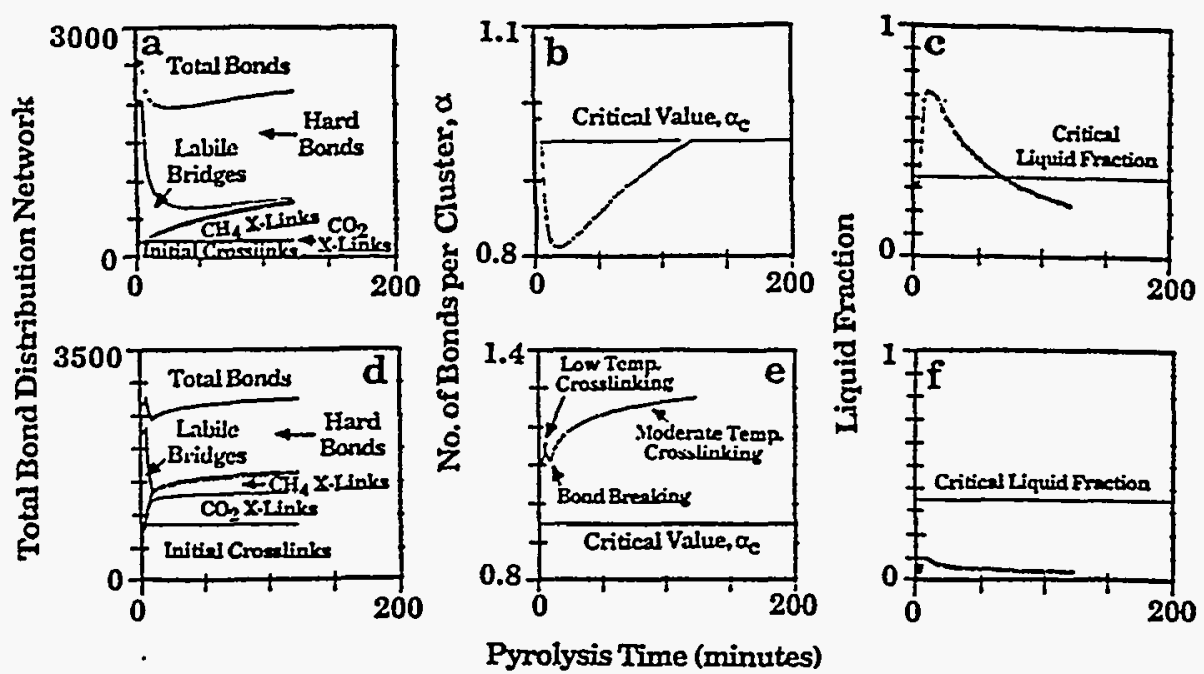

Figure 3. Comparison of the variation in the unbroken bond concentration, bonds/cluster, $\alpha$, and liquid fraction with time at constant temperature of $440^{\circ} \mathrm{C}$ for (a-c) Upper Freeport bituminous coal and (d-f) Wyodak subbituminous coal.

smaller the fragments. Thus, $m_{0}$ determines the ratio of small to large fragments.

To illustrate the model's predictions, we consider in Figure 3 the break up of the network as a function of time at a temperature of $440^{\circ} \mathrm{C}$. Figure 3 compares, for a subbituminous and a bituminous coal, the variation with time at constant temperature in the total number of unbroken bonds (bridges and cross-links), the number of unbroken bonds per cluster $\alpha$, and the liquid fraction. Also shown are the individual contributions to the total bond count. Both networks experience a minimum in $\alpha$ as bonds are cleaved and then reconnected through cross-linking reactions. This leads to a maximum in the liquid fraction shown in Figure 3, $c$ and $f$. As discussed below, the viscosity is related to the liquid fraction produced during the break-up of the network. The liquid fraction consists of all molecules detached from the starting macromolecular network (the solid fraction). Operationally, we count the largest three molecules as belonging to the solid fraction and all others as belonging to the liquid fraction. This is a convenient approximation which gives results that agree reasonably well with experiment and do not depend on the molecular weight of the starting computer molecule. We have also used just the largest molecule as the solid fraction and found that computed results are similar but noisier.

An important feature of a network model is that the break-up or solidification of the network occurs near a "gel point" where the number of unbroken bonds per ring cluster (monomer), $\alpha$, reaches a critical low value. In a homogeneous network model, this gel point for a polymerizing melt occurs at the first appearance of the solid (or in the case of a decomposing network when the liquid fraction goes to 1.0), since the solid in a homogeneous melt extends throughout the entire melt. For most network geometries, the break-up of the network occurs between $\alpha=1.0$ and $\alpha=0.8$.

For an inhomogeneous melt (such as expected for a liquefying powdered coal sample), the solids can appear as isolated particles (instead of extending across the whole sample as in a homogeneous melt), so the gel point occurs at a higher solid fraction (lower liquid fraction). Based on the inhomogeneous model of fluidity discussed below, the liquid fraction must only exceed 0.35 before there is sufficient liquid to separate the solid particles, and appreciable fluidity can occur. With the network geometry assumed for this model, this minimum liquid fraction is achieved at a critical value $\alpha_{c}$ of approximately 0.95 . For bituminous coals, this critical value can be achieved in pyrolysis and the coal melts and becomes fluid as illustrated in Figure 3c. For low-rank coals, the effect of lowtemperature cross-linking is to increase $\alpha$ (see Figure 3e), so that in some cases the network cannot come apart by normal pyrolytic reactions. For the subbituminous coal, shown in Figure 3, the value of $\alpha$ has a sharp increase at low temperatures due to $\mathrm{CO}_{2}$ related cross-linking. This can be seen by the appearance of $\mathrm{CO}_{2}$ related bonds in Figure 3d. These bonds are a significant fraction of the total bonds connecting the network. With this large number of additional bonds, the value of $\alpha$ never achieves the critical value $\left(\alpha_{c}=0.95\right)$ and the solid fraction of the coal remains too high to liquefy (see Figure $3 f$ ). On the other hand, $\alpha$ for the bituminous coal is not increased by $\mathrm{CO}_{2}$ related cross-linking. The value of $\alpha$ falls below the critical value and sufficient liquid is produced for the network to disintegrate and become fluid.

The Viscosity Model. Because of the inhomogeneous and complicated nature of coals, the viscosity model which we developed is purely phenomenological. The previous literature was employed to suggest the important parameters and appropriate relationships, but the final choice of the model was arrived at by finding the simplest expressions capable of fitting the available data. The viscosity model used here has terms that depend on the coal liquid temperature, and the volume fraction of solids, with on inhomogeneous gel-point.

In common with previous studies of coal viscosity, a two-phase model is used. The particular equation chosen is that put forward by Mooney ${ }^{17,32}$

$$
\ln \left(\eta / \eta_{\mathrm{liq}}\right)=\left[\frac{k_{\mathrm{E}} \phi_{\mathrm{s}}}{1-\phi_{s} / \phi_{\mathrm{c}}}\right]
$$

where $\eta$ is the viscosity of the suspension, $\eta_{\text {iq }}$ the viscosity of the liquid, $k_{\mathrm{E}}$ is the Einstein coefficient, and $\phi_{\mathrm{g}}$ is the volume fraction of solids, having a critical value $\phi_{c}$ at which $\eta$ goes to $\infty$.

The viscosity of the liquid phase is given by the Andrade equation

$$
\eta_{\text {liq }}=C \exp \left(E_{\eta} / R T^{*}\right)
$$

which on combining with eq 1 leads to

$$
\eta=C \exp \left(E_{\eta} / R T^{*}\right) \exp \left[\frac{k_{\mathrm{E}} \phi_{\mathrm{s}}}{1-\phi_{\mathrm{s}} / \phi_{\mathrm{c}}}\right]
$$

The constants used in the viscosity theory are as follows: 

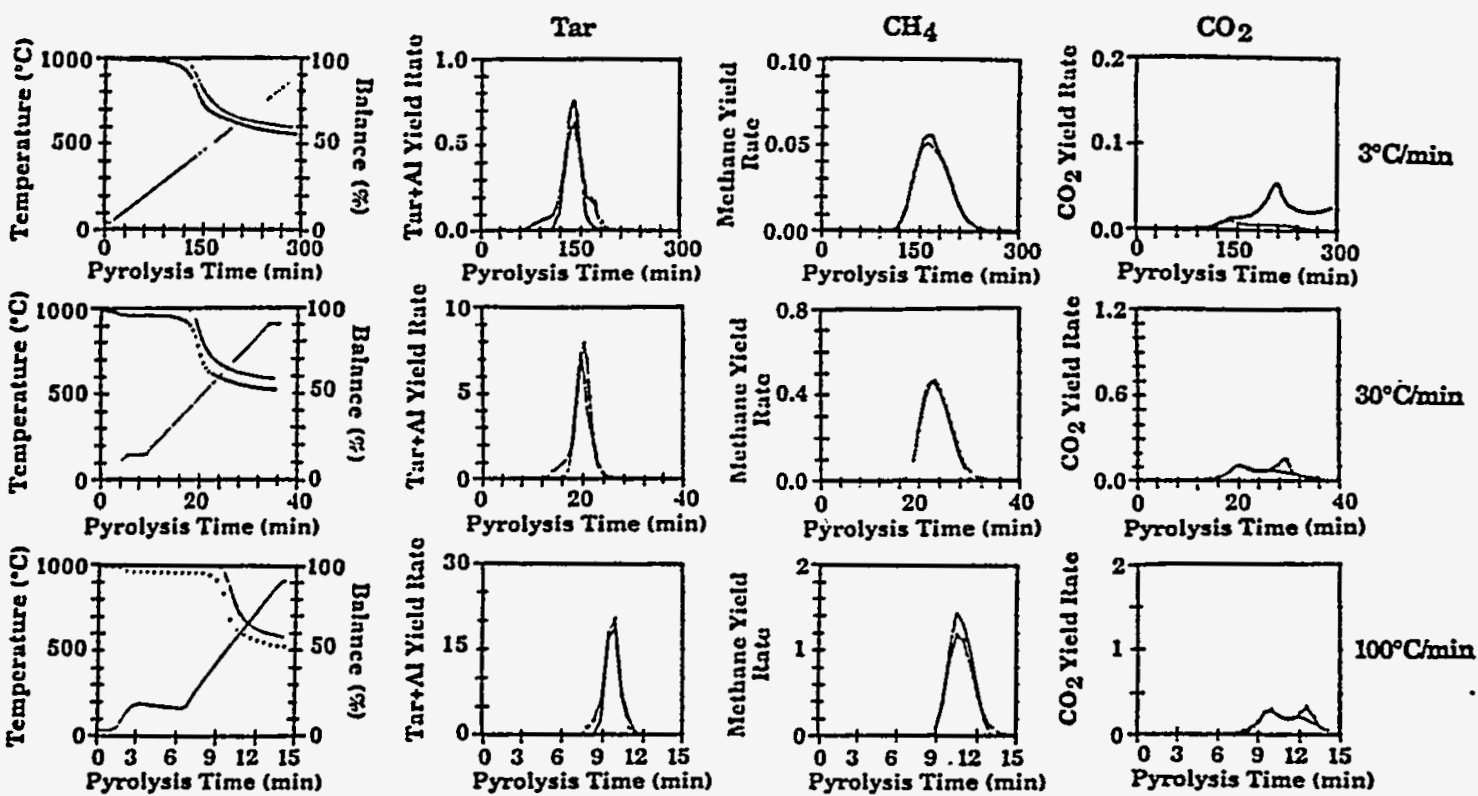

Figure 4. Kinetic analysis for Utah Blind Canyon coal for weight loss, tar evolution, methane evolution, and $\mathrm{CO}_{2}$ evolution at three heating rates. Experiment (*) and theory (solid line) using the FG-DVC Model ${ }^{4-43}$ with the kinetic rates reported in Table I.

$k_{E}$, Einstein coefficient, $k_{E}=5.0 ; \phi_{e}$ volume fraction of the solid phase at the gel-point, $\phi_{c}=0.65 ; \eta_{\text {liqu }}$, viscosity of the liquid phase; $\eta_{\text {iq }}=C_{1} \exp \left(E_{71} / R T^{*}\right)=7.5 \times 10^{-31} \exp -$ $\left(100000 / R T^{*}\right)$ for $T \leq 708 \mathrm{~K}$, and $\eta_{\mathrm{bia}}=C_{2} \exp \left(E_{\eta_{2}} / R T^{*}\right)$ $=1.623 \times 10^{-15} \exp \left(50000 / R T^{*}\right)$ for $T \geq 708 \mathrm{~K} ; T^{*}: \mathrm{Ab}$ solute temperature, cutoff at $T^{*}=750 \mathrm{~K}$, i.e., $T^{*}=T$ for $T \leq T^{\mathrm{k}} \mathrm{K}$, and $T^{*}=T^{\mathrm{c}}$ for $T>T^{ } \mathrm{K} ; R$ : gas constant, $R$ $=1.98 \mathrm{cal} \mathrm{mol}^{-1} \mathrm{~K}^{-1}$.

The value of $k_{\mathrm{E}}=5$ was arrived at empirically, i.e., to fit the data. This value, however, matches the situation in which liquid is entrapped within large agglomerates of solid. 63 The value of the activation energies for coal was chosen based on measured liquid viscosities (from $\mathbf{5 0}$ to $100 \mathrm{kcal} / \mathrm{mol}$ ) determined by Waters. ${ }^{50}$ It should be noted that this is higher than that typically used for polymers and is probably describing chemical reactions and instrument-dependent effects as well as true viscosity. The use of a cutoff temperature $T$ follows the concept introduced by $\mathrm{Oh}$ et al. ${ }^{18}$

\section{Results}

Volatile Evolution. There are two aspects to validating the model by comparison with data. The first is that the FG-DVC model should provide good predictions for pyrolysis products. This validates the coal-dependent kinetic rates for bond breaking and cross-linking and the amount of cross-linking. The second comparison of predictions is to fluidity data. Extensive comparisons of the FG and FG-DVC models have been made by Solomon et al. and Serio et al. ${ }^{39-11}$ These comparisons show reasonable agreement between theory and experiment using rank-independent kinetics for the Argonne coals and several other coals. There are, however, some variations of kinetic rates with rank 59 and these differences particularly affect the viscosity predictions. We have consequently adopted rank-dependent kinetics for the FG-DVC model in making the fluidity predictions. We have employed the volatile evolution profiles from the TG-FTIR analysis to determine the rates by adjusting the rate constants to make the theory fit the data. Figure 4 compares the observed and predicted weight loss, tar evolution, methane evolution, and $\mathrm{CO}_{2}$ evolution profiles at heating rates of $0.05,0.5$, and

(63) Lewis, T. B.; Nielsen, L. E. Trans. Soc. Rheol. 1968, 12, 421.
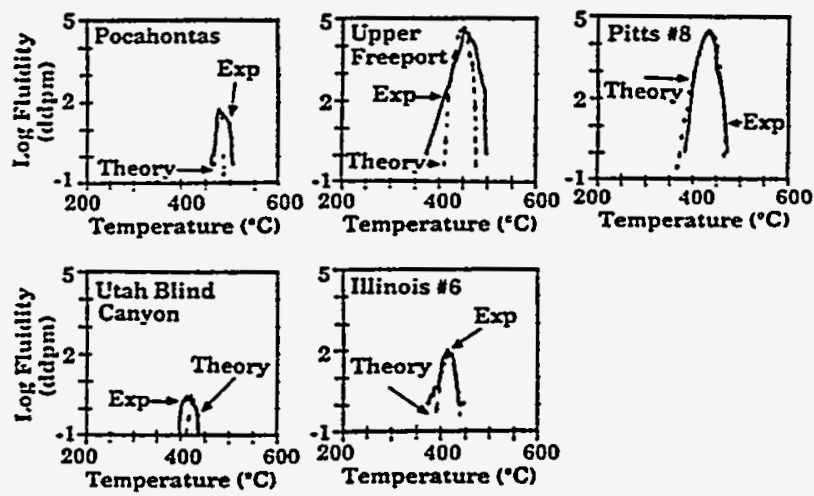

Figure 5. Comparison of measured and predicted fluidity for five Argonne coals: $\left(^{*}\right)$ experiment; $(--)$ theory.

$1.67^{\circ} \mathrm{C} / \mathrm{s}$ for Utah bituminous coal. The agreement is good for all three heating rates. The same procedure was employed for all eight Argonne coals. ${ }^{43,59,50}$ A discussion of the procedure and a complete tabulation of rates appears elsewhere. ${ }^{43,60}$ For PSOC 815 the results were fit - from the $30^{\circ} \mathrm{C} / \mathrm{min}$ data only, assuming the same preexponential values used for the Argonne coals. The results are presented in Table $I$ for $E, k_{0}$, and $\sigma$ which are the parameters of rate equations of the form

$$
k_{n}=k_{0} \exp (-((E / R) \pm(\sigma / R)) / T)
$$

with $k_{0}$ in $\mathrm{s}^{-1}, E / R$ in $\mathrm{K}$, and, $\sigma / R$ in $\mathrm{K}^{41,42}$ The accuracy of the activation energies is estimated to be within $\pm 2 \mathrm{kcal}$. With a fixed activation energy, the accuracy of the preexponential $k_{0}$ is $\pm 25 \%$ and of $\sigma$ is $\pm 10 \%$. These rates appear to provide good predictions when applied to fit data taken at much higher heating rates $\left(5000^{\circ} \mathrm{C} / \mathrm{s}\right)$ as well ${ }^{43,50}$ The bond-breaking rates are in reasonable agreement with the tar evolution rates derived by Burnham et al.

Viscosity Predictions for the Argonne Coals. All the viscosity data were fitted using the same viscosity equations and same viscosity model constants. The kinetic rates for bond breaking and the amount and rates of $\mathrm{CO}_{2}$ and $\mathrm{CH}_{4}$ evolution are determined from the TG-FTIR analysis. The only adjustable parameters for each coal

(64) Burnham, A. K.; Oh, M. S.; Crawford, R. W.; Samoun, A. M. Energy Fuels 1989, 3, 42 

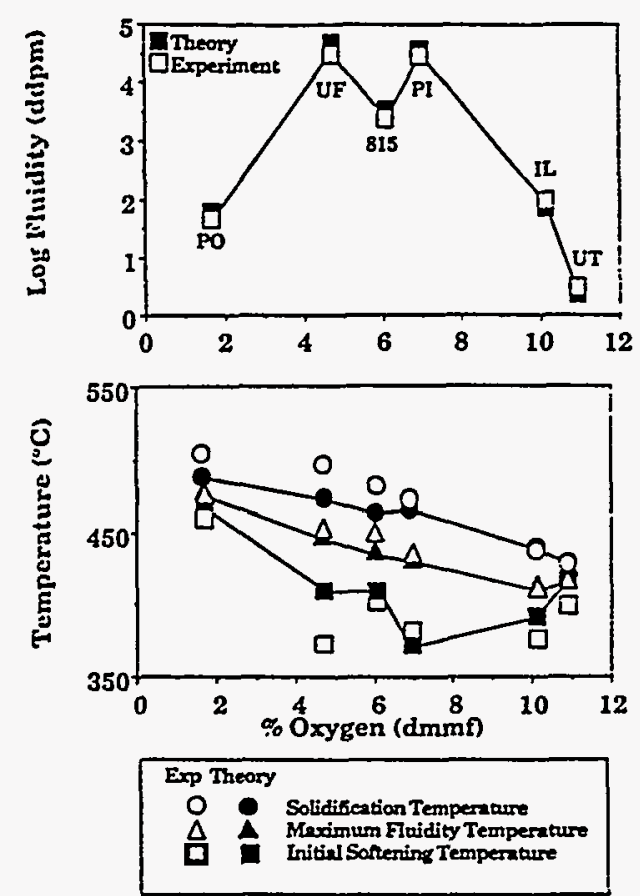

Figure 6. Fluidity behavior as a function of coal rank: theory and experiment. (a) Maximum fluidity values and (b) fluidity temperatures.

were the number of initial cross-links per monomer, $m_{0}$, and the percent of donatable hydrogen $H$ (al). The value of $l$ is obtained from the extracted amount and the value chosen for $m_{0}$. The cross-linking efficiency for $\mathrm{CH}_{4}$ and $\mathrm{CO}_{2}$ is one cross-link formed per $\mathrm{CH}_{4}$ or $\mathrm{CO}_{2}$ evolved.

Figure 5 compares the theoretical and experimental Gieseler plastometer curves ${ }^{53}$ for the five Argonne coals for which data were available. The low-rank Argonne coals, Zap and Wyodak, showed no fluidity and the LewisStockton coal was not measured. Figure 6 compares the maximum fluidity, solidification temperature, maximum fluidity temperature, and initial softening temperature as a function of the coal's oxygen concentration. In general, the data are in good agreement with the model. The agreement is the worst for the Upper Freeport initial softening and solidification temperatures. The maximum fluidities as a function of carbon concentration match the trends summarized by Whitehurst, ${ }^{65}$ with maximum fluidity achieved between 4-8\% 0 DMMF (84 and 88\% C).

Considering that there are only two adjustable parameters required to fit the Gieseler curve, it appears that the model is more than just curve fitting. Further comparisons which allow predictions of other conditions lend further credence to the model's accuracy in simulating the chemical and physical processes in fluidity. One such comparison is of the predicted liquid yield compared with $M_{2 T}$ values derived by Lynch et al.6 The $M_{2 \mathrm{~T}}$ parameter is a measure of the hydrogens attached to nonmobile molecules. As coal is heated $M_{2 \mathrm{~T}}$ is observed to decrease as pyrolysis begins, reaching a minimum value (which coincides with the maximum fluidity temperature), and then to increase. We find that our predicted solid fractions have a similar dependence on temperature when compared to the $M_{2 \mathrm{~T}}$ data for the Argonne coals. ${ }^{67}$ Comparison of our

(65) Whitehurst, D. D. Mitchell, T. O. Farcasiu, M. Coal Liquefaction: The Chemistry and Technology of Thermal Processes; Academic Press: New York, 1980.

(66) Lynch, L. J.; Webster, D. S.; Sakurovs, R.; Barton, W. A.; Maher, T. P. Fuel 1988, 67, 579.

(67) Sakurovs, Richard. Private communication.
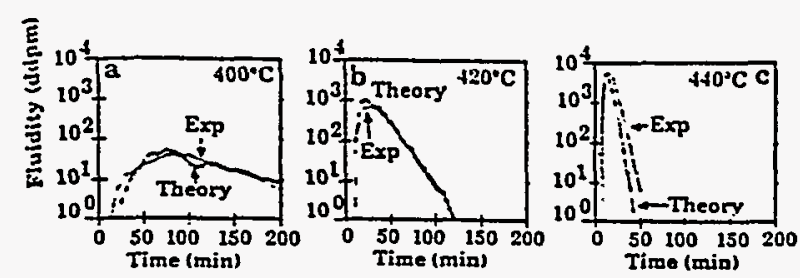

Figure 7. Comparison of experiment and theory for fluidity of a high-rank coal at constant temperatures of (a) $400^{\circ} \mathrm{C}$, (b) 420 ${ }^{\circ} \mathrm{C}$, and $(\mathrm{c}) 440^{\circ} \mathrm{C}$. The experiment is for a coal which has $30 \%$ volatile matter content ${ }^{+8}$ and theory is for Upper Kanawha (see Tables I and II).
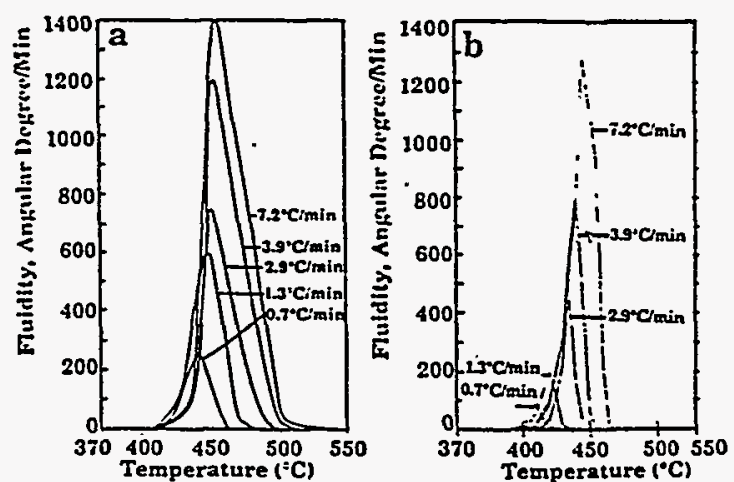

Figure 8. Fluidity at constant heating rate: (a) experimental data of van Krevelen ${ }^{1}$ for $24 \%$ volatile coal and (b) theory for Upper Freeport coal (see Tables I and II).

model with the $M_{2 T}$ data will be the subject of a future publication. Other fluidity conditions which can be compared to the model are discussed below.

Viscosity Predictions for Literature Data. The first example of the application of the model to predict fluidity for literature data is shown in Figure 7. The data are from Oxley and Pitt ${ }^{+8}$ obtained by heating to constant temperatures of 400,420 , and $440^{\circ} \mathrm{C}$. The coal is believed to be closest in behavior to the Lewis-Stockton since it has comparable volatile weight loss, and the parameters for this coal, except for a different amount of $\mathrm{CH}_{4}$ and slightly different network parameters, were employed (see Table II). The agreement is excellent in following the increase and decrease in fluidity due to bond breaking and crosslinking, suggesting that these processes are accurately modeled. The magnitude of the fluidity is accurately predicted at all temperatures.

Results for a higher rank coal studied by van Krevelen ${ }^{1}$ at constant heating rates are presented in Figure 8a. Considering fluidity data and volatile matter, this coal's rank seemed to be between Upper Freeport and Pocahontas coals. The coal composition and kinetic parameters picked to represent the coal were that of Upper Freeport. The bond-breaking rate was, however, chosen to be the same as for Pocahontas in order to match the amount of fluidity (see Table II). The theoretical predictions in Figure $8 \mathrm{~b}$ are in reasonable agreement with the data. The data, however, shows a narrower range of initial softening than the simulation, suggesting a greater influence of melting for this coal.

Data from isothermal experiments for the same Van Krevelen coal are presented in Figure 9a. The theory in Figure 9b show the right increase in fluidity with temperature. The model, however, does not predict the very early rise in fluidity at low temperature $\left(400\right.$ and $\left.408^{\circ} \mathrm{C}\right)$, which is probably related to the breakage of hydrogen bonds and consequent melting. The loss of fluidity is also predicted to occur sooner than is indicated by the data. Additional effort to determine the sources of error was not made because the coals were not available to accurately 


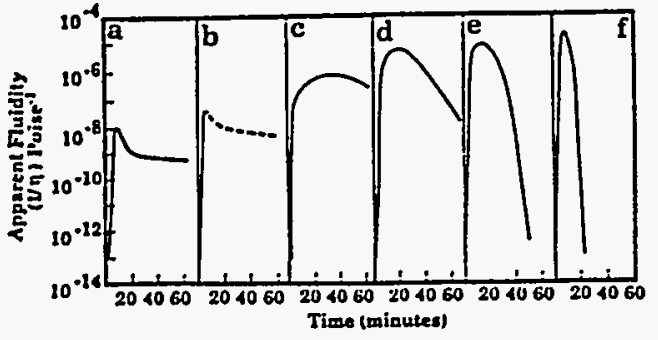

a

Measured

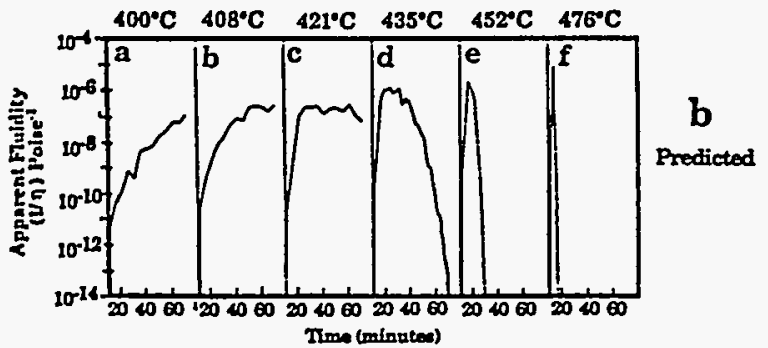

Figure 9. Fluidity at constant temperature: (a) experimental data of van Krevelen ${ }^{1}$ for $24 \%$ volatile coal and (b) theory for Upper Freeport coal (see Tables I and II).

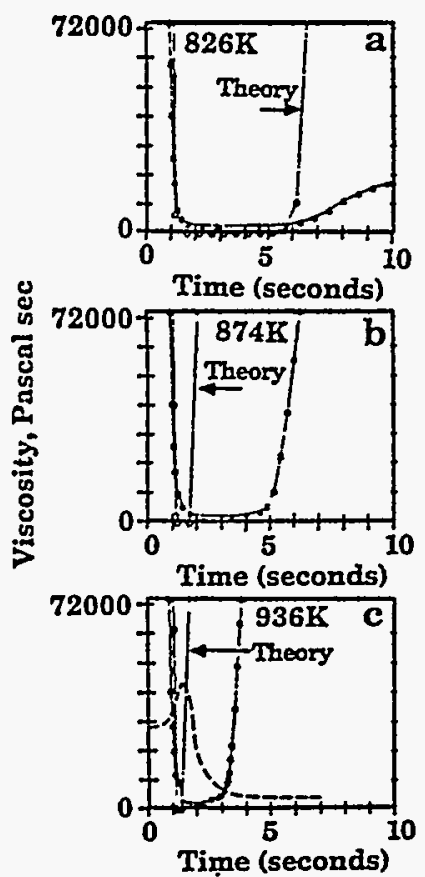

Figure 10. Viscosity at high temperatures. Symbols connected by line are data of Fong et al. ${ }^{27}$ Lines without symbols are theory. Dashed line in $(c)$ is the extract yield.

determine the kinetic and composition parameters.

Results for a Pittsburgh Seam coal obtained at high heating rates by Fong et al. ${ }^{17}$ are presented in Figure 10 as the symbols connected by lines. The theory is shown as the solid lines. There is good agreement between theory and experiment for the onset of fluidity. The maximum fluidity is overpredicted by about 1 order of magnitude, possibly because the model based the prediction on the Argonne Pittsburgh No. 8, instead of the Pittsburgh coal from Fong's experiment (see Table II). Another possibility is that Fong's calibration relating disk rotation velocity to fluidity is not fully consistent with the calibration made for the Gieseler plastometer by Stevens ${ }^{56}$ and Soth and Russel..$^{57}$ Since the model constants were calibrated using Gieseler plastometer data in which rotation is measured at constant torque, the discrepancy may suggest that there are non-Newtonian effects or consequences due to bubbles

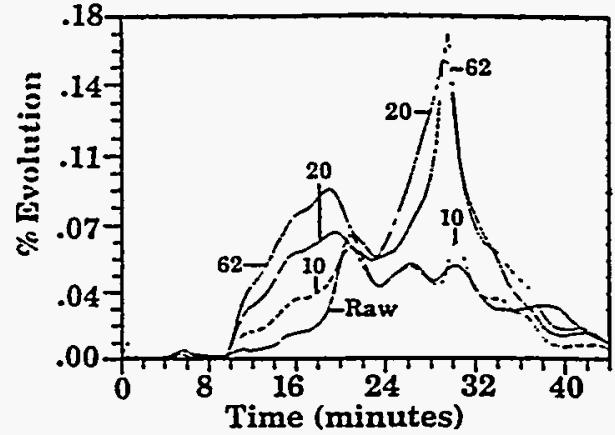

Figure 11. $\mathrm{CO}_{2}$ evolution curves for raw coal and 10, 20, and 62 days oxidized coals.

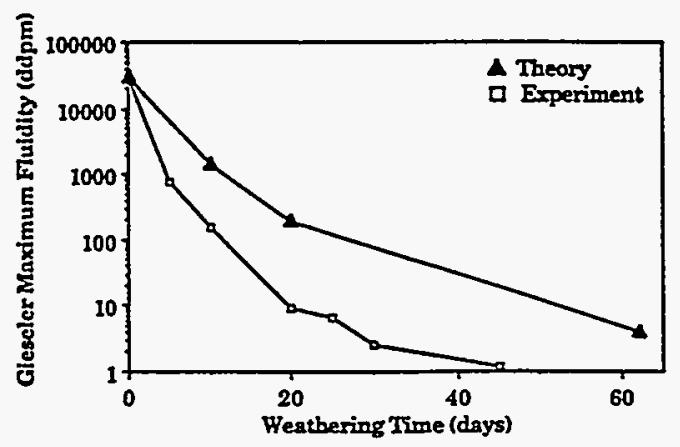

Figure 12. Comparison of predicted and measured fluidity for oxidized Pittsburgh coal (data from $\mathrm{Wu}$ et $\mathrm{al}^{52}$ ).

or ash. The loss of fluidity is also predicted to occur much sooner than expected. The extract yield obtained by Fong et al. ${ }^{17}$ in a heated grid experiment is also shown as a dashed line. The disappearance of fluidity predicted by our model does appear to coincide with the disappearance of the extract yield. This is as expected, since the model is dependent on the liquid fraction. The fact that the fluidity and extract data do not agree may suggest that there are differences in temperature between the two apparatuses employed by Fong.

Effect of Oxidation on Fluidity. It is well known that oxidation of coal rapidly destroys its fluidity. A systematic study of the effect of coal weathering on fluidity was recently reported by Wu et al ${ }^{52}$ The maximum fluidity for a Pittsburgh Seam coal was reduced from 30000 DDPM to a little over 1 DDPM by oxidation for 40 days at a temperature of $80^{\circ} \mathrm{C}$ and to a little over $10 \mathrm{DDPM}$ by weathering for 380 days at $50^{\circ} \mathrm{C}$.

Oxidation of a Pittsburgh Seam coal in our laboratory was performed at $80^{\circ} \mathrm{C}$ for 10,20 , and 62 days. In our model, the loss of fluidity with increasing orygen concentration is related to the increase in $\mathrm{CO}_{2}$ evolution and, hence, increases in low-temperature cross-linking. To determine the $\mathrm{CO}_{2}$ evolution at low temperatures, measurements were made in the TG-FTIR. ${ }^{59}$ The data in Figure 11 show that the low-temperature $\mathrm{CO}_{2}$ evolution was significantly increased, becoming comparable to that for Illinois No. 6 after 10 days of oxidation, and becoming larger than that for the Utah bituminous coal after 20 days. After 3 months at $110^{\circ} \mathrm{C}$ the $\mathrm{CO}_{2}$ evolution was comparable to that of a lignite. When these increased $\mathrm{CO}_{2}$ yields were incorporated in the simulation for the oxidized Pittsburgh Seam coal's fluidity, the maximum fluidity was reduced.

We compare our predicted maximum fluidity with the measurement of $W u$ et al $^{52}$ for comparable coal and oxidation treatment in Figure 12. The agreement is quite reasonable considering the difference in coal samples. Also, the work of Wu et al. suggests that some of the loss of 


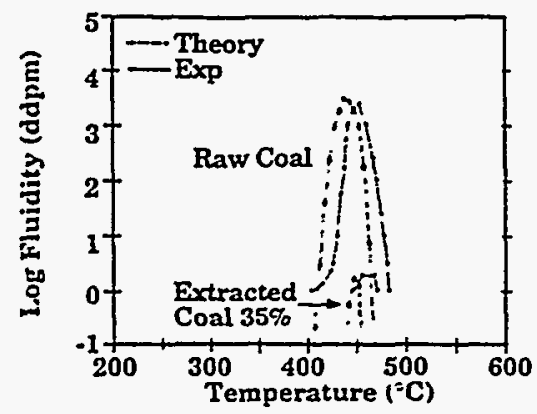

Figure 13. Comparison of predicted and measured fluidity for raw PSOC 815 coal and $35 \%$ extracted coal (data from Seki et al. ${ }^{\text {II). }}$.

fluidity is caused by weathering of the pyrite which is not included in our model.

Effect of Extraction on Fluidity. The extraction of coal with organic solvents has been shown to affect the caking property of the coal residue. ${ }^{68}$ To study this effect, Seki et al. measured the fluidity of a coal and a set of residua after extractions in a mix of $\mathrm{CS}_{2}$-pyridine and $\mathrm{CS}_{2}-\mathrm{NMP}$ solvents.51 Their results in Figure 13 show that extracting the coal reduces the fluidity.

In our model, extraction of the coal reduces the fluid fraction and hence the fluidity. To model their results, we obtained a sample of PSOC 815 and determined the composition and kinetic parameters for the model by performing TG-FTIR and solvent swelling analysis experiments. Based on these data, the computed liquid percentage of the room temperature sample of raw PSOC 815 is $40 \%$. The predicted fluidity for the raw coal is shown as the dashed line in Figure 13. The agreement with the data is good. To model the residua after extraction, the oligomer length $l$ was varied to reduce the liquid fraction in the starting residue by the amount extracted. For the case in which $30 \%$ of the coal was extracted, the starting liquid amount was reduced from $40 \%$ to $10 \%$. The fluidity calculated for the $30 \%$ extract yield case shown in Figure 13 agrees well with the measured values.

\section{Discussion}

The comparisons presented above demonstrate that the model which we have developed fits fluidity data for all ranks of coal and predicts the proper trends with heating rate, weathering, or extraction. In evaluating the usefulness of any model, there are, however, a number of additional questions which should be addressed. (1) Does the model extrapolate to other conditions? (2) Are the number of adjustable parameters manageable? (3) Is the model really describing the physical and chemical processes, or simply curve fitting? (4) How unique is the model?

With regard to the first question, all of the kinetic rates were derived from low heating rate data, but the one comparison to high heating rate fluidity ${ }^{17}$ seems reasonable. Also, extrapolations of the model to predict tar and gas yields at high heating rates are good.60,69,70

With regard to the second question, the fluidity part of the model has seven parameters: $C=C_{1}$ or $C_{2}$ depending on temperature, $K_{\mathrm{E}} ; E_{\eta}=E_{\eta 1}$ or $E_{\eta 2}$ depending on temperature; $\phi_{c}$, and $T^{c}$, but these are fixed for all coals and conditions. The FG-DVC model has many coal compo-

(68) Dormans, H. N. M.; van Krevelen, D. W. Fuel 1960, 39, 273.

(69) Serio, M. A.; Solomon, P. R.; Yu, Z. Z; Bassilakis, R. Prepr. Pap.-Am. Chem. Soc., Div. Fuel Chem. 1989, 34 (4), 1324.

(70) Solomon, P. R.; Serio, M. A.; Markham, J. R. Kinetics of Coal Pyrolysis Int. Conf. Coal Sci., Proc., IEA, Tokyo, Jpn. October 23-27; 1989, 575.
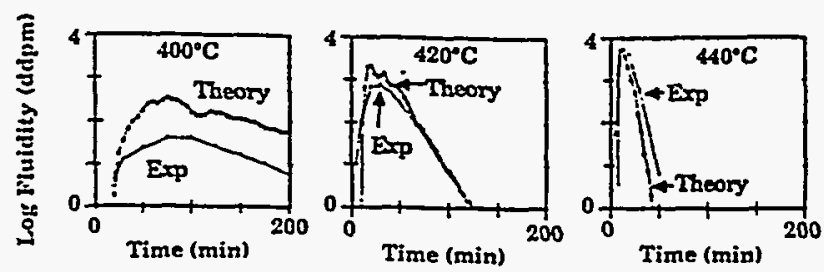

Figure 14. Comparison of Oxley and Pitt Datats with predictions using $E_{\mathrm{q}}=50 \mathrm{kcal}$.

sition and kinetic parameters, but these are fixed based on the TG-FTIR analysis except for three parameters related to the macromolecular network geometry $\left(m_{0}, l\right.$, and $H(a l))$. Two of these can be independently adjusted to fit the fluidity, tar evolution, and solvent extraction data. We believe that two adjustable parameters is reasonable.

With regard to the third question, the description of the decomposition and resolidification of the macromolecular network appears sound in concept, since the model fits fluidity, extract yields, tar evolution, gas evolution, and $M_{2 T}$ measurements. ${ }^{6,67}$ The network model appears to provide a good parameter, the solid fraction, $\phi_{3}$, upon which the fluidity equations are based. This parameter incorporates all of the rank-dependent bond breaking and cross-linking phenomena and the effects of weathering or extraction. On the other hand, the fluidity equations are empirical and do not have any obvious relationships to physical phenomena. Their functional form is consistent with those employed in previous investigations.

A further test is whether the network parameters make any sense. This is related to the fourth question of how unique is the choice of these parameters. To address these issues we have performed a sensitivity analysis, considered the variation in the parameters with rank, and considered the meaning of the parameters. These issues are discussed below.

Sensitivity Analysis. A sensitivity analysis was conducted in which the predictions of the model were determined using variations in the values of the fluidity model parameters, $k_{E}, E_{\eta}, \phi_{c}$, and $T$, and the coal network parameters $H(a), m_{0}$, and $l$. The parameter $C$ is simply a proportionality constant.

Variations in $E_{\eta}$ and $T^{c}$. Variations in the assumed value of $E_{\eta}$ were made between the extremes reported by Waters of 50-100 kcal $/ \mathrm{mol}$. Also, $T^{k}$ (cutoff temperature) was varied between 708 and $750 \mathrm{~K}$. The network parameters were readjusted in each case to get the best fit. Good fits were achieved for all the Argonne coals for values of 50,80 , and $100 \mathrm{kcal} / \mathrm{mol}$. In this case, since the data were taken for a single heating rate, there is only one value of the temperature for maximum fluidity for each coal. Potential errors in choosing the wrong value of $E$ can be overcome by adjusting the network parameters. Thus, if just the Argonne data at $3^{\circ} \mathrm{C} / \mathrm{min}$ are used, then the model is insensitive to the choices of $E_{\eta}$ and $T^{\mathrm{c}}$.

1 The same comparisons at 50, 80, and $100 \mathrm{kcal}$ and variable $T^{*}$ were made in simulating the data of Oxley and Pitt and van Krevelen which provide data for several temperatures of maximum fluidity for each coal. Figure 14 presents the Oxley and Pitt predictions for $E_{\eta}=50 \mathrm{kcal}$ with the critical temperature $T^{e}=750 \mathrm{~K}$. The highest value of $E_{\eta}(100 \mathrm{kcal} / \mathrm{mol})$ in Figure 7 gave a better fit, especially for low temperatures, suggesting a high value of $E_{r}$.

The Orley and Pitt data were insensitive to the value of the critical temperatures $T^{c}$, since all the experiments were done at temperature lower than $713 \mathrm{~K}$. The van Krevelen data, which describe the behavior of a coal up 


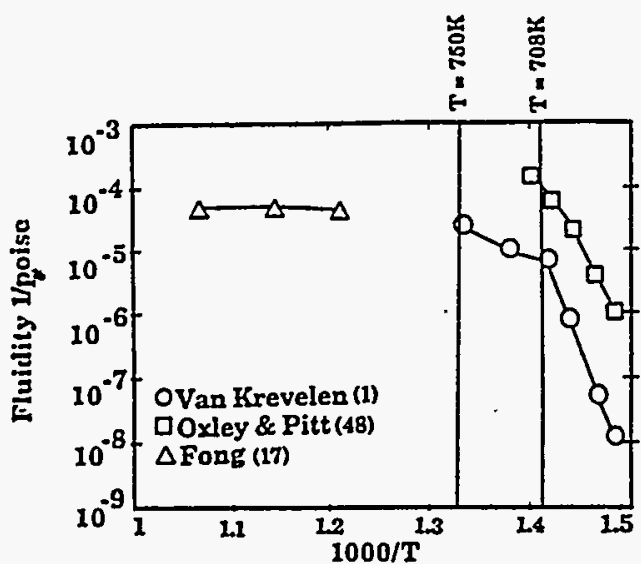

Figure 15. Marimum fluidity for three experiments.

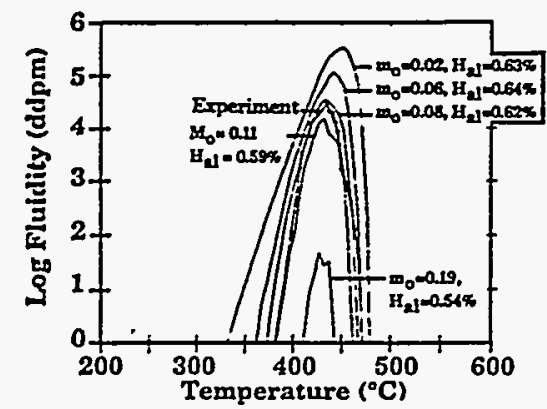

Figure 16. Comparison of measured fluidity for Pittsburgh No. 8 conl with predictions using different values of $m_{0}$ (initial cross-link density).

to $749 \mathrm{~K}$, were also well predicted using $E_{\eta}=100 \mathrm{kcal}$ at low temperatures (up to $708 \mathrm{~K}$ ). However, at higher temperatures the fluidity was overpredicted. Figure 15 which presents the maximum fluidity achieved as a function of temperature seems to indicate a change in $E_{n}$ at about 708 K. To take this into account we reduced $E$, from 100 to $50 \mathrm{kcal}$ for temperatures between 708 and $750 \mathrm{~K}$ and adjusted $C$ accordingly. This change does not have any influence on Argonne coal fluidity predictions and Oxley and Pitt predictions, since in these cases fluidity is present at temperatures generally lower than $708 \mathrm{~K}$. While this choice of reducing $E_{\eta}$ is only based on one experiment, it gave good predictions for all available data including van Krevelen's. Further experiments on variations in fluidity with temperature should be performed and compared.

Variations in $k_{\mathrm{E}}$. We varied $k_{\mathrm{E}}$ between 2.5 and $5 . k_{\mathrm{E}}$ $=5$ gave a better fit by allowing a wide range of fluidity values since it emphasizes the role of the amount of liquid fraction. This helped us fit low-rank coals like Ilinois No. 6, which have a large tar amount but low fluidity.

Variations in $\phi_{c}$ As mentioned earlier in the Introduction, the critical solid volume fraction $\phi_{c}$ has a value of 0.64 for randomly close packed spheres and is thought to range between 0.5 and 0.9 for other systems. As a first approrimation, we chose $\phi_{c}$ to be 0.65 and were able to fit all the available data. We believe that other parameters (initial cross-link density and the proportionally constant for the viscosity) can compensate for the choice of $\phi_{\mathrm{c}}$ and values between 0.60 and 0.70 could be used equally well.

Variations with $m_{0}$ and $H(a l)$ and 1 . Having fixed the parameters in the fluidity model, tests were made of the sensitivity of the model to the assumed values of $m_{0}$, $H(\mathrm{al})$, and $l$. Figure 16 presents predictions for the fluidity of the Pittsburgh Seam coal for different values of $m_{0}$. The simulations were performed with the following rules: (1) a value of $m_{0}$ was picked, (2) $H$ (al) was adjusted to give the correct tar yield in the TG/plus experiment, (3) $l$ was
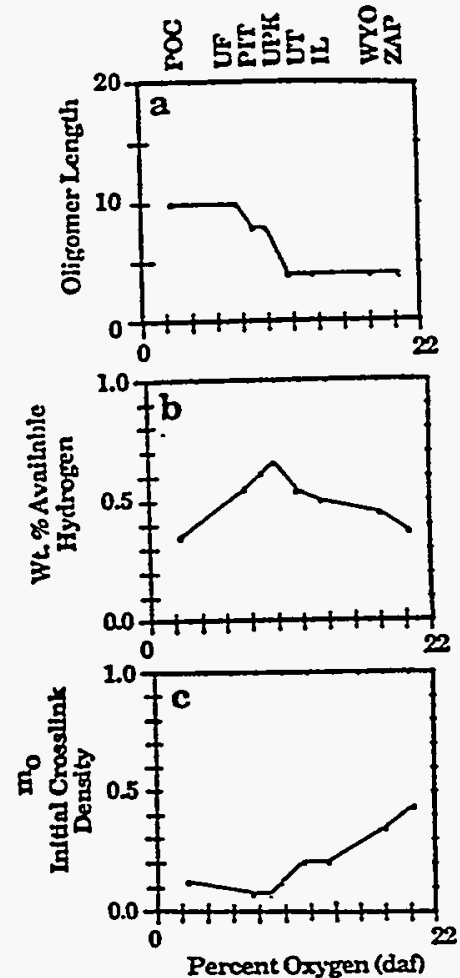

Figure 17. Variation of network parameters with rank: (a) starting oligomer length $l$; (b) weight percent hydrogen $H(a)$; and (c) initial cross-link density, $m_{0}$. Cross-links/cluster (note: one cross-link connects two monomers).

picked to give the correct extract yield for the starting coal. The fluidity predictions were then compared with the data. Figure 16 demonstrates that the model requires a unique value of $m_{0}$ and $H(\mathrm{al})$. The simulation required a value of $m_{0}=0.08 \pm 0.01$, a value of $l=8 \pm 2$, and a value for $H(\mathrm{all})$ of $0.62 \%$. This value of $m_{0}$ will be chemically meaningful if the viscosity parameters (especially $E_{n}$ ) have been correctly chosen. The same result is achieved if $H(a l)$ is varied and $m_{0}$ and $l$ are adjusted to give the correct tar yield and extract yield.

Variation of Network Parameters with Rank. The three parameters of the model, $l, H(a l)$, and $m_{0}$, have been determined for the Argonne coals. These are plotted in Figure $17 a-c$ as a function of the oxygen in the coal. Also plotted in Figure 18 is the molecular weight between cross-links $M_{c}$ derived from $m_{0}$ and the average molecular weight of a ring cluster, the measured solvent swelling ratio, and the extract yield.

The network parameters show systematic variations with rank. The molecular weight between cross-links and the oligomer length both decrease with decreasing rank. This suggests the low-rank cosls are more highly branched (large $m_{0}$ ) to yield low extractable amounts while the highest rank coals have fewer branches but longer chains (large $l$ ) to yield low extractable amounts. The high fluidity coals must have low values of $m_{0}$ to yield a high fluid fraction, but also must have small amounts of low-temperature cross-linking so that the amount of branching remains low prior to cross-linking. The starting cross-link density for low-rank coals (Zap, Wyodak) presents some uncertainty, since there is no apparent fluidity to better define the network parameters.

Figure $18 \mathrm{~b}$ presents the molecular weight between cross-links, $M_{\mathrm{c}}$, determined by our model,

$$
M_{\mathrm{c}}=M_{\mathrm{avg}} / 2 m_{0}
$$

where $M_{\text {avg }}$ is the average molecular weight of a cluster. 


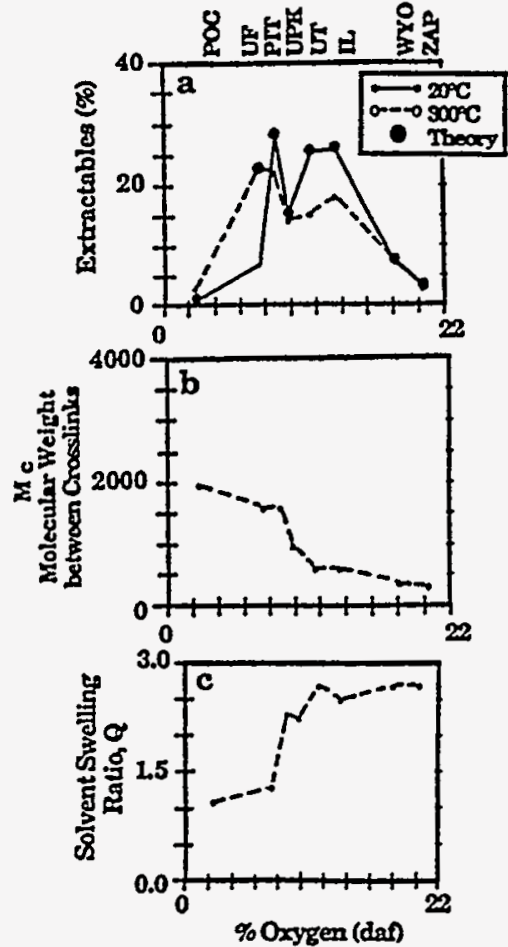

Figure 18. Variation of network parameters with rank: (a) extractables, (b) molecular weight between cross-links; (c) solvent swelling ratio, $Q$.

The factor of 2 occurs because each cross-link connects two clusters. We have not determined $M_{c}$ from solvent swelling theorieg ${ }^{71,72}$ because of the uncertainty in the proper way to calculate the solvent swelling and uncertainties in the values of the interaction parameter $\chi$. Our values of $M_{c}$ for Pittsburgh, Lewis Stockton, Illinois No. 6, and Utah coals are within the range of those predicted from current theories of solvent swelling. ${ }^{71,72}$ Our values for Pocahontas and Upper Freeport are, however, much higher than what would be calculated from their very low solvent swelling ratios (1.12 and 1.32, respectively) using any of the theories for $M_{c}$ and acceptable $\chi$ values. ${ }^{71,72}$ It is probable, however, that van der Waals forces or $\pi-\pi$ bonding in these coals (not cross-linking) limit the swelling. ${ }^{73}$ When heated to $400^{\circ} \mathrm{C}$, for example, the solvent swelling of the cooled Upper Freeport increases from 1.32 to $2.13 .^{57}$ Little chemistry has occurred, but the $\pi-\pi$ bonding may have been disrupted.

The problems of $\pi-\pi$ bonding may also affect the determination of the value of the $l$ parameter. For the six lowest rank Argonne coals, pyridine swells the coal between a factor of 2 and 3 . With the coals swollen to roughly the same large amount, it is expected that most of the loose molecules which are soluble in pyridine can be removed. On the other hand, the Upper Freeport and Pocahontas coals only have $Q$ values of 1.32 and 1.12 , and extract yields of $10 \%$ and $1 \%$. The question is: are these coals covalently cross-linked, or is pyridine not able to break other noncovalent bonds such as the stacking interactions, or $\pi-\pi$ bonds discussed by Larsen. ${ }^{73}$ If the latter were the case, much of the loose material may be trapped. One indication that this is the case is the observation that after heating to $300^{\circ} \mathrm{C}$ the extract yield from the more easily swollen Upper Freeport went from 10 to $23 \%$. We have, therefore, chosen to employ the extract yield after heating to $300^{\circ} \mathrm{C}$

(71) Larsen, J. W.; Kovac. J. ACS Symp. Ser. 1978, 71, 36.

(72) Lucht, L. M.; Peppas, N. A. ACS Symp. Ser. 1981, 169, 43.

(73) Larsen, J. W. Prepr. Pap.-Am. Chem. Soc., Div. Fuel Chem. 1988, $33(1), 400$.
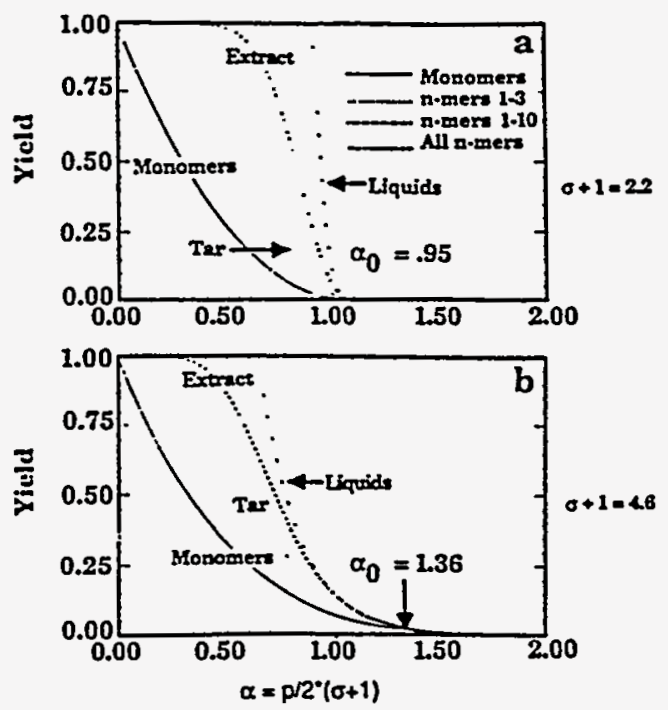

Figure 19. Percolation theory predictions for pyrolysis products (monomers, tar, extracts, and total liquids) for two values of coordination number: (a) $\sigma+1=2.2$ and (b) $\sigma+1=4.6$.

to determine a better value of $l$. This procedure may not give the complete extractable, so the value of $l$ may still be in error. Also, it is possible that $\pi-\pi$ bonding could affect the viscosity itself, so that $C_{1}$ and $C_{2}$ could be rank dependent. These effects couid explain why the temperature predictions for Upper Freeport in Figure 6 are the worst of all the coals.

The values of $H(\mathrm{al})$ are between 0.3 and $0.7 \%$. This is within the range of expected concentrations for donatable hydrogen (the total aliphatic or hydroaromatic hydrogen $(2-3 \%)$ minus the hydrogen in methyl groups $(\sim 1 \%)$ and other aliphatic peripheral groups or guest molecules ( 1\%).

Meaning of the Network Parameters. To understand the relationship between the network parameters and the pyrolysis behavior, it is useful to consider an alternative to the Monte Carlo method to compute the molecular weight distributions. The alternative is percolation theory employed by several research groups to describe the network fragmentation. ${ }^{43-47}$ The percolation theory has the advantage that it is much faster to calculate than the Monte Carlo method, since the molecular weights are predicted by closed form analytical expressions. In addition, the relationship between the network parameters and the pyrolysis behavior is more transparent for the percolation theory than it is for the Monte Carlo method.

Figure 19 compares the predictions of the $n$-mer distribution (proportional to molecular weight distributions) for two networks with coordination numbers of 2.2 and 4.6. The coordination number designated by $(\sigma+1)$ is the number of possible attachments per ring cluster. A linear polymer has $\sigma+1=2$, while a "fish net" has $\sigma+1=4$. The actual number of bridges per ring cluster $\alpha$ is defined by the probability $p$ that bridges are unbroken, where $\alpha$ $=(p / 2)(\sigma+1)$.

The importance of the coordination number is its effect on the n-mer distribution as a function of $\alpha$. For low values of $\sigma+1$, the liquid fraction is a strong function of $\alpha$. For the $\sigma+1=2.2$ network, there are large changes in the liquid yield for small change in $\alpha$. Also, the ratio of liquids (all $n$-mers) to monomers, to $n$-mers up to 3 (representing the tar for a ring cluster molecular weight of 300 ), and to $n$-mers up to 10 (representing the extracts) is large. In contrast, for $\sigma+1=4.6$ the liquid yield goes up more slowly with decreasing $\alpha$ and the ratio of liquids to the other products is much smaller. 
By eramining the percolation theory it is possible to see how the three parameters $l, H(a l)$, and $m_{0}$ are constrained. The ratio of liquid yield (to match the fluidity) to the measured tar yield constrains the value of $\sigma+1$. In the Monte Carlo model, this is equivalent to constraining $m_{0}$. A high ratio of liquids to tar requires a low $\sigma+1$ and a low $m_{0}$. Thus, referring to the rank dependence of the parameters in Figures 17 and 18, it is the decrease in the cross-linking during the process of maturation and the reduction in the low-temperature cross-linking which keeps the coordination number low prior to bond breaking. That allows the coals to become fluid.

From this discussion it is now possible to understand why weathering (oxidation) is so effective in reducing fluidity. It has always been puzzling why a small amount of oxygen uptake (which sometimes cannot even be measured) leads to large changes in fluidity. The apparent reason is that oxidation leads to low-temperature crosslinking, thus increasing the coordination number and reducing the liquid yield for the same number of bonds broken.

\section{Conclusion}

1. An empirical model for fluidity of coal has been developed based on a previously published macromolecular network model for coal pyrolysis.

2. The network model is used to predict the fraction of liquids under the combined effects of bond breaking and cross-linking.

3. The empirical model for an inhomogeneous melt assumes the fluidity to depend on the liquid fraction in the melt, and on the viscosity of the liquid.
4. Good agreement is obtained with fluidity data for eight coals which covers 5 orders of magnitude in fluidity and several hundred degrees in temperature. This agreement is obtained with fixed parameters in the empirical fluidity equations, with kinetics and volatile amounts fixed by TG-FTIR data, but with two adjustable parameters, the number of initial cross-links per monomer, $m_{0}$, and the percent of donatable hydrogens $H(a)$. The third parameter $l$ is fixed by the extract yield.

5. The model predicts the observed trends to lower fluidity with oxidation or extraction.

6. A sensitivity analysis suggests that the model is most sensitive to the three network parameters: the cross-link density $m_{0}$, the amount of donatable hydrogen $H(a)$, and the oligomer length of $l$. These parameters appear to have some physical and chemical significance.

7. The effect of $\pi \pi$ bonding appears to be important for high-rank coals, reducing the accuracy of the predictions. Additional study is required to account for these effects.

8. Additional tests of the model using fluidity data derived for the Argonne coals over a wider range of heating rates would be important to further improve the model.

Acknowledgment. We gratefully acknowledge the support for the work provided by the Morgantown Energy Technology Center of the Department of Energy under Contract DE-AC21-86MC23075, Dr. Richard Johnson, Contract Monitor. We thank Mr. George Engelke from Commercial Testing and Engineering Co. who provided us with the Gieseler plastometer data for the Argonne coals. 
Appendix N

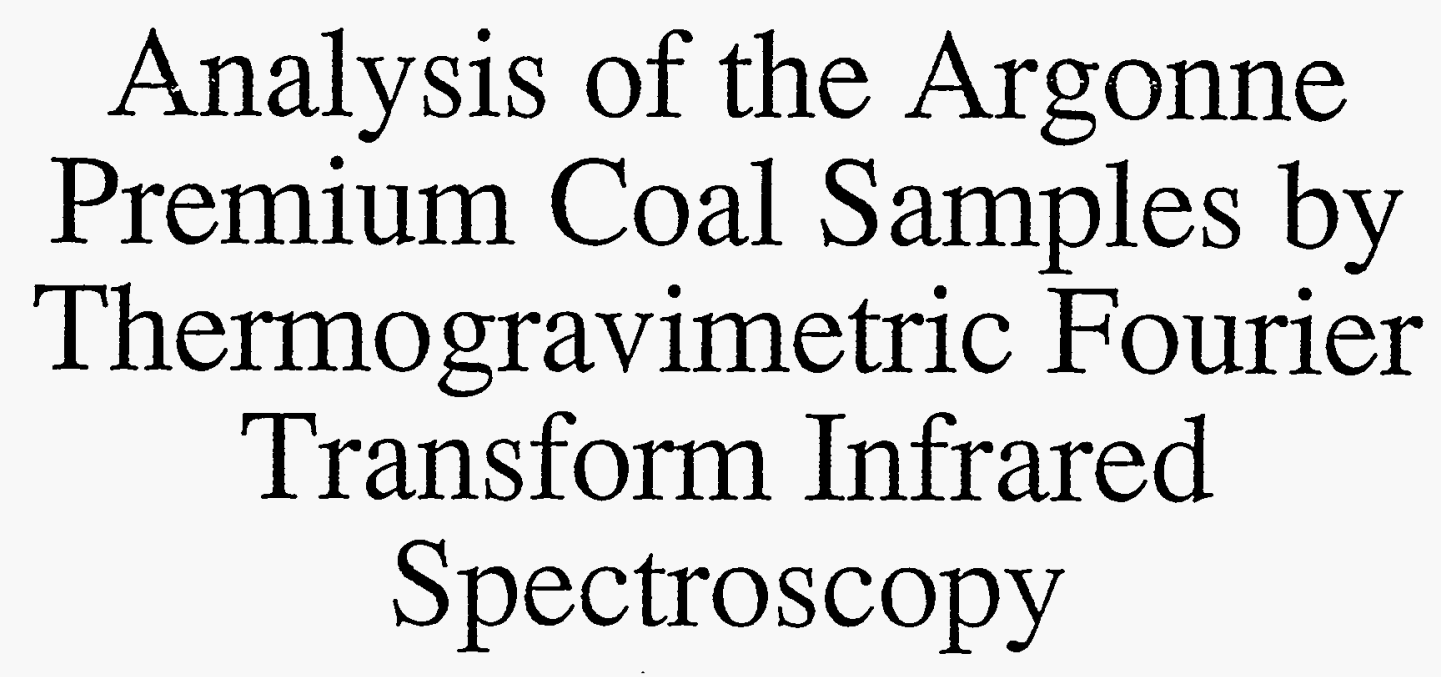


Analysis of the Argonne Premium Coal Samples by Thermogravimetric Fourier Transform Infrared Spectroscopy 
Reprinted from ENERGY \& FUELS. 1990, 4.

Copyright $\odot 1990$ by the American Chemical Society and reprinted by permission of the copyright owner.

\title{
Analysis of the Argonne Premium Coal Samples by Thermogravimetric Fourier Transform Infrared Spectroscopy
}

\author{
P. R. Solomon,* M. A. Serio, R. M. Carangelo, and R. Bassilakis \\ Advanced Fuel Research, Inc., 87 Church Street, East Hartford, Connecticut 06108 \\ D. Gravel, M. Baillargeon, F. Baudais, and G. Vail \\ Bomem, Inc., 450 rue St. Baptiste, Quebec City, Quebec, Canada .
}

Received October 19, 1989. Revised Manuscript Received April 2, 1990

\begin{abstract}
We have developed a TG-FTIR instrument that combines thermogravimetric analysis (TGA) with evolved product analysis by Fourier transform infrared (FT-IR) spectroscopy. FT-IR analysis of evolved products has advantages over mass spectroscopy in allowing analysis of very heavy products and over gas chromatography in speed. This paper describes the most recent improvements in the apparatus and presents its application in characterizing the Argonne premium coal samples. The TG-FTIR apparatus for pyrolysis, oxidation of pyrolysis products, and oxidation of the sample is described. To analyze coal, a sequence of drying, pyrolysis, and combustion is employed to obtain proximate analysis, volatile composition, volatile kinetics, and relative char reactivity. Pyrolysis results are presented for the eight Argonne coals, several demineralized coals, and two oxidized samples of Pittsburgh Seam coal. A kinetic analysis was applied to species evolution data collected at several different heating rates. There is a systematic variation in rate with rank. The rate for tar evolution from Pittsburgh Seam coal is in good agreement with that of Burnham et al. using a similar set of data. Analysis of the amounts of evolved products also show a systematic variation with rank consistent with the coal's elemental and functional group comositions. Postoxidation of the volatile products has been successful in providing elemental composition information on the volatile products as well as showing the evolution of $\mathrm{H}_{2}$, which is not infrared active, and $\mathrm{H}_{2} \mathrm{~S}$ (in the postoxidized $\mathrm{SO}_{2}$ profile), which is a weak infrared absorber. Oxidation of the char yields an ash amount as well as two measures of the char's reactivity, the oxygen absorbed by the char and the temperature at which significant oxidation of the char occurs.
\end{abstract}

\section{Introduction}

Thermogravimetric analysis (TGA) has been employed in coal science to perform a number of characterizations including proximate analysis, ${ }^{1}$ kinetics of weight loss, ${ }^{2,3}$ char reactivity, ${ }^{\text {t-9 }}$ and gas adsorption measurements. ${ }^{10} \mathrm{~A}$ complimentary technique, evolved-product analysis, has been employed to study pyrolysis product distributions 


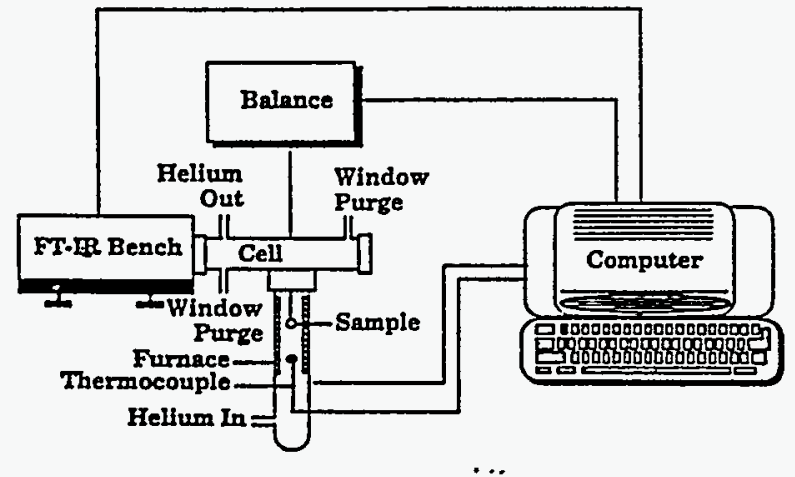

Figure 1. Schematic of $T G /$ plus.

and kinetics, ${ }^{11-18}$ functional group compositions, ${ }^{14,19-21}$ and temperature-programmed desorption. ${ }^{22-24}$ We have developed a TG-FTR instrument which combines TGA with evolved-product analysis by Fourier transform infrared (FT-IR) spectroscopy. FT-IR analysis of evolved products has advantages over mass spectroscopy in allowing analysis of very heavy products and over gas chromatography in speed.

The application of TG-FTIR to coal and petroleum source rock has recently been described. ${ }^{25,26}$ To analyze coal, a sequence of drying, pyrolysis, and combustion is employed to obtain proximate analysis, volatile composition, volatile kinetics, and relative char reactivity. By

(1) Ottaway, W. Fuel 1982, 61,713.

(2) Ciuryia, V. T.; Weimer, R. F.; Bivans, A.; Motika, S. A. Fuel 1979, $58,748$.

(3) van Krevelen, D. W.; van Heerden, C.; Huntjens, F. J. Fuel 1951, 30,253

(4) Solomon P. R. Serio, M. A. Heninger, S. G. Prepr Pap-Am. Chem. Soc Div. Fuel Chem. 1986, 31 (3), 200

(5) Best, P. E; Solomon, P. R.; Serio, M. A.; Suuberg, E. M.; Mott, W. R, Jr.; Bassilakis, R. Prepr. Pap-Am. Chem. Soc., Div. Fuel Chem. 1987, 32 (4) 138.

(6) Serio, M. A.; Solomon, P. R.; Bassilakis, R.; Suuberg, E. M. Prepr. Pap-Am. Chem. Soc., Div. Fuel Chem. 1989, 34 (1), 9.

(7) Serio, M. A.; Solomon, P. R.; Suuberg, E.M. The Variation in Char Reactivity with Pyrolysis Conditions and Coal Type. To be submitted.

(8) Jenkins, R. G.; Nandi, S. P.; Walker, P. L., Jr. Fuel 1973, 52, 228.

(9) Sahu, R.; Levendis, Y. A.; Flogan, R. C.; Gavales, G. R. Fuel 1988, 67,275 .

(10) Suuberg, E. M.; Calo, J. M.; Wojtowicz, W. Prepr. Pap.-Am. Chem. Soc., Div. Fuel Chem. 1986, 31 (3), 186.

(11) Juntgen, H.; van Heek, K H. Fuel Process. Technol. 1979, $2,261$.

(12) Winans, R. E.; McBeth, R. I.: Neill, P. H. Prepr. Pap.-Am. Chem. Soe. Div. Fuel Chem. 1988, 33 (3), 85.

(13) Chakravarty, T.; Windig, W.; Hill, G. R.; Meuzelaar, H.L.C. Energy Fuels 1988, 2, 400.

(14) Solomon, P. R; Hamblen, D. G. Finding Order in Coal Pyrolysis Kinetics; Topical Report submitted to U.S. Department of Energy under Contract No. DE-AC21-81FE05122, 1983; also Prog. Energy Combust. Sci. 1983, 9, 323.

(15) Fitzgerald, D. van Krevelen, D. W. Fuel 1959, 38, 17.

(16) Campbell, J. H. Fuel 1978, 57, 217

(17) Burnham, A. K.; Oh, M. S.; Crawford, R. W.; Samoun, A. M. Energy Fuels 1989, 3, 42.

(18). Weimer, R. F.; Ngan, D. Y. Prepr. Pap.-Am. Chem. Soc., Div. Fuel Chem. 1979, 24 (3), 129.

(19) Attar, A.; Dupuis, F. Prepr. Pap.-Am. Chem. Soc., Div. Fuel Chem. 1978, $23(2), 44$.

(20) Attar, A.; Hendrickson, G. G. Coal Structure; Meyers, R. A., Ed.; Academic Press: New York, 1982; Chapter 5, p 131.

(21) LaCount, R. B.; Anderson, R. R.; Friedman, S.; Blaustein, B. D. Fuel 1987, 66, 873 .

(22) Hall, P. J.; Calo, J. M. Energy Fuels 1989, 3, 370.

(23) Zhang, Z. G.; Kyotani, T.; Tomita, A. Energy Fuels 1989, 3, 566.

(24) Kyotani, T.; Zhang, Z. G.; Hayashi, S.; Tomita, A. Energy Fuels $1988,2,1136$.

(25) Carangelo, R. M.; Solomon, P. R.; Gerson, D. G. Fuel 1987, 66,

(26) Whelan, J. K.; Solomon, P. R.; Deshpande, G. V.; Carangelo, R. M. Energy Fuels 1988, 2, 65.
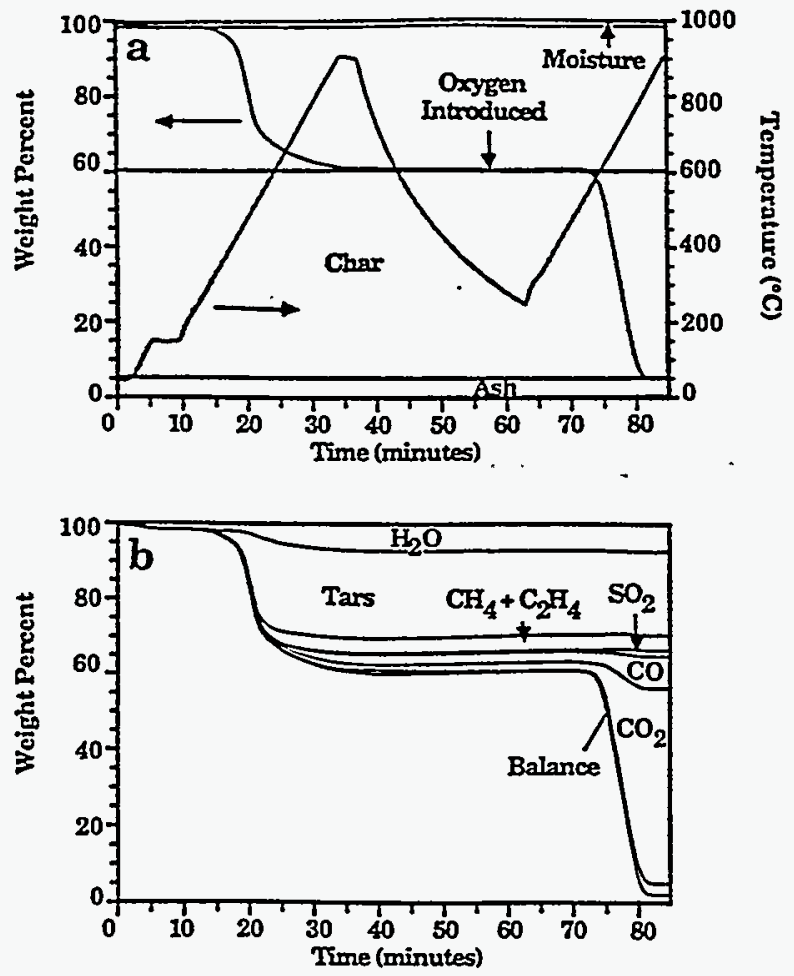

Figure 2. TG-FTIR analysis of Pittsburgh Seam coal. (a) Temperature history and weight loss. (b) Species contributions to weight loss.
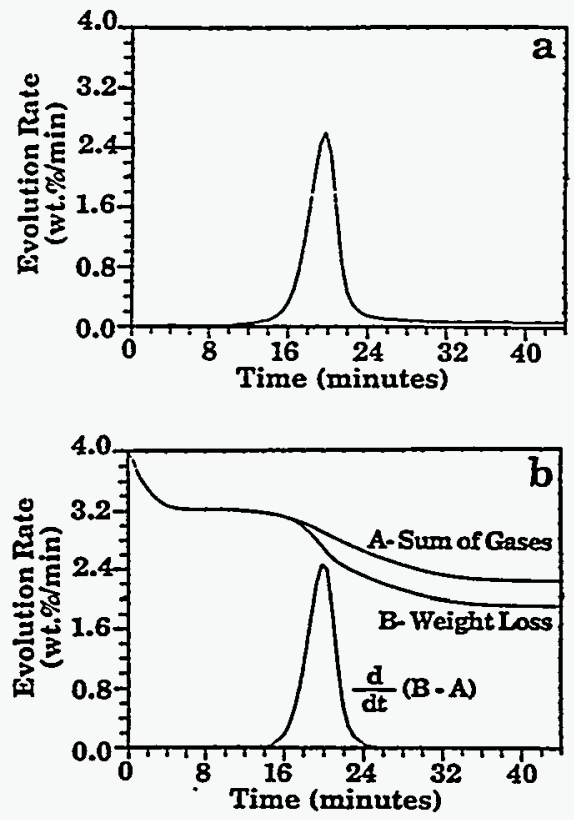

Figure 3. Calibration of tar absorptivity. (a) Tar absorption profile. (b) Weight loss minus the sum of gases.

employing several different heating rates, kinetic rate constants have been obtained. ${ }^{27.23}$ The apparatus has been very useful in studying the effects of pretreatments such

(27) Serio, M. A.; Solomon, P. R.; Yu, Z. Z,; Deshpande, G. V. An Improved Model of Coal Devolatilization. In International Conference on Coal Science Proceedings; IEA: Tokyo, Japan, 1989; p 209.

(28) Serio, M. A.; Solomon, P. R. Yu, Z Z; Bassilakis, R.; Markham, J. R.; Klapheke, J. G. Prepr. Pap.-Am. Chem. Soc., Div. Fuel Chem. 1989,34 (4), 1324. 

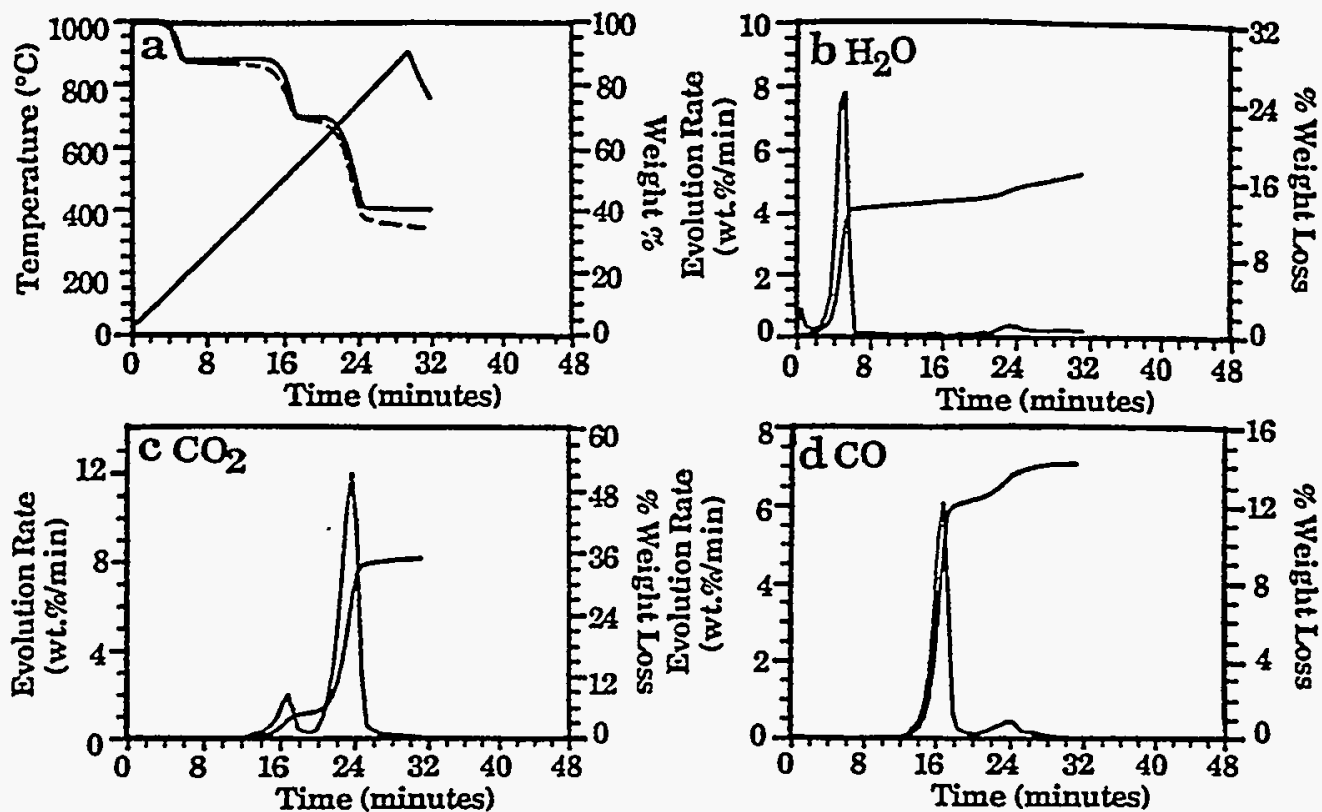

Figure 4. Pyrolysis of calcium oxylate. (a) Weight loss (solid), sum of evolved products (dashed), and temperature profile. (b) $\mathrm{H}_{2} \mathrm{O}$ evolution and integrated amount evolved. (c) $\mathrm{CO}_{2}$ evolution and integrated amount evolved. (d) $\mathrm{CO}$ evolution and integrated amount evolved.
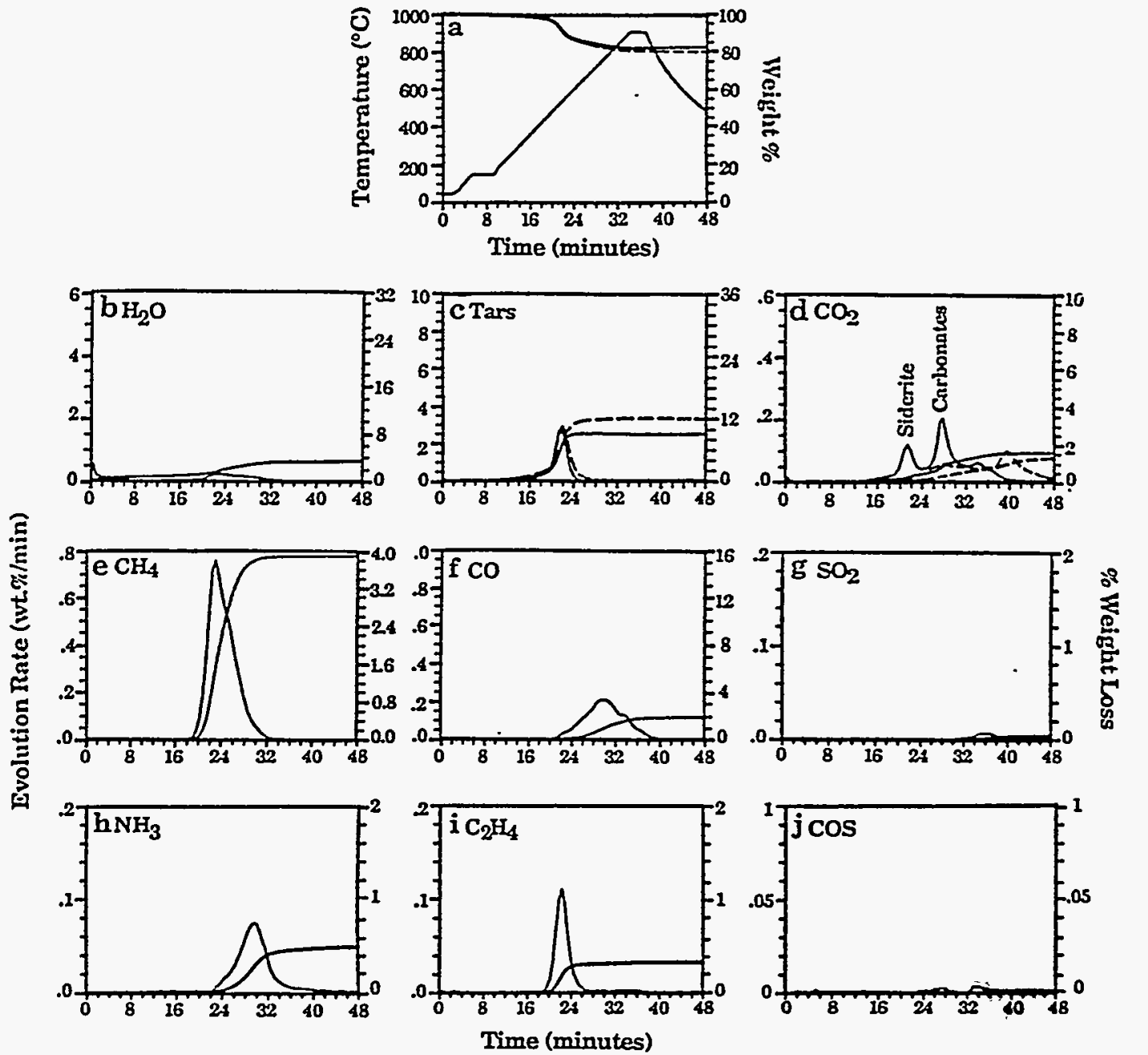

Figure 5. TG-FTIR analysis of raw and demineralized Pocahontas coal during the pyrolysis cycle. (a) weight loss (solid), sum of evolved products (dashed), and temperature profile. (b) $\mathrm{H}_{2} \mathrm{O}$ evolution rate and integrated amount evolved. (c) Tar evolution rate and integrated amount evolved (raw coal (solid line); demineralized coal (dashed line)). (d) $\mathrm{CO}_{2}$ evolution rate and integrated amount evolved (raw coal (solid line); demineralized coal (dashed line)). (e) Methane evolution rate and integrated amount evolved. (f) CO evolution rate and integrated amount evolved. (g) $\mathrm{SO}_{2}$ evolution rate and integrated amount evolved. (h) $\mathrm{NH}_{3}$ evolution rate and integrated amount evolved. (i) $\mathrm{C}_{2} \mathrm{H}_{4}$ evolution rate and integrated amount evolved. (j) COS evolution rate and integrated amount evolved. All weight losses are on an as received basis. 

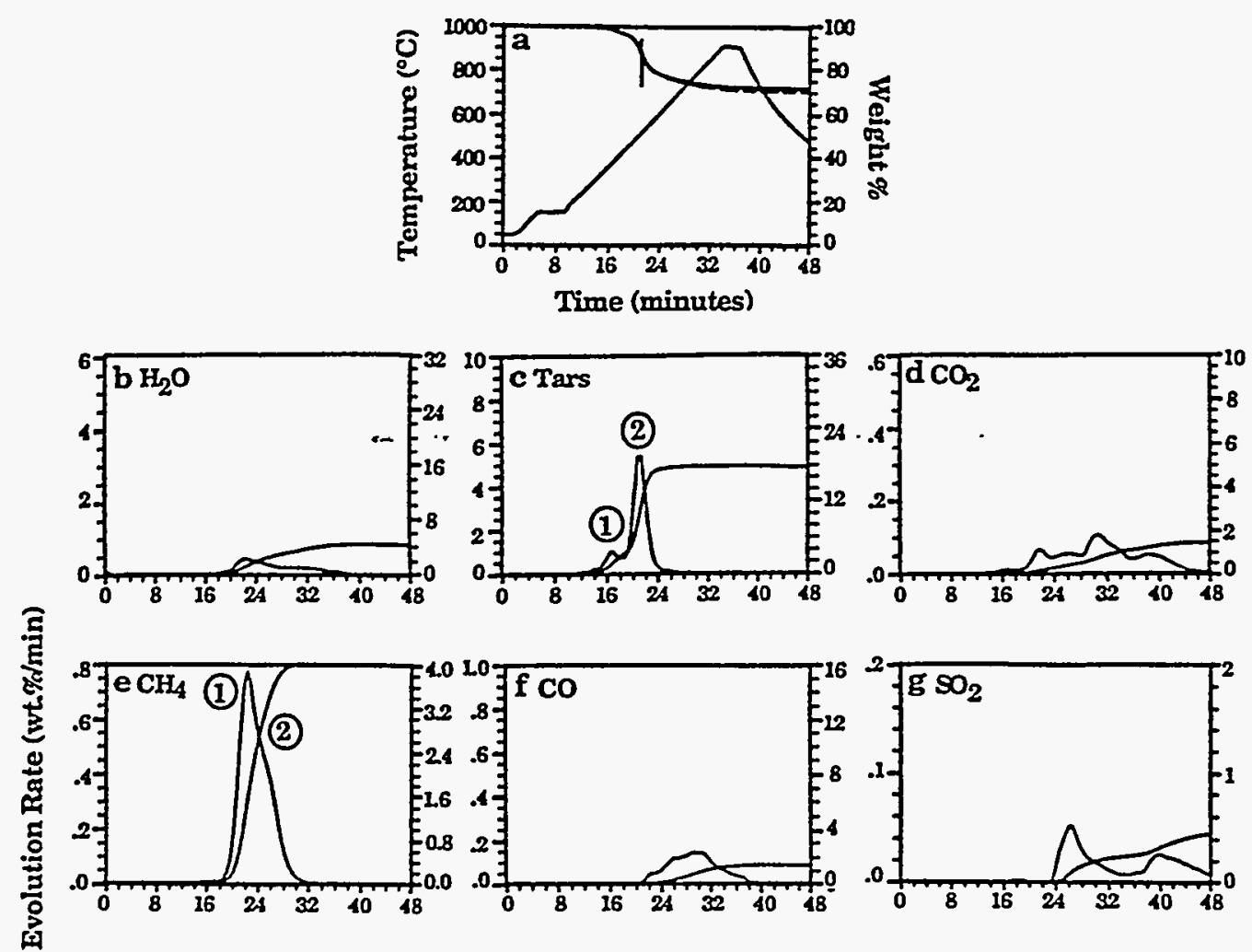

总

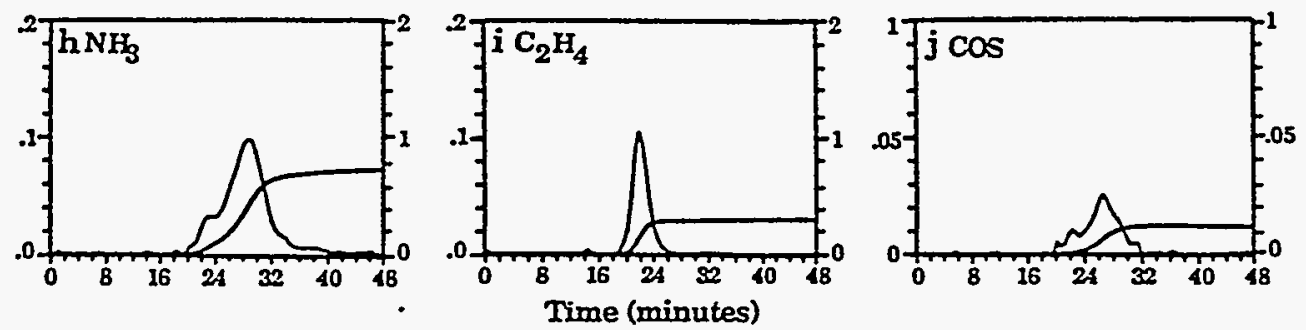

Figure 6. TG-FTIR analysis of Upper Freeport coal during the pyrolysis cycle. (a) Weight loss (solid), sum of evolved products (dashed), and temperature profile. (b) $\mathrm{H}_{2} \mathrm{O}$ evolution rate and integrated amount evolved. (c) Tar evolution rate and integrated amount evolved. (d) $\mathrm{CO}_{2}$ evolution rate and integrated amount evolved. (e) Methane evolution rate and integrated amount evolved. (f) $\mathrm{CO}$ evolution rate and integrated amount evolved. $(\mathrm{g}) \mathrm{SO}_{2}$ evolution rate and integrated amount evolved. (h) $\mathrm{NH}_{3}$ evolution rate and integrated amount evolved. (i) $\mathrm{C}_{2} \mathrm{H}_{4}$ evolution rate and integrated amount evolved. (j) COS evolution rate and integrated amount evolved. All weight losses are on an as received basis.

as catalytic preliquefaction, ${ }^{29}$ demineralization, ${ }^{30}$ ion exchange, ${ }^{30}$ methylation, ${ }^{30,31}$ or oxidation ${ }^{31}$ on the volatile product distribution. Temperature-programmed desorption has also been performed to study oxidation and gasification mechanisms. ${ }^{32}$ The purpose of this paper is to describe the most recent improvements in the apparatus and present its application in characterizing the Argonne premium coal samples. The technique is being employed to obtain the kinetic and composition parameters to be employed in a recently developed general model of coal pyrolysis. ${ }^{33}$

(29) Solomon, P. R.; Serio, M. A.; Deshpande, G. V.; Kroo, E.; Schobert, H.; Burgess, C. Prepr. Pap.-Am. Chem. Soc, Div. Fuel Chem. 1989, $34(3), 803$

(30) Serio, M. A.; Solomon, P. R.; Kroo, E.; Bassilakis, R.; Malhotra, R; McMillen, D. Prepr. Pap.-Am. Chem. Soc., Div. Fuel Chem. 1990, 35 (1), 61.

(3i) Solomon, P. R; Serio, M. A.; Deshpande, G. V.; Kroo, E. Energy Fuels 1990, 1, 42.

(32) Serio, M. A; Solomon, P. R; Bassilakis, R.; Suuberg, E. M. The Effects of Minerals on Coal Reactivity. In International Conference on Coal Science Proceedings; IEA: Tokyo, Japan, 1989; p 341.

(33) Solomon, P. R.; Hamblen, D. G.; Carangelo, R. M.; Serio, M. A.; Deshpande, G. V. Energy Fuels 1988, 2, 405.
Table I. Normalized Adsorptivity for Coal Tar

\begin{tabular}{lcc}
\multicolumn{1}{c}{ coal } & \% oxygen (maf) & $\begin{array}{c}\text { rel } \\
\text { absorptivity } \\
\text { at 30 } \\
{ }^{\circ} \mathrm{C} / \mathrm{min}\end{array}$ \\
\hline Pocahontas No. 3 & $\mathbf{2 . 4 7}$ & 0.60 \\
Upper Freeport & 7.51 & 0.82 \\
Pittsburgh No. 8 & 8.83 & 0.95 \\
Upper Kanawha & 9.83 & 0.87 \\
Utah Blind Canyon & 11.58 & 1.04 \\
Illinoid No. 6 & 13.51 & 0.70 \\
Wyodak & 18.02 & 1.32 \\
Beulah Zap & 20.34 & 1.28
\end{tabular}

'Relative to a Pittsburgh No. 8 Seam tar spectrum.

\section{Experimental Section}

Apparatus. A schematic of the instrument is presented in Figure 1. Its components are as follows: a DuPont 951 TGA; a hardware interface (including a furnace power supply); an Infrared Analysis 16 pass gas cell with transfer optics; a MICHELSON 110 FT-IR; (resolution, $4 \mathrm{~cm}^{-1}$; detector, MCT). A helium sweep gas $\left(250 \mathrm{~cm}^{3} / \mathrm{s}\right)$ is employed to bring evolved products from the TGA directly into the gas cell. A window purge 

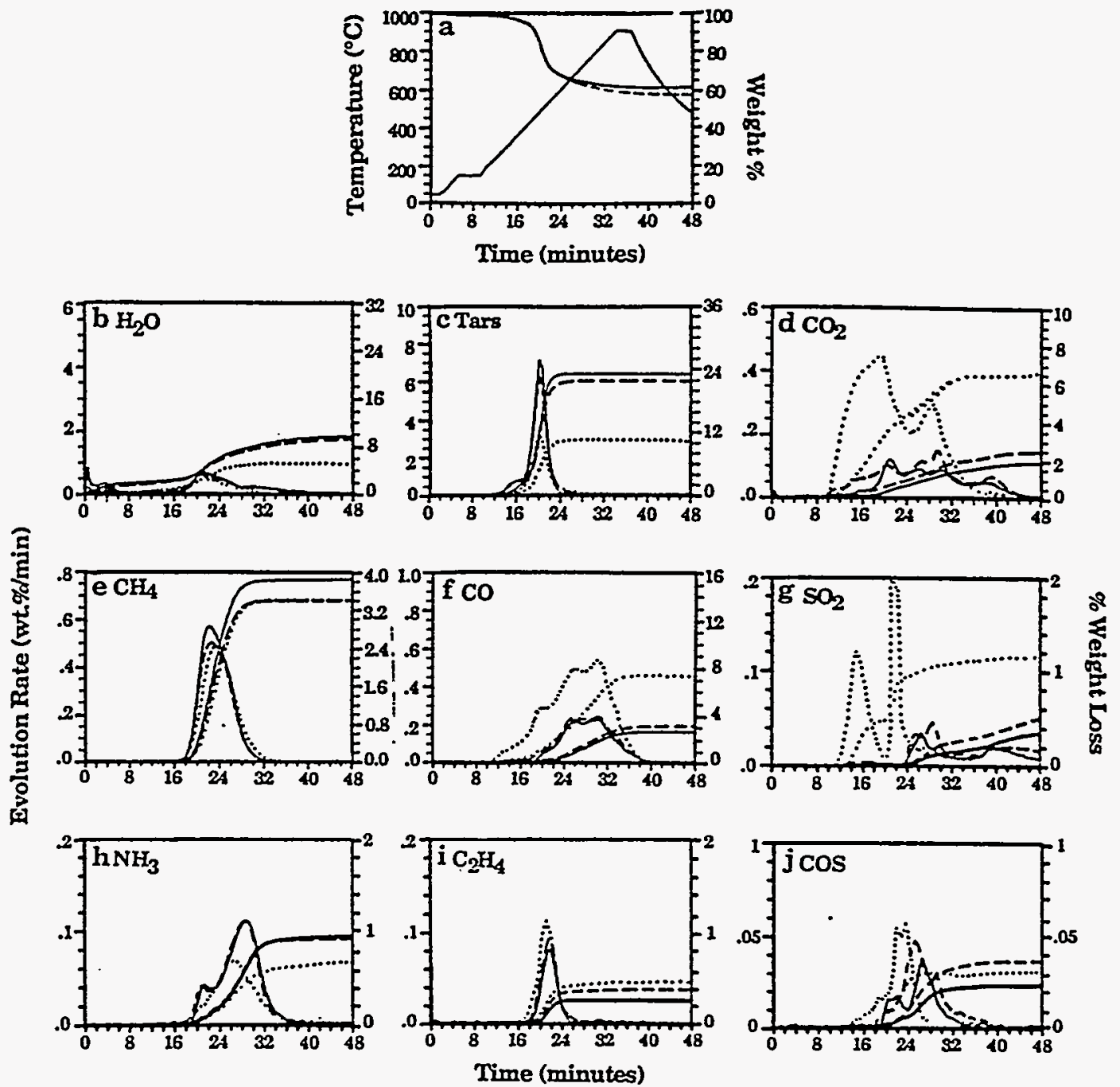

Figure 7. TG-FTIR analysis of raw and oxidized Pittsburgh No. 8 coal during the pyrolysis cycle: solid line, raw coal; dashed line, oxidized 3 days; and dotted line, oxidized 3 months. (a) Weight loss for raw coal (solid), sum of evolved products for raw coal (dashed), and temperature profile. (b) $\mathrm{H}_{2} \mathrm{O}$ evolution rate and integrated amount evolved. (c) Tar evolution rate and integrated amount evolved. (d) $\mathrm{CO}_{2}$ evolution rate and integrated amount evolved. (e) Methane evolution rate and integrated amount evolved. (f) $\mathrm{CO}$ evolution rate and integrated amount evolved. (g) $\mathrm{SO}_{2}$ evolution rate and integrated amount evolved. (h) $\mathrm{NH}_{3}$ evolution rate and integrated amount evolved. (i) $\mathrm{C}_{2} \mathrm{H}_{4}$ evolution rate and integrated amount evoived. (j) $\mathrm{COS}$ evolution rate and integrated amount evolved. All weight losses are on an as received basis.

of $700 \mathrm{~cm}^{3} / \mathrm{s}$ was employed at each end of the cell. The system is operated at atmospheric pressure. This instrument package is now available commercially as the TG/plus from Bomem, Inc.

The most difficult volatiles to analyze are the heavy decomposition products that condense at room temperature, such as tars from coal. In the TG/plus, the high-conductivity helium sweep gas and the rapid cooling cause these products to form an aerosol that is fine enough to follow the gas through the analysis cell. The cell is connected without restrictions to the sample area. The aerosol is also fine enough that there is only a little scattering of the infrared beam, and it is thus attenuated almost as though the tar was in the gas phase. On the basis of the aerosol's Rayleigh scattering of infrared radiation, the diameter of the aerosol droplets is less than $1.0 \mu \mathrm{m}$.

Procedure. As an example of the analysis procedure, the pyrolysis and oxidation of a coal is described. More detail can be found in refs 25 and 26 . Figure $2 a$ illustrates the weight loss from this sample and the temperature history. A 35-mg sample of Pittsburgh Seam coal, loaded in the sample basket of the DuPont 951 , is taken on a $30^{\circ} \mathrm{C} / \mathrm{min}$ temperature excursion in the helium sweep gas, first to $150^{\circ} \mathrm{C}$ to dry for $240 \mathrm{~s}$ and then at $30^{\circ} \mathrm{C} / \mathrm{min}$ to $900^{\circ} \mathrm{C}$ for pyrolysis. Upon reaching $900^{\circ} \mathrm{C}$, the sample is immediately cooled to $250^{\circ} \mathrm{C}$ over a 20 -min period. After cooling, a small flow of $\mathrm{O}_{2}\left(0.3 \mathrm{~cm}^{3} / \mathrm{s}\right)$ is added to the helium sweep gas at the 57-min mark and the temperature is ramped to $700^{\circ} \mathrm{C}$ at $30^{\circ} \mathrm{C} / \mathrm{min}$ (or as high as $1000^{\circ} \mathrm{C}$ ) for oxidation. During this excursion, infrared spectra are obtained once every $41 \mathrm{~s}$. As discussed previously, ${ }^{25.28}$ the spectra show absorption bands for $\mathrm{CO}, \mathrm{CO}_{2}, \mathrm{CH}_{4}, \mathrm{H}_{2} \mathrm{O}, \mathrm{SO}_{2}, \mathrm{COS}, \mathrm{C}_{2} \mathrm{H}_{4}$, and $\mathrm{NH}_{3}$. The spectra above $250^{\circ} \mathrm{C}$ also show aliphatic, aromatic, hydroxyl, carbonyl, and ether bands from tar. The evolution of gases derived from the IR absorbance spectra are obtained by a quantitative analysis program that employs a database of integration regions and calibration spectra for different compounds. The routine employs regions of each calibration spectrum that permit the best quantitation with the least interferences. The routine is fast, so the product analysis is displayed on the computer screen during the actual experiment.

Figure $2 \mathrm{~b}$ illustrates the integral of the evolution curves to obtain cumulative evolved-product amounts. Because the data are quantitative, the sum of these curves matches the weight loss as determined by the TGA balance. Discrepancies occur in this match because of missing components such as $\mathrm{H}_{2}$ which cannot be seen by IR. Also, when $\mathrm{O}_{2}$ is introduced, the balance shows a net gain in weight due to $\mathrm{O}_{2}$ chemisorption.

Calibration. To calibrate the instrument, known flows of calibration gases were mixed with a fixed flow of sweep gas and passed through the gas cell. Reference spectra were collected and the flow rate was varied to provide spectra over the range of expected concentrations. For water calibration, a TGA pan was 

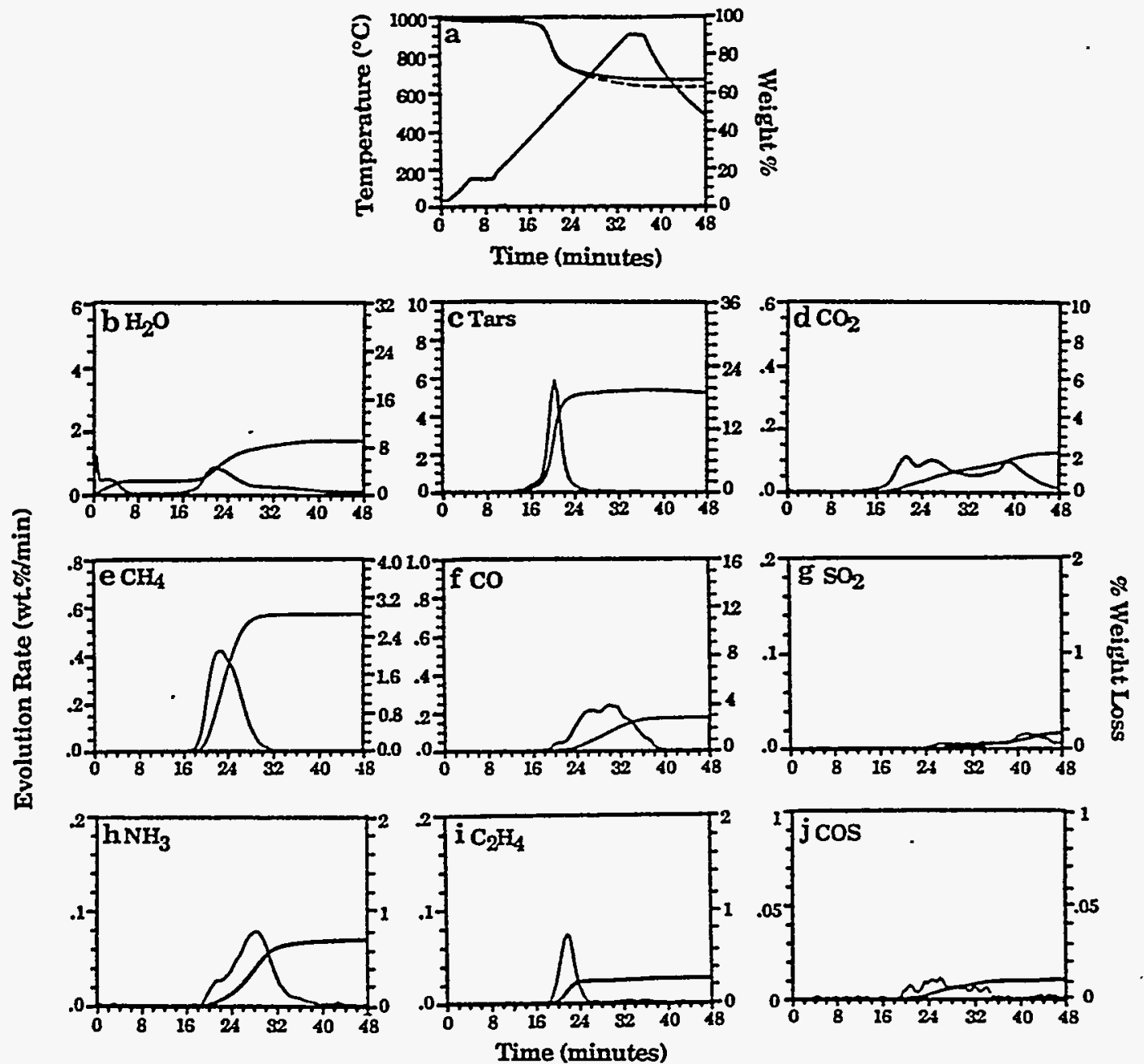

Figure 8. TG-FTIR analysis of Lewis-Stockton Seam coal during the pyrolysis cycle. (a) Weight loss (solid), sum of evolved products (dashed), and temperature profile. (b) $\mathrm{H}_{2} \mathrm{O}$ evolution rate and integrated amount evolved. (c) Tar evolution rate and integrated amount evolved. (d) $\mathrm{CO}_{2}$ evolution rate and integrated amount evolved. (e) Methane evolution rate and integrated amount evolved. (f) $\mathrm{CO}$ evolution rate and integrated amount evolved. (g) $\mathrm{SO}_{2}$ evolution rate and integrated amount evolved. (h) $\mathrm{NH}_{3}$ evolution rate and integrated amount evolved. (i) $\mathrm{C}_{2} \mathrm{H}_{4}$ evolution rate and integrated amount evolved. (j) $\mathrm{COS}$ evolution rate and integrated amount evolved. All weight losses are on an as received basis.

filled with water and the furnace temperature set to obtain the desired flow rate as measured by the TGA weight loss. The quantitative analysis program employs the spectrum that most closely matches the experimental amplitudes, since Beer's law (absorption is proportional to concentration) is not valid for many light gases.

Calibration spectra cannot be employed in the same way for tar since the absorptivity of any band varies with the tar compositions. Instead, the evolution of tar is derived by using the spectrum of a Pittsburgh Seam coal tar as a calibration standard. This coal tar has all the functional group features characteristic of coal tars. Its use as a reference spectrum, therefore, employs the important tar functional group regions whose amplitudes provide a qualitative tar evolution profile for other coals. The tar's evolution determined in this manner typically exhibits a sharply peaked function with increasing temperature as shown in Figure $3 \mathrm{a}$.

To quantitatively determine the tar loss, it is assumed that the qualitative tar evolution profile is proportional to the rate of loss of tar. This will be true when the functional group composition of the tar does not change with temperature, a condition that holds over most of the tar evolution profile as shown by comparison of the infrared spectra at different temperatures. To determine the constant of proportionality for each coal sample, the tar loss profile is compared to the rate of weight loss from the balance minus the rate of weight loss for all the gases. This difference is presented in Figure $3 \mathrm{~b}$. The proportionality constant is picked by performing a least-squares fit between the two curves over the region of tar evolution, except for temperatures where other gases are evolving quickly and might introduce error. Table I compares the absorptivities for the eight coals normalized by the absorptivity of the reference Pittsburgh Seam tar. The measured-spectral areas divided by the absorptivities yield the concentration of tar. The absorptivities are averages over several runs at $0.5^{\circ} \mathrm{C} / \mathrm{s}$. There is a systematic trend of increasing absorptivity with decreasing rank. There are two factors that affect the absorptivity. One is that the oxygen functionalities, which are highly absorbing, become more important with decreasing rank. The second is that the aliphatic peak in tar generally increases with decreasing rank. The exception to the trend is the absorptivity for Illinois No. 6 coal. The Illinois No. 6 tar absorbance spectra indicate that its $\mathrm{C}_{\mathrm{e}} / \mathrm{C}_{\mathrm{al}}$ resembles that of a higher rank coal.

Routine calibration of the instrument is performed on a monthly basis by use of calcium oxalate. A typical evolution profile is presented in Figure 4. The calcium oxalate has three weight loss regions yielding $\mathrm{H}_{2} \mathrm{O}, \mathrm{CO}_{2}$, and $\mathrm{CO}$. When the gas calibration constants and the sweep gas flow rate are correct, then the sum of gases matches the weight loss. The temperature of gas evolution peaks compared to a known reference validates the accuracy of the thermocouple temperature measurement. To check for possible leaks in the system and the absence of oxygen in the helium sweep gas, graphite is run periodically. If there are no leaks and the helium is of high purity, no appreciable weight loss or $\mathrm{CO}_{2}$ evolution is experienced during the pyrolysis cycle. 

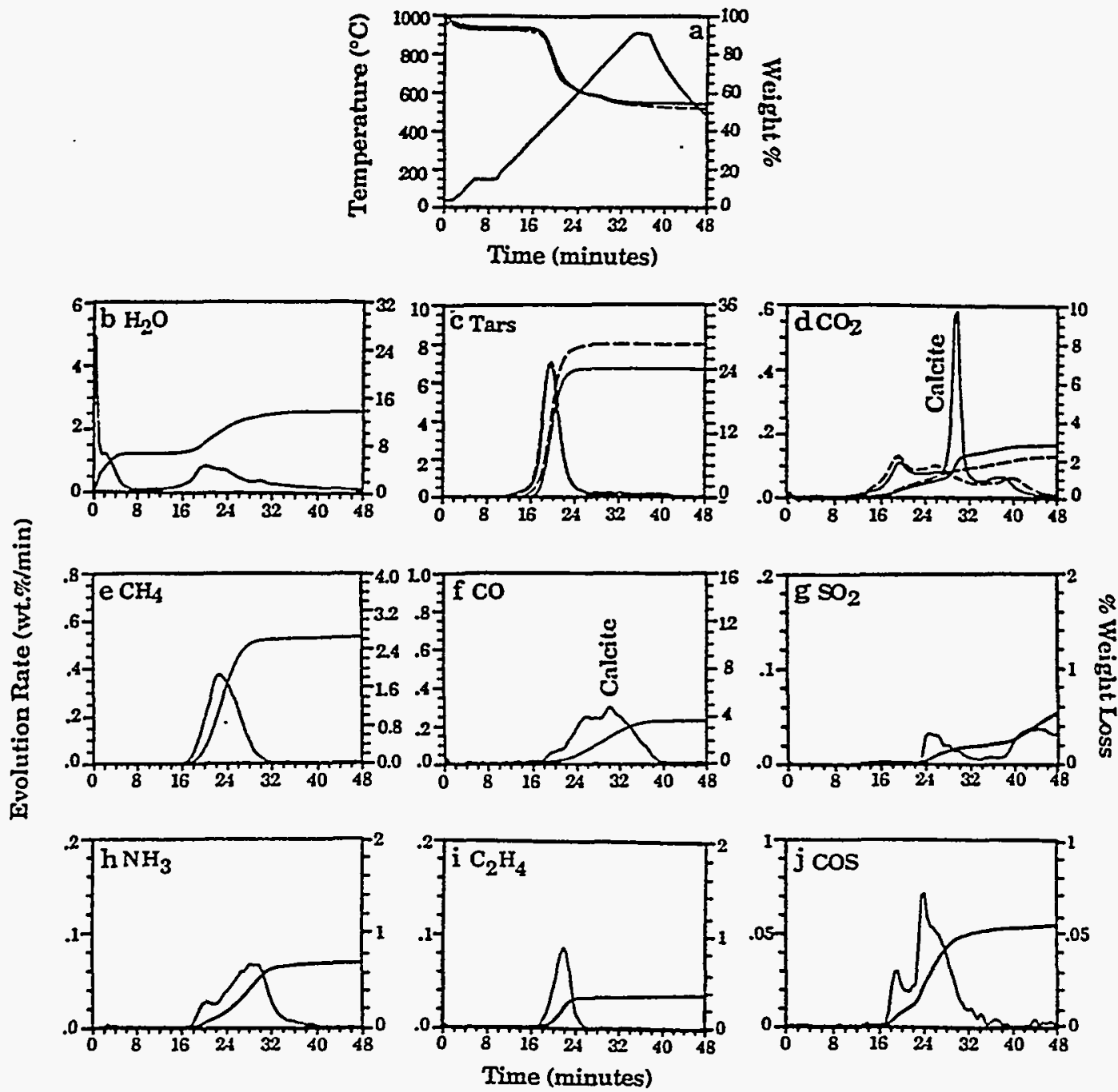

Figure 9. TG-FTIR analysis of raw and demineralized Mlinois No. 6 coal during the pyrolysis cycle. (a) Weight loss (solid), sum of evolved products (dashed), and temperature profile. (b) $\mathrm{H}_{2} \mathrm{O}$ evolution rate and integrated amount evolved. (c) Tar evolution rate and integrated amount evolved (raw coal (solid line); demineralized coal (dashed line)). (d) $\mathrm{CO}_{2}$ evolution rate and integrated amount evolved (raw coal (solid line); demineralized coal (dashed line)). (e) Methane evolution rate and integrated amount evolved. (f) CO evolution rate and integrated amount evolved. (g) $\mathrm{SO}_{2}$ evolution rate and integrated amount evolved. (h) $\mathrm{NH}_{3}$ evolution rate and integrated amount evolved. (i) $\mathrm{C}_{2} \mathrm{H}_{4}$ evolution rate and integrated amount evolved. (j) COS evolution rate and integrated amount evolved. All weight losses are on an as received basis.

Samples. The coals analyzed were Argonne premium coal samples. The characterization of these samples has appeared elsewhere. ${ }^{34}$ In addition, demineralized samples of the same coals were produced according to the technique of Bishop and Ward. ${ }^{35}$ This technique removes both discrete minerals as well as organically bound alkali and alkaline-earth metals. Oxidized samples were prepared in air in an oven at $110^{\circ} \mathrm{C}$ or at room temperature.

\section{Results and Discussion}

Analysis of Volatile Products from Eight Argonne Coals. The results of eight Argonne coals for the pyrolysis cycle are presented in Figures 5-12. Figures 5a-12a present the weight losses and temperature profiles. Also presented (dashed line) is the sum of species (tar, $\mathrm{CH}_{4}$, $\mathrm{H}_{2} \mathrm{O}, \mathrm{CO}_{2}, \mathrm{CO}, \mathrm{SO}_{2}, \mathrm{NH}_{3}, \mathrm{C}_{2} \mathrm{H}_{4}$, and $\mathrm{COS}$ ). In general, the sum of species is within a few percent of the weight loss.

Water evolution is presented in Figures $5 b-12 b$. Water

(34) Vorres, K. S. Users Handbook for the Argonne Premium Coal Sample Program; Supported by US DOE, Contract No. W-31-109-ENG238, 1989.

(35) Bishop, M.; Ward, D. L. Fuel 1958, 37, 191. appears first at low temperature when the coal's moisture is evolved. For all coals, a prominent water peak also occurs simultaneously with the tar peak. This suggests that the chemistry responsible for this peak is related to either the free radicals produced or the increase in fluidity (and hence mobility for bimolecular interactions), both of which occur during tar formation. Burnham et al. ${ }^{17}$ report the coincidence of the tar peak with the low-temperature peaks for $\mathrm{CO}_{2}$ and $\mathrm{H}_{2} \mathrm{~S}$. There is also a higher temperature $\mathrm{H}_{2} \mathrm{O}$ peak and a lower temperature peak or shoulder accompanying $\mathrm{CO}_{2}$ evolution in low rank coals. In Figure $11 \mathrm{~b}$, these peaks are labeled 1 (moisture), 2 (water extra loose, associated with early $\mathrm{CO}_{2}$ evolution), 3 (water loose, associated with tar evolution), and 4 (water tight).

The evolution of tar and aliphatic gases is presented in Figures 5c-12c. These tar evolution profiles typically consist of a low-temperature peak or shoulder followed by a narrow larger peak. The low-temperature peak is believed to be due to the evaporation of unattached "guest" molecules (or the term used by Marzec, ${ }^{36}$ the "molecular

(36) Marzec, A. Fuel 1989, 68, 1104. 

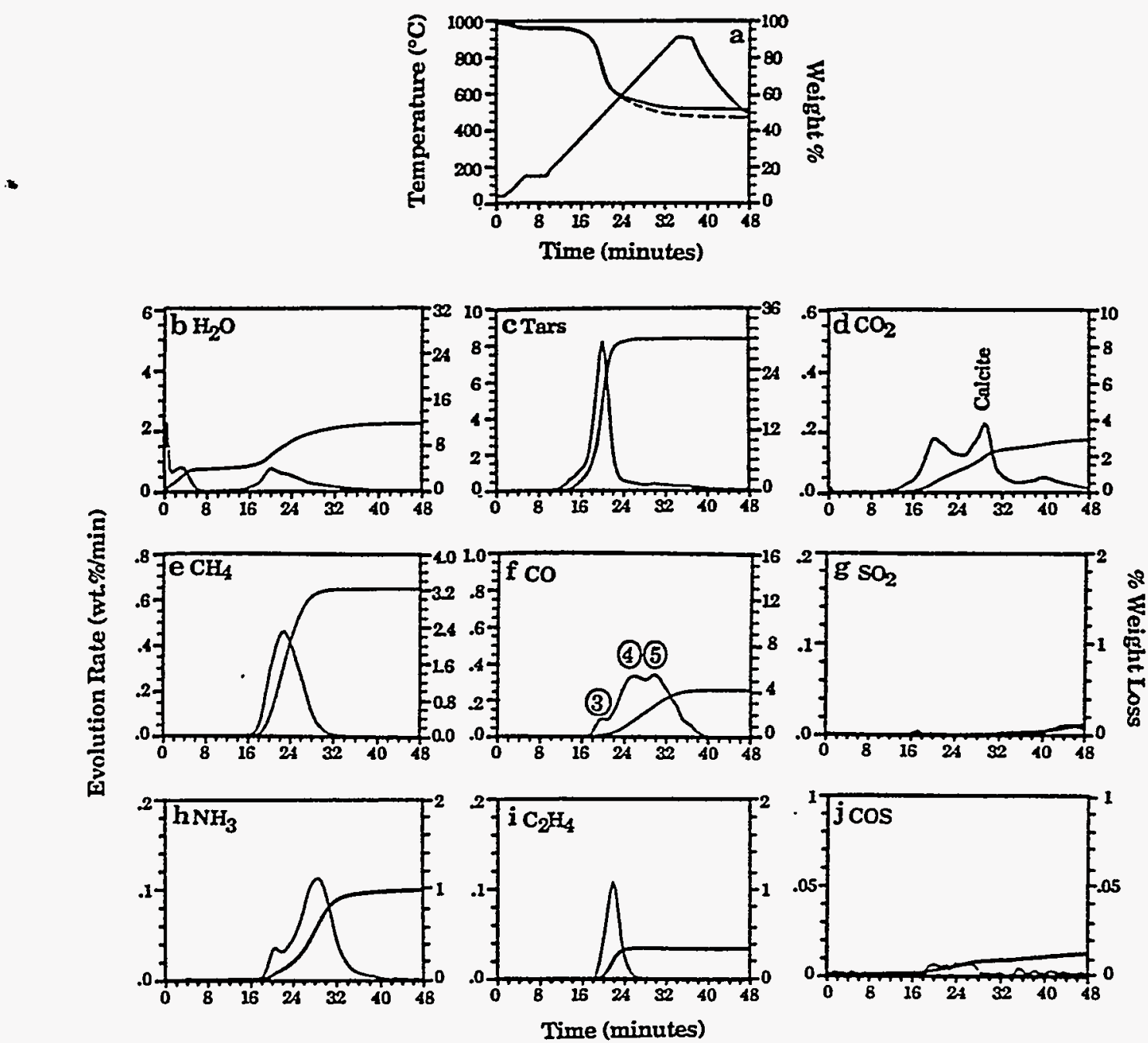

Figure 10. TG-FTIR analysis of Utah Blind Canyon coal during the pyrolysis cycle. (a) Weight loss (solid), sum of evolved products (dashed), and temperature profile. (b) $\mathrm{H}_{2} \mathrm{O}$ evolution rate and integrated amount evolved. (c) Tar evolution rate and integrated amount evolved. (d) $\mathrm{CO}_{2}$ evolution rate and integrated amount evolved. (e) Methane evolution rate and integrated amount evolved. (f) $\mathrm{CO}$ evolution rate and integrated amount evolved. (g) $\mathrm{SO}_{2}$ evolution rate and integrated amount evolved. (h) $\mathrm{NH}_{3}$ evolution rate and integrated amount evolved. (i) $\mathrm{C}_{2} \mathrm{H}_{4}$ evolution rate and integrated amount evolved. (j) $\mathrm{COS}$ evolution rate and integrated amount evolved. All weight losses are on an as received basis.

phase"). This early tar evolution peak is also evident in the data of Burnham et al. ${ }^{17}$ using the Rock-Eval and Meuzelaar et al ${ }^{37}$ using pyrolysis-FIMS. The higher temperature peak is due to the release of coal fragments by bond breaking, evaporation and transport. ${ }^{33}$ In Figure $6 c$, these peaks are labeled 1 (molecular phase) and 2 (pyrolysis).

Figures $5 \mathrm{~d}-12 \mathrm{~d}$ present the results for $\mathrm{CO}_{2}$. Wyodak (Figure 11d), which is typical of low-rank coals, shows three peaks between 200 and $900^{\circ} \mathrm{C}$ labeled $2\left(\mathrm{CO}_{2}\right.$ extra loose associated with $\left.\mathrm{H}_{2} \mathrm{O}\right), 3\left(\mathrm{CO}_{2}\right.$ loose associated with tar evolution and $\mathrm{H}_{2} \mathrm{O}$ evolution), and $4\left(\mathrm{CO}_{2}\right.$ tight). There is also a very low temperature peak labeled 1 (occurring only for the lowest rank coals) whose origin appears to be the low-temperature tail of the $\mathrm{CO}_{2}$ extra loose. The peak occurs because of the constant temperature of the drying cycle. Higher rank coals usually have peaks 3 and 4 but not peak 2 unless they are oxidized. Peak 2 is one of the regions affected most by oxidation. In addition, the evolution of $\mathrm{CO}_{2}$ is often complicated in high-rank coals by

(37) Meuzelaar, H. L. C.; Yun, Y.; Simmleit, N.; Schulten, H. R. Prepr. Pap.-Am. Chem. Soc., Div. Fuel Chem. 1989, 34 (3), 693. the evolution of $\mathrm{CO}_{2}$ from carbonates such as calcite (Figure 9d) and siderite (Figure 5d).

Methane evolution is presented in Figures 5e-12e. Methane evolution occurs in two closely spaced peaks. The low-temperature peak is initiated coincident with the initiation of tar evolution but reaches a maximum at a slightly higher temperature than the maximum tar evolution rate. The temperature for the maximum evolution varies little with rank, but the temperature of initiation of methane evolution decreases with decreasing rank. It has been suggested that the low-temperature evolution of methane occurs by ipso-substitution reactions involving free radicals on macromolecular fragments or hydrogen free radicals, both of which are produced during the decomposition of the macromolecular network. 31,3840 This is consistent with the observation that the early methane evolution peak is initiated coincident with the tar peak.

(38) Solomon, P. R.; Best, P. E.; Yu, Z. Z.; Deshpande, G. V. Prepr. Pap.-Am. Chem. Soc. Div. Fuel Chem. 1989, 34 (3), 895.

(39) Squire, K. R.; Solomon, P. R.; Carangelo, R. M.; DiTaranto, M. B. Fuel 1986, 65, 833 .

(40) Squire, K. R.; Solomon, P. R.; DiTaranto, M. B.; Carangelo, R. M. Prepr. Pap.-Am. Chem. Soc., Div. Fuel Chem. 1985, 30 (1), 386. 

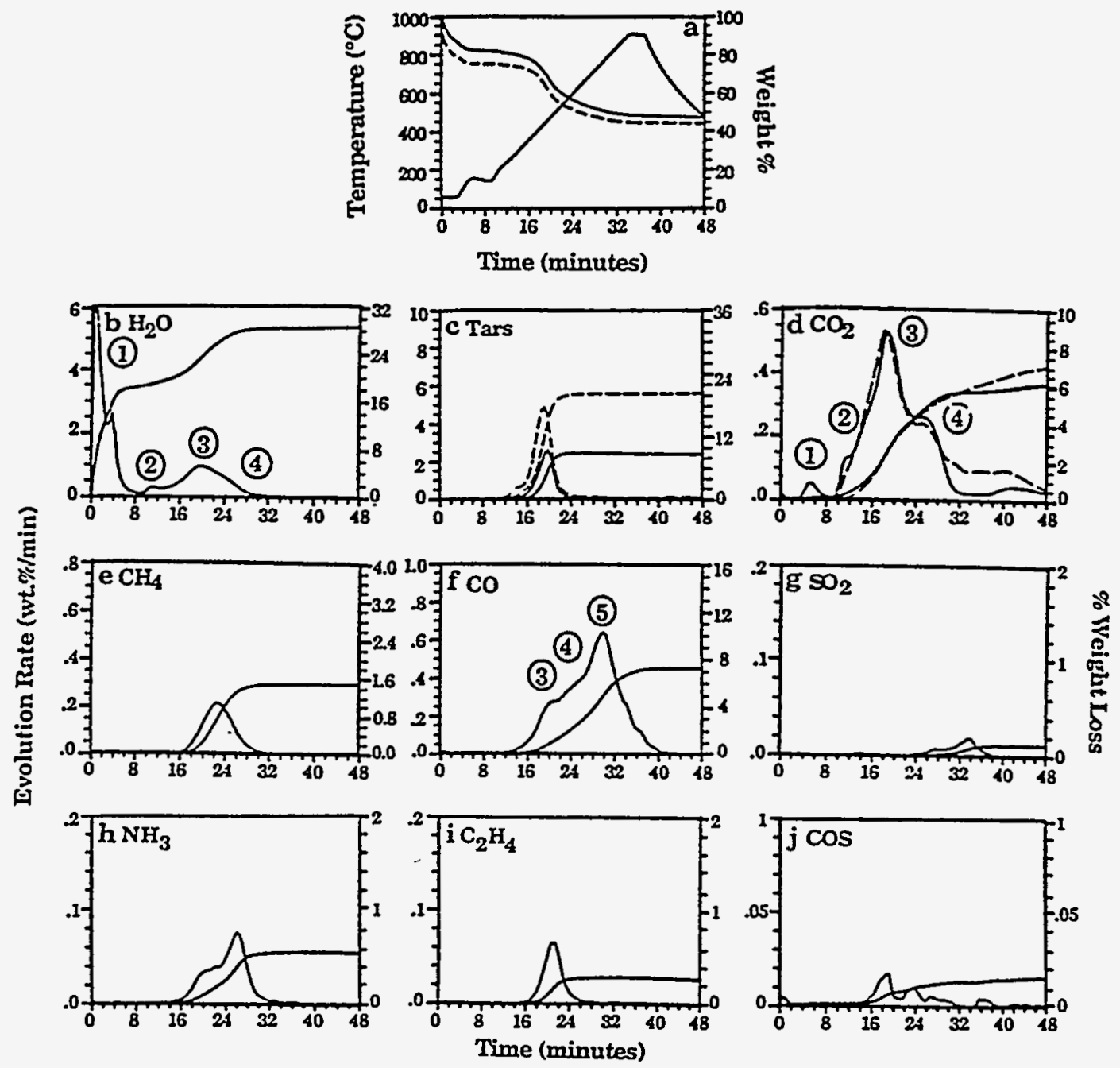

Figure 11. TG-FTIR analysis of raw and demineralized Wyodak subbituminous coal during the pyrolysis cycle. (a) Weight loss (solid), sum of evolved products (dashed), and temperature profile. (b) $\mathrm{H}_{2} \mathrm{O}$ evolution rate and integrated amount evolved. (c) Tar evolution rate and integrated amount evolved (raw coal (solid line); demineralized coal (dashed line)). (d) $\mathrm{CO}_{2}$ evolution rate and integrated amount evolved (raw coal (solid line); demineralized coal (dashed line)). (e) Methane evolution rate and integrated amount evolved. (f) $\mathrm{CO}$ evolution rate and integrated amount evolved. (g) $\mathrm{SO}_{2}$ evolution rate and integrated amount evolved. (h) $\mathrm{NH}_{3}$ evolution rate and integrated amount evolved. (i) $\mathrm{C}_{2} \mathrm{H}_{4}$ evolution rate and integrated amount evolved. (j) $\mathrm{COS}$ evolution rate and integrated amount evolved. All weight losses are on an as received basis.

The second peak appears as a shoulder on the high-temperature side of the first peak. This peak may be due to methane formed by homolytic cleavage of methyl groups or by ipso substitution associated with $\mathrm{H}$ radicals released during ring condensation. In Figure $6 \mathrm{e}$, these peaks are labeled 1 (methane loose) and 2 (methane tight).

The evolution of $\mathrm{CO}$ is presented in Figures $5 \mathrm{f}-12 \mathrm{f}$. Low-rank coals exhibit three peaks labeled 3,4 , and 5 as shown in Figure 10f. Peaks 3 and 4 coincide with the $\mathrm{CO}_{2}$ peaks 3 and 4, while peak 5 has no accompanying peaks for $\mathrm{H}_{2} \mathrm{O}$ or $\mathrm{CO}_{2}$. $\mathrm{CO}$ peaks also can be seen accompanying the $\mathrm{CO}_{2}$ calcite peak (see Figure 9). High-rank coals appear to have only the high-temperature peak 5 .

Results for other gases are presented in parts $g-i$ of Figures 5-12. The $\mathrm{C}_{2} \mathrm{H}_{2}$ evolution shown in Figures 5g-12g occurs as a narrow peak which lags the tar peak, but which precedes the methane. The ammonia evolution in Figures $5 \mathrm{~h}-12 \mathrm{~h}$ appears to coincide with the start of $\mathrm{CO}$ evolution (Figures 5f-12f). The $\mathrm{SO}_{2}$ peak near $28 \mathrm{~min}$ (Figures 5i12i) appears to coincide with one of the $\operatorname{COS}$ peaks (Figures $5 \mathrm{j}-12 \mathrm{j})$.

The evolution peaks in Figures 5-12 are currently being examined to elucidate the chemistry responsible for their formation. For example, there are certain evolution peaks that match in shape and temperature for more than one species, suggesting that there is common chemistry responsible for the evolution of these species. Also, certain evolution peaks (i.e., $\mathrm{CH}_{4}$ and $\mathrm{CO}_{2}$ ) are observed to coincide with cross-linking ${ }^{31.33}$ and certain peaks show correlations such as decreases in tar yield with increases in $\mathrm{CO}_{2}$ due to oxidation ${ }^{31}$ or increases in tar yield associated with decreases in $\mathrm{CO}_{2}$ due to demineralization ${ }^{30}$ (as discussed below) or preliquefaction. ${ }^{29}$

Analysis of Argonne Chars in the Combination Cycle. Sample results for the combustion cycle are presented in Figures 13 and 14. Since oxygen is added, the reported weight loss is for the elements $C, H$, and $S$, not the oxide. This will make the sum of the elements (C, $\mathrm{H}$, and S) lost less than the total measured weight loss, the difference being the oxygen in the char. The combustion cycle is dominated by the evolution of $\mathrm{CO}_{2}, \mathrm{CO}$, and $\mathrm{SO}_{2}$. The sum of the $\mathrm{C}, \mathrm{H}$, and $\mathrm{S}$ in these species is in reasonable agreement with the weight loss. As discussed below, the reactivity of the char affects the weight gain when oxygen is introduced and also affects the temperature of initiation of the combustion reactions. The higher the reactivity, the 

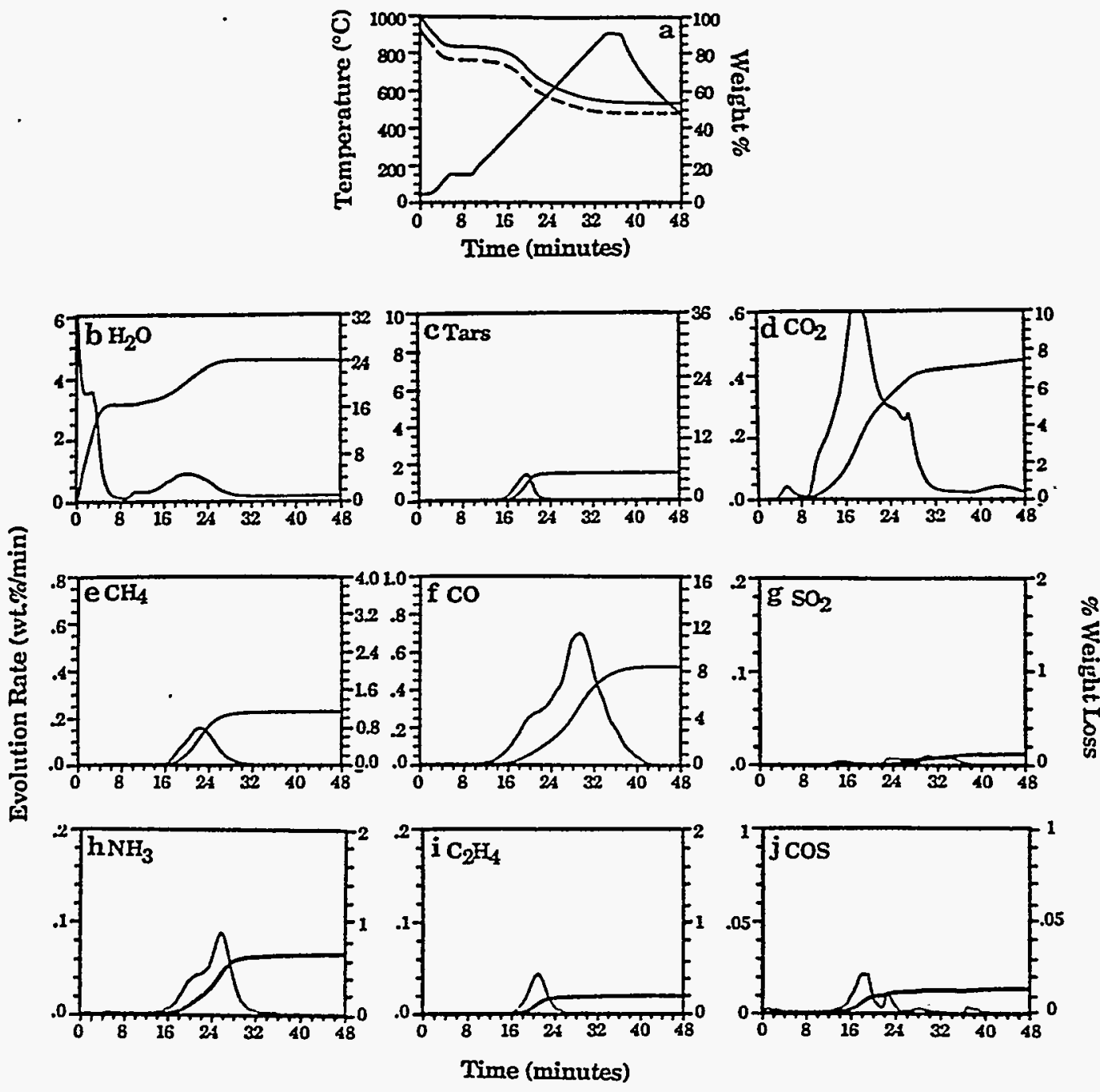

Figure 12. TG-FTIR analysis of Zap lignite during the pyrolysis cycle. (a) Weight loss (solid), sum of evolved products (dashed), and temperature profile. (b) $\mathrm{H}_{2} \mathrm{O}$ evolution rate and integrated amount evolved. (c) Tar evolution rate and integrated amount evolved. (d) $\mathrm{CO}_{2}$ evolution rate and integrated amount evolved. (e) Methane evolution rate and integrated amount evolved. (f) $\mathrm{CO}$ evolution rate and integrated amount evolved. (g) $\mathrm{SO}_{2}$ evolution rate and integrated amount evolved. (h) $\mathrm{NH}_{3}$ evolution rate and integrated amount evolved. (i) $\mathrm{C}_{2} \mathrm{H}_{4}$ evolution rate and integrated amount evolved. (j) $\mathrm{COS}$ evolution rate and integrated amount evolved. All weight losses are on an as received basis.

more oxygen is adsorbed and the lower is the reaction temperature.

Analysis of Minerals. The identification of evolution peaks due to minerals was made by performing TG/plus analysis of reference minerals and demineralized coals. Figure 15 presents the data for calcite. The major reaction is the evolution of $\mathrm{CO}_{2}$ near $800^{\circ} \mathrm{C}$. There are also small amounts of $\mathrm{CO}$ and $\mathrm{H}_{2} \mathrm{O}$ evolved. Reference spectra for kaolin (now shown) exhibit a 14\% weight loss caused by a large water evolution peak at $550^{\circ} \mathrm{C}$ and small $\mathrm{CO}_{2}$ peaks at 518 and $884^{\circ} \mathrm{C}$. Evolution profiles for montmorillonite and illite (not shown) exhibit smaller weight losses (6-8\%) primarily due to water loss near $650^{\circ} \mathrm{C}$ and smaller $\mathrm{CO}_{2}$ losses.

The $\mathrm{CO}_{2}$ peak exhibited by calcite is almost identical in shape and position with that exhibited by the Illinois No. 6 coal in Figure 9d. When the Illinois coal wes demineralized (dashed line in Figure 9d) the $800^{\circ} \mathrm{C} \mathrm{CO}_{2}$ peak disappeared. Several high-rank coals (Pocahontas, Upper Freeport, and Pittsburgh) showed $\mathrm{CO}_{2}$ peaks at about 525 ${ }^{\circ} \mathrm{C}$ which disappeared with demineralization. Figure 5, which shows the evolution profiles for demineralized Pocahontas (dashed line), is an example, showing removal of the $\mathrm{CO}_{2}$ peaks at 520 and $760^{\circ} \mathrm{C}$. The $520^{\circ} \mathrm{C}$ peak appears to be due to siderite on the basis of the discussion by Raask. ${ }^{41}$ However, the TG/plus results on a reference siderite sample obtained from Copper Lake in Nova Scotia showed the $\mathrm{CO}_{2}$ peak at $580^{\circ} \mathrm{C}$. Another possible source is dolomite, which decomposes in this range. The hightemperature $\mathrm{CO}_{2}$ peak is most likely a carbonate, although it is at a different temperature from the reference calcite.

Effects of Minerals on Other Volatile Products. Besides the changes observed in the $\mathrm{CO}_{2}$ yields, demineralization also affects the tar yield. Figures $5 c$ and $9 c$ show 20-30\% increases in tar yield for Pocahontas and Illinois No. 6 upon demineralization. This is larger than can be attributed to the weight percent adjustment from an as-received basis to a daf basis. More than a $100 \%$ increase in the tar was observed for Wyodak coal (Figure 11c). Similar results were obtained for Zap lignite. ${ }^{30}$ The tar increases in both the low-rank coals were accompanied by reductions in the early release of $\mathrm{CO}_{2}$ as discussed by

(41) Raask, E Mineral Impurities in Coal Combustion: Behavior, Problems, and Remedial Measures; Hemisphere: New York, 1985. 

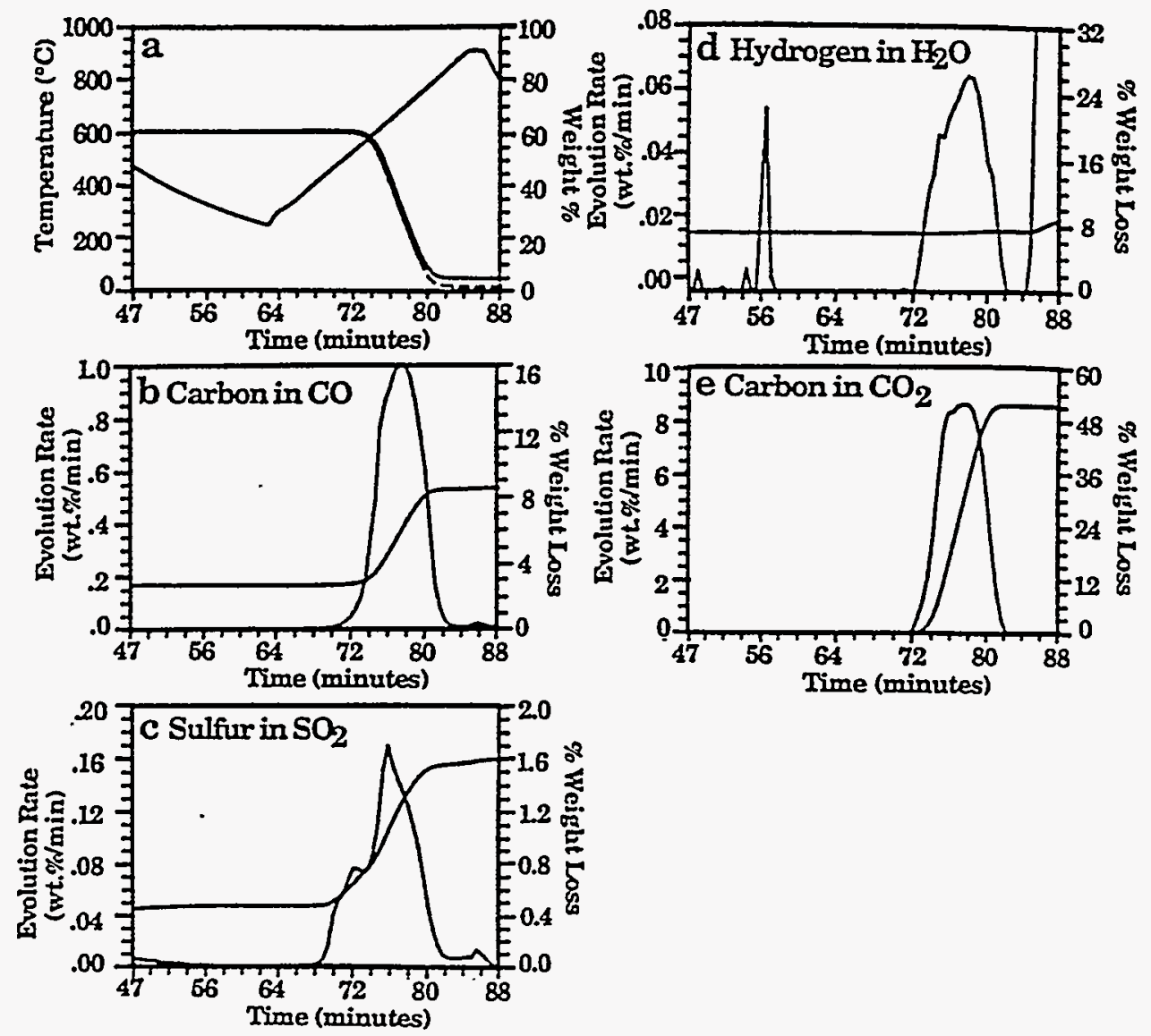

Figure 13. TG-FTIR analysis of Pittsburgh Seam coal during the combustion cycle. (a) Weight loss (solid), sum of evolved products (dashed), and temperature profile. (b) Carbon in $\mathrm{CO}$ evolution rate and integrated amount evolved. (c) Sulfur in $\mathrm{SO}_{2}$ evolution rate and integrated amount evolved. (d) Hydrogen in $\mathrm{H}_{2} \mathrm{O}$ evolution rate and integrated amount evolved. (e) Carbon in $\mathrm{CO}_{2}$ evolution rate and integrated amount evolved.

Serio et al..$^{30}$ This result is further confirmation of the concept of low-temperature cross-linking related to $\mathrm{CO}_{2}$ evolution. ${ }^{31}$ For low-rank coals, changes are also observed in the $\mathrm{CO}$ profile with demineralization, but little change was observed in any of the other species. The effect of demineralization and other pretreatments on the evolution profiles is discussed in more detail by Serio et al. . $^{30}$

Effect of Coal Oxidation on Volatile Evolution. The effect of coal oxidation on the volatile product evolution was also examined. Figure 7 compares the evolution curves for a raw Pittsburgh Seam bituminous (solid line) to those for samples oxidized in air at $110^{\circ} \mathrm{C}$ for 3 days (dashed line) and 3 months (dotted lines). The 3-day oxidation resulted in minor changes in the evolution curves. A difference can be seen in increased $\mathrm{CO}_{2}$ released at low temperatures. Major changes occur for the 3-month sample. Large increases are observed in the $\mathrm{CO}_{2}, \mathrm{CO}$, and $\mathrm{SO}_{2}$ yields while a large reduction (50\%) is seen in the tar. Similar results on the increase in early $\mathrm{CO}_{2}$ evolution and reduction in tar yield have been presented as evidence for low-temperature cross-linking associated with $\mathrm{CO}_{2}$ evolution. ${ }^{31}$ The reduction in tar yield upon oxidation is consistent with the usual observation of reduced fluidity and deterioration of liquefaction behavior with oxidation. Of interest is the surprising reduction in the $\mathrm{H}_{2} \mathrm{O}$ yield. Smaller changes are observed for other species.

Proximate Analysis. The cycle of drying, pyrolysis, and oxidation, illustrated in Figure $2 \mathrm{a}$, simulates the conditions for the proximate analysis. Table II compares the moisture (measured at the end of the drying period), the volatile matter (measured at the end of the pyrolysis
Table II. Proximate Analysis by TG/plus; ASTM Values in Parentheses

\begin{tabular}{lcccc}
\hline \multicolumn{1}{c}{ coal } & moisture & $\begin{array}{c}\text { volatile } \\
\text { matter }\end{array}$ & $\begin{array}{c}\text { fixed } \\
\text { carbon }\end{array}$ & ash \\
\hline Pocahontas No. & $0.2(0.7)$ & $20.6(18.5)$ & $73.4(76.1)$ & $6.3(4.7)$ \\
$\quad 3$ & & & & \\
Upper Freeport & $0.4(1.1)$ & $25.2(27.1)$ & $60.0(58.7)$ & $14.4(13.0)$ \\
Pittsburgh No. 8 & $1.1(1.7)$ & $35.9(37.2)$ & $49.5(52.1)$ & $13.4(9.1)$ \\
Stockton & $1.5(2.4)$ & $31.8(29.4)$ & $43.2(48.8)$ & $23.4(19.4)$ \\
Illinois No. 6 & $2.0(8.0)$ & $39.3(36.9)$ & $43.4(40.9)$ & $15.3(14.3)$ \\
Utah & $2.3(4.6)$ & $43.5(43.7)$ & $47.8(47.2)$ & $6.3(4.5)$ \\
Wyodak & $25.7(28.1)$ & $33.0(32.2)$ & $34.8(33.4)$ & $6.5(6.3)$ \\
Beulah Zap & $30.6(32.2)$ & $28.4(30.5)$ & $33.7(30.7)$ & $7.3(6.6)$
\end{tabular}

period), and the ash (measured at the end of the combustion cycle) with the proximate analysis values published by Argonne National Laboratory. ${ }^{34}$ The agreement is within $1 \%$ or $2 \%$ except for moisture, which varies with the laboratory conditions, and ash, which may vary because of the small sample size ( $35 \mathrm{mg}$ ) used in the TG/plus analysis.

Volatile Kinetics. The TG-FTIR analysis can be used to study product evolution kinetics. Since tar and hydrocarbon gases are the most important contributors to the weight loss, we have compared, in Figure $16 \mathrm{a}$, the temperature for the maximum hyrocarbon evolution rate for the Argonne coals as a function of rank at $0.05,0.5,0.83$, and $1.67^{\circ} \mathrm{C} / \mathrm{s}$. Duplicate runs were all within $\pm 4^{\circ} \mathrm{C}$. The peak temperatures as well as the shape of the tar peaks are in good agreement with the results of Burnham et al. ${ }^{17}$ obtained by using a Rock-Eval analyzer and a triple- 

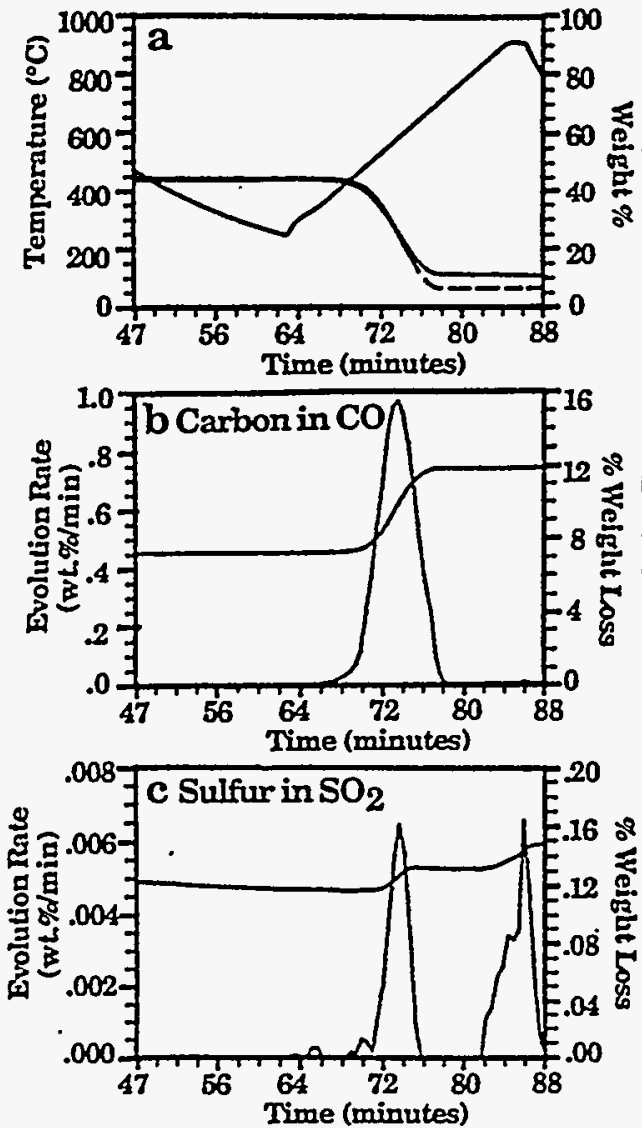
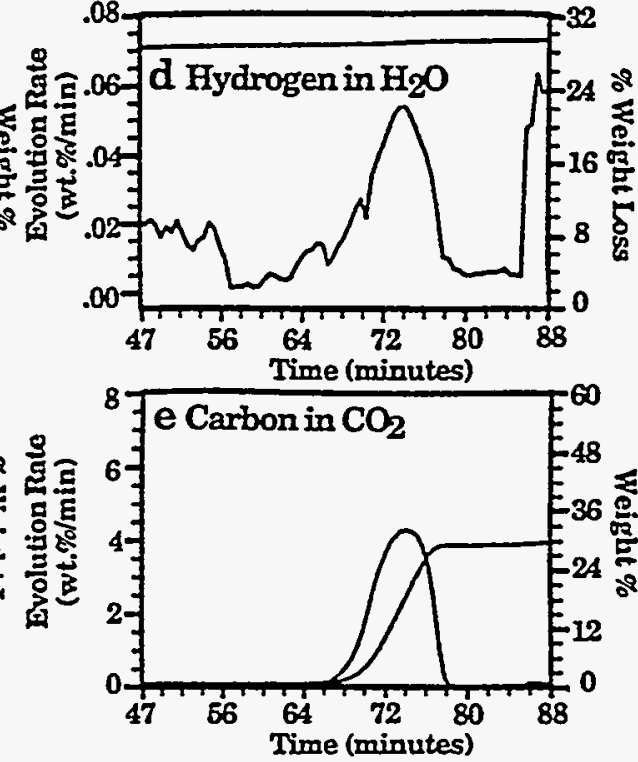

Figure 14. TG-FTIR analysis of Wyodak subbituminous coal during the combustion cycle. (a) Weight loss (solid), sum of evolved products (dashed), and temperature profile. (b) Carbon in $\mathrm{CO}$ evolution rate and integrated amount evolved. (c) $\mathrm{Sulfur}_{\text {in }} \mathrm{SO}_{2}$ evolution rate and integrated amount evolved. (d) Hydrogen in $\mathrm{H}_{2} \mathrm{O}$ evolution rate and integrated amount evolved. (e) Carbon in $\mathrm{CO}_{2}$ evolution rate and integrated amount evolved.

quadrupole mass spectrometer (TQMS) at similar heating rates. The kinetic rate for hydrocarbon evolution shows a systematic variation with rank. The variation in the rate of thermal decomposition is in part responsible for the variation of ignition behavior with rank. ${ }^{42}$

The peak shift with heating rate has been employed by Serio et al. to derive rates for tar evolution. ${ }^{228}$ The 50-65 ${ }^{\circ} \mathrm{C}$ shift in temperatures with heating rate corresponds to activation energies approximately between 45 and 60 $\mathrm{kcal} / \mathrm{mol}$. Recently, we have employed the TG/plus volatile evolution profiles to determine rank-dependent rates to employ in the FG-DVC model of coal pyrolysis. ${ }^{33}$ The rates are determined by adjusting the rate constants to make the theory fit the data. Figure 17 compares the observed and predicted weight loss, tar evolution, and methane evolution profiles at heating rates of $0.05,0.5$, and $1.67^{\circ} \mathrm{C} / \mathrm{s}$ for Pittsburgh Seam coal. The agreement is good for all three heating rates. To obtain these fits, the following rates were employed for the Pittsburgh Seam coal.

$$
\begin{aligned}
k_{\text {bond breaking }} & =1.28 \times 10^{12} \exp (-(24000 \pm 1200) / T) \mathrm{s}^{-1} \\
k_{\mathrm{CH}_{4} \text { loose }} & =7.95 \times 10^{13} \exp (-(29000 \pm 1500) / T) \mathrm{s}^{-1} \\
k_{\mathrm{CH}_{4} \text { tight }} & =4.5 \times 10^{12} \exp (-(30000 \pm 2000) / T) \mathrm{s}^{-1}
\end{aligned}
$$

The rate equation is of the form $k_{n}=k_{0} \exp (-((E / R) \pm$ $(\sigma / R)) / T$, with $k_{0}$ in $\mathrm{s}^{-1}, E / R$ in $\mathrm{K}$, and $\sigma / R$ in $\mathrm{K}$. The

(42) Solomon, P. R; Chien, P. L.; Carangelo, R. M.; Best, P. E.; Markham, J. R. Twenty-Second Symposium (International) on Combustion; The Combustion Institute: Pittsburgh, PA, (1988); 211. accuracy of the activation energies is estimated to be within $\pm 2 \mathrm{kcal}$. With a fixed activation energy, the accuracy of the preexponential is $\pm 25 \%$ and that of $\sigma$ is $\pm 10 \%$. These rates appear to provide good predictions when applied to fit data taken at much higher heating rates $\left(5000^{\circ} \mathrm{C} / \mathrm{s}\right)$ as well. The bond-breaking rate is in good agreement with the approximate rate of $3.1 \times 10^{12} \mathrm{exp}(-(24942 \pm 1250) / T)$ derived by Burnham et al..$^{17}$ The above rates can be compared to the rank-independent rates employed previously: $:^{33}$

$$
\begin{gathered}
k_{\text {bond breaking }}=8.6 \times 10^{15} \exp (-(27700 \pm 1500) / T) \mathrm{s}^{-1} \\
k_{\mathrm{CH}_{6} \text { loose }}=7.5 \times 10^{13} \mathrm{exp}(-(30000 \pm 2000) / T) \mathrm{s}^{-1} \\
k_{\mathrm{CH}_{4} \text { tight }}=3.4 \times 10^{11} \exp (-(3000 \pm 2000) / T) \mathrm{s}^{-1}
\end{gathered}
$$

The activation energy for bond breaking is smaller, the rate for $\mathrm{CH}_{4}$ loose is similar, and the rate for $\mathrm{CH}_{4}$ tight is about a factor of 10 faster.

Functional Group Composition. The TG-FTIR analysis provides information on the coal's functional group composition since it is the functional group composition that gives rise to the variation in gas yields. Figure $16 \mathrm{~b}$ shows the variation in oxygen-containing products with rank. Low-rank coals have a high content of oxygen functional groups and thus produce higher yields of oxygenated species. The trends are in agreement with the data of Burnham et al. ${ }^{17}$ The results of the present work are, however, higher for high-rank cosls and lower for low-rank coals. 

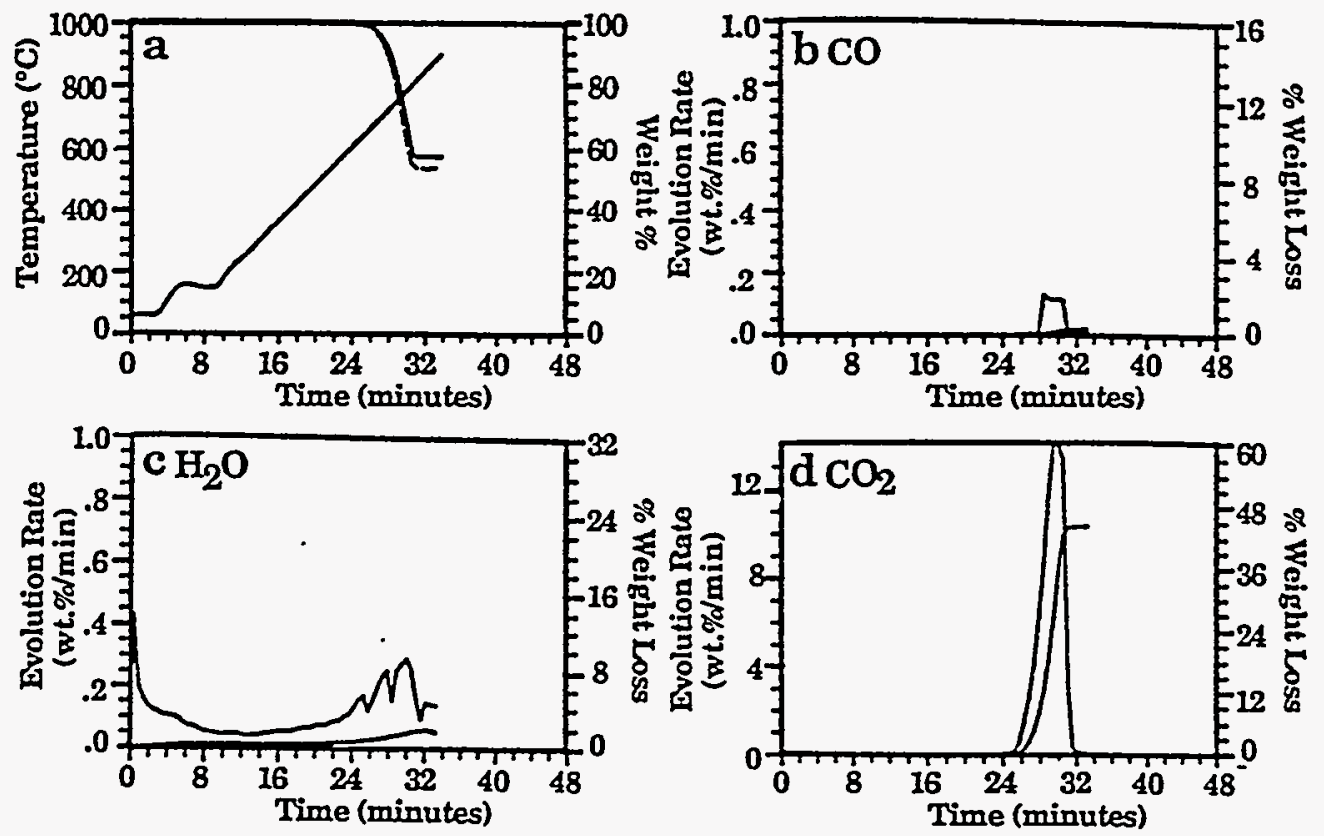

Figure 15. TG-FTIR analysis of calcium carbonate during the pyrolysis cycle. (a) Weight loss (solid), sum of evolved products (dashed), and temperature profile. (b) Tar evolution rate and integrated amount evolved. (c) Methane evolution rate and integrated amount evolved. (d) $\mathrm{H}_{2} \mathrm{O}$ evolution rate and integrated amount evolved. (e) $\mathrm{CO}_{2}$ evolution rate and integrated amount evolved. (f) $\mathrm{CO}$ evolution rate and integrated amount evolved.
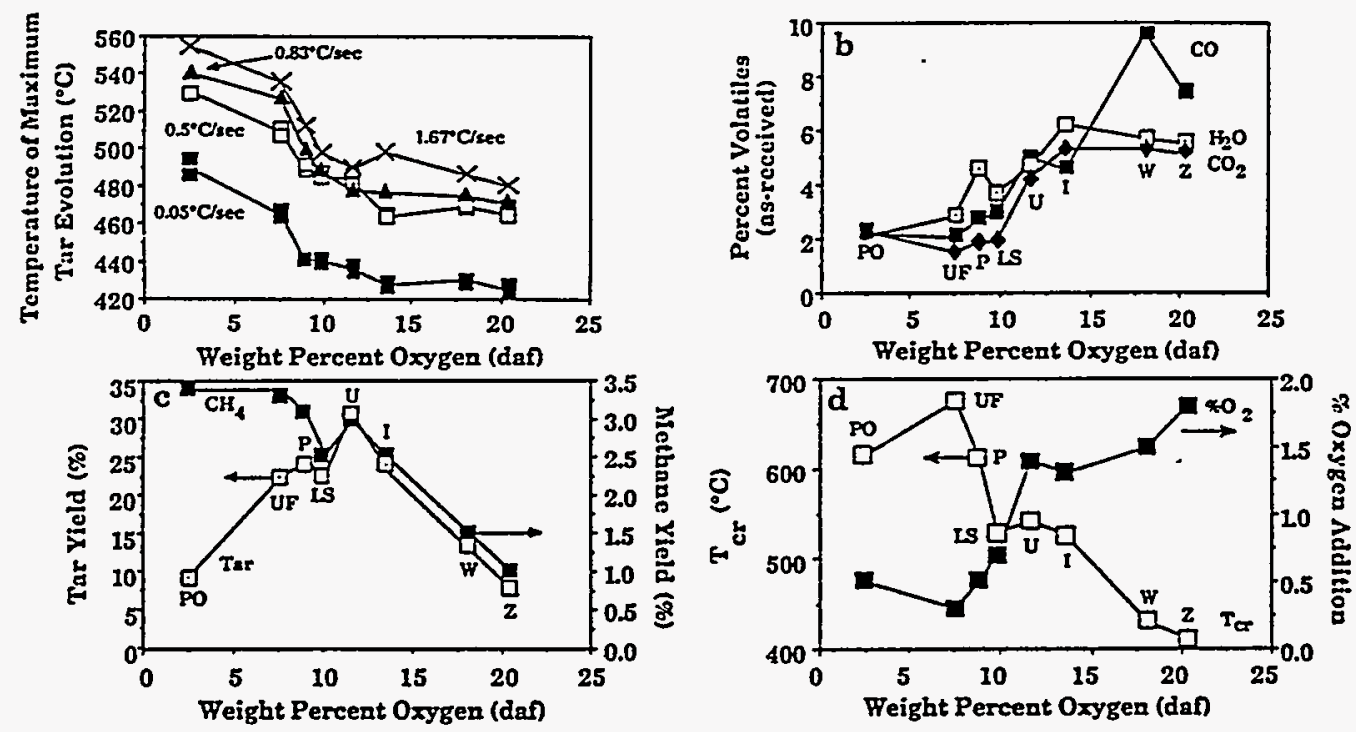

Figure 16. Variation of coal pyrolysis properties with rank. (a) Rank variation of tar evolution temperature. (b) Rank variation of oxygenated gases $\mathrm{CO}$ and $\mathrm{CO}_{2}$ and pyrolysis water. (c) Rank variation of $\mathrm{CH}_{4}$ and hydrocarbons. (d) Rank variation of $T_{\mathrm{c}}$ and oxygen chemisorption. Results are on an as received basis.

Figure $16 \mathrm{c}$ presents the data for tar and $\mathrm{CH}_{4}$ yields. Methane increases systematically with increasing rank. The trend also agrees with the results of Burnham et al. ${ }^{17}$ The amounts are comparable for the high-rank coals, but our results for low-rank coals are lower than those of Burnham et al. For example, we get 1.0 wt \% for Zap lignite while Burnham et al. get 2.4 wt \%.

Tar yields are low for both high- and low-rank coals, with high-volatile bituminous coals having the most tar. The tar amounts are in good quantitative agreement with the $S_{2}$ peak reported by Burnham et al..$^{17}$ from Rock-Eval analysis. Tar yields are related to soot formation in combustion, ${ }^{42}$ to fluidity, ${ }^{38}$ and to liquid yields in liquefaction ${ }^{30}$ or mild gasification. ${ }^{43}$ The tar functional group compo-

(43) Khan, M. R.; Serio, M. A.; Malhotra, R.; Solomon, P. R. Prepr. Pap.-Am. Chem. Soc., Div. Fuel Chem. 1989, 34 (4), 1054. sition can also be obtained from the infrared spectra during tar evolution.

Char Reactivity. As discussed above, the TG-FTIR ahalysis provides two measurements related to char reactivity. The first is the weight gain of the char which occurs when oxygen is added early in the combustion cycle (see Figure 2b). This weight gain is proportional to the concentration of active sites that are accessible to $\mathrm{O}_{2}$. The second measurement is of the temperature required to produce a specified rate of weight loss during the oxidation cycle. As discussed previously, this critical temperature $T_{\mathrm{a}}$ is related to the reactivity. ${ }^{47}$ The higher the reactivity, the lower is $T_{c r}$

Figure 16d compares both the oxygen chemisorbed and $T_{c r}$ as functions of the oxygen in the coal. There is a systematic decrease in $T_{c t}$ and an increase in oxygen chemisorbed with increasing oxygen. The interplay of 

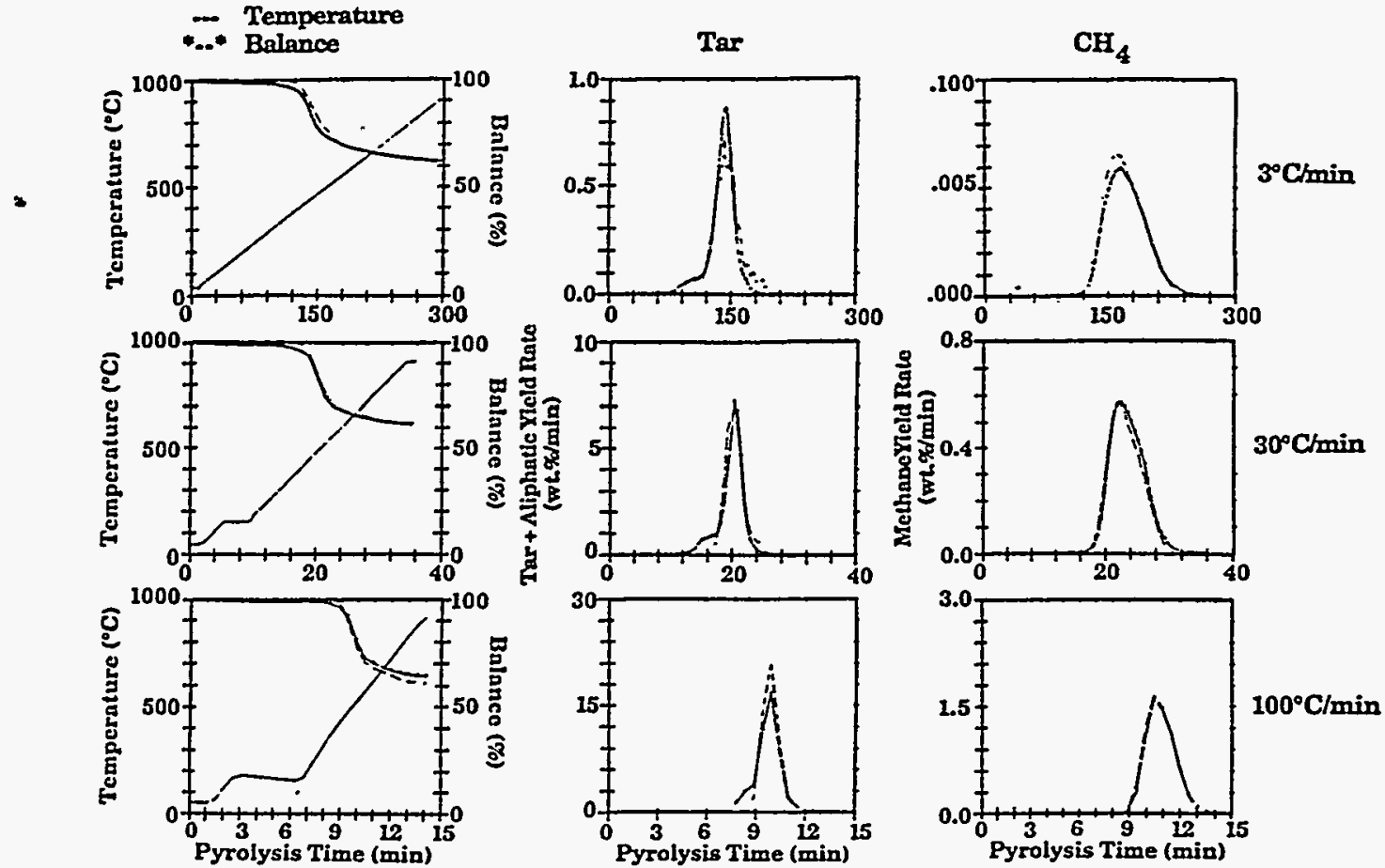

Figure 17. Kinetic analysis for Pittsburgh Seam coal for weight loss, tar evolution, and methane evolution at three heating rates. Experiment (solid line) and theory (dashed line) using the FG-DVC Model 33 with the kinetic rates reported in this paper.
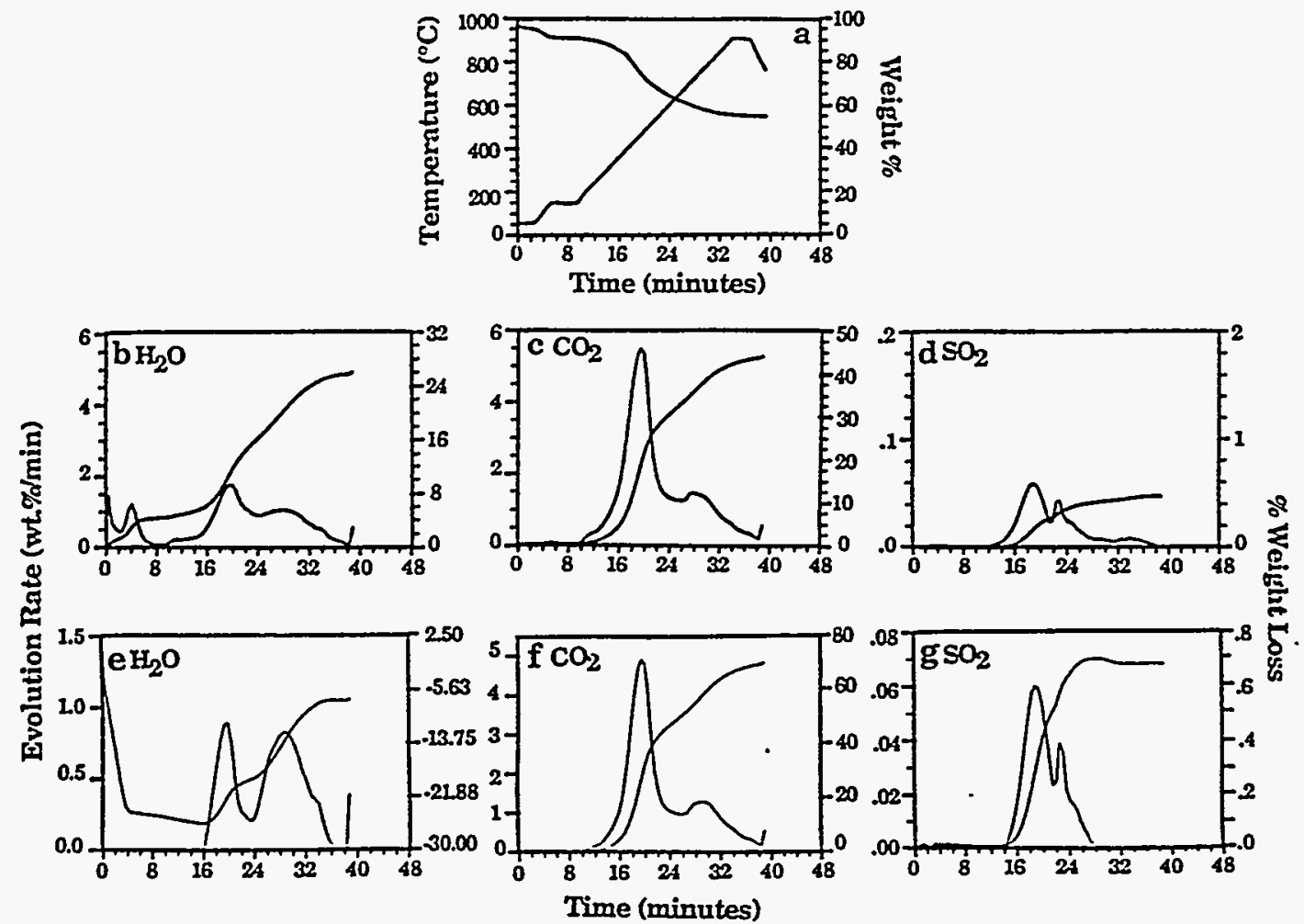

Figure 18. TG-FTIR analysis with postoxidizer. (a) Weight loss and temperature profile. (b) $\mathrm{H}_{2} \mathrm{O}$ evolution rate and integrated amount evolved. (c) $\mathrm{CO}_{2}$ evolution rate and integrated amount evolved. (d) $\mathrm{SO}_{2}$ evolution rate and integrated amount evolved. (e) $\mathrm{H}_{2} \mathrm{O}$ postoxidizer profile minus pyrolysis profile. (f) $\mathrm{CO}_{2}$ postoxidizer profile minus pyrolysis profile. (g) $\mathrm{SO}_{2}$ postozidizer profile minus pyrolysis profile.

reactivity and decomposition kinetics control the ignition behavior and burnout in combustion or gasification. ${ }^{42}$

Postoxidation. It is possible to perform a (C, H, N, S) elemental analysis of the volatile products by adding heat and oxygen to the product stream after the furnace but before the analysis cell. This step has the added benefit of indicating the presence of products like $\mathrm{H}_{2}$ that are not infrared active or $\mathrm{H}_{2} \mathrm{~S}$ that are very weak infrared absorbers.

The hardware modification is made by inserting a quartz tube section about $3 \mathrm{~cm}$ long between the quartz tube leaving the furnace section and the multipass cell. The 
insert has an input line for oxygen addition and a platinum heater. An oxygen flow of $10 \mathrm{~cm}^{3} / \mathrm{min}$ and a temperature of about $800^{\circ} \mathrm{C}$ in the platinum heater were employed.

Results are presented for a Zap lignite in Figure 18. Figure 18a presents the weight loss, which is almost identical with that in Figure 12a. Parts b, $c$, and $d$ of Figure 18 present the yields of $\mathrm{H}_{2} \mathrm{O}, \mathrm{CO}_{2}$, and $\mathrm{SO}_{2}$, which are the primary oxidation products of the volatile species. We could not see significant amounts of NO in the combustion products. The other volatile species ( $\operatorname{tar}, \mathrm{CH}_{4}$, $\mathrm{C}_{2} \mathrm{H}_{4}, \mathrm{CO}, \mathrm{NH}_{3}$, and $\mathrm{COS}$ ) have also been oxidized and are thus absent from the spectra.

To analyze the results, the $\mathrm{H}_{2} \mathrm{O}, \mathrm{CO}_{2}$, and $\mathrm{SO}_{2}$ observed in pyrolysis (Figure 12) have been subtracted from the curves in Figure 18b-d and the results presented in Figure 18e-g. The $\mathrm{H}_{2} \mathrm{O}, \mathrm{CO}_{2}$, and $\mathrm{SO}_{2}$ difference curves now have peaks that match the tar evolution peak in Figure 12c. These data determine the $\mathrm{C}, \mathrm{H}$, and $\mathrm{S}$ composition of the tar. In addition, the $\mathrm{H}_{2} \mathrm{O}$ evolution profile has a wide peak at elevated temperatures believed to result from the oxidation of hydrogen. The $\mathrm{CO}_{2}$ has a high-temperature peak from the oxidation of $\mathrm{CO}$. The $\mathrm{SO}_{2}$ has peaks at 449 and $565^{\circ} \mathrm{C}$ related to the release of $\mathrm{H}_{2} \mathrm{~S}$. The peak at $565^{\circ} \mathrm{C}$ is close to that for a sample of pure pyrite run in the TG-FTIR.

Thus, the postoxidation section adds significant new information to the analysis.

\section{Summary and Conclusions}

A single TG-FTIR analysis provides an extensive characterization of coal with regard to the decomposition kinetics, char reactivity, functional group compositions, and conversion behavior. The paper presents the following:

1. The TG-FTIR apparatus for pyrolysis, oxidation of pyrolysis products, and oxidation of the sample is described.
2. Pyrolysis results are presented for the eight Argonne coals, several demineralized coals, and two oxidized samples of Pittsburgh Seam coal.

3. There are certain evolution peaks that match in shape and temperature for more than one species, suggesting common chemistry responsible for the evolution of these species.

4. Several peaks in the $\mathrm{CO}_{2}$ evolution have been identified with mineral decomposition.

5. Increases in tar yield are observed for the demineralized coals (especially low-rank coals) while decreases in tar yield are observed for the oxidized samples.

6. A kinetic analysis was applied to species evolution data collected at several different heating rates. There is a systematic variation in rate with rank. The rate for tar evolution from Pittsburgh Seam coal is in good agreement with that of Burnham et al..$^{17}$ using a similar set of data.

7. Analyses of the amounts of evolved products also show a systematic variation with rank consistent with the coal's elemental and functional group compositions.

8. Postoxidation of the volatile products has been successful in providing elemental composition information on the volatile products as well as showing the evolution of $\mathrm{H}_{2}$, which is not infrared active, and $\mathrm{H}_{2} \mathrm{~S}$ (in the postoxidized $\mathrm{SO}_{2}$ profile), which is a weak infrared absorber.

9. Oxidation of the char vields the ash amount as well as two measures of the cricrs reactivity, the oxygen absorbed by the char. and the remperature at which significant oxidation of the char occurs.

Acknowledgment. This work was supported by the U.S. Department of Energy, Morgantown Energy Technology Center, under Contract No. DE-AC21-86MC23075 and the National Science Foundation, Grant No. ISI8703520 . We acknowledge the contributions of Z. Z. Yu and S. Charpenay on the modeling of the TG-FTIR data. We also thank Jean Whelan of Woods Hole Oceanographic Institute for supplying the siderite sample. 
Appendix $O$

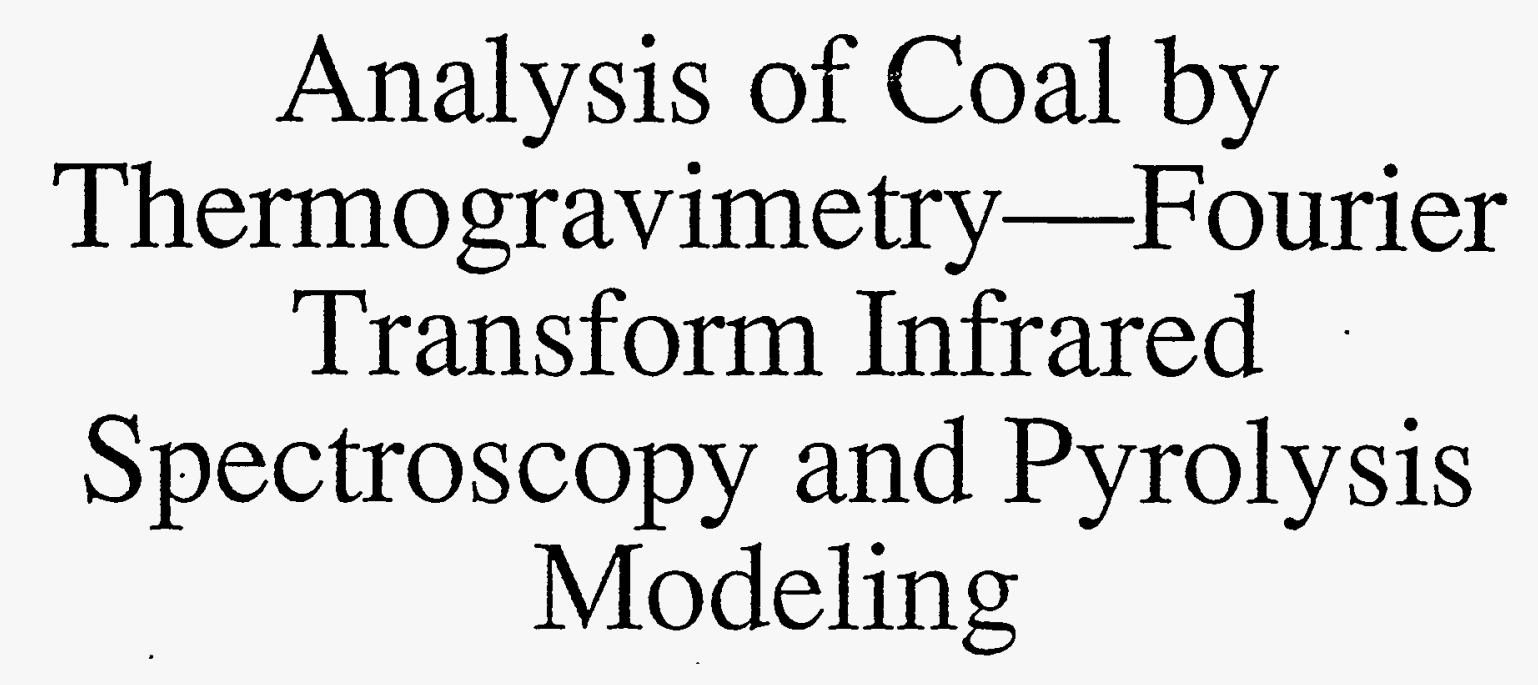


Analysis of Coal by Thermogravimetry-Fourier Transform Infrared Spectroscopy and Pyrolysis Modeling 


\title{
Analysis of coal by thermogravimetry-Fourier transform infrared spectroscopy and pyrolysis modeling
}

\author{
P.R. Solomon ${ }^{\text {a.* }}$, M.A. Serio a, R.M. Carangelo a, R. Bassilakis a, Z.Z. \\ $\mathrm{Yu}^{\mathrm{a}}$, S. Charpenay ${ }^{\mathrm{a}}$ and J. Whelan ${ }^{\mathrm{b}}$ \\ advanced Fuel Research, Inc., 87 Church Street, East Hartford. CT 06108 (U.S.A.) \\ 'Woods Hole Oceanographic Institute, Woods Hole, MA 02543 (U.S.A.)
}

(Received June 29, 1990; accepted in final form February 8. 1991)

\begin{abstract}
We have developed a TG-FT-IR instrument which combines thermogravimetric analysis (TGA) with evolved product analysis by Fourier Transform Infrared (FT-IR) spectroscopy. FT-IR analysis of evolved products has an advantage over mass spectroscopy in allowing analysis of very heavy products, and over gas chromatography in speed. This paper describes the most recent improvements in the apparatus and presents its application in characterizing coal. The emphasis in this work is on employing the TG-FT-IR system to obtain kinetic rates for species evolution under easily obtained laboratory conditions. These rates can then be extrapolated to predict the conversion behavior of the hydrocarbon at higher heating rates and temperatures in practical conversion processes or at lower heating rates and temperatures in geological conversion processes. For several coal samples, a kinetic analysis was applied to species evolution data collected at several different heating rates. For coal. there is a systematic variation in rate with rank for almost all volatile species. Extrapolation to high temperatures shows excellent agreement with high temperature coal pyroloysis data. Extrapolation to low temperatures is consistent with the expected changes induced by bed temperatures over geological times.
\end{abstract}

Argonne coals; coal; Fourier transform infrared spectroscopy; pyrolysis; thermogravimetric analysis.

\section{INTRODUCTION}

For the characterization of hydrocarbons. thermogravimetric (TG) analysis techniques have proved to be very useful. However, TG analysis by itself does not identify the decomposition products. When TG analysis is coupled with evolved product analysis, a great deal of additional information regard- 
ing the composition of pyrolysis and oxidation products can be obtained. We have recently employed Fourier transform infrared (FT-IR) spectroscopy to perform such evolved product analysis. FT-IR has an advantage over mass spectroscopy in allowing analysis of very heavy products, and over gas chromatography in speed. The application of TG-FT-IR to coal and petroleum source rock has been described in two recent publications $[1,2]$.

The effluents most difficult to analyze are the heavy decomposition products which condense at room temperature. These condensates, such as tars from coal, are the major evolved products from most hydrocarbons. A recently designed TG-FT-IR apparatus, the Bomem TG/plus allows these products to be analyzed by condensing them into an aerosol which is fine enough to follow the gas through the infrared analysis cell, which is connected without restrictions to the sample area. The aerosol is also fine enough so that there is only a little scattering of the infrared beam and it is thus attenuated almost as though the tar was in the gas phase. The quantitative analysis of both gases and tars allows the mass balance to be closed within a few percent.

The TG/plus apparatus has been applied to the analysis of the Argonne premium coal samples to determine their pyrolysis behavior [3]. In addition, kinetic rate coefficients for the evolution of specific products have been obtained by employing several heating rates between $0.05 \mathrm{~K} \mathrm{~s}^{-1}$ and $1.67 \mathrm{~K}$ $s^{-1}$ [4]. These rate coefficients are employed in the FG-DVC model for coal devolatilization [5-9] to allow predictions of pyrolysis at other heating rates and temperatures. This general model combines a functional group (FG) model for gas evolution [10.11] and a statistical depolymerization, vaporization, and crosslinking (DVC) model for tar formation [12-14]. The FG model describes the evolution of gases from sources in the coal, char and tar. The DVC model describes the decomposition and condensation of a macromolecular network under the influence of bond breaking and crosslinking to predict (using statistical methods) the molecular weight distribution of the network fragments. Both Monte Carlo [5,8] and percolation statistics [9] have been employed. Tar is formed from the light fraction of the network fragments which vaporizes and is transported by the light gases. The crosslinking reactions are related to the evolution of $\mathrm{CO}_{2}$ at low temperature and $\mathrm{CH}_{4}$ at moderate temperature [5,14,15].

In this paper, we explore how well the kinetic rate coefficients determined at moderate heating rates can be extrapolated to conversion processes at high heating rates $\left(\approx 40000 \mathrm{~K} \mathrm{~s}^{-1}\right)$ and natural maturation processes at 10 $\mathrm{K}$ per million years. The kinetic rate coefficients and composition parameters derived using the TG/plus applied to the Argonne coals [3,4] were employed in the FG-DVC model which was used to simulate high heating rate pyrolysis experiments and natural maturation. The simulations for coal are compared with high heating rate data previously obtained for Illinois 
No. 6 coal [11] and with the natural maturation represented by the Argonne premium sample collection.

\section{EXPERIMENTAL}

The TG/plus apparatus has been described previously [3,16]. Its components are as follows: a Du Pont ${ }^{\text {TM }} 951$ TGA; an Infrared Analysis 16 pass gas cell with transfer optics; a MICHELSON 110 FT-IR; (resolution: 4 $\mathrm{cm}^{-1}$, detector: $\mathrm{MCT}$ ). A typical analysis is performed as follows: a sample (typically $5-200 \mathrm{mg}$ ) is taken on a $30^{\circ} \mathrm{C} \mathrm{min}^{-1}$ temperature excursion in the helium sweep gas, first to $150^{\circ} \mathrm{C}$ to dry for $240 \mathrm{~s}$, then at $30^{\circ} \mathrm{C} \mathrm{min}-1$ to $900^{\circ} \mathrm{C}$ for pyrolysis. Upon reaching $900^{\circ} \mathrm{C}$, the sample is cooled to $250^{\circ} \mathrm{C}$ over a 20 minute period. After cooling, a small flow of $\mathrm{O}_{2}\left(0.3 \mathrm{cc} \mathrm{s}^{-1}\right)$ is added to the helium sweep gas and the temperature is ramped to $700^{\circ} \mathrm{C}$ (or as high as $1000^{\circ} \mathrm{C}$ ) at $30^{\circ} \mathrm{C} \mathrm{min}^{-1}$ for oxidation.

During this excursion, IR spectra are obtained once every 41 s. As discussed previously [1-3,15], the spectra show absorption bands for $\mathrm{CO}$, $\mathrm{CO}_{2}, \mathrm{CH}_{4}, \mathrm{H}_{2} \mathrm{O}, \mathrm{SO}_{2}, \mathrm{COS}, \mathrm{C}_{2} \mathrm{H}_{4}$ and $\mathrm{NH}_{3}$. The spectra above $250^{\circ} \mathrm{C}$ also show aliphatic, aromatic, hydroxyl, carbonyl and ether bands from tar. The evolution of gases derived from the IR absorbance spectra are obtained by a quantitative analysis program. Gas calibrations were performed using known flows of individual gases. The calibration for $\mathrm{CO}_{2}, \mathrm{CO}$, and $\mathrm{H}_{2} \mathrm{O}$ is checked on a routine basis using a sample of calcium oxalate, as described previously $[3,16]$. The tar absorptivity for each run is obtained by comparing a qualitative evolution curve for the appearance of tar functional groups with the sample weight loss minus the sum of gases.

\section{Samples}

The coals analyzed were Argonne premium coal samples. Table 1 presents their ultimate and proximate analysis. More complete characterization of these samples has appeared elsewhere [17].

\section{RESULTS}

\section{Determination of kinetic rate coefficients}

The TG-FT-IR results for the Illinois No. 6 coal at three heating rates are presented in Fig. 1. The broken lines are the prediction of the FG-DVC model [5-9] while the experimental data are plotted as asterisks connected by solid lines. The left-hand set of curves is for the cumulative weight loss from the balance. Superimposed on each of these plots is the time-tempera- 
TABLE 1

Analytical data: ultimate (elemental) and proximate analyses

\begin{tabular}{lrrrrrrrr}
\hline Coal & \multicolumn{1}{c}{ UF } & \multicolumn{1}{c}{ WY } & \multicolumn{1}{c}{ IL } & PITT & POC & \multicolumn{1}{c}{ UT } & WV & \multicolumn{1}{c}{ ND } \\
\hline AR H H O & 1.13 & 28.09 & 7.97 & 1.65 & 0.65 & 4.63 & 2.42 & 32.24 \\
AR Ash & 13.03 & 6.31 & 14.25 & 9.10 & 4.74 & 4.49 & 19.36 & 6.59 \\
Dry Ash & 13.18 & 8.77 & 15.48 & 9.25 & 4.77 & 4.71 & 19.84 & 9.72 \\
Dry VM & 27.45 & 44.73 & 40.05 & 37.82 & 18.60 & 45.84 & 30.17 & 44.94 \\
Dry S & 2.32 & 0.63 & 4.83 & 2.19 & 0.66 & 0.62 & 0.71 & 0.80 \\
Pyritic S & 1.77 & 0.17 & 2.81 & 1.37 & 0.15 & 0.24 & 0.16 & 0.14 \\
Sulfate S & 0.01 & 0.03 & 0.01 & 0.01 & 0.03 & 0.03 & 0.03 & 0.03 \\
Organic S & 0.54 & 0.43 & 2.01 & 0.81 & 0.48 & 0.35 & 0.52 & 0.63 \\
MAF C & 85.50 & 75.01 & 77.67 & 83.20 & 91.05 & 80.69 & 82.58 & 72.94 \\
MAF H & 4.70 & 5.35 & 5.00 & 5.32 & 4.44 & 5.76 & 5.25 & 4.83 \\
MAF N & 1.55 & 1.12 & 1.37 & 1.64 & 1.33 & 1.57 & 1.56 & 1.15 \\
MAF Org S & 0.74 & 0.47 & 2.38 & 0.89 & 0.50 & 0.37 & 0.65 & 0.70 \\
MAF O & 7.51 & 18.02 & 13.51 & 8.83 & 2.47 & 11.58 & 9.83 & 20.34 \\
\hline
\end{tabular}

ture profile: Except for very low heating rates, the coal is heated first to $150^{\circ} \mathrm{C}$ for drying before heating at the designated rate to $900^{\circ} \mathrm{C}$. The agreement between the experimental and predicted weight predictions is quite good at each of the three heating rates. The predicted weight loss is the sum of the tar evolution and the major gases $\left(\mathrm{CO}, \mathrm{CO}_{2}, \mathrm{H}_{2}, \mathrm{H}_{2} \mathrm{O}, \mathrm{CH}_{4}\right.$, paraffins, olefins) which are included in the FG-DVC model. The theoretical curves have been shifted downwards to account for the moisture loss which is not modeled.

The second set of curves in Fig. 1 is for the tar evolution. The prediction of tar evolution is based on the breaking of weak linkages between an assumed polymeric structure for coal followed by transport of the molecule out of the coal if it meets the volatility criteria [5-9]. The position and shape of the main tar peak is predicted very well.

The evolution of $\mathrm{CH}_{4}$ is also well predicted. The $\mathrm{CH}_{4}$ evolution is modeled using two sources which evolve in a manner such that the peaks are usually merged into a single peak [6,11]. The evolution of $\mathrm{CO}_{2}$ is reasonably well predicted at the highest heating rates, but a slight oxidation at the lowest heating rate produces more $\mathrm{CO}_{2}$ than predicted. Note that the large $\mathrm{CO}_{2}$ peak observed at high temperatures is due to calcite, not the organic portion of the coal.

The evolution profiles show a systematic shift with increasing heating rate. The change in the temperature for the maximum evolution rate $\left(T_{\max }\right)$ with temperature can be used in a preliminary analysis to derive kinetic parameters $[18,19]$. We have used this approach to obtain a preliminary estimate of the mean values of the distributed activation energy parameters. The parameters are further refined by using the FG-DVC model to best fit the complete evolution profiles at each of four heating rates. A similar 
E

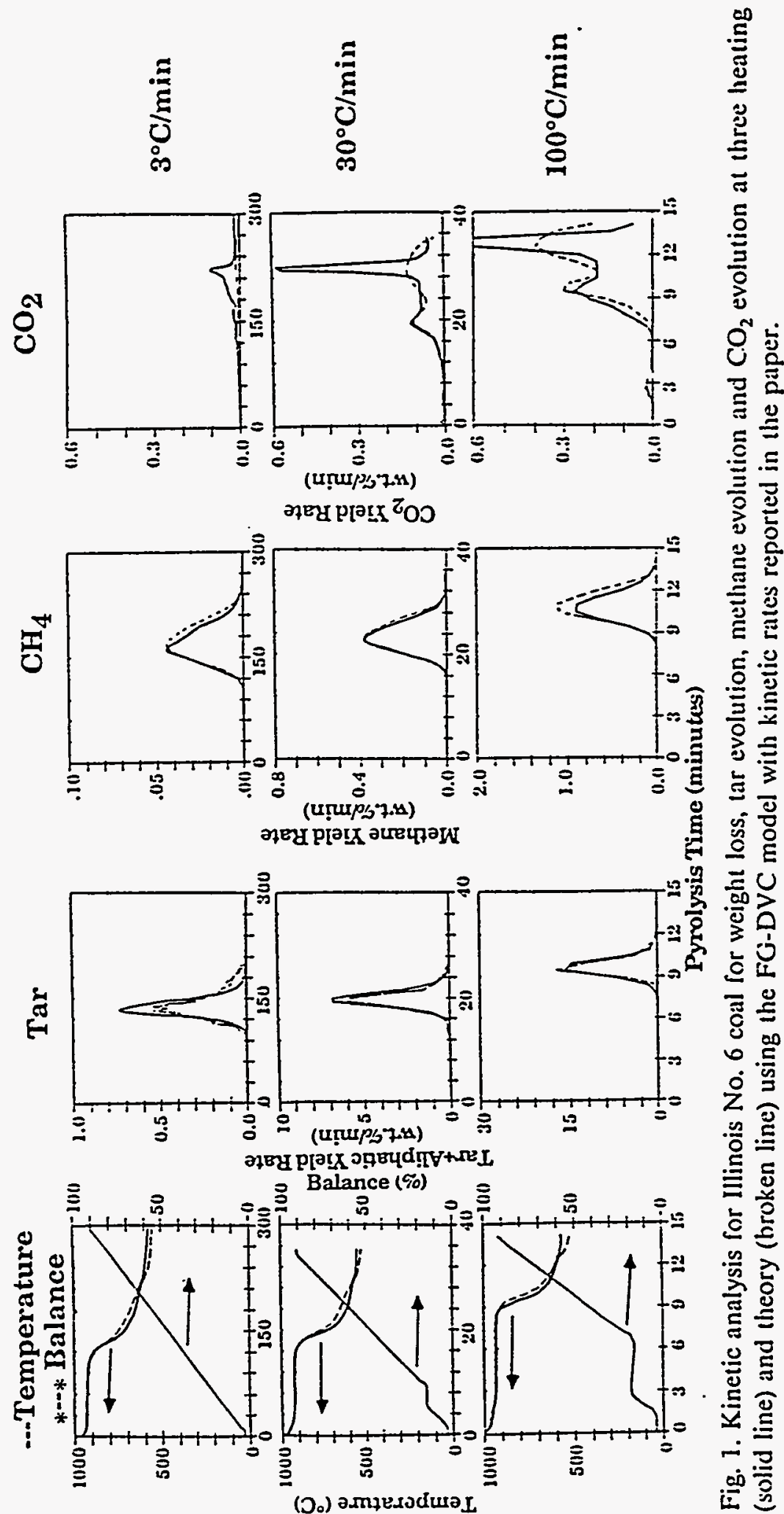


analysis was performed for the Zap lignite [4]. The kinetic rate coefficients determined for bond breaking, methane, and $\mathrm{CO}_{2}$ evolution for Illinois No. 6 and Zap lignite are as follows:

$$
\begin{aligned}
& \text { Illinois No. } 6 \\
& k_{\text {bond breaking }}=1.6 \times 10^{13} \exp [-(25000 \pm 1250) / T] \mathrm{s}^{-1} \\
& k_{\mathrm{CH}_{4} \text {-loose }}=1.5 \times 10^{14} \exp [-(30000 \pm 2000) / T] \mathrm{s}^{-1} \\
& k_{\mathrm{CH}_{4} \text {-light }}=1.9 \times 10^{12} \exp [-(30000 \pm 2000) / T] \mathrm{s}^{-1} \\
& k_{\mathrm{CO}_{2} \text { extra loose }}=2.0 \times 10^{13} \exp [-(22500 \pm 3000) / T] \mathrm{s}^{-1} \\
& k_{\mathrm{CO}_{2} \text { loose }}=8.0 \times 10^{17} \exp [-(33850 \pm 2000) / T] \mathrm{s}^{-1} \\
& k_{\mathrm{CO}_{2} \text { light }}=1.25 \times 10^{14} \exp [-(38315 \pm 5000) / T] \mathrm{s}^{-1}
\end{aligned}
$$

\section{Zap Lignite}

$$
\begin{aligned}
& 5 \times 10^{15} \exp [-(30000 \pm 1500) / T] \mathrm{s}^{-1} \\
& 7 \times 10^{13} \exp [-(30000 \pm 3000) / T] \mathrm{s}^{-1} \\
& 2.1 \times 10^{12} \exp [-(30000 \pm 3000) / T] \mathrm{s}^{-1} \\
& 6.2 \times 10^{12} \exp [-(22500 \pm 3000) / T] \mathrm{s}^{-1} \\
& 1.6 \times 10^{17} \exp [-(33850 \pm 3000) / T] \mathrm{s}^{-1} \\
& 2.1 \times 10^{16} \exp [-(38315 \pm 3000) / T] \mathrm{s}^{-1}
\end{aligned}
$$

The rate equation is of the form $k_{\mathrm{n}}=k_{0} \exp \{-[(E / R) \pm(\sigma / R)] / T\}$, with $k_{0}$ in reciprocal seconds, $E / R$ in kelvins, and $\sigma / R$ in kelvins. The accuracy of the activation energies is estimated to be within $\pm 2 \mathrm{kcal}$. With a fixed activation energy, the accuracy of the pre-exponential factor is $\pm 25 \%$ and of $\sigma$ is $\pm 10 \%$. The bond breaking rate is in good agreement with the approximate rate of $3.1 \times 10^{12} \exp [-(24,942 \pm 1250) / T]$ derived by Burnham et al. [20].

\section{Simulation of high heating rate experiments}

A good test of the validity of the kinetic parameters derived using the TG-FT-IR over a range of low heating rates is the ability to use these parameters to extrapolate to high heating rate conditions. An example is shown in Fig. 2, where the kinetic parameters for the Illinois No. 6 coal were used to simulate previously obtained high heating rate $\left(\approx 40000 \mathrm{~K} \mathrm{~s}^{-1}\right)$ data for pyrolysis of another Illinois No. 6 coal with somewhat higher oxygen content [11]. The agreement is excellent except for the amount of $\mathrm{CO}_{2}$. The contribution from calcite (not included in the model) and the higher oxygen content in this sample (16.5\% compared to $11 \%$ of the Argonne coal) are the problem. 

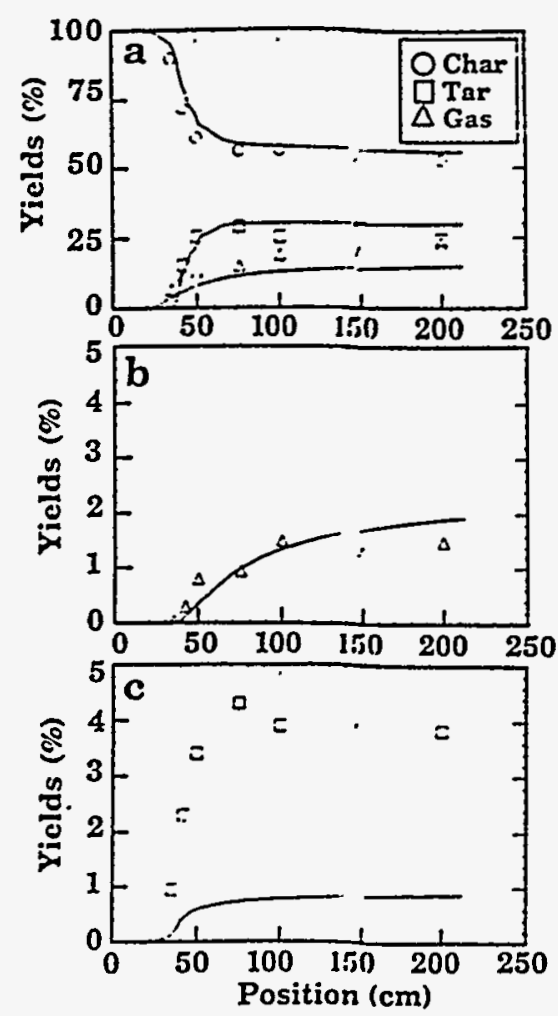

Fig. 2. Pyrolysis of Illinois No. $6 \mathrm{coal}$ in a heated tube reactor at a heating rate of $40000 \mathrm{~K}$ $\mathrm{s}^{-1}$; (a) char (O), tar (口), and gas ( $\Delta$ ), (b) $\mathrm{CH}_{4}$, and (c) $\mathrm{CO}_{2}$.

Variations in kinetic rates and compositions with rank

TG/plus analyses have been performed for the other seven Argonne coals to determine their product evolution profile and kinetic rate coefficients $[3,4]$. Results for the evolution curves for the major evolved species, tar, $\mathrm{CH}_{4}, \mathrm{CO}_{2}, \mathrm{CO}$, and $\mathrm{H}_{2} \mathrm{O}$, are presented in Figs. 3(a)-7(a). For most evolution curves there is a systematic shift in the evolution curves to higher temperatures with increasing rank. There are also systematic decreases in the amounts of $\mathrm{CO}_{2}, \mathrm{CO}$, and $\mathrm{H}_{2} \mathrm{O}$, and a systematic increase in the amount of methane found with increasing rank. With increasing rank, tar increases reaching a maximum for Pittsburgh Seam coal and then decreases.

\section{Simulation of natural maturation}

The FG-DVC model was employed to determine whether the shifts in the evolution curves as a function of rank could be predicted by the FG-DVC model. We assume that coals of increasing rank are the products from lower rank coals pyrolyzed under natural conditions at increased times and temperatures. Based on the work of Welte et al. on profiling of the deep basin in Western Canada [21], it is assumed that high rank coals have been subjected to temperatures up to $180^{\circ} \mathrm{C}$ while low rank coals have not been 

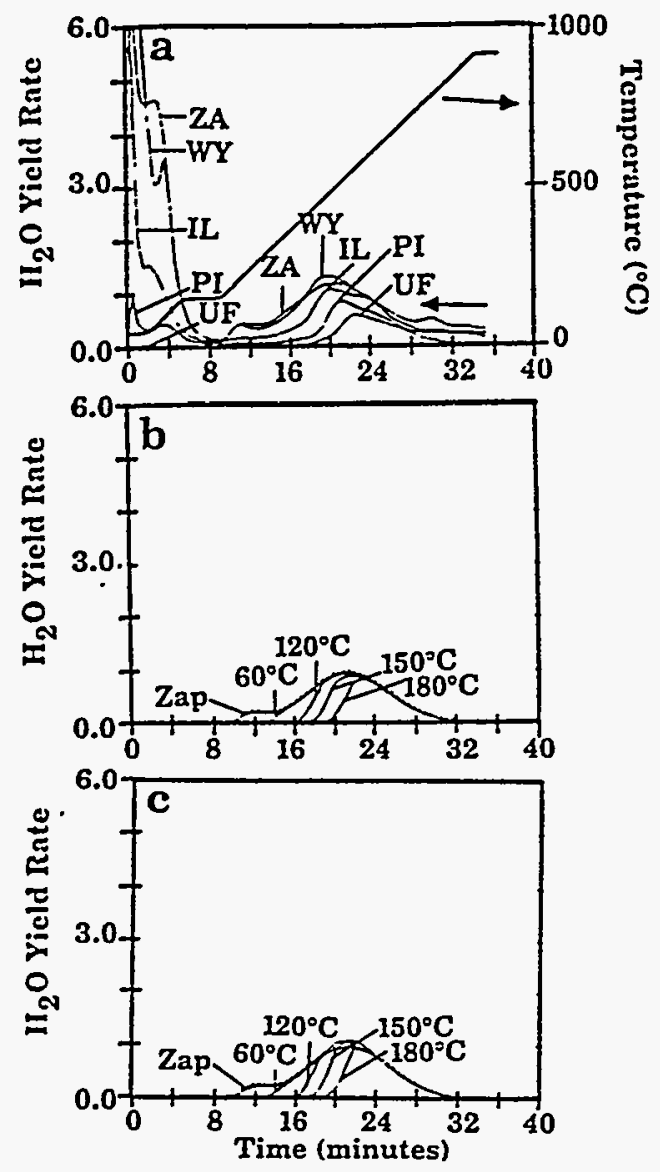

Fig. 3. Evolution curves for $\mathrm{H}_{2} \mathrm{O}$ for five coals from the Argonne premium samples collected at $30^{\circ} \mathrm{C} \mathrm{min}$ min $^{-1}$ (UF, Upper Freeport; PI, Pittsburgh; IL. Illinois: WY, Wyodak: ZA, Zap Lignite); (a) measured in TG/Plus, (b) predicted using FG-DVC pyrolysis model, and (c) predicted using FG-DVC maturation model.

elevated above $100^{\circ} \mathrm{C}$. This is also the range suggested by van Krevelen [22]. The pressures were assumed to be $100 \mathrm{~atm}$. during pyrolysis.

The evolution curves assuming a constant heating rate of $10^{\circ} \mathrm{C}$ per $10^{6}$ years to temperatures of $60,120,150$, and $180^{\circ} \mathrm{C}$ are presented in Figs. 3 (b) -7 (b). The starting coal (i.e. the coal that we employ in the simulation of natural pyrolysis) was the Zap lignite. The predicted evolution curves for water (Fig. 3(b)) show reasonable agreement with the trends measured for water. The $\mathrm{CO}$ and $\mathrm{CO}_{2}$ in Figs. 4(b) and 5(b), however, do not show sufficient removal compared to the natural sequence. The removal of total oxygen is also too slow compared to the removal of hydrogen. This is illustrated in Fig. 8, on a plot of $\mathrm{H} / \mathrm{C}$ vs. $\mathrm{O} / \mathrm{C}$ for the maturation prediction compared to the natural maturation. In addition, the model does not predict the increase in tar (Fig. 6(b)) or methane yield (Fig. 7(b)) with maturation. Clearly, the simulation based on the pyrolytic reaction derived using the TG/plus does not represent the natural occurrence.

The question of simulating natural maturation has been discussed by Lewen [23] and Landais and co-workers [24-26]. They observe that labora- 

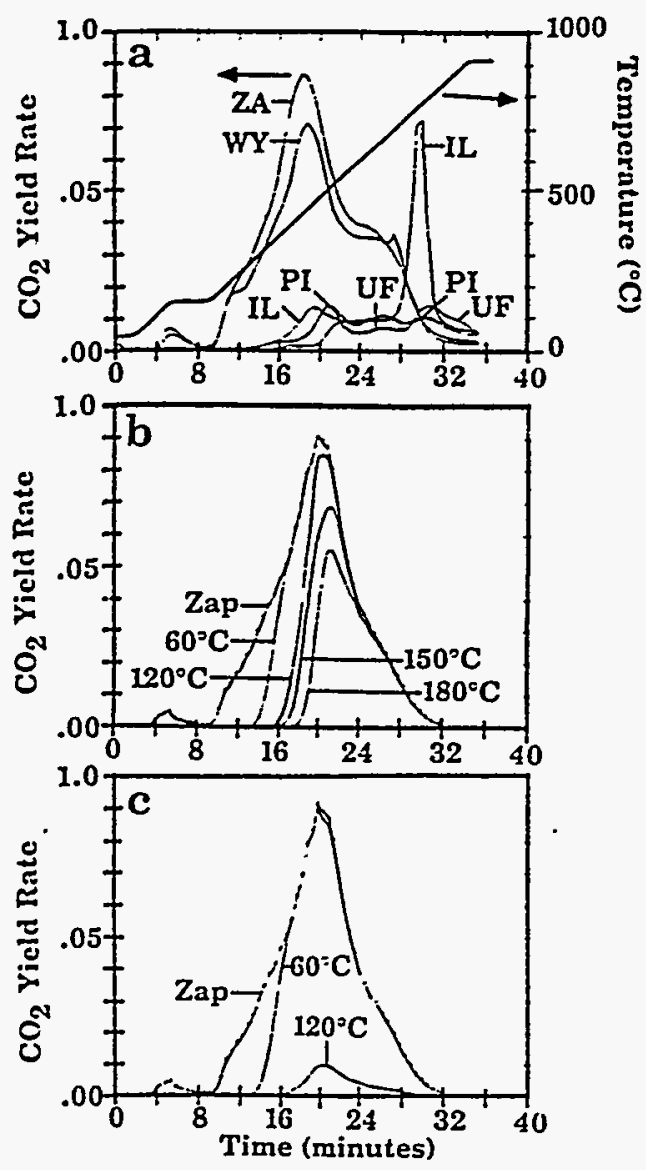

Fig. 4. Evolution curves for $\mathrm{CO}_{2}$ for five coals from the Argonne premium samples collected at $30{ }^{\circ} \mathrm{C} \mathrm{min}{ }^{-1}$ (UF, Upper Freeport; PI, Pittsburgh; IL. Illinois; WY, Wyodak: ZA, Zap Lignite); (a) measured in TG/Plus. (b) predicted using FG-DVC pyrolysis model, and (c) predicted using FG-DVC maturation model.

tory pyrolysis under conditions where the pyrolysis products can escape (open system pyrolysis) (such as in the TG/plus) does not represent natural maturation. Landais has proposed that a system termed "confined pyrolysis" is a good simulation of natural maturation. In confined pyrolysis, the coal is subjected to high pressures in a tightly confined tube without dead volume. Lewen has proposed that the hydrous pyrolysis in which the sample is confined with water at high pressure is a better laboratory representation of natural maturation.

These results suggest that the FG-DVC model derived from open system laboratory data should not accurately simulate natural maturation, and so we have explored what changes might be made in the model to simulate the chemistry of the natural system. Based on the TG/plus analysis, we have assumed that the functional group sources for $\mathrm{CO}$ and $\mathrm{CO}_{2}$ are removed by a higher rate process not observed in the TG/plus experiment, but which may occur in confined systems at high pressures in the presence of water. This assumption is consistent with the observation of Lewen [23] that large amounts of $\mathrm{CO}_{2}$ are produced in hydrous pyrolysis. It is assumed that under 

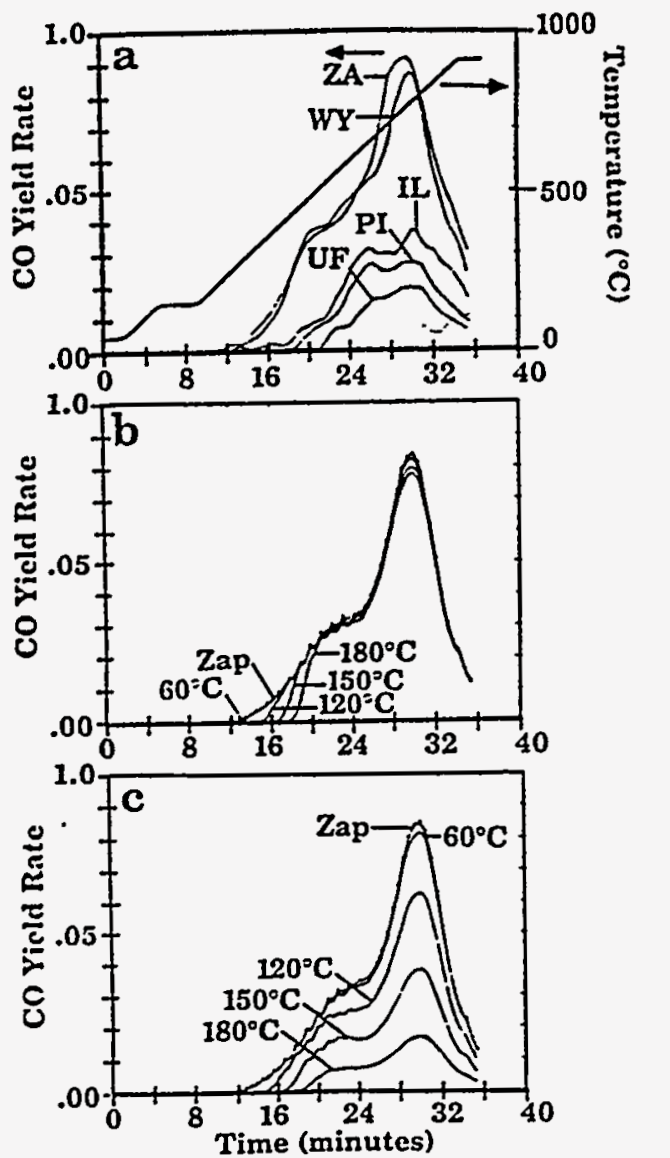

Fig. 5. Evolution curves for $\mathrm{CO}$ for five coals from the Argonne premium samples collected at $30^{\circ} \mathrm{C} \min ^{-1}$ (UF, Upper Freeport; PI, Pittsburgh; IL. Illinois; WY, Wyodak; ZA, Zap Lignite); (a) measured in TG/Plus, (b) predicted using FG-DVC pyrolysis model, and (c) predicted using FG-DVC maturation model.

these conditions, all the $\mathrm{CO}$ sources are reduced at a kinetic rate $3.5 \times$ $\left.10^{14} \exp [-(27500 \pm 2500) / T)\right]$ while the $\mathrm{CO}_{2}$ sources are reduced at a rate $1.5 \times 10^{12} \exp [-(22500 \pm 750) / T]$. These rates are chosen to fit the $\mathrm{CO}$ and $\mathrm{CO}_{2}$ profiles in Figs. 4(a) and 5(a). Based on the values of water $T_{\max }$, the temperature maximum in the basin for each coal was assumed to be 60,120 , 150 , and $180^{\circ} \mathrm{C}$ for the Wyodak, Illinois, Pittsburgh, and Upper Freeport coals, respectively. It is also assumed that neither process involves the formation of crosslinks. Under these assumptions, the curves in Fig. 3(c)-7(c) are obtained, as well as the broken curve in Fig. 8. As can be seen, these changes in the assumed chemistry for natural maturation give a much better simulation of the natural maturation sequence for $\mathrm{CO}$ and $\mathrm{CO}_{2}$ (as expected), but, in addition, improvements are made in the predictions for the $\operatorname{tar}$, the $\mathrm{O} / \mathrm{C}$ vs. $\mathrm{H} / \mathrm{C}$ ratio, and the systematic variation in kinetic rates with coal rank.

These results suggest that natural maturation provides an additional mechanism for the removal of carboxyl groups and ether linkages (which are believed to be the sources for $\mathrm{CO}_{2}$ and $\left.\mathrm{CO}[5,10]\right)$ which is faster than that 


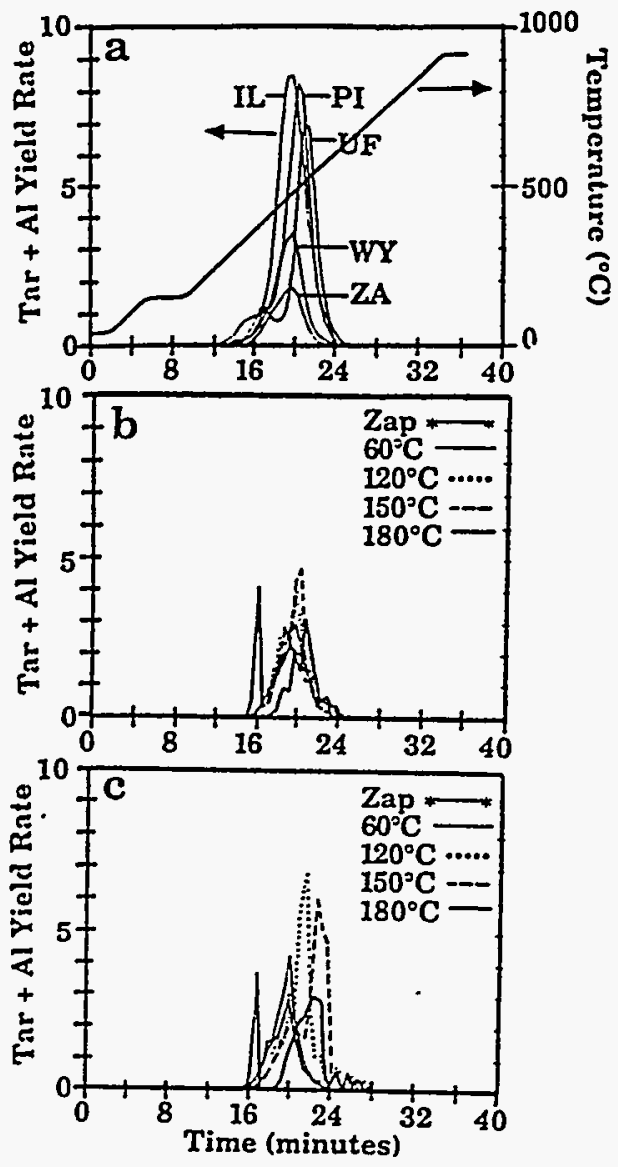

Fig. 6. Evolution curves for tar for five coals from the Argonne premium samples collected at $30^{\circ} \mathrm{C}$ min $^{-1}$ (UF, Upper Freeport; PI, Pittsburgh; IL, Illinois: WY, Wyodak: ZA, Zap Lignite); (a) measured in TG/Plus. (b) predicted using FG-DVC pyrolysis model, and (c) predicted using FG-DVC maturation model.

observed under laboratory conditions in an open system. These reactions do not result in the formation of crosslinks as is observed to accompany $\mathrm{CO}_{2}$ formation under open laboratory conditions. While the rates we have picked are highly speculative, it is clear that some reactions of the kind described do occur in the natural aging process, but not in open system pyrolysis.

There are, however, still some problems with the model. The increase in methane is not predicted. It appears that maturation chemistry must include some process for adding methyl groups. Also, the shift in the $T_{\max }$ for tar are somewhat too large $\left(\approx 70^{\circ} \mathrm{C}\right)$ compared to what is observed $\left(\approx 50^{\circ} \mathrm{C}\right)$. This may be due to the fact that the $T_{\max }$ values for the low rank coals (Zap and Wyodak) are too high due to the domination in these peaks of polymethylenes.

\section{CONCLUSIONS}

The TG/plus provides an extensive analysis of the pyrolysis behavior of coals. 

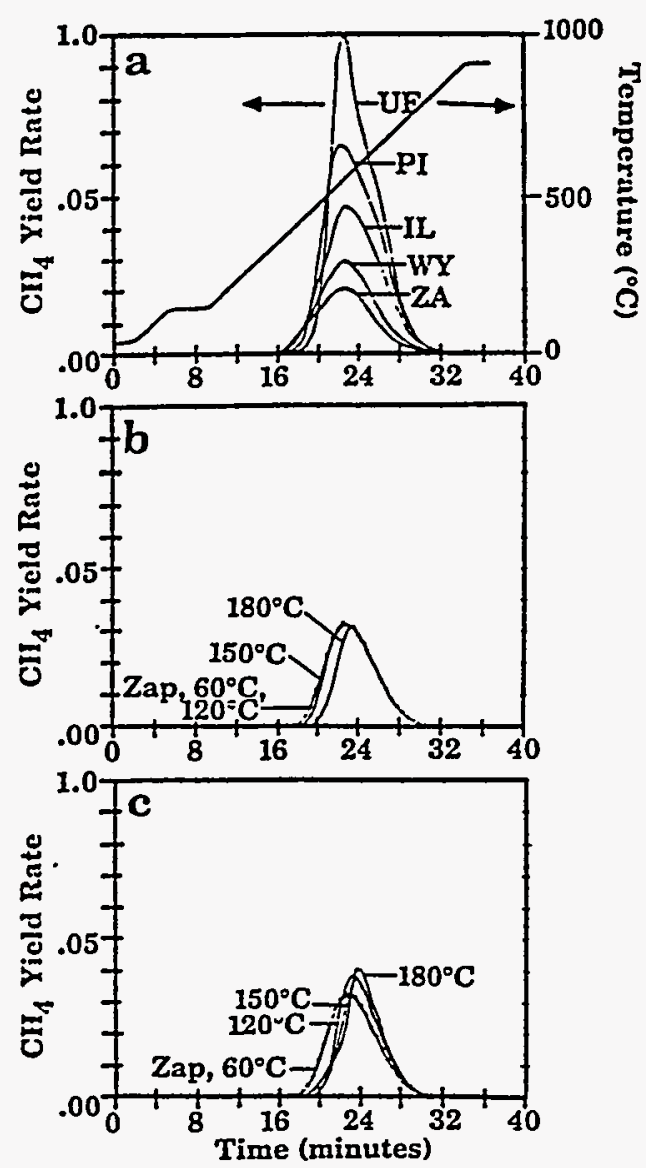

Fig. 7. Evolution curves for $\mathrm{CH}_{4}$ for five coals from the Argonne premium samples collected

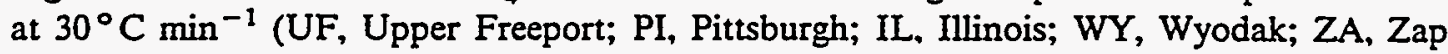
Lignite); (a) measured in TG/Plus. (b) predicted using FG-DVC pyrolysis model, and (c) predicted using FG-DVC maturation model.

Kinetic rate coefficients have been derived for the evolution of $\operatorname{tar}, \mathrm{CH}_{4}$, $\mathrm{CO}_{2}, \mathrm{CO}$, and $\mathrm{H}_{2} \mathrm{O}$ for an Illinois No. 6 bituminous coal and a Zap lignite using data from the TG/plus at $0.05,0.5,0.83$, and 1.67 at $\mathrm{K} \mathrm{s}^{-1}$.

The kinetic rate coefficients for Illinois No. 6 were employed in the FG-DVC coal pyrolysis model to simulate pyrolysis of Illinois No. 6 coal at $20000 \mathrm{~K} \mathrm{~s}^{-1}$. There is good agreement with the data.

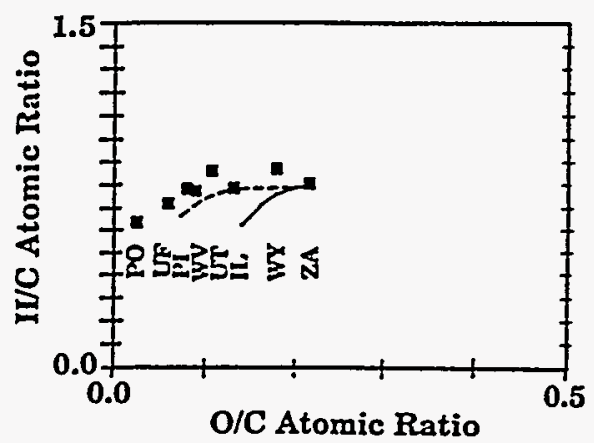

Fig. 8. Maturation of coal. Comparison of predictions for FG-DVC pyrolysis model (solid), FG, DVC maturation model (broken) and the data for the Argonne coals (symbols). 
The kinetic rate coefficients for Zap lignite were employed in the FG-DVC model to simulate natural maturation at $10 \mathrm{~K}$ per $10^{6}$ years. Except for the $\mathrm{H}_{2} \mathrm{O}$ prediction, the simulations based on the chemical reactions determined from experiments performed in the laboratory in open systems do not match the variations in evolution curves with rank for the Argonne premium sample collection.

Two changes were made in the FG-DVC model to account for the possible differences between the latoratcry open pyrolysis and confined natural maturation in the presence of $\mathrm{H}_{2} \mathrm{O}$ at high pressure: (a) a decarboxylation reaction is assumed to occur which removes the sources of $\mathrm{CO}_{2}$ at a higher rate than originally predicted and without the formation of crosslinks usually associated with $\mathrm{CO}_{2}$ evolutions; and (b) ether groups are removed (thus removing the source for $\mathrm{CO}$ evolution) at a higher rate than originally predicted.

With these two changes made and the rates for these reactions picked so that the predicted $\mathrm{CO}_{2}$ and $\mathrm{CO}$ evolution profiles match the natural sequence, the model predicts the proper variation in $\mathrm{H} / \mathrm{C}$ ratio vs. $\mathrm{O} / \mathrm{C}$ ratio, the correct variation with rank of the tar yield and the nearly correct variation with rank in kinetic rates for product evolution. The increase in methane with increasing rank is not properly predicted, suggesting some additional chemistry in the natural sequence which forms methyl groups.

\section{ACKNOWLEDGEMENT}

Support from NSF Grant No. ISI-8703520 and DOE Contract Nos. DE-AC21-86MC23075 and DE-AC22-89PC89878 is gratefully acknowledged.

\section{REFERENCES}

1 R.M. Carangelo. P.R. Solomon and D.J. Gerson, Fuel, 66 (1987) 960.

2 J.K. Whelan, P.R. Solomon. G.V. Deshpande and R.M. Carangelo. Energy and Fuels, 2 (1988) 65.

3 P.R. Solomon, M.A. Serio, R.M. Carangelo, R. Bassilakis, D. Gravel, M. Baillargeon. F. Baudais and G. Vail. Energy \& Fuels, 4 (3) Y1990) 319.

4 M.A. Serio, P.R. Solomon. S. Charpenay, Z.Z. Yu and R. Bassilakis, Kinetics of Volatile Product Evolution from the Argonne Premium Coals, ACS Div. Fuel Chem. Preprints, Washington, DC, 35 (1990) to be presented.

5 P.R. Solomon, D.G. Hamblen. R.M. Carangelo. M.A. Serio and G.V. Deshpande. Energy and Fuel. 2 (1988) 405.

6 P.R. Solomon, M.A. Serio, D.G. Hamblen, Z.Z. Yu and S. Charpenay, ACS Div. Fuel Chem. Preprints, 35 (2) (1990) 479.

7 P.R. Solomon, D.G. Hamblen, Z.Z. Yu and M.A. Serio, Fuel, 69 (1990) 754. 
8 M.A. Serio, P.R. Solomon, Z.Z. Yu and G.V. Deshpande, An Improved Model of Coal Devolatilization, Int. Conf. on Coal Science Proceedings, IEA, October 23-27, 1989 , Tokyo, Japan, p. 209.

9 P.R. Solomon, M.A. Serio, D.G. Hamblen, Z.Z. Yu and S. Charpenay, ACS Div. Fuel Chem. Preprints, 35 (2) (1990) 479.

10 P.R. Solomon and D.G. Hamblen, "Pyrolysis", in R.H. Schlosberg (Editor), Chemistry of Coal Conversion, Plenum, New York, NY, (1985) pp. 121-251.

11 M.A. Serio, D.G. Hamblen, J.R. Markham. P.R. Solomon, Energy and Fuel, 1 (1987) 138.

12 P.R. Solomon and H.H. King, Fuel, 63 (1984) 1302.

13 K.R. Squire, R.M. Carangelo, M.B. DiTaranto, and P.R. Solomon, Fuel, 65 (1986). 833.

14 P.R. Solomon, D.G. Hamblen. R.M. Carangelo. M.A. Serio and G.V. Deshpande. Combustion and Flame, 71 (1988) 137.

15 P.R. Solomon, M.A. Serio, G.V. Deshpande and E. Kroo, Energy and Fuels, 4 (1) (1990) 42.

16 R.M. Carangelo, P.R. Solomon, R. Bassilakis, D. Gravel, M. Baillargeon, F. Baudais and G. Vail, Applications of TG-FT-IR in the Analytical Lab. American Laboratory, (1990) p. 51.

17 .K. Vorres, Am. Chem. Soc. Div. Fuel Chem. Preprints. 32 (4) (1987) 221, see also Users Handbook for the Argonne Premium Coal Sample Program, Argonne National Lab. (September 1989).

18 H. Jüntgen, and K.H. Van Heek, Fuel, 47 (1968) 103; Progress Made in the Research of Bituminous Coal, paper given at the annual meeting of the DGMK, Salzburg, (1968), Translated by Belov and Associates, Denver, CO, APTIC-TR-0779 (1970).

19 R.L. Braun, and A.K. Burnham. Energy and Fuels. 1 (1987) 153.

20 A.K. Burnham, M.S. Oh, R.W. Crawford, and A.M. Samoun. Energy and Fuels, 3 (1989) 42.

21 D.H. Welte, R.G. Schaefer, W. Stoessinger, and W. Radke. Mitt, Geol. Palaontol Inst. Univ. Hamb. 56 (1984) 263.

22 van Krevelen, Coal, Elsevier, Amsterdam 1961.

23 M.D. Lewen, in M.H. Engel and S.A. Macko, (Editor), Organic Geochemistry, Plenum. NY, Chapter 24, to be published.

24 P. Landais, R. Michels, and B. Poty, J. Anal. and Appl. Pyrol. 16 (1989) 103.

25 P. Landais. J.C. Monin, M. Monthioux. B. Poty, and P. Zaugg, C.R. Acad. Sci. Paris, t, 308, Serie II, 1989 p. 1161.

26 M. Monthioux, and P. Landais, Energy and Fuels. 2 (1988) 794. 


\section{User's Forum}

You are invited to submit your comments concerning the clarity, accuracy, and usefulness of this manual. Constructive critical comments are most welcome and will help us continue our efforts to generate quality user documentation. Please list the page number for questions and comments. Use other side if necessary.

From:

Name:

Company

Address and Phone No.

Mail to:

Dr. Andy M. Eaton

45 Crabtree Building

Brigham Young University

Provo, UT 84602
Title

Date 


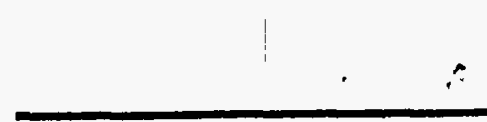

User's Forum

$m$ 